\title{
Cleanup Verification Package for the 118-C-1, 105-C Solid Waste Burial Ground
}

\author{
Prepared for the U.S. Department of Energy \\ by Washington Closure Hanford
}

July 2007 


\section{EXECUTIVE SUMMARY}

This cleanup verification package documents completion of remedial action for the 118-C-1, 105-C Solid Waste Burial Ground. The 118-C-1 site is located within the 100-BC-2 Operable Unit in the 100-B/C Area of the Hanford Site in southeastern Washington State. This waste site was the primary burial ground for general wastes from the operation of the 105-C Reactor. The burial ground was in operation between 1953 and 1969 and received process tubes, aluminum fuel spacers, control rods, reactor hardware, spent nuclear fuel and soft wastes. The site was located approximately $150 \mathrm{~m}(500 \mathrm{ft}$ ) southeast of the 105-C Reactor.

Remedial action at the 118-C-1 site began on February 2, 2004, and was completed on May 27, 2006. Remedial action activities involved removing the uncontaminated overburden, the buried contaminated debris and the underlying contaminated soil. All contaminated materials were disposed at the Environmental Restoration Disposal Facility, with the exception of spent nuclear fuel, which will be transferred to the $100-\mathrm{K}$ Basins for interim storage prior to final packaging and disposal. Results of the verification sampling, laboratory analyses, and data evaluations for the 118-C-1 site (which includes the remediation footprint, the overburden/below cleanup level soil, and the waste staging pile areas) indicate that all remedial action objectives and goals for direct exposure, protection of groundwater, and protection of the Columbia River have been met (see Table ES-1).

The site meets cleanup standards and has been reclassified as "interim closed out" in accordance with the Hanford Federal Facility Agreement and Consent Order (Ecology et al. 1989) and the Waste Site Reclassification Guideline TPA-MP-14 (RL-TPA-90-0001) (DOE-RL 1998). A copy of the waste site reclassification form is included as Attachment ES-1. 


\section{Table ES-1. Summary of Cleanup Verification Results for the 118-C-1 Burial Ground. (2 Pages)}

\begin{tabular}{|c|c|c|c|c|}
\hline $\begin{array}{l}\text { Regulatory } \\
\text { Requirement }\end{array}$ & Remedial Action Goals & Results & $\begin{array}{l}\text { Remedial } \\
\text { Action } \\
\text { Objectives } \\
\text { Attained? }\end{array}$ & Ref. \\
\hline $\begin{array}{l}\text { Direct Exposure - } \\
\text { Radionuclides }\end{array}$ & $\begin{array}{l}\text { 1. Attain } 15 \mathrm{mrem} / \mathrm{yr} \text { dose rate } \\
\text { above background over } \\
1,000 \text { years. }\end{array}$ & $\begin{array}{l}\text { 1. Maximum predicted dose rates for } \\
\text { each sampling area at the } \\
118-\mathrm{C}-1 \text { site are below } 15 \\
\text { mrem/yr. Inclusion of one } \\
\text { carbon-14 result for the staging } \\
\text { pile area resulted in an elevated } \\
\text { calculated dose rate; therefore, } \\
\text { soil associated with this result } \\
\text { was backfilled to deep zone } \\
\text { portions of the site per agreement } \\
\text { with the EPA Project Manager. }\end{array}$ & Yes & e \\
\hline $\begin{array}{l}\text { Direct Exposure - } \\
\text { Nonradionuclides }\end{array}$ & 1. Attain individual COC RAGs. & $\begin{array}{l}\text { 1. Polycyclic aromatic hydrocarbons } \\
\text { were detected above the direct } \\
\text { exposure RAGs in a verification } \\
\text { sample at } 118-C-1 \text { staging pile } \\
\text { area, but determined to be the } \\
\text { result of asphalt cross- } \\
\text { contamination. Asphalt that has } \\
\text { been used for structural and } \\
\text { construction purposes is not listed } \\
\text { as a CERCLA hazardous } \\
\text { substance, and does not present } \\
\text { a health risk for this waste site. } \\
\text { All other individual COC/COPC } \\
\text { concentrations are below the } \\
\text { direct exposure criteria. }\end{array}$ & Yes & $f$ \\
\hline \multirow{4}{*}{$\begin{array}{l}\text { Meet } \\
\text { Nonradionuclide } \\
\text { Risk } \\
\text { Requirements }\end{array}$} & $\begin{array}{l}\text { 1. Hazard quotient of }<1 \text { for } \\
\text { noncarcinogens. }\end{array}$ & $\begin{array}{l}\text { 1. The hazard quotients for } \\
\text { individual nonradionuclide } \\
\text { COCs/COPCs are less than } 1 .\end{array}$ & \multirow{2}{*}{ Yes } & $g$ \\
\hline & $\begin{array}{l}\text { 2. Cumulative hazard quotient of }<1 \\
\text { for noncarcinogens. }\end{array}$ & $\begin{array}{l}\text { 2. The cumulative hazard quotient } \\
\text { for all sampling areas }\left(1.6 \times 10^{-1}\right) \\
\text { is less than } 1 \text {. }\end{array}$ & & $g$ \\
\hline & $\begin{array}{l}\text { Excess cancer risk of }<1 \times 10^{-6} \\
\text { for individual carcinogens. }\end{array}$ & $\begin{array}{l}\text { 3. Excess cancer risk values for } \\
\text { individual nonradionuclide } \\
\text { COCs/COPCs are less than } \\
1 \times 10^{-6} \text {. }\end{array}$ & Yes & g \\
\hline & $\begin{array}{l}\text { 4. Attain a total excess cancer risk } \\
\text { of }<1 \times 10^{-5} \text { for carcinogens. }\end{array}$ & $\begin{array}{l}\text { 4. The total excess carcinogenic risk } \\
\text { for all sampling areas }\left(1.6 \times 10^{-6}\right) \\
\text { is less than } 1 \times 10^{-5} \text {. }\end{array}$ & Yes & $g$ \\
\hline \multirow[t]{2}{*}{$\begin{array}{l}\text { Groundwater/ } \\
\text { River Protection - } \\
\text { Radionuclides }\end{array}$} & $\begin{array}{l}\text { 1. Attain single COC groundwater } \\
\text { and river protection RAGs. }\end{array}$ & $\begin{array}{l}\text { 1. Among the radionuclide COCs, } \\
\text { cesium-137, cobalt-60, nickel- } 63 \text {, } \\
\text { strontium-90 and tritium are } \\
\text { calculated to reach groundwater } \\
\text { at concentrations significantly } \\
\text { below the RAGs. }\end{array}$ & \multirow[t]{2}{*}{ Yes } & $\mathrm{h}$ \\
\hline & $\begin{array}{l}\text { 2. Attain National Primary Drinking } \\
\text { Water Standards: } 4 \mathrm{mrem} / \mathrm{yr} \\
\text { (beta/gamma) dose rate to target } \\
\text { receptor/organs. }\end{array}$ & $\begin{array}{l}\text { 2. The maximum calculated drinking } \\
\text { water dose from beta/gamma- } \\
\text { emitting radionuclides is less than } \\
4 \mathrm{mrem} / \mathrm{yr} \text {. }\end{array}$ & & $\mathrm{h}$ \\
\hline
\end{tabular}


CVP-2006-00011

Rev. 0

\section{Table ES-1. Summary of Cleanup Verification Results for the 118-C-1 Burial Ground. (2 Pages)}

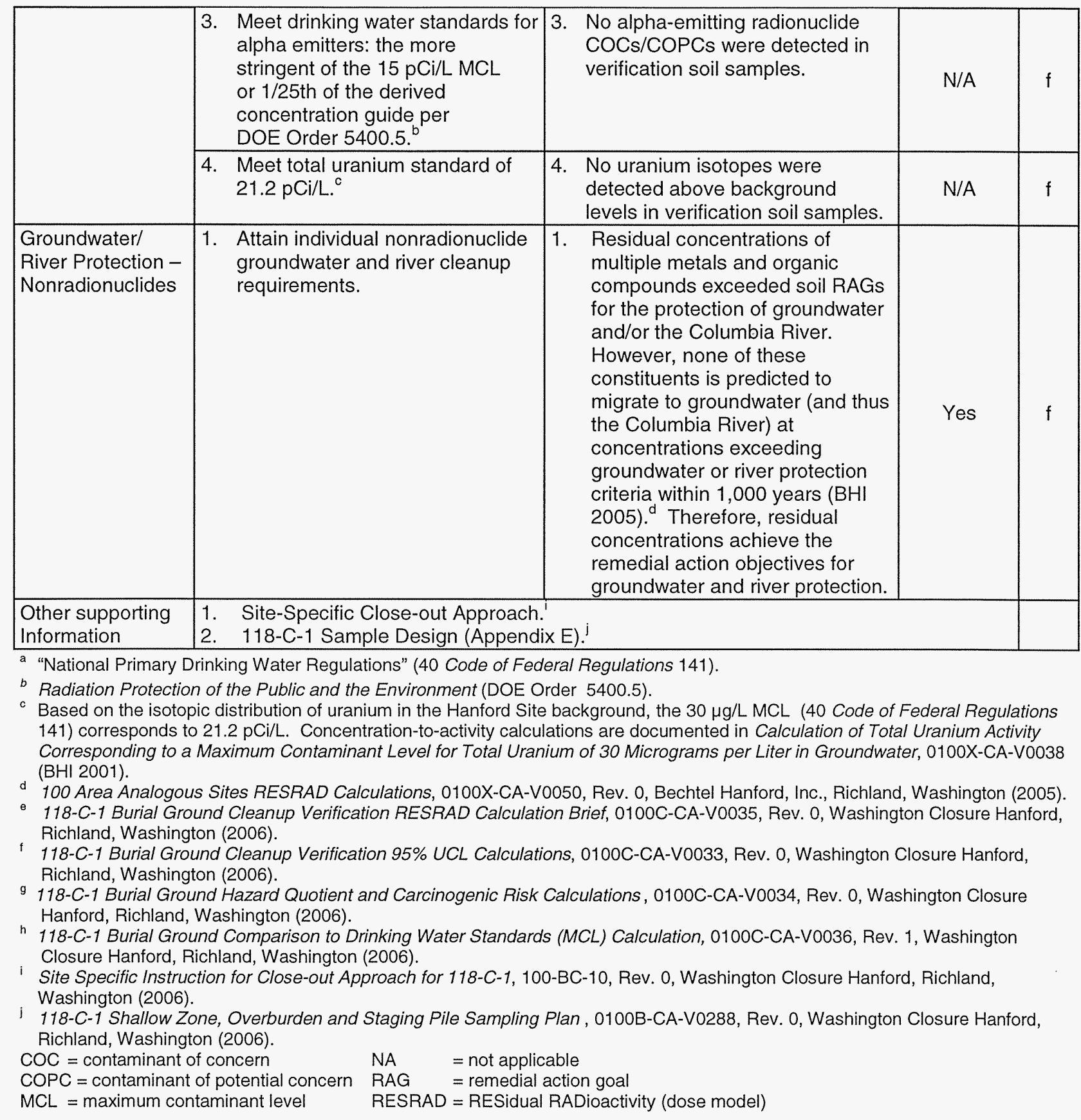


CVP-2006-00011

Rev. 0

ES-4 
CVP-2006-00011

Rev. 0

\section{Attachment ES-1}

\section{Waste Site Reclassification Form}

Date Submitted: $6 / 21 / 2007$

Originator: L. M. Dittmer

Phone: $\quad 372-9227$

\section{WASTE SITE RECLASSIFICATION FORM}

Operable Unit(s): 100-BC-2

Waste Site Code: $\quad 118-\mathrm{C}-1$

Type of Reclassification Action:

Closed Out $\square \quad$ Interim Closed Out $\square$ No Action $\square$

RCRA Postclosure $\square \quad$ Rejected $\square$ Consolidated $\square$

This form documents agreement among parties listed authorizing classification of the subject unit as Closed Out, Interim Closed Out, No Action, RCRA Postclosure, Rejected, or Consolidated. This form also authorizes backfill of the waste management unit, if appropriate, for Closed Out and Interim Closed Out units. Final removal from the NPL of No Action and Closed Out waste management units will occur at a future date.

Description of current waste site condition:

Remedial action at this site has been performed in accordance with remedial action objectives and goals established by the U.S. Environmental Protection Agency and the U.S. Department of Energy, Richland Operations Office, in concurrence with the Washington State Department of Ecology. The selected remedial action involves (1) excavating the site to the extent required to meet specified soil cleanup levels, (2) disposing contaminated excavation materials at the Environmental Restoration Disposal Facility at the 200 Area of the Hanford Site, and (3) backfilling the site with clean soil to adjacent grade elevations. The excavation and disposal activities have been completed.

Basis for reclassification:

The results of verification sampling of the soils at the 118-C-1 waste site demonstrated that residual contaminant concentrations do not preclude any future uses (as bounded by the rural-residential scenario) and allow for unrestricted use of shallow zone soils (i.e., surface to $4.6 \mathrm{~m}$ [15 $\mathrm{ft}$ ] deep). The results also showed that residual contaminant concentrations are protective of groundwater and the Columbia River. The waste site has a deep zone; therefore, institutional controls to prevent uncontrolled drilling or excavation into the deep zone are required. The basis for reclassification is described in detail in the Cleanup Verification Package for the 118-C-1,105-C Solid Waste Burial Ground

(CVP-2006-00011), Washington Closure Hanford, Richland, Washington.

Waste Site Controls:

Engineered Controls: Yes $\square$ No $\square \quad$ Institutional Controls: Yes $\bigotimes$ No $\square \quad$ O\&M requirements: Yes $\square$ No $\square$ Deed restriction to prevent uncontrolled drilling or excavation into the deep zone (greater than $4.6 \mathrm{~m}$ [15 ft] below ground surface).

S. L. Charboneau
DOE Federal Project Director (printed)
D. A. Faulk
EPA Project Manager (printed)


CVP-2006-00011

Rev. 0

ES-6 


\section{CONTENTS}

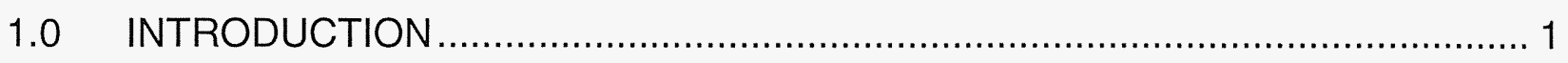

2.0 SITE DESCRIPTION AND SUPPORTING INFORMATION .............................. 2 2.1 CONTAMINANTS OF CONCERN/CONTAMINANTS OF POTENTIAL

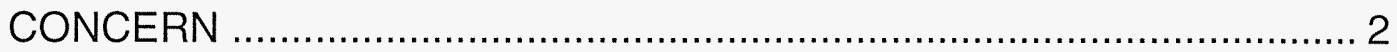

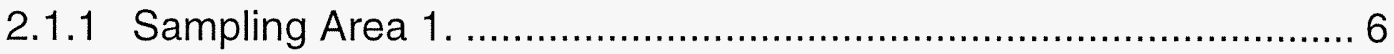

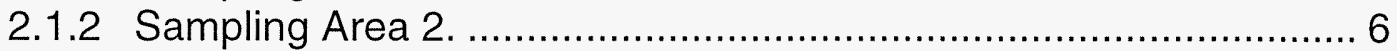

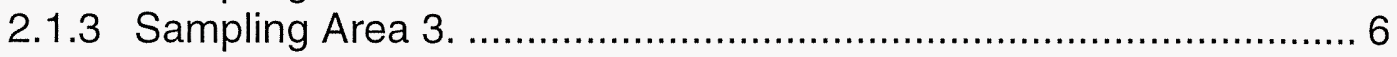

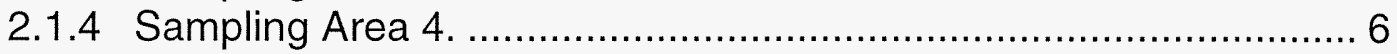

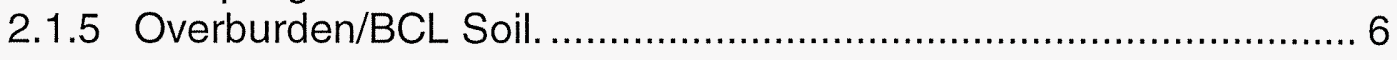

2.1.6 Waste Staging Pile Area. ...................................................... 6

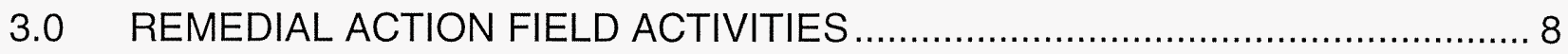

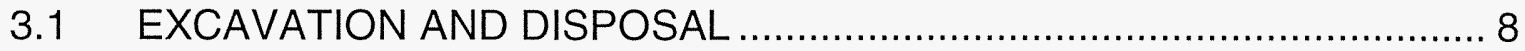

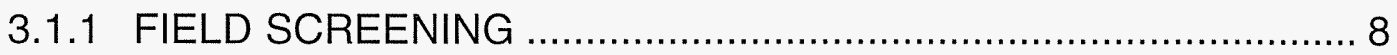

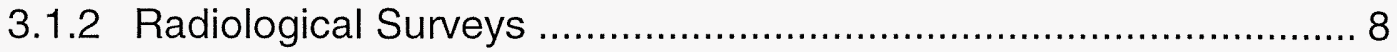

3.2 BIASED SAMPLING AND ANALYSIS .......................................... 9

3.3 CLEANUP VERIFICATION SAMPLING AND ANALYSIS ................... 11

4.0 CLEANUP VERIFICATION DATA EVALUATION …...................................... 12

4.1 DATA QUALITY ASSESSMENT PROCESS .................................. 13

4.2 CONTAMINANTS OF CONCERN 95\% UPPER CONFIDENCE LIMIT ... 13

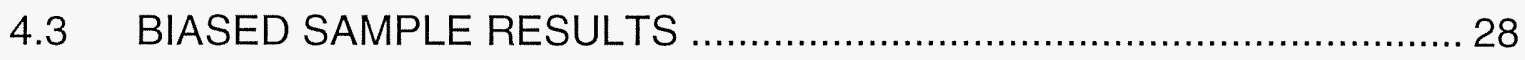

4.3.1 Biased Sample Data Evaluation.............................................. 30

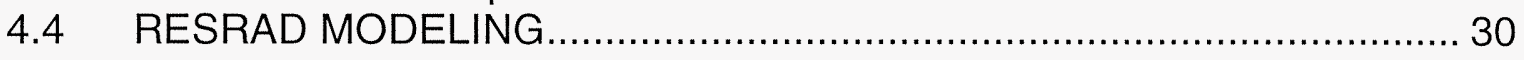

5.0 EVALUATION OF REMEDIAL ACTION GOAL ATTAINMENT …...................... 31

5.1 DIRECT EXPOSURE SOIL REMEDIAL ACTION GOALS ATTAINED.... 31

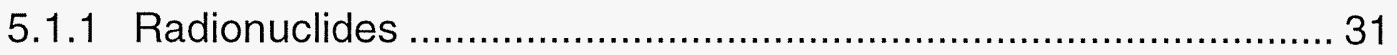

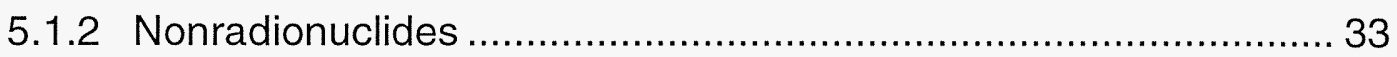

5.2 GROUNDWATER REMEDIAL ACTION GOALS ATTAINED ................ 34

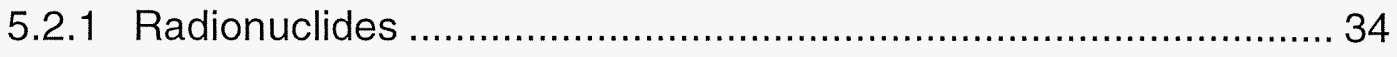

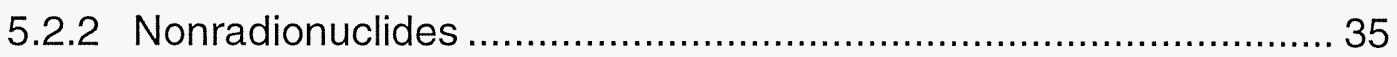

5.3 COLUMBIA RIVER REMEDIAL ACTION GOALS ATTAINED ............... 36

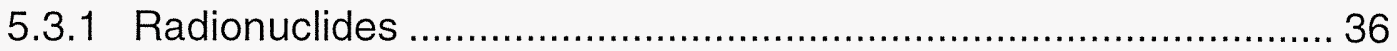

5.3.2 Nonradionuclides ............................................................. 36

5.4 WAC 173-340 THREE-PART TEST FOR NONRADIONUCLIDES ......... 36

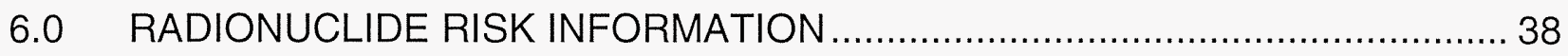

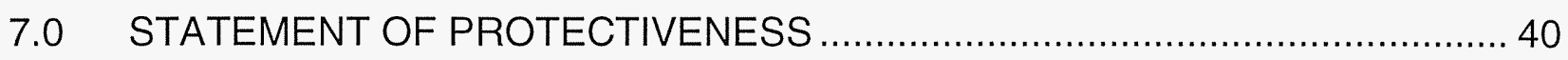

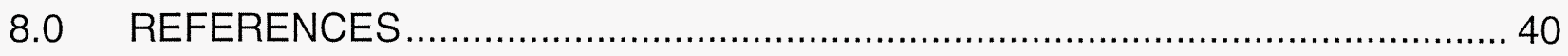




\section{APPENDICES}

A SUMMARY OF VERIFICATION SOIL SAMPLING AND ANALYTICAL RESULTS

B 1.18-C-1 WASTE SITE REMEDIATION PHOTOGRAPHS B-i

C DATA QUALITY ASSESSMENT

D RADIOLOGICAL SURVEY MAPS D-i

E RESRAD INPUT PARAMETERS AND CALCULATION BRIEF EXCERPTS .... E-i

E1 118-C-1 Shallow Zone, Overburden and Staging Pile Sampling Plan, 0100B-CA-V0288

E2 118-C-1 Burial Ground Hazard Quotient and Carcinogenic Risk Calculations, 0100C-CA-V0034

E3 118-C-1 Burial Ground Cleanup Verification 95\% UCL Calculations, 0100C-CA-V0033 E-103

E4 118-C-1 Burial Ground Comparison to Drinking Water Standards (MCL) Calculation, 0100C-CA-V0036

E5 118-C-1 Burial Ground Cleanup Verification RESRAD Calculation Brief, 0100C-CA-V0035

\section{FIGURES}

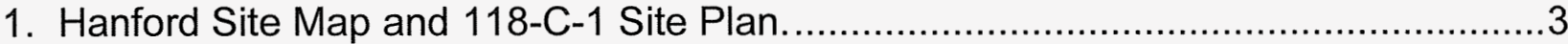

2. 118-C-1 Burial Ground Post-Excavation Topographic Boundary ........................... 4

3. Remediation Footprint Sampling Area Boundaries............................................. 5

4. 118-C-1 Biased Sampling Locations within Remediation Footprint. .......................10

5. RESRAD Analysis for the Remediation Footprint-All Radionuclides,

All-Pathways Dose Rate Estimate 32

6. RESRAD Analysis for the Overburden/BCL soil- All Radionuclides, 32

7. RESRAD Analysis for the Waste Staging Pile Area- All Radionuclides,

All-Pathways Dose Rate Estimate.

8. Dose Rate to Organs from Groundwater.

9. RESRAD Analysis - Radionuclide Risk, All Pathways -

Remediation Footprint.

10. RESRAD Analysis - Radionuclide Risk, All Pathways -

Overburden/BCL Soil.

11. RESRAD Analysis - Radionuclide Risk, All Pathways Waste Staging Pile Footprint. 


\section{TABLES}

1. Summary of the 118-C-1 Waste Site COCs/COPCs for the Six Sampling Subunits...7

2. 118-C-1 Biased Samples ..............................................................................11

3. Number of Verification Samples for the 118-C-1 Burial Ground. ...........................12

4. Comparison of Statistical Contaminant Concentrations to Action Levels for Verification Sampling of the 118-C-1 Sample Area 1. ...................................14

5. Comparison of Statistical Contaminant Concentrations to Action Levels for Verification Sampling of the 118-C-1 Sample Area 2. .....................................16

6. Comparison of Statistical Contaminant Concentrations to Action Levels for Verification Sampling of the 118-C-1 Sample Area 3.

7. Comparison of Statistical Contaminant Concentrations to Action Levels for Verification Sampling of the 118-C-1 Sample Area 4. )

8. Comparison of Statistical Contaminant Concentrations to Action Levels for Verification Sampling of the 118-C-1 Sample Overburden/BCL Material...........22

9. Comparison of Statistical Contaminant Concentrations to Action Levels for Verification Sampling of the 118-C-1 Staging Pile Area.

10. Comparison of 118-C-1 Staging Pile Area Statistical Results to a Known Asphalt

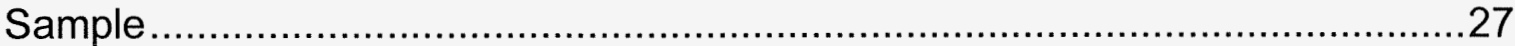

11. Comparison of Maximum Biased Sample Concentrations to Cleanup Levels. .....28

12. Estimated Peak Radionuclide Groundwater Concentrations in the Soils for 118-C-1.

13. Summary of the WAC 173-340 Three-Part Test for the 118-C-1 Burial Ground. ..37 


\section{ACRONYMS AND ABBREVIATIONS}

$\begin{array}{ll}\text { BCL } & \text { below cleanup level } \\ \text { COC } & \text { contaminant of concern } \\ \text { COPC } & \text { contaminant of potential concern } \\ \text { DOE-RL } & \text { U.S. Department of Energy, Richland Operations Office } \\ \text { DQA } & \text { data quality assessment } \\ \text { EPA } & \text { U.S. Environmental Protection Agency } \\ \text { ERDF } & \text { Environmental Restoration Disposal Facility } \\ \text { PAH } & \text { polycyclic aromatic hydrocarbon } \\ \text { PCBS } & \text { polychlorinated biphenyls } \\ \text { RAG } & \text { remedial action goal } \\ \text { RDR/RAWP } & \text { remedial design report/remedial action work plan } \\ \text { RESRAD } & \text { RESidual RADioactivity dose assessment model } \\ \text { ROD } & \text { record of decision } \\ \text { SAP } & \text { sampling and analysis plan } \\ \text { SNF } & \text { spent nuclear fuel } \\ \text { SSI } & \text { site-specific instruction } \\ \text { SVOA } & \text { semi-volatile organic analysis } \\ \text { TPH } & \text { total petroleum hydrocarbons } \\ \text { UCL } & \text { upper confidence limit } \\ \text { VOA } & \text { volatile organic analysis } \\ \text { WAC } & \text { Washington Administrative Code }\end{array}$




\subsection{INTRODUCTION}

The purpose of this cleanup verification package is to document that the 118-C-1 Solid Waste Burial Ground site was remediated in accordance with the Record of Decision for the 100-BC-1, 100-BC-2, 100-DR-1, 100-DR-2, 100-FR-2, 100-HR-2, and 100-KR-2 Operable Units, Hanford Site (100 Area Burial Grounds), Benton County, Washington (ROD) (EPA 2000). Remedial action objectives and goals for the 118-C-1 site were established by the U.S. Environmental Protection Agency (EPA) and the U.S. Department of Energy, Richland Operations Office (DOE-RL), in concurrence with the Washington State Department of Ecology. These goals and objectives are documented in the 100 Area Burial Grounds ROD (EPA 2000) and the Remedial Design Report/Remedial Action Work Plan for the 100 Area (RDR/RAWP) (DOE-RL 2005). The ROD (EPA 2000) provides the U.S. Department of Energy, Richland Operations Office the authority, guidance, and objectives to conduct this remedial action.

The preferred remedy specified in the ROD (EPA 2000) and conducted for the 118-C-1 site included (1) excavating the site to the extent required to meet specified soil cleanup levels, (2) disposing of contaminated excavation materials at the Environmental Restoration Disposal Facility (ERDF) at the 200 Area of the Hanford Site, and (3) backfilling the site with overburden and clean soil to average adjacent grade elevation. Excavation was driven by remedial action objectives for direct exposure, protection of groundwater, and protection of the Columbia River.

Waste site contaminants of concern (COCs) and contaminants of potential concern (COPCs) were identified in the Remedial Design Report/Remedial Action Work Plan for the 100 Area (DOE-RL 2005) and include tritium, carbon-14, cesium-137, cobalt-60, nickel-63, strontium-90, silver-108m, europium-152, europium-154, cadmium, lead and mercury. Additional waste site COCs/COPCs were identified for this site based on the observed waste forms found during remediation activities and the results of waste characterization sampling as documented in the Site Specific Instruction (SSI) for Close-Out Approach for 118-C-1 (118-C-1 SSI) (WCH 2006). The 118-C-1 SSI documents the agreements between the Department of Energy Richland Operations Office (DOE-RL) and the Environmental Protection Agency (EPA) regarding waste site close-out for the118-C-1 Burial Ground. The additional COCs/COPCs identified in the 118-C-1 SSI (WCH 2006) include americium-241, plutonium-238, plutonium-239/240, uranium-233/234, uranium-235, uranium-238, the expanded list of inductively coupled plasma (ICP) metals (arsenic, antimony, barium, beryllium, boron, cadmium, chromium, lead, cobalt, copper, manganese, molybdenum, nickel, selenium, silver, vanadium, and zinc), hexavalent chromium, polychlorinated biphenyls (PCBs), total petroleum hydrocarbons (TPH), semi-volatile organic compounds, and volatile organic compounds. The COCs/COPCs detected at the 118-C-1 Burial Ground and their respective points of compliance, the remedial action goals (RAGs), are summarized in Tables 4 through 9 (Section 4.2) of this CVP.

Soil cleanup levels were established in the interim action ROD based on a limited ecological risk assessment. Although not required by the ROD (EPA 2000), a 
comparison against ecological risk screening levels has been made for the site COCs/COPCs. Screening levels were not exceeded for the site constituents, with the exception of barium, boron, copper, lead, and molybdenum. Exceedance of screening values does not necessarily indicate the existence of risk to ecological receptors. It is believed that the presence of these constituents does not pose a risk to ecological receptors as concentrations of barium, copper, and lead are within the range of Hanford Site background levels and boron and molybdenum concentrations are consistent with those seen elsewhere at the Hanford Site (no established background value is available for boron or molybdenum). A baseline risk assessment for the river corridor portion of the Hanford Site began in 2004, which includes a more complete quantitative ecological risk assessment. That baseline risk assessment will be used as part of the final closeout decision for this site.

\subsection{SITE DESCRIPTION AND SUPPORTING INFORMATION}

The 118-C-1 site is located in the 100-BC-2 Operable Unit of the 100-BC Area

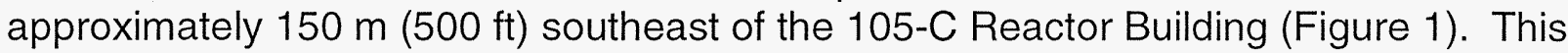
waste site was the primary burial ground for general wastes from the operation of the 105-C Reactor. The burial ground was in operation between 1953 and 1969 and received process tubes, aluminum fuel spacers, control rods, reactor hardware, spent nuclear fuel (SNF) and soft wastes. The C-Area Land Burial Log (1962-1965) identifies additional waste including trash, poison splines, dummies, hot laundry, fan filters, irradiated balls, ceramic samples, thimbles, gun barrels, and hoses that were deposited in the site (WCH 2006). The 118-C-1, 105-C Solid Waste Burial Ground was estimated to contain 86 metric tons (94.8 tons) of boron, 1.1 metric tons (1.2 tons) of graphite, 0.51 metric tons ( 0.56 tons) of lead, 21.6 metric tons (23.8 tons) of lead/cadmium, and 96 metric tons (105.9 tons) of other materials.

\subsection{CONTAMINANTS OF CONCERN/CONTAMINANTS OF POTENTIAL CONCERN}

The 118-C-1 Burial Ground contains ten trenches, eight oriented in a north-south direction and the other two oriented east-west (Figure 2 and Figure 3). For close-out verification sampling, the trenches and surrounding land between the trenches were grouped into four sampling decision units. The four sampling decision units were grouped based on similarity of the waste forms observed during remediation (WCH 2006). The four different decision units were sampled only for the COCs/COPCs pertinent to the waste forms identified within that area. The waste forms discovered within the four sampling decision units are discussed below and the COCs/COPCs associated with each sampling decision unit are provided in Table 1. The 118-C-1 waste site also includes an overburden/below cleanup level $(B C L)$ decision unit and a waste staging pile area decision unit. Both of these decision units were sampled for all waste site COCs/COPCs identified for the 118-C-1 waste site, as shown in Table 1. 
Figure 1. Hanford Site Map and 118-C-1 Site Plan.

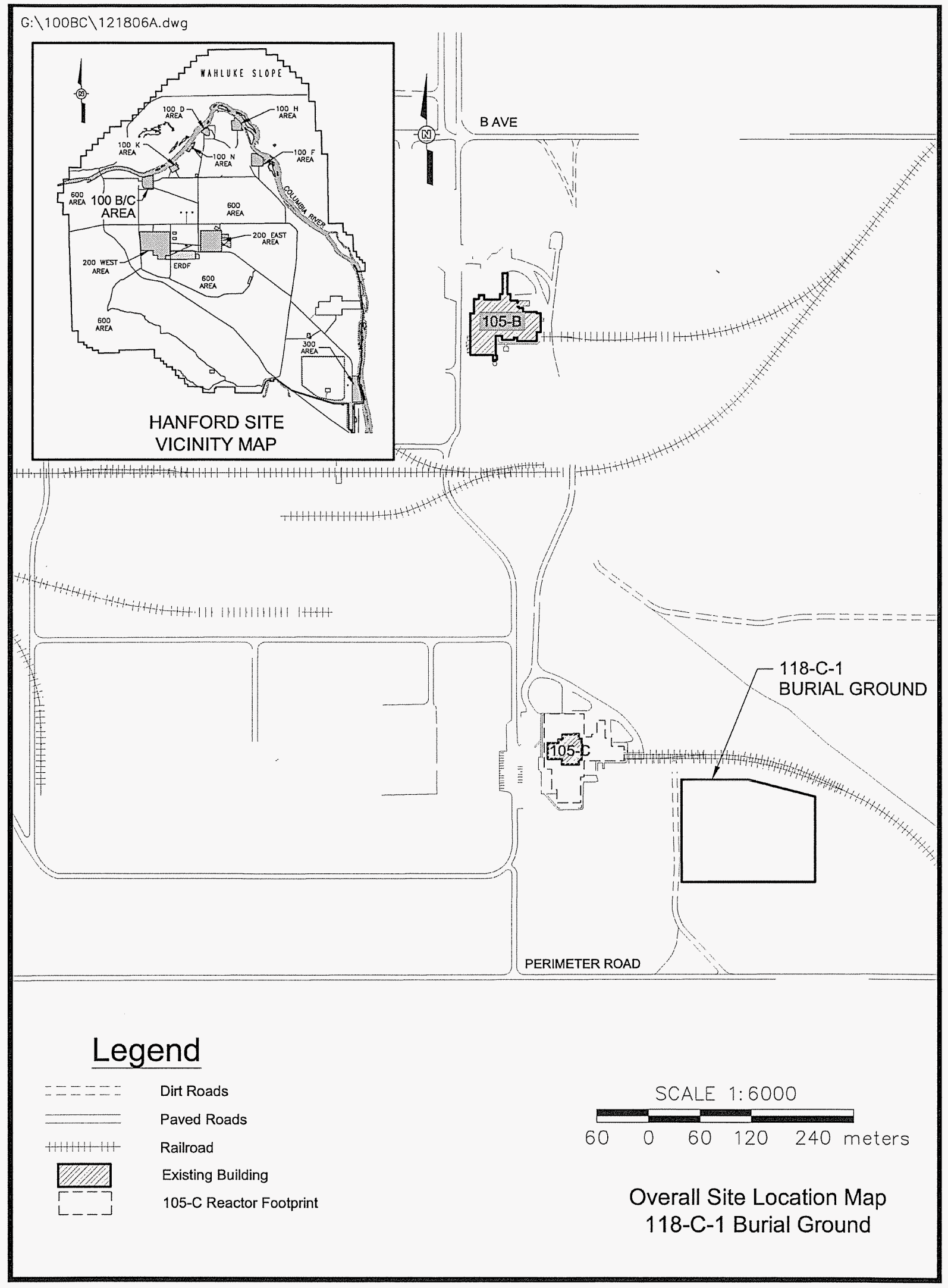


Rev. 0

Figure 2. 118-C-1 Burial Ground Post-Excavation Topographic Boundary.

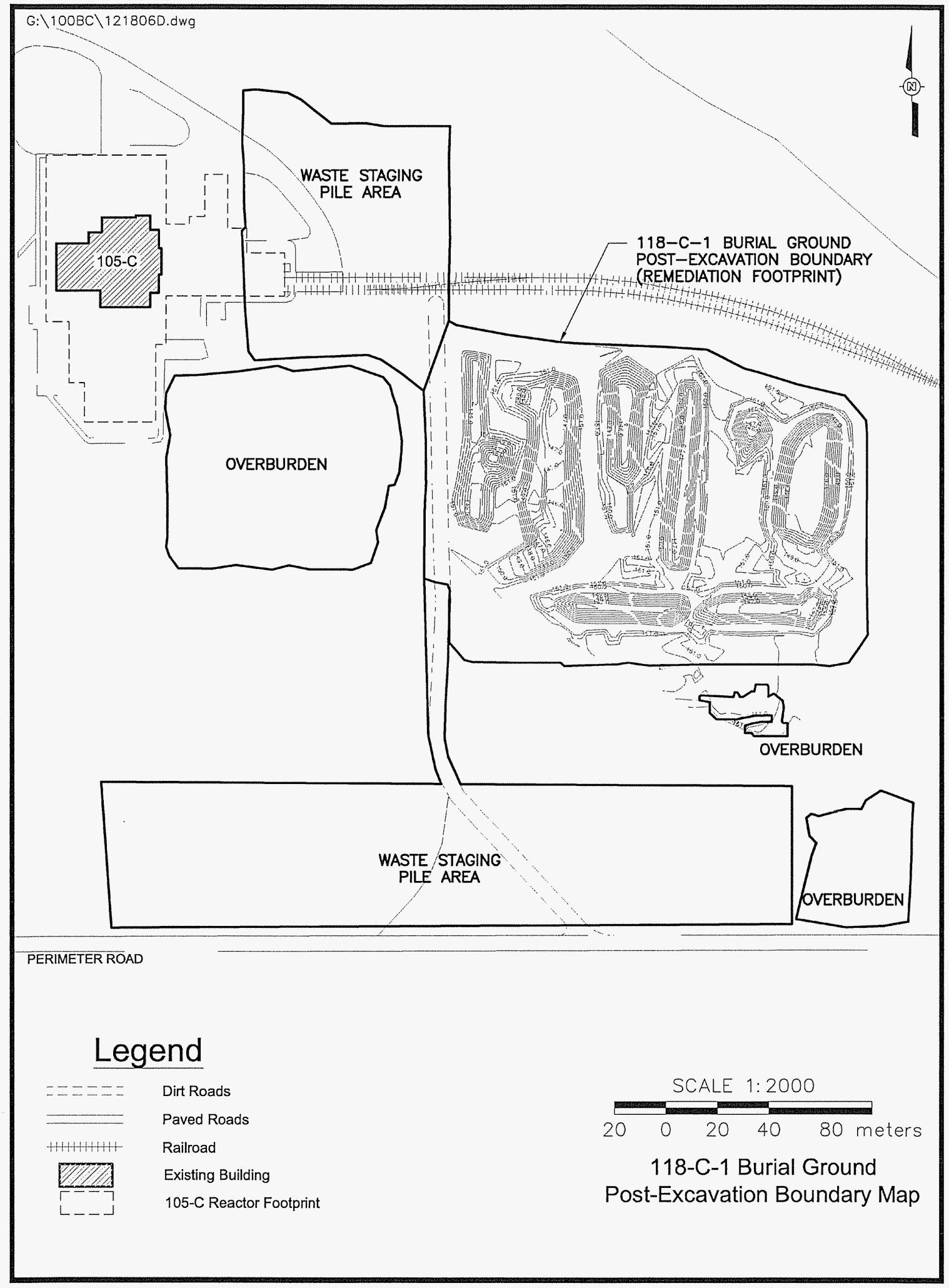


Figure 3. Remediation Footprint Sampling Area Boundaries.

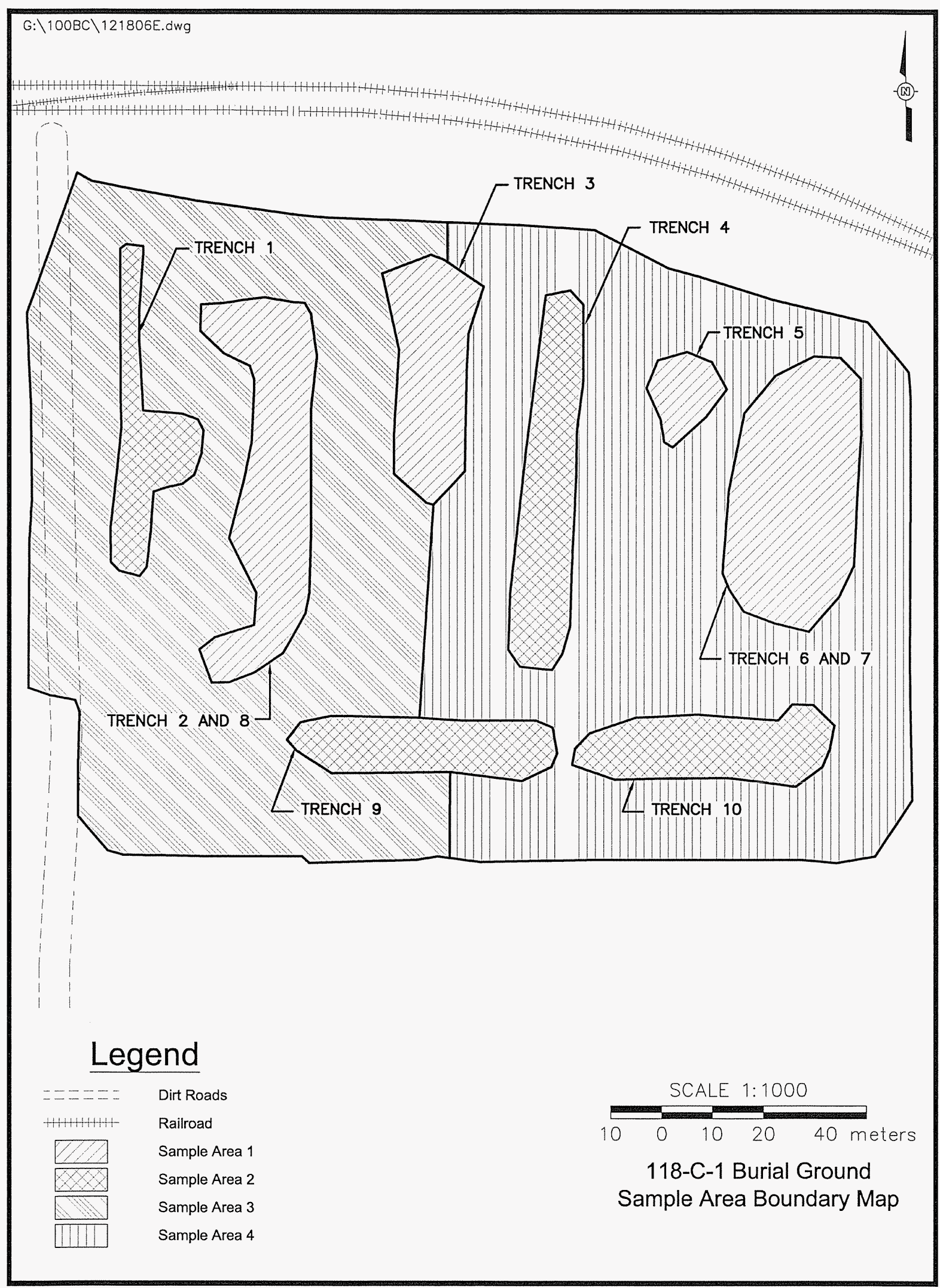




\subsubsection{Sampling Area 1.}

Sampling Area 1 is composed of Trenches 2, 3, 5, 6, 7, and 8 (Figure 3). The waste forms observed within these trenches included general debris, perforated and non-perforated spacers, mercury tubes, splines, lead, sheet metal, vertical control rods, boron balls, vent pipes, and SNF. Because of the presence of SNF, additional radionuclide COCs were added to this sampling area for close-out purposes. The COCs/COPCs associated with this area are provided in Table 1.

\subsubsection{Sampling Area 2.}

Sampling Area 2 is composed of Trenches 1, 4, 9, and 10 (Figure 2). The waste forms observed within these trenches included general debris, perforated and non-perforated spacers, vertical control rods, lead, boron balls, paint, piping, wax, tar, tar paper, casks, and concrete blocks. The COCs/COPCs associated with this area are provided in Table 1.

\subsubsection{Sampling Area 3.}

Sampling Area 3 consists of all the land between the targeted trenches on the west side of the burial ground. This sampling area includes all COCs/COPCs associated with Sampling Areas 1 and 2 . The COCs/COPCs associated with this area are provided in Table 1.

\subsubsection{Sampling Area 4.}

Sampling Area 4 consists of all the land between the targeted trenches on the east side of the burial ground. This sampling area includes all COCs/COPCs associated with Sampling Areas 1 and 2. The COCs/COPCs associated with this area are provided in Table 1.

\subsubsection{Overburden/BCL Soil.}

The three overburden/BCL soil piles are considered one decision unit (Figure 2). This decision unit contains all COCs/COPCs identified for the remediation footprint, as shown in Table 1.

\subsubsection{Waste Staging Pile Area.}

The 118-C-1 waste site contained two waste staging pile footprints (Figure 2). Excavated waste material from the 118-C-1 Burial Ground was initially transported to the northern waste staging pile area for sorting and sampling. Subsequent sorting of excavated waste material was performed in the southern waste staging area, which utilized process cells (trenches) for the waste sorting operations. The two staging pile area footprints will collectively be referred to as the waste staging pile area and will be closed out as one decision unit. The waste staging pile area includes all COCs/COPCs identified for the remediation footprint, as shown in Table 1. 


\section{Table 1. Summary of the 118-C-1 Waste Site COCs/COPCs for the Six Sampling Subunits.}

\begin{tabular}{|c|c|c|c|c|c|c|}
\hline COCs/COPCs & $\begin{array}{c}\text { Sampling } \\
\text { Area } 1\end{array}$ & $\begin{array}{c}\text { Sampling } \\
\text { Area } 2\end{array}$ & $\begin{array}{l}\text { Sampling } \\
\text { Area } 3\end{array}$ & $\begin{array}{c}\text { Sampling } \\
\text { Area } 4\end{array}$ & $\begin{array}{c}\text { Overburden/ } \\
\text { BCL }\end{array}$ & $\begin{array}{c}\text { Waste Staging } \\
\text { Pile Area }\end{array}$ \\
\hline \multicolumn{7}{|c|}{ Radionuclides } \\
\hline Carbon-14 & $x$ & $x$ & $x$ & $x$ & $x$ & $x$ \\
\hline Cesium-137 & $X$ & $x$ & $x$ & $X$ & $X$ & $x$ \\
\hline Cobalt-60 & $x$ & $x$ & $x$ & $x$ & $x$ & $x$ \\
\hline Europium-152 & $x$ & $x$ & $x$ & $x$ & $x$ & $x$ \\
\hline Europium-154 & $x$ & $x$ & $x$ & $x$ & $x$ & $x$ \\
\hline Nickel-63 & $x$ & $x$ & $x$ & $x$ & $x$ & $x$ \\
\hline Strontium-90 & $x$ & $x$ & $x$ & $x$ & $x$ & $x$ \\
\hline Silver-108m & $x$ & $x$ & $x$ & $x$ & $x$ & $x$ \\
\hline Tritium & $x$ & $x$ & $x$ & $x$ & $x$ & $x$ \\
\hline Americium-241 & $x$ & & $x$ & $x$ & $x$ & $x$ \\
\hline Plutonium-238 & $x$ & & $x$ & $x$ & $x$ & $x$ \\
\hline Plutonium-239/240 & $x$ & & $x$ & $x$ & $x$ & $x$ \\
\hline Plutonium-241 & $x$ & & $x$ & $x$ & $x$ & $x$ \\
\hline Uranium-233/234 & $x$ & & $x$ & $x$ & $x$ & $x$ \\
\hline Uranium-235 & $x$ & & $x$ & $x$ & $x$ & $x$ \\
\hline Uranium-238 & $x$ & & $x$ & $x$ & $x$ & $x$ \\
\hline \multicolumn{7}{|c|}{ Nonradionuclides } \\
\hline ICP metals ${ }^{a}$ & $x$ & $x$ & $x$ & $x$ & $x$ & $x$ \\
\hline Mercury & $x$ & $x$ & $x$ & $x$ & $x$ & $x$ \\
\hline $\begin{array}{l}\text { Hexavalent } \\
\text { chromium }\end{array}$ & $x$ & $x$ & $x$ & $x$ & $x$ & $x$ \\
\hline PCBs & & $x$ & $x$ & $x$ & $x$ & $x$ \\
\hline SVOA & & $x$ & $x$ & $x$ & $x$ & $x$ \\
\hline TPH & & $x$ & $x$ & $x$ & $x$ & $x$ \\
\hline VOA & & $x$ & $x$ & $x$ & $x$ & $x$ \\
\hline
\end{tabular}

${ }^{2}$ The expanded list of ICP metals analysis was performed (arsenic, antimony, barium, beryllium, boron, cadmium, chromium, lead, cobalt, copper, manganese, molybdenum, nickel, selenium, silver, vanadium, and zinc).

$\mathrm{BCL}=$ below cleanup level $\quad \mathrm{TPH}=$ total petroleum hydrocarbons

$I C P=$ inductively coupled plasma $\quad V O A=$ volatile organic analysis

$\mathrm{PCBs}=$ polychlorinated biphenyls

$S V O A=$ semi-volatile organic analysis 


\subsection{REMEDIAL ACTION FIELD ACTIVITIES}

\subsection{EXCAVATION AND DISPOSAL}

Remedial action at the 118-C-1 site began on February 2, 2004. Excavation of the site involved removing the uncontaminated overburden, the buried contaminated debris, and the underlying contaminated soil. On March 31, 2004, excavation and sorting of the burial ground was initiated. Suspect SNF was discovered during the waste debris sorting operation on September 27, 2004. Subsequent characterization of the suspect SNF confirmed it to be SNF. All remedial activities associated with the 118-C-1 Burial Ground were suspended until the Authorization Basis could be properly assessed and revised to include SNF (WCH 2006).

Load-out operations were re-established on April 11, 2005, for previously sorted and segregated material. All remedial activities (excavation, sorting, and load-out) resumed on October 25, 2005 and were completed on May 27, 2006. Over 75,300 metric tons (83,000 tons) of waste and contaminated soil from the 118-C-1 Burial Ground was disposed at ERDF and approximately 9,300 bank cubic meters (BCM) of overburden soil was removed for subsequent backfill. The post-remediation topographic map is shown in Figure 2 and the remediation footprint is shown in Figure 3. At the conclusion of excavation activities, the elevation at the deepest part of the remedial excavation was

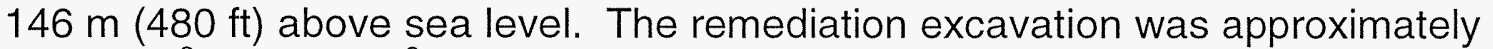
$20,500 \mathrm{~m}^{2}\left(220,600 \mathrm{ft}^{2}\right)$ in area with a maximum depth of approximately $5 \mathrm{~m}(17 \mathrm{ft})$.

Visual observations of the buried waste confirmed that the following items were disposed of in the 118-C-1 Burial Ground during its operation: several thousand perforated and nonperforated spacers, piping and tubing, vertical control rods, sheet metal, boron balls, boron ball vacuums, bismuth, paint, high-dose piping, wax, casks, tar, tar paper, miscellaneous metal, mercury tubes, lead items, reactor parts and hardware, SNF, hydraulic hoses and parts, degraded drums, glassware, concrete, electrical components, and other miscellaneous debris. Photographs of the waste site debris and remediation activities are provided in Appendix B.

The SNF discovered during remediation activities was staged in five bunkers within the 118-C-1 northern waste staging pile area. In total, nine intact SNF rods and four fractional pieces of SNF were discovered and characterized at the 118-C-1 waste site. All SNF is awaiting transfer to the 100-K fuel storage basins for interim storage prior to final packaging and disposal, pending completion of shipping and transfer documentation.

\subsubsection{FIELD SCREENING}

\subsubsection{Radiological Surveys}

Radiological field screening was conducted during the site remedial actions as specified in the SAP (DOE-RL 2001). Field screening was used to guide the excavation to 
quickly assess the presence and level of contamination. Field screening at the site included using a radiological data mapping system survey. The radiological mapping survey was performed over more than $50 \%$ of the site excavation surface area using a Global Positioning Environmental Radiological Surveyor (GPERS) with instrumentation specific to the detection of radiation associated with gamma-emitting radionuclides.

The radiological surveys are provided in Appendix D. Several radiological "hot spots" requiring additional remediation were identified in the trenches in Sampling Areas 1 and 2 as well as in the waste staging area process cells. The "hot spots" were targeted and an additional 454 metric tons (500 tons) of soil containing scattered metal debris were removed. These areas were resurveyed to confirm that waste site excavation was complete. Both the initial and final GPERS surveys for the remediation footprint are provided in Appendix D.

The overburden/BCL material was surveyed for radioactivity using GPERS throughout the remedial process. No contamination was identified in the final GPERS survey, and therefore, no further remedial action was required prior to the collection of verification samples.

\subsection{BIASED SAMPLING AND ANALYSIS}

Biased samples are collected to provide confidence for the absence of contamination in residual soil beneath locations exhibiting visual soil stains, buried liquid wastes or equipment, mercury-containing piping, or large inventories of dangerous/hazardous wastes (e.g., lead bricks). Additionally, biased samples may be collected from locations where process knowledge indicates the potential for elevated concentrations of alphaor beta-emitter contamination, or where waste characterization sampling results indicate elevated concentrations of chemical contaminants above the RAGs.

Less than twenty unique anomalous waste forms were discovered during excavation and sorting at the 118-C-1 Burial Ground. Anomalous items included: casks, multiple small glass and plastic containers, SNF, sealed metal pipes, filter traps, boron ball vacuums, paraffin wax, high dose metal items, vertical control rods, and mercury tubes. Photographs of anomalous waste debris items are provided in Appendix B.

Twelve discrete soil verification samples (i.e., biased samples) were collected, in addition to the statistical cleanup verification samples, based on agreement with the EPA and DOE-RL (WCH 2006). Seven of the biased samples were collected at locations where anomalous waste items were found within the remediation footprint (Figure 4). Five of the biased samples were collected from soil underlying the SNF bunkers located in the waste staging pile area. Table 2 provides a description of the biased samples along with the associated COCs. The results of the biased verification soil samples are included with the statistical soil verification assays provided in Appendix A. 
Figure 4. 118-C-1 Biased Sampling Locations within Remediation Footprint.

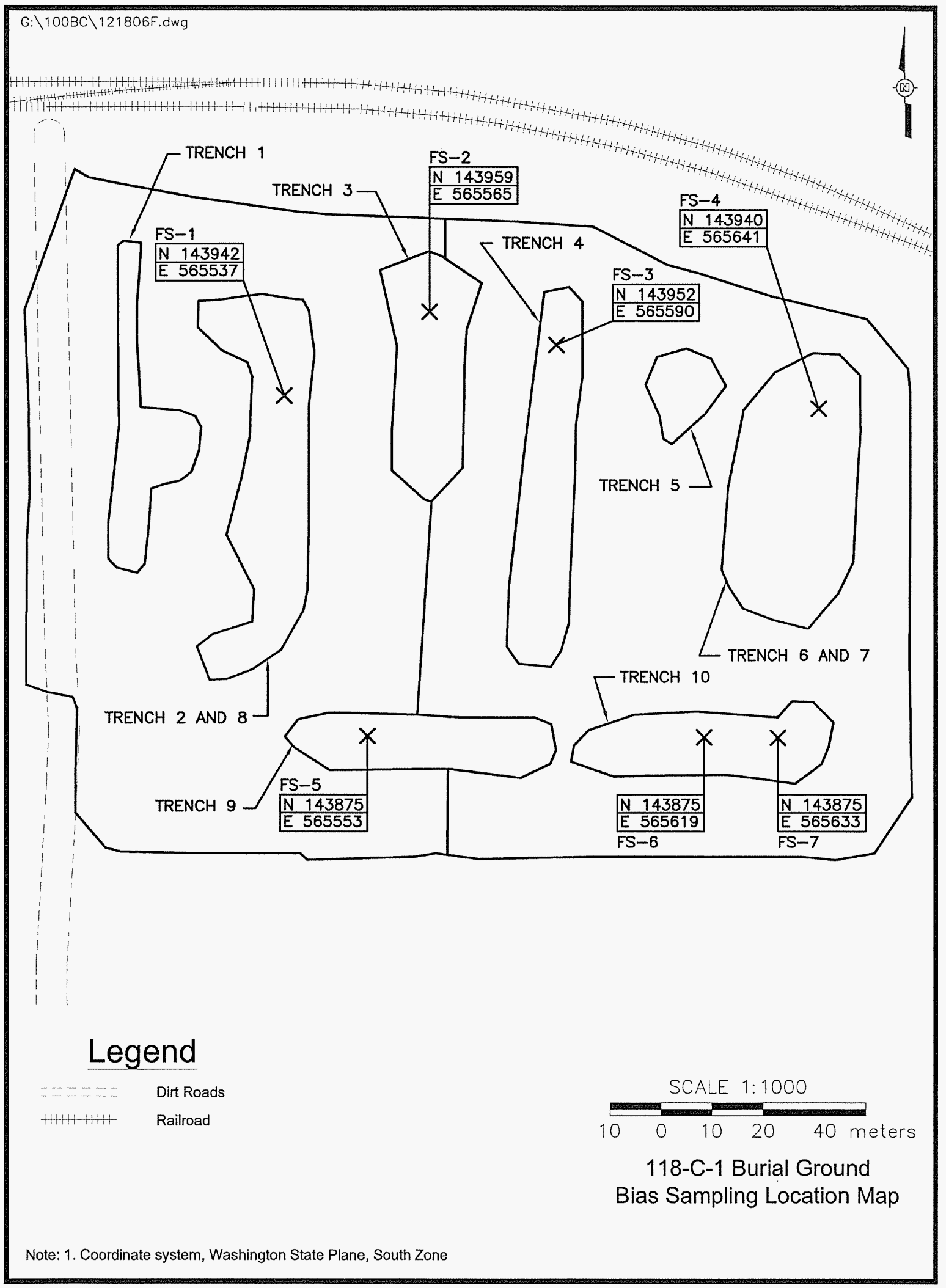


Table 2. 118-C-1 Biased Samples

\begin{tabular}{|c|c|c|c|}
\hline $\begin{array}{l}\text { Biased } \\
\text { Sample }\end{array}$ & Location & Associated Anomaly & coCs \\
\hline FS-1 & $\begin{array}{l}\text { Area 1, } \\
\text { Trench } 2 \\
\end{array}$ & Cache of lead bricks & ICP Metals ${ }^{a}$ \\
\hline FS-2 & $\begin{array}{l}\text { Area 1, } \\
\text { Trench } 3\end{array}$ & Cache of lead bricks & ICP Metals ${ }^{a}$ \\
\hline FS-3 & $\begin{array}{l}\text { Area 2, } \\
\text { Trench } 4\end{array}$ & Miscellaneous lead debris & ICP Metals ${ }^{a}$ \\
\hline FS-4 & $\begin{array}{l}\text { Area 1, } \\
\text { Trench } 7\end{array}$ & $\begin{array}{l}\text { Instrumentation bundle containing } \\
\text { high levels of bismuth }\end{array}$ & ICP Metals ${ }^{a}$ \\
\hline FS-5 & $\begin{array}{l}\text { Area 2, } \\
\text { Trench } 9\end{array}$ & Cache of paint products & $\begin{array}{l}\text { ICP Metals }{ }^{a} \text {, SVOA, VOA, } \\
\text { PCBs, TPH }\end{array}$ \\
\hline FS-6 & $\begin{array}{l}\text { Area 2, } \\
\text { Trench } 10\end{array}$ & Cache of tar and wax products & SVOA, VOA, PCBs, TPH \\
\hline FS-7 & $\begin{array}{l}\text { Area 2, } \\
\text { Trench } 10\end{array}$ & Cache of boron ball vacuums & ICP Metals ${ }^{\mathrm{a}}$, GEA \\
\hline FS-8 & $\begin{array}{c}\text { Waste Staging Pile } \\
\text { Area }\end{array}$ & SNF Bunker 1 & $\begin{array}{l}\text { Americium-241, } \\
\text { Isotopic Plutonium, } \\
\text { Plutonium-241, Isotopic } \\
\text { Uranium, GEA }\end{array}$ \\
\hline FS-9 & $\begin{array}{c}\text { Waste Staging Pile } \\
\text { Area }\end{array}$ & SNF Bunker 2 & $\begin{array}{l}\text { Americium-241, Isotopic } \\
\text { Plutonium, Plutonium-241, } \\
\text { Isotopic Uranium, GEA }\end{array}$ \\
\hline FS-10 & $\begin{array}{c}\text { Waste Staging Pile } \\
\text { Area }\end{array}$ & SNF Bunker 3 & $\begin{array}{l}\text { Americium-241, Isotopic } \\
\text { Plutonium, Plutonium-241, } \\
\text { Isotopic Uranium, GEA }\end{array}$ \\
\hline FS-11 & $\begin{array}{c}\text { Waste Staging Pile } \\
\text { Area }\end{array}$ & SNF Bunker 4 & $\begin{array}{l}\text { Americium-241, Isotopic } \\
\text { Plutonium, Plutonium-241, } \\
\text { Isotopic Uranium, GEA }\end{array}$ \\
\hline FS-12 & $\begin{array}{l}\text { Waste Staging Pile } \\
\text { Area }\end{array}$ & SNF Bunker 5 & $\begin{array}{l}\text { Americium-241, Isotopic } \\
\text { Plutonium, Plutonium-241, } \\
\text { Isotopic Uranium, GEA }\end{array}$ \\
\hline
\end{tabular}

${ }^{a}$ The expanded list of ICP metals analysis was performed (arsenic, antimony, barium, beryllium, boron, cadmium, chromium, lead, cobalt, copper, manganese, molybdenum, nickel, selenium, silver, vanadium, and zinc).

GEA = gamma energy analysis $\mathrm{SNF}=$ spent nuclear fuel

$I C P=$ inductively coupled plasma

$\mathrm{TPH}=$ total petroleum hydrocarbons

$\mathrm{PCBs}=$ polychlorinated biphenyls

$\mathrm{VOA}=$ volatile organic analysis

SVOA $=$ semi-volatile organic analysis

\subsection{CLEANUP VERIFICATION SAMPLING AND ANALYSIS}

Final cleanup verification samples were collected in August and September, 2006 and May 2007 to confirm acceptability of residual contaminant concentrations in the soil at the 118-C-1 waste site. Each statistical verification sample was a composite formed by combining soil collected at four randomly selected nodes within each sampling decision 
subunit. The verification samples were submitted to offsite laboratories for analysis using approved EPA analytical methods, as required per the 100 Area Burial Grounds Remedial Action Sampling and Analysis Plan (SAP) (DOE-RL 2001). The sample design methodology and sample location figures are presented in the calculation brief for verification sample design in Appendix E.

For close-out verification sampling of the 118-C-1 remediation footprint, the ten trenches and surrounding land were grouped into four separate sampling decision units based on similarity of the waste forms observed during remediation. In addition to these four sampling decision units, the 118-C-1 waste site includes one decision unit for the overburden/BCL piles and one decision unit for the waste staging pile area (Figure 3). All six sampling decision units will be closed-out using the more restrictive shallow zone cleanup criteria, even though the remediation footprint does contain a deep zone

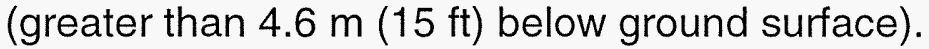

The number of decision subunits is determined by the overall footprint area of the decision unit (DOE-RL 2001). The default number of verification samples for each decision unit is four (4) composite samples. The required number of verification samples for each of the decision units associated with the 118-C-1 Burial Ground waste site are listed in Table 3.

Table 3. Number of Verification Samples for the 118-C-1 Burial Ground.

\begin{tabular}{|c|c|c|c|c|}
\hline $\begin{array}{c}\text { Decision } \\
\text { Unit }\end{array}$ & $\begin{array}{c}\text { Waste Site } \\
\text { Footprint }\left(\mathrm{ft}^{2}\right)\end{array}$ & $\begin{array}{c}\text { Size Classification } \\
\left(\mathrm{ft}^{2}\right)\end{array}$ & $\begin{array}{c}\text { Decision } \\
\text { Subunits }\end{array}$ & $\begin{array}{c}\text { Verification } \\
\text { Samples }\end{array}$ \\
\hline Sampling Area 1 & 32,500 & Small $(<100,000)$ & 1 & 4 composite \\
\hline Sampling Area 2 & 25,200 & Small $(<100,000)$ & 1 & 4 composite \\
\hline Sampling Area 3 & 82,500 & Small $(<100,000)$ & 1 & 4 composite \\
\hline Sampling Area 4 & 82,500 & Small $(<100,000)$ & 1 & 4 composite \\
\hline Overburden/BCL pile & 97,000 & Small $(<100,000)$ & 1 & 4 composite \\
\hline Waste Staging Pile Area & 250,00 & $\begin{array}{c}\text { Medium } \\
(>100,000 \text { to } \\
400,000)\end{array}$ & 4 & 16 composite \\
\hline
\end{tabular}

$\mathrm{BCL}=$ below cleanup level

\subsection{CLEANUP VERIFICATION DATA EVALUATION}

This section presents the evaluation and modeling of the 118-C-1 cleanup verification data for comparison with the data quality criteria and RAGs. 


\subsection{DATA QUALITY ASSESSMENT PROCESS}

A data quality assessment (DQA) is performed to compare the verification sampling approach and resulting analytical data with the sampling and data quality requirements specified by the project objectives and performance specifications.

The DQA for the 118-C-1 site established that the data are of the right type, quality, and quantity to support site verification decisions within specified error tolerances. All analytical data were found to be acceptable for decision-making purposes. The evaluation verified that the sample design was sufficient for the purpose of clean site verification. The cleanup verification sample analytical data are stored in the Environmental Restoration (ENRE) project-specific database for data evaluation prior to submittal for archival in the Hanford Environmental Information System (HEIS) and are summarized in Appendix A. The detailed DQA is presented in Appendix C.

\subsection{CONTAMINANTS OF CONCERN 95\% UPPER CONFIDENCE LIMIT}

The primary statistical calculation to support cleanup verification is the $95 \%$ upper confidence limit (UCL) on the arithmetic mean of the data. The $95 \%$ UCL values for each COC/COPC are computed for each decision unit. Prior to calculating the $95 \%$ $\mathrm{UCL}$, the individual sample results are reviewed and, as appropriate, adjusted per the SAP (DOE-RL 2001) and RDR/RAWP (DOE-RL 2005). Statistical evaluations were not performed on data sets with no reported detections. This process is summarized below:

- Radionuclides: The laboratory reported value is used in the calculation of the $95 \%$ UCL. In cases where the laboratory does not report a value for data qualified with a "U" (i.e., less than the detection limit), half of the minimum detectable activity is used in the calculation of the $95 \% \mathrm{UCL}$.

- Nonradionuclides: For data flagged with a "U" (i.e., less than detection), a value equal to one-half the practical quantitation limit is used in the calculation of the $95 \%$ UCL, as required by Washington State Department of Ecology regulations (Washington Administrative Code [WAC] 173-340-740[7][g]). If greater than half of the sample results for a given $C O C$ are below detection, then the statistical value is set equal to the maximum concentration detected (i.e., versus computing a $95 \% \mathrm{UCL}$ ).

Statistical calculations are presented in the 118-C-1 cleanup verification 95\% UCL calculation brief (Appendix E). Comparisons of the statistical results for waste site COCs/COPCs and the site RAGs are summarized in Tables 4 through 9. Calculated cleanup levels are not presented in the Cleanup Levels and Risk Calculations (CLARC) Database (Ecology 2005) under the Washington Administrative Code (WAC) 173-340-740(3) for aluminum, calcium, iron, magnesium, potassium, silicon, and sodium; therefore, these constituents are not considered site COCs/COPCs. Potassium-40, radium-226, radium-228, thorium-228, and thorium-232 were detected in samples collected at the site, but are not considered within the statistical calculations as these isotopes are not related to the operational history of the site. All individual sample 
cleanup verification results are presented in Appendix A. The cleanup verification data set, summarized in Tables 4 through 9, was used for the RESidual RADioactivity (RESRAD) modeling discussed in Section 5.0.

Initial statistical data results from the waste staging pile area indicated the presence of arsenic and TPHs at concentrations exceeding the cleanup criteria. Consequently, additional soil was removed from these areas and the areas re-sampled and analyzed for ICP metals and TPHs. The second set of analyses for ICP metals and TPH were used to perform the $95 \%$ UCL calculations for the waste staging pile area, the results of which are presented in Table 9.

Carbon-14 was detected in a single verification sample collected from the waste staging pile area (Table 9). This detection was in exceedance of the carbon-14 direct exposure dose-equivalence lookup value. Evaluation of radionuclide data from the waste staging pile area indicated that statistical evaluation and dose modeling using this sample would result in an elevated dose estimate due to the presence of the carbon-14. Therefore, this sample was not considered in the statistical calculations for residual radionuclide activities at the waste staging pile area, and instead a sample-specific dose evaluation was performed, using RESRAD (Appendix E). Based on this evaluation, it has been determined that the soil containing carbon-14 from the waste staging pile area will be used as backfill in the deep zone.

Table 4. Comparison of Statistical Contaminant Concentrations to Action Levels for Verification Sampling of the 118-C-1 Sample Area 1. (2 Pages)

\begin{tabular}{|c|c|c|c|c|c|c|}
\hline \multirow[b]{2}{*}{ COC/COPC } & \multirow[b]{2}{*}{$\begin{array}{l}\text { Statistical } \\
\text { Result } \\
\text { (pCi/g) }\end{array}$} & \multicolumn{3}{|c|}{ Generic Site Lookup Values $^{\mathrm{a}}(\mathrm{pCi} / \mathrm{g})$} & \multirow{2}{*}{$\begin{array}{l}\text { Does the } \\
\text { Statistical } \\
\text { Result } \\
\text { Exceed } \\
\text { Lookup } \\
\text { Values? }\end{array}$} & \multirow[b]{2}{*}{$\begin{array}{l}\text { Does the } \\
\text { Statistical } \\
\text { Result Pass } \\
\text { RESRAD } \\
\text { Modeling? }\end{array}$} \\
\hline & & $\begin{array}{l}\text { Shallow } \\
\text { Zone } \\
\text { Lookup } \\
\text { Value }^{b}\end{array}$ & $\begin{array}{l}\text { Groundwater } \\
\text { Protection } \\
\text { Lookup } \\
\text { Value }\end{array}$ & $\begin{array}{l}\text { River } \\
\text { Protection } \\
\text { Lookup } \\
\text { Value }\end{array}$ & & \\
\hline Cesium-137 & 0.185 & 6.2 & $1,465^{c}$ & $1,465^{c}$ & No & \multirow{2}{*}{ Yes $^{d}$} \\
\hline Europium-152 & 0.121 & 3.3 & $-{ }^{e}$ & $--^{e}$ & No & \\
\hline Uranium-233/234 & $0.506(<B G)$ & $1.1^{f}$ & $1.1^{f}$ & $1.1^{t}$ & No & -- \\
\hline Uranium-235 & $0.084(<B G)$ & 0.84 & $0.5^{9}$ & $0.5^{9}$ & No & -- \\
\hline Uranium-238 & $0.637(<B G)$ & $1.1^{f}$ & $1.1^{f}$ & $1.1^{f}$ & No & - \\
\hline \multirow[b]{2}{*}{ COC/COPC } & \multirow[b]{2}{*}{$\begin{array}{l}\text { Statistical } \\
\text { Result } \\
\text { (mg/kg) }\end{array}$} & \multicolumn{3}{|c|}{ Remedial Action Goals ${ }^{\mathrm{a}}(\mathrm{mg} / \mathrm{kg})$} & \multirow[b]{2}{*}{$\begin{array}{l}\text { Does the } \\
\text { Statistical } \\
\text { Data Set } \\
\text { Exceed } \\
\text { RAGs? }\end{array}$} & \multirow[b]{2}{*}{$\begin{array}{l}\text { Does the } \\
\text { Statistical } \\
\text { Result Pass } \\
\text { RESRAD } \\
\text { Modeling? }\end{array}$} \\
\hline & & $\begin{array}{c}\text { Direct } \\
\text { Exposure }\end{array}$ & $\begin{array}{l}\text { Soil Cleanup } \\
\text { Level for } \\
\text { Groundwater } \\
\text { Protection }\end{array}$ & $\begin{array}{c}\text { Soil } \\
\text { Cleanup } \\
\text { Level for } \\
\text { River } \\
\text { Protection }\end{array}$ & & \\
\hline Arsenic & $3.0(<B G)$ & 20 & 20 & 20 & No & -- \\
\hline Barium & $54.3(<B G)$ & $5,600^{h}$ & $132^{\mathrm{i}, \mathrm{j}}$ & $224^{k}$ & No & -- \\
\hline Beryllium & $0.84(<B G)$ & $10.4^{1}$ & $1.51^{j}$ & $1.51^{j}$ & No & -- \\
\hline
\end{tabular}


Table 4. Comparison of Statistical Contaminant Concentrations to Action Levels for Verification Sampling of the 118-C-1 Sample Area 1. (2 Pages)

\begin{tabular}{|l|c|c|c|c|c|c|}
\hline Boron $^{\mathrm{m}}$ & 1.9 & 16,000 & 320 & $-^{\mathrm{n}}$ & No & -- \\
\hline Chromium (total) & $7.9(<\mathrm{BG})$ & $80,000^{\mathrm{h}}$ & $18.5^{\mathrm{j}}$ & $18.5^{\mathrm{j}}$ & No & -- \\
\hline Cobalt & $7.9(<\mathrm{BG})$ & 1,600 & 32 & $--^{\mathrm{n}}$ & No & -- \\
\hline Copper & $13.7(<\mathrm{BG})$ & 2,960 & 59.2 & $22.0^{\mathrm{j}}$ & No & -- \\
\hline Lead & $4.7(<\mathrm{BG})$ & 353 & $10.2^{\mathrm{j}}$ & $10.2^{\mathrm{j}}$ & No & -- \\
\hline Manganese & $339(<\mathrm{BG})$ & 11,200 & $512^{\mathrm{j}}$ & $512^{\mathrm{j}}$ & No & -- \\
\hline Nickel & $10.3(<\mathrm{BG})$ & 1,600 & $19.1^{\mathrm{j}}$ & 27.4 & No & -- \\
\hline Vanadium & $39.8(<\mathrm{BG})$ & 560 & $85.1^{\mathrm{j}}$ & $--^{\mathrm{n}}$ & No & -- \\
\hline Zinc & $37.3(<\mathrm{BG})$ & 24,000 & 480 & $67.8^{\mathrm{j}}$ & No & -- \\
\hline
\end{tabular}

a Lookup values and remedial action goals (RAGs) obtained from the Remedial Design Report/Remedial Action Work Plan for the 100 Area (RDR/RAWP) (DOE-RL 2005) or calculated per Washington Administrative Code (WAC) 173-340-720, 730, and 740, Method B, 1996, unless otherwise noted.

b Activity corresponding to a single-radionuclide $15 \mathrm{mrem} / \mathrm{yr}$ exposure as calculated using a generic RESidual RADioactivity (RESRAD) model (DOE-RL 2005).

c Revised lookup value per 100 Area Radionuclide and Nonradionuclide Lookup Values for the 1995 Interim Remedial Action Record of Decision (BHI 2004).

d 118-C-1 Burial Ground Cleanup Verification RESRAD Calculation Brief (Appendix E).

- No value-RESRAD modeling predicts the contaminant will not reach groundwater within 1,000 years.

$f$ The calculated lookup value is below the Hanford-specific statistical soil background activity. The value presented is the Hanfordspecific statistical soil background activity.

$\mathrm{g}$ The lookup value is below the required minimum detectable activity (MDA). The value presented is the required MDA.

h Noncarcinogenic cleanup level calculated from WAC 173-340-740(3), 1996 (Method B for soils) (as presented in the RDR/RAWP [DOE-RL 2005]). Updated oral reference dose values (as provided in the Integrated Risk Information System [IRIS]) yield Method B direct exposure RAG values of $16,000 \mathrm{mg} / \mathrm{kg}$ and $120,000 \mathrm{mg} / \mathrm{kg}$ for barium and chromium, respectively.

i Barium soil cleanup level for groundwater protection calculated from WAC 173-340-740(3)(a)(ii)(A), 1996 ("100 times rule") and WAC 173-340-720(3), 1996 (Method B for groundwater) is $112 \mathrm{mg} / \mathrm{kg}$ (as presented in the RDR/RAWP [DOE-RL 2005]). The updated oral reference dose value (as provided in IRIS) yields a Method B groundwater cleanup criteria of $7 \mathrm{mg} / \mathrm{L}$, as compared to the more restrictive maximum contaminant level of $2 \mathrm{mg} / \mathrm{L}$ (40 Code of Federal Regulations [CFR] 141). Per WAC 173-340-740(3)(a)(ii)(A), 1996 ("100 times rule"), the most restrictive updated soil cleanup level for groundwater protection would be 200 mg/kg.

j Where cleanup levels are less than background, cleanup levels default to background (WAC 173-340-700[4][d]) (1996).

k Barium soil cleanup level for river protection calculated from WAC 173-340-740(3)(a)(ii)(A), 1996 ("100 times rule"), a dilutionattenuation factor of 2, and WAC 173-340-720(3), 1996 (Method B for groundwater) is $224 \mathrm{mg} / \mathrm{kg}$ (as presented in the RDR/RAWP [DOE-RL 2005]). No surface water bioconcentration factor is available for barium and no ambient water quality criteria value exists separate from the previous drinking water standard; therefore no WAC 173-340-730(3), 1996 (Method B for surface waters) value can be determined.

1 Carcinogenic cleanup level calculated based on the inhalation exposure pathway per WAC 173-340-750[3], 1996 (Method B for air quality) and an airborne particulate mass loading rate of $0.0001 \mathrm{~g} / \mathrm{m}^{3}$ (WDOH 1997).

$m$ No Hanford Site-specific or Washington State background value available.

$n$ No parameters (bioconcentration factors or ambient water quality criteria values) are available from the Ecology Cleanup Levels and Risk Calculations database (Ecology 2005) or other databases to calculate cleanup levels (WAC 173-340-730(3)(a)(iii), 1996 [Method $B$ for surface waters]).
= not applicable
BG = background (from DOE-RL [1996, 2001], unless otherwise noted)
COC = contaminant of concern
$\begin{array}{ll}\text { COPC } & =\text { contaminant of potential concern } \\ \text { RAG } & =\text { remedial action goal } \\ \text { RESRAD } & =\text { RESidual RADioactivity }\end{array}$ 
Table 5. Comparison of Statistical Contaminant Concentrations to Action Levels for Verification Sampling of the 118-C-1 Sample Area 2. (2 Pages )

\begin{tabular}{|c|c|c|c|c|c|c|}
\hline \multirow[b]{2}{*}{$\mathrm{COC} / \mathrm{COPC}$} & \multirow[b]{2}{*}{$\begin{array}{c}\text { Statistical } \\
\text { Result } \\
(\mathrm{pCi} / \mathrm{g})\end{array}$} & \multicolumn{3}{|c|}{ Generic Site Lookup Values $^{a}(\mathrm{pCi} / \mathrm{g})$} & \multirow{2}{*}{$\begin{array}{l}\text { Does the } \\
\text { Statistical } \\
\text { Result } \\
\text { Exceed } \\
\text { Lookup } \\
\text { Values? }\end{array}$} & \multirow[b]{2}{*}{$\begin{array}{l}\text { Does the } \\
\text { Statistical } \\
\text { Result Pass } \\
\text { RESRAD } \\
\text { Modeling? }\end{array}$} \\
\hline & & $\begin{array}{l}\text { Shallow } \\
\text { Zone } \\
\text { Lookup } \\
\text { Value }^{\mathrm{b}}\end{array}$ & $\begin{array}{l}\text { Groundwater } \\
\text { Protection } \\
\text { Lookup } \\
\text { Value }\end{array}$ & $\begin{array}{l}\text { River } \\
\text { Protection } \\
\text { Lookup } \\
\text { Value }\end{array}$ & & \\
\hline Carbon-14 & $8.0^{c}$ & 5.16 & $--^{d}$ & $--^{d}$ & Yes & \multirow{6}{*}{ Yes $^{f}$} \\
\hline Cesium-137 & 0.140 & 6.2 & $1,465^{\mathrm{e}}$ & $1,465^{\mathrm{e}}$ & No & \\
\hline Cobalt-60 & 0.99 & 1.4 & $13,900^{e}$ & $13,900^{\mathrm{e}}$ & No & \\
\hline Europium-152 & 0.199 & 3.3 & $--^{d}$ & $--^{d}$ & No & \\
\hline Nickel-63 & 26.1 & $4,013^{e}$ & $83^{e}$ & $83^{e}$ & No & \\
\hline Tritium & 4.76 & $459^{e}$ & $12.6^{\mathrm{e}}$ & $12.6^{e}$ & No & \\
\hline \multirow[b]{2}{*}{ COC/COPC } & \multirow[b]{2}{*}{$\begin{array}{c}\text { Statistical } \\
\text { Result } \\
(\mathrm{mg} / \mathrm{kg})\end{array}$} & \multicolumn{3}{|c|}{ Remedial Action Goals ${ }^{\mathrm{a}}(\mathrm{mg} / \mathrm{kg})$} & \multirow[b]{2}{*}{$\begin{array}{l}\text { Does the } \\
\text { Statistical } \\
\text { Data Set } \\
\text { Exceed } \\
\text { RAGs? }\end{array}$} & \multirow[b]{2}{*}{$\begin{array}{c}\text { Does the } \\
\text { Statistical } \\
\text { Result Pass } \\
\text { RESRAD } \\
\text { Modeling? }\end{array}$} \\
\hline & & $\begin{array}{c}\text { Direct } \\
\text { Exposure }\end{array}$ & $\begin{array}{l}\text { Soil Cleanup } \\
\text { Level for } \\
\text { Groundwater } \\
\text { Protection }\end{array}$ & $\begin{array}{c}\text { Soil } \\
\text { Cleanup } \\
\text { Level for } \\
\text { River } \\
\text { Protection }\end{array}$ & & \\
\hline Arsenic & $2.7(<B G)$ & 20 & 20 & 20 & No & - \\
\hline Barium & $51.9(<B G)$ & $5,600^{9}$ & $132^{h, i}$ & $224^{j}$ & No & -- \\
\hline Beryllium & $0.88(<B G)$ & $10.4^{k}$ & $1.51^{i}$ & $1.51^{i}$ & No & -- \\
\hline Boron' & 2.1 & 16,000 & 320 & $-{ }^{m}$ & No & -- \\
\hline Chromium (total) & $6.1(<B G)$ & $80,000^{h}$ & $18.5^{i}$ & $18.5^{i}$ & No & -- \\
\hline Chromium (hexavalent) & 0.24 & 2.1 & $4.8^{n}$ & 2 & No & -- \\
\hline Cobalt & $7.7(<B G)$ & 1,600 & 32 & $\ldots-1$ & No & -- \\
\hline Copper & $13.6(<B G)$ & 2,960 & 59.2 & $22.0^{h}$ & No & -- \\
\hline Lead & $4.1(<B G)$ & 353 & $10.2^{h}$ & $10.2^{h}$ & No & -- \\
\hline Manganese & $340(<B G)$ & 11,200 & $512^{\mathrm{h}}$ & $512^{h}$ & No & -- \\
\hline Nickel & $9.8(<B G)$ & 1,600 & $19.1^{h}$ & 27.4 & No & -- \\
\hline Vanadium & $46.2(<B G)$ & 560 & $85.1^{\mathrm{h}}$ & -1 & No & -- \\
\hline Zinc & $39.3(<B G)$ & 24,000 & 480 & $67.8^{i}$ & No & -- \\
\hline
\end{tabular}




\section{Table 5. Comparison of Statistical Contaminant Concentrations to Action Levels for Verification Sampling of the 118-C-1 Sample Area 2. (2 Pages )}

\begin{tabular}{|c|c|c|c|c|c|c|}
\hline \multirow[b]{2}{*}{$\mathrm{COC} / \mathrm{COPC}$} & \multirow[b]{2}{*}{$\begin{array}{c}\text { Statistical } \\
\text { Result } \\
\text { (mg/kg) }\end{array}$} & \multicolumn{3}{|c|}{ Remedial Action Goals ${ }^{\mathrm{a}}$ (mg/kg) } & \multirow[b]{2}{*}{$\begin{array}{l}\text { Does the } \\
\text { Statistical } \\
\text { Data Set } \\
\text { Exceed } \\
\text { RAGs? }\end{array}$} & \multirow[b]{2}{*}{$\begin{array}{l}\text { Does the } \\
\text { Statistical } \\
\text { Result Pass } \\
\text { RESRAD } \\
\text { Modeling? }\end{array}$} \\
\hline & & $\begin{array}{c}\text { Direct } \\
\text { Exposure }\end{array}$ & $\begin{array}{l}\text { Soil Cleanup } \\
\text { Level for } \\
\text { Groundwater } \\
\text { Protection }\end{array}$ & $\begin{array}{l}\text { Soil } \\
\text { Cleanup } \\
\text { Level for } \\
\text { River } \\
\text { Protection }\end{array}$ & & \\
\hline $\begin{array}{l}\text { bis }(2-\text {-Ethylhexyl) } \\
\text { phthalate }\end{array}$ & 0.28 & 71.4 & 0.625 & 0.36 & Yes $^{\circ}$ & Yes $^{p}$ \\
\hline Di-n-butylphthalate & 0.035 & 8,000 & 160 & 540 & No & -- \\
\hline Methylene chloride & 0.012 & 133 & 0.5 & 0.94 & No & -- \\
\hline
\end{tabular}

a Lookup values and remedial action goals (RAGs) obtained from the Remedial Design Report/Remedial Action Work Plan for the 100 Area (RDR/RAWP) (DOE-RL 2005) or calculated per Washington Administrative Code (WAC) 173-340-720, 730, and 740, Method B, 1996, unless otherwise noted.

b Activity corresponding to a single-radionuclide $15 \mathrm{mrem} / \mathrm{yr}$ exposure as calculated using a generic RESidual RADioactivity (RESRAD) model (DOE-RL 2005).

c The statistical value for the carbon-14 activity in Sample Area $2(8 \mathrm{pCi} / \mathrm{g})$ is greater than the shallow zone lookup value (5.16 pCi/g) because the lookup value was calculated for a generic site using the RESRAD model with a carbon-14 distribution coefficient (Kd value) of $0 \mathrm{~mL} / \mathrm{g}$ while 100 Area RESRAD evaluations use a Kd value of $200 \mathrm{~mL} / \mathrm{g}$, per Table $\mathrm{E}-1$ of the 100 Area RDR/RAWP (DOE-RL 2005). Using a Kd value of $200 \mathrm{~mL} / \mathrm{g}$ the shallow zone lookup value is calculated to be $8.69 \mathrm{pCi} / \mathrm{g}$.

d No value-RESRAD modeling predicts the contaminant will not reach groundwater within 1,000 years.

e Revised lookup value per 100 Area Radionuclide and Nonradionuclide Lookup Values for the 1995 Interim Remedial Action Record of Decision (BHI 2004).

118-C-1 Burial Ground Cleanup Verification RESRAD Calculation Brief (Appendix E).

9 Noncarcinogenic cleanup level calculated from WAC 173-340-740(3), 1996 (Method B for soils) (as presented in the RDR/RAWP [DOE-RL 2005]). Updated oral reference dose values (as provided in the Integrated Risk Information System [IRIS]) yield Method B direct exposure RAG values of $16,000 \mathrm{mg} / \mathrm{kg}$ and $120,000 \mathrm{mg} / \mathrm{kg}$ for barium and chromium, respectively.

h Barium soil cleanup level for groundwater protection calculated from WAC 173-340-740(3)(a)(ii)(A), 1996 ("100 times rule") and WAC 173-340-720(3), 1996 (Method B for groundwater) is $112 \mathrm{mg} / \mathrm{kg}$ (as presented in the RDR/RAWP [DOE-RL 2005]). The updated oral reference dose value (as provided in IRIS) yields a Method B groundwater cleanup criteria of $7 \mathrm{mg} / \mathrm{L}$, as compared to the more restrictive maximum contaminant level of $2 \mathrm{mg} / \mathrm{L}$ (40 Code of Federal Regulations [CFR] 141). Per WAC 173-340-740(3)(a)(ii)(A), 1996 ("100 times rule"), the most restrictive updated soil cleanup level for groundwater protection would be $200 \mathrm{mg} / \mathrm{kg}$.

i Where cleanup levels are less than background, cleanup levels default to background (WAC 173-340-700[4][d]) (1996).

i Barium soil cleanup level for river protection calculated from WAC 173-340-740(3)(a)(ii)(A), 1996 ("100 times rule"), a dilutionattenuation factor of 2, and WAC 173-340-720(3), 1996 (Method B for groundwater) is $224 \mathrm{mg} / \mathrm{kg}$ (as presented in the RDR/RAWP [DOE-RL 2005]). No surface water bioconcentration factor is available for barium and no ambient water quality criteria value exists separate from the previous drinking water standard; therefore no WAC 173-340-730(3), 1996 (Method B for surface waters) value can be determined.

k Carcinogenic cleanup level calculated based on the inhalation exposure pathway per WAC 173-340-750[3], 1996 (Method B for air quality) and an airborne particulate mass loading rate of $0.0001 \mathrm{~g} / \mathrm{m}^{3}$ (WDOH 1997).

1 No Hanford Site-specific or Washington State background value available.

m No parameters (bioconcentration factors or ambient water quality criteria values) are available from the Ecology Cleanup Levels and Risk Calculations database (Ecology 2005) or other databases to calculate cleanup levels (WAC 173-340-730(3)(a)(iii), 1996 [Method B for surface waters]).

" Calculated cleanup level (per WAC 173-340-720(3), 1996 [Method B for groundwater] and WAC 173-340-740(3)(a)(ii)(A), 1996 ["100 times rule"]) presented is lower than that presented in the RDR/RAWP (DOE-RL 2005), based on updated oral reference dose value (as provided in IRIS).

- The statistical verification data set fails the second and/or third component of the WAC 173-340-740(7)(e) 3-part test in comparison against soil RAGs for groundwater and/or river protection (one or more sample concentrations exceeds twice the soil RAG value and/or more than $10 \%$ of the data set exceeds the soil RAG value).

$\mathrm{P}$ Based on the 100 Area Analogous Sites RESRAD Calculations (BHI 2005) and contaminant-specific soil portioning coefficient ( $\left.\mathrm{K}_{\mathrm{d}}\right)$ value, contaminant will not migrate vertically more than $3 \mathrm{~m}(10 \mathrm{ft})$ in 1,000 years. As the vadose zone underlying the site is greater than $25 \mathrm{~m}$ (82 ft) thick, the contaminant will not reach groundwater (and thus the Columbia River) in 1,000 years.

= not applicable

BG = background (obtained from DOE-RL [1996] and DOE-RL [2001], unless otherwise noted)

COC = contaminant of concern

$\mathrm{COPC}=$ contaminant of potential concern

RAG = remedial action goal

RESRAD = RESidual RADioactivity (dose-assessment model) 
Table 6. Comparison of Statistical Contaminant Concentrations to Action Levels for Verification Sampling of the 118-C-1 Sample Area 3. (2 Pages)

\begin{tabular}{|c|c|c|c|c|c|c|}
\hline \multirow[b]{2}{*}{ COC/COPC } & \multirow[b]{2}{*}{$\begin{array}{c}\text { Statistical } \\
\text { Result } \\
\text { (pCi/g) }\end{array}$} & \multicolumn{3}{|c|}{ Generic Site Lookup Values $^{a}(\mathrm{pCi} / \mathrm{g})$} & \multirow{2}{*}{$\begin{array}{c}\text { Does the } \\
\text { Statistical } \\
\text { Result } \\
\text { Exceed } \\
\text { Lookup } \\
\text { Values? }\end{array}$} & \multirow{2}{*}{$\begin{array}{c}\text { Does the } \\
\text { Statistical } \\
\text { Result } \\
\text { Pass } \\
\text { RESRAD } \\
\text { Modeling? }\end{array}$} \\
\hline & & $\begin{array}{l}\text { Shallow } \\
\text { Zone } \\
\text { Lookup } \\
\text { Value }^{b}\end{array}$ & $\begin{array}{l}\text { Groundwater } \\
\text { Protection } \\
\text { Lookup } \\
\text { Value }\end{array}$ & $\begin{array}{l}\text { River } \\
\text { Protection } \\
\text { Lookup } \\
\text { Value }\end{array}$ & & \\
\hline Cesium-137 & 0.709 & 6.2 & $1,465^{\mathrm{C}}$ & $1,465^{\mathrm{C}}$ & No & \multirow{4}{*}{ Yes $^{d}$} \\
\hline Cobalt-60 & 0.037 & 1.4 & $13,900^{c}$ & $13,900^{c}$ & No & \\
\hline Europium-152 & 0.140 & 3.3 & $-\mathrm{e}^{\mathrm{e}}$ & $-\mathrm{e}^{\mathrm{e}}$ & No & \\
\hline Strontium-90 & 0.268 & 4.5 & $27.6^{c}$ & $27.6^{c}$ & No & \\
\hline Uranium-233/234 & $0.83(<B G)$ & $1.1^{f}$ & $1.1^{f}$ & $1.1^{f}$ & No & -- \\
\hline Uranium-238 & $0.666(<B G)$ & $1.1^{f}$ & $1.1^{f}$ & $1.1^{f}$ & No & -- \\
\hline \multirow[b]{2}{*}{ COC/COPC } & \multirow[b]{2}{*}{$\begin{array}{c}\text { Statistical } \\
\text { Result } \\
\text { (mg/kg) }\end{array}$} & \multicolumn{3}{|c|}{ Remedial Action Goals ${ }^{\mathrm{a}}(\mathrm{mg} / \mathrm{kg})$} & \multirow[b]{2}{*}{$\begin{array}{l}\text { Does the } \\
\text { Statistical } \\
\text { Data Set } \\
\text { Exceed } \\
\text { RAGs? }\end{array}$} & \multirow[b]{2}{*}{$\begin{array}{c}\text { Does the } \\
\text { Statistical } \\
\text { Result } \\
\text { Pass } \\
\text { RESRAD } \\
\text { Modeling? }\end{array}$} \\
\hline & & $\begin{array}{c}\text { Direct } \\
\text { Exposure }\end{array}$ & $\begin{array}{l}\text { Soil Cleanup } \\
\text { Level for } \\
\text { Groundwater } \\
\text { Protection }\end{array}$ & $\begin{array}{l}\text { Soil } \\
\text { Cleanup } \\
\text { Level for } \\
\text { River } \\
\text { Protection }\end{array}$ & & \\
\hline Arsenic & $4.5(<B G)$ & 20 & 20 & 20 & No & -- \\
\hline Barium & $75.3(<\mathrm{BG})$ & $5,600^{g}$ & $132^{h, i}$ & $224^{j}$ & No & -- \\
\hline Beryllium & $0.64(<B G)$ & $10.4^{k}$ & $1.51^{i}$ & $1.51^{i}$ & No & -- \\
\hline Boron' & 2.7 & 16,000 & 320 & $--^{m}$ & No & - \\
\hline Cadmium $^{n}$ & $0.12(<B G)$ & 13.9 & $0.81^{i}$ & $0.81^{i}$ & No & -- \\
\hline Chromium (total) & $8.7(<B G)$ & $80,000^{9}$ & $18.5^{i}$ & $18.5^{\mathrm{i}}$ & No & -- \\
\hline Cobalt & $8.8(<B G)$ & 1,600 & 32 & $--^{m}$ & No & -- \\
\hline Copper & 38.6 & 2,960 & 59.2 & $22.0^{i}$ & Yes & $\mathrm{Yes}^{\circ}$ \\
\hline Lead & 18.6 & 353 & $10.2^{i}$ & $10.2^{i}$ & Yes & $\mathrm{Yes}^{\circ}$ \\
\hline Manganese & $349(<B G)$ & 11,200 & $512^{i}$ & $512^{i}$ & No & -- \\
\hline Molybdenum' & 4.5 & 400 & 8 & $--^{m}$ & No & -- \\
\hline Nickel & $12.0(<B G)$ & 1,600 & $19.1^{i}$ & 27.4 & No & -- \\
\hline Vanadium & $50.6(<B G)$ & 560 & $85.1^{i}$ & $--^{m}$ & No & -- \\
\hline Zinc & 74.8 & 24,000 & 480 & $67.8^{i}$ & Yes & $\mathrm{Yes}^{\circ}$ \\
\hline Aroclor-1254 & 0.054 & 0.5 & $0.017^{p}$ & $0.017^{p}$ & Yes & Yes $^{\circ}$ \\
\hline Benzo(a)anthracene & 0.18 & $1.37^{q}$ & $0.33^{p}$ & $0.33^{p}$ & No & -- \\
\hline Benzo(a)pyrene & 0.12 & $0.33^{p}$ & $0.33^{p}$ & $0.33^{p}$ & No & -- \\
\hline Benzo(b)fluoranthene & 0.14 & $1.37^{q}$ & $0.33^{p}$ & $0.33^{p}$ & No & -- \\
\hline Benzo(g,h,i)perylene ${ }^{r}$ & 0.051 & 2,400 & 48 & 192 & No & -- \\
\hline Benzo(k)fluoranthene & 0.14 & $13.7^{q}$ & $0.33^{p}$ & $0.33^{p}$ & No & -- \\
\hline bis(2-Ethylhexyl)phthalate & 0.22 & 71.4 & 0.625 & 0.36 & No & -- \\
\hline Butylbenzylphthalate & 0.038 & 16,000 & 320 & 250 & No & -- \\
\hline Chrysene & 0.21 & $137^{q}$ & $1.2^{q}$ & $0.33^{p}$ & No & -- \\
\hline
\end{tabular}




\section{Table 6. Comparison of Statistical Contaminant Concentrations to Action Levels for Verification Sampling of the 118-C-1 Sample Area 3. (2 Pages)}

\begin{tabular}{|c|c|c|c|c|c|c|}
\hline \multirow[b]{2}{*}{ COC/COPC } & \multirow[b]{2}{*}{$\begin{array}{c}\text { Statistical } \\
\text { Result } \\
(\mathrm{mg} / \mathrm{kg})\end{array}$} & \multicolumn{3}{|c|}{ Remedial Action Goals ${ }^{\mathrm{a}}(\mathrm{mg} / \mathrm{kg})$} & \multirow[b]{2}{*}{$\begin{array}{c}\text { Does the } \\
\text { Statistical } \\
\text { Data Set } \\
\text { Exceed } \\
\text { RAGs? }\end{array}$} & \multirow{2}{*}{$\begin{array}{c}\text { Does the } \\
\text { Statistical } \\
\text { Result } \\
\text { Pass } \\
\text { RESRAD } \\
\text { Modeling? }\end{array}$} \\
\hline & & $\begin{array}{c}\text { Direct } \\
\text { Exposure }\end{array}$ & $\begin{array}{l}\text { Soil Cleanup } \\
\text { Level for } \\
\text { Groundwater } \\
\text { Protection }\end{array}$ & $\begin{array}{l}\text { Soil } \\
\text { Cleanup } \\
\text { Level for } \\
\text { River } \\
\text { Protection }\end{array}$ & & \\
\hline Di-n-butylphthalate & 0.13 & 8,000 & 160 & 540 & No & -- \\
\hline Fluoranthene & 0.32 & 3,200 & 64 & 18 & No & -- \\
\hline Indeno(1,2,3-cd)pyrene & 0.046 & 1.37 & $0.33^{p}$ & $0.33^{p}$ & No & -- \\
\hline Phenanthrene $^{r}$ & 0.055 & 24,000 & 240 & 1,920 & No & -- \\
\hline Pyrene & 0.32 & 2,400 & 48 & 192 & No & -- \\
\hline Acetone & 0.011 & 72,000 & 720 & $--^{m}$ & No & -- \\
\hline Methylene chloride & 0.011 & 133 & 0.5 & 0.94 & No & -- \\
\hline
\end{tabular}

a Lookup values and remedial action goals (RAGs) obtained from the Remedial Design Report/Remedial Action Work Plan for the 100 Area (RDR/RAWP) (DOE-RL 2005) or calculated per Washington Administrative Code (WAC) 173-340-720, 730, and 740, Method B, 1996, unless otherwise noted.

b Activity corresponding to a single-radionuclide $15 \mathrm{mrem} / \mathrm{yr}$ exposure as calculated using a generic RESidual RADioactivity (RESRAD) model (DOE-RL 2005).

c Revised lookup value per 100 Area Radionuclide and Nonradionuclide Lookup Values for the 1995 Interim Remedial Action Record of Decision (BHI 2004).

d 118-C-1 Burial Ground Cleanup Verification RESRAD Calculation Brief (Appendix E).

e No value-RESRAD modeling predicts the contaminant will not reach groundwater within 1,000 years.

f The calculated lookup value is below the Hanford-specific statistical soil background activity. The value presented is the Hanford-specific statistical soil background activity.

g Noncarcinogenic cleanup level calculated from WAC 173-340-740(3), 1996 (Method B for soils) (as presented in the RDR/RAWP [DOE-RL 2005]). Updated oral reference dose values (as provided in the Integrated Risk Information System [IRIS]) yield Method B direct exposure RAG values of $16,000 \mathrm{mg} / \mathrm{kg}$ and $120,000 \mathrm{mg} / \mathrm{kg}$ for barium and chromium, respectively.

h Barium soil cleanup level for groundwater protection calculated from WAC 173-340-740(3)(a)(ii)(A), 1996 ("100 times rule") and WAC 173-340-720(3), 1996 (Method B for groundwater) is $112 \mathrm{mg} / \mathrm{kg}$ (as presented in the RDR/RAWP [DOE-RL 2005]). The updated oral reference dose value (as provided in IRIS) yields a Method B groundwater cleanup criteria of $7 \mathrm{mg} / \mathrm{L}$, as compared to the more restrictive maximum contaminant level of $2 \mathrm{mg} / \mathrm{L}$ (40 Code of Federal Regulations [CFR] 141). Per WAC 173-340-740(3)(a)(ii)(A), 1996 ("100 times rule"), the most restrictive updated soil cleanup level for groundwater protection would be $200 \mathrm{mg} / \mathrm{kg}$.

Where cleanup levels are less than background, cleanup levels default to background (WAC 173-340-700[4][d]) (1996).

i Barium soil cleanup level for river protection calculated from WAC 173-340-740(3)(a)(ii)(A), 1996 ("100 times rule"), a dilution-attenuation factor of 2, and WAC 173-340-720(3), 1996 (Method B for groundwater) is $224 \mathrm{mg} / \mathrm{kg}$ (as presented in the RDR/RAWP [DOE-RL 2005]). No surface water bioconcentration factor is available for barium and no ambient water quality criteria value exists separate from the previous drinking water standard; therefore no WAC 173-340-730(3), 1996 (Method B for surface waters) value can be determined.

k Carcinogenic cleanup level calculated based on the inhalation exposure pathway per WAC 173-340-750[3], 1996 (Method B for air quality) and an airborne particulate mass loading rate of $0.0001 \mathrm{~g} / \mathrm{m}^{3}$ (WDOH 1997).

1 No Hanford Site-specific or Washington State background value available.

m No parameters (bioconcentration factors or ambient water quality criteria values) are available from the Ecology Cleanup Levels and Risk Calculations database (Ecology 2005) or other databases to calculate cleanup levels (WAC 173-340-730(3)(a)(iii), 1996 [Method B for surface waters]).

n Hanford Site-specific background value is not available; not evaluated during background study. Value used is from Natural Background Soil Metals Concentrations in Washington State (Ecology 1994).

- Based on the 100 Area Analogous Sites RESRAD Calculations (BHI 2005) and contaminant-specific soil portioning coefficient (K $\mathrm{K}_{\mathrm{d}}$ ) value, contaminant will not migrate vertically more than $3 \mathrm{~m} \mathrm{(10} \mathrm{ft)} \mathrm{in} \mathrm{1,000} \mathrm{years.} \mathrm{As} \mathrm{the} \mathrm{vadose} \mathrm{zone} \mathrm{underlying} \mathrm{the} \mathrm{site} \mathrm{is} \mathrm{greater} \mathrm{than} 25 \mathrm{~m}$ $(82 \mathrm{ft})$ thick, the contaminant will not reach groundwater (and thus the Columbia River) in 1,000 years.

$\mathrm{p}$ Where cleanup levels are less than the required detection limit, cleanup levels default to the required detection limit (WAC 173-340707[2], 1996 and DOE-RL 2005).

q RAG value listed in the RDR/RAWP (DOE-RL 2005) is based on the use of benzo(a)pyrene as a surrogate. Compound-specific carcinogenic cleanup level calculated per WAC 173-340-740(3), 1996 (Method B for soils) using Oak Ridge National Laboratory oral cancer potency factors.

$r$ Toxicity data for this chemical are not available. RAGs for benzo(g,h,i)perylene and phenanthrene are based on the surrogate chemicals pyrene and anthracene, respectively.

= not applicable

BG = background (from DOE-RL [1996, 2001], unless otherwise noted)

$\mathrm{COC}=$ contaminant of concern

COPC = contaminant of potential concern

RAG = remedial action goal

RESRAD = RESidual RADioactivity 
Table 7. Comparison of Statistical Contaminant Concentrations to Action Levels for Verification Sampling of the 118-C-1 Sample Area 4. (2 Pages)

\begin{tabular}{|c|c|c|c|c|c|c|}
\hline \multirow[b]{2}{*}{ COC/COPC } & \multirow[b]{2}{*}{$\begin{array}{c}\text { Statistical } \\
\text { Result } \\
\text { (pCi/g) }\end{array}$} & \multicolumn{3}{|c|}{ 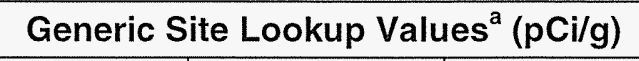 } & \multirow{2}{*}{$\begin{array}{l}\text { Does the } \\
\text { Statistical } \\
\text { Result } \\
\text { Exceed } \\
\text { Lookup } \\
\text { Values? }\end{array}$} & \multirow[b]{2}{*}{$\begin{array}{c}\text { Does the } \\
\text { Statistical } \\
\text { Result Pass } \\
\text { RESRAD } \\
\text { Modeling? }\end{array}$} \\
\hline & & $\begin{array}{l}\text { Shallow } \\
\text { Zone } \\
\text { Lookup } \\
\text { Value }^{b}\end{array}$ & $\begin{array}{c}\text { Groundwater } \\
\text { Protection } \\
\text { Lookup } \\
\text { Value }\end{array}$ & $\begin{array}{c}\text { River } \\
\text { Protection } \\
\text { Lookup } \\
\text { Value }\end{array}$ & & \\
\hline Cesium-137 & 0.81 & 6.2 & $1,465^{\mathrm{c}}$ & $1,465^{\mathrm{C}}$ & No & \multirow{2}{*}{$Y_{e s}^{d}$} \\
\hline Europium-152 & 0.114 & 3.3 & $-\mathrm{e}^{\mathrm{e}}$ & $-{ }^{e}$ & No & \\
\hline Uranium-233/234 & $0.479(<B G)$ & $1.1^{f}$ & $1.1^{f}$ & $1.1^{f}$ & No & -- \\
\hline Uranium-238 & $0.636(<B G)$ & $1.1^{f}$ & $1.1^{f}$ & $1.1^{f}$ & No & -- \\
\hline \multirow[b]{2}{*}{ COC/COPC } & \multirow[b]{2}{*}{$\begin{array}{c}\text { Statistical } \\
\text { Result } \\
\text { (mg/kg) }\end{array}$} & \multicolumn{3}{|c|}{ Remedial Action Goals ${ }^{a}$ (mg/kg) } & \multirow[b]{2}{*}{$\begin{array}{l}\text { Does the } \\
\text { Statistical } \\
\text { Data Set } \\
\text { Exceed } \\
\text { RAGs? }\end{array}$} & \multirow[b]{2}{*}{$\begin{array}{c}\text { Does the } \\
\text { Statistical } \\
\text { Result Pass } \\
\text { RESRAD } \\
\text { Modeling? }\end{array}$} \\
\hline & & $\begin{array}{c}\text { Direct } \\
\text { Exposure }\end{array}$ & $\begin{array}{l}\text { Soil Cleanup } \\
\text { Level for } \\
\text { Groundwater } \\
\text { Protection }\end{array}$ & $\begin{array}{l}\text { Soil } \\
\text { Cleanup } \\
\text { Level for } \\
\text { River } \\
\text { Protection }\end{array}$ & & \\
\hline Arsenic & $2.4(<B G)$ & 20 & 20 & 20 . & No & -- \\
\hline Barium & 206 & $5,600^{9}$ & $132^{h, i}$ & $224^{\mathrm{j}}$ & Yes & Yes $^{k}$ \\
\hline Beryllium & $0.58(<B G)$ & $10.4^{1}$ & $1.51^{i}$ & $1.51^{\mathrm{i}}$ & No & -- \\
\hline Boron $^{m}$ & 3.4 & 16,000 & 320 & $--^{n}$ & No & -- \\
\hline Chromium (total) & $6.5(<B G)$ & $80,000^{9}$ & $18.5^{i}$ & $18.5^{i}$ & No & -- \\
\hline Cobalt & $8.7(<B G)$ & 1,600 & 32 & $--^{n}$ & No & -- \\
\hline Copper & $15.0(<\mathrm{BG})$ & 2,960 & 59.2 & $22.0^{i}$ & No & -- \\
\hline Lead & $5.0(<B G)$ & 353 & $10.2^{i}$ & $10.2^{i}$ & No & -- \\
\hline Manganese & $340(<B G)$ & 11,200 & $512^{i}$ & $512^{i}$ & No & -- \\
\hline Nickel & $9.9(<B G)$ & 1,600 & $19.1^{i}$ & 27.4 & No & -- \\
\hline Vanadium & $51.6(<\mathrm{BG})$ & 560 & $85.1^{i}$ & $--^{n}$ & No & -- \\
\hline Zinc & 41.0 (<BG) & 24,000 & 480 & $67.8^{i}$ & No & -- \\
\hline Aroclor-1254 & 0.013 & 0.5 & $0.017^{\circ}$ & $0.017^{\circ}$ & No & -- \\
\hline Benzo(a)anthracene & 0.13 & $1.37^{p}$ & $0.33^{\circ}$ & $0.33^{\circ}$ & No & -- \\
\hline Benzo(a)pyrene & 0.083 & $0.33^{\circ}$ & $0.33^{\circ}$ & $0.33^{\circ}$ & No & -- \\
\hline Benzo(b)fluoranthene & 0.11 & $1.37^{\mathrm{p}}$ & $0.33^{\circ}$ & $0.33^{s}$ & No & -- \\
\hline Benzo(g,h,i)perylene ${ }^{q}$ & 0.037 & 2,400 & 48 & 192 & No & -- \\
\hline Benzo(k)fluoranthene & 0.092 & $13.7^{p}$ & $0.33^{\circ}$ & $0.33^{\circ}$ & No & -- \\
\hline $\begin{array}{l}\text { bis(2- } \\
\text { Ethylhexyl)phthalate }\end{array}$ & 0.017 & 71.4 & 0.625 & 0.36 & No & -- \\
\hline Chrysene & 0.17 & $137^{p}$ & $1.2^{p}$ & $0.33^{\circ}$ & No & -- \\
\hline Di-n-butylphthalate & 0.026 & 8,000 & 160 & 540 & No & - \\
\hline Fluoranthene & 0.33 & 3,200 & 64 & 18 & No & -- \\
\hline Indeno(1,2,3-cd)pyrene & 0.035 & 1.37 & $0.33^{\circ}$ & $0.33^{\circ}$ & No & -- \\
\hline
\end{tabular}


Table 7. Comparison of Statistical Contaminant Concentrations to Action Levels for Verification Sampling of the 118-C-1 Sample Area 4. (2 Pages)

\begin{tabular}{|c|c|c|c|c|c|c|}
\hline \multirow[b]{2}{*}{ COC/COPC } & \multirow[b]{2}{*}{$\begin{array}{c}\text { Statistical } \\
\text { Result } \\
\text { (mg/kg) }\end{array}$} & \multicolumn{3}{|c|}{ Remedial Action Goals ${ }^{a}(\mathrm{mg} / \mathrm{kg})$} & \multirow[b]{2}{*}{$\begin{array}{l}\text { Does the } \\
\text { Statistical } \\
\text { Data Set } \\
\text { Exceed } \\
\text { RAGs? }\end{array}$} & \multirow[b]{2}{*}{$\begin{array}{c}\text { Does the } \\
\text { Statistical } \\
\text { Result Pass } \\
\text { RESRAD } \\
\text { Modeling? }\end{array}$} \\
\hline & & $\begin{array}{c}\text { Direct } \\
\text { Exposure }\end{array}$ & $\begin{array}{l}\text { Soil Cleanup } \\
\text { Level for } \\
\text { Groundwater } \\
\text { Protection }\end{array}$ & $\begin{array}{c}\text { Soil } \\
\text { Cleanup } \\
\text { Level for } \\
\text { River } \\
\text { Protection }\end{array}$ & & \\
\hline Phenanthrene ${ }^{q}$ & 0.097 & 24,000 & 240 & 1,920 & No & -- \\
\hline Pyrene & 0.35 & 2,400 & 48 & 192 & No & -- \\
\hline Acetone & 0.012 & 72,000 & 720 & $--^{n}$ & No & -- \\
\hline Benzene & 0.001 & 18 & 0.080 & 0.24 & No & -- \\
\hline Methylene chloride & 0.014 & 133 & 0.5 & 0.94 & No & -- \\
\hline Xylene (total) & 0.001 & 16,000 & 160 & $--^{n}$ & No & - \\
\hline
\end{tabular}

a Lookup values and remedial action goals (RAGs) obtained from the Remedial Design Report/Remedial Action Work Plan for the 100 Area (RDR/RAWP) (DOE-RL 2005) or calculated per Washington Administrative Code (WAC) 173-340-720, 730, and 740, Method B, 1996, unless otherwise noted.

b Activity corresponding to a single-radionuclide $15 \mathrm{mrem} / \mathrm{yr}$ exposure as calculated using a generic RESidual RADioactivity (RESRAD) model (DOE-RL 2005).

c Revised lookup value per 100 Area Radionuclide and Nonradionuclide Lookup Values for the 1995 Interim Remedial Action Record of Decision (BHI 2004).

d 118-C-1 Burial Ground Cleanup Verification RESRAD Calculation Brief (Appendix E).

e No value-RESRAD modeling predicts the contaminant will not reach groundwater within 1,000 years.

f The calculated lookup value is below the Hanford-specific statistical soil background activity. The value presented is the Hanfordspecific statistical soil background activity.

9 Noncarcinogenic cleanup level calculated from WAC 173-340-740(3), 1996 (Method B for soils) (as presented in the RDR/RAWP [DOE-RL 2005]). Updated oral reference dose values (as provided in the Integrated Risk Information System [IRIS]) yield Method B direct exposure RAG values of $16,000 \mathrm{mg} / \mathrm{kg}$ and $120,000 \mathrm{mg} / \mathrm{kg}$ for barium and chromium, respectively.

h Barium soil cleanup level for groundwater protection calculated from WAC 173-340-740(3)(a)(ii)(A), 1996 ("100 times rule") and WAC 173-340-720(3), 1996 (Method B for groundwater) is $112 \mathrm{mg} / \mathrm{kg}$ (as presented in the RDR/RAWP [DOE-RL 2005]). The updated oral reference dose value (as provided in IRIS) yields a Method $B$ groundwater cleanup criteria of $7 \mathrm{mg} / \mathrm{L}$, as compared to the more restrictive maximum contaminant level of $2 \mathrm{mg} / \mathrm{L}$ (40 Code of Federal Regulations [CFR] 141). Per WAC 173-340-740(3)(a)(ii)(A), 1996 ("100 times rule"), the most restrictive updated soil cleanup level for groundwater protection would be $200 \mathrm{mg} / \mathrm{kg}$.

i Where cleanup levels are less than background, cleanup levels default to background (WAC 173-340-700[4][d]) (1996).

I Barium soil cleanup level for river protection calculated from WAC 173-340-740(3)(a)(ii)(A), 1996 ("100 times rule"), a dilutionattenuation factor of 2, and WAC 173-340-720(3), 1996 (Method B for groundwater) is $224 \mathrm{mg} / \mathrm{kg}$ (as presented in the RDR/RAWP [DOE-RL 2005]). No surface water bioconcentration factor is available for barium and no ambient water quality criteria value exists separate from the previous drinking water standard; therefore no WAC 173-340-730(3), 1996 (Method B for surface waters) value can be determined.

k Based on the 100 Area Analogous Sites RESRAD Calculations (BHI 2005) and contaminant-specific soil portioning coefficient (K $\left.\mathrm{K}_{\mathrm{d}}\right)$ value, contaminant will not migrate vertically more than $3 \mathrm{~m}$ (10 ft) in 1,000 years. As the vadose zone underlying the site is greater than $25 \mathrm{~m}$ (82 ft) thick, the contaminant will not reach groundwater (and thus the Columbia River) in 1,000 years.

1 Carcinogenic cleanup level calculated based on the inhalation exposure pathway per WAC 173-340-750[3], 1996 (Method B for air quality) and an airborne particulate mass loading rate of $0.0001 \mathrm{~g} / \mathrm{m}^{3}$ (WDOH 1997).

$m$ No Hanford Site-specific or Washington State background value available.

n No parameters (bioconcentration factors or ambient water quality criteria values) are available from the Ecology Cleanup Levels and Risk Calculations database (Ecology 2005) or other databases to calculate cleanup levels (WAC 173-340-730(3)(a)(iii), 1996 [Method B for surface waters]).

- Where cleanup levels are less than the required detection limit, cleanup levels default to the required detection limit (WAC 173-340707[2], 1996 and DOE-RL 2005).

p RAG value listed in the RDR/RAWP (DOE-RL 2005) is based on the use of benzo(a)pyrene as a surrogate. Compound-specific carcinogenic cleanup level calculated per WAC 173-340-740(3), 1996 (Method B for soils) using Oak Ridge National Laboratory oral cancer potency factors.

q Toxicity data for this chemical are not available. RAGs for benzo(g,h,i)perylene and phenanthrene are based on the surrogate chemicals pyrene and anthracene, respectively.
- $\quad$ = not applicable
BG = background (from DOE-RL [1996, 2001], unless otherwise noted)
COPC = contaminant of potential concern
COC $=$ contaminant of concern
RAG = remedial action goal
RESRAD = RESidual RADioactivity 
Table 8. Comparison of Statistical Contaminant Concentrations to Action Levels for Verification Sampling of the 118-C-1 Sample Overburden/BCL Material. (2 Pages)

\begin{tabular}{|c|c|c|c|c|c|c|}
\hline \multirow[b]{2}{*}{ COC/COPC } & \multirow[b]{2}{*}{$\begin{array}{c}\text { Statistical } \\
\text { Result } \\
\text { (pci/g) }\end{array}$} & \multicolumn{3}{|c|}{ 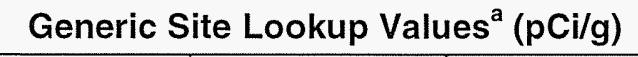 } & \multirow{2}{*}{$\begin{array}{l}\text { Does the } \\
\text { Statistical } \\
\text { Result } \\
\text { Exceed } \\
\text { Lookup } \\
\text { Values? }\end{array}$} & \multirow[b]{2}{*}{$\begin{array}{l}\text { Does the } \\
\text { Statistical } \\
\text { Result Pass } \\
\text { RESRAD } \\
\text { Modeling? }\end{array}$} \\
\hline & & $\begin{array}{l}\text { Shallow } \\
\text { Zone } \\
\text { Lookup } \\
\text { Value }^{b}\end{array}$ & $\begin{array}{l}\text { Groundwater } \\
\text { Protection } \\
\text { Lookup } \\
\text { Value }\end{array}$ & $\begin{array}{l}\text { River } \\
\text { Protection } \\
\text { Lookup } \\
\text { Value }\end{array}$ & & \\
\hline Cesium-137 & $0.352(<B G)$ & 6.2 & $1,465^{\mathrm{C}}$ & $1,465^{\mathrm{c}}$ & No & -- \\
\hline Strontium-90 & 1.41 & 4.5 & $27.6^{\mathrm{c}}$ & $27.6^{c}$ & No & $Y_{e s}^{d}$ \\
\hline Uranium-233/234 & $0.523(<\mathrm{BG})$ & $1.1^{\mathrm{e}}$ & $1.1^{\mathrm{e}}$ & $1.1^{\mathrm{e}}$ & No & -- \\
\hline Uranium-238 & $0.505(<B G)$ & $1.1^{\mathrm{e}}$ & $1.1^{\mathrm{e}}$ & $1.1^{\mathrm{e}}$ & No & -- \\
\hline \multirow[b]{2}{*}{ COC/COPC } & \multirow[b]{2}{*}{$\begin{array}{l}\text { Statistical } \\
\text { Result } \\
(\mathrm{mg} / \mathrm{kg})\end{array}$} & \multicolumn{3}{|c|}{ Remedial Action Goals ${ }^{a}(\mathrm{mg} / \mathrm{kg})$} & \multirow[b]{2}{*}{$\begin{array}{l}\text { Does the } \\
\text { Statistical } \\
\text { Data Set } \\
\text { Exceed } \\
\text { RAGs? }\end{array}$} & \multirow[b]{2}{*}{$\begin{array}{c}\text { Does the } \\
\text { Statistical } \\
\text { Result Pass } \\
\text { RESRAD } \\
\text { Modeling? }\end{array}$} \\
\hline & & $\begin{array}{c}\text { Direct } \\
\text { Exposure }\end{array}$ & $\begin{array}{l}\text { Soil Cleanup } \\
\text { Level for } \\
\text { Groundwater } \\
\text { Protection }\end{array}$ & $\begin{array}{c}\text { Soil } \\
\text { Cleanup } \\
\text { Level for } \\
\text { River } \\
\text { Protection }\end{array}$ & & \\
\hline Arsenic & $2.4(<B G)$ & 20 & 20 & 20 & No & -- \\
\hline Barium & $55.2(<B G)$ & $5,600^{f}$ & $132^{g, h}$ & $224^{i}$ & No & -- \\
\hline Beryllium & $0.68(<\mathrm{BG})$ & $10.4^{\mathrm{i}}$ & $1.51^{n}$ & $1.51^{h}$ & No & -- \\
\hline Chromium (total) & $6.7(<B G)$ & $80,000^{f}$ & $18.5^{h}$ & $18.5^{h}$ & No & -- \\
\hline Chromium (hexavalent) & 0.24 & 2.1 & $4.8^{k}$ & 2 & No & -- \\
\hline Cobalt & $7.7(<B G)$ & 1,600 & 32 & -1 & No & -- \\
\hline Copper & $15.4(<B G)$ & 2,960 & 59.2 & $22.0^{h}$ & No & -- \\
\hline Lead & $4.9(<B G)$ & 353 & $10.2^{h}$ & $10.2^{h}$ & No & -- \\
\hline Manganese & $325(<B G)$ & 11,200 & $512^{h}$ & $512^{h}$ & No & -- \\
\hline Nickel & $9.5(<B G)$ & 1,600 & $19.1^{h}$ & 27.4 & No & -- \\
\hline Vanadium & $44.7(<B G)$ & 560 & $85.1^{h}$ & -1 & No & -- \\
\hline Zinc & $37.5(<B G)$ & 24,000 & 480 & $67.8^{h}$ & No & -- \\
\hline Aroclor-1254 & 0.0065 & 0.5 & $0.017^{\mathrm{m}}$ & $0.017^{\mathrm{m}}$ & No & - \\
\hline Benzo(a)anthracene & 0.038 & $1.37^{n}$ & $0.33^{m}$ & $0.33^{m}$ & No & -- \\
\hline Benzo(a)pyrene & 0.024 & $0.33^{m}$ & $0.33^{m}$ & $0.33^{m}$ & No & -- \\
\hline Benzo(b)fluoranthene & 0.033 & $1.37^{n}$ & $0.33^{m}$ & $0.33^{m}$ & No & -- \\
\hline Benzo(k)fluoranthene & 0.029 & $13.7^{n}$ & $0.33^{m}$ & $0.33^{m}$ & No & -- \\
\hline Chrysene & 0.052 & $137^{n}$ & $1.2^{n}$ & $0.33^{m}$ & No & -- \\
\hline Fluoranthene & 0.11 & 3,200 & 64 & 18 & No & -- \\
\hline Phenanthrene $^{\circ}$ & 0.037 & 24,000 & 240 & 1,920 & No & -. \\
\hline Pyrene & 0.057 & 2,400 & 48 & 192 & No & -- \\
\hline Acetone & 0.011 & 72,000 & 720 & -1 & No & -- \\
\hline Methylene chloride & 0.012 & 133 & 0.5 & 0.94 & No & -- \\
\hline
\end{tabular}




\section{Table 8. Comparison of Statistical Contaminant Concentrations to Action Levels for Verification Sampling of the 118-C-1 Sample Overburden/BCL Material. (2 Pages)}

a Lookup values and remedial action goals (RAGs) obtained from the Remedial Design Report/Remedial Action Work Plan for the 100 Area (RDR/RAWP) (DOE-RL 2005) or calculated per Washington Administrative Code (WAC) 173-340-720, 730, and 740, Method B, 1996, unless otherwise noted.

b Activity corresponding to a single-radionuclide $15 \mathrm{mrem} / \mathrm{yr}$ exposure as calculated using a generic RESidual RADioactivity (RESRAD) model (DOE-RL 2005).

c Revised lookup value per 100 Area Radionuclide and Nonradionuclide Lookup Values for the 1995 Interim Remedial Action Record of Decision (BHI 2004).

d 118-C-1 Burial Ground Cleanup Verification RESRAD Calculation Brief (Appendix E).

a The calculated lookup value is below the Hanford-specific statistical soil background activity. The value presented is the Hanfordspecific statistical soil background activity.

f Noncarcinogenic cleanup level calculated from WAC 173-340-740(3), 1996 (Method B for soils) (as presented in the RDR/RAWP [DOE-RL 2005]). Updated oral reference dose values (as provided in the Integrated Risk Information System [IRIS]) yield Method B direct exposure RAG values of $16,000 \mathrm{mg} / \mathrm{kg}$ and $120,000 \mathrm{mg} / \mathrm{kg}$ for barium and chromium, respectively.

9 Barium soil cleanup level for groundwater protection calculated from WAC 173-340-740(3)(a)(ii)(A), 1996 ("100 times rule") and WAC 173-340-720(3), 1996 (Method B for groundwater) is $112 \mathrm{mg} / \mathrm{kg}$ (as presented in the RDR/RAWP [DOE-RL 2005]). The updated oral reference dose value (as provided in IRIS) yields a Method $B$ groundwater cleanup criteria of $7 \mathrm{mg} / \mathrm{L}$, as compared to the more restrictive maximum contaminant level of $2 \mathrm{mg} / \mathrm{L}$ (40 Code of Federal Regulations [CFR] 141). Per WAC 173-340-740(3)(a)(ii)(A), 1996 ("100 times rule"), the most restrictive updated soil cleanup level for groundwater protection would be $200 \mathrm{mg} / \mathrm{kg}$.

h Where cleanup levels are less than background, cleanup levels default to background (WAC 173-340-700[4][d]) (1996).

i Barium soil cleanup level for river protection calculated from WAC 173-340-740(3)(a)(ii)(A), 1996 ("100 times rule"), a dilutionattenuation factor of 2, and WAC 173-340-720(3), 1996 (Method B for groundwater) is $224 \mathrm{mg} / \mathrm{kg}$ (as presented in the RDR/RAWP [DOE-RL 2005]). No surface water bioconcentration factor is available for barium and no ambient water quality criteria value exists separate from the previous drinking water standard; therefore no WAC 173-340-730(3), 1996 (Method B for surface waters) value can be determined.

i Carcinogenic cleanup level calculated based on the inhalation exposure pathway per WAC 173-340-750[3], 1996 (Method B for air quality) and an airborne particulate mass loading rate of $0.0001 \mathrm{~g} / \mathrm{m}^{3}$ (WDOH 1997).

k Calculated cleanup level (per WAC 173-340-720(3), 1996 [Method B for groundwater] and WAC 173-340-740(3)(a)(ii)(A), 1996 [“100 times rule"]) presented is lower than that presented in the RDR/RAWP (DOE-RL 2005), based on updated oral reference dose value (as provided in IRIS).

1 No parameters (bioconcentration factors or ambient water quality criteria values) are available from the Ecology Cleanup Levels and Risk Calculations database (Ecology 2005) or other databases to calculate cleanup levels (WAC 173-340-730(3)(a)(iii), 1996 [Method B for surface waters]).

$m$ Where cleanup levels are less than the required detection limit, cleanup levels default to the required detection limit (WAC 173-340707[2], 1996 and DOE-RL 2005).

n RAG value listed in the RDR/RAWP (DOE-RL 2005) is based on the use of benzo(a)pyrene as a surrogate. Compound-specific carcinogenic cleanup level calculated per WAC 173-340-740(3), 1996 (Method B for soils) using Oak Ridge National Laboratory oral cancer potency factors.

- Toxicity data for this chemical are not available. RAGs for benzo(g,h,i)perylene and phenanthrene are based on the surrogate chemicals pyrene and anthracene, respectively.

= not applicable

BG = background (obtained from DOE-RL [1996] and DOE-RL [2001], unless otherwise noted)

$\mathrm{COC}=$ contaminant of concern

COPC = contaminant of potential concern

RAG = remedial action goal

RESRAD = RESidual RADioactivity (dose-assessment model) 
Table 9. Comparison of Statistical Contaminant Concentrations to Action Levels for Verification Sampling of the 118-C-1 Staging Pile Area. (3 Pages)

\begin{tabular}{|c|c|c|c|c|c|c|}
\hline \multirow[b]{2}{*}{$\mathrm{COC} / \mathrm{COPC}$} & \multirow[b]{2}{*}{$\begin{array}{l}\text { Statistical } \\
\text { Result } \\
\text { (pCi/g) }\end{array}$} & \multicolumn{3}{|c|}{ Generic Site Lookup Values ${ }^{a}(\mathrm{pCi} / \mathrm{g})$} & \multirow{2}{*}{$\begin{array}{l}\text { Does the } \\
\text { Statistical } \\
\text { Result } \\
\text { Exceed } \\
\text { Lookup } \\
\text { Values? }\end{array}$} & \multirow[b]{2}{*}{$\begin{array}{c}\text { Does the } \\
\text { Statistical } \\
\text { Result Pass } \\
\text { RESRAD } \\
\text { Modeling? }\end{array}$} \\
\hline & & $\begin{array}{l}\text { Shallow } \\
\text { Zone } \\
\text { Lookup } \\
\text { Value }^{b}\end{array}$ & $\begin{array}{l}\text { Groundwater } \\
\text { Protection } \\
\text { Lookup } \\
\text { Value }\end{array}$ & $\begin{array}{l}\text { River } \\
\text { Protection } \\
\text { Lookup } \\
\text { Value }\end{array}$ & & \\
\hline Carbon-14 & $182^{c}$ & 5.16 & $-{ }^{8}$ & $--^{e}$ & Yes & \multirow{5}{*}{ Yes $^{d}$} \\
\hline Cesium-137 & 1.03 & 6.2 & $1,465^{\dagger}$ & $1,465^{f}$ & No & \\
\hline Cobalt-60 & 0.029 & 1.4 & $13,900^{f}$ & $13,900^{f}$ & No & \\
\hline Europium-152 & 0.177 & 3.3 & $-\mathrm{e}^{\mathrm{e}}$ & $-{ }^{e}$ & No & \\
\hline Strontium-90 & 0.334 & 4.5 & $27.6^{f}$ & $27.6^{f}$ & No & \\
\hline Uranium-233/234 & $0.489(<B G)$ & $1.1^{\mathrm{g}}$ & $1.1^{9}$ & $1.1^{\mathrm{g}}$ & No & -- \\
\hline Uranium-235 & $0.038(<B G)$ & 0.84 & $0.5^{\mathrm{h}}$ & $0.5^{\mathrm{h}}$ & No & -- \\
\hline Uranium-238 & $0.573(<B G)$ & $1.1^{\mathrm{g}}$ & $1.1^{\mathrm{g}}$ & $1.1^{\mathrm{g}}$ & No & -- \\
\hline \multirow[b]{2}{*}{$\mathrm{COC} / \mathrm{COPC}$} & \multirow[b]{2}{*}{$\begin{array}{c}\text { Statistical } \\
\text { Result } \\
(\mathrm{mg} / \mathrm{kg})\end{array}$} & \multicolumn{3}{|c|}{ Remedial Action Goals ${ }^{a}(\mathrm{mg} / \mathrm{kg})$} & \multirow[b]{2}{*}{$\begin{array}{l}\text { Does the } \\
\text { Statistical } \\
\text { Data Set } \\
\text { Exceed } \\
\text { RAGs? }\end{array}$} & \multirow[b]{2}{*}{$\begin{array}{c}\text { Does the } \\
\text { Statistical } \\
\text { Result Pass } \\
\text { RESRAD } \\
\text { Modeling? }\end{array}$} \\
\hline & & $\begin{array}{c}\text { Direct } \\
\text { Exposure }\end{array}$ & $\begin{array}{l}\text { Soil Cleanup } \\
\text { Level for } \\
\text { Groundwater } \\
\text { Protection }\end{array}$ & $\begin{array}{c}\text { Soil } \\
\text { Cleanup } \\
\text { Level for } \\
\text { River } \\
\text { Protection }\end{array}$ & & \\
\hline Arsenic & $2.8(<B G)$ & 20 & 20 & 20 & No & -- \\
\hline Barium & $130(<B G)$ & $5,600^{i}$ & $132^{\mathrm{j}, \mathrm{k}}$ & $224^{\prime}$ & Yes $^{m}$ & Yes $^{n}$ \\
\hline Beryllium & $0.43(<\mathrm{BG})$ & $10.4^{\circ}$ & $1.51^{k}$ & $1.51^{k}$ & No & -- \\
\hline Boron $^{\mathrm{p}}$ & 2.8 & 16,000 & 320 & $--^{9}$ & No & -- \\
\hline Chromium (total) & $8.2(<B G)$ & $80,000^{i}$ & $18.5^{k}$ & $18.5^{\mathrm{k}}$ & No & -- \\
\hline Chromium (hexavalent) & 0.5 & 2.1 & $4.8^{r}$ & 2 & No & - \\
\hline Cobalt & $7.0(<B G)$ & 1,600 & 32 & $\ldots 9$ & No & -- \\
\hline Copper & 36 & 2,960 & 59.2 & $22.0^{k}$ & Yes & Yes $^{n}$ \\
\hline Lead & 10.7 & 353 & $10.2^{k}$ & $10.2^{k}$ & Yes & Yes $^{n}$ \\
\hline Manganese & $323(<B G)$ & 11,200 & $512^{k}$ & $512^{k}$ & No & -- \\
\hline Mercury & $0.06(<B G)$ & 24 & $0.33^{k}$ & $0.33^{k}$ & No & -- \\
\hline Molybdenum ${ }^{p}$ & 0.62 & 400 & 8 & $--^{9}$ & No & -- \\
\hline Nickel & $10.6(<B G)$ & 1,600 & $19.1^{k}$ & 27.4 & No & -- \\
\hline Selenium $^{\text {s }}$ & 0.80 & 400 & 5 & 1 & No & -. \\
\hline Vanadium & $46.6(<B G)$ & 560 & $85.1^{k}$ & $--^{9}$ & No & -- \\
\hline Zinc & 46.1 (<BG) & 24,000 & 480 & $67.8^{k}$ & No & -- \\
\hline $\begin{array}{l}\text { Total petroleum } \\
\text { hydrocarbons }\end{array}$ & 146 & -- & $200^{t}$ & $200^{t}$ & No & -- \\
\hline Aroclor-1254 & 0.015 & 0.5 & $0.017^{\mathrm{u}}$ & $0.017^{\mathrm{u}}$ & $Y_{e s}{ }^{m}$ & $Y_{e s^{n}}$ \\
\hline Aroclor-1260 & 0.061 & 0.5 & $0.017^{\mathrm{u}}$ & $0.017^{\mathrm{u}}$ & Yes & $Y_{e s}^{n}$ \\
\hline
\end{tabular}


Table 9. Comparison of Statistical Contaminant Concentrations to Action Levels for Verification Sampling of the 118-C-1 Staging Pile Area. (3 Pages)

\begin{tabular}{|c|c|c|c|c|c|c|}
\hline \multirow[b]{2}{*}{ COC/COPC } & \multirow[b]{2}{*}{$\begin{array}{c}\text { Statistical } \\
\text { Result } \\
(\mathrm{mg} / \mathrm{kg})\end{array}$} & \multicolumn{3}{|c|}{ Remedial Action Goals ${ }^{\mathrm{a}}(\mathrm{mg} / \mathrm{kg})$} & \multirow[b]{2}{*}{$\begin{array}{l}\text { Does the } \\
\text { Statistical } \\
\text { Data Set } \\
\text { Exceed } \\
\text { RAGs? }\end{array}$} & \multirow[b]{2}{*}{$\begin{array}{c}\text { Does the } \\
\text { Statistical } \\
\text { Result Pass } \\
\text { RESRAD } \\
\text { Modeling? }\end{array}$} \\
\hline & & $\begin{array}{l}\text { Direct } \\
\text { Exposure }\end{array}$ & $\begin{array}{l}\text { Soil Cleanup } \\
\text { Level for } \\
\text { Groundwater } \\
\text { Protection }\end{array}$ & $\begin{array}{c}\text { Soil } \\
\text { Cleanup } \\
\text { Level for } \\
\text { River } \\
\text { Protection }\end{array}$ & & \\
\hline Acenaphthene & 0.041 & 4,800 & 96 & 129 & No & -- \\
\hline Anthracene & 0.26 & 24,000 & 240 & 1,920 & No & -- \\
\hline Benzo(a)anthracene & 0.5 & $1.37^{v}$ & $0.33^{\mathrm{u}}$ & $0.33^{\mathrm{u}}$ & $\mathrm{No}^{\mathrm{w}}$ & -- \\
\hline Benzo(a)pyrene & 0.48 & $0.33^{\mathrm{u}}$ & $0.33^{\mathrm{u}}$ & $0.33^{\mathrm{u}}$ & $\mathrm{No}^{\mathrm{w}}$ & -- \\
\hline Benzo(b)fluoranthene & 0.21 & $1.37^{v}$ & $0.33^{\mathrm{u}}$ & $0.33^{\mathrm{u}}$ & $\mathrm{No}^{\mathrm{w}}$ & -- \\
\hline Benzo(g,h,i)perylene ${ }^{x}$ & 0.26 & 2,400 & 48 & 192 & No & -- \\
\hline Benzo(k)fluoranthene & 0.20 & $13.7^{v}$ & $0.33^{\mathrm{u}}$ & $0.33^{u}$ & Now & -- \\
\hline $\begin{array}{l}\text { bis(2- } \\
\text { Ethylhexyl)phthalate }\end{array}$ & 0.052 & 71.4 & 0.625 & 0.36 & No & -- \\
\hline Butylbenzylphthalate & 0.017 & 16,000 & 320 & 250 & No & -- \\
\hline Carbazole & 0.24 & 50 & 0.438 & $-{ }^{9}$ & No & -- \\
\hline Chrysene & 0.4 & $137^{v}$ & $1.2^{v}$ & $0.33^{\mathrm{u}}$ & Now & -- \\
\hline Di-n-butylphthalate & 0.11 & 8,000 & 160 & 540 & No & -- \\
\hline Dibenz(a,h)anthracene & 0.18 & $0.33^{\mathrm{u}}$ & $0.33^{\mathrm{u}}$ & $0.33^{u}$ & No & -- \\
\hline Dibenzofuran & 0.034 & 160 & 3.2 & $--^{9}$ & No & -- \\
\hline Fluoranthene $^{y}$ & 0.19 & 3,200 & 64 & 18 & No & -- \\
\hline Fluorene & 0.043 & 3,200 & 64 & 260 & No & -- \\
\hline Indeno(1,2,3-cd)pyrene & 0.25 & 1.37 & $0.33^{\mathrm{u}}$ & $0.33^{u}$ & No & -- \\
\hline Phenanthrene $^{x}$ & 1.3 & 24,000 & 240 & 1,920 & No & -- \\
\hline Phenol & 0.024 & 24,000 & 480 & 4,200 & No & -- \\
\hline Pyrene & 0.5 & 2,400 & 48 & 192 & No & -- \\
\hline 2-Butanone & 0.004 & 48,000 & 480 & $--^{9}$ & No & -- \\
\hline Acetone & 0.015 & 72,000 & 720 & $--^{9}$ & No & -- \\
\hline Methylene chloride & 0.023 & 133 & 0.5 & 0.94 & No & -- \\
\hline Styrene & 0.004 & 33.3 & 0.15 & $--^{9}$ & No & -- \\
\hline Toluene & 0.002 & 6,400 & 64 & 1,360 & No & -- \\
\hline Xylene (total) & 0.001 & 16,000 & 160 & $--^{9}$ & No & -- \\
\hline
\end{tabular}




\section{Table 9. Comparison of Statistical Contaminant Concentrations to Action Levels for Verification Sampling of the 118-C-1 Staging Pile Area. (3 Pages)}

a Lookup values and remedial action goals (RAGs) obtained from the Remedial Design Report/Remedial Action Work Plan for the 100 Area (RDR/RAWP) (DOE-RL 2005) or calculated per Washington Administrative Code (WAC) 173-340-720, 730, and 740, Method B, 1996, unless otherwise noted.

b Activity corresponding to a single-radionuclide $15 \mathrm{mrem} / \mathrm{yr}$ exposure as calculated using a generic RESidual RADioactivity (RESRAD) model (DOE-RL 2005).

c Carbon-14 value presented is the result for a single sample from the staging pile area; this isotope was not detected in other samples collected at the staging pile area. Initial dose evaluation indicated that including this result in the statistical data set for the entire staging pile area would result in an elevated dose estimate. Material at this location will be backfilled into deep zone portions of the site, and this sample was omitted from statistical calculations for the staging pile area and a sample-specific evaluation was performed using RESRAD (Appendix E).

d 118-C-1 Burial Ground Cleanup Verification RESRAD Calculation Brief (Appendix E).

e No value-RESRAD modeling predicts the contaminant will not reach groundwater within 1,000 years.

(Revised lookup value per 100 Area Radionuclide and Nonradionuclide Lookup Values for the 1995 Interim Remedial Action Record of Decision (BHI 2004).

$g$ The calculated lookup value is below the Hanford-specific statistical soil background activity. The value presented is the Hanfordspecific statistical soil background activity.

$\mathrm{h}$ The lookup value is below the required minimum detectable activity (MDA). The value presented is the required MDA.

i Noncarcinogenic cleanup level calculated from WAC 173-340-740(3), 1996 (Method B for soils) (as presented in the RDR/RAWP [DOE-RL 2005]). Updated oral reference dose values (as provided in the Integrated Risk Information System [IRIS]) yield Method B direct exposure RAG values of $16,000 \mathrm{mg} / \mathrm{kg}$ and $120,000 \mathrm{mg} / \mathrm{kg}$ for barium and chromium, respectively.

i Barium soil cleanup level for groundwater protection calculated from WAC 173-340-740(3)(a)(ii)(A), 1996 ("100 times rule") and WAC 173-340-720(3), 1996 (Method B for groundwater) is $112 \mathrm{mg} / \mathrm{kg}$ (as presented in the RDR/RAWP [DOE-RL 2005]). The updated oral reference dose value (as provided in IRIS) yields a Method B groundwater cleanup criteria of $7 \mathrm{mg} / \mathrm{L}$, as compared to the more restrictive maximum contaminant level of $2 \mathrm{mg} / \mathrm{L}$ (40 Code of Federal Regulations [CFR] 141). Per WAC 173-340-740(3)(a)(ii)(A), 1996 ("100 times rule"), the most restrictive updated soil cleanup level for groundwater protection would be $200 \mathrm{mg} / \mathrm{kg}$.

$k$ Where cleanup levels are less than background, cleanup levels default to background (WAC 173-340-700[4][d]) (1996).

'Barium soil cleanup level for river protection calculated from WAC 173-340-740(3)(a)(ii)(A), 1996 ("100 times rule"), a dilutionattenuation factor of 2, and WAC 173-340-720(3), 1996 (Method B for groundwater) is $224 \mathrm{mg} / \mathrm{kg}$ (as presented in the RDR/RAWP [DOE-RL 2005]). No surface water bioconcentration factor is available for barium and no ambient water quality criteria value exists separate from the previous drinking water standard; therefore no WAC 173-340-730(3), 1996 (Method B for surface waters) value can be determined.

$m$ The statistical verification data set fails the second and/or third component of the WAC 173-340-740(7)(e) 3-part test in comparison against soil RAGs for groundwater and/or river protection (one or more sample concentrations exceeds twice the soil RAG value and/or more than $10 \%$ of the data set exceeds the soil RAG value).

n Based on the 100 Area Analogous Sites RESRAD Calculations (BHI 2005) and contaminant-specific soil portioning coefficient ( $\mathrm{K}_{\mathrm{d}}$ ) value, contaminant will not migrate vertically more than $3 \mathrm{~m}(10 \mathrm{ft})$ in 1,000 years. As the vadose zone underlying the site is greater than $25 \mathrm{~m}$ ( $82 \mathrm{ft}$ ) thick, the contaminant will not reach groundwater (and thus the Columbia River) in 1,000 years.

- Carcinogenic cleanup level calculated based on the inhalation exposure pathway per WAC 173-340-750[3], 1996 (Method B for air quality) and an airborne particulate mass loading rate of $0.0001 \mathrm{~g} / \mathrm{m}^{3}$ (WDOH 1997).

$p$ No Hanford Site-specific or Washington State background value available.

q No parameters (bioconcentration factors or ambient water quality criteria values) are available from the Ecology Cleanup Levels and Risk Calculations database (Ecology 2005) or other databases to calculate cleanup levels (WAC 173-340-730(3)(a)(iii), 1996 [Method B for surface waters]).

' Calculated cleanup level (per WAC 173-340-720(3), 1996 [Method B for groundwater] and WAC 173-340-740(3)(a)(ii)(A), 1996 ["100 times rule"]) presented is lower than that presented in the RDR/RAWP (DOE-RL 2005), based on updated oral reference dose value (as provided in IRIS).

$s$ Hanford Site-specific background value is not available; not evaluated during background study. Value used is from Natural Background Soil Metals Concentrations in Washington State (Ecology 1994).

1 RAG values for total petroleum hydrocarbons obtained from WAC 173-340-740(2), 1996 (Method A for soils).

"Where cleanup levels are less than the required detection limit, cleanup levels default to the required detection limit (WAC 173-340707[2], 1996 and DOE-RL 2005).

$\checkmark$ RAG value listed in the RDR/RAWP (DOE-RL 2005) is based on the use of benzo(a)pyrene as a surrogate. Compound-specific carcinogenic cleanup level calculated per WAC 173-340-740(3), 1996 (Method B for soils) using Oak Ridge National Laboratory oral cancer potency factors.

w Constituent is the result of asphalt cross-contamination of the sample matrix. Asphalt that has been used for structural and construction purposes is excluded from consideration as a dangerous waste by WAC 173-303-071(3)(e), 2004, is listed as an inert waste in WAC 173-350-990(2)(b), 2005, and does not present a significant human health risk.

$x$ Toxicity data for this chemical are not available. RAGs for benzo(g,h,i)perylene and phenanthrene are based on the surrogate chemicals pyrene and anthracene, respectively.

y A portion of the fluoranthene data for the staging pile area was rejected based on data quality deficiencies; the value presented is the maximum detected accepted value. Because polycyclic aromatic hydrocarbons in the staging pile area were determined to be the result of asphalt cross-contamination of the sample matrix, and because fluoranthene is not a significant risk-driver as compared to other polycyclic aromatic hydrocarbons, no further analysis was performed.

$\quad=$ not applicable

BG = background (from DOE-RL [1996, 2001], unless otherwise noted)

$\mathrm{COC}=$ contaminant of concern
COPC = contaminant of potential concern
RAG = remedial action goal
RESRAD = RESidual RADioactivity


Several polycyclic aromatic hydrocarbons (PAHs) were detected above the RAGs in statistical samples collected from the waste staging pile area (Table 9). The PAHs were determined to be the result of asphalt cross-contamination. The elevated detections of PAHs within the staging pile area occurred at locations formerly overlain by asphalt paving. Because the area previously contained several paved surfaces and was used as a location for temporary structures and staging during construction of the 105-C Reactor Building, the likelihood for the presence of asphalt debris is high. Asphalt that has been used for structural and construction purposes is excluded from consideration as a dangerous waste in WAC 173-303-071(3)(e), is listed as an inert waste in WAC 173-350-990(2)(b), and does not present a significant risk to human health or the environment. A comparison of the statistical PAH concentrations in the verification data set to a known asphalt sample (Table 10) shows a good correlation, as indicated by the ratio column.

Table 10. Comparison of 118-C-1 Staging Pile Area
Statistical Results to a Known Asphalt Sample

\begin{tabular}{|l|c|c|c|}
\hline \multicolumn{1}{|c|}{ Analyte } & $\begin{array}{c}\text { Asphalt } \\
\text { Sample Result } \\
\text { (mg/kg) }\end{array}$ & $\begin{array}{c}\text { Statistical } \\
\text { Verification } \\
\text { Result } \\
(\mathbf{m g} / \mathbf{k g})\end{array}$ & $\begin{array}{c}\text { Ratio } \\
\left(\mathbf{x ~ 1 0} \mathbf{~}^{-4}\right)\end{array}$ \\
\hline 2-Methylnaphthalene & 394 & $\mathrm{ND}$ & -- \\
\hline Acenaphthene & 1,783 & 0.041 & 2.3 \\
\hline Anthracene & 3,699 & 0.26 & 7.0 \\
\hline Benzo(a)anthracene & 5,792 & 0.5 & 8.6 \\
\hline Benzo(a)pyrene & 5,533 & 0.48 & 8.7 \\
\hline Benzo(b)fluoranthene & 4,619 & 0.21 & 4.5 \\
\hline Benzo(g,h,i)perylene & 2,839 & 0.26 & 9.2 \\
\hline Benzo(k)fluoranthene & 4,527 & 0.20 & 4.4 \\
\hline Carbazole & 2,049 & 0.24 & 11.7 \\
\hline Chrysene & 5,580 & 0.4 & 7.2 \\
\hline Dibenzo(a,h)anthracene & 1,531 & 0.18 & 11.8 \\
\hline Dibenzofuran & 1,135 & 0.034 & 3.0 \\
\hline Fluoranthene ${ }^{a}$ & 10,665 & 0.19 & 1.8 \\
\hline Fluorene & 1,756 & 0.043 & 2.4 \\
\hline Indeno(1,2,3-cd) pyrene & 2,751 & 0.25 & 9.1 \\
\hline Naphthalene & 1,917 & $\mathrm{ND}$ & -- \\
\hline Phenanthrene & 10,975 & 1.3 & 11.8 \\
\hline Pyrene & 10,205 & 0.5 & 4.9 \\
\hline Nanet & & & \\
\hline
\end{tabular}

$\mathrm{ND}=$ not detected

${ }^{a}$ A portion of the fluoranthene data for the staging pile area was rejected due to data quality deficiencies, including the portion where the highest detections of other polycyclic aromatic hydrocarbons occurred. The value presented is the maximum accepted detected value, but is low relative to other polycyclic aromatic hydrocarbon results due to the exclusion of some data. 


\subsection{BIASED SAMPLE RESULTS}

Twelve biased samples were collected from the 118-C-1 waste site. The biased samples were collected from soil underlying anomalous media found within the trenches during the remedial excavations or from soil underlying the SNF interim storage bunkers in the waste staging pile area. Statistical analysis (e.g., calculation of a $95 \% \mathrm{UCL}$ value) is inappropriate for evaluation of biased samples; therefore, the sample results for each biased sample are evaluated using the maximum detected activity for each $\mathrm{COC/COPC}$ and comparing the value directly to the cleanup level. Table 11 provides a comparison of the maximum result of the twelve biased samples against the cleanup criteria. Individual sample results are provided in Appendix A.

Table 11. Comparison of Maximum Biased Sample Concentrations to Cleanup Levels. (2 Pages)

\begin{tabular}{|c|c|c|c|c|c|c|}
\hline \multirow[b]{2}{*}{ COC/COPC } & \multirow[b]{2}{*}{$\begin{array}{l}\text { Maximum } \\
\text { Result } \\
(\mathrm{pCi} / \mathrm{g})\end{array}$} & \multicolumn{3}{|c|}{ Generic Site Lookup Values ${ }^{a}(\mathrm{pCi} / \mathrm{g})$} & \multirow{2}{*}{$\begin{array}{c}\text { Does the } \\
\text { Statistical } \\
\text { Result } \\
\text { Exceed } \\
\text { Lookup } \\
\text { Values? }\end{array}$} & \multirow{2}{*}{$\begin{array}{c}\text { Does the } \\
\text { Statistical } \\
\text { Result } \\
\text { Pass } \\
\text { RESRAD } \\
\text { Modeling? }\end{array}$} \\
\hline & & $\begin{array}{l}\text { Shallow } \\
\text { Zone } \\
\text { Lookup } \\
\text { Value }^{\mathrm{b}}\end{array}$ & $\begin{array}{c}\text { Groundwater } \\
\text { Protection } \\
\text { Lookup } \\
\text { Value }\end{array}$ & $\begin{array}{l}\text { River } \\
\text { Protection } \\
\text { Lookup } \\
\text { Value }\end{array}$ & & \\
\hline Cesium-137 & 0.437 & 6.2 & $1,465^{c}$ & $1,465^{c}$ & No & -- \\
\hline Plutonium-239/240 & 0.204 & 33.9 & $--^{d}$ & $--^{d}$ & No & -- \\
\hline Uranium-233/234 & $0.681(<B G)$ & $1.1^{e}$ & $1.1^{\mathrm{e}}$ & $1.1^{\mathrm{e}}$ & No & -- \\
\hline Uranium-238 & $0.483(<B G)$ & $1.1^{\mathrm{e}}$ & $1.1^{e}$ & $1.1^{e}$ & No & -- \\
\hline \multirow[b]{2}{*}{ COC/COPC } & \multirow[b]{2}{*}{$\begin{array}{c}\text { Maximum } \\
\text { Result } \\
(\mathrm{mg} / \mathrm{kg})\end{array}$} & \multicolumn{3}{|c|}{ Remedial Action Goals ${ }^{\mathrm{a}}(\mathrm{mg} / \mathrm{kg})$} & \multirow[b]{2}{*}{$\begin{array}{c}\text { Does the } \\
\text { Maximum } \\
\text { Result } \\
\text { Exceed } \\
\text { RAGs? }\end{array}$} & \multirow{2}{*}{$\begin{array}{c}\text { Does the } \\
\text { Maximum } \\
\text { Result } \\
\text { Pass } \\
\text { RESRAD } \\
\text { Modeling? }\end{array}$} \\
\hline & & $\begin{array}{c}\text { Direct } \\
\text { Exposure }\end{array}$ & $\begin{array}{c}\text { Soil Cleanup } \\
\text { Level for } \\
\text { Groundwater } \\
\text { Protection }\end{array}$ & $\begin{array}{c}\text { Soil Cleanup } \\
\text { Level for } \\
\text { River } \\
\text { Protection }\end{array}$ & & \\
\hline Arsenic & $5.4(<B G)$ & 20 & 20 & 20 & No & -- \\
\hline Barium & $80.2(<B G)$ & $5,600^{f}$ & $132^{g, h}$ & $224^{i}$ & No & - \\
\hline Beryllium & $0.34(<\mathrm{BG})$ & $10.4^{\mathrm{j}}$ & $1.51^{h}$ & $1.51^{h}$ & No & -- \\
\hline Boron $^{k}$ & 3.2 & 16,000 & 320 & -1 & No & -- \\
\hline Chromium (total) & $10.2(<B G)$ & $80,000^{f}$ & $18.5^{h}$ & $18.5^{h}$ & No & -- \\
\hline Cobalt & $10.5(<\mathrm{BG})$ & 1,600 & 32 & -1 & No & -- \\
\hline Copper & 23.3 & 2,960 & 59.2 & $22.0^{h}$ & Yes & Yes $^{m}$ \\
\hline Lead & $7.2(<\mathrm{BG})$ & 353 & $10.2^{h}$ & $10.2^{h}$ & No & -- \\
\hline Manganese & $446(<B G)$ & 11,200 & $512^{h}$ & $512^{h}$ & No & -- \\
\hline Mercury & $0.04(<B G)$ & 24 & $0.33^{h}$ & $0.33^{h}$ & No & - \\
\hline Nickel & $14.1(<B G)$ & 1,600 & $19.1^{h}$ & 27.4 & No & -- \\
\hline Vanadium & $46.4(<B G)$ & 560 & $85.1^{\mathrm{h}}$ & -1 & No & -- \\
\hline Zinc & $45.1(<B G)$ & 24,000 & 480 & $67.8^{\text {h }}$ & No & -- \\
\hline Aroclor-1254 & 0.022 & 0.5 & $0.017^{n}$ & $0.017^{n}$ & Yes & $Y_{e s}^{m}$ \\
\hline
\end{tabular}




\section{Table 11. Comparison of Maximum Biased Sample Concentrations to Cleanup Levels. (2 Pages)}

\begin{tabular}{|c|c|c|c|c|c|c|}
\hline \multirow[b]{2}{*}{ COC/COPC } & \multirow[b]{2}{*}{$\begin{array}{l}\text { Maximum } \\
\text { Result } \\
(\mathrm{mg} / \mathrm{kg})\end{array}$} & \multicolumn{3}{|c|}{ Remedial Action Goals ${ }^{\mathrm{a}}(\mathrm{mg} / \mathrm{kg})$} & \multirow[b]{2}{*}{$\begin{array}{l}\text { Does the } \\
\text { Maximum } \\
\text { Result } \\
\text { Exceed } \\
\text { RAGs? }\end{array}$} & \multirow{2}{*}{$\begin{array}{c}\text { Does the } \\
\text { Maximum } \\
\text { Result } \\
\text { Pass } \\
\text { RESRAD } \\
\text { Modeling? }\end{array}$} \\
\hline & & $\begin{array}{c}\text { Direct } \\
\text { Exposure }\end{array}$ & $\begin{array}{l}\text { Soil Cleanup } \\
\text { Level for } \\
\text { Groundwater } \\
\text { Protection }\end{array}$ & $\begin{array}{l}\text { Soil Cleanup } \\
\text { Level for } \\
\text { River } \\
\text { Protection }\end{array}$ & & \\
\hline $\begin{array}{l}\text { bis(2- } \\
\text { Ethylhexyl)phthalate }\end{array}$ & 0.067 & 71.4 & 0.625 & 0.36 & No & -- \\
\hline Di-n-butylphthalate & 0.055 & 8,000 & 160 & 540 & No & -- \\
\hline Diethylphthalate & 0.022 & 64,000 & 1,280 & 4,600 & No & -- \\
\hline Phenol & 0.036 & 24,000 & 480 & 4,200 & No & -- \\
\hline Acetone & 0.024 & 72,000 & 720 & --1 & No & -- \\
\hline Carbon tetrachloride & 0.039 & 7.7 & 0.034 & 0.05 & Yes & Yes $^{\circ}$ \\
\hline Methylene chloride & 0.019 & 133 & 0.5 & 0.94 & No & -- \\
\hline
\end{tabular}

a Lookup values and remedial action goals (RAGs) obtained from the Remedial Design Report/Remedial Action Work Plan for the 100 Area (RDR/RAWP) (DOE-RL 2005) or calculated per Washington Administrative Code (WAC) 173-340-720, 730, and 740, Method B, 1996, unless otherwise noted.

b Activity corresponding to a single-radionuclide $15 \mathrm{mrem} / \mathrm{yr}$ exposure as calculated using a generic RESidual RADioactivity (RESRAD) model (DOE-RL 2005).

c Revised lookup value per 100 Area Radionuclide and Nonradionuclide Lookup Values for the 1995 Interim Remedial Action Record of Decision (BHI 2004).

d No value-RESRAD modeling predicts the contaminant will not reach groundwater within 1,000 years.

e The calculated lookup value is below the Hanford-specific statistical soil background activity. The value presented is the Hanfordspecific statistical soil background activity.

f Noncarcinogenic cleanup level calculated from WAC 173-340-740(3), 1996 (Method B for soils) (as presented in the RDR/RAWP [DOE-RL 2005]). Updated oral reference dose values (as provided in the Integrated Risk Information System [IRIS]) yield Method B direct exposure RAG values of $16,000 \mathrm{mg} / \mathrm{kg}$ and $120,000 \mathrm{mg} / \mathrm{kg}$ for barium and chromium, respectively.

9 Barium soil cleanup level for groundwater protection calculated from WAC 173-340-740(3)(a)(ii)(A), 1996 ("100 times rule") and WAC 173-340-720(3), 1996 (Method B for groundwater) is $112 \mathrm{mg} / \mathrm{kg}$ (as presented in the RDR/RAWP [DOE-RL 2005]). The updated oral reference dose value (as provided in IRIS) yields a Method B groundwater cleanup criteria of $7 \mathrm{mg} / \mathrm{L}$, as compared to the more restrictive maximum contaminant level of $2 \mathrm{mg} / \mathrm{L}$ (40 Code of Federal Regulations [CFR] 141). Per WAC 173-340-740(3)(a)(ii)(A), 1996 ("100 times rule"), the most restrictive updated soil cleanup level for groundwater protection would be $200 \mathrm{mg} / \mathrm{kg}$.

h Where cleanup levels are less than background, cleanup levels default to background (WAC 173-340-700[4][d]) (1996).

'Barium soil cleanup level for river protection calculated from WAC 173-340-740(3)(a)(ii)(A), 1996 ("100 times rule"), a dilutionattenuation factor of 2, and WAC 173-340-720(3), 1996 (Method B for groundwater) is $224 \mathrm{mg} / \mathrm{kg}$ (as presented in the RDR/RAWP [DOE-RL 2005]). No surface water bioconcentration factor is available for barium and no ambient water quality criteria value exists separate from the previous drinking water standard; therefore no WAC 173-340-730(3), 1996 (Method B for surface waters) value can be determined.

i Carcinogenic cleanup level calculated based on the inhalation exposure pathway per WAC 173-340-750[3], 1996 (Method B for air quality) and an airborne particulate mass loading rate of $0.0001 \mathrm{~g} / \mathrm{m}^{3}$ (WDOH 1997).

k No Hanford Site-specific or Washington State background value available.

1 No parameters (bioconcentration factors or ambient water quality criteria values) are available from the Ecology Cleanup Levels and Risk Calculations database (Ecology 2005) or other databases to calculate cleanup levels (WAC 173-340-730(3)(a)(iii), 1996 [Method B for surface waters]).

m Based on the 100 Area Analogous Sites RESRAD Calculations (BHI 2005) and contaminant-specific soil portioning coefficient ( $\left.\mathrm{K}_{\mathrm{d}}\right)$

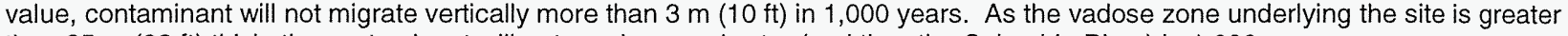
than $25 \mathrm{~m}$ (82 ft) thick, the contaminant will not reach groundwater (and thus the Columbia River) in 1,000 years.

$n$ Where cleanup levels are less than the required detection limit, cleanup levels default to the required detection limit (WAC 173-340707[2], 1996 and DOE-RL 2005).

- The total mass of carbon tetrachloride present is not expected to be sufficient to migrate to groundwater and result in a concentration exceeding the WAC 173-340-720(3), 1996 (Method B for groundwater) carcinogenic groundwater cleanup level (0.34 $\mu \mathrm{g} / \mathrm{L})$.

$--\quad=$ not applicable

BG = background (obtained from DOE-RL [1996] and DOE-RL [2001], unless otherwise noted)

COC = contaminant of concern

$\mathrm{COPC}=$ contaminant of potential concern

RAG = remedial action goal

RESRAD = RESidual RADioactivity (dose-assessment model) 


\subsubsection{Biased Sample Data Evaluation}

All biased sample results are below the direct exposure RAGs. The maximum values detected in the biased samples for copper and aroclor-1254 exceed the soil RAG for the protection of groundwater and/or river protection (Table 11). Copper and aroclor-1254 are not predicted to reach groundwater based on RESRAD modeling using their soil partitioning coefficients (BHI 2005). Data were not collected showing the vertical extent of residual contamination; however, given the soil-partitioning coefficients of $22 \mathrm{~mL} / \mathrm{g}$ and $75.6 \mathrm{~mL} / \mathrm{g}$ for copper and aroclor-1254, respectively, the RESRAD modeling predicts these constituents will not migrate vertically more than $3 \mathrm{~m}(10 \mathrm{ft})$ in 1,000

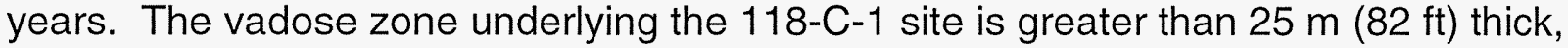
so these contaminants are not predicted to reach groundwater (and therefore the Columbia River) in 1,000 years.

The maximum value detected in the biased samples for carbon tetrachloride $(0.039 \mathrm{mg} / \mathrm{kg})$ exceeds the soil RAG for the protection of groundwater $(0.034 \mathrm{mg} / \mathrm{kg})$. However, because the maximum concentration represents a single sample at a point source, the total mass of carbon tetrachloride present at the site is not expected to be sufficient to migrate to groundwater and result in a concentration exceeding the groundwater cleanup level of $0.34 \mu \mathrm{g} / \mathrm{L}$. As such, the nonradionuclide soil RAGs for the protection of groundwater and the Columbia River have been attained for the biased samples.

\subsection{RESRAD MODELING}

The individual radionuclide cleanup verification statistical values (Tables 4 through 9) were entered into the RESRAD computer code, Version 6.30 (ANL 2005), to estimate the dose rate and the impact on groundwater and the river from residual $\mathrm{COC}$ concentrations. Separate RESRAD runs were performed for the four individual sampling areas in addition to the overburden/BCL decision unit and the waste staging pile area. A RESRAD run was also performed using the maximum $95 \% \mathrm{UCL}$ values from the four individual remediation sampling areas in order to provide a combined dose for the entire remediation footprint. To be conservative, the combined dose for the four individual sampling areas (referred to collectively as the remediation footprint) will be presented in Section 5.0. The individual dose contributions from each of the four sampling areas are provided in the RESRAD calculation brief (Appendix E).

Carbon-14 was detected in a single verification sample collected from the waste staging pile area (Table 9). The soil containing carbon-14 will be used as backfill into the deep zone areas of the 118-C-1 waste site, therefore, the contaminated soil was evaluated in RESRAD as deep zone material (with $4.6 \mathrm{~m}[15 \mathrm{ft}]$ of clean soil cover). All RESRAD modeling methodologies, results, and input values are included in the RESRAD calculation brief (Appendix E). The RESRAD derived groundwater radionuclide concentrations as compared to groundwater remedial action goals and maximum contaminant levels (MCLs) are provided in the MCL calculation brief (Appendix E). 
Specific results from the calculations are discussed in the RAG evaluation section (Section 5.0).

\subsection{EVALUATION OF REMEDIAL ACTION GOAL ATTAINMENT}

This section demonstrates that remedial actions at the 118-C-1 site have achieved the applicable RAGs. Sections 5.1, 5.2, and 5.3 address attainment of direct exposure RAGs, groundwater protection RAGs, and Columbia River protection RAGs, respectively. Section 5.4 summarizes the results of the WAC 173-340 three-part test performed in the $95 \%$ UCL calculation brief (Appendix E). This test is required for nonradionuclide COCs only and is based on the most restrictive RAG (defined as the lowest of the direct exposure, groundwater protection, and river protection RAGs).

\subsection{DIRECT EXPOSURE SOIL REMEDIAL ACTION GOALS ATTAINED}

\subsubsection{Radionuclides}

The results of the 118-C-1 RESRAD dose rate estimates for the all-pathways scenarios for the remediation footprint (sampling areas 1 through 4), the overburden/BCL, and the staging pile area are shown in Figures 5 through 7 . These dose rates represent the dose contributions from soils at relevant time periods. The 2018 date corresponds to the 30-year site cleanup schedule of the Hanford Federal Facility Agreement and Consent Order (Ecology et al. 1989). All dose rate estimates are less than the 15 $\mathrm{mrem} / \mathrm{yr}$ RAG. The RESRAD computations are shown in detail in the calculation briefs presented in Appendix E.

5.1.1.1 118-C-1 Remediation Footprint. The estimated maximum total all-pathways dose rate from the 118-C-1 Burial Ground remediation footprint is $12.8 \mathrm{mrem} / \mathrm{yr}$ at year zero (2006) and decreases to $1.01 \times 10^{-4} \mathrm{mrem} / \mathrm{yr}$ in 1,000 years (Figure 5). The estimated dose rate in the year 2018 is $3.89 \mathrm{mrem} / \mathrm{yr}$. The all-pathways dose rate estimate remains below the direct exposure RAG. 
Figure 5. RESRAD Analysis for the Remediation Footprint- All Radionuclides, All-Pathways Dose Rate Estimate.

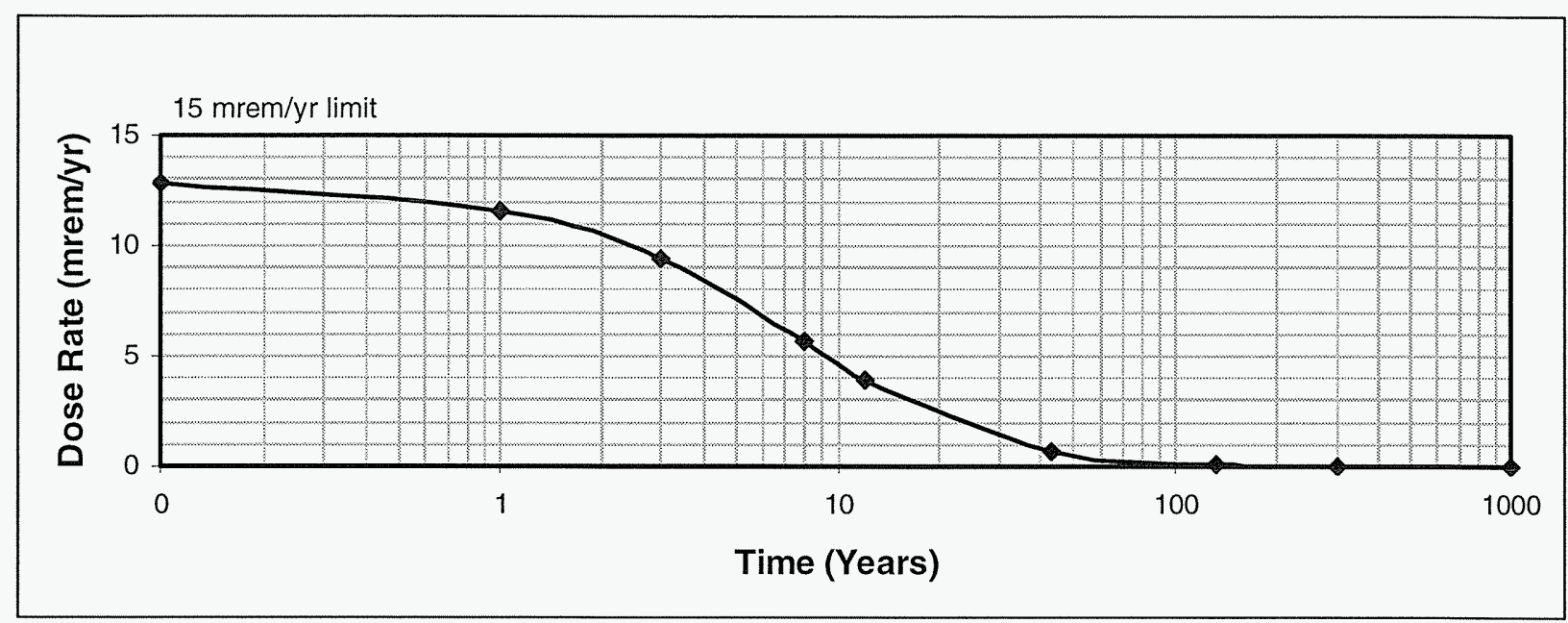

5.1.1.2 118-C-1 Overburden/BCL Soil. The estimated maximum total all-pathways dose rate from the 118-C-1 Burial Ground overburden/BCL soil is $4.01 \mathrm{mrem} / \mathrm{yr}$ at year zero (2006) and decreases to $2.47 \times 10^{-10} \mathrm{mrem} / \mathrm{yr}$ in 1,000 years (Figure 6). The estimated dose rate in the year 2018 is $3.04 \mathrm{mrem} / \mathrm{yr}$. The all-pathways dose rate estimate remains below the direct exposure RAG.

Figure 6. RESRAD Analysis for the Overburden/BCL soil- All Radionuclides, All-Pathways Dose Rate Estimate.

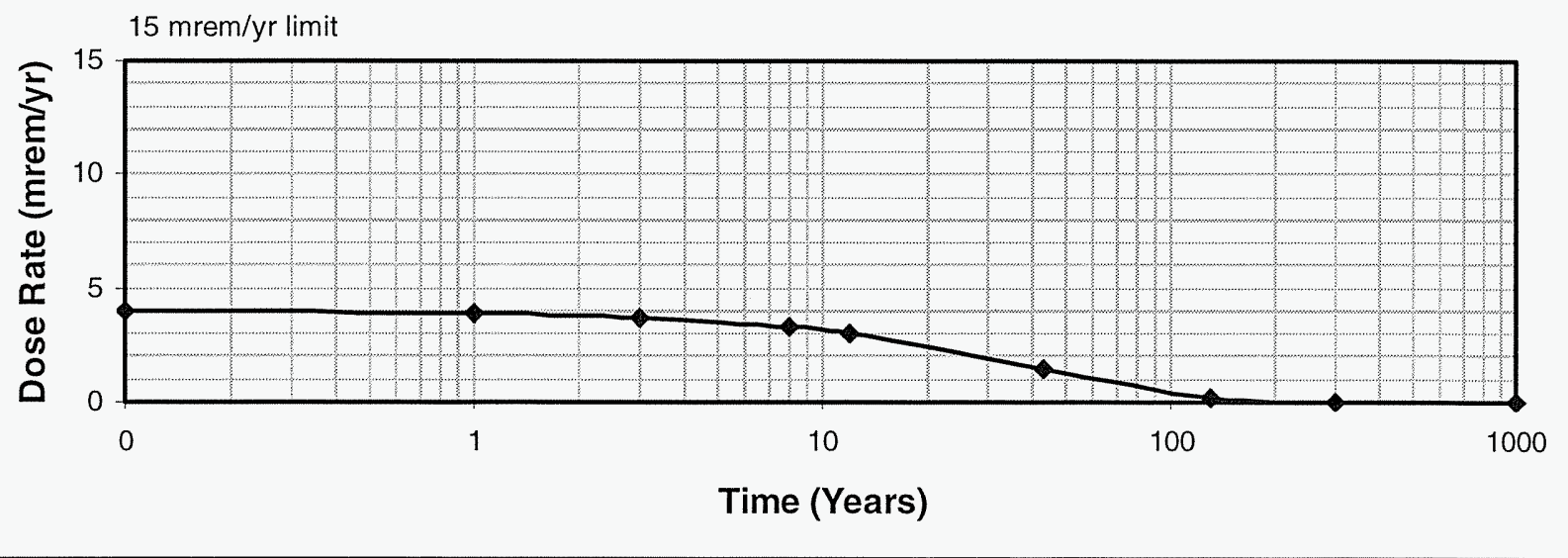


5.1.1.3 118-C-1 Waste Staging Pile Area. The estimated maximum total all-pathways dose rate from the 118-C-1 Burial Ground waste staging pile area is $12.4 \mathrm{mrem} / \mathrm{yr}$ at year zero (2006) and decreases to $2.42 \times 10^{-10} \mathrm{mrem} / \mathrm{yr}$ in 1,000 years (Figure 7 ). The estimated dose rate in the year 2018 is $7.38 \mathrm{mrem} / \mathrm{yr}$. The all-pathways dose rate estimate remains below the direct exposure RAG.

Figure 7. RESRAD Analysis for the Waste Staging Pile Area- All Radionuclides, All-Pathways Dose Rate Estimate.

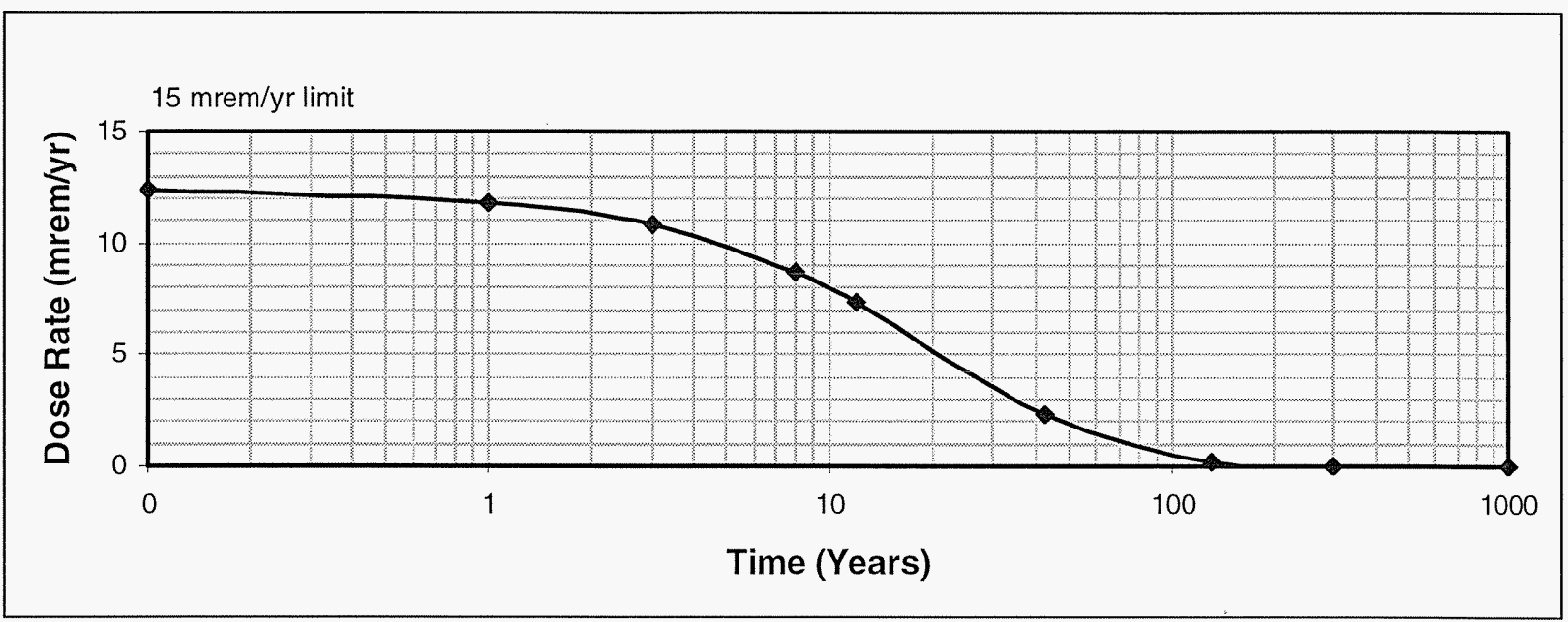

\subsubsection{Nonradionuclides}

5.1.2.1 Direct Comparison to RAGs. Tables 4 through 9 compare the cleanup verification statistical values to the direct exposure RAGs. All nonradionuclides are below the direct exposure cleanup criteria and, therefore, all applicable RAGs are met.

5.1.2.2 Noncarcinogenic Hazard Quotient RAG Attained. For noncarcinogenic COCs, WAC 173-340-745(a) and (b) specify the evaluation of the hazard quotient which is given as a daily intake divided by a reference dose. Both individual and cumulative hazard quotient values must be less than 1.0. Hazard quotient calculations were performed for the 118-C-1 Burial Ground using the highest statistical value from all sampling areas or individual detections in the biased samples (locations 1 through 7).

The details and results of the hazard quotient calculations are provided in the hazard quotient and carcinogenic risk calculation brief (Appendix E). Results of the hazard quotient calculations show that all individual hazard quotients are less than one and the cumulative hazard quotient for the 118-C-1 waste site is $1.6 \times 10^{-1}$ (Appendix E). Therefore, the noncarcinogenic hazard quotient RAG has been attained for the 118-C-1 waste site. 
5.1.2.3 Carcinogenic Risk RAG Attained. For individual nonradionuclide carcinogenic COCs, the WAC 173-340 Method B cleanup limits are based on an incremental cancer risk of $1 \times 10^{-6}$. For nonradionuclide carcinogenic COCs, the total excess cancer risk must be less than $1 \times 10^{-5}$. Carcinogenic risk calculations were performed for the 118-C-1 Burial Ground using the highest statistical value from all sampling areas or individual detections in the biased samples (locations 1 through 7 ) for the nonradionuclide carcinogenic COCs. The details and results of the hazard quotient calculations are provided in the hazard quotient and carcinogenic risk calculation brief (Appendix E).

Results of the carcinogenic risk calculations show that all individual nonradionuclide carcinogenic COCs have an incremental cancer risk below $1 \times 10^{-6}$ (Appendix $\mathrm{E}$ ). The calculated total excess cancer risk for the $118-\mathrm{C}-1$ waste site is $1.6 \times 10^{-6}$, which is below the RAO of $1 \times 10^{-5}$. The nonradionuclide carcinogenic risk RAG has been attained for the 118-C-1 waste site.

\subsection{GROUNDWATER REMEDIAL ACTION GOALS ATTAINED}

\subsubsection{Radionuclides}

The estimated groundwater concentrations for all of the radionuclide COCs contributed by the site soils are shown in the RESRAD calculation brief (Appendix E). Among the radionuclide contaminants of concern, only cesium-137, cobalt-60, nickel-63, strontium-90, and tritium are calculated to reach groundwater in 1,000 years at concentrations significantly below the RAGs. Table 12 gives the total peak concentrations predicted for each radionuclide COC along with the individual RAGs for comparison. The peak concentration for each $\mathrm{COC}$ is the maximum value, as predicted by RESRAD modeling, from the sampling areas (i.e., remediation footprint, overburden/BCL, and waste staging pile area). No COC concentration is predicted to exceed the RAGs; therefore, the RAOs are attained.

Figure 8 shows the individual organ dose rate for beta- and gamma-emitting radionuclides predicted over 1,000 years as shown in the comparison to drinking water standards calculation brief ( $M C L$ calculation brief in Appendix E). The cumulative dose for each total body, bone, liver, and gastrointestinal tract for all beta- and gammaemitting COCs is less than the $4 \mathrm{mrem} / \mathrm{yr}$ standard over 1,000 years. Results of the RESRAD calculations are shown in the MCL calculation brief (Appendix E).

No non-uranium alpha-emitting radionuclide COCs/COPCs were detected in the verification soil samples and no uranium isotopes were detected above background levels in the verification soil samples. Therefore, the drinking water concentration standard for non-uranium alpha emitters and the uranium standard of $30 \mu \mathrm{g} / \mathrm{L}$ have been met. 
Table 12. Estimated Peak Radionuclide Groundwater Concentrations in the Soils for 118-C-1.

\begin{tabular}{|l|c|c|c|}
\hline \multicolumn{1}{|c|}{ Radionuclide } & $\begin{array}{c}\text { Peak Concentration } \\
(\mathrm{pCi} / \mathrm{L})^{\mathrm{a}}\end{array}$ & $\begin{array}{c}\text { RAG } \\
(\mathrm{pCi} / \mathrm{L})\end{array}$ & $\begin{array}{c}\text { RAGs Attained? } \\
\text { (Yes/No) }\end{array}$ \\
\hline Cobalt-60 & 0.00712 & $100^{\mathrm{b}}$ & Yes \\
\hline Cesium-137 & 0.0290 & $60^{\mathrm{b}}$ & Yes \\
\hline Nickel-63 & 15.7 & $50^{\mathrm{b}}$ & Yes \\
\hline Strontium-90 & 0.357 & $8^{\mathrm{c}}$ & Yes \\
\hline Tritium & 5,430 & $20,000^{\mathrm{c}}$ & Yes \\
\hline
\end{tabular}

a The peak concentration is the maximum predicted value from the three sampling areas (remediation footprint, overburden/BCL, and waste staging pile area). All results are presented in the $\mathrm{MCL}$ calculation brief (Appendix E).

${ }^{b}$ Lookup value corresponding to a dose rate of $4 \mathrm{mrem} / \mathrm{yr}$.

c EPA drinking water promulgated MCL (40 CFR 141.66).

CFR = Code of Federal Regulations

EPA $=$ U.S. Environmental Protection Agency

$\mathrm{MCL} \quad=$ maximum contaminant level

RAG = remedial action goal

Figure 8. Dose Rate to Organs from Groundwater.

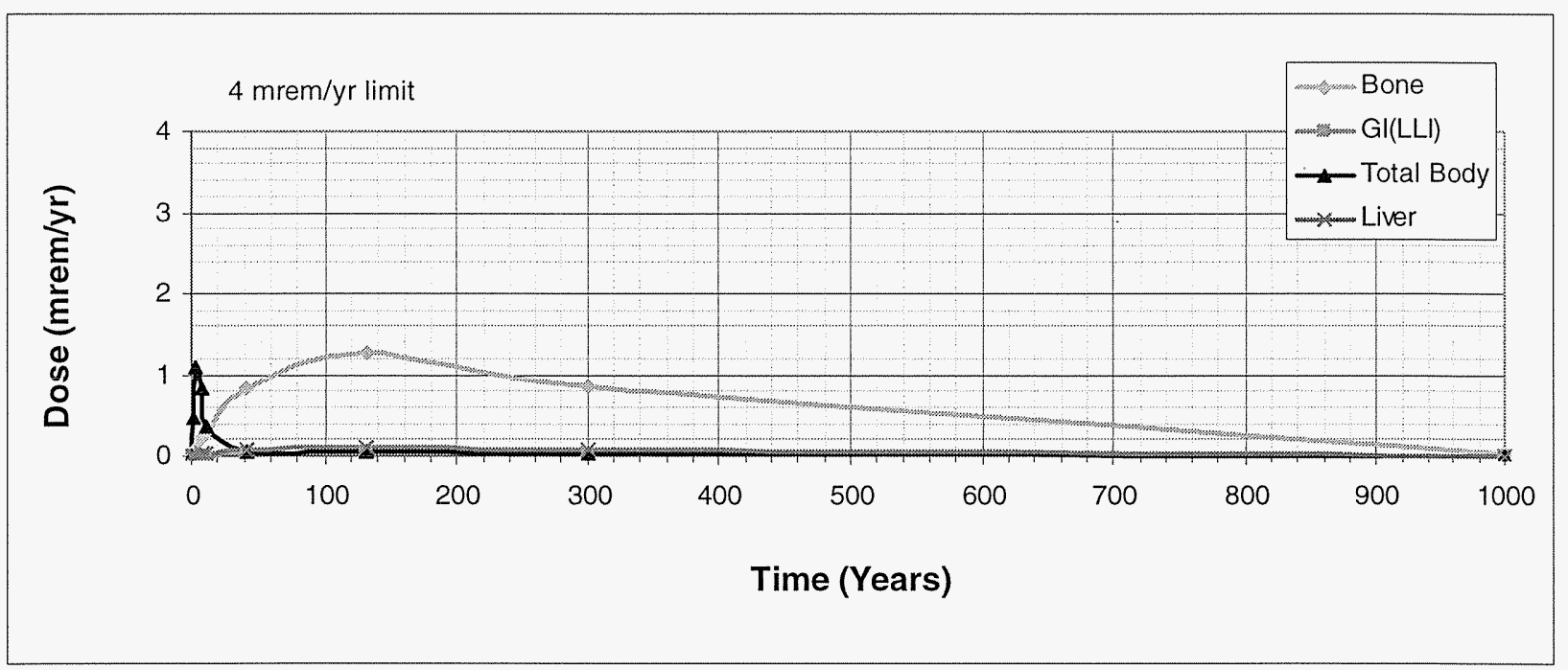

\subsubsection{Nonradionuclides}

Tables 4 through 9 compare the statistical verification results to the groundwater protection RAGs for all sampling areas. The tables show that the statistical results for barium, lead, aroclor-1254, and aroclor-1260 exceed the soil RAGs for the protection of groundwater. The residual concentrations of these constituents, however, are not predicted to reach groundwater (and, therefore, the Columbia River) in 1,000 years based on the 100 Area Analogous Sites RESRAD Calculations (BHI 2005) and their contaminant-specific soil partitioning coefficient $\left(\mathrm{K}_{d}\right)$ values. Given the soil-partitioning coefficients of barium $(25 \mathrm{~mL} / \mathrm{g})$, lead $(30 \mathrm{~mL} / \mathrm{g})$, aroclor- $1254(75.6 \mathrm{~mL} / \mathrm{g})$, and 
aroclor-1260 $(530 \mathrm{~mL} / \mathrm{g})$, the results of RESRAD modeling $(\mathrm{BHI} 2005)$ indicate that these constituents will not migrate vertically more than $3 \mathrm{~m}(10 \mathrm{ft})$ in 1,000 years. The vadose zone underlying the 118-C-1 site is greater than $25 \mathrm{~m}$ ( $82 \mathrm{ft}$ ) thick and, as such, these contaminants will not reach groundwater (and, therefore, the Columbia River) in 1,000 years.

\subsection{COLUMBIA RIVER REMEDIAL ACTION GOALS ATTAINED}

\subsubsection{Radionuclides}

The river protection RAGs for radionuclides are identical to the groundwater protection RAGs. The RESRAD modeling results are compared to the groundwater protection RAGs in Table 12. The results of RESRAD modeling indicate that radionuclides are not predicted to reach groundwater (and by extension, not predicted to reach the Columbia River) at levels that exceed the RAGs; therefore, the Columbia River protection RAOs have been attained.

\subsubsection{Nonradionuclides}

Tables 4 through 9 compare the statistical verification results to the Columbia River protection RAGs for all sampling areas. The tables show that the statistical results for copper, lead, zinc, aroclor-1254, and aroclor-1260 exceed the soil RAGs for the protection of groundwater. The residual concentrations of these constituents, however, are not predicted to reach groundwater (and, therefore, the Columbia River) in 1,000 years based on the 100 Area Analogous Sites RESRAD Calculations (BHI 2005) and their contaminant-specific soil-partitioning coefficient values. Given the soil-partitioning coefficients of copper $(22 \mathrm{~mL} / \mathrm{g})$, lead $(30 \mathrm{~mL} / \mathrm{g})$, zinc $(30 \mathrm{~mL} / \mathrm{g})$, aroclor-1254 $(75.6 \mathrm{~mL} / \mathrm{g})$, and aroclor $-1260(530 \mathrm{~mL} / \mathrm{g})$, the results of the RESRAD modeling (BHI 2005 ) indicate that these constituents will not migrate vertically more than $3 \mathrm{~m}(10 \mathrm{ft})$ in 1,000 years. The vadose zone underlying the $118-\mathrm{C}-1$ site is greater than $25 \mathrm{~m}(82 \mathrm{ft})$ thick and, as such, these contaminants will not reach groundwater (and, therefore, the Columbia River) in 1,000 years.

\subsection{WAC 173-340 THREE-PART TEST FOR NONRADIONUCLIDES}

Sections 5.1, 5.2, and 5.3 looked separately at compliance with direct exposure RAGs, groundwater protection soil RAGs, and Columbia River protection soil RAGs, respectively. When using a statistical sampling approach, a RAG requirement for nonradionuclides is the WAC 173-340-740(7)(e) three-part test. The WAC 173-340 three-part test consists of the following criteria: 1) the cleanup verification statistical value must be less than the cleanup level, 2) no single detection can exceed two times the cleanup criteria, and 3) the percentage of samples exceeding the cleanup criteria must be less than $10 \%$. 
The application of the three-part test for the 118-C-1 waste site is included in the $95 \%$ UCL calculation brief (Appendix E) and summarized in Table 13. Only the COCs/COPCs that failed one or more parts of the WAC 173-340 three-part test are provided in Table 13. The table lists the most restrictive RAG, the maximum detected value, the total number of samples collected, and the percentage of samples exceeding the RAG. The final column of the table describes the result of applying the three WAC 173-340-740(7)(e) criteria using the values listed in the preceding columns.

Residual concentrations of bis(2-ethylhexyl)phthalate, barium, copper, lead, zinc, aroclor-1254, and aroclor-1260 fail the three-part test in comparison against the soil RAGs for the protection of groundwater and/or the Columbia River. Based on RESRAD modeling (BHI 2005) and contaminant-specific soil-partitioning coefficient values, these constituents will not vertically migrate more than $3 \mathrm{~m}(10 \mathrm{ft})$ in 1,000 years. Given a vadose zone thickness greater than $25 \mathrm{~m}$ (82 ft) thick underlying the 118-C-1 waste site, residual concentrations are not predicted to reach groundwater (and, therefore, the Columbia River) within 1,000 years. As such, all non-radionuclide statistical verification data sets pass the WAC 173-340 three part test.

Table 13. Summary of the WAC 173-340 Three-Part Test for the 118-C-1 Burial Ground. ${ }^{a}$ (2 Pages)

\begin{tabular}{|c|c|c|c|c|c|c|}
\hline COC/COPC & $\begin{array}{c}\text { Most } \\
\text { Restrictive } \\
\text { Applicable } \\
\text { RAG }\end{array}$ & $\begin{array}{c}\text { Statistical } \\
\text { Cleanup } \\
\text { Verification } \\
\text { Value } \\
(\mathrm{mg} / \mathrm{kg})^{\mathrm{b}}\end{array}$ & $\begin{array}{l}\text { Maximum } \\
\text { Detected } \\
\text { Cleanup } \\
\text { Verification } \\
\text { Value } \\
(\mathrm{mg} / \mathrm{kg})^{c}\end{array}$ & $\begin{array}{c}\text { Total } \\
\text { Number of } \\
\text { Samples }^{d}\end{array}$ & $\begin{array}{c}\text { Percentage } \\
\text { of Cleanup } \\
\text { Verification } \\
\text { Data Set } \\
\text { Exceeding } \\
\text { RAG }^{\text {E }}\end{array}$ & $\begin{array}{c}\text { Cleanup } \\
\text { Criteria } \\
\text { Attained? }\end{array}$ \\
\hline \multicolumn{7}{|c|}{ Remediation Footprint (Sample Area 2) } \\
\hline $\begin{array}{l}\text { bis(2- } \\
\text { Ethylhexyl)phthalate }\end{array}$ & 0.36 & 0.28 & 0.40 & 5 & $60 \%$ & Yes $^{f}$ \\
\hline \multicolumn{7}{|c|}{ Remediation Footprint (Sample Area 3) } \\
\hline Copper & $22.0^{9}$ & 38.6 & 45.2 & 4 & $50 \%$ & Yes $^{f}$ \\
\hline Lead & $10.2^{9}$ & 18.6 & 23.1 & 4 & $50 \%$ & Yes $^{f}$ \\
\hline Zinc & $67.8^{9}$ & 74.8 & 77.1 & 4 & $50 \%$ & Yes $^{f}$ \\
\hline Aroclor-1254 & $0.017^{h}$ & 0.054 & 0.062 & 4 & $75 \%$ & Yes $^{f}$ \\
\hline \multicolumn{7}{|c|}{ Remediation Footprint (Sample Area 4) } \\
\hline Barium & $132^{\mathrm{i}, \mathrm{g}}$ & 206 & 286 & 4 & $25 \%$ & Yes $^{f}$ \\
\hline \multicolumn{7}{|c|}{ Waste Staging Pile Area } \\
\hline Barium & $132^{i, g}$ & $130(<\mathrm{BG})$ & 495 & 16 & $6 \%$ & Yes $^{f}$ \\
\hline Copper & $22.0^{9}$ & 36 & 144 & 16 & $6 \%$ & Yes $^{f}$ \\
\hline Lead & $10.2^{g}$ & 10.7 & 25.8 & 16 & $12.5 \%$ & Yes $^{\dagger}$ \\
\hline Aroclor-1254 & $0.017^{h}$ & 0.015 & 0.025 & 16 & $19 \%$ & Yes $^{f}$ \\
\hline Aroclor-1260 & $0.017^{h}$ & 0.061 & 0.061 & 16 & $12.5 \%$ & Yes $^{f}$ \\
\hline
\end{tabular}




\section{Table 13. Summary of the WAC 173-340 Three-Part Test for the 118-C-1 Burial Ground. a (2 Pages)}

a Only the COCs/COPCs that failed the WAC 173-340 Three-Part Test are presented.

b Criterion is statistical value cannot exceed most restrictive applicable RAG.

c Criterion is no single detection can exceed two times the most restrictive applicable RAG.

d Total number of samples in the decision unit may include field duplicate samples, which are included in the evaluation as separate samples.

e Criterion is percentage of data set exceeding the most restrictive applicable RAG cannot exceed $10 \%$.

I Based on the 100 Area Analogous Sites RESRAD Calculations (BHI 2005) and contaminant-specific soil-portioning coefficient $\left(K_{d}\right)$ value, contaminant will not migrate vertically more than $3 \mathrm{~m}(10 \mathrm{ft})$ in 1,000 years. As the vadose zone underlying the site is greater than $25 \mathrm{~m}(82 \mathrm{ft}$ ) thick, the contaminant will not reach groundwater (and thus the Columbia River) in 1,000 years.

g Where cleanup levels are less than background, cleanup levels default to background (WAC 173-340-700[4][d]) (1996).

h Where cleanup levels are less than the required detection limit, cleanup levels default to the required detection limit (WAC 173340-707[2], 1996 and DOE-RL 2005).

i Barium soil cleanup level for groundwater protection calculated from WAC 173-340-740(3)(a)(ii)(A), 1996 ("100 times rule") and WAC 173-340-720(3), 1996 (Method B for groundwater) is $112 \mathrm{mg} / \mathrm{kg}$ (as presented in the RDR/RAWP [DOE-RL 2005]). The updated oral reference dose value (as provided in IRIS) yields a Method B groundwater cleanup criteria of $7 \mathrm{mg} / \mathrm{L}$, as compared to the more restrictive maximum contaminant level of $2 \mathrm{mg} / \mathrm{L}$ (40 Code of Federal Regulations [CFR] 141). Per WAC 173-340$740(3)(a)(i i)(A), 1996$ ("100 times rule"), the most restrictive updated soil cleanup level for groundwater protection would be 200 $\mathrm{mg} / \mathrm{kg}$.

$\mathrm{COC}=$ contaminant of concern

COPC = contaminant of potential concern

IRIS = Integrates Risk Information System

RAG = remedial action goal

RESRAD = RESidual Radioactivity (dose model)

RDR/RAWP = remedial design report/remedial action work plan

WAC $=$ Washington Administrative Code

\subsection{RADIONUCLIDE RISK INFORMATION}

The radionuclide RAG for direct exposure is derived from the ROD (EPA 2000) and is expressed in terms of an allowable radiation dose rate above background (i.e., $15 \mathrm{mrem} / \mathrm{yr}$ ). The RAG evaluation (Section 5.0) involved using the RESRAD model to estimate total annual radiation dose rates for 1,000 years for comparison to the RAG. Radiation presents a carcinogenic risk, and the RESRAD model also calculates the excess lifetime cancer risk (ELCR) associated with the estimated radiation dose rates using the EPA's Health Effects Assessment Summary Tables (update dated April 16, 2001, "Update of Radionuclide Carcinogenicity Slope Factors," available on the Internet at www.epa.gov/radiation/heast). The "National Oil and Hazardous Substances Pollution Contingency Plan" (40 Code of Federal Regulations 300) presents a target range for residual risk of $10^{-4}$ to $10^{-6}$.

Figures 9 through 11 illustrate ELCR for the remediation footprint, overburden/BCL, and waste staging pile area as estimated using the RESRAD model. Because of radioactive decay, the risk decreases over time. The maximum predicted ELCR occurs at present (year 2006). The maximum ELCR is $9.95 \times 10^{-5}$ for the remediation footprint, $5.42 \times 10^{-5}$ for the overburden/BCL soil and $1.55 \times 10^{-4}$ for the waste staging pile area. The ELCR predicted for the year 2018 is $3.79 \times 10^{-5}$ for the remediation footprint, $4.10 \times 10^{-5}$ for the overburden/BCL soil and $9.57 \times 10^{-5}$ for the waste staging pile footprint. All three sampling areas meet the target risk range of $10^{-4}$ to $10^{-6}$. 
Figure 9. RESRAD Analysis - Radionuclide Risk, All Pathways - Remediation Footprint.

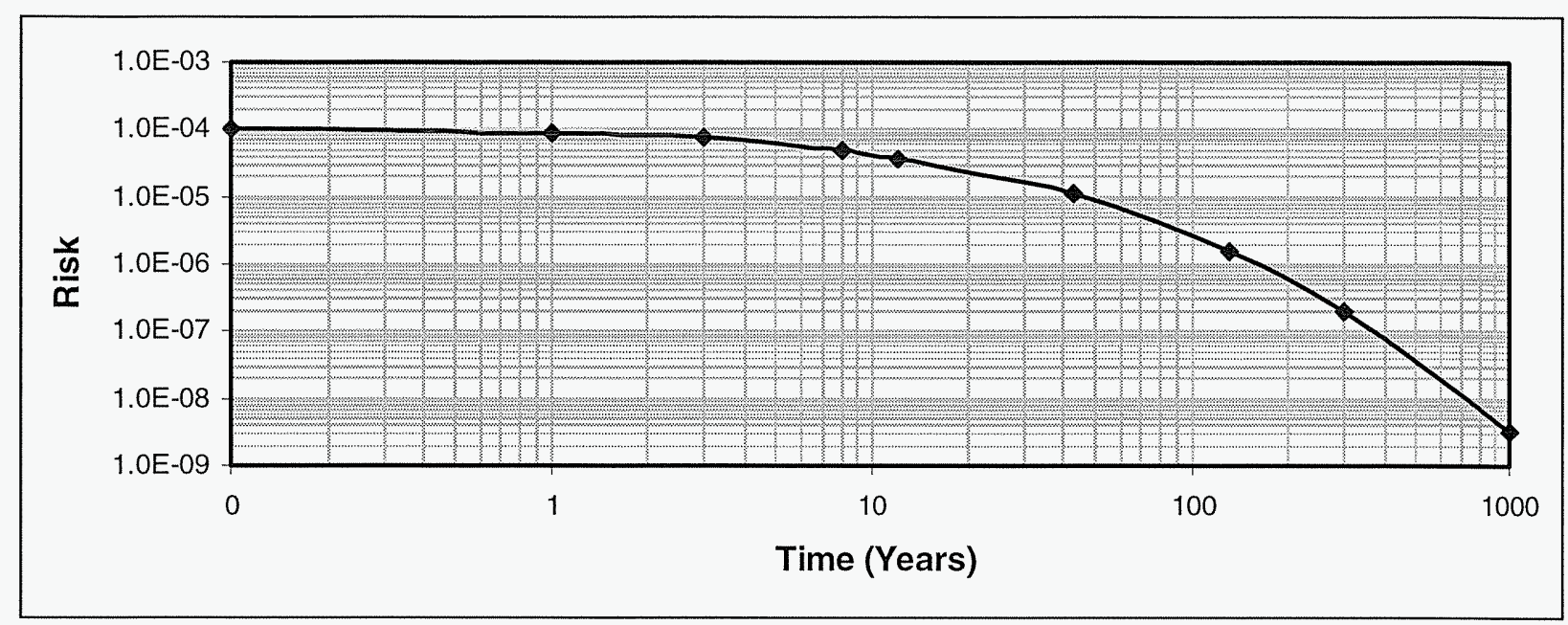

Figure 10. RESRAD Analysis - Radionuclide Risk, All Pathways Overburden/BCL Soil.

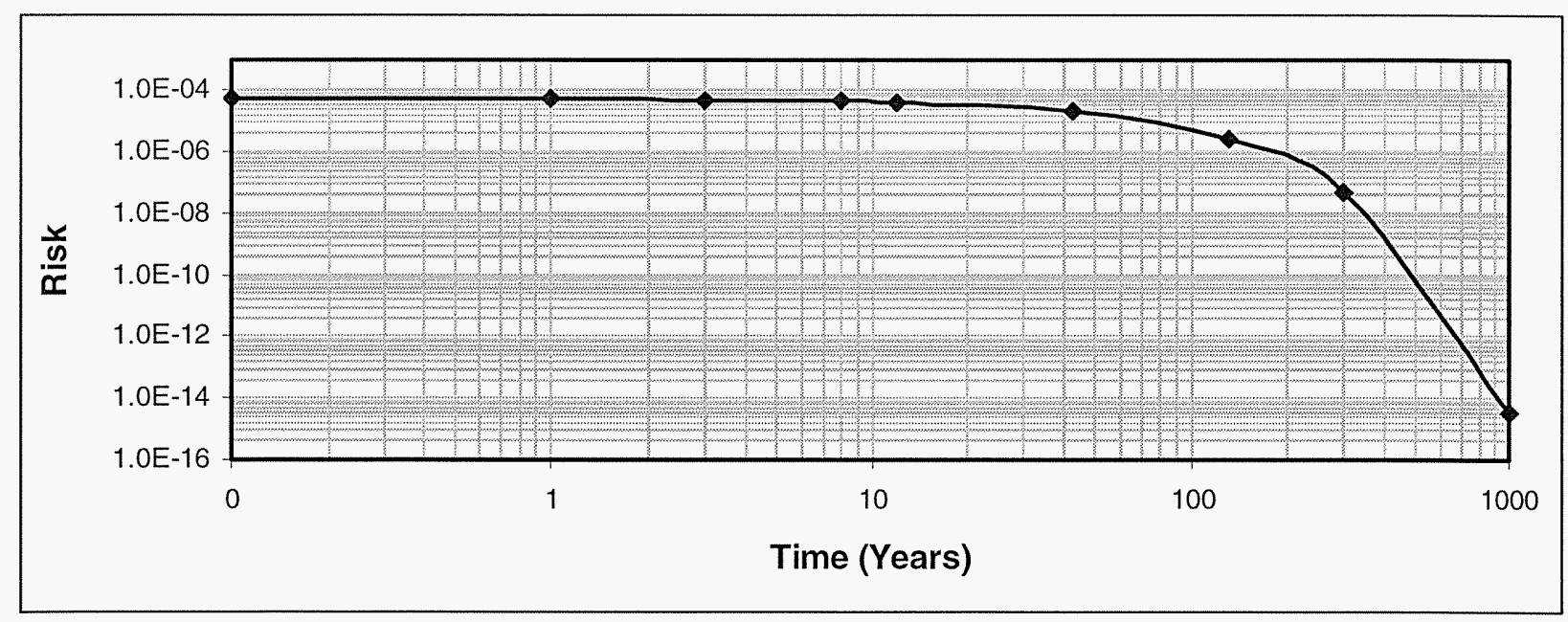


Figure 11. RESRAD Analysis - Radionuclide Risk, All Pathways - Waste Staging Pile Footprint.

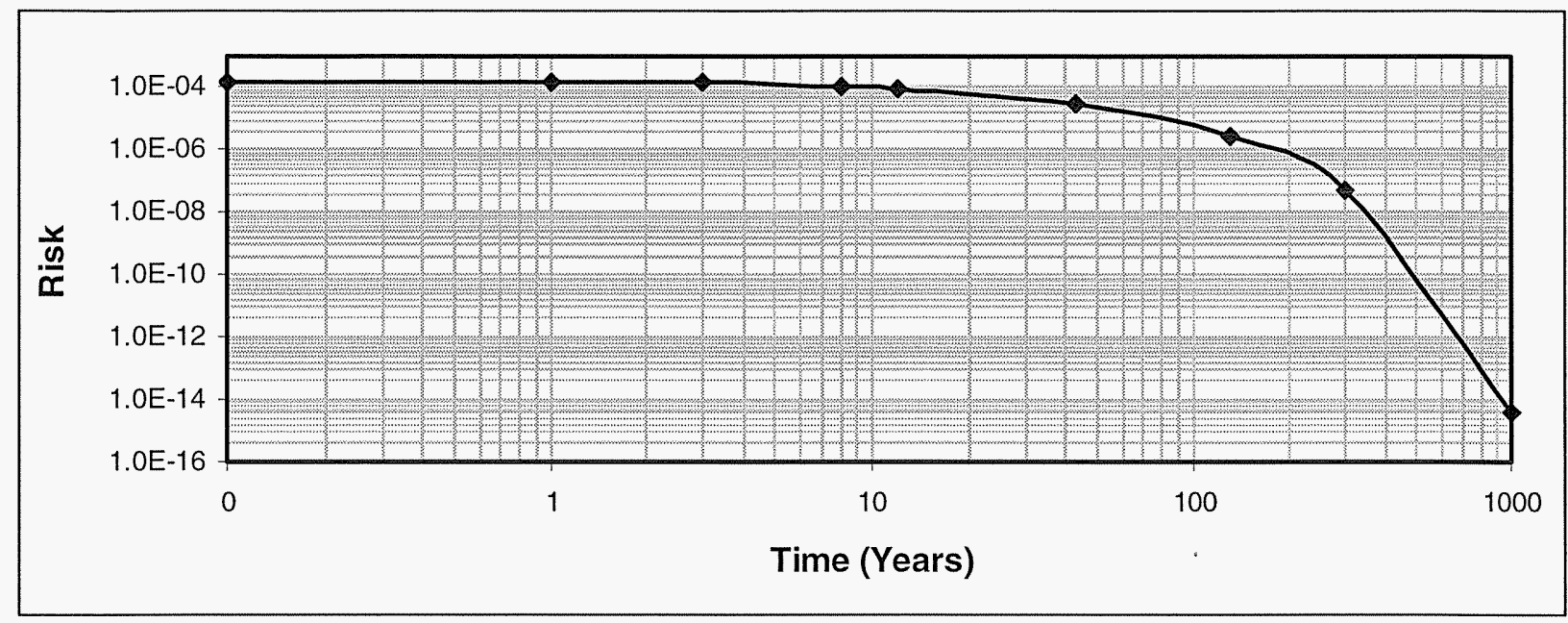

\subsection{STATEMENT OF PROTECTIVENESS}

This cleanup verification package demonstrates that remedial action at the 118-C-1 site has achieved the remedial action objectives and corresponding RAGs established in the ROD (EPA 2000) and RDR/RAWP (DOE-RL 2005). The remaining soils at the 118-C-1 site have been sampled, analyzed, and modeled. The results of this effort indicate that the materials from the 118-C-1 site containing COCs at concentrations exceeding RAGs have been excavated and disposed at ERDF. These results also indicate that residual concentrations will support future land uses that can be represented (or bounded) by a rural-residential scenario and that residual concentrations throughout the site pose no threat to groundwater or the Columbia River. Institutional controls are required for the site to prevent uncontrolled drilling or excavation into deep zone soils. The 118-C-1 site is verified to be remediated in accordance with the ROD (EPA 2000) and may be backfilled.

\subsection{REFERENCES}

40 CFR 141, "National Primary Drinking Water Regulations," Code of Federal Regulations, as amended.

40 CFR 300, "National Oil and Hazardous Substances Pollution Contingency Plan," Code of Federal Regulations, as amended.

ANL, 2005, RESRAD for Windows, Version 6.30, Argonne National Laboratory, Environmental Assessment Division, Argonne, Illinois. 
BHI, 2001, Calculation of Total Uranium Activity Corresponding to a Maximum Contaminant Level for Total Uranium of 30 Micrograms per Liter in Groundwater, 0100X-CA-V0038, Rev. 0, Bechtel Hanford, Inc., Richland, Washington.

BHI, 2004, 100 Area Radionuclide and Nonradionuclide Lookup Values for the 1995 Interim Remedial Action Record of Decision, 0100X-CA-V0046, Rev. 0, Bechtel Hanford Inc., Richland, Washington.

BHI, 2005, 100 Area Analogous Sites RESRAD Calculations, Calculation No. 0100X-CA-V0050, Rev. 0, Bechtel Hanford, Inc., Richland, Washington.

DOE Order 5400.5, Radiation Protection of the Public and the Environment, U.S. Department of Energy, Washington, D.C.

DOE-RL, 1996, Hanford Site Background: Part 2, Soil Background for Radionuclides, DOE/RL-96-12, Rev. 0, U.S. Department of Energy, Richland Operations Office, Richland, Washington.

DOE-RL, 1998, Tri-Party Agreement Handbook Management Procedures, RL-TPA-90-0001, Guideline Number TPA-MP-14, "Maintenance of the Waste Information Data System (WIDS)," U.S. Department of Energy, Richland Operations Office, Richland, Washington.

DOE-RL, 2001, 100 Area Burial Grounds Remedial Action Sampling and Analysis Plan, DOE/RL-2001-35, Rev. 0, U.S. Department of Energy, Richland Operations Office, Richland, Washington.

DOE-RL, 2005, Remedial Design Report/Remedial Action Work Plan for the 100 Area, DOE/RL-96-17, Rev. 5, U.S. Department of Energy, Richland Operations Office, Richland, Washington.

Ecology, EPA, and DOE, 1989, Hanford Federal Facility Agreement and Consent Order, 2 vols., as amended, Washington State Department of Ecology,

U.S. Environmental Protection Agency, and U.S. Department of Energy, Olympia, Washington.

Ecology, 1994, Natural Background Soil Metals Concentrations in Washington State, Publication No. 94-115, Washington State Department of Ecology, Olympia, Washington.

Ecology, 2005, Cleanup Levels and Risk Calculations (CLARC) Database, Washington State Department of Ecology, Olympia, Washington, $<$ https://fortress.wa.gov/ecy/clarc/CLARCHome.aspx>.

EPA, 1994, Guidance Manual for the Integrated Exposure Uptake Biokinetic Model for Lead in Children, EPA/540/R-93/081, Publication No. 9285.7, U.S. Environmental Protection Agency, Washington, D.C. 
EPA, 2000, Record of Decision for the 100-BC-1, 100-BC-2, 100-DR-1, 100-DR-2, 100-FR-2, 100-HR-2, and 100-KR-2 Operable Units, Hanford Site (100 Area Burial Grounds), Benton County, Washington, U.S. Environmental Protection Agency, Region 10, Seattle, Washington.

WAC 173-303, 2004, "Dangerous Waste Regulations," Washington Administrative Code.

WAC 173-340, 1996, "Model Toxics Control Act - Cleanup," Washington Administrative Code.

WAC 173-350, 2005, "Solid Waste Handling Standards," Washington Administrative Code.

WDOH, 1997, State of Washington Department of Health Interim Regulatory Guidance: Hanford Guidance for Radiological Cleanup, WDOH/320-015, Rev. 1, Washington Department of Health, Richland, Washington.

WCH, 2006, Site Specific Instruction for Close-out Approach for 118-C-1, Rev. 0, SSI No. 100-BC-10, CCN 129632, Washington Closure Hanford, Richland, Washington. 
CVP-2006-00011

Rev. 0

APPENDIX A

SUMMARY OF VERIFICATION SOIL SAMPLING

AND ANALYTICAL RESULTS 
CVP-2006-00011

Rev. 0

A-ii 
118-C-1 Verification Sampling Results

\begin{tabular}{|c|c|c|c|c|c|c|c|c|c|c|c|c|c|c|c|c|c|}
\hline \multirow{2}{*}{ Sample Location } & \multirow{2}{*}{$\begin{array}{l}\text { Sample } \\
\text { Number }\end{array}$} & \multirow{2}{*}{$\begin{array}{c}\text { Sample } \\
\text { Date }\end{array}$} & \multicolumn{3}{|c|}{ Americium-241 } & \multicolumn{3}{|c|}{ Americium-241 GEA } & \multicolumn{3}{|c|}{ Carbon-14 } & \multicolumn{3}{|c|}{ Cesium-137 } & \multicolumn{3}{|c|}{ Cobalt-60 } \\
\hline & & & $\mathrm{pCi} / \mathrm{g}$ & $Q$ & MDA & $\mathrm{pCi} / \mathrm{g}$ & $Q$ & MDA & $\mathrm{pCi} / \mathrm{g}$ & $Q$ & MDA & $\mathrm{pCi} / \mathrm{g}$ & $Q$ & MDA & $\mathrm{pCi} / \mathrm{g}$ & $Q$ & MDA \\
\hline Area $1 \mathrm{~A} 1$ & $\mathrm{~J} 134 \mathrm{Y} 7$ & $8 / 17 / 06$ & 0.073 & $\mathrm{U}$ & 0.28 & 0.42 & $\mathrm{U}$ & 0.42 & -0.292 & $\mathrm{U}$ & 3.0 & 0.090 & $\mathrm{U}$ & 0.090 & 0.048 & $\vec{U}$ & 0.048 \\
\hline Duplicate of J134Y7 & J134Y9 & $8 / 17 / 06$ & -0.036 & $\mathrm{U}$ & 0.27 & 0.24 & $\mathrm{U}$ & 0.24 & -0.395 & $\mathrm{U}$ & 3.0 & 0.299 & & 0.032 & 0.046 & $\mathrm{U}$ & 0.046 \\
\hline Split of J134Y7 & $\mathrm{J} 13504$ & $8 / 17 / 06$ & 0.0311 & $\mathrm{U}$ & 0.0211 & & & & 0.418 & $\mathrm{U}$ & 0.720 & 0.391 & & 0.0162 & 0.00841 & $\mathrm{U}$ & 0.0217 \\
\hline Area $1 \mathrm{~A} 2$ & $\mathrm{~J} 13500$ & $8 / 17 / 06$ & 0 & $\mathrm{U}$ & 0.34 & 0.18 & $U$ & 0.18 & 1.24 & $\mathrm{U}$ & 3.4 & 0.129 & & 0.021 & 0.020 & $\mathrm{U}$ & 0.020 \\
\hline Area $1 \mathrm{~A} 3$ & J134Y8 & $8 / 17 / 06$ & -0.041 & $\mathrm{U}$ & 0.32 & 0.15 & $\mathrm{U}$ & 0.15 & -1.60 & $\mathrm{U}$ & 3.2 & 0.178 & & 0.025 & 0.026 & $\mathrm{U}$ & 0.026 \\
\hline Area $1 \mathrm{~A} 4$ & $\mathrm{~J} 13501$ & $8 / 17 / 06$ & 0.036 & $\mathrm{U}$ & 0.28 & 0.056 & $\mathrm{U}$ & 0.056 & 1.64 & $\mathrm{U}$ & 2.9 & 0.018 & & 0.009 & 0.009 & $\mathrm{U}$ & 0.009 \\
\hline Area $2 \mathrm{~A} 1$ & $\mathrm{~J} 13502$ & $8 / 17 / 06$ & & & & 0.32 & $\mathrm{U}$ & 0.32 & -0.488 & $U$ & 2.9 & 0.128 & & 0.034 & 0.036 & $\bar{U}$ & 0.036 \\
\hline Duplicate of J13502 & $\mathrm{J} 13508$ & $8 / 17 / 06$ & & & & 0.32 & $U$ & 0.32 & 0.048 & $U$ & 3.5 & 0.092 & & 0.040 & 0.034 & $\mathrm{U}$ & 0.034 \\
\hline Split of J13502 & $\mathrm{J} 13505$ & $8 / 17 / 06$ & & & & & & & 0.407 & $\mathrm{U}$ & 0.719 & 0.0464 & & 0.0148 & 0.00506 & $\mathrm{U}$ & 0.0174 \\
\hline Area $2 \mathrm{~A} 2$ & $\mathrm{~J} 13503$ & $8 / 17 / 06$ & & & & 0.16 & $\mathrm{U}$ & 0.16 & 0.827 & $\mathrm{U}$ & 2.8 & 0.149 & & 0.029 & 0.087 & $\mathrm{U}$ & 0.087 \\
\hline Area $2 \mathrm{~A} 3$ & $\mathrm{~J} 13506$ & $8 / 17 / 06$ & & & & 0.43 & $\mathrm{U}$ & 0.43 & 1.66 & $\mathrm{U}$ & 2.9 & 0.063 & $\mathrm{U}$ & 0.063 & 0.076 & $\mathrm{U}$ & 0.076 \\
\hline Area 2 A4 & $\mathrm{J} 13507$ & $8 / 17 / 06$ & & & & 0.24 & $\mathrm{U}$ & 0.24 & 11.7 & & 3.1 & 0.107 & & 0.047 & 1.48 & & 0.045 \\
\hline Area $3 \mathrm{A1}$ & $\mathrm{J} 13510$ & $8 / 21 / 06$ & -0.009 & $U$ & 0.064 & 0.20 & $\mathrm{U}$ & 0.20 & 0.660 & $U$ & 2.8 & 0.195 & & 0.033 & 0.034 & $\mathrm{U}$ & 0.034 \\
\hline Area $3 \mathrm{~A} 2$ & $\mathrm{~J} 13511$ & $8 / 21 / 06$ & 0.007 & $\mathrm{U}$ & 0.041 & & & & 0.636 & $\mathrm{U}$ & 2.9 & 0.240 & & 0.020 & 0.042 & & 0.025 \\
\hline Area $3 \mathrm{~A} 3$ & $\mathrm{~J} 13512$ & $8 / 21 / 06$ & 0.027 & $U$ & 0.042 & 0.44 & $\mathrm{U}$ & 0.44 & -0.369 & $\mathrm{U}$ & 3.0 & 0.958 & & 0.049 & 0.061 & $\mathrm{U}$ & 0.061 \\
\hline Area 3 A4 & $\mathrm{J} 13513$ & $8 / 21 / 06$ & 0.039 & $\mathrm{U}$ & 0.048 & & & & -0.866 & $\mathrm{U}$ & 2.8 & 0.227 & & 0.027 & 0.024 & $\mathrm{U}$ & 0.024 \\
\hline Area 4 A1 & $\mathrm{J} 13514$ & $8 / 21 / 06$ & -0.031 & $\mathrm{U}$ & 0.063 & 0.22 & $\bar{U}$ & 0.22 & 1.72 & $\mathrm{U}$ & 2.8 & 0.065 & & 0.031 & 0.035 & $\mathrm{U}$ & 0.035 \\
\hline Area 4 A2 & $\mathrm{J} 13515$ & $8 / 21 / 06$ & -0.014 & $\mathrm{U}$ & 0.068 & 1.7 & $\mathrm{U}$ & 1.7 & 0.560 & $\mathrm{U}$ & 2.8 & 1.19 & & 0.036 & 0.050 & $\mathrm{U}$ & 0.050 \\
\hline Area $4 \mathrm{~A} 3$ & $\mathrm{~J} 13516$ & $8 / 21 / 06$ & -0.008 & $\mathrm{U}$ & 0.050 & & & & 0.360 & $\mathrm{U}$ & 3.0 & 0.044 & & 0.020 & 0.016 & $\mathrm{U}$ & 0.016 \\
\hline Area 4 A4 & $\mathrm{J} 13517$ & $8 / 21 / 06$ & 0.053 & $\mathrm{U}$ & 0.13 & 0.14 & $\mathrm{U}$ & 0.14 & -0.307 & $\mathrm{U}$ & 2.9 & 0.082 & & 0.024 & 0.022 & $\mathrm{U}$ & 0.022 \\
\hline $\mathrm{OB} / \mathrm{BCL} \mathrm{A} 1$ & $\mathrm{~J} 13518$ & $8 / 22 / 06$ & 0.123 & $\mathrm{U}$ & 0.31 & 0.25 & $\mathrm{U}$ & 0.25 & -0.903 & $\mathrm{U}$ & 2.9 & 0.220 & & 0.035 & 0.030 & $\mathrm{U}$ & 0.030 \\
\hline $\mathrm{OB} / \mathrm{BCL} \mathrm{A} 2$ & $J 13519$ & $8 / 22 / 06$ & 0.036 & $\mathrm{U}$ & 0.28 & 0.20 & $\mathrm{U}$ & 0.20 & -0.592 & $\mathrm{U}$ & 2.9 & 0.059 & & 0.033 & 0.030 & $\mathrm{U}$ & 0.030 \\
\hline OB/BCL A3 & $\mathrm{J} 13520$ & $8 / 22 / 06$ & 0.169 & $\mathrm{U}$ & 0.43 & 0.13 & $\mathrm{U}$ & 0.13 & -0.345 & $\mathrm{U}$ & 2.9 & 0.437 & & 0.018 & 0.015 & $\mathrm{U}$ & 0.015 \\
\hline $\mathrm{OB} / \mathrm{BCL} \mathrm{A} 4$ & $\mathrm{~J} 135 \mathrm{~N} 5$ & $8 / 22 / 06$ & 0.007 & $\mathrm{U}$ & 0.11 & 0.37 & $\mathrm{U}$ & 0.37 & 0.472 & $\mathrm{U}$ & 3.3 & 0.172 & & 0.046 & 0.040 & $U$ & 0.040 \\
\hline
\end{tabular}

Note: The following abbreviations apply to all Appendix A tables.

Note: Data qualified with $B, B^{*}, C, D, N$, or $J$ are considered acceptable values.

$\mathrm{B}=$ method blank contamination $\quad \mathrm{OB}=$ overburden

$\mathrm{B}^{*}=$ estimated

$\mathrm{BCL}=$ below cleanup levels

$\mathrm{C}=$ method blank contamination

$\mathrm{MDA}=$ minimum detectable activity

$\mathrm{N}=$ spiked analyte recovery outside control limits

$\mathrm{D}=$ diluted

$\mathrm{PQL}=$ practical quantitation limit

$\mathrm{FS}=$ focused sample

$\mathrm{Q}=$ qualifier

GEA = gamma energy analysis $\quad$ SPA = staging pile area

$\mathrm{J}=$ estimated

$\mathrm{U}=$ undetected 
118-C-1 Verification Sampling Results.

\begin{tabular}{|c|c|c|c|c|c|c|c|c|c|c|c|c|c|c|c|c|c|}
\hline \multirow{2}{*}{ Sample Location } & \multirow{2}{*}{$\begin{array}{c}\text { Sample } \\
\text { Number }\end{array}$} & \multirow{2}{*}{$\begin{array}{c}\text { Sample } \\
\text { Date }\end{array}$} & \multicolumn{3}{|c|}{ Americium-241 } & \multicolumn{3}{|c|}{ Americium-241 GEA } & \multicolumn{3}{|c|}{ Carbon-14 } & \multicolumn{3}{|c|}{ Cesium-137 } & \multicolumn{3}{|c|}{ Cobalt-60 } \\
\hline & & & $\mathrm{pCi} / \mathrm{g}$ & $Q$ & MDA & $\mathrm{pCi} / \mathrm{g}$ & $Q$ & MDA & $\mathrm{pCi} / \mathrm{g}$ & $Q$ & MDA & $\mathrm{pCi} / \mathrm{g}$ & $Q$ & MDA & $\mathrm{pCi} / \mathrm{g}$ & $Q$ & MDA \\
\hline SPA A1 & $\mathrm{J} 13522$ & $9 / 11 / 06$ & 0 & $\mathrm{U}$ & 0.24 & 0.056 & $\mathrm{U}$ & 0.056 & -1.24 & $\mathrm{U}$ & 3.3 & 0.424 & & 0.050 & 0.040 & $\mathrm{U}$ & 0.040 \\
\hline SPA A2 & $\mathrm{J} 13523$ & $9 / 11 / 06$ & 0.056 & $U$ & 0.21 & 0.26 & $U$ & 0.26 & -1.19 & $\mathrm{U}$ & 2.8 & 0.141 & & 0.035 & 0.031 & $\mathrm{U}$ & 0.031 \\
\hline SPA A3 & $\mathrm{J} 135 \mathrm{P} 1$ & $9 / 12 / 06$ & 0 & $\mathrm{UJ}$ & 1.1 & 0.39 & $\mathrm{U}$ & 0.39 & -0.524 & $\mathrm{U}$ & 2.1 & 3.00 & & 0.041 & 0.036 & $\mathrm{U}$ & 0.036 \\
\hline SPA A4 & $\mathrm{J} 135 \mathrm{P} 0$ & $9 / 12 / 06$ & -0.068 & $\mathrm{U}$ & 0.22 & 0.045 & $\mathrm{U}$ & 0.045 & -0.403 & $\mathrm{U}$ & 2.5 & 0.826 & & 0.039 & 0.029 & $\mathrm{U}$ & 0.029 \\
\hline SPA B5 & $\mathrm{J} 13524$ & $9 / 11 / 06$ & 0.034 & $\mathrm{U}$ & 0.26 & 0.15 & $\mathrm{U}$ & 0.15 & -1.58 & $\mathrm{U}$ & 2.9 & 0.494 & & 0.028 & 0.026 & $\mathrm{U}$ & 0.026 \\
\hline SPA B6 & $\mathrm{J} 13525$ & $9 / 11 / 06$ & 0 & $\mathrm{U}$ & 0.22 & 0.47 & $\mathrm{U}$ & 0.47 & -1.05 & $\mathrm{U}$ & 3.1 & 0.830 & & 0.049 & 0.044 & $\mathrm{U}$ & 0.044 \\
\hline SPA B7 & J135N8 & $9 / 11 / 06$ & 0 & $\mathrm{U}$ & 0.23 & 0.025 & $\mathrm{U}$ & 0.025 & -1.27 & $\mathrm{U}$ & 2.7 & 0.160 & & 0.024 & 0.022 & $\mathrm{U}$ & 0.022 \\
\hline SPA B8 & J135N9 & $9 / 11 / 06$ & 0.060 & $\mathrm{U}$ & 0.23 & 0.051 & $\mathrm{U}$ & 0.051 & -1.53 & $\mathrm{U}$ & 2.8 & 0.383 & & 0.048 & 0.034 & $\mathrm{U}$ & 0.034 \\
\hline SPA C9 & $\mathrm{J} 135 \mathrm{P} 2$ & $9 / 12 / 06$ & 0.030 & $\mathrm{U}$ & 0.23 & 0.11 & $\mathrm{U}$ & 0.11 & -0.600 & $\mathrm{U}$ & 2.6 & 0.290 & & 0.020 & 0.023 & $\mathrm{U}$ & 0.023 \\
\hline SPA C10 & J135P3 & $9 / 12 / 06$ & 0.271 & $\mathrm{U}$ & 0.52 & 0.042 & $\mathrm{U}$ & 0.042 & -0.647 & $U$ & 2.1 & 0.979 & & 0.035 & 0.055 & & 0.033 \\
\hline SPA C1 & J135P4 & $9 / 12 / 06$ & 0 & $\mathrm{U}$ & 0.23 & 0.49 & $\mathrm{U}$ & 0.49 & -0.487 & $\mathrm{U}$ & 2.4 & 1.16 & & 0.046 & 0.050 & $\mathrm{U}$ & 0.050 \\
\hline SPA C2 & J135P5 & $9 / 12 / 06$ & -0.033 & $\mathrm{U}$ & 0.25 & 0.57 & $\mathrm{U}$ & 0.57 & -0.935 & $\mathrm{U}$ & 2.1 & 0.644 & & 0.055 & 0.055 & $\mathrm{U}$ & 0.055 \\
\hline SPA D3 & $\mathrm{J} 135 \mathrm{P} 6$ & $9 / 12 / 06$ & 0.063 & $\mathrm{U}$ & 0.24 & 0.27 & $\mathrm{U}$ & 0.27 & -0.356 & $\mathrm{U}$ & 2.1 & 0.071 & & 0.033 & 0.034 & $\mathrm{U}$ & 0.034 \\
\hline SPA D4 & J135P7 & $9 / 12 / 06$ & 0.085 & $\mathrm{U}$ & 0.22 & 0.16 & $\mathrm{U}$ & 0.16 & 182 & & 2.1 & 0.091 & & 0.033 & 0.030 & $\mathrm{U}$ & 0.030 \\
\hline SPA D5 & J135P8 & $9 / 12 / 06$ & 0 & $\mathrm{U}$ & 0.59 & 0.067 & $\mathrm{U}$ & 0.067 & 0.362 & $\mathrm{U}$ & 2.2 & 1.18 & & 0.053 & 0.063 & & 0.051 \\
\hline SPAD6 & J135P9 & $9 / 12 / 06$ & 0.059 & $\mathrm{U}$ & 0.23 & 0.020 & $\mathrm{U}$ & 0.020 & -1.06 & $\mathrm{U}$ & 2.3 & 0.198 & & 0.017 & 0.014 & $\mathrm{U}$ & 0.015 \\
\hline FS-7 & $\mathrm{J} 12 \mathrm{XM} 4$ & $7 / 26 / 06$ & & & & 0.040 & $\mathrm{U}$ & 0.040 & & & & 0.045 & $U$ & 0.045 & 0.062 & $\mathrm{U}$ & 0.062 \\
\hline FS-8 & $\mathrm{J} 14 \mathrm{Y} 70$ & $5 / 15 / 07$ & -0.0334 & $\bar{U}$ & 0.11 & 0.26 & $\mathrm{U}$ & 0.26 & & & & 0.437 & & 0.038 & 0.033 & $\bar{U}$ & 0.033 \\
\hline FS-9 & $\mathrm{J} 14 \mathrm{Y} 71$ & $5 / 15 / 07$ & 0 & $\mathrm{U}$ & 0.11 & 0.085 & $\mathrm{U}$ & 0.085 & & & & 0.346 & & 0.026 & 0.025 & $\mathrm{U}$ & 0.025 \\
\hline FS-10 & $\mathrm{J} 14 \mathrm{Y} 72$ & $5 / 15 / 07$ & 0 & $\mathrm{U}$ & 0.089 & 0.029 & $\mathrm{U}$ & 0.029 & & & & 0.305 & & 0.030 & 0.025 & $\mathrm{U}$ & 0.025 \\
\hline FS-11 & $\mathrm{J} 14 \mathrm{Y} 73$ & $5 / 15 / 07$ & 0.0355 & $\mathrm{U}$ & 0.10 & 0.14 & $\mathrm{U}$ & 0.14 & & & & 0.321 & & 0.026 & 0.021 & $\mathrm{U}$ & 0.021 \\
\hline FS-12 & $\mathrm{J} 14 \mathrm{Y} 74$ & $5 / 15 / 07$ & 0.0100 & $\mathrm{U}$ & 0.061 & 0.057 & $\bar{U}$ & 0.057 & & & & 0.552 & & 0.017 & 0.019 & $\mathrm{U}$ & 0.019 \\
\hline
\end{tabular}

\begin{tabular}{|c|c|c|c|c|c|c|c|c|c|c|c|c|c|c|c|c|c|}
\hline \multirow{2}{*}{ Sample Location } & \multirow{2}{*}{$\begin{array}{c}\text { Sample } \\
\text { Number }\end{array}$} & \multirow{2}{*}{$\begin{array}{c}\text { Sample } \\
\text { Date }\end{array}$} & \multicolumn{3}{|c|}{ Europium-152 } & \multicolumn{3}{|c|}{ Europium-154 } & \multicolumn{3}{|c|}{ Europium-155 } & \multicolumn{3}{|c|}{ Nickel-63 } & \multicolumn{3}{|c|}{ Plutonium-238 } \\
\hline & & & $\mathrm{pCi} / \mathrm{g}$ & $Q$ & MDA & $\mathrm{pCi} / \mathrm{g}$ & $Q$ & MDA & $\mathrm{pCi} / \mathrm{g}$ & $Q$ & MDA & $\mathrm{pCi} / \mathrm{g}$ & $Q$ & MDA & $\mathrm{pCi} / \mathrm{g}$ & $Q$ & MDA \\
\hline Area $1 \mathrm{~A} 1$ & J134Y7 & $8 / 17 / 06$ & 0.161 & & 0.097 & 0.15 & U & 0.15 & 0.16 & $\mathrm{U}$ & 0.16 & 2.49 & $\mathrm{U}$ & 2.9 & -0.025 & $\mathrm{U}$ & 0.30 \\
\hline Duplicate of J134Y7 & J134Y9 & $8 / 17 / 06$ & 0.142 & & 0.083 & 0.16 & $\mathrm{U}$ & 0.16 & 0.12 & $\mathrm{U}$ & 0.12 & 1.42 & $\mathrm{U}$ & 3.1 & -0.074 & $\mathrm{U}$ & 0.57 \\
\hline Area $1 \mathrm{~A} 2$ & $\mathrm{~J} 13500$ & $8 / 17 / 06$ & 0.083 & & 0.044 & 0.069 & $\mathrm{U}$ & 0.069 & 0.068 & $\mathrm{U}$ & 0.068 & 0.441 & $\mathrm{U}$ & 2.9 & 0.186 & $\mathrm{U}$ & 0.71 \\
\hline Area $1 \mathrm{~A} 3$ & $\mathrm{~J} 134 \mathrm{Y} 8$ & $8 / 17 / 06$ & 0.064 & $\mathrm{U}$ & 0.064 & 0.082 & $\mathrm{U}$ & 0.082 & 0.092 & $\mathrm{U}$ & 0.092 & -0.194 & $\mathrm{U}$ & 3.1 & -0.110 & $\mathrm{U}$ & 0.84 \\
\hline Area $1 \mathrm{~A} 4$ & $\mathrm{~J} 13501$ & & 0.024 & $\mathrm{U}$ & 0.024 & 0.032 & $\mathrm{U}$ & 0.032 & 0.051 & $\mathrm{U}$ & 0.051 & 0.086 & $\mathrm{U}$ & 2.8 & 0 & $\mathrm{U}$ & 0.56 \\
\hline Duplicate of J13502 & $\mathrm{J} 13508$ & $8 / 17 / 06$ & 0.085 & $\mathrm{U}$ & 0.085 & 0.11 & $\mathrm{U}$ & 0.11 & 0.12 & $\mathrm{U}$ & 0.12 & 1.49 & $\mathrm{U}$ & 2.8 & & & \\
\hline Split of J13502 & $\mathrm{J} 13505$ & $8 / 17 / 06$ & 0.00852 & $\mathrm{U}$ & 0.0396 & 0.0175 & $\mathrm{U}$ & 0.0522 & 0.0255 & $\mathrm{U}$ & 0.0411 & 3.57 & $\mathrm{U}$ & 5.58 & & & \\
\hline Area 2 A2 & $\mathrm{J} 13503$ & $8 / 17 / 06$ & 0.055 & $\mathrm{U}$ & 0.055 & 0.075 & $\mathrm{U}$ & 0.075 & 0.073 & $\mathrm{U}$ & 0.073 & 12.4 & & 2.8 & & & \\
\hline Area 2 A3 & $\mathrm{J} 13506$ & $8 / 17 / 06$ & 0.281 & & 0.099 & 0.15 & $U$ & 0.15 & 0.14 & $\mathrm{U}$ & 0.14 & 1.37 & $\bar{U}$ & 2.8 & & & \\
\hline Area 2 A4 & $\mathrm{J} 13507$ & $8 / 17 / 06$ & 0.087 & $\mathrm{U}$ & 0.087 & 0.11 & $\mathrm{U}$ & 0.11 & 0.11 & $\mathrm{U}$ & 0.11 & 35.8 & & 2.9 & & & \\
\hline
\end{tabular}


118-C-1 Verification Sampling Results.

\begin{tabular}{|c|c|c|c|c|c|c|c|c|c|c|c|c|c|c|c|c|c|}
\hline \multirow{2}{*}{ Sample Location } & \multirow{2}{*}{$\begin{array}{c}\text { Sample } \\
\text { Number }\end{array}$} & \multirow{2}{*}{$\begin{array}{c}\text { Sample } \\
\text { Date }\end{array}$} & \multicolumn{3}{|c|}{ Europium-152 } & \multicolumn{3}{|c|}{ Europium-154 } & \multicolumn{3}{|c|}{ Europium-155 } & \multicolumn{3}{|c|}{ Nickel-63 } & \multicolumn{3}{|c|}{ Plutonium-238 } \\
\hline & & & $\mathrm{pCi} / \mathrm{g}$ & $Q$ & MDA & $\mathrm{pCi} / \mathrm{g}$ & $Q$ & MDA & $\mathrm{pCi} / \mathrm{g}$ & $Q$ & MDA & $\mathrm{pCi} / \mathrm{g}$ & $Q$ & MDA & $\mathrm{pCi} / \mathrm{g}$ & $Q$ & MDA \\
\hline Area $3 \mathrm{~A} 1$ & $\mathrm{~J} 13510$ & $8 / 21 / 06$ & 0.069 & $\mathrm{U}$ & 0.069 & 0.090 & $\mathrm{U}$ & 0.090 & 0.095 & $\mathrm{U}$ & 0.095 & -1.60 & $\mathrm{U}$ & 3.6 & 0 & UJ & 0.31 \\
\hline Area 3 A2 & $J 13511$ & $8 / 21 / 06$ & 0.138 & & 0.053 & 0.071 & $\bar{U}$ & 0.071 & 0.081 & $\bar{U}$ & 0.081 & -0.913 & $\mathrm{U}$ & 3.6 & 0.009 & $\mathrm{U}$ & 0.11 \\
\hline Area $3 \mathrm{~A} 3$ & $\mathrm{~J} 13512$ & $8 / 21 / 06$ & 0.140 & & 0.097 & 0.14 & $\bar{U}$ & 0.14 & 0.16 & $\bar{U}$ & 0.16 & -0.975 & $\mathrm{U}$ & 3.5 & 0.030 & $\mathrm{U}$ & 0.097 \\
\hline Area 3 A4 & $J 13513$ & $8 / 21 / 06$ & 0.082 & & 0.046 & 0.068 & $\bar{U}$ & 0.068 & 0.078 & $\bar{U}$ & 0.078 & -1.31 & $\mathrm{U}$ & 3.6 & 0.176 & UJ & 0.67 \\
\hline Area 4 A1 & $J 13514$ & $8 / 21 / 06$ & 0.070 & $\bar{U}$ & 0.070 & 0.11 & $\bar{U}$ & 0.11 & 0.098 & $\bar{U}$ & 0.098 & -0.912 & $\bar{U}$ & $\overline{3.4}$ & 0 & UJ & 0.79 \\
\hline Area 4 A2 & $J 13515$ & $8 / 21 / 06$ & 0.159 & & 0.074 & 0.095 & $\bar{U}$ & 0.095 & 0.11 & $\bar{U}$ & 0.11 & -1.91 & $\mathrm{U}$ & 3.5 & 0 & UJ & 0.56 \\
\hline Area 4 A3 & $J 13516$ & $8 / 21 / 06$ & 0.038 & $\bar{U}$ & 0.038 & 0.049 & $\bar{U}$ & 0.049 & 0.075 & $\mathrm{U}$ & 0.075 & -2.31 & $\mathrm{U}$ & 3.6 & -0.050 & $\mathrm{U}$ & 0.39 \\
\hline Area 4 A4 & J13517 & $8 / 21 / 06$ & 0.048 & $\mathrm{U}$ & 0.048 & 0.070 & $\bar{U}$ & 0.070 & 0.097 & $\bar{U}$ & 0.097 & -0.187 & $\mathrm{U}$ & 3.4 & 0 & $\mathrm{U}$ & 0.62 \\
\hline$\overline{\mathrm{OB} / \mathrm{BCL} \mathrm{A} 1}$ & $\mathrm{~J} 13518$ & $8 / 22 / 06$ & 0.080 & $\mathrm{U}$ & 0.080 & 0.097 & $\bar{U}$ & 0.097 & 0.13 & $\bar{U}$ & 0.13 & 0.290 & $\bar{U}$ & 2.4 & 0.037 & UJ & 0.28 \\
\hline OB/BCL A2 & $\mathrm{J} 13519$ & $8 / 22 / 06$ & 0.065 & $\mathrm{U}$ & 0.065 & 0.10 & $\mathrm{U}$ & 0.10 & 0.094 & $\bar{U}$ & 0.094 & 0.236 & $\bar{U}$ & 2.4 & 0 & $U$ & 0.18 \\
\hline $\mathrm{OB} / \mathrm{BCL} \mathrm{A} 3$ & $J 13520$ & $8 / 22 / 06$ & 0.041 & $\mathrm{U}$ & 0.041 & 0.050 & $\bar{U}$ & 0.050 & 0.083 & $\bar{U}$ & 0.083 & -0.523 & $\mathrm{U}$ & 2.3 & -0.076 & $\mathrm{U}$ & 0.27 \\
\hline $\mathrm{OB} / \mathrm{BCL} \mathrm{A} 4$ & $\mathrm{~J} 135 \mathrm{~N} 5$ & $8 / 22 / 06$ & 0.10 & $\mathrm{U}$ & 0.10 & 0.12 & $\mathrm{U}$ & 0.12 & 0.14 & $\mathrm{U}$ & 0.14 & -0.556 & $\mathrm{U}$ & 2.4 & 0 & $\mathrm{U}$ & 0.14 \\
\hline SPA A1 & $\mathrm{J} 13522$ & $9 / 11 / 06$ & 0.11 & $\mathrm{U}$ & 0.11 & 0.12 & $\mathrm{U}$ & 0.12 & 0.088 & $\mathrm{U}$ & 0.088 & 0.760 & $\mathrm{U}$ & 2.5 & -0.070 & $\mathrm{U}$ & 0.53 \\
\hline$\overline{\text { SPA A2 }}$ & $J 13523$ & $9 / 11 / 06$ & 0.075 & $\mathrm{U}$ & 0.075 & 0.12 & $\bar{U}$ & 0.12 & 0.10 & $\bar{U}$ & 0.10 & 0.208 & $\mathrm{U}$ & 2.6 & 0.060 & $\mathrm{U}$ & 0.46 \\
\hline SPA A3 & J135P1 & $9 / 12 / 06$ & 0.483 & & 0.11 & 0.13 & $\bar{U}$ & 0.13 & 0.15 & $\mathrm{U}$ & 0.15 & -0.735 & $\mathrm{U}$ & 3.7 & 0.168 & UJ & 0.43 \\
\hline SPA A4 & J135P0 & $9 / 12 / 06$ & 0.214 & & 0.085 & 0.095 & $\mathrm{U}$ & 0.095 & 0.068 & $\mathrm{U}$ & 0.068 & -0.821 & $\mathrm{U}$ & 3.1 & 0 & $\mathrm{U}$ & 0.51 \\
\hline SPA B5 & $J 13524$ & $9 / 11 / 06$ & 0.112 & & 0.064 & 0.080 & $U$ & 0.080 & 0.15 & $\mathrm{U}$ & 0.15 & 0.382 & $\mathrm{U}$ & 2.7 & -0.061 & $\mathrm{U}$ & 0.47 \\
\hline SPA B6 & $\mathrm{J} 13525$ & $9 / 11 / 06$ & 0.13 & $\mathrm{U}$ & 0.13 & 0.16 & $\mathrm{U}$ & 0.16 & 0.17 & $\mathrm{U}$ & 0.17 & 1.55 & $\mathrm{U}$ & 2.6 & 0.073 & $\mathrm{U}$ & 0.56 \\
\hline SPA B7 & $\mathrm{J} 135 \mathrm{~N} 8$ & $9 / 11 / 06$ & 0.046 & $\mathrm{U}$ & 0.046 & 0.078 & $\mathrm{U}$ & 0.078 & 0.049 & $\bar{U}$ & 0.049 & -0.340 & $\bar{U}$ & 2.7 & 0 & $\mathrm{U}$ & 0.49 \\
\hline SPA B8 & $\mathrm{J} 135 \mathrm{~N} 9$ & $9 / 11 / 06$ & 0.093 & $\bar{U}$ & 0.093 & 0.10 & $\bar{U}$ & 0.10 & 0.079 & $\bar{U}$ & 0.079 & 0.215 & $\bar{U}$ & 2.7 & 0 & $\mathrm{U}$ & 0.50 \\
\hline SPA C9 & $\mathrm{J} 135 \mathrm{P} 2$ & $9 / 12 / 06$ & 0.049 & $\mathrm{U}$ & 0.049 & 0.054 & $\mathrm{U}$ & 0.054 & 0.065 & $\mathrm{U}$ & 0.065 & 2.36 & $\mathrm{U}$ & 3.6 & 0 & $\mathrm{U}$ & 0.45 \\
\hline SPA C10 & $\mathrm{J} 135 \mathrm{P} 3$ & $9 / 12 / 06$ & 0.363 & & 0.079 & 0.089 & U & 0.089 & 0.064 & $\mathrm{U}$ & 0.064 & 0.238 & $\mathrm{U}$ & 3.4 & 0 & $\mathrm{U}$ & 0.46 \\
\hline SPA C1 & $\mathrm{J} 135 \mathrm{P} 4$ & $9 / 12 / 06$ & 0.156 & & 0.12 & 0.16 & $\bar{U}$ & 0.16 & 0.18 & $\bar{U}$ & 0.18 & 0.826 & $\bar{U}$ & 3.5 & 0 & $\mathrm{U}$ & 0.44 \\
\hline SPA C2 & J135P5 & $9 / 12 / 06$ & 0.16 & $\mathrm{U}$ & 0.16 & 0.19 & U & 0.19 & 0.20 & $\mathrm{U}$ & 0.20 & 0.462 & $\mathrm{U}$ & 3.6 & 0.104 & $\mathrm{U}$ & 0.50 \\
\hline SPA D3 & J135P6 & $9 / 12 / 06$ & 0.077 & $\mathrm{U}$ & 0.077 & 0.11 & $\bar{U}$ & 0.11 & 0.11 & $\mathrm{U}$ & 0.11 & -0.569 & $\mathrm{U}$ & 3.5 & 0 & $\mathrm{U}$ & 0.51 \\
\hline SPA D4 & $\mathrm{J} 135 \mathrm{P} 7$ & $9 / 12 / 06$ & 0.071 & $\mathrm{U}$ & 0.071 & 0.087 & U & 0.087 & 0.095 & $\mathrm{U}$ & 0.095 & 0 & $\mathrm{U}$ & 3.5 & 0.075 & $\mathrm{U}$ & 0.57 \\
\hline SPA D5 & J135P8 & $9 / 12 / 06$ & 0.14 & $\mathrm{U}$ & 0.14 & 0.14 & $\bar{U}$ & 0.14 & 0.10 & $\bar{U}$ & 0.10 & 1.66 & $\bar{U}$ & 4.0 & -0.063 & $\mathrm{U}$ & 0.48 \\
\hline SPA D6 & J135P9 & $9 / 12 / 06$ & 0.035 & U & 0.035 & 0.041 & U & 0.041 & 0.035 & $\mathrm{U}$ & 0.035 & -0.594 & $\mathrm{U}$ & 3.6 & 0.020 & $\mathrm{U}$ & 0.076 \\
\hline$\overline{\text { FS-7 }}$ & $\mathrm{J} 12 \mathrm{XM} 4$ & $7 / 26 / 06$ & 0.070 & $\bar{U}$ & 0.070 & 0.17 & $\bar{U}$ & 0.17 & 0.041 & $\bar{U}$ & 0.041 & & & & & & \\
\hline FS-8 & $\mathrm{J} 14 \mathrm{Y} 70$ & $5 / 15 / 07$ & 0.084 & $\mathrm{U}$ & 0.084 & 0.12 & $\bar{U}$ & 0.12 & 0.098 & $\bar{U}$ & 0.098 & & & & 0.0358 & $\mathrm{U}$ & 0.16 \\
\hline FS-9 & J14Y71 & $5 / 15 / 07$ & 0.068 & $\mathrm{U}$ & 0.068 & 0.086 & $U$ & 0.086 & 0.070 & $\mathrm{U}$ & 0.070 & & & & 0.101 & $\mathrm{U}$ & 0.34 \\
\hline FS-10 & $\mathrm{J} 14 \mathrm{Y} 72$ & $5 / 15 / 07$ & 0.093 & U & 0.093 & 0.083 & $\mathrm{U}$ & 0.083 & 0.059 & $\mathrm{U}$ & 0.059 & & & & 0.121 & $\mathrm{U}$ & 0.15 \\
\hline FS-11 & $\mathrm{J} 14 \mathrm{Y} 73$ & $5 / 15 / 07$ & 0.0355 & $\mathrm{U}$ & 0.10 & 0.075 & $\mathrm{U}$ & 0.075 & 0.076 & $\mathrm{U}$ & 0.076 & & & & 0.0226 & $\mathrm{U}$ & 0.35 \\
\hline FS-12 & J14Y74 & $5 / 15 / 07$ & 0.10 & $\mathrm{U}$ & 0.10 & 0.051 & $\mathrm{U}$ & 0.051 & 0.047 & $\mathrm{U}$ & 0.047 & & & & -0.0137 & $\mathrm{U}$ & 0.13 \\
\hline
\end{tabular}


118-C-1 Verification Sampling Results

\begin{tabular}{|c|c|c|c|c|c|c|c|c|c|c|c|c|c|c|c|c|c|}
\hline \multirow{2}{*}{ Sample Location } & \multirow{2}{*}{$\begin{array}{l}\text { Sample } \\
\text { Number }\end{array}$} & \multirow{2}{*}{$\begin{array}{c}\text { Sample } \\
\text { Date }\end{array}$} & \multicolumn{3}{|c|}{ Plutonium-239/240 } & \multicolumn{3}{|c|}{ Plutonium-241 } & \multicolumn{3}{|c|}{ Potassium-40 } & \multicolumn{3}{|c|}{ Radium-226 } & \multicolumn{3}{|c|}{ Radium-228 } \\
\hline & & & $\mathrm{pCi} / \mathrm{g}$ & $\mathbf{Q}$ & MDA & $\mathrm{pCi} / \mathrm{g}$ & $Q$ & MDA & $\mathrm{pCi} / \mathrm{g}$ & $\mathbf{Q}$ & MDA & $\mathrm{pCi} / \mathrm{g}$ & $\mathbf{Q}$ & MDA & $\mathrm{pCi} / \mathrm{g}$ & $\mathbf{Q}$ & MDA \\
\hline Area $1 \mathrm{~A} 1$ & $\mathrm{~J} 134 \mathrm{Y} 7$ & $8 / 17 / 06$ & 0.025 & $\mathrm{U}$ & 0.19 & 1.16 & $\mathrm{U}$ & 24 & 21.6 & & 0.37 & 0.754 & & 0.082 & 1.04 & & 0.19 \\
\hline $\begin{array}{c}\text { Duplicate of } \\
\text { J134Y7 }\end{array}$ & $\mathrm{J} 134 \mathrm{Y9}$ & $8 / 17 / 06$ & 0 & $\mathrm{U}$ & 0.57 & 4.91 & $U$ & 21 & 11.4 & & 0.28 & 0.401 & & 0.063 & 0.530 & & 0.15 \\
\hline Split of J134Y7 & $\mathrm{J} 13504$ & $8 / 17 / 06$ & 0.0461 & & 0.0250 & 1.26 & $\mathrm{U}$ & 2.87 & & & & & & & & & \\
\hline Area $1 \mathrm{~A} 2$ & $\mathrm{~J} 13500$ & $8 / 17 / 06$ & 0 & $\mathrm{U}$ & 0.71 & 14.1 & $\mathrm{U}$ & 21 & 23.8 & & 0.18 & 0.843 & & 0.032 & 1.34 & & 0.080 \\
\hline Area $1 \mathrm{~A} 3$ & J134Y8 & $8 / 17 / 06$ & 0 & $\mathrm{U}$ & 0.84 & 6.40 & $\mathrm{U}$ & 22 & 13.1 & & 0.27 & 0.566 & & 0.048 & 0.873 & & 0.11 \\
\hline Area $1 \mathrm{~A} 4$ & $\mathrm{~J} 13501$ & $8 / 17 / 06$ & 0 & $\mathrm{U}$ & 0.56 & 8.14 & $\mathrm{U}$ & 22 & 11.5 & & 0.10 & 0.409 & & 0.021 & 0.638 & & 0.040 \\
\hline Area $2 \mathrm{~A} 1$ & $\mathrm{~J} 13502$ & $8 / 17 / 06$ & & & & & & & 21.3 & & 0.29 & 0.757 & & 0.061 & 1.17 & & 0.13 \\
\hline Duplicate of J13502 & $\mathrm{J} 13508$ & $8 / 17 / 06$ & & & & & & & 21.4 & & 0.33 & 0.758 & & 0.064 & 1.13 & & 0.14 \\
\hline Split of J13502 & $\mathrm{J} 13505$ & $8 / 17 / 06$ & & & & & & & & & & & & & & & \\
\hline Area 2 A2 & $\mathrm{J} 13503$ & $8 / 17 / 06$ & & & & & & & 10.7 & & 0.26 & 0.414 & & 0.046 & 0.656 & & 0.096 \\
\hline Area $2 \mathrm{~A} 3$ & $\mathrm{~J} 13506$ & $8 / 17 / 06$ & & & & & & & 21.9 & & 0.38 & 0.832 & & 0.089 & 1.15 & & 0.17 \\
\hline Area 2 A4 & $\mathrm{J} 13507$ & $8 / 17 / 06$ & & & & & & & 10.5 & & 0.32 & 0.382 & & 0.064 & 0.680 & & 0.25 \\
\hline Area $3 \mathrm{~A} 1$ & $\mathrm{~J} 13510$ & $8 / 21 / 06$ & -0.023 & UJ & 0.22 & -0.542 & $\mathrm{U}$ & 23 & 11.3 & & 0.23 & 0.524 & & 0.059 & 0.781 & & 0.14 \\
\hline Area $3 \mathrm{~A} 2$ & $\mathrm{~J} 13511$ & $8 / 21 / 06$ & 0.036 & $\mathrm{U}$ & 0.068 & -1.91 & $\mathrm{U}$ & 22 & 12.3 & & 0.21 & 0.833 & & 0.042 & 0.844 & & 0.094 \\
\hline Area $3 \mathrm{~A} 3$ & $\mathrm{~J} 13512$ & $8 / 21 / 06$ & 0 & $\mathrm{U}$ & 0.077 & -10.6 & $\mathrm{U}$ & 18 & 20.2 & & 0.41 & 0.827 & & 0.086 & 1.14 & & 0.18 \\
\hline Area 3 A4 & $\mathrm{J} 13513$ & $8 / 21 / 06$ & 0 & UJ & 0.67 & -10.6 & $\mathrm{U}$ & 20 & 11.7 & & 0.22 & 0.411 & & 0.042 & 0.644 & & 0.097 \\
\hline Area $4 \mathrm{~A} 1$ & $\mathrm{~J} 13514$ & $8 / 21 / 06$ & 0 & $\mathrm{UJ}$ & 0.79 & -4.03 & $\mathrm{U}$ & 24 & 10.5 & & 0.32 & 0.441 & & 0.051 & 0.607 & & 0.15 \\
\hline Area 4 A2 & $\mathrm{J} 13515$ & $8 / 21 / 06$ & 0.073 & UJ & 0.56 & -7.73 & $\mathrm{U}$ & 27 & 20.2 & & 0.32 & 0.981 & & 0.056 & 1.25 & & 0.13 \\
\hline Area 4 A3 & $\mathrm{J} 13516$ & $8 / 21 / 06$ & 0.050 & $\mathrm{U}$ & 0.39 & -3.48 & $\mathrm{U}$ & 22 & 12.0 & & 0.16 & 0.434 & & 0.032 & 0.649 & & 0.076 \\
\hline Area 4 A4 & $\mathrm{J} 13517$ & $8 / 21 / 06$ & 0.081 & $U$ & 0.62 & -5.31 & $\mathrm{U}$ & 21 & 10.4 & & 0.19 & 0.399 & & 0.036 & 0.596 & & 0.085 \\
\hline $\mathrm{OB} / \mathrm{BCL} \mathrm{A} 1$ & $\mathrm{~J} 13518$ & $8 / 22 / 06$ & 0.037 & UJ & 0.14 & 14.2 & $\mathrm{U}$ & 28 & 22.0 & & 0.36 & 0.724 & & 0.062 & 1.31 & & 0.15 \\
\hline $\mathrm{OB} / \mathrm{BCL} \mathrm{A} 2$ & $\mathrm{~J} 13519$ & $8 / 22 / 06$ & 0 & U & 0.11 & 7.02 & $\mathrm{U}$ & 28 & 11.1 & & 0.25 & 0.417 & & 0.052 & 0.670 & & 0.13 \\
\hline $\mathrm{OB} / \mathrm{BCL} \mathrm{A} 3$ & $\mathrm{~J} 13520$ & $8 / 22 / 06$ & 0.045 & $\mathrm{U}$ & 0.12 & 6.27 & $\mathrm{U}$ & 27 & 21.7 & & 0.15 & 0.710 & & 0.030 & 1.11 & & 0.068 \\
\hline $\mathrm{OB} / \mathrm{BCL} \mathrm{A} 4$ & J135N5 & $8 / 22 / 06$ & 0 & $\mathrm{U}$ & 0.069 & 6.19 & $\mathrm{U}$ & 27 & 25.8 & & 0.35 & 0.911 & & 0.076 & 1.34 & & 0.14 \\
\hline
\end{tabular}


118-C-1 Verification Sampling Results.

\begin{tabular}{|c|c|c|c|c|c|c|c|c|c|c|c|c|c|c|c|c|c|}
\hline \multirow{2}{*}{ Sample Location } & \multirow{2}{*}{$\begin{array}{c}\text { Sample } \\
\text { Number }\end{array}$} & \multirow{2}{*}{$\begin{array}{c}\text { Sample } \\
\text { Date }\end{array}$} & \multicolumn{3}{|c|}{ Plutonium-239/240 } & \multicolumn{3}{|c|}{ Plutonium-241 } & \multicolumn{3}{|c|}{ Potassium-40 } & \multicolumn{3}{|c|}{ Radium-226 } & \multicolumn{3}{|c|}{ Radium-228 } \\
\hline & & & $\mathrm{pCi} / \mathrm{g}$ & $Q$ & MDA & $\mathrm{pCi} / \mathrm{g}$ & $Q$ & MDA & $\mathrm{pCi} / \mathrm{g}$ & $\bar{Q}$ & MDA & $\mathrm{pCi} / \mathrm{g}$ & $Q$ & MDA & $\mathrm{pCi} / \mathrm{g}$ & $\bar{Q}$ & MDA \\
\hline SPA A1 & $\mathrm{J} 13522$ & $9 / 11 / 06$ & 0 & $\mathrm{U}$ & 0.53 & 13.7 & $\mathrm{U}$ & 19 & 12.4 & & 0.38 & 0.503 & & 0.078 & 0.824 & & 0.17 \\
\hline SPA A2 & $\mathrm{J} 13523$ & 9/11/06 & 0 & $\mathrm{U}$ & 0.46 & 5.29 & $\mathrm{U}$ & 19 & 12.0 & & 0.34 & 0.376 & & 0.063 & 0.598 & & 0.15 \\
\hline SPA A3 & $\mathrm{J} 135 \mathrm{P} 1$ & 9/12/06 & 0.279 & UJ & 0.43 & 5.82 & $\mathrm{U}$ & 18 & 1.3 & $\mathrm{U}$ & 1.3 & 0.740 & & 0.062 & 1.08 & & 0.15 \\
\hline SPA A4 & $\mathrm{J} 135 \mathrm{PO}$ & $9 / 12 / 06$ & 0 & $\mathrm{U}$ & 0.51 & 1.71 & $\mathrm{U}$ & 21 & 12.5 & & 0.26 & 0.438 & & 0.068 & 0.765 & & 0.12 \\
\hline SPA B5 & $\mathrm{J} 13524$ & $9 / 11 / 06$ & 0.061 & $\mathrm{U}$ & 0.47 & 11.9 & $\mathrm{U}$ & 18 & 14.1 & & 0.26 & 0.412 & & 0.053 & 0.822 & & 0.10 \\
\hline SPA B6 & $\mathrm{J} 13525$ & 9/11/06 & 0.145 & $\mathrm{U}$ & 0.56 & 2.92 & $\mathrm{U}$ & 18 & 22.7 & & 0.42 & 0.799 & & 0.076 & 1.33 & & 0.18 \\
\hline SPA B7 & $\mathrm{J} 135 \mathrm{~N} 8$ & $9 / 11 / 06$ & 0.064 & $\mathrm{U}$ & 0.49 & 7.08 & $\mathrm{U}$ & 16 & 13.0 & & 0.18 & 0.446 & & 0.043 & 0.728 & & 0.10 \\
\hline SPA B8 & J135N9 & 9/11/06 & 0 & $\mathrm{U}$ & 0.50 & 9.76 & $\mathrm{U}$ & 21 & 13.6 & & 0.33 & 0.561 & & 0.072 & 1.09 & & 0.16 \\
\hline SPA C9 & $\mathrm{J} 135 \mathrm{P} 2$ & $9 / 12 / 06$ & 0 & $\mathrm{U}$ & 0.45 & -1.19 & $\mathrm{U}$ & 18 & 13.0 & & 0.17 & 0.670 & & 0.036 & 0.927 & & 0.082 \\
\hline SPA C10 & $\mathrm{J} 135 \mathrm{P} 3$ & $9 / 12 / 06$ & 0.179 & $\mathrm{U}$ & 0.46 & 4.53 & $\mathrm{U}$ & 21 & 12.6 & & 0.25 & 0.501 & & 0.061 & 0.698 & & 0.14 \\
\hline SPA C1 & $\mathrm{J} 135 \mathrm{P} 4$ & $9 / 12 / 06$ & 0.115 & $\mathrm{U}$ & 0.44 & 6.88 & $\mathrm{U}$ & 22 & 27.4 & & 1.7 & 0.835 & & 0.078 & 1.37 & & 0.21 \\
\hline SPA C2 & J135P5 & $9 / 12 / 06$ & 0.157 & $\mathrm{U}$ & 0.40 & 6.23 & $\mathrm{U}$ & 20 & 23.1 & & 0.34 & 0.878 & & 0.100 & 1.17 & & 0.24 \\
\hline SPA D3 & J135P6 & $9 / 12 / 06$ & 0.106 & $\mathrm{U}$ & 0.41 & 5.04 & $\mathrm{U}$ & 19 & 11.4 & & 0.34 & 0.499 & & 0.069 & 0.729 & & 0.13 \\
\hline SPA D4 & J135P7 & $9 / 12 / 06$ & 0 & $\mathrm{U}$ & 0.57 & 12.5 & $\mathrm{U}$ & 20 & 13.1 & & 0.30 & 0.491 & & 0.053 & 0.769 & & 0.10 \\
\hline SPA D5 & J135P8 & $9 / 12 / 06$ & 0 & $\mathrm{U}$ & 0.48 & 0.953 & $\mathrm{U}$ & 20 & 13.1 & & 0.42 & 0.547 & & 0.100 & 0.779 & & 0.20 \\
\hline SPA D6 & J135P9 & $9 / 12 / 06$ & 0.010 & $\mathrm{U}$ & 0.076 & 4.26 & $\mathrm{U}$ & 19 & 13.1 & & 0.13 & 0.563 & & 0.027 & 0.824 & & 0.062 \\
\hline FS-7 & $\mathrm{J} 12 \mathrm{XM} 4$ & $7 / 26 / 06$ & & & & & & & 7.49 & & 0.58 & 0.256 & & 0.078 & 0.357 & & 0.21 \\
\hline FS-8 & $\mathrm{J} 14 \mathrm{Y} 70$ & $5 / 15 / 07$ & 0.0239 & $\mathrm{U}$ & 0.091 & 7.21 & $\mathrm{U}$ & 27 & 13.2 & & 0.32 & 0.471 & & 0.061 & 0.560 & & 0.14 \\
\hline FS-9 & $\mathrm{J} 14 \mathrm{Y} 7 \mathrm{I}$ & $5 / 15 / 07$ & 0 & $\mathrm{U}$ & 0.28 & 4.00 & $\mathrm{U}$ & 24 & 11.8 & & 0.26 & 0.431 & & 0.035 & 0.636 & & 0.096 \\
\hline FS-10 & $\mathrm{J} 14 \mathrm{Y} 72$ & $5 / 15 / 07$ & 0.0202 & $\mathrm{U}$ & 0.15 & 14.0 & $U$ & 29 & 12.6 & & 0.24 & 0.449 & & 0.044 & 0.670 & & 0.080 \\
\hline FS-11 & $\mathrm{J} 14 \mathrm{Y} 73$ & $5 / 15 / 07$ & 0.204 & & 0.17 & 11.8 & $U$ & 28 & 11.9 & & 0.24 & 0.440 & & 0.044 & 0.744 & & 0.095 \\
\hline FS-12 & $\mathrm{J} 14 \mathrm{Y} 74$ & $5 / 15 / 07$ & 0.0957 & $\mathrm{U}$ & 0.10 & 11.7 & $\mathrm{U}$ & 26 & 12.0 & & 0.16 & 0.441 & & 0.036 & 0.667 & & 0.063 \\
\hline
\end{tabular}

\begin{tabular}{|c|c|c|c|c|c|c|c|c|c|c|c|c|c|c|c|c|c|}
\hline \multirow{2}{*}{ Sample Location } & \multirow{2}{*}{$\begin{array}{c}\text { Sample } \\
\text { Number }\end{array}$} & \multirow{2}{*}{$\begin{array}{l}\text { Sample } \\
\text { Date }\end{array}$} & \multicolumn{3}{|c|}{ Silver-108m } & \multicolumn{3}{|c|}{ Thorium-228 } & \multicolumn{3}{|c|}{ Thorium-232 } & \multicolumn{3}{|c|}{$\begin{array}{c}\text { Total beta } \\
\text { Radiostrontium }\end{array}$} & \multicolumn{3}{|c|}{ Tritium } \\
\hline & & & $\mathrm{pCi} / \mathrm{g}$ & $Q$ & MDA & $\mathrm{pCi} / \mathrm{g}$ & $\mathbf{Q}$ & MDA & $\mathrm{pCi} / \mathrm{g}$ & $\mathbf{Q}$ & MDA & $\mathrm{pCi} / \mathrm{g}$ & Q & MDA & $\mathrm{pCi} / \mathrm{g}$ & $Q$ & MDA \\
\hline Area $1 \mathrm{Al}$ & $34 \mathrm{Y} 7$ & $8 / 17 / 06$ & 0.034 & $\overrightarrow{\mathrm{U}}$ & 0.034 & 1.09 & & 0.063 & 1.04 & & 0.19 & 0.148 & $\mathrm{U}$ & 0.24 & -2.44 & $\mathrm{U}$ & 2.7 \\
\hline Duplicate of J134Y7 & $4 Y 9$ & $7 / 06$ & 0.025 & $\mathrm{U}$ & 0.025 & 0.622 & & 0.049 & 0.530 & & 0.15 & 0.115 & $\mathrm{U}$ & 0.22 & -1.10 & $\mathrm{U}$ & 2.7 \\
\hline Split of J134Y7 & 113504 & $7 / 06$ & \begin{tabular}{|c|}
-0.00909 \\
\end{tabular} & $\mathrm{U}$ & 0.0130 & & & & & & & 0.323 & & 0.149 & 0.00726 & $\mathrm{U}$ & 0.044 \\
\hline Area 1 & 500 & $7 / 06$ & 0.014 & $\mathrm{U}$ & 0.014 & 1.26 & & 5 & 1.34 & & 080 & 0.048 & $\mathrm{U}$ & 0.27 & -1.42 & $\mathrm{U}$ & 3.1 \\
\hline Area $1 \mathrm{~A} 3$ & $\mathrm{~J} 134 \mathrm{Y} 8$ & $8 / 17 / 06$ & 0.018 & $\mathrm{U}$ & 0.018 & 0.705 & & 0.034 & 0.873 & & 0.11 & 0.136 & $\mathrm{U}$ & 0.21 & -1.21 & $\mathrm{U}$ & 2.9 \\
\hline Area $1 \mathrm{~A} 4$ & 3501 & $17 / 06$ & 0.006 & $\mathrm{U}$ & 0.006 & 0.549 & & 0.012 & 0.638 & & 0.040 & 0.049 & $\mathrm{U}$ & 0.24 & -1.56 & $\mathrm{U}$ & 2.7 \\
\hline & & & 0.024 & $\mathrm{U}$ & & 1.05 & & & 1.17 & & 0.1 & 0.009 & $\mathrm{U}$ & 27 & -0.857 & $\mathrm{U}$ & 2.8 \\
\hline Duplic & & & 0.023 & $\mathrm{U}$ & $\overline{0}$ & 1.08 & & 0.043 & 1.13 & & 0.1 & 0.065 & \begin{tabular}{|l|l}
$\mathrm{U}$ \\
\end{tabular} & 0.22 & -1.10 & $\mathrm{U}$ & 3.1 \\
\hline Split of J13502 & 505 & $7 / 06$ & 0.00243 & $\mathrm{U}$ & 0.0112 & & & & & & & 0.0128 & \begin{tabular}{|l|l}
$\mathrm{U}$ \\
\end{tabular} & 0.157 & -0.0323 & $\mathrm{U}$ & 0.0492 \\
\hline Area 2 A2 & 503 & $17 / 06$ & 0.017 & $\mathrm{U}$ & 0.017 & 0.646 & & 0.043 & 0.656 & & 0.096 & 0.114 & \begin{tabular}{|l|}
$\mathrm{U}$ \\
\end{tabular} & 0.22 & -0.836 & $\overline{\mathrm{U}}$ & 2.7 \\
\hline & 3506 & $8 / 17706$ & 0.029 & $\overline{\mathrm{U}}$ & 0.029 & 1.10 & & 0.055 & 1.15 & & 0.1 & 0.035 & \begin{tabular}{|l|}
$\mathrm{U}$ \\
\end{tabular} & 0.2 & -1.24 & $\mathrm{U}$ & 2.7 \\
\hline Area 2 A4 & $\mathrm{J} 13507$ & $8 / 17 / 06$ & 0.026 & $\mathrm{U}$ & 0.026 & 0.647 & & 0.073 & 0.680 & & 0.25 & 0.094 & $\mathrm{U}$ & 0.18 & 7.71 & & 2.8 \\
\hline
\end{tabular}


118-C-1 Verification Sampling Results.

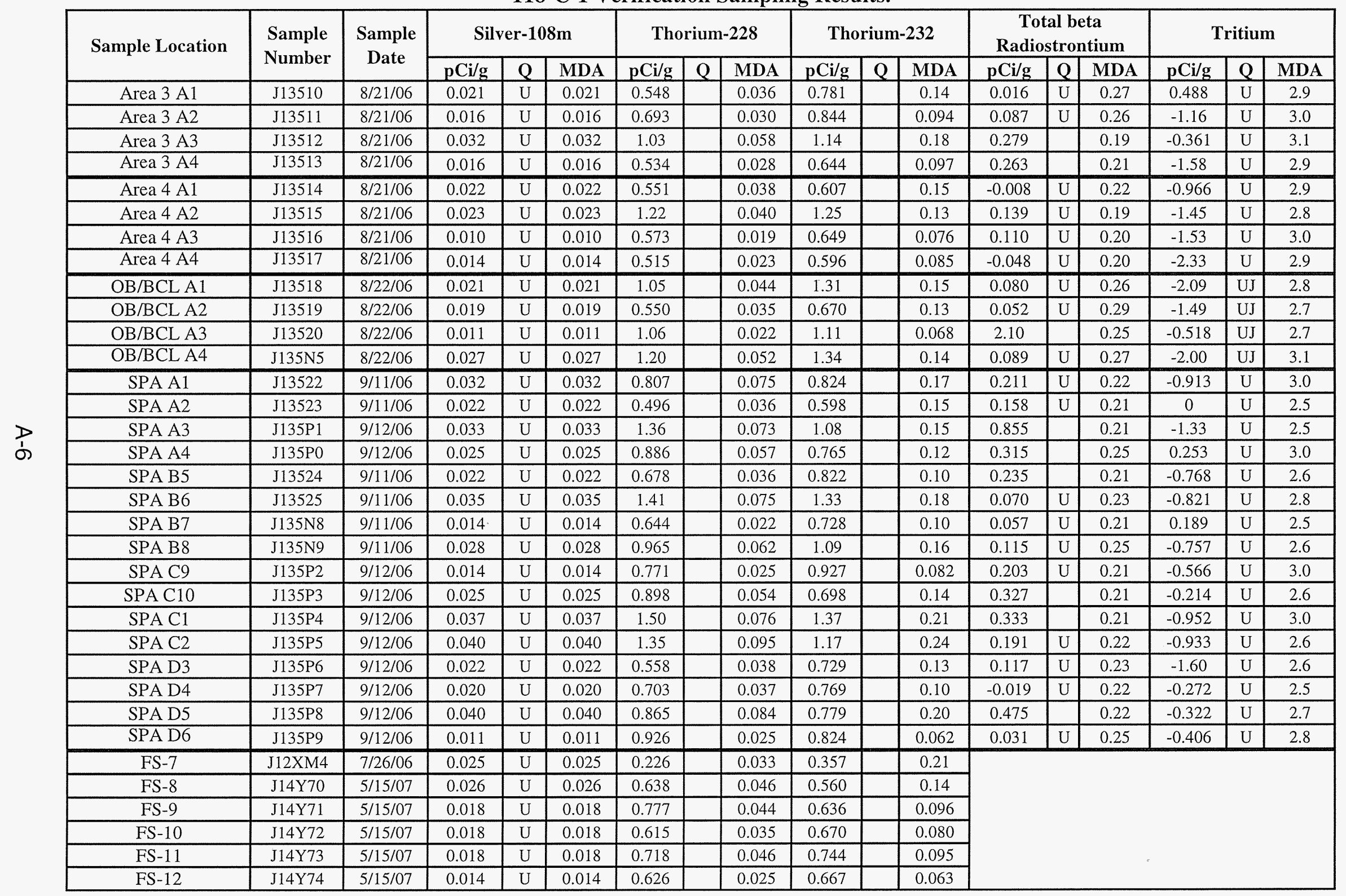


118-C-1 Verification Sampling Results.

\begin{tabular}{|c|c|c|c|c|c|c|c|c|c|c|c|c|c|c|c|c|c|}
\hline \multirow{2}{*}{ Sample Location } & \multirow{2}{*}{$\begin{array}{c}\text { Sample } \\
\text { Number }\end{array}$} & \multirow{2}{*}{$\begin{array}{c}\text { Sample } \\
\text { Date }\end{array}$} & \multicolumn{3}{|c|}{ Uranium-233/234 } & \multicolumn{3}{|c|}{ Uranium-235 } & \multicolumn{3}{|c|}{ Uranium-235 GEA } & \multicolumn{3}{|c|}{ Uranium-238 } & \multicolumn{3}{|c|}{ Uranium-238 GEA } \\
\hline & & & $\mathrm{pCi} / \mathrm{g}$ & $\mathbf{Q}$ & MDA & $\mathrm{pCi} / \mathrm{g}$ & $Q$ & MDA & $\mathrm{pCi} / \mathrm{g}$ & $Q$ & MDA & $\mathrm{pCi} / \mathrm{g}$ & $Q$ & MDA & $\mathrm{pCi} / \mathrm{g}$ & $\mathbf{Q}$ & MDA \\
\hline Area $1 \mathrm{~A} 1$ & $\mathrm{~J} 134 \mathrm{Y7}$ & $8 / 17 / 06$ & 0.338 & & 0.022 & 0.010 & $\bar{U}$ & 0.027 & 0.18 & $\mathrm{U}$ & 0.18 & 0.389 & & 0.022 & 5.3 & $\mathrm{U}$ & 5.3 \\
\hline Duplicate of J134Y7 & J134Y9 & $8 / 17 / 06$ & 0.387 & & 0.026 & 0.027 & & 0.025 & 0.20 & $U$ & 0.20 & 0.415 & & 0.034 & 4.2 & $\mathrm{U}$ & 4.2 \\
\hline Split of J134Y7 & $\mathrm{J} 13504$ & $8 / 17 / 06$ & 0.545 & & 0.0170 & 0.0225 & & 0.0354 & & & & 0.575 & & 0.0300 & & & \\
\hline Area $1 \mathrm{~A} 2$ & $\mathrm{~J} 13500$ & $8 / 17 / 06$ & 0.420 & & 0.031 & 0.024 & $U$ & 0.026 & 0.10 & $U$ & 0.10 & 0.422 & & 0.027 & 2.3 & $\mathrm{U}$ & 2.3 \\
\hline Area $1 \mathrm{~A} 3$ & $\mathrm{~J} 134 \mathrm{Y} 8$ & $8 / 17 / 06$ & 0.527 & & 0.029 & 0.025 & $\mathrm{U}$ & 0.028 & 0.12 & $\mathrm{U}$ & 0.12 & 0.479 & & 0.029 & 2.7 & $\mathrm{U}$ & 2.7 \\
\hline Area $1 \mathrm{~A} 4$ & $\mathrm{~J} 13501$ & $8 / 17 / 06$ & 0.480 & & 0.24 & 0.116 & $\mathrm{U}$ & 0.30 & 0.044 & $\mathrm{U}$ & 0.044 & 0.736 & & 0.24 & 1.1 & $\mathrm{U}$ & 1.1 \\
\hline Area 2 A1 & $\mathrm{J} 13502$ & $8 / 17 / 06$ & & & & & & & 0.13 & U & 0.13 & & & & 4.0 & $\mathrm{U}$ & 4.0 \\
\hline Duplicate of J13502 & $\mathrm{J} 13508$ & $8 / 17 / 06$ & & & & & & & 0.16 & $\mathrm{U}$ & 0.16 & & & & 3.8 & $\mathrm{U}$ & 3.8 \\
\hline Split of J13502 & $\mathrm{J} 13505$ & $8 / 17 / 06$ & & & & & & & & & & & & & & & \\
\hline Area $2 \mathrm{~A} 2$ & $\mathrm{~J} 13503$ & $8 / 17 / 06$ & & & & & & & 0.10 & $\mathrm{U}$ & 0.10 & & & & 2.8 & $\mathrm{U}$ & 2.8 \\
\hline Area 2 A3 & $\mathrm{J} 13506$ & $8 / 17 / 06$ & & & & & & & 0.18 & $\mathrm{U}$ & 0.18 & & & & 5.4 & $\mathrm{U}$ & 5.4 \\
\hline Area 2 A4 & $\mathrm{J} 13507$ & $8 / 17 / 06$ & & & & & & & 0.15 & $\mathrm{U}$ & 0.15 & & & & 5.4 & $\mathrm{U}$ & 5.4 \\
\hline Area $3 \mathrm{~A} 1$ & $\mathrm{~J} 13510$ & $8 / 21 / 06$ & 0.364 & & 0.25 & 0.040 & $\mathrm{U}$ & 0.31 & 0.14 & $\mathrm{U}$ & 0.14 & 0.265 & & 0.25 & 3.4 & $\mathrm{U}$ & 3.4 \\
\hline Area 3 A2 & $\mathrm{J} 13511$ & $8 / 21 / 06$ & 1.09 & & 0.20 & 0.129 & $\mathrm{U}$ & 0.25 & 0.11 & $\mathrm{U}$ & 0.11 & 0.825 & & 0.20 & 2.5 & $\mathrm{U}$ & 2.5 \\
\hline Area $3 \mathrm{~A} 3$ & $\mathrm{~J} 13512$ & $8 / 21 / 06$ & 0.235 & $\mathrm{U}$ & 0.26 & 0.041 & $\mathrm{U}$ & 0.31 & 0.21 & $\mathrm{U}$ & 0.21 & 0.269 & & 0.26 & 4.8 & $\mathrm{U}$ & 4.8 \\
\hline Area 3 A4 & $J 13513$ & $8 / 21 / 06$ & 0.328 & & 0.21 & 0 & $\mathrm{U}$ & 0.25 & 0.099 & $U$ & 0.099 & 0.438 & & 0.21 & 2.1 & $\bar{U}$ & 2.1 \\
\hline Area 4 A1 & $\mathrm{J} 13514$ & $8 / 21 / 06$ & 0.231 & & 0.22 & 0.070 & $U$ & 0.27 & 0.14 & $\mathrm{U}$ & 0.14 & 0.607 & & 0.22 & 3.5 & $\mathrm{U}$ & 3.5 \\
\hline Area 4 A2 & $\mathrm{J} 13515$ & $8 / 21 / 06$ & 0.211 & $\mathrm{U}$ & 0.23 & 0 & $\mathrm{U}$ & 0.28 & 0.19 & $\mathrm{U}$ & 0.19 & 0.362 & & 0.23 & 3.3 & $\mathrm{U}$ & 3.3 \\
\hline Area $4 \mathrm{~A} 3$ & $\mathrm{~J} 13516$ & $8 / 21 / 06$ & 0.537 & & 0.26 & 0.041 & $\mathrm{U}$ & 0.31 & 0.069 & $\mathrm{U}$ & 0.069 & 0.671 & & 0.26 & 1.7 & $\mathrm{U}$ & 1.7 \\
\hline Area 4 A4 & $\mathrm{J} 13517$ & $8 / 21 / 06$ & 0.422 & & 0.29 & 0 & $\mathrm{U}$ & 0.36 & 0.091 & $\mathrm{U}$ & 0.091 & 0.268 & $\mathrm{U}$ & 0.29 & 2.2 & $\mathrm{U}$ & 2.2 \\
\hline OB/BCL A1 & $\mathrm{J} 13518$ & $8 / 22 / 06$ & 0.430 & & 0.13 & 0.020 & U & 0.15 & 0.19 & $\mathrm{U}$ & 0.19 & 0.480 & & 0.13 & 3.4 & $\mathrm{U}$ & 3.4 \\
\hline $\mathrm{OB} / \mathrm{BCL} \mathrm{A} 2$ & $\mathrm{~J} 13519$ & $8 / 22 / 06$ & 0.428 & & 0.13 & 0.020 & $\mathrm{U}$ & 0.15 & 0.13 & $\mathrm{U}$ & 0.13 & 0.478 & & 0.13 & 3.4 & $\mathrm{U}$ & 3.4 \\
\hline $\mathrm{OB} / \mathrm{BCL} \mathrm{A} 3$ & $\mathrm{~J} 13520$ & $8 / 22 / 06$ & 0.575 & & 0.13 & 0 & $\mathrm{U}$ & 0.16 & 0.072 & $\mathrm{U}$ & 0.072 & 0.508 & & 0.13 & 4.4 & $\mathrm{U}$ & 4.4 \\
\hline OB/BCL A4 & J135N5 & $8 / 22 / 06$ & 0.383 & & 0.12 & 0 & $\mathrm{U}$ & 0.14 & 0.19 & $U$ & 0.19 & 0.399 & & 0.12 & 4.7 & $\bar{U}$ & 4.7 \\
\hline
\end{tabular}


118-C-1 Verification Sampling Results.

\begin{tabular}{|c|c|c|c|c|c|c|c|c|c|c|c|c|c|c|c|c|c|}
\hline \multirow{2}{*}{ Sample Location } & \multirow{2}{*}{$\begin{array}{l}\text { Sample } \\
\text { Number }\end{array}$} & \multirow{2}{*}{$\begin{array}{c}\text { Sample } \\
\text { Date }\end{array}$} & \multicolumn{3}{|c|}{ Uranium-233/234 } & \multicolumn{3}{|c|}{ Uranium-235 } & \multicolumn{3}{|c|}{ Uranium-235 GEA } & \multicolumn{3}{|c|}{ Uranium-238 } & \multicolumn{3}{|c|}{ Uranium-238 GEA } \\
\hline & & & $\mathrm{pCi} / \mathrm{g}$ & $Q$ & MDA & $\mathrm{pCi} / \mathrm{g}$ & $Q$ & MDA & $\mathrm{pCi} / \mathrm{g}$ & $Q$ & MDA & $\mathrm{pCi} / \mathrm{g}$ & $\mathbf{Q}$ & MDA & $\mathrm{pCi} / \mathrm{g}$ & $\mathbf{Q}$ & MDA \\
\hline SPA A1 & $\mathrm{J} 13522$ & $9 / 11 / 06$ & 0.415 & & 0.21 & 0.067 & $\mathrm{U}$ & 0.26 & 0.15 & $\mathrm{U}$ & 0.15 & 0.663 & & 0.21 & 4.5 & $\mathrm{U}$ & 4.5 \\
\hline SPA A2 & $\mathrm{J} 13523$ & $9 / 11 / 06$ & 0.364 & & 0.25 & 0.040 & $\mathrm{U}$ & 0.31 & 0.14 & $\mathrm{U}$ & 0.14 & 0.398 & & 0.25 & 4.2 & $\mathrm{U}$ & 4.2 \\
\hline SPA A3 & $\mathrm{J} 135 \mathrm{P} 1$ & $9 / 12 / 06$ & 0.406 & & 0.26 & 0 & $\mathrm{U}$ & 0.31 & 0.18 & $\mathrm{U}$ & 0.18 & 0.541 & & 0.26 & 4.2 & $\mathrm{U}$ & 4.2 \\
\hline SPA A4 & $\mathrm{J} 135 \mathrm{P} 0$ & $9 / 12 / 06$ & 0.387 & & 0.25 & 0.039 & $\mathrm{U}$ & 0.30 & 0.11 & $\mathrm{U}$ & 0.11 & 0.646 & & 0.25 & 3.7 & $\mathrm{U}$ & 3.7 \\
\hline SPA B5 & J13524 & $9 / 11 / 06$ & 0.448 & & 0.21 & 0 & $\mathrm{U}$ & 0.26 & 0.14 & $\mathrm{U}$ & 0.14 & 0.811 & & 0.21 & 3.0 & $U$ & 3.0 \\
\hline SPA B6 & $\mathrm{J} 13525$ & $9 / 11 / 06$ & 0.415 & & 0.23 & 0 & $\mathrm{U}$ & 0.27 & 0.22 & $\mathrm{U}$ & 0.22 & 0.237 & & 0.23 & 5.1 & $U$ & 5.1 \\
\hline SPA B7 & J135N8 & $9 / 11 / 06$ & 0.276 & & 0.26 & 0.042 & $\mathrm{U}$ & 0.32 & 0.072 & $\mathrm{U}$ & 0.072 & 0.518 & & 0.26 & 2.7 & $\mathrm{U}$ & 2.7 \\
\hline SPA B8 & J135N9 & $9 / 11 / 06$ & 0.320 & & 0.22 & 0.035 & $\mathrm{U}$ & 0.27 & 0.13 & $\mathrm{U}$ & 0.13 & 0.640 & & 0.22 & 4.1 & $\mathrm{U}$ & 4.1 \\
\hline SPA C9 & $\mathrm{J} 135 \mathrm{P} 2$ & $9 / 12 / 06$ & 0.626 & & 0.039 & 0.045 & & 0.038 & 0.12 & $\mathrm{U}$ & 0.12 & 0.498 & & 0.039 & 2.0 & $\mathrm{U}$ & 2.0 \\
\hline SPA C10 & $\mathrm{J} 135 \mathrm{P} 3$ & $9 / 12 / 06$ & 0.523 & & 0.033 & 0.047 & & 0.040 & 0.11 & $\mathrm{U}$ & 0.11 & 0.554 & & 0.033 & 3.3 & $\mathrm{U}$ & 3.3 \\
\hline SPA C1 & J135P4 & $9 / 12 / 06$ & 0.467 & & 0.042 & 0.011 & $\mathrm{U}$ & 0.041 & 0.23 & $\mathrm{U}$ & 0.23 & 0.370 & & 0.034 & 5.6 & $\mathrm{U}$ & 5.6 \\
\hline SPA C2 & J135P5 & $9 / 12 / 06$ & 0.467 & & 0.032 & 0.035 & $\mathrm{U}$ & 0.039 & 0.26 & $\mathrm{U}$ & 0.26 & 0.396 & & 0.032 & 6.4 & $\mathrm{U}$ & 6.4 \\
\hline SPA D3 & J135P6 & $9 / 12 / 06$ & 0.552 & & 0.047 & 0.036 & $\mathrm{U}$ & 0.045 & 0.15 & $\mathrm{U}$ & 0.15 & 0.489 & & 0.047 & 4.2 & $\mathrm{U}$ & 4.2 \\
\hline SPA D4 & $\mathrm{J} 135 \mathrm{P} 7$ & $9 / 12 / 06$ & 0.524 & & 0.038 & 0.043 & & 0.037 & 0.14 & $\mathrm{U}$ & 0.14 & 0.497 & & 0.030 & 3.2 & $\mathrm{U}$ & 3.2 \\
\hline SPA D5 & $\mathrm{J} 135 \mathrm{P} 8$ & $9 / 12 / 06$ & 0.484 & & 0.066 & 0.042 & & 0.040 & 0.17 & $\mathrm{U}$ & 0.17 & 0.510 & & 0.033 & 5.4 & $\mathrm{U}$ & 5.4 \\
\hline SPA D6 & J135P9 & $9 / 12 / 06$ & 0.577 & & 0.19 & 0 & $\mathrm{U}$ & 0.23 & 0.077 & $\mathrm{U}$ & 0.077 & 0.426 & & 0.19 & 1.6 & $\mathrm{U}$ & 1.6 \\
\hline FS-7 & $\mathrm{J} 12 \mathrm{XM} 4$ & $7 / 26 / 06$ & & & & & & & 0.068 & $\mathrm{U}$ & 0.068 & & & & 6.4 & $\mathrm{U}$ & 6.4 \\
\hline FS-8 & $\mathrm{J} 14 \mathrm{Y} 70$ & $5 / 15 / 07$ & 0.483 & & 0.18 & 0.0278 & U & 0.21 & 0.15 & $\mathrm{U}$ & 0.15 & 0.483 & & 0.18 & 4.0 & $\mathrm{U}$ & 4.0 \\
\hline FS-9 & J14Y71 & $5 / 15 / 07$ & 0.339 & & 0.24 & 0.0373 & $U$ & 0.29 & 0.16 & $\mathrm{U}$ & 0.16 & 0.462 & & 0.24 & 2.9 & $\mathrm{U}$ & 2.9 \\
\hline FS-10 & $\mathrm{J} 14 \mathrm{Y} 72$ & $5 / 15 / 07$ & 0.499 & & 0.20 & 0.0636 & $U$ & 0.24 & 0.11 & $\mathrm{U}$ & 0.11 & 0.473 & & 0.20 & 3.0 & $\mathrm{U}$ & 3.0 \\
\hline FS-11 & $\mathrm{J} 14 \mathrm{Y} 73$ & $5 / 15 / 07$ & 0.306 & & 0.23 & 0 & $\mathrm{U}$ & 0.28 & 0.11 & $\mathrm{U}$ & 0.11 & 0.428 & & 0.23 & 2.6 & $\mathrm{U}$ & 2.6 \\
\hline FS-12 & $\mathrm{J} 14 \mathrm{Y} 74$ & $5 / 15 / 07$ & 0.681 & & 0.24 & 0.0750 & & 0.29 & 0.076 & $\mathrm{U}$ & 0.076 & 0.434 & & 0.24 & 2.1 & $\mathrm{U}$ & 2.1 \\
\hline
\end{tabular}


118-C-1 Verification Sampling Results.

\begin{tabular}{|c|c|c|c|c|c|c|c|c|c|c|c|c|c|c|c|c|c|c|c|c|}
\hline \multirow{2}{*}{ Sample Location } & \multirow{2}{*}{$\begin{array}{c}\text { Sample } \\
\text { Number }\end{array}$} & \multirow{2}{*}{$\begin{array}{c}\text { Sample } \\
\text { Date }\end{array}$} & \multicolumn{3}{|c|}{ Aluminum } & \multicolumn{3}{|c|}{ Antimony } & \multicolumn{3}{|c|}{ Arsenic } & \multicolumn{3}{|c|}{ Barium } & \multicolumn{3}{|c|}{ Beryllium } & \multicolumn{3}{|c|}{ Boron } \\
\hline & & & $\mathrm{mg} / \mathrm{kg}$ & Q & PQL & $\mathrm{mg} / \mathrm{kg}$ & Q & PQL & $\mathrm{mg} / \mathrm{kg}$ & $Q$ & PQL & $\mathrm{mg} / \mathrm{kg}$ & Q & PQL & $\mathrm{mg} / \mathrm{kg}$ & $Q$ & PQL & $\mathrm{mg} / \mathrm{kg}$ & Q & PQL \\
\hline Area $1 \mathrm{~A} 1$ & $\mathrm{~J} 134 \mathrm{Y} 7$ & $8 / 17 / 06$ & 3880 & & 8.0 & 1.2 & $\mathrm{U}$ & 1.2 & 2.7 & & 1.7 & 48.1 & & 0.06 & 0.78 & & 0.06 & 1.3 & & 0.66 \\
\hline Duplicate of J134Y7 & J134Y9 & $8 / 17 / 06$ & 4500 & & 7.9 & 1.2 & $\mathrm{U}$ & 1.2 & 2.3 & & 1.7 & 51.5 & & 0.05 & 0.82 & & 0.05 & 1.7 & & 0.66 \\
\hline Split of J134Y7 & $\mathrm{J} 13504$ & $8 / 17 / 06$ & 6610 & $\mathrm{~N}$ & 6.3 & 1.2 & $\mathrm{~N}$ & 0.33 & 2.6 & & 0.28 & 57.5 & & 0.50 & 0.34 & $\mathrm{~B}^{*}$ & 0.071 & 1.6 & $\mathrm{~B}^{*}$ & 1.5 \\
\hline Area $1 \mathrm{~A} 2$ & $\mathrm{~J} 13500$ & $8 / 17 / 06$ & 4130 & & 8.0 & 1.2 & $\mathrm{U}$ & 1.2 & 1.9 & & 1.7 & 52.0 & & 0.06 & 0.69 & & 0.06 & 1.6 & & 0.66 \\
\hline Area $1 \mathrm{~A} 3$ & $\mathrm{~J} 134 \mathrm{Y} 8$ & $8 / 17 / 06$ & 4570 & & 8.0 & 1.2 & $\mathrm{U}$ & 1.2 & 2.3 & & 1.7 & 56.0 & & 0.06 & 0.69 & & 0.06 & 2.1 & & 0.67 \\
\hline Area $1 \mathrm{~A} 4$ & $\mathrm{~J} 13501$ & $8 / 17 / 06$ & 4460 & & 7.9 & 1.2 & $\mathrm{U}$ & 1.2 & 3.3 & & 1.7 & 48.7 & & 0.06 & 0.88 & & 0.06 & 1.6 & & 0.66 \\
\hline Area 2 A1 & $\mathrm{J} 13502$ & $8 / 17 / 06$ & 4690 & & 7.9 & 1.2 & $\mathrm{U}$ & 1.2 & 2.3 & & 1.7 & 48.1 & & 0.06 & 0.70 & & 0.06 & 1.9 & & 0.66 \\
\hline Duplicate of J13502 & $\mathrm{J} 13508$ & $8 / 17 / 06$ & 4090 & & 8.0 & 1.2 & $\mathrm{U}$ & 1.2 & 2.4 & & 1.7 & 48.4 & & 0.06 & 0.86 & & 0.06 & 1.7 & & 0.67 \\
\hline Split of J13502 & $\mathrm{J} 13505$ & $8 / 17 / 06$ & 6210 & $\mathrm{~N}$ & 6.3 & 1.5 & $\mathrm{~N}$ & 0.33 & 3.1 & & 0.28 & 54.7 & & 0.50 & 0.34 & $\mathrm{~B}^{*}$ & 0.071 & 1.9 & $\mathrm{~B}^{*}$ & 1.5 \\
\hline Area $2 \mathrm{~A} 2$ & $\mathrm{~J} 13503$ & $8 / 17 / 06$ & 4310 & & 8.0 & 1.2 & $\mathrm{U}$ & 1.2 & 2.8 & & 1.7 & 45.6 & & 0.06 & 0.78 & & 0.06 & 2.0 & & 0.66 \\
\hline Area $2 \mathrm{~A} 3$ & $\mathrm{~J} 13506$ & $8 / 17 / 06$ & 4050 & & 8.0 & 1.2 & $\mathrm{U}$ & 1.2 & 2.0 & & 1.7 & 44.4 & & 0.06 & 0.68 & & 0.06 & 2.2 & & 0.66 \\
\hline Area 2 A4 & $\mathrm{J} 13507$ & $8 / 17 / 06$ & 3990 & & 7.9 & 1.2 & $\mathrm{U}$ & 1.2 & 2.4 & & 1.7 & 54.5 & & 0.05 & 0.93 & & 0.05 & 1.5 & & 0.66 \\
\hline Area $3 \mathrm{Al}$ & $\mathrm{J} 13510$ & $8 / 21 / 06$ & 5540 & & 7.9 & 1.2 & $\mathrm{U}$ & 1.2 & 1.8 & & 1.7 & 63.8 & & 0.06 & 0.70 & & 0.06 & 1.6 & $J$ & 0.66 \\
\hline Area $3 \mathrm{A2}$ & $\mathrm{J} 13511$ & $8 / 21 / 06$ & 4550 & & 8.0 & 4.3 & & 1.2 & 33.9 & & 1.7 & 132 & & 0.06 & 0.50 & & 0.06 & 4.6 & $\mathrm{~J}$ & 0.66 \\
\hline Area 3 A2 resample & J13HN3 & $9 / 29 / 06$ & 4950 & $\mathrm{C}$ & 7.0 & 1.1 & $\mathrm{U}$ & 1.1 & 5.8 & & 1.1 & 71.3 & $\mathrm{C}$ & 0.06 & 0.28 & & 0.03 & 2.1 & & 1.1 \\
\hline Area $3 \mathrm{A3}$ & $\mathrm{J} 13512$ & $8 / 21 / 06$ & 5040 & & 7.9 & 1.2 & $\mathrm{U}$ & 1.2 & 2.2 & & 1.7 & 78.4 & & 0.06 & 0.51 & & 0.06 & 3.2 & $\mathrm{~J}$ & 0.66 \\
\hline Area $3 \mathrm{A4}$ & $\mathrm{J} 13513$ & $8 / 21 / 06$ & 4700 & & 7.9 & 1.2 & $\mathrm{U}$ & 1.2 & 2.0 & & 1.7 & 55.5 & & 0.05 & 0.52 & & 0.05 & 1.5 & $\mathrm{~J}$ & 0.66 \\
\hline Area 4 Al & $\mathrm{J13514}$ & $8 / 21 / 06$ & 4880 & & 7.9 & 1.2 & $\mathrm{U}$ & 1.2 & 2.1 & & 1.7 & 48.6 & & 0.05 & 0.58 & & 0.05 & 1.0 & $\mathrm{~J}$ & 0.66 \\
\hline Area 4 A2 & $\mathrm{J} 13515$ & $8 / 21 / 06$ & 5100 & & 7.9 & 1.2 & $\mathrm{U}$ & 1.2 & 2.3 & & 1.7 & 286 & & 0.05 & 0.58 & & 0.05 & 4.9 & $\mathrm{~J}$ & 0.66 \\
\hline Area 4 A3 & $\mathrm{J} 13516$ & $8 / 21 / 06$ & 4080 & & 7.9 & 1.2 & $\mathrm{U}$ & 1.2 & 2.4 & & 1.7 & 49.9 & & 0.05 & 0.37 & & 0.05 & 0.65 & UJ & 0.65 \\
\hline Area 4 A4 & $\mathrm{J} 13517$ & $8 / 21 / 06$ & 4070 & & 7.9 & 1.2 & $U$ & 1.2 & 2.2 & & 1.7 & 49.2 & & 0.06 & 0.43 & & 0.06 & 0.66 & UJ & 0.66 \\
\hline OB/BCL A1 & $\mathrm{J} 13518$ & $8 / 22 / 06$ & 4660 & & 8.3 & 1.3 & UJ & 1.3 & 2.2 & & 1.8 & 48.7 & & 0.06 & 0.65 & & 0.06 & 2.1 & UJ & 0.69 \\
\hline $\mathrm{OB} / \mathrm{BCL} \mathrm{A} 2$ & $\mathrm{~J} 13519$ & $8 / 22 / 06$ & 4740 & & 8.4 & 1.3 & $\mathrm{UJ}$ & 1.3 & 2.2 & & 1.8 & 50.0 & & 0.06 & 0.69 & & 0.06 & 1.8 & UJ & 0.70 \\
\hline $\mathrm{OB} / \mathrm{BCL} \mathrm{A} 3$ & $\mathrm{~J} 13520$ & $8 / 22 / 06$ & 3690 & & 8.4 & 1.3 & UJ & 1.3 & 1.8 & $\mathrm{U}$ & 1.8 & 46.0 & & 0.06 & 0.60 & & 0.06 & 1.6 & UJ & 0.70 \\
\hline $\mathrm{OB} / \mathrm{BCL} \mathrm{A} 4$ & J135N5 & $8 / 22 / 06$ & 4760 & & 8.4 & 1.3 & $\mathrm{UJ}$ & 1.3 & 2.3 & & 1.8 & 58.4 & & 0.06 & 0.52 & & 0.06 & 2.0 & UJ & 0.70 \\
\hline SPA A1 & $\mathrm{J} 13522$ & $9 / 11 / 06$ & 5120 & & 6.6 & 1.0 & $\mathrm{U}$ & 1.0 & 3.0 & & 1.1 & 52.9 & & 0.06 & 0.43 & & 0.03 & 1.2 & & 1.1 \\
\hline SPA A2 & $\mathrm{J} 13523$ & $9 / 11 / 06$ & 5020 & & 6.6 & 1.0 & $\mathrm{U}$ & 1.0 & 2.5 & & 1.1 & 53.4 & & 0.06 & 0.31 & & 0.03 & 1.1 & $\mathrm{U}$ & 1.1 \\
\hline SPA A3 & $\mathrm{J} 135 \mathrm{P} 1$ & $9 / 12 / 06$ & 5300 & & 6.5 & 1.0 & $\mathrm{U}$ & 1.0 & 2.3 & & 1.1 & 53.3 & & 0.06 & 0.37 & & 0.03 & 1.7 & & 1.1 \\
\hline SPA A4 & $\mathrm{J} 135 \mathrm{P} 0$ & $9 / 12 / 06$ & 4600 & & 6.5 & 1.0 & $\mathrm{U}$ & 1.0 & 3.3 & & 1.1 & 53.5 & & 0.06 & 0.31 & & 0.03 & 2.0 & & 1.1 \\
\hline SPA B5 & $\mathrm{J} 13524$ & $9 / 11 / 06$ & 5130 & & 6.6 & 1.0 & $\mathrm{U}$ & 1.0 & 2.4 & & 1.1 & 55.6 & & 0.06 & 0.31 & & 0.03 & 1.1 & $\mathrm{U}$ & 1.1 \\
\hline SPA B6 & $\mathrm{J} 13525$ & $9 / 11 / 06$ & 4830 & & 6.6 & 1.0 & $\mathrm{U}$ & 1.0 & 2.3 & & 1.1 & 54.9 & & 0.06 & 0.34 & & 0.03 & 1.1 & $\mathrm{U}$ & 1.1 \\
\hline SPA B7 & $\mathrm{J} 135 \mathrm{~N} 8$ & $9 / 11 / 06$ & 5310 & & 6.6 & 1.0 & $\mathrm{U}$ & 1.0 & 2.4 & & 1.1 & 54.7 & & 0.06 & 0.35 & & 0.03 & 1.1 & $\mathrm{U}$ & 1.1 \\
\hline SPA B8 & J135N9 & $9 / 11 / 06$ & 4900 & & 6.5 & 1.0 & $\mathrm{U}$ & 1.0 & 2.9 & & 1.1 & 60.1 & & 0.06 & 0.30 & & 0.03 & 1.1 & $\mathrm{U}$ & 1.1 \\
\hline SPA C9 & $\mathrm{J} 135 \mathrm{P} 2$ & $9 / 12 / 06$ & 6390 & & 6.5 & 1.0 & $U$ & 1.0 & 1.8 & & 1.1 & 495 & & 0.06 & 0.57 & & 0.03 & 10.7 & & 1.1 \\
\hline SPA C10 & $\mathrm{J} 135 \mathrm{P} 3$ & $9 / 12 / 06$ & 4580 & & 6.6 & 1.0 & $\mathrm{U}$ & 1.0 & 2.0 & & 1.1 & 60.0 & & 0.06 & 0.36 & & 0.03 & 1.1 & $\mathrm{U}$ & 1.1 \\
\hline SPA C1 & $\mathrm{J} 135 \mathrm{P} 4$ & $9 / 12 / 06$ & 5100 & & 6.5 & 1.0 & $\mathrm{U}$ & 1.0 & 2.8 & & 1.1 & 58.2 & & 0.06 & 0.41 & & 0.03 & 2.4 & & 1.1 \\
\hline SPA C2 & J135P5 & $9 / 12 / 06$ & 4840 & & 6.6 & 1.0 & $\mathrm{U}$ & 1.0 & 2.6 & & 1.1 & 62.9 & & 0.06 & 0.41 & & 0.03 & 2.6 & & 1.1 \\
\hline SPAD3 & J135P6 & $9 / 12 / 06$ & 5000 & & 6.5 & 1.0 & $\mathrm{U}$ & 1.0 & 3.0 & & 1.1 & 63.8 & & 0.06 & 0.44 & & 0.03 & 1.5 & & 1.1 \\
\hline SPA D4 & $\mathrm{J} 135 \mathrm{P} 7$ & $9 / 12 / 06$ & 4870 & & 6.6 & 1.0 & $\mathrm{U}$ & 1.0 & 2.0 & & 1.1 & 56.0 & & 0.06 & 0.42 & & 0.03 & 1.1 & & 1.1 \\
\hline SPA D5 & $\mathrm{J} 135 \mathrm{P} 8$ & $9 / 12 / 06$ & 5540 & & 6.5 & 1.0 & $\mathrm{U}$ & 1.0 & 2.5 & & 1.1 & 59.4 & & 0.06 & 0.47 & & 0.03 & 1.1 & $\mathrm{U}$ & 1.1 \\
\hline SPA D6 & $\mathrm{J} 135 \mathrm{P} 9$ & $9 / 12 / 06$ & 5540 & & 6.5 & 1.0 & $\mathrm{U}$ & 1.0 & 2.9 & & 1.1 & 64.8 & & 0.06 & 0.49 & & 0.03 & 1.4 & & 1.1 \\
\hline
\end{tabular}


118-C-1 Verification Sampling Results.

\begin{tabular}{|c|c|c|c|c|c|c|c|c|c|c|c|c|c|c|c|c|c|c|c|c|}
\hline \multirow{2}{*}{ Sample Location } & \multirow{2}{*}{$\begin{array}{c}\text { Sample } \\
\text { Number }\end{array}$} & \multirow{2}{*}{$\begin{array}{c}\text { Sample } \\
\text { Date }\end{array}$} & \multicolumn{3}{|c|}{ Aluminum } & \multicolumn{3}{|c|}{ Antimony } & \multicolumn{3}{|c|}{ Arsenic } & \multicolumn{3}{|c|}{ Barium } & \multicolumn{3}{|c|}{ Beryllium } & \multicolumn{3}{|c|}{ Boron } \\
\hline & & & $\mathrm{mg} / \mathrm{kg}$ & $Q$ & PQL & $\mathrm{mg} / \mathrm{kg}$ & Q & PQL & $\mathrm{mg} / \mathrm{kg}$ & Q & PQL & $\mathrm{mg} / \mathrm{kg}$ & Q & PQL & $\mathrm{mg} / \mathrm{kg}$ & Q & PQL & $\mathrm{mg} / \mathrm{kg}$ & $\mathbf{Q}$ & PQL \\
\hline FS-1 & $\mathrm{J} 12 \mathrm{XL} 8$ & $7 / 26 / 06$ & 5510 & & 8.3 & 1.3 & $\mathrm{U}$ & 1.3 & 2.2 & & 1.8 & 59.6 & $\mathrm{C}$ & 0.06 & 0.23 & & 0.06 & 1.9 & & 0.69 \\
\hline FS-2 & J12XL9 & $7 / 26 / 06$ & 4040 & & 8.3 & 1.3 & $\mathrm{U}$ & 1.3 & 3.5 & & 1.8 & 40.5 & $\mathrm{C}$ & 0.06 & 0.18 & & 0.06 & 1.4 & & 0.69 \\
\hline FS-3 & $\mathrm{J} 12 \mathrm{XM0}$ & $7 / 26 / 06$ & 3630 & & 8.2 & 1.3 & $\mathrm{U}$ & 1.3 & 2.5 & & 1.7 & 41.8 & C & 0.06 & 0.16 & & 0.06 & 1.2 & & 0.69 \\
\hline FS-4 & $\mathrm{J} 12 \mathrm{XM} 1$ & $7 / 26 / 06$ & 5670 & & 8.4 & 1.3 & $\mathrm{U}$ & 1.3 & 5.4 & & 1.8 & 47.7 & $\mathrm{C}$ & 0.06 & 0.19 & & 0.06 & 1.9 & & 0.70 \\
\hline FS-5 & $\mathrm{J} 12 \mathrm{XM} 2$ & $7 / 26 / 06$ & 7520 & & 8.3 & 1.3 & $\mathrm{U}$ & 1.3 & 3.4 & & 1.8 & 80.2 & $\mathrm{C}$ & 0.06 & 0.34 & & 0.06 & 3.2 & & 0.69 \\
\hline FS-7 & $\mathrm{J} 12 \mathrm{XM} 4$ & $7 / 26 / 06$ & 3160 & & 8.4 & 1.3 & $\mathrm{U}$ & 1.3 & 1.8 & $\mathrm{U}$ & 1.8 & 34.3 & C & 0.06 & 0.12 & & 0.06 & 2.5 & & 0.70 \\
\hline Equipment blank & $\mathrm{J} 13509$ & $8 / 17 / 06$ & 39.4 & & 2.6 & 0.40 & $\mathrm{U}$ & 0.40 & 0.56 & $\mathrm{U}$ & 0.56 & 1.1 & & 0.02 & 0.02 & & 0.02 & 0.42 & & 0.22 \\
\hline
\end{tabular}

\begin{tabular}{|c|c|c|c|c|c|c|c|c|c|c|c|c|c|c|c|c|c|c|c|c|}
\hline \multirow[t]{2}{*}{ Sample Location } & \multirow{2}{*}{$\begin{array}{c}\text { Sample } \\
\text { Number }\end{array}$} & \multirow{2}{*}{$\begin{array}{c}\text { Sample } \\
\text { Date }\end{array}$} & \multicolumn{3}{|c|}{ Cadmium } & \multicolumn{3}{|c|}{ Calcium } & \multicolumn{3}{|c|}{ Chromium } & \multicolumn{3}{|c|}{ Cobalt } & \multicolumn{3}{|c|}{ Copper } & \multicolumn{3}{|c|}{$\begin{array}{l}\text { Hexavalent } \\
\text { Chromium }\end{array}$} \\
\hline & & & $\mathrm{mg} / \mathrm{kg}$ & $Q$ & PQL & $\mathrm{mg} / \mathrm{kg}$ & $Q$ & PQL & $\mathrm{mg} / \mathrm{kg}$ & $Q$ & PQL & $\mathrm{mg} / \mathrm{kg}$ & $Q$ & PQL & $\mathrm{mg} / \mathrm{kg}$ & $Q$ & PQL & $\mathrm{mg} / \mathrm{kg}$ & $Q$ & PQL \\
\hline Area $1 \mathrm{Al}$ & $\mathrm{J} 134 \mathrm{Y7}$ & $8 / 17 / 06$ & 0.19 & $\mathrm{U}$ & 0.19 & 4380 & & 4.5 & 5.6 & $\mathrm{C}$ & 0.36 & 7.0 & & 0.39 & 11.7 & & 0.33 & 0.20 & $\mathrm{U}$ & 0.20 \\
\hline Duplicate of J134Y7 & J134Y9 & $8 / 17 / 06$ & 0.19 & $\mathrm{U}$ & 0.19 & 4570 & & 4.5 & 6.6 & $\mathrm{C}$ & 0.36 & 7.9 & & 0.38 & 12.7 & & 0.33 & 0.20 & $\mathrm{U}$ & 0.20 \\
\hline Split of J134Y7 & $\mathrm{J} 13504$ & $8 / 17 / 06$ & 0.14 & UN & 0.14 & 5080 & $\mathrm{CN}$ & 8.6 & 9.1 & $\mathrm{~N}$ & 0.36 & 11.8 & & 0.50 & 13.2 & & 0.30 & 0.350 & $\mathrm{U}$ & 0.350 \\
\hline Area $1 \mathrm{~A} 2$ & $\mathrm{~J} 13500$ & $8 / 17 / 06$ & 0.19 & $\mathrm{U}$ & 0.19 & 3820 & & 4.5 & 8.6 & $\mathrm{C}$ & 0.36 & 5.9 & & 0.39 & 9.9 & & 0.33 & 0.20 & $\mathrm{U}$ & 0.20 \\
\hline Area $1 \mathrm{~A} 3$ & $\mathrm{~J} 134 \mathrm{Y} 8$ & $8 / 17 / 06$ & 0.20 & $\mathrm{U}$ & 0.20 & 6890 & & 4.6 & 6.8 & $\mathrm{C}$ & 0.36 & 5.7 & & 0.39 & 11.3 & & 0.33 & 0.20 & $\mathrm{U}$ & 0.20 \\
\hline Area $1 \mathrm{~A} 4$ & $\mathrm{~J} 13501$ & $8 / 17 / 06$ & 0.19 & $\mathrm{U}$ & 0.19 & 8720 & & 4.5 & 6.0 & $\mathrm{C}$ & 0.36 & 8.4 & & 0.39 & 14.7 & & 0.33 & 0.20 & $\mathrm{U}$ & 0.20 \\
\hline Area $2 \mathrm{Al}$ & $\mathrm{J} 13502$ & $8 / 17 / 06$ & 0.19 & $\mathrm{U}$ & 0.19 & 6960 & & 4.5 & 6.2 & $\mathrm{C}$ & 0.36 & 7.6 & & 0.39 & 13.4 & & 0.33 & 0.20 & $\mathrm{U}$ & 0.20 \\
\hline Duplicate of J13502 & $\mathrm{J} 13508$ & $8 / 17 / 06$ & 0.20 & $\mathrm{U}$ & 0.20 & 6310 & & 4.6 & 5.8 & $\mathrm{C}$ & 0.36 & 7.5 & & 0.39 & 13.4 & & 0.34 & 0.21 & & 0.20 \\
\hline Split of J13502 & $\mathrm{J} 13505$ & $8 / 17 / 06$ & 0.14 & UN & 0.14 & 7420 & $\mathrm{CN}$ & 8.6 & 8.5 & $\mathrm{~N}$ & 0.36 & 12.9 & & 0.50 & 14.8 & & 0.30 & 0.350 & $\mathrm{U}$ & 0.350 \\
\hline Area $2 \mathrm{~A} 2$ & $\mathrm{~J} 13503$ & $8 / 17 / 06$ & 0.19 & $\mathrm{U}$ & 0.09 & 6810 & & 4.5 & 6.2 & $\mathrm{C}$ & 0.36 & 7.3 & & 0.39 & 12.8 & & 0.33 & 0.24 & & 0.20 \\
\hline Area $2 \mathrm{~A} 3$ & $\mathrm{~J} 13506$ & $8 / 17 / 06$ & 0.19 & $\mathrm{U}$ & 0.19 & 3560 & & 4.5 & 5.4 & $\mathrm{C}$ & 0.36 & 6.8 & & 0.39 & 10.5 & & 0.33 & 0.20 & $\mathrm{U}$ & 0.20 \\
\hline Area 2 A4 & $\mathrm{J} 13507$ & $8 / 17 / 06$ & 0.19 & $\mathrm{U}$ & 0.19 & 5160 & & 4.5 & 4.9 & $\mathrm{C}$ & 0.36 & 7.7 & & 0.38 & 13.2 & & 0.33 & 0.23 & & 0.20 \\
\hline Area $3 \mathrm{~A} 1$ & $\mathrm{~J} 13510$ & $8 / 21 / 06$ & 0.19 & $\mathrm{U}$ & 0.19 & 4550 & & 4.5 & 8.0 & $\mathrm{C}$ & 0.36 & 8.9 & & 0.39 & 18.1 & & 0.33 & 0.20 & $\mathrm{U}$ & 0.20 \\
\hline Area 3 A2 & $\mathrm{J} 13511$ & $8 / 21 / 06$ & 0.19 & $\mathrm{U}$ & 0.19 & 5450 & & 4.5 & 8.1 & $\mathrm{C}$ & 0.36 & 13.1 & & 0.39 & 286 & & 0.33 & 0.20 & $\mathrm{U}$ & 0.20 \\
\hline Area 3 A2 resample & $\mathrm{J} 13 \mathrm{HN} 3$ & $9 / 29 / 06$ & 0.12 & & 0.09 & 5080 & $\mathrm{C}$ & 2.6 & 7.2 & $\mathrm{C}$ & 0.22 & 8.6 & & 0.15 & 45.2 & & 0.25 & & & \\
\hline Area $3 \mathrm{~A} 3$ & $\mathrm{~J} 13512$ & $8 / 21 / 06$ & 0.19 & $\mathrm{U}$ & 0.19 & 14100 & & 4.5 & 9.2 & $\mathrm{C}$ & 0.36 & 7.7 & & 0.39 & 30.6 & & 0.33 & 0.20 & $\mathrm{U}$ & 0.20 \\
\hline Area 3 A4 & $\mathrm{J} 13513$ & $8 / 21 / 06$ & 0.19 & $\mathrm{U}$ & 0.19 & 5580 & & 4.5 & 7.4 & $\mathrm{C}$ & 0.36 & 8.5 & & 0.38 & 17.9 & & 0.33 & 0.20 & $\mathrm{U}$ & 0.20 \\
\hline Area $4 \mathrm{Al}$ & $\mathrm{J} 13514$ & $8 / 21 / 06$ & 0.19 & $\bar{U}$ & 0.19 & 5600 & & 4.5 & 6.6 & $\mathrm{C}$ & 0.36 & 8.8 & & 0.38 & 14.7 & & 0.33 & 0.20 & $\mathrm{U}$ & 0.20 \\
\hline Area $4 \mathrm{~A} 2$ & $\mathrm{~J} 13515$ & $8 / 21 / 06$ & 0.19 & $\mathrm{U}$ & 0.19 & 7040 & & 4.5 & 6.2 & $\mathrm{C}$ & 0.35 & 8.5 & & 0.38 & 15.1 & & 0.33 & 0.20 & $\mathrm{U}$ & 0.20 \\
\hline Area $4 \mathrm{A3}$ & $J 13516$ & $8 / 21 / 06$ & 0.19 & $\mathrm{U}$ & 0.19 & 8360 & & 4.5 & 6.0 & $\mathrm{C}$ & 0.35 & 7.2 & & 0.38 & 14.2 & & 0.33 & 0.20 & $\mathrm{U}$ & 0.20 \\
\hline Area 4 A4 & $\mathrm{J} 13517$ & $8 / 21 / 06$ & 0.19 & $\mathrm{U}$ & 0.19 & 5450 & & 4.5 & 5.3 & $\mathrm{C}$ & 0.36 & 8.1 & & 0.39 & 14.8 & & 0.33 & 0.20 & $\mathrm{U}$ & 0.20 \\
\hline $\mathrm{OB} / \mathrm{BCL} \mathrm{A} 1$ & $\mathrm{~J} 13518$ & $8 / 22 / 06$ & 0.20 & $\mathrm{U}$ & 0.20 & 5120 & & 4.7 & 6.1 & & 0.38 & 7.7 & & 0.40 & 15.8 & & 0.35 & 0.20 & $\mathrm{U}$ & 0.20 \\
\hline $\mathrm{OB} / \mathrm{BCL} \mathrm{A} 2$ & $\mathrm{~J} 13519$ & $8 / 22 / 06$ & 0.21 & $\mathrm{U}$ & 0.21 & 5110 & & 4.8 & 6.4 & & 0.38 & 7.8 & & 0.41 & 15.0 & & 0.35 & 0.20 & $\mathrm{U}$ & 0.20 \\
\hline $\mathrm{OB} / \mathrm{BCL} \mathrm{A} 3$ & $\mathrm{~J} 13520$ & $8 / 22 / 06$ & 0.20 & $\mathrm{U}$ & 0.20 & 4200 & & 4.8 & 5.3 & & 0.38 & 6.9 & & 0.41 & 12.8 & & 0.35 & 0.24 & & 0.20 \\
\hline $\mathrm{OB} / \mathrm{BCL} \mathrm{A} 4$ & J135N5 & $8 / 22 / 06$ & 0.20 & $\mathrm{U}$ & 0.20 & 4710 & & 4.8 & 6.8 & & 0.38 & 7.1 & & 0.41 & 11.9 & & 0.35 & 0.20 & $\mathrm{U}$ & 0.20 \\
\hline
\end{tabular}


118-C-1 Verification Sampling Results.

\begin{tabular}{|c|c|c|c|c|c|c|c|c|c|c|c|c|c|c|c|c|c|c|c|c|}
\hline \multirow[t]{2}{*}{ Sample Location } & \multirow{2}{*}{$\begin{array}{c}\text { Sample } \\
\text { Number }\end{array}$} & \multirow{2}{*}{$\begin{array}{c}\text { Sample } \\
\text { Date }\end{array}$} & \multicolumn{3}{|c|}{ Cadmium } & \multicolumn{3}{|c|}{ Calcium } & \multicolumn{3}{|c|}{ Chromium } & \multicolumn{3}{|c|}{ Cobalt } & \multicolumn{3}{|c|}{ Copper } & \multicolumn{3}{|c|}{$\begin{array}{l}\text { Hexavalent } \\
\text { Chromium }\end{array}$} \\
\hline & & & $\mathrm{mg} / \mathrm{kg}$ & $Q$ & PQL & $\mathrm{mg} / \mathrm{kg}$ & $Q$ & PQL & $\mathrm{mg} / \mathrm{kg}$ & $Q$ & PQL & $\mathrm{mg} / \mathrm{kg}$ & $Q$ & PQL & $\mathrm{mg} / \mathrm{kg}$ & $Q$ & $\mathbf{P Q L}$ & $\mathrm{mg} / \mathrm{kg}$ & $Q$ & PQL \\
\hline SPA A1 & $\mathrm{J} 13522$ & $9 / 11 / 06$ & 0.09 & $\mathrm{U}$ & 0.09 & 5340 & & 2.5 & 7.5 & & 0.20 & 7.6 & & 0.14 & 15.3 & & 0.23 & 0.20 & $\mathrm{U}$ & 0.20 \\
\hline SPA A2 & $\mathrm{J} 13523$ & $9 / 11 / 06$ & 0.09 & $\mathrm{U}$ & 0.09 & 4730 & & 2.5 & 8.1 & & 0.20 & 6.2 & & 0.14 & 15.6 & & 0.23 & 0.20 & $\mathrm{U}$ & 0.20 \\
\hline SPA A3 & $\mathrm{J} 135 \mathrm{P} 1$ & $9 / 12 / 06$ & 0.09 & $\mathrm{U}$ & 0.09 & 6010 & & 2.5 & 8.0 & & 0.20 & 7.0 & & 0.14 & 15.8 & & 0.23 & 0.20 & & 0.20 \\
\hline SPA A4 & $\mathrm{J} 135 \mathrm{P} 0$ & $9 / 12 / 06$ & 0.09 & $\mathrm{U}$ & 0.09 & 5390 & & 2.5 & 7.0 & & 0.20 & 6.7 & & 0.14 & 15.7 & & 0.23 & 0.30 & & 0.20 \\
\hline SPA B5 & $\mathrm{J} 13524$ & $9 / 11 / 06$ & 0.09 & $\mathrm{U}$ & 0.09 & 4420 & & 2.5 & 8.8 & & 0.20 & 6.4 & & 0.14 & 16.4 & & 0.23 & 1.9 & & 0.20 \\
\hline SPA B6 & $\mathrm{J} 13525$ & $9 / 11 / 06$ & 0.09 & $\mathrm{U}$ & 0.09 & 4330 & & 2.5 & 9.3 & & 0.20 & 7.2 & & 0.14 & 15.9 & & 0.23 & 0.20 & $\mathrm{U}$ & 0.20 \\
\hline SPA B7 & $\mathrm{J} 135 \mathrm{~N} 8$ & $9 / 11 / 06$ & 0.09 & $\mathrm{U}$ & 0.09 & 4610 & & 2.5 & 8.0 & & 0.20 & 7.0 & & 0.14 & 14.9 & & 0.23 & 0.20 & $\mathrm{U}$ & 0.20 \\
\hline SPA B8 & J135N9 & $9 / 11 / 06$ & 0.09 & $\mathrm{U}$ & 0.09 & 4090 & & 2.5 & 7.6 & & 0.20 & 6.7 & & 0.14 & 13.9 & & 0.23 & 0.20 & $\mathrm{U}$ & 0.20 \\
\hline SPA C9 & $\mathrm{J} 135 \mathrm{P} 2$ & $9 / 12 / 06$ & 0.09 & $\mathrm{U}$ & 0.09 & 8710 & & 2.5 & 6.8 & & 0.20 & 5.6 & & 0.14 & 144 & & 0.23 & 0.20 & $\mathrm{U}$ & 0.20 \\
\hline SPA C10 & $\mathrm{J} 135 \mathrm{P} 3$ & $9 / 12 / 06$ & 0.09 & $\mathrm{U}$ & 0.09 & 4870 & & 2.5 & 6.5 & & 0.20 & 6.5 & & 0.14 & 13.3 & & 0.23 & 0.29 & & 0.20 \\
\hline SPA C1 & $\mathrm{J} 135 \mathrm{P} 4$ & $9 / 12 / 06$ & 0.09 & $\mathrm{U}$ & 0.09 & 3800 & & 2.5 & 7.7 & & 0.20 & 6.4 & & 0.14 & 14.4 & & 0.23 & 0.20 & & 0.20 \\
\hline SPA C2 & J135P5 & 9/12/06 & 0.09 & U & 0.09 & 4920 & & 2.5 & 7.6 & & 0.20 & 6.8 & & 0.14 & 15.2 & & 0.23 & 0.20 & $\mathrm{U}$ & 0.20 \\
\hline SPA D3 & J135P6 & $9 / 12 / 06$ & 0.09 & $\mathrm{U}$ & 0.09 & 5000 & & 2.5 & 7.7 & & 0.20 & 6.6 & & 0.14 & 12.7 & & 0.23 & 0.26 & & 0.20 \\
\hline SPA D4 & J135P7 & $9 / 12 / 06$ & 0.09 & $\mathrm{U}$ & 0.09 & 5170 & & 2.5 & 7.2 & & 0.20 & 6.6 & & 0.14 & 12.6 & & 0.23 & 0.34 & & 0.20 \\
\hline SPA D5 & J135P8 & $9 / 12 / 06$ & 0.09 & $\mathrm{U}$ & 0.09 & 4860 & & 2.5 & 9.1 & & 0.20 & 7.5 & & 0.14 & 14.3 & & 0.23 & 0.20 & U & 0.20 \\
\hline SPA D6 & J135P9 & $9 / 12 / 06$ & 0.09 & $\mathrm{U}$ & 0.09 & 5180 & & 2.5 & 8.2 & & 0.20 & 7.8 & & 0.14 & 14.1 & & 0.23 & 0.30 & & 0.20 \\
\hline FS-1 & $\mathrm{J} 12 \mathrm{XL} 8$ & $7 / 26 / 06$ & 0.20 & $\mathrm{U}$ & 0.20 & 3880 & & 4.7 & 8.6 & & 0.38 & 7.3 & & 0.41 & 13.8 & & 0.35 & & & \\
\hline FS-2 & J12XL9 & $7 / 26 / 06$ & 0.20 & $\mathrm{U}$ & 0.20 & 9250 & & 4.7 & 6.3 & & 0.38 & 7.9 & & 0.40 & 13.6 & & 0.35 & & & \\
\hline FS-3 & J12XM0 & $7 / 26 / 06$ & 0.20 & $\mathrm{U}$ & 0.20 & 6320 & & 4.7 & 4.8 & & 0.37 & 6.1 & & 0.40 & 10.5 & & 0.34 & & & \\
\hline FS-4 & $\mathrm{J} 12 \mathrm{XM1}$ & $7 / 26 / 06$ & 0.20 & $\mathrm{U}$ & 0.20 & 11400 & & 4.8 & 7.2 & & 0.38 & 8.6 & & 0.41 & 17.4 & & 0.35 & & & \\
\hline FS-5 & $\mathrm{J} 12 \mathrm{XM} 2$ & $7 / 26 / 06$ & 0.20 & $\mathrm{U}$ & 0.20 & 6130 & & 4.7 & 10.2 & & 0.38 & 10.5 & & 0.40 & 23.3 & & 0.35 & & & \\
\hline FS-7 & $\mathrm{J} 12 \mathrm{XM} 4$ & $7 / 26 / 06$ & 0.20 & $\mathrm{U}$ & 0.20 & 13900 & & 4.8 & 4.0 & & 0.38 & 7.0 & & 0.41 & 16.7 & & 0.35 & & & \\
\hline Equipment blank & $\mathrm{J} 13509$ & $8 / 17 / 06$ & 0.06 & $\mathrm{U}$ & 0.06 & 22.1 & & 1.5 & 0.70 & $\mathrm{C}$ & 0.12 & 0.13 & $\mathrm{U}$ & 0.13 & 0.11 & $\mathrm{U}$ & 0.11 & & & \\
\hline
\end{tabular}

\begin{tabular}{|c|c|c|c|c|c|c|c|c|c|c|c|c|c|c|c|c|c|c|c|c|}
\hline \multirow{2}{*}{ Sample Location } & \multirow{2}{*}{$\begin{array}{c}\text { Sample } \\
\text { Number } \\
\end{array}$} & \multirow{2}{*}{$\begin{array}{c}\text { Sample } \\
\text { Date } \\
\end{array}$} & \multicolumn{3}{|c|}{ Iron } & \multicolumn{3}{|c|}{ Lead } & \multicolumn{3}{|c|}{ Magnesium } & \multicolumn{3}{|c|}{ Manganese } & \multicolumn{3}{|c|}{ Mercury } & \multicolumn{3}{|c|}{ Molybdenum } \\
\hline & & & $\mathrm{mg} / \mathrm{kg}$ & $Q$ & PQL & $\mathrm{mg} / \mathrm{kg}$ & $Q$ & PQL & $\mathrm{mg} / \mathrm{kg}$ & $Q$ & PQL & $\mathrm{mg} / \mathrm{kg}$ & $\mathbf{Q}$ & PQL & $\mathrm{mg} / \mathrm{kg}$ & $Q$ & PQL & $\mathrm{mg} / \mathrm{kg}$ & $Q$ & PQL \\
\hline Area $1 \mathrm{Al}$ & $\mathrm{J} 134 \mathrm{Y} 7$ & $8 / 17 / 06$ & 15700 & & 9.7 & 4.5 & & 0.86 & 3170 & & 2.7 & 296 & & 0.08 & 0.02 & $\mathrm{U}$ & 0.02 & 0.80 & $\mathrm{U}$ & 0.80 \\
\hline Duplicate of J134Y7 & J134Y9 & $8 / 17 / 06$ & 18100 & & 9.6 & 4.5 & & 0.85 & 3630 & & 2.7 & 328 & & 0.08 & 0.01 & $\mathrm{U}$ & 0.01 & 0.80 & $\mathrm{U}$ & 0.80 \\
\hline Split of J134Y7 & $\mathrm{J} 13504$ & $8 / 17 / 06$ & 22600 & $\mathrm{~N}$ & 2.4 & 4.2 & & 0.15 & 4570 & & 12.2 & 342 & $\mathrm{~N}$ & 0.10 & 0.0067 & $\mathrm{U}$ & 0.0067 & 1.2 & $\mathrm{U}$ & 1.2 \\
\hline Area $1 \mathrm{~A} 2$ & $\mathrm{~J} 13500$ & $8 / 17 / 06$ & 13900 & & 9.6 & 4.1 & & 0.86 & 3270 & & 2.7 & 260 & & 0.08 & 0.02 & $\mathrm{U}$ & 0.02 & 0.80 & $\mathrm{U}$ & 0.80 \\
\hline Area $1 \mathrm{~A} 3$ & $\mathrm{~J} 134 \mathrm{Y} 8$ & $8 / 17 / 06$ & 14000 & & 9.7 & 3.5 & & 0.86 & 3250 & & 2.7 & 259 & & 0.08 & 0.02 & $\mathrm{U}$ & 0.02 & 0.81 & $\mathrm{U}$ & 0.81 \\
\hline Area $1 \mathrm{~A} 4$ & $\mathrm{~J} 13501$ & $8 / 17 / 06$ & 19100 & & 9.6 & 4.9 & & 0.86 & 4620 & & 2.7 & 362 & & 0.08 & 0.02 & $\mathrm{U}$ & 0.02 & 0.80 & $\mathrm{U}$ & 0.80 \\
\hline Area $2 \mathrm{~A} 1$ & $\mathrm{~J} 13502$ & $8 / 17 / 06$ & 18900 & & 9.6 & 3.7 & & 0.85 & 3970 & & 2.7 & 317 & & 0.08 & 0.01 & $\mathrm{U}$ & 0.01 & 0.80 & $\mathrm{U}$ & 0.80 \\
\hline Duplicate of J13502 & $\mathrm{J} 13508$ & $8 / 17 / 06$ & 17800 & & 9.7 & 3.7 & & 0.87 & 3680 & & 2.7 & 314 & & 0.08 & 0.01 & $\mathrm{U}$ & 0.01 & 0.81 & $\mathrm{U}$ & 0.81 \\
\hline Split of J13502 & $\mathrm{J} 13505$ & $8 / 17 / 06$ & 23700 & $\mathrm{~N}$ & 2.4 & 4.8 & & 0.15 & 4800 & & 12.2 & 340 & $\mathrm{~N}$ & 0.10 & 0.0067 & $\mathrm{U}$ & 0.0067 & 1.2 & $\mathrm{U}$ & 1.2 \\
\hline Area 2 A2 & $\mathrm{J} 13503$ & $8 / 17 / 06$ & 18400 & & 9.7 & 3.8 & & 0.86 & 3760 & & 2.7 & 285 & & 0.08 & 0.01 & $\mathrm{U}$ & 0.01 & 0.80 & $\mathrm{U}$ & 0.80 \\
\hline Area $2 \mathrm{~A} 3$ & $\mathrm{J13506}$ & $8 / 17 / 06$ & 15900 & & 9.7 & 4.3 & & 0.86 & 3150 & & 2.7 & 279 & & 0.08 & 0.02 & $\mathrm{U}$ & 0.02 & 0.80 & $\mathrm{U}$ & 0.80 \\
\hline Area $2 \mathrm{~A} 4$ & $\mathrm{~J} 13507$ & $8 / 17 / 06$ & 19000 & & 9.6 & 3.2 & & 0.85 & 3660 & & 2.7 & 359 & & 0.08 & 0.02 & $\mathrm{U}$ & 0.02 & 0.80 & $\mathrm{U}$ & 0.80 \\
\hline
\end{tabular}


118-C-1 Verification Sampling Results.

\begin{tabular}{|c|c|c|c|c|c|c|c|c|c|c|c|c|c|c|c|c|c|c|c|c|}
\hline \multirow{2}{*}{ Sample Location } & \multirow{2}{*}{$\begin{array}{c}\text { Sample } \\
\text { Number }\end{array}$} & \multirow{2}{*}{$\begin{array}{c}\text { Sample } \\
\text { Date }\end{array}$} & \multicolumn{3}{|c|}{ Iron } & \multicolumn{3}{|c|}{ Lead } & \multicolumn{3}{|c|}{ Magnesium } & \multicolumn{3}{|c|}{ Manganese } & \multicolumn{3}{|c|}{ Mercury } & \multicolumn{3}{|c|}{ Molybdenum } \\
\hline & & & $\mathrm{mg} / \mathrm{kg}$ & Q & PQL & $\mathrm{mg} / \mathrm{kg}$ & $Q$ & PQL & $\mathrm{mg} / \mathrm{kg}$ & $Q$ & PQL & $\mathrm{mg} / \mathrm{kg}$ & $Q$ & PQL & $\mathrm{mg} / \mathrm{kg}$ & $Q$ & PQL & $\mathrm{mg} / \mathrm{kg}$ & $Q$ & PQL \\
\hline Area $3 \mathrm{~A} 1$ & $\mathrm{~J} 13510$ & $8 / 21 / 06$ & 22300 & & 9.6 & 8.3 & & 0.85 & 4170 & & 2.7 & 357 & & 0.08 & 0.02 & $\mathrm{U}$ & 0.02 & 0.80 & $\mathrm{U}$ & 0.80 \\
\hline Area $3 \mathrm{~A} 2$ & $\mathrm{~J} 13511$ & $8 / 21 / 06$ & 28400 & & 9.7 & 130 & & 0.86 & 3350 & & 2.7 & 313 & & 0.08 & 0.05 & & 0.01 & 54.9 & & 0.80 \\
\hline Area $3 \mathrm{~A} 2$ resample & $\mathrm{J} 13 \mathrm{HN} 3$ & $9 / 29 / 06$ & 21000 & & 7.5 & 23.1 & & 0.37 & 3740 & $\mathrm{C}$ & 2.2 & 338 & & 0.06 & 0.02 & U & 0.02 & 4.5 & & 0.31 \\
\hline Area $3 \mathrm{~A} 3$ & $\mathrm{~J} 13512$ & $8 / 21 / 06$ & 21200 & & 9.6 & 11.8 & & 0.86 & 3620 & & 2.7 & 331 & & 0.08 & 0.02 & $\mathrm{U}$ & 0.02 & 0.80 & $\mathrm{U}$ & 0.80 \\
\hline Area 3 A4 & $\mathrm{J} 13513$ & $8 / 21 / 06$ & 20900 & & 9.5 & 6.9 & & 0.85 & 4030 & & 2.7 & 320 & & 0.08 & 0.01 & $\mathrm{U}$ & 0.01 & 0.79 & $\mathrm{U}$ & 0.79 \\
\hline Area 4 A1 & $\mathrm{J} 13514$ & $8 / 21 / 06$ & 23200 & & 9.6 & 5.0 & & 0.85 & 4490 & & 2.7 & 323 & & 0.08 & 0.01 & $\mathrm{U}$ & 0.01 & 0.80 & $\mathrm{U}$ & 0.80 \\
\hline Area 4 A2 & $\mathrm{J} 13515$ & $8 / 21 / 06$ & 20400 & & 9.5 & 4.7 & & 0.85 & 3970 & & 2.6 & 350 & & 0.08 & 0.02 & U & 0.02 & 0.79 & $\mathrm{U}$ & 0.79 \\
\hline Area $4 \mathrm{A3}$ & $\mathrm{J} 13516$ & $8 / 21 / 06$ & 17600 & & 9.5 & 3.1 & & 0.85 & 3610 & & 2.6 & 269 & & 0.08 & 0.01 & $\mathrm{U}$ & 0.01 & 0.79 & $\mathrm{U}$ & 0.79 \\
\hline Area 4 A4 & $\mathrm{J} 13517$ & $8 / 21 / 06$ & 19600 & & 9.6 & 4.5 & & 0.85 & 3820 & & 2.7 & 306 & & 0.08 & 0.02 & $\mathrm{U}$ & 0.02 & 0.80 & $\mathrm{U}$ & 0.80 \\
\hline $\mathrm{OB} / \mathrm{BCL} \mathrm{A} 1$ & $\mathrm{~J} 13518$ & $8 / 22 / 06$ & 18300 & & 10.1 & 5.2 & & 0.90 & 3900 & & 2.8 & 311 & & 0.09 & 0.01 & $\mathrm{U}$ & 0.01 & 0.84 & $\mathrm{U}$ & 0.84 \\
\hline $\mathrm{OB} / \mathrm{BCL} \mathrm{A} 2$ & J13519 & $8 / 22 / 06$ & 18800 & & 10.2 & 4.5 & & 0.91 & 4010 & & 2.8 & 329 & & 0.09 & 0.02 & $\mathrm{U}$ & 0.02 & 0.85 & $\mathrm{U}$ & 0.85 \\
\hline $\mathrm{OB} / \mathrm{BCL} \mathrm{A} 3$ & $\mathrm{~J} 13520$ & $8 / 22 / 06$ & 15900 & & 10.2 & 3.8 & & 0.91 & 3370 & & 2.8 & 272 & & 0.09 & 0.02 & U & 0.02 & 0.85 & $\mathrm{U}$ & 0.85 \\
\hline $\mathrm{OB} / \mathrm{BCL} \mathrm{A} 4$ & J135N5 & $8 / 22 / 06$ & 17100 & & 10.2 & 4.3 & & 0.90 & 3680 & & 2.8 & 308 & & 0.09 & 0.01 & U & 0.01 & 0.84 & U & 0.84 \\
\hline SPA A1 & $\mathrm{J} 13522$ & $9 / 11 / 06$ & 21300 & & 7.0 & 5.7 & & 0.35 & 4170 & & 2.1 & 339 & & 0.06 & 0.02 & $\mathrm{U}$ & 0.02 & 0.29 & $\mathrm{U}$ & 0.29 \\
\hline SPA A2 & J13523 & $9 / 11 / 06$ & 16500 & & 7.0 & 4.4 & & 0.35 & 3760 & & 2.1 & 298 & & 0.06 & 0.02 & $\mathrm{U}$ & 0.02 & 0.29 & $\mathrm{U}$ & 0.29 \\
\hline SPA A3 & $\mathrm{J} 135 \mathrm{P} 1$ & $9 / 12 / 06$ & 19100 & & 7.0 & 5.7 & & 0.34 & 4350 & & 2.1 & 320 & & 0.06 & 0.02 & & 0.01 & 0.29 & $\mathrm{U}$ & 0.29 \\
\hline SPA A4 & J135P0 & $9 / 12 / 06$ & 18400 & & 7.0 & 7.5 & & 0.34 & 3850 & & 2.1 & 305 & & 0.06 & 0.02 & $\mathrm{U}$ & 0.02 & 0.29 & $\mathrm{U}$ & 0.29 \\
\hline SPA B5 & $J 13524$ & $9 / 11 / 06$ & 16900 & & 7.0 & 25.8 & & 0.35 & 3930 & & 2.1 & 292 & & 0.06 & 0.02 & $\mathrm{U}$ & 0.02 & 0.29 & $\mathrm{U}$ & 0.29 \\
\hline SPA B6 & $\mathrm{J} 13525$ & $9 / 11 / 06$ & 20000 & & 7.0 & 6.4 & & 0.35 & 3690 & & 2.1 & 292 & & 0.06 & 0.02 & $\mathrm{U}$ & 0.02 & 0.29 & $\mathrm{U}$ & 0.29 \\
\hline SPA B7 & J135N8 & $9 / 11 / 06$ & 19200 & & 7.0 & 5.5 & & 0.35 & 3830 & & 2.1 & 308 & & 0.06 & 0.06 & & 0.01 & 0.29 & $\mathrm{U}$ & 0.29 \\
\hline SPA B8 & J135N9 & $9 / 11 / 06$ & 18300 & & 6.9 & 7.2 & & 0.34 & 3500 & & 2.1 & 303 & & 0.06 & 0.02 & $\mathrm{U}$ & 0.02 & 0.45 & & 0.29 \\
\hline SPA C9 & $\mathrm{J} 135 \mathrm{P} 2$ & $9 / 12 / 06$ & 16500 & & 7.0 & 20.8 & & 0.34 & 3440 & & 2.1 & 334 & & 0.06 & 0.02 & & 0.01 & 0.62 & & 0.29 \\
\hline SPA C10 & $\mathrm{J} 135 \mathrm{P} 3$ & $9 / 12 / 06$ & 17300 & & 7.0 & 5.7 & & 0.35 & 3600 & & 2.1 & 300 & & 0.06 & 0.02 & $\mathrm{U}$ & 0.02 & 0.29 & $\mathrm{U}$ & 0.29 \\
\hline SPA C1 & $\mathrm{J} 135 \mathrm{P} 4$ & $9 / 12 / 06$ & 18300 & & 7.0 & 7.0 & & 0.34 & 3820 & & 2.1 & 302 & & 0.06 & 0.02 & $\mathrm{U}$ & 0.02 & 0.34 & & 0.29 \\
\hline SPA C2 & J135P5 & $9 / 12 / 06$ & 18400 & & 7.0 & 8.4 & & 0.35 & 3730 & & 2.1 & 297 & & 0.06 & 0.06 & & 0.02 & 0.29 & $\mathrm{U}$ & 0.29 \\
\hline SPA D3 & J135P6 & $9 / 12 / 06$ & 18100 & & 7.0 & 6.2 & & 0.34 & 3630 & & 2.1 & 314 & & 0.06 & 0.01 & $\mathrm{U}$ & 0.01 & 0.44 & & 0.29 \\
\hline SPA D4 & J135P7 & $9 / 12 / 06$ & 17100 & & 7.0 & 5.5 & & 0.35 & 3540 & & 2.1 & 306 & & 0.06 & 0.01 & $\mathrm{U}$ & 0.01 & 0.29 & $\mathrm{U}$ & 0.29 \\
\hline SPA D5 & J135P8 & $9 / 12 / 06$ & 20500 & & 6.9 & 4.7 & & 0.34 & 3980 & & 2.1 & 350 & & 0.06 & 0.02 & $\mathrm{U}$ & 0.02 & 0.29 & $\mathrm{U}$ & 0.29 \\
\hline SPA D6 & J135P9 & $9 / 12 / 06$ & 21100 & & 6.9 & 5.7 & & 0.34 & 4020 & & 2.1 & 367 & & 0.06 & 0.01 & $\mathrm{U}$ & 0.01 & 0.45 & & 0.29 \\
\hline$\overline{F S-1}$ & J12XL8 & $7 / 26 / 06$ & 18200 & & 10.1 & 4.4 & & 0.90 & 3880 & & 2.8 & 311 & & 0.09 & 0.02 & $\mathrm{U}$ & 0.02 & 0.84 & $\mathrm{U}$ & 0.84 \\
\hline FS-2 & J12XL9 & $7 / 26 / 06$ & 20300 & & 10.1 & 5.2 & & 0.89 & 4760 & & 2.8 & 312 & & 0.09 & 0.02 & $\mathrm{U}$ & 0.02 & 0.84 & $\mathrm{U}$ & 0.84 \\
\hline FS-3 & $\mathrm{J} 12 \mathrm{XM0}$ & $7 / 26 / 06$ & 16100 & & 10 & 2.9 & & 0.89 & 3510 & & 2.8 & 248 & & 0.09 & 0.01 & $\mathrm{U}$ & 0.01 & 0.83 & $\mathrm{U}$ & 0.83 \\
\hline FS-4 & $\mathrm{J} 12 \mathrm{XM1}$ & $7 / 26 / 06$ & 20600 & & 10.1 & 7.2 & & 0.90 & 5720 & & 2.8 & 346 & & 0.09 & 0.02 & $\mathrm{U}$ & 0.02 & 0.84 & $\mathrm{U}$ & 0.84 \\
\hline FS-5 & J12XM2 & $7 / 26 / 06$ & 21900 & & 10.1 & 7.2 & & 0.90 & 5080 & & 2.8 & 446 & & 0.09 & 0.04 & & 0.01 & 0.84 & $\mathrm{U}$ & 0.84 \\
\hline FS-7 & $\mathrm{J} 12 \mathrm{XM} 4$ & $7 / 26 / 06$ & 17800 & & 10.1 & 2.5 & & 0.90 & 3830 & & 2.8 & 241 & & 0.09 & 0.02 & $\mathrm{U}$ & 0.02 & 0.84 & $\mathrm{U}$ & 0.84 \\
\hline Equipment blank & $\mathrm{J} 13509$ & $8 / 17 / 06$ & 106 & & 3.2 & 0.28 & $\mathrm{U}$ & 0.28 & 6.4 & & 0.89 & 3.3 & & 0.03 & 0.01 & $\mathrm{U}$ & 0.01 & 0.27 & $\mathrm{U}$ & 0.27 \\
\hline
\end{tabular}


118-C-1 Verification Sampling Results.

\begin{tabular}{|c|c|c|c|c|c|c|c|c|c|c|c|c|c|c|c|c|c|c|c|c|}
\hline \multirow{2}{*}{ Sample Location } & \multirow{2}{*}{$\begin{array}{c}\text { Sample } \\
\text { Number } \\
\end{array}$} & \multirow{2}{*}{$\begin{array}{c}\text { Sample } \\
\text { Date }\end{array}$} & \multicolumn{3}{|c|}{ Nickel } & \multicolumn{3}{|c|}{ Potassium } & \multicolumn{3}{|c|}{ Selenium } & \multicolumn{3}{|c|}{ Silicon } & \multicolumn{3}{|c|}{ Silver } & \multicolumn{3}{|c|}{ Sodium } \\
\hline & & & $\mathrm{mg} / \mathrm{kg}$ & $Q$ & PQL & $\mathrm{mg} / \mathrm{kg}$ & Q & $\mathrm{PQL}$ & $\mathrm{mg} / \mathrm{kg}$ & $\mathrm{Q}$ & $\mathrm{PQL}$ & $\mathrm{mg} / \mathrm{kg}$ & $Q$ & PQL & $\mathrm{mg} / \mathbf{k g}$ & $Q$ & PQL & $\mathrm{mg} / \mathrm{kg}$ & $Q$ & PQL \\
\hline Area $1 \mathrm{~A} 1$ & $\mathrm{~J} 134 \mathrm{Y} 7$ & $8 / 17 / 06$ & 8.5 & & 0.66 & 847 & & 6.3 & 1.3 & UC & 1.3 & 540 & \begin{tabular}{|l|l}
$\mathrm{R}$ \\
\end{tabular} & 6.3 & 0.19 & $\frac{x}{U}$ & 0.19 & 86.3 & & 2.1 \\
\hline Duplicate of J134Y7 & J134Y9 & $8 / 17 / 06$ & 9.5 & & 0.66 & 920 & & 6.2 & 1.3 & UC & 1.3 & 525 & \begin{tabular}{|l|l}
$\mathrm{R}$ \\
\end{tabular} & 6.2 & 0.19 & $\mathrm{U}$ & 0.19 & 112 & & 2.1 \\
\hline Split of J134Y7 & $\mathrm{J} 13504$ & $8 / 17 / 06$ & 11.6 & & 0.76 & 1210 & & 50.4 & 1.1 & $B * C$ & 0.17 & 635 & \begin{tabular}{|l|}
$\mathrm{N}$ \\
\end{tabular} & 4.0 & 0.20 & UN & 0.20 & 166 & & 10.1 \\
\hline Area 1 A2 & $\mathrm{J} 13500$ & $8 / 17 / 06$ & 10.1 & & 0.66 & 858 & & 6.3 & 1.3 & $\mathrm{UC}$ & 1.3 & 550 & \begin{tabular}{|l|}
$\mathrm{R}$ \\
\end{tabular} & 6.3 & 0.19 & $\mathrm{U}$ & 0.19 & 85.3 & & 2.1 \\
\hline Area $1 \mathrm{~A} 3$ & $\mathrm{~J} 134 \mathrm{Y} 8$ & $8 / 17 / 06$ & 9.9 & & 0.67 & 916 & & 6.3 & 1.3 & $\mathrm{UC}$ & 1.3 & 560 & \begin{tabular}{|l|l}
$R$ \\
\end{tabular} & 6.3 & 0.20 & $U$ & 0.20 & 122 & & 2.1 \\
\hline Area $1 \mathrm{~A} 4$ & $\mathrm{~J} 13501$ & $8 / 17 / 06$ & 10.2 & & 0.66 & 957 & & 6.3 & 1.3 & $\mathrm{UC}$ & 1.3 & 544 & \begin{tabular}{|l|l}
$R$ \\
\end{tabular} & 6.3 & 0.19 & $\bar{U}$ & 0.19 & 143 & & 2.1 \\
\hline Area 2 A1 & $\mathrm{J} 13502$ & $8 / 17 / 06$ & 10.4 & & 0.66 & 813 & & 6.3 & 1.3 & UC & 1.3 & 668 & \begin{tabular}{|l|l}
$R$ \\
\end{tabular} & 6.3 & 0.19 & $\bar{U}$ & 0.19 & 113 & & 2.1 \\
\hline Duplicate of J13502 & $\mathrm{J} 13508$ & $8 / 17 / 06$ & 10.1 & & 0.67 & 770 & & 6.3 & 1.3 & $\mathrm{UC}$ & 1.3 & 559 & \begin{tabular}{|l|l}
$R$ \\
\end{tabular} & 6.3 & 0.20 & $\mathrm{U}$ & 0.20 & 95.7 & & 2.1 \\
\hline Split of J13502 & $\mathrm{J} 13505$ & $8 / 17 / 06$ & 13.0 & & 0.76 & 968 & & 50.4 & 0.89 & $B * C$ & 0.17 & 593 & $\mathrm{~N}$ & 4.0 & 0.20 & UN & 0.20 & 171 & & 10.1 \\
\hline Area $2 \mathrm{~A} 2$ & $\mathrm{~J} 13503$ & $8 / 17 / 06$ & 9.2 & & 0.66 & 805 & & 6.3 & 1.3 & UC & 1.3 & 506 & \begin{tabular}{|l|l}
$R$ \\
\end{tabular} & 6.3 & 0.19 & U & 0.19 & 141 & & 2.1 \\
\hline Area 2 A3 & $\mathrm{J} 13506$ & $8 / 17 / 06$ & 7.9 & & 0.66 & 862 & & 6.3 & 1.3 & $\mathrm{UC}$ & 1.3 & 557 & \begin{tabular}{|l|l|}
$R$ \\
\end{tabular} & 6.3 & 0.19 & $\mathrm{U}$ & 0.19 & 126 & & 2.1 \\
\hline Area $2 \mathrm{~A} 4$ & $\mathrm{~J} 13507$ & $8 / 17 / 06$ & 8.7 & & 0.66 & 803 & & 6.2 & 1.3 & $\mathrm{UC}$ & 1.3 & 530 & \begin{tabular}{|l|l}
$\mathrm{R}$ & $\mathrm{P}$ \\
\end{tabular} & 6.2 & 0.19 & $\mathrm{U}$ & 0.19 & 127 & & 2.1 \\
\hline Area 3 A1 & $\mathrm{J} 13510$ & $8 / 21 / 06$ & 11.0 & & 0.66 & 1120 & & 6.3 & 1.3 & $\mathrm{UC}$ & 1.3 & 593 & \begin{tabular}{|l|l|l}
$R$ \\
\end{tabular} & 6.3 & 0.19 & $\mathrm{U}$ & 0.19 & 141 & & 2.1 \\
\hline Area $3 \mathrm{~A} 2$ & $\mathrm{~J} 13511$ & $8 / 21 / 06$ & 9.3 & & 0.66 & 1050 & & 6.3 & 1.3 & $\mathrm{UC}$ & 1.3 & 612 & \begin{tabular}{l|l}
$R$ \\
\end{tabular} & 6.3 & 0.19 & $\mathrm{U}$ & 0.19 & 190 & & 2.1 \\
\hline Area 3 A2 resample & J13HN3 & $9 / 29 / 06$ & 10.2 & & 0.40 & 1080 & $\mathrm{C}$ & 6.5 & 0.80 & $\mathrm{U}$ & 0.80 & 550 & $\mathrm{CJ}$ & 1.2 & 0.34 & $\mathrm{U}$ & 0.34 & 123 & $\begin{array}{ll}\mathrm{C} \\
\end{array}$ & 0.65 \\
\hline Area $3 \mathrm{~A} 3$ & $\mathrm{~J} 13512$ & $8 / 21 / 06$ & 12.7 & & 0.66 & 1140 & & 6.3 & 1.3 & $\mathrm{UC}$ & 1.3 & 660 & \begin{tabular}{|l|l|l}
$R$ \\
\end{tabular} & 6.3 & 0.19 & $\mathrm{U}$ & 0.19 & 228 & & 2.1 \\
\hline Area 3 A4 & $\mathrm{J} 13513$ & $8 / 21 / 06$ & 9.6 & & 0.66 & 919 & & 6.2 & 1.3 & $\mathrm{UC}$ & 1.3 & 465 & \begin{tabular}{|l|l|}
$R$ \\
\end{tabular} & 6.2 & 0.19 & $\mathrm{U}$ & 0.19 & 143 & & 2.1 \\
\hline Area 4A1 & $\mathrm{J} 13514$ & $8 / 21 / 06$ & 10.1 & & 0.66 & 933 & & 6.2 & 1.3 & $\mathrm{UC}$ & 1.3 & 439 & \begin{tabular}{|l|l|}
$\mathrm{R}$ & \\
\end{tabular} & 6.2 & 0.19 & $\mathrm{U}$ & 0.19 & 147 & & 2.1 \\
\hline Area 4 A2 & $\mathrm{J} 13515$ & $8 / 21 / 06$ & 9.3 & & 0.66 & 873 & & 6.2 & 1.3 & UC & 1.3 & 516 & \begin{tabular}{|l|l|}
$\mathrm{R}$ \\
\end{tabular} & 6.2 & 0.19 & $\mathrm{U}$ & 0.19 & 160 & & 2.1 \\
\hline Area 4 A3 & $\mathrm{J} 13516$ & $8 / 21 / 06$ & 9.7 & & 0.65 & 774 & & 6.2 & 1.3 & $\mathrm{UC}$ & 1.3 & 527 & \begin{tabular}{|l|l|}
$\mathrm{R}$ \\
\end{tabular} & 6.2 & 0.19 & $\mathrm{U}$ & 0.19 & 113 & & 2.1 \\
\hline Area 4 A4 & $\mathrm{J} 13517$ & $8 / 21 / 06$ & 8.7 & & 0.66 & 853 & & 6.3 & 1.3 & UC & 1.3 & 534 & $\begin{array}{ll} \\
\end{array}$ & 6.3 & 0.19 & $\mathrm{U}$ & 0.19 & 114 & & 2.1 \\
\hline$\overline{\mathrm{OB} / \mathrm{BCL} \mathrm{Al}}$ & $J 13518$ & $8 / 22 / 06$ & 9.0 & & 0.69 & 917 & & 6.6 & 1.4 & $\bar{U}$ & 1.4 & 527 & $\mathrm{~J}$ & 6.6 & 0.20 & $\bar{U}$ & 0.20 & 110 & & 2.2 \\
\hline $\mathrm{OB} / \mathrm{BCL} \mathrm{A} 2$ & J13519 & $8 / 22 / 06$ & 9.3 & & 0.70 & 960 & & 6.7 & 1.4 & $\mathrm{U}$ & 1.4 & 510 & \begin{tabular}{|l|l|}
$J$ \\
\end{tabular} & 6.7 & 0.21 & $\mathrm{U}$ & 0.21 & 113 & & 2.2 \\
\hline $\mathrm{OB} / \mathrm{BCL} \mathrm{A} 3$ & $\mathrm{~J} 13520$ & $8 / 22 / 06$ & 9.6 & & 0.70 & 763 & & 6.6 & 1.4 & $\mathrm{U}$ & 1.4 & 540 & \begin{tabular}{|l|l}
$\mathrm{J}$ \\
\end{tabular} & 6.6 & 0.20 & $\mathrm{U}$ & 0.20 & 89.3 & & 2.2 \\
\hline $\mathrm{OB} / \mathrm{BCL} \mathrm{A} 4$ & J135N5 & $8 / 22 / 06$ & 9.0 & & 0.70 & 1080 & & 6.6 & 1.4 & $\mathrm{U}$ & 1.4 & 568 & \begin{tabular}{|l|l|}
$J$ \\
\end{tabular} & 6.6 & 0.20 & $\mathrm{U}$ & 0.20 & 104 & & 2.2 \\
\hline SPA A1 & $J 13522$ & $9 / 11 / 06$ & 10.5 & & 0.38 & 886 & & 6.1 & 0.75 & $\mathrm{U}$ & 0.75 & 450 & $\mathrm{~J}$ & 1.2 & 0.32 & $\bar{U}$ & 0.32 & 148 & & 0.61 \\
\hline SPA A2 & $\mathrm{J} 13523$ & $9 / 11 / 06$ & 10.1 & & 0.38 & 776 & & 6.1 & 0.75 & $\mathrm{U}$ & 0.75 & 373 & \begin{tabular}{|l|}
$\mathrm{J}$ \\
\end{tabular} & 1.2 & 0.32 & $\mathrm{U}$ & 0.32 & 138 & & 0.61 \\
\hline SPA A3 & $\mathrm{J} 135 \mathrm{PI}$ & $9 / 12 / 06$ & 10.6 & & 0.37 & 967 & & 6.1 & 0.74 & $\mathrm{U}$ & 0.74 & 366 & \begin{tabular}{|l|l|}
$\mathrm{J}$ \\
\end{tabular} & 1.1 & 0.32 & $\mathrm{U}$ & 0.32 & 182 & & 0.60 \\
\hline SPA A4 & $\mathrm{J} 135 \mathrm{P} 0$ & $9 / 12 / 06$ & 9.5 & & 0.37 & 932 & & 6.1 & 0.75 & $\mathrm{U}$ & 0.75 & 389 & \begin{tabular}{|l|l|}
$\mathrm{J}$ \\
\end{tabular} & 1.1 & 0.32 & $\mathrm{U}$ & 0.32 & 145 & & 0.60 \\
\hline SPA B5 & $\mathrm{J} 13524$ & $9 / 11 / 06$ & 11.0 & & 0.37 & 836 & & 6.1 & 0.75 & $\mathrm{U}$ & 0.75 & 417 & \begin{tabular}{|l|l|}
$J$ \\
\end{tabular} & 1.2 & 0.32 & $\mathrm{U}$ & 0.32 & 128 & & 0.61 \\
\hline SPA B6 & $\mathrm{J} 13525$ & $9 / 11 / 06$ & 13.7 & & 0.38 & 991 & & 6.1 & 0.75 & $\mathrm{U}$ & 0.75 & 377 & \begin{tabular}{|l|}
$\mathrm{J}$ \\
\end{tabular} & 1.2 & 0.32 & $\mathrm{U}$ & 0.32 & 156 & & 0.61 \\
\hline SPA B7 & J135N8 & $9 / 11 / 06$ & 9.7 & & 0.37 & 1100 & & 6.1 & 0.75 & $\mathrm{U}$ & 0.75 & 461 & \begin{tabular}{|l|} 
\\
\end{tabular} & 1.2 & 0.32 & $\mathrm{U}$ & 0.32 & 148 & & 0.61 \\
\hline SPA B 8 & $\mathrm{~J} 135 \mathrm{~N} 9$ & $9 / 11 / 06$ & 8.7 & & 0.37 & 1130 & & 6.1 & 0.74 & $\mathrm{U}$ & 0.74 & 415 & \begin{tabular}{|l|l|} 
\\
\end{tabular} & 1.1 & 0.31 & $\mathrm{U}$ & 0.31 & 139 & & 0.60 \\
\hline SPA C9 & $\mathrm{J} 135 \mathrm{P} 2$ & $9 / 12 / 06$ & 8.3 & & 0.37 & 1060 & & 6.1 & 0.74 & $\mathrm{U}$ & 0.74 & 632 & \begin{tabular}{|l|}
$\mathrm{J}$ \\
\end{tabular} & 1.1 & 0.31 & $\mathrm{U}$ & 0.31 & 274 & & 0.60 \\
\hline SPAC10 & $\mathrm{J} 135 \mathrm{P} 3$ & $9 / 12 / 06$ & 8.7 & & 0.38 & 923 & & 6.1 & 0.75 & $\mathrm{U}$ & 0.75 & 406 & \begin{tabular}{|l|}
$\mathrm{J}$ \\
\end{tabular} & 1.2 & 0.32 & $\mathrm{U}$ & 0.32 & 139 & & 0.61 \\
\hline SPAC1 & $\mathrm{J} 135 \mathrm{P} 4$ & $9 / 12 / 06$ & 9.3 & & 0.37 & 1030 & & 6.1 & 0.74 & $\mathrm{U}$ & 0.74 & 472 & \begin{tabular}{|l|l|}
$\mathrm{J}$ \\
\end{tabular} & 1.1 & 0.31 & $\mathrm{U}$ & 0.31 & 111 & & 0.60 \\
\hline SPAC2 & J135P5 & $9 / 12 / 06$ & 9.4 & & 0.38 & 943 & & 6.1 & 0.75 & $\mathrm{U}$ & 0.75 & 435 & \begin{tabular}{|l|l|}
$\mathrm{J}$ \\
\end{tabular} & 1.2 & 0.32 & $\mathrm{U}$ & 0.32 & 170 & & 0.61 \\
\hline SPA D3 & J135P6 & $9 / 12 / 06$ & 10.6 & & 0.37 & 997 & & 6.1 & 0.80 & & 0.74 & 530 & \begin{tabular}{|l|} 
\\
\end{tabular} & 1.1 & 0.31 & $\mathrm{U}$ & 0.31 & 142 & & 0.60 \\
\hline SPA D4 & J135P7 & $9 / 12 / 06$ & 9.4 & & 0.38 & 967 & & 6.1 & 0.75 & $\mathrm{U}$ & 0.75 & 459 & \begin{tabular}{|l|}
$\mathrm{J}$ \\
\end{tabular} & 1.2 & 0.32 & $\mathrm{U}$ & 0.32 & 115 & & 0.61 \\
\hline SPA D5 & J135P8 & $9 / 12 / 06$ & 10.8 & & 0.37 & 1050 & & 6.1 & 0.74 & $\mathrm{U}$ & 0.74 & 584 & \begin{tabular}{|l|l|}
$J$ \\
\end{tabular} & 1.1 & 0.31 & $\mathrm{U}$ & 0.31 & 126 & & 0.60 \\
\hline SPA D6 & J135P9 & $9 / 12 / 06$ & 10.7 & & 0.37 & 1070 & & 6.1 & 0.74 & $\mathrm{U}$ & 0.74 & 449 & \begin{tabular}{|l|l|}
$J$ \\
\end{tabular} & 1.1 & 0.31 & $\mathrm{U}$ & 0.31 & 165 & & 0.60 \\
\hline
\end{tabular}


118-C-1 Verification Sampling Results.

\begin{tabular}{|c|c|c|c|c|c|c|c|c|c|c|c|c|c|c|c|c|c|c|c|c|}
\hline \multirow{2}{*}{ Sample Location } & \multirow{2}{*}{$\begin{array}{c}\text { Sample } \\
\text { Number }\end{array}$} & \multirow{2}{*}{$\begin{array}{c}\text { Sample } \\
\text { Date }\end{array}$} & \multicolumn{3}{|c|}{ Nickel } & \multicolumn{3}{|c|}{ Potassium } & \multicolumn{3}{|c|}{ Selenium } & \multicolumn{3}{|c|}{ Silicon } & \multicolumn{3}{|c|}{ Silver } & \multicolumn{3}{|c|}{ Sodium } \\
\hline & & & $\mathrm{mg} / \mathrm{kg}$ & $Q$ & PQL & $\mathrm{mg} / \mathrm{kg}$ & $\mathbf{Q}$ & PQL & $\mathrm{mg} / \mathrm{kg}$ & $Q$ & PQL & $\mathrm{mg} / \mathrm{kg}$ & Q & PQL & $\mathrm{mg} / \mathrm{kg}$ & Q & PQL & $\mathrm{mg} / \mathrm{kg}$ & $\mathbf{Q}$ & PQL \\
\hline FS-1 & J12XL8 & $7 / 26 / 06$ & 10.7 & & 0.69 & 1180 & & 6.6 & 1.4 & $\mathrm{U}$ & 1.4 & 597 & $\mathrm{~J}$ & 6.6 & 0.20 & $\mathrm{U}$ & 0.20 & 105 & & 2.2 \\
\hline FS-2 & J12XL9 & $7 / 26 / 06$ & 10.1 & & 0.69 & 828 & & 6.6 & 1.4 & $\mathrm{U}$ & 1.4 & 564 & $\mathrm{~J}$ & 6.6 & 0.20 & $\mathrm{U}$ & 0.20 & 122 & & 2.2 \\
\hline FS-3 & $\mathrm{J} 12 \mathrm{XM} 0$ & $7 / 26 / 06$ & 9.6 & & 0.69 & 619 & & 6.5 & 1.3 & $\mathrm{U}$ & 1.3 & 618 & $\mathrm{~J}$ & 6.5 & 0.20 & $\mathrm{U}$ & 0.20 & 101 & & 2.2 \\
\hline FS-4 & $\mathrm{J} 12 \mathrm{XM} 1$ & $7 / 26 / 06$ & 11.1 & & 0.70 & 1120 & & 6.6 & 1.4 & $\mathrm{U}$ & 1.4 & 670 & $\mathrm{~J}$ & 6.6 & 0.20 & $\mathrm{U}$ & 0.20 & 185 & & 2.2 \\
\hline FS-5 & $\mathrm{J} 12 \mathrm{XM} 2$ & $7 / 26 / 06$ & 14.1 & & 0.69 & 1640 & & 6.6 & 1.4 & $\mathrm{U}$ & 1.4 & 697 & $\mathrm{~J}$ & 6.6 & 0.20 & $\mathrm{U}$ & 0.20 & 153 & & 2.2 \\
\hline FS-7 & J12XM4 & $7 / 26 / 06$ & 9.9 & & 0.70 & 573 & & 6.6 & 1.4 & $\mathrm{U}$ & 1.4 & 533 & $\mathrm{~J}$ & 6.6 & 0.20 & $\mathrm{U}$ & 0.20 & 135 & & 2.2 \\
\hline Equipment blank & $\mathrm{J} 13509$ & $8 / 17 / 06$ & 0.26 & & 0.22 & 16.6 & & 2.1 & 0.43 & $\mathrm{UC}$ & 0.43 & 30.7 & $\mathrm{R}$ & 2.1 & 0.06 & $U$ & 0.06 & 7.1 & & 0.70 \\
\hline
\end{tabular}

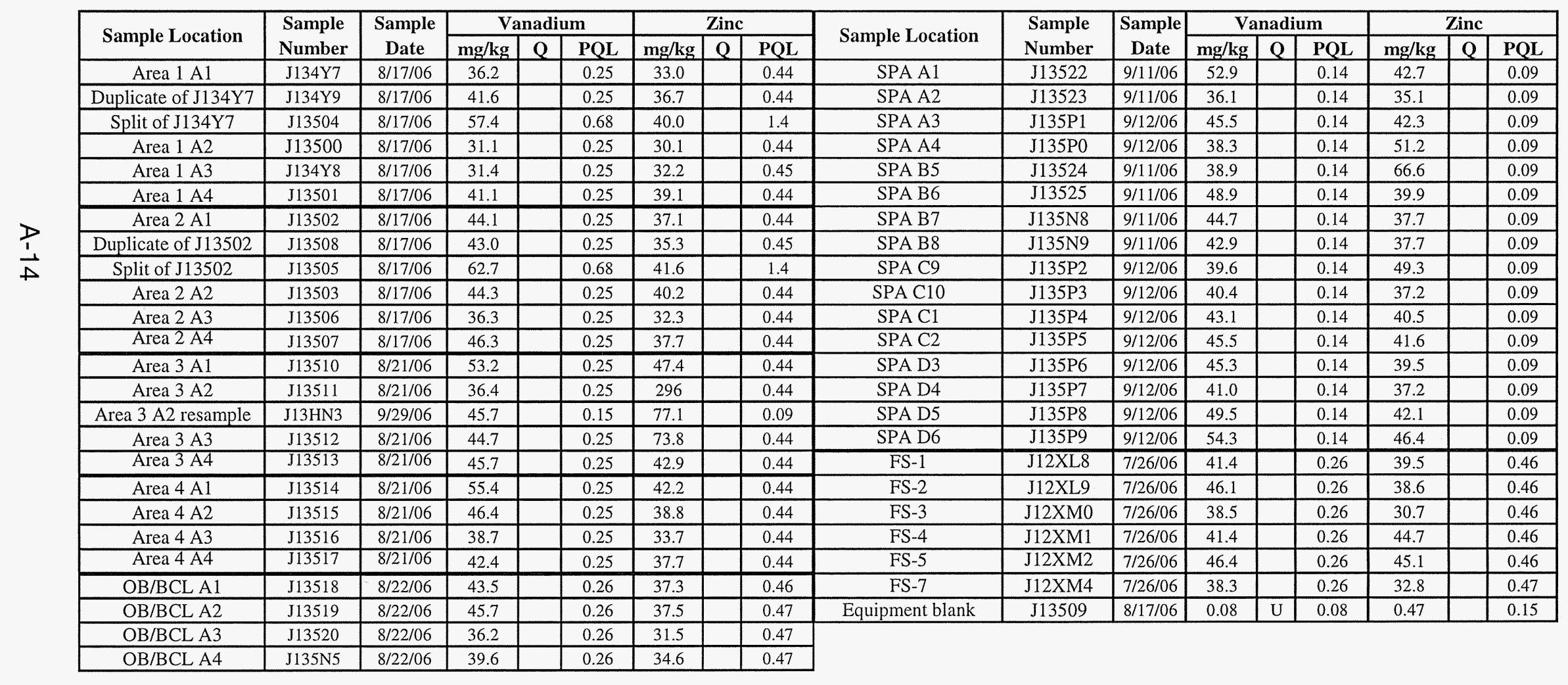


118-C-1 Verification Sampling Results.

\begin{tabular}{|c|c|c|c|c|c|c|c|c|c|c|c|}
\hline \multirow[t]{2}{*}{ Sample Location } & \multirow{2}{*}{$\begin{array}{l}\text { Sample } \\
\text { Number }\end{array}$} & \multirow{2}{*}{$\begin{array}{c}\text { Sample } \\
\text { Date }\end{array}$} & \multicolumn{3}{|c|}{$\begin{array}{l}\text { Total Petroleum } \\
\text { Hydrocarbons }\end{array}$} & \multirow[t]{2}{*}{ Sample Location } & \multirow{2}{*}{$\begin{array}{l}\text { Sample } \\
\text { Number }\end{array}$} & \multirow{2}{*}{$\begin{array}{c}\text { Sample } \\
\text { Date }\end{array}$} & \multicolumn{3}{|c|}{$\begin{array}{l}\text { Total Petroleum } \\
\text { Hydrocarbons }\end{array}$} \\
\hline & & & $\mathrm{mg} / \mathrm{kg}$ & $\mathbf{Q}$ & PQL & & & & $\mathrm{mg} / \mathrm{kg}$ & $Q$ & PQL \\
\hline Area $2 \mathrm{~A} 1$ & J135R0 & $8 / 28 / 06$ & 137 & $\mathrm{U}$ & 137 & SPA A1 & $\mathrm{J} 13522$ & $9 / 11 / 06$ & 134 & $U$ & 134 \\
\hline Duplicate of J135R0 & J135T6 & $8 / 28 / 06$ & 141 & $U$ & 141 & SPA A2 & $\mathrm{J} 13523$ & $9 / 11 / 06$ & 132 & $\mathrm{U}$ & 132 \\
\hline Split of J135R0 & $\mathrm{J} 135 \mathrm{~T} 7$ & $8 / 28 / 06$ & 173 & UN & 173 & SPA A3 & J135P1 & $9 / 12 / 06$ & 133 & $\mathrm{UC}$ & 133 \\
\hline Area $2 \mathrm{~A} 2$ & $\mathrm{~J} 135 \mathrm{R} 1$ & $8 / 28 / 06$ & 137 & U & 137 & SPA A4 & $\mathrm{J} 135 \mathrm{P} 0$ & $9 / 12 / 06$ & 135 & $\mathrm{CJ}$ & 134 \\
\hline Area $2 \mathrm{~A} 3$ & $\mathrm{~J} 135 \mathrm{R} 2$ & $8 / 28 / 06$ & 139 & $\mathrm{U}$ & 139 & SPA B5 & $\mathrm{J} 13524$ & $9 / 11 / 06$ & 132 & $\mathrm{U}$ & 132 \\
\hline Area 2 A4 & J135R3 & $8 / 28 / 06$ & 149 & $\mathrm{U}$ & 149 & SPA B6 & $\mathrm{J} 13525$ & $9 / 11 / 06$ & 133 & $U$ & 133 \\
\hline Area $3 \mathrm{A1}$ & J135R4 & $8 / 28 / 06$ & 135 & $\mathrm{U}$ & 135 & SPA B7 & $\mathrm{J} 135 \mathrm{~N} 8$ & $9 / 11 / 06$ & 133 & $\mathrm{U}$ & 133 \\
\hline Area 3 A2 & J135R5 & $8 / 28 / 06$ & 133 & $\mathrm{U}$ & 133 & SPA B 8 & J135N9 & $9 / 11 / 06$ & 133 & U & 133 \\
\hline Area $3 \mathrm{~A} 3$ & J135R6 & $8 / 28 / 06$ & 135 & $\mathrm{U}$ & 135 & SPA C9 & $\mathrm{J} 135 \mathrm{P} 2$ & $9 / 12 / 06$ & 132 & UC & 132 \\
\hline Area $3 \mathrm{A4}$ & J135R7 & $8 / 28 / 06$ & 348 & & 133 & SPA C10 & $\mathrm{J} 135 \mathrm{P} 3$ & $9 / 12 / 06$ & 133 & UC & 133 \\
\hline Area 3 A4 resample & J13HN4 & 9/29/06 & 135 & $\mathrm{U}$ & 135 & SPA C1 & $\mathrm{J} 135 \mathrm{P} 4$ & $9 / 12 / 06$ & 133 & UC & 133 \\
\hline Area $4 \mathrm{Al}$ & $\mathrm{J} 135 \mathrm{R} 8$ & $8 / 28 / 06$ & 143 & $\mathrm{U}$ & 143 & SPA C2 & J135P5 & $9 / 12 / 06$ & 133 & UC & 133 \\
\hline Area 4 A2 & J135R9 & $8 / 28 / 06$ & 133 & $\mathrm{U}$ & 133 & SPA D3 & $\mathrm{J} 135 \mathrm{P} 6$ & $9 / 12 / 06$ & 133 & $\mathrm{UC}$ & 133 \\
\hline Area 4 A3 & $\mathrm{J} 135 \mathrm{~T} 0$ & \begin{tabular}{|l|}
$8 / 28 / 06$ \\
\end{tabular} & 144 & $\mathrm{U}$ & 144 & SPA D4 & $\mathrm{J} 135 \mathrm{P} 7$ & $9 / 12 / 06$ & 146 & $\mathrm{CJ}$ & 134 \\
\hline Area 4 A4 & $\mathrm{J} 135 \mathrm{~T} 1$ & $8 / 28 / 06$ & 142 & $\mathrm{U}$ & 142 & SPA D5 & J135P8 & $9 / 12 / 06$ & 133 & UC & 133 \\
\hline $\mathrm{OB} / \mathrm{BCL} \mathrm{A} 1$ & $\mathrm{~J} 135 \mathrm{~T} 2$ & $8 / 28 / 06$ & 133 & $\mathrm{U}$ & 133 & SPA D6 & J135P9 & $9 / 12 / 06$ & 133 & UC & 133 \\
\hline $\mathrm{OB} / \mathrm{BCL} \mathrm{A} 2$ & $\mathrm{~J} 135 \mathrm{~T} 3$ & $8 / 28 / 06$ & 146 & $\mathrm{U}$ & 146 & FS-5 & J12XM2 & $7 / 26 / 06$ & 133 & $\mathrm{U}$ & 133 \\
\hline $\mathrm{OB} / \mathrm{BCL} \mathrm{A} 3$ & $\mathrm{~J} 135 \mathrm{~T} 4$ & $8 / 28 / 06$ & 138 & $\mathrm{U}$ & 138 & FS-6 & J12XM3 & $7 / 26 / 06$ & 135 & $\mathrm{U}$ & 135 \\
\hline
\end{tabular}


CVP-2006-00011

Rev. 0

118-C-1 Verification Sampling Results.

\begin{tabular}{|c|c|c|c|c|c|c|c|c|c|c|c|c|}
\hline \multirow[t]{2}{*}{ Constituents } & \multicolumn{3}{|c|}{\begin{tabular}{|c|}
$\mathrm{J} 13502$ \\
Area $2 \mathrm{~A} 1$ \\
Sample Date $8 / 17 / 06$ \\
\end{tabular}} & \multicolumn{3}{|c|}{\begin{tabular}{|c|}
$\mathbf{J 1 3 5 0 8}$ \\
Duplicate of $\mathbf{J 1 3 5 0 2}$ \\
Sample Date $8 / 17 / 06$ \\
\end{tabular}} & \multicolumn{3}{|c|}{$\begin{array}{c}\mathrm{J} 13505 \\
\text { Split of J13502 } \\
\text { Sample Date 8/17/06 }\end{array}$} & \multicolumn{3}{|c|}{$\begin{array}{c}\mathbf{J 1 3 5 0 3} \\
\text { Area } 2 \text { A2 } \\
\text { Sample Date 8/17/06 } \\
\end{array}$} \\
\hline & $\mu \mathrm{g} / \mathrm{kg}$ & $\mathbf{Q}$ & PQL & $\mu \mathrm{g} / \mathrm{kg}$ & $Q$ & PQL & $\mu \mathrm{g} / \mathrm{kg}$ & $\mathbf{Q}$ & PQL & $\mu \mathrm{g} / \mathrm{kg}$ & Q & PQL \\
\hline \multicolumn{13}{|c|}{ Polychlorinated Biphenyls } \\
\hline Aroclor-1016 & 13 & $\mathrm{U}$ & 13 & 14 & $\mathrm{U}$ & 14 & 17 & $\mathrm{U}$ & 17 & 13 & $\mathrm{U}$ & 13 \\
\hline Aroclor-1221 & 13 & $\mathrm{U}$ & 13 & 14 & $\mathrm{U}$ & 14 & 17 & $\mathrm{U}$ & 17 & 13 & $\mathrm{U}$ & 13 \\
\hline Aroclor-1232 & 13 & $\mathrm{U}$ & 13 & 14 & $\mathrm{U}$ & 14 & 17 & $\mathrm{U}$ & 17 & 13 & $\mathrm{U}$ & 13 \\
\hline Aroclor-1242 & 13 & $\mathrm{U}$ & 13 & 14 & $\mathrm{U}$ & 14 & 17 & $\mathrm{U}$ & 17 & 13 & $\mathrm{U}$ & 13 \\
\hline Aroclor-1248 & 13 & $\mathrm{U}$ & 13 & 14 & $\mathrm{U}$ & 14 & 17 & $\mathrm{U}$ & 17 & 13 & $\mathrm{U}$ & 13 \\
\hline Aroclor-1254 & 13 & $\mathrm{U}$ & 13 & 14 & $\mathrm{U}$ & 14 & 17 & $\mathrm{U}$ & 17 & 13 & $\mathrm{U}$ & 13 \\
\hline Aroclor- 1260 & 13 & $\mathrm{U}$ & 13 & 14 & $\mathrm{U}$ & 14 & 17 & $\mathrm{U}$ & 17 & 13 & $\mathrm{U}$ & 13 \\
\hline \multicolumn{13}{|c|}{ Semivolatile Organic Compounds } \\
\hline 1,2,4-Trichlorobenzene & 340 & $\mathrm{U}$ & 340 & 340 & $\mathrm{U}$ & 340 & 330 & $\mathrm{U}$ & 330 & 340 & $\mathrm{U}$ & 340 \\
\hline 1,2-Dichlorobenzene & 340 & $\mathrm{U}$ & 340 & 340 & $\mathrm{U}$ & 340 & 330 & $\mathrm{U}$ & 330 & 340 & $\mathrm{U}$ & 340 \\
\hline 1,3-Dichlorobenzene & 340 & $\mathrm{U}$ & 340 & 340 & $\mathrm{U}$ & 340 & 330 & $\mathrm{U}$ & 330 & 340 & $\mathrm{U}$ & 340 \\
\hline 1,4-Dichlorobenzene & 340 & $\mathrm{U}$ & 340 & 340 & $\mathrm{U}$ & 340 & 330 & $\mathrm{U}$ & 330 & 340 & $\mathrm{U}$ & 340 \\
\hline 2,4,5-Trichlorophenol & 840 & $\mathrm{U}$ & 840 & 840 & $\mathrm{U}$ & 840 & 330 & $\mathrm{U}$ & 330 & 840 & $\mathrm{U}$ & 840 \\
\hline 2,4,6-Trichlorophenol & 340 & $U$ & 340 & 340 & $\mathrm{U}$ & 340 & 330 & $\mathrm{U}$ & 330 & 340 & $\mathrm{U}$ & 340 \\
\hline 2,4-Dichlorophenol & 340 & $\mathrm{U}$ & 340 & 340 & $\mathrm{U}$ & 340 & 330 & $\mathrm{U}$ & 330 & 340 & $\mathrm{U}$ & 340 \\
\hline 2,4-Dimethylphenol & 340 & $\mathrm{U}$ & 340 & 340 & $\mathrm{U}$ & 340 & 330 & $\mathrm{U}$ & 330 & 340 & $\mathrm{U}$ & 340 \\
\hline 2,4-Dinitrophenol & 840 & $\mathrm{U}$ & 840 & 840 & $\mathrm{U}$ & 840 & 1600 & UNR & 1600 & 840 & $\mathrm{U}$ & 840 \\
\hline 2,4-Dinitrotoluene & 340 & $\mathrm{U}$ & 340 & 340 & $\mathrm{U}$ & 340 & 330 & $\mathrm{U}$ & 330 & 340 & $\mathrm{U}$ & 340 \\
\hline 2,6-Dinitrotoluene & 340 & $\mathrm{U}$ & 340 & 340 & $\mathrm{U}$ & 340 & 330 & $\mathrm{U}$ & 330 & 340 & $\mathrm{U}$ & 340 \\
\hline 2-Chloronaphthalene & 340 & $\mathrm{U}$ & 340 & 340 & $\mathrm{U}$ & 340 & 330 & $\mathrm{U}$ & 330 & 340 & $\mathrm{U}$ & 340 \\
\hline 2-Chlorophenol & 340 & $\mathrm{U}$ & 340 & 340 & $\mathrm{U}$ & 340 & 330 & $\mathrm{U}$ & 330 & 340 & $\mathrm{U}$ & 340 \\
\hline 2-Methylnaphthalene & 340 & $\mathrm{U}$ & 340 & 340 & $\mathrm{U}$ & 340 & 330 & $\mathrm{U}$ & 330 & 340 & $\mathrm{U}$ & 340 \\
\hline 2-Methylphenol (cresol, o-) & 340 & $\mathrm{U}$ & 340 & 340 & $\mathrm{U}$ & 340 & 330 & $\mathrm{U}$ & 330 & 340 & $\mathrm{U}$ & 340 \\
\hline 2-Nitroaniline & 840 & $\mathrm{U}$ & 840 & 840 & $\mathrm{U}$ & 840 & 1600 & $\mathrm{U}$ & 1600 & 840 & $\mathrm{U}$ & 840 \\
\hline 2-Nitrophenol & 340 & $\mathrm{U}$ & 340 & 340 & $\mathrm{U}$ & 340 & 330 & $\mathrm{U}$ & 330 & 340 & $\mathrm{U}$ & 340 \\
\hline 3,3'-Dichlorobenzidine & 340 & $\mathrm{U}$ & 340 & 340 & $\mathrm{U}$ & 340 & 1600 & $\mathrm{U}$ & 1600 & 340 & $\mathrm{U}$ & 340 \\
\hline 3-Nitroaniline & 840 & $\mathrm{U}$ & 840 & 840 & $\mathrm{U}$ & 840 & 1600 & $\mathrm{U}$ & 1600 & 840 & $\mathrm{U}$ & 840 \\
\hline 4,6-Dinitro-2-methylphenol & 840 & $\mathrm{U}$ & 840 & 840 & $\mathrm{U}$ & 840 & 1600 & $\mathrm{U}$ & 1600 & 840 & $\mathrm{U}$ & 840 \\
\hline 4-Bromophenyl-phenylether & 340 & $\mathrm{U}$ & 340 & 340 & $\mathrm{U}$ & 340 & 330 & $\mathrm{U}$ & 330 & 340 & $\mathrm{U}$ & 340 \\
\hline 4-Chloro-3-methylphenol & 340 & $\mathrm{U}$ & 340 & 340 & $\mathrm{U}$ & 340 & 330 & $\mathrm{U}$ & 330 & 340 & $\mathrm{U}$ & 340 \\
\hline 4-Chloroaniline & 340 & $\mathrm{U}$ & 340 & 340 & $\mathrm{U}$ & 340 & 330 & $\mathrm{U}$ & 330 & 340 & $\mathrm{U}$ & 340 \\
\hline 4-Chlorophenyl-phenylether & 340 & $\mathrm{U}$ & 340 & 340 & $\mathrm{U}$ & 340 & 330 & $\mathrm{U}$ & 330 & 340 & $\mathrm{U}$ & 340 \\
\hline 4-Methylphenol (p-cresol) & 340 & $\mathrm{U}$ & 340 & 340 & $\mathrm{U}$ & 340 & 670 & $\mathrm{U}$ & 670 & 340 & $\mathrm{U}$ & 340 \\
\hline 4-Nitroaniline & 840 & $\mathrm{U}$ & 840 & 840 & $\mathrm{U}$ & 840 & 1600 & $\mathrm{U}$ & 1600 & 840 & $\mathrm{U}$ & 840 \\
\hline 4-Nitrophenol & 840 & $\mathrm{U}$ & 840 & 840 & $\mathrm{U}$ & 840 & 1600 & $\mathrm{U}$ & 1600 & 840 & $\mathrm{U}$ & 840 \\
\hline Acenaphthene & 340 & $\mathrm{U}$ & 340 & 340 & $\mathrm{U}$ & 340 & 330 & $\mathrm{U}$ & 330 & 340 & $\mathrm{U}$ & 340 \\
\hline Acenaphthylene & 340 & $\mathrm{U}$ & 340 & 340 & $\mathrm{U}$ & 340 & 330 & $\mathrm{U}$ & 330 & 340 & $\mathrm{U}$ & 340 \\
\hline Anthracene & 340 & $\mathrm{U}$ & 340 & 340 & $\mathrm{U}$ & 340 & 330 & $\mathrm{U}$ & 330 & 340 & $\mathrm{U}$ & 340 \\
\hline Benzo(a)anthracene & 340 & $\mathrm{U}$ & 340 & 340 & $\mathrm{U}$ & 340 & 330 & $\mathrm{U}$ & 330 & 340 & $\mathrm{U}$ & 340 \\
\hline Benzo(a)pyrene & 340 & $\mathrm{U}$ & 340 & 340 & $\mathrm{U}$ & 340 & 330 & $\mathrm{U}$ & 330 & 340 & $\mathrm{U}$ & 340 \\
\hline Benzo(b)fluoranthene & 340 & $\mathrm{U}$ & 340 & 340 & $\mathrm{U}$ & 340 & 330 & $\mathrm{U}$ & 330 & 340 & $\mathrm{U}$ & 340 \\
\hline Benzo(g,h,i)perylene & 340 & $\mathrm{U}$ & 340 & 340 & $\mathrm{U}$ & 340 & 330 & $\mathrm{U}$ & 330 & 340 & $\mathrm{U}$ & 340 \\
\hline Benzo(k)fluoranthene & 340 & $\mathrm{U}$ & 340 & 340 & $\mathrm{U}$ & 340 & 330 & $\mathrm{U}$ & 330 & 340 & $\mathrm{U}$ & 340 \\
\hline bis(2-Chloro-1-methylethyl)ether & 340 & $\mathrm{U}$ & 340 & 340 & $\mathrm{U}$ & 340 & 330 & $\mathrm{U}$ & 330 & 340 & $\mathrm{U}$ & 340 \\
\hline bis(2-Chloroethoxy)methane & 340 & $\mathrm{U}$ & 340 & 340 & $\mathrm{U}$ & 340 & 330 & $\mathrm{U}$ & 330 & 340 & $\mathrm{U}$ & 340 \\
\hline bis(2-Chloroethyl)ether & 340 & $\mathrm{U}$ & 340 & 340 & $\mathrm{U}$ & 340 & 330 & $\mathrm{U}$ & 330 & 340 & $\mathrm{U}$ & 340 \\
\hline bis(2-Ethylhexyl)phthalate & 38 & $\mathrm{JB}$ & 340 & 36 & $\mathrm{JB}$ & 340 & 330 & $\mathrm{U}$ & 330 & 38 & $\mathrm{JB}$ & 340 \\
\hline
\end{tabular}


CVP-2006-00011

Rev. 0

118-C-1 Verification Sampling Results.

\begin{tabular}{|c|c|c|c|c|c|c|c|c|c|c|c|c|}
\hline \multirow[t]{2}{*}{ Constituents } & \multicolumn{3}{|c|}{$\begin{array}{c}\mathrm{J13502} \\
\text { Area } 2 \mathrm{A1} \\
\text { Sample Date } 8 / 17 / 06\end{array}$} & \multicolumn{3}{|c|}{$\begin{array}{c}\mathrm{J13508} \\
\text { Duplicate of } \mathrm{J13502} \\
\text { Sample Date } 8 / 17 / 06 \\
\end{array}$} & \multicolumn{3}{|c|}{$\begin{array}{c}\mathrm{J} 13505 \\
\text { Split of } \mathrm{J13502} \\
\text { Sample Date 8/17/06 } \\
\end{array}$} & \multicolumn{3}{|c|}{$\begin{array}{c}\text { J13503 } \\
\text { Area } 2 \text { A2 } \\
\text { Sample Date 8/17/06 } \\
\end{array}$} \\
\hline & $\mu \mathrm{g} / \mathrm{kg}$ & $\mathbf{Q}$ & PQL & $\mu \mathrm{g} / \mathrm{kg}$ & $Q$ & $\mathrm{PQL}$ & $\mu \mathrm{g} / \mathrm{kg}$ & $\mathbf{Q}$ & MDL & $\mu \mathrm{g} / \mathrm{kg}$ & $Q$ & $\mathrm{PQL}$ \\
\hline \multicolumn{13}{|c|}{ Semivolatile Organic Compounds (continued) } \\
\hline Butylbenzylphthalate & 340 & $\mathrm{U}$ & 340 & 340 & $\mathrm{U}$ & 340 & 330 & $\mathrm{U}$ & 330 & 340 & $\mathrm{U}$ & 340 \\
\hline Carbazole & 340 & $\mathrm{U}$ & 340 & 340 & $\mathrm{U}$ & 340 & 330 & $\mathrm{U}$ & 330 & 340 & $\mathrm{U}$ & 340 \\
\hline Chrysene & 340 & $\mathrm{U}$ & 340 & 340 & $\mathrm{U}$ & 340 & 330 & $\mathrm{U}$ & 330 & 340 & $\mathrm{U}$ & 340 \\
\hline Di-n-butylphthalate & 22 & $\mathrm{JB}$ & 340 & 35 & $\mathrm{JB}$ & 340 & 330 & $\mathrm{U}$ & 330 & 340 & $\mathrm{U}$ & 340 \\
\hline Di-n-octylphthalate & 340 & $\mathrm{U}$ & 340 & 340 & $\mathrm{U}$ & 340 & 330 & $\mathrm{U}$ & 330 & 340 & $\mathrm{U}$ & 340 \\
\hline Dibenz $(\mathrm{a}, \mathrm{h})$ anthracene & 340 & $\mathrm{U}$ & 340 & 340 & $\mathrm{U}$ & 340 & 330 & $\mathrm{U}$ & 330 & 340 & $\mathrm{U}$ & 340 \\
\hline \begin{tabular}{|l} 
Dibenzofuran \\
\end{tabular} & 340 & $\mathrm{U}$ & 340 & 340 & $\mathrm{U}$ & 340 & 330 & $\mathrm{U}$ & 330 & 340 & $\mathrm{U}$ & 340 \\
\hline Diethylphthalate & 340 & $\mathrm{U}$ & 340 & 340 & $\mathrm{U}$ & 340 & 330 & $\mathrm{U}$ & 330 & 340 & $\mathrm{U}$ & 340 \\
\hline Dimethylphthalate & 340 & $\mathrm{U}$ & 340 & 340 & U & 340 & 330 & $\mathrm{U}$ & 330 & 340 & $\mathrm{U}$ & 340 \\
\hline Fluoranthene & 340 & $\mathrm{U}$ & 340 & 340 & $\mathrm{U}$ & 340 & 330 & $\mathrm{U}$ & 330 & 340 & $\mathrm{U}$ & 340 \\
\hline Fluorene & 340 & $\mathrm{U}$ & 340 & 340 & $\mathrm{U}$ & 340 & 330 & $\mathrm{U}$ & 330 & 340 & $\mathrm{U}$ & 340 \\
\hline Hexachlorobenzene & 340 & $\mathrm{U}$ & 340 & 340 & $\mathrm{U}$ & 340 & 330 & $\mathrm{U}$ & 330 & 340 & $\mathrm{U}$ & 340 \\
\hline Hexachlorobutadiene & 340 & $\mathrm{U}$ & 340 & 340 & $\mathrm{U}$ & 340 & 330 & $\mathrm{U}$ & 330 & 340 & $\mathrm{U}$ & 340 \\
\hline Hexachlorocyclopentadiene & 340 & $\mathrm{U}$ & 340 & 340 & $\mathrm{U}$ & 340 & 1600 & $\mathrm{U}$ & 1600 & 340 & $\mathrm{U}$ & 340 \\
\hline Hexachloroethane & 340 & $\mathrm{U}$ & 340 & 340 & $\mathrm{U}$ & 340 & 330 & $\mathrm{U}$ & 330 & 340 & $\mathrm{U}$ & 340 \\
\hline Indeno(1,2,3-cd)pyrene & 340 & $\mathrm{U}$ & 340 & 340 & $\mathrm{U}$ & 340 & 330 & $\mathrm{U}$ & 330 & 340 & $\mathrm{U}$ & 340 \\
\hline Isophorone & 340 & $\mathrm{U}$ & 340 & 340 & $\mathrm{U}$ & 340 & 330 & $\mathrm{U}$ & 330 & 340 & $\mathrm{U}$ & 340 \\
\hline N-Nitroso-di-n-dipropylamine & 340 & $\mathrm{U}$ & 340 & 340 & $\mathrm{U}$ & 340 & 330 & $\mathrm{U}$ & 330 & 340 & $\mathrm{U}$ & 340 \\
\hline \begin{tabular}{|l} 
N-Nitrosodiphenylamine \\
\end{tabular} & 340 & $\mathrm{U}$ & 340 & 340 & $\mathrm{U}$ & 340 & 330 & $\mathrm{U}$ & 330 & 340 & $\mathrm{U}$ & 340 \\
\hline Naphthalene & 340 & $\mathrm{U}$ & 340 & 340 & $\mathrm{U}$ & 340 & 330 & $\mathrm{U}$ & 330 & 340 & $\mathrm{U}$ & 340 \\
\hline \begin{tabular}{|l} 
Nitrobenzene \\
\end{tabular} & 340 & $\mathrm{U}$ & 340 & 340 & U & 340 & 330 & $\mathrm{U}$ & 330 & 340 & $\mathrm{U}$ & 340 \\
\hline \begin{tabular}{|l|} 
Pentachlorophenol \\
\end{tabular} & 840 & $\mathrm{U}$ & 840 & 840 & $\mathrm{U}$ & 840 & 1600 & $\mathrm{U}$ & 1600 & 840 & $\mathrm{U}$ & 840 \\
\hline Phenanthrene & 340 & $\mathrm{U}$ & 340 & 340 & $\mathrm{U}$ & 340 & 330 & $\mathrm{U}$ & 330 & 340 & $\mathrm{U}$ & 340 \\
\hline Phenol & 340 & $\mathrm{U}$ & 340 & 340 & $\mathrm{U}$ & 340 & 330 & $\mathrm{U}$ & 330 & 340 & $\mathrm{U}$ & 340 \\
\hline Pyrene & 340 & $\mathrm{U}$ & 340 & 340 & $\mathrm{U}$ & 340 & 330 & $\mathrm{U}$ & 330 & 340 & $\mathrm{U}$ & 340 \\
\hline
\end{tabular}


118-C-1 Verification Sampling Results.

\begin{tabular}{|c|c|c|c|c|c|c|c|c|c|c|c|c|}
\hline \multirow[t]{2}{*}{ Constituents } & \multicolumn{3}{|c|}{$\begin{array}{c}\text { J135R0 } \\
\text { Area } 2 \text { A1 } \\
\text { Sample Date } 8 / 28 / 06\end{array}$} & \multicolumn{3}{|c|}{$\begin{array}{c}\text { J135T6 } \\
\text { Duplicate of J135R0 } \\
\text { Sample Date 8/28/06 }\end{array}$} & \multicolumn{3}{|c|}{$\begin{array}{c}\text { J135T7 } \\
\text { Split of J135R0 } \\
\text { Sample Date 8/28/06 }\end{array}$} & \multicolumn{3}{|c|}{$\begin{array}{c}\text { J135R1 } \\
\text { Area 2 A2 } \\
\text { Sample Date 8/28/06 } \\
\end{array}$} \\
\hline & $\mu \mathrm{g} / \mathrm{kg}$ & $Q$ & PQL & $\mu \mathrm{g} / \mathrm{kg}$ & $\mathbf{Q}$ & PQL & $\mu \mathrm{g} / \mathrm{kg}$ & $\mathbf{Q}$ & MDL & $\mu \mathrm{g} / \mathrm{kg}$ & $\mathbf{Q}$ & PQL \\
\hline \multicolumn{13}{|c|}{ Volatile Organic Compounds } \\
\hline 1,1,1-Trichloroethane & 5 & $\mathrm{U}$ & 5 & 5 & $\mathrm{U}$ & 5 & 5.1 & $\mathrm{U}$ & 5.1 & 5 & $\mathrm{U}$ & 5 \\
\hline 1,1,2,2-Tetrachloroethane & 5 & $\mathrm{UJ}$ & 5 & 5 & UJ & 5 & 5.1 & $\mathrm{U}$ & 5.1 & 5 & UJ & 5 \\
\hline 1,1,2-Trichloroethane & 5 & $\mathrm{U}$ & 5 & 5 & $\mathrm{U}$ & 5 & 5.1 & $\mathrm{U}$ & 5.1 & 5 & $\mathrm{U}$ & 5 \\
\hline 1,1-Dichloroethane & 5 & $\mathrm{U}$ & 5 & 5 & $\mathrm{U}$ & 5 & 5.1 & $\mathrm{U}$ & 5.1 & 5 & $\mathrm{U}$ & 5 \\
\hline 1,1-Dichloroethene & 5 & $\mathrm{U}$ & 5 & 5 & $\mathrm{U}$ & 5 & 5.1 & $\mathrm{U}$ & 5.1 & 5 & $\mathrm{U}$ & 5 \\
\hline 1,2-Dichloroethane & 5 & $\mathrm{U}$ & 5 & 5 & $\mathrm{U}$ & 5 & 5.1 & $\mathrm{U}$ & 5.1 & 5 & $\mathrm{U}$ & 5 \\
\hline 1,2-Dichloroethene (total) & 5 & $\mathrm{U}$ & 5 & 5 & $\mathrm{U}$ & 5 & 10 & $\mathrm{U}$ & 10 & 5 & $\mathrm{U}$ & 5 \\
\hline 1,2-Dichloropropane & 5 & $\mathrm{U}$ & 5 & 5 & $\mathrm{U}$ & 5 & 5.1 & $\mathrm{U}$ & 5.1 & 5 & $\mathrm{U}$ & 5 \\
\hline 2-Butanone & 9 & $\mathrm{U}$ & 9 & 10 & $\mathrm{U}$ & 10 & 10 & $\mathrm{U}$ & 10 & 10 & $\mathrm{U}$ & 10 \\
\hline 2-Hexanone & 9 & $\mathrm{U}$ & 9 & 10 & $\mathrm{U}$ & 10 & 20 & $\mathrm{U}$ & 20 & 10 & $\mathrm{U}$ & 10 \\
\hline 4-Methyl-2-pentanone & 9 & $\mathrm{U}$ & 9 & 10 & $\mathrm{U}$ & 10 & 10 & $\mathrm{U}$ & 10 & 10 & $\mathrm{U}$ & 10 \\
\hline Acetone & 9 & $\mathrm{U}$ & 9 & 10 & $\mathrm{U}$ & 10 & 20 & $U$ & 20 & 10 & $\mathrm{U}$ & 10 \\
\hline Benzene & 5 & $\mathrm{U}$ & 5 & 5 & $\mathrm{U}$ & 5 & 5.1 & $\mathrm{U}$ & 5.1 & 5 & $\mathrm{U}$ & 5 \\
\hline Bromodichloromethane & 5 & $\mathrm{U}$ & 5 & 5 & $\mathrm{U}$ & 5 & 5.1 & $\mathrm{U}$ & 5.1 & 5 & $\mathrm{U}$ & 5 \\
\hline Bromoform & 5 & $\mathrm{U}$ & 5 & 5 & $\mathrm{U}$ & 5 & 5.1 & $\mathrm{U}$ & 5.1 & 5 & $\mathrm{U}$ & 5 \\
\hline Bromomethane & 9 & $\mathrm{U}$ & 9 & 10 & $\mathrm{U}$ & 10 & 10 & $\mathrm{U}$ & 10 & 10 & $\mathrm{U}$ & 10 \\
\hline Carbon disulfide & 5 & $\mathrm{U}$ & 5 & 5 & $\mathrm{U}$ & 5 & 5.1 & $\mathrm{U}$ & 5.1 & 5 & $\mathrm{U}$ & 5 \\
\hline Carbon tetrachloride & 5 & $\mathrm{U}$ & 5 & 5 & $\mathrm{U}$ & 5 & 5.1 & $\mathrm{U}$ & 5.1 & 5 & $\mathrm{U}$ & 5 \\
\hline Chlorobenzene & 5 & $\mathrm{U}$ & 5 & 5 & $U$ & 5 & 5.1 & $U$ & 5.1 & 5 & $\mathrm{U}$ & 5 \\
\hline Chloroethane & 9 & $\mathrm{U}$ & 9 & 10 & $\mathrm{U}$ & 10 & 10 & $\mathrm{U}$ & 10 & 10 & $\mathrm{U}$ & 10 \\
\hline Chloroform & 5 & $\mathrm{U}$ & 5 & 5 & $\mathrm{U}$ & 5 & 5.1 & $\mathrm{U}$ & 5.1 & 5 & $\mathrm{U}$ & 5 \\
\hline Chloromethane & 9 & $\mathrm{U}$ & 9 & 10 & $\mathrm{U}$ & 10 & 10 & $\mathrm{U}$ & 10 & 10 & $\mathrm{U}$ & 10 \\
\hline cis-1,2-Dichloroethylene & 5 & $\mathrm{U}$ & 5 & 5 & $\mathrm{U}$ & 5 & & & & 5 & $\mathrm{U}$ & 5 \\
\hline cis-1,3-Dichloropropene & 5 & $\mathrm{U}$ & 5 & 5 & $\mathrm{U}$ & 5 & 5.1 & $\mathrm{U}$ & 5.1 & 5 & $\mathrm{U}$ & 5 \\
\hline Dibromochloromethane & 5 & $\mathrm{U}$ & 5 & 5 & $\mathrm{U}$ & 5 & 5.1 & $\mathrm{U}$ & 5.1 & 5 & $\mathrm{U}$ & 5 \\
\hline Ethylbenzene & 5 & $\mathrm{U}$ & 5 & 5 & $\mathrm{U}$ & 5 & 5.1 & $\mathrm{U}$ & 5.1 & 5 & $\mathrm{U}$ & 5 \\
\hline Methylene chloride & 5 & $\mathrm{~B}$ & 5 & 11 & $\mathrm{~B}$ & 5 & 5.1 & $\mathrm{U}$ & 5.1 & 6 & $\mathrm{~B}$ & 5 \\
\hline Styrene & 5 & $\mathrm{U}$ & 5 & 5 & $\mathrm{U}$ & 5 & 5.1 & $\mathrm{U}$ & 5.1 & 5 & $\mathrm{U}$ & 5 \\
\hline Tetrachloroethene & 5 & $\mathrm{U}$ & 5 & 5 & $\mathrm{U}$ & 5 & 5.1 & UN & 5.1 & 5 & $\mathrm{U}$ & 5 \\
\hline Toluene & 5 & $\mathrm{U}$ & 5 & 5 & $\mathrm{U}$ & 5 & 5.1 & $\mathrm{U}$ & 5.1 & 5 & $\mathrm{U}$ & 5 \\
\hline trans-1,2-Dichloroethylene & 5 & $\mathrm{U}$ & 5 & 5 & $\mathrm{U}$ & 5 & & & & 5 & $\mathrm{U}$ & 5 \\
\hline trans-1,3-Dichloropropene & 5 & $\mathrm{U}$ & 5 & 5 & $\mathrm{U}$ & 5 & 5.1 & $\mathrm{U}$ & 5.1 & 5 & $\mathrm{U}$ & 5 \\
\hline Trichloroethene & 5 & $\mathrm{U}$ & 5 & 5 & $\mathrm{U}$ & 5 & 5.1 & $\bar{U}$ & 5.1 & 5 & $\mathrm{U}$ & 5 \\
\hline Vinyl chloride & 9 & $\mathrm{U}$ & 9 & 10 & $\mathrm{U}$ & 10 & 5.1 & $\mathrm{U}$ & 5.1 & 10 & $\mathrm{U}$ & 10 \\
\hline Xylenes (total) & 5 & $\mathrm{U}$ & 5 & 5 & $\mathrm{U}$ & 5 & 10 & $\mathrm{U}$ & 10 & 5 & $\mathrm{U}$ & 5 \\
\hline
\end{tabular}


CVP-2006-00011

Rev. 0

118-C-1 Verification Sampling Results.

\begin{tabular}{|c|c|c|c|c|c|c|c|c|c|c|c|c|}
\hline \multirow[t]{2}{*}{ Constituents } & \multicolumn{3}{|c|}{$\begin{array}{c}\text { J13506 } \\
\text { Area } 2 \text { A3 } \\
\text { Sample Date 8/17/06 }\end{array}$} & \multicolumn{3}{|c|}{$\begin{array}{c}\text { J13507 } \\
\text { Area } 2 \mathrm{A4} \\
\text { Sample Date } 8 / 17 / 06\end{array}$} & \multicolumn{3}{|c|}{$\begin{array}{c}\text { J13510 } \\
\text { Area } 3 \text { A1 } \\
\text { Sample Date 8/21/06 }\end{array}$} & \multicolumn{3}{|c|}{$\begin{array}{c}\mathbf{J 1 3 5 1 1} \\
\text { Area } 3 \mathrm{~A} 2 \\
\text { Sample Date 8/21/06 }\end{array}$} \\
\hline & $\mu \mathrm{g} / \mathrm{kg}$ & $\mathbf{Q}$ & $\mathrm{PQL}$ & $\mu \mathrm{g} / \mathrm{kg}$ & $Q$ & PQL & $\mu \mathrm{g} / \mathrm{kg}$ & Q & PQL & $\mu \mathrm{g} / \mathrm{kg}$ & $Q$ & $\mathrm{PQL}$ \\
\hline \multicolumn{13}{|c|}{ Polychlorinated Biphenyls } \\
\hline Aroclor-1016 & 13 & $\mathrm{U}$ & 13 & 13 & $\mathrm{U}$ & 13 & 13 & $\bar{U}$ & 13 & 13 & $\mathrm{U}$ & 13 \\
\hline Aroclor-1221 & 13 & $\mathrm{U}$ & 13 & 13 & $\mathrm{U}$ & 13 & 13 & $\mathrm{U}$ & 13 & 13 & $\mathrm{U}$ & 13 \\
\hline Aroclor-1232 & 13 & $\mathrm{U}$ & 13 & 13 & $\mathrm{U}$ & 13 & 13 & $\mathrm{U}$ & 13 & 13 & $\mathrm{U}$ & 13 \\
\hline Aroclor-1242 & 13 & $\mathrm{U}$ & 13 & 13 & $\mathrm{U}$ & 13 & 13 & $\mathrm{U}$ & 13 & 13 & $\mathrm{U}$ & 13 \\
\hline Aroclor-1248 & 13 & $\mathrm{U}$ & 13 & 13 & $\mathrm{U}$ & 13 & 13 & $\mathrm{U}$ & 13 & 13 & $\mathrm{U}$ & 13 \\
\hline Aroclor-1254 & 13 & $\mathrm{U}$ & 13 & 13 & $\mathrm{U}$ & 13 & 14 & & 13 & 46 & & 13 \\
\hline Aroclor-1260 & 13 & $\mathrm{U}$ & 13 & 13 & $\mathrm{U}$ & 13 & 13 & $\mathrm{U}$ & 13 & 13 & $\mathrm{U}$ & 13 \\
\hline \multicolumn{13}{|c|}{ Semivolatile Organic Compounds } \\
\hline 1,2,4-Trichlorobenzene & 340 & $U$ & 340 & 340 & $\mathrm{U}$ & 340 & 330 & U & 330 & 330 & $\mathrm{U}$ & 330 \\
\hline 1,2-Dichlorobenzene & 340 & $\mathrm{U}$ & 340 & 340 & $\mathrm{U}$ & 340 & 330 & $\mathrm{U}$ & 330 & 330 & $\mathrm{U}$ & 330 \\
\hline 1,3-Dichlorobenzene & 340 & $\mathrm{U}$ & 340 & 340 & $\mathrm{U}$ & 340 & 330 & $\mathrm{U}$ & 330 & 330 & $\mathrm{U}$ & 330 \\
\hline 1,4-Dichlorobenzene & 340 & $\mathrm{U}$ & 340 & 340 & $\mathrm{U}$ & 340 & 330 & $\mathrm{U}$ & 330 & 330 & $\mathrm{U}$ & 330 \\
\hline 2,4,5-Trichloropl & 840 & $\mathrm{U}$ & 840 & 840 & $\mathrm{U}$ & 840 & 840 & $\mathrm{U}$ & 840 & 840 & $\mathrm{U}$ & 840 \\
\hline 2,4,6-Trichlorophenol & 340 & $\mathrm{U}$ & 340 & 340 & $\mathrm{U}$ & 340 & 330 & $\mathrm{U}$ & 330 & 330 & $\mathrm{U}$ & 330 \\
\hline 2,4-Dichlorophenol & 340 & $\mathrm{U}$ & 340 & 340 & $\mathrm{U}$ & 340 & 330 & $\mathrm{U}$ & 330 & 330 & $\mathrm{U}$ & 330 \\
\hline 2,4-Dimethylphenol & 340 & $\mathrm{U}$ & 340 & 340 & $\mathrm{U}$ & 340 & 330 & $\mathrm{U}$ & 330 & 330 & $\mathrm{U}$ & 330 \\
\hline 2,4-Dinitrophenol & 840 & $\mathrm{U}$ & 840 & 840 & $\mathrm{U}$ & 840 & 840 & $\mathrm{UJ}$ & 840 & 840 & UJ & 840 \\
\hline 2,4-Dinitrotoluene & 340 & $\mathrm{U}$ & 340 & 340 & $\mathrm{U}$ & 340 & 330 & $\mathrm{U}$ & 330 & 330 & $\mathrm{U}$ & 330 \\
\hline 2,6-Dinitrotoluene & 340 & $\mathrm{U}$ & 340 & 340 & $\mathrm{U}$ & 340 & 330 & $\mathrm{U}$ & 330 & 330 & $\mathrm{U}$ & 330 \\
\hline 2-Chloronaphthalen & 340 & $\mathrm{U}$ & 340 & 340 & $\mathrm{U}$ & 340 & 330 & $\mathrm{U}$ & 330 & 330 & $\mathrm{U}$ & 330 \\
\hline 2-Chlorophenol & 340 & $\mathrm{U}$ & 340 & 340 & $\mathrm{U}$ & 340 & 330 & $\mathrm{U}$ & 330 & 330 & $\mathrm{U}$ & 330 \\
\hline 2-MethyInaphthalene & 340 & $\mathrm{U}$ & 340 & 340 & $\mathrm{U}$ & 340 & 330 & $\mathrm{U}$ & 330 & 330 & $\mathrm{U}$ & 330 \\
\hline 2-Methylphenol (cresol, o-) & 340 & $\mathrm{U}$ & 340 & 340 & $\mathrm{U}$ & 340 & 330 & $\mathrm{U}$ & 330 & 330 & $\mathrm{U}$ & 330 \\
\hline 2-Nitroaniline & 840 & $\mathrm{U}$ & 840 & 840 & $\mathrm{U}$ & 840 & 840 & $\mathrm{U}$ & 840 & 840 & $\mathrm{U}$ & 840 \\
\hline 2-Nitrophenol & 340 & $\mathrm{U}$ & 340 & 340 & $\mathrm{U}$ & 340 & 330 & $\mathrm{U}$ & 330 & 330 & $\mathrm{U}$ & 330 \\
\hline 3,3'-Dichlorobenzidine & 340 & $\mathrm{U}$ & 340 & 340 & $\mathrm{U}$ & 340 & 330 & $\mathrm{U}$ & 330 & 330 & $\mathrm{U}$ & 330 \\
\hline 3-Nitroaniline & 840 & $\mathrm{U}$ & 840 & 840 & $\mathrm{U}$ & 840 & 840 & $\mathrm{U}$ & 840 & 840 & $\mathrm{U}$ & 840 \\
\hline 4,6-Dinitro-2-methylphenol & 840 & $\mathrm{U}$ & 840 & 840 & $\mathrm{U}$ & 840 & 840 & $\mathrm{U}$ & 840 & 840 & $\mathrm{U}$ & 840 \\
\hline 4-Bromophenyl-phenylether & 340 & $\mathrm{U}$ & 340 & 340 & $\mathrm{U}$ & 340 & 330 & $\mathrm{U}$ & 330 & 330 & $\mathrm{U}$ & 330 \\
\hline 4-Chloro-3-methylphenol & 340 & $\mathrm{U}$ & 340 & 340 & $\mathrm{U}$ & 340 & 330 & $\mathrm{U}$ & 330 & 330 & $\mathrm{U}$ & 330 \\
\hline 4-Chloroaniline & 340 & $\mathrm{U}$ & 340 & 340 & $\mathrm{U}$ & 340 & 330 & $\mathrm{U}$ & 330 & 330 & $\mathrm{U}$ & 330 \\
\hline 4-Chlorophenyl-phenylether & 340 & $\mathrm{U}$ & 340 & 340 & $\mathrm{U}$ & 340 & 330 & $\mathrm{U}$ & 330 & 330 & $\mathrm{U}$ & 330 \\
\hline 4-Methylphenol (p-cresol) & 340 & $\mathrm{U}$ & 340 & 340 & $\mathrm{U}$ & 340 & 330 & $\mathrm{U}$ & 330 & 330 & $\mathrm{U}$ & 330 \\
\hline 4-Nitroaniline & 840 & $\mathrm{U}$ & 840 & 840 & $\mathrm{U}$ & 840 & 840 & $\mathrm{U}$ & 840 & 840 & $\mathrm{U}$ & 840 \\
\hline 4-Nitrophenol & 840 & $\mathrm{U}$ & 840 & 840 & $\mathrm{U}$ & 840 & 840 & $\mathrm{U}$ & 840 & 840 & $\mathrm{U}$ & 840 \\
\hline Acenaphthene & 340 & $\mathrm{U}$ & 340 & 340 & $\mathrm{U}$ & 340 & 330 & $\mathrm{U}$ & 330 & 330 & $\mathrm{U}$ & 330 \\
\hline Acenaphthylene & 340 & $\mathrm{U}$ & 340 & 340 & $\mathrm{U}$ & 340 & 330 & $\mathrm{U}$ & 330 & 330 & $\mathrm{U}$ & 330 \\
\hline Anthrac & 340 & $\mathrm{U}$ & 340 & 340 & $\mathrm{U}$ & 340 & 330 & $\mathrm{U}$ & 330 & 330 & $\mathrm{U}$ & 330 \\
\hline Benzo(a)anthracene & 340 & $\mathrm{U}$ & 340 & 340 & $\mathrm{U}$ & 340 & 180 & $\mathrm{~J}$ & 330 & 330 & $\mathrm{U}$ & 330 \\
\hline Benzo(a)pyrene & 340 & $\mathrm{U}$ & 340 & 340 & $\mathrm{U}$ & 340 & 120 & $\mathrm{~J}$ & 330 & 330 & $\mathrm{U}$ & 330 \\
\hline Benzo(b)fluoranthene & 340 & $\mathrm{U}$ & 340 & 340 & $\mathrm{U}$ & 340 & 140 & $\mathrm{~J}$ & 330 & 330 & $\mathrm{U}$ & 330 \\
\hline perylene & 340 & $\mathrm{U}$ & 340 & 340 & $\mathrm{U}$ & 340 & 51 & $\mathrm{~J}$ & 330 & 330 & $\mathrm{U}$ & 330 \\
\hline Benzo(k)fluoranthene & 340 & $\mathrm{U}$ & 340 & 340 & $\mathrm{U}$ & 340 & 140 & $\mathrm{~J}$ & 330 & 330 & $\mathrm{U}$ & 330 \\
\hline bis(2-Chloro-1-methylethyl)ether & 340 & $\mathrm{U}$ & 340 & 340 & $\mathrm{U}$ & 340 & 330 & $\mathrm{U}$ & 330 & 330 & U & 330 \\
\hline bis(2-Chloroethoxy)methane & 340 & $\mathrm{U}$ & 340 & 340 & $\mathrm{U}$ & 340 & 330 & $\mathrm{U}$ & 330 & 330 & $\mathrm{U}$ & 330 \\
\hline bis(2-Chloroethyl)ether & 340 & $\mathrm{U}$ & 340 & 340 & $\mathrm{U}$ & 340 & 330 & $\mathrm{U}$ & 330 & 330 & $\mathrm{U}$ & 330 \\
\hline bis(2-Ethylhexyl)phthalate & 20 & $\mathrm{JB}$ & 340 & 400 & $\mathrm{~B}$ & 340 & 330 & $\mathrm{U}$ & 330 & 30 & $\mathrm{~J}$ & 330 \\
\hline
\end{tabular}


118-C-1 Verification Sampling Results.

\begin{tabular}{|c|c|c|c|c|c|c|c|c|c|c|c|c|}
\hline \multirow[t]{2}{*}{ Constituents } & \multicolumn{3}{|c|}{$\begin{array}{c}\text { J13506 } \\
\text { Area 2 A3 } \\
\text { Sample Date 8/17/06 }\end{array}$} & \multicolumn{3}{|c|}{$\begin{array}{c}\text { J13507 } \\
\text { Area } 2 \text { A4 } \\
\text { Sample Date 8/17/06 }\end{array}$} & \multicolumn{3}{|c|}{$\begin{array}{c}\text { J13510 } \\
\text { Area } 3 \text { A1 } \\
\text { Sample Date 8/21/06 }\end{array}$} & \multicolumn{3}{|c|}{$\begin{array}{c}\text { J13511 } \\
\text { Area } 3 \mathrm{A2} \\
\text { Sample Date 8/21/06 }\end{array}$} \\
\hline & $\mu \mathrm{g} / \mathrm{kg}$ & $\mathbf{Q}$ & PQL & $\mu \mathrm{g} / \mathrm{kg}$ & Q & PQL & $\mu \mathrm{g} / \mathrm{kg}$ & $\mathbf{Q}$ & PQL & $\mu \mathrm{g} / \mathrm{kg}$ & $\mathbf{Q}$ & PQL \\
\hline \multicolumn{13}{|c|}{ Semivolatile Organic Compounds (continued) } \\
\hline Butylbenzylphthalate & 340 & $\mathrm{U}$ & 340 & 340 & $\mathrm{U}$ & 340 & 330 & $\mathrm{U}$ & 330 & 330 & $\mathrm{U}$ & 330 \\
\hline Carbazole & 340 & $\mathrm{U}$ & 340 & 340 & $\mathrm{U}$ & 340 & 330 & $\mathrm{U}$ & 330 & 330 & $\mathrm{U}$ & 330 \\
\hline Chrysene & 340 & $\mathrm{U}$ & 340 & 340 & $\mathrm{U}$ & 340 & 210 & $\mathrm{~J}$ & 330 & 330 & $\mathrm{U}$ & 330 \\
\hline Di-n-butylphthalate & 340 & $\mathrm{U}$ & 340 & 340 & $\mathrm{U}$ & 340 & 51 & $\mathrm{~J}$ & 330 & 330 & $\mathrm{U}$ & 330 \\
\hline Di-n-octylphthalate & 340 & $\mathrm{U}$ & 340 & 340 & $\mathrm{U}$ & 340 & 330 & $\mathrm{U}$ & 330 & 330 & $U$ & 330 \\
\hline Dibenz(a,h)anthracene & 340 & $\mathrm{U}$ & 340 & 340 & $\mathrm{U}$ & 340 & 330 & $\mathrm{U}$ & 330 & 330 & $\mathrm{U}$ & 330 \\
\hline Dibenzofuran & 340 & $\mathrm{U}$ & 340 & 340 & $\mathrm{U}$ & 340 & 330 & $\mathrm{U}$ & 330 & 330 & $\mathrm{U}$ & 330 \\
\hline Diethylphthalate & 340 & $\mathrm{U}$ & 340 & 340 & $\mathrm{U}$ & 340 & 330 & $\mathrm{U}$ & 330 & 330 & $\mathrm{U}$ & 330 \\
\hline Dimethylphthalate & 340 & $\mathrm{U}$ & 340 & 340 & $\mathrm{U}$ & 340 & 330 & $\mathrm{U}$ & 330 & 330 & U & 330 \\
\hline Fluoranthene & 340 & $\mathrm{U}$ & 340 & 340 & $\bar{U}$ & 340 & 320 & $\mathrm{~J}$ & 330 & 330 & $\mathrm{U}$ & 330 \\
\hline Fluorene & 340 & $\mathrm{U}$ & 340 & 340 & $\mathrm{U}$ & 340 & 330 & $\mathrm{U}$ & 330 & 330 & $\mathrm{U}$ & 330 \\
\hline Hexachlorobenzene & 340 & $\mathrm{U}$ & 340 & 340 & $\mathrm{U}$ & 340 & 330 & $\mathrm{U}$ & 330 & 330 & $\mathrm{U}$ & 330 \\
\hline Hexachlorobutadiene & 340 & $\mathrm{U}$ & 340 & 340 & $\mathrm{U}$ & 340 & 330 & $\mathrm{U}$ & 330 & 330 & $\mathrm{U}$ & 330 \\
\hline Hexachlorocyclopentadiene & 340 & $\mathrm{U}$ & 340 & 340 & $\mathrm{U}$ & 340 & 330 & $\mathrm{U}$ & 330 & 330 & $\overline{\mathrm{U}}$ & 330 \\
\hline Hexachloroethane & 340 & $\mathrm{U}$ & 340 & 340 & $\mathrm{U}$ & 340 & 330 & $\mathrm{U}$ & 330 & 330 & $\mathrm{U}$ & 330 \\
\hline Indeno( $1,2,3$-cd)pyrene & 340 & $\mathrm{U}$ & 340 & 340 & $\mathrm{U}$ & 340 & 46 & $\mathrm{~J}$ & 330 & 330 & $\mathrm{U}$ & 330 \\
\hline Isophorone & 340 & $\mathrm{U}$ & 340 & 340 & $\mathrm{U}$ & 340 & 330 & $\mathrm{U}$ & 330 & 330 & $\mathrm{U}$ & 330 \\
\hline $\mathrm{N}$-Nitroso-di-n-dipropylamine & 340 & $\mathrm{U}$ & 340 & 340 & $\mathrm{U}$ & 340 & 330 & $\mathrm{U}$ & 330 & 330 & $\mathrm{U}$ & 330 \\
\hline N-Nitrosodiphenylamine & 340 & $\mathrm{U}$ & 340 & 340 & $\mathrm{U}$ & 340 & 330 & $\mathrm{U}$ & 330 & 330 & $\mathrm{U}$ & 330 \\
\hline Naphthalene & 340 & $\mathrm{U}$ & 340 & 340 & $\mathrm{U}$ & 340 & 330 & $\mathrm{U}$ & 330 & 330 & $\mathrm{U}$ & 330 \\
\hline Nitrobenzene & 340 & $\mathrm{U}$ & 340 & 340 & $\mathrm{U}$ & 340 & 330 & $\mathrm{U}$ & 330 & 330 & $\mathrm{U}$ & 330 \\
\hline Pentachlorophenol & 840 & $\mathrm{U}$ & 840 & 840 & $\mathrm{U}$ & 840 & 840 & $\mathrm{U}$ & 840 & 840 & $\mathrm{U}$ & 840 \\
\hline Phenanthrene & 340 & $\mathrm{U}$ & 340 & 340 & $\mathrm{U}$ & 340 & 55 & $\mathrm{~J}$ & 330 & 330 & $\mathrm{U}$ & 330 \\
\hline Phenol & 340 & $\mathrm{U}$ & 340 & 340 & $\mathrm{U}$ & 340 & 330 & $\mathrm{U}$ & 330 & 330 & $\mathrm{U}$ & 330 \\
\hline Pyrene & 340 & $\mathrm{U}$ & 340 & 340 & $\mathrm{U}$ & 340 & 320 & $\mathrm{~J}$ & 330 & 330 & $\mathrm{U}$ & 330 \\
\hline
\end{tabular}


118-C-1 Verification Sampling Results.

\begin{tabular}{|c|c|c|c|c|c|c|c|c|c|c|c|c|}
\hline \multirow[t]{2}{*}{ Constituents } & \multicolumn{3}{|c|}{$\begin{array}{c}\text { J135R2 } \\
\text { Area } 2 \text { A3 } \\
\text { Sample Date } 8 / 28 / 06\end{array}$} & \multicolumn{3}{|c|}{$\begin{array}{c}\text { J135R3 } \\
\text { Area } 2 \text { A4 } \\
\text { Sample Date 8/28/06 }\end{array}$} & \multicolumn{3}{|c|}{$\begin{array}{c}\text { J135R4 } \\
\text { Area } 3 \text { A1 } \\
\text { Sample Date } 8 / 21 / 06\end{array}$} & \multicolumn{3}{|c|}{$\begin{array}{c}\text { J135R5 } \\
\text { Area } 3 \text { A2 } \\
\text { Sample Date 8/28/06 }\end{array}$} \\
\hline & $\mu \mathrm{g} / \mathrm{kg}$ & $\mathbf{Q}$ & PQL & $\mu \mathrm{g} / \mathrm{kg}$ & $\mathbf{Q}$ & PQL & $\mu \mathrm{g} / \mathrm{kg}$ & $\mathbf{Q}$ & PQL & $\mu g / \mathrm{kg}$ & $\mathbf{Q}$ & $\mathrm{PQL}$ \\
\hline \multicolumn{13}{|c|}{ Volatile Organic Compounds } \\
\hline 1,1,1-Trichloroethane & 6 & $\mathrm{U}$ & 6 & 6 & $\mathrm{U}$ & 6 & 5 & $\mathrm{U}$ & 5 & 5 & $\mathrm{U}$ & 5 \\
\hline 1,1,2,2-Tetrachloroethane & 6 & UJ & 6 & 6 & UJ & 6 & 5 & UJ & 5 & 5 & UJ & 5 \\
\hline 1,1,2-Trichloroethane & 6 & $\mathrm{U}$ & 6 & 6 & $\mathrm{U}$ & 6 & 5 & $\mathrm{U}$ & 5 & 5 & $\mathrm{U}$ & 5 \\
\hline 1,1-Dichloroethane & 6 & $\mathrm{U}$ & 6 & 6 & $\mathrm{U}$ & 6 & 5 & $\mathrm{U}$ & 5 & 5 & $\mathrm{U}$ & 5 \\
\hline 1,1-Dichloroethene & 6 & $\mathrm{U}$ & 6 & 6 & $\mathrm{U}$ & 6 & 5 & $\mathrm{U}$ & 5 & 5 & $\mathrm{U}$ & 5 \\
\hline 1,2-Dichloroethane & 6 & $\mathrm{U}$ & 6 & 6 & $\mathrm{U}$ & 6 & 5 & $\mathrm{U}$ & 5 & 5 & $\mathrm{U}$ & 5 \\
\hline 1,2-Dichloroethene (total) & 6 & $\mathrm{U}$ & 6 & 6 & $\mathrm{U}$ & 6 & 5 & $\mathrm{U}$ & 5 & 5 & $\mathrm{U}$ & 5 \\
\hline 1,2-Dichloropropane & 6 & $\mathrm{U}$ & 6 & 6 & $\mathrm{U}$ & 6 & 5 & $\mathrm{U}$ & 5 & 5 & $\mathrm{U}$ & 5 \\
\hline 2-Butanone & 11 & $\mathrm{U}$ & 11 & 11 & $\mathrm{U}$ & 11 & 10 & $\mathrm{U}$ & 10 & 10 & $\mathrm{U}$ & 10 \\
\hline 2-Hexanone & 11 & $\mathrm{U}$ & 11 & 11 & $\mathrm{U}$ & 11 & 10 & $\mathrm{U}$ & 10 & 10 & $\mathrm{U}$ & 10 \\
\hline 4-Methyl-2-pentanone & 11 & $\mathrm{U}$ & 11 & 11 & $\mathrm{U}$ & 11 & 10 & $\mathrm{U}$ & 10 & 10 & $\mathrm{U}$ & 10 \\
\hline Acetone & 11 & $\mathrm{U}$ & 11 & 11 & $\mathrm{U}$ & 11 & 10 & $\mathrm{U}$ & 10 & 10 & $\mathrm{U}$ & 10 \\
\hline Benzene & 6 & $\mathrm{U}$ & 6 & 6 & $\mathrm{U}$ & 6 & 5 & $\mathrm{U}$ & 5 & 5 & $\mathrm{U}$ & 5 \\
\hline Bromodichloromethane & 6 & $\mathrm{U}$ & 6 & 6 & $\mathrm{U}$ & 6 & 5 & $\mathrm{U}$ & 5 & 5 & $\mathrm{U}$ & 5 \\
\hline Bromoform & 6 & $\mathrm{U}$ & 6 & 6 & $\mathrm{U}$ & 6 & 5 & $\mathrm{U}$ & 5 & 5 & $\mathrm{U}$ & 5 \\
\hline Bromomethane & 11 & $\mathrm{U}$ & 11 & 11 & $\mathrm{U}$ & 11 & 10 & $\mathrm{U}$ & 10 & 10 & $\mathrm{U}$ & 10 \\
\hline Carbon disulfide & 6 & $\mathrm{U}$ & 6 & 6 & $\mathrm{U}$ & 6 & 5 & $\mathrm{U}$ & 5 & 5 & $\mathrm{U}$ & 5 \\
\hline Carbon tetrachloride & 6 & $\mathrm{U}$ & 6 & 6 & $\mathrm{U}$ & 6 & 5 & $U$ & 5 & 5 & $\mathrm{U}$ & 5 \\
\hline Chlorobenzene & 6 & $\mathrm{U}$ & 6 & 6 & $\mathrm{U}$ & 6 & 5 & $\mathrm{U}$ & 5 & 5 & $\mathrm{U}$ & 5 \\
\hline Chloroethane & 11 & $\mathrm{U}$ & 11 & 11 & $\mathrm{U}$ & 11 & 10 & $\mathrm{U}$ & 10 & 10 & $\mathrm{U}$ & 10 \\
\hline Chloroform & 6 & $\mathrm{U}$ & 6 & 6 & $\mathrm{U}$ & 6 & 5 & $\mathrm{U}$ & 5 & 5 & U & 5 \\
\hline Chloromethane & 11 & $\mathrm{U}$ & 11 & 11 & $\mathrm{U}$ & 11 & 10 & $\mathrm{U}$ & 10 & 10 & $\mathrm{U}$ & 10 \\
\hline cis-1,2-Dichloroethylene & 6 & $\mathrm{U}$ & 6 & 6 & $\mathrm{U}$ & 6 & 5 & $\mathrm{U}$ & 5 & 5 & $\mathrm{U}$ & 5 \\
\hline cis-1,3-Dichloropropene & 6 & $\mathrm{U}$ & 6 & 6 & $\mathrm{U}$ & 6 & 5 & $\mathrm{U}$ & 5 & 5 & $\mathrm{U}$ & 5 \\
\hline Dibromochloromethane & 6 & $\mathrm{U}$ & 6 & 6 & $\mathrm{U}$ & 6 & 5 & $\mathrm{U}$ & 5 & 5 & $\mathrm{U}$ & 5 \\
\hline Ethylbenzene & 6 & $\mathrm{U}$ & 6 & 6 & $\mathrm{U}$ & 6 & 5 & $\mathrm{U}$ & 5 & 5 & $\mathrm{U}$ & 5 \\
\hline Methylene chloride & 9 & $\mathrm{~B}$ & 6 & 14 & $\mathrm{~B}$ & 6 & 12 & $\mathrm{~B}$ & 5 & 8 & $B$ & 5 \\
\hline Styrene & 6 & $\mathrm{U}$ & 6 & 6 & $\mathrm{U}$ & 6 & 5 & $\mathrm{U}$ & 5 & 5 & $\mathrm{U}$ & 5 \\
\hline Tetrachloroethene & 6 & $\mathrm{U}$ & 6 & 6 & $\mathrm{U}$ & 6 & 5 & $\mathrm{U}$ & 5 & 5 & $\mathrm{U}$ & 5 \\
\hline Toluene & 6 & $\mathrm{U}$ & 6 & 6 & $\mathrm{U}$ & 6 & 5 & $\mathrm{U}$ & 5 & 5 & $\mathrm{U}$ & 5 \\
\hline trans-1,2-Dichloroethylene & 6 & $\mathrm{U}$ & 6 & 6 & $\mathrm{U}$ & 6 & 5 & $\mathrm{U}$ & 5 & 5 & $\mathrm{U}$ & 5 \\
\hline trans-1,3-Dichloropropene & 6 & $\mathrm{U}$ & 6 & 6 & $\mathrm{U}$ & 6 & 5 & $\mathrm{U}$ & 5 & 5 & $\mathrm{U}$ & 5 \\
\hline Trichloroethene & 6 & $\mathrm{U}$ & 6 & 6 & $\mathrm{U}$ & 6 & 5 & $\mathrm{U}$ & 5 & 5 & $\mathrm{U}$ & 5 \\
\hline Vinyl chloride & 11 & $\mathrm{U}$ & 11 & 11 & $\mathrm{U}$ & 11 & 10 & $\mathrm{U}$ & 10 & 10 & $\mathrm{U}$ & 10 \\
\hline Xylenes (total) & 6 & $\mathrm{U}$ & 6 & 6 & $\mathrm{U}$ & 6 & 5 & $\mathrm{U}$ & 5 & 5 & $\mathrm{U}$ & 5 \\
\hline
\end{tabular}


118-C-1 Verification Sampling Results.

\begin{tabular}{|c|c|c|c|c|c|c|c|c|c|c|c|c|}
\hline \multirow[t]{2}{*}{ Constituents } & \multicolumn{3}{|c|}{$\begin{array}{c}\mathrm{J13512} \\
\text { Area } 3 \mathrm{A3} \\
\text { Sample Date } 8 / 21 / 06\end{array}$} & \multicolumn{3}{|c|}{$\begin{array}{c}\text { J13513 } \\
\text { Area } 3 \mathrm{A4} \\
\text { Sample Date } 8 / 21 / 06\end{array}$} & \multicolumn{3}{|c|}{$\begin{array}{c}\text { J13514 } \\
\text { Area } 4 \text { A1 } \\
\text { Sample Date } 8 / 21 / 06\end{array}$} & \multicolumn{3}{|c|}{$\begin{array}{c}\text { J13515 } \\
\text { Area } 4 \text { A2 } \\
\text { Sample Date 8/21/06 }\end{array}$} \\
\hline & $\mu \mathrm{g} / \mathrm{kg}$ & $Q$ & PQL & $\mu \mathrm{g} / \mathrm{kg}$ & $Q$ & PQL & $\mu \mathrm{g} / \mathrm{kg}$ & $Q$ & PQL & $\mu \mathrm{g} / \mathrm{kg}$ & $Q$ & PQL \\
\hline \multicolumn{13}{|c|}{ Polychlorinated Biphenyls } \\
\hline Aroclor-1016 & 13 & $\mathrm{U}$ & 13 & 13 & $\mathrm{U}$ & 13 & 13 & $\mathrm{U}$ & 13 & 13 & $\mathrm{U}$ & 13 \\
\hline Aroclor-1221 & 13 & $\bar{U}$ & 13 & 13 & $\mathrm{U}$ & 13 & 13 & $\mathrm{U}$ & 13 & 13 & $\mathrm{U}$ & 13 \\
\hline Aroclor-1232 & 13 & $\bar{U}$ & 13 & 13 & $\bar{U}$ & 13 & 13 & $\mathrm{U}$ & 13 & 13 & $\mathrm{U}$ & 13 \\
\hline Aroclor-1242 & 13 & $\bar{U}$ & 13 & 13 & $\bar{U}$ & 13 & 13 & $\mathrm{U}$ & 13 & 13 & $\mathrm{U}$ & 13 \\
\hline Aroclor-1248 & 13 & $\mathrm{U}$ & 13 & 13 & $\mathrm{U}$ & 13 & 13 & $\mathrm{U}$ & 13 & 13 & $\mathrm{U}$ & 13 \\
\hline Aroclor-1254 & 62 & & 13 & 21 & & 13 & 13 & $\mathrm{U}$ & 13 & 6.4 & $\mathrm{~J}$ & 13 \\
\hline Aroclor-1260 & 13 & $\bar{U}$ & 13 & 13 & $\bar{U}$ & 13 & 13 & $\mathrm{U}$ & 13 & 13 & $\mathrm{U}$ & 13 \\
\hline \multicolumn{13}{|c|}{ Semivolatile Organic Compounds } \\
\hline 1,2,4-Trichlorobenzene & 340 & $\mathrm{U}$ & 340 & 330 & $\mathrm{U}$ & 330 & 330 & $\mathrm{U}$ & 330 & 330 & $\mathrm{U}$ & 330 \\
\hline 1,2-Dichlorobenzene & 340 & $\mathrm{U}$ & 340 & 330 & $\mathrm{U}$ & 330 & 330 & $\mathrm{U}$ & 330 & 330 & $\mathrm{U}$ & 330 \\
\hline 1,3-Dichlorobenzene & 340 & $\mathrm{U}$ & 340 & 330 & $\bar{U}$ & 330 & 330 & $U$ & 330 & 330 & $\mathrm{U}$ & 330 \\
\hline 1,4-Dichlorobenzene & 340 & $\mathrm{U}$ & 340 & 330 & $\bar{U}$ & 330 & 330 & $\mathrm{U}$ & 330 & 330 & $\mathrm{U}$ & 330 \\
\hline 2,4,5-Trichlorophenol & 840 & $\mathrm{U}$ & 840 & 840 & $\mathrm{U}$ & 840 & 840 & $\mathrm{U}$ & 840 & 840 & $\mathrm{U}$ & 840 \\
\hline 2,4,6-Trichlorophenol & 340 & $\mathrm{U}$ & 340 & 330 & $\mathrm{U}$ & 330 & 330 & $\mathrm{U}$ & 330 & 330 & $\mathrm{U}$ & 330 \\
\hline 2,4-Dichlorophenol & 340 & $\mathrm{U}$ & 340 & 330 & $\mathrm{U}$ & 330 & 330 & $\mathrm{U}$ & 330 & 330 & $\mathrm{U}$ & 330 \\
\hline 2,4-Dimethylphenol & 340 & $\mathrm{U}$ & 340 & 330 & $\mathrm{U}$ & 330 & 330 & $\mathrm{U}$ & 330 & 330 & $U$ & 330 \\
\hline 2,4-Dinitrophenol & 840 & UJ & 840 & 840 & UJ & 840 & 840 & UJ & 840 & 840 & $\mathrm{UJ}$ & 840 \\
\hline 2,4-Dinitrotoluene & 340 & $\mathrm{U}$ & 340 & 330 & $\mathrm{U}$ & 330 & 330 & $\mathrm{U}$ & 330 & 330 & $\mathrm{U}$ & 330 \\
\hline 2,6-Dinitrotoluene & 340 & $\bar{U}$ & 340 & 330 & $\bar{U}$ & 330 & 330 & $U$ & 330 & 330 & $\mathrm{U}$ & 330 \\
\hline 2-Chloronaphthalene & 340 & $\mathrm{U}$ & 340 & 330 & $\mathrm{U}$ & 330 & 330 & $\mathrm{U}$ & 330 & 330 & $\mathrm{U}$ & 330 \\
\hline 2-Chlorophenol & 340 & $\bar{U}$ & 340 & 330 & $\bar{U}$ & 330 & 330 & $\mathrm{U}$ & 330 & 330 & $\mathrm{U}$ & 330 \\
\hline 2-Methylnaphthalene & 340 & $\mathrm{U}$ & 340 & 330 & $\bar{U}$ & 330 & 330 & $\mathrm{U}$ & 330 & 330 & $\mathrm{U}$ & 330 \\
\hline 2-Methylphenol (cresol, o-) & 340 & $\mathrm{U}$ & 340 & 330 & $\mathrm{U}$ & 330 & 330 & $\mathrm{U}$ & 330 & 330 & $\mathrm{U}$ & 330 \\
\hline 2-Nitroaniline & 840 & $\mathrm{U}$ & 840 & 840 & $\mathrm{U}$ & 840 & 840 & $\mathrm{U}$ & 840 & 840 & $\mathrm{U}$ & 840 \\
\hline 2-Nitrophenol & 340 & $\mathrm{U}$ & 340 & 330 & $\mathrm{U}$ & 330 & 330 & $\mathrm{U}$ & 330 & 330 & $\mathrm{U}$ & 330 \\
\hline 3,3'-Dichlorobenzidine & 340 & $\mathrm{U}$ & 340 & 330 & $\mathrm{U}$ & 330 & 330 & $\mathrm{U}$ & 330 & 330 & $\mathrm{U}$ & 330 \\
\hline 3-Nitroaniline & 840 & $\mathrm{U}$ & 840 & 840 & $\mathrm{U}$ & 840 & 840 & $\mathrm{U}$ & 840 & 840 & $\mathrm{U}$ & 840 \\
\hline 4,6-Dinitro-2-methylphenol & 840 & $\mathrm{U}$ & 840 & 840 & $\mathrm{U}$ & 840 & 840 & $\mathrm{U}$ & 840 & 840 & $\mathrm{U}$ & 840 \\
\hline 4-Bromophenyl-phenylether & 340 & $\mathrm{U}$ & 340 & 330 & $\mathrm{U}$ & 330 & 330 & $\mathrm{U}$ & 330 & 330 & $\mathrm{U}$ & 330 \\
\hline 4-Chloro-3-methylphenol & 340 & $\mathrm{U}$ & 340 & 330 & $\mathrm{U}$ & 330 & 330 & $\mathrm{U}$ & 330 & 330 & $\bar{U}$ & 330 \\
\hline 4-Chloroaniline & 340 & $\mathrm{U}$ & 340 & 330 & $\mathrm{U}$ & 330 & 330 & $\mathrm{U}$ & 330 & 330 & $\mathrm{U}$ & 330 \\
\hline 4-Chlorophenyl-phenylether & 340 & $\mathrm{U}$ & 340 & 330 & $\mathrm{U}$ & 330 & 330 & $\mathrm{U}$ & 330 & 330 & $\mathrm{U}$ & 330 \\
\hline 4-Methylphenol (p-cresol) & 340 & $\mathrm{U}$ & 340 & 330 & $\mathrm{U}$ & 330 & 330 & $\mathrm{U}$ & 330 & 330 & $\mathrm{U}$ & 330 \\
\hline 4-Nitroaniline & 840 & $\mathrm{U}$ & 840 & 840 & $\mathrm{U}$ & 840 & 840 & $\mathrm{U}$ & 840 & 840 & $\mathrm{U}$ & 840 \\
\hline 4-Nitrophenol & 840 & U & 840 & 840 & $\bar{U}$ & 840 & 840 & $\mathrm{U}$ & 840 & 840 & $\mathrm{U}$ & 840 \\
\hline Acenaphthene & 340 & $\mathrm{U}$ & 340 & 330 & $\mathrm{U}$ & 330 & 330 & $\mathrm{U}$ & 330 & 330 & $\mathrm{U}$ & 330 \\
\hline Acenaphthylene & 340 & $\mathrm{U}$ & 340 & 330 & $\mathrm{U}$ & 330 & 330 & $\mathrm{U}$ & 330 & 330 & $\mathrm{U}$ & 330 \\
\hline Anthracene & 340 & $\mathrm{U}$ & 340 & 330 & $\mathrm{U}$ & 330 & 330 & $\mathrm{U}$ & 330 & 330 & $\mathrm{U}$ & 330 \\
\hline Benzo(a)anthracene & 340 & $\bar{U}$ & 340 & 330 & $\mathrm{U}$ & 330 & 330 & $\mathrm{U}$ & 330 & 130 & $\mathrm{~J}$ & 330 \\
\hline Benzo(a)pyrene & 340 & $\mathrm{U}$ & 340 & 330 & $\mathrm{U}$ & 330 & 330 & $\mathrm{U}$ & 330 & 83 & $\mathrm{~J}$ & 330 \\
\hline Benzo(b)fluoranthene & 340 & $\mathrm{U}$ & 340 & 330 & $\mathrm{U}$ & 330 & 330 & $\mathrm{U}$ & 330 & 110 & $\mathrm{~J}$ & 330 \\
\hline Benzo(g,h,i)perylene & 340 & $\mathrm{U}$ & 340 & 330 & $\mathrm{U}$ & 330 & 330 & $\mathrm{U}$ & 330 & 37 & $\mathrm{~J}$ & 330 \\
\hline Benzo(k)fluoranthene & 340 & $\mathrm{U}$ & 340 & 330 & $\mathrm{U}$ & 330 & 330 & $\mathrm{U}$ & 330 & 92 & $\mathrm{~J}$ & 330 \\
\hline bis(2-Chloro-1-methylethyl)ether & 340 & $\mathrm{U}$ & 340 & 330 & $\mathrm{U}$ & 330 & 330 & $\mathrm{U}$ & 330 & 330 & $\mathrm{U}$ & 330 \\
\hline bis(2-Chloroethoxy)methane & 340 & $\mathrm{U}$ & 340 & 330 & $\mathrm{U}$ & 330 & 330 & $\mathrm{U}$ & 330 & 330 & $\mathrm{U}$ & 330 \\
\hline bis(2-Chloroethyl)ether & 340 & $\mathrm{U}$ & 340 & 330 & $U$ & 330 & 330 & $\mathrm{U}$ & 330 & 330 & $\mathrm{U}$ & 330 \\
\hline bis(2-Ethylhexyl)phthalate & 340 & $\bar{U}$ & 340 & 240 & $\mathrm{~J}$ & 330 & 17 & $\mathrm{~J}$ & 330 & 330 & $\mathrm{U}$ & 330 \\
\hline
\end{tabular}


CVP-2006-00011

Rev. 0

118-C-1 Verification Sampling Results.

\begin{tabular}{|c|c|c|c|c|c|c|c|c|c|c|c|c|}
\hline \multirow[t]{2}{*}{ Constituents } & \multicolumn{3}{|c|}{$\begin{array}{c}\mathrm{J13512} \\
\text { Area } 3 \text { A3 } \\
\text { Sample Date 8/21/06 }\end{array}$} & \multicolumn{3}{|c|}{$\begin{array}{c}\mathrm{J13513} \\
\text { Area } 3 \mathrm{A4} \\
\text { Sample Date } 8 / 21 / 06 \\
\end{array}$} & \multicolumn{3}{|c|}{$\begin{array}{c}\text { J13514 } \\
\text { Area } 4 \text { A1 } \\
\text { Sample Date } 8 / 21 / 06\end{array}$} & \multicolumn{3}{|c|}{$\begin{array}{c}\mathrm{J13515} \\
\text { Area } 4 \mathrm{A2} \\
\text { Sample Date 8/21/06 } \\
\end{array}$} \\
\hline & $\mu \mathrm{g} / \mathrm{kg}$ & $Q$ & PQL & $\mu \mathrm{g} / \mathrm{kg}$ & $\mathbf{Q}$ & PQL & $\mu \mathrm{g} / \mathrm{kg}$ & $Q$ & PQL & $\mu \mathrm{g} / \mathrm{kg}$ & $Q$ & PQL \\
\hline \multicolumn{13}{|c|}{ Semivolatile Organic Compounds (continued) } \\
\hline Butylbenzylphthalate & 340 & $\mathrm{U}$ & 340 & 38 & $\mathrm{~J}$ & 330 & 330 & $\mathrm{U}$ & 330 & 330 & $\mathrm{U}$ & 330 \\
\hline Carbazole & 340 & $\mathrm{U}$ & 340 & 330 & $\mathrm{U}$ & 330 & 330 & $\mathrm{U}$ & 330 & 330 & $\mathrm{U}$ & 330 \\
\hline Chrysene & 340 & $\mathrm{U}$ & 340 & 330 & $\mathrm{U}$ & 330 & 330 & $\mathrm{U}$ & 330 & 170 & $\mathrm{~J}$ & 330 \\
\hline Di-n-butylphthalate & 25 & $\mathrm{~J}$ & 340 & 63 & $\mathrm{~J}$ & 330 & 330 & $\mathrm{U}$ & 330 & 330 & $\mathrm{U}$ & 330 \\
\hline Di-n-octylphthalate & 340 & $\mathrm{U}$ & 340 & 330 & $\mathrm{U}$ & 330 & 330 & $\mathrm{U}$ & 330 & 330 & $\mathrm{U}$ & 330 \\
\hline Dibenz(a,h)anthracene & 340 & $\mathrm{U}$ & 340 & 330 & $\mathrm{U}$ & 330 & 330 & $\mathrm{U}$ & 330 & 330 & $\mathrm{U}$ & 330 \\
\hline Dibenzofuran & 340 & $\mathrm{U}$ & 340 & 330 & $\mathrm{U}$ & 330 & 330 & $\mathrm{U}$ & 330 & 330 & $\mathrm{U}$ & 330 \\
\hline Diethylphthalate & 340 & $\mathrm{U}$ & 340 & 330 & $\mathrm{U}$ & 330 & 330 & $\mathrm{U}$ & 330 & 330 & $\mathrm{U}$ & 330 \\
\hline Dimethylphthalate & 340 & $\mathrm{U}$ & 340 & 330 & $\mathrm{U}$ & 330 & 330 & $\mathrm{U}$ & 330 & 330 & $\mathrm{U}$ & 330 \\
\hline Fluoranthene & 340 & $\mathrm{U}$ & 340 & 330 & $\mathrm{U}$ & 330 & 330 & $\mathrm{U}$ & 330 & 330 & & 330 \\
\hline Fluorene & 340 & $\mathrm{U}$ & 340 & 330 & $\mathrm{U}$ & 330 & 330 & $\mathrm{U}$ & 330 & 330 & $\mathrm{U}$ & 330 \\
\hline Hexachlorobenzene & 340 & $\mathrm{U}$ & 340 & 330 & $\mathrm{U}$ & 330 & 330 & $\mathrm{U}$ & 330 & 330 & $\mathrm{U}$ & 330 \\
\hline Hexachlorobutadiene & 340 & $\mathrm{U}$ & 340 & 330 & $\mathrm{U}$ & 330 & 330 & $\mathrm{U}$ & 330 & 330 & $\mathrm{U}$ & 330 \\
\hline Hexachlorocyclopentadiene & 340 & $\mathrm{U}$ & 340 & 330 & $\mathrm{U}$ & 330 & 330 & $\mathrm{U}$ & 330 & 330 & $\mathrm{U}$ & 330 \\
\hline Hexachloroethane & 340 & $\mathrm{U}$ & 340 & 330 & $U$ & 330 & 330 & $\mathrm{U}$ & 330 & 330 & $\mathrm{U}$ & 330 \\
\hline Indeno(1,2,3-cd)pyrene & 340 & $\mathrm{U}$ & 340 & 330 & $\mathrm{U}$ & 330 & 330 & $\mathrm{U}$ & 330 & 35 & $\mathrm{~J}$ & 330 \\
\hline Isophorone & 340 & $\mathrm{U}$ & 340 & 330 & $\mathrm{U}$ & 330 & 330 & $\mathrm{U}$ & 330 & 330 & $\mathrm{U}$ & 330 \\
\hline N-Nitroso-di-n-dipropylamine & 340 & $\mathrm{U}$ & 340 & 330 & $\mathrm{U}$ & 330 & 330 & $\mathrm{U}$ & 330 & 330 & $\mathrm{U}$ & 330 \\
\hline N-Nitrosodiphenylamine & 340 & $\mathrm{U}$ & 340 & 330 & $\mathrm{U}$ & 330 & 330 & $\mathrm{U}$ & 330 & 330 & $\mathrm{U}$ & 330 \\
\hline Naphthalene & 340 & $\mathrm{U}$ & 340 & 330 & $\mathrm{U}$ & 330 & 330 & $\mathrm{U}$ & 330 & 330 & $\mathrm{U}$ & 330 \\
\hline Nitrobenzene & 340 & $\mathrm{U}$ & 340 & 330 & $\mathrm{U}$ & 330 & 330 & $\mathrm{U}$ & 330 & 330 & $\mathrm{U}$ & 330 \\
\hline Pentachlorophenol & 840 & $\mathrm{U}$ & 840 & 840 & $\mathrm{U}$ & 840 & 840 & $\mathrm{U}$ & 840 & 840 & $\mathrm{U}$ & 840 \\
\hline Phenanthrene & 340 & $\mathrm{U}$ & 340 & 330 & $\mathrm{U}$ & 330 & 330 & $\mathrm{U}$ & 330 & 97 & $\mathrm{~J}$ & 330 \\
\hline Phenol & 340 & $\mathrm{U}$ & 340 & 330 & $\mathrm{U}$ & 330 & 330 & $\mathrm{U}$ & 330 & 330 & $\mathrm{U}$ & 330 \\
\hline Pyrene & 340 & $\mathrm{U}$ & 340 & 330 & $\mathrm{U}$ & 330 & 330 & $\mathrm{U}$ & 330 & 350 & & 330 \\
\hline
\end{tabular}


CVP-2006-00011

Rev. 0

118-C-1 Verification Sampling Results.

\begin{tabular}{|c|c|c|c|c|c|c|c|c|c|c|c|c|}
\hline \multirow[t]{2}{*}{ Constituents } & \multicolumn{3}{|c|}{$\begin{array}{c}\text { J135R6 } \\
\text { Area } 3 \text { A3 } \\
\text { Sample Date 8/28/06 }\end{array}$} & \multicolumn{3}{|c|}{$\begin{array}{c}\text { J135R7 } \\
\text { Area } 3 \text { A4 } \\
\text { Sample Date 8/28/06 }\end{array}$} & \multicolumn{3}{|c|}{$\begin{array}{c}\text { J135R8 } \\
\text { Area } 4 \text { A1 } \\
\text { Sample Date } 8 / 28 / 06\end{array}$} & \multicolumn{3}{|c|}{$\begin{array}{c}\text { J135R9 } \\
\text { Area } 4 \text { A2 } \\
\text { Sample Date } 8 / 28 / 06\end{array}$} \\
\hline & $\mu \mathrm{g} / \mathrm{kg}$ & $\mathbf{Q}$ & PQL & $\mu \mathrm{g} / \mathrm{kg}$ & $\mathbf{Q}$ & PQL & $\mu \mathrm{g} / \mathrm{kg}$ & $\mathbf{Q}$ & PQL & $\mu \mathrm{g} / \mathrm{kg}$ & $\mathbf{Q}$ & PQL \\
\hline \multicolumn{13}{|c|}{ Volatile Organic Compounds } \\
\hline 1,1,1-Trichloroethane & 5 & $\mathrm{U}$ & 5 & 5 & $\mathrm{U}$ & 5 & 5 & $\mathrm{U}$ & 5 & 5 & $\mathrm{U}$ & 5 \\
\hline $1,1,2,2$-Tetrachloroethane & 5 & UJ & 5 & 5 & UJ & 5 & 5 & UJ & 5 & 5 & UJ & 5 \\
\hline 1,1,2-Trichloroethane & 5 & $\mathrm{U}$ & 5 & 5 & $\mathrm{U}$ & 5 & 5 & $\mathrm{U}$ & 5 & 5 & $\mathrm{U}$ & 5 \\
\hline 1,1-Dichloroethane & 5 & $\mathrm{U}$ & 5 & 5 & $\mathrm{U}$ & 5 & 5 & $\mathrm{U}$ & 5 & 5 & $\mathrm{U}$ & 5 \\
\hline 1,1-Dichloroethene & 5 & $\mathrm{U}$ & 5 & 5 & $\mathrm{U}$ & 5 & 5 & $\mathrm{U}$ & 5 & 5 & $\mathrm{U}$ & 5 \\
\hline 1,2-Dichloroethane & 5 & $\mathrm{U}$ & 5 & 5 & $\mathrm{U}$ & 5 & 5 & $\mathrm{U}$ & 5 & 5 & $\mathrm{U}$ & 5 \\
\hline 1,2-Dichloroethene (total) & 5 & $\mathrm{U}$ & 5 & 5 & $\mathrm{U}$ & 5 & 5 & $\mathrm{U}$ & 5 & 5 & $\mathrm{U}$ & 5 \\
\hline 1,2-Dichloropropane & 5 & $\mathrm{U}$ & 5 & 5 & $\mathrm{U}$ & 5 & 5 & $\mathrm{U}$ & 5 & 5 & $\mathrm{U}$ & 5 \\
\hline 2-Butanone & 10 & $\mathrm{U}$ & 10 & 10 & $\mathrm{U}$ & 10 & 10 & $\mathrm{U}$ & 10 & 10 & $\mathrm{U}$ & 10 \\
\hline 2-Hexanone & 10 & $\mathrm{U}$ & 10 & 10 & $\mathrm{U}$ & 10 & 10 & $\mathrm{U}$ & 10 & 10 & $\mathrm{U}$ & 10 \\
\hline 4-Methyl-2-pentanone & 10 & $\mathrm{U}$ & 10 & 10 & $\mathrm{U}$ & 10 & 10 & $\mathrm{U}$ & 10 & 10 & $\mathrm{U}$ & 10 \\
\hline Acetone & 10 & $\mathrm{U}$ & 10 & 11 & $\mathrm{~J}$ & 10 & 13 & $\mathrm{~J}$ & 10 & 10 & $\mathrm{U}$ & 10 \\
\hline Benzene & 5 & $\mathrm{U}$ & 5 & 5 & $\mathrm{U}$ & 5 & 5 & $\mathrm{U}$ & 5 & 1 & $\mathrm{~J}$ & 5 \\
\hline Bromodichloromethane & 5 & $\mathrm{U}$ & 5 & 5 & $\mathrm{U}$ & 5 & 5 & $\mathrm{U}$ & 5 & 5 & $\mathrm{U}$ & 5 \\
\hline Bromoform & 5 & $\mathrm{U}$ & 5 & 5 & $\mathrm{U}$ & 5 & 5 & $\mathrm{U}$ & 5 & 5 & $\mathrm{U}$ & 5 \\
\hline Bromomethane & 10 & $\mathrm{U}$ & 10 & 10 & $\mathrm{U}$ & 10 & 10 & $\mathrm{U}$ & 10 & 10 & $\mathrm{U}$ & 10 \\
\hline Carbon disulfide & 5 & $\mathrm{U}$ & 5 & 5 & $\mathrm{U}$ & 5 & 5 & $\mathrm{U}$ & 5 & 5 & $\mathrm{U}$ & 5 \\
\hline Carbon tetrachloride & 5 & $\mathrm{U}$ & 5 & 5 & $\mathrm{U}$ & 5 & 5 & $\mathrm{U}$ & 5 & 5 & $\mathrm{U}$ & 5 \\
\hline Chlorobenzene & 5 & $\mathrm{U}$ & 5 & 5 & $\mathrm{U}$ & 5 & 5 & $\mathrm{U}$ & 5 & 5 & $\mathrm{U}$ & 5 \\
\hline Chloroethane & 10 & $\mathrm{U}$ & 10 & 10 & $\mathrm{U}$ & 10 & 10 & $\mathrm{U}$ & 10 & 10 & $\mathrm{U}$ & 10 \\
\hline Chloroform & 5 & $\mathrm{U}$ & 5 & 5 & $\mathrm{U}$ & 5 & 5 & $\mathrm{U}$ & 5 & 5 & $\mathrm{U}$ & 5 \\
\hline Chloromethane & 10 & $\mathrm{U}$ & 10 & 10 & $\mathrm{U}$ & 10 & 10 & $\mathrm{U}$ & 10 & 10 & $\mathrm{U}$ & 10 \\
\hline cis-1,2-Dichloroethylene & 5 & $\mathrm{U}$ & 5 & 5 & $\mathrm{U}$ & 5 & 5 & $\mathrm{U}$ & 5 & 5 & $\mathrm{U}$ & 5 \\
\hline cis-1,3-Dichloropropene & 5 & $\mathrm{U}$ & 5 & 5 & $\mathrm{U}$ & 5 & 5 & $\bar{U}$ & 5 & 5 & $\mathrm{U}$ & 5 \\
\hline Dibromochloromethane & 5 & $\mathrm{U}$ & 5 & 5 & $\mathrm{U}$ & 5 & 5 & $\mathrm{U}$ & 5 & 5 & $\mathrm{U}$ & 5 \\
\hline Ethylbenzene & 5 & $\mathrm{U}$ & 5 & 5 & $\mathrm{U}$ & 5 & 5 & $\mathrm{U}$ & 5 & 5 & $\mathrm{U}$ & 5 \\
\hline Methylene chloride & 9 & $\mathrm{~B}$ & 5 & 10 & $B$ & 5 & 13 & $\mathrm{~B}$ & 5 & 12 & $\mathrm{~B}$ & 5 \\
\hline Styrene & 5 & $\mathrm{U}$ & 5 & 5 & $\mathrm{U}$ & 5 & 5 & $\mathrm{U}$ & 5 & 5 & $\mathrm{U}$ & 5 \\
\hline Tetrachloroethene & 5 & $\mathrm{U}$ & 5 & 5 & $\mathrm{U}$ & 5 & 5 & $\mathrm{U}$ & 5 & 5 & $\mathrm{U}$ & 5 \\
\hline Toluene & 5 & $\mathrm{U}$ & 5 & 5 & $\mathrm{U}$ & 5 & 5 & $\mathrm{U}$ & 5 & 5 & $\mathrm{U}$ & 5 \\
\hline trans-1,2-Dichloroethylene & 5 & $\mathrm{U}$ & 5 & 5 & $\mathrm{U}$ & 5 & 5 & $\mathrm{U}$ & 5 & 5 & $\mathrm{U}$ & 5 \\
\hline trans-1,3-Dichloropropene & 5 & $\mathrm{U}$ & 5 & 5 & $\mathrm{U}$ & 5 & 5 & $\mathrm{U}$ & 5 & 5 & $\mathrm{U}$ & 5 \\
\hline Trichloroethene & 5 & $\mathrm{U}$ & 5 & 5 & $\mathrm{U}$ & 5 & 5 & $\mathrm{U}$ & 5 & 5 & $\mathrm{U}$ & 5 \\
\hline Vinyl chloride & 10 & $\mathrm{U}$ & 10 & 10 & $\mathrm{U}$ & 10 & 10 & $\mathrm{U}$ & 10 & 10 & $\mathrm{U}$ & 10 \\
\hline Xylenes (total) & 5 & $\mathrm{U}$ & 5 & 5 & $\mathrm{U}$ & 5 & 5 & $\mathrm{U}$ & 5 & 1 & $\mathrm{~J}$ & 5 \\
\hline
\end{tabular}


118-C-1 Verification Sampling Results.

\begin{tabular}{|c|c|c|c|c|c|c|c|c|c|c|c|c|}
\hline \multirow[t]{2}{*}{ Constituents } & \multicolumn{3}{|c|}{$\begin{array}{c}\text { J13516 } \\
\text { Area } 4 \text { A3 } \\
\text { Sample Date 8/21/06 }\end{array}$} & \multicolumn{3}{|c|}{$\begin{array}{c}\text { J13517 } \\
\text { Area } 4 \text { A4 } \\
\text { Sample Date } 8 / 21 / 06\end{array}$} & \multicolumn{3}{|c|}{$\begin{array}{c}\text { J13518 } \\
\text { OB/BCL A1 } \\
\text { Sample Date } 8 / 22 / 06\end{array}$} & \multicolumn{3}{|c|}{$\begin{array}{c}\text { J13519 } \\
\text { OB/BCL A2 } \\
\text { Sample Date } 8 / 22 / 06 \\
\end{array}$} \\
\hline & $\mu \mathrm{g} / \mathrm{kg}$ & $Q$ & PQL & $\mu \mathrm{g} / \mathrm{kg}$ & $Q$ & PQL & $\mu \mathrm{g} / \mathrm{kg}$ & $Q$ & PQL & $\mu \mathrm{g} / \mathrm{kg}$ & $Q$ & PQL \\
\hline \multicolumn{13}{|c|}{ Polychlorinated Biphenyls } \\
\hline Aroclor-1016 & 13 & $\mathrm{U}$ & 13 & 13 & $\mathrm{U}$ & 13 & 13 & $\mathrm{U}$ & 13 & 13 & $\mathrm{U}$ & 13 \\
\hline Aroclor-1221 & 13 & $\mathrm{U}$ & 13 & 13 & $\mathrm{U}$ & 13 & 13 & $\mathrm{U}$ & 13 & 13 & $\mathrm{U}$ & 13 \\
\hline Aroclor-1232 & 13 & $\mathrm{U}$ & 13 & 13 & $\mathrm{U}$ & 13 & 13 & $\mathrm{U}$ & 13 & 13 & $\mathrm{U}$ & 13 \\
\hline Aroclor -1242 & 13 & $\mathrm{U}$ & 13 & 13 & $\mathrm{U}$ & 13 & 13 & $\mathrm{U}$ & 13 & 13 & $\mathrm{U}$ & 13 \\
\hline Aroclor- 1248 & 13 & $\mathrm{U}$ & 13 & 13 & $\mathrm{U}$ & 13 & 13 & $\mathrm{U}$ & 13 & 13 & $\mathrm{U}$ & 13 \\
\hline Aroclor-1254 & 13 & $\mathrm{U}$ & 13 & 16 & & 13 & 13 & $\mathrm{U}$ & 13 & 4.9 & $\mathrm{~J}$ & 13 \\
\hline Aroclor-1260 & 13 & $\mathrm{U}$ & 13 & 13 & $\mathrm{U}$ & 13 & 13 & $\mathrm{U}$ & 13 & 13 & $\mathrm{U}$ & 13 \\
\hline \multicolumn{13}{|c|}{ Semivolatile Organic Compounds } \\
\hline 1,2,4-Trichlorobenzene & 330 & $\mathrm{U}$ & 330 & 330 & $\mathrm{U}$ & 330 & 330 & $\mathrm{U}$ & 330 & 340 & $\mathrm{U}$ & 340 \\
\hline 1,2-Dichlorobenzene & 330 & $\mathrm{U}$ & 330 & 330 & $\mathrm{U}$ & 330 & 330 & $\mathrm{U}$ & 330 & 340 & $\mathrm{U}$ & 340 \\
\hline 1,3-Dichlorobenzene & 330 & $\bar{U}$ & 330 & 330 & $\mathrm{U}$ & 330 & 330 & $\mathrm{U}$ & 330 & 340 & $\mathrm{U}$ & 340 \\
\hline 1,4-Dichlorobenzene & 330 & $\mathrm{U}$ & 330 & 330 & $\mathrm{U}$ & 330 & 330 & $\mathrm{U}$ & 330 & 340 & $\mathrm{U}$ & 340 \\
\hline 2,4,5-Trichlorophenol & 840 & $\mathrm{U}$ & 840 & 840 & $\mathrm{U}$ & 840 & 840 & $\mathrm{U}$ & 840 & 840 & $\mathrm{U}$ & 840 \\
\hline 2,4,6-Trichlorophenol & 330 & $\mathrm{U}$ & 330 & 330 & $\mathrm{U}$ & 330 & 330 & $\mathrm{U}$ & 330 & 340 & $\mathrm{U}$ & 340 \\
\hline 2,4-Dichlorophenol & 330 & $\mathrm{U}$ & 330 & 330 & $\mathrm{U}$ & 330 & 330 & $\mathrm{U}$ & 330 & 340 & $\mathrm{U}$ & 340 \\
\hline 2,4-Dimethylphenol & 330 & $\mathrm{U}$ & 330 & 330 & $\mathrm{U}$ & 330 & 330 & $\mathrm{U}$ & 330 & 340 & $\mathrm{U}$ & 340 \\
\hline 2,4-Dinitrophenol & 840 & UJ & 840 & 840 & $\mathrm{UJ}$ & 840 & 840 & $\mathrm{U}$ & 840 & 840 & $\mathrm{U}$ & 840 \\
\hline 2,4-Dinitrotoluene & 330 & $\mathrm{U}$ & 330 & 330 & $\mathrm{U}$ & 330 & 330 & $\mathrm{U}$ & 330 & 340 & $\mathrm{U}$ & 340 \\
\hline 2,6-Dinitrotoluene & 330 & $\mathrm{U}$ & 330 & 330 & $\mathrm{U}$ & 330 & 330 & $\mathrm{U}$ & 330 & 340 & $\mathrm{U}$ & 340 \\
\hline 2-Chloronaphthalene & 330 & $\mathrm{U}$ & 330 & 330 & $\mathrm{U}$ & 330 & 330 & $\mathrm{U}$ & 330 & 340 & $\mathrm{U}$ & 340 \\
\hline 2-Chlorophenol & 330 & $\mathrm{U}$ & 330 & 330 & $\mathrm{U}$ & 330 & 330 & $\mathrm{U}$ & 330 & 340 & $\mathrm{U}$ & 340 \\
\hline 2-Methylnaphthalene & 330 & $\mathrm{U}$ & 330 & 330 & $\mathrm{U}$ & 330 & 330 & $\mathrm{U}$ & 330 & 340 & $\mathrm{U}$ & 340 \\
\hline 2-Methylphenol (cresol, o-) & 330 & $\mathrm{U}$ & 330 & 330 & $\mathrm{U}$ & 330 & 330 & $\mathrm{U}$ & 330 & 340 & $\mathrm{U}$ & 340 \\
\hline 2-Nitroaniline & 840 & $\mathrm{U}$ & 840 & 840 & $\mathrm{U}$ & 840 & 840 & $\mathrm{U}$ & 840 & 840 & $\mathrm{U}$ & 840 \\
\hline 2-Nitrophenol & 330 & $\mathrm{U}$ & 330 & 330 & $\mathrm{U}$ & 330 & 330 & $\mathrm{U}$ & 330 & 340 & $\mathrm{U}$ & 340 \\
\hline 3,3'-Dichlorobenzidine & 330 & $\mathrm{U}$ & 330 & 330 & $\mathrm{U}$ & 330 & 330 & $\mathrm{U}$ & 330 & 340 & $\mathrm{U}$ & 340 \\
\hline 3-Nitroaniline & 840 & $\mathrm{U}$ & 840 & 840 & $\mathrm{U}$ & 840 & 840 & $\mathrm{U}$ & 840 & 840 & $\mathrm{U}$ & 840 \\
\hline 4,6-Dinitro-2-methylphenol & 840 & $\mathrm{U}$ & 840 & 840 & $\mathrm{U}$ & 840 & 840 & $\mathrm{U}$ & 840 & 840 & $\mathrm{U}$ & 840 \\
\hline 4-Bromophenyl-phenylether & 330 & $\mathrm{U}$ & 330 & 330 & $\mathrm{U}$ & 330 & 330 & $\mathrm{U}$ & 330 & 340 & $\mathrm{U}$ & 340 \\
\hline 4-Chloro-3-methylphenol & 330 & $\mathrm{U}$ & 330 & 330 & $\mathrm{U}$ & 330 & 330 & $\mathrm{U}$ & 330 & 340 & $\mathrm{U}$ & 340 \\
\hline 4-Chloroaniline & 330 & $\mathrm{U}$ & 330 & 330 & $\mathrm{U}$ & 330 & 330 & $\mathrm{U}$ & 330 & 340 & $\mathrm{U}$ & 340 \\
\hline 4-Chlorophenyl-phenylether & 330 & $\mathrm{U}$ & 330 & 330 & $\mathrm{U}$ & 330 & 330 & $\mathrm{U}$ & 330 & 340 & $\mathrm{U}$ & 340 \\
\hline 4-Methylphenol (p-cresol) & 330 & $\mathrm{U}$ & 330 & 330 & $\mathrm{U}$ & 330 & 330 & $\mathrm{U}$ & 330 & 340 & $\mathrm{U}$ & 340 \\
\hline 4-Nitroaniline & 840 & $\mathrm{U}$ & 840 & 840 & $\mathrm{U}$ & 840 & 840 & $\mathrm{U}$ & 840 & 840 & $\mathrm{U}$ & 840 \\
\hline 4-Nitrophenol & 840 & $\mathrm{U}$ & 840 & 840 & $\mathrm{U}$ & 840 & 840 & $\mathrm{U}$ & 840 & 840 & $\mathrm{U}$ & 840 \\
\hline Acenaphthene & 330 & $\mathrm{U}$ & 330 & 330 & $\mathrm{U}$ & 330 & 330 & $\mathrm{U}$ & 330 & 340 & $\mathrm{U}$ & 340 \\
\hline Acenaphthylene & 330 & $\mathrm{U}$ & 330 & 330 & $\mathrm{U}$ & 330 & 330 & $\mathrm{U}$ & 330 & 340 & $\mathrm{U}$ & 340 \\
\hline Anthracene & 330 & $\mathrm{U}$ & 330 & 330 & $\mathrm{U}$ & 330 & 330 & $\mathrm{U}$ & 330 & 340 & $\mathrm{U}$ & 340 \\
\hline Benzo(a)anthracene & 330 & $\mathrm{U}$ & 330 & 330 & $\mathrm{U}$ & 330 & 330 & $\mathrm{U}$ & 330 & 340 & $\mathrm{U}$ & 340 \\
\hline Benzo(a)pyrene & 330 & $\mathrm{U}$ & 330 & 330 & $\mathrm{U}$ & 330 & 330 & $\mathrm{U}$ & 330 & 340 & $\mathrm{U}$ & 340 \\
\hline Benzo(b)fluoranthene & 330 & $\mathrm{U}$ & 330 & 330 & $\mathrm{U}$ & 330 & 330 & $\mathrm{U}$ & 330 & 340 & $\mathrm{U}$ & 340 \\
\hline Benzo $(\mathrm{g}, \mathrm{h}, \mathrm{i})$ perylene & 330 & $\mathrm{U}$ & 330 & 330 & $\mathrm{U}$ & 330 & 330 & $\mathrm{U}$ & 330 & 340 & $\mathrm{U}$ & 340 \\
\hline Benzo(k)fluoranthene & 330 & $\bar{U}$ & 330 & 330 & $\mathrm{U}$ & 330 & 330 & $\mathrm{U}$ & 330 & 340 & $\mathrm{U}$ & 340 \\
\hline bis(2-Chloro-1-methylethyl)ether & 330 & $\mathrm{U}$ & 330 & 330 & $\mathrm{U}$ & 330 & 330 & $\mathrm{U}$ & 330 & 340 & $\mathrm{U}$ & 340 \\
\hline bis(2-Chloroethoxy)methane & 330 & $\mathrm{U}$ & 330 & 330 & $\mathrm{U}$ & 330 & 330 & $\mathrm{U}$ & 330 & 340 & $\mathrm{U}$ & 340 \\
\hline bis(2-Chloroethyl)ether & 330 & $\mathrm{U}$ & 330 & 330 & $\mathrm{U}$ & 330 & 330 & $\mathrm{U}$ & 330 & 340 & $\mathrm{U}$ & 340 \\
\hline bis(2-Ethylhexyl)phthalate & 330 & $\mathrm{U}$ & 330 & 330 & $\mathrm{U}$ & 330 & 330 & $\mathrm{U}$ & 330 & 330 & $\mathrm{U}$ & 340 \\
\hline
\end{tabular}


118-C-1 Verification Sampling Results.

\begin{tabular}{|c|c|c|c|c|c|c|c|c|c|c|c|c|}
\hline \multirow[t]{2}{*}{ Constituents } & \multicolumn{3}{|c|}{$\begin{array}{c}J 13516 \\
\text { Area } 4 \mathrm{A3} \\
\text { Sample Date 8/21/06 } \\
\end{array}$} & \multicolumn{3}{|c|}{$\begin{array}{c}\mathrm{J13517} \\
\text { Area 4 A4 } \\
\text { Sample Date 8/21/06 } \\
\end{array}$} & \multicolumn{3}{|c|}{$\begin{array}{c}\text { J13518 } \\
\text { OB/BCL A1 } \\
\text { Sample Date } 8 / 22 / 06 \\
\end{array}$} & \multicolumn{3}{|c|}{$\begin{array}{c}\text { J13519 } \\
\text { OB/BCL A2 } \\
\text { Sample Date 8/22/06 } \\
\end{array}$} \\
\hline & $\mu \mathrm{g} / \mathrm{kg}$ & Q & PQL & $\mu \mathrm{g} / \mathrm{kg}$ & $\mathbf{Q}$ & PQL & $\mu \mathrm{g} / \mathrm{kg}$ & $\mathbf{Q}$ & PQL & $\mu \mathrm{g} / \mathrm{kg}$ & Q & $\mathrm{PQL}$ \\
\hline \multicolumn{13}{|c|}{ Semivolatile Organic Compounds (continued) } \\
\hline Butylbenzylphthalate & 330 & $\mathrm{U}$ & 330 & 330 & $\mathrm{U}$ & 330 & 330 & $\mathrm{U}$ & 330 & 340 & $\mathrm{U}$ & 340 \\
\hline Carbazole & 330 & $\mathrm{U}$ & 330 & 330 & $\mathrm{U}$ & 330 & 330 & $\mathrm{U}$ & 330 & 340 & $\mathrm{U}$ & 340 \\
\hline \begin{tabular}{|l} 
Chrysene \\
\end{tabular} & 330 & $\mathrm{U}$ & 330 & 330 & $\mathrm{U}$ & 330 & 330 & $\mathrm{U}$ & 330 & 340 & $\mathrm{U}$ & 340 \\
\hline Di-n-butylphthalate & 330 & $\mathrm{U}$ & 330 & 26 & $\mathrm{~J}$ & 330 & 330 & $\mathrm{U}$ & 330 & 330 & $\mathrm{U}$ & 340 \\
\hline \begin{tabular}{|l} 
Di-n-octylphthalate \\
\end{tabular} & 330 & $\mathrm{U}$ & 330 & 330 & $\mathrm{U}$ & 330 & 330 & $\mathrm{U}$ & 330 & 340 & $\mathrm{U}$ & 340 \\
\hline Dibenz(a,h)anthracene & 330 & $\mathrm{U}$ & 330 & 330 & $\mathrm{U}$ & 330 & 330 & $\mathrm{U}$ & 330 & 340 & $\mathrm{U}$ & 340 \\
\hline \begin{tabular}{|l} 
Dibenzofuran \\
\end{tabular} & 330 & $\mathrm{U}$ & 330 & 330 & $\mathrm{U}$ & 330 & 330 & $\mathrm{U}$ & 330 & 340 & $\mathrm{U}$ & 340 \\
\hline Diethylphthalate & 330 & $\mathrm{U}$ & 330 & 330 & $\mathrm{U}$ & 330 & 330 & $\mathrm{U}$ & 330 & 340 & $\mathrm{U}$ & 340 \\
\hline Dimethylphthalate & 330 & $\mathrm{U}$ & 330 & 330 & $\mathrm{U}$ & 330 & 330 & $\mathrm{U}$ & 330 & 340 & $\mathrm{U}$ & 340 \\
\hline Fluoranthene & 330 & $\mathrm{U}$ & 330 & 330 & $\mathrm{U}$ & 330 & 330 & UJ & 330 & 340 & $\overline{U J}$ & 340 \\
\hline Fluorene & 330 & $\mathrm{U}$ & 330 & 330 & $\mathrm{U}$ & 330 & 330 & $\mathrm{U}$ & 330 & 340 & $\mathrm{U}$ & 340 \\
\hline Hexachlorobenzene & 330 & $\mathrm{U}$ & 330 & 330 & $\mathrm{U}$ & 330 & 330 & $\mathrm{U}$ & 330 & 340 & $\mathrm{U}$ & 340 \\
\hline Hexachlorobutadiene & 330 & $\mathrm{U}$ & 330 & 330 & $\mathrm{U}$ & 330 & 330 & $\mathrm{U}$ & 330 & 340 & $\mathrm{U}$ & 340 \\
\hline Hexachlorocyclopentadiene & 330 & $\mathrm{U}$ & 330 & 330 & $\mathrm{U}$ & 330 & 330 & $\mathrm{U}$ & 330 & 340 & $\mathrm{U}$ & 340 \\
\hline \begin{tabular}{|l|} 
Hexachloroethane \\
\end{tabular} & 330 & $\mathrm{U}$ & 330 & 330 & $\mathrm{U}$ & 330 & 330 & $\mathrm{U}$ & 330 & 340 & $\mathrm{U}$ & 340 \\
\hline Indeno(1,2,3-cd)pyrene & 330 & $\mathrm{U}$ & 330 & 330 & $\mathrm{U}$ & 330 & 330 & $\mathrm{U}$ & 330 & 340 & $\mathrm{U}$ & 340 \\
\hline \begin{tabular}{|l} 
Isophorone \\
\end{tabular} & 330 & $\mathrm{U}$ & 330 & 330 & $\mathrm{U}$ & 330 & 330 & $\mathrm{U}$ & 330 & 340 & $\mathrm{U}$ & 340 \\
\hline N-Nitroso-di-n-dipropylamine & 330 & $\mathrm{U}$ & 330 & 330 & $\mathrm{U}$ & 330 & 330 & $\mathrm{U}$ & 330 & 340 & $\mathrm{U}$ & 340 \\
\hline N-Nitrosodiphenylamine & 330 & $\mathrm{U}$ & 330 & 330 & $\mathrm{U}$ & 330 & 330 & $\mathrm{U}$ & 330 & 340 & $\mathrm{U}$ & 340 \\
\hline Naphthalene & 330 & $\mathrm{U}$ & 330 & 330 & $\mathrm{U}$ & 330 & 330 & $\mathrm{U}$ & 330 & 340 & $\mathrm{U}$ & 340 \\
\hline Nitrobenzene & 330 & $\mathrm{U}$ & 330 & 330 & U & 330 & 330 & $\mathrm{U}$ & 330 & 340 & $\mathrm{U}$ & 340 \\
\hline Pentachlorophenol & 840 & $\mathrm{U}$ & 840 & 840 & $\mathrm{U}$ & 840 & 840 & $\mathrm{U}$ & 840 & 840 & $\mathrm{U}$ & 840 \\
\hline \begin{tabular}{|l} 
Phenanthrene \\
\end{tabular} & 330 & $\mathrm{U}$ & 330 & 330 & $\mathrm{U}$ & 330 & 330 & UJ & 330 & 340 & $\mathrm{UJ}$ & 340 \\
\hline \begin{tabular}{|l|} 
Phenol \\
\end{tabular} & 330 & $\mathrm{U}$ & 330 & 330 & $\mathrm{U}$ & 330 & 330 & $\mathrm{U}$ & 330 & 340 & $\mathrm{U}$ & 340 \\
\hline Pyrene & 330 & $\mathrm{U}$ & 330 & 330 & $\mathrm{U}$ & 330 & 330 & UJ & 330 & 340 & $\mathrm{UJ}$ & 340 \\
\hline
\end{tabular}


CVP-2006-00011

Rev. 0

118-C-1 Verification Sampling Results.

\begin{tabular}{|c|c|c|c|c|c|c|c|c|c|c|c|c|}
\hline \multirow[t]{2}{*}{ Constituents } & \multicolumn{3}{|c|}{$\begin{array}{c}\text { J135T0 } \\
\text { Area } 4 \text { A3 } \\
\text { Sample Date 8/28/06 }\end{array}$} & \multicolumn{3}{|c|}{$\begin{array}{c}\text { J135T1 } \\
\text { Area } 4 \text { A4 } \\
\text { Sample Date } 8 / 28 / 06\end{array}$} & \multicolumn{3}{|c|}{$\begin{array}{c}\text { J135T2 } \\
\text { OB/BCL A1 } \\
\text { Sample Date } 8 / 28 / 06\end{array}$} & \multicolumn{3}{|c|}{$\begin{array}{c}\text { J135T3 } \\
\text { OB/BCL A2 } \\
\text { Sample Date } 8 / 28 / 06\end{array}$} \\
\hline & $\mu \mathrm{g} / \mathrm{kg}$ & $Q$ & PQL & $\mu \mathrm{g} / \mathrm{kg}$ & $Q$ & PQL & $\mu \mathrm{g} / \mathrm{kg}$ & $Q$ & PQL & $\mu \mathrm{g} / \mathrm{kg}$ & $\mathbf{Q}$ & PQL \\
\hline \multicolumn{13}{|c|}{ Volatile Organic Compounds } \\
\hline 1,1,1-Trichloroethane & 6 & $\mathrm{U}$ & 6 & 6 & $\mathrm{U}$ & 6 & 5 & $\mathrm{U}$ & 5 & 6 & $\mathrm{U}$ & 6 \\
\hline 1,1,2,2-Tetrachloroethane & 6 & UJ & 6 & 6 & $\mathrm{UJ}$ & 6 & 5 & $\mathrm{UJ}$ & 5 & 6 & UJ & 6 \\
\hline 1,1,2-Trichloroethane & 6 & $\mathrm{U}$ & 6 & 6 & $\mathrm{U}$ & 6 & 5 & $\mathrm{U}$ & 5 & 6 & $\mathrm{U}$ & 6 \\
\hline 1,1-Dichloroethane & 6 & $\bar{U}$ & 6 & 6 & $\mathrm{U}$ & 6 & 5 & $\mathrm{U}$ & 5 & 6 & $\mathrm{U}$ & 6 \\
\hline 1,1-Dichloroethene & 6 & $\mathrm{U}$ & 6 & 6 & $\mathrm{U}$ & 6 & 5 & $\mathrm{U}$ & 5 & 6 & $\mathrm{U}$ & 6 \\
\hline 1,2-Dichloroethane & 6 & $\mathrm{U}$ & 6 & 6 & $\mathrm{U}$ & 6 & 5 & $\mathrm{U}$ & 5 & 6 & $\mathrm{U}$ & 6 \\
\hline 1,2-Dichloroethene (total) & 6 & $\mathrm{U}$ & 6 & 6 & $\mathrm{U}$ & 6 & 5 & $\mathrm{U}$ & 5 & 6 & $\mathrm{U}$ & 6 \\
\hline 1,2-Dichloropropane & 6 & $\bar{U}$ & 6 & 6 & $\mathrm{U}$ & 6 & 5 & $\mathrm{U}$ & 5 & 6 & $\mathrm{U}$ & 6 \\
\hline 2-Butanone & 11 & $\mathrm{U}$ & 11 & 11 & $\mathrm{U}$ & 11 & 10 & $\mathrm{U}$ & 10 & 11 & $\mathrm{U}$ & 11 \\
\hline 2-Hexanone & 11 & $\mathrm{U}$ & 11 & 11 & $\mathrm{U}$ & 11 & 10 & $\mathrm{U}$ & 10 & 11 & $\mathrm{U}$ & 11 \\
\hline 4-Methyl-2-pentanone & 11 & $\mathrm{U}$ & 11 & 11 & $\mathrm{U}$ & 11 & 10 & $\mathrm{U}$ & 10 & 11 & $\mathrm{U}$ & 11 \\
\hline Acetone & 9 & $\mathrm{~J}$ & 11 & 9 & $\mathrm{~J}$ & 11 & 10 & $\mathrm{U}$ & 10 & 11 & $\bar{U}$ & 11 \\
\hline Benzene & 6 & $\mathrm{U}$ & 6 & 6 & $\mathrm{U}$ & 6 & 5 & $\mathrm{U}$ & 5 & 6 & $\mathrm{U}$ & 6 \\
\hline Bromodichloromethane & 6 & $\mathrm{U}$ & 6 & 6 & $\mathrm{U}$ & 6 & 5 & $\mathrm{U}$ & 5 & 6 & $\mathrm{U}$ & 6 \\
\hline Bromoform & 6 & $\mathrm{U}$ & 6 & 6 & $\mathrm{U}$ & 6 & 5 & $\mathrm{U}$ & 5 & 6 & $\mathrm{U}$ & 6 \\
\hline Bromomethane & 11 & $\mathrm{U}$ & 11 & 11 & $\mathrm{U}$ & 11 & 10 & $\mathrm{U}$ & 10 & 11 & $\mathrm{U}$ & 11 \\
\hline Carbon disulfide & 6 & $\mathrm{U}$ & 6 & 6 & $\mathrm{U}$ & 6 & 5 & $\mathrm{U}$ & 5 & 6 & $\mathrm{U}$ & 6 \\
\hline Carbon tetrachloride & 6 & $\mathrm{U}$ & 6 & 6 & $\mathrm{U}$ & 6 & 5 & $\mathrm{U}$ & 5 & 6 & $\mathrm{U}$ & 6 \\
\hline Chlorobenzene & 6 & $\mathrm{U}$ & 6 & 6 & $\mathrm{U}$ & 6 & 5 & $\mathrm{U}$ & 5 & 6 & $\mathrm{U}$ & 6 \\
\hline Chloroethane & 11 & $\mathrm{U}$ & 11 & 11 & $\mathrm{U}$ & 11 & 10 & $\mathrm{U}$ & 10 & 11 & $\mathrm{U}$ & 11 \\
\hline Chloroform & 6 & $U$ & 6 & 6 & $\mathrm{U}$ & 6 & 5 & $\mathrm{U}$ & 5 & 6 & $\mathrm{U}$ & 6 \\
\hline Chloromethane & 11 & $\mathrm{U}$ & 11 & 11 & $\mathrm{U}$ & 11 & 10 & $\mathrm{U}$ & 10 & 11 & $\mathrm{U}$ & 11 \\
\hline cis-1,2-Dichloroethylene & 6 & $\mathrm{U}$ & 6 & 6 & $\mathrm{U}$ & 6 & 5 & $\mathrm{U}$ & 5 & 6 & $\mathrm{U}$ & 6 \\
\hline cis-1,3-Dichloropropene & 6 & $\mathrm{U}$ & 6 & 6 & $\mathrm{U}$ & 6 & 5 & $\mathrm{U}$ & 5 & 6 & $\mathrm{U}$ & 6 \\
\hline Dibromochloromethane & 6 & $\mathrm{U}$ & 6 & 6 & $\mathrm{U}$ & 6 & 5 & $\mathrm{U}$ & 5 & 6 & $\mathrm{U}$ & 6 \\
\hline Ethylbenzene & 6 & $U$ & 6 & 6 & $\mathrm{U}$ & 6 & 5 & $\mathrm{U}$ & 5 & 6 & $\mathrm{U}$ & 6 \\
\hline Methylene chloride & 14 & $\mathrm{~B}$ & 6 & 11 & $\mathrm{~B}$ & 6 & 9 & $\mathrm{~B}$ & 5 & 8 & $\mathrm{~B}$ & 6 \\
\hline \begin{tabular}{|l} 
Styrene \\
\end{tabular} & 6 & $\mathrm{U}$ & 6 & 6 & $\mathrm{U}$ & 6 & 5 & $\mathrm{U}$ & 5 & 6 & $\mathrm{U}$ & 6 \\
\hline Tetrachloroethene & 6 & $\mathrm{U}$ & 6 & 6 & $\mathrm{U}$ & 6 & 5 & $\mathrm{U}$ & 5 & 6 & $\mathrm{U}$ & 6 \\
\hline Toluene & 6 & $\mathrm{U}$ & 6 & 6 & $\mathrm{U}$ & 6 & 5 & $\mathrm{U}$ & 5 & 6 & $\mathrm{U}$ & 6 \\
\hline trans-1,2-Dichloroethylene & 6 & $\mathrm{U}$ & 6 & 6 & $\mathrm{U}$ & 6 & 5 & $\mathrm{U}$ & 5 & 6 & $\mathrm{U}$ & 6 \\
\hline trans-1,3-Dichloropropene & 6 & $\mathrm{U}$ & 6 & 6 & $\mathrm{U}$ & 6 & 5 & $\mathrm{U}$ & 5 & 6 & $U$ & 6 \\
\hline Trichloroethene & 6 & $\mathrm{U}$ & 6 & 6 & $\mathrm{U}$ & 6 & 5 & $\mathrm{U}$ & 5 & 6 & $\mathrm{U}$ & 6 \\
\hline Vinyl chloride & 11 & $\mathrm{U}$ & 11 & 11 & $\mathrm{U}$ & 11 & 10 & $\mathrm{U}$ & 10 & 11 & $\mathrm{U}$ & 11 \\
\hline Xylenes (total) & 6 & $\mathrm{U}$ & 6 & 6 & $\mathrm{U}$ & 6 & 5 & $\mathrm{U}$ & 5 & 6 & $\mathrm{U}$ & 6 \\
\hline
\end{tabular}


118-C-1 Verification Sampling Results.

\begin{tabular}{|c|c|c|c|c|c|c|c|c|c|c|c|c|}
\hline \multirow[t]{2}{*}{ Constituents } & \multicolumn{3}{|c|}{$\begin{array}{c}\text { J13520 } \\
\text { OB/BCL A3 } \\
\text { Sample Date } 8 / 22 / 06\end{array}$} & \multicolumn{3}{|c|}{$\begin{array}{c}\text { J135N5 } \\
\text { OB/BCL A4 } \\
\text { Sample Date } 8 / 22 / 06\end{array}$} & \multicolumn{3}{|c|}{$\begin{array}{c}\mathrm{J13522} \\
\text { SPA A1 } \\
\text { Sample Date } 9 / 11 / 06\end{array}$} & \multicolumn{3}{|c|}{$\begin{array}{c}\text { J13523 } \\
\text { SPA A2 } \\
\text { Sample Date 9/11/06 }\end{array}$} \\
\hline & $\mu \mathrm{g} / \mathrm{kg}$ & $\mathbf{Q}$ & PQL & $\mu \mathrm{g} / \mathrm{kg}$ & $\mathbf{Q}$ & PQL & $\mu \mathrm{g} / \mathrm{kg}$ & $Q$ & PQL & $\mu \mathrm{g} / \mathrm{kg}$ & $\mathbf{Q}$ & PQL \\
\hline \multicolumn{13}{|c|}{ Polychlorinated Biphenyls } \\
\hline Aroclor-1016 & 13 & $\mathrm{U}$ & 13 & 13 & $\mathrm{U}$ & 13 & 13 & $\bar{U}$ & 13 & 13 & $\mathrm{U}$ & 13 \\
\hline Aroclor-1221 & 13 & $\mathrm{U}$ & 13 & 13 & $\mathrm{U}$ & 13 & 13 & $\bar{U}$ & 13 & 13 & $\bar{U}$ & 13 \\
\hline Aroclor-1232 & 13 & $\mathrm{U}$ & 13 & 13 & $\mathrm{U}$ & 13 & 13 & $\bar{U}$ & 13 & 13 & $\bar{U}$ & 13 \\
\hline Aroclor-1242 & 13 & $\mathrm{U}$ & 13 & 13 & $\mathrm{U}$ & 13 & 13 & $\bar{U}$ & 13 & 13 & $\mathrm{U}$ & 13 \\
\hline Aroclor-1248 & 13 & $\mathrm{U}$ & 13 & 13 & $\mathrm{U}$ & 13 & 13 & $\mathrm{U}$ & 13 & 13 & $\mathrm{U}$ & 13 \\
\hline Aroclor-1254 & 5.3 & $\mathrm{~J}$ & 13 & 13 & $\mathrm{U}$ & 13 & 13 & $\mathrm{U}$ & 13 & 13 & $\mathrm{U}$ & 13 \\
\hline Aroclor-1260 & 13 & $\mathrm{U}$ & 13 & 13 & $\mathrm{U}$ & 13 & 3.7 & $\mathrm{~J}$ & 13 & 13 & $\mathrm{U}$ & 13 \\
\hline \multicolumn{13}{|c|}{ Semivolatile Organic Compounds } \\
\hline 1,2,4-Trichlorobenzene & 330 & $\mathrm{U}$ & 330 & 330 & $\mathrm{U}$ & 330 & 330 & UJ & 330 & 330 & UJ & 330 \\
\hline 1,2-Dichlorobenzene & 330 & $\mathrm{U}$ & 330 & 330 & $U$ & 330 & 330 & $\bar{U}$ & 330 & 330 & $\mathrm{U}$ & 330 \\
\hline 1,3-Dichlorobenzene & 330 & $\mathrm{U}$ & 330 & 330 & $\mathrm{U}$ & 330 & 330 & $\mathrm{U}$ & 330 & 330 & $\mathrm{U}$ & 330 \\
\hline 1,4-Dichlorobenzene & 330 & $\mathrm{U}$ & 330 & 330 & $\mathrm{U}$ & 330 & 330 & $\bar{U}$ & 330 & 330 & $U$ & 330 \\
\hline 2,4,5-Trichlorophenol & 840 & $\mathrm{U}$ & 840 & 830 & $\mathrm{U}$ & 830 & 840 & $\mathrm{U}$ & 840 & 840 & $\mathrm{U}$ & 840 \\
\hline 2,4,6-Trichlorophenol & 330 & $\bar{U}$ & 330 & 330 & $\mathrm{U}$ & 330 & 330 & $\mathrm{U}$ & 330 & 330 & $\mathrm{U}$ & 330 \\
\hline 2,4-Dichlorophenol & 330 & $\mathrm{U}$ & 330 & 330 & $\mathrm{U}$ & 330 & 330 & $\mathrm{UJ}$ & 330 & 330 & $\mathrm{UJ}$ & 330 \\
\hline 2,4-Dimethylphenol & 330 & $\mathrm{U}$ & 330 & 330 & $\mathrm{U}$ & 330 & 330 & $\mathrm{UJ}$ & 330 & 330 & $\mathrm{UJ}$ & 330 \\
\hline 2,4-Dinitrophenol & 840 & $\mathrm{U}$ & 840 & 830 & $\mathrm{U}$ & 830 & 840 & UR & 840 & 840 & UR & 840 \\
\hline 2,4-Dinitrotoluene & 330 & $\mathrm{U}$ & 330 & 330 & $\mathrm{U}$ & 330 & 330 & $\mathrm{U}$ & 330 & 330 & $\mathrm{U}$ & 330 \\
\hline 2,6-Dinitrotoluene & 330 & $\mathrm{U}$ & 330 & 330 & $\mathrm{U}$ & 330 & 330 & $\mathrm{U}$ & 330 & 330 & $\mathrm{U}$ & 330 \\
\hline 2-Chloronaphthalene & 330 & $\mathrm{U}$ & 330 & 330 & $\mathrm{U}$ & 330 & 330 & $\mathrm{U}$ & 330 & 330 & $\mathrm{U}$ & 330 \\
\hline 2-Chlorophenol & 330 & $\mathrm{U}$ & 330 & 330 & $\mathrm{U}$ & 330 & 330 & $\mathrm{U}$ & 330 & 330 & $\mathrm{U}$ & 330 \\
\hline 2-Methylnaphthalene & 330 & $\mathrm{U}$ & 330 & 330 & $\mathrm{U}$ & 330 & 330 & UJ & 330 & 330 & UJ & 330 \\
\hline 2-Methylphenol (creso & 330 & $\mathrm{U}$ & 330 & 330 & $\mathrm{U}$ & 330 & 330 & UJ & 330 & 330 & UJ & 330 \\
\hline 2-Nitroaniline & 840 & $\mathrm{U}$ & 840 & 830 & $\mathrm{U}$ & 830 & 840 & $\mathrm{U}$ & 840 & 840 & $\mathrm{U}$ & 840 \\
\hline 2-Nitrophenol & 330 & $\mathrm{U}$ & 330 & 330 & $\mathrm{U}$ & 330 & 330 & UJ & 330 & 330 & UJ & 330 \\
\hline 3,3'-Dichlorobenzidine & 330 & $\mathrm{U}$ & 330 & 330 & $\bar{U}$ & 330 & 330 & $\bar{U}$ & 330 & 330 & $\mathrm{U}$ & 330 \\
\hline 3-Nitroaniline & 840 & $\mathrm{U}$ & 840 & 830 & $\mathrm{U}$ & 830 & 840 & $\mathrm{U}$ & 840 & 840 & $\mathrm{U}$ & 840 \\
\hline 4,6-Dinitro-2-methylphenol & 840 & $\bar{U}$ & 840 & 830 & $\mathrm{U}$ & 830 & 840 & $\bar{U}$ & 840 & 840 & $\bar{U}$ & 840 \\
\hline 4-Bromophenyl-phenylether & 330 & $\mathrm{U}$ & 330 & 330 & $\mathrm{U}$ & 330 & 330 & $\mathrm{U}$ & 330 & 330 & $\mathrm{U}$ & 330 \\
\hline 4-Chloro-3-methylphenol & 330 & $\mathrm{U}$ & 330 & 330 & $\mathrm{U}$ & 330 & 330 & UJ & 330 & 330 & $\mathrm{UJ}$ & 330 \\
\hline 4-Chloroaniline & 330 & $\mathrm{U}$ & 330 & 330 & $\mathrm{U}$ & 330 & 330 & $\mathrm{U}$ & 330 & 330 & $\mathrm{U}$ & 330 \\
\hline 4-Chlorophenyl-phenylether & 330 & $\mathrm{U}$ & 330 & 330 & $\mathrm{U}$ & 330 & 330 & $\mathrm{U}$ & 330 & 330 & $\mathrm{U}$ & 330 \\
\hline 4-Methylphenol (p-cresol) & 330 & $\mathrm{U}$ & 330 & 330 & $\mathrm{U}$ & 330 & 330 & UJ & 330 & 330 & UJ & 330 \\
\hline 4-Nitroaniline & 840 & $U$ & 840 & 830 & $\mathrm{U}$ & 830 & 840 & $\mathrm{U}$ & 840 & 840 & $\mathrm{U}$ & 840 \\
\hline 4-Nitrophenol & 840 & $\mathrm{U}$ & 840 & 830 & $\mathrm{U}$ & 830 & 840 & $\mathrm{U}$ & 840 & 840 & $\mathrm{U}$ & 840 \\
\hline Acenaphthene & 330 & $\mathrm{U}$ & 330 & 330 & $\mathrm{U}$ & 330 & 330 & $U$ & 330 & 330 & $\mathrm{U}$ & 330 \\
\hline Acenaphthylene & 330 & $\bar{U}$ & 330 & 330 & $\mathrm{U}$ & 330 & 330 & $\bar{U}$ & 330 & 330 & $\bar{U}$ & 330 \\
\hline Anthracene & 330 & $\mathrm{U}$ & 330 & 330 & $\mathrm{U}$ & 330 & 330 & $\mathrm{U}$ & 330 & 330 & $\mathrm{U}$ & 330 \\
\hline Benzo(a)anthracene & 330 & $\mathrm{U}$ & 330 & 38 & $\mathrm{~J}$ & 330 & 46 & $\mathrm{~J}$ & 330 & 33 & $\mathrm{~J}$ & 330 \\
\hline Benzo(a)pyrene & 330 & $\mathrm{U}$ & 330 & 24 & $\mathrm{~J}$ & 330 & 19 & $\mathrm{~J}$ & 330 & 30 & $\mathrm{~J}$ & 330 \\
\hline Benzo(b)fluoranthene & 330 & $\mathrm{U}$ & 330 & 33 & $\mathrm{~J}$ & 330 & 43 & $\mathrm{~J}$ & 330 & 32 & $\mathrm{~J}$ & 330 \\
\hline Benzo(g,h,i)perylene & 330 & $\bar{U}$ & 330 & 330 & $\mathrm{U}$ & 330 & 19 & $\mathrm{~J}$ & 330 & 22 & & 330 \\
\hline Benzo(k)fluoranthene & 330 & $\mathrm{U}$ & 330 & 29 & $\mathrm{~J}$ & 330 & 41 & $\mathrm{~J}$ & 330 & 31 & $\mathrm{~J}$ & 330 \\
\hline bis(2-Chloro-1-methylethyl)ether & 330 & $\mathrm{U}$ & 330 & 330 & $\mathrm{U}$ & 330 & 330 & $\mathrm{U}$ & 330 & 330 & $\mathrm{U}$ & 330 \\
\hline bis(2-Chloroethoxy)methane & 330 & $\mathrm{U}$ & 330 & 330 & $\bar{U}$ & 330 & 330 & $\mathrm{U}$ & 330 & 330 & $\bar{U}$ & 330 \\
\hline bis(2-Chloroethyl)ether & 330 & $\mathrm{U}$ & 330 & 330 & $\mathrm{U}$ & 330 & 330 & $\mathrm{U}$ & 330 & 330 & $\mathrm{U}$ & 330 \\
\hline bis(2-Ethylhexyl)phthalate & 330 & $\mathrm{U}$ & 330 & 330 & $\mathrm{U}$ & 330 & 71 & JB & 330 & 36 & JB & 330 \\
\hline
\end{tabular}


CVP-2006-00011

Rev. 0

118-C-1 Verification Sampling Results.

\begin{tabular}{|c|c|c|c|c|c|c|c|c|c|c|c|c|}
\hline \multirow[t]{2}{*}{ Constituents } & \multicolumn{3}{|c|}{$\begin{array}{c}\text { J13520 } \\
\text { OB/BCL A3 } \\
\text { Sample Date } 8 / 22 / 06\end{array}$} & \multicolumn{3}{|c|}{$\begin{array}{c}\text { J135N5 } \\
\text { OB/BCL A4 } \\
\text { Sample Date 8/22/06 }\end{array}$} & \multicolumn{3}{|c|}{$\begin{array}{c}\text { J13522 } \\
\text { SPA A1 } \\
\text { Sample Date } 9 / 11 / 06\end{array}$} & \multicolumn{3}{|c|}{$\begin{array}{c}\text { J13523 } \\
\text { SPA A2 } \\
\text { Sample Date } 9 / 11 / 06\end{array}$} \\
\hline & $\mu \mathrm{g} / \mathrm{kg}$ & $Q$ & PQL & $\mu \mathrm{g} / \mathrm{kg}$ & $\mathbf{Q}$ & PQL & $\mu \mathrm{g} / \mathrm{kg}$ & $Q$ & PQL & $\mu \mathrm{g} / \mathrm{kg}$ & $Q$ & PQL \\
\hline \multicolumn{13}{|c|}{ Semivolatile Organic Compounds (continued) } \\
\hline Butylbenzylphthalate & 330 & $\mathrm{U}$ & 330 & 330 & $\mathrm{U}$ & 330 & 330 & $\mathrm{U}$ & 330 & 330 & $\mathrm{U}$ & 330 \\
\hline Carbazole & 330 & $\mathrm{U}$ & 330 & 330 & $\mathrm{U}$ & 330 & 330 & $\mathrm{U}$ & 330 & 330 & $\mathrm{U}$ & 330 \\
\hline Chrysene & 330 & $\mathrm{U}$ & 330 & 52 & $\mathrm{~J}$ & 330 & 76 & $\mathrm{~J}$ & 330 & 40 & $\mathrm{~J}$ & 330 \\
\hline Di-n-butylphthalate & 330 & $\mathrm{U}$ & 330 & 330 & $\mathrm{U}$ & 330 & 23 & $\mathrm{JB}$ & 330 & 27 & $\mathrm{JB}$ & 330 \\
\hline Di-n-octylphthalate & 330 & $\mathrm{U}$ & 330 & 330 & $\mathrm{U}$ & 330 & 330 & $\mathrm{U}$ & 330 & 330 & $\mathrm{U}$ & 330 \\
\hline Dibenz(a,h)anthracene & 330 & $\mathrm{U}$ & 330 & 330 & $\mathrm{U}$ & 330 & 330 & $\mathrm{U}$ & 330 & 330 & $\mathrm{U}$ & 330 \\
\hline Dibenzofuran & 330 & $\mathrm{U}$ & 330 & 330 & $\mathrm{U}$ & 330 & 330 & $\mathrm{U}$ & 330 & 330 & $\mathrm{U}$ & 330 \\
\hline Diethylphthalate & 330 & $\mathrm{U}$ & 330 & 330 & $\mathrm{U}$ & 330 & 330 & $\mathrm{U}$ & 330 & 330 & $U$ & 330 \\
\hline Dimethylphthalate & 330 & $\mathrm{U}$ & 330 & 330 & $\mathrm{U}$ & 330 & 330 & $\mathrm{U}$ & 330 & 330 & $\mathrm{U}$ & 330 \\
\hline Fluoranthene & 330 & UJ & 330 & 110 & $\mathrm{~J}$ & 330 & 110 & $\mathrm{~J}$ & 330 & 56 & $\mathrm{~J}$ & 330 \\
\hline Fluorene & 330 & $\mathrm{U}$ & 330 & 330 & $\mathrm{U}$ & 330 & 330 & $\mathrm{U}$ & 330 & 330 & $\mathrm{U}$ & 330 \\
\hline Hexachlorobenzene & 330 & $\mathrm{U}$ & 330 & 330 & $\mathrm{U}$ & 330 & 330 & $\mathrm{U}$ & 330 & 330 & $\mathrm{U}$ & 330 \\
\hline Hexachlorobutadiene & 330 & $\mathrm{U}$ & 330 & 330 & $\mathrm{U}$ & 330 & 330 & $\mathrm{U}$ & 330 & 330 & $\mathrm{U}$ & 330 \\
\hline Hexachlorocyclopenta & 330 & $U$ & 330 & 330 & $\mathrm{U}$ & 330 & 330 & $\mathrm{U}$ & 330 & 330 & $\mathrm{U}$ & 330 \\
\hline Hexachloroethane & 330 & $\mathrm{U}$ & 330 & 330 & $\mathrm{U}$ & 330 & 330 & $\mathrm{U}$ & 330 & 330 & $\mathrm{U}$ & 330 \\
\hline Indeno(1,2,3-cd)pyrene & 330 & $\mathrm{U}$ & 330 & 330 & $\mathrm{U}$ & 330 & 17 & $\mathrm{~J}$ & 330 & 330 & $\mathrm{U}$ & 330 \\
\hline Isophorone & 330 & $\mathrm{U}$ & 330 & 330 & $\mathrm{U}$ & 330 & 330 & UJ & 330 & 330 & UJ & 330 \\
\hline N-Nitroso-di-n-dipropylamine & 330 & $\mathrm{U}$ & 330 & 330 & $\mathrm{U}$ & 330 & 330 & $\mathrm{U}$ & 330 & 330 & $\mathrm{U}$ & 330 \\
\hline N-Nitrosodiphenylamine & 330 & $\mathrm{U}$ & 330 & 330 & $\mathrm{U}$ & 330 & 330 & $\mathrm{U}$ & 330 & 330 & $\mathrm{U}$ & 330 \\
\hline Naphthalene & 330 & $\mathrm{U}$ & 330 & 330 & $\mathrm{U}$ & 330 & 330 & $\mathrm{U}$ & 330 & 330 & $\mathrm{U}$ & 330 \\
\hline Nitrobenzene & 330 & $\mathrm{U}$ & 330 & 330 & $\mathrm{U}$ & 330 & 330 & UJ & 330 & 330 & UJ & 330 \\
\hline Pentachlorophenol & 840 & $\mathrm{U}$ & 840 & 830 & $\mathrm{U}$ & 830 & 840 & $\mathrm{U}$ & 840 & 840 & $\mathrm{U}$ & 840 \\
\hline Phenanthrene & 330 & $\mathrm{UJ}$ & 330 & 37 & $\mathrm{~J}$ & 330 & 27 & $\mathrm{~J}$ & 330 & 330 & $\mathrm{U}$ & 330 \\
\hline Phenol & 330 & $\mathrm{U}$ & 330 & 330 & $\mathrm{U}$ & 330 & 330 & $\mathrm{U}$ & 330 & 330 & $\mathrm{U}$ & 330 \\
\hline Pyrene & 330 & UJ & 330 & 57 & $\mathrm{~J}$ & 330 & 78 & $\mathrm{~J}$ & 330 & 57 & $\mathrm{~J}$ & 330 \\
\hline
\end{tabular}


118-C-1 Verification Sampling Results.

\begin{tabular}{|c|c|c|c|c|c|c|c|c|c|c|c|c|}
\hline \multirow[t]{2}{*}{ Constituents } & \multicolumn{3}{|c|}{$\begin{array}{c}\text { J135T4 } \\
\text { OB/BCL A3 } \\
\text { Sample Date } 8 / 28 / 06\end{array}$} & \multicolumn{3}{|c|}{$\begin{array}{c}\text { J135T5 } \\
\text { OB/BCL A4 } \\
\text { Sample Date } 8 / 28 / 06\end{array}$} & \multicolumn{3}{|c|}{$\begin{array}{c}\mathrm{J13522} \\
\text { SPA A1 } \\
\text { Sample Date } 9 / 11 / 06\end{array}$} & \multicolumn{3}{|c|}{$\begin{array}{c}\text { J13523 } \\
\text { SPA A2 } \\
\text { Sample Date } 9 / 11 / 06\end{array}$} \\
\hline & $\mu \mathrm{g} / \mathrm{kg}$ & $\mathbf{Q}$ & PQL & $\mu \mathrm{g} / \mathrm{kg}$ & $\mathbf{Q}$ & PQL & $\mu \mathrm{g} / \mathrm{kg}$ & $Q$ & PQL & $\mu \mathrm{g} / \mathrm{kg}$ & $Q$ & PQL \\
\hline \multicolumn{13}{|c|}{ Volatile Organic Compounds } \\
\hline 1,1,1-Trichloroethane & 6 & $\mathrm{U}$ & 6 & 5 & $\mathrm{U}$ & 5 & 6 & $\mathrm{U}$ & 6 & 5 & $\mathrm{U}$ & 5 \\
\hline 1,1,2,2-Tetrachloroethane & 6 & UJ & 6 & 5 & UJ & 5 & 6 & $\mathrm{UJ}$ & 6 & 5 & UJ & 5 \\
\hline 1,1,2-Trichloroethane & 6 & $\mathrm{U}$ & 6 & 5 & $\mathrm{U}$ & 5 & 6 & $\mathrm{U}$ & 6 & 5 & $U$ & 5 \\
\hline 1,1-Dichloroethane & 6 & $\mathrm{U}$ & 6 & 5 & $\mathrm{U}$ & 5 & 6 & $\mathrm{U}$ & 6 & 5 & $\mathrm{U}$ & 5 \\
\hline 1,1-Dichloroethene & 6 & $\mathrm{U}$ & 6 & 5 & $\mathrm{U}$ & 5 & 6 & $\mathrm{U}$ & 6 & 5 & $\mathrm{U}$ & 5 \\
\hline 1,2-Dichloroethane & 6 & $\mathrm{U}$ & 6 & 5 & $\mathrm{U}$ & 5 & 6 & $\mathrm{U}$ & 6 & 5 & $\mathrm{U}$ & 5 \\
\hline 1,2-Dichloroethene (total) & 6 & $\mathrm{U}$ & 6 & 5 & $\bar{U}$ & 5 & 6 & $\mathrm{U}$ & 6 & 5 & $U$ & 5 \\
\hline 1,2-Dichloropropane & 6 & $\mathrm{U}$ & 6 & 5 & $\mathrm{U}$ & 5 & 6 & $\mathrm{U}$ & 6 & 5 & $\mathrm{U}$ & 5 \\
\hline 2-Butanone & 11 & $\mathrm{U}$ & 11 & 10 & $\mathrm{U}$ & 10 & 11 & $\mathrm{U}$ & 11 & 10 & $\mathrm{U}$ & 10 \\
\hline 2-Hexanone & 11 & $\mathrm{U}$ & 11 & 10 & $\mathrm{U}$ & 10 & 11 & $\mathrm{U}$ & 11 & 10 & $\mathrm{U}$ & 10 \\
\hline 4-Methyl-2-pentanone & 11 & $\mathrm{U}$ & 11 & 10 & $\mathrm{U}$ & 10 & 11 & $\mathrm{U}$ & 11 & 10 & $\mathrm{U}$ & 10 \\
\hline Acetone & 12 & $\mathrm{~J}$ & 11 & 11 & $\mathrm{~J}$ & 10 & 25 & $\mathrm{~J}$ & 11 & 10 & $\mathrm{U}$ & 10 \\
\hline Benzene & 6 & $\mathrm{U}$ & 6 & 5 & $\mathrm{U}$ & 5 & 6 & $\mathrm{U}$ & 6 & 5 & $\mathrm{U}$ & 5 \\
\hline Bromodichloromethane & 6 & $\mathrm{U}$ & 6 & 5 & $\mathrm{U}$ & 5 & 6 & $\mathrm{U}$ & 6 & 5 & $\mathrm{U}$ & 5 \\
\hline Bromoform & 6 & $\mathrm{U}$ & 6 & 5 & $\mathrm{U}$ & 5 & 6 & $\mathrm{U}$ & 6 & 5 & $\mathrm{U}$ & 5 \\
\hline Bromomethane & 11 & $\mathrm{U}$ & 11 & 10 & $\mathrm{U}$ & 10 & 11 & $\mathrm{U}$ & 11 & 10 & $\mathrm{U}$ & 10 \\
\hline Carbon disulfide & 6 & $U$ & 6 & 5 & $\mathrm{U}$ & 5 & 6 & $\mathrm{U}$ & 6 & 5 & $\mathrm{U}$ & 5 \\
\hline Carbon tetrachloride & 6 & $\mathrm{U}$ & 6 & 5 & $\mathrm{U}$ & 5 & 6 & $\mathrm{U}$ & 6 & 5 & $\mathrm{U}$ & 5 \\
\hline Chlorobenzene & 6 & $\mathrm{U}$ & 6 & 5 & $\mathrm{U}$ & 5 & 6 & $\mathrm{U}$ & 6 & 5 & $\mathrm{U}$ & 5 \\
\hline Chloroethane & 11 & $\mathrm{U}$ & 11 & 10 & $\mathrm{U}$ & 10 & 11 & $\mathrm{U}$ & 11 & 10 & $\mathrm{U}$ & 10 \\
\hline Chloroform & 6 & $\mathrm{U}$ & 6 & 5 & $\mathrm{U}$ & 5 & 6 & $\mathrm{U}$ & 6 & 5 & $\mathrm{U}$ & 5 \\
\hline Chloromethane & 11 & $\mathrm{U}$ & 11 & 10 & $\mathrm{U}$ & 10 & 11 & $\mathrm{U}$ & 11 & 10 & $U$ & 10 \\
\hline cis-1,2-Dichloroethylene & 6 & $\mathrm{U}$ & 6 & 5 & $\mathrm{U}$ & 5 & 6 & $\mathrm{U}$ & 6 & 5 & $\mathrm{U}$ & 5 \\
\hline cis-1,3-Dichloropropene & 6 & $\mathrm{U}$ & 6 & 5 & $\mathrm{U}$ & 5 & 6 & $\mathrm{U}$ & 6 & 5 & $\mathrm{U}$ & 5 \\
\hline Dibromochloromethane & 6 & $U$ & 6 & 5 & $\mathrm{U}$ & 5 & 6 & $\mathrm{U}$ & 6 & 5 & $\mathrm{U}$ & 5 \\
\hline Ethylbenzene & 6 & $\mathrm{U}$ & 6 & 5 & $\mathrm{U}$ & 5 & 6 & $\mathrm{U}$ & 6 & 5 & $\mathrm{U}$ & 5 \\
\hline Methylene chloride & 12 & $\mathrm{~B}$ & 6 & 13 & $\mathrm{~B}$ & 5 & 11 & $\mathrm{~B}$ & 6 & 8 & $\mathrm{~B}$ & 5 \\
\hline Styrene & 6 & $\mathrm{U}$ & 6 & 5 & $\mathrm{U}$ & 5 & 6 & $\mathrm{U}$ & 6 & 5 & $\mathrm{U}$ & 5 \\
\hline Tetrachloroethene & 6 & $\mathrm{U}$ & 6 & 5 & $\mathrm{U}$ & 5 & 6 & $\mathrm{U}$ & 6 & 5 & $\mathrm{U}$ & 5 \\
\hline Toluene & 6 & $\mathrm{U}$ & 6 & 5 & $\mathrm{U}$ & 5 & 1 & $\mathrm{~J}$ & 6 & 5 & $\mathrm{U}$ & 5 \\
\hline trans-1,2-Dichloroethylene & 6 & U & 6 & 5 & $\mathrm{U}$ & 5 & 6 & $\mathrm{U}$ & 6 & 5 & $\mathrm{U}$ & 5 \\
\hline trans-1,3-Dichloropropene & 6 & $\mathrm{U}$ & 6 & 5 & $\mathrm{U}$ & 5 & 6 & $\mathrm{U}$ & 6 & 5 & $U$ & 5 \\
\hline Trichloroethene & 6 & $\mathrm{U}$ & 6 & 5 & $\mathrm{U}$ & 5 & 6 & $\mathrm{U}$ & 6 & 5 & $\mathrm{U}$ & 5 \\
\hline Vinyl chloride & 11 & $\mathrm{U}$ & 11 & 10 & $\mathrm{U}$ & 10 & 11 & $\mathrm{U}$ & 11 & 10 & $\mathrm{U}$ & 10 \\
\hline Xylenes (total) & 6 & $\mathrm{U}$ & 6 & 5 & $\mathrm{U}$ & 5 & 6 & $\mathrm{U}$ & 6 & 5 & $\mathrm{U}$ & 5 \\
\hline
\end{tabular}


CVP-2006-00011

Rev. 0

118-C-1 Verification Sampling Results.

\begin{tabular}{|c|c|c|c|c|c|c|c|c|c|c|c|c|}
\hline \multirow[t]{2}{*}{ Constituents } & \multicolumn{3}{|c|}{$\begin{array}{c}\text { J135P1 } \\
\text { SPA A3 } \\
\text { Sample Date 9/12/06 }\end{array}$} & \multicolumn{3}{|c|}{$\begin{array}{c}\text { J135P0 } \\
\text { SPA A4 } \\
\text { Sample Date } 9 / 12 / 06\end{array}$} & \multicolumn{3}{|c|}{$\begin{array}{c}\text { J13524 } \\
\text { SPA B5 } \\
\text { Sample Date 9/11/06 }\end{array}$} & \multicolumn{3}{|c|}{$\begin{array}{c}\text { J13525 } \\
\text { SPA B6 } \\
\text { Sample Date 9/11/06 }\end{array}$} \\
\hline & $\mu \mathrm{g} / \mathrm{kg}$ & $Q$ & PQL & $\mu \mathrm{g} / \mathrm{kg}$ & $Q$ & PQL & $\mu \mathrm{g} / \mathrm{kg}$ & Q & PQL & $\mu \mathrm{g} / \mathrm{kg}$ & $\mathbf{Q}$ & PQL \\
\hline \multicolumn{13}{|c|}{ Polychlorinated Biphenyls } \\
\hline Aroclor-1016 & 13 & $\mathrm{U}$ & 13 & 13 & $\mathrm{U}$ & 13 & 13 & $\mathrm{U}$ & 13 & 13 & $\mathrm{U}$ & 13 \\
\hline Aroclor-1221 & 13 & $U$ & 13 & 13 & $\mathrm{U}$ & 13 & 13 & $\mathrm{U}$ & 13 & 13 & $\mathrm{U}$ & 13 \\
\hline Aroclor-1232 & 13 & $\mathrm{U}$ & 13 & 13 & $\mathrm{U}$ & 13 & 13 & $\mathrm{U}$ & 13 & 13 & $\mathrm{U}$ & 13 \\
\hline Aroclor- 1242 & 13 & $\mathrm{U}$ & 13 & 13 & $\mathrm{U}$ & 13 & 13 & $\mathrm{U}$ & 13 & 13 & $\mathrm{U}$ & 13 \\
\hline Aroclor-1248 & 13 & $\mathrm{U}$ & 13 & 13 & $\mathrm{U}$ & 13 & 13 & $\mathrm{U}$ & 13 & 13 & $\mathrm{U}$ & 13 \\
\hline Aroclor-1254 & 13 & $\mathrm{U}$ & 13 & 13 & $\mathrm{U}$ & 13 & 13 & $\mathrm{U}$ & 13 & 13 & $\mathrm{~J}$ & 13 \\
\hline Aroclor-1260 & 13 & $\mathrm{U}$ & 13 & 31 & & 13 & 61 & & 13 & 13 & $\mathrm{U}$ & 13 \\
\hline \multicolumn{13}{|c|}{ Semivolatile Organic Compounds } \\
\hline 1,2,4-Trichlorobenzene & 330 & UJ & 330 & 670 & UDJ & 670 & 330 & UJ & 330 & 330 & UJ & 330 \\
\hline 1,2-Dichlorobenzene & 330 & UJ & 330 & 670 & UDJ & 670 & 330 & $\mathrm{U}$ & 330 & 330 & $\mathrm{U}$ & 330 \\
\hline 1,3-Dichlorobenzene & 330 & UJ & 330 & 670 & UDJ & 670 & 330 & $\mathrm{U}$ & 330 & 330 & $\mathrm{U}$ & 330 \\
\hline 1,4-Dichlorobenzene & 330 & UJ & 330 & 670 & UDJ & 670 & 330 & $\mathrm{U}$ & 330 & 330 & $\mathrm{U}$ & 330 \\
\hline 2,4,5-Trichlorophenol & 840 & $\mathrm{U}$ & 840 & 1700 & UD & 1700 & 840 & $\mathrm{U}$ & 840 & 840 & $\mathrm{U}$ & 840 \\
\hline 2,4,6-Trichlorophenol & 330 & $\mathrm{U}$ & 330 & 670 & UD & 670 & 330 & $\mathrm{U}$ & 330 & 330 & $\mathrm{U}$ & 330 \\
\hline 2,4-Dichlorophenol & 330 & $\mathrm{U}$ & 330 & 670 & UD & 670 & 330 & UJ & 330 & 330 & $\mathrm{UJ}$ & 330 \\
\hline 2,4-Dimethylphenol & 330 & UJ & 330 & 670 & UDJ & 670 & 330 & UJ & 330 & 330 & $\mathrm{UJ}$ & 330 \\
\hline 2,4-Dinitrophenol & 840 & UJ & 840 & 1700 & UDJ & 1700 & 840 & UR & 840 & 840 & UR & 840 \\
\hline 2,4-Dinitrotoluene & 330 & $\mathrm{U}$ & 330 & 670 & UD & 670 & 330 & $\mathrm{U}$ & 330 & 330 & $\mathrm{U}$ & 330 \\
\hline 2,6-Dinitrotoluene & 330 & $\mathrm{U}$ & 330 & 670 & UD & 670 & 330 & $\mathrm{U}$ & 330 & 330 & $\mathrm{U}$ & 330 \\
\hline 2-Chloronaphthalene & 330 & $\mathrm{U}$ & 330 & 670 & UD & 670 & 330 & $\mathrm{U}$ & 330 & 330 & $\mathrm{U}$ & 330 \\
\hline 2-Chlorophenol & 330 & $\mathrm{UJ}$ & 330 & 670 & UDJ & 670 & 330 & $\mathrm{U}$ & 330 & 330 & $U$ & 330 \\
\hline 2-Methylnaphthalene & 330 & $\mathrm{UJ}$ & 330 & 670 & UDJ & 670 & 330 & UJ & 330 & 330 & UJ & 330 \\
\hline 2-Methylphenol (cresol, o-) & 330 & UJ & 330 & 670 & UDJ & 670 & 330 & UJ & 330 & 330 & $\mathrm{UJ}$ & 330 \\
\hline 2-Nitroaniline & 840 & $\mathrm{U}$ & 840 & 1700 & UD & 1700 & 840 & $\mathrm{U}$ & 840 & 840 & $\mathrm{U}$ & 840 \\
\hline 2-Nitrophenol & 330 & UJ & 330 & 670 & UDJ & 670 & 330 & UJ & 330 & 330 & $\mathrm{UJ}$ & 330 \\
\hline 3,3'-Dichlorobenzidine & 330 & $\mathrm{U}$ & 330 & 670 & UD & 670 & 330 & $\mathrm{U}$ & 330 & 330 & $\mathrm{U}$ & 330 \\
\hline 3-Nitroaniline & 840 & $\mathrm{U}$ & 840 & 1700 & UD & 1700 & 840 & $\mathrm{U}$ & 840 & 840 & $\mathrm{U}$ & 840 \\
\hline 4,6-Dinitro-2-methylphenol & 840 & $\mathrm{U}$ & 840 & 1700 & UD & 1700 & 840 & $\mathrm{U}$ & 840 & 840 & $\mathrm{U}$ & 840 \\
\hline 4-Bromophenyl-phenylether & 330 & $\mathrm{U}$ & 330 & 670 & UD & 670 & 330 & $\mathrm{U}$ & 330 & 330 & $\mathrm{U}$ & 330 \\
\hline 4-Chloro-3-methylphenol & 330 & UJ & 330 & 670 & UDJ & 670 & 330 & UJ & 330 & 330 & UJ & 330 \\
\hline 4-Chloroaniline & 330 & $\mathrm{U}$ & 330 & 670 & UD & 670 & 330 & $\mathrm{U}$ & 330 & 330 & $\mathrm{U}$ & 330 \\
\hline 4-Chlorophenyl-phenylether & 330 & $\mathrm{U}$ & 330 & 670 & UD & 670 & 330 & $\mathrm{U}$ & 330 & 330 & $\mathrm{U}$ & 330 \\
\hline 4-Methylphenol (p-cresol) & 330 & UJ & 330 & 670 & UDJ & 670 & 330 & UJ & 330 & 330 & UJ & 330 \\
\hline 4-Nitroaniline & 840 & $\mathrm{U}$ & 840 & 1700 & UD & 1700 & 840 & $\mathrm{U}$ & 840 & 840 & $\mathrm{U}$ & 840 \\
\hline 4-Nitrophenol & 840 & $\mathrm{U}$ & 840 & 1700 & UD & 1700 & 840 & $\mathrm{U}$ & 840 & 840 & $\mathrm{U}$ & 840 \\
\hline Acenaphthene & 330 & $\mathrm{U}$ & 330 & 670 & UD & 670 & 330 & $\mathrm{U}$ & 330 & 330 & $\mathrm{U}$ & 330 \\
\hline Acenaphthylene & 330 & $\mathrm{U}$ & 330 & 670 & UD & 670 & 330 & $\mathrm{U}$ & 330 & 330 & $\mathrm{U}$ & 330 \\
\hline Anthracene & 330 & UJ & 330 & 170 & JD & 670 & 330 & $\mathrm{U}$ & 330 & 330 & $\mathrm{U}$ & 330 \\
\hline Benzo(a)anthrace & 37 & $\mathrm{~J}$ & 330 & 660 & JD & 670 & 25 & $\mathrm{~J}$ & 330 & 27 & $\mathrm{~J}$ & 330 \\
\hline Benzo(a)pyrene & 330 & UJ & 330 & 320 & JD & 670 & 330 & $\mathrm{U}$ & 330 & 20 & $\mathrm{~J}$ & 330 \\
\hline Benzo(b)fluoranthene & 25 & $\mathrm{~J}$ & 330 & 390 & JD & 670 & 27 & $\mathrm{~J}$ & 330 & 27 & $\mathrm{~J}$ & 330 \\
\hline Benzo(g,h,i)perylene & 330 & $\mathrm{U}$ & 330 & 140 & JD & 670 & 330 & $\bar{U}$ & 330 & 330 & $\mathrm{U}$ & 330 \\
\hline Benzo(k)fluoranthene & 27 & $\mathrm{~J}$ & 330 & 360 & $\mathrm{JD}$ & 670 & 24 & $\mathrm{~J}$ & 330 & 23 & $J$ & 330 \\
\hline bis(2-Chloro-1-methylethyl)ether & 330 & $\mathrm{U}$ & 330 & 670 & UD & 670 & 330 & $\mathrm{U}$ & 330 & 330 & $\mathrm{U}$ & 330 \\
\hline bis(2-Chloroethoxy)methane & 330 & $\mathrm{U}$ & 330 & 670 & UD & 670 & 330 & $\mathrm{U}$ & 330 & 330 & $\mathrm{U}$ & 330 \\
\hline bis(2-Chloroethyl)ether & 330 & $\mathrm{U}$ & 330 & 670 & UD & 670 & 330 & $\mathrm{U}$ & 330 & 330 & $\mathrm{U}$ & 330 \\
\hline bis(2-Ethylhexyl)phthalate & 42 & JB & 330 & 50 & JBD & 670 & 35 & JB & 330 & 59 & JB & 330 \\
\hline
\end{tabular}


CVP-2006-00011

Rev. 0

118-C-1 Verification Sampling Results.

\begin{tabular}{|c|c|c|c|c|c|c|c|c|c|c|c|c|}
\hline \multirow[t]{2}{*}{ Constituents } & \multicolumn{3}{|c|}{$\begin{array}{c}\text { J135P1 } \\
\text { SPA A3 } \\
\text { Sample Date 9/12/06 }\end{array}$} & \multicolumn{3}{|c|}{$\begin{array}{c}\text { J135P0 } \\
\text { SPA A4 } \\
\text { Sample Date } 9 / 12 / 06 \\
\end{array}$} & \multicolumn{3}{|c|}{$\begin{array}{c}\text { J13524 } \\
\text { SPA B5 } \\
\text { Sample Date } 9 / 11 / 06 \\
\end{array}$} & \multicolumn{3}{|c|}{$\begin{array}{c}\text { J13525 } \\
\text { SPA B6 } \\
\text { Sample Date 9/11/06 } \\
\end{array}$} \\
\hline & $\mu \mathrm{g} / \mathrm{kg}$ & $\mathbf{Q}$ & PQL & $\mu \mathrm{g} / \mathrm{kg}$ & $\mathbf{Q}$ & PQL & $\mu \mathrm{g} / \mathrm{kg}$ & $\mathbf{Q}$ & PQL & $\mu \mathrm{g} / \mathrm{kg}$ & $Q$ & $\mathrm{PQL}$ \\
\hline \multicolumn{13}{|c|}{ Semivolatile Organic Compounds (continued) } \\
\hline Butylbenzylphthalate & 17 & $\mathrm{~J}$ & 330 & 670 & UD & 670 & 330 & $\mathrm{U}$ & 330 & 330 & $\mathrm{U}$ & 330 \\
\hline Carbazole & 330 & $\mathrm{UJ}$ & 330 & 47 & JD & 670 & 330 & $\mathrm{U}$ & 330 & 330 & $\mathrm{U}$ & 330 \\
\hline Chrysene & 51 & $\mathrm{~J}$ & 330 & 790 & JD & 670 & 43 & $\mathrm{~J}$ & 330 & 39 & $\mathrm{~J}$ & 330 \\
\hline Di-n-butylphthalate & 27 & $\mathrm{JB}$ & 330 & 670 & UD & 670 & 27 & JB & 330 & 39 & $\mathrm{JB}$ & 330 \\
\hline Di-n-octylphthalate & 330 & $\mathrm{U}$ & 330 & 670 & UD & 670 & 330 & $\mathrm{U}$ & 330 & 330 & $\mathrm{U}$ & 330 \\
\hline Dibenz(a,h)anthracene & 330 & $\mathrm{U}$ & 330 & 75 & JD & 670 & 330 & $\mathrm{U}$ & 330 & 330 & $\mathrm{U}$ & 330 \\
\hline Dibenzofuran & 330 & $\mathrm{UJ}$ & 330 & 670 & UDJ & 670 & 330 & $\mathrm{U}$ & 330 & 330 & $\mathrm{U}$ & 330 \\
\hline Diethylphthalate & 330 & $\mathrm{U}$ & 330 & 670 & UD & 670 & 330 & $\mathrm{U}$ & 330 & 330 & $\mathrm{U}$ & 330 \\
\hline Dimethylphthalate & 330 & $\mathrm{U}$ & 330 & 670 & UD & 670 & 330 & $\mathrm{U}$ & 330 & 330 & $\mathrm{U}$ & 330 \\
\hline Fluoranthene & 73 & $\mathrm{JR}$ & 330 & 1400 & DR & 670 & 48 & $\mathrm{~J}$ & 330 & 59 & $\mathrm{~J}$ & 330 \\
\hline Fluorene & 330 & UJ & 330 & 670 & UDJ & 670 & 330 & $\mathrm{U}$ & 330 & 330 & $\mathrm{U}$ & 330 \\
\hline Hexachlorobenzene & 330 & $\mathrm{U}$ & 330 & 670 & UD & 670 & 330 & $\mathrm{U}$ & 330 & 330 & $\mathrm{U}$ & 330 \\
\hline Hexachlorobutadiene & 330 & $\mathrm{U}$ & 330 & 670 & UD & 670 & 330 & $\mathrm{U}$ & 330 & 330 & $\mathrm{U}$ & 330 \\
\hline Hexachlorocyclopentadiene & 330 & $\mathrm{U}$ & 330 & 670 & UD & 670 & 330 & $\mathrm{U}$ & 330 & 330 & $\mathrm{U}$ & 330 \\
\hline Hexachloroethane & 330 & $\mathrm{UJ}$ & 330 & 670 & UDJ & 670 & 330 & $\mathrm{U}$ & 330 & 330 & $\mathrm{U}$ & 330 \\
\hline Indeno(1,2,3-cd)pyrene & 330 & UJ & 330 & 150 & JD & 670 & 330 & $\mathrm{U}$ & 330 & 330 & $\mathrm{U}$ & 330 \\
\hline Isophorone & 330 & $\mathrm{UJ}$ & 330 & 670 & UDJ & 670 & 330 & UJ & 330 & 330 & UJ & 330 \\
\hline N-Nitroso-di-n-dipropylamine & 330 & UJ & 330 & 670 & UDJ & 670 & 330 & $\mathrm{U}$ & 330 & 330 & U & 330 \\
\hline N-Nitrosodiphenylamine & 330 & $\mathrm{U}$ & 330 & 670 & UD & 670 & 330 & $\mathrm{U}$ & 330 & 330 & $\mathrm{U}$ & 330 \\
\hline Naphthalene & 330 & $\mathrm{U}$ & 330 & 670 & UD & 670 & 330 & $\mathrm{U}$ & 330 & 330 & $\mathrm{U}$ & 330 \\
\hline Nitrobenzene & 330 & UJ & 330 & 670 & UDJ & 670 & 330 & $\mathrm{UJ}$ & 330 & 330 & UJ & 330 \\
\hline Pentachlorophenol & 840 & $\mathrm{U}$ & 840 & 1700 & UD & 1700 & 840 & $\mathrm{U}$ & 840 & 840 & $\mathrm{U}$ & 840 \\
\hline Phenanthrene & 26 & $\mathrm{~J}$ & 330 & 710 & $\mathrm{D}$ & 670 & 330 & $\mathrm{U}$ & 330 & 19 & $\mathrm{~J}$ & 330 \\
\hline Phenol & 330 & $\mathrm{UJ}$ & 330 & 670 & UDJ & 670 & 24 & $\mathrm{~J}$ & 330 & 330 & $\mathrm{U}$ & 330 \\
\hline Pyrene & 60 & $\mathrm{~J}$ & 330 & 1700 & $D$ & 670 & 38 & $\mathrm{~J}$ & 330 & 51 & $\mathrm{~J}$ & 330 \\
\hline
\end{tabular}


118-C-1 Verification Sampling Results.

\begin{tabular}{|c|c|c|c|c|c|c|c|c|c|c|c|c|}
\hline \multirow[t]{2}{*}{ Constituents } & \multicolumn{3}{|c|}{\begin{tabular}{|c|} 
J135P1 \\
SPA A3 \\
Sample Date 9/12/06 \\
\end{tabular}} & \multicolumn{3}{|c|}{$\begin{array}{c}\text { J135P0 } \\
\text { SPA A4 } \\
\text { Sample Date 9/12/06 }\end{array}$} & \multicolumn{3}{|c|}{$\begin{array}{c}\text { J13524 } \\
\text { SPA B5 } \\
\text { Sample Date } 9 / 11 / 06 \\
\end{array}$} & \multicolumn{3}{|c|}{$\begin{array}{c}\text { J13525 } \\
\text { SPA B6 } \\
\text { Sample Date 9/11/06 } \\
\end{array}$} \\
\hline & $\mu \mathrm{g} / \mathrm{kg}$ & Q & PQL & $\mu \mathrm{g} / \mathrm{kg}$ & $\mathbf{Q}$ & PQL & $\mu \mathrm{g} / \mathrm{kg}$ & $Q$ & $\mathrm{PQL}$ & $\mu \mathrm{g} / \mathrm{kg}$ & $Q$ & PQL \\
\hline \multicolumn{13}{|c|}{ Volatile Organic Compounds } \\
\hline 1,1,1-Trichloroethane & 5 & $\mathrm{U}$ & 5 & 5 & $\mathrm{U}$ & 5 & 5 & $\mathrm{U}$ & 5 & 5 & $\mathrm{U}$ & 5 \\
\hline 1,1,2,2-Tetrachloroethane & 5 & $\mathrm{U}$ & 5 & 5 & $\mathrm{U}$ & 5 & 5 & UJ & 5 & 5 & $\mathrm{UJ}$ & 5 \\
\hline 1,1,2-Trichloroethane & 5 & $\mathrm{U}$ & 5 & 5 & $\mathrm{U}$ & 5 & 5 & $\mathrm{U}$ & 5 & 5 & $\mathrm{U}$ & 5 \\
\hline 1,1-Dichloroethane & 5 & $\mathrm{U}$ & 5 & 5 & $\mathrm{U}$ & 5 & 5 & $\mathrm{U}$ & 5 & 5 & $\mathrm{U}$ & 5 \\
\hline 1,1-Dichloroethene & 5 & $\mathrm{U}$ & 5 & 5 & $\mathrm{U}$ & 5 & 5 & $\mathrm{U}$ & 5 & 5 & $\mathrm{U}$ & 5 \\
\hline 1,2-Dichloroethane & 5 & $\mathrm{U}$ & 5 & 5 & $\mathrm{U}$ & 5 & 5 & $\mathrm{U}$ & 5 & 5 & $\mathrm{U}$ & 5 \\
\hline 1,2-Dichloroethene (total) & 5 & $\mathrm{U}$ & 5 & 5 & $\mathrm{U}$ & 5 & 5 & $\mathrm{U}$ & 5 & 5 & $\mathrm{U}$ & 5 \\
\hline 1,2-Dichloropropane & 5 & $\mathrm{U}$ & 5 & 5 & $\mathrm{U}$ & 5 & 5 & $\mathrm{U}$ & 5 & 5 & $\mathrm{U}$ & 5 \\
\hline 2-Butanone & 4 & $\mathrm{~J}$ & 10 & 10 & $\mathrm{U}$ & 10 & 10 & $\mathrm{U}$ & 10 & 10 & $\mathrm{U}$ & 10 \\
\hline 2-Hexanone & 10 & $\mathrm{U}$ & 10 & 10 & $\mathrm{U}$ & 10 & 10 & $\mathrm{U}$ & 10 & 10 & $\mathrm{U}$ & 10 \\
\hline 4-Methyl-2-pentanone & 10 & $\mathrm{U}$ & 10 & 10 & $\mathrm{U}$ & 10 & 10 & $\mathrm{U}$ & 10 & 10 & $\mathrm{U}$ & 10 \\
\hline Acetone & 14 & & 10 & 9 & $\mathrm{~J}$ & 10 & 10 & $\mathrm{U}$ & 10 & 10 & $\mathrm{U}$ & 10 \\
\hline Benzene & 5 & $\mathrm{U}$ & 5 & 5 & $\mathrm{U}$ & 5 & 5 & $\mathrm{U}$ & 5 & 5 & $\mathrm{U}$ & 5 \\
\hline Bromodichloromethane & 5 & $\mathrm{U}$ & 5 & 5 & $\mathrm{U}$ & 5 & 5 & $\mathrm{U}$ & 5 & 5 & $\mathrm{U}$ & 5 \\
\hline Bromoform & 5 & $\mathrm{U}$ & 5 & 5 & $\mathrm{U}$ & 5 & 5 & $\mathrm{U}$ & 5 & 5 & $\mathrm{U}$ & 5 \\
\hline Bromomethane & 10 & $\mathrm{U}$ & 10 & 10 & $\mathrm{U}$ & 10 & 10 & $\mathrm{U}$ & 10 & 10 & $\mathrm{U}$ & 10 \\
\hline Carbon disulfide & 5 & $\mathrm{U}$ & 5 & 5 & $\mathrm{U}$ & 5 & 5 & $U$ & 5 & 5 & $\mathrm{U}$ & 5 \\
\hline Carbon tetrachloride & 5 & $\mathrm{U}$ & 5 & 5 & $\mathrm{U}$ & 5 & 5 & $\mathrm{U}$ & 5 & 5 & $\mathrm{U}$ & 5 \\
\hline Chlorobenzene & 5 & $\mathrm{U}$ & 5 & 5 & $\mathrm{U}$ & 5 & 5 & $\mathrm{U}$ & 5 & 5 & $\mathrm{U}$ & 5 \\
\hline Chloroethane & 10 & $\mathrm{U}$ & 10 & 10 & $\mathrm{U}$ & 10 & 10 & $\mathrm{U}$ & 10 & 10 & $\mathrm{U}$ & 10 \\
\hline Chloroform & 5 & $\mathrm{U}$ & 5 & 5 & $\mathrm{U}$ & 5 & 5 & $\mathrm{U}$ & 5 & 5 & $\mathrm{U}$ & 5 \\
\hline Chloromethane & 10 & $\mathrm{U}$ & 10 & 10 & $\mathrm{U}$ & 10 & 10 & $\mathrm{U}$ & 10 & 10 & $\mathrm{U}$ & 10 \\
\hline cis-1,2-Dichloroethylene & 5 & $\mathrm{U}$ & 5 & 5 & $\mathrm{U}$ & 5 & 5 & $\mathrm{U}$ & 5 & 5 & $\mathrm{U}$ & 5 \\
\hline cis-1,3-Dichloropropene & 5 & $\mathrm{U}$ & 5 & 5 & $\mathrm{U}$ & 5 & 5 & $\mathrm{U}$ & 5 & 5 & $\mathrm{U}$ & 5 \\
\hline Dibromochloromethane & 5 & $\mathrm{U}$ & 5 & 5 & $\mathrm{U}$ & 5 & 5 & $\mathrm{U}$ & 5 & 5 & $\mathrm{U}$ & 5 \\
\hline Ethylbenzene & 5 & $\mathrm{U}$ & 5 & 5 & $\mathrm{U}$ & 5 & 5 & $\mathrm{U}$ & 5 & 5 & $\mathrm{U}$ & 5 \\
\hline Methylene chloride & 23 & $\mathrm{~B}$ & 5 & 17 & $\mathrm{~B}$ & 5 & 11 & $\mathrm{~B}$ & 5 & 14 & $\mathrm{~B}$ & 5 \\
\hline Styrene & 5 & $\mathrm{U}$ & 5 & 5 & $\mathrm{U}$ & 5 & 5 & $\mathrm{U}$ & 5 & 5 & $\mathrm{U}$ & 5 \\
\hline Tetrachloroethene & 5 & $\mathrm{U}$ & 5 & 5 & $\mathrm{U}$ & 5 & 5 & $\mathrm{U}$ & 5 & 5 & $\mathrm{U}$ & 5 \\
\hline \begin{tabular}{|l} 
Toluene \\
\end{tabular} & 2 & $\mathrm{~J}$ & 5 & 5 & $\mathrm{U}$ & 5 & 5 & $\mathrm{U}$ & 5 & 5 & $\mathrm{U}$ & 5 \\
\hline trans-1,2-Dichloroethylene & 5 & $\mathrm{U}$ & 5 & 5 & $\mathrm{U}$ & 5 & 5 & $\mathrm{U}$ & 5 & 5 & $\mathrm{U}$ & 5 \\
\hline trans-1,3-Dichloropropene & 5 & $\mathrm{U}$ & 5 & 5 & $\mathrm{U}$ & 5 & 5 & $\mathrm{U}$ & 5 & 5 & $\mathrm{U}$ & 5 \\
\hline Trichloroethene & 5 & $\mathrm{U}$ & 5 & 5 & $\mathrm{U}$ & 5 & 5 & $\mathrm{U}$ & 5 & 5 & $\mathrm{U}$ & 5 \\
\hline \begin{tabular}{|l} 
Vinyl chloride \\
\end{tabular} & 10 & $\mathrm{U}$ & 10 & 10 & $\mathrm{U}$ & 10 & 10 & $\mathrm{U}$ & 10 & 10 & $\mathrm{U}$ & 10 \\
\hline Xylenes (total) & 5 & $\mathrm{U}$ & 5 & 5 & $\mathrm{U}$ & 5 & 5 & $\mathrm{U}$ & 5 & 5 & $\mathrm{U}$ & 5 \\
\hline
\end{tabular}


CVP-2006-00011

Rev. 0

118-C-1 Verification Sampling Results.

\begin{tabular}{|c|c|c|c|c|c|c|c|c|c|c|c|c|}
\hline \multirow[t]{2}{*}{ Constituents } & \multicolumn{3}{|c|}{\begin{tabular}{|c|} 
J135N8 \\
SPA B7 \\
Sample Date 9/11/06 \\
\end{tabular}} & \multicolumn{3}{|c|}{$\begin{array}{c}\text { J135N9 } \\
\text { SPA B8 } \\
\text { Sample Date 9/11/06 } \\
\end{array}$} & \multicolumn{3}{|c|}{$\begin{array}{c}\text { J135P2 } \\
\text { SPA C9 } \\
\text { Sample Date 9/12/06 } \\
\end{array}$} & \multicolumn{3}{|c|}{$\begin{array}{c}\text { J135P3 } \\
\text { SPA C10 } \\
\text { Sample Date 9/12/06 } \\
\end{array}$} \\
\hline & $\mu \mathrm{g} / \mathrm{kg}$ & $\mathbf{Q}$ & PQL & $\mu \mathrm{g} / \mathrm{kg}$ & $\mathbf{Q}$ & PQL & $\mu \mathrm{g} / \mathrm{kg}$ & $Q$ & PQL & $\mu \mathrm{g} / \mathrm{kg}$ & $\mathbf{Q}$ & PQL \\
\hline \multicolumn{13}{|c|}{ Polychlorinated Biphenyls } \\
\hline Aroclor-1016 & 13 & $\mathrm{U}$ & 13 & 13 & $U$ & 13 & 13 & $\mathrm{U}$ & 13 & 13 & $\mathrm{U}$ & 13 \\
\hline Aroclor-1221 & 13 & $\mathrm{U}$ & 13 & 13 & $U$ & 13 & 13 & $\mathrm{U}$ & 13 & 13 & $\mathrm{U}$ & 13 \\
\hline Aroclor-1232 & 13 & $\mathrm{U}$ & 13 & 13 & $\mathrm{U}$ & 13 & 13 & $\mathrm{U}$ & 13 & 13 & $\mathrm{U}$ & 13 \\
\hline Aroclor-1242 & 13 & $\mathrm{U}$ & 13 & 13 & $\mathrm{U}$ & 13 & 13 & $\mathrm{U}$ & 13 & 13 & $\mathrm{U}$ & 13 \\
\hline Aroclor-1248 & 13 & $\mathrm{U}$ & 13 & 13 & $\bar{U}$ & 13 & 13 & $\mathrm{U}$ & 13 & 13 & $\mathrm{U}$ & 13 \\
\hline Aroclor-1254 & 9.5 & $\mathrm{~J}$ & 13 & 19 & & 13 & 17 & & 13 & 6.5 & $\mathrm{~J}$ & 13 \\
\hline Aroclor- 1260 & 13 & $\mathrm{U}$ & 13 & 13 & $\mathrm{U}$ & 13 & 13 & $\mathrm{U}$ & 13 & 13 & $\mathrm{U}$ & 13 \\
\hline \multicolumn{13}{|c|}{ Semivolatile Organic Compounds } \\
\hline 1,2,4-Trichlorobenzene & 330 & UJ & 330 & 330 & UJ & 330 & 330 & UJ & 330 & 330 & UJ & 330 \\
\hline 1,2-Dichlorobenzene & 330 & $\mathrm{U}$ & 330 & 330 & $\mathrm{U}$ & 330 & 330 & UJ & 330 & 330 & UJ & 330 \\
\hline 1,3-Dichlorobenzene & 330 & $\mathrm{U}$ & 330 & 330 & $\mathrm{U}$ & 330 & 330 & UJ & 330 & 330 & UJ & 330 \\
\hline 1,4-Dichlorobenzene & 330 & $\mathrm{U}$ & 330 & 330 & $\mathrm{U}$ & 330 & 330 & UJ & 330 & 330 & UJ & 330 \\
\hline 2,4,5-Trichlorophenol & 840 & $\mathrm{U}$ & 840 & 840 & $\mathrm{U}$ & 840 & 840 & $\mathrm{U}$ & 840 & 840 & $\mathrm{U}$ & 840 \\
\hline 2,4,6-Trichlorophenol & 330 & $\mathrm{U}$ & 330 & 330 & $\mathrm{U}$ & 330 & 330 & $\mathrm{U}$ & 330 & 330 & $\mathrm{U}$ & 330 \\
\hline 2,4-Dichlorophenol & 330 & $\mathrm{UJ}$ & 330 & 330 & UJ & 330 & 330 & $\mathrm{U}$ & 330 & 330 & $\mathrm{U}$ & 330 \\
\hline 2,4-Dimethylphenol & 330 & UJ & 330 & 330 & UJ & 330 & 330 & UJ & 330 & 330 & $\mathrm{UJ}$ & 330 \\
\hline 2,4-Dinitrophenol & 840 & UR & 840 & 840 & UR & 840 & 840 & UJ & 840 & 840 & $\mathrm{UJ}$ & 840 \\
\hline 2,4-Dinitrotoluene & 330 & $\mathrm{U}$ & 330 & 330 & $\mathrm{U}$ & 330 & 330 & $\mathrm{U}$ & 330 & 330 & $\mathrm{U}$ & 330 \\
\hline 2,6-Dinitrotoluene & 330 & $\mathrm{U}$ & 330 & 330 & $\mathrm{U}$ & 330 & 330 & $\mathrm{U}$ & 330 & 330 & $\mathrm{U}$ & 330 \\
\hline 2-Chloronaphthalene & 330 & $\mathrm{U}$ & 330 & 330 & $\mathrm{U}$ & 330 & 330 & $\mathrm{U}$ & 330 & 330 & $\mathrm{U}$ & 330 \\
\hline 2-Chlorophenol & 330 & $\mathrm{U}$ & 330 & 330 & $\mathrm{U}$ & 330 & 330 & UJ & 330 & 330 & UJ & 330 \\
\hline 2-Methylnaphthalene & 330 & UJ & 330 & 330 & UJ & 330 & 330 & UJ & 330 & 330 & $\mathrm{UJ}$ & 330 \\
\hline 2-Methylphenol (cresol, o-) & 330 & UJ & 330 & 330 & UJ & 330 & 330 & UJ & 330 & 330 & UJ & 330 \\
\hline 2-Nitroaniline & 840 & $\mathrm{U}$ & 840 & 840 & $\mathrm{U}$ & 840 & 840 & $\mathrm{U}$ & 840 & 840 & $\mathrm{U}$ & 840 \\
\hline 2-Nitrophenol & 330 & $\mathrm{UJ}$ & 330 & 330 & UJ & 330 & 330 & UJ & 330 & 330 & UJ & 330 \\
\hline 3,3'-Dichlorobenzidine & 330 & $\mathrm{U}$ & 330 & 330 & $\mathrm{U}$ & 330 & 330 & $\mathrm{U}$ & 330 & 330 & $\mathrm{U}$ & 330 \\
\hline 3-Nitroaniline & 840 & $\mathrm{U}$ & 840 & 840 & $\mathrm{U}$ & 840 & 840 & $\mathrm{U}$ & 840 & 840 & $\mathrm{U}$ & 840 \\
\hline 4,6-Dinitro-2-methylphenol & 840 & $\mathrm{U}$ & 840 & 840 & $\mathrm{U}$ & 840 & 840 & $\mathrm{U}$ & 840 & 840 & $\mathrm{U}$ & 840 \\
\hline 4-Bromophenyl-phenylether & 330 & $\mathrm{U}$ & 330 & 330 & $\mathrm{U}$ & 330 & 330 & $\mathrm{U}$ & 330 & 330 & $\mathrm{U}$ & 330 \\
\hline 4-Chloro-3-methylphenol & 330 & UJ & 330 & 330 & UJ & 330 & 330 & UJ & 330 & 330 & UJ & 330 \\
\hline 4-Chloroaniline & 330 & $\mathrm{U}$ & 330 & 330 & $\mathrm{U}$ & 330 & 330 & $\mathrm{U}$ & 330 & 330 & $\mathrm{U}$ & 330 \\
\hline 4-Chlorophenyl-phenylether & 330 & $\mathrm{U}$ & 330 & 330 & $\mathrm{U}$ & 330 & 330 & $\mathrm{U}$ & 330 & 330 & $\mathrm{U}$ & 330 \\
\hline 4-Methylphenol (p-cresol) & 330 & $\mathrm{UJ}$ & 330 & 330 & UJ & 330 & 330 & UJ & 330 & 330 & $\mathrm{UJ}$ & 330 \\
\hline 4-Nitroaniline & 840 & $\mathrm{U}$ & 840 & 840 & $\mathrm{U}$ & 840 & 840 & $\mathrm{U}$ & 840 & 840 & $\mathrm{U}$ & 840 \\
\hline 4-Nitrophenol & 840 & $\mathrm{U}$ & 840 & 840 & $\mathrm{U}$ & 840 & 840 & $\mathrm{U}$ & 840 & 840 & $\mathrm{U}$ & 840 \\
\hline Acenaphthene & 330 & $\mathrm{U}$ & 330 & 330 & $\mathrm{U}$ & 330 & 330 & $\mathrm{U}$ & 330 & 330 & $\mathrm{U}$ & 330 \\
\hline Acenaphthylene & 330 & $\mathrm{U}$ & 330 & 330 & $\mathrm{U}$ & 330 & 330 & $\mathrm{U}$ & 330 & 330 & $\mathrm{U}$ & 330 \\
\hline Anthracene & 330 & $\mathrm{U}$ & 330 & 330 & $\mathrm{U}$ & 330 & 330 & UJ & 330 & 330 & UJ & 330 \\
\hline Benzo(a)anthracene & 330 & $\mathrm{U}$ & 330 & 61 & $\mathrm{~J}$ & 330 & 330 & UJ & 330 & 330 & $\overline{U J}$ & 330 \\
\hline Benzo(a)pyrene & 330 & $\mathrm{U}$ & 330 & 38 & $\mathrm{~J}$ & 330 & 330 & UJ & 330 & 330 & $\mathrm{UJ}$ & 330 \\
\hline Benzo(b)fluoranthene & 330 & $\mathrm{U}$ & 330 & 45 & $\mathrm{~J}$ & 330 & 330 & $\mathrm{UJ}$ & 330 & 330 & $\mathrm{U}$ & 330 \\
\hline Benzo(g,h,i)perylene & 330 & $\mathrm{U}$ & 330 & 22 & $\mathrm{~J}$ & 330 & 330 & $\mathrm{U}$ & 330 & 330 & $\mathrm{U}$ & 330 \\
\hline Benzo(k)fluoranthene & 330 & $\mathrm{U}$ & 330 & 43 & $\mathrm{~J}$ & 330 & 330 & UJ & 330 & 330 & UJ & 330 \\
\hline bis(2-Chloro-1-methylethyl)ether & 330 & $\mathrm{U}$ & 330 & 330 & $\mathrm{U}$ & 330 & 330 & $\mathrm{U}$ & 330 & 330 & $\mathrm{U}$ & 330 \\
\hline bis(2-Chloroethoxy)methane & 330 & $\mathrm{U}$ & 330 & 330 & $\mathrm{U}$ & 330 & 330 & $\mathrm{U}$ & 330 & 330 & $\mathrm{U}$ & 330 \\
\hline bis(2-Chloroethyl)ether & 330 & $\mathrm{U}$ & 330 & 330 & $\mathrm{U}$ & 330 & 330 & $\mathrm{U}$ & 330 & 330 & $\mathrm{U}$ & 330 \\
\hline bis(2-Ethylhexyl)phthalate & 36 & $\mathrm{JB}$ & 330 & 35 & $\mathrm{JB}$ & 330 & 30 & $\mathrm{JB}$ & 330 & 48 & $\mathrm{JB}$ & 330 \\
\hline
\end{tabular}


Rev. 0

118-C-1 Verification Sampling Results.

\begin{tabular}{|c|c|c|c|c|c|c|c|c|c|c|c|c|}
\hline \multirow[t]{2}{*}{ Constituents } & \multicolumn{3}{|c|}{$\begin{array}{c}\text { J135N8 } \\
\text { SPA B7 } \\
\text { Sample Date 9/11/06 }\end{array}$} & \multicolumn{3}{|c|}{$\begin{array}{c}\text { J135N9 } \\
\text { SPA B8 } \\
\text { Sample Date } 9 / 11 / 06\end{array}$} & \multicolumn{3}{|c|}{$\begin{array}{c}\text { J135P2 } \\
\text { SPA C9 } \\
\text { Sample Date } 9 / 12 / 06\end{array}$} & \multicolumn{3}{|c|}{$\begin{array}{c}\text { J135P3 } \\
\text { SPA C10 } \\
\text { Sample Date 9/12/06 }\end{array}$} \\
\hline & $\mu \mathrm{g} / \mathrm{kg}$ & $Q$ & PQL & $\mu \mathrm{g} / \mathrm{kg}$ & Q & PQL & $\mu \mathrm{g} / \mathrm{kg}$ & Q & PQL & $\mu \mathrm{g} / \mathrm{kg}$ & $Q$ & PQL \\
\hline \multicolumn{13}{|c|}{ Semivolatile Organic Compounds (continued) } \\
\hline Butylbenzylphthalate & 330 & $\mathrm{U}$ & 330 & 330 & $\bar{U}$ & 330 & 330 & $\bar{U}$ & 330 & 330 & $\bar{U}$ & 330 \\
\hline Carbazole & 330 & $\mathrm{U}$ & 330 & 330 & $\mathrm{U}$ & 330 & 330 & UJ & 330 & 330 & UJ & 330 \\
\hline Chrysene & 330 & $\mathrm{U}$ & 330 & 81 & $\mathrm{~J}$ & 330 & 330 & $\overline{\mathrm{UJ}}$ & 330 & 330 & UJ & 330 \\
\hline Di-n-butylphthalate & 32 & JB & 330 & 330 & $\mathrm{U}$ & 330 & 19 & $\mathrm{JB}$ & 330 & 17 & $\mathrm{JB}$ & 330 \\
\hline Di-n-octylphthalate & 330 & $\mathrm{U}$ & 330 & 330 & $\mathrm{U}$ & 330 & 330 & $\mathrm{U}$ & 330 & 330 & $\mathrm{U}$ & 330 \\
\hline Dibenz(a,h)anthracene & 330 & $\mathrm{U}$ & 330 & 330 & $\bar{U}$ & 330 & 330 & $\bar{U}$ & 330 & 330 & $\mathrm{U}$ & 330 \\
\hline Dibenzofuran & 330 & $\mathrm{U}$ & 330 & 330 & $\mathrm{U}$ & 330 & 330 & $\mathrm{UJ}$ & 330 & 330 & UJ & 330 \\
\hline Diethylphthalate & 330 & $\mathrm{U}$ & 330 & 330 & $\mathrm{U}$ & 330 & 330 & $\mathrm{U}$ & 330 & 330 & $\mathrm{U}$ & 330 \\
\hline Dimethylphthalate & 330 & $\mathrm{U}$ & 330 & 330 & $\bar{U}$ & 330 & 330 & $\mathrm{U}$ & 330 & 330 & $U$ & 330 \\
\hline Fluoranthene & 24 & $\mathrm{~J}$ & 330 & 190 & $\mathrm{~J}$ & 330 & 330 & $\mathrm{U}$ & 330 & 330 & $\mathrm{U}$ & 330 \\
\hline Fluorene & 330 & $\mathrm{U}$ & 330 & 330 & $\bar{U}$ & 330 & 330 & $\overline{\mathrm{UJ}}$ & 330 & 330 & UJ & 330 \\
\hline Hexachlorobenzene & 330 & $\mathrm{U}$ & 330 & 330 & $\mathrm{U}$ & 330 & 330 & $\mathrm{U}$ & 330 & 330 & $U$ & 330 \\
\hline Hexachlorobutadiene & 330 & $\mathrm{U}$ & 330 & 330 & $\mathrm{U}$ & 330 & 330 & $\mathrm{U}$ & 330 & 330 & $\mathrm{U}$ & 330 \\
\hline Hexachlorocyclopentadiene & 330 & $\mathrm{U}$ & 330 & 330 & $\mathrm{U}$ & 330 & 330 & $\mathrm{U}$ & 330 & 330 & $\bar{U}$ & 330 \\
\hline Hexachloroethane & 330 & $\mathrm{U}$ & 330 & 330 & $\mathrm{U}$ & 330 & 330 & UJ & 330 & 330 & UJ & 330 \\
\hline Indeno( $1,2,3-\mathrm{cd})$ pyrene & 330 & $\mathrm{U}$ & 330 & 19 & $\mathrm{~J}$ & 330 & 330 & UJ & 330 & 330 & UJ & 330 \\
\hline Isophorone & 330 & $\overline{\mathrm{UJ}}$ & 330 & 330 & $\overline{\mathrm{UJ}}$ & 330 & 330 & $\overline{\mathrm{UJ}}$ & 330 & 330 & UJ & 330 \\
\hline N-Nitroso-di-n-dipropylamine & 330 & $\mathrm{U}$ & 330 & 330 & $\mathrm{U}$ & 330 & 330 & UJ & 330 & 330 & UJ & 330 \\
\hline N-Nitrosodiphenylamine & 330 & $\mathrm{U}$ & 330 & 330 & $\mathrm{U}$ & 330 & 330 & $\mathrm{U}$ & 330 & 330 & $U$ & 330 \\
\hline Naphthalene & 330 & $\bar{U}$ & 330 & 330 & $\mathrm{U}$ & 330 & 330 & $\bar{U}$ & 330 & 330 & $\bar{U}$ & 330 \\
\hline Nitrobenzene & 330 & $\mathrm{UJ}$ & 330 & 330 & $\mathrm{UJ}$ & 330 & 330 & UJ & 330 & 330 & UJ & 330 \\
\hline Pentachlorophenol & 840 & $\mathrm{U}$ & 840 & 840 & $\mathrm{U}$ & 840 & 840 & $\mathrm{U}$ & 840 & 840 & $U$ & 840 \\
\hline Phenanthrene & 330 & $\mathrm{U}$ & 330 & 71 & $\mathrm{~J}$ & 330 & 330 & UJ & 330 & 330 & UJ & 330 \\
\hline Phenol & 330 & $\mathrm{U}$ & 330 & 330 & $\mathrm{U}$ & 330 & 330 & $\mathrm{U}$ & 330 & 330 & $U$ & 330 \\
\hline Pyrene & 21 & $\mathrm{~J}$ & 330 & 130 & $\mathrm{~J}$ & 330 & 330 & $\mathrm{U}$ & 330 & 330 & $U$ & 330 \\
\hline
\end{tabular}


CVP-2006-00011

Rev. 0

118-C-1 Verification Sampling Results.

\begin{tabular}{|c|c|c|c|c|c|c|c|c|c|c|c|c|}
\hline \multirow[t]{2}{*}{ Constituents } & \multicolumn{3}{|c|}{\begin{tabular}{|c|} 
J135N8 \\
SPA B7 \\
Sample Date 9/11/06 \\
\end{tabular}} & \multicolumn{3}{|c|}{$\begin{array}{c}\text { J135N9 } \\
\text { SPA B8 } \\
\text { Sample Date 9/11/06 }\end{array}$} & \multicolumn{3}{|c|}{$\begin{array}{c}\text { J135P2 } \\
\text { SPA C9 } \\
\text { Sample Date 9/12/06 } \\
\end{array}$} & \multicolumn{3}{|c|}{$\begin{array}{c}\text { J135P3 } \\
\text { SPA C10 } \\
\text { Sample Date 9/12/06 } \\
\end{array}$} \\
\hline & $\mu \mathrm{g} / \mathrm{kg}$ & $Q$ & PQL & $\mu \mathrm{g} / \mathrm{kg}$ & $Q$ & PQL & $\mu \mathrm{g} / \mathrm{kg}$ & $\mathbf{Q}$ & PQL & $\mu \mathrm{g} / \mathrm{kg}$ & $\mathbf{Q}$ & PQL \\
\hline \multicolumn{13}{|c|}{ Volatile Organic Compounds } \\
\hline 1,1,1-Trichloroethane & 5 & $\mathrm{U}$ & 5 & 5 & $\mathrm{U}$ & 5 & 5 & $\mathrm{U}$ & 5 & 5 & $\mathrm{U}$ & 5 \\
\hline $1,1,2,2$-Tetrachloroethane & 5 & $\mathrm{UJ}$ & 5 & 5 & UJ & 5 & 5 & $\mathrm{U}$ & 5 & 5 & $\mathrm{U}$ & 5 \\
\hline 1,1,2-Trichloroethane & 5 & $\mathrm{U}$ & 5 & 5 & $\mathrm{U}$ & 5 & 5 & $\mathrm{U}$ & 5 & 5 & $\mathrm{U}$ & 5 \\
\hline 1,1-Dichloroethane & 5 & $\mathrm{U}$ & 5 & 5 & $\mathrm{U}$ & 5 & 5 & $\mathrm{U}$ & 5 & 5 & $\mathrm{U}$ & 5 \\
\hline 1,1-Dichloroethene & 5 & $\mathrm{U}$ & 5 & 5 & $\mathrm{U}$ & 5 & 5 & $\mathrm{U}$ & 5 & 5 & $\mathrm{U}$ & 5 \\
\hline 1,2-Dichloroethane & 5 & $\mathrm{U}$ & $\overline{5}$ & 5 & $\mathrm{U}$ & 5 & 5 & $\mathrm{U}$ & 5 & 5 & $\mathrm{U}$ & 5 \\
\hline 1,2-Dichloroethene (total) & 5 & $\mathrm{U}$ & 5 & 5 & $\mathrm{U}$ & 5 & 5 & $\mathrm{U}$ & 5 & 5 & $\mathrm{U}$ & 5 \\
\hline 1,2-Dichloropropane & 5 & $\mathrm{U}$ & 5 & 5 & $\mathrm{U}$ & 5 & 5 & $\mathrm{U}$ & 5 & 5 & $\mathrm{U}$ & 5 \\
\hline 2-Butanone & 10 & $\mathrm{U}$ & 10 & 10 & $\mathrm{U}$ & 10 & 4 & $\mathrm{~J}$ & 10 & 10 & $\mathrm{U}$ & 10 \\
\hline 2-Hexanone & 10 & $\mathrm{U}$ & 10 & 10 & $\mathrm{U}$ & 10 & 10 & $\mathrm{U}$ & 10 & 10 & $\mathrm{U}$ & 10 \\
\hline 4-Methyl-2-pentanone & 10 & $\mathrm{U}$ & 10 & 10 & $\mathrm{U}$ & 10 & 10 & $\mathrm{U}$ & 10 & 10 & $\mathrm{U}$ & 10 \\
\hline Acetone & 25 & $\mathrm{~J}$ & 10 & 28 & $\mathrm{~J}$ & 10 & 12 & & 10 & 7 & $\mathrm{~J}$ & 10 \\
\hline Benzene & 5 & $\mathrm{U}$ & 5 & 5 & $\mathrm{U}$ & 5 & 5 & $\mathrm{U}$ & 5 & 5 & $\mathrm{U}$ & 5 \\
\hline Bromodichloromethane & 5 & $\mathrm{U}$ & 5 & 5 & $\mathrm{U}$ & 5 & 5 & $U$ & 5 & 5 & $\mathrm{U}$ & 5 \\
\hline Bromoform & 5 & $\mathrm{U}$ & 5 & 5 & $\mathrm{U}$ & 5 & 5 & $U$ & 5 & 5 & $\mathrm{U}$ & 5 \\
\hline Bromomethane & 10 & $\mathrm{U}$ & 10 & 10 & $\mathrm{U}$ & 10 & 10 & $\mathrm{U}$ & 10 & 10 & $\mathrm{U}$ & 10 \\
\hline Carbon disulfide & 5 & $\mathrm{U}$ & 5 & 5 & $\mathrm{U}$ & 5 & 5 & $\mathrm{U}$ & 5 & 5 & $\mathrm{U}$ & 5 \\
\hline Carbon tetrachloride & 5 & $\mathrm{U}$ & 5 & 5 & $\mathrm{U}$ & 5 & 5 & $\mathrm{U}$ & 5 & 5 & $\mathrm{U}$ & 5 \\
\hline Chlorobenzene & 5 & $\mathrm{U}$ & 5 & 5 & $\mathrm{U}$ & 5 & 5 & $\mathrm{U}$ & 5 & 5 & $\mathrm{U}$ & 5 \\
\hline Chloroethane & 10 & $\mathrm{U}$ & 10 & 10 & $\mathrm{U}$ & 10 & 10 & $\mathrm{U}$ & 10 & 10 & $\mathrm{U}$ & 10 \\
\hline Chloroform & 5 & $\mathrm{U}$ & 5 & 5 & $\mathrm{U}$ & 5 & 5 & $\mathrm{U}$ & 5 & 5 & $\mathrm{U}$ & 5 \\
\hline Chloromethane & 10 & $\mathrm{U}$ & 10 & 10 & $\mathrm{U}$ & 10 & 10 & $\mathrm{U}$ & 10 & 10 & $\mathrm{U}$ & 10 \\
\hline cis-1,2-Dichloroethylene & 5 & $\mathrm{U}$ & 5 & 5 & $\mathrm{U}$ & 5 & 5 & $\mathrm{U}$ & 5 & 5 & $\mathrm{U}$ & 5 \\
\hline cis-1,3-Dichloropropene & 5 & $\mathrm{U}$ & 5 & 5 & $\mathrm{U}$ & 5 & 5 & $\mathrm{U}$ & 5 & 5 & $\mathrm{U}$ & 5 \\
\hline Dibromochloromethane & 5 & $\mathrm{U}$ & 5 & 5 & $\mathrm{U}$ & 5 & 5 & $\mathrm{U}$ & 5 & 5 & $\mathrm{U}$ & 5 \\
\hline Ethylbenzene & 5 & $\mathrm{U}$ & 5 & 5 & $\mathrm{U}$ & 5 & 5 & $\mathrm{U}$ & 5 & 5 & $\mathrm{U}$ & 5 \\
\hline Methylene chloride & 14 & $\mathrm{~B}$ & 5 & 15 & $\mathrm{~B}$ & 5 & 21 & $\mathrm{~B}$ & 5 & 24 & $\mathrm{~B}$ & 5 \\
\hline Styrene & 5 & $\mathrm{U}$ & 5 & 5 & $\mathrm{U}$ & 5 & 5 & $\mathrm{U}$ & 5 & 5 & $\mathrm{U}$ & 5 \\
\hline Tetrachloroethene & 5 & $\mathrm{U}$ & 5 & 5 & $\mathrm{U}$ & 5 & 5 & $\mathrm{U}$ & 5 & 5 & $\mathrm{U}$ & 5 \\
\hline Toluene & 2 & $\mathrm{~J}$ & 5 & 1 & $\mathrm{~J}$ & 5 & 5 & $\mathrm{U}$ & 5 & 1 & $\mathrm{~J}$ & 5 \\
\hline trans-1,2-Dichloroethylene & 5 & $\mathrm{U}$ & 5 & 5 & $U$ & 5 & 5 & $U$ & 5 & 5 & $\mathrm{U}$ & 5 \\
\hline trans-1,3-Dichloropropene & 5 & $\mathrm{U}$ & 5 & 5 & $\mathrm{U}$ & 5 & 5 & $\mathrm{U}$ & 5 & 5 & $\mathrm{U}$ & 5 \\
\hline Trichloroethene & 5 & $\mathrm{U}$ & 5 & 5 & $U$ & 5 & 5 & $\mathrm{U}$ & 5 & 5 & $\mathrm{U}$ & 5 \\
\hline Vinyl chloride & 10 & $\mathrm{U}$ & 10 & 10 & $\mathrm{U}$ & 10 & 10 & $\bar{U}$ & 10 & 10 & $\mathrm{U}$ & 10 \\
\hline Xylenes (total) & 5 & $\mathrm{U}$ & 5 & 5 & $\mathrm{U}$ & 5 & 5 & $\mathrm{U}$ & 5 & 5 & $\mathrm{U}$ & 5 \\
\hline
\end{tabular}


CVP-2006-00011

Rev. 0

118-C-1 Verification Sampling Results.

\begin{tabular}{|c|c|c|c|c|c|c|c|c|c|c|c|c|}
\hline \multirow[t]{2}{*}{ Constituents } & \multicolumn{3}{|c|}{$\begin{array}{c}\text { J135P4 } \\
\text { SPA C1 } \\
\text { Sample Date 9/12/06 }\end{array}$} & \multicolumn{3}{|c|}{$\begin{array}{c}\text { J135P5 } \\
\text { SPA C2 } \\
\text { Sample Date 9/12/06 }\end{array}$} & \multicolumn{3}{|c|}{\begin{tabular}{|c|} 
J135P6 \\
SPA D3 \\
Sample Date 9/12/06 \\
\end{tabular}} & \multicolumn{3}{|c|}{$\begin{array}{c}\text { J135P7 } \\
\text { SPA D4 } \\
\text { Sample Date 9/12/06 } \\
\end{array}$} \\
\hline & $\mu \mathrm{g} / \mathrm{kg}$ & $Q$ & PQL & $\mu \mathrm{g} / \mathrm{kg}$ & $\mathbf{Q}$ & PQL & $\mu \mathrm{g} / \mathrm{kg}$ & $\mathbf{Q}$ & PQL & $\mu \mathrm{g} / \mathrm{kg}$ & $Q$ & PQL \\
\hline \multicolumn{13}{|c|}{ Polychlorinated Biphenyls } \\
\hline Aroclor-1016 & 13 & $\mathrm{U}$ & 13 & 13 & $\mathrm{U}$ & 13 & 13 & $\mathrm{U}$ & 13 & 13 & $\mathrm{U}$ & 13 \\
\hline Aroclor-1221 & 13 & $\mathrm{U}$ & 13 & 13 & $\mathrm{U}$ & 13 & 13 & $\mathrm{U}$ & 13 & 13 & $\mathrm{U}$ & 13 \\
\hline Aroclor-1232 & 13 & $\mathrm{U}$ & 13 & 13 & $\mathrm{U}$ & 13 & 13 & $\mathrm{U}$ & 13 & 13 & $\mathrm{U}$ & 13 \\
\hline Aroclor-1242 & 13 & $\mathrm{U}$ & 13 & 13 & $\mathrm{U}$ & 13 & 13 & $\mathrm{U}$ & 13 & 13 & $\mathrm{U}$ & 13 \\
\hline Aroclor-1248 & 13 & $\mathrm{U}$ & 13 & 13 & $\mathrm{U}$ & 13 & 13 & $\mathrm{U}$ & 13 & 13 & $\mathrm{U}$ & 13 \\
\hline Aroclor-1254 & 14 & & 13 & 25 & & 13 & 20 & & 13 & 7.9 & $\mathrm{~J}$ & 13 \\
\hline Aroclor- 1260 & 13 & $\mathrm{U}$ & 13 & 13 & $\mathrm{U}$ & 13 & 13 & $\mathrm{U}$ & 13 & 13 & $\mathrm{U}$ & 13 \\
\hline \multicolumn{13}{|c|}{ Semivolatile Organic Compounds } \\
\hline 1,2,4-Trichlorobenzene & 330 & UJ & 330 & 330 & UJ & 330 & 330 & UJ & 330 & 330 & UJ & 330 \\
\hline 1,2-Dichlorobenzene & 330 & UJ & 330 & 330 & UJ & 330 & 330 & UJ & 330 & 330 & UJ & 330 \\
\hline 1,3-Dichlorobenzene & 330 & UJ & 330 & 330 & UJ & 330 & 330 & UJ & 330 & 330 & UJ & 330 \\
\hline 1,4-Dichlorobenzene & 330 & UJ & 330 & 330 & UJ & 330 & 330 & UJ & 330 & 330 & UJ & 330 \\
\hline 2,4,5-Trichlorophenol & 840 & $\mathrm{U}$ & 840 & 840 & $\mathrm{U}$ & 840 & 840 & $\mathrm{U}$ & 840 & 840 & $\mathrm{U}$ & 840 \\
\hline 2,4,6-Trichlorophenol & 330 & $\mathrm{U}$ & 330 & 330 & $\mathrm{U}$ & 330 & 330 & $\mathrm{U}$ & 330 & 330 & $\mathrm{U}$ & 330 \\
\hline 2,4-Dichlorophenol & 330 & $\mathrm{U}$ & 330 & 330 & $\mathrm{U}$ & 330 & 330 & $\mathrm{U}$ & 330 & 330 & $\mathrm{U}$ & 330 \\
\hline 2,4-Dimethylphenol & 330 & UJ & 330 & 330 & UJ & 330 & 330 & UJ & 330 & 330 & UJ & 330 \\
\hline 2,4-Dinitrophenol & 840 & UJ & 840 & 840 & $\mathrm{UJ}$ & 840 & 840 & UJ & 840 & 840 & UJ & 840 \\
\hline 2,4-Dinitrotoluene & 330 & $\mathrm{U}$ & 330 & 330 & $\mathrm{U}$ & 330 & 330 & $\mathrm{U}$ & 330 & 330 & $\mathrm{U}$ & 330 \\
\hline 2,6-Dinitrotoluene & 330 & $\mathrm{U}$ & 330 & 330 & $\mathrm{U}$ & 330 & 330 & $\mathrm{U}$ & 330 & 330 & $\mathrm{U}$ & 330 \\
\hline 2-Chloronaphthalene & 330 & $\mathrm{U}$ & 330 & 330 & $\mathrm{U}$ & 330 & 330 & $\mathrm{U}$ & 330 & 330 & $\mathrm{U}$ & 330 \\
\hline 2-Chlorophenol & 330 & UJ & 330 & 330 & UJ & 330 & 330 & UJ & 330 & 330 & UJ & 330 \\
\hline 2-Methylnaphthalene & 330 & UJ & 330 & 330 & UJ & 330 & 330 & $\mathrm{UJ}$ & 330 & 330 & UJ & 330 \\
\hline 2-Methylphenol (cresol, o-) & 330 & UJ & 330 & 330 & UJ & 330 & 330 & UJ & 330 & 330 & UJ & 330 \\
\hline 2-Nitroaniline & 840 & $\mathrm{U}$ & 840 & 840 & $\mathrm{U}$ & 840 & 840 & $\mathrm{U}$ & 840 & 840 & $\mathrm{U}$ & 840 \\
\hline 2-Nitrophenol & 330 & UJ & 330 & 330 & UJ & 330 & 330 & UJ & 330 & 330 & UJ & 330 \\
\hline 3,3'-Dichlorobenzidine & 330 & $\mathrm{U}$ & 330 & 330 & $\mathrm{U}$ & 330 & 330 & $\mathrm{U}$ & 330 & 330 & $\mathrm{U}$ & 330 \\
\hline 3-Nitroaniline & 840 & $\mathrm{U}$ & 840 & 840 & $\mathrm{U}$ & 840 & 840 & $\mathrm{U}$ & 840 & 840 & $\mathrm{U}$ & 840 \\
\hline 4,6-Dinitro-2-methylphenol & 840 & $\mathrm{U}$ & 840 & 840 & $\bar{U}$ & 840 & 840 & $\mathrm{U}$ & 840 & 840 & $\mathrm{U}$ & 840 \\
\hline 4-Bromophenyl-phenylether & 330 & $\mathrm{U}$ & 330 & 330 & $\mathrm{U}$ & 330 & 330 & $\mathrm{U}$ & 330 & 330 & $\mathrm{U}$ & 330 \\
\hline 4-Chloro-3-methylphenol & 330 & UJ & 330 & 330 & UJ & 330 & 330 & UJ & 330 & 330 & UJ & 330 \\
\hline 4-Chloroaniline & 330 & $\mathrm{U}$ & 330 & 330 & $\mathrm{U}$ & 330 & 330 & $\mathrm{U}$ & 330 & 330 & $\mathrm{U}$ & 330 \\
\hline 4-Chlorophenyl-phenylether & 330 & $\mathrm{U}$ & 330 & 330 & $\mathrm{U}$ & 330 & 330 & $\mathrm{U}$ & 330 & 330 & $\mathrm{U}$ & 330 \\
\hline 4-Methylphenol (p-cresol) & 330 & UJ & 330 & 330 & $\mathrm{UJ}$ & 330 & 330 & UJ & 330 & 330 & UJ & 330 \\
\hline 4-Nitroaniline & 840 & $\mathrm{U}$ & 840 & 840 & $\mathrm{U}$ & 840 & 840 & $\mathrm{U}$ & 840 & 840 & $\mathrm{U}$ & 840 \\
\hline 4-Nitrophenol & 840 & $\mathrm{U}$ & 840 & 840 & $\mathrm{U}$ & 840 & 840 & $\mathrm{U}$ & 840 & 840 & $\mathrm{U}$ & 840 \\
\hline Acenaphthene & 330 & $\mathrm{U}$ & 330 & 330 & $\mathrm{U}$ & 330 & 330 & $\mathrm{U}$ & 330 & 41 & $\mathrm{~J}$ & 330 \\
\hline Acenaphthylene & 330 & $\mathrm{U}$ & 330 & 330 & $\mathrm{U}$ & 330 & 330 & $\mathrm{U}$ & 330 & 330 & $\mathrm{U}$ & 330 \\
\hline Anthracene & 330 & UJ & 330 & 330 & UJ & 330 & 330 & UJ & 330 & 260 & $\mathrm{~J}$ & 330 \\
\hline Benzo(a)anthracene & 330 & UJ & 330 & 27 & $\mathrm{~J}$ & 330 & 330 & UJ & 330 & 1100 & $\mathrm{~J}$ & 330 \\
\hline Benzo(a)pyrene & 330 & UJ & 330 & 28 & $\mathrm{~J}$ & 330 & 330 & UJ & 330 & 480 & $\mathrm{~J}$ & 330 \\
\hline Benzo(b)fluoranthene & 330 & UJ & 330 & 26 & $\mathrm{~J}$ & 330 & 330 & UJ & 330 & 600 & $\mathrm{~J}$ & 330 \\
\hline Benzo(g,h,i)perylene & 330 & $\mathrm{U}$ & 330 & 21 & $\mathrm{~J}$ & 330 & 330 & $\mathrm{U}$ & 330 & 260 & $J$ & 330 \\
\hline Benzo(k)fluoranthene & 330 & UJ & 330 & 28 & $\mathrm{~J}$ & 330 & 330 & $\mathrm{UJ}$ & 330 & 540 & $\mathrm{~J}$ & 330 \\
\hline bis(2-Chloro-1-methylethyl)ether & 330 & $\mathrm{U}$ & 330 & 330 & $\mathrm{U}$ & 330 & 330 & $\mathrm{U}$ & 330 & 330 & $\mathrm{U}$ & 330 \\
\hline bis(2-Chloroethoxy)methane & 330 & $\mathrm{U}$ & 330 & 330 & $\mathrm{U}$ & 330 & 330 & $\mathrm{U}$ & 330 & 330 & $\mathrm{U}$ & 330 \\
\hline bis(2-Chloroethyl)ether & 330 & $\mathrm{U}$ & 330 & 330 & $\mathrm{U}$ & 330 & 330 & $U$ & 330 & 330 & $U$ & 330 \\
\hline bis(2-Ethylhexyl)phthalate & 23 & $\mathrm{JB}$ & 330 & 66 & $\mathrm{JB}$ & 330 & 56 & $\mathrm{JB}$ & 330 & 27 & $\mathrm{JB}$ & 330 \\
\hline
\end{tabular}


Rev. 0

118-C-1 Verification Sampling Results.

\begin{tabular}{|c|c|c|c|c|c|c|c|c|c|c|c|c|}
\hline \multirow[t]{2}{*}{ Constituents } & \multicolumn{3}{|c|}{\begin{tabular}{|c|} 
J135P4 \\
SPA C1 \\
Sample Date 9/12/06 \\
\end{tabular}} & \multicolumn{3}{|c|}{\begin{tabular}{|c|} 
J135P5 \\
SPA C2 \\
Sample Date 9/12/06 \\
\end{tabular}} & \multicolumn{3}{|c|}{$\begin{array}{c}\text { J135P6 } \\
\text { SPA D3 } \\
\text { Sample Date 9/12/06 }\end{array}$} & \multicolumn{3}{|c|}{$\begin{array}{c}\text { J135P7 } \\
\text { SPA D4 } \\
\text { Sample Date } 9 / 12 / 06\end{array}$} \\
\hline & $\mu \mathrm{g} / \mathrm{kg}$ & Q & PQL & $\mu \mathrm{g} / \mathrm{kg}$ & $\mathbf{Q}$ & PQL & $\mu \mathrm{g} / \mathrm{kg}$ & $\mathbf{Q}$ & PQL & $\mu \mathrm{g} / \mathrm{kg}$ & $Q$ & PQL \\
\hline \multicolumn{13}{|c|}{ Semivolatile Organic Compounds (continued) } \\
\hline Butylbenzylphthalate & 330 & $\mathrm{U}$ & 330 & 330 & $\mathrm{U}$ & 330 & 330 & $\mathrm{U}$ & 330 & 330 & $\mathrm{U}$ & 330 \\
\hline Carbazole & 330 & $\mathrm{UJ}$ & 330 & 330 & UJ & 330 & 330 & UJ & 330 & 240 & $\mathrm{~J}$ & 330 \\
\hline Chrysene & 330 & UJ & 330 & 43 & $\mathrm{~J}$ & 330 & 330 & UJ & 330 & 1300 & $\mathrm{~J}$ & 330 \\
\hline Di-n-butylphthalate & 330 & $\mathrm{U}$ & 330 & 31 & $\mathrm{JB}$ & 330 & 32 & $\mathrm{JB}$ & 32 & 17 & $\mathrm{JB}$ & 330 \\
\hline Di-n-octylphthalate & 330 & $\mathrm{U}$ & 330 & 330 & $\mathrm{U}$ & 330 & 330 & $\mathrm{U}$ & 330 & 330 & $\mathrm{U}$ & 330 \\
\hline Dibenz(a,h)anthracene & 330 & $\mathrm{U}$ & 330 & 330 & $\mathrm{U}$ & 330 & 330 & $\mathrm{U}$ & 330 & 180 & $\mathrm{~J}$ & 330 \\
\hline Dibenzofuran & 330 & UJ & 330 & 330 & UJ & 330 & 330 & UJ & 330 & 34 & $\mathrm{~J}$ & 330 \\
\hline Diethylphthalate & 330 & $\mathrm{U}$ & 330 & 330 & $\mathrm{U}$ & 330 & 330 & $\mathrm{U}$ & 330 & 330 & $\mathrm{U}$ & 330 \\
\hline Dimethylphthalate & 330 & $\mathrm{U}$ & 330 & 330 & $\mathrm{U}$ & 330 & 330 & $\mathrm{U}$ & 330 & 330 & $\mathrm{U}$ & 330 \\
\hline Fluoranthene & 330 & $\mathrm{U}$ & 330 & 52 & $\mathrm{JR}$ & 330 & 330 & UR & 330 & 2100 & $\mathrm{R}$ & 330 \\
\hline Fluorene & 330 & $\mathrm{UJ}$ & 330 & 330 & UJ & 330 & 330 & UJ & 330 & 43 & $\mathrm{~J}$ & 330 \\
\hline Hexachlorobenzene & 330 & $\mathrm{U}$ & 330 & 330 & $\mathrm{U}$ & 330 & 330 & $\mathrm{U}$ & 330 & 330 & $\mathrm{U}$ & 330 \\
\hline Hexachlorobutadiene & 330 & $\mathrm{U}$ & 330 & 330 & $\mathrm{U}$ & 330 & 330 & $\mathrm{U}$ & 330 & 330 & $\mathrm{U}$ & 330 \\
\hline Hexachlorocyclopentadiene & 330 & $\mathrm{U}$ & 330 & 330 & $\mathrm{U}$ & 330 & 330 & $\mathrm{U}$ & 330 & 330 & $\mathrm{U}$ & 330 \\
\hline Hexachloroethane & 330 & UJ & 330 & 330 & $\mathrm{UJ}$ & 330 & 330 & UJ & 330 & 330 & UJ & 330 \\
\hline Indeno(1,2,3-cd)pyrene & 330 & UJ & 330 & 18 & $\mathrm{~J}$ & 330 & 330 & UJ & 330 & 250 & $\mathrm{~J}$ & 330 \\
\hline Isophorone & 330 & UJ & 330 & 330 & $\mathrm{UJ}$ & 330 & 330 & UJ & 330 & 330 & UJ & 330 \\
\hline N-Nitroso-di-n-dipropylamine & 330 & UJ & 330 & 330 & $\mathrm{UJ}$ & 330 & 330 & UJ & 330 & 330 & $\mathrm{UJ}$ & 330 \\
\hline N-Nitrosodiphenylamine & 330 & $\mathrm{U}$ & 330 & 330 & $\mathrm{U}$ & 330 & 330 & $U$ & 330 & 330 & $\mathrm{U}$ & 330 \\
\hline Naphthalene & 330 & $\mathrm{U}$ & 330 & 330 & $\mathrm{U}$ & 330 & 330 & $\mathrm{U}$ & 330 & 330 & $\mathrm{U}$ & 330 \\
\hline Nitrobenzene & 330 & UJ & 330 & 330 & UJ & 330 & 330 & $\mathrm{UJ}$ & 330 & 330 & $\mathrm{UJ}$ & 330 \\
\hline Pentachlorophenol & 840 & $\mathrm{U}$ & 840 & 840 & $\mathrm{U}$ & 840 & 840 & $\mathrm{U}$ & 840 & 840 & $\mathrm{U}$ & 840 \\
\hline \begin{tabular}{|l} 
Phenanthrene \\
\end{tabular} & 330 & UJ & 330 & 19 & $\mathrm{~J}$ & 330 & 330 & $\mathrm{UJ}$ & 330 & 1300 & $\mathrm{~J}$ & 330 \\
\hline Phenol & 330 & $\mathrm{U}$ & 330 & 330 & $\mathrm{U}$ & 330 & 330 & $\mathrm{U}$ & 330 & 330 & $\mathrm{U}$ & 330 \\
\hline Pyrene & 330 & U & 330 & 57 & $\mathrm{~J}$ & 330 & 330 & $\mathrm{U}$ & 330 & 1600 & & 330 \\
\hline
\end{tabular}


CVP-2006-00011

Rev. 0

118-C-1 Verification Sampling Results.

\begin{tabular}{|c|c|c|c|c|c|c|c|c|c|c|c|c|}
\hline \multirow[t]{2}{*}{ Constituents } & \multicolumn{3}{|c|}{\begin{tabular}{|c|} 
J135P4 \\
SPA C1 \\
Sample Date 9/12/06 \\
\end{tabular}} & \multicolumn{3}{|c|}{$\begin{array}{c}\text { J135P5 } \\
\text { SPA C2 } \\
\text { Sample Date } 9 / 12 / 06\end{array}$} & \multicolumn{3}{|c|}{$\begin{array}{c}\text { J135P6 } \\
\text { SPA D3 } \\
\text { Sample Date 9/12/06 } \\
\end{array}$} & \multicolumn{3}{|c|}{$\begin{array}{c}\text { J135P7 } \\
\text { SPA D4 } \\
\text { Sample Date 9/12/06 } \\
\end{array}$} \\
\hline & $\mu \mathrm{g} / \mathrm{kg}$ & $\mathbf{Q}$ & PQL & $\mu \mathrm{g} / \mathrm{kg}$ & $\mathbf{Q}$ & PQL & $\mu \mathrm{g} / \mathrm{kg}$ & $\mathbf{Q}$ & PQL & $\mu \mathrm{g} / \mathrm{kg}$ & $Q$ & PQL \\
\hline \multicolumn{13}{|c|}{ Volatile Organic Compounds } \\
\hline 1,1,1-Trichloroethane & 5 & $\mathrm{U}$ & 5 & 6 & $\mathrm{U}$ & 6 & 5 & $\mathrm{U}$ & 5 & 5 & $\bar{U}$ & 5 \\
\hline 1,1,2,2-Tetrachloroethane & 5 & $\mathrm{U}$ & 5 & 6 & $\mathrm{U}$ & 6 & 5 & $\mathrm{U}$ & 5 & 5 & $\mathrm{U}$ & 5 \\
\hline 1,1,2-Trichloroethane & 5 & $\mathrm{U}$ & 5 & 6 & $\mathrm{U}$ & 6 & 5 & $\mathrm{U}$ & 5 & 5 & $\mathrm{U}$ & 5 \\
\hline 1,1-Dichloroethane & 5 & $\mathrm{U}$ & 5 & 6 & $\mathrm{U}$ & 6 & 5 & $\mathrm{U}$ & 5 & 5 & $\mathrm{U}$ & 5 \\
\hline 1,1-Dichloroethene & 5 & $\mathrm{U}$ & 5 & 6 & $\mathrm{U}$ & 6 & 5 & $\mathrm{U}$ & 5 & 5 & $\mathrm{U}$ & 5 \\
\hline 1,2-Dichloroethane & 5 & $\mathrm{U}$ & 5 & 6 & $\mathrm{U}$ & 6 & 5 & $\mathrm{U}$ & 5 & 5 & $\mathrm{U}$ & 5 \\
\hline 1,2-Dichloroethene (total) & 5 & $\mathrm{U}$ & 5 & 6 & $\mathrm{U}$ & 6 & 5 & $\mathrm{U}$ & 5 & 5 & $\mathrm{U}$ & 5 \\
\hline 1,2-Dichloropropane & 5 & $\mathrm{U}$ & 5 & 6 & $\mathrm{U}$ & 6 & 5 & $\mathrm{U}$ & 5 & 5 & $\mathrm{U}$ & 5 \\
\hline 2-Butanone & 10 & $\mathrm{U}$ & 10 & 11 & $\mathrm{U}$ & 11 & 10 & $\mathrm{U}$ & 10 & 9 & $\mathrm{U}$ & 9 \\
\hline 2-Hexanone & 10 & $\mathrm{U}$ & 10 & 11 & $\mathrm{U}$ & 11 & 10 & $\mathrm{U}$ & 10 & 9 & $\mathrm{U}$ & 9 \\
\hline 4-Methyl-2-pentanone & 10 & $\mathrm{U}$ & 10 & 11 & $\mathrm{U}$ & 11 & 10 & $\mathrm{U}$ & 10 & 9 & $\mathrm{U}$ & 9 \\
\hline Acetone & 7 & $\mathrm{~J}$ & 10 & 8 & $\mathrm{~J}$ & 11 & 7 & $\mathrm{~J}$ & 10 & 11 & & 9 \\
\hline Benzene & 5 & $\mathrm{U}$ & 5 & 6 & $\mathrm{U}$ & 6 & 5 & $\mathrm{U}$ & 5 & 5 & $\mathrm{U}$ & 5 \\
\hline Bromodichloromethane & 5 & $\mathrm{U}$ & 5 & 6 & $\mathrm{U}$ & 6 & 5 & $\mathrm{U}$ & 5 & 5 & $\mathrm{U}$ & 5 \\
\hline Bromoform & 5 & $\mathrm{U}$ & 5 & 6 & $\mathrm{U}$ & 6 & 5 & $\mathrm{U}$ & 5 & 5 & $\mathrm{U}$ & 5 \\
\hline Bromomethane & 10 & $\mathrm{U}$ & 10 & 11 & $\mathrm{U}$ & 11 & 10 & $\mathrm{U}$ & 10 & 9 & $\mathrm{U}$ & 9 \\
\hline Carbon disulfide & 5 & $\mathrm{U}$ & 5 & 6 & $\mathrm{U}$ & 6 & 5 & $\mathrm{U}$ & 5 & 5 & $\mathrm{U}$ & 5 \\
\hline Carbon tetrachloride & 5 & $\mathrm{U}$ & 5 & 6 & $\mathrm{U}$ & 6 & 5 & $\mathrm{U}$ & 5 & 5 & $\mathrm{U}$ & 5 \\
\hline Chlorobenzene & 5 & $\mathrm{U}$ & 5 & 6 & $\mathrm{U}$ & 6 & 5 & $\mathrm{U}$ & 5 & 5 & $\mathrm{U}$ & 5 \\
\hline Chloroethane & 10 & $\mathrm{U}$ & 10 & 11 & $\mathrm{U}$ & 11 & 10 & $\mathrm{U}$ & 10 & 9 & $\mathrm{U}$ & 9 \\
\hline Chloroform & 5 & $\mathrm{U}$ & 5 & 6 & $\mathrm{U}$ & 6 & 5 & $\mathrm{U}$ & 5 & 5 & $U$ & 5 \\
\hline Chloromethane & 10 & $\mathrm{U}$ & 10 & 11 & $\mathrm{U}$ & 11 & 10 & $\mathrm{U}$ & 10 & 9 & $\mathrm{U}$ & 9 \\
\hline cis-1,2-Dichloroethylene & 5 & $\mathrm{U}$ & 5 & 6 & $\mathrm{U}$ & 6 & 5 & $\mathrm{U}$ & 5 & 5 & $\mathrm{U}$ & 5 \\
\hline cis-1,3-Dichloropropene & 5 & $\mathrm{U}$ & 5 & 6 & $\mathrm{U}$ & 6 & 5 & $\mathrm{U}$ & 5 & 5 & $\mathrm{U}$ & 5 \\
\hline Dibromochloromethane & 5 & $\mathrm{U}$ & 5 & 6 & $\mathrm{U}$ & 6 & 5 & $\mathrm{U}$ & 5 & 5 & $\mathrm{U}$ & 5 \\
\hline Ethylbenzene & 5 & $\mathrm{U}$ & 5 & 6 & $\mathrm{U}$ & 6 & 5 & $\mathrm{U}$ & 5 & 5 & $\mathrm{U}$ & 5 \\
\hline Methylene chloride & 24 & $\mathrm{~B}$ & 5 & 27 & $\mathrm{~B}$ & 6 & 26 & $\mathrm{~B}$ & 5 & 18 & $\mathrm{~B}$ & 5 \\
\hline Styrene & 5 & $\mathrm{U}$ & 5 & 4 & $\mathrm{~J}$ & 6 & 5 & $\mathrm{U}$ & 5 & 5 & $\mathrm{U}$ & 5 \\
\hline Tetrachloroethene & 5 & $\mathrm{U}$ & 5 & 6 & $\mathrm{U}$ & 6 & 5 & $\mathrm{U}$ & 5 & 5 & $\mathrm{U}$ & 5 \\
\hline Toluene & 5 & $\mathrm{U}$ & 5 & 1 & $\mathrm{~J}$ & 6 & 1 & $\mathrm{~J}$ & 5 & 5 & $\mathrm{U}$ & 5 \\
\hline trans-1,2-Dichloroethylene & 5 & $\mathrm{U}$ & 5 & 6 & $\mathrm{U}$ & 6 & 5 & $\mathrm{U}$ & 5 & 5 & $\mathrm{U}$ & 5 \\
\hline trans-1,3-Dichloropropene & 5 & $\mathrm{U}$ & 5 & 6 & $\mathrm{U}$ & 6 & 5 & $\mathrm{U}$ & 5 & 5 & $\mathrm{U}$ & 5 \\
\hline Trichloroethene & 5 & $\mathrm{U}$ & 5 & 6 & $\mathrm{U}$ & 6 & 5 & $\mathrm{U}$ & 5 & 5 & $\mathrm{U}$ & 5 \\
\hline Vinyl chloride & 10 & $\mathrm{U}$ & 10 & 11 & $\mathrm{U}$ & 11 & 10 & $\mathrm{U}$ & 10 & 9 & $\mathrm{U}$ & 9 \\
\hline Xylenes (total) & 5 & $\mathrm{U}$ & 5 & 6 & $\mathrm{U}$ & 6 & 5 & $\mathrm{U}$ & 5 & 5 & $\mathrm{U}$ & 5 \\
\hline
\end{tabular}


CVP-2006-00011

Rev. 0

118-C-1 Verification Sampling Results.

\begin{tabular}{|c|c|c|c|c|c|c|c|c|c|c|c|c|}
\hline \multirow[t]{2}{*}{ Constituents } & \multicolumn{3}{|c|}{$\begin{array}{c}\text { J135P8 } \\
\text { SPA D5 } \\
\text { Sample Date } 9 / 12 / 06\end{array}$} & \multicolumn{3}{|c|}{$\begin{array}{c}\text { J135P9 } \\
\text { SPA D6 } \\
\text { Sample Date 9/12/06 }\end{array}$} & \multicolumn{3}{|c|}{$\begin{array}{c}\text { J12XM2 } \\
\text { FS-5 } \\
\text { Sample Date } 7 / 26 / 06\end{array}$} & \multicolumn{3}{|c|}{$\begin{array}{c}\text { J12XM3 } \\
\text { FS-6 } \\
\text { Sample Date } 7 / 26 / 06\end{array}$} \\
\hline & $\mu \mathrm{g} / \mathrm{kg}$ & $\mathbf{Q}$ & PQL & $\mu \mathrm{g} / \mathrm{kg}$ & $Q$ & PQL & $\mu \mathrm{g} / \mathrm{kg}$ & $Q$ & PQL & $\mu \mathrm{g} / \mathrm{kg}$ & $Q$ & PQL \\
\hline \multicolumn{13}{|c|}{ Polychlorinated Biphenyls } \\
\hline Aroclor-1016 & 13 & $\mathrm{U}$ & 13 & 13 & $\mathrm{U}$ & 13 & 13 & $\mathrm{U}$ & 13 & 14 & U & 14 \\
\hline Aroclor-1221 & 13 & $\mathrm{U}$ & 13 & 13 & $\mathrm{U}$ & 13 & 13 & $\mathrm{U}$ & 13 & 14 & $\mathrm{U}$ & 14 \\
\hline Aroclor-1232 & 13 & $\mathrm{U}$ & 13 & 13 & $\mathrm{U}$ & 13 & 13 & $\mathrm{U}$ & 13 & 14 & $\mathrm{U}$ & 14 \\
\hline Aroclor-1242 & 13 & $\mathrm{U}$ & 13 & 13 & $\mathrm{U}$ & 13 & 13 & $\mathrm{U}$ & 13 & 14 & $\mathrm{U}$ & 14 \\
\hline Aroclor-1248 & 13 & $\mathrm{U}$ & 13 & 13 & $\mathrm{U}$ & 13 & 13 & $\mathrm{U}$ & 13 & 14 & $\mathrm{U}$ & 14 \\
\hline Aroclor-1254 & 3.7 & $\mathrm{~J}$ & 13 & 4.7 & $\mathrm{~J}$ & 13 & 22 & & 13 & 14 & $\mathrm{U}$ & 14 \\
\hline Aroclor-1260 & 13 & $\mathrm{U}$ & 13 & 13 & $\mathrm{U}$ & 13 & 13 & $\mathrm{U}$ & 13 & 14 & $\mathrm{U}$ & 14 \\
\hline \multicolumn{13}{|c|}{ Semivolatile Organic Compounds } \\
\hline 1,2,4-Trichlorobenzene & 330 & UJ & 330 & 330 & UJ & 330 & 330 & $\mathrm{U}$ & 330 & 340 & $\mathrm{U}$ & 340 \\
\hline 1,2-Dichlorobenzene & 330 & UJ & 330 & 330 & UJ & 330 & 330 & $\mathrm{U}$ & 330 & 340 & U & 340 \\
\hline 1,3-Dichlorobenzene & 330 & UJ & 330 & 330 & UJ & 330 & 330 & $\mathrm{U}$ & 330 & 340 & $\mathrm{U}$ & 340 \\
\hline 1,4-Dichlorobenzene & 330 & UJ & 330 & 330 & UJ & 330 & 330 & $\mathrm{U}$ & 330 & 340 & $\mathrm{U}$ & 340 \\
\hline 2,4,5-Trichlorophenol & 840 & $\mathrm{U}$ & 840 & 840 & $\mathrm{U}$ & 840 & 840 & $\mathrm{U}$ & 840 & 850 & U & 850 \\
\hline 2,4,6-Trichlorophenol & 330 & $\mathrm{U}$ & 330 & 330 & $\mathrm{U}$ & 330 & 330 & $\mathrm{U}$ & 330 & 340 & $\mathrm{U}$ & 340 \\
\hline 2,4-Dichlorophenol & 330 & $\mathrm{U}$ & 330 & 330 & $\mathrm{U}$ & 330 & 330 & $\mathrm{U}$ & 330 & 340 & $\mathrm{U}$ & 340 \\
\hline 2,4-Dimethylphenol & 330 & UJ & 330 & 330 & UJ & 330 & 330 & $\mathrm{U}$ & 330 & 340 & $\mathrm{U}$ & 340 \\
\hline 2,4-Dinitrophenol & 840 & $\mathrm{UJ}$ & 840 & 840 & UJ & 840 & 840 & $\mathrm{U}$ & 840 & 850 & $\mathrm{U}$ & 850 \\
\hline 2,4-Dinitrotoluene & 330 & $\mathrm{U}$ & 330 & 330 & $\mathrm{U}$ & 330 & 330 & $\mathrm{U}$ & 330 & 340 & $\mathrm{U}$ & 340 \\
\hline 2,6-Dinitrotoluene & 330 & $\mathrm{U}$ & 330 & 330 & $\mathrm{U}$ & 330 & 330 & $\mathrm{U}$ & 330 & 340 & $\mathrm{U}$ & 340 \\
\hline 2-Chloronaphthalene & 330 & $\mathrm{U}$ & 330 & 330 & $\mathrm{U}$ & 330 & 330 & $\mathrm{U}$ & 330 & 340 & $\mathrm{U}$ & 340 \\
\hline 2-Chlorophenol & 330 & UJ & 330 & 330 & UJ & 330 & 330 & $\mathrm{U}$ & 330 & 340 & $\mathrm{U}$ & 340 \\
\hline 2-Methylnaphthalene & 330 & UJ & 330 & 330 & $\mathrm{U}$ & 330 & 330 & $\mathrm{U}$ & 330 & 340 & $\mathrm{U}$ & 340 \\
\hline 2-Methylphenol (cresol, o-) & 330 & $\mathrm{UJ}$ & 330 & 330 & $\mathrm{UJ}$ & 330 & 330 & $\mathrm{U}$ & 330 & 340 & $\mathrm{U}$ & 340 \\
\hline 2-Nitroaniline & 840 & $\mathrm{U}$ & 840 & 840 & $\mathrm{U}$ & 840 & 840 & $\mathrm{U}$ & 840 & 850 & $\mathrm{U}$ & 850 \\
\hline 2-Nitrophenol & 330 & UJ & 330 & 330 & UJ & 330 & 330 & $\mathrm{U}$ & 330 & 340 & $\mathrm{U}$ & 340 \\
\hline 3,3'-Dichlorobenzidine & 330 & $\mathrm{U}$ & 330 & 330 & $\mathrm{U}$ & 330 & 330 & $\mathrm{U}$ & 330 & 340 & $\mathrm{U}$ & 340 \\
\hline 3-Nitroaniline & 840 & $\mathrm{U}$ & 840 & 840 & $\mathrm{U}$ & 840 & 840 & $\mathrm{U}$ & 840 & 850 & $\mathrm{U}$ & 850 \\
\hline 4,6-Dinitro-2-methylphenol & 840 & $\mathrm{U}$ & 840 & 840 & $\mathrm{U}$ & 840 & 840 & $\mathrm{U}$ & 840 & 850 & $\mathrm{U}$ & 850 \\
\hline 4-Bromophenyl-phenylether & 330 & $\mathrm{U}$ & 330 & 330 & $\mathrm{U}$ & 330 & 330 & $\mathrm{U}$ & 330 & 340 & $\mathrm{U}$ & 340 \\
\hline 4-Chloro-3-methylphenol & 330 & $\mathrm{UJ}$ & 330 & 330 & $\mathrm{UJ}$ & 330 & 330 & $\mathrm{U}$ & 330 & 340 & $\mathrm{U}$ & 340 \\
\hline 4-Chloroaniline & 330 & $\mathrm{U}$ & 330 & 330 & $\mathrm{U}$ & 330 & 330 & $\mathrm{U}$ & 330 & 340 & $\mathrm{U}$ & 340 \\
\hline 4-Chlorophenyl-phenylether & 330 & $\mathrm{U}$ & 330 & 330 & $\mathrm{U}$ & 330 & 330 & $\mathrm{U}$ & 330 & 340 & $\mathrm{U}$ & 340 \\
\hline 4-Methylphenol (p-cresol) & 330 & $\mathrm{UJ}$ & 330 & 330 & UJ & 330 & 330 & $\mathrm{U}$ & 330 & 340 & $\mathrm{U}$ & 340 \\
\hline 4-Nitroaniline & 840 & $\mathrm{U}$ & 840 & 840 & $\mathrm{U}$ & 840 & 840 & $\mathrm{U}$ & 840 & 850 & $\mathrm{U}$ & 850 \\
\hline 4-Nitrophenol & 840 & $\mathrm{U}$ & 840 & 840 & $\mathrm{U}$ & 840 & 840 & $\mathrm{U}$ & 840 & 850 & $\mathrm{U}$ & 850 \\
\hline Acenaphthene & 330 & $\mathrm{U}$ & 330 & 330 & $\mathrm{U}$ & 330 & 330 & $\mathrm{U}$ & 330 & 340 & $\mathrm{U}$ & 340 \\
\hline Acenaphthylene & 330 & $\mathrm{U}$ & 330 & 330 & $\mathrm{U}$ & 330 & 330 & $\mathrm{U}$ & 330 & 340 & $\mathrm{U}$ & 340 \\
\hline Anthracene & 330 & UJ & 330 & 330 & UJ & 330 & 330 & $\mathrm{U}$ & 330 & 340 & $\mathrm{U}$ & 340 \\
\hline Benzo(a)anthracene & 330 & UJ & 330 & 18 & $\mathrm{~J}$ & 330 & 330 & $\mathrm{U}$ & 330 & 340 & $U$ & 340 \\
\hline Benzo(a)pyrene & 330 & $\mathrm{UJ}$ & 330 & 330 & UJ & 330 & 330 & $\mathrm{U}$ & 330 & 340 & $\mathrm{U}$ & 340 \\
\hline Benzo(b)fluoranthene & 330 & UJ & 330 & 330 & UJ & 330 & 330 & $\mathrm{U}$ & 330 & 340 & $\mathrm{U}$ & 340 \\
\hline Benzo(g,h,i)perylene & 330 & $\mathrm{U}$ & 330 & 330 & $\mathrm{U}$ & 330 & 330 & $\mathrm{U}$ & 330 & 340 & $\mathrm{U}$ & 340 \\
\hline Benzo(k)fluoranthene & 330 & $\mathrm{UJ}$ & 330 & 330 & UJ & 330 & 330 & $\mathrm{U}$ & 330 & 340 & $\mathrm{U}$ & 340 \\
\hline bis(2-Chloro-1-methylethyl)ether & 330 & $\mathrm{U}$ & 330 & 330 & $\mathrm{U}$ & 330 & 330 & $\mathrm{U}$ & 330 & 340 & $\mathrm{U}$ & 340 \\
\hline bis(2-Chloroethoxy)methane & 330 & $\mathrm{U}$ & 330 & 330 & $\mathrm{U}$ & 330 & 330 & $\mathrm{U}$ & 330 & 340 & $\mathrm{U}$ & 340 \\
\hline bis(2-Chloroethyl)ether & 330 & $\mathrm{U}$ & 330 & 330 & $\mathrm{U}$ & 330 & 330 & $\mathrm{U}$ & 330 & 340 & $\mathrm{U}$ & 340 \\
\hline bis(2-Ethylhexyl)phthalate & 50 & $\mathrm{JB}$ & 330 & 41 & $\mathrm{JB}$ & 330 & 39 & JB & 330 & 67 & $\mathrm{JB}$ & 340 \\
\hline
\end{tabular}


CVP-2006-00011

Rev. 0

118-C-1 Verification Sampling Results.

\begin{tabular}{|c|c|c|c|c|c|c|c|c|c|c|c|c|}
\hline \multirow[t]{2}{*}{ Constituents } & \multicolumn{3}{|c|}{$\begin{array}{c}\text { J135P8 } \\
\text { SPA D5 } \\
\text { Sample Date 9/12/06 }\end{array}$} & \multicolumn{3}{|c|}{$\begin{array}{c}\text { J135P9 } \\
\text { SPA D6 } \\
\text { Sample Date 9/12/06 }\end{array}$} & \multicolumn{3}{|c|}{$\begin{array}{c}\text { J12XM2 } \\
\text { FS-5 } \\
\text { Sample Date } 7 / 26 / 06 \\
\end{array}$} & \multicolumn{3}{|c|}{$\begin{array}{c}\text { J12XM3 } \\
\text { FS } 6 \\
\text { Sample Date } 7 / 26 / 06 \\
\end{array}$} \\
\hline & $\mu \mathrm{g} / \mathrm{kg}$ & $\mathbf{Q}$ & PQL & $\mu \mathrm{g} / \mathrm{kg}$ & $\mathbf{Q}$ & PQL & $\mu \mathrm{g} / \mathrm{kg}$ & $\mathbf{Q}$ & PQL & $\mu \mathrm{g} / \mathrm{kg}$ & $\mathbf{Q}$ & $\mathrm{PQL}$ \\
\hline \multicolumn{13}{|c|}{ Semivolatile Organic Compounds (continued) } \\
\hline Butylbenzylphthalate & 330 & $\mathrm{U}$ & 330 & 330 & $\mathrm{U}$ & 330 & 330 & $\mathrm{U}$ & 330 & 340 & $\mathrm{U}$ & 340 \\
\hline Carbazole & 330 & UJ & 330 & 330 & UJ & 330 & 330 & $\mathrm{U}$ & 330 & 340 & $\mathrm{U}$ & 340 \\
\hline Chrysene & 330 & UJ & 330 & 27 & $\mathrm{~J}$ & 330 & 330 & $\mathrm{U}$ & 330 & 340 & $\mathrm{U}$ & 340 \\
\hline Di-n-butylphthalate & 29 & $\mathrm{JB}$ & 330 & 330 & $\mathrm{U}$ & 330 & 55 & $\mathrm{JB}$ & 330 & 49 & $\mathrm{JB}$ & 340 \\
\hline Di-n-octylphthalate & 330 & $\mathrm{U}$ & 330 & 330 & $\mathrm{U}$ & 330 & 330 & $\mathrm{U}$ & 330 & 340 & $\mathrm{U}$ & 340 \\
\hline Dibenz $(a, h)$ anthracene & 330 & $\mathrm{U}$ & 330 & 330 & $\mathrm{U}$ & 330 & 330 & $\mathrm{U}$ & 330 & 340 & $\mathrm{U}$ & 340 \\
\hline Dibenzofuran & 330 & UJ & 330 & 330 & UJ & 330 & 330 & $\mathrm{U}$ & 330 & 340 & $\mathrm{U}$ & 340 \\
\hline Diethylphthalate & 330 & $\mathrm{U}$ & 330 & 330 & $\mathrm{U}$ & 330 & 22 & $\mathrm{~J}$ & 330 & 340 & $\mathrm{U}$ & 340 \\
\hline Dimethylphthalate & 330 & $\mathrm{U}$ & 330 & 330 & $\mathrm{U}$ & 330 & 330 & $\mathrm{U}$ & 330 & 340 & $\mathrm{U}$ & 340 \\
\hline Fluoranthene & 18 & $\mathrm{JR}$ & 330 & 34 & JR & 330 & 330 & $\mathrm{U}$ & 330 & 340 & $\mathrm{U}$ & 340 \\
\hline Fluorene & 330 & $\mathrm{UJ}$ & 330 & 330 & $\mathrm{UJ}$ & 330 & 330 & $\mathrm{U}$ & 330 & 340 & $\mathrm{U}$ & 340 \\
\hline Hexachlorobenzene & 330 & $\mathrm{U}$ & 330 & 330 & $\mathrm{U}$ & 330 & 330 & $\mathrm{U}$ & 330 & 340 & $\mathrm{U}$ & 340 \\
\hline Hexachlorobutadiene & 330 & $\mathrm{U}$ & 330 & 330 & $\mathrm{U}$ & 330 & 330 & $\mathrm{U}$ & 330 & 340 & $\mathrm{U}$ & 340 \\
\hline Hexachlorocyclopentadiene & 330 & $\mathrm{U}$ & 330 & 330 & $\mathrm{U}$ & 330 & 330 & $\mathrm{U}$ & 330 & 340 & $\mathrm{U}$ & 340 \\
\hline Hexachloroethane & 330 & UJ & 330 & 330 & UJ & 330 & 330 & $\mathrm{U}$ & 330 & 340 & $\mathrm{U}$ & 340 \\
\hline Indeno(1,2,3-cd)pyrene & 330 & UJ & 330 & 330 & $\mathrm{UJ}$ & 330 & 330 & $\mathrm{U}$ & 330 & 340 & $\mathrm{U}$ & 340 \\
\hline Isophorone & 330 & $\mathrm{UJ}$ & 330 & 330 & $\mathrm{UJ}$ & 330 & 330 & $\mathrm{U}$ & 330 & 340 & $\mathrm{U}$ & 340 \\
\hline $\mathrm{N}$-Nitroso-di-n-dipropylamine & 330 & UJ & 330 & 330 & UJ & 330 & 330 & $\mathrm{U}$ & 330 & 340 & $\mathrm{U}$ & 340 \\
\hline N-Nitrosodiphenylamine & 330 & $\mathrm{U}$ & 330 & 330 & $\mathrm{U}$ & 330 & 330 & $\mathrm{U}$ & 330 & 340 & $U$ & 340 \\
\hline Naphthalene & 330 & $\mathrm{U}$ & 330 & 330 & $\mathrm{U}$ & 330 & 330 & $\mathrm{U}$ & 330 & 340 & $\mathrm{U}$ & 340 \\
\hline Nitrobenzene & 330 & UJ & 330 & 330 & UJ & 330 & 330 & $\mathrm{U}$ & 330 & 340 & $\mathrm{U}$ & 340 \\
\hline Pentachlorophenol & 840 & $\mathrm{U}$ & 840 & 840 & $\mathrm{U}$ & 840 & 840 & $\mathrm{U}$ & 840 & 850 & $\bar{U}$ & 850 \\
\hline Phenanthrene & 330 & UJ & 330 & 330 & $\mathrm{UJ}$ & 330 & 330 & $\mathrm{U}$ & 330 & 340 & $\mathrm{U}$ & 340 \\
\hline Phenol & 330 & $\mathrm{U}$ & 330 & 330 & $\mathrm{U}$ & 330 & 36 & JB & 330 & 33 & $\mathrm{JB}$ & 340 \\
\hline Pyrene & 330 & $\mathrm{U}$ & 330 & 27 & $\mathrm{~J}$ & 330 & 330 & $\mathrm{U}$ & 330 & 340 & $\mathrm{U}$ & 340 \\
\hline
\end{tabular}


118-C-1 Verification Sampling Results.

\begin{tabular}{|c|c|c|c|c|c|c|c|c|c|c|c|c|}
\hline \multirow[t]{2}{*}{ Constituents } & \multicolumn{3}{|c|}{$\begin{array}{c}\text { J135P8 } \\
\text { SPA D5 } \\
\text { Sample Date 9/12/06 }\end{array}$} & \multicolumn{3}{|c|}{$\begin{array}{c}\text { J135P9 } \\
\text { SPA D6 } \\
\text { Sample Date 9/12/06 }\end{array}$} & \multicolumn{3}{|c|}{$\begin{array}{c}\text { J12XM2 } \\
\text { FS-5 } \\
\text { Sample Date 7/26/06 }\end{array}$} & \multicolumn{3}{|c|}{$\begin{array}{c}\text { J12XM3 } \\
\text { FS-6 } \\
\text { Sample Date } 7 / 26 / 06 \\
\end{array}$} \\
\hline & $\mu \mathrm{gg} / \mathrm{kg}$ & $\mathbf{Q}$ & PQL & $\mu \mathrm{g} / \mathrm{kg}$ & $Q$ & PQL & $\mu \mathrm{g} / \mathrm{kg}$ & $\mathbf{Q}$ & PQL & $\mu \mathrm{g} / \mathrm{kg}$ & Q & PQL \\
\hline \multicolumn{13}{|c|}{ Volatile Organic Compounds } \\
\hline 1,1,1-Trichloroethane & 6 & $\mathrm{U}$ & 6 & 5 & $\mathrm{U}$ & 5 & 5 & $\mathrm{U}$ & 5 & 5 & $\mathrm{U}$ & 5 \\
\hline 1,1,2,2-Tetrachloroethane & 6 & $\mathrm{U}$ & 6 & 5 & $\mathrm{U}$ & 5 & 5 & $\mathrm{U}$ & 5 & 5 & $\mathrm{U}$ & 5 \\
\hline 1,1,2-Trichloroethane & 6 & $\mathrm{U}$ & 6 & 5 & $\mathrm{U}$ & 5 & 5 & $\mathrm{U}$ & 5 & 5 & $\mathrm{U}$ & 5 \\
\hline 1,1-Dichloroethane & 6 & $\mathrm{U}$ & 6 & 5 & $\mathrm{U}$ & 5 & 5 & $\mathrm{U}$ & 5 & 5 & $\mathrm{U}$ & 5 \\
\hline 1,1-Dichloroethene & 6 & $\mathrm{U}$ & 6 & 5 & $\mathrm{U}$ & 5 & 5 & $\mathrm{U}$ & 5 & 5 & $\mathrm{U}$ & 5 \\
\hline 1,2-Dichloroethane & 6 & $\mathrm{U}$ & 6 & 5 & $\mathrm{U}$ & 5 & 5 & $\mathrm{U}$ & 5 & 5 & $\mathrm{U}$ & 5 \\
\hline 1,2-Dichloroethene (total) & 6 & $\mathrm{U}$ & 6 & 5 & $\mathrm{U}$ & 5 & 5 & $\mathrm{U}$ & 5 & 5 & $\mathrm{U}$ & 5 \\
\hline 1,2-Dichloropropane & 6 & $\mathrm{U}$ & 6 & 5 & U & 5 & 5 & $\mathrm{U}$ & 5 & 5 & $\mathrm{U}$ & 5 \\
\hline 2-Butanone & 11 & $\mathrm{U}$ & 11 & 10 & $\mathrm{U}$ & 10 & 10 & $\mathrm{U}$ & 10 & 10 & $\mathrm{U}$ & 10 \\
\hline 2-Hexanone & 11 & $\mathrm{U}$ & 11 & 10 & $\mathrm{U}$ & 10 & 10 & $\mathrm{U}$ & 10 & 10 & $\mathrm{U}$ & 10 \\
\hline 4-Methyl-2-pentanone & 11 & $\mathrm{U}$ & 11 & 10 & $\mathrm{U}$ & 10 & 10 & $\mathrm{U}$ & 10 & 10 & $\mathrm{U}$ & 10 \\
\hline Acetone & 11 & $\mathrm{U}$ & 11 & 8 & $\mathrm{~J}$ & 10 & 24 & & 10 & 10 & $\mathrm{~J}$ & 10 \\
\hline Benzene & 6 & $\mathrm{U}$ & 6 & 5 & $\mathrm{U}$ & 5 & 5 & $\mathrm{U}$ & 5 & 5 & $\mathrm{U}$ & 5 \\
\hline Bromodichloromethane & 6 & $\mathrm{U}$ & 6 & 5 & $\mathrm{U}$ & 5 & 5 & $\mathrm{U}$ & 5 & 5 & $\mathrm{U}$ & 5 \\
\hline Bromoform & 6 & $\mathrm{U}$ & 6 & 5 & $\mathrm{U}$ & 5 & 5 & $\mathrm{U}$ & 5 & 5 & $\mathrm{U}$ & 5 \\
\hline Bromomethane & 11 & $\mathrm{U}$ & 11 & 10 & $\mathrm{U}$ & 10 & 10 & $\mathrm{U}$ & 10 & 10 & $\mathrm{U}$ & 10 \\
\hline Carbon disulfide & 6 & $\mathrm{U}$ & 6 & 5 & $\mathrm{U}$ & 5 & 5 & $\mathrm{U}$ & 5 & 5 & $\mathrm{U}$ & 5 \\
\hline Carbon tetrachloride & 6 & $\mathrm{U}$ & 6 & 5 & $\mathrm{U}$ & 5 & 39 & & 5 & 10 & & 5 \\
\hline Chlorobenzene & 6 & $\mathrm{U}$ & 6 & 5 & $\mathrm{U}$ & 5 & 5 & $\mathrm{U}$ & 5 & 5 & $\mathrm{U}$ & 5 \\
\hline Chloroethane & 11 & $\mathrm{U}$ & 11 & 10 & $\mathrm{U}$ & 10 & 10 & $\mathrm{U}$ & 10 & 10 & $\mathrm{U}$ & 10 \\
\hline Chloroform & 6 & $\mathrm{U}$ & 6 & 5 & $\mathrm{U}$ & 5 & 5 & $\mathrm{U}$ & 5 & 5 & $\mathrm{U}$ & 5 \\
\hline Chloromethane & 11 & $\mathrm{U}$ & 11 & 10 & $\mathrm{U}$ & 10 & 10 & $\mathrm{U}$ & 10 & 10 & $\mathrm{U}$ & 10 \\
\hline cis-1,2-Dichloroethylene & 6 & $\mathrm{U}$ & 6 & 5 & $\mathrm{U}$ & 5 & 5 & $\mathrm{U}$ & 5 & 5 & $\mathrm{U}$ & 5 \\
\hline cis-1,3-Dichloropropene & 6 & $\mathrm{U}$ & 6 & 5 & $\mathrm{U}$ & 5 & 5 & $\mathrm{U}$ & 5 & 5 & $\mathrm{U}$ & 5 \\
\hline Dibromochloromethane & 6 & $\mathrm{U}$ & 6 & 5 & $\mathrm{U}$ & 5 & 5 & $\mathrm{U}$ & 5 & 5 & $\mathrm{U}$ & 5 \\
\hline Ethylbenzene & 6 & $\mathrm{U}$ & 6 & 5 & $\mathrm{U}$ & 5 & 5 & $\mathrm{U}$ & 5 & 5 & U & 5 \\
\hline Methylene chloride & 23 & $\mathrm{~B}$ & 6 & 30 & $\mathrm{~B}$ & 5 & 19 & & 5 & 5 & $\mathrm{U}$ & 5 \\
\hline Styrene & 6 & $\mathrm{U}$ & 6 & 1 & $\mathrm{~J}$ & 5 & 5 & $\mathrm{U}$ & 5 & 5 & $\mathrm{U}$ & 5 \\
\hline Tetrachloroethene & 6 & $\mathrm{U}$ & 6 & 5 & $\mathrm{U}$ & 5 & 5 & $\mathrm{U}$ & 5 & 5 & $\mathrm{U}$ & 5 \\
\hline Toluene & 6 & $\mathrm{U}$ & 6 & 1 & $\mathrm{~J}$ & 5 & 5 & $\mathrm{U}$ & 5 & 5 & $\mathrm{U}$ & 5 \\
\hline trans-1,2-Dichloroethylene & 6 & $\mathrm{U}$ & 6 & 5 & $\mathrm{U}$ & 5 & 5 & $\mathrm{U}$ & 5 & 5 & $\mathrm{U}$ & 5 \\
\hline trans-1,3-Dichloropropene & 6 & $\mathrm{U}$ & 6 & 5 & $\mathrm{U}$ & 5 & 5 & $\mathrm{U}$ & 5 & 5 & $\mathrm{U}$ & 5 \\
\hline Trichloroethene & 6 & $\mathrm{U}$ & 6 & 5 & $\mathrm{U}$ & 5 & 5 & $\mathrm{U}$ & 5 & 5 & $\mathrm{U}$ & 5 \\
\hline Vinyl chloride & 11 & $\mathrm{U}$ & 11 & 10 & $\mathrm{U}$ & 10 & 10 & $\mathrm{U}$ & 10 & 10 & $\mathrm{U}$ & 10 \\
\hline Xylenes (total) & 6 & $\mathrm{U}$ & 6 & 1 & $\mathrm{~J}$ & 5 & 5 & $\mathrm{U}$ & 5 & 5 & $\mathrm{U}$ & 5 \\
\hline
\end{tabular}


CVP-2006-00011

Rev. 0

118-C-1 Verification Sampling Results.

\begin{tabular}{|c|c|c|c|c|c|c|c|}
\hline \multirow[t]{2}{*}{ Constituents } & \multicolumn{3}{|c|}{$\begin{array}{c}\text { J13509 } \\
\text { Equipment Blank } \\
\text { Sample Date 8/17/06 } \\
\end{array}$} & \multirow[t]{2}{*}{ Constituents } & \multicolumn{3}{|c|}{$\begin{array}{c}\text { J13509 } \\
\text { Equipment Blank } \\
\text { Sample Date 8/17/06 }\end{array}$} \\
\hline & $\mu \mathrm{g} / \mathrm{kg}$ & $\mathbf{Q}$ & PQL & & $\mu \mathrm{g} / \mathrm{kg}$ & $\mathbf{Q}$ & PQL \\
\hline \multicolumn{4}{|c|}{ Semivolatile Organic Compounds } & \multicolumn{4}{|c|}{ Semivolatile Organic Compounds (continued) } \\
\hline 1,2,4-Trichlorobenzene & 330 & $\mathrm{U}$ & 330 & Dimethylphthalate & 330 & $\mathrm{U}$ & 330 \\
\hline 1,2-Dichlorobenzene & 330 & $\mathrm{U}$ & 330 & Fluoranthene & 330 & $\mathrm{U}$ & 330 \\
\hline 1,3-Dichlorobenzene & 330 & $\mathrm{U}$ & 330 & \begin{tabular}{|l|} 
Fluorene \\
\end{tabular} & 330 & $\mathrm{U}$ & 330 \\
\hline 1,4-Dichlorobenzene & 330 & $\mathrm{U}$ & 330 & Hexachlorobenzene & 330 & $\mathrm{U}$ & 330 \\
\hline 2,4,5-Trichlorophenol & 840 & $\mathrm{U}$ & 840 & Hexachlorobutadiene & 330 & $\mathrm{U}$ & 330 \\
\hline 2,4,6-Trichlorophenol & 330 & $\mathrm{U}$ & 330 & Hexachlorocyclopentadiene & 330 & $\mathrm{U}$ & 330 \\
\hline 2,4-Dichlorophenol & 330 & $\mathrm{U}$ & 330 & Hexachloroethane & 330 & $\mathrm{U}$ & 330 \\
\hline 2,4-Dimethylphenol & 330 & $\mathrm{U}$ & 330 & Indeno(1,2,3-cd)pyrene & 330 & $\mathrm{U}$ & 330 \\
\hline 2,4-Dinitrophenol & 840 & $\mathrm{U}$ & 840 & Isophorone & 330 & $\mathrm{U}$ & 330 \\
\hline 2,4-Dinitrotoluene & 330 & $\mathrm{U}$ & 330 & N-Nitroso-di-n-dipropylamine & 330 & $\mathrm{U}$ & 330 \\
\hline 2,6-Dinitrotoluene & 330 & $\mathrm{U}$ & 330 & N-Nitrosodiphenylamine & 330 & $\mathrm{U}$ & 330 \\
\hline 2-Chloronaphthalene & 330 & $\mathrm{U}$ & 330 & \begin{tabular}{|l} 
Naphthalene \\
\end{tabular} & 330 & $\mathrm{U}$ & 330 \\
\hline 2-Chlorophenol & 330 & $\mathrm{U}$ & 330 & \begin{tabular}{|l|} 
Nitrobenzene \\
\end{tabular} & 330 & $\mathrm{U}$ & 330 \\
\hline 2-Methylnaphthalene & 330 & $\mathrm{U}$ & 330 & Pentachlorophenol & 840 & $\mathrm{U}$ & 840 \\
\hline 2-Methylphenol (cresol, o-) & 330 & $\mathrm{U}$ & 330 & Phenanthrene & 330 & $\mathrm{U}$ & 330 \\
\hline 2-Nitroaniline & 840 & $\mathrm{U}$ & 840 & Phenol & 330 & $\mathrm{U}$ & 330 \\
\hline 2-Nitrophenol & 330 & $\mathrm{U}$ & 330 & Pyrene & 330 & $\mathrm{U}$ & 330 \\
\hline
\end{tabular}

\begin{tabular}{|l|c|c|c|}
\hline 3,3-Dichlorobenzidine & 330 & $\mathrm{U}$ & 330 \\
\hline 3-Nitroaniline & 840 & $\mathrm{U}$ & 840 \\
\hline 4,6-Dinitro-2-methylphenol & 840 & $\mathrm{U}$ & 840 \\
\hline 4-Bromophenyl-phenylether & 330 & $\mathrm{U}$ & 330 \\
\hline 4-Chloro-3-methylphenol & 330 & $\mathrm{U}$ & 330 \\
\hline 4-Chloroaniline & 330 & $\mathrm{U}$ & 330 \\
\hline 4-Chlorophenyl-phenylether & 330 & $\mathrm{U}$ & 330 \\
\hline 4-Methylphenol (p-cresol) & 330 & $\mathrm{U}$ & 330 \\
\hline 4-Nitroaniline & 840 & $\mathrm{U}$ & 840 \\
\hline 4-Nitrophenol & 840 & $\mathrm{U}$ & 840 \\
\hline Acenaphthene & 330 & $\mathrm{U}$ & 330 \\
\hline Acenaphthylene & 330 & $\mathrm{U}$ & 330 \\
\hline Anthracene & 330 & $\mathrm{U}$ & 330 \\
\hline Benzo(a)anthracene & 330 & $\mathrm{U}$ & 330 \\
\hline Benzo(a)pyrene & 330 & $\mathrm{U}$ & 330 \\
\hline Benzo(b)fluoranthene & 330 & $\mathrm{U}$ & 330 \\
\hline Benzo(g,h,i)perylene & 330 & $\mathrm{U}$ & 330 \\
\hline Benzo(k)fluoranthene & 330 & $\mathrm{U}$ & 330 \\
\hline bis(2-Chloro-1-methylethyl)ether & 330 & $\mathrm{U}$ & 330 \\
\hline bis(2-Chloroethoxy)methane & 330 & $\mathrm{U}$ & 330 \\
\hline bis(2-Chloroethyl)ether & 330 & $\mathrm{U}$ & 330 \\
\hline bis(2-Ethylhexyl)phthalate & 27 & JB & 330 \\
\hline Butylbenzylphthalate & 330 & $\mathrm{U}$ & 330 \\
\hline Carbazole & 330 & $\mathrm{U}$ & 330 \\
\hline Chrysene & 330 & $\mathrm{U}$ & 330 \\
\hline Di-n-butylphthalate & 33 & JB & 330 \\
\hline Di-n-octylphthalate & 330 & $\mathrm{U}$ & 330 \\
\hline Dibenz(a,h)anthracene & 330 & $\mathrm{U}$ & 330 \\
\hline Dibenzofuran & 330 & $\mathrm{U}$ & 330 \\
\hline Diethylphthalate & $\mathrm{U}$ & 330 \\
\hline
\end{tabular}


CVP-2006-00011

Rev. 0

A-44 
CVP-2006-00011

Rev. 0

APPENDIX B

118-C-1 WASTE SITE REMEDIATION PHOTOGRAPHS

$B-i$ 
CVP-2006-00011

Rev. 0

B-ii 
Photograph B-1. Aerial View of the 118-C-1 Burial Ground and Surrounding Area (2003).

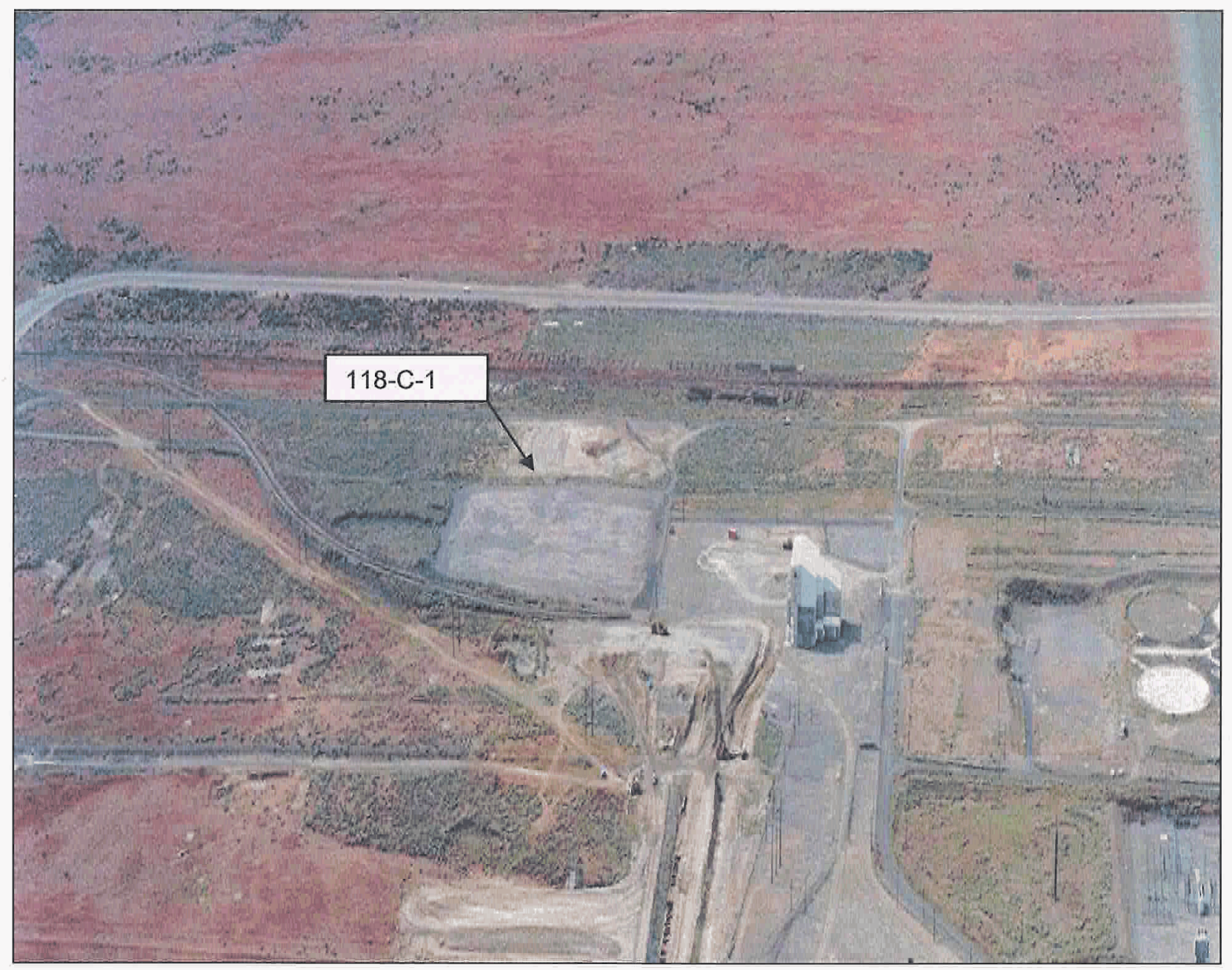


CVP-2006-00011

Rev. 0

Photograph B-2. 118-C-1 Burial Ground (Beginning of Remedial Activities).

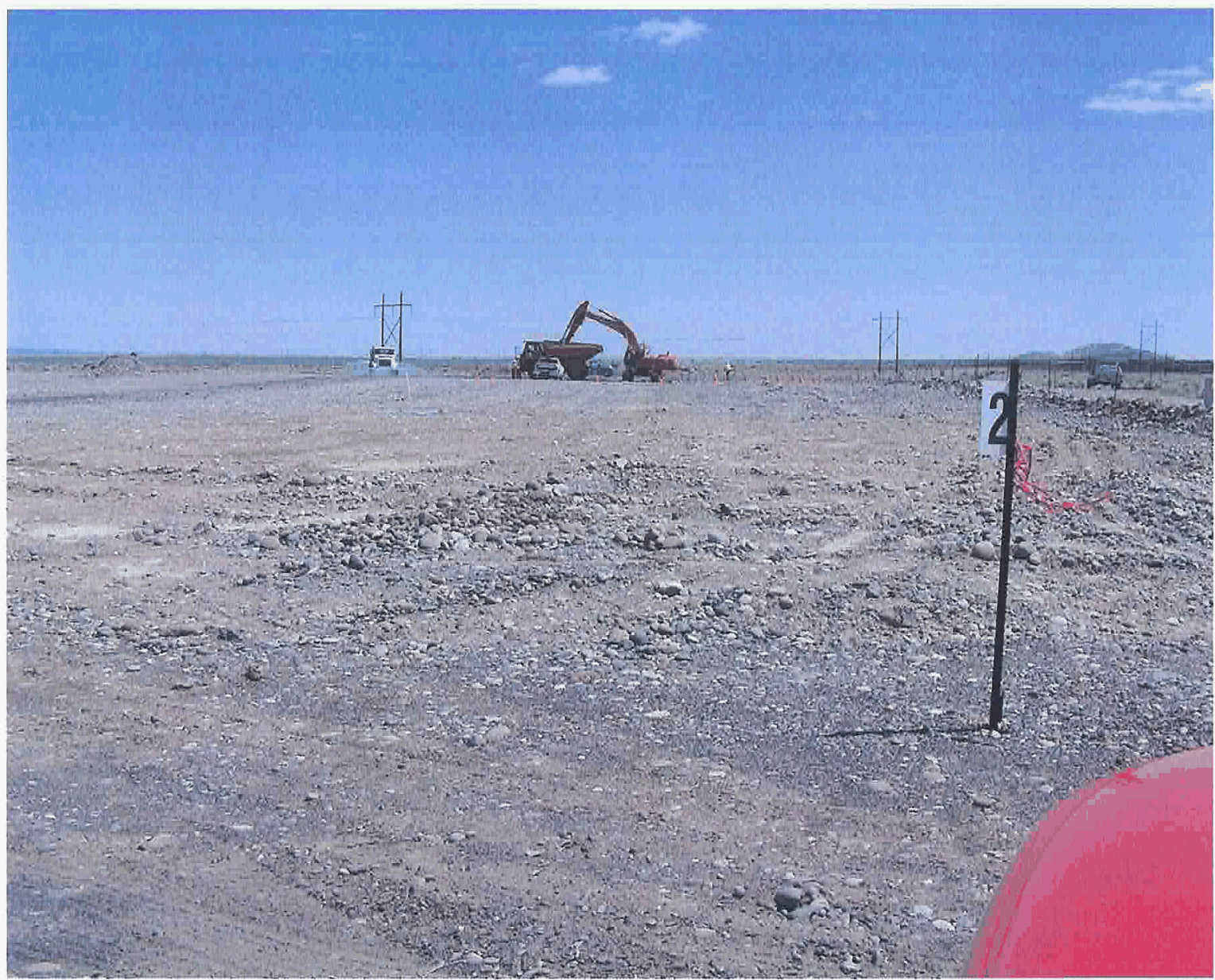




\section{Photograph B-3. Reactor Hardware.}

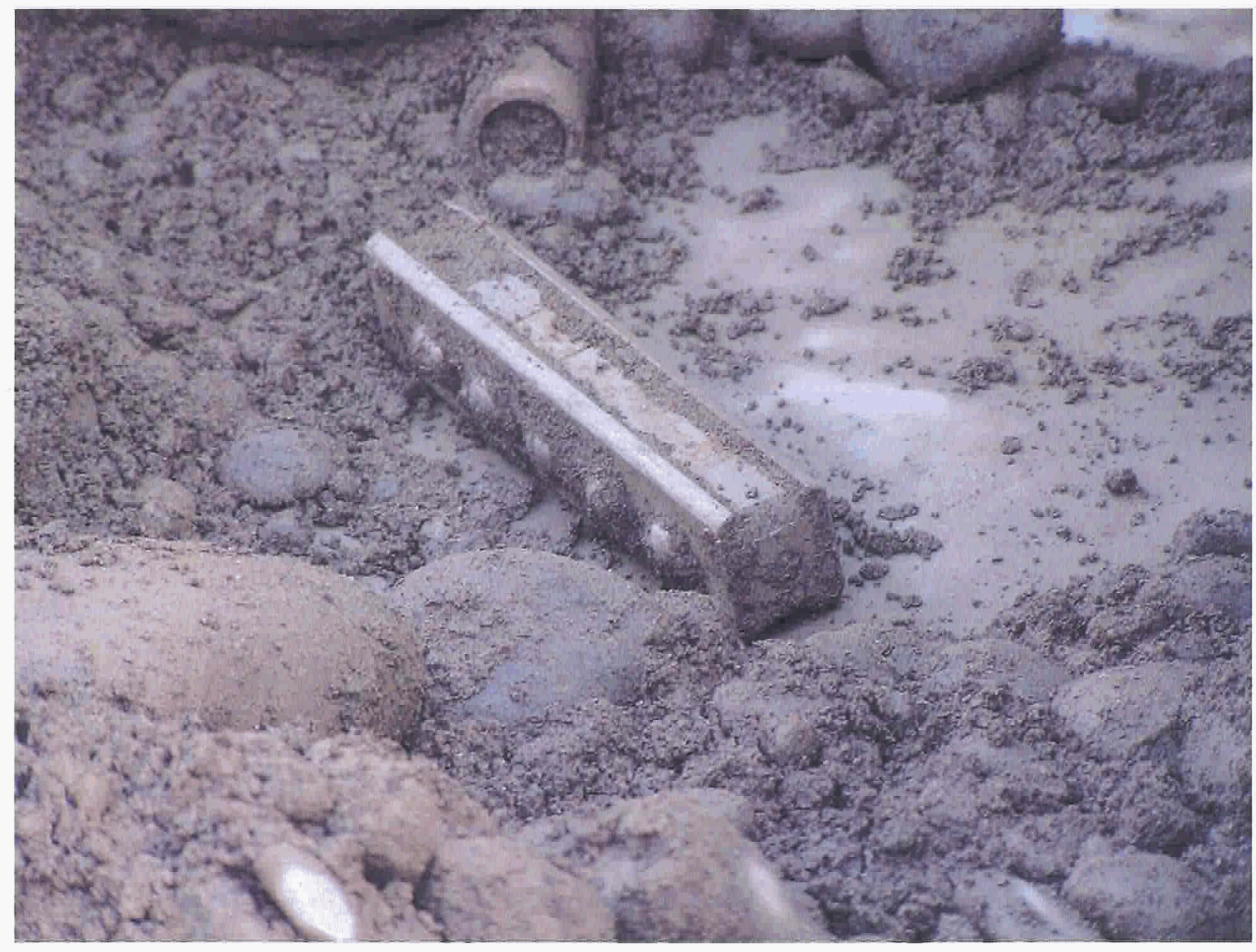


Photograph B-4. Perforated Spacers.

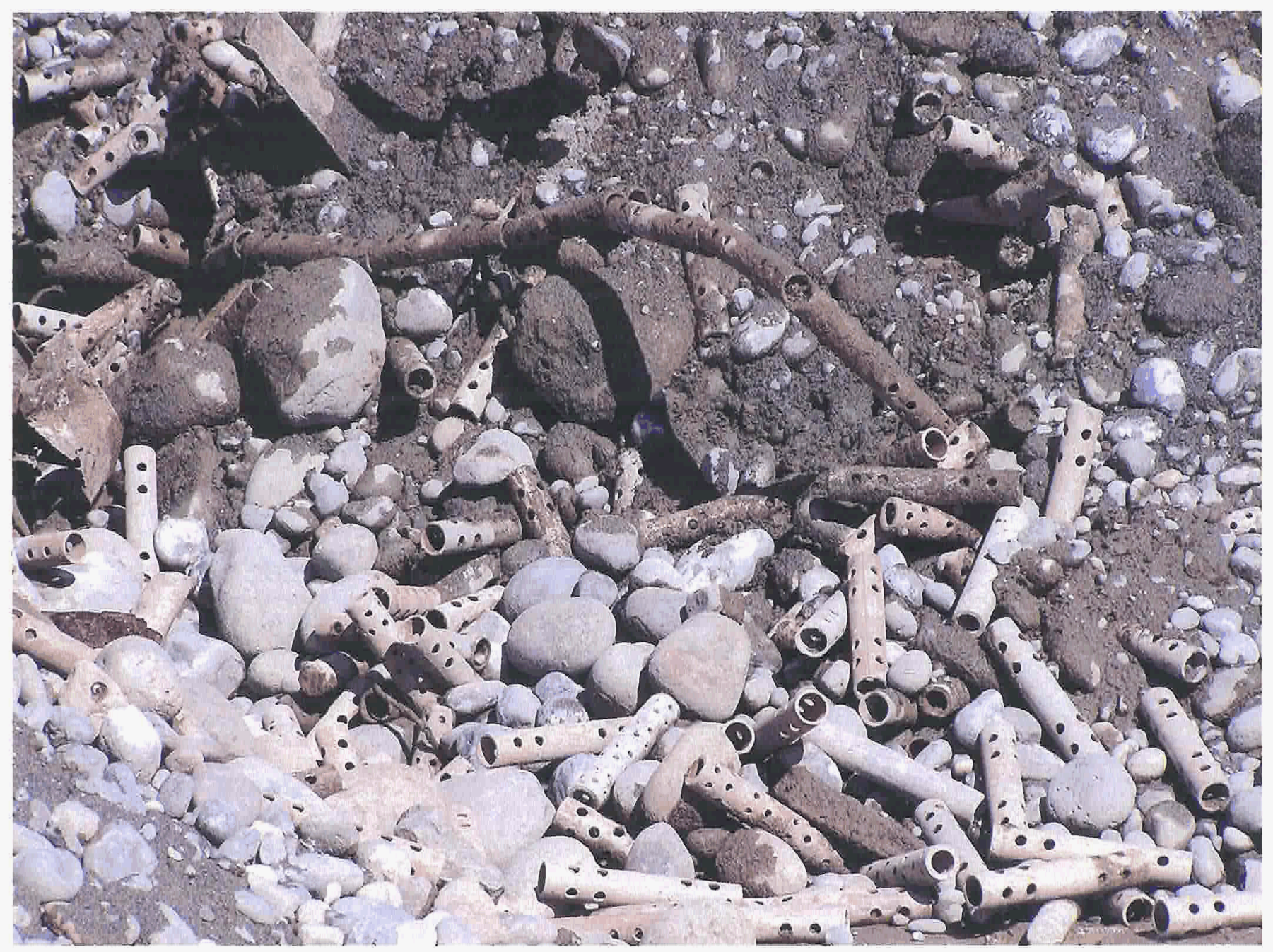


CVP-2006-00011

Rev. 0

\section{Photograph B-5. Vacuum Drum for Boron Balls.}

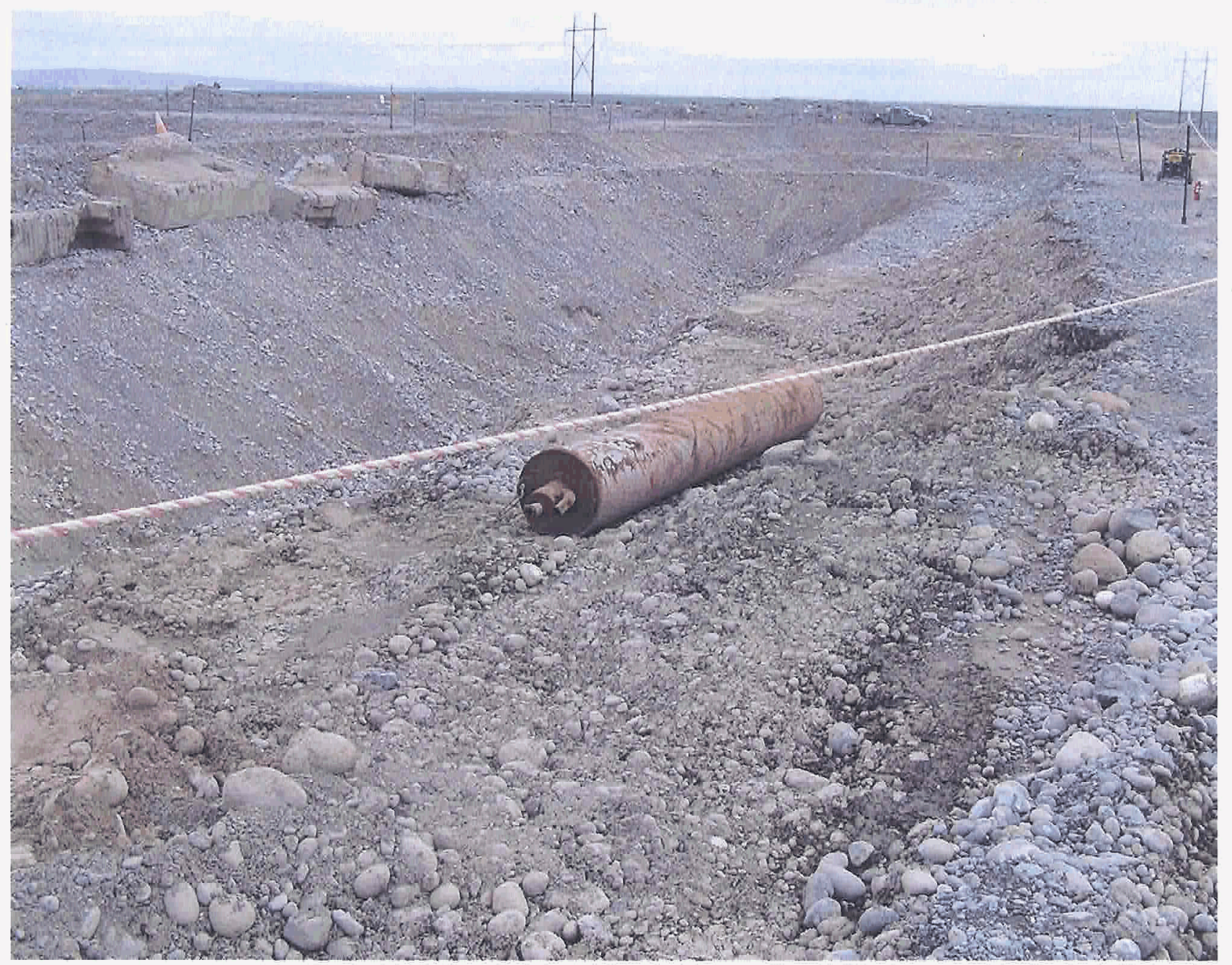


CVP-2006-00011

Rev. 0

Photograph B-6. Excavation of Spacers and Soils at the 118-C-1 Burial Ground.

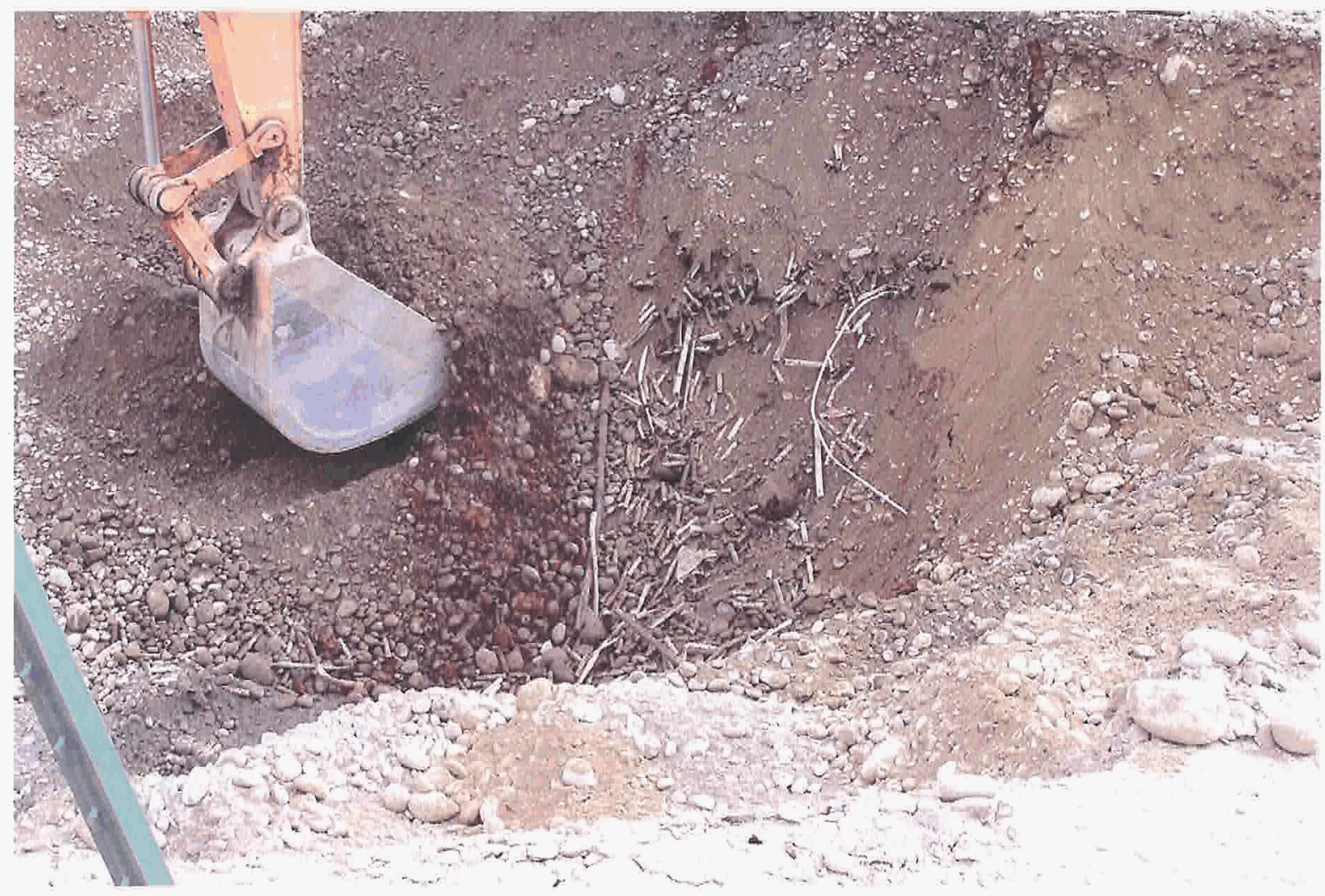


CVP-2006-00011

Rev. 0

Photograph B-7. Staged Waste at 118-C-1 Burial Ground.

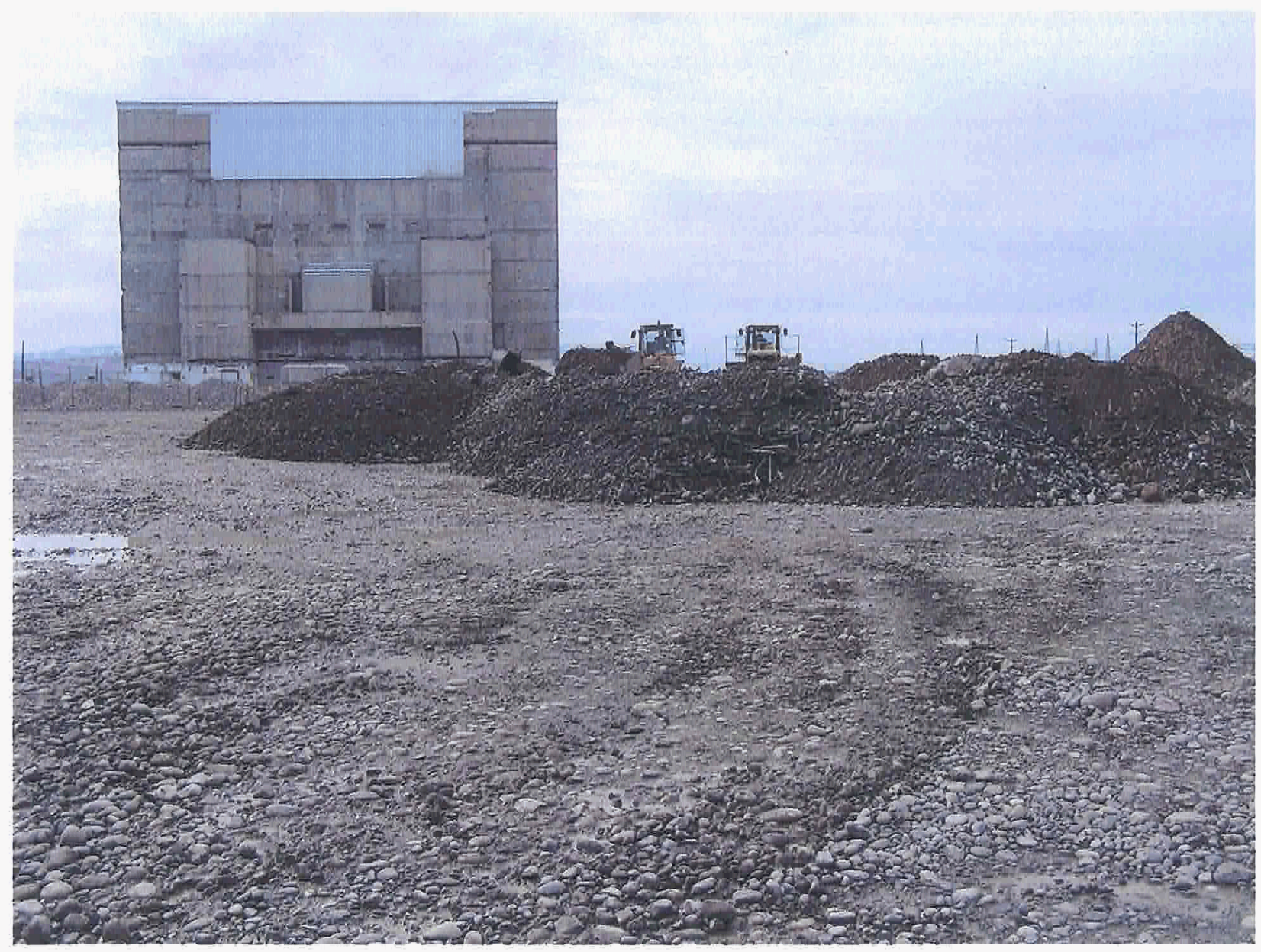


CVP-2006-00011

Rev. 0

Photograph B-8. Perforated and Non-Perforated Spacers.

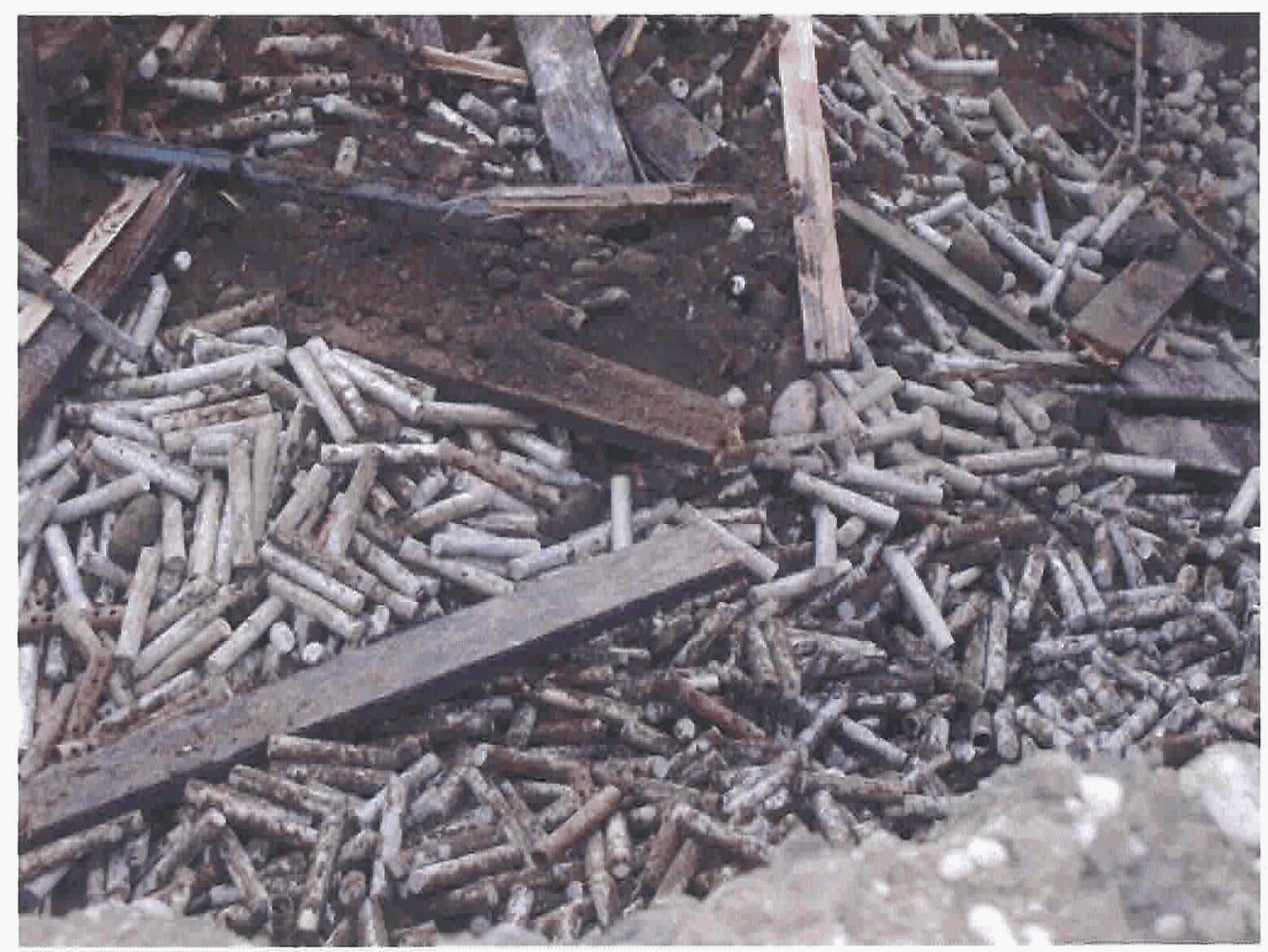


CVP-2006-00011

Rev. 0

Photograph B-9. Spent Nuclear Fuel.

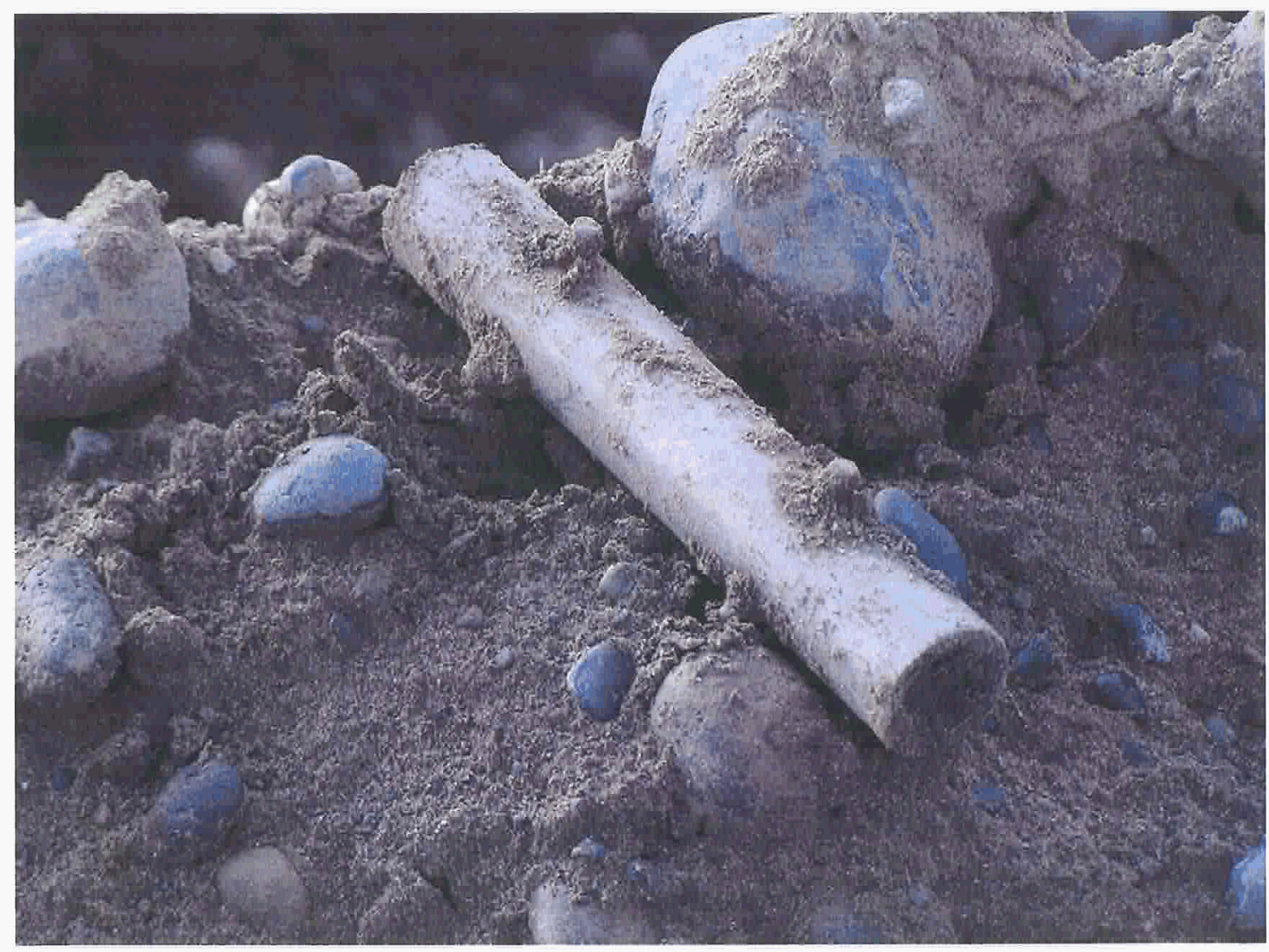


CVP-2006-00011

Rev. 0

Photograph B-10. Shield Plug.

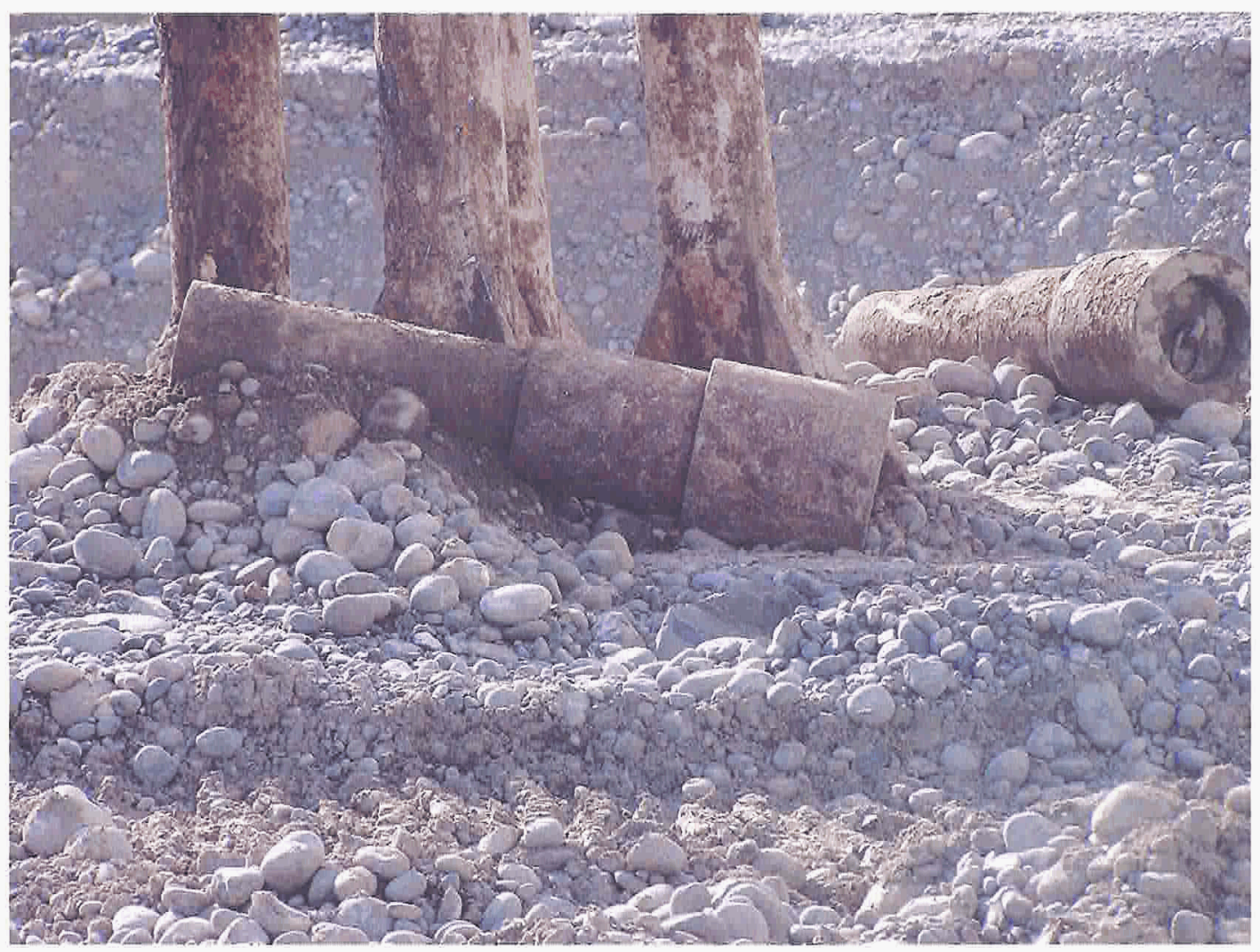


Photograph B-11. Boron Balls.

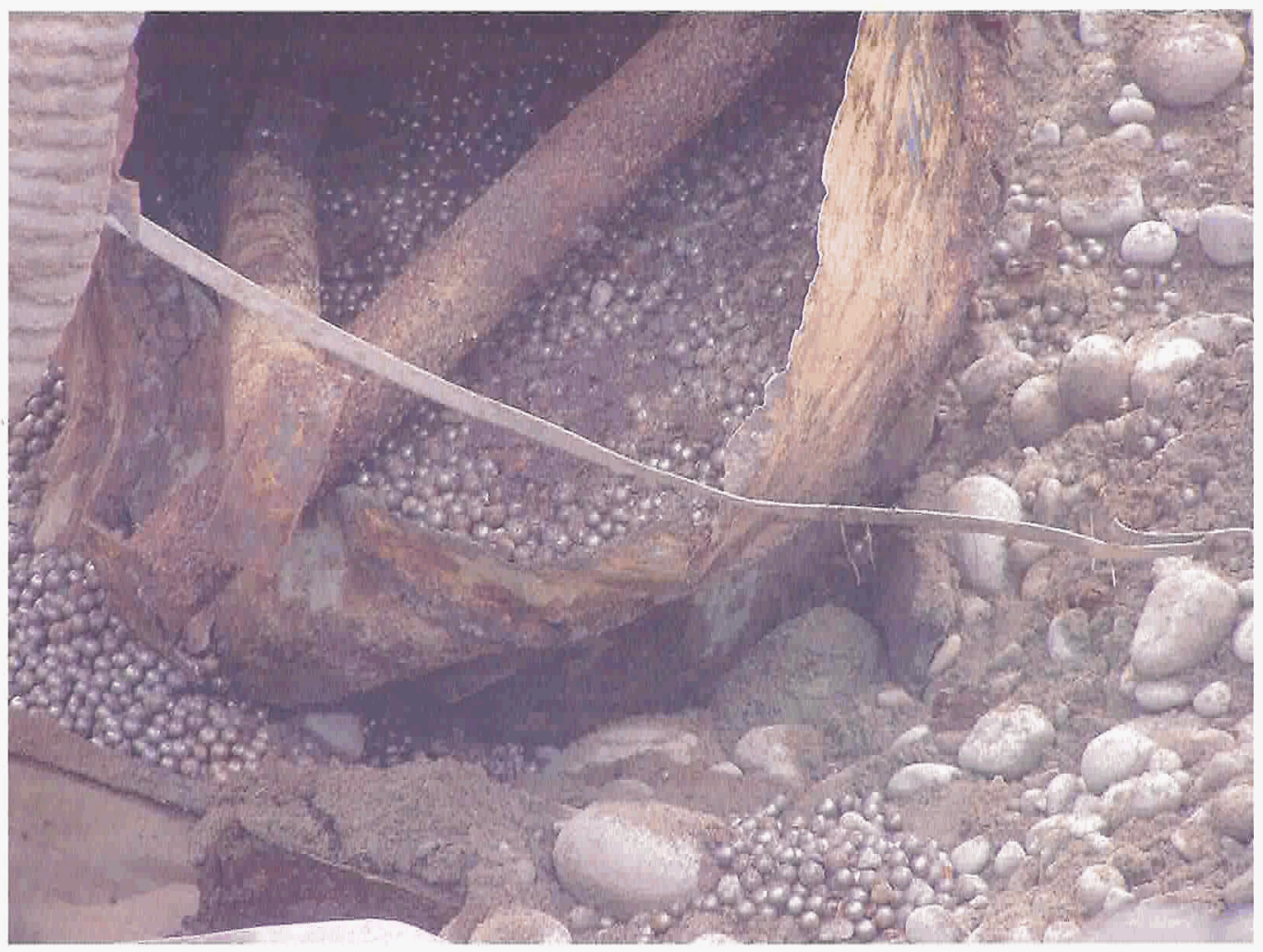




\section{Photograph B-12. Vertical Control Rods.}

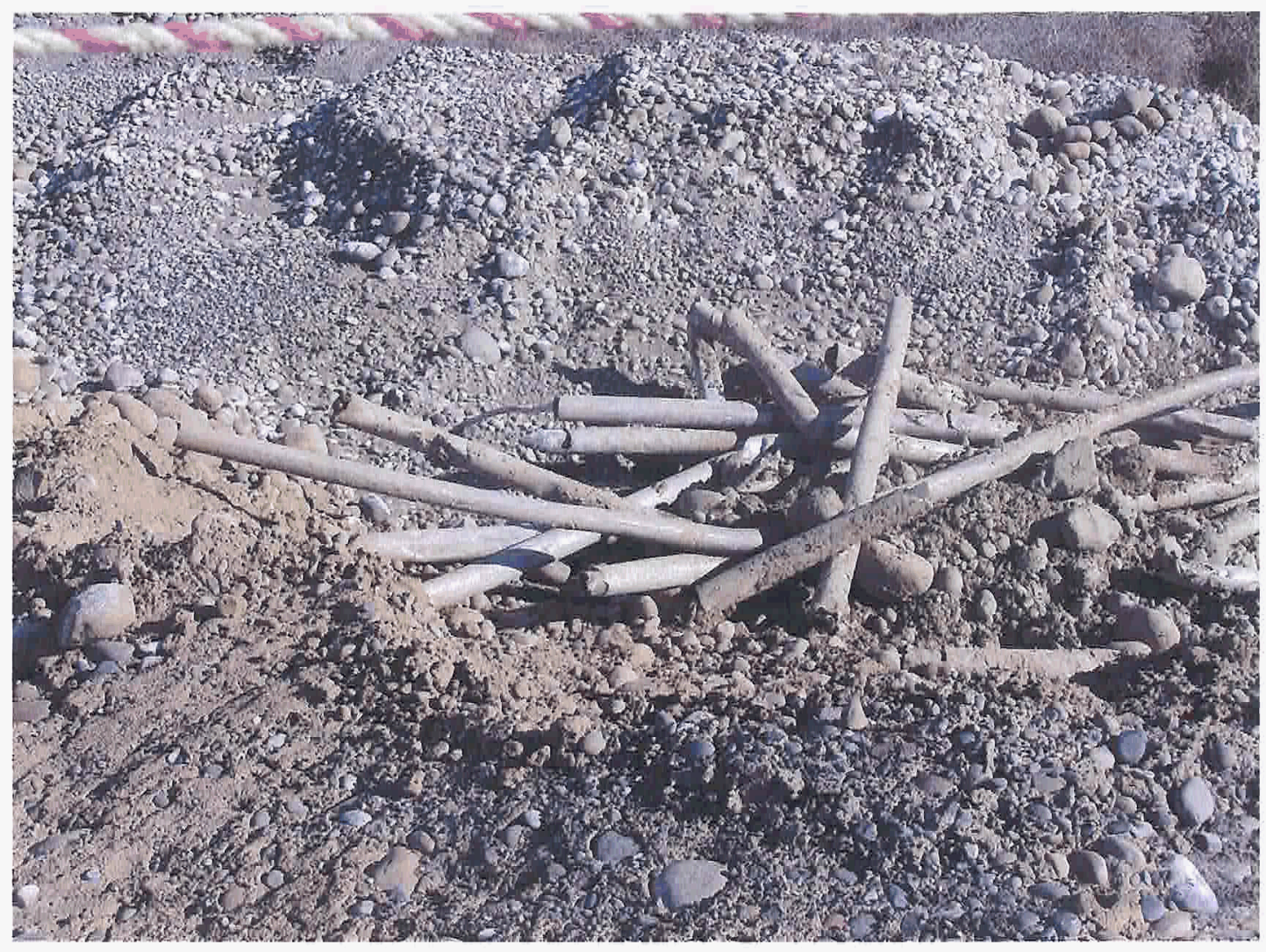




\section{Photograph B-13. Mercury Tubes.}

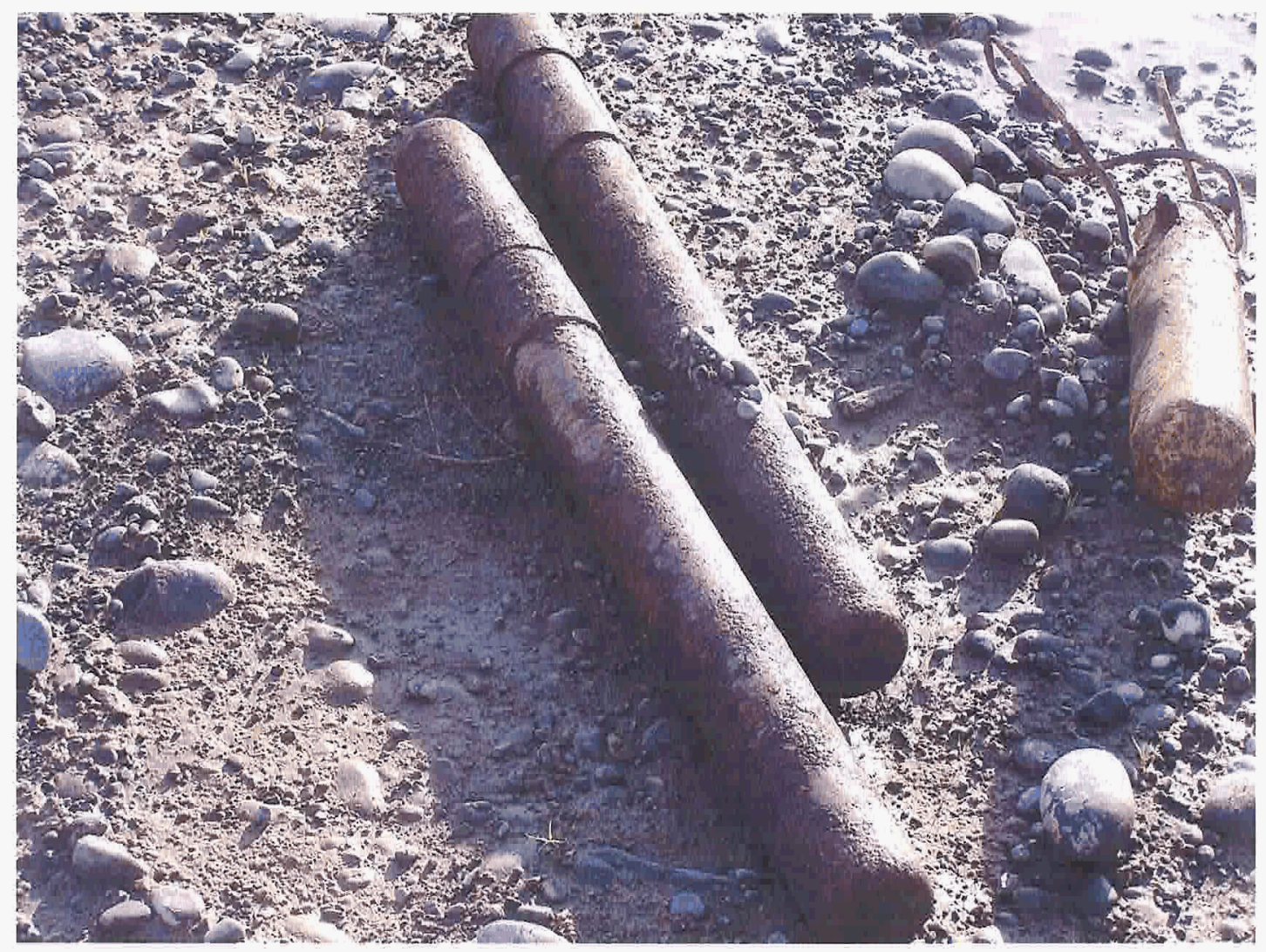


CVP-2006-00011

Rev. 0

B-14 
CVP-2006-00011

Rev. 0

APPENDIX C

DATA QUALITY ASSESSMENT

C-i 
CVP-2006-00011

Rev. 0

C-ii 


\section{C1.0 DATA QUALITY ASSESSMENT FOR THE 118-C-1 BURIAL GROUND}

\section{C1.1 OVERVIEW}

The data quality assessment (DQA) completes the data life cycle (i.e., planning, implementation, and assessment) that was initiated by the data quality objectives process. The DQA includes a review of the field logbook information (WCH 2006b, WCH 2006c) to verify sample location, date, and time. It also involves a scientific and statistical evaluation of the data to determine if they are of the right type, quality, and quantity to support their intended use for closeout decisions (EPA 2000).

This DQA was performed in accordance with data quality objectives found in the 100 Area Remedial Action Sampling and Analysis Plan (SAP) (DOE-RL 2001). The DQA is based on the guidelines presented in Guidance for Data Quality Assessment (EPA 2000). Statistical tests used in this DQA were performed as specified in the SAP and the Remedial Design Report/Remedial Action Work Plan for the 100 Area (RDR/RAWP) (DOE-RL 2005).

Prior to performing statistical tests, the field logbooks (WCH 2006b, WCH 2006c), the Site Specific Instruction for Close-out Approach for 118-C-1 (WCH 2006a), and the sample analytical data were evaluated. A portion of the cleanup verification sample analytical data was validated for compliance requirements (DOE-RL 2005). An evaluation is performed to determine if the laboratory carried out all steps required by the SAP and the laboratory contract governing the conduct of analysis and reporting of the data. Third-party data validation, in accordance with validation procedures specified in Data Validation Procedure for Chemical Analysis (BHI 2000a) and in Data Validation Procedure for Radiochemical Analysis (BHI 2000b), is performed as part of data evaluation. After validation and data evaluation, the appropriate statistical analyses are performed on the analytical data (Appendix E) to determine statistical values, as appropriate, for each contaminant. The cleanup verification sample analytical data are stored in the Environmental Restoration (ENRE) project-specific database prior to being submitted for inclusion in the Hanford Environmental Information System (HEIS) database and are also summarized in Appendix A.

\section{C1.2 LABORATORY QUALITY MEASURES}

All verification samples are subject to laboratory-specific quality assurance (QA) requirements, including instrument procurement, maintenance, calibration, and operation. Additional laboratory quality control (QC) checks are performed, as appropriate, for the analytical method at a rate of 1 per sample delivery group (SDG), or 1 per 20 samples, whichever is more frequent. Laboratory internal QC checks include the following: 
- Laboratory Contamination. Each analytical batch contains a laboratory (method) blank (material of similar composition as the samples with known/minimal concentrations of the analytes of interest) carried through the complete analytical process. The method blank is used to evaluate samples for false-positive results due to contamination at the laboratory.

- Analytical Accuracy. For most analyses, a known quantity of representative analytes of interest are added to a separate aliquot of a sample from the analytical batch, known as the matrix spike/matrix spike duplicate (MS/MSD). The recovery percentages of the added MS are used to evaluate analytical accuracy. For analyses not amenable to MS techniques (e.g., gamma energy analysis) or where analytical recovery is corrected via internal standards (e.g., alpha spectral analyses), accuracy is evaluated from recovery of the QC reference sample (i.e., laboratory control sample [LCS] or blank spike sample).

- Analytical Precision. Separate aliquots removed from the same sample container are analyzed for each analytical batch, and referred to as the laboratory duplicate or replicate. The replicate sample results (evaluated by relative percent differences [RPDs]) are used to assess the analytical precision achieved at the laboratory. However, natural heterogeneities often found in soil matrix samples also play a significant part in the RPD result.

- QC Reference Samples. A QC reference sample is prepared from an independent standard at a concentration other than that used for calibration, but within the calibration range. Reference samples provide an independent check on analytical technique and methodology.

Laboratories are also subject to periodic and random assessments of the laboratory performance, systems, and overall program. These assessments are performed by the Washington Closure Hanford QA group to ensure that the laboratories are performing within laboratory contract requirements.

\section{C1.3 DATA VALIDATION}

After sampling was completed, all of the fixed laboratory data from SDG K0528 were submitted for third-party Level $C$ validation. Level $C$ validation procedures are specified in Data Validation Procedure for Radiochemical Analysis (BHI 2000b) and Data Validation Procedure for Chemical Analysis (BHI 2000a).

Level $\mathrm{C}$ validation procedures were used to review and qualify the data for the following parameters:

- Sample holding times

- Method blanks

- MS/MSD recovery 
- Surrogate recovery

- Sample replicates (duplicates)

- Laboratory control sample results

- Data package completeness

- Achievement of required detection limits (RDLs) or contract required quantitation limits.

Data qualified as rejected (i.e., " $R$ " flagged) indicate that the associated analytical result is tainted by a major deficiency in the quality of the data. Rejected data are unsuitable for decision-making purposes. Data qualified as estimated (i.e., "J" flagged) indicate that the data is estimated, but may be used for decision-making purposes. Data qualified as undetected (i.e., "U" flagged) indicate the analyte was analyzed for but not detected. For nonradionuclides, nondetected results are reported at the practical quantitation limit (PQL). For radionuclides, nondetected results are reported at the actual value obtained from analysis (positive or negative but less than the minimum detected activity [MDA]), except for limited analyses where no value can be calculated and the analytes are reported nondetected at the MDA. All other validated results are considered accurate within the standard errors associated with the methods.

The adequacy of laboratory QA/QC was evaluated for precision, accuracy, completeness, and RDLs pursuant to the SAP (DOE-RL 2001). The organization performing the data validation reported that, of the data given formal validation, the laboratory met the standards for performance for precision $( \pm 30 \%)$, accuracy $( \pm 30 \%)$, and completeness (>90\%).

\section{SDG K0528}

This SDG comprises four samples (J135N5, J13518, J13519, and J13520) analyzed for carbon-14, tritium, nickel-63, isotopic plutonium, plutonium-241, americium-241, isotopic uranium, total strontium, gamma scan analytes (gamma spectroscopy), semivolatile organic compounds (SVOA), polychlorinated biphenyls (PCB), hexavalent chromium, and by inductively coupled plasma (ICP) analysis for metals including mercury. The samples were collected from the 118-C-1 overburden and the below contaminant level (BCL) staging piles. SDG K0528 was evaluated through a formal third-party validation process. A summary of the validation follows:

Radionuclides. No major deficiencies were found in the SDG K0528 radiological data. Minor deficiencies are as follows:

Due to a lack of MS analysis, third-party validation qualified all tritium results in SDG K0528 as estimates with "J" flags. Matrix spikes for some radionuclides are not typically performed by the laboratory. Other accuracy measures, such as blind audit samples, are used to assess laboratory accuracy for these radionuclides. Estimated data are useable for decision-making purposes. 
The radiochemical yield in sample $\mathrm{J} 13518$ was outside the QC limits at $18 \%$. Thirdparty validation qualified all of the plutonium-238 and plutonium-239/240 results in sample J13D61 as estimated with "J" flags. Estimated data are useable for decisionmaking purposes.

Nonradionuclides. No major or minor deficiencies were found in the SDG K0528 nonradiological data.

Third-party validation qualified all of the boron results, in the ICP metals analysis of SDG K0528, as estimated nondetects with "UJ" flags due to method blank (MB) contamination. Estimated data are useable for decision-making purposes.

Third-party validation qualified all of the antimony results, in the ICP metals analysis of SDG K0528, as estimated with "J" flags due to a low matrix spike (MS) recovery of $63.8 \%$. Estimated data are useable for decision-making purposes.

Third-party validation qualified all of the phenanthrene, pyrene, benzo(a)anthracene, and chrysene results, in the SVOA of SDG K0528, as estimated with "J" flags due to high matrix spike duplicate (MSD) recoveries of $207 \%, 168 \%, 142 \%$, and $168 \%$, respectively. Estimated data are useable for decision-making purposes.

Third-party validation qualified all of the fluoranthene results, in the SVOA of SDG K0528, as estimated with "J" flags due to high MS/MSD recoveries of $171 \%$ and $270 \%$, respectively. Estimated data are useable for decision-making purposes.

Third-party validation qualified all of the phenanthrene, fluoranthene, and pyrene results, in the SVOA of SDG K0528, as estimated with "J" flags due to elevated relative percent differences (RPDs) of $52 \%, 45 \%$, and $33 \%$, respectively. Estimated data are useable for decision-making purposes.

Third-party validation qualified all of the silicon results, in the SVOA of SDG K0528, as estimated with "J" flags due to a low LCS result of 50.5\%. Estimated data are useable for decision-making purposes.

Due to MB contamination, third-party validation qualified all of the detected bis(2-ethylhexyl)phthalate and di-n-butylphthalate results, in the SVOA of SDG K0528. The detected results for these two analytes, were reported at the required detection limit $(R Q L)$ (aka the contract required quantitation limit [CRQL]) as nondetected with " $U$ " flags. Estimated data are useable for decision-making purposes.

With the exception of the MB contamination and the LCS deficiencies, all of the quality control (QC) issues listed above involve the use of field collected soils. Soil samples are known for heterogeneity, which is known to cause difficulties in the evaluation of QC samples. 
Limited, random, or sample matrix-specific influenced batch QC issues, such as those identified in the radionuclide and nonradionuclide SDG K0528 data, are potential issues for any analysis. The number and types seen in SDG K0528 are within expectations for the matrix types and analyses performed. All of the data in SDG K0528 are useable for decision-making purposes.

\section{C1.4 LABORATORY DATA EVALUATION}

The following paragraphs include a data evaluation of the remaining verification sample SDGs (J00094, J00095, K0495, K0526, K0527, K0549, K0554, K0559, K0590, K0593, and K0790) for the 118-C-1 waste site. Comments on the comparability of the samples to the project splits are presented in section C1.5.

\section{SDG J00094}

This data set comprises two field samples (J13504, J13505). Samples J13504 and J13505 are project field splits of the Area 1 (J134Y7) and the Area 2 (J13502) "A1" samples. Sample J13505 was analyzed for carbon-14, tritium, nickel-63, isotopic plutonium, plutonium-241, americium-241, isotopic uranium, total strontium, gamma scan analytes (gamma spectroscopy), SVOAs, PCBs, hexavalent chromium, and by inductively coupled plasma (ICP) analysis for metals including mercury. Sample J13504 was only analyzed for the radiological contaminants listed above.

Radionuclides. No major or minor deficiencies were found in the SDG J00094 radiological data.

Nonradionuclides. One major deficiency was identified in the SDG J00094 nonradiological data. Major and minor deficiencies are as follows:

A major deficiency was identified for the analyte 2,4-dinitrophenol in SDG J00094. The MS and MSD recoveries of 2,4-dinitrophenol were both $0 \%$. Furthermore, the LCS recovery was below the acceptance criteria at $19 \%$ and the analyte was not detected in the field sample (J13505). The 2,4-dinitrophenol data for the field split sample J13505 is not acceptable for decision-making purposes. The project has qualified this result as rejected with an " $\mathrm{R}$ " flag.

Recoveries of hexavalent chromium in the associated MS and MSD are below the acceptance criteria at $41 \%$ and $54 \%$, respectively. The sample preparation and analytical system are shown to be acceptable by the associated LCS recovery at $105 \%$. The MS/MSD results are attributed to a hostile reduction/oxidation environment within the sample matrix that caused the added hexavalent chromium spike to convert to trivalent chromium. Under these conditions no hexavalent chromium is expected, nor observed, in the sample matrix (J13505) from which the MS and MSD were prepared. The data are useable for decision-making purposes. 
The MS and/or MSD recoveries for aluminum, iron, and manganese are outside the acceptance criteria. Because the added spike concentrations of these analytes are less than $1 / 4^{\text {th }}$ of the native concentration in the sample, the resulting percent recovery information is not representative of the method performance. The method performance is demonstrated by the LCS result. The data are useable for decision-making purposes.

The MS and/or MSD recoveries for antimony, calcium, silicon, silver, and cadmium are outside the acceptance criteria. Relative percent differences (RPDs) and LCS recoveries are within the acceptance criteria. A matrix interference may be indicated in this data. The data may be considered estimated but useable for decision-making purposes.

\section{SDG J00095}

This data set contains one field sample (J135T7). Sample J135T7 is a project field split of the overburden/BCL "A1" sample (J135R0). Sample J135T7 was analyzed for volatile organic compounds (VOCs) and total petroleum hydrocarbons (TPH).

Radionuclides. No major or minor deficiencies were found in the SDG J00095 radiological data.

Nonradionuclides. No major deficiencies were found in the SDG J00095 chemical data. Minor deficiencies are as follows:

In the VOC analysis, the MS/MSD recoveries for tetrachloroethene are above the acceptance criteria at $161 \%$ and $168 \%$, respectively. The laboratory has qualified the data with an "N" flag. A high bias is suggested in the field data for tetrachloroethene. Furthermore, the analyte was not detected in the only sample (J135T7); therefore, a high bias is of no consequence. The data are acceptable for decision-making purposes.

\section{SDG K0495}

This data set comprises seven field samples (J12XL8, J12XL9, and J12XM0 through J12XM4). Samples J12XL8, J12XL9, and J12XM0 through J12XM4 are the focused samples designated FS-1 through FS-7, respectively. All but sample J12XM3 were analyzed for ICP metals. Samples J12XM2 and J12XM3 were also analyzed for VOA, SVOAs, PCBs, and TPH. Additionally, sample J12XM4 was analyzed by gamma spectroscopy.

Radionuclides. No major deficiencies were found in the SDG K0495 radiological data. Minor deficiencies are as follows: 
The RPD calculated for thorium-228, in the laboratory duplicate, is above the acceptance criteria (less than $30 \%$ ) at $39 \%$. Elevated RPDs are attributed to sample matrix heterogeneities commonly found in environmental soil samples. The data are useable for decision-making purposes.

Nonradionuclides. No major deficiencies were found in the SDG K0495 nonradiological data. Minor deficiencies are as follows:

In the VOA, 33 of $77 \mathrm{MS}$ or MSD recoveries were above the acceptance criteria. Three of 35 LCS recoveries were above the acceptance criteria. The continuing calibration check for bromomethane was out of criteria, also high. All of these deficiencies suggest a high bias in the VOA data for SDG K0495. High biased data are acceptable for decision-making purposes.

In the SVOA, the MS and/or MSD recoveries for isophorone, nitrobenzene, 2,4-dinitrophenol, 1,2,4-trichlorobenzene, 4-chloro-3-methylphenol, and 2-methyl naphthalene were below the acceptance criteria in the range of $41 \%$ to $59 \%$. The LCS recoveries for these analytes indicate that the analytical system was functioning correctly. A matrix interference is possible. The field data for these analytes in SDG K0495 may be considered estimated. Estimated data are useable for decision-making purposes.

In the ICP metals analysis, the LCS recovery for silicon is below the acceptance criteria at $59 \%$. The project has qualified all associated silicon data in SDG K0495 as estimated with "J" flags for detected results and "UJ" flags for nondetected results. Estimated data are useable for decision-making purposes.

In the ICP metals analysis, the MS recoveries for aluminum, iron, antimony, and silicon are outside the acceptance criteria. The added spike concentrations for these analytes are much less than the concentrations found in the original samples. Therefore, the spike concentrations were overwhelmed by the typical analytical variation in the original sample concentrations, resulting in matrix spike recoveries that are not representative of the method performance. Method performance was confirmed by serial dilutions and PDSs at more meaningful concentrations. The PDSs for these analytes all recovered within the acceptance criteria, in the range of $88.8 \%$ to $108 \%$. The data are useable for decision-making purposes.

\section{SDG K0526}

This data set comprises eleven field samples (J13500 through J13503, J13506 through $\mathrm{J} 13509$, and J134Y7 through J135Y9). Samples J134Y9 and J13508 are field duplicates of the Area 1 and Area 2 "A1" samples J134Y7 and J13502, respectively. Sample J13509 is the equipment blank for the site. All of the samples except J13509 were analyzed for carbon-14, tritium, nickel-63, isotopic plutonium, plutonium-241, americium-241, isotopic uranium, total strontium, and by gamma spectroscopy. 
Samples J13502, J13503, and J13506 through J13508 were analyzed for SVOCs, PCBs, ICP metals, and hexavalent chromium. Sample J13509 was analyzed for SVOCs, ICP metals, and hexavalent chromium. Samples J13500, J13501, and J134Y7 through J135Y9 were analyzed for ICP metals.

Radionuclides. No major deficiencies were found in the SDG K0526 radiological data. Minor deficiencies are as follows:

The radiochemical yield for the isotopic plutonium analysis in sample J135P1 was outside the QC limits at $16 \%$. The project has qualified all of the plutonium-238 and plutonium-239/240 results in sample J135P1 as estimated and applied "J" flags to the data. Estimated data are useable for decision-making purposes.

Nonradionuclides. One major deficiency was identified in the SDG K0526 nonradiological data. Major and minor deficiencies are as follows:

In the ICP metals analysis, a major deficiency was identified for the analyte silicon. The LCS result for silicon is well below the acceptance criteria at $2.8 \%$. The project has qualified the silicon results for all samples in SDG K0526 as rejected and applied "R" flags to the data (applies to samples J13500 through J13503, J13506 through J13509, and J134Y7 through J135Y9).

In the SVOC analysis, the common laboratory contaminants bis(2-ethylhexyl)phthalate and di-n-butylphthalate were found in the MB at concentrations below the CRQL. These low-level detections have no impact on the field sample data. The data are useable for decision-making purposes.

In the ICP metals analysis, the MS results for aluminum, calcium, iron, magnesium, antimony, and silicon are outside the acceptance criteria. The added spike concentrations for these analytes were inappropriate compared to the original sample concentrations such that the matrix spike recoveries are not representative of the method performance. Method performance was confirmed by serial dilutions and PDSs at more meaningful concentrations. The PDSs for these analytes all recovered within the acceptance criteria in the range of $91.4 \%$ to $102.6 \%$. The data are useable for decision-making purposes.

\section{SDG K0527}

This data set comprises eight field samples (J13510 through J13517), analyzed for carbon-14, tritium, nickel-63, isotopic plutonium, plutonium-241, americium-241, isotopic uranium, total strontium, SVOAs, PCBs, ICP metals (including mercury) and by gamma spectroscopy.

Radionuclides. No major deficiencies were found in the SDG K0527 radiological data. Minor deficiencies are as follows: 
Tracer yields in the isotopic plutonium analysis for samples $\mathrm{J} 13510, \mathrm{~J} 13513, \mathrm{J13514}$, and $\mathrm{J} 13515$ were below the $20 \%$ criteria in the range of $13-19 \%$. The project has qualified the plutonium-238 and plutonium 239/240 results in samples J13510, J13513, $\mathrm{J} 13514$, and $\mathrm{J} 13515$ as estimated and applied "J" flags to the data. Estimated data are useable for decision making purposes.

Minimum detection activities (MDAs) for plutonium-241 are above the CRQL (15pCi/g) in the range of $18 \mathrm{pCi} / \mathrm{g}$ to $27 \mathrm{pCi} / \mathrm{g}$. These results are all well below the lookup value for plutonium-241 (1061 pCi/g) and are, therefore, acceptable. The data are useable for decision making purposes.

Nonradionuclides. One major deficiency was identified in the SDG K0527 nonradiological data. Major and minor deficiencies are as follows:

In the ICP metals analysis, a major deficiency was identified for the analyte silicon. The LCS result for silicon is well below the acceptance criteria at $2.8 \%$. The project has qualified the silicon results for all samples in SDG K0527 as rejected and applied "R" flags to the data (applies to samples J13510 through J13517).

One of $128 \mathrm{MS} / \mathrm{MSD}$ results, in the SVOC analysis, is below the acceptance criteria. The MSD result for 2,4-dimethylphenol is $49 \%$. The project has qualified the 2,4-dimethylpehol results in SDG K0527 as estimated and applied "J" flags to the data. Estimated data are useable for decision-making purposes.

In the ICP metals analysis, the MS results for aluminum, iron, magnesium, lead, antimony, and silicon are outside the acceptance criteria. The added spike concentrations for these analytes were inappropriate compared to the original sample concentrations because the matrix spike recoveries are not representative of the method performance. Method performance was confirmed by serial dilutions and PDSs at more meaningful concentrations. The PDSs for these analytes all recovered within the acceptance criteria in the range of $98.5 \%$ to $109.5 \%$. The data are useable for decision-making purposes.

The RPD result for boron in the ICP metals analysis is above the acceptance criteria (greater than $30 \%$ ) at $52.3 \%$. The project has qualified the boron results in SDG K0527 as estimated and applied "J" flags to the data. Estimated data are useable for decision making purposes.

\section{SDG K0549}

This data set comprises seventeen field samples (J135R0 through J135R9, and J135T0 through J135T6), analyzed for VOA and TPH. 
Radionuclides. The SDG K0549 samples were not analyzed for radiological contaminants.

Nonradionuclides. No major deficiencies are identified in the SDG K0549 nonradiological data. Minor deficiencies are as follows:

Four of 70 MS recoveries in the VOA were outside the acceptance criteria. MS and MSD results for 1,1,2,2-tetrachloroethane are below the acceptance criteria: both at $58 \%$. MS results for carbon disulfide and the common laboratory contaminant acetone are above the acceptance criteria at $172 \%$ and $311 \%$, respectively. The project has qualified the 1,1,2,2-tetrachloroethane, carbon disulfide, and acetone results in SDG K0549 as estimated and applied "J" flags to the data. Estimated data are useable for decision-making purposes.

\section{SDG K0554}

This data set comprises six field samples (J13522 through J13525, J135N8, and J135N9) analyzed for carbon-14, tritium, nickel-63, isotopic plutonium, plutonium-241, americium-241, isotopic uranium, total strontium, VOAs, SVOAs, PCBs, ICP metals (including mercury), hexavalent chromium, and by gamma spectroscopy.

Radionuclides. No major deficiencies were found in the SDG K0554 radiological data. Minor deficiencies are as follows:

Minimum detection activities (MDAs) for plutonium-241 are above the CRQL (15pCi/g) in the range of $16 \mathrm{pCi} / \mathrm{g}$ to $21 \mathrm{pCi} / \mathrm{g}$. These results are all well below the lookup value for plutonium-241 (1061 pCi/g) and are, therefore, acceptable. The data are useable for decision-making purposes.

Nonradionuclides. One major deficiency was identified in the SDG K0554 nonradiological data. Major and minor deficiencies are as follows:

The analyte 2,4-dinitrophenol recovered at $6 \%$ in the MS. This result is considered a major deficiency. The project has qualified all of the 2,4-dinitrophenol results in SDG K0554 as rejected with " $R$ " flags. Rejected data are not useable for decision-making purposes.

Three of 70 MS recoveries in the VOA were outside the acceptance criteria. The MS result for 1,1,2,2-tetrachloroethane is below the acceptance criteria, both at $59 \%$. MS results for bromoform and acetone are above the acceptance criteria at $146 \%$ and $418 \%$, respectively. The LCS result for acetone was also above the acceptance criteria at $321 \%$. The project has qualified the 1,1,2,2-tetrachloroethane results and the detected bromoform and acetone results in SDG K0554 as estimated with "J" flags applied to the data. Estimated data are useable for decision-making purposes. 
The common laboratory contaminant methylene chloride was identified below the $\mathrm{CRQL}$ in the VOA's MB. This low-level detection has no impact on the field sample data for methylenechloride. The data are useable for decision-making purposes.

The common laboratory contaminants bis(2-ethylhexyl)phthalate and di-n-butylphthalate were identified below the PQL in the MB for the SVOA, at $31 \mathrm{mg} / \mathrm{kg}$ and $18 \mathrm{mg} / \mathrm{kg}$, respectively. The project has qualified results for these analytes as estimated, applied "J" flags to the data. Estimated data are useable for decision-making purposes.

The SVOA analysis has multiple analytes with MS and/or LCS recoveries below the acceptance criteria, in the range of $37 \%$ to $56 \%$. The project has qualifed these results as estimated and applied "J" flags to the data. Estimated data are useable for decisionmaking purposes. The affected analytes in SDG K0554 are: 2-methylphenol, 4-methylphenol, nitrobenzene, isophorone, 2-nitrophenol, 2,4dimethylphenol, 2,4-dichlorophenol, 1,2,4-trichlorobenzene, 4-chloro-3-methylphenol, and 2-methylnaphthalene.

In the ICP metals analysis, the LCS result for silicon is below the acceptance criteria at $14.5 \%$. The project has qualified the all of the silicon results in SDG K0554 as estimated and applied "J" flags to the data. Estimated data are useable for decisionmaking purposes.

In the ICP metals analysis, the MS results for aluminum, iron, mangnesium, and antimony are outside the acceptance criteria. The added spike concentrations for these analytes were inappropriate compared to the original sample concentrations because the matrix spike recoveries are not representative of the method performance. Method performance was confirmed by serial dilutions and PDSs at acceptable concentrations. The PDSs for these analytes all recovered within the acceptance criteria in the range of $96.4 \%$ to $100.7 \%$. The data are useable for decision-making purposes.

\section{SDG K0559}

This data set comprises 10 field samples (J135P0 through J135P9) analyzed for carbon-14, tritium, nickel-63, isotopic plutonium, plutonium-241, americium-241, isotopic uranium, total strontium, VOAs, SVOAs, PCBs, ICP metals (including mercury), hexavalent chromium, and by gamma spectroscopy.

Radionuclides. No major deficiencies were found in the SDG K0559 radiological data. Minor deficiencies are as follows:

Minimum detection activities (MDAs) for plutonium-241 are above the CRQL (15pCi/g) in the range of $18 \mathrm{pCi} / \mathrm{g}$ to $22 \mathrm{pCi} / \mathrm{g}$. These results are all well below the lookup value for plutonium-241 (1061 pCi/g) and are, therefore, acceptable. The data are useable for decision-making purposes. 
The radiochemical yield for americium-241 in sample J135P1 was outside the QC limits at $16 \%$. The low tracer yield also raised the MDA to $1.1 \mathrm{pCi} / \mathrm{g}$, which is just above the $\mathrm{RDL}$ of $1.0 \mathrm{pCi} / \mathrm{g}$. The project has qualified all of the americium-241 results in sample J135P1 as estimated and applied "J" flags to the data. Estimated data are useable for decision-making purposes.

Nonradionuclides. One major deficiency was identified in the SDG K0559 nonradiological data. Major and minor deficiencies are as follows:

In the SVOC analysis, the analyte fluoroanthene has MS/MSD recoveries of $6 \%$ and $4 \%$, respectively. This result is considered a major deficiency. The project has qualified all of the fluroanthene results in SDG K0559 as rejected with "R" flags. Rejected data are not useable for decision-making purposes.

The common laboratory contaminant methylenechloride was identified below the CRQL in the VOA's MB. This low-level detection has no impact on the field sample data for methylenechloride. The data are useable for decision-making purposes.

Due to an equipment problem at the laboratory, the VOA was performed 3 days past the 14 day hold time. The samples were all refrigerated and stored in sealed containers after collection. The additional 3 days will have no impact on the field sample data. The data are useable for decision-making purposes.

The common laboratory contaminants bis(2-ethylhexyl)phthalate and di-n-butylphthalate were identified below the PQL in the MB for the SVOA, at $31 \mathrm{mg} / \mathrm{kg}$ and $18 \mathrm{mg} / \mathrm{kg}$, respectively. The project has qualified these results as estimated, applied "J" flags to the data. Estimated data are useable for decision-making purposes.

The SVOA analysis has multiple analytes with MS and/or LCS recoveries below the acceptance criteria, in the range of $37 \%$ to $59 \%$. The project has qualifed these results as estimated and applied "J" flags to the data. Estimated data are useable for decision making purposes. The affected analytes in SDG K0559 are: 2-methylphenol, 4-methylphenol, nitrobenzene, isophorone, 2-nitrophenol, 2,4-dimethylphenol, 1,2,4-trichlorobenzene, 4-chloro-3-methylphenol, 2-methylnaphthalene, 2-chlorophenol, 1,3-dichlorobenzene, 1,4-dichlorobenzene, 1,2-dichlorobenzene, n-nitroso-di-npropylamine, hexachloroethane, 2,4-dinitrophenol, dibenzofuran, fluorene, phenanthrene, anthracene, carbazole, benzo(a)anthracene, chrysene, benzo(b)fluoranthene, benzo(k)fluoranthene, benzo(a)pyrene, and indeno(1,2,3,-cd)pyrene.

In the ICP metals analysis, the LCS recovery for silicon is low at $14.5 \%$. The project has qualified all associated silicon data in SDG K0559 as estimated with "J" flags for detected results and "UJ" flags for nondetected results. Estimated data are useable for decision-making purposes. 
In the ICP metals analysis, the MS results for aluminum, iron, magnesium, and antimony are outside the acceptance criteria. The added spike concentrations for these analytes were inappropriate compared to the original sample concentrations because the matrix spike recoveries are not representative of the method performance. Method performance was confirmed by serial dilutions and PDSs at a more meaningful concentrations. The PDSs for these analytes all recovered within the acceptance criteria in the range of $100.9 \%$ to $109.0 \%$. The data are useable for decision making purposes.

The RPDs calculated for arsenic and boron, in the ICP metals laboratory duplicate, are above the acceptance criteria (less than $30 \%$ ) at $35.7 \%$ and $35.3 \%$, respectively. Elevated RPDs are attributed to sample matrix heterogeneities commonly found in environmental soil samples. The data are useable for decision-making purposes.

In the TPH analysis the MB result $(177 \mathrm{mg} / \mathrm{kg}$ ) was greater than the PQL. This suggests a high bias in the field sample results. All of the samples that had detections for TPH in SDG K0559 were less than the MB result. The project has qualified the detected TPH results in SDG K0559 as estimated and applied "J" flags to the data. Estimated and/or high biased data are useable for decision making purposes.

\section{SDG K0590}

This data set comprises two field samples (J13HN3, J13HN4) analyzed for TPH and ICP metals (including mercury)

Radionuclides. The SDG K0590 samples were not analyzed for radiological contaminants.

Nonradionuclides. No major deficiencies were identified in the SDG K0590 nonradiological data. Minor deficiencies are as follows:

In the ICP metals analysis, the LCS recovery for silicon is low at $19.8 \%$. The project has qualified all associated silicon data in SDG K0590 as estimated with "J" flags for detected results and "UJ" flags for nondetected results. Estimated data are useable for decision-making purposes.

In the ICP metals analysis, the MS results for aluminum, iron, mangnesium, and antimony are outside the acceptance criteria. The added spike concentrations for these analytes were inappropriate compared to the original sample concentrations because the matrix spike recoveries are not representative of the method performance. Method performance was confirmed by serial dilutions and PDSs at appropriate concentrations. The PDSs for these analytes all recovered within the acceptance criteria in the range of $94.2 \%$ to $107.4 \%$. The data are useable for decision making purposes. 
The RPDs calculated for calcium and molybdenum, in the ICP metals laboratory duplicate, are above the acceptance criteria (less than $30 \%$ ) at $48.6 \%$ and $53.7 \%$, respectively. Elevated RPDs are attributed to sample matrix heterogeneities commonly found in environmental soil samples. The data are useable for decision-making purposes.

\section{SDG K0790}

This data set comprises five field samples (J14Y70 through J14Y74, from the spent nuclear fuel bunker footprints) analyzed for isotopic plutonium, plutonium-241, americium-241, isotopic uranium, and by gamma spectroscopy.

Radionuclides. No major deficiencies were identified in the SDG K0790 nonradiological data. Minor deficiencies are as follows:

Minimum detection activities (MDAs) for plutonium-241 are above the CRQL (15pCi/g) in the range of $24 \mathrm{pCi} / \mathrm{g}$ to $32 \mathrm{pCi} / \mathrm{g}$. These results are all well below the lookup value for plutonium-241 $(1,061 \mathrm{pCi} / \mathrm{g})$ and are therefore acceptable. The data are useable for decision-making purposes.

Nonradionuclides. The SDG K0790 samples were not analyzed for nonradiological contaminants.

\section{C1.4.1 MAJOR DEFICIENCIES}

Any data anomaly that causes final data to be qualified as rejected (" $R$ " flagged) is considered a major deficiency. Major deficiencies were identified in SDGs J00094, K0526, K0527, K0554, and K0559. Associated data has been rejected.

\section{C1.4.2 MINOR DEFICIENCIES}

Sample Holding Times. The method-specific holding times were met for all samples in the 118-C-1 verification data set, except for the VOA samples in SDG K0559.

Method Blanks. The method blank is used to evaluate false-positive results in samples due to contamination during handling at the laboratory.

Radionuclides. All of the radionuclide method blank results were within the acceptance criteria.

Nonradionuclides. Minor method blank deficiencies were identified in SDGs K0526, K0528, K0554, and K0559 (see above). Some data are qualified as estimated. Estimated data are useable for decision-making purposes. 
MS/MSDs Recoveries. Recoveries of spiked analytes in the MS/MSD pair(s) are used to evaluate method efficiency and the effect of the sample matrix on the environmental sample results.

Radionuclides. A minor deficiency is identified in SDG K0528. Some data are qualified as estimated. Estimated data are useable for decision-making purposes.

Nonradionuclides. Minor deficiencies in the MS/MSD recoveries are identified in SDGs J00094, J00095, K0495, K0526, K0527, K0528, K0549, K0554, K0559, and K0590. Some data are qualified as estimated. Estimated data are useable for decisionmaking purposes.

RDL Comparison. Reported analytical detection levels for nondetected analytes were compared to the RDLs (required detection levels) specified in the SAP (DOE-RL 2001). Where detected results are obtained, an evaluation of detection limits is not performed.

Radionuclides. All of the reported contaminant of concern (COC) MDAs are sufficiently low for decision-making purposes. Minor deficiencies in SDGs K0527, $\mathrm{K} 0554, \mathrm{~K} 0559$, and $\mathrm{K} 0790$ are identified. All values meet the site cleanup criteria as demonstrated in the calculation briefs (Appendix E) and discussed in this cleanup verification package.

Nonradionuclides. All of the reported MDLs are sufficiently low for decision-making purposes. All values meet the site cleanup criteria as discussed in this cleanup verification package.

Precision and Accuracy Evaluation. RPD evaluation of the main sample(s) versus the laboratory duplicate(s) are routinely performed by the laboratory, and any deficiencies in those calculations are reported by SDG in section C1.4.

\section{C1.5 FIELD QUALITY ASSURANCE/QUALITY CONTROL}

Field QA/QC measures were used to assess potential sources of error and cross contamination of soil samples that could bias results. Field QA/QC samples listed in the field logbook (WCH 2006b, WCH 2006c) are summarized in Table C-1. The main and QA/QC sample results are presented in Appendix A.

Table C-1. Summary of Field Quality Control Samples.

\begin{tabular}{|l|c|c|c|}
\hline Sample location (designation) & Main Sample & Duplicate Sample & Split Sample \\
\hline Area 1(A1) & $\mathrm{J} 134 \mathrm{Y} 7$ & $\mathrm{~J} 134 \mathrm{Y} 9$ & $\mathrm{~J} 13504$ \\
\hline Area 2(A1) & $\mathrm{J} 13502$ & $\mathrm{~J} 13508$ & $\mathrm{~J} 13505$ \\
\hline
\end{tabular}


Field duplicate samples are collected to provide a relative measure of the local heterogeneity in the sampling medium, unlike laboratory duplicates that are used to evaluate precision in the analytical process. The field duplicates are evaluated by computing the RPD of the duplicate samples for each COC. Only analytes with values above five times the detection limits for both the main and duplicate samples are calculated and evaluated. The 95\% upper confidence limit (UCL) calculation brief in Appendix $E$ provides details on duplicate pair evaluation and RPD calculation. The data are suitable for the intended purpose of cleanup verification.

Split samples are collected to provide a relative measure of the variability in the sampling, sample handling, and analytical techniques used by commercial laboratories. The field main and split samples are evaluated by computing the RPD of the split samples for each COC to determine the usability of the verification data. The U.S. EPA Contract Laboratory Program duplicate sample comparison methodology, USEPA Contract Laboratory Program National Functional Guidelines for Inorganic Data Review (EPA 1994), is used as an initial test of the data from the splits. Only analytes that had values above five times the contractual RDL for both the main and split sample were compared. The $95 \%$ UCL calculation briefs in Appendix E provide details on split pair RPD calculation. The acceptance criteria RPD for project-split samples is $\leq 30 \%$ (less than or equal to $30 \%$ ).

\section{Radionuclides.}

- The RPDs calculated for the Area 1 field duplicate sample (J134Y9) analyte potassium-40 is above the acceptance criteria (less than $30 \%$ ) at $62 \%$. Elevated RPDs, such as this, in the analysis of environmental soil samples, are largely attributed to heterogeneities in the soil matrix and only in small part attributed to precision and accuracy issues at the laboratory. The data are useable for decisionmaking purposes.

\section{Nonradionuclides.}

- The RPD calculated for the Area1 project split sample (J13504) analytes aluminum, chromium, iron, vanadium and magnesium are above the acceptance criteria (30\%) at $52 \%, 48 \%, 36 \%, 45 \%$, and $36 \%$, respectively. The RPD calculated for the Area 2 project split sample (J13505) analyte vanadium is above the acceptance criteria at $35 \%$. These RPD results are attributed to heterogeneities in the soil matrix. The data are useable for decision making purposes.

RPDs for the remaining radionuclides and nonradionuclide analytes were either within the acceptance criteria or were not calculated because an evaluation of the data shows the analytes were not detected in both the main and duplicate (or main and split) sample at more than 5 times the target detection limit. RPDs of analytes detected at low concentrations (less than five times the detection limit) are not considered to be indicative of the analytical system performance. The data are useable for decisionmaking purposes. 
A secondary check of the data variability is used when one or both of the samples being evaluated (main and duplicate or main and split) is less than 5 times the target detection limit (TDL), including undetected analytes. In these cases, a control limit of \pm 2 times the TDL is used (Appendix E) to indicate that a visual check of the data is required by the reviewer. The Area 1 cesium-137, radium-226, radium-228, and cobalt results, as well as the Area 2 cobalt results required this check. These results are all attributed to heterogeneities in the sample matrix from which the samples were collected. A visual inspection of all of the data is also performed. No additional major or minor deficiencies were noted.

\section{C1.6 SUITABILITY OF DATA}

The DQA for the 118-C-1 waste site determined that the sample design and data are of the right type, quality, and quantity to support site cleanup verification decisions, within specified error tolerances.

\section{C2.0 REFERENCES}

BHI, 2000a, Data Validation Procedure for Chemical Analysis, BHI-01435, Rev. 0, Bechtel Hanford, Inc., Richland, Washington.

BHI, 2000b, Data Validation Procedure for Radiochemical Analysis, BHI-01433, Rev. 0, Bechtel Hanford, Inc., Richland, Washington.

DOE-RL, 2001, 100 Area Burial Grounds Remedial Action Sampling and Analysis Plan, DOE/RL-2001-35, Rev. 0, U.S. Department of Energy, Richland Operations Office, Richland, Washington.

DOE-RL, 2005, Remedial Design Report/Remedial Action Work Plan for the 100 Area, DOE/RL-96-17, Rev. 5, U.S. Department of Energy, Richland Operations Office, Richland, Washington.

EPA, 1994, USEPA Contract Laboratory Program National Functional Guidelines for Inorganic Data Review, EPA 540/R-94/013, U.S. Environmental Protection Agency, Washington, D.C.

EPA, 2000, Guidance for Data Quality Assessment, EPA QA/G-9, QA00 Update, U.S. Environmental Protection Agency, Office of Environmental Information, Washington, D.C.

WCH, 2006a, Site Specific Instruction for Close-out Approach for 118-C-1, CCN 129632, Washington Closure Hanford, Richland, Washington. 
WCH, 2006b, Remedial Sampling, Logbook EFL-1173-8, Washington Closure Hanford, Richland, Washington.

WCH, 2006c, Remedial Sampling, Logbook EFL-1173-9, Washington

Closure Hanford, Richland, Washington. 
CVP-2006-00011

Rev. 0

APPENDIX D

118-C-1 RADIOLOGICAL SURVEY MAPS

D-i 
CVP-2006-00011

Rev. 0

D-ii 


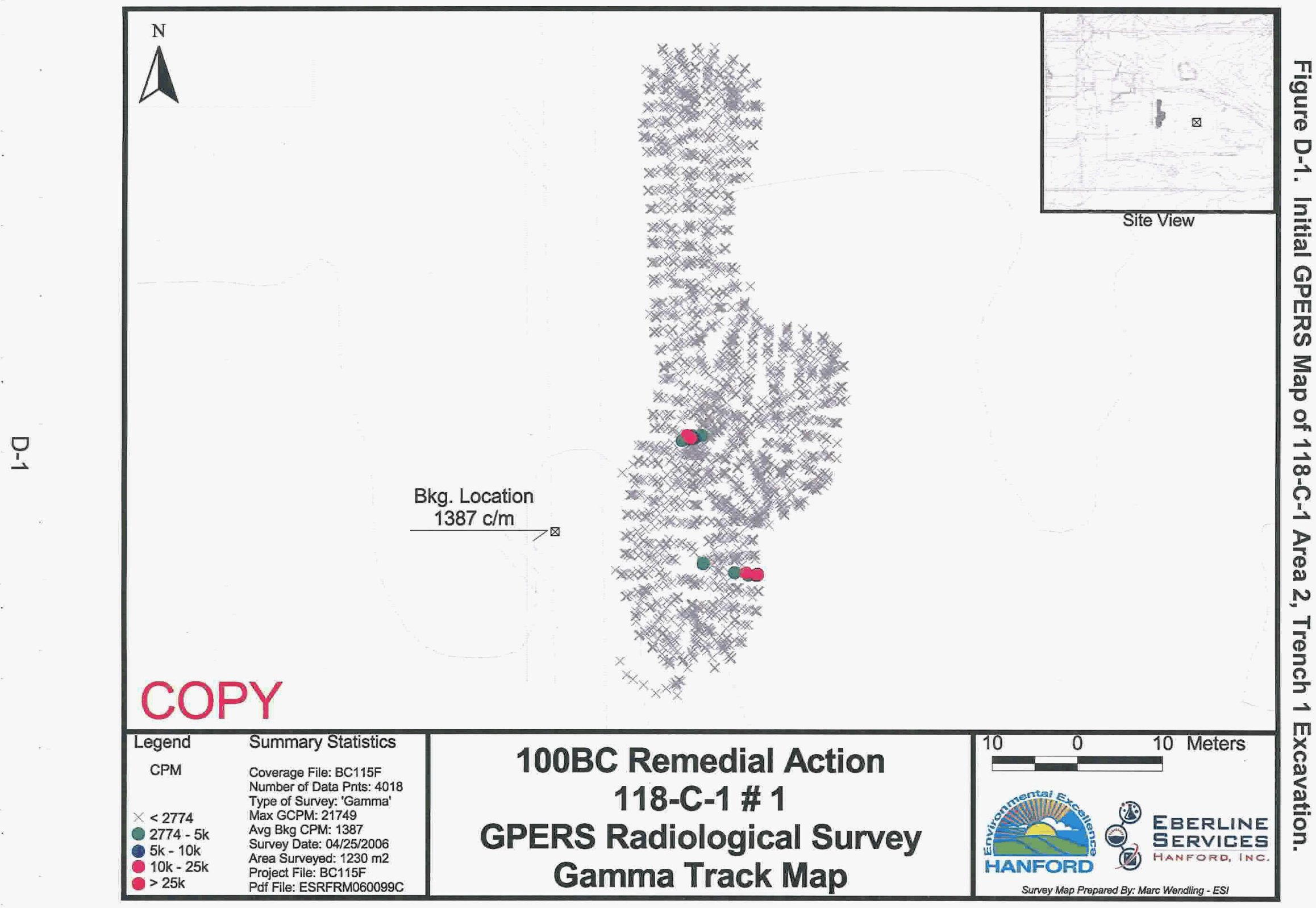




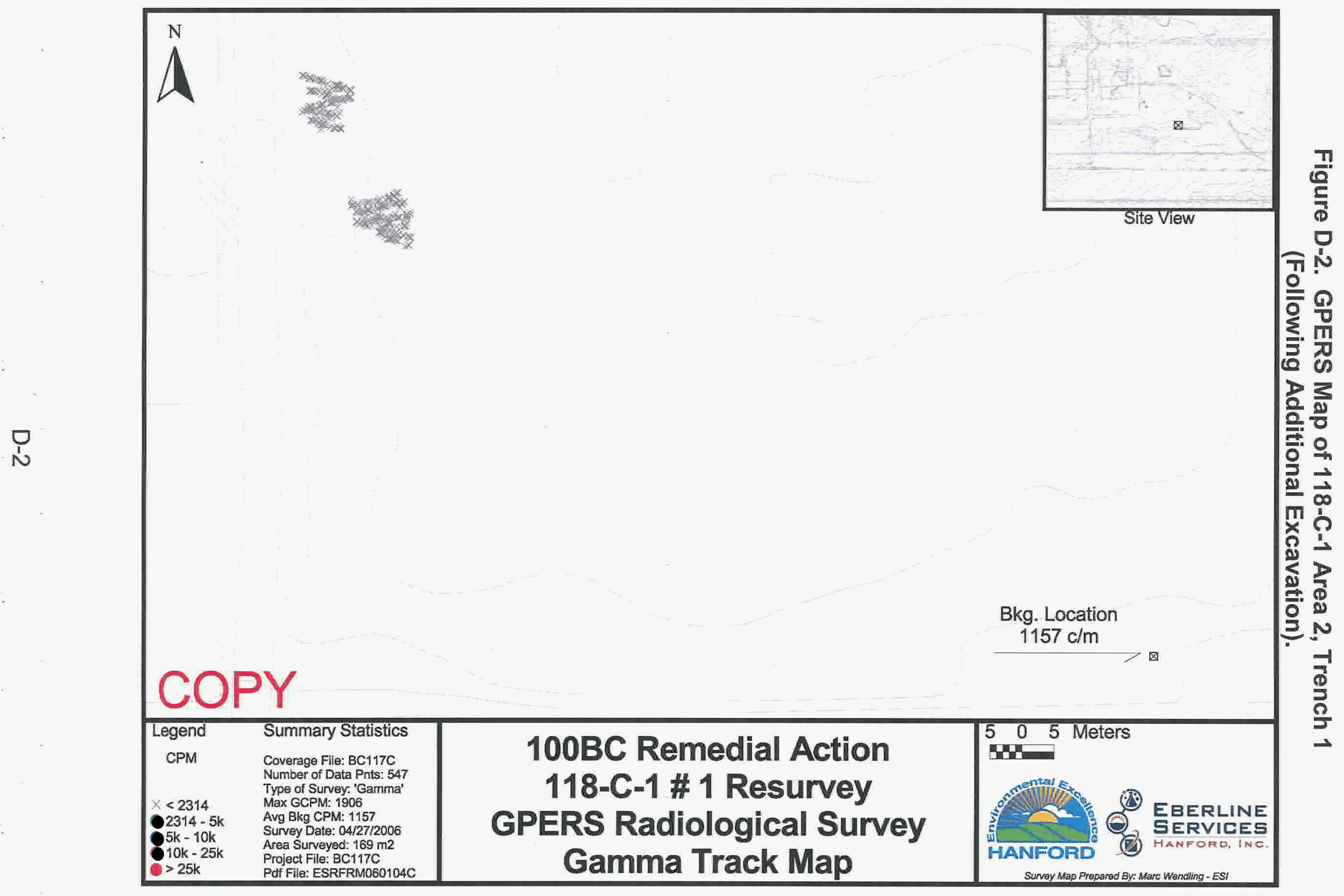




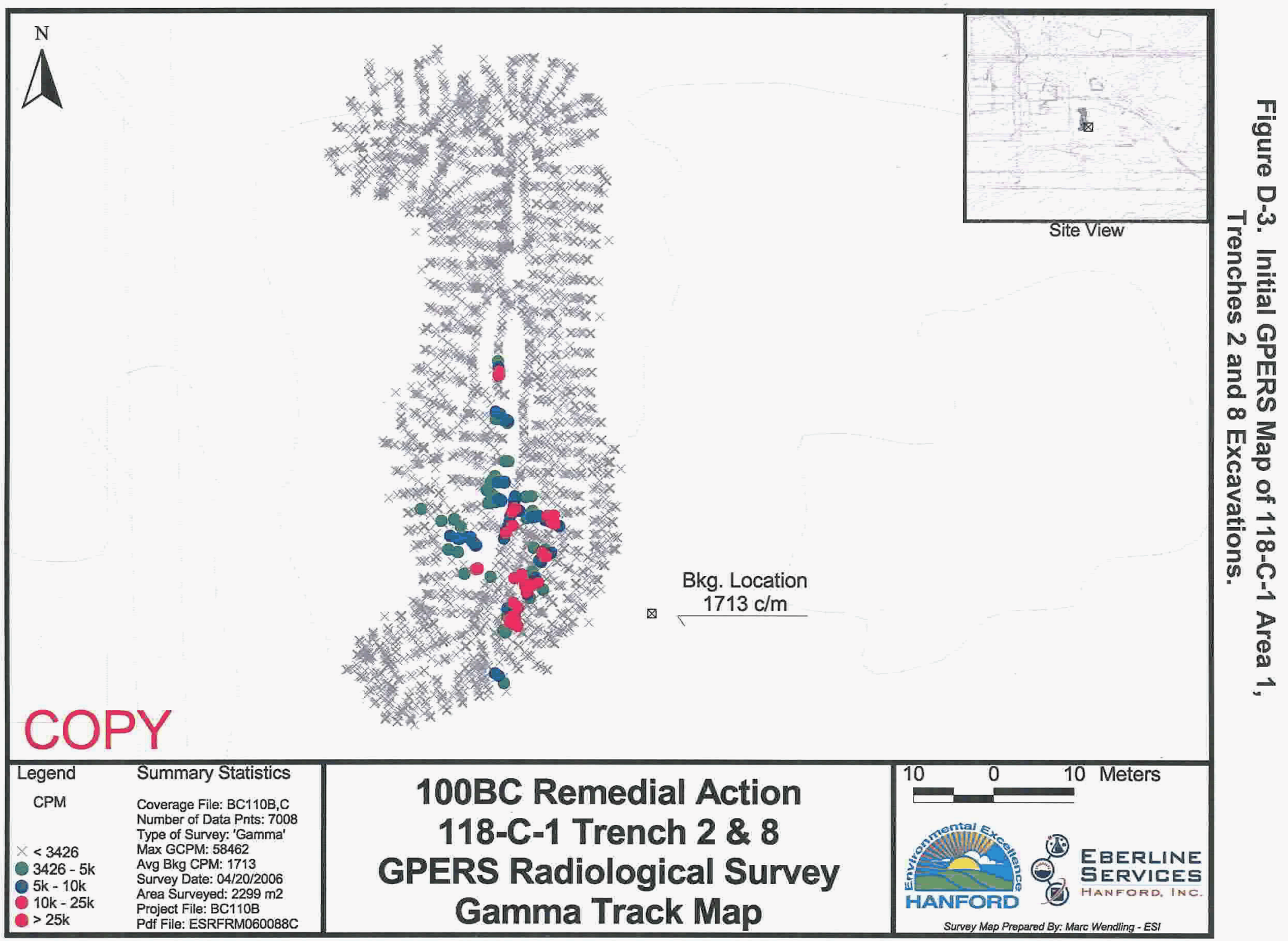

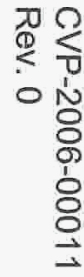




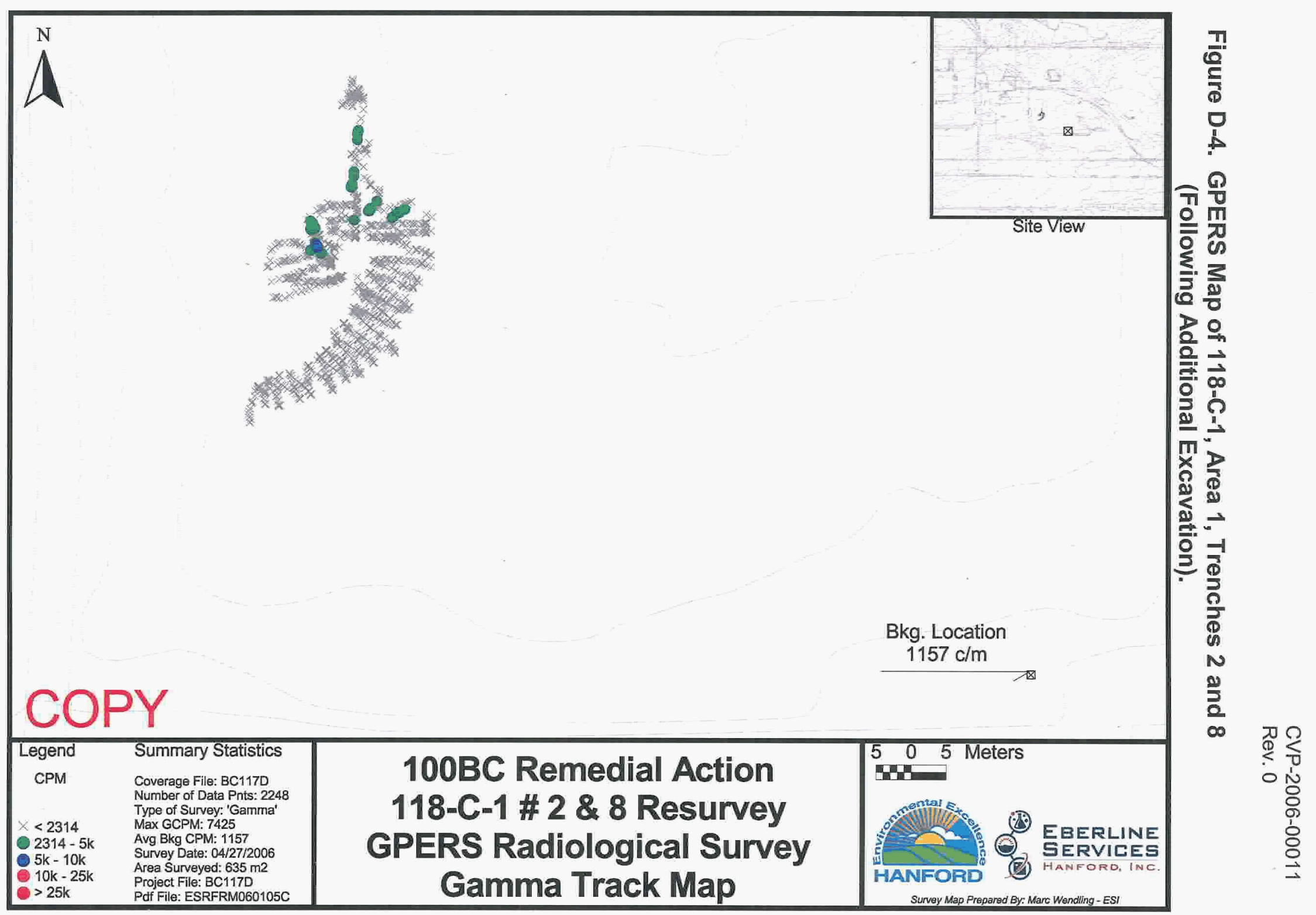




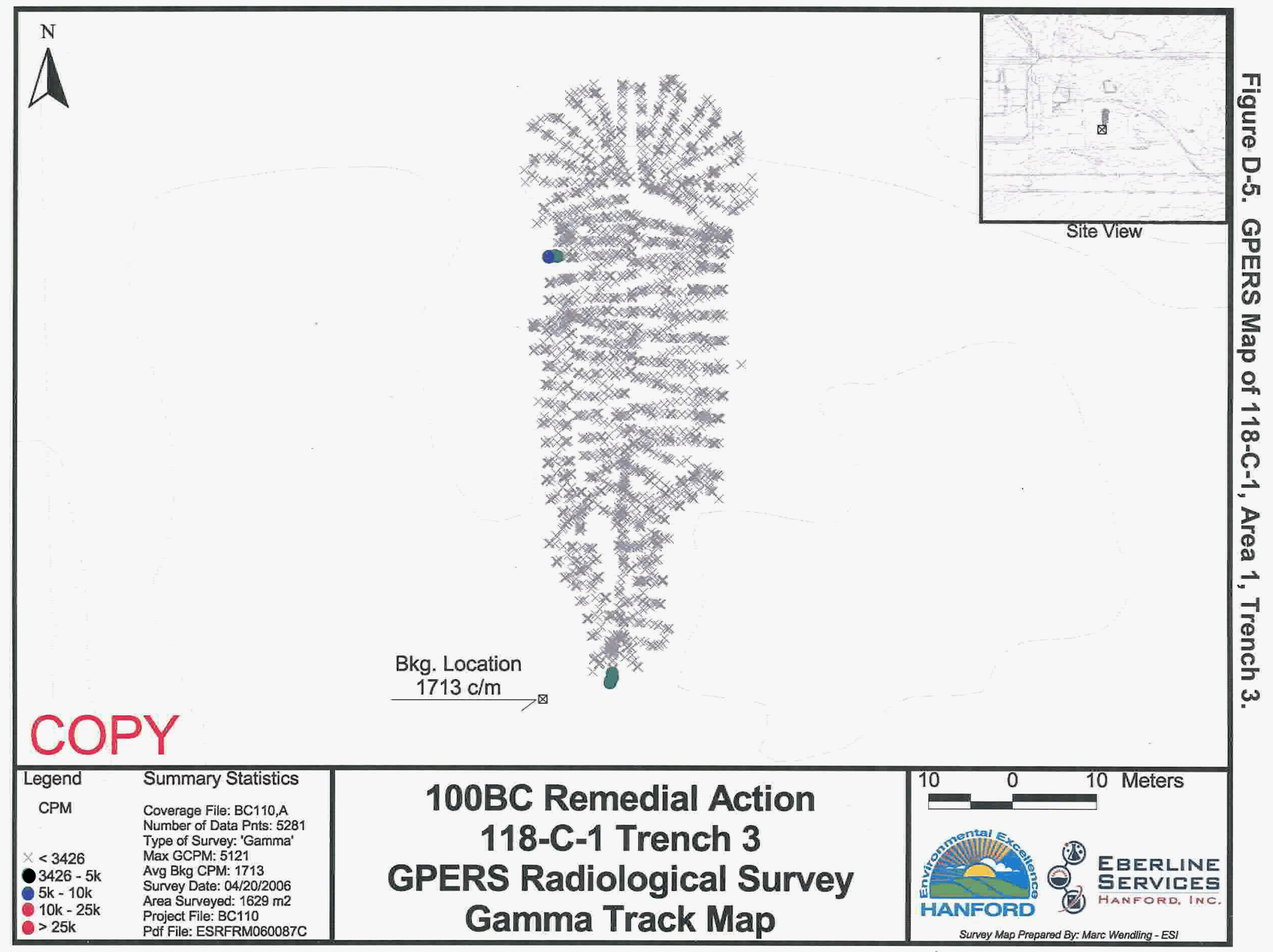




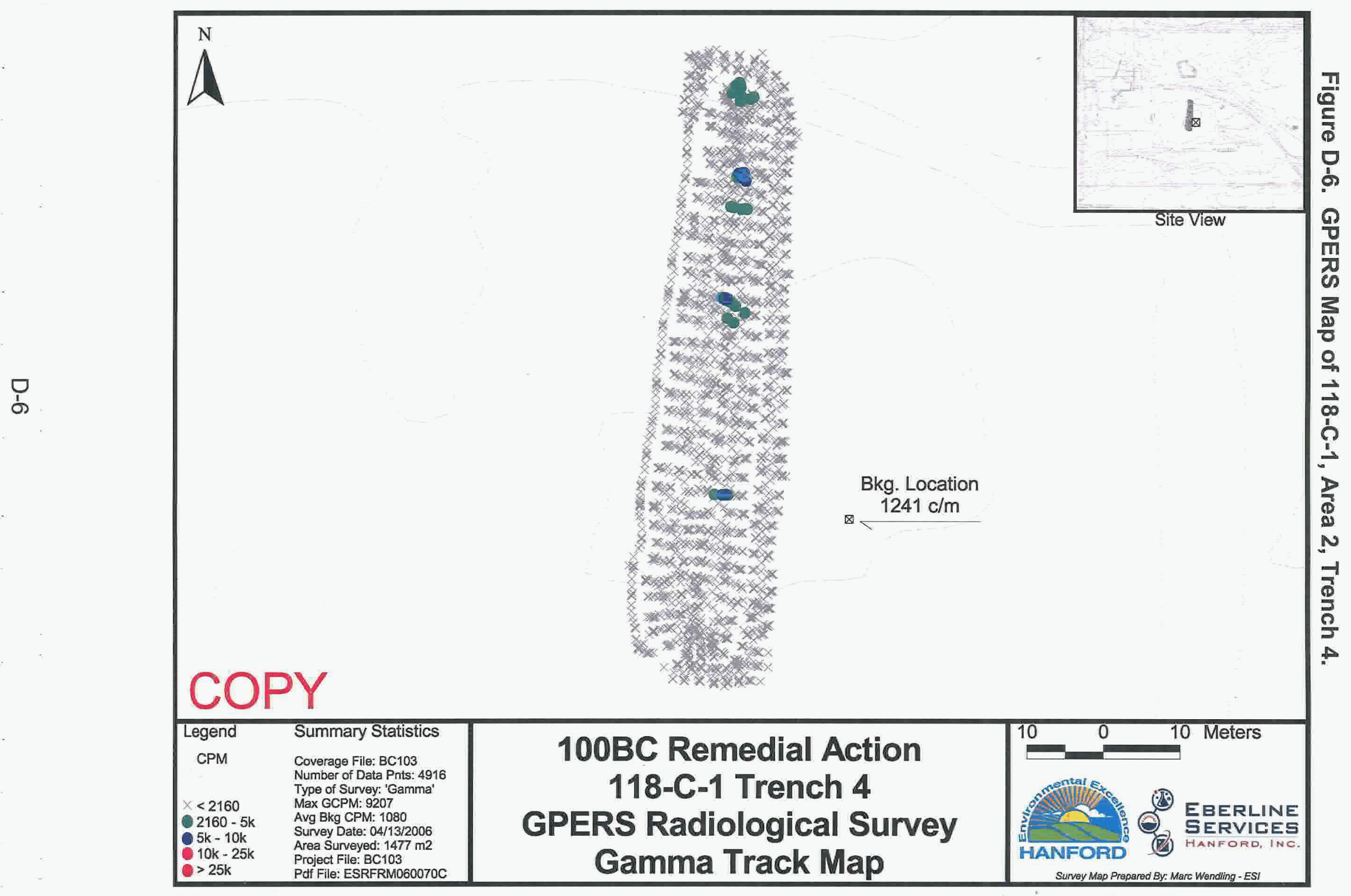




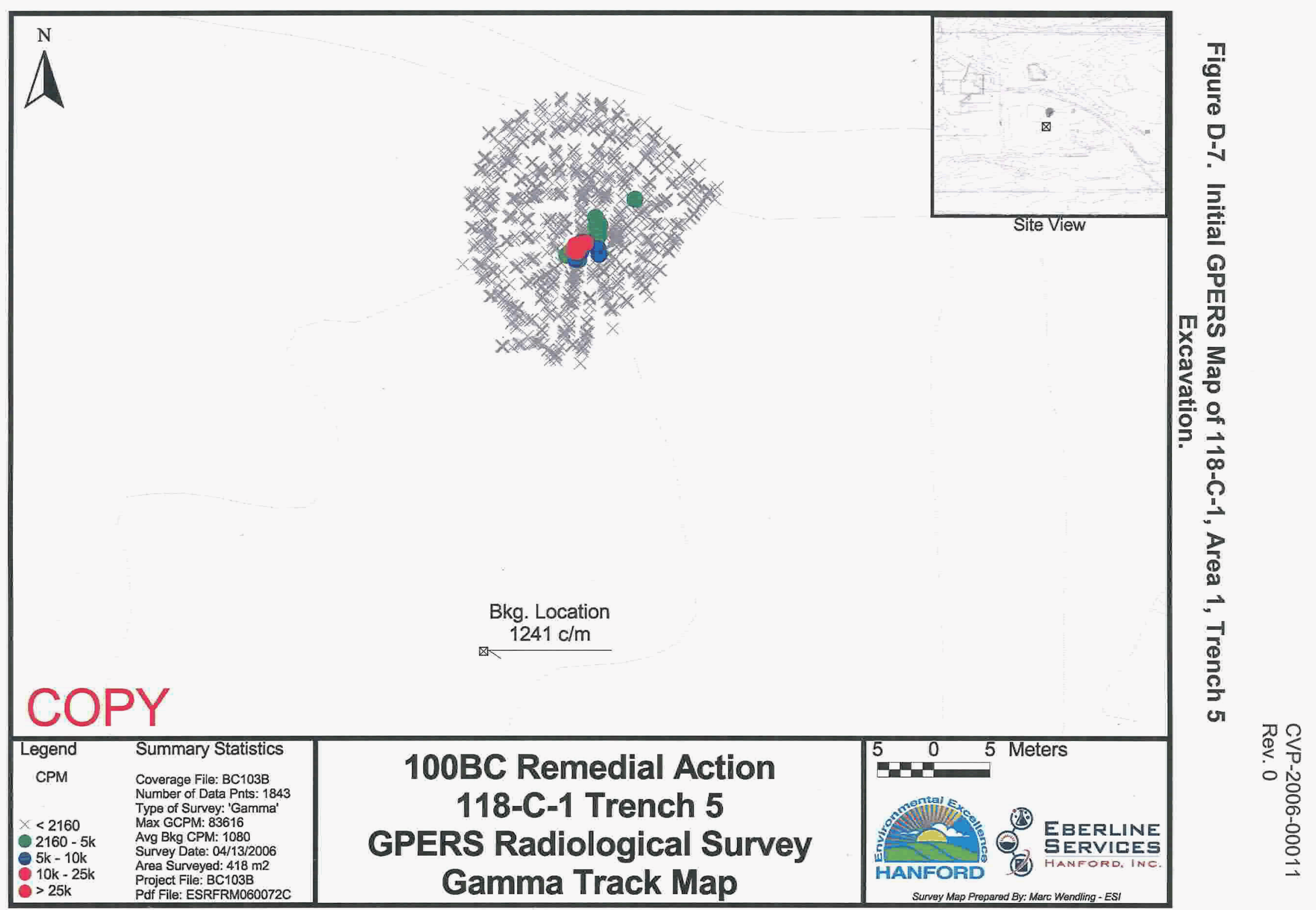




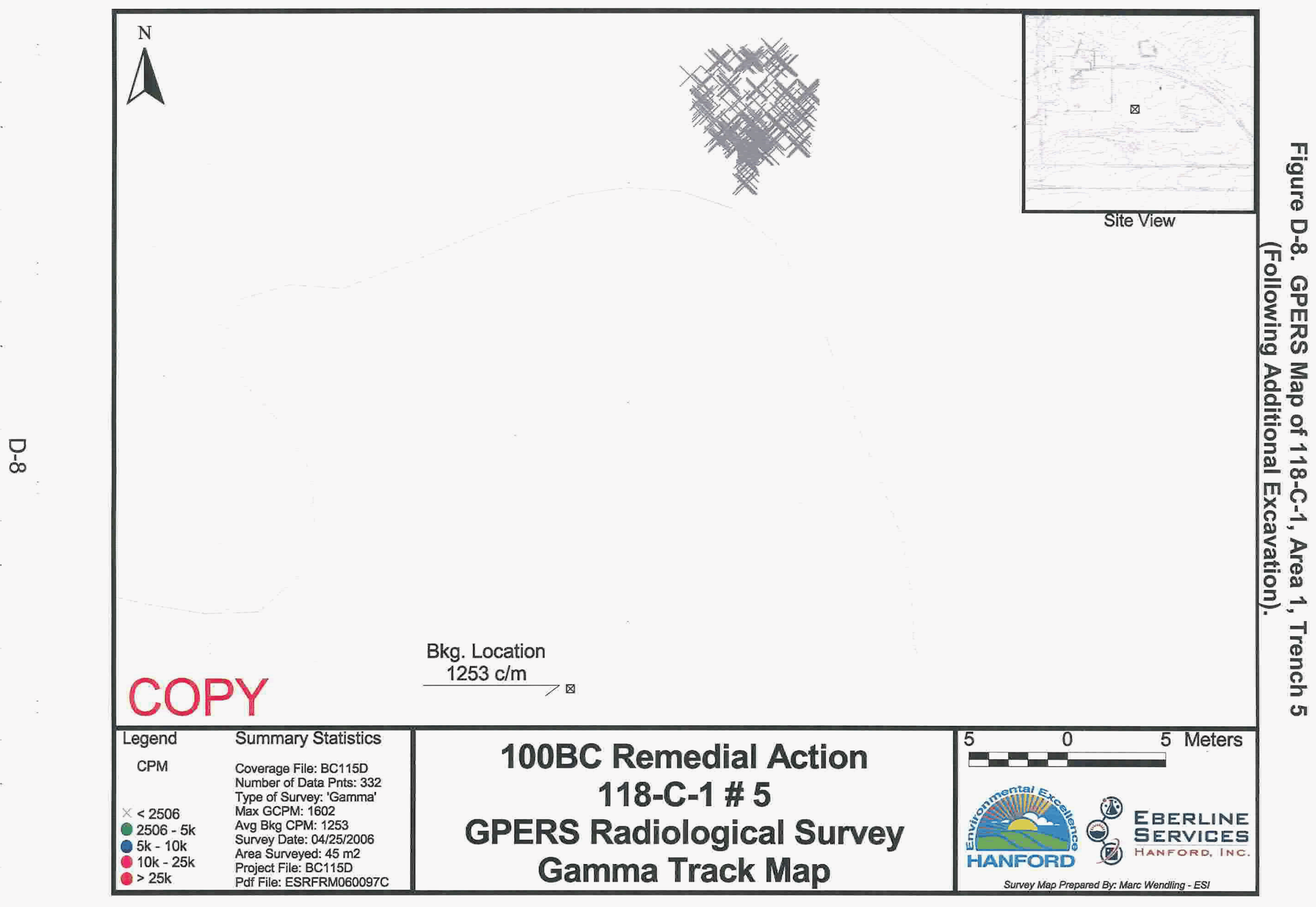




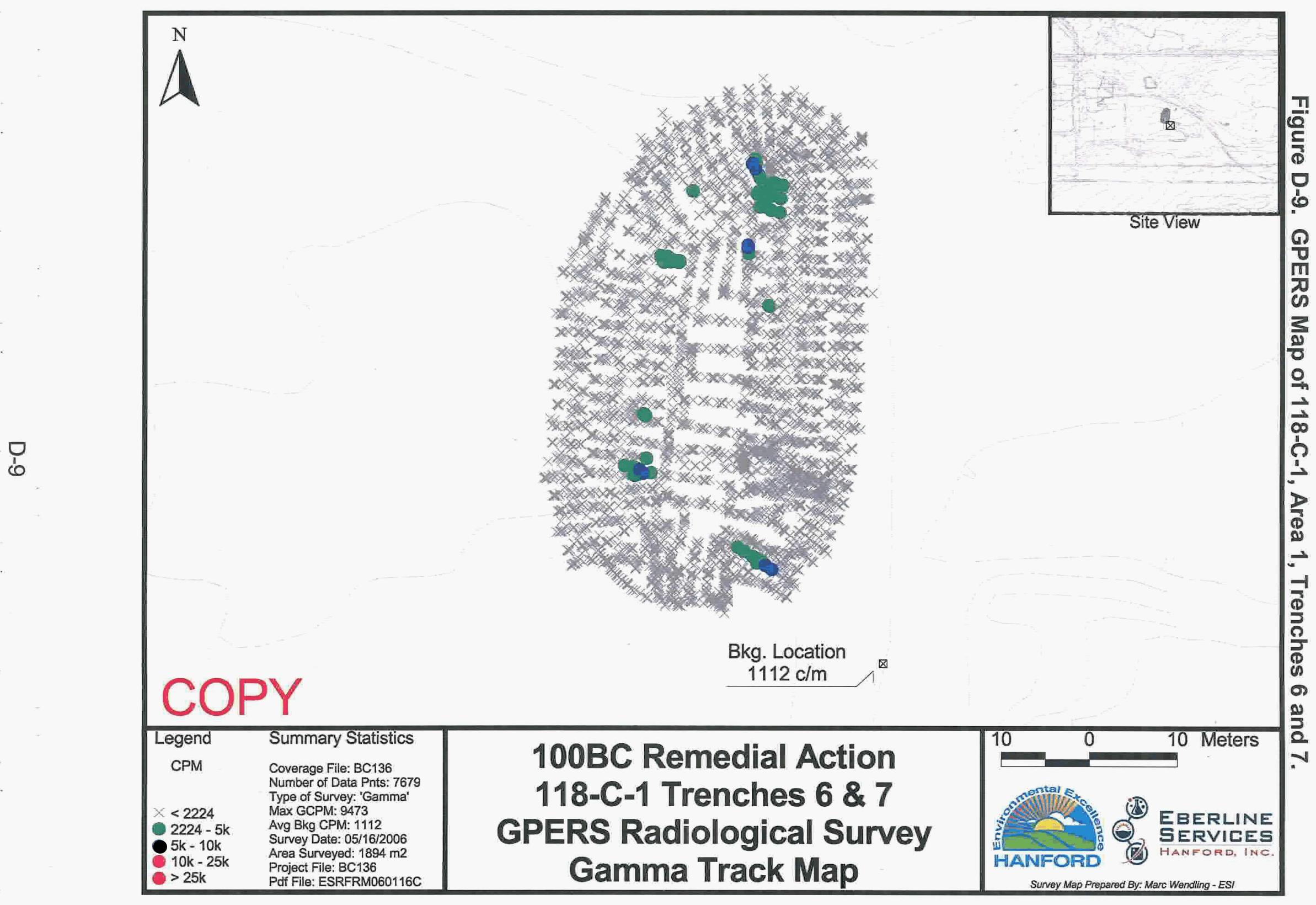




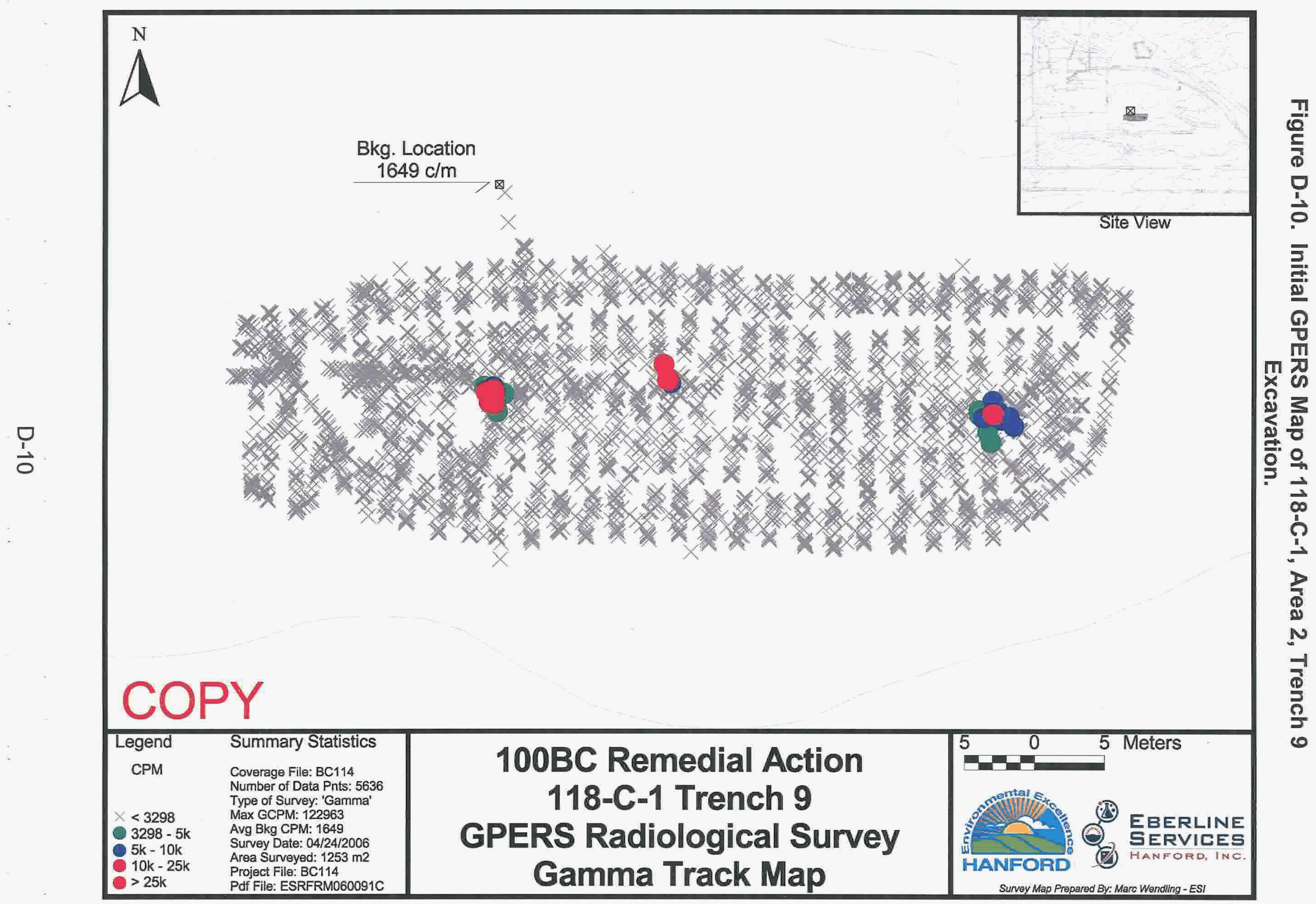




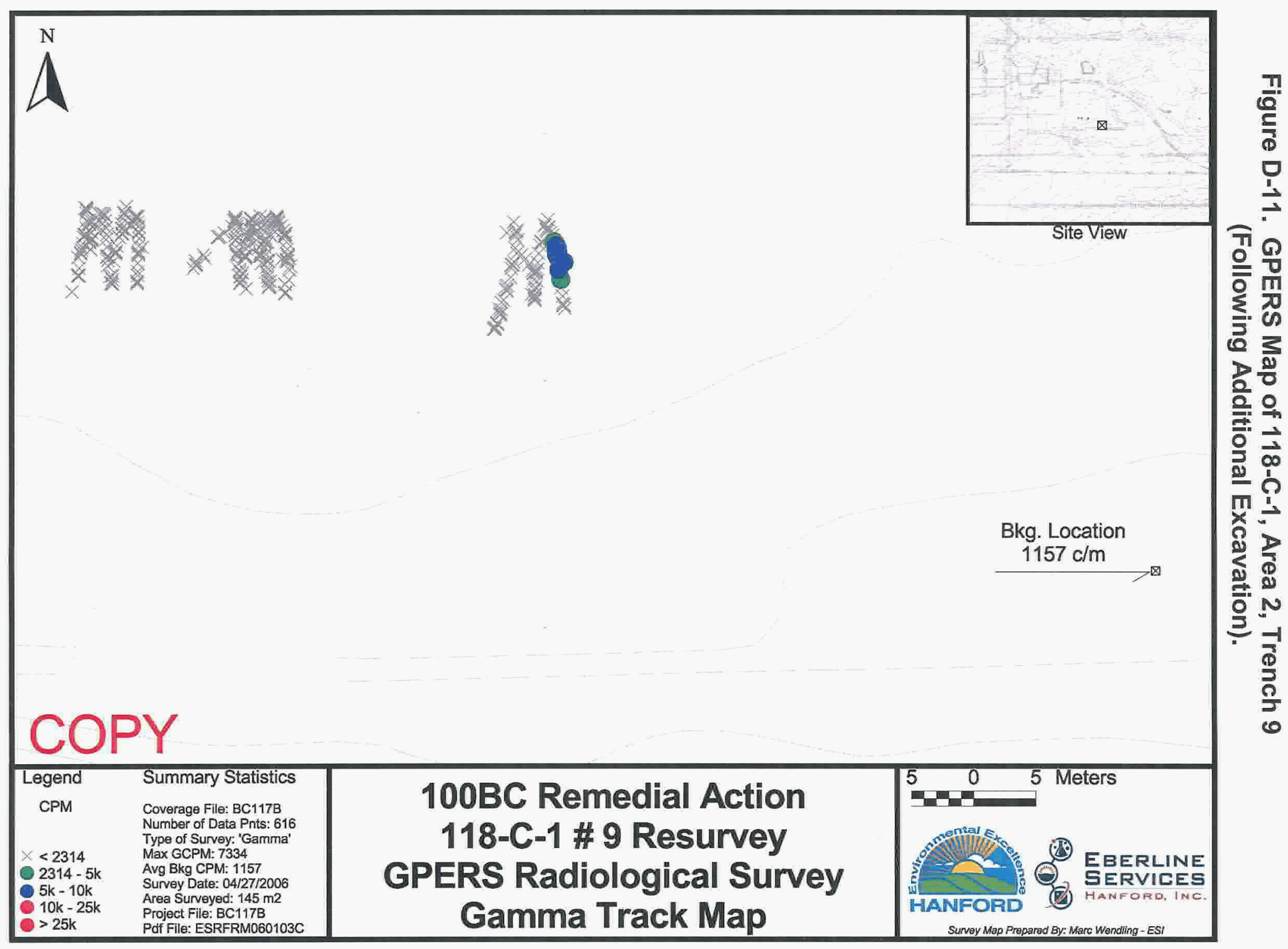




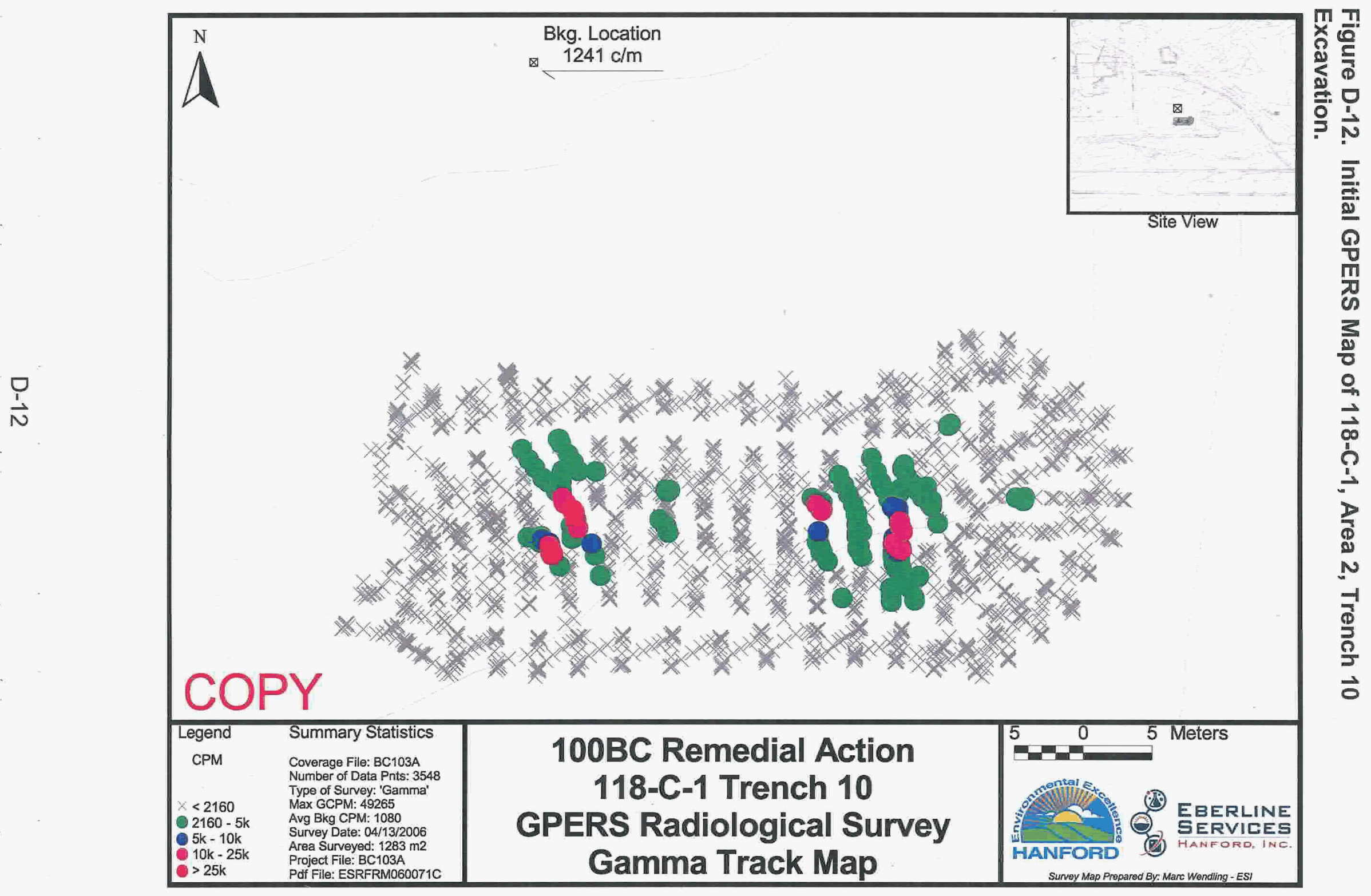




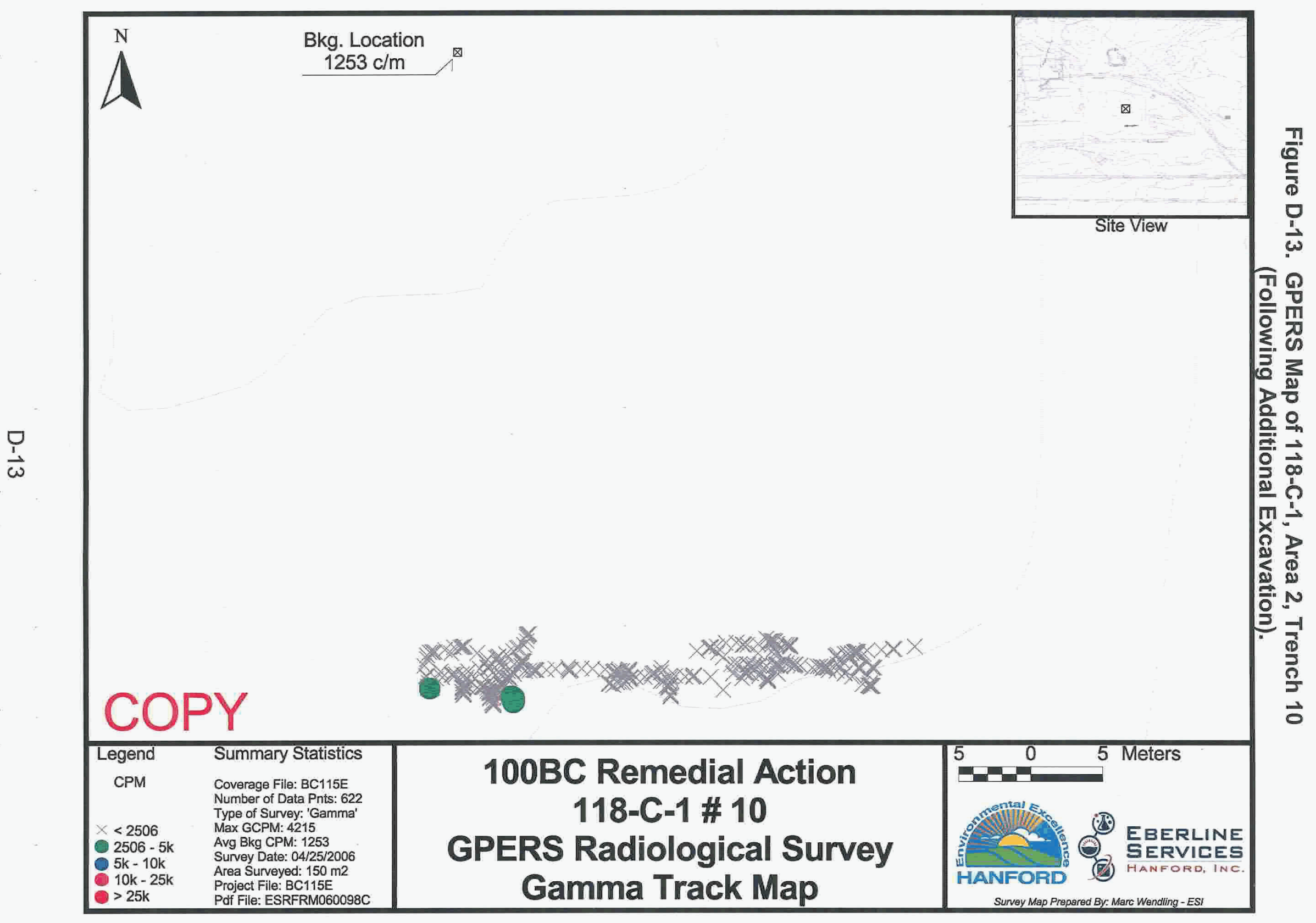




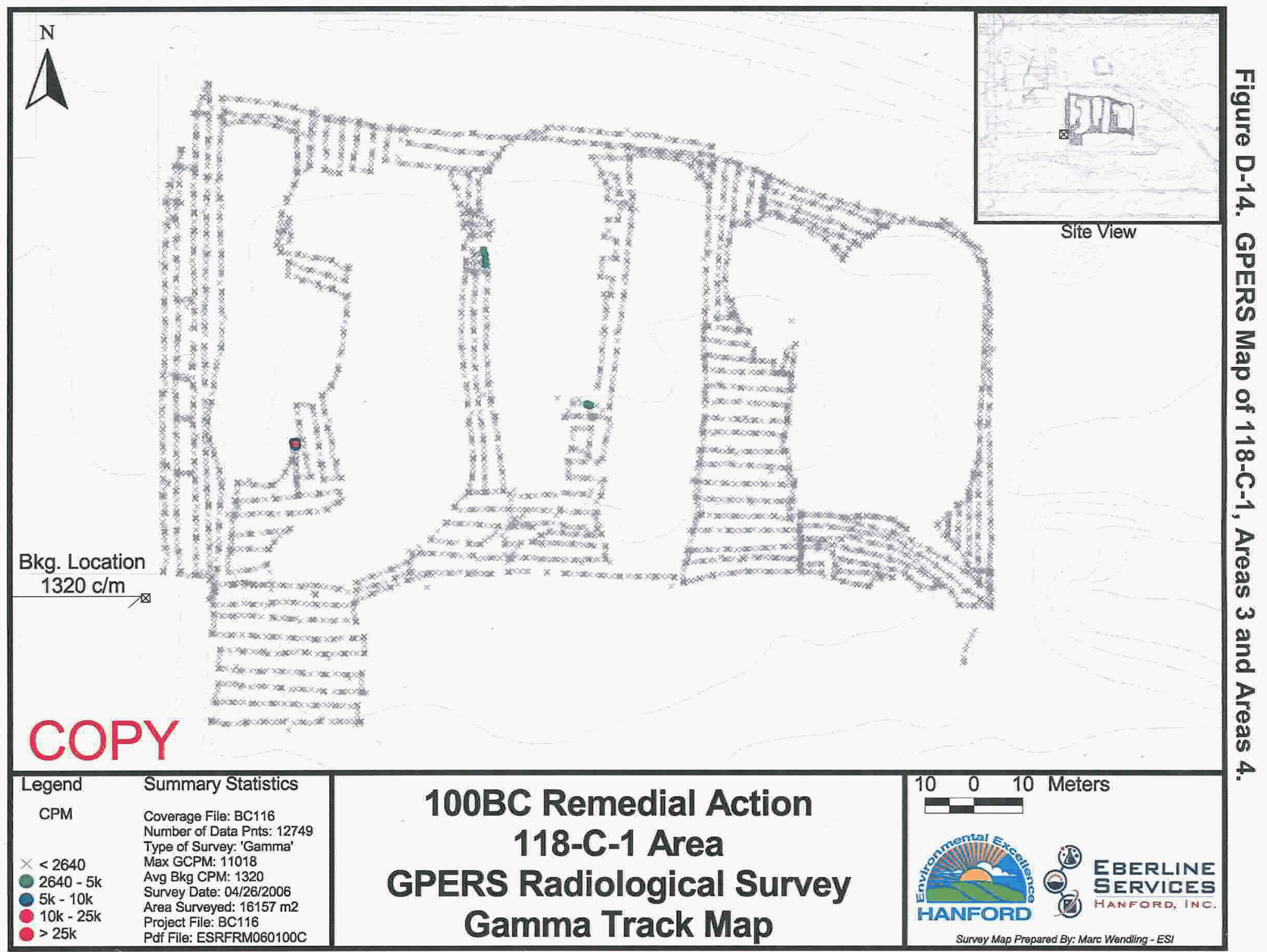

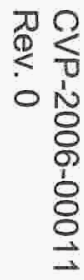




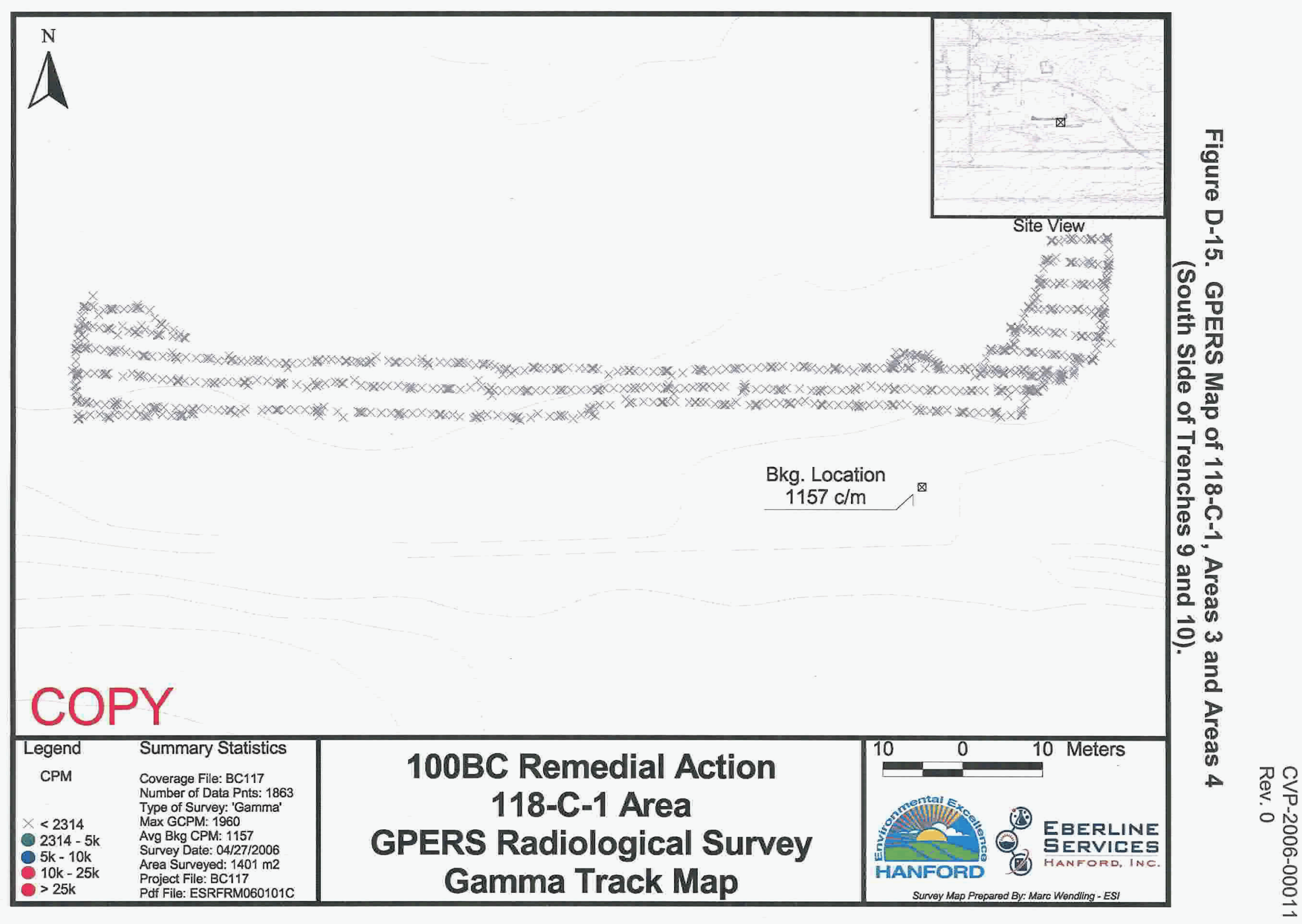


CVP-2006-00011

Rev. 0

D-16 


\section{APPENDIX E}

RESRAD INPUT PARAMETERS

AND CALCULATION BRIEF EXCERPTS 
CVP-2006-00011

Rev. 0

$E-i i$ 


\section{CALCULATION COVER SHEET}

Project Title 118-C-1 Burial Ground Cleanup Verification Job No.

14655

Area $100 \mathrm{~B} / \mathrm{C}$ Area

Discipline Environmental

*Calc. No. 0100C-CA-V0035

Subject 118-C-1 Burial Ground Cleanup Verification RESRAD Calculation Brief

Computer Program RESRAD

Program No. Version 6.30

The attached calculations have been generated to document compliance with established cleanup levels.

These calculations should be used in conjunction with other relevant documents in the administrative record.

Committed Calculation $\otimes \quad$ Preliminary $\square \quad$ Superseded $\square \quad$ Voided

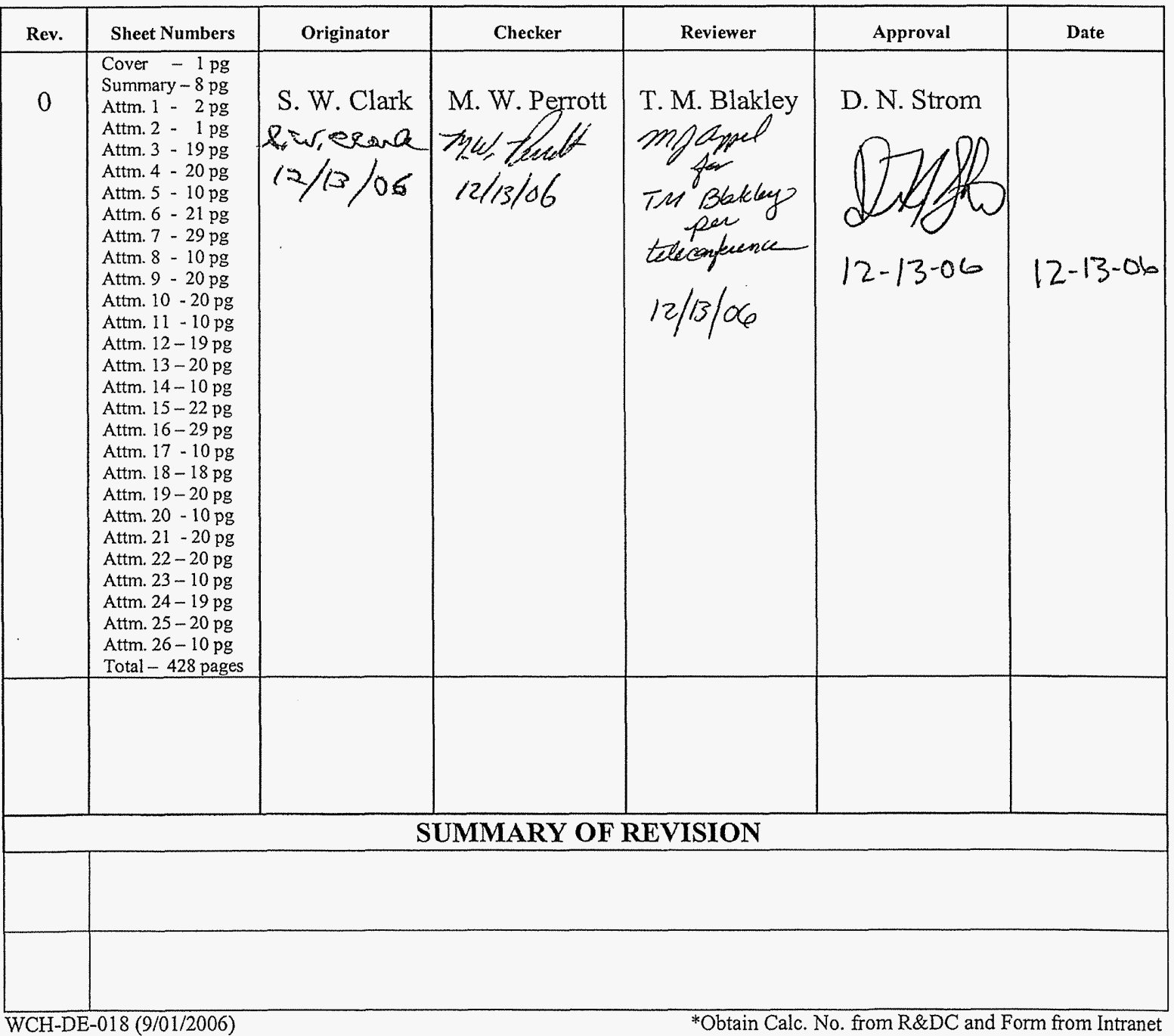


Washington Closure Hanford CALCULATION SHEET

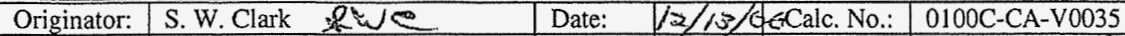

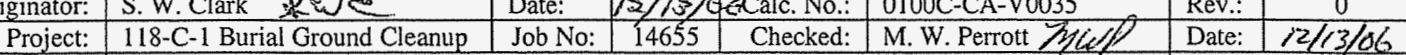

Subject: 118-C-1 Burial Ground Cleanup Verification RESRAD Calculation Brief

Sheet No 1 of 8

\section{PURPOSE:}

2

Calculate the soil and groundwater concentrations, dose, and risk contributions from remaining radionuclide contaminants in remediated 118-C-1 Burial Ground areas, in overburden/below cleanup level soil, and in staging pile (stockpile) areas over a period of 1,000 years.

\section{GIVEN/REFERENCES:}

1) Cleanup verification data from 118-C-1 Burial Ground 95\% UCL Calculation, Calculation No. 0100C-CA-V0033, Rev. 0, Washington Closure Hanford, Richland, Washington.

2) Remedial Design Report/Remedial Action Work Plan for the 100 Area (RDR/RAWP), DOE/RL-96-17, Rev. 5, U.S. Department of Energy, Richland Operations Office, Richland, Washington.

3) Radioactive and nonradioactive contaminants of concern from the 100 Area Burial Grounds Remedial Action Sampling and Analysis Plan, DOE/RL-2001-35, Rev. 0, U.S. Department of Energy, Richland Operations Office, Richland, Washington. For the purpose of these RESRAD calculations, the radioactive contaminants of potential concern (COPCs) are americium-241, carbon-14, cesium-137, cobalt-60, europium-152, europium-154, europium-155, nickel-63, plutonium-238, plutonium-239/240, plutonium-241, silver-108m, strontium-90, tritium ( $\mathrm{H}-3$ ), uranium-233/234, uranium-235, and uranium-238. The nonradionuclide contaminants of concern are cadmium, hexavalent chromium, mercury, PCBs, SVOAs, TPH, and VOAs.

4) RESidual RADioactivity (RESRAD) computer code, version 6.30, to calculate compliance with residual radioactivity guidelines, developed for the U.S. Department of Energy by the Environmental Assessment Division of Argonne National Laboratory, Argonne, Illinois.

5) Sample design data from the 118-C-1 Burial Ground Sampling Plan, Calculation No. 0100B-CA-V0288, Rev. 0, Washington Closure Hanford, Richland, Washington..

6) Groundwater elevation from Hanford Groundwater Monitoring for Fiscal Year 2005, PNNL-15670, Pacific Northwest National Laboratory, Richland, Washington.

\section{SOLUTION:}

1) Separate RESRAD runs were performed for the 118-C-1 Burial Ground trenches with spent nuclear fuel (Area 1), other trenches (Area 2), west interstitial area (Area 3), east interstitial area (Area 4), combined Areas 1, 2, 3, and 4, overburden/below cleanup level (OB/BCL) material, and staging pile (stockpile) area (SPA). The RESRAD run for the combined Areas 1, 2, 3, and 4 was done using using the maximum 95\% UCL values from the individual Areas. Table 1 shows the elevations (NAVD88) and thickness of each soil horizon. The average surrounding grade elevation and the maximum excavation depth are used to represent the burial ground excavation. Attachment 1 shows representative dimensions of soil horizons and contaminant pathways considered for dose, risk, and groundwater protection. Attachment 2 shows a schematic of the 118-C-1 Burial Ground area with the pieces making up the individual units labeled. Input factors for each run are shown in the "Summary" section of the RESRAD "Mixture Sums and Single Radionuclide Guidelines" printouts in the Attachments to this Calculation Summary. 
Washington Closure Hanford CALCULATION SHEET

Originator: S.W Clark Wue

Date: / ti / 3 /o \& Calc. No.: $0100 \mathrm{C}-\mathrm{CA}-\mathrm{V} 0035$

Project: 118 -C-1 Burial Ground Cleanup Job No: 14655 Checked: M. W. Perrott 3 i

Subject: $118-\mathrm{C}-1$ Burial Ground Cleanup Verification RESRAD Calculation Brief

Date: $/ 2 / 13 / a 6$

Sheet No. 2 of 8

1

\begin{tabular}{|c|c|c|c|c|c|c|c|c|c|}
\hline Parameter & Units & Area 1 & Area 2 & Area 3 & Area 4 & $\begin{array}{c}\text { Combined } \\
\text { Areas }\end{array}$ & $\mathrm{OB} / \mathrm{BCL}$ & \begin{tabular}{|c|} 
Shallow \\
Zone SPA
\end{tabular} & $\begin{array}{c}\text { Deep } \\
\text { Zone SPA }\end{array}$ \\
\hline \multicolumn{10}{|c|}{ Contaminated Zone Dimensions } \\
\hline Cover Depth & $\mathrm{m}$ & 0 & 0 & 0 & 0 & 0 & 0 & 0 & 4.6 \\
\hline $\begin{array}{l}\text { Area of Contaminated } \\
\text { Zone (CZ) }\end{array}$ & $\mathrm{m}^{2}$ & 3,018 & 2,339 & 7,576 & 7,576 & 20,509 & 9,006 & 23,230 & 23,230 \\
\hline $\begin{array}{l}\text { Length Parallel to } \\
\text { Aquifer Flow }\end{array}$ & $\mathrm{m}$ & 75 & 85 & 130 & 130 & 150 & 80 & 240 & 240 \\
\hline \multicolumn{10}{|c|}{ Elevations of Vadose Zone Horizons } \\
\hline Elevation: Surface & $\mathrm{m}$ & 150.0 & 149.0 & 151.0 & 151.0 & 151.0 & 151.5 & 148.5 & 148.5 \\
\hline $\begin{array}{l}\text { Elevation: Bottom of } \\
\text { Excavation }\end{array}$ & $\mathrm{m}$ & 145.5 & 146.0 & 146.0 & 146.0 & 146.0 & - & -- & - \\
\hline Elevation: Groundwater & $\mathrm{m}$ & 121.8 & 121.8 & 121.8 & 121.8 & 121.8 & 121.8 & 121.8 & 121.8 \\
\hline $\begin{array}{l}\text { Thickness: } \\
\text { Contaminated Zone }\end{array}$ & $\mathrm{m}$ & 28.2 & 27.2 & 29.2 & 29.2 & 29.2 & 29.7 & 4.6 & 22.1 \\
\hline $\begin{array}{l}\text { Thickness: Unsaturated } \\
\text { Zone }\end{array}$ & $\mathrm{m}$ & 0 & 0 & 0 & 0 & 0 & 0 & 22.1 & 0 \\
\hline
\end{tabular}

2

2) The year where the peak dose (or concentration) occurs from each individual radionuclide COC and layer is determined by a preliminary run. This year is then added for all horizons for the final RESRAD runs. For the direct exposure pathway (i.e. soil ingestion and inhalation and external radiation), the peak year occurred at year zero (year 2006) for all COCs. For the water pathways (i.e. drinking water and food ingestion) the peak yearr was year 3 for tritium, year 8 for cobalt- 60 , year 43 for cesium- 137 and strontium-90, and year 131 for nickel-63. The 3, 8, 43, and 131-year time periods were added to all RESRAD runs.

To be conservative, shallow zone evaluations were performed for the 118-C-1 Burial Ground areas assuming that the residual contamination levels from the shallow zone sample data sets extend uniformly from the surface to groundwater (Attachment 1). For Area 2 and the combined Areas 1, 2,3, and 4 this evaluation is too conservative and carbon-14 in the pathways affected by plant ingestion caused the total all pathways dose to be greater than 15 $\mathrm{mrem} / \mathrm{yr}$. Because the residual carbon-14 is deeper than the root penetration zone (10 ft), and not accessible to plants, the RESRAD inputs for "contaminated fractions" in the plant ingestion input parameters for Area 2 and the combined Areas 1,2,3, and 4 are set equal to zero.

For the staging pile area (SPA) the maximum carbon-14 concentration $(182 \mathrm{pCi} / \mathrm{g})$ is above the direct exposure lookup value (5.16 pCi/g per the 100 Area RDR/RAWP) while carbon-14 in all other SPA sampling grid areas was undetected. The soil that contains carbon-14 above lookup values will be used as backfill in deep zone areas and has been evaluated in RESRAD as SPA deep zone material (with $4.6 \mathrm{~m}$ [15 ft] of cover; see Table 2). 
1

\section{METHODOLOGY:}

1) Runs of RESRAD version 6.30 were completed for the 118-C-1 Burial Ground trenches with spent nuclear fuel (Area 1), other trenches (Area 2), west interstitial area (Area 3), east interstitial area (Area 4), the combined Areas 1,2,3, and 4, overburden/below cleanup level $(\mathrm{OB} / \mathrm{BCL})$ material, and staging pile area (SPA) soils using the radionuclide concentrations shown in Table 2. RESRAD numerical output reports for dose, risk, and concentration for the shallow zone, deep zone, and overburden are presented in the Attachments to this calculation summary.

\begin{tabular}{|c|c|c|c|c|c|c|c|c|}
\hline \multicolumn{9}{|c|}{ Table 2. Cleanup Verification Data Set ${ }^{a}$} \\
\hline COPCs & Area 1 & Area 2 & Area 3 & Area 4 & $\begin{array}{c}\text { Combined } \\
\text { Areas }\end{array}$ & $\mathrm{OB} / \mathrm{BCL}$ & $\begin{array}{c}\text { Shallow } \\
\text { Zone SPA }\end{array}$ & $\begin{array}{c}\text { Deep } \\
\text { Zone SPA }\end{array}$ \\
\hline \multicolumn{9}{|c|}{ Radionuclide Activity ( $\mathrm{pCi} / \mathrm{g}$ ) } \\
\hline Am-241 & - & - & - & -- & -- & - & - & - \\
\hline $\mathrm{C}-14$ & - & 8.0 & -- & - & 8.0 & -- & - & 182 \\
\hline Cs- 137 & 0.185 & 0.140 & 0.709 & 0.81 & 0.140 & $0(<\mathrm{BG})$ & 1.03 & 0.091 \\
\hline Co-60 & & 0.99 & 0.037 & & 0.99 & -- & 0.029 & -- \\
\hline Eu-152 & 0.121 & 0.199 & 0.140 & 0.114 & 0.199 & - & 0.177 & - \\
\hline Eu-154 & -- & - & - & $\ldots$ & - & - & - & -- \\
\hline Eu-155 & - & -- & - & - & - & -- & -- & - \\
\hline $\mathrm{Ni}-63$ & & 26.1 & & & 26.1 & - & & - \\
\hline $\mathrm{Pu}-238$ & - & -- & $\ldots$ & $\ldots$ & - & - & $\ldots$ & -- \\
\hline $\mathrm{Pu}-239 / 240$ & -- & -- & - & -- & - & -- & -- & - \\
\hline $\mathrm{Pu}-241$ & - & -- & -- & - & -- & - & - & -- \\
\hline \begin{tabular}{|l} 
Silver $(\mathrm{Ag}-108 \mathrm{~m})$ \\
\end{tabular} & -- & -- & - & -- & -- & - & -- & -- \\
\hline $\mathrm{Sr}-90$ & -- & - & 0.268 & -- & 0.268 & 1.23 & 0.334 & $\ldots$ \\
\hline Tritium (H-3) & & 4.76 & $\ldots$ & - & 4.76 & -- & - & - \\
\hline $\mathrm{U}-233 / 234$ & $O(<B G)$ & $\therefore$ & $0(<B G)$ & $O(<B G)$ & $0(<B G)$ & $0(<\mathrm{BG})$ & $0(<B G)$ & $\ldots$ \\
\hline $\mathrm{U}-235$ & $O(<B G)$ & - & - & $\ldots$ & $0(<B G)$ & -- & $\mathrm{O}(<\mathrm{BG})$ & - \\
\hline $\mathrm{U}-238$ & $0(<B G)$ & - & $0(<B G)$ & $O(<B G)$ & $0(<B G)$ & $0(<\mathrm{BG})$ & $O(<B G)$ & - \\
\hline
\end{tabular}

$--=$ Not evaluated by laboratory analysis or not detected (for all samples in the data set). $\mathrm{OB} / \mathrm{BCL}=$ Overburden/Below Cleanup Levels SPA $=$ Staging Pile (Stockpile) Area

${ }^{a}$ Soil concentration values are from 118-C-1 Burial Ground 95\% UCL Calculation, Calculation No. 0100C-CA-V0033, Rev. 0, Washington Closure Hanford, Richland, Washington. Refer to the $95 \%$ UCL for nonradionuclide concentrations. Nonradionuclides are not evaluated in this RESRAD calculation. 
Washington Closure Hanford

CALCULATION SHEET

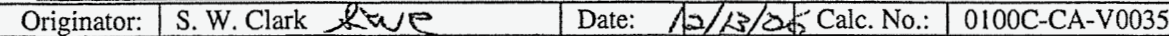

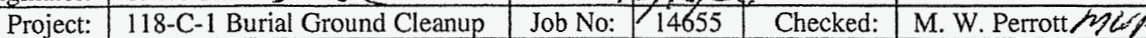

Subject: $118-\mathrm{C}-1$ Burial Ground Cleanup Verification RESRAD Calculation Brief

1 RESULTS:

2

1) Radionuclide "All Pathways" Dose Rate

The "all pathways" (maximum) dose rates are shown in Table 3. The maximum all pathways dose rate from the 118-C-1 Burial Ground combined Areas 1, 2, 3, and 4 is $12.8 \mathrm{mrem} / \mathrm{yr}$ at year zero (2006). The maximum all-pathways dose rate for the overburden (OB/BCL) soil is 4.01 $\mathrm{mrem} / \mathrm{yr}$ at year zero (2006). For the staging pile area (SPA) the total maximum all-pathways dose rate for the shallow and deep zones is $12.4 \mathrm{mrem} / \mathrm{yr}$ at year zero (2006).

\begin{tabular}{|c|c|c|c|c|c|c|c|c|c|c|}
\hline \multicolumn{70}{|c|}{ Table 3. All Pathways Dose Rate (mrem/yr) } \\
\hline $\begin{array}{c}\text { RESRAD } \\
\text { Run }\end{array}$ & $\begin{array}{c}\text { Vadose Zone } \\
\text { Horizons }\end{array}$ & \multicolumn{7}{c|}{ "All Pathways" Dose Contributions in mrem/yr at Each Time Slice (yr) } \\
\cline { 3 - 11 } & 0 & 1 & 3 & 8 & 12 & 43 & 131 & 300 & 1000 \\
\hline Area 1 & Shallow Zone & $9.45 \mathrm{E}-01$ & $9.09 \mathrm{E}-01$ & $8.41 \mathrm{E}-01$ & $6.95 \mathrm{E}-01$ & $6.00 \mathrm{E}-01$ & $2.12 \mathrm{E}-01$ & $2.10 \mathrm{E}-02$ & $4.13 \mathrm{E}-04$ & $4.01 \mathrm{E}-11$ \\
\hline Area 2 & Shallow Zone & $1.11 \mathrm{E}+01$ & $9.91 \mathrm{E}+00$ & $7.95 \mathrm{E}+00$ & $4.36 \mathrm{E}+00$ & $2.77 \mathrm{E}+00$ & $2.40 \mathrm{E}-01$ & $2.23 \mathrm{E}-02$ & $4.91 \mathrm{E}-03$ & $5.61 \mathrm{E}-05$ \\
\hline Area 3 & Shallow Zone & $3.54 \mathrm{E}+00$ & $3.41 \mathrm{E}+00$ & $3.16 \mathrm{E}+00$ & $2.65 \mathrm{E}+00$ & $2.33 \mathrm{E}+00$ & $1.01 \mathrm{E}+00$ & $1.24 \mathrm{E}-01$ & $2.50 \mathrm{E}-03$ & $2.22 \mathrm{E}-10$ \\
\hline Area 4 & Shallow Zone & $2.43 \mathrm{E}+00$ & $2.36 \mathrm{E}+00$ & $2.23 \mathrm{E}+00$ & $1.94 \mathrm{E}+00$ & $1.73 \mathrm{E}+00$ & $7.69 \mathrm{E}-01$ & $9.46 \mathrm{E}-02$ & $1.91 \mathrm{E}-03$ & $1.88 \mathrm{E}-10$ \\
\hline Combined Areas & $1.28 \mathrm{E}+01$ & $1.16 \mathrm{E}+01$ & $9.46 \mathrm{E}+00$ & $5.70 \mathrm{E}+00$ & $3.89 \mathrm{E}+00$ & $7.26 \mathrm{E}-01$ & $8.82 \mathrm{E}-02$ & $6.59 \mathrm{E}-03$ & $1.01 \mathrm{E}-04$ \\
\hline OB/BCL & Shallow Zone & $4.01 \mathrm{E}+00$ & $3.92 \mathrm{E}+00$ & $3.74 \mathrm{E}+00$ & $3.34 \mathrm{E}+00$ & $3.04 \mathrm{E}+00$ & $1.49 \mathrm{E}+00$ & $1.95 \mathrm{E}-01$ & $3.91 \mathrm{E}-03$ & $2.47 \mathrm{E}-10$ \\
\hline SPA & Shallow Zone & $1.24 \mathrm{E}+01$ & $1.18 \mathrm{E}+01$ & $1.08 \mathrm{E}+01$ & $8.72 \mathrm{E}+00$ & $7.38 \mathrm{E}+00$ & $2.31 \mathrm{E}+00$ & $1.91 \mathrm{E}-01$ & $3.39 \mathrm{E}-03$ & $2.39 \mathrm{E}-10$ \\
\hline SPA & Deep Zone & $0.00 \mathrm{E}+00$ & $2.94 \mathrm{E}-05$ & $9.38 \mathrm{E}-05$ & $2.30 \mathrm{E}-04$ & $3.16 \mathrm{E}-04$ & $5.58 \mathrm{E}-04$ & $2.23 \mathrm{E}-04$ & $1.03 \mathrm{E}-05$ & $3.22 \mathrm{E}-12$ \\
\hline $\begin{array}{c}\text { SPA Total All Pathway } \\
\text { Dose Rate (mrem/yr) }\end{array}$ & $1.24 \mathrm{E}+01$ & $1.18 \mathrm{E}+01$ & $1.08 \mathrm{E}+01$ & $8.72 \mathrm{E}+00$ & $7.38 \mathrm{E}+00$ & $2.31 \mathrm{E}+00$ & $1.91 \mathrm{E}-01$ & $3.40 \mathrm{E}-03$ & $2.42 \mathrm{E}-10$ \\
\hline
\end{tabular}

$\mathrm{OB} / \mathrm{BCL}=$ Overburden/Below Cleanup Levels

SPA = Staging Pile (Stockpile) Area

10

11 2) Radionuclide Excess Lifetime Cancer Risk

12 The radionuclide excess lifetime cancer risk (ELCR) results are shown in Table 4. The

13 maximum ELCR for the 118-C-1 Burial Ground combined Areas 1, 2, 3, and 4 is $9.95 \times 10^{-5}$ at

14 year zero (2006). The maximum ELCR for the overburden (OB/BCL) soil is $5.42 \times 10^{-5}$ which

15 also occurs at year zero (2006). For the staging pile area (SPA) the total maximum all-pathways

16 ELRC for the shallow and deep zones is $1.55 \times 10^{-4}$ at year zero (2006).

17

Table 4. Radionuclide Excess Lifetime Cancer Risk

\begin{tabular}{|c|c|c|c|c|c|c|c|c|c|c|}
\hline \multicolumn{8}{|c|}{ Table 4. Radionuclide Excess Lifetime Cancer Risk } \\
\hline $\begin{array}{c}\text { RESRAD } \\
\text { Run }\end{array}$ & $\begin{array}{c}\text { Vadose Zone } \\
\text { Horizons }\end{array}$ & \multicolumn{7}{c|}{ Excess Cancer Risk at Each Time Slice (yr) } \\
\cline { 3 - 11 } & 0 & 1 & 3 & 8 & 12 & 43 & 131 & 300 & 1000 \\
\hline Area 1 & Shallow Zone & $1.28 \mathrm{E}-05$ & $1.24 \mathrm{E}-05$ & $1.15 \mathrm{E}-05$ & $9.63 \mathrm{E}-06$ & $8.38 \mathrm{E}-06$ & $3.16 \mathrm{E}-06$ & $3.36 \mathrm{E}-07$ & $6.68 \mathrm{E}-09$ & $6.45 \mathrm{E}-16$ \\
\hline Area 2 & Shallow Zone & $7.35 \mathrm{E}-05$ & $6.60 \mathrm{E}-05$ & $5.34 \mathrm{E}-05$ & $3.12 \mathrm{E}-05$ & $2.11 \mathrm{E}-05$ & $3.24 \mathrm{E}-06$ & $5.05 \mathrm{E}-07$ & $1.62 \mathrm{E}-07$ & $1.80 \mathrm{E}-09$ \\
\hline Area 3 & Shallow Zone & $4.81 \mathrm{E}-05$ & $4.66 \mathrm{E}-05$ & $4.38 \mathrm{E}-05$ & $3.77 \mathrm{E}-05$ & $3.37 \mathrm{E}-05$ & $1.51 \mathrm{E}-05$ & $1.89 \mathrm{E}-06$ & $3.78 \mathrm{E}-08$ & $3.37 \mathrm{E}-15$ \\
\hline Area 4 & Shallow Zone & $3.70 \mathrm{E}-05$ & $3.60 \mathrm{E}-05$ & $3.41 \mathrm{E}-05$ & $2.98 \mathrm{E}-05$ & $2.68 \mathrm{E}-05$ & $1.22 \mathrm{E}-05$ & $1.53 \mathrm{E}-06$ & $3.09 \mathrm{E}-08$ & $3.02 \mathrm{E}-15$ \\
\hline Combined Areas & $9.95 \mathrm{E}-05$ & $9.07 \mathrm{E}-05$ & $7.60 \mathrm{E}-05$ & $5.04 \mathrm{E}-05$ & $3.79 \mathrm{E}-05$ & $1.11 \mathrm{E}-05$ & $1.57 \mathrm{E}-06$ & $1.97 \mathrm{E}-07$ & $3.32 \mathrm{E}-09$ \\
\hline OB/BCL & Shallow Zone & $5.42 \mathrm{E}-05$ & $5.29 \mathrm{E}-05$ & $5.05 \mathrm{E}-05$ & $4.50 \mathrm{E}-05$ & $4.10 \mathrm{E}-05$ & $2.00 \mathrm{E}-05$ & $2.59 \mathrm{E}-06$ & $5.09 \mathrm{E}-08$ & $3.14 \mathrm{E}-15$ \\
\hline SPA & Shallow Zone & $1.55 \mathrm{E}-04$ & $1.49 \mathrm{E}-04$ & $1.37 \mathrm{E}-04$ & $1.12 \mathrm{E}-04$ & $9.57 \mathrm{E}-05$ & $3.20 \mathrm{E}-05$ & $2.88 \mathrm{E}-06$ & $5.20 \mathrm{E}-08$ & $3.74 \mathrm{E}-15$ \\
\hline SPA & Deep Zone & $7.15 \mathrm{E}-09$ & $7.51 \mathrm{E}-09$ & $8.18 \mathrm{E}-09$ & $9.55 \mathrm{E}-09$ & $1.04 \mathrm{E}-08$ & $1.13 \mathrm{E}-08$ & $3.80 \mathrm{E}-09$ & $1.66 \mathrm{E}-10$ & $5.05 \mathrm{E}-17$ \\
\hline SPA Total ELCR & $1.55 \mathrm{E}-04$ & $1.49 \mathrm{E}-04$ & $1.37 \mathrm{E}-04$ & $1.12 \mathrm{E}-04$ & $9.57 \mathrm{E}-05$ & $3.20 \mathrm{E}-05$ & $2.89 \mathrm{E}-06$ & $5.21 \mathrm{E}-08$ & $3.79 \mathrm{E}-15$ \\
\hline
\end{tabular}

$\mathrm{OB} / \mathrm{BCL}=$ Overburden/Below Cleanup Levels

SPA $=$ Staging Pile (Stockpile) Area 
Washington Closure Hanford

CALCULATION SHEET

\begin{tabular}{|c|c|c|c|c|c|c|}
\hline Origir & S. W. Clark & Date: & Calc. No. & 0100 & Rev.: & 0 \\
\hline & 118-C-1 Burial Ground Cleanup & Job No: 146 & & M.W. Perrott mefef & Date: & 2 \\
\hline & \multicolumn{6}{|c|}{ 118-C-1 Burial Ground Cleanup Verification RESRAD Calculation Brief } \\
\hline
\end{tabular}

1

2 3) Radionuclide Groundwater Protection

3 The radionuclide concentrations in groundwater calculated by the RESRAD model are

4 summarized in Table 5. Among the radionuclide contaminants of concern only cesium-137,

5 cobalt-60, nickel-63, strontium-90, and tritium are calculated to reach groundwater in the 1,000

6 years of the RESRAD model run. The organ specific dose via the groundwater (and river)

7 pathway is presented in a separate calculation brief [118-C-1 Burial Ground Comparison To

8 Drinking Water Standards (MCL) Calculation, Calculation No. 0100C-CA-V0036, Rev. 0,

9 Washington Closure Hanford, Richland, Washington]. Only concentrations are presented here. 10

Table 5. Predicted Groundwater (Well Water/Drinking Water) Concentrations (2 pages)

\begin{tabular}{|c|c|c|c|c|c|c|c|c|c|c|c|}
\hline \multirow{2}{*}{$\begin{array}{l}\text { Radio- } \\
\text { nuclides }\end{array}$} & \multirow{2}{*}{$\begin{array}{c}\text { Vadose Zone } \\
\text { Horizon }\end{array}$} & \multicolumn{9}{|c|}{ Ground water Concentrations in $\mathrm{pCi} / \mathrm{L}$ at Each Time Slice (yr) } & \multirow{2}{*}{$\begin{array}{l}\text { RAGs } \\
\mathrm{pCi} / \mathrm{L}\end{array}$} \\
\hline & & 0 & 1 & 3 & 8 & 12 & 43 & 131 & 300 & 1000 & \\
\hline \multirow{9}{*}{ Cs- 137} & Area $1 \mathrm{SZ}$ & 0 & 4.65E-04 & $1.33 \mathrm{E}-03$ & $3.17 \mathrm{E}-03$ & $4.33 \mathrm{E}-03$ & $7.58 \mathrm{E}-03$ & $3.02 \mathrm{E}-03$ & 1.39E-04 & $4.34 \mathrm{E}-11$ & \multirow{9}{*}{60} \\
\hline & Area $2 \mathrm{SZ}$ & 0 & $3.52 \mathrm{E}-04$ & $1.01 \mathrm{E}-03$ & $2.40 \mathrm{E}-03$ & $3.28 \mathrm{E}-03$ & $5.74 \mathrm{E}-03$ & $2.29 \mathrm{E}-03$ & $1.05 \mathrm{E}-04$ & $3.29 \mathrm{E}-11$ & \\
\hline & Area $3 \mathrm{SZ}$ & 0 & $1.78 \mathrm{E}-03$ & $5.11 \mathrm{E}-03$ & $1.21 \mathrm{E}-02$ & $1.66 \mathrm{E}-02$ & $2.91 \mathrm{E}-02$ & $1.16 \mathrm{E}-02$ & $5.33 \mathrm{E}-04$ & $1.67 \mathrm{E}-10$ & \\
\hline & Area $4 \mathrm{SZ}$ & 0 & $2.04 \mathrm{E}-03$ & $5.84 \mathrm{E}-03$ & $1.39 \mathrm{E}-02$ & $1.90 \mathrm{E}-02$ & $3.32 \mathrm{E}-02$ & $1.32 \mathrm{E}-02$ & $6.09 \mathrm{E}-04$ & $1.90 \mathrm{E}-10$ & \\
\hline & Comb. Areas & 0 & $1.78 \mathrm{E}-03$ & $5.11 \mathrm{E}-03$ & $1.21 \mathrm{E}-02$ & $1.66 \mathrm{E}-02$ & $2.90 \mathrm{E}-02$ & $1.16 \mathrm{E}-02$ & $5.33 \mathrm{E}-04$ & $1.66 \mathrm{E}-10$ & \\
\hline & $\mathrm{OB} / \mathrm{BCL}$ & 0 & 0 & 0 & 0 & 0 & 0 & 0 & 0 & 0 & \\
\hline & SPA SZ & 0 & 0 & 0 & 0 & 0 & 0 & 0 & 0 & 0 & \\
\hline & SPA DZ & 0 & $2.29 \mathrm{E}-04$ & $6.56 \mathrm{E}-04$ & $1.56 \mathrm{E}-03$ & $2.13 \mathrm{E}-03$ & $3.73 \mathrm{E}-03$ & $1.49 \mathrm{E}-03$ & $6.85 \mathrm{E}-05$ & $2.15 \mathrm{E}-11$ & \\
\hline & SPA Total & 0 & $2.29 \mathrm{E}-04$ & $6.56 \mathrm{E}-04$ & $1.56 \mathrm{E}-03$ & $2.13 \mathrm{E}-03$ & $3.73 \mathrm{E}-03$ & $1.49 \mathrm{E}-03$ & $6.85 \mathrm{E}-05$ & $2.15 \mathrm{E}-11$ & \\
\hline \multirow{9}{*}{ Co-60 } & Area $1 \mathrm{SZ}$ & 0 & 0 & 0 & 0 & 0 & 0 & 0 & 0 & 0 & \multirow{9}{*}{100} \\
\hline & Area $2 \mathrm{SZ}$ & 0 & $2.24 \mathrm{E}-03$ & $5.15 \mathrm{E}-03$ & 7.12E-03 & $6.31 \mathrm{E}-03$ & $3.84 \mathrm{E}-04$ & $1.10 \mathrm{E}-08$ & $5.60 \mathrm{E}-18$ & 0 & \\
\hline & Area $3 \mathrm{SZ}$ & 0 & $8.35 \mathrm{E}-05$ & $1.93 \mathrm{E}-04$ & $2.66 \mathrm{E}-04$ & $2.36 \mathrm{E}-04$ & $1.43 \mathrm{E}-05$ & $4.11 \mathrm{E}-10$ & $2.10 \mathrm{E}-19$ & 0 & \\
\hline & Area $4 \mathrm{SZ}$ & 0 & 0 & 0 & 0 & 0 & 0 & 0 & 0 & 0 & \\
\hline & Comb. Areas & 0 & $2.24 \mathrm{E}-03$ & 5.15E-03 & 7.12E-03 & $6.31 \mathrm{E}-03$ & 3.84E-04 & 1.10E-08 & $5.60 \mathrm{E}-18$ & $2.24 \mathrm{E}-03$ & \\
\hline & $\mathrm{OB} / \mathrm{BCL}$ & 0 & 0 & 0 & 0 & 0 & 0 & 0 & 0 & 0 & \\
\hline & SPA SZ & 0 & 0 & 0 & 0 & 0 & 0 & 0 & 0 & 0 & \\
\hline & SPA DZ & 0 & 0 & 0 & 0 & 0 & 0 & 0 & 0 & 0 & \\
\hline & SPA Total & 0 & 0 & 0 & 0 & 0 & 0 & 0 & 0 & 0 & \\
\hline \multirow{9}{*}{$\mathrm{Ni}-63$} & Area $1 \mathrm{SZ}$ & 0 & 0 & 0 & 0 & 0 & 0 & 0 & 0 & 0 & \multirow{9}{*}{50} \\
\hline & Area $2 \mathrm{SZ}$ & 0 & $3.08 \mathrm{E}-01$ & $9.11 \mathrm{E}-01$ & $2.34 \mathrm{E}+00$ & $3.41 \mathrm{E}+00$ & $9.77 \mathrm{E}+00$ & $1.57 \mathrm{E}+01$ & $1.06 \mathrm{E}+01$ & $1.30 \mathrm{E}-01$ & \\
\hline & Area $3 \mathrm{SZ}$ & 0 & 0 & 0 & 0 & 0 & 0 & 0 & 0 & 0 & \\
\hline & Area $4 \mathrm{SZ}$ & 0 & 0 & 0 & 0 & 0 & 0 & 0 & 0 & 0 & \\
\hline & Comb. Areas & 0 & $3.08 \mathrm{E}-01$ & 9.11E-01 & $2.34 \mathrm{E}+00$ & $3.41 \mathrm{E}+00$ & $9.77 \mathrm{E}+00$ & $1.57 \mathrm{E}+01$ & $1.06 \mathrm{E}+01$ & $2.20 \mathrm{E}-01$ & \\
\hline & $\mathrm{OB} / \mathrm{BCL}$ & 0 & 0 & 0 & 0 & 0 & 0 & 0 & 0 & 0 & \\
\hline & SPA SZ & 0 & 0 & 0 & 0 & 0 & 0 & 0 & 0 & 0 & \\
\hline & SPA DZ & 0 & 0 & 0 & 0 & 0 & 0 & 0 & 0 & 0 & \\
\hline & SPA Total & 0 & 0 & 0 & 0 & 0 & 0 & 0 & 0 & 0 & \\
\hline
\end{tabular}


Washington Closure Hanford

CALCULATION SHEET

Originator: S. W. Clark Xwe

Date: A/ $3 / 86$ Calc. No.: $0100 \mathrm{C}-\mathrm{CA}-\mathrm{V} 0035$

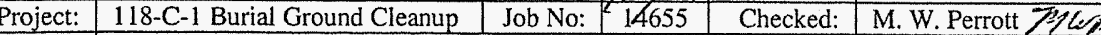

Subject: $118-\mathrm{C}-1$ Burial Ground Cleanup Verification RESRAD Calculation Brief

Table 5. Predicted Groundwater (Well Water/Drinking Water) Concentrations (2 pages)

\begin{tabular}{|c|c|c|c|c|c|c|c|c|c|c|c|}
\hline \multirow{2}{*}{$\begin{array}{l}\text { Radio- } \\
\text { nuclides }\end{array}$} & \multirow{2}{*}{$\begin{array}{c}\text { Vadose Zone } \\
\text { Horizon }\end{array}$} & \multicolumn{9}{|c|}{ Groundwater Concentrations in $\mathrm{pCi} / \mathrm{L}$ at Each Time Slice (yr) } & \multirow{2}{*}{$\begin{array}{l}\text { RAGs, } \\
\mathrm{pCi} / \mathrm{L}\end{array}$} \\
\hline & & 0 & 1 & 3 & 8 & 12 & 43 & 131 & 300 & 1000 & \\
\hline \multirow{9}{*}{ Sr-90 } & Area $1 \mathrm{SZ}$ & 0 & 0 & 0 & 0 & 0 & 0 & 0 & 0 & 0 & \multirow{9}{*}{8} \\
\hline & Area $2 \mathrm{SZ}$ & 0 & 0 & 0 & 0 & 0 & 0 & 0 & 0 & 0 & \\
\hline & Area $3 \mathrm{SZ}$ & 0 & $4.92 \mathrm{E}-03$ & $1.41 \mathrm{E}-02$ & $3.33 \mathrm{E}-02$ & $4.54 \mathrm{E}-02$ & 7.77E-02 & $2.91 \mathrm{E}-02$ & $1.18 \mathrm{E}-03$ & $1.53 \mathrm{E}-10$ & \\
\hline & Area $4 \mathrm{SZ}$ & 0 & 0 & 0 & 0 & 0 & 0 & 0 & 0 & 0 & \\
\hline & Comb. Areas & 0 & 4.92E-03 & $1.41 \mathrm{E}-02$ & $3.33 \mathrm{E}-02$ & $4.54 \mathrm{E}-02$ & $7.77 \mathrm{E}-02$ & $2.90 \mathrm{E}-02$ & $1.18 \mathrm{E}-03$ & $1.77 \mathrm{E}-10$ & \\
\hline & $\mathrm{OB} / \mathrm{BCL}$ & 0 & $2.26 \mathrm{E}-02$ & $6.46 \mathrm{E}-02$ & $1.53 \mathrm{E}-01$ & $2.08 \mathrm{E}-01$ & $3.57 \mathrm{E}-01$ & $1.33 \mathrm{E}-01$ & $5.44 \mathrm{E}-03$ & $5.10 \mathrm{E}-10$ & \\
\hline & SPA SZ & 0 & 0 & 0 & 0 & 0 & 0 & 0 & 0 & 0 & \\
\hline & SPA DZ & 0 & 0 & 0 & 0 & 0 & 0 & 0 & 0 & 0 & \\
\hline & SPA Total & 0 & 0 & 0 & 0 & 0 & 0 & 0 & 0 & 0 & \\
\hline \multirow{9}{*}{ Tritium (H-3) } & Area $1 \mathrm{SZ}$ & 0 & 0 & 0 & 0 & 0 & 0 & 0 & 0 & 0 & \multirow{9}{*}{20,000} \\
\hline & Area $2 \mathrm{SZ}$ & 0 & $2.37 \mathrm{E}+03$ & $5.43 \mathrm{E}+03$ & $1.82 \mathrm{E}+03$ & $7.42 \mathrm{E}+02$ & $6.93 \mathrm{E}-01$ & $1.64 \mathrm{E}-09$ & $3.46 \mathrm{E}-26$ & 0 & \\
\hline & Area $3 \mathrm{SZ}$ & 0 & 0 & 0 & 0 & 0 & 0 & 0 & 0 & 0 & \\
\hline & Area $4 \mathrm{SZ}$ & 0 & 0 & 0 & 0 & 0 & 0 & 0 & 0 & 0 & \\
\hline & Comb. Areas & 0 & $2.37 E+03$ & $5.43 \mathrm{E}+03$ & $4.02 \mathrm{E}+03$ & $1.64 \mathrm{E}+03$ & $1.53 \mathrm{E}+00$ & 3.62E-09 & $7.68 \mathrm{E}-26$ & 0 & \\
\hline & $\mathrm{OB} / \mathrm{BCL}$ & 0 & 0 & 0 & 0 & 0 & 0 & 0 & 0 & 0 & \\
\hline & SPA SZ & 0 & 0 & 0 & 0 & 0 & 0 & 0 & 0 & 0 & \\
\hline & SPADZ & 0 & 0 & 0 & 0 & 0 & 0 & 0 & 0 & 0 & \\
\hline & SPA Total & 0 & 0 & 0 & 0 & 0 & 0 & 0 & 0 & 0 & \\
\hline
\end{tabular}

RAGs = Remedial action goals from the 100 Area RDR/RAWP

$\mathrm{OB} / \mathrm{BCL}=$ Overburden/Below Cleanup Levels

$\mathrm{SPA}=$ Staging Pile (Stockpile) Area

1

2

3

4

5

6

\section{CONCLUSIONS:}

- The "all pathways" (maximum) dose rates are shown in Table 3. The maximum all pathways dose rate from the 118-C-1 Burial Ground excavated combined Areas 1, 2, 3, and 4 is 12.8 $\mathrm{mrem} / \mathrm{yr}$ at year zero (2006). The maximum all-pathways dose rate for the overburden (OB/BCL) soil is $4.01 \mathrm{mrem} / \mathrm{yr}$ at year zero (2006). For the staging pile area (SPA) the total maximum all-pathways dose rate for the shallow and deep zones is $12.4 \mathrm{mrem} / \mathrm{yr}$ at year zero (2006).

- The dominant pathway for the dose rate for most of the RESRAD evaluations is direct external exposure (external gamma). See Table 6, below.

- The primary radionuclide contributing to the direct exposure pathway for the majority of the evaluations is cesium-137. The primary radionuclide contributing to the direct exposure pathway for the combined Areas 1,2,3, and 4 is cobalt-60. See Table 6, below.

- None of the site COCs are projected to exceed remedial action goals (RAGs). 
1

- The radionuclide excess lifetime cancer risk (ELCR) results are shown in Table 4. The maximum ELCR from the 118-C-1 Burial Ground areas combined Areas 1,2, 3, and 4 is $9.95 \times 10^{-5}$ at year zero (2006). The maximum ELCR for the overburden (OB/BCL) soil $\left(5.42 \times 10^{-5}\right)$ also occurs at year zero (2006). The total maximum all-pathways ELRC for the staging pile area (SPA) shallow and deep zones is $1.55 \times 10^{-4}$ at year zero (2006).

\begin{tabular}{|c|c|c|c|}
\hline \multicolumn{5}{|c|}{ Table 6. Dominant Pathways and Primary Radionuclides for RESRAD Evaluations } \\
\hline RESRAD Run & Vadose Zone Horizon & Dominant Pathway & Primary Radionuclide \\
\hline Area 1 & Shallow Zone & External Gamma & Eu-152 \\
\hline Area 2 & Shallow Zone & External Gamma & Co-60 \\
\hline Area 3 & Shallow Zone & External Gamma & Cs-137 \\
\hline Area 4 & Shallow Zone & External Gamma & Cs-137 \\
\hline Combined Areas & Shallow Zone & External Gamma & Co-60 \\
\hline OB/BCL & Shallow Zone & Plant Ingestion & Sr-90 \\
\hline SPA & Shallow Zone & External Gamma & Eu-152 \\
\hline SPA & Deep Zone & Fish & Cs-137 \\
\hline
\end{tabular}

$\mathrm{OB} / \mathrm{BCL}=$ Overburden/Below Cleanup Levels

SPA = Staging Pile (Stockpile) Area

- Among the radionuclide contaminants of concern, cesium-137, cobalt-60, nickel-63, strontium-90, and tritium are calculated to reach groundwater in the 1,000 years of the RESRAD model run. These radionuclides are predicted to reach groundwater at concentrations significantly below the RAGs.

\section{ATTACHMENTS:}

1. Graphic showing 118-C-1 Cleanup Verification Model (2 pages)

2. Schematic of the 118-C-1 Burial Ground Remediation Areas (1 page)

3. RESRAD Output: 118-C-1 Area 1 Shallow Zone Radionuclides - Mixture Sums and Single Radionuclide Guidelines (19 pages)

4. RESRAD Output: 118-C-1 Area 1 Shallow Zone Radionuclides - Intake Quantities and Health Risk Factors (20 pages)

5. RESRAD Output: 118-C-1 Area 1 Shallow Zone Radionuclides - Concentration of Radionuclides, (10 pages)

6. RESRAD Output: 118-C-1 Area 2 Shallow Zone Radionuclides - Mixture Sums and Single Radionuclide Guidelines (21 pages)

7. RESRAD Output: 118-C-1 Area 2 Shallow Zone Radionuclides - Intake Quantities and Health Risk Factors (29 pages)

8. RESRAD Output: 118-C-1 Area 2 Shallow Zone Radionuclides - Concentration of Radionuclides (10 pages)

9. RESRAD Output: 118-C-1 Area 3 Shallow Zone Radionuclides - Mixture Sums and Single Radionuclide Guidelines (20 pages) 
10. RESRAD Output: 118-C-1 Area 3 Shallow Zone Radionuclides - Intake Quantities and Health Risk Factors (20 pages)

11. RESRAD Output: 118-C-1 Area 3 Shallow Zone Radionuclides - Concentration of Radionuclides (10 pages)

12. RESRAD Output: 118-C-1 Area 4 Shallow Zone Radionuclides - Mixture Sums and Single Radionuclide Guidelines (19 pages)

13. RESRAD Output: 118-C-1 Area 4 Shallow Zone Radionuclides - Intake Quantities and Health Risk Factors (20 pages)

14. RESRAD Output: 118-C-1 Area 4 Shallow Zone Radionuclides - Concentration of Radionuclides (10 pages)

15. RESRAD Output: 118-C-1 Combined Areas 1, 2, 3, and 4 Shallow Zone Radionuclides Mixture Sums and Single Radionuclide Guidelines (22 pages)

16. RESRAD Output: 118-C-1 Combined Areas 1, 2, 3, and 4 Shallow Zone Radionuclides Intake Quantities and Health Risk Factors (29 pages)

17. RESRAD Output: 118-C-1 Combined Areas 1, 2, 3, and 4 Shallow Zone Radionuclides Concentration of Radionuclides (10 pages)

18. RESRAD Output: 118-C-1 Overburden/Below Cleanup Levels Radionuclides - Mixture Sums and Single Radionuclide Guidelines (18 pages)

19. RESRAD Output: 118-C-1 Overburden/Below Cleanup Levels Radionuclides - Intake Quantities and Health Risk Factors (20 pages)

20. RESRAD Output: 118-C-1 Overburden/Below Cleanup Levels Radionuclides Concentration of Radionuclides (10 pages)

21. RESRAD Output: 118-C-1 Staging Pile (Stockpile) Area Shallow Zone Radionuclides Mixture Sums and Single Radionuclide Guidelines (20 pages)

22. RESRAD Output: 118-C-1 Staging Pile (Stockpile) Area Shallow Zone Radionuclides Intake Quantities and Health Risk Factors (20 pages)

23. RESRAD Output: 118-C-1 Staging Pile (Stockpile) Area Shallow Zone Radionuclides Concentration of Radionuclides (10 pages)

24. RESRAD Output: 118-C-1 Staging Pile (Stockpile) Area Deep Zone Radionuclides Mixture Sums and Single Radionuclide Guidelines (19 pages)

25. RESRAD Output: 118-C-1 Staging Pile (Stockpile) Area Deep Zone Radionuclides - Intake Quantities and Health Risk Factors (20 pages)

26. RESRAD Output: 118-C-1 Staging Pile (Stockpile) Area Deep Zone Radionuclides Concentration of Radionuclides (10 pages) 
CVP-2006-00011

Rev. 0

E-10 
Rev. 0

\section{ATTACHMENT 1}

118-C-1 Burial Ground Cleanup Verification Model (2 Pages)

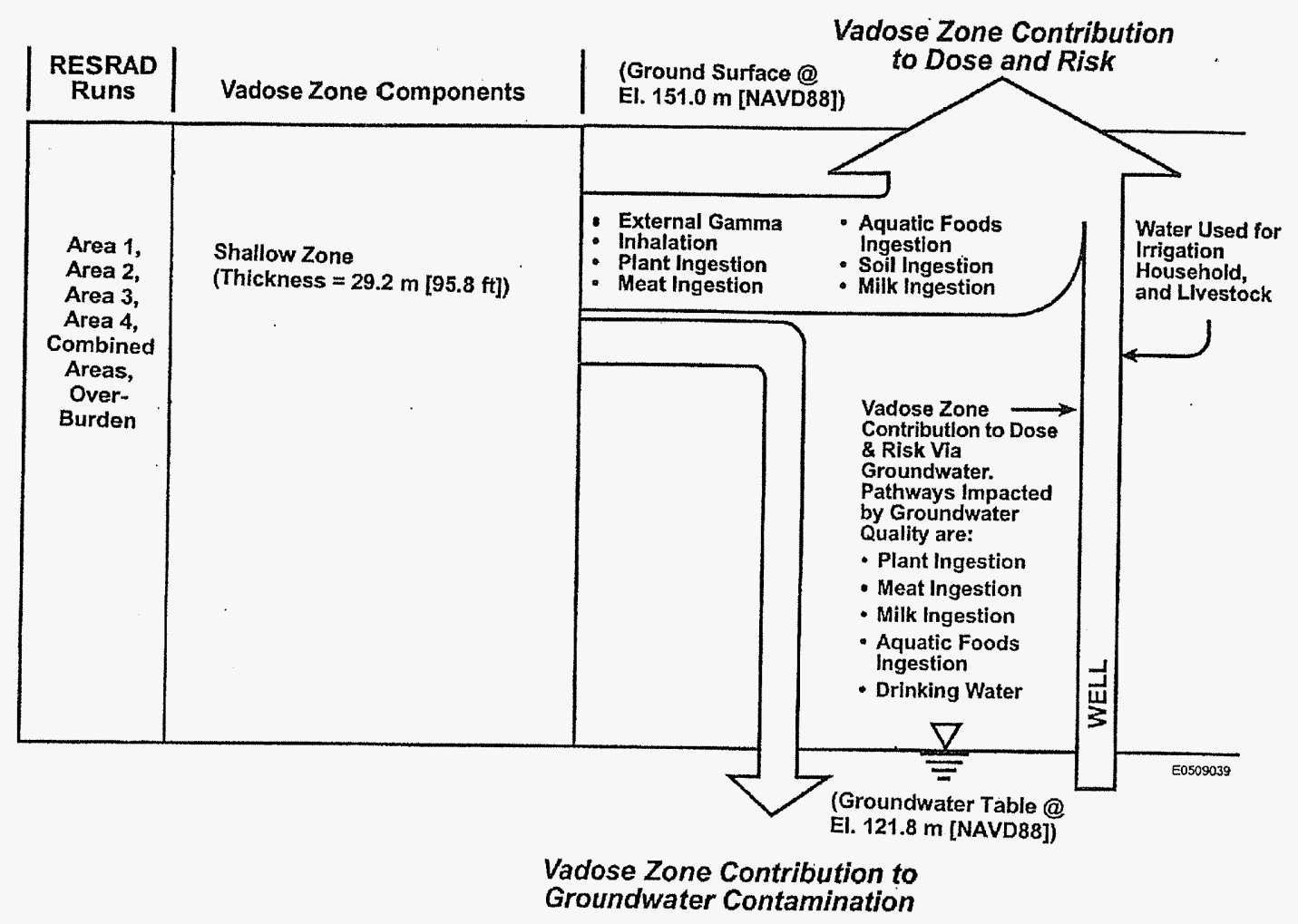

Attachment

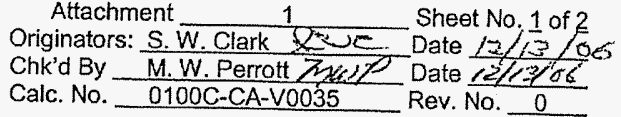


Rev. 0

ATTACHMENT 1

118-C-1 Burial Ground Cleanup Verification Model (2 Pages)
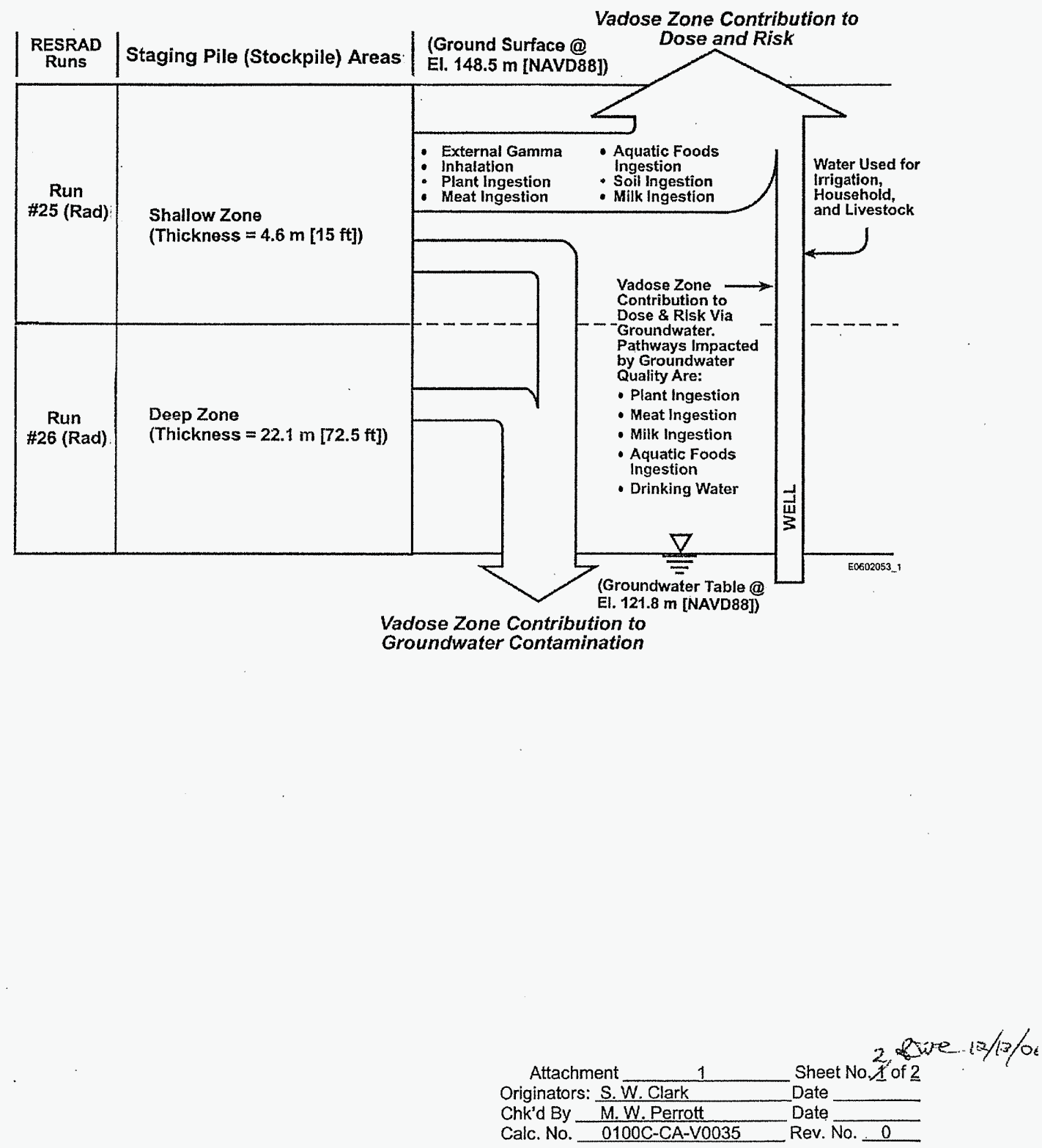


\section{ATTACHMENT 2}

\section{Schematic of the 118-C-1 Burial Ground Remediation Areas Sampled for Cleanup Verification}

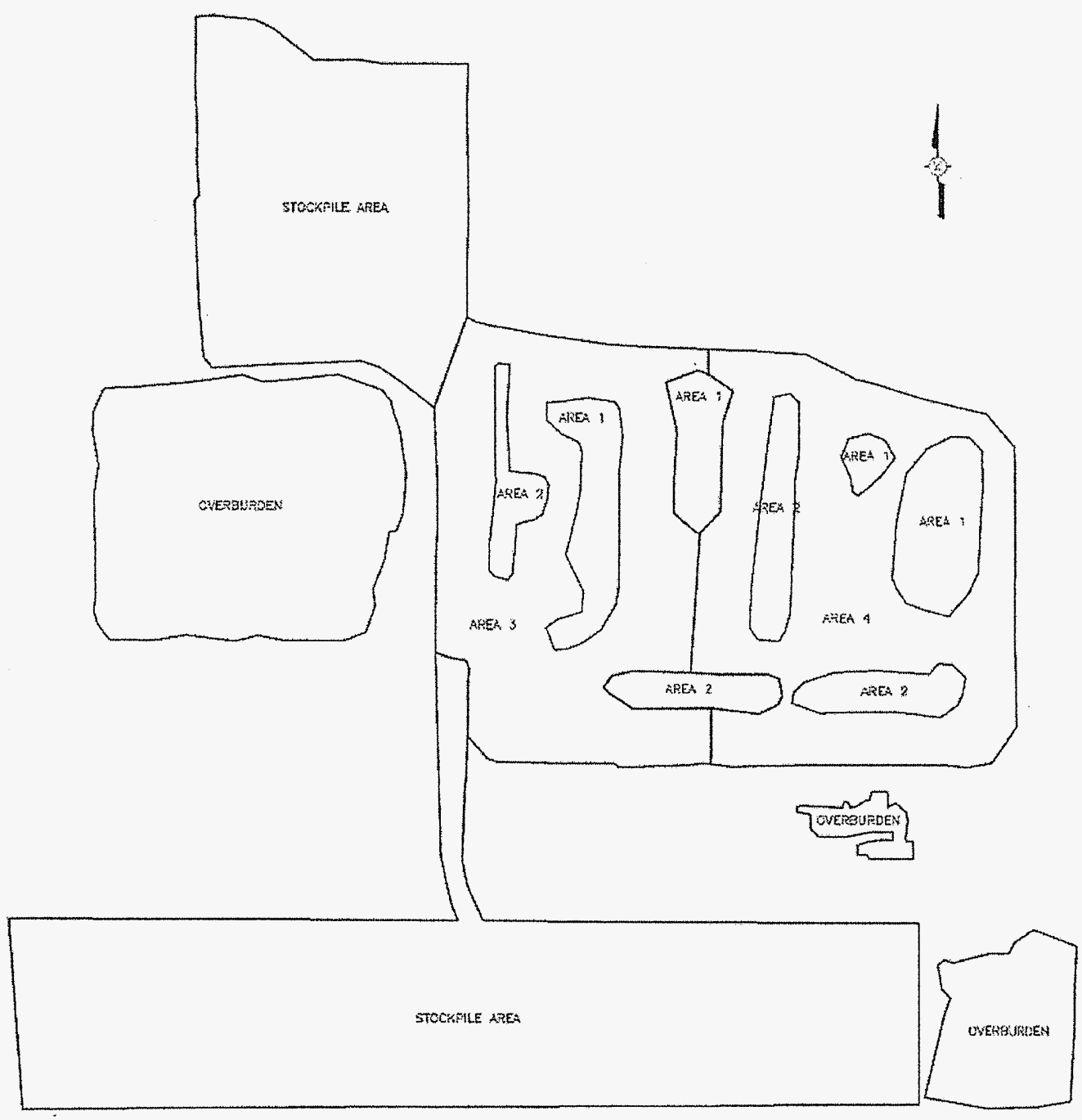

-Attachment

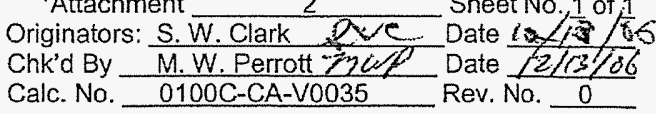


CVP-2006-00011

Rev. 0

E-14 
Rev. 0

\section{ATTACHMENT 3}

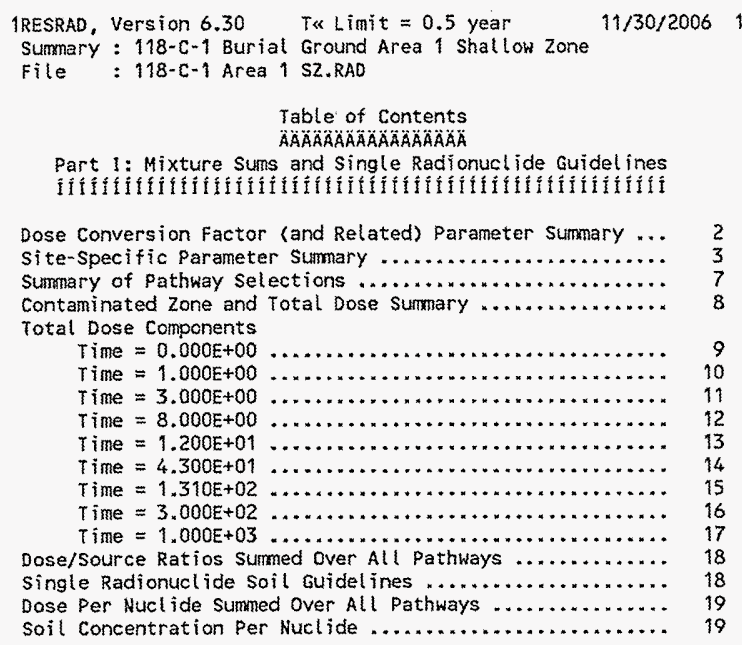

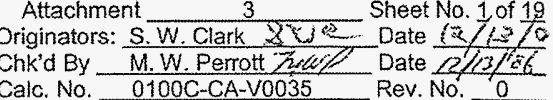




\section{ATTACHMENT 3}

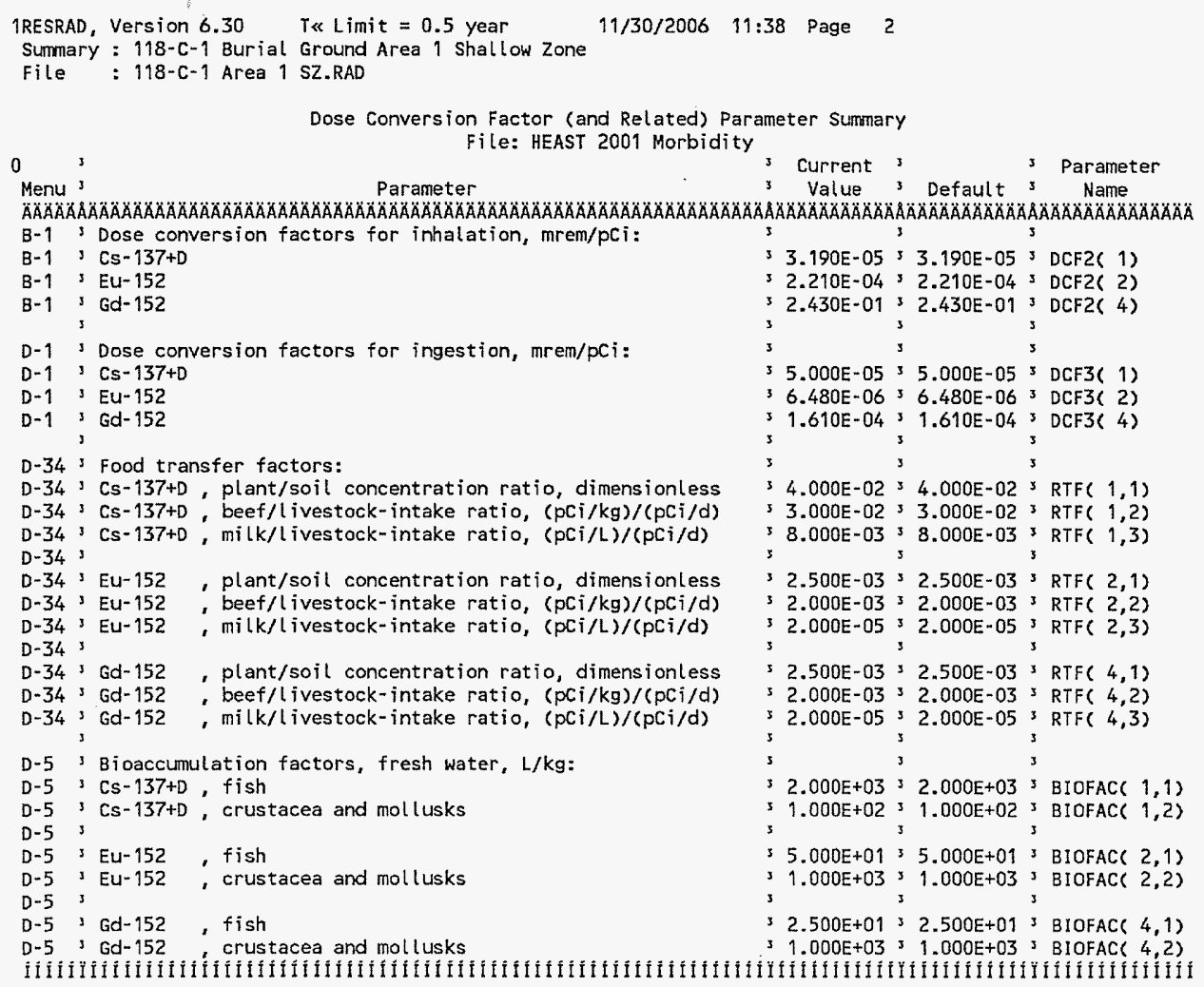

Attachment

3 Sheet No. 2 of 19 


\section{ATTACHMENT 3}

1RESRAD, Version 6.30 T\& Limit $=0.5$ year $11 / 30 / 2006 \quad 11: 38$ Page 3 Summary : 118-C-1 Burial Ground Area 1 shallow Zone

file: $118-C-1$ Area 1 SZ.RAD

MenU ${ }^{3}$

Site-Specific Parameter Summary 3 Used by RESRAD I Parameter

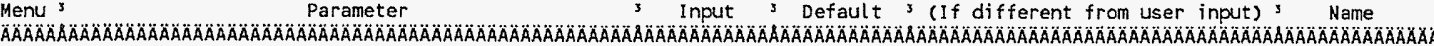
RO11 3 A R011 Area of contaminated zone $\left(m^{* * 2}\right)$ R011 3. Thickness of contaminated zone (m) R011' Length parallel to aquifer flow (m) R011 3 Basic radiation dose limit (mrem/yr) R011 s Time since placement of material (yr) R011' Times for calculations $(y r)$ $33.018 \mathrm{E}+03 ; 1.000 \mathrm{E}+04$

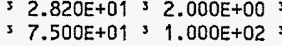
$1.500 E+01^{3} 2.500 E+01$ $0.000 \mathrm{E}+00=0.000 \mathrm{E}+00$ $1.000 E+0031.000 E+00$ $3.000 E+00>3.000 E+00$ $8.000 E+0031.000 E+01$ R011 Times for calculations $(y r)$ R011 Times for calculations $(y r)$ R011, Times for calculations (yr) R011 3 Times for calculations (yr) $1.200 \mathrm{E}+0133.000 \mathrm{E}+01$ $4.300 \mathrm{E}+01,1.000 \mathrm{E}+02$ $31.310 E+02 ; 3.000 E+02$ $33.000 E+023,1.000 E+03$ $31.000 E+03=0.000 E+00$ R011 3 Times for calculations (yr) 3 not used $30.000 \mathrm{E}+00$ R011 S Times for calculations $(y r)$ R011 ' Times for calculations (yr)

3 $31.850 E-01^{3} 0.000 E+00$ s

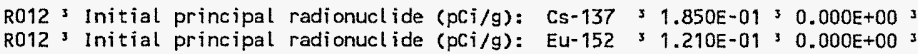
R012 : Concentration in groundwater (pCi/L): Cs-137 s not used s $0.000 E+00$ R012: Concentration in groundwater (pCi/L): Eu-152; not used; $0.000 E+00$ s R013 3 Cover depth (m) R013 * Density of cover material $\left(\mathrm{g} / \mathrm{cm}^{* * 3}\right)$ R013 Cover depth erosion rate $(\mathrm{m} / \mathrm{yr})$ R013 3 Density of contaminated zone (g/cm**3) $30.000 E+00 ; 0.000 E+00$ not used $31.500 E+00$ 3 not used $11.000 E-03$ $31.600 E+0031.500 E+00$ $31.000 E-03,1.000 E-03$

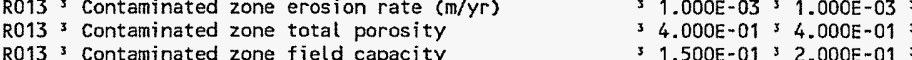

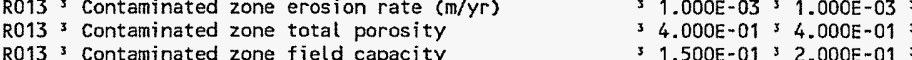
R013 Contaminated zone field capacity $1.500 E-0132.000 E-01$ R013 Contaminated zone hydraulic conductivity $(\mathrm{m} / \mathrm{yr})=3.500 \mathrm{E}+02: 1.000 \mathrm{E}+01$

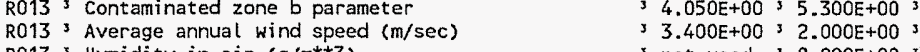
R013 Humidity in air $\left(\mathrm{g} / \mathrm{m}^{* \star 3}\right)$

R013 'Evapotranspiration coefficient R013 3 Precipitation $(\mathrm{m} / \mathrm{yr})$

R013 I Irrigation (m/yr) R013 I irrigation mode R013 Runoff coefficient R013' Watershed area for nearby stream or pond $\left(m^{* * 2}\right.$ R013 * Accuracy for water/soil computations

$$
\text { , }
$$

not Used $8.000 E+00$

$9.100 \mathrm{E}-0135.000 \mathrm{E}-01$ $1.600 E-01>1.000 E+00$ $7.600 \mathrm{E}-01^{3} 2.000 \mathrm{E}-01$ averhead 3 overhead 3 $32.000 E-0132.000 E-01$ $31.000 E+06^{3} 1.000 E+063$

R014 3 Density of saturated zone $\left(\mathrm{g} / \mathrm{cm}^{* * 3}\right)$ R014 3 Saturated zone total porosity R014 S Saturated zone effective porosity R014 3 Saturated zone field capacity R014 3 Saturated zone hydraulic conductivity (m/yr) R014 3 Saturated zone hydraulic gradient R014 Saturated zone b parameter R014 3 Water table drop rate $(\mathrm{m} / \mathrm{yr}$ ) R014 'Well pump intake depth (m below water table) R014 3 Model: Nondispersion (ND) or Mass-Balance (MB) R014 3 Well pumping rate $\left(m^{\star *} 3 / y r\right)$

$1.000 \mathrm{E}-03$ ? $1.000 \mathrm{E}-03$ s

3 $1.600 E+00^{3} 1.500 E+00^{3}$ $34.000 \mathrm{E}-0134.000 \mathrm{E}-013$ $32.500 E-0132.000 E-01$ 3. $1.00 \mathrm{E}-01,2.000 \mathrm{E}-01$, $5.530 \mathrm{E}+03,3,000 \mathrm{E}+02$ 3 $1.250 \mathrm{E}-03,2.000 \mathrm{E}-02$ . $1.000 \mathrm{E}-03$, $1.000 \mathrm{E}-03$ $3.600 E+00 ? 1.000 E+01$ ? ND

$32.500 E+02 ; 2.500 E+023$

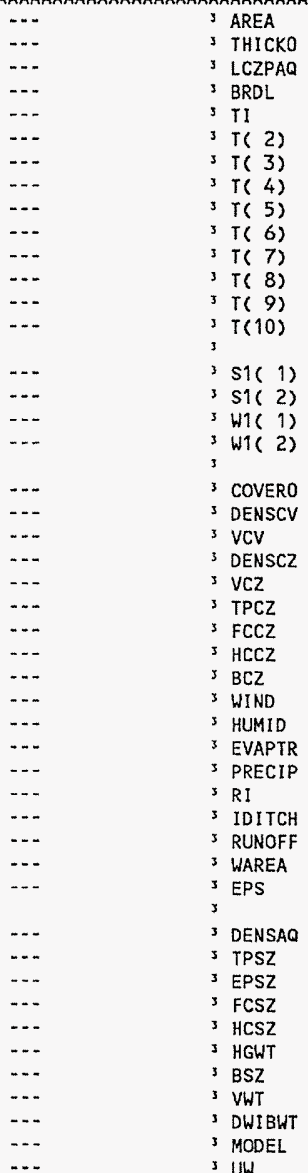

Attachment Originators: S. W. Clark 3 Chk'd By M.W. Perrott Calc. No. 0100C-CA-V0035 Date Date Rev. No. 0 


\section{ATTACHMENT 3}

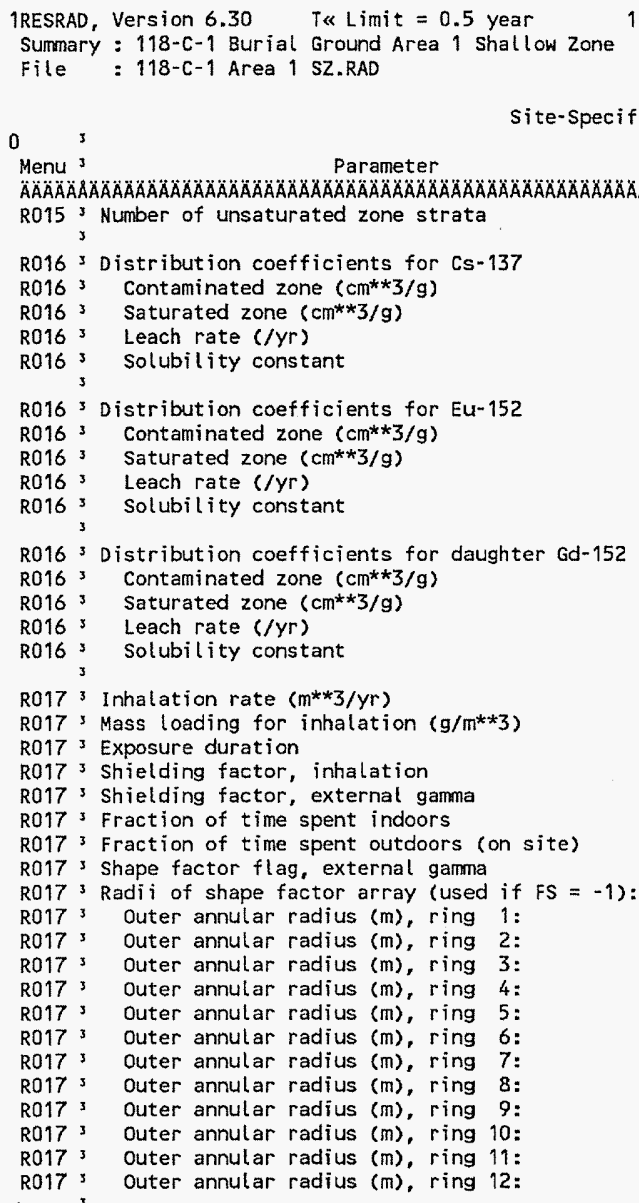

$>0$ shows circular AREA.

\begin{tabular}{|c|c|}
\hline$\cdots$ & 3 RAD SHAPE ( 1$)$ \\
\hline-0 & IRAD_SHAPE( 2) \\
\hline -.. & ${ }^{3}$ RAD_SHAPE ( 3) \\
\hline & $\checkmark$ RAD_SHAPE( 4) \\
\hline & $=$ RAD_SHAPE ( 5) \\
\hline & ${ }^{3}$ RAD_SHAPE ( 6) \\
\hline & 3 RAD_SHAPE ( 7) \\
\hline & $=$ RAD_SHAPE ( 8) \\
\hline & ${ }^{3}$ RAD_SHAPE $(9)$ \\
\hline & 3 RAD_SHAPE $(10)$ \\
\hline & ${ }^{3}$ RAD_SHAPE (11) \\
\hline & 3 RAD_SHAPE(12) \\
\hline
\end{tabular}

$\begin{array}{ll}\text { Attachment } \frac{3}{\text { S. Clark }} & \text { Sheet No. } 4 \text { of } 19 \\ \text { Originators: } \frac{\text { S.W. }}{\text { M.W. Perrott }} & \text { Date } \\ \text { Chk'd By } & \text { Rev. No. } 0 \\ \text { Calc. No. } & 0100 \text { C-CA-V0035 }\end{array}$




\section{ATTACHMENT 3}

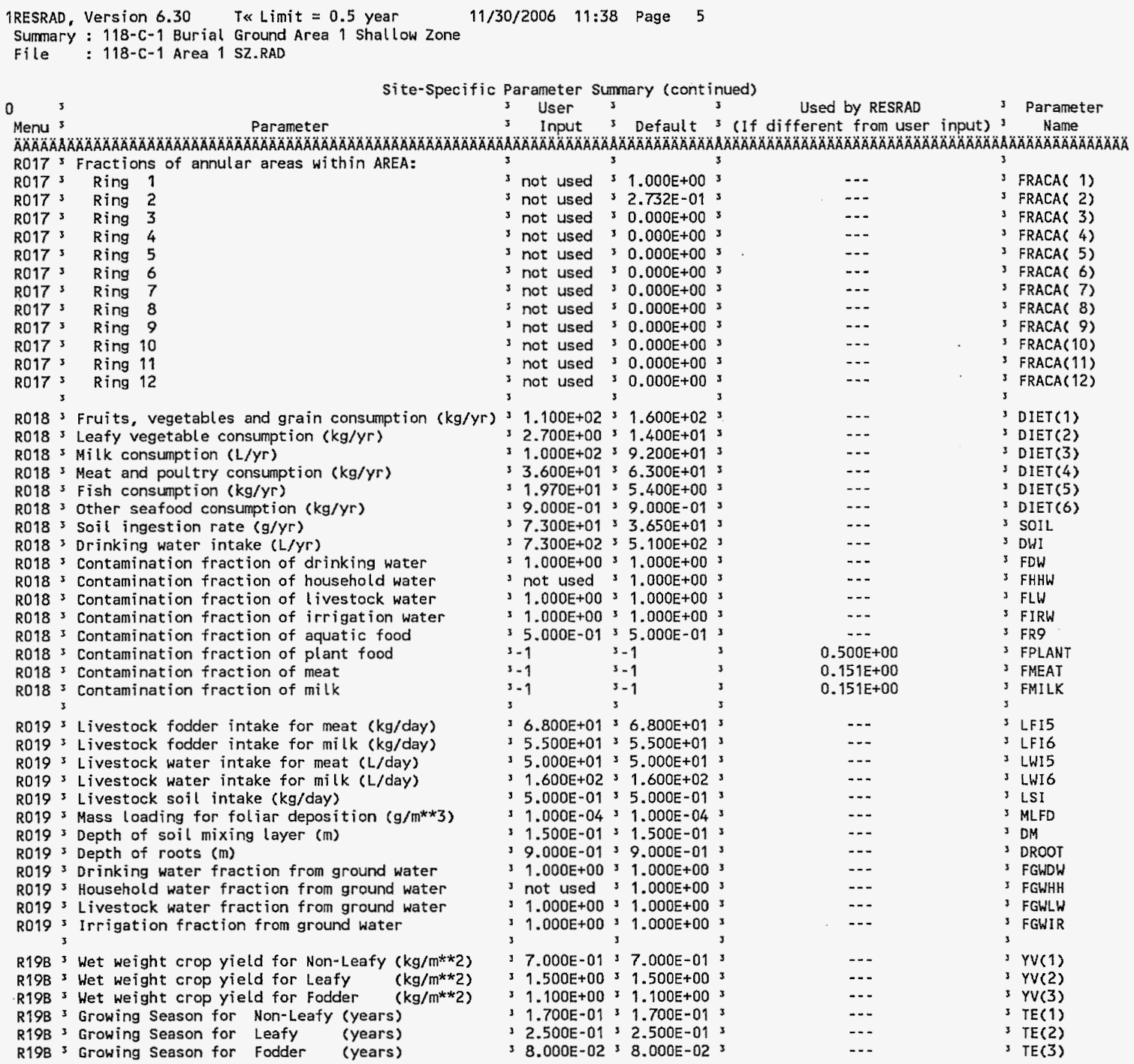

Attachment Originators: S.W. Clark Chk'd By M.W. Perrott Calc. No. $0100 \mathrm{C}-\mathrm{CA}$-V0035
Sheet No. $\underline{5}$ of 19 Date

Rev. No 0 


\section{ATTACHMENT 3}

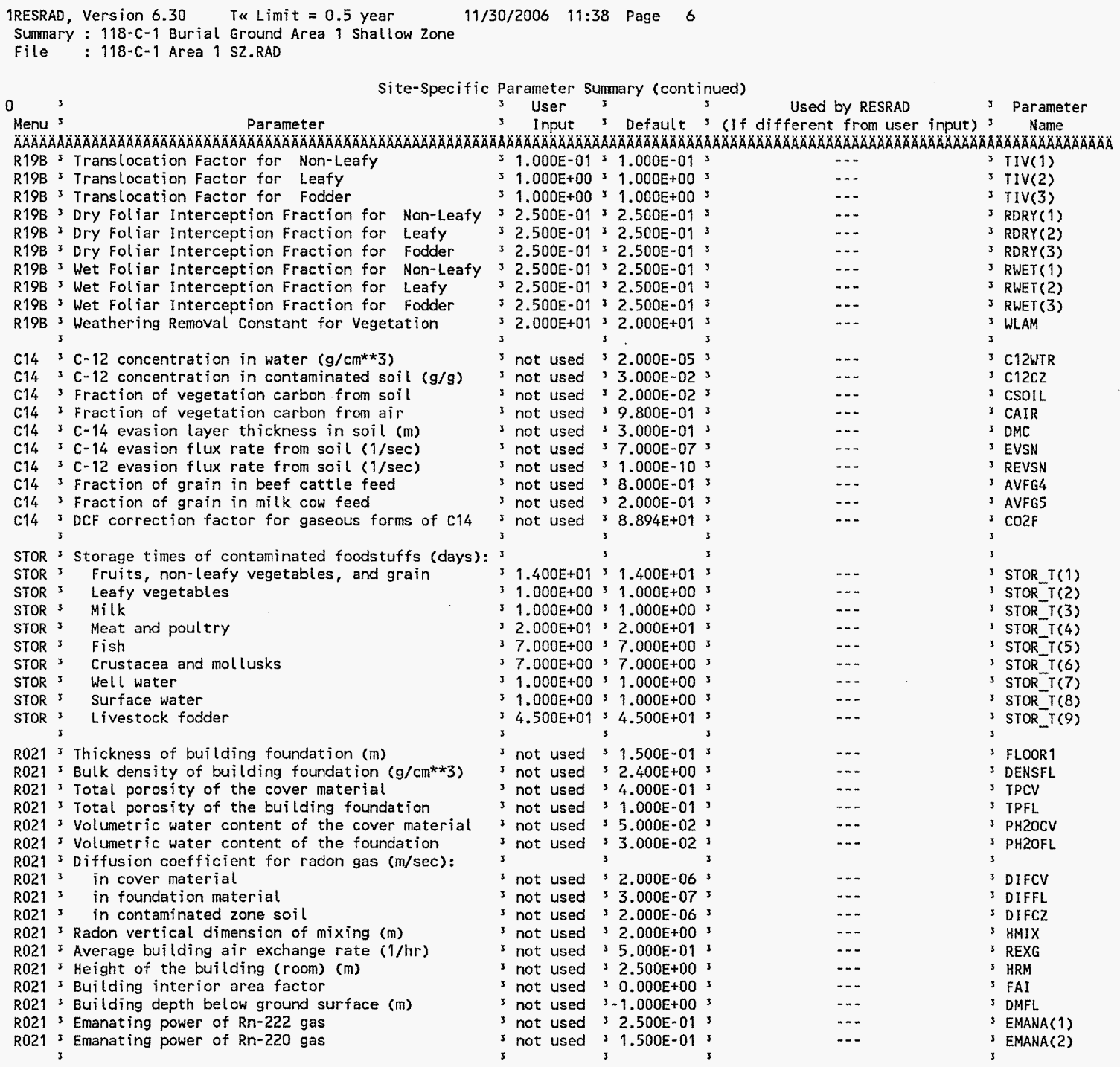




\section{ATTACHMENT 3}

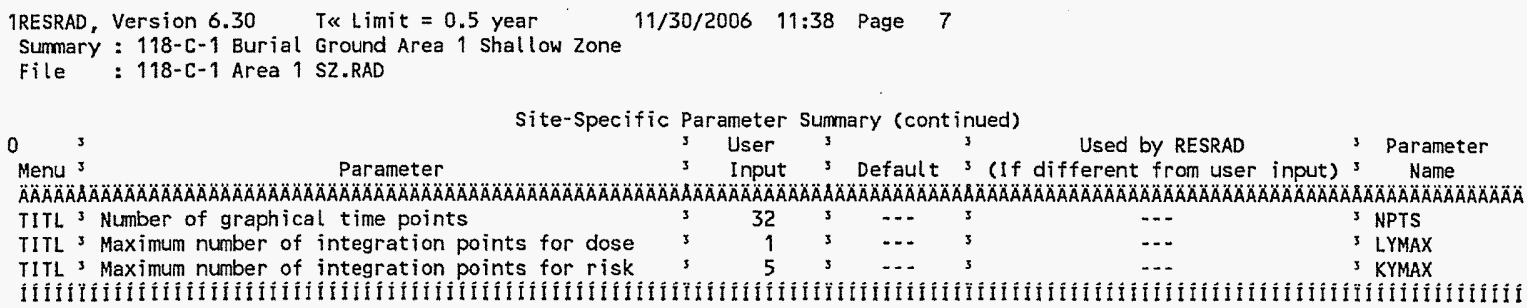




\section{ATTACHMENT 3}

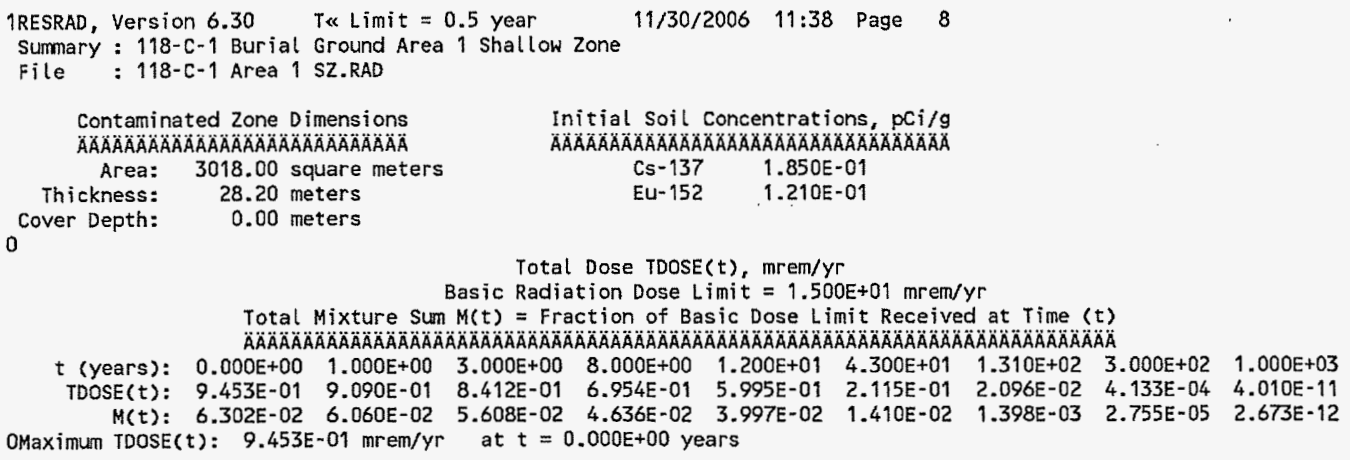

$t$ (years): $\begin{array}{cccccccccc}0.000 E+00 & 1.000 E+00 & 3.000 E+00 & 8.000 E+00 & 1.200 E+01 & 4.300 E+01 & 1.310 E+02 & 3.000 E+02 & 1.000 E+03\end{array}$

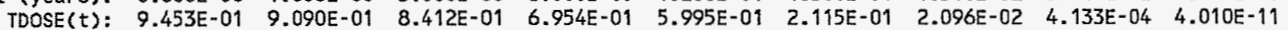

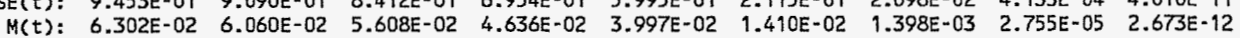
OMaximun TDOSE $(t): 9.453 \mathrm{E}-01 \mathrm{mrem} / \mathrm{yr}$ at $t=0.000 \mathrm{E}+00$ years 


\section{ATTACHMENT 6}

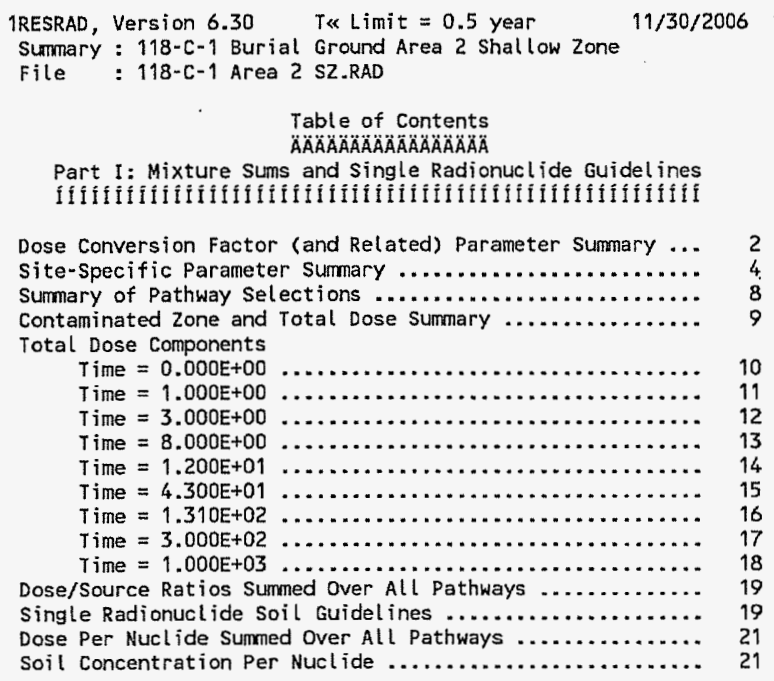

Attachment

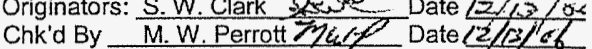
Calc. No. $0100 \mathrm{C}$-CA-V0035 


\section{ATTACHMENT 6}

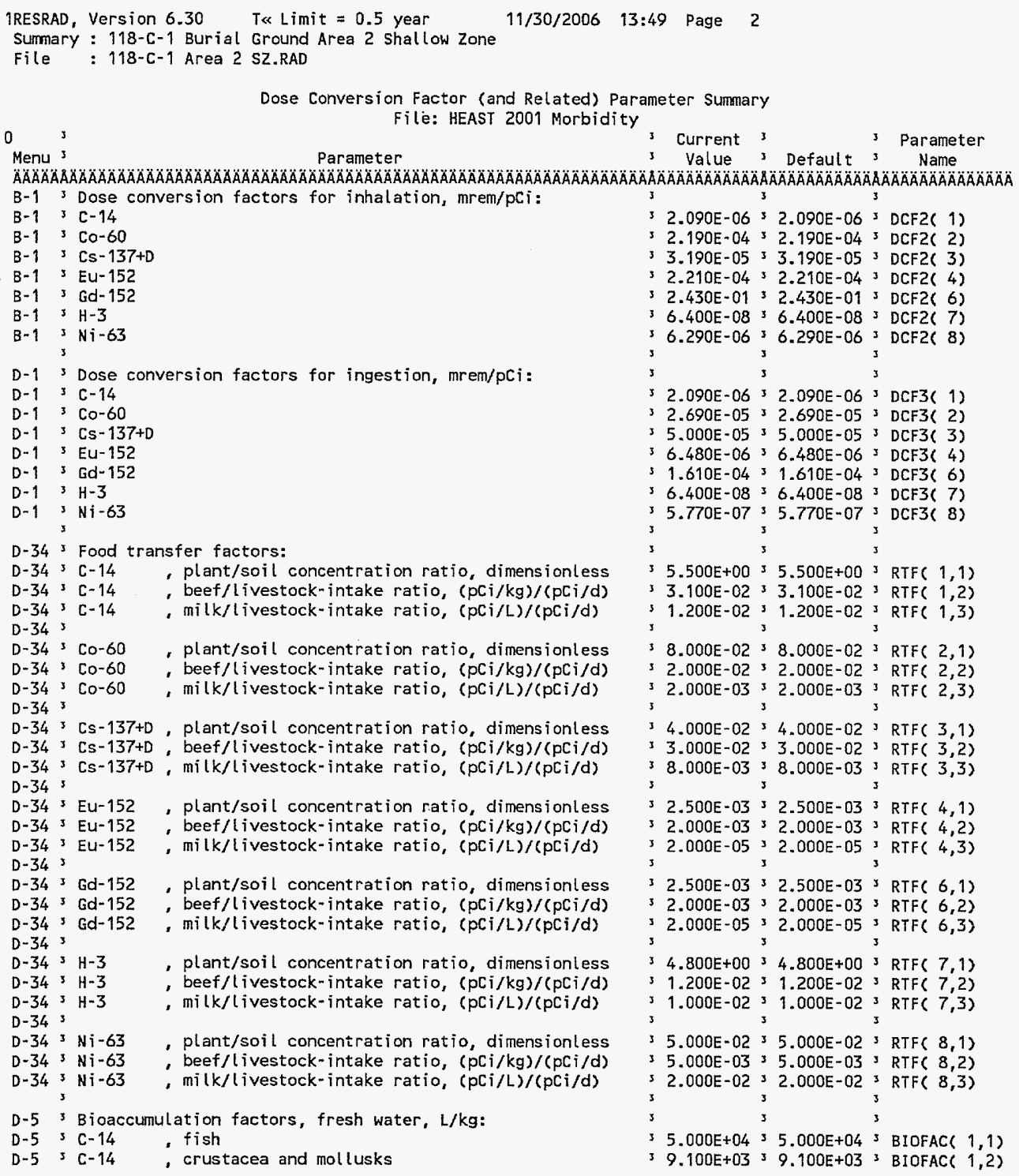




\section{ATTACHMENT 6}

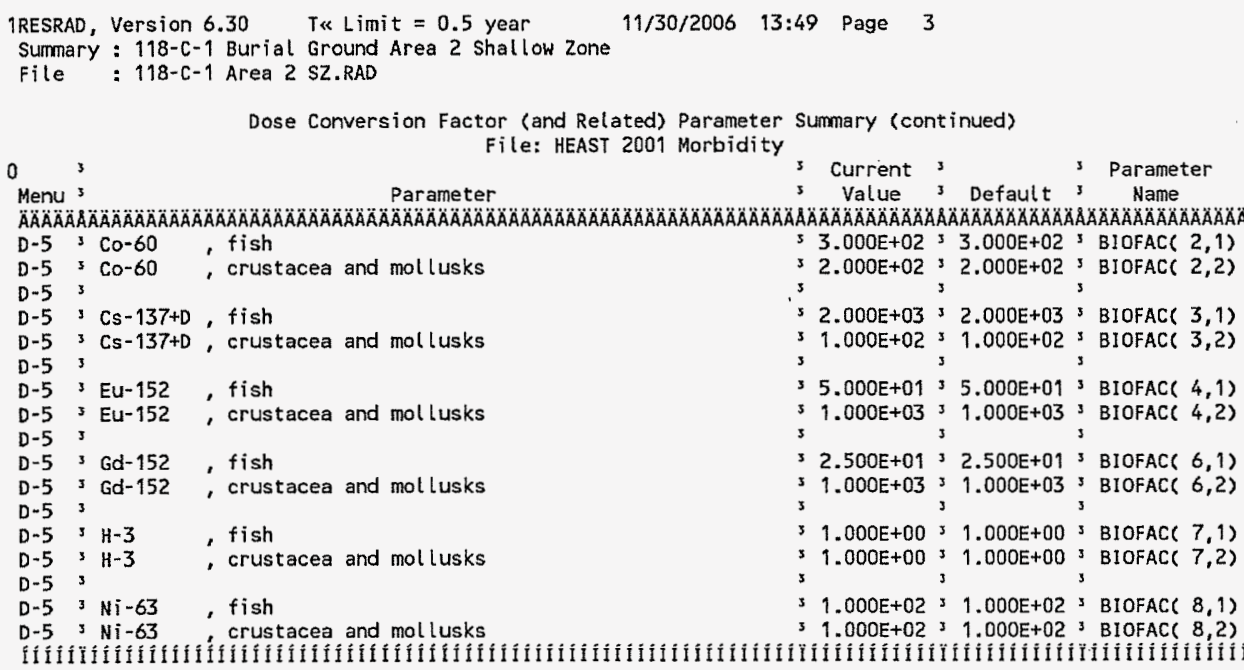

\begin{tabular}{|c|c|c|}
\hline \multicolumn{3}{|c|}{ Attachment $\quad \frac{6}{8} \times \alpha^{2}$ Sheet No. 3 of 21} \\
\hline Originators: & S. W. Clark & \\
\hline & M. W. Perrott & \\
\hline & $0100 \mathrm{C}-\mathrm{CA}$ & No. \\
\hline
\end{tabular}


Rev. 0

\section{ATTACHMENT 6}

IRESRAD, Version $6.30 \quad T \ll$ Limit $=0.5$ year Summary: 118- C-1 Burial Ground Area 2 shallow Zone File: 118-C-1 Area 2 SZ.RAD

Site-Specific Parameter Summary Used by RESRAD Is Parameter

${ }^{3}$

Parameter 3 Input 3 Default s (If different from user input) 3 Name

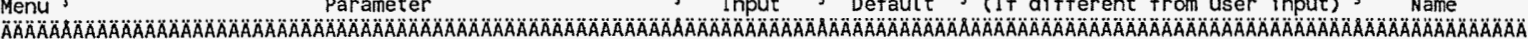

$32.339 E+03: 1.000 E+04$

$3.720 \mathrm{E}+01 ; 2.000 \mathrm{E}+00$

$3.500 \mathrm{E}+0131.000 \mathrm{E}+02,3$

$1.500 E+0132.500 E+01$

$30.000 E+00 \div 0.000 E+003$

$31.000 E+00 \div 1.000 E+00$

$33.000 E+0033.000 E+003$

$3.000+00$ S $3.000 E+00$

$8.000 E+0031.000 E+01$

$31.200 \mathrm{E}+0133.000 \mathrm{E}+01$

$3.300 E+013 \quad 1.000 E+02$

$31.310 E+0233.000 E+02$ ?

$33.000 E+0231.000 E+03$

$31.000 E+0330.000 E+003$

3 not used; $0.000 E+00$;

$8.000 E+0030.000 E+00$ R011 3 Times for calculations $(y r)$ R011' Times for calculations (yr)

R012 3 Initial principal radionuclide (PCi/g): $C^{-14}$ R012, Initial principal radionuctide $(\mathrm{pCi} / \mathrm{g}):$ Co-60, $9.900 \mathrm{E}-0130.000 \mathrm{E}+00$ R012 Initial principal radionuclide (pCi/g): Cs-137 $31.400 E-01: 0.000 E+00$ R012 I Initial principal radionuclide $(\mathrm{pCi} / \mathrm{g})$ : Eu-152 $31.990 \mathrm{E}-0130.000 \mathrm{E}+00$ R012 I Initial principal radionuclide (pCi/g): $\mathrm{H}-3$ 3 $4.760 \mathrm{E}+00^{3} 0.000 \mathrm{E}+00^{3}$ R012 3 Initial principal radionuclide (PCi/g): $\mathrm{Ni}-63$; $2.610 \mathrm{E}+01,0.000 \mathrm{E}+00$ R012 I Initial $\mathrm{principal}$ radionuclide ( $\mathrm{PCi} / \mathrm{g}$ ): $\mathrm{Ni}-63$, $2.610 \mathrm{C}+\mathrm{O}, 0.00 \mathrm{C}+00$

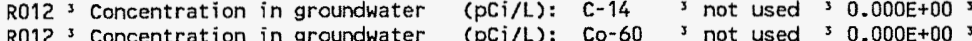

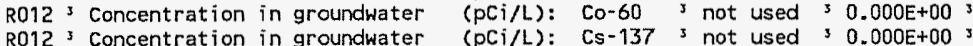
R012 Concentration in groundwater (pCi/L): Cs-137 3 not used $30.000 E+00$ R012' Concentration in groundwater (pCi/L): Eu-152 3 not used $30.000 E+00$ R012 3 Concentration in groundwater $(\mathrm{pCi} / \mathrm{L}): \mathrm{H}-3$ ' 3 not used $30.000 \mathrm{E}+00$ R012 Concentration in groundwater (pCi/L): $\mathrm{Ni}-63$

R013 3 Cover depth (m)

R013 s Density of cover material $\left(\mathrm{g} / \mathrm{cm}^{* * 3}\right)$

2013 3 Cover depth erosion rate $(\mathrm{m} / \mathrm{yr})$

$\mathrm{R} 013$ Density of contaminated zone $\left(\mathrm{g} / \mathrm{cm}^{* * 3}\right)$

R013 3 Contaminated zone erosion rate $(\mathrm{m} / \mathrm{yr})$

R013 3 Contaminated zone total porosity

R013 3 Contaminated zone field capacity

R013 S Contaminated zone hydraulic conductivity (m/yr)

R013' Contaminated zone b parameter

R013 Average annual wind speed $(\mathrm{m} / \mathrm{sec})$

R013 3 Humidity in air $(\mathrm{g} / \mathrm{m} * * 3)$

R013 Evapotranspiration coefficient

R013' Precipitation $(\mathrm{m} / \mathrm{yr})$

R013 3 Irrigation $(\mathrm{m} / \mathrm{yr})$

R013 3 Irrigation mode

R013 s Runoff coefficient

R013 S Watershed area for nearby stream or pond $\left(m^{* * 2}\right)$

R013 I ACcuracy for water/soil computations

3

R014 3 Density of saturated zone $\left(\mathrm{g} / \mathrm{cm}^{\star *} 3\right)$

R014 I Saturated zone total porosity

R014 S Saturated zone effective porosity
3

$0.0005+00,3,0.000 E^{2}+00$

3 not used $31.500 E+00$

not used, $1.000 \mathrm{E}-03$

$1.600 \mathrm{E}+00 ; 1.500 \mathrm{E}+00$

3 1.000E-03 $>1.000 E-03$

3 4.000E-01 3 4.000E-01

$31.500 E-0132.000 E-01$

$2.500 \mathrm{E}+0231.000 \mathrm{E}+01$

s. $4.050 E+00$; $5.300 E+00$;

$3.400 E+0032.000 E+00$

$8.000 E+0038.000 E+00$

$9.100 \mathrm{E}-0135.000 \mathrm{E}-01$

$37.600 \mathrm{E}-01^{3} 2.000 \mathrm{E}-01$

3 overhead 3 overhead

overhead s overhead

$31.000 E+06=1.000 E+06$

$31.000 \mathrm{E}-03,1.000 \mathrm{E}-03$;

s $1.600 \mathrm{E}+00$ ? $1.500 \mathrm{E}+00$

3 4.000E-01 3 4.000E-01

$32.500 \mathrm{E}-0132.000 \mathrm{E}-01 \mathrm{~J}$
$=1.600 \mathrm{E}-01 \times 1.000 \mathrm{E}+00$
-.. 5 AREA

-.. 3 THICKO

-.. 3 LCZPAO

...

..- 3 T 2 ( 2$)$

$\cdots+T(3)$

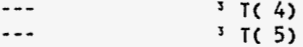

$\ldots 3(6)$

$\ldots+1(7)$

$\ldots$
$\ldots$

...

... $\quad 351(1)$

... $\quad 351(2)$

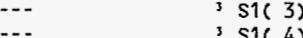

3 s1 7 (

$\ldots \quad s 1(8)$

-- SW1( 1$)$

-.. 3 W1( 2 )

... $31(4)$

... 3 W1 7 (

... W1( 8 )

... 3 COVERO

...

-.. $\quad 3$ VCV

3 DENSCZ

VCZ

‥

-.. $3 \mathrm{FCCZ}$

$\begin{array}{ll}\ldots- & 3 \mathrm{HCCZ} \\ \ldots- & 3 \mathrm{BCZ}\end{array}$

-. 3 WIND

-.. 3 HUMID

a.

-..

-.. 3 IDITCH

-.. 3 RUNOF

-.- 3 WAREA

I

-.. 3 DENSAD

... 3 TPSZ
Attachment

Originators: S. W Clark

Chk'd By M.W. Perrott

Calc. No.

0100C-CA-V0035 Date Date Rev. No. 0 


\section{ATTACHMENT 6}

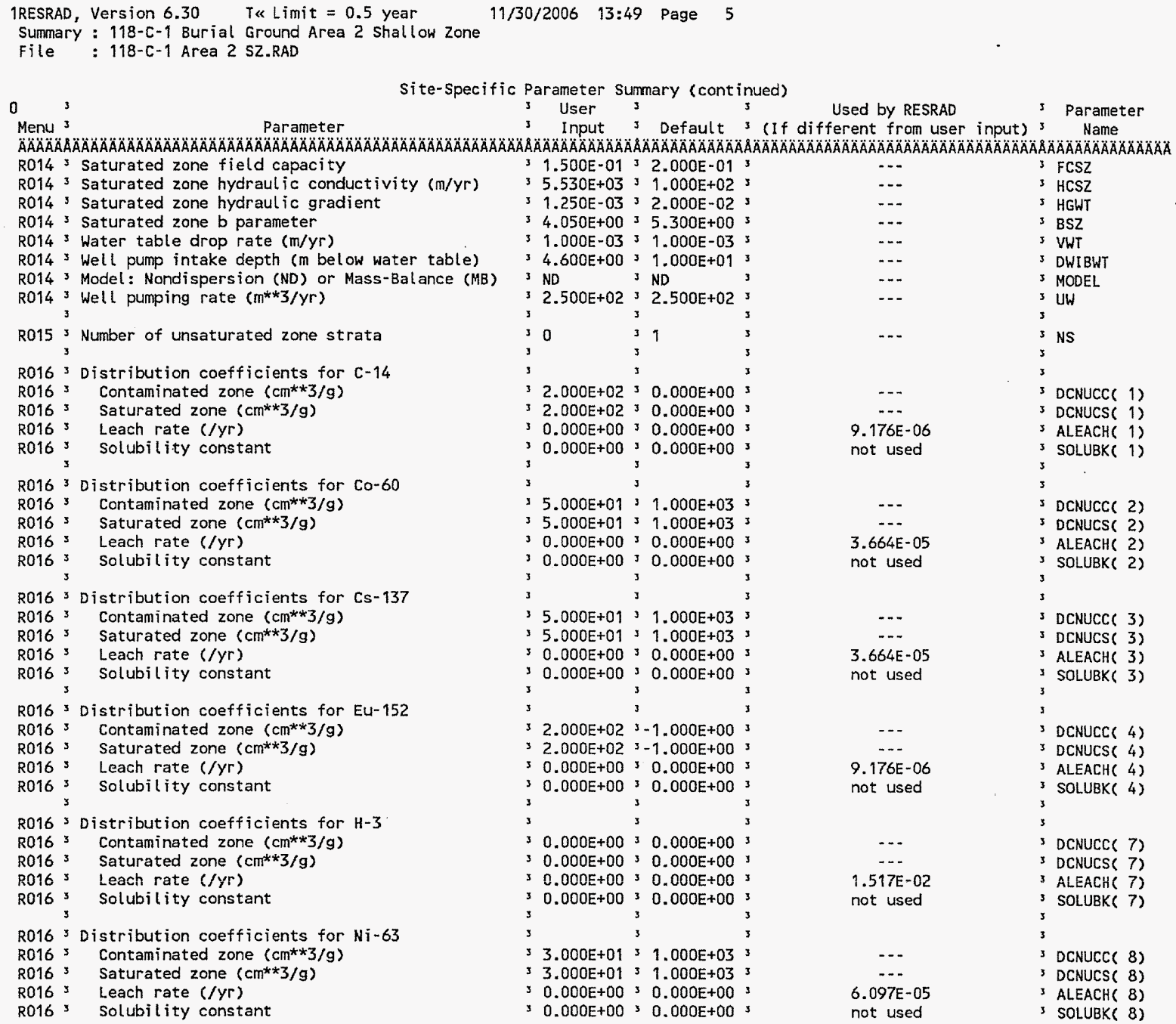

Attachment 6.8 \& 8 S Sheet No. 5 of 21 Originators: $S$.W. Clark Date Chk'd By M.W. Perrott Date Calc. No. $0100 \mathrm{C}$-CA-V0035 Rev. No. _ 0 


\section{ATTACHMENT 6}

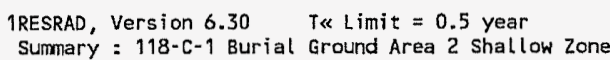

\section{8. $249 E+02$ \\ $8.249 E+02$ \\ $2.226 \mathrm{E}-06$} not used

…

-..

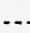

$-\cdot$

-.-

3 DCNUCC (6)

3 DCNUCS( 6)

3 ALEACH ( 6)

SOLUBK( 6)

INHALR

MLINH

SED

SHF3

3 SHF

$>0$ shows circular AREA.

RAD SHAPES (1)

$+-$

... 5 RAD SHAPE( 3 )

-.- 3 RAD SHAPE( 4)

... 3 RAD SHAPE ( 5)

... 3 RAD_SHAPE( 6 )

... 3 RAD_SHAPE( 7)

... 3 RAD_SHAPE( 8)

-..

... 3 RAD SHAPE(11)

-.. 3 RAD_SHAPE(12)

3 FRACAC (1)

3 FRACA( 2)

3 FRACA( 3)

FRACA( 4)

3 FRACA( 5)

FRACAC 6)

FRACAC 7)

FRACAC 8)

3 FRACAC 9)

FRACA(10)

3 FRACAC11)

3 FRACA (12)

$1.100 E+0231.600 \mathrm{E}+02$ $2.700 E+00,1.400 E+01$ $1.000 E+02=9.200 E+01$ $3.600 E+0136.300 E+01$ $1.970 E+0135.400 E+00$ $39.000 E-0139.000 E-01$

$>7.300 E+01>3.650 E+01$

$37.300 E+02 \times 5.100 E+02$
$\mathrm{R} 018$; Leafy vegetable consumption $(\mathrm{kg} / \mathrm{yr})$

R018: Milk consumption ( $L / Y r)$

Reat and pouttry consumption ( $\mathrm{kg} / \mathrm{yr}$

R018 Fish consumption ( $\mathrm{kg} / \mathrm{yr}$ )

R018 Other seafood consumption $(\mathrm{kg} / \mathrm{yr}$

R018 Soil ingestion rate (g/yr) 


\section{ATTACHMENT 6}

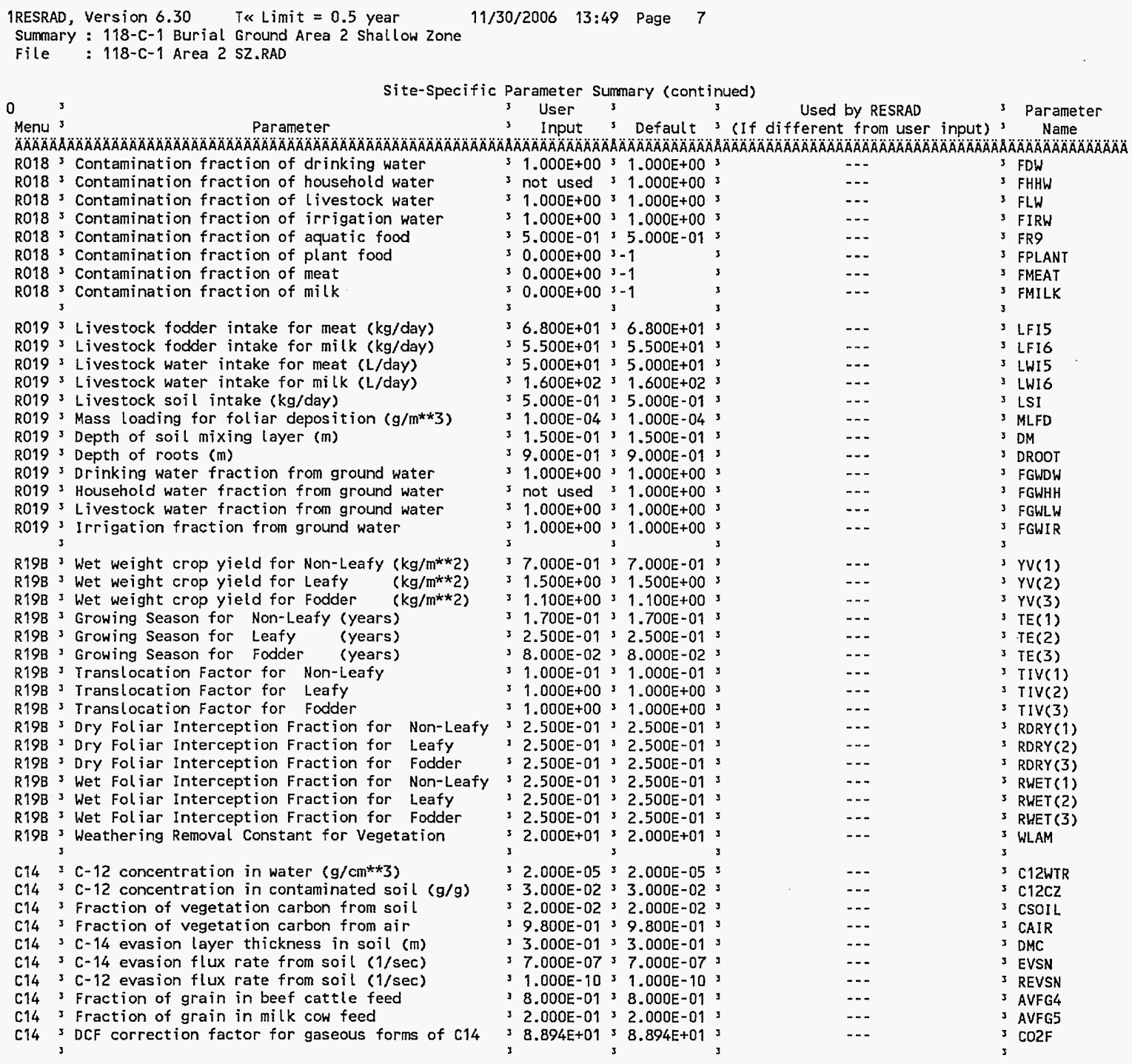




\section{ATTACHMENT 6}

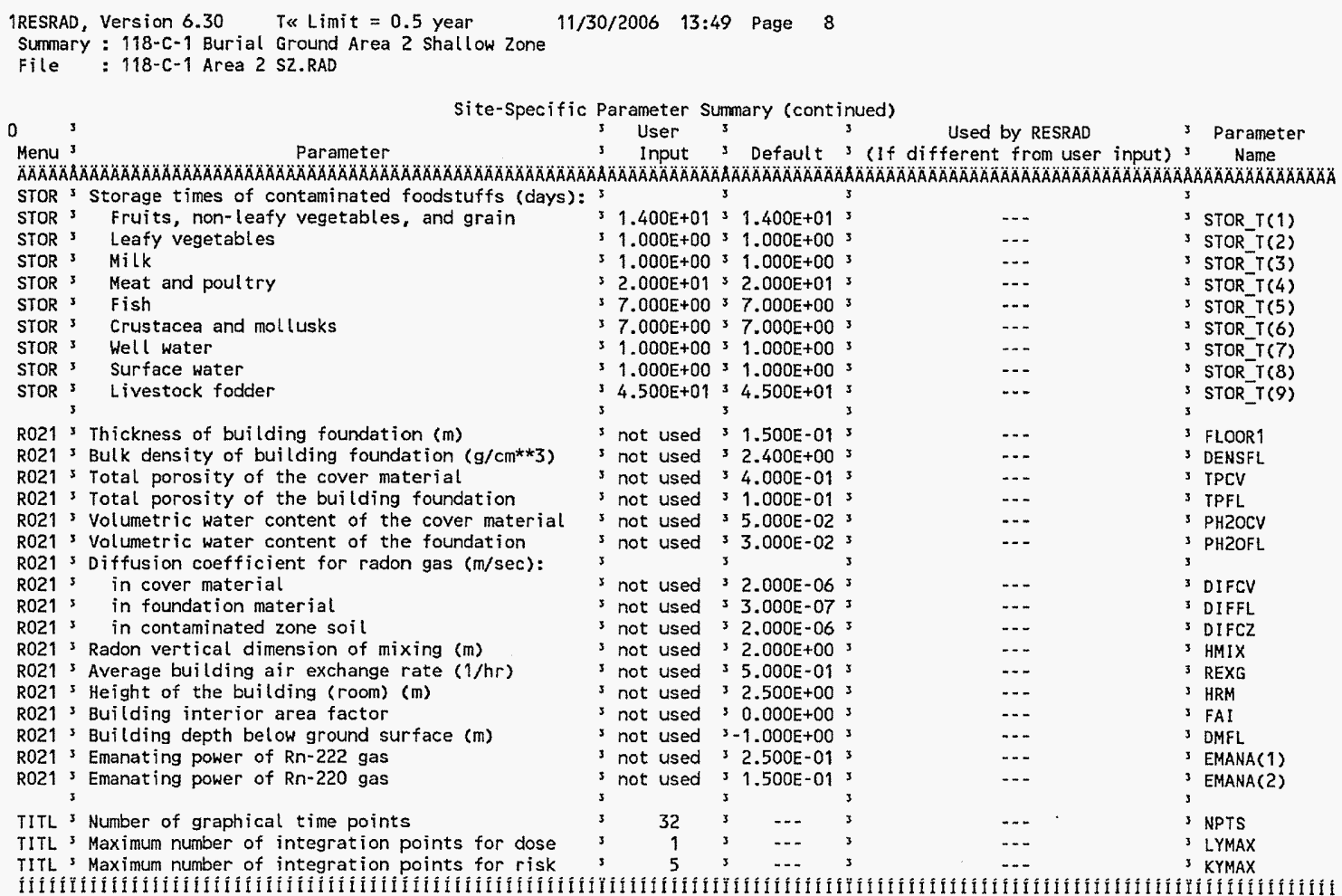

Surmary of Pathway Selections

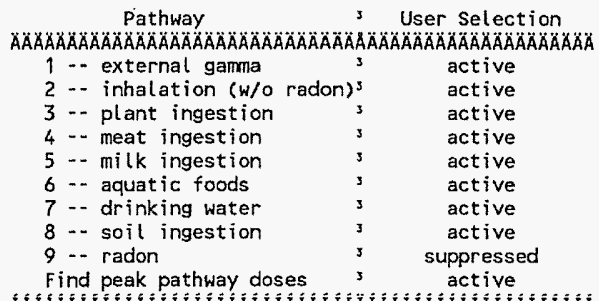

Attachment 6.86 Sve Sheet No. 8 of 21
Originators: $\frac{\text { S.W. Clark }}{\text { Date }}$
Chk'd By $\frac{\text { Mate }}{\text { M. Perrott }}$ Rev. No. 0
Calc. No. $\frac{0100 \text { C-CA-V0035 }}{\text { Reve }}$




\section{ATTACHMENT 6}

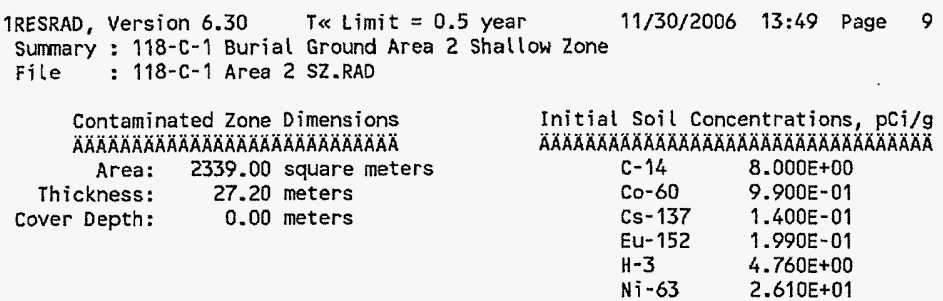

Total Dose TDOSE $(t)$, mrem/yr

Basic Radiation Dose Limit $=1.500 \mathrm{E}+01 \mathrm{mrem} / \mathrm{yr}$

Total Mixture Sum $M(t)=$ Fraction of Basic Dose Limit Received at Time $(t)$

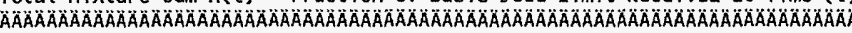

$t$ (years): $\begin{array}{cccccccccc}0.000 E+00 & 1.000 E+00 & 3.000 E+00 & 8.000 E+00 & 1.200 E+01 & 4.300 E+01 & 1.310 E+02 & 3.000 E+02 & 1.000 E+03\end{array}$

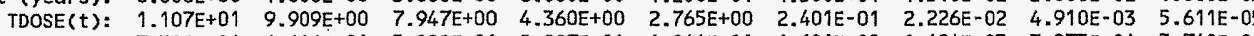

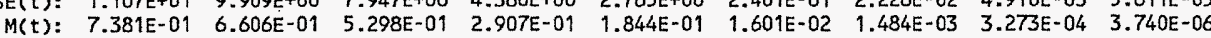
OMaximum TDOSE $(t): 1.107 \mathrm{E}+01 \mathrm{mrem} / \mathrm{yr}$ at $t=0.000 \mathrm{E}+00$ years

Attachment $6 \&$ Q $<5$ Sheet No. 9 of 21 


\section{ATTACHMENT 9}

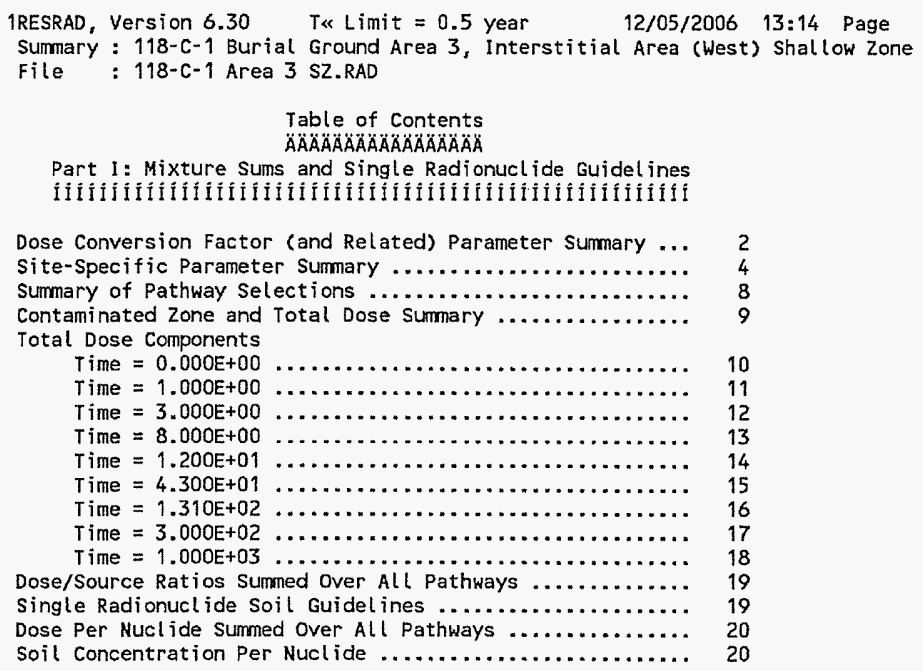

Dose Conversion Factor (and Related) Parameter Sumary ... 2

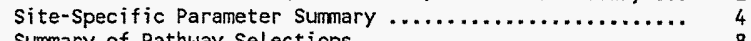

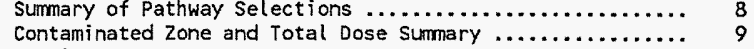

Total Dose Components

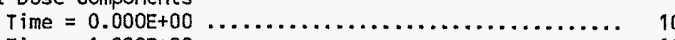

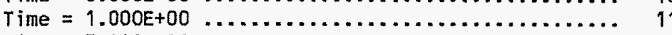

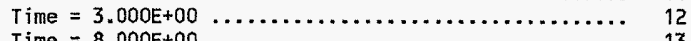

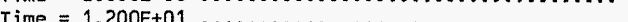

$T$ Time $=4.300 E+01$.

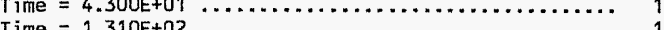

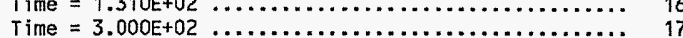

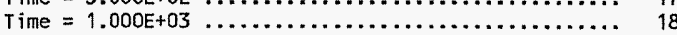

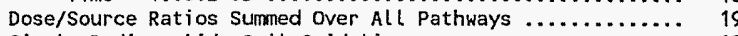

single Radionuclide Soil Guidel ines .................... 19

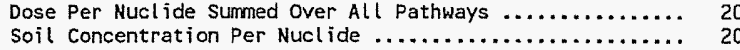

Attachment

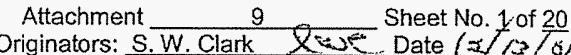

Chk'd By M.W. Perrott / and Date $\frac{1}{2 / 3 / 3 / s}$

Calc. No. $0100 \mathrm{C}$-CA-V0035 Rev. No. 0 


\section{ATTACHMENT 9}

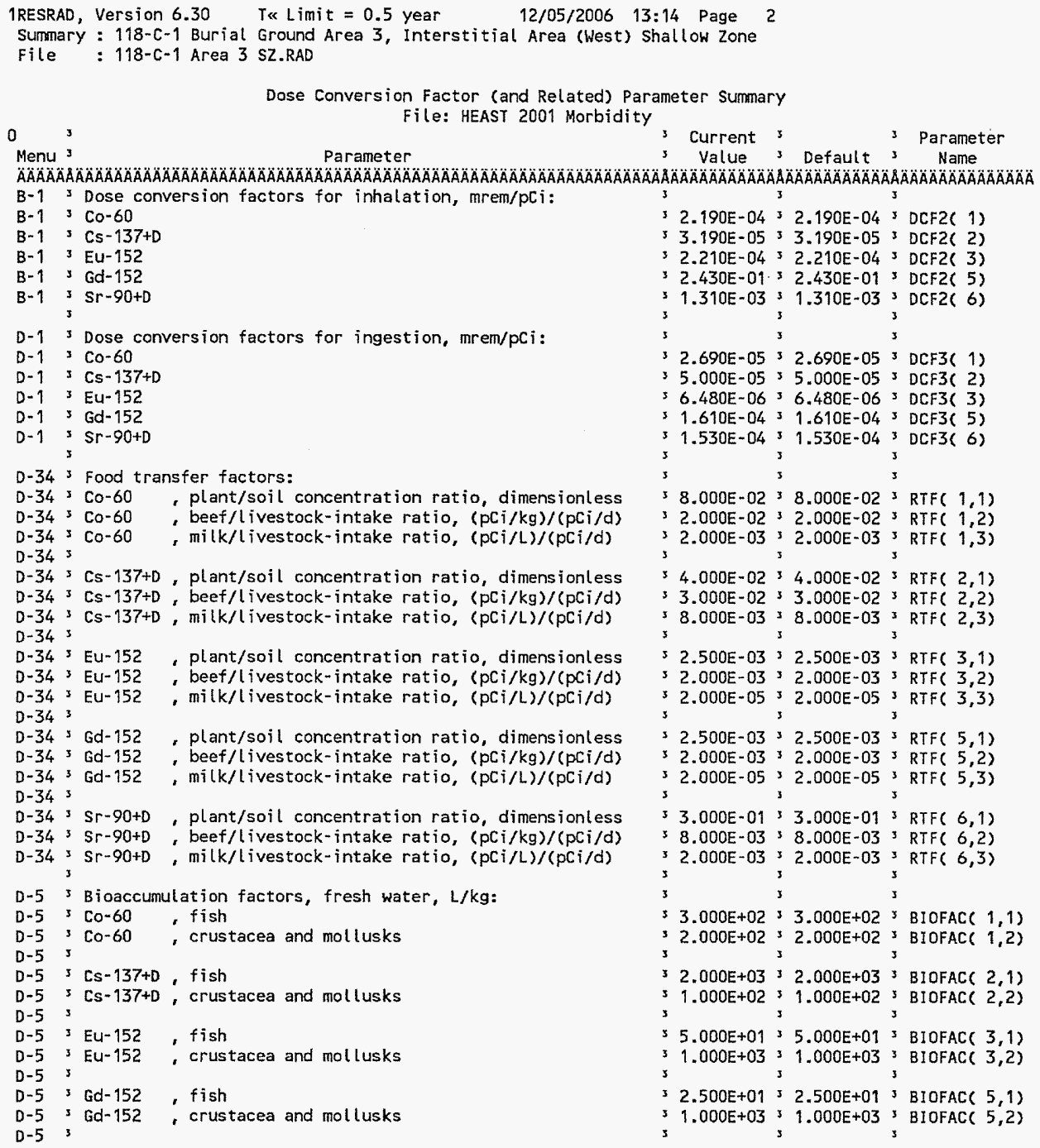


Rev. 0

\section{ATTACHMENT 9}

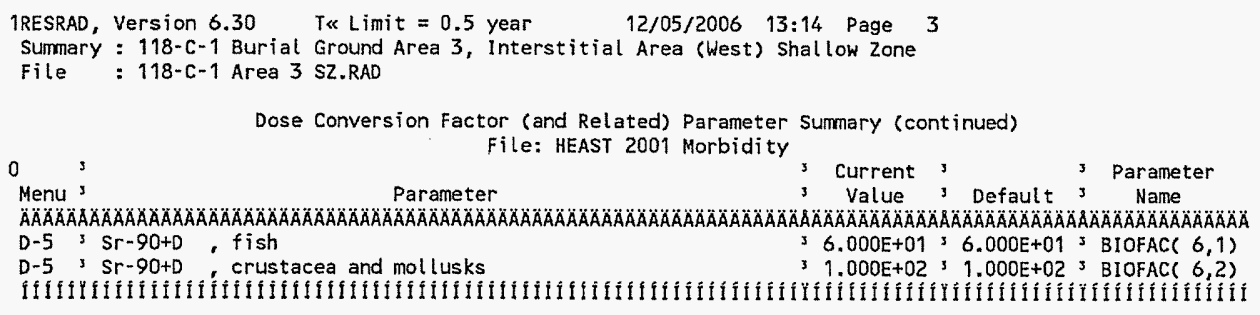

Attachment 


\section{ATTACHMENT 9}

IRESRAD, Version 6.30 T« Limit $=0.5$ year $12 / 05 / 2006 \quad 13: 14$ Page 4 Surmary : 118-C-1 Burial Ground Area 3, Interstitial Area (West) Shallow Zone file: $118-c-1$ Area 3 SZ.RAD

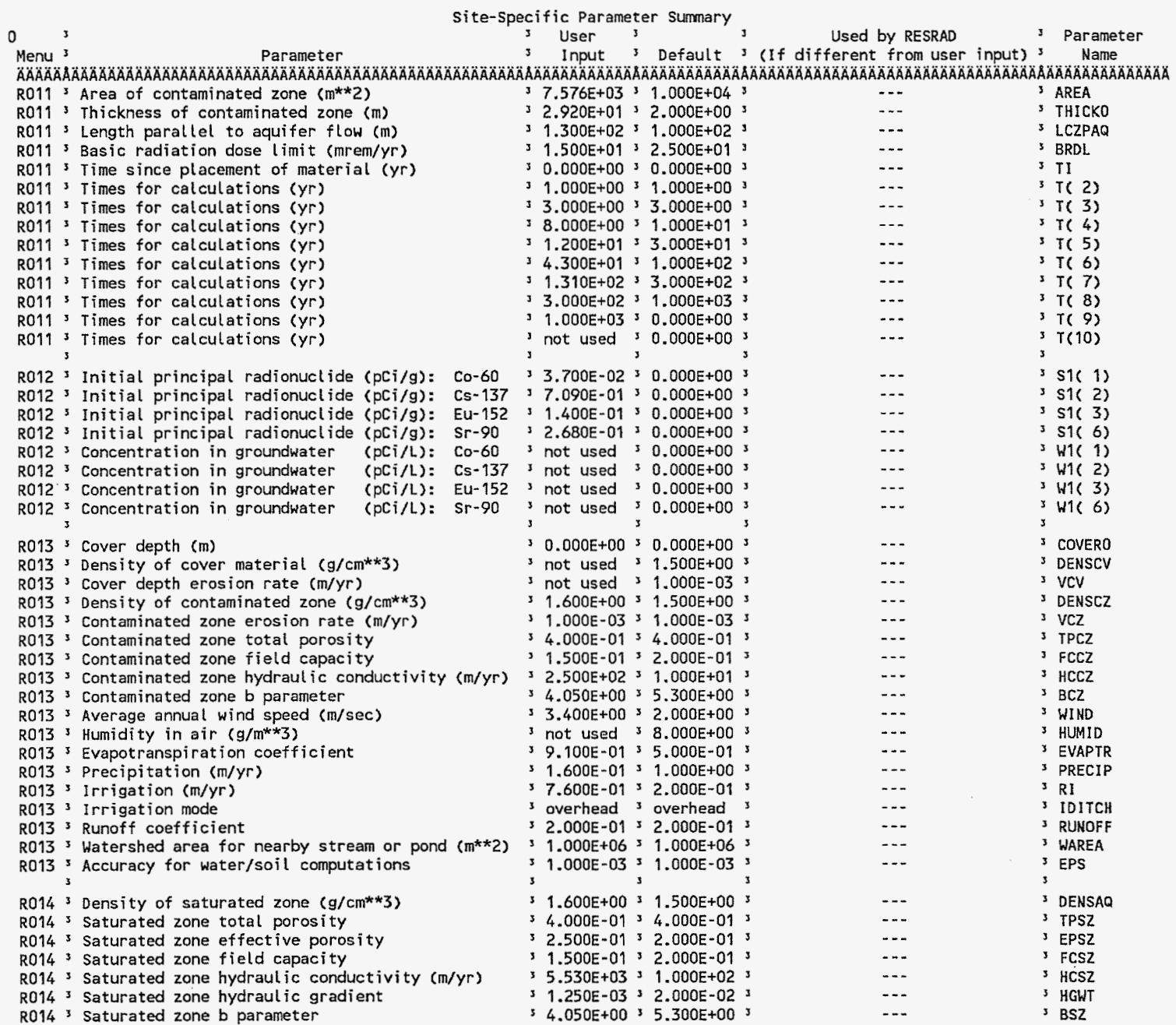

Attachment Originators: S.W. Clark 9 Sheet No. 4 of 20 Chk'd By M.W. Perrott Date

Calc. No. 0100C-CA-V0035

Date

Rev. No. 0




\section{ATTACHMENT 9}

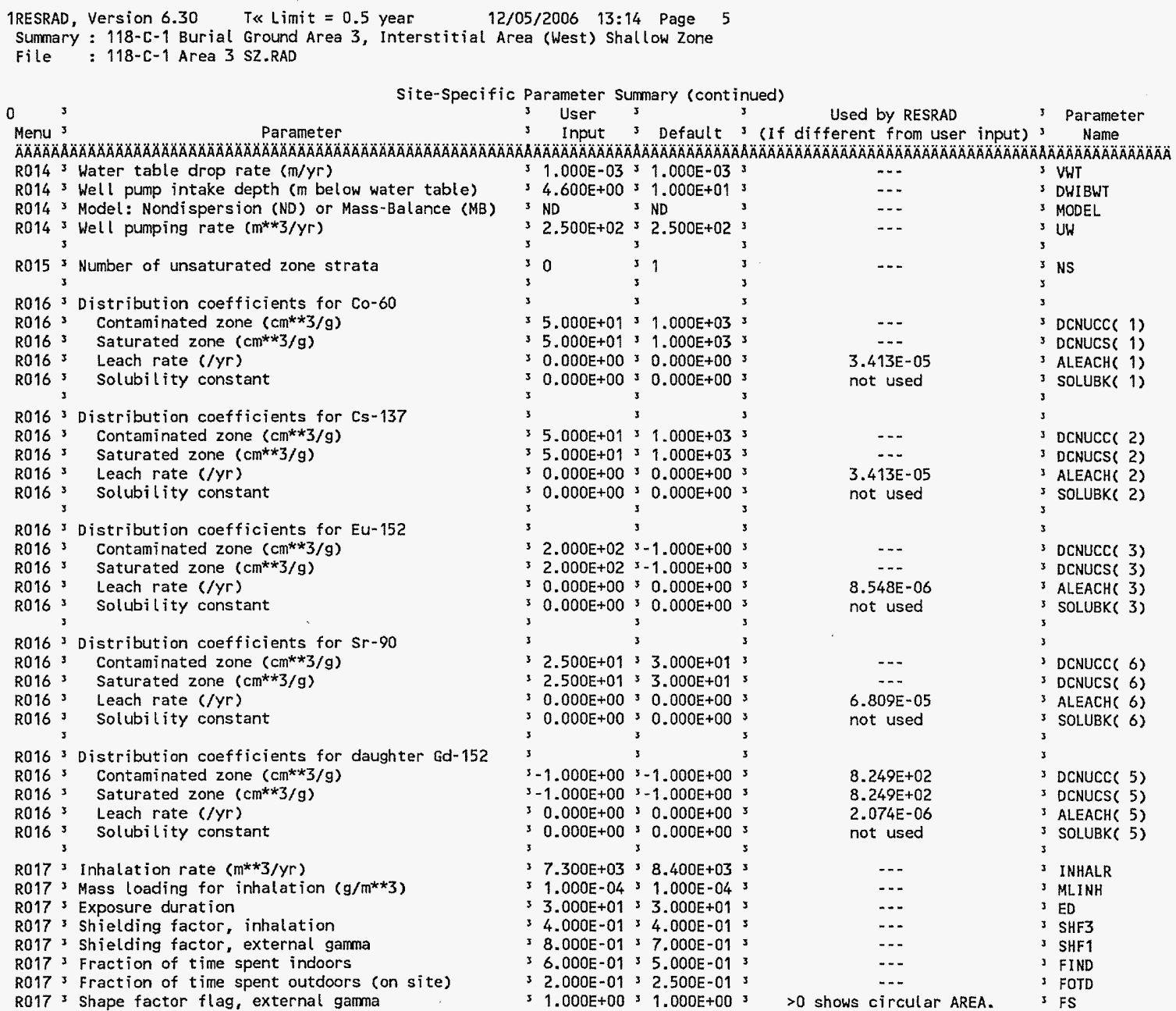

Attachment

9

Chk'd By M.W. Perrott Sheet No. $\underline{5}$ of $\underline{20}$ 


\section{ATTACHMENT 9}

1RESRAD, Version 6.30 T« Limit $=0.5$ year $12 / 05 / 2006 \quad 13: 14$ Page 6

Summary : 118-C-1 Burial Ground Area 3, Interstitial Area (West) Shallow zone

file : 118-C-1 Area 3 SZ.RAD

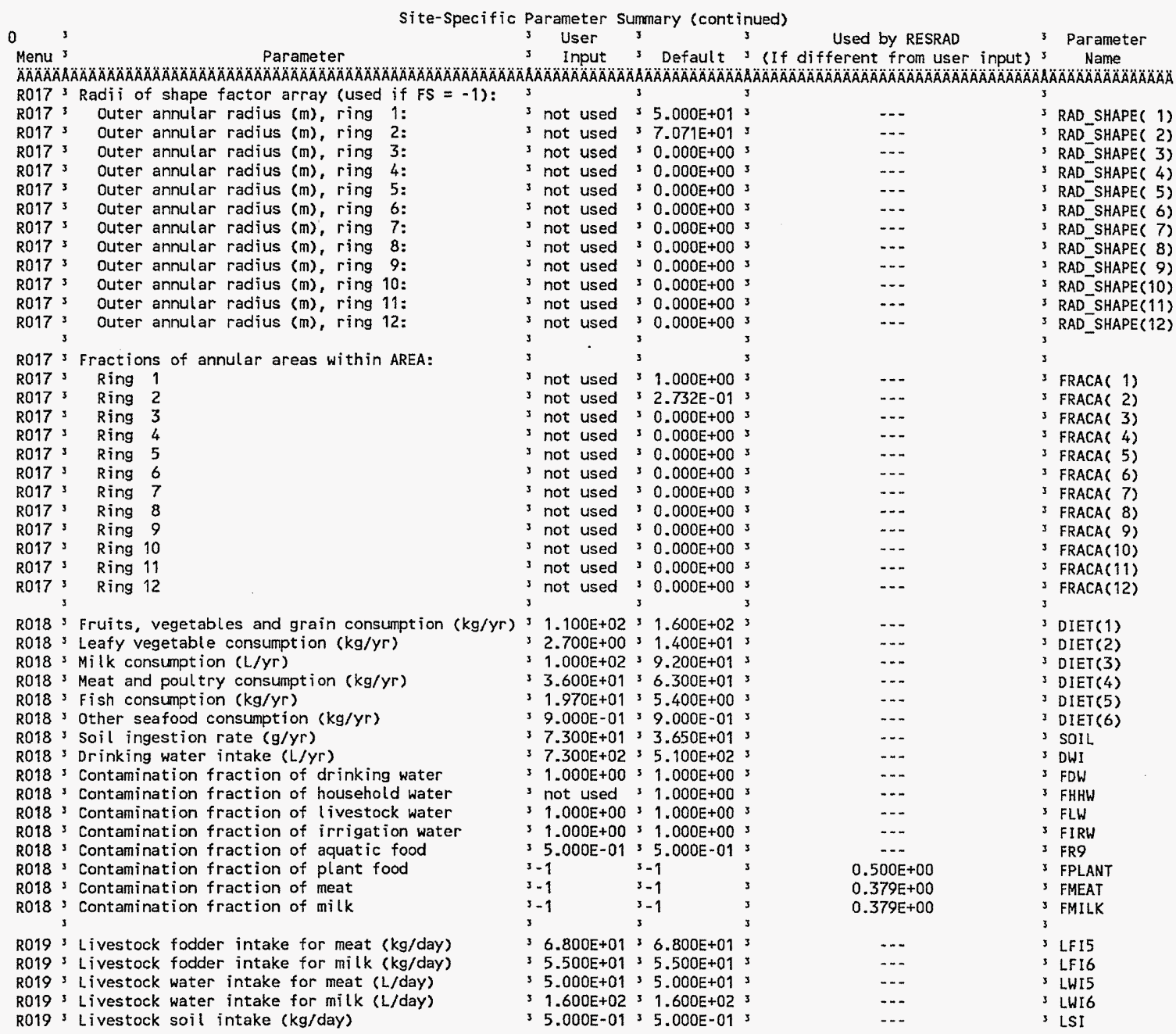

Attachment 9 Sheet No. $\underline{6}$ of $\underline{20}$ 


\section{ATTACHMENT 9}

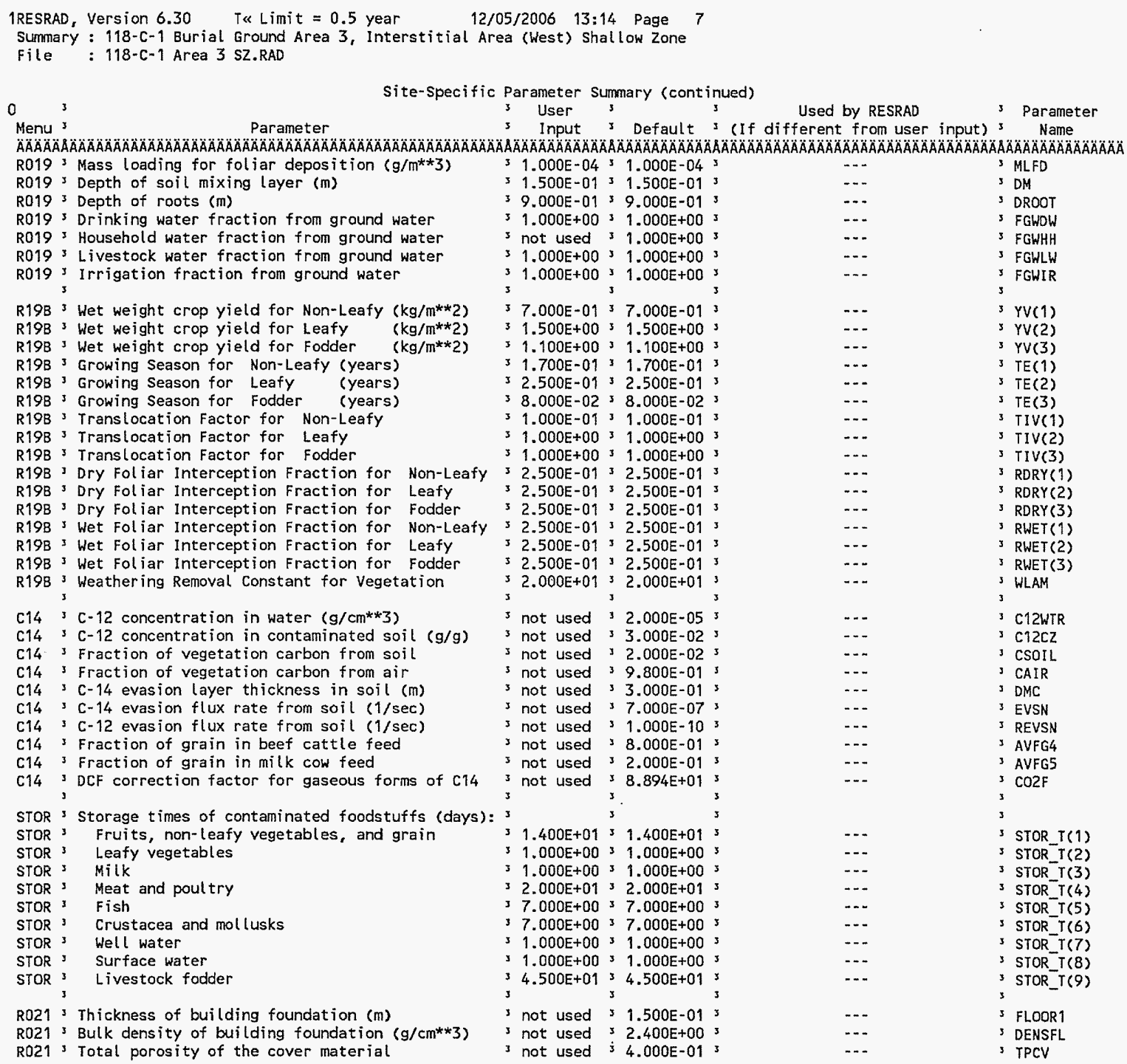

Attachment Originators: S. W. Clark Chk'd By M.W. Perrott Calc. No. 0100C-CA-V0035
Sheet No. 7 of 20 Date Date Rev. No. 0 


\section{ATTACHMENT 9}

1RESRAD, Version 6.30 Te Limit $=0.5$ year $12 / 05 / 2006 \quad 13: 14$ Page 8

Summary : 118-C-1 Burial Ground Area 3, Interstitial Area (West) Shallow Zone

File: $118-C-1$ Area 3 SZ.RAD

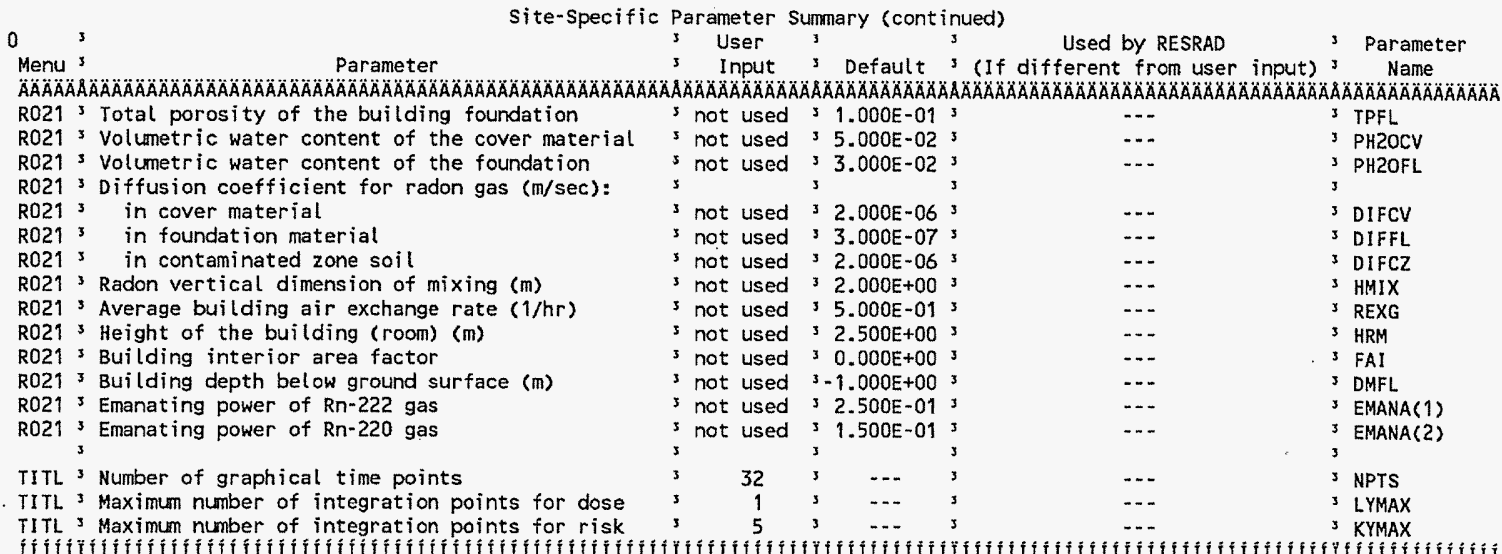

Summary of Pathway Selections

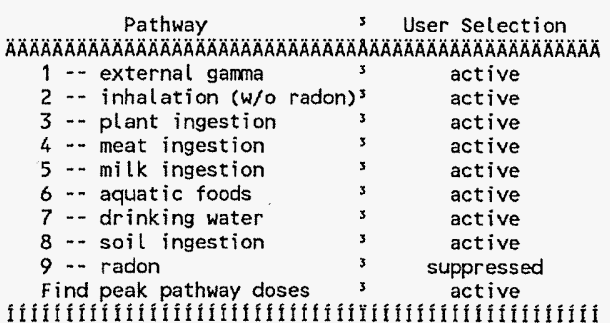

Attachment Originators: S.W Clark

Chk'd By M.W. Perrott

Calc. No. $0100 \mathrm{C}$-CA-V0035
Sheet No. 8 of 20

Date Date Rev. No. 
Rev. 0

\section{ATTACHMENT 9}

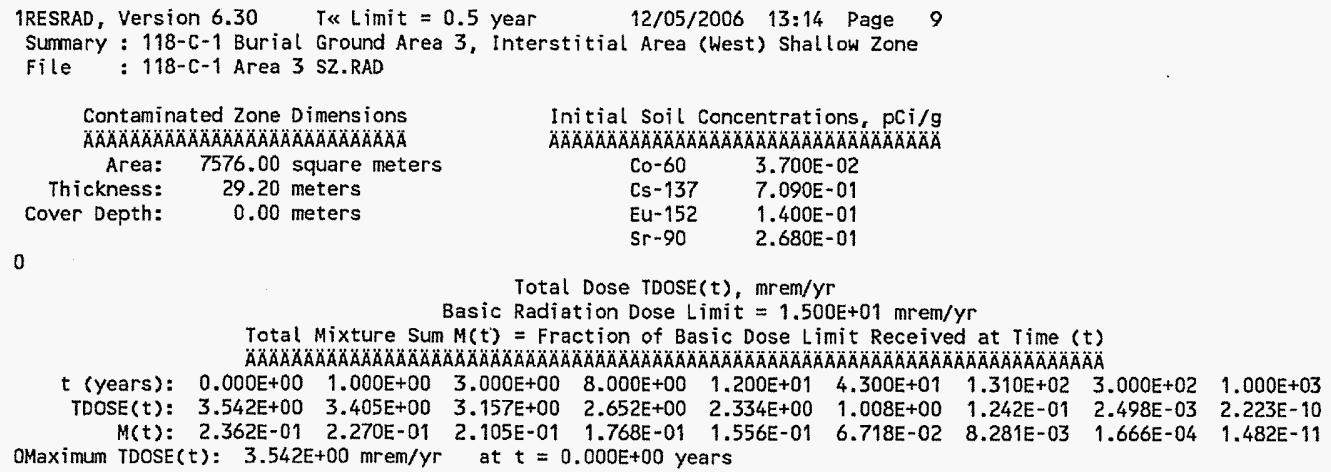

Attachment 


\section{ATTACHMENT 12}

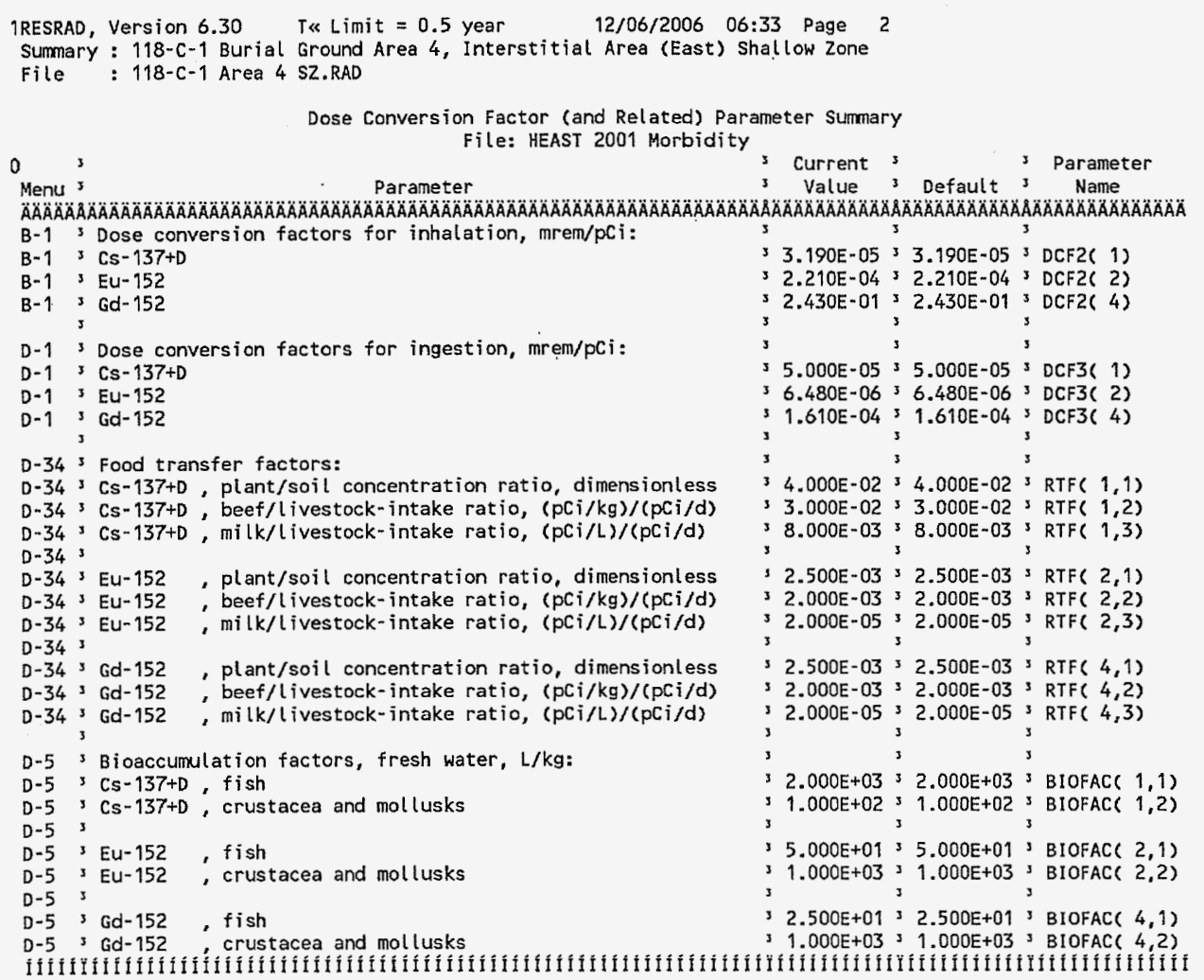

\begin{tabular}{|c|c|}
\hline Attachment & Sheet No. 2 of 19 \\
\hline Originators: S.W. Clark & Date \\
\hline Chk'd By M.W. Perrott & Date \\
\hline $0100 C-C A-V 0035$ & Rev. No. 0 \\
\hline
\end{tabular}




\section{ATTACHMENT 12}

IRESRAD, Version 6.30 T\& Limit $=0.5$ year 12/06/2006 06:33 Page 3 Summary : 118-C-1 Burial Ground Area 4, Interstitial Area (East) Shallow Zone File: $118-C-1$ Area 4 SZ.RAD

$\begin{array}{ccc} & \text { Site-Specific Parameter summary } \\ 0 & 3 & \text { User } 3\end{array}$

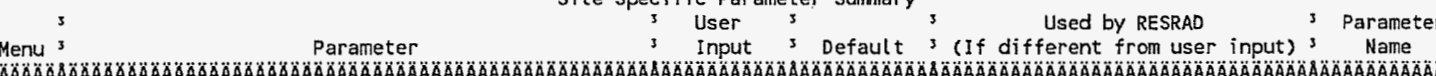
RO11 3 Area of contaminated zone. ( $m^{* * 2}$ ) 011 , Area R011 Thickness of contaminated zone (m) R011 Length parallel to aquifer flow (m) R011 Basic radiation dose limit (mrem/yr) R011 Time since placement of material (yr) R011' Times for calculations ( $y r$ ) R011' Times for calculations (yr) R011's Times for calculations $(y r)$ R011' Times for calculations (yr) R011 3 Times for calculations $(y r)$ R011 Times for calculations ( $y r$ ) R011' Times for calculations (yr) R011 Times for calculations ( $y r$ ) R011 3 Times for calculations (yr) $37.576 \mathrm{E}+033 \mathrm{3} 1.000 \mathrm{E}+04$ $3.570 E+0132.000+04$ $1.300 \mathrm{E}+02$ 3 $1.000 \mathrm{E}+02$ \& $1.300 E+02$ S $1.000 E+02$ $1.500 \mathrm{E}+01,2.500 \mathrm{E}+01$ $0.000 E+0030.000 E+00$ $3.1 .000 \mathrm{E}+00>1.000 \mathrm{E}+00^{3}$ $3.000 \mathrm{E}+00 \div 3.000 \mathrm{E}+003$ $3.000 \mathrm{E}+0031.000 \mathrm{E}+01$ $31.200 E+0133.000 E+013$ $34.300 \mathrm{E}+01^{3} 1.000 \mathrm{E}+02^{3}$ $31310 E+0233.000 E+02$ $3.310 E+02$ s $3.000 E+023$ 1.000E+02 $1.000 E+03$ $1.000 E+0330.000 E+00$ , not used $30.000 \mathrm{E}+00^{3}$ R012 s Initial principal radionuclide $(\mathrm{pCi} / \mathrm{g}): \mathrm{Cs}-137$ 3 $8.100 \mathrm{E}-0130.000 \mathrm{E}+00$ R012 Initial principal radionuclide (pCi/g): Eu-152 $\$ 1.140 \mathrm{E}-01$ s $0.000 \mathrm{E}+00$ R012 3 Concentration in groundwater ( $\mathrm{pCi} / \mathrm{L}$ ): $\mathrm{Cs}-137$ s not used $30.000 \mathrm{E}+00$ $R 012$ Concentration in groundwater ( $\mathrm{pCi} / \mathrm{L}$ ): Eu-152,s not used s $0.000 \mathrm{E}+00^{3}$

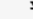

R013 3 Cover depth (m)

R013 S Density of cover material $\left(\mathrm{g} / \mathrm{cm}^{* * 3}\right)$

R013' Cover depth erosion rate (m/yr)

$R 013$ ' Density of contaminated zone ( $\left.\mathrm{g} / \mathrm{cm}^{\star *} 3\right)$

R013 3 Contaminated zone erosion rate $(\mathrm{m} / \mathrm{yr})$

R013 3 Contaminated zone total porosity

R013 3 Contaminated zone field capacity

R013 3 Contaminated zone hydraul ic conductivity $(\mathrm{m} / \mathrm{yr})$

R013 3 Contaminated

R013 3 Average annual wind speed $(\mathrm{m} / \mathrm{sec})$

R013 3 Humidity in air $\left(\mathrm{g} / \mathrm{m}^{* * 3}\right)$

0013 s Evapotranspiration coefficient

R013 3 Precipitation $(\mathrm{m} / \mathrm{yr})$

R013 3 Irrigation $(m / y r)$

R013 3 Irrigation mode

R013 sunoff coefficient

R013 $s$ Watershed area for nearby stream or pond $\left(m^{* *}\right.$ )

R013 Accuracy for water/soil computations

3

R014 3 Density of saturated zone $\left(\mathrm{g} / \mathrm{cm}^{* * 3}\right)$

R014 3 Saturated zone total porosity

R014 Saturated zone effective porosity

R014 S Saturated zone field capacity

R014's Saturated zone hydraulic conductivity (m/yr)

R014's Saturated zone hydraulic gradient

R014 3 Saturated zone b parameter

R014 3 Water table drop rate (m/yr)

R014 3 Well pump intake depth (m below water table)

R014 3 Model: Nondispersion (ND) or Mass-Balance (MB)

R014 3 Hell pumping rate $\left(m^{* * 3 / y r}\right)$

$30.000 E+0030.000 E+00$

3 not used $31.500 E+00$

not used $31.500 E+00$

not used $31.000 \mathrm{E}-03$

1.600E+00 $31.500 E+00$

s $1.000 \mathrm{E}-03>1.000 \mathrm{E}-03$

$34.000 E-0134.000 E-01$

$3.500 \mathrm{E}+0231.000 \mathrm{E}+01$

$34.050 E+0035.300 E+00$ s

$33.400 E+0033.000 E+00$

$3.400 E+0032.000 E+00$

not used $8.000 E+00$

$9.100 E-01$ a $5.000 E-01$

$E+00$

$7.600 E-0132.000 E-01$

overhead 3 overhead

2.000E-01 $32.000 E-01$

$1.000 E \div 0631.000 E+06$

$1.000 E-03=1.000 E-03$

$1.600 E+0031.500 E+00$

$4.600 E+00,1.500 E+00$

$4.000 E-0134.000 E-01$

$2.500 E-0132.000 E-01$
$1.500 E-01,2.000 E-01$

$5.530 E+03,1.000 E+02$

$1.250 E-0332.000 E-02$

s $4.050 E+00>5.300 E+00$

$31.000 E-0331.000 E-03$

3 $4.600 \mathrm{E}+00^{3} 1.000 \mathrm{E}+01^{3}$

$3 \mathrm{ND}$

$3 \mathrm{ND}$

, $2.500 E+02,2.500 E+02$

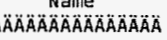

3 AREA

3 THICKO

...

-.. 3 BRDL

$\begin{array}{ll}-- & 3 \\ -. & 3\end{array}$

.- $\quad 3(3)$

J $T(4)$

- $3(5)$

$\cdots+1(6)$

$3 \pi(7)$

...

... $\quad$ s $s 1(1)$

$s$ s1( 2$)$

$\cdots \quad 3$ WI( 1$)$

-

... 3 COVERO

-.. DENSCV

... 3 VCV

" DENSCZ

3 VC2

...

s $\mathrm{HCCZ}$

-.. $\quad \mathrm{BCZ}$

.. SWIND

-. 3 HUMID

... 3 EVAPTR

...

3 IDITCH

‥ 3 RUNOFF

- WAREA

-.

-.. 3 DENSAO

-.- 3 TPSZ

--- 3 EPSZ

-. 3 FCSZ

$+-$

-.

...

3 DWIBWT

-- 3 MODEL

3 UH

Attachment

12 Sheet No. 3 of 19 Date

Calc. No. 0100C-CA-V0035

Rev. No. 0 


\section{ATTACHMENT 12}

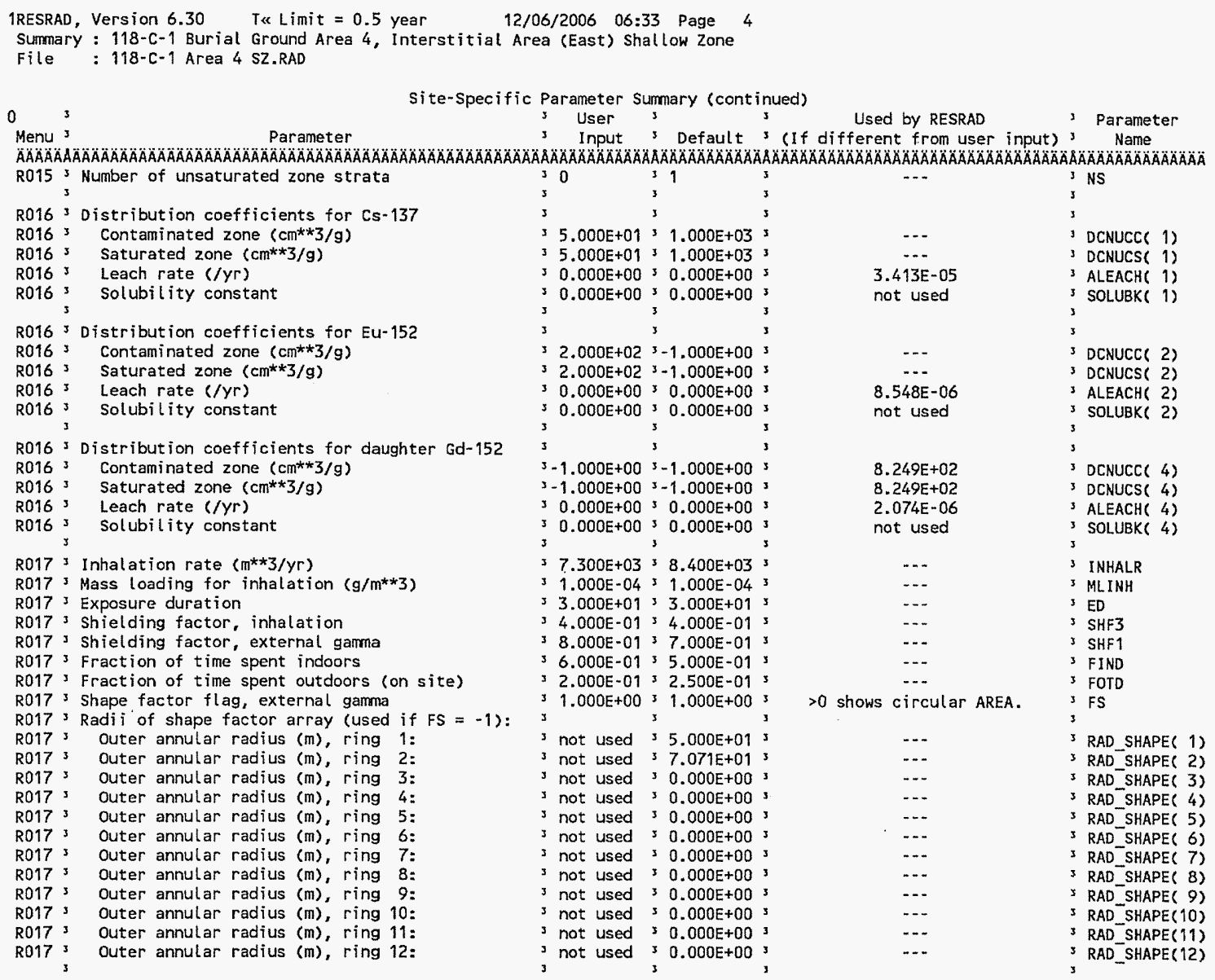

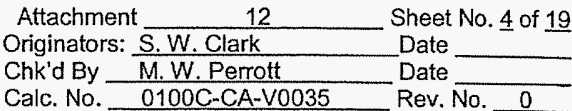


Rev. 0

\section{ATTACHMENT 12}

IRESRAD, Version 6.30 T\& Limit $=0.5$ year 12/06/2006 06:33 Page 5 Summary : 118-C-1 Burial Ground Area 4, Interstitial Area (East) Shallow Zone File : 118-C-1 Area 4 SZ.RAD

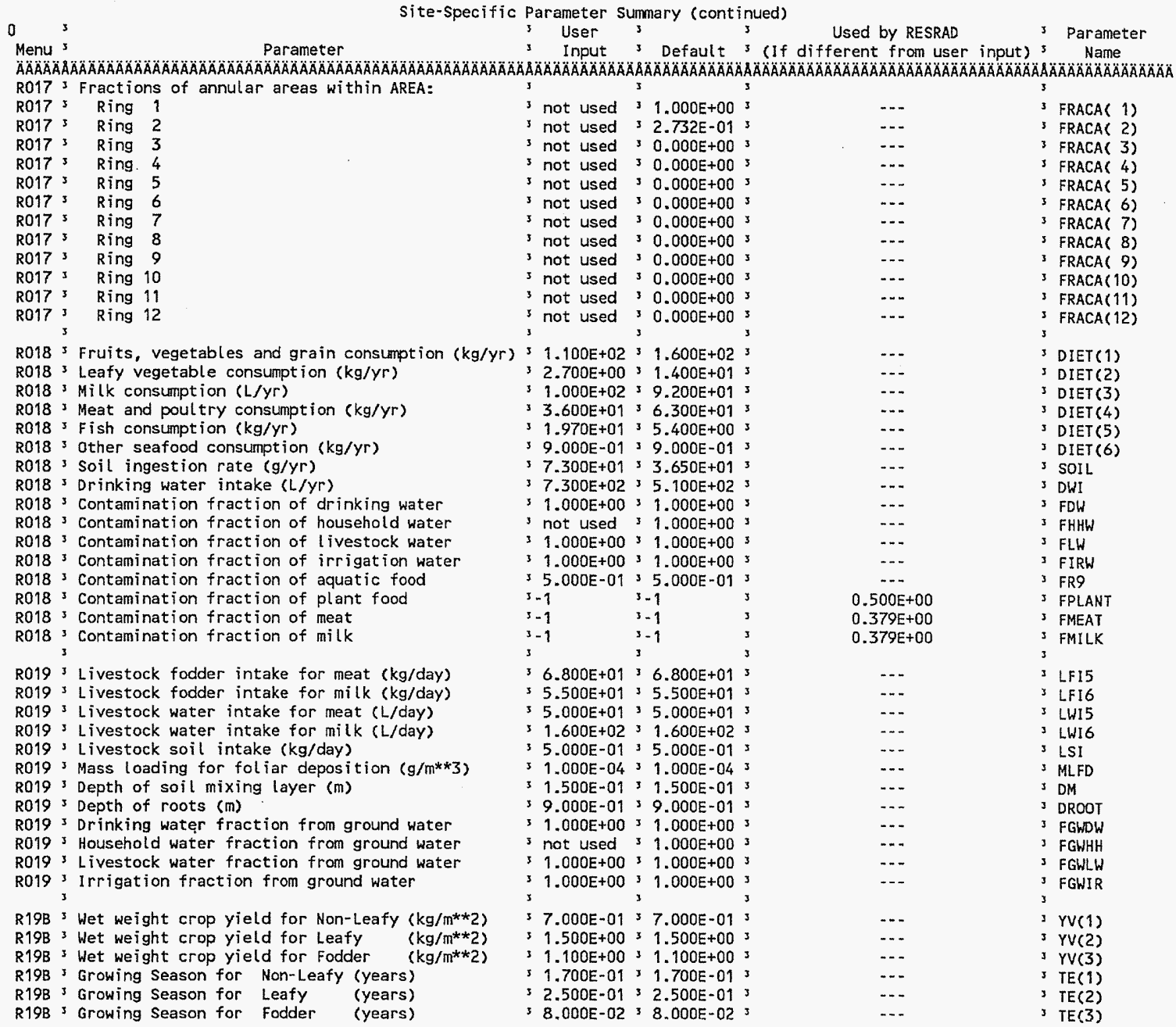

Attachment

12 Sheet No. $\underline{5}$ of 19 Chk'd By $\frac{\text { S.W. Clark }}{\text { M. Perroth }}$ Date

Calc. No. 0100C-CA-V0035

Rev No. 


\section{ATTACHMENT 12}

1RESRAD, Version 6.30 T\& Limit $=0.5$ year 12/06/2006 06:33 Page 6 Summary : $118-c-1$ Burial Ground Area 4, Interstitial Area (East) Shallow Zone File : 118-C-1 Area 4 SZ.RAD

0

Site-Specific Parameter Summary (cont inued)

(1)

Used by RESRAD I Parameter

' Input 3 Default ${ }^{3}$ (If different from user input) 3 Name

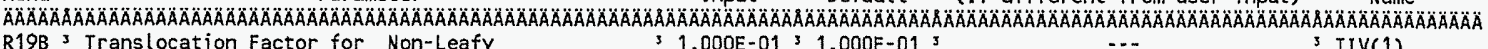

R19B 3 Translocation Factor for Non-Leafy $31.000 E-0131.000 E-01^{3}$

R19B T Translocation Factor for Leafy $31.000 \mathrm{E}+00^{3} 1.000 \mathrm{E}+00$

R19B $:$ Translocation Factor for Fodder

R19B 3 Dry Foliar Interception Fraction for Non-Leafy $32.500 E-0132.500 E-01$

R19B 3 Dry Foliar Interception Fraction for Leafy $32.500 \mathrm{E}-0132.500 \mathrm{E}-01$

R19B 3 Dry Foliar Interception Fraction for Fodder $32.500 E-0132.500 E-01$

$R 19 B$ s Wet Foliar Interception Fraction for Non-

R19B 3 Wet foliar Interception Fraction for Leafy $32.500 \mathrm{E}-0132.500 \mathrm{E}-01$

R19B 3 Wet Foliar Interception Fraction for Fodder $32.500 \mathrm{E}-0132.500 \mathrm{E}-01$

$R 19 B$ " Weathering Removal Constant for Vegetation 3 $2.000 E+0132.000 E+013$

3

C14 $5 \mathrm{C}-12$ concentration in water $(\mathrm{g} / \mathrm{cm} * * 3)$

C14 $3 \mathrm{C}-12$ concentration in contaminated soil $(\mathrm{g} / \mathrm{g})$ ' not used $33.000 \mathrm{E}-02$

C14 3 Fraction of vegetation carbon from soil

143 Fraction of vegetation carbon from air

$\mathrm{C14}_{3} 3 \mathrm{C}-14$ evasion layer thickness in soil (m)

C14 3 $\mathrm{C}-14$ evasion flux rate from soil (1/sec)

C14 $5 \mathrm{c}-12$ evasion flux rate from soil (1/sec) s not used $31.000 \mathrm{E}-10$

C14 3 Fraction of grain in beef cattle feed 3 not used $38.000 E-01$

C14 F Fraction of grain in milk cow feed s not used $32.000 E-01$

C14 3 DCF correction factor for gaseous forms of C14 3 not used $38.894 \mathrm{E}+01$

STOR ${ }^{3}$ Storage times of contaminated foodstuffs (days):

STOR 3 Fruits, non-leafy vegetables, and grain

STOR ${ }^{3}$ Leafy vegetables

STOR 3 Milk

STOR 3 Meat and poultry

STOR ${ }^{3}$ Fish

STOR ${ }^{3}$ Crustacea and mollusks

STOR ${ }^{3}$ Well water

STOR ${ }^{3}$ Surface water

STOR ${ }^{3}$ Livestock fodder

R021 3 Thickness of building foundation $(\mathrm{m})$

R021 Bulk density of building foundation $\left(\mathrm{g} / \mathrm{cm}^{\star * 3}\right)$

R021 Total porosity of the cover material

RO21 3 Total porosity of the building foundation

R021 Volumetric water content of the cover material

R021 3 Volumetric water content of the foundation

R021 ${ }^{3}$ Diffusion coefficient for radon gas $(\mathrm{m} / \mathrm{sec})$ :

$\mathrm{RO}^{3} 1^{3}$ in cover material

$R 021^{3}$ in foundation material

$R 021$ s in contaminated zone soit

R021 3 Radon vertical dimension of mixing (m)

R021 Average building air exchange rate $(1 / \mathrm{hr})$

R021 Height of the building (room) (m)

RO21 s Building interior area factor

R021' Building depth below ground surface $(\mathrm{m})$

R021 Emanating power of Rn-222 gas

$R 021$ Emanating power of $R n-220$ gas

3

3 not used 32.000 E-05

:

$\begin{array}{ll}3 \\ \cdots-. & 3 \text { TIV(1) } \\ --- & \text { TIV(2) }\end{array}$

- 3 TIV(3)

-.. $3 \operatorname{RDRY}(1)$

-.. $\quad$ RDRY(2)

-.. $R D R Y(3)$

3 RWET(1)

3 RWET(2)

… $\quad 3$ RWET(3)

3 WLAM

-.. 3 C12WTR

-.. $3 \mathrm{C12C2}$

-.. 3 CSOIL

3 CAlR

3 DMC

$\ldots$ EVSN

...

-.- 3 AVFG5

$+\cdots$

(3)

$1.400 E+01=1.400 E+01$

$31.000 \mathrm{E}+00 \times 1.000 \mathrm{E}+00$

s $1.000 \mathrm{E}+00 ; 1.000 \mathrm{E}+00$

$32.000 E+0132.000 E+01$

$7.000 E+00 \times 7.000 E+00$

$7.000 \mathrm{E}+00 \div 7.000 \mathrm{E}+00$

s $1.000 \mathrm{E}+00=1.000 \mathrm{E}+00$ s

$>1.000 \mathrm{E}+00$ \& $1.000 \mathrm{E}+00$

$4.500 E+0134.500 E+01$

not used $51.500 \mathrm{E}-01$

not used $32.400 E+00$

, not used 3 4.000E-01.

not used $31.000 E-01$

3 not used $35.000 E-02$

3 not used 3.000 - 02 ,

3 not used $32.000 \mathrm{E}-06$

3 not used 3 3.000E-07.

3 not used $32.000 E-06$

3 not used $32.000 E+00$

not used 3 5.000E-01

3 not used $32.500 \mathrm{E}+00$

not used $30.000 E+00$

3 not used $3.1 .000 E+00$

3 not used $32.500 E-01$

3 not used s 1.500E-01'
-

STOR_T(1)

3 STORTT(3)

3 STORTT(4)

3 STOR_T(5)

3 STOR T(6)

3 STOR T(7)

3 STORT(B)

... $\quad$ STOR_T(9)

… 3 FLOOR1

... 3 DENSFL

3 TPCV

...

3 PH2OF

... 3 DIFCY

...

-.. 3 DIFCZ

-..

-. 3 REXG

-. 3 HRM

...

...

...

\begin{tabular}{|c|c|}
\hline Attachment & et No. $\underline{6}$ of 19 \\
\hline Originators: S.W. Clark & Date \\
\hline M. W. Perrott & Date \\
\hline $0100 C-C A-V 0035$ & Rev. No. \\
\hline
\end{tabular}




\section{ATTACHMENT 12}

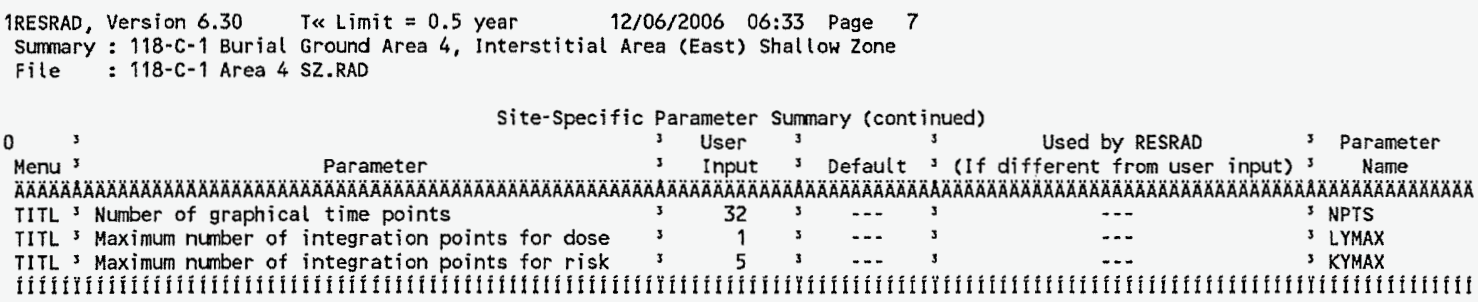

Summary of Pathway Selections

Pathway 3 User selection

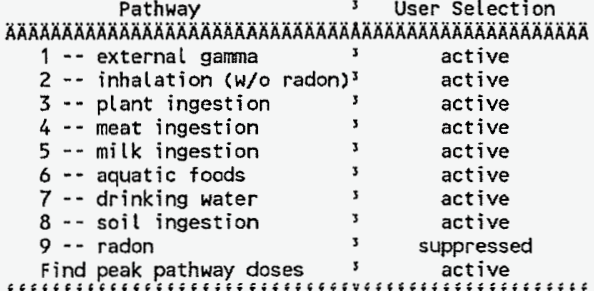

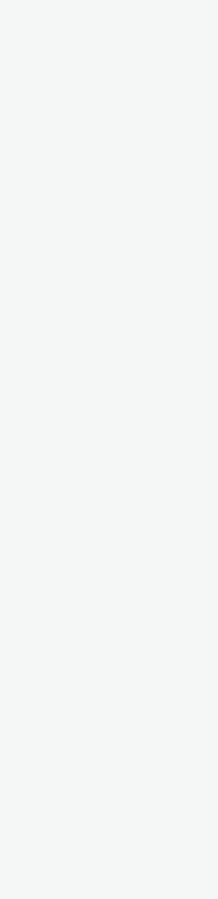

Attachment 12 


\section{ATTACHMENT 12}

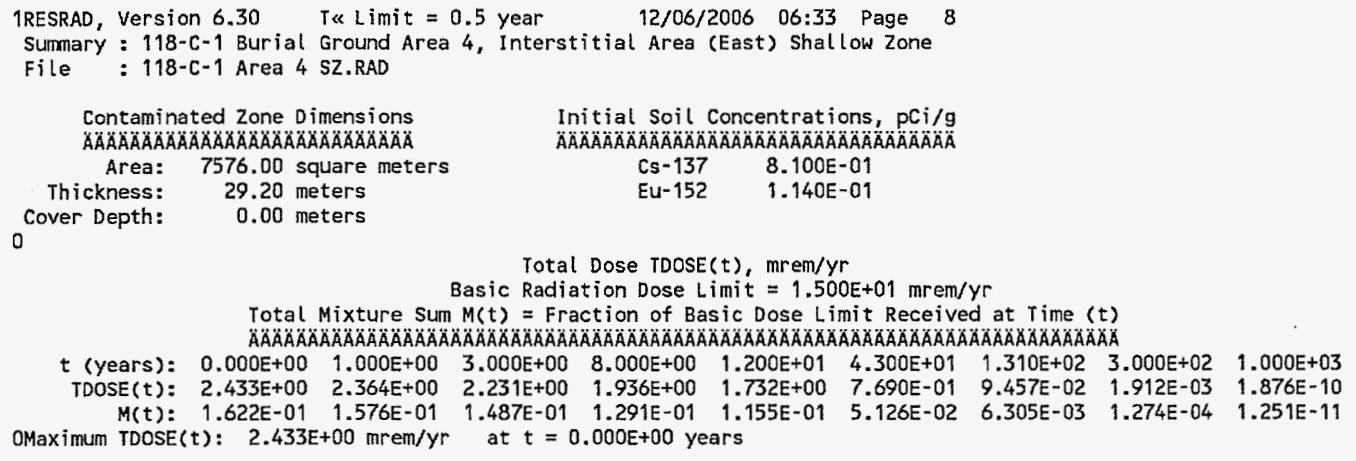

$t$ (years): $\begin{array}{llllllllll}0.000 E+00 & 1.000 E+00 & 3.000 E+00 & 8.000 E+00 & 1.200 E+01 & 4.300 E+01 & 1.310 E+02 & 3.000 E+02 & 1.000 E+03\end{array}$

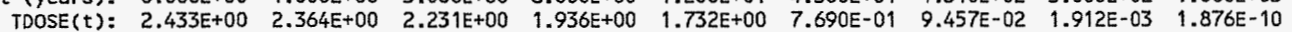
$M(t): \begin{array}{llllllllll}1.622 E-01 & 1.576 E-01 & 1.487 E-01 & 1.291 E-01 & 1.155 E-01 & 5.126 E-02 & 6.305 E-03 & 1.274 E-04 & 1.251 E-11\end{array}$ OMaximum $\operatorname{TDOSE}(t): 2.433 E+00 \mathrm{mrem} / \mathrm{yr}$ at $t=0.000 E+00$ years

\begin{tabular}{|c|c|}
\hline Attachment & Sheet No. 8 of 19 \\
\hline Originators: $\mathrm{S.W}$. Clark & Date \\
\hline M.W. Perrott & Date \\
\hline $0100 C-C A-V 0035$ & Rev. No. \\
\hline
\end{tabular}




\section{ATTACHMENT 15}

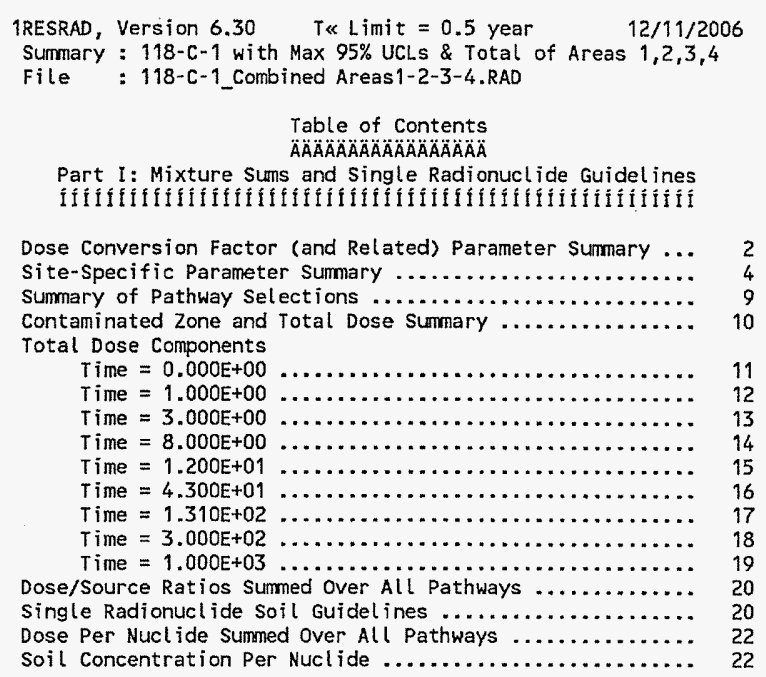

Attachment 15 Sheet No. 1 of 22 Chk'dors: S.W. Clark 25ave Date $13 / 13 / 00$ Calc. No. $0100 \mathrm{C}-\mathrm{CA}$-V0035 $\mathrm{Rev.No}$ No 


\section{ATTACHMENT 15}

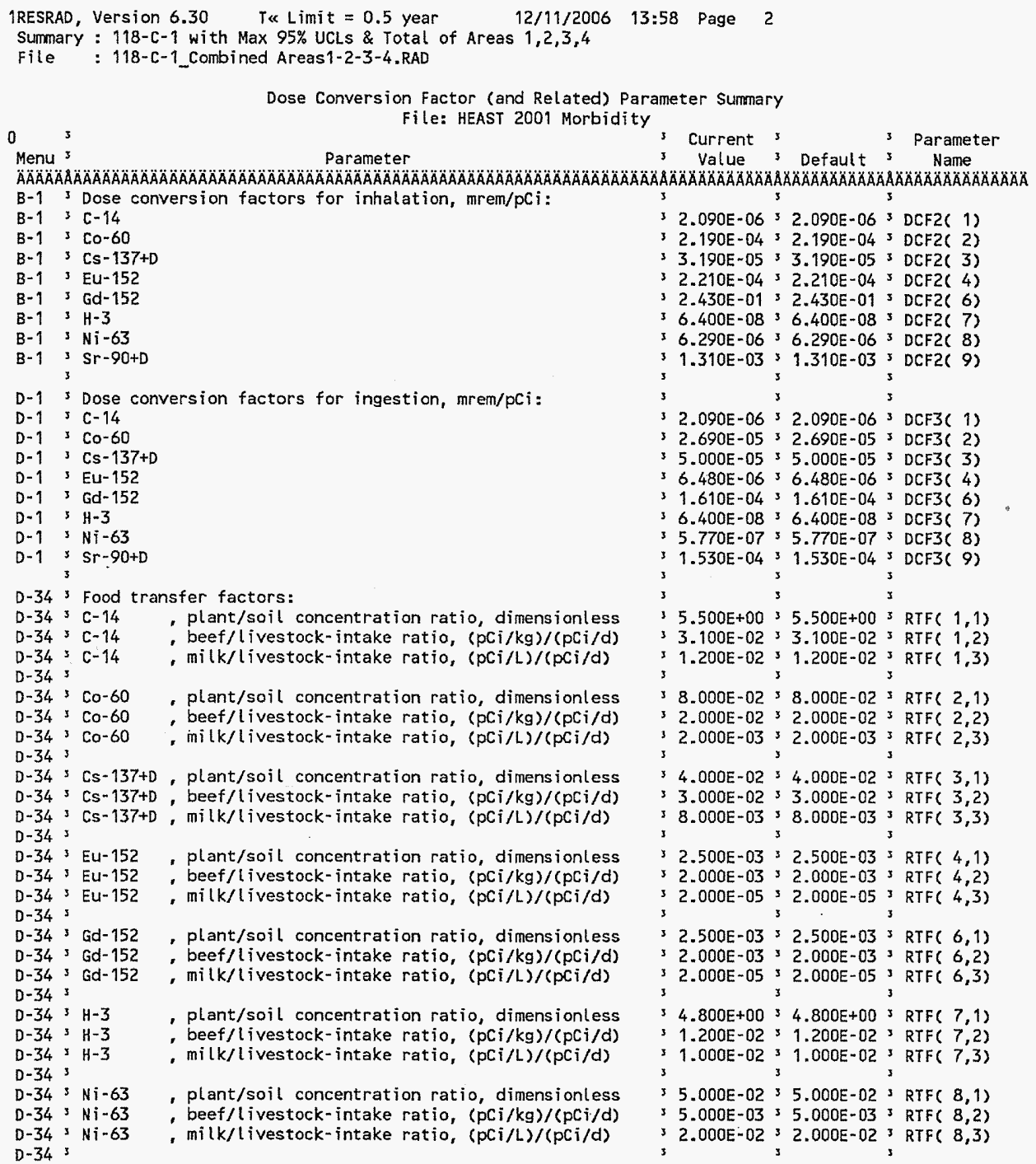




\section{ATTACHMENT 15}

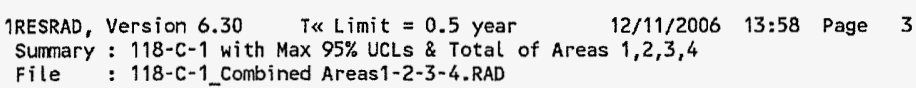

\begin{tabular}{|c|c|}
\hline Attachment & Sheet No. $\underline{3}$ of 22 \\
\hline Originators: $\mathrm{S.W.Clark}$ & Date \\
\hline M.W. Perrott & Date \\
\hline $0100 C-C A-V 0035$ & Rev. No. 0 \\
\hline
\end{tabular}




\section{ATTACHMENT 15}

IRESRAD, Version 6.30 T\& Limit $=0.5$ year $12 / 11 / 2006 \quad 13: 58$ Page 4 Surmary : $118-C-1$ with Max $95 \%$ UCLs \& Total of Areas $1,2,3,4$

File: 118-C-1_combined Areas?-2-3-4.RAD

Site-Specific Parameter Summary

3 User 3 Used by RESRAD 3 Parameter

0

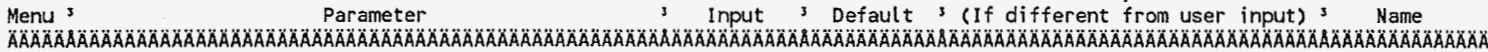

Menu ${ }^{3}$ Parameter 3 Input s Default s (If different from user input) ${ }^{3}$ Name

RO11 3 Area of contaminated zone $\left(m^{\star \star} 2\right)$

R011 3 Thickness of contaminated zone (m)

RO11'3 Length parallel to aquifer flow (m)

R011 3 Basic radiation dose limit (mrem/yr)

R011 Time since placement of material (yr)

$32.051 \mathrm{E}+04^{3} 1.000 \mathrm{E}+04$

$32.720 \mathrm{E}+01>2.000 \mathrm{E}+00$

$1.500 \mathrm{E}+02,1.000 \mathrm{E}+02$

$1.500 E+0132.500 E+0\}$ s

$0.000 E+00>0.000 E+00$

$1.000 E+00^{3} 1.000 E+00$

$3.000 E+00 ; 3.000 E+00$

R011 Times for calculations $(y r)$

R011'Times for calculations $(y r)$

3 $8.000 E+00,1.000 E+01$

$31.200 \mathrm{E}+01,3.000 \mathrm{E}+01$

$4.300 E+0131.000 E+02$

$1.310 E+02,3.000 E+02$

$3.000 E+02,1.000 E+03$

$1.000 E+03=0.000 E+00$

not used $0.000 E+003$

RO11 Times for calculations (yr)

R011' Times for calculations ( $y r)$

R011 S Times for calculations (yr)

3

R012 I Initial principal radionuclide $(\mathrm{pCi} / \mathrm{g})=\mathrm{c}-14,8.000 \mathrm{E}+00,0.000 \mathrm{E}+00$

$\mathrm{R} 0123$ Initial principal radionuclide $(\mathrm{pCi} / \mathrm{g}): \mathrm{CO}_{2}, 60,9.900 \mathrm{E}-0130.000 \mathrm{E}+00$,

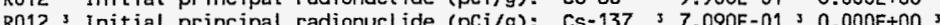

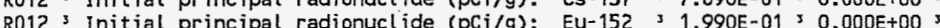

R012, Initial principal radionuclide (pCi/g): $\mathrm{H}-3,34.760 \mathrm{E}+00,0.000 \mathrm{E}+003$

R0123 Initial principal radionuclide $(\mathrm{pCi} / \mathrm{g})$ : $\mathrm{Ni}-63 ; 2.610 \mathrm{E}+01 ; 0.000 \mathrm{E}+00$

R012 s Initial principal radionuclide $(\mathrm{pCi} / \mathrm{g}): \mathrm{sr}-90$ > $2.680 \mathrm{E}-01$ > $0.000 \mathrm{E}+00$

R012 3 Concentration in groundwater (pCi/L): $C-14$ s not used $30.000 \mathrm{E}+00$

R012 s Concentration in groundwater (pCi/L): $\operatorname{Co-60} 3$ not used $30.000 E+00$

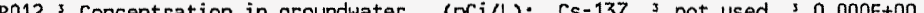

( $\mathrm{pC} \mathrm{i} / \mathrm{L}): \operatorname{Eu} 152$ s not

R012 3 Concentration in groundwater ( $\mathrm{PCi} / \mathrm{L}$ ): $\mathrm{H}-3$ s not used $30.000 \mathrm{~B}+00$

$\mathrm{R} 012$ 3 Concentration in groundwater (pCi/L): $\mathrm{Ni}-63$ s not used $30.000 \mathrm{E}+00$,

R012. Concentration in groundwater (pCi/L): $\mathrm{Sr}-90$

not used $30.000 E+00$

R013 Cover depth (m)

R013 s Density of cover material $\left(\mathrm{g} / \mathrm{cm}^{\star *} 3\right)$

R013' Cover depth erosion rate $(\mathrm{m} / \mathrm{yr})$

R013 Density of contaminated zone $(\mathrm{g} / \mathrm{cm} * * 3)$

R013 ? Contaminated zone erosion rate $(\mathrm{m} / \mathrm{yr})$

R013 ' Contaminated zone total porosity

R013 'Contaminated zone field capacity

$30.000 E+00=0.000 E+00$

3 not used $31.500 E+00$

not used $11.000 \mathrm{E}-03$

$1.600 E+0031.500 E+00$

$1.000 E-03$, $1.000 E-03$

$4.000-03,1.000 E-03$ s

$1.500 \mathrm{E}-01,2.000 \mathrm{~T}-01$

$1.500 E-01$ 2.000E-01

R013 3 Contaminated zone hydraulic conductivity $(\mathrm{m} / \mathrm{yr})$ s

R013 ' Contaminated zone b parameter

R013' Average annual wind speed $(\mathrm{m} / \mathrm{sec})$

$4.050 E+00^{3} 5.300 E+00$

$3.400 E+00 \div 2.000 E+00$

$8.000 E+00: 8.000 E+00$

$9.100 \mathrm{E}-0135.000 \mathrm{E}-01$

$1.600 E-0131.000 E+00$

$7.600 \mathrm{E}-01,2.000 \mathrm{E}-01$

overhead overhead

R013 3 Precipitation $(\mathrm{m} / \mathrm{Yr}$

R013 I Irrigation ( $\mathrm{m} / \mathrm{yr}$ )

R013 3 Irrigation mode

R013 s Runoff coefficient

(ODE-01, $3.000 \mathrm{E}-01$

R013 3 Watershed area for nearby stream or pond $\left(m^{* *} 2\right)$, $1.000 E+06,1.000 E+06$

R013 Accuracy for water/soit computations

$1.000 E-0331.000 E-03$

R014 3 Density of saturated zone $\left(\mathrm{g} / \mathrm{cm}^{* * 3}\right)$

s $1.600 \mathrm{E}+00,1.500 \mathrm{E}+00$

-

3 THICKO

-.. 3 LCZPAQ

- 3 BRDL

3 TI

$3 T(2)$

- 1 T $T(3)$

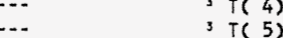

.. 3 T $T(6)$

..

T $T(8)$

-.. 3 T $T(9)$

$+-$

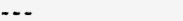

$\ldots \quad s \operatorname{sic}(2)$

... $\quad 351(3)$

$\cdots \quad 3 \sin (4)$

3 s1( 7 )

$\ldots+31(8)$

3 W1( 1$)$

3 W1( 2)

J W1( 3 )

‥ $W 1(4)$

-.. $\quad 3 W 1(7)$

... 3 WI( 8 )

.. $31(9)$

... 3 COVERO

-...

$3 \mathrm{VCV}$

3 DENSCZ

-.. $\quad 3$ VCZ

-.. $\quad$ I TPCZ

-.. 3 FCCZ

... 3 HCCZ

..- $\mathrm{BCZ}$

3 WIND

3 HUMID

... 3 EVAPTR

-

... 5 IDITC

$\ldots$ RUNOFF

... 3 HAREA

... 3 EPS

... I DENSAQ

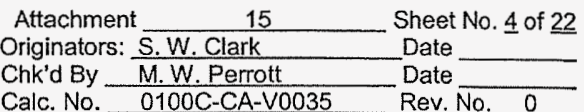




\section{ATTACHMENT 15}

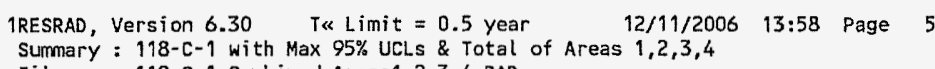

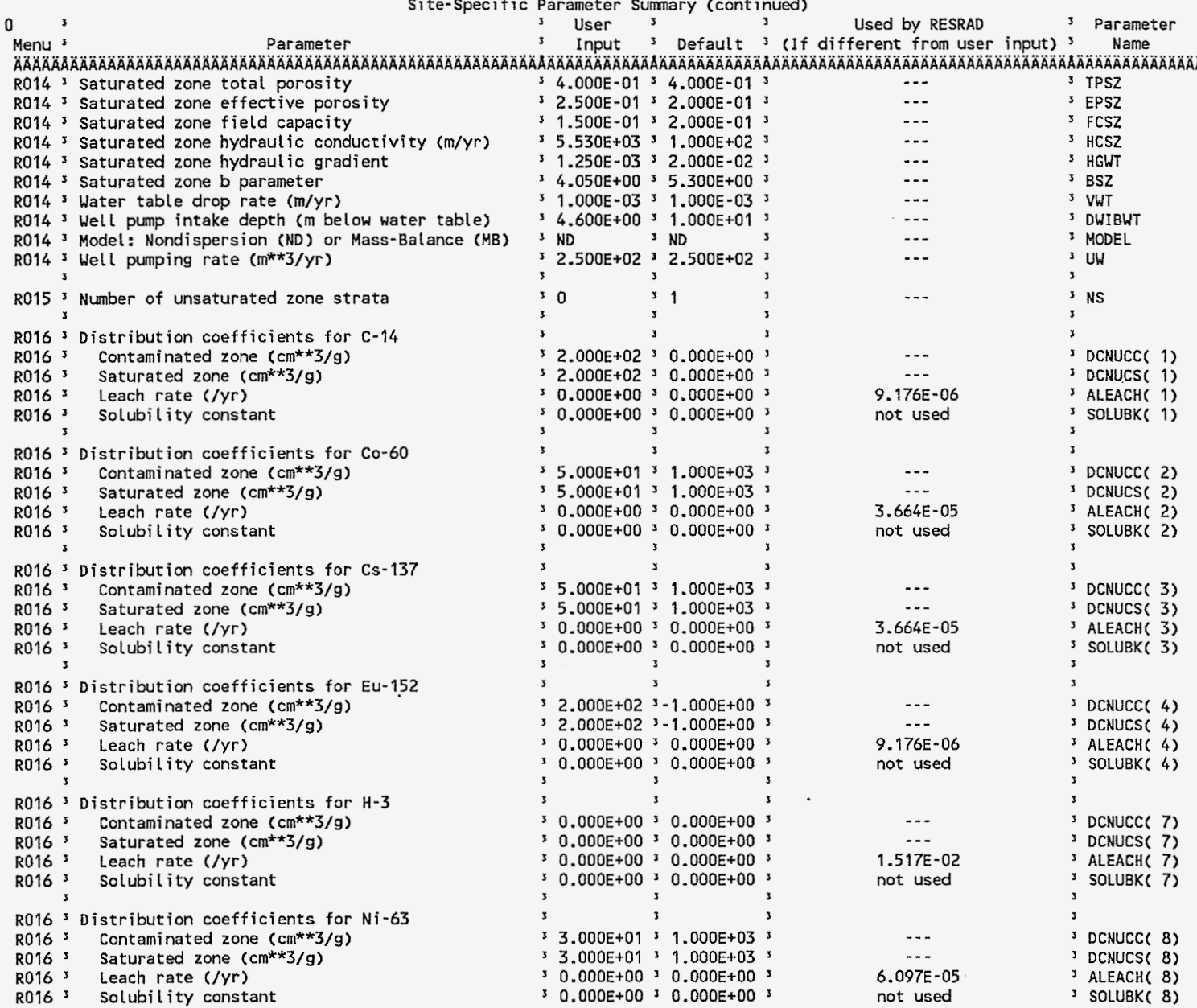

\begin{tabular}{|c|c|}
\hline Attachment & et No. $\underline{5}$ of $\underline{22}$ \\
\hline Originators: S.W. Clark & Date \\
\hline Chk'd By M.W. Perrott & Date \\
\hline Calc. No. $0100 \mathrm{C}-\mathrm{CA}-\mathrm{V} 0035$ & Rev. \\
\hline
\end{tabular}




\section{ATTACHMENT 15}

1RESRAD, Version $6.30 \quad T \ll$ Limit $=0.5$ year $12 / 11 / 2006 \quad 13: 58$ Page 6

file : $118-c-1$ Combined Areas1-2-3-4.RAD

Site-Specific Parameter Sumary (continued)

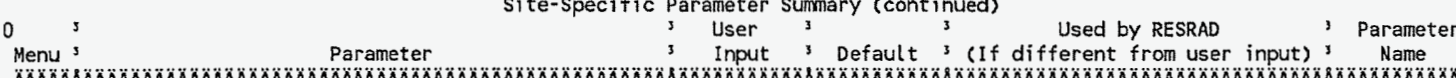

Ä

R016 ${ }^{3}$ Distribution coefficients for $\mathrm{Sr}-90$

R016 3 Contaminated zone $\left(\mathrm{cm}^{* * 3 / g}\right)$

R016 3 Saturated zone $(\mathrm{cm} * * 3 / \mathrm{g})$

(1)

R016's Leach rate (/yr)

R016; Solubility constant

R016 Distribution coefficients for daughter Gd-152

R016 3 Contaminated zone $(\mathrm{cm} * * 3 / \mathrm{g})$

R016 3 Saturated zone $\left(\mathrm{cm}^{* * 3 / \mathrm{g})}\right.$

R016' Leach rate (/yr)

R016 Solubility constant

R017: Inhalation rate $\left(m^{* * 3 / y r)}\right.$

R017 Mass loading for inhalation $\left(\mathrm{g} / \mathrm{m}^{* *}\right)$

R017' Exposure duration

R017' Shielding factor, inhalation

R017'shielding factor, external gamma

R017' Fraction of time spent indoors

R017' Fraction of time spent outdoors (on site)

R017' Shape factor flag, external gamma

R017' Radi i of shape factor array (used if FS $=-1$ ).

R017 3 Outer annular radius (m), ring 1

$R 017$, Outer annular radius $(m)$, ring 2 :

R017 s Outer annular radius (m), ring 3 :

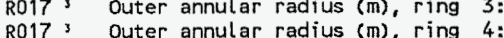

R017: Outer annular radius $(\mathrm{m})$, ring 5 :

$2.500 E+0133.000 E+013$

$2.500 E+0133.000 E+013$

$0.000 E+0030.000 E+003$

s $0.000 \mathrm{E}+00 \times 0.000 \mathrm{E}+00$ s

3

$3-1.000 E+00^{3}-1.000 E+00^{3}$

$3-1.000 E+003-1.000 E+00$

$30.000 E+00=0.000 E+003$

$30.000 E+00 ; 0.000 E+00$,

s $7.300 \mathrm{E}+03$ 3 $8.400 E+03$ 3

$1.000 E-04^{3} 1.000 E-04^{3}$

$3.000 E+01,3.000 E+01$;

$3.000 E-0134.000 E-013$

s $8.000 E-0137.000 E-01$ s

$36.000 E-01: 5.000 E-013$

3 $2.000 E-0132.500 E-01$

$31.000 E+0031.000 E+00^{3}$

3 not used $35.000 E+01$

not used $37.071 \mathrm{E}+01$

not used $30.000 \mathrm{E}+00$

not used 0.000 to

not used $0.000 E+00$

not used 0.000 t

not used $30.000 E+00$

3 not used $30.000 E+00$

outer annular radius (m), ring 6 :

3 not used $30.000 \mathrm{E}+00$

3 not used $30.000 E+00$

Outer annular radius (m), ring 9:

$s$ not used $s 0.000 E+00$

3 not used $30.000 \mathrm{E}+00$

Outer annular radius (m), ring 10

3 not used $0.000 \mathrm{E}+00$

R017, Outer annular radius (m), ring 11

R017 Fractions of annular areas with in AREA:

R017' Ring 1

R017 ' Ring 2

R017' Ring 3

R017: Ring 4

$\mathrm{RO17}^{3}$ Ring 5

$\mathrm{R}_{017^{3}}{ }^{3}$ Ring 6

R017 s Ring 7

R017'sing 8

R017 3 Ring 9

not used $31.000 E+00$

3 not used $32.732 E-01$

not used $: 0.000 E+00$

not used $30.000 E+00$

not used $\$ 0.000 E+00$

not used $30.000 E+00$

not used $30.000 E+00$

not used $30.000 E+00$

not used $>0.000 E+00$

not used $0.000 E+00$

not used $30.000 E+00$

3 not used, $0.000 \mathrm{E}+00^{3}$

R017, Ring 11

R017 Bing 12

R018 Fruits, vegetables and grain consumption $(\mathrm{kg} / \mathrm{yr})=1.100 \mathrm{E}+02=1.600 \mathrm{E}+02$ R018' Leafy vegetable consumption $(\mathrm{kg} / \mathrm{yr})$

$3.700 E+0031.400 E+01$

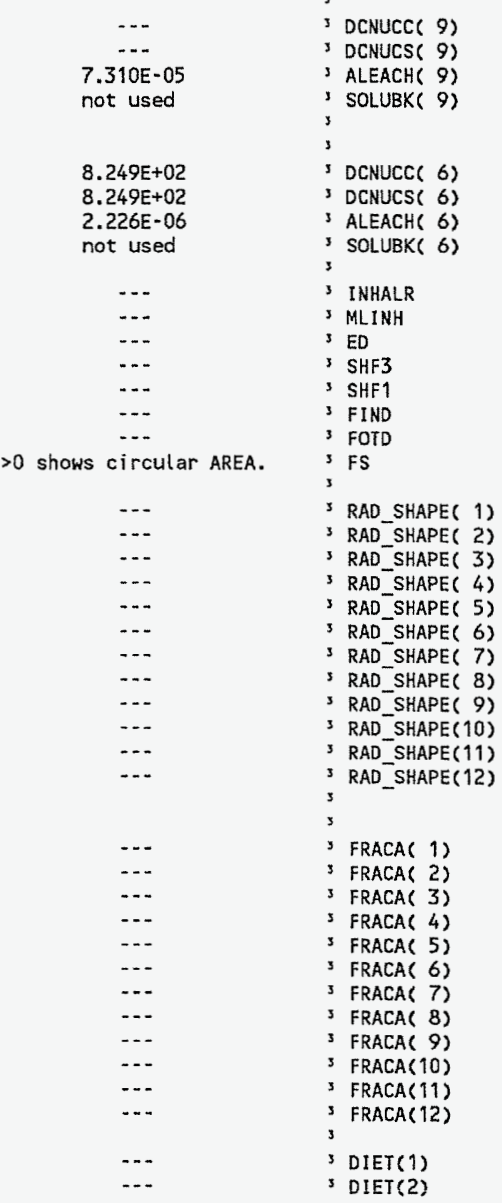

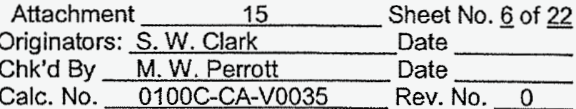




\section{ATTACHMENT 15}

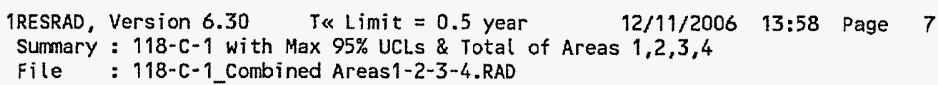

Site-Specific Parameter sumary (continued)

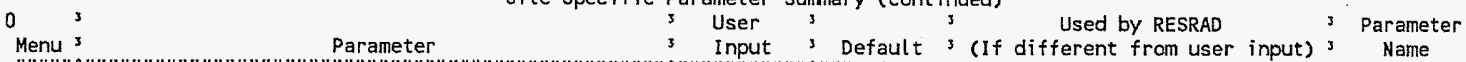

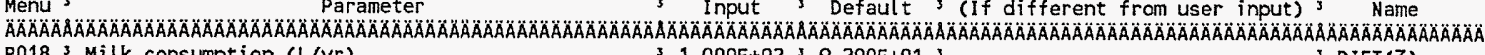

R018 3 Milk consumption ( $L / Y r$ )

(kg/Yr) $3.600 \mathrm{E}+01^{3} 6.300 \mathrm{E}+01$

sumption $(\mathrm{kg} / \mathrm{yr})$

ingestion rate $(g / y r)$

R018 3 Drinking water intake ( $L / Y r)$

R018 s Contamination fraction of drinking water

R018 Contamination fraction of household water

R018 S Contamination fraction of livestock water

R018 S Contamination fraction of irrigation water

R018 3 Contamination fraction of aquatic food

R018 3 Contamination fraction of plant food

R018 3 Contamination fraction of meat

R018 ' Contamination fraction of milk

3

1.970E+01, $5.400 E+00$

$3.300 \mathrm{E}+0133.650 \mathrm{E}+013$

$3.300 E+02 \times 5.100 E+02$

$31.000 E+00>1.000 E+00$

3 not used $\$ 1.000 E+00$,

$1.000 E+00>1.000 E+00$

$1.000 E+0031.000 E+00$

5.000E-01? 5.000E-01

$30.000 E+003-1$

$0.000 E+003-1$

$0.000 E+003-1$

R019 3 Livestock fodder intake for meat ( $\mathrm{kg} /$ day)

$R 0193$ Livestock fodder intake for $\mathrm{mi}(\mathrm{k}$ ( $\mathrm{kg} /$ day)

$\mathrm{R} 0193$ Livestock water intake for meat (L/day)

R019 3 Livestock water intake for milk (L/day)

$\mathrm{R} 019^{3}$ Livestock soil intake ( $\mathrm{kg} /$ day)

R019 * Mass loading for foliar deposition $\left(\mathrm{g} / \mathrm{m}^{* *} 3\right)$

R019 3 Depth of soil mixing layer (m)

R019' Depth of roots (m)

R019: Drinking water fraction from ground water

R019 3 Household water fraction from ground water

R019 : Livestock water fraction from ground water

R019: Irrigation fraction from ground water

$\left.36.800 E^{3}+01,6.800 E^{3}+01\right)^{3}$

$5.500 E+01,5.500 E+01$

$5.000 E+0135.000 E+01$

$31.600 \mathrm{E}+02 ; 1.600 \mathrm{E}+02$

3 5. $000 \mathrm{E}-0135.000 \mathrm{E}-01$

$31.000 E-043 \quad 1.000 E-04$

$31.500 E-01>1.500 E-01$

\%

$9.000 E-01,9.000 E-01$

1.000E+00 3 $1.000 E+00$

.00E +00

$1.000 E+00$ ? $1.000 E+00$

$31.000 E+00 ; 1.000 E+00$;

$3.000 E-0137.000 E-01$

R19B * Wet weight crop yield for Leafy (kg/m**2)

$31.500 \mathrm{E}+00,3.000 \mathrm{E}-01$

$31.100 E+00 ; 1.100 E+00$

$=1.700 \mathrm{E}-0131.700 \mathrm{E}-01$

3 2.500E-01, $2.500 \mathrm{E}-01$

$38.000 \mathrm{E}-02,8.000 \mathrm{E}-02$

$31.000 \mathrm{E}-01+1.000 \mathrm{E}-01$

$1.000 E+00 ; 1.000 E+00$

$1.000 E+00,1.000 E+00$

R19B 3 Growing Season for Fodder (years)

R19B Translocation Factor for Non-Leafy

R198 3 Translocation Factor for Leafy

3 2.500E-01 $32.500 \mathrm{E}-01$

R19B 3 Dry Foliar Interception Fraction for Non-Leafy 2.500

R19B 3 Dry Foliar Interception Fraction for Leafy ${ }^{3} 2.500 E$

R19B ' Dry Foliar Interception Fraction for Fodder 32.500 -01 $32.500 E-01$

R19B 3 Wet Foliar Interception Fraction for Non-Leafy $32.500 E-0132.500 E-01$

$R 19 B$ s Wet Foliar interception Fraction for Leafy, $2.500 E-01$, 2.500E-01,

R19B 3 Wet Foliar interception fraction for fodder $32.500 \mathrm{E}-0132.500 \mathrm{E}-01$

3

$2.500 \mathrm{E}-0132.500 \mathrm{E}-01$

s.

C14 $3 \mathrm{C}-12$ concentration in water $\left(\mathrm{g} / \mathrm{cm}^{* *} 3\right)$

$\mathrm{C} 14{ }^{3} \mathrm{C}-12$ concentration in contaminated soil (g/g)

C14 Fraction of vegetation carbon from soi!

C14 3 Fraction of vegetation carbon from air

$\mathrm{C} 143 \mathrm{C}-14$ evasion layer thickness in soil (m)

$32.000 \mathrm{E}-05 ; 2.000 \mathrm{E}-05$

$33.000 \mathrm{E}-02,3.000 \mathrm{E}-02$

$32.000 \mathrm{E}-02 \times 2.000 \mathrm{E}-02$

$9.800 E-0139.800 E-01$

3 3.000E-01 > 3.000E-01

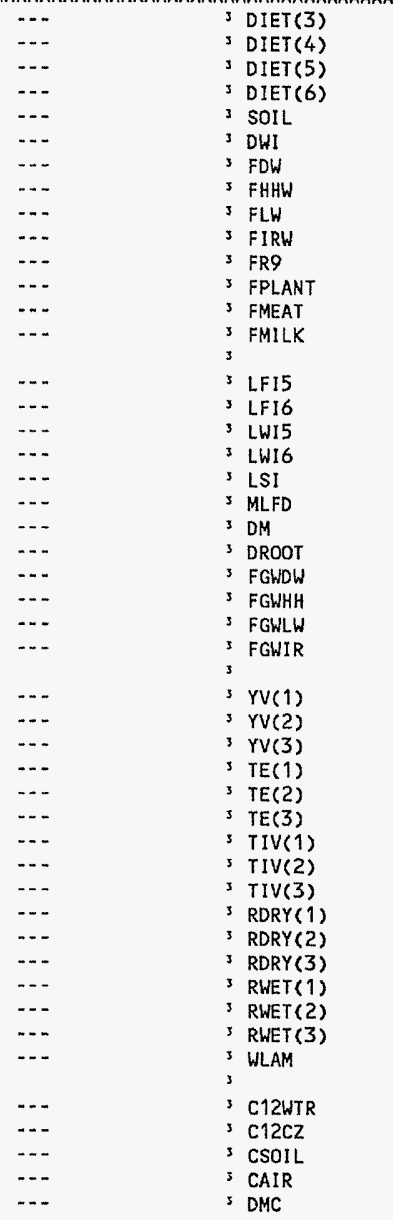

Attachment

15 Sheet No. 7 of 22 Chk'd B

S.W. Clark
Date

Calc. No. 0100C-CA-V0035

M. W. Perrott

Date

Rev. No. 


\section{ATTACHMENT 15}

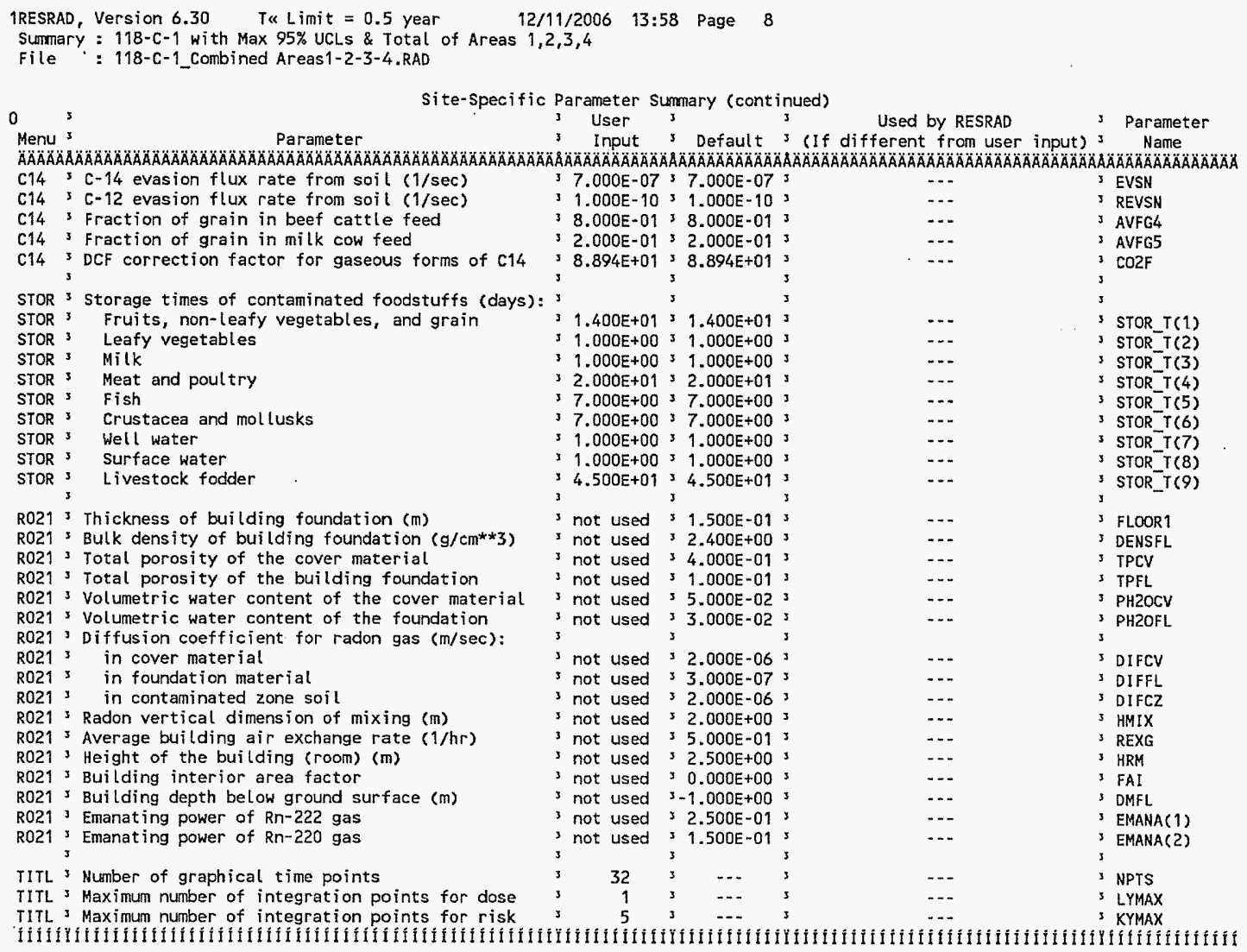

$\begin{array}{ll}\text { Attachment } \frac{15}{\text { Ot.W. Clark }} & \text { Sheet No. } \underline{8} \text { of } \underline{22} \\ \text { Originators: } & \text { Date } \\ \text { Chk'd By } \frac{\text { M.W. Perrott }}{\text { Date }} & \\ \text { Calc. No. } & \text { Rev. No. } 0\end{array}$


Rev. 0

\title{
ATTACHMENT 15
}

\author{
IRESRAD, Version 6.30 T* Limit $=0.5$ year $12 / 11 / 2006 \quad 13: 58$ Page 9 \\ Summary : $118-\mathrm{C}-1$ with Max $95 \%$ UCLs \& Total of Areas $1,2,3,4$ \\ File: 118-C-1 Combined Areas1-2-3-4-RAD
}

Surmary of Pathway Selections

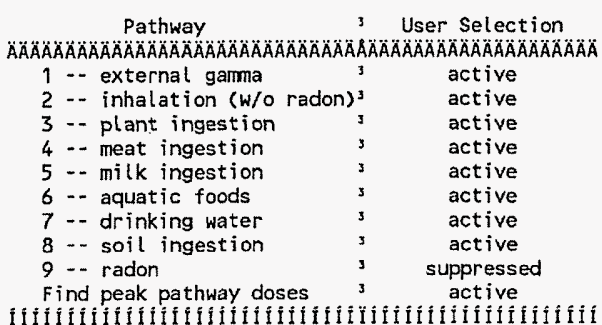

Attachment Sheet No. $\underline{9}$ of 22

Originators: S. W. Clark Date

Chk'd By M.W. Perrott.

Date

Caic. No. 0100C-CA-V0035

Rev. No. 0 


\section{ATTACHMENT 15}

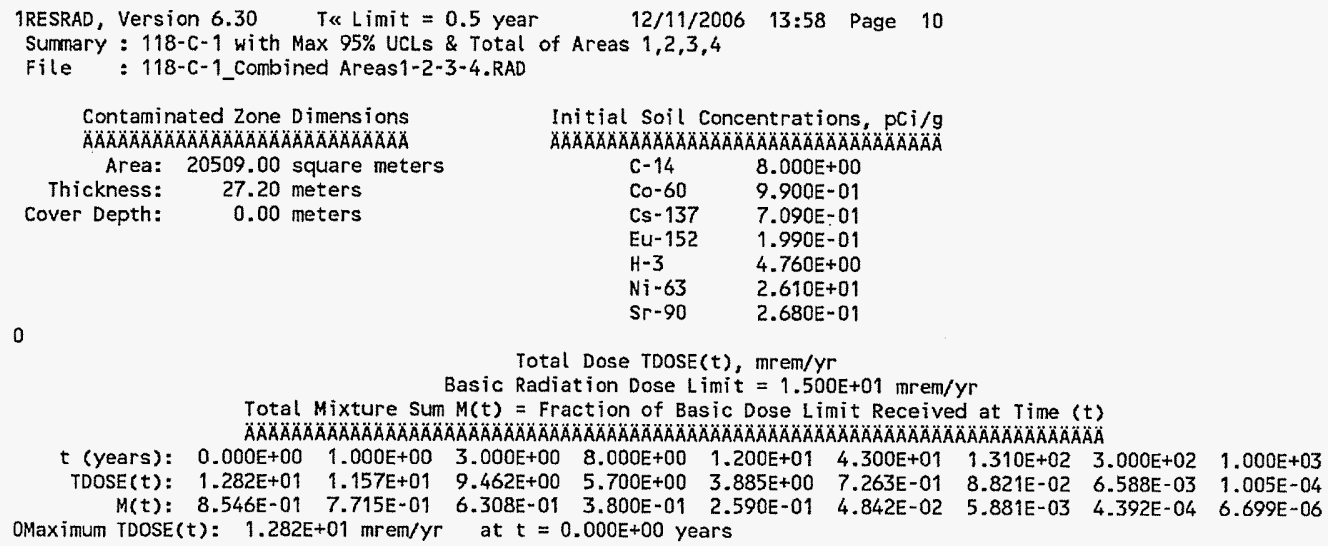

Total Dose TDOSE( $t$ ), mrem/yr

Basic Radiation Dose Limit $=1.500 \mathrm{E}+01 \mathrm{mrem} / \mathrm{yr}$

Total Mixture sum $M(t)=$ Fraction of Basic Dose Limit Received at Time $(t)$

Totat Mixture Sum $M(t)=$ Fraction of Basic Dose $L$ imit Received at $T$ ime $(t)$

$t$ (years): $\begin{array}{ccccccccc}0.000 E+00 & 1.000 E+00 & 3.000 E+00 & 8.000 E+00 & 1.200 E+01 & 4.300 E+01 & 1.310 E+02 & 3.000 E+02 & 1.000 E+03\end{array}$

TDOSE $(t): \begin{array}{lllllllll}1.282 E+01 & 1.157 E+01 & 9.462 E+00 & 5.700 E+00 & 3.885 E+00 & 7.263 E-01 & 8.821 E-02 & 6.588 E-03 & 1.005 E-04\end{array}$

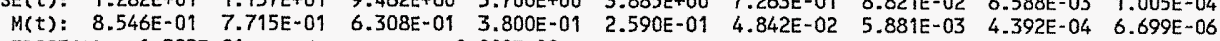
OMaximum TDOSE $(t): 1.282 E+01 \mathrm{mrem} / \mathrm{yr}$ at $t=0.000 E+00$ years

Attachment $\frac{15}{\text { Originators: S. W. Clark }}$

Sheet No. 10 of 22

Chk'd By MW Perrott Date

Calc. No. 0100C-CA-V0035 


\section{ATTACHMENT 18}

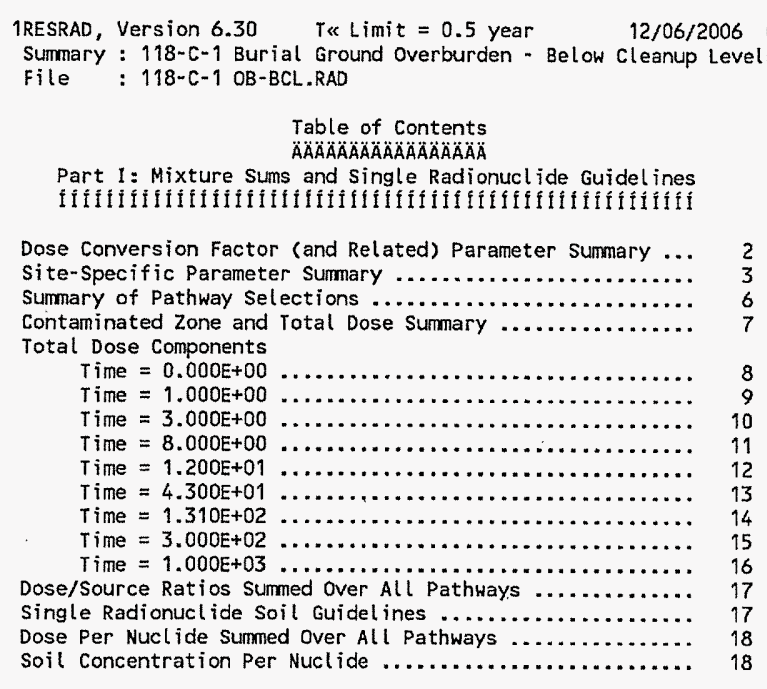

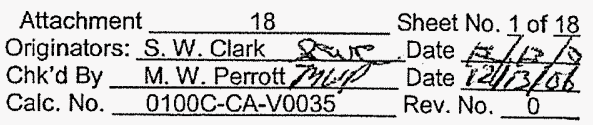




\section{ATTACHMENT 18}

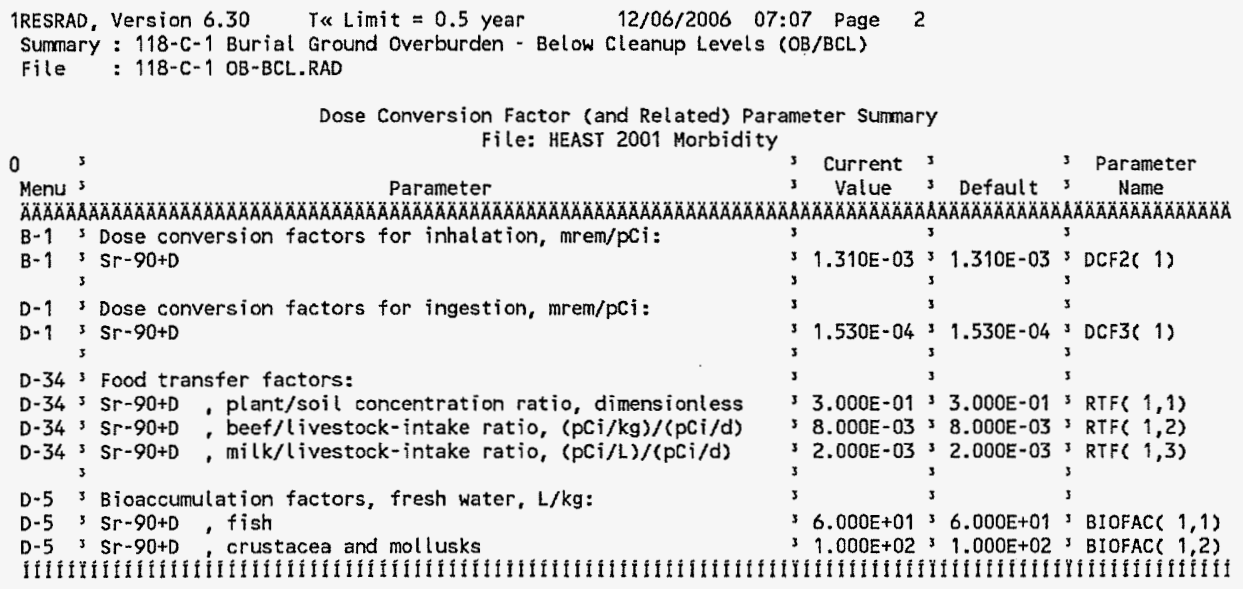

Attachment 


\section{ATTACHMENT 18}

IRESRAD, Version $6.30 \quad T \ll$ Limit $=0.5$ year 12/06/2006 07:07 Page 3 Summary : 118-C-1 Burial Ground Overburden - Below Cleanup Levels (OB/BCL) file: $118-C-1$ OB-BCL.RAD Site-Specific Parameter Sumary

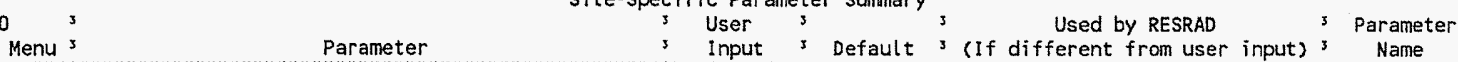

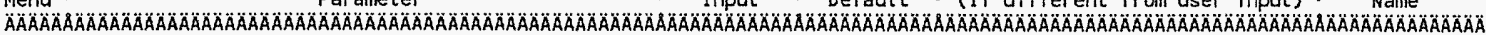
R011 3 Area of contaminated zone (m**2)

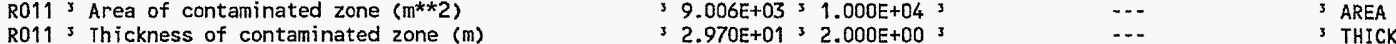
R011 ' Thickness of contaminated zone (m)

R011 s Length parallel to aquifer flow $(m)$ linit (mrem/yr) $\quad 39.500 E+01 ; 1.000 E+02$ R011's Times for calculations $(y r)$

R011: Times for calculations $(y r)$

> $1.000 E+00>1.000 E+00$

$3.000 E+00^{3} 3.000 E+00^{3}$

$38.000 E+00 \times 1.000 E+013$

$31.200 E+0133.000 E+013$

> $4.300 \mathrm{E}+01 \times 1.000 \mathrm{E}+02$

$31.310 E+02=3.000 E+02 ;$

$3.000 E+02 ; 1.000 E+03$;

$31.000 E+03 \div 0.000 E+00$

not used $30.000 E+00^{3}$

R011

R011 3 Times for calculations (yr)

$1.230 E+00=0.000 E+00$ 3

R012 3 Initial principal radionuclide ( $\mathrm{pC} i / g): \quad s r-90$

$\mathrm{R}^{2} 12^{3}$ Concentration in groundwater ( $\mathrm{pC} \mathrm{i} / \mathrm{L}$ ): $\mathrm{Sr}-90$

not used $30.000 E+00$

$0.000 \mathrm{E}+00$ s $0.000 \mathrm{E}+00,3$

R013 s Cover depth $(\mathrm{m})$

R013 Density of cover material $\left(\mathrm{g} / \mathrm{cm}^{* * 3}\right)$

$R 013^{3}$ Cover depth erosion rate $(\mathrm{m} / \mathrm{yr})$

R013 Density of contaminated zone $\left(\mathrm{g} / \mathrm{cm}^{* * 3}\right)$

3 not used $3,1.500 \mathrm{E}+00$

not used $31.5000 \mathrm{E}+003$

$1.600 \mathrm{E}+00$ ? $1.500 \mathrm{E}+00$

$1.000 E-03>1.000 E-03$

4.000E-01 $>4.000 E-01$

R013 Contaminated zone total porosity

R013' Contaminated zone field capacity

$31.500 \mathrm{E}-01$ ? $2.000 \mathrm{E}-013$

0133 Contaminated zone hydraulic conductivity $(\mathrm{m} / \mathrm{yr}) 32.500 \mathrm{E}+0231.000 \mathrm{E}+01$

R013 3 Contaminated zone b parameter

R013 3 Average annual wind speed $(\mathrm{m} / \mathrm{sec}) \quad 33.400 \mathrm{E}+0032.000 \mathrm{E}+00$ 3

R013 3 Humidity in air $\left(\mathrm{g} / \mathrm{m}^{* * 3}\right)$, not used $38.000 E+00$.

R013 3 Evapotranspiration coefficient $39.100 E-0135.000 E-013$

R013 3 Precipitation (m/yr) $\quad, 1.600 \mathrm{E}-0131.000 \mathrm{E}+00^{3}$

$37.600 E-0132.000 E-01$

R013 I Irrigation mode

R013' Runoff coefficient

3 overhead 's overhead

$32.000 E-01^{3} 2.000 E-013$

R013 Watershed area for nearby stream or pond $\left(m^{* * 2}\right)$

$1.000 E+06 ; 1.000 E+06$;

R013 Accuracy for water/soil computations

R014 3 Density of saturated zone $\left(\mathrm{g} / \mathrm{cm}^{* * 3}\right)$

R014 Saturated zone total porosity

R014 3 Saturated zone effective porosity

R014 3 Saturated zone field capacity

R014 3 Saturated zone hydraulic conductivity $(\mathrm{m} / \mathrm{yr})$

R014'Saturated zone hydraulic gradient

R014' Saturated zone b parameter

R014 3 Water table drop rate (m/Yr)

R014 Well pump intake depth (m below water table)

R014 Model: Nondispersion (ND) or Mass-Balance (MB) R014 3 Well pumping rate $\left(m^{* *} 3 / \mathrm{yr}\right)$

R015 N Number of unsaturated zone strata
1.000E-03 $>1.000 E-03$,

$1.600 E+00>1.500 E+00$

$4.000 \mathrm{E}-0134.000 \mathrm{E}-01$

$2.500 \mathrm{E}-0132.000 \mathrm{E}-01$

3 $1.500 \mathrm{E}-01^{3} 2.000 \mathrm{E}-01^{3}$

$5.530 \mathrm{E}+0331.000 \mathrm{E}+02$

, $1.250 \mathrm{E}-03$ ? $2.000 \mathrm{E}-02$ ?

\& $4.050 \mathrm{E}+0035.300 \mathrm{E}+00^{3}$

$31.000 E-0331.000 E-03$

$34.600 \mathrm{E}+00$ ? $1.000 \mathrm{E}+\mathrm{D} 1$ ?

3 ND

$2.500 E+02$
3

-.- 3 LCZPAO

3 BRDL

-.

${ }^{3} T(2)$

$\cdots$

$3 T(4)$

‥ 3 T 6 ( 6 )

-.. $3 \mathrm{~T}(7)$

$3 T(8)$

...

… $351(1)$

3 W1( 1$)$

COVERO

3 DENSCV

3 VCV

3 DENSCZ

I VCZ

I TPCZ

3 FCCZ

$3 \mathrm{HCCZ}$

$3 \mathrm{BCZ}$

BCZ

HUMID

EVAPTR

3 PRECIP

3 RI

3 IDITCH

WAREA

DENSAQ

3 TPSZ

3 TPSZ

3 EPSZ

3 HCSZ

3 HGWT

BSZ

VWT

$\begin{array}{lll}\ldots & \text { WWT } \\ \ldots & 3 \text { DWIBWT } \\ \ldots & 3 \text { MODEL }\end{array}$

...

...

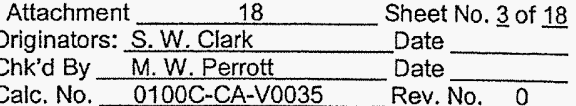




\section{ATTACHMENT 18}

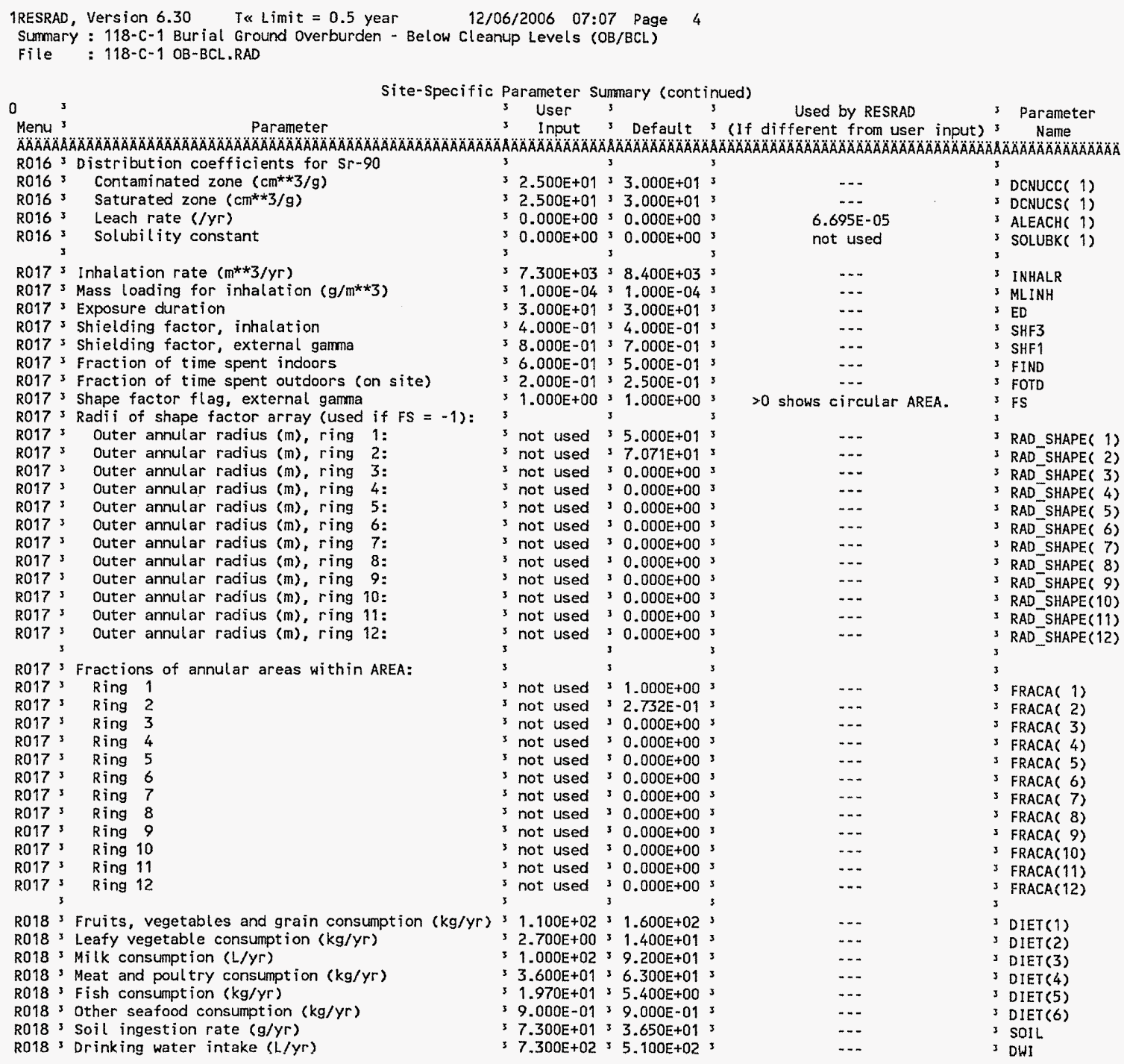

\begin{tabular}{|c|c|}
\hline Attachment & Sheet No. 4 of 18 \\
\hline Originators: S. W. Clark & Date. \\
\hline Chk'd By M.W. Perrott & Date \\
\hline Calc. No. $0100 \mathrm{C}-\mathrm{CA}-\mathrm{V} 0035$ & Rev. No. 0 \\
\hline
\end{tabular}




\section{ATTACHMENT 18}

1RESRAD, Version 6.30 T\& Limit $=0.5$ year 12/06/2006 07:07 Page 5 summary : $118-C-1$ Burial Ground Overburden - Below cleanup Levels (OB/BCL) File: 118-C-1 OB-BCL.RAD

0 Site-Specific Parameter Summary (continued)

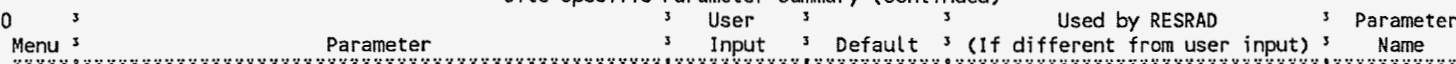

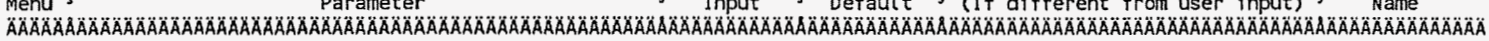
AAAAAAAAAAAAAAAAAAAAAAAAAAAAAAAAAAAAAANARAKAAAAAAAAAAAAAAAAAAAAAAAAAAAAAAAAAAAAAAAAAAAAAAAAAAAAAAAAAAAAAAAAAAAAAAAAAAAAAAAA

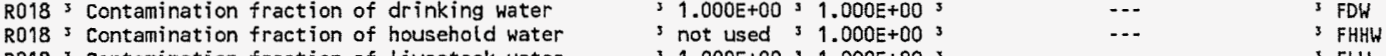
RD18 3 Contamination fraction of livestock water $31.000 \mathrm{E}+00^{3} 1.000 \mathrm{E}+00^{3} \quad \ldots$

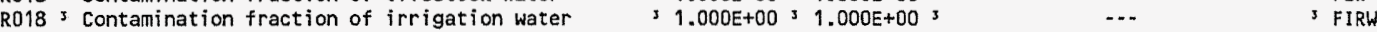
R018 Contamination fraction of aquatic food $3.000 E-0135.000 E-01$ R018 3 Contamination fraction of plant food R018 3 Contamination fraction of meat R018 3 Contamination fraction of milk 3 $=-1,3-1$ $\begin{array}{lll}3-1 & 3-1 \\ 3-1 & 3-1\end{array}$ 3

$\begin{array}{ll}0.500 E+00 & 3 \text { FPLANT } \\ 0.450 E+00 & 3 \text { FMEAT }\end{array}$

R019 3 Livestock fodder intake for meat ( $\mathrm{kg} /$ day) R019 s Livestock fodder intake for $m i(k)(\mathrm{kg} /$ day) R019 ' Livestock water intake for meat (L/day)

$36.800 E+0136.800 E+01$ $5.500 E+01 ; 5.500 E+01$ $5.000 \mathrm{E}+01>5.000 \mathrm{E}+01$ $1.600 E+0231.600 E+02$ 3 5.000E-01 3 $5.000 \mathrm{E}-01^{3}$ R019 3 Livestock soil intake ( $\mathrm{kg} /$ day) R019' Mass Loading for foliar deposition $\left(\mathrm{g} / \mathrm{m}^{* *} 3\right)$ R019 'Depth of soil mixing layer (m)

R019 ' Depth of roots (m)

R019 : Drinking water fraction from ground water R019' Household water fraction from ground water R019 3 Livestock water fraction from ground water R019: Irrigation fraction from ground water s s $1.000 E-0431.000 E-04$ $31.500 E-0131.500 E-01$ $39.000 E-01$ S $9.000 E-01$

$31.000 \mathrm{E}+00^{3} \quad 1.000 \mathrm{E}+00$

$1.000 E+00,1.000+00$

$1.000 E+00,1.000 E+00$ $1.000 E+00,1.000 E+00$ $31.000 E+0031.000 E+003$ $R 19 B$ Wet weight crop yield for Non-Leafy $\left(\mathrm{kg} / \mathrm{m}^{* * 2}\right)$ 7.000E-01:7.000E-01 $R 19 B$ s Wet weight crop yield for Leafy $\left(\mathrm{kg} / \mathrm{m}^{* \star 2}\right)$ \& $1.500 \mathrm{E}+00 ; 1.500 \mathrm{E}+00$ R19B 3 Wet weight crop yield for fodder $\left(\mathrm{kg} / \mathrm{m}^{* * 2}\right){ }^{3} 1.100 E+00^{3} 1.100 \mathrm{E}+00$ R19B 3 Growing Season for Non-Leafy (years) $31.700 E-01 ; 1.700 E-01$; R19B 3 Growing Season for Leafy (years) $32.500 E-0132.500 E-013$ R19B 3 Growing Season for fodder (years) $38.000 E-0238.000 E-02$, R19B 3 Growing Season for fodder (years) $31.000 \mathrm{E}-0131.000 \mathrm{E}-01$, R19B 3 Translocation Factor for Non-Leafy 3 , $1.000 E+0031.000 E+00$

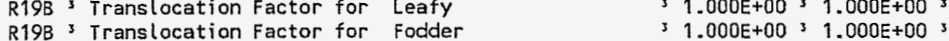

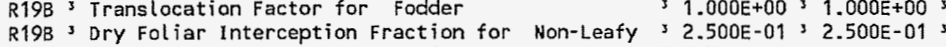
R19B 3 Dry Foliar Interception Fraction for Leafy $32.500 E-0132.500$ E-013 R198 3 Dry Foliar Interception Fraction for Fodder $32.500 E-0132.500 E-013$ R19B 3 Wet Foliar Interception Fraction for Non-Leafy $32.500 E-0132.500 E-013$ R198 3 Wet Foliar Interception Fraction for Leafy s 2.500E-01 3 2.500E-01 R19B Wet Foliar Interception Fraction for Fodder $33^{2.500 E-013} 3.5005-01$ R198 3 Weathering Removal Constant for Vegetation 3

C14 $3 \mathrm{C}-12$ concentration in water $(\mathrm{g} / \mathrm{cm} * * 3)$

C14 $3 \mathrm{C}-12$ concentration in contaminated soil $(\mathrm{g} / \mathrm{g})$

C14 Fraction of vegetation carbon from soil

C14 3 Fraction of vegetation carbon from air

$\mathrm{C14} 3 \mathrm{C}-14$ evasion layer thickness in soil (m)

$\mathrm{C14} 3 \mathrm{c}-14$ evasion flux rate from soil (1/sec)

C14 $3 \mathrm{c}-12$ evasion flux rate from soi! ( $1 / \mathrm{sec})$

C14 3 Fraction of grain in beef cattle feed

C14 3 Fraction of grain in milk cow feed

C14 3 DCF correction factor for gaseous forms of $\mathrm{C14}$

$32.000 E+0132.000 E+013$

not used 3 2.000E-05

not used $33.000 E-02$

not used 3 2.000E-02

not used 3 9.800E-01

not used 3.000E-01

not used $37.000 E-07$

3 not used $1.000 \mathrm{E}^{10}$

not used 1 .OOE-10

not used s.000E-01

not used $3.894 \mathrm{E}+01$,

$0.450 E+00$ S FMIK

$S$

-.. 3 LFI5

$\begin{array}{ll}\cdots- & \text { s LFI6 } \\ \cdots & \text { s LWI5 }\end{array}$

$\begin{array}{ll}\ldots- & \text { s LWI6 } \\ -.- & \text { s LWI5 }\end{array}$

... 3 LSI

-.. 3 MLFD

$\begin{array}{ll}3 \\ \ldots & 3 \mathrm{DM} \\ \ldots & 3 \mathrm{DROO}\end{array}$

$n$

-.. 3 FGWHH

-.- 3 FGWLW

...

I $P(1)$

-.. $\quad 3 Y Y(2)$

YV(3)

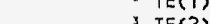

TE(2)

-.- 3 TIV(1)

-.. 3 TIV(2)

$3 \operatorname{TIV}(3)$

$\begin{array}{ll}\ldots & \text { RDRY(1) } \\ \ldots & 3 \text { RDRY(2) }\end{array}$

3 RDRY (3)

( RWET( (1)

3 RWET(2)

RWET(

-.. 3

-. 3 CI2WTR

-.. 3 C12C2

...

...

EVSN

REVS

, AVFG5

$\begin{array}{ll}\cdots & 3 \text { AVFG5 } \\ \cdots & 3 \\ \cdots\end{array}$

Attachment

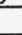




\section{ATTACHMENT 18}

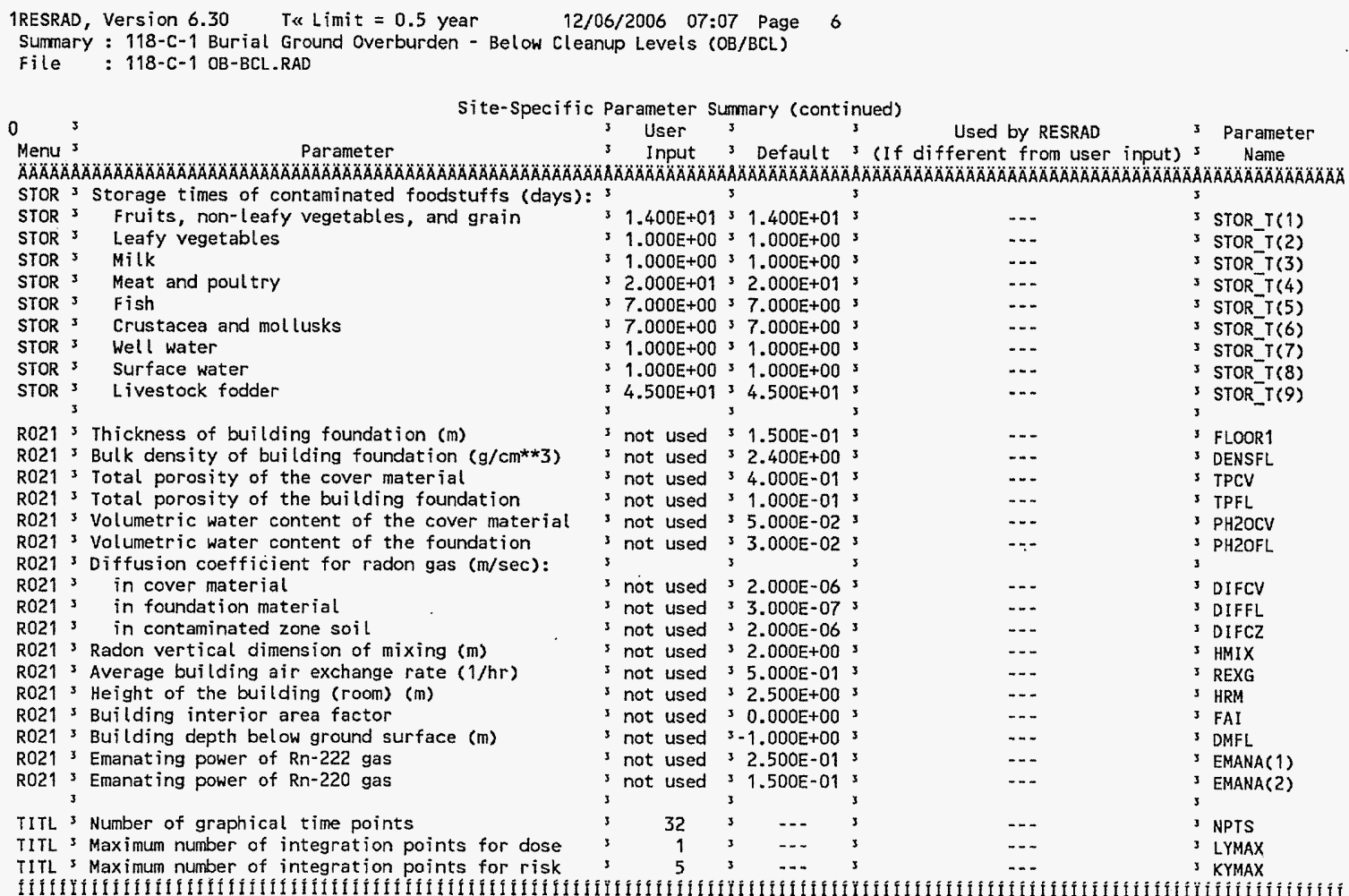

Surmary of Pathway Selections

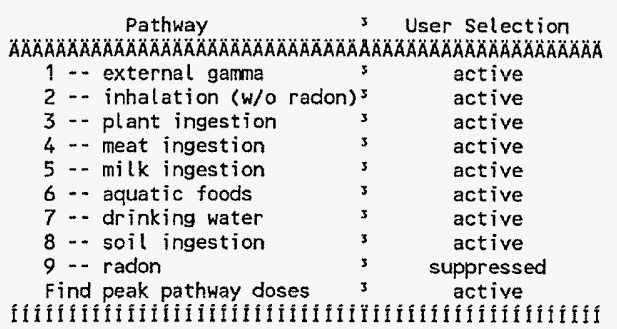

\begin{tabular}{|c|c|}
\hline \multirow{2}{*}{\multicolumn{2}{|c|}{$\begin{array}{cc}\text { Attachment } & 18 \\
\text { Originators: Sh. W. Clark } & \text { Sheet No. } \underline{6} \text { of } 18 \\
\end{array}$}} \\
\hline & \\
\hline M. W. Perrott & Date \\
\hline $0100 C-C A-V 0035$ & No. \\
\hline
\end{tabular}


Rev. 0

\section{ATTACHMENT 18}

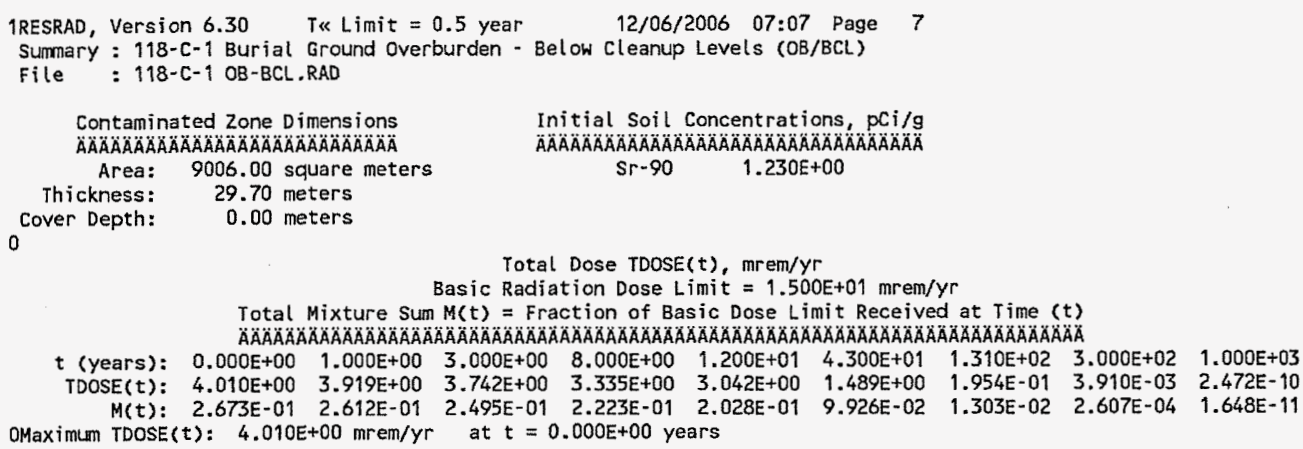

$t$ (years): $\begin{array}{lllllllllll}0.000 E+00 & 1.000 E+00 & 3.000 E+00 & 8.000 E+00 & 1.200 E+01 & 4.300 E+01 & 1.310 E+02 & 3.000 E+02 & 1.000 E+03\end{array}$

$\operatorname{TDOSE}(t): \quad 4.010 E+00 \quad 3.919 E+00 \quad 3.742 E+00 \quad 3.335 E+00 \quad 3.042 E+00 \quad 1.489 E+00 \quad 1.954 E-01 \quad 3.910 E-03 \quad 2.472 E-10$

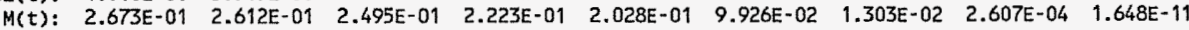
OMaximum TDOSE $(t): 4.010 E+00 \mathrm{mrem} / \mathrm{yr}$ at $t=0.000 \mathrm{E}+00$ years

$\begin{array}{ll}\text { Attachment } \frac{18}{\text { S.W. Clark }} & \text { Sheet No. } 7 \text { of } 18 \\ \text { Originators: } \frac{\text { S.W. }}{\text { M. W. Perrott }} & \text { Date } \\ \text { Chk'd By } & \text { Rev. No. } 0 \\ \text { Calc. No. } 0100 \text { C-CA-V0035 } & \text { Rev. No }\end{array}$




\section{ATTACHMENT 21}

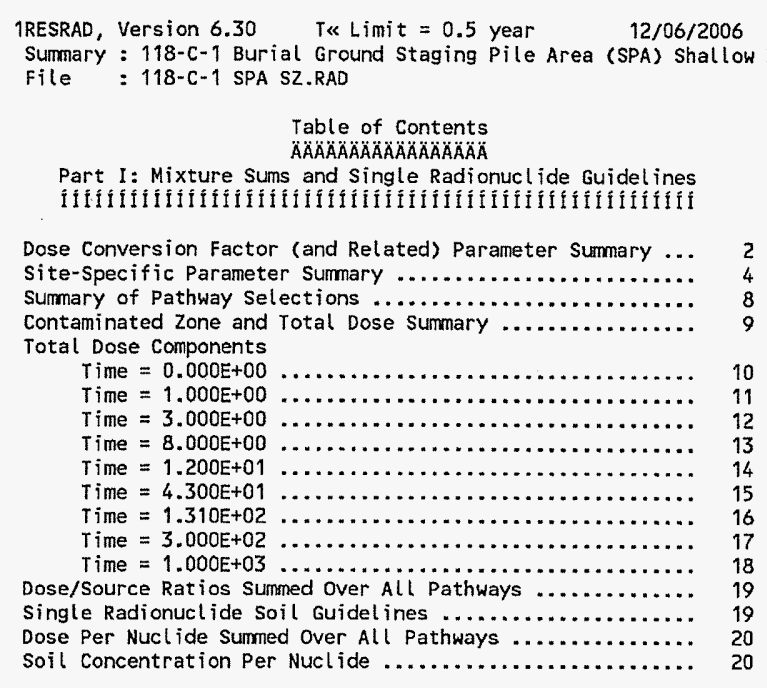

\begin{tabular}{|c|c|c|}
\hline \multirow{2}{*}{\multicolumn{2}{|c|}{$\begin{array}{l}\text { Attachment } \frac{21}{\text { Originators: S.W. Clark Rue }} \\
\text { S. We }\end{array}$}} & \multirow{2}{*}{ Sheet No. 1 of 20} \\
\hline & S.W. Clark Reuse & \\
\hline & M. W. Perrott 7htic & Date $12 / 13 / a t$ \\
\hline & $0100 \mathrm{C}-\mathrm{CA}-\mathrm{V} 0035$ & No. \\
\hline
\end{tabular}




\section{ATTACHMENT 21}

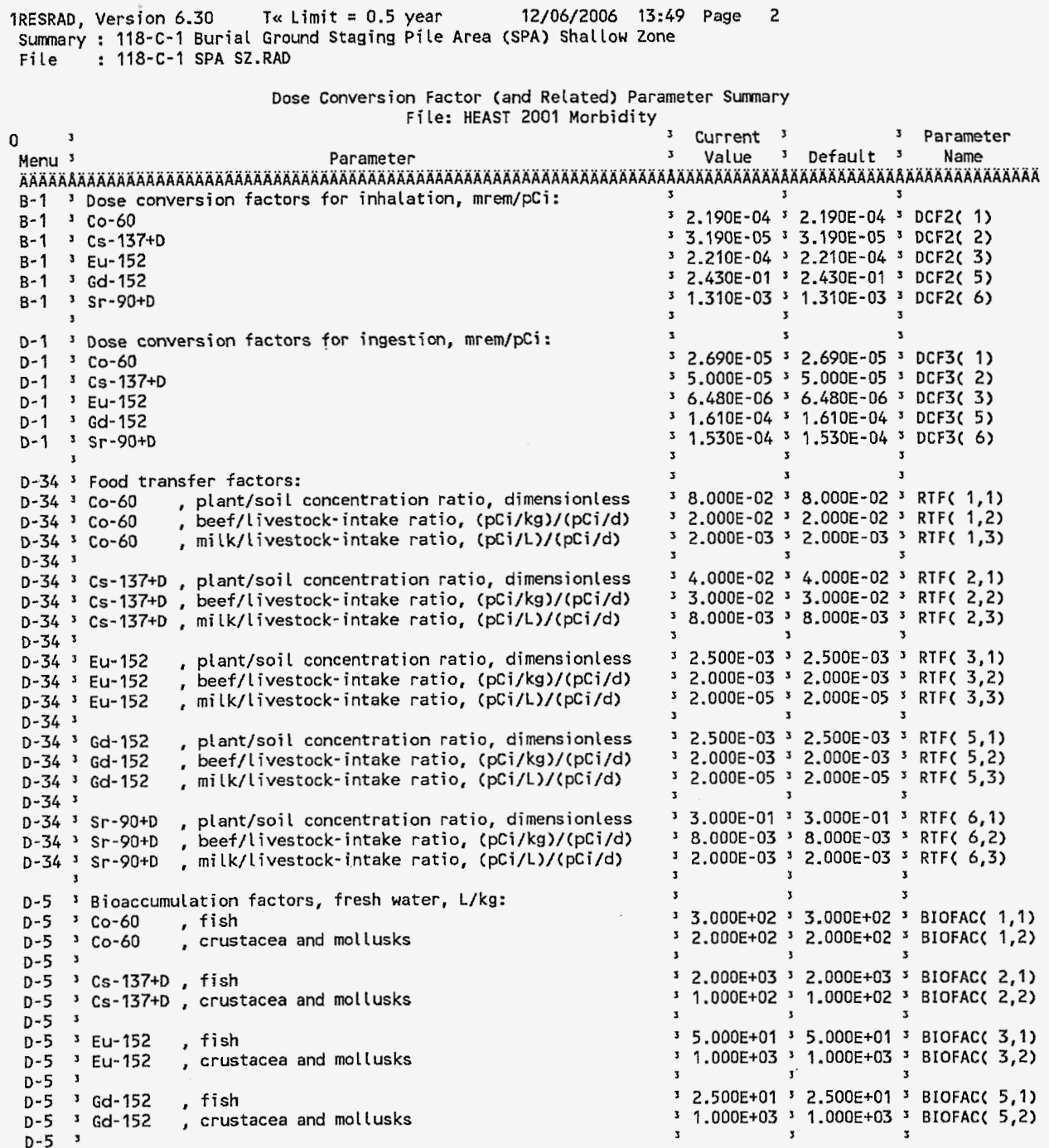




\section{ATTACHMENT 21}

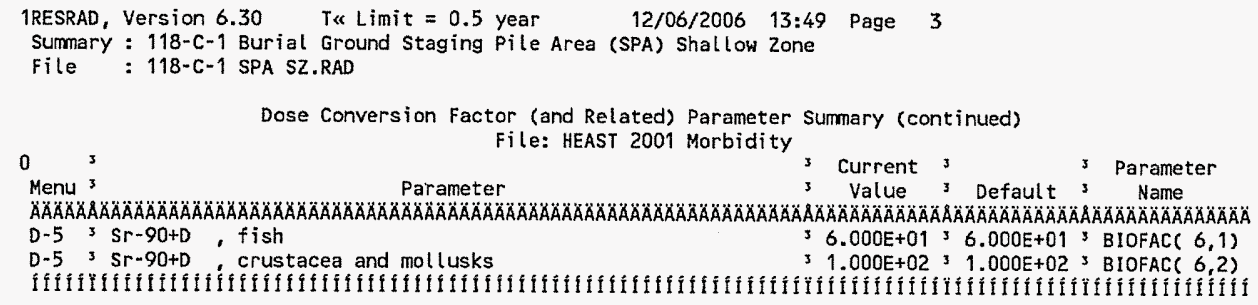




\section{ATTACHMENT 21}

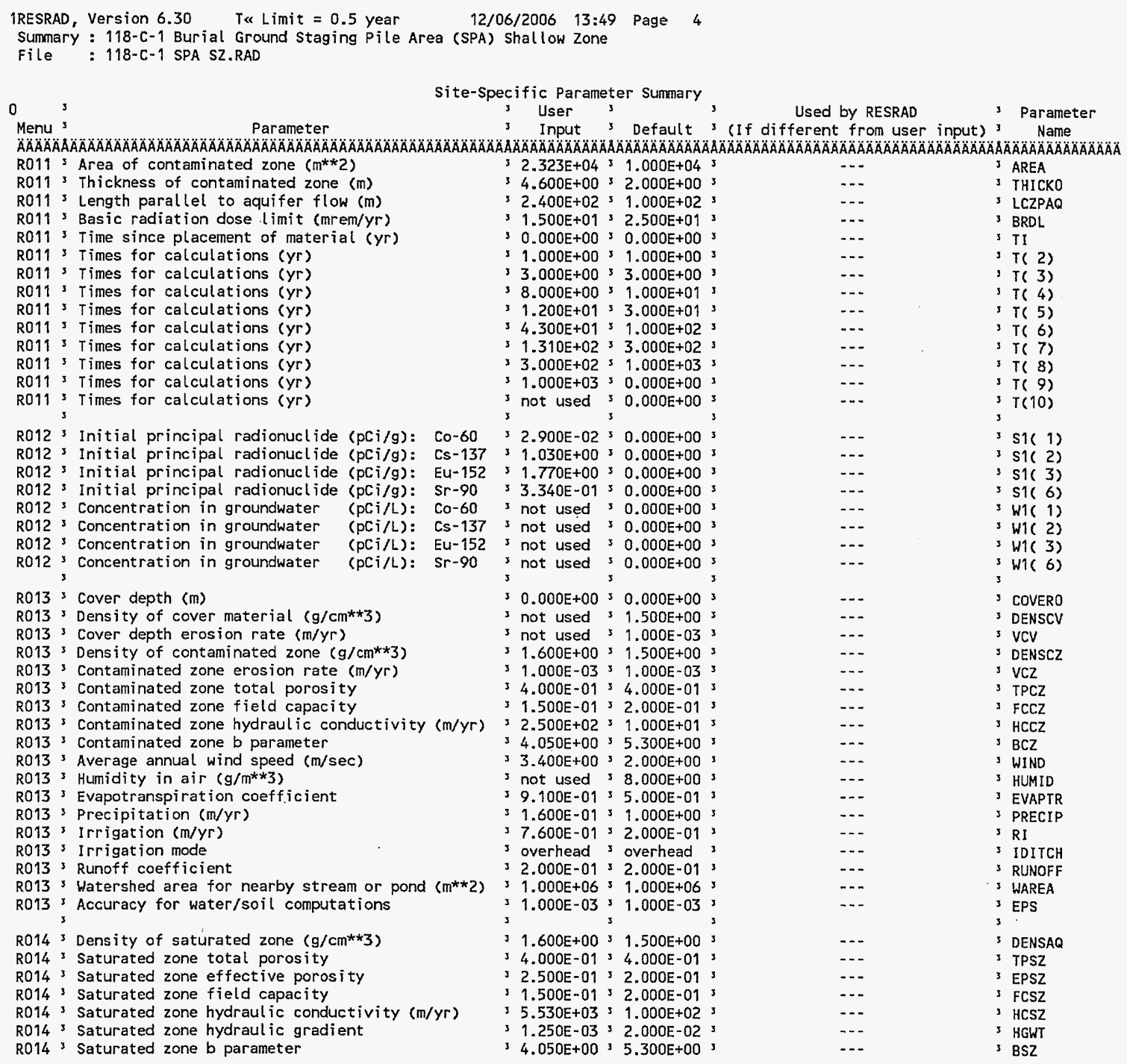

\begin{tabular}{|c|c|}
\hline \multirow{2}{*}{\multicolumn{2}{|c|}{ 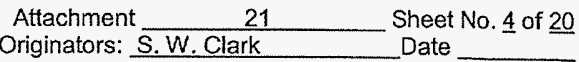 }} \\
\hline & \\
\hline Chk'd By M.W. Perrott & Date \\
\hline $0100 \mathrm{C}-\mathrm{CA}-\mathrm{V} 0035$ & Rev. No. 0 \\
\hline
\end{tabular}




\section{ATTACHMENT 21}

1RESRAD, Version 6.30 T«Limit $=0.5$ year $12 / 06 / 2006 \quad 13: 49$ Page 5 Surmary : 118-C-1 Burial Ground Staging Pile Area (SPA) Shallow Zone file : 118-C-1 SPA SZ.RAD

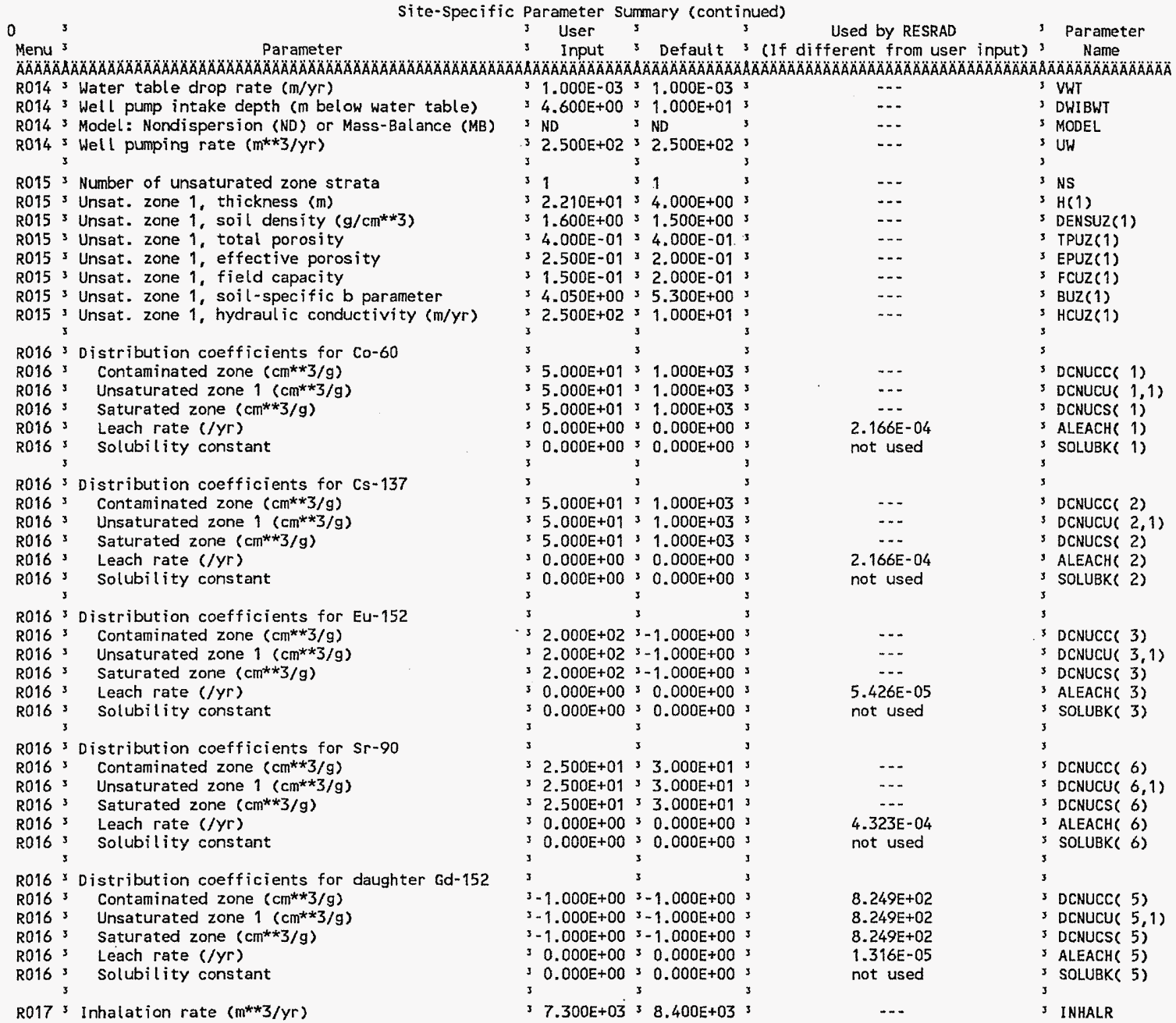




\section{ATTACHMENT 21} 1RESRAD, Version $6.30 \quad$ T\& Limit $=0.5$ year $\quad 12 / 06 / 2006 \quad 13: 49$ Page $\quad 7$
Summary : $118-c-1$ Burial Ground Staging Pile Area (SPA) Shallow Zone Fite : 118-C-1 SPA SZ.RAD

0 Site-Specific Parameter Summary (continued)

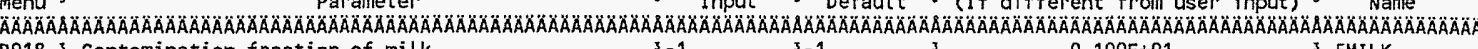
R018 3 Contamination fraction of milk 3

R019' Livestock fodder intake for meat ( $\mathrm{kg} /$ day) R019' Livestock fodder intake for $m i l k(\mathrm{~kg} /$ day) RO19 3 Livestock water intake for meat (L/day) RO19, Livestock water intake for milk (L/day) R019 3 Livestock soil intake ( $\mathrm{kg} /$ day) R019 3 Mass loading for foliar deposition $\left(\mathrm{g} / \mathrm{m}^{* * 3}\right)$ R019 S Depth of soil mixing layer $(\mathrm{m})$ R019' Depth of roots $(\mathrm{m})$

R019 3 Drinking water fraction from ground water R019' Household water fraction from ground water R019 3 Livestock water fraction from ground water R019' Irrigation fraction from ground water

$\begin{array}{lllll}3-1 & 3-1 & 3 & 0.100 E+01 & 3 \\ 3 & 3 & 3 & 3 & 3\end{array}$

$6.800 E+01^{3} 6.800 E+01^{3}$ $5.500 E+0135.500 E+01$
$5.000 E+0135$ $35.000 E+01>5.000 E+01$ ?

$31.600 \mathrm{E}+0231.600 \mathrm{E}+023$

$35.000 E-01$ s $5.0005-013$

5.000E-01 3 S.000E-01

$1.000 E-04$ 3 $1.000 E-04$

$1.500 \mathrm{E}-0131.500 \mathrm{E}-01$
$3.000 \mathrm{~s}-013$
3

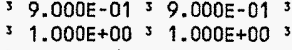

not used $31.000 E+00$

$1.000 E+00$ \& $1.000 E+00$

$31.000 \mathrm{E}+00 \div 1.000 \mathrm{E}+00$

198 Wet weight crop yield for Non-Leafy $\left(\mathrm{kg} / \mathrm{m}^{* *} 2\right)$

$R 19 B$, Wet weight crop yield for Leafy $\left(\mathrm{kg} / \mathrm{m}^{* * 2}\right)$

R19B Wet weight crop yield for Fodder $\left(\mathrm{kg} / \mathrm{m}^{* *} 2\right)$

R19B 3 Growing Season for Non-Leafy (years)

R19B " Growing Season for Leafy (years)

R198 3 Growing Season for Fodder (years)

R19B 3 Translocation Factor for Non-Leafy

R19B S Translocation Factor for Leafy

$7.000 E-01 ; 7.000 E-01$

$1.500 E+0031.500 E+00$

$1.100 E+00=1.100 E+00$

$1.700 E-01 ; 1.700 E-01$,

$32.500 E-0132.500 E-013$

$38.000 E-02 \div 8.000 E-02$

$1.000 E-01>1.000 E-01$

$1.000 E+00=1.000 E+00$

$31.000 E+00^{3} 1.000 E+00$

R19B 3 Dry Foliar Interception Fraction for Non-Leafy $32.500 E-0132.500 E-01$

R19B 3 Dry Foliar interception Fraction for Leafy $32.500 E-0132.500 E-01$

R19B 3 Dry Foliar Interception Fraction for Fodder $32.500 E-0132.500 E-013$

R19B s Wet Foliar Interception Fraction for Non-Leafy s $2.500 E-01$

R19B 3 Wet Foliar Interception Fraction for Leafy $32.500 E-0132.500 E-01$.

R19B s Wet Foliar Interception Fraction for Fodder $32.500 \mathrm{E}-0132.500 \mathrm{E}-01$

R19B 3 Weathering Renoval Constant for Vegetation

3

C14 $\mathrm{C}-12$ concentration in water $(\mathrm{g} / \mathrm{cm} * * 3)$

C14 $3 \mathrm{C}-12$ concentration in contaminated soil $(\mathrm{g} / \mathrm{g})$

C14 3 Fraction of vegetation carbon from soil

C14 3 Fraction of vegetation carbon from air

c14 $\mathrm{c}-14$ evasion layer thickness in soil $(\mathrm{m})$

C14, $\mathrm{c}-14$ evasion flux rate from soil $(1 / \mathrm{sec})$

c14 $3-12$ evasion flux rate from soil $(1 / \mathrm{sec})$

C14 3 Fraction of grain in beef cattle feed

$\begin{array}{lll}2.500 E-01 & 3 & 2.500 E-01 \\ 2.000 E+01 & 3 & 2.000 E+01\end{array}$

3 not used $2.000 E-05$

not used $33,000 E-02$

not used $32.000 \mathrm{E}^{-02}$

3 not used $3.8 .8005-01$

not used $3.0000^{3}$

not used 3.000 E 01

not used 7.000 - 07

not used $1.000 E-10$

not used $8.000 E-01$

3

not used $8.894 E+01$

STOR Storage times of contaminated foodstuffs (days):

STOR 3 Fruits, non-leafy vegetables, and grain

STOR 3 Leafy vegetables

STOR L Leafy

STOR $=$ Meat and poultry

STOR 3 Fish

STOR $^{3}$ Crustacea and mollusks

$: 3$

$31.400 E+01+1.400 E+01$

$31.000 E+00>1.000 E+00$

$31.000 \mathrm{E}+0031.000 \mathrm{E}+00$

$32.000 E+01,2.000 E+01$

$7.000 E+00>7.000 E+00$

$7.000 \mathrm{E}+0037.000 \mathrm{E}+00$

\begin{tabular}{|c|c|c|}
\hline$\cdots$ & 3 & LFI5 \\
\hline$\cdots$ & 3 & LFI6 \\
\hline$\cdots$ & 3 & LWI5 \\
\hline$\cdots$ & 3 & LWI6 \\
\hline$\cdots$ & 3 & LSI \\
\hline$\cdots$ & 3 & MLFD \\
\hline$\cdots$ & 3 & DM \\
\hline$\cdots$ & 3 & DROOT \\
\hline$\cdots$ & 3 & FGWDW \\
\hline$\cdots$ & 3 & FGWHH \\
\hline$\cdots$ & s & FGWLW \\
\hline$\cdots$ & $\begin{array}{l}3 \\
3\end{array}$ & FGWIR \\
\hline- & s & $Y V(1)$ \\
\hline$\cdots$ & 3 & $Y V(2)$ \\
\hline$\cdots$ & s & $Y V(3)$ \\
\hline$\cdots$ & 3 & $T E(1)$ \\
\hline$\cdots$ & 3 & $T E(2)$ \\
\hline$\ldots$ & 3 & $T E(3)$ \\
\hline- & 3 & $\operatorname{rIV}(1)$ \\
\hline$\cdots$ & 3 & TIV(2) \\
\hline$\cdots$ & 3 & $\operatorname{TIV}(3)$ \\
\hline & 3 & $\operatorname{RDRY}(1)$ \\
\hline$\cdots$ & 3 & RDRY (2) \\
\hline$\cdots$ & s & RDRY (3) \\
\hline & 3 & RWET (1) \\
\hline$\cdots$ & 3 & RWET (2) \\
\hline$\cdots$ & 3 & RWET (3) \\
\hline$\cdots$ & 3 & WLAM \\
\hline & 3 & \\
\hline$\cdots$ & 3 & C12WTR \\
\hline & s & $\mathrm{C12C2}$ \\
\hline$\cdots$ & 3 & CSOIL \\
\hline$\cdots$ & 3 & CAIR \\
\hline$\cdots$ & 3 & DMC \\
\hline$\cdot$ & 3 & EVSN \\
\hline- & s & REVSN \\
\hline$\cdots$ & 3 & AVFG4 \\
\hline$\cdots$ & 3 & AVFG5 \\
\hline$\cdots$ & 3 & $\mathrm{CO} 2 \mathrm{~F}$ \\
\hline & $s$ & \\
\hline & 3 & \\
\hline- & 3 & STOR_T(1) \\
\hline & 3 & STOR_T(2) \\
\hline -. & s & STOR_T (3) \\
\hline & 3 & STOR_T(4) \\
\hline 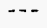 & 3 & STOR_T(5) \\
\hline & $\mathrm{s}$ & STOR_T( 6 ) \\
\hline
\end{tabular}

Attachment 21

tors: S. W. Clark

Chk'd By

M. W. Perrott

$100 \mathrm{C}-\mathrm{CA}-\mathrm{V} 0035$
Sheet No. 7 of 20

Date

Date

Rev. No. 0 


\section{ATTACHMENT 21}

1RESRAD, Version 6.30 T $\quad$ Limit $=0.5$ year $\quad 12 / 06 / 2006 \quad 13: 49$ Page 8 Sumary : 118-C-1 Burial Ground Staging Pile Area (SPA) Shallow Zone File : 118-C-1 SPA SZ.RAD

0 s

Site-Specific Parameter Sumary (cont inued)

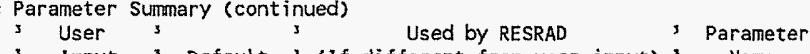

Menu s Parameter ${ }^{3}$ Input ${ }^{3}$ Default ${ }^{3}$ (1f different from user input) 3 Name

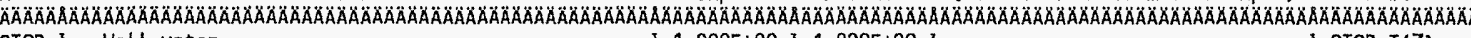
STOR ${ }^{3}$ Well water

STOR 3 Surface water

STOR 3 Livestock fodder

R021 3 Thickness of building foundation $(\mathrm{m})$

R021 3 Bulk density of building foundation $\left(\mathrm{g} / \mathrm{cm}^{* *} 3\right)$

RO21 Total porosity of the cover material

R021 Total porosity of the cover material

$\mathrm{RO21}$ s Volumetric water content of the cover material

R021 Volumetric water content of the foundation

R021 3 Diffusion coefficient for radon gas $(\mathrm{m} / \mathrm{sec})$ :

R021's in cover material

R021 3 in foundation material

$\mathrm{RO21} 3$ in contaminated zone soil

021 s Radon vertical dimension of mixing (m)

$31.000 E+00^{3} 1.000 \mathrm{E}+00^{3}$

$31.000 E+00^{3} 1.000 E+003$

; $4.500 \mathrm{E}+01: 4.500 \mathrm{E}+013$

3 not used $31.500 \mathrm{E}-01$ s

s not used $32.400 \mathrm{E}+00$,

3 not used 3 4.000E-01?

3 not used 1 1.000E-01,

not used $35.000 \mathrm{E}-02$

3 not used $3.000 \mathrm{E}-02$;

not used 3.000 E-06

not used $3.000 E-07$

3 not used $32.000 E-06$

3 not used $32.000 E+00$ s

not used s $5.0000^{-01}$

not. used s s.000E-01

not used 2.500 t +00

not used $0.000 E+00$

not used $3-1.000 E+00$

3 not used 32.500 E- 0 ?

RO21 Building interior area factor

RD21 Building depth below ground surface (m)

$\mathrm{R} 0213$ Emanating power of $\mathrm{Rn}-222$ gas

3 not used 3 1.500E-01

s

TIIL 3 Number of graphical time points

TITL 3 Maximum number of integration points for dose

$\begin{array}{rrrr}32 & 3 & \cdots & 3 \\ 1 & 3 & \cdots & 3\end{array}$

TITL 3 Maximum number of integration points for risk

53 - 3

\begin{tabular}{|c|c|}
\hline$\cdots$ & STOR T(7) \\
\hline$\cdots$ & ${ }^{3}$ STOR_T $_{-}^{-T}(8)$ \\
\hline$\cdots$ & , STOR_T(9) \\
\hline$\cdots$ & FLOOR 1 \\
\hline- & 3 DENSFL \\
\hline & ' TPCV \\
\hline & ${ }^{3}$ TPFL \\
\hline$\cdots$ & $3 \mathrm{PH} 2 \mathrm{OCV}$ \\
\hline- & s PH2OFL \\
\hline- & ' DIFCV \\
\hline & DIFFL \\
\hline & 'DIFCZ \\
\hline- & ${ }^{3}$ HMIX \\
\hline & 'REXG \\
\hline & $=\mathrm{HRM}$ \\
\hline & 3 FAI \\
\hline & 3 DMFL \\
\hline & 3 EMANA(1) \\
\hline & ${ }_{3}^{5}$ EMANA(2) \\
\hline & N NPTS \\
\hline & s LYMAX \\
\hline & ${ }^{3}$ KYMAX \\
\hline
\end{tabular}

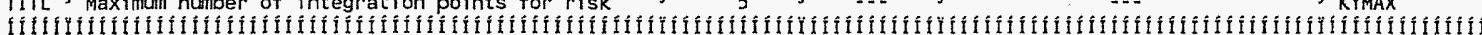

Summary of Pathway Selections

$$
\text { Pathway }
$$

3 User Selection

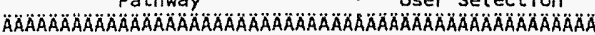

1 - external gamma active

3 .- plant ingestion

4 - plant ingestion

4 -

5 .- milk ingestion

6 .. aquat ic foods

7 -. drinking water

8 ... soil ingestion

9 -. radon

ilifilifififfilitififilifilitifiliti

$\begin{array}{ll}\text { Attachment } \frac{21}{\text { O. W. Clark }} & \text { Sheet No. } \underline{8} \text { of } \underline{20} \\ \text { Originators: } \frac{\text { Sate }}{\text { M.W. Perrott }} & \text { Date } \\ \text { Chk'd By } & \text { Rev. No. } 0 \\ \text { Calc. No. } 0100 \text { C-CA-V0035 } & \text { Re }\end{array}$




\section{ATTACHMENT 21}

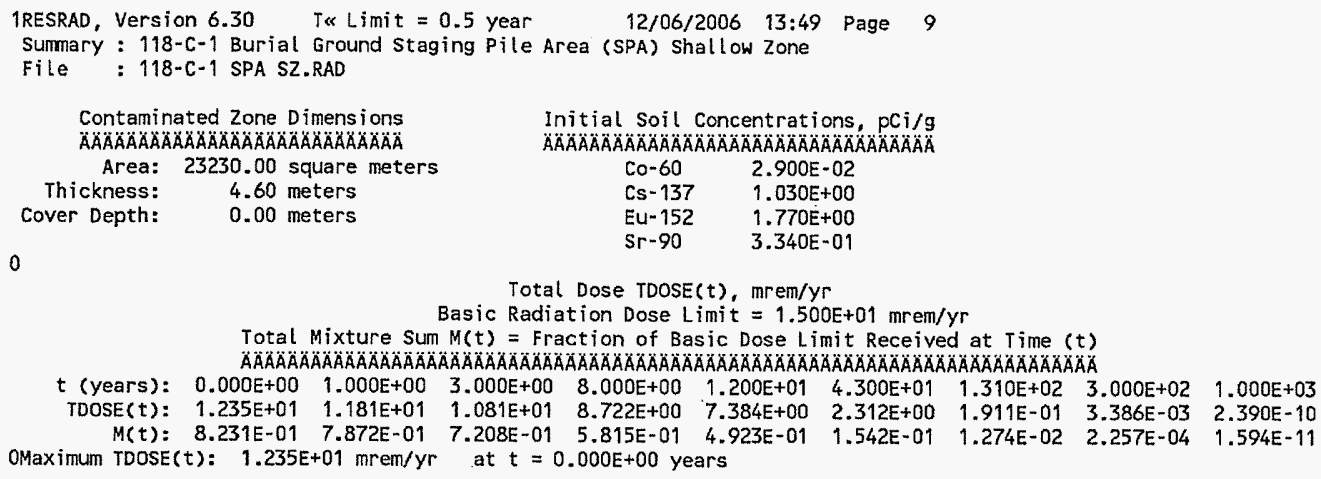

0

Total Dose TDOSE( $t), \mathrm{mrem} / \mathrm{yr}$

Basic Radiation Dose Limit $=1.500 \mathrm{E}+01 \mathrm{mrem} / \mathrm{yr}$

Total Mixture Sum $M(t)=$ Fraction of Basic Dose Limit Received at Time $(t)$

$t$ (years): $\begin{array}{llllllllll}0.000 E+00 & 1.000 E+00 & 3.000 E+00 & 8.000 E+00 & 1.200 E+01 & 4.300 E+01 & 1.310 E+02 & 3.000 E+02 & 1.000 E+03\end{array}$

$\operatorname{rDOSE}(t): \begin{array}{lllllllll}1.235 E+01 & 1.181 \mathrm{E}+01 & 1.081 \mathrm{E}+01 & 8.722 \mathrm{E}+00 & 7.384 \mathrm{E}+00 & 2.312 \mathrm{E}+00 & 1.911 \mathrm{E}-01 & 3.386 \mathrm{E}-03 & 2.390 \mathrm{E}-10\end{array}$

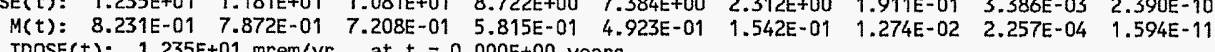
OMaximum TDOSE $(t): \quad 1.235 E+01 \mathrm{mrem} / \mathrm{yr}$ at $t=0.000 E+00$ years

\begin{tabular}{|c|c|}
\hline Attachment & Sheet No. 9 of 20 \\
\hline Originators: S. W. Clark & Date \\
\hline M. W. Perrott & Date \\
\hline $0100 \mathrm{C}-\mathrm{CA}-\mathrm{V} 0035$ & Rev. No. 0 \\
\hline
\end{tabular}




\title{
ATTACHMENT 24
}

\author{
1RESRAD, Version 6.30 T\& Limit $=0.5$ year $12 / 06 / 2006 \quad 13: 53$ Page 1 \\ Summary : 118-C-1 Burial Ground Staging Pile Area (SPA) Deep Zone \\ File : 118-C-1 SPA DZ.RAD \\ Table of Contents \\ ÄÄÄÄÄA ÄÄÄÄÄÄA \\ Part I: Mixture Sums and Single Radionucl ide Guidel ines

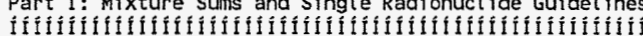 \\ Dose Conversion Factor (and Related) Parameter Summary ...

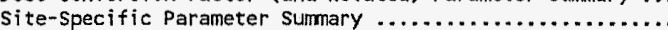

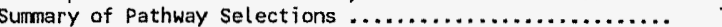 \\ Contaminated Zone and Total Dose Summary ............... 8 \\ Total Dose Components

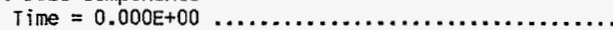 \\ Time $=3.000 \mathrm{E}+00$ \\ Time $=8.000 E+00$ \\ Time $=4.300 \mathrm{E}+01$ \\ Time $=3.000 E+02$ \\ Time $=1.000 E+03$

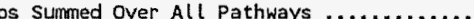 \\ ingle Radionucl ide Soil Guidel ines

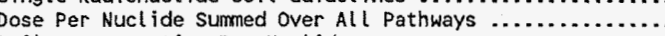

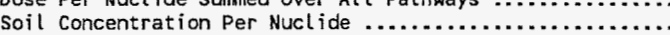




\section{ATTACHMENT 24}

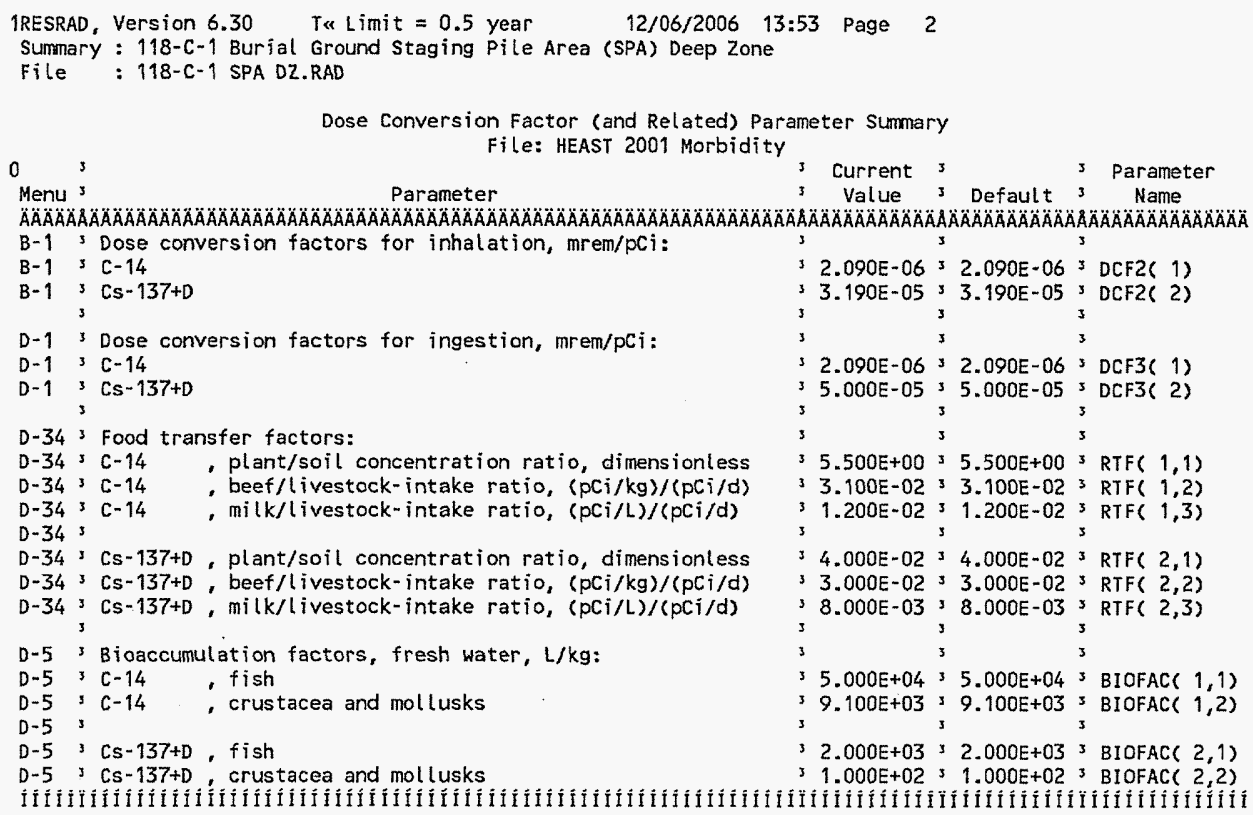

\begin{tabular}{|c|c|}
\hline \multirow{2}{*}{\multicolumn{2}{|c|}{ Attachment $\quad \frac{24}{\text { Oriainators: }}$}} \\
\hline & Date \\
\hline M. W. Perrott & Date \\
\hline $0100 C-C A-V 0035$ & Rev. No. \\
\hline
\end{tabular}




\section{ATTACHMENT 24}

TRESRAD, Version $6.30 \quad$ T\& Limit $=0.5$ year $12 / 06 / 2006 \quad 13: 53$ Page 3 Summary : 118-C-1 Burial Ground Staging Pile Area (SPA) Deep Zone

File: 118-C-1 SPA DZ.RAD

0

Site-Specific Parameter Summary

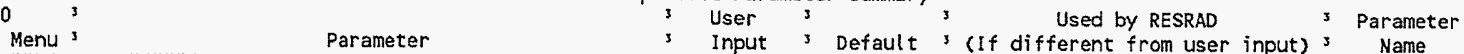

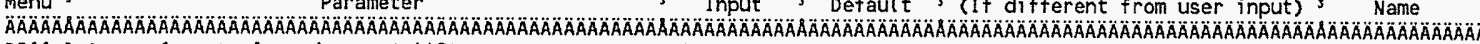
RO11 3 Area of contaminated zone $\left(m^{* * 2}\right)$

R011 Thickness of contaminated zone $(\mathrm{m})$

RO11 ${ }^{3}$ Length parallel to aquifer flow $(\mathrm{m})$

R011' Basic radiation dose limit (mrem/yr)

R011 Time since placement of material $(\mathrm{yr})$

$2.323 E+0431.000 E+04$

$2.210 E+0132.000 E+00$

$32.400 E+02+1.000 E+02$,

$31.500 E+0132.500 E+01$ s

$30.000 \mathrm{E}+0030.000 \mathrm{E}+00$

$31.000 E+0031.000 E+00$

3.000200 S $1.000 E+00$

$3.000 E+00,3.000 E+00$

DOE+01

$31.200 \mathrm{E}+0133.000 \mathrm{E}+01$ s

$34.300 E+0131.000 E+02$

$31.310 E+02 ? 3.000 E+02$

$3.000 E+0231.000 E+03$

$31.000 E+03=0.000 E+00$

not used $0.000 \mathrm{E}+00$

R011'3 Times for calculations (yr)
R011'3 Times for calculations (yr)

R011' Times for calculations $(\gamma r)$

3

R012 Initial principal radionuclide $(\mathrm{pCi} / \mathrm{g}): \mathrm{C}-14$, $1.820 \mathrm{E}+02,0.000 \mathrm{E}+00$

R012 I Initial principal radionuclide (pCi/g): $\mathrm{Cs}-137,9.100 \mathrm{E}-02$, $0.000 \mathrm{E}+00$

R012 3 Concentration in groundwater (pCi/L): $C-14$ s not used $30.000 E+00$

R012 Concentration in groundwater (pCi/L): Cs-137, not used s $0.000 E+00$

R013' Cover depth (m)

R013 Density of cover material $\left(\mathrm{g} / \mathrm{cm}^{* *} 3\right)$

R013 ' Cover depth erosion rate $(\mathrm{m} / \mathrm{yr})$

$R 013$ S Density of contaminated zone $\left(\mathrm{g} / \mathrm{cm}^{\star *} 3\right)$

$R 013$, Contaminated zone erosion rate $(\mathrm{m} / \mathrm{yr})$

R013 Contaminated zone total porosity

R013 Contaminated zone field capacity

R013 Average annual wind speed ( $\mathrm{m} / \mathrm{sec})$

R013 Humidity in air $\left(\mathrm{g} / \mathrm{m}^{* * 3}\right)$

R013 3 Evapotranspiration coefficient

R013 Precipitation $(\mathrm{m} / \mathrm{yr})$

R013' Irrigation (m/yr)

R013 3 Irrigation mode

R013 3 Runoff coefficient

I $4.600 E+0030.000 E+00$

$1.600 \mathrm{E}+0031.500 \mathrm{E}+00$

$1.000 E-03^{3} 1.000 E-03$

$31.600 \mathrm{E}+0031.500 \mathrm{E}+00$

$2.500 \mathrm{E}+02,4.050 \mathrm{E}+00 ; 5.300 \mathrm{E}+00$
$3.400 \mathrm{E}+00 ; 2.000 \mathrm{E}+00$

not used $8.000 E+00$

$1.600 \mathrm{C}, 1.000 \mathrm{C}-0 \mathrm{~T}$

$7.600 E-01,1.000 E+00$

$7.600 E-01$ 2.000E-01

overhead 'overhead

2.000E-01 $+2.000 \mathrm{E}-01$

$\mathrm{R} 013$ ' Watershed area for nearby stream or pond $\left(\mathrm{m}^{* * 2}\right), 1.000 \mathrm{E}+06,1.000 \mathrm{E}+06$

R013 Accuracy for water/soil computations

$1.000 \mathrm{E}-03 ; 1.000 \mathrm{E}-03$ ?

s $1.600 \mathrm{E}+00,1.500 \mathrm{E}+00$

R014 Density of saturated zone $\left(\mathrm{g} / \mathrm{cm}^{* * 3}\right)$

R014' Saturated zone total porosity

$4.000 E-01 ; 4.000 E-01$

$3.500 \mathrm{E}-013 \mathrm{~S}, 000 \mathrm{C}-01$

2.500E-01 2.000E-01

$1.500 \mathrm{E}-0132.000 \mathrm{E}-01$

$5.530 E+03>1.000 E+02$

$1.250 E-03$ ? $2.000 E-02$ ?

$=4.050 \mathrm{E}+00$ > $5.300 \mathrm{E}+00$

$1.000 \mathrm{E}-03 ; 1.000 \mathrm{E}-03$

R014'3 Saturated zone hydraulic gradient

R014 Saturated zone b parameter

R014 3 Water table drop rate (m/yr)

$34.600 E+00 ; 1.000 E+013$

$32.500 E+0232.500 E+02$ s

$\begin{array}{ll}\ldots . . & \text { 3 AREA } \\ \cdots & \text {, THICKO }\end{array}$

-.. $\quad$ LCZPAO

-.. 3 BRDL

$3 \mathrm{TI}$

... 3 T $T(2)$

$-\cdot$

3 T( 4)

$\ldots-3(5)$

-..

$3 \mathrm{~T}(8)$

3 T( 9$)$

$+\infty$

ज $\operatorname{W1}(1)$

3 Wi( 2)

-.. 3 COVERO

S DENSCY

-.. 3 VCV

-.. 3 DENSCZ

-.. 3 VCZ

-.

$\cdots$

3 HCCZ

$3 \mathrm{BCZ}$

- WIND

S HUMID

-.. 3 EVAPTR

-.. 3 PRECIP

... 3 RI

3 IDITCH

3 RUNOFF

3 WAREA

... 3 EPS

... 3 DENSAQ

...

... 3 EPSZ

a..

...

3 HGWT

$\checkmark$ BSZ

3 VWT

..- $\quad 3$ DWIBWT

... 3 MODEL

3 UW

R014 Well pumping rate $\left(m^{* *} 3 / y r\right)$

\begin{tabular}{|c|c|}
\hline Attachment & Sheet No. $\underline{3}$ of 19 \\
\hline Originators: S.W. Clark & Date \\
\hline M. W. Perrott & Date \\
\hline $0100 C-C A-V 0035$ & Rev. No. \\
\hline
\end{tabular}




\section{ATTACHMENT 24}

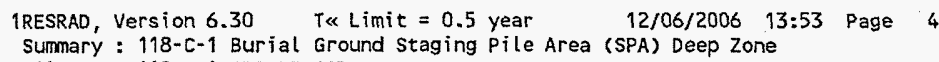

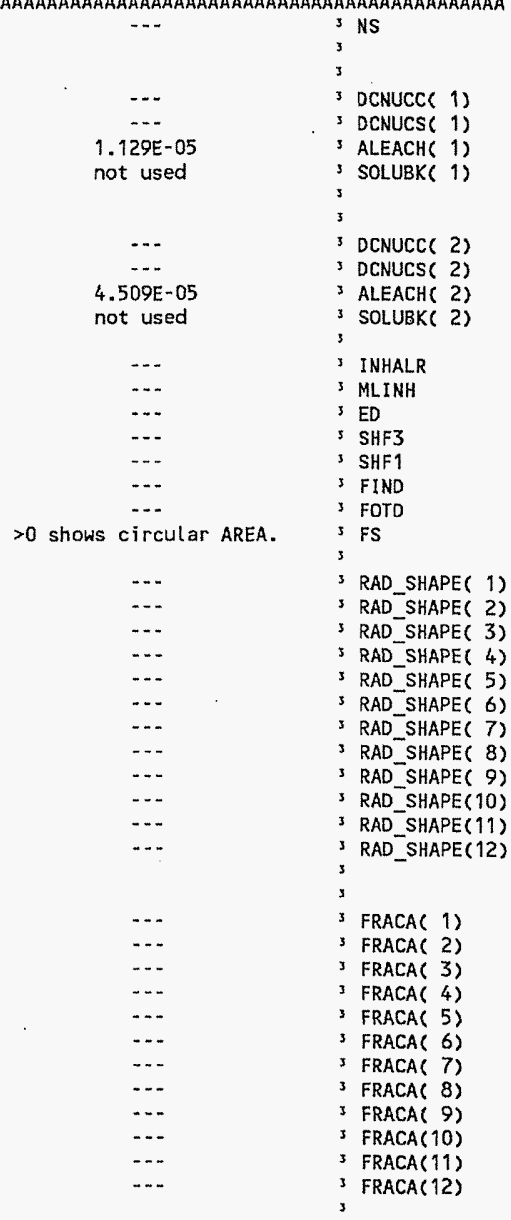

Attachment 24 


\section{ATTACHMENT 24}

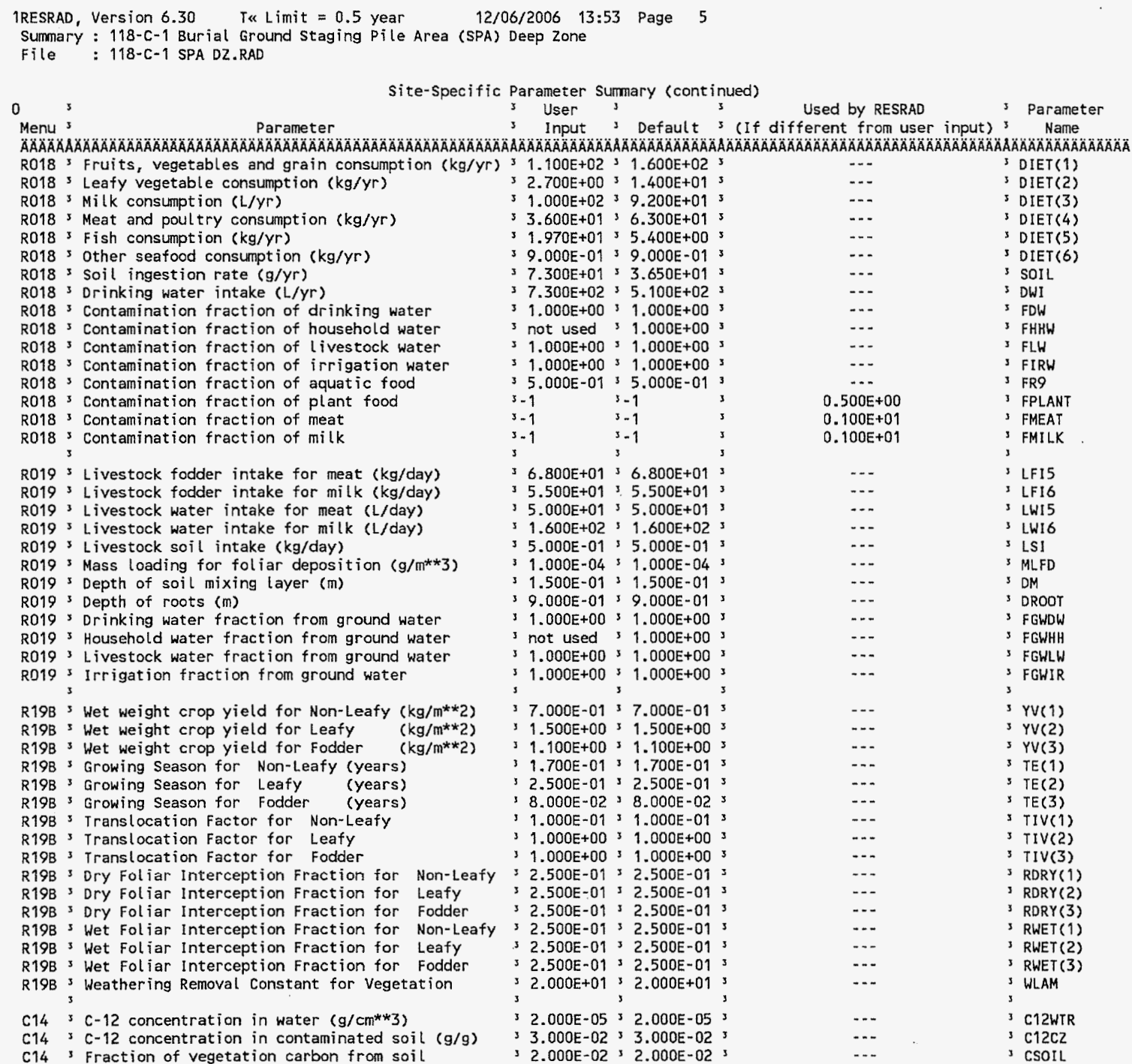

\begin{tabular}{|c|c|}
\hline Attachment & Sheet No. $\underline{5}$ of 19 \\
\hline Originators: S.W. Clark & Date \\
\hline Chk'd By M.W. Perrott & Date \\
\hline $0100 \mathrm{C}-\mathrm{CA}-\mathrm{V} 0035$ & Rev. No. 0 \\
\hline
\end{tabular}




\section{ATTACHMENT 24}

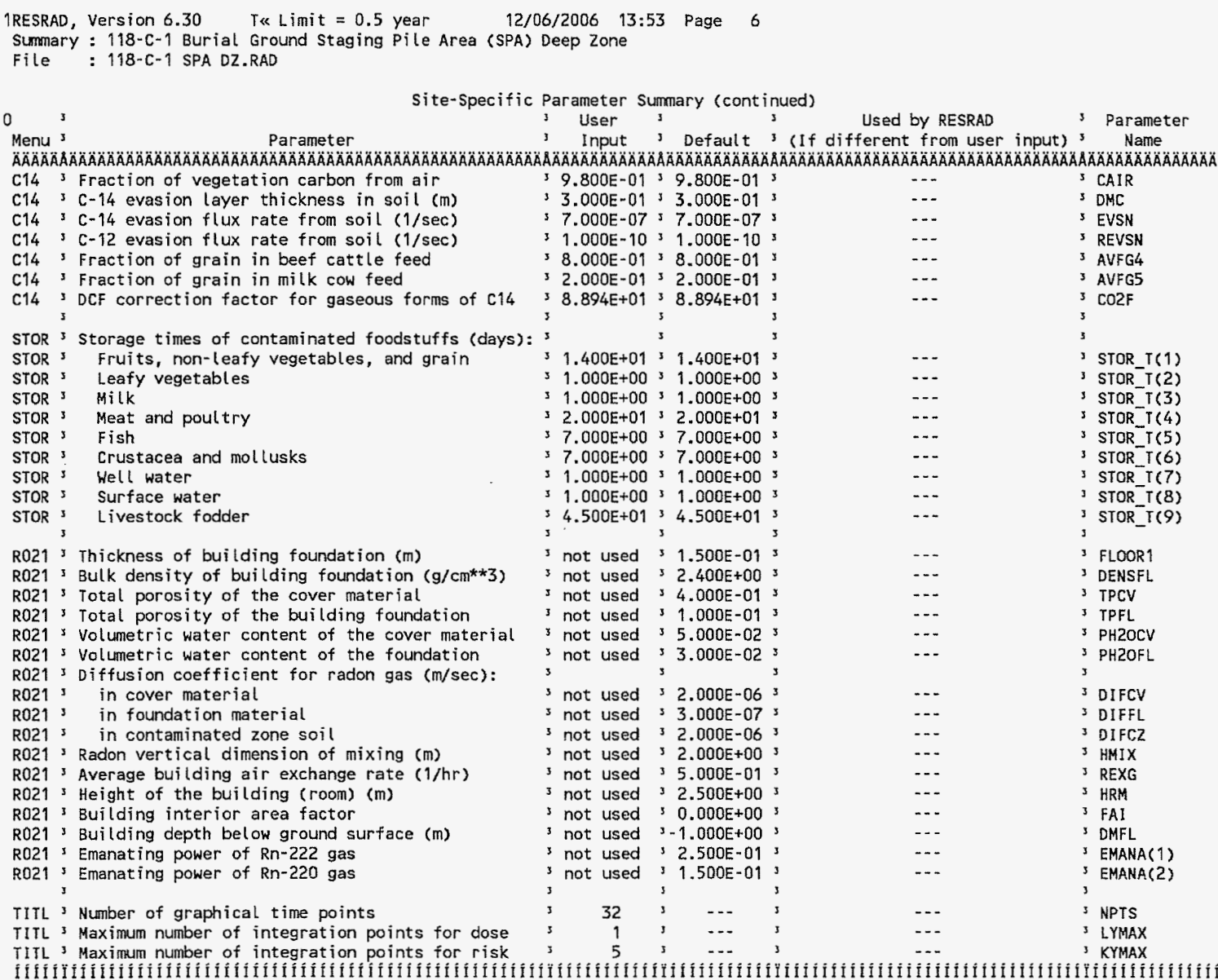

iffififílificiumer of integration points for risk

Attachment

24

Sheet No. $\underline{6}$ of 19

Originators: S.W. Clark

Date

Chk'd By M.W. Perrott

Date

Calc. No. 0100C-CA-V0035

Rev No 0


Rev. 0

ATTACHMENT 24

1RESRAD, Version $6.30 \quad$ T\& Limit $=0.5$ year $\quad 12 / 06 / 2006 \quad 13: 53$ Page 7 Summary : $118-C-1$ Burial Ground Staging Pile Area (SPA) Deep Zone

file : 118-C-1 SPA DZ.RAD

Summary of Pathway Selections

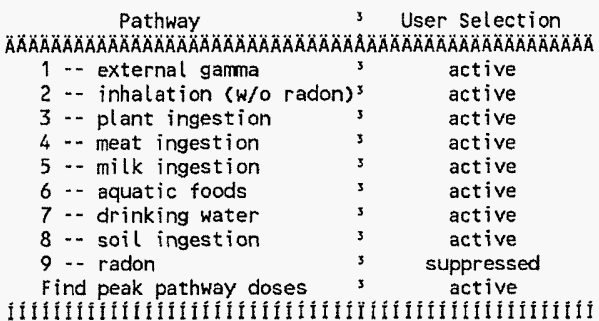

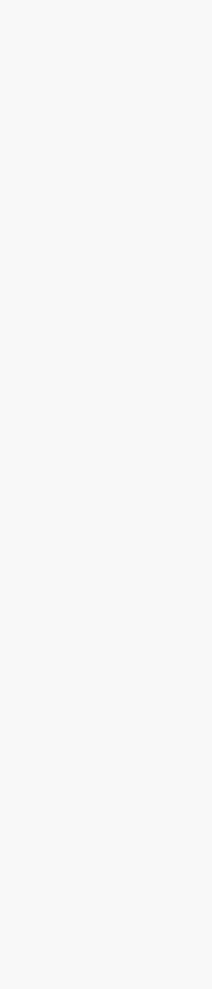

Attachment

Originators: S.W. Clark

24

Sheet No. 7 of 19

Chk'd By M. W. Perrott

Date

Calc. No. O100C-CA-V0035

Rev. No. $\mathrm{O}$ 


\section{ATTACHMENT 24}

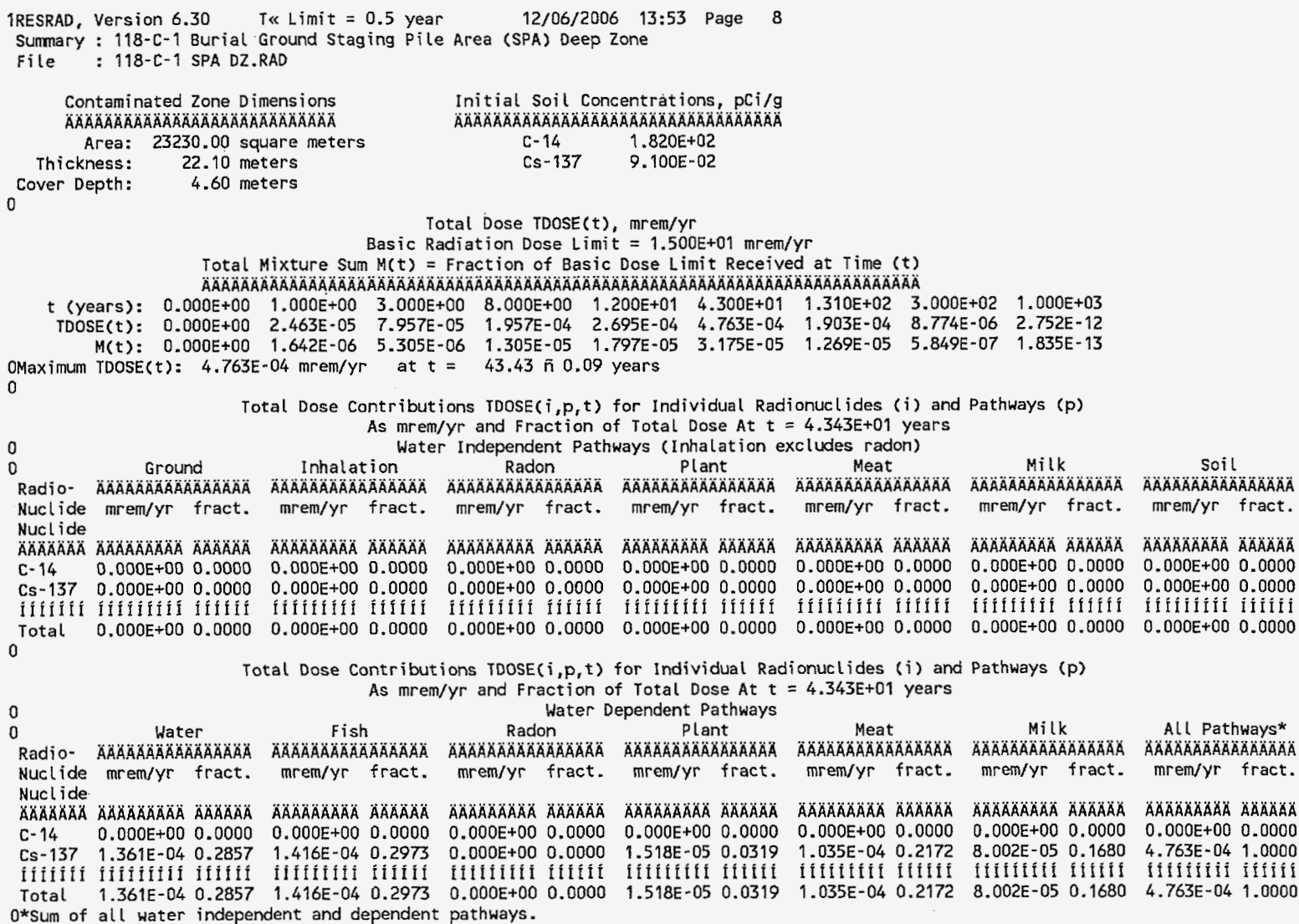

$\begin{array}{ll}\text { Attachment } \frac{24}{\text { S.W.Clark }} & \text { Sheet No. } 8 \text { of } 19 \\ \text { Originators: } & \text { Date } \\ \text { Chk'd By M.W. Perrott } & \text { Date } \\ \text { Calc. No. } \quad \text { O100C-CA-V0035 Rev. No. } 0\end{array}$


CVP-2006-00011

Rev. 0

CALCULATION BRIEF EXCERPTS 
CVP-2006-00011

Rev. 0

\section{DISCLAIMER FOR CALCULATIONS}

The calculations that are provided in the following appendix have been generated to document compliance with established cleanup levels. These calculations should be used in conjunction with other relevant documents in the administrative record. 


\section{CALCULATION BRIEFS}

The following calculation briefs have been prepared in accordance with ENG-1, Engineering Services,, ENG-1-4.5, "Project Calculations," Washington Closure Hanford, Richland, Washington.

118-C-1 Shallow Zone, Overburden and Staging Pile Sampling Plan, 0100B-CAV0288, Rev. 0, Washington Closure Hanford, Richland, Washington (2006).

118-C-1 Burial Ground Hazard Quotient and Carcinogenic Risk Calculations, 0100CCA-V0034, Rev. 0, Washington Closure Hanford, Richland, Washington (2006).

118-C-1 Burial Ground Cleanup Verification 95\% UCL Calculations, 0100C-CA-V0033, Rev. 0, Washington Closure Hanford, Richland, Washington (2006).

118-C-1 Burial Ground Comparison to Drinking Water Standards (MCL) Calculation, 0100C-CA-V0036, Rev. 1, Washington Closure Hanford, Richland, Washington (2006).

118-C-1 Burial Ground Cleanup Verification RESRAD Calculation Brief, 0100C-CAV0035, Rev. 0, Washington Closure Hanford, Richland, Washington.

NOTE: The calculation briefs referenced in this appendix are kept in the active Washington Closure Hanford project files and are available upon request. When the project is completed, the files will be stored in a U.S. Department of Energy, Richland Operations Office repository. Only excerpts of the calculation briefs are included in this appendix. 
CVP-2006-00011

Rev. 0

\section{E-85}


CVP-2006-00011

Rev. 0

\section{CALCULATION COVER SHEET}

Project Title:

Area

Discipline

Subject

Computer Program
118-C-1 Burial Ground Sample Design

Job No.

14655
Environmental Engineering *Calc. No. 0100B-CA-V0288

118-C-1 Shallow Zone, Overburden, and Staging Pile Sampling Plan

Excel Program No. Excel 2003

The attached calculations have been generated to document compliance with established cleanup levels. These calculations should be used in conjuction with other relevent documents in the administrative record.

\section{Committed Calculation $\quad x \quad$ Preliminary $\square \quad$ Superseded $\square \quad$ Voided $\square$}

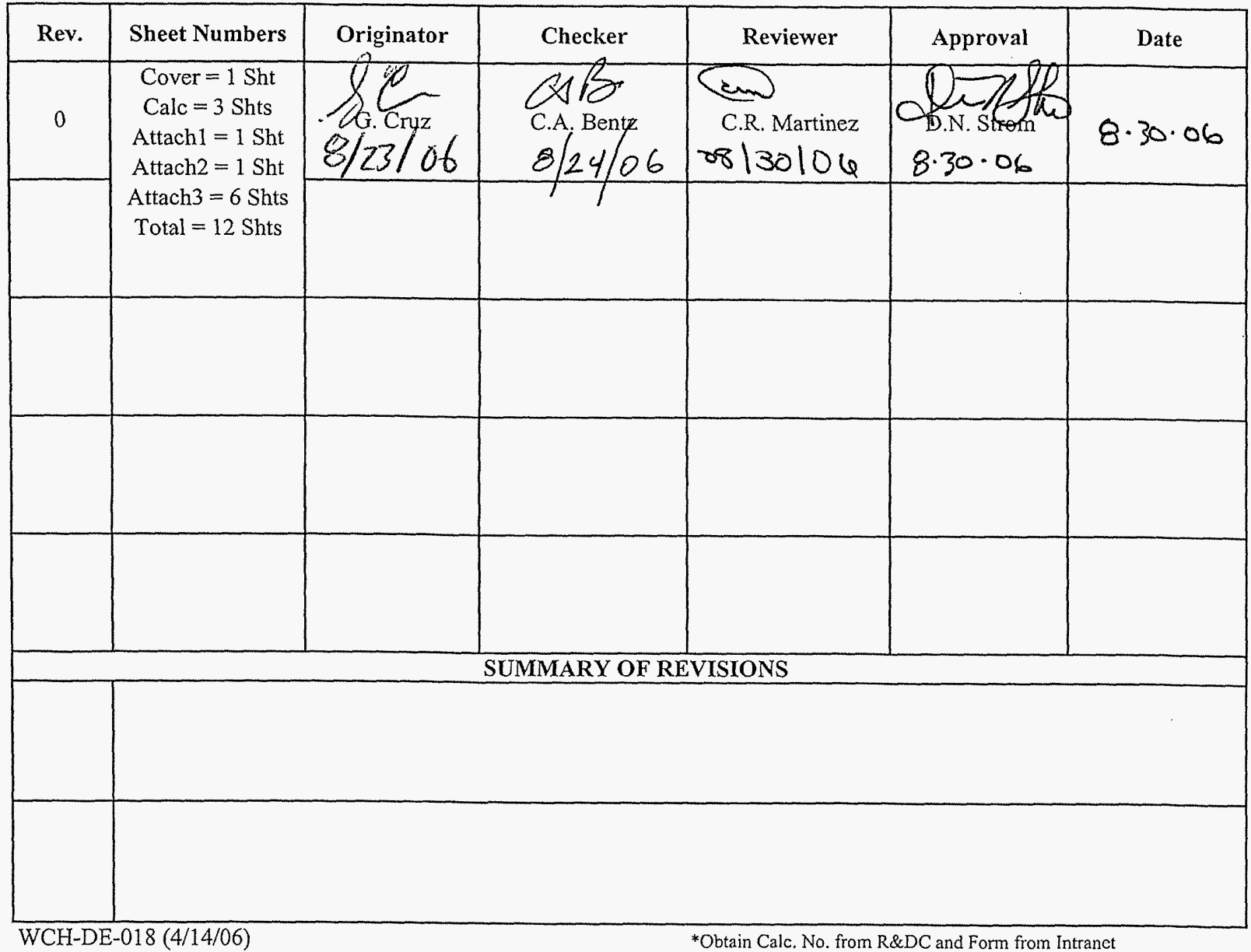




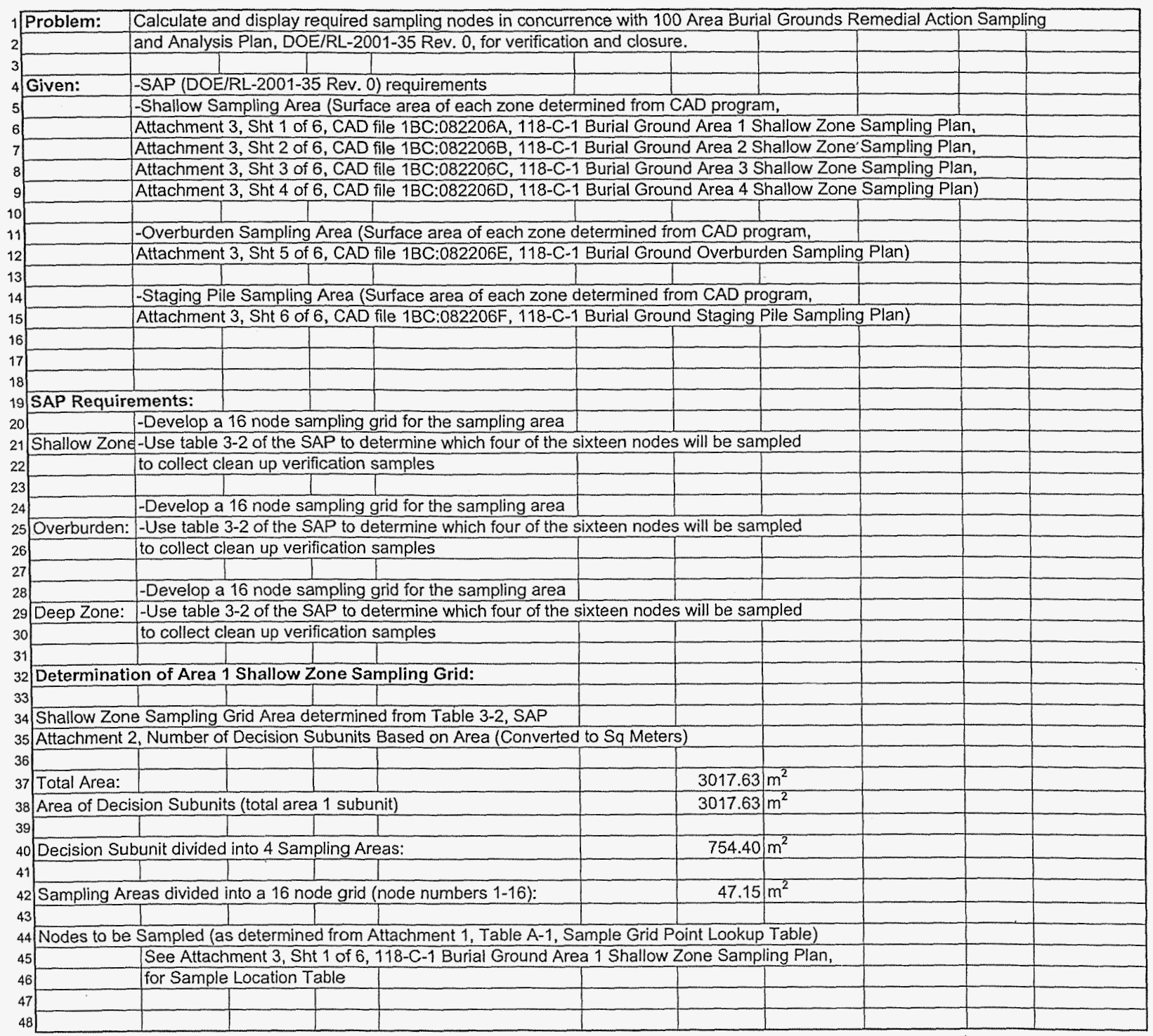




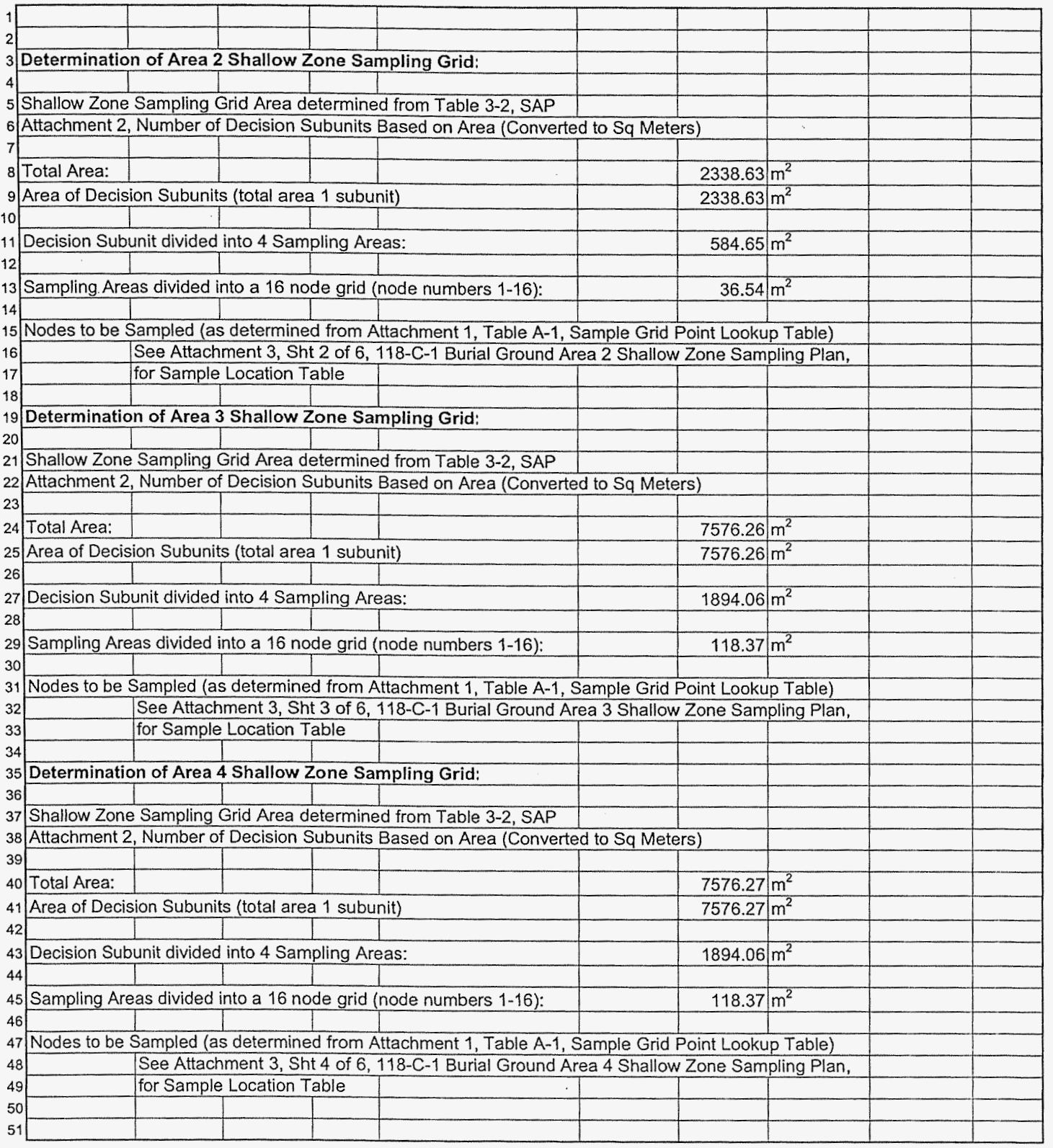


CVP-2006-00011

Rev. 0

Washington Closure Hanford

CALCULATION SHEET

Originator G. Cryz Date 8/22/2006

Calc. No. 0100B-CA-V0288 Rev. No. 0

Project 118-C-1 Burial Ground Sample Design

Job No. $\frac{14655 \text { Checked }}{\text { CSB }} \frac{\text { Date } 8 / 24 / 06}{\text { Sheet No. } 3 \text { of3 }}$

Subject 118-C-1 Shallow Zone, Overburden, and Staging Pile Sampling Plan

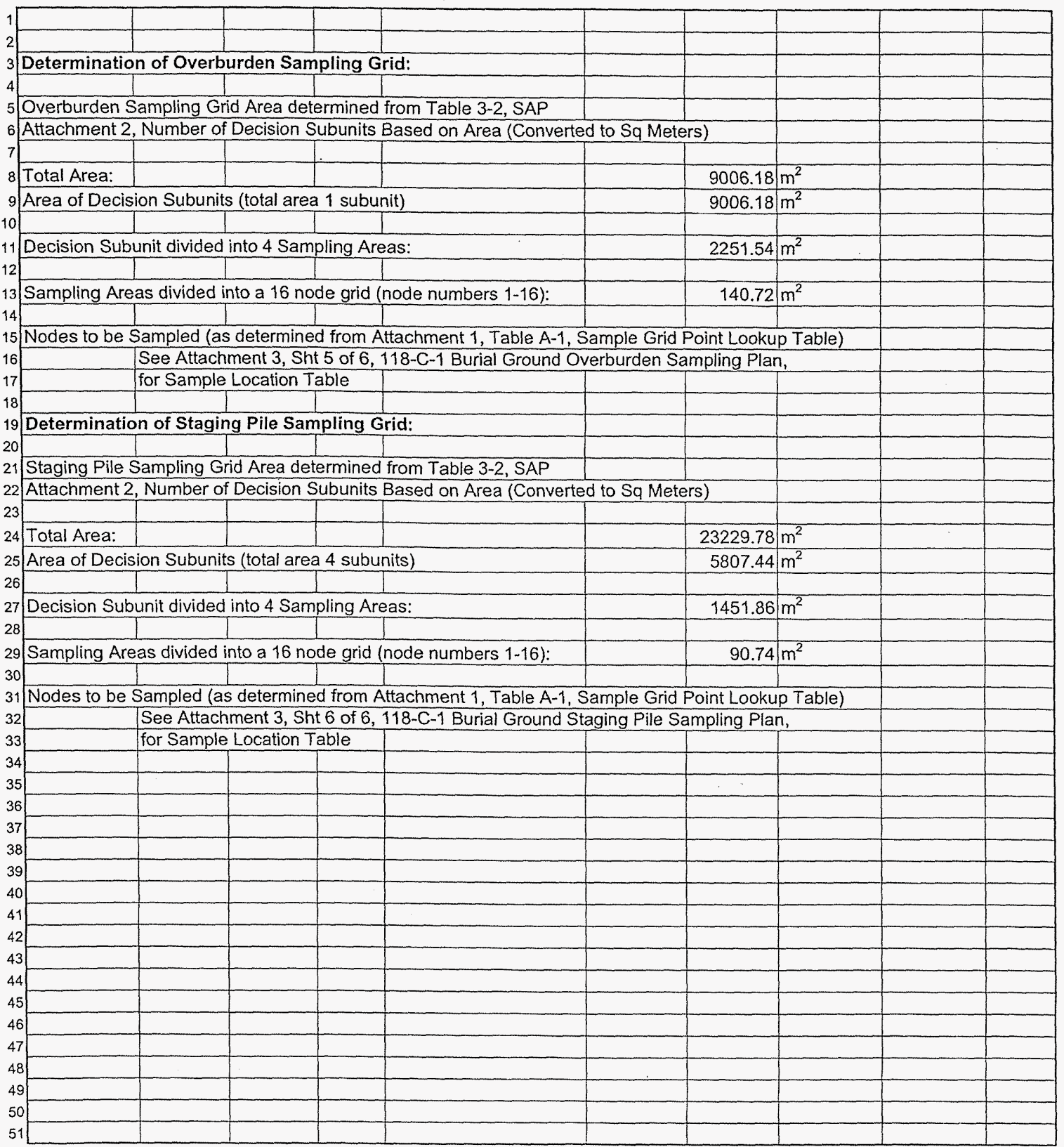


CVP-2006-00011

Rev. 0

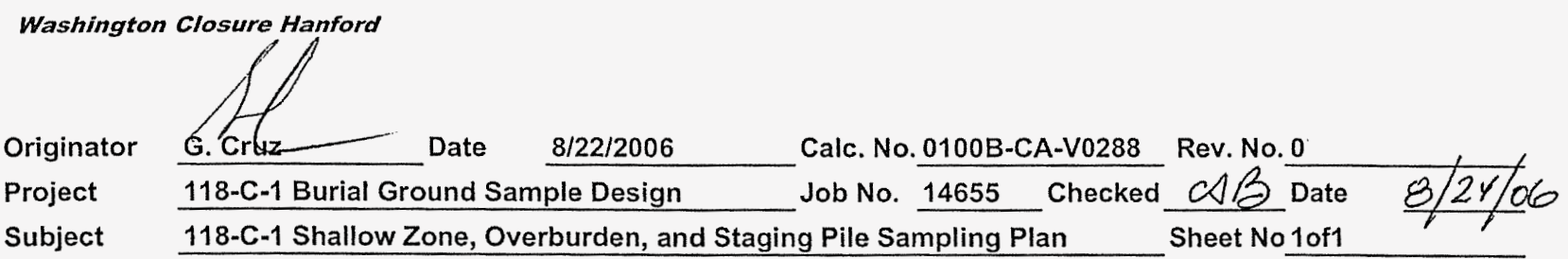

I ATTACHMENT 1

2

${ }_{3}$ Sample Grid Point Lookup Table.

4

\begin{tabular}{|c|c|c|c|c|c|c|c|c|c|c|}
\hline Default Plan & $\begin{array}{l}\text { Sampling } \\
\text { Area } 1\end{array}$ & $\begin{array}{c}\text { Sampling } \\
\text { Area } 2\end{array}$ & $\begin{array}{c}\text { Sampling } \\
\text { Area } 3\end{array}$ & $\begin{array}{c}\text { Sampling } \\
\text { Area } 4\end{array}$ & $\begin{array}{l}\text { Sampling } \\
\text { Area } 5\end{array}$ & $\begin{array}{c}\text { Sampling } \\
\text { Area } 5\end{array}$ & $\begin{array}{c}\text { Sampling } \\
\text { Area } 7\end{array}$ & $\begin{array}{c}\text { Sampling } \\
\text { Area } 8\end{array}$ & $\begin{array}{c}\text { Sampling } \\
\text { Area } 9\end{array}$ & $\begin{array}{c}\text { Sampling } \\
\text { Area to }\end{array}$ \\
\hline Closeout & 3 & 6 & 1 & 4 & 5 & 1 & 3 & 3 & 4 & 16 \\
\hline Closeout & 4 & 7 & 11 & 3 & 15 & 15 & 5 & 13 & 10 & 10 \\
\hline Closeout & 16 & 3 & 2 & 7 & 7 & 10 & 11 & 4 & 3 & 14 \\
\hline Closeout & 10 & 15 & 4 & 12 & 1 & 13 & 4 & 8 & 16 & 4 \\
\hline Not Sampling & 2 & 14 & 5 & 9 & 13 & 12 & 8 & 2 & 14 & 8 \\
\hline Not Sampling & 13 & 10 & 9 & 13 & 2 & 16 & 1 & 12 & 5 & 3 \\
\hline Not Sampling & 6 & 1 & 10 & 8 & 14 & 4 & 16 & 5 & 8 & 6 \\
\hline Not Sampling & 1 & 9 & 13 & 1 & 10 & 5 & 12 & 1 & 1 & 15 \\
\hline Not Sampling & 9 & 12 & 7 & 5 & 6 & 2 & 6 & 7 & 15 & 9 \\
\hline Not Sampling & 15 & 16 & 15 & 14 & 16 & 6 & 2 & 15 & 11 & 1 \\
\hline Not Sampling & 8 & 13 & 8 & 10 & 12 & 11 & 13 & 14 & 2 & 12 \\
\hline Not Sampling & 5 & 2 & 3 & 11 & 4 & 3 & 9 & 10 & 7 & 11 \\
\hline Not Sampling & 7 & 11 & 14 & 15 & 11 & 14 & 14 & 6 & 13 & 2 \\
\hline Not Sampling & 11 & 4 & 6 & 2 & 9 & 7 & 7 & 11 & 9 & 7 \\
\hline 1 Not Sampling & 12 & 8 & 16 & 16 & 3 & 8 & 15 & 9 & 6 & 13 \\
\hline Not Sampling & 14 & 5 & 12 & 6 & 8 & 9 & 10 & 16 & 12 & 5 \\
\hline
\end{tabular}

${ }_{24}$ the nodes in the northwesternmost node. Then number consecutively left to right. 
Originator $\mathrm{G}$. Cruz

Date $8 / 22 / 2006$

Calc. No. 0100B-CA-V0288 Rev. No. 0

Project 118-C-1 Burial Ground Sample Design Job No. 14655 Checked CSB Date

Subject 118-C-1 Shallow Zone, Overburden, and Staging Pile Sampling Plan Sheet No. 1of1

\begin{tabular}{|c|c|c|c|c|c|c|}
\hline \multicolumn{7}{|c|}{${ }_{4}$ Number of Decision Subunits Based on Area. } \\
\hline $\begin{array}{l}7 \\
8\end{array}$ & \multicolumn{6}{|c|}{ Site Verification Sampling Frequencies Based on Area. } \\
\hline 10 & Decision Unit ${ }^{2}$ & Waste Site Size & $\begin{array}{l}\text { Decision } \\
\text { Subunits }\end{array}$ & Blocks $^{c}$ & $\begin{array}{l}\text { Discrete } \\
\text { Samples }\end{array}$ & $\begin{array}{c}\text { Composite } \\
\text { Samples }\end{array}$ \\
\hline $\begin{array}{l}11 \\
12\end{array}$ & \multirow{3}{*}{$\begin{array}{l}\text { Shallow zone - } \\
01015 \mathrm{ft}\end{array}$} & Small: $<100,000 \mathrm{ft}^{2}$ & 1 & 4 & 16 & 4 \\
\hline $\begin{array}{l}12 \\
13\end{array}$ & & Medium: $>100,000 \mathrm{ft}^{2}<400,000 \mathrm{ft}^{2}$ & 4 & 16 & 64 & 16 \\
\hline 14 & & Large: $>400,000 \mathrm{ft}^{2}$ & 8 & 32 & 128 & 32 \\
\hline 15 & \multirow{3}{*}{$\begin{array}{l}\text { Deep Zone - } \\
>15 \mathrm{ft}\end{array}$} & Small: $<100,000 \mathrm{ft}^{2}$ & 1 & 4 & 16 & 4 \\
\hline 16 & & Medium: $>100,000 \mathrm{ft}^{2}<400,000 \mathrm{ft}^{2}$ & 4 & 16 & 64 & 16 \\
\hline 17 & & Large: $>400,000 \mathrm{ft}^{2}$ & 8 & 32 & 128 & 32 \\
\hline 18 & \multirow{3}{*}{$\begin{array}{l}\text { Overburen/layback } \\
\text { stockpiles }\end{array}$} & Small: $<100,000 \mathrm{ft}^{2}$ & 1 & 4 & 16 & 4 \\
\hline 19 & & Medium: $>100,000 \mathrm{ft}^{2}<400,000 \mathrm{ft}^{2}$ & 4 & 16 & 64 & 16 \\
\hline 20 & & Large: $>400,000 \mathrm{ft}^{2}$ & 8 & 32 & 128 & 32 \\
\hline 21 & \multirow{3}{*}{$\begin{array}{l}\text { Staging pile areas } \\
\text { (residual soil) }\end{array}$} & Small: $<100,000 \mathrm{ft}^{2}$ & 1 & 4 & 16 & 4 \\
\hline 22 & & Medium: $>100,000 \mathrm{ft}^{2}<400,000 \mathrm{ft}^{2}$ & 4 & 16 & 64 & 16 \\
\hline & & Large: $>400,000 \mathrm{ft}^{2}$ & 8 & 32 & 128 & 32 \\
\hline
\end{tabular}

" The shallow zone, deep zone, overburden stockpile, and staging pile areas cach represent single decision units. The total number of decision - units will vary because individual waste sites may not have a decp zone, overburden stockpile, and/or staging pile areas.

- Area of exposed surface after excavation or arca of stockpile base (as applicable)
Decision subunits are divided into four blocks to ensure that random sampling locations are not bunched together in one area 
CVP-2006-00011

Rev. 0

\section{E-92}




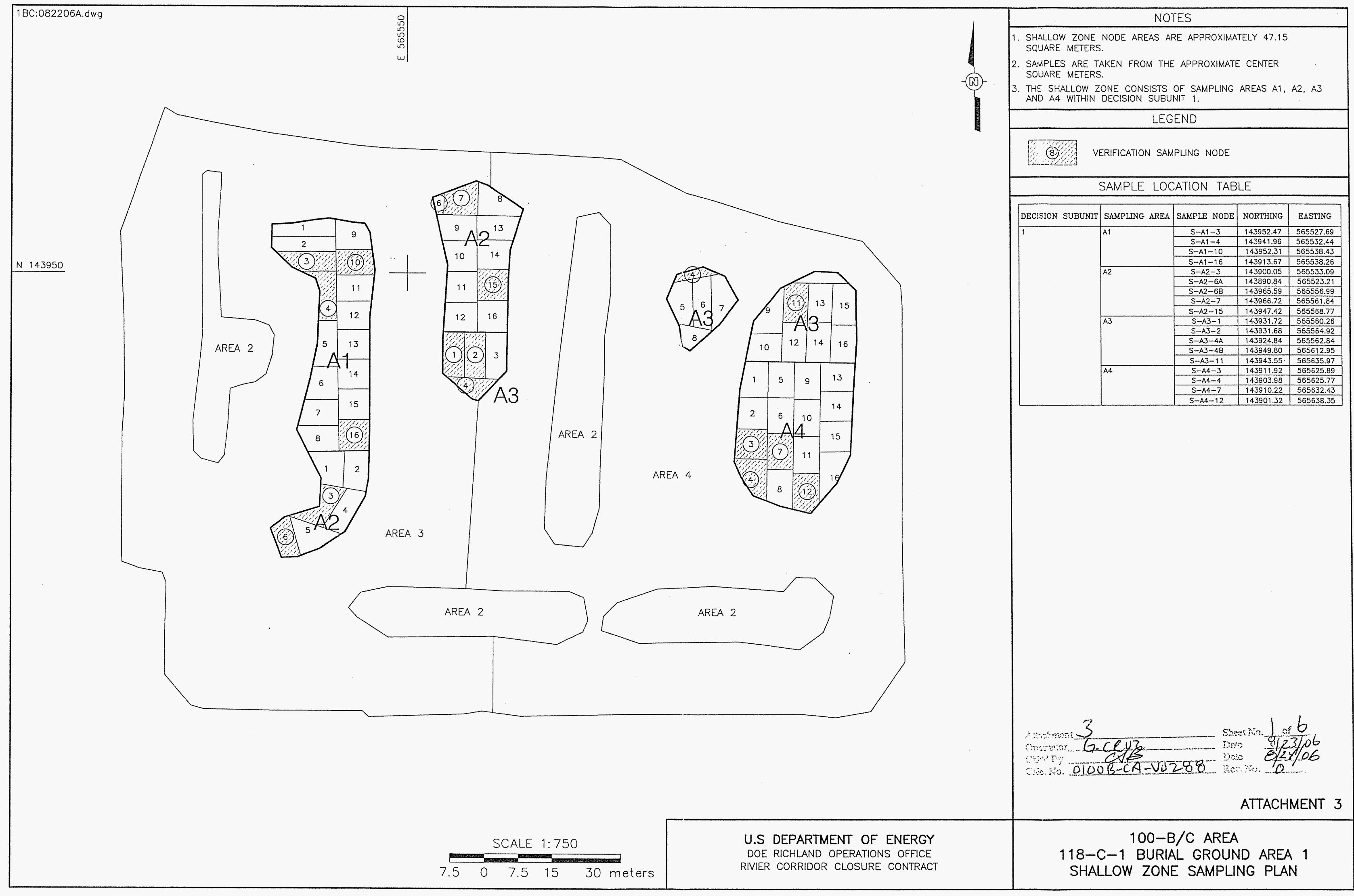




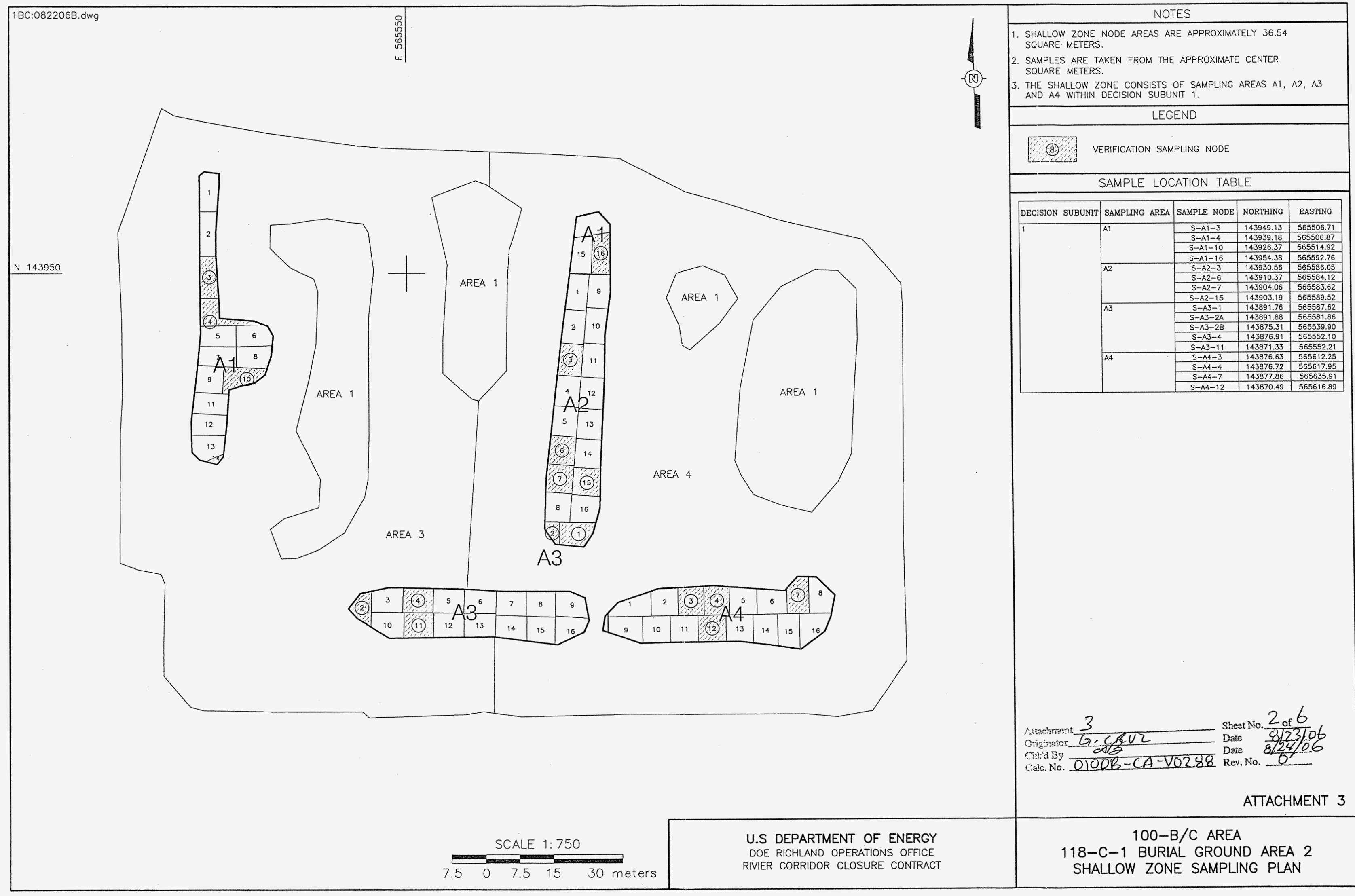




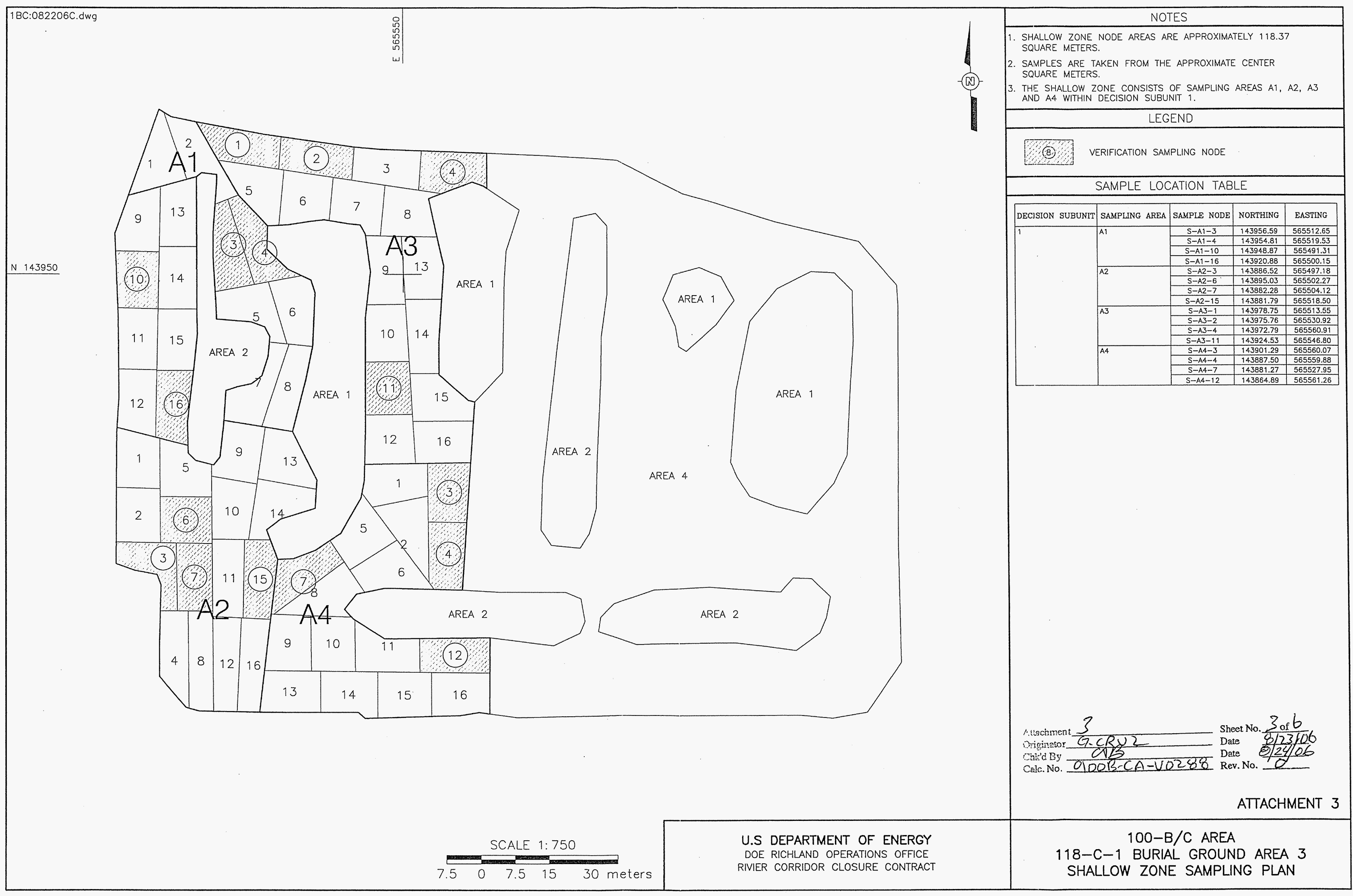




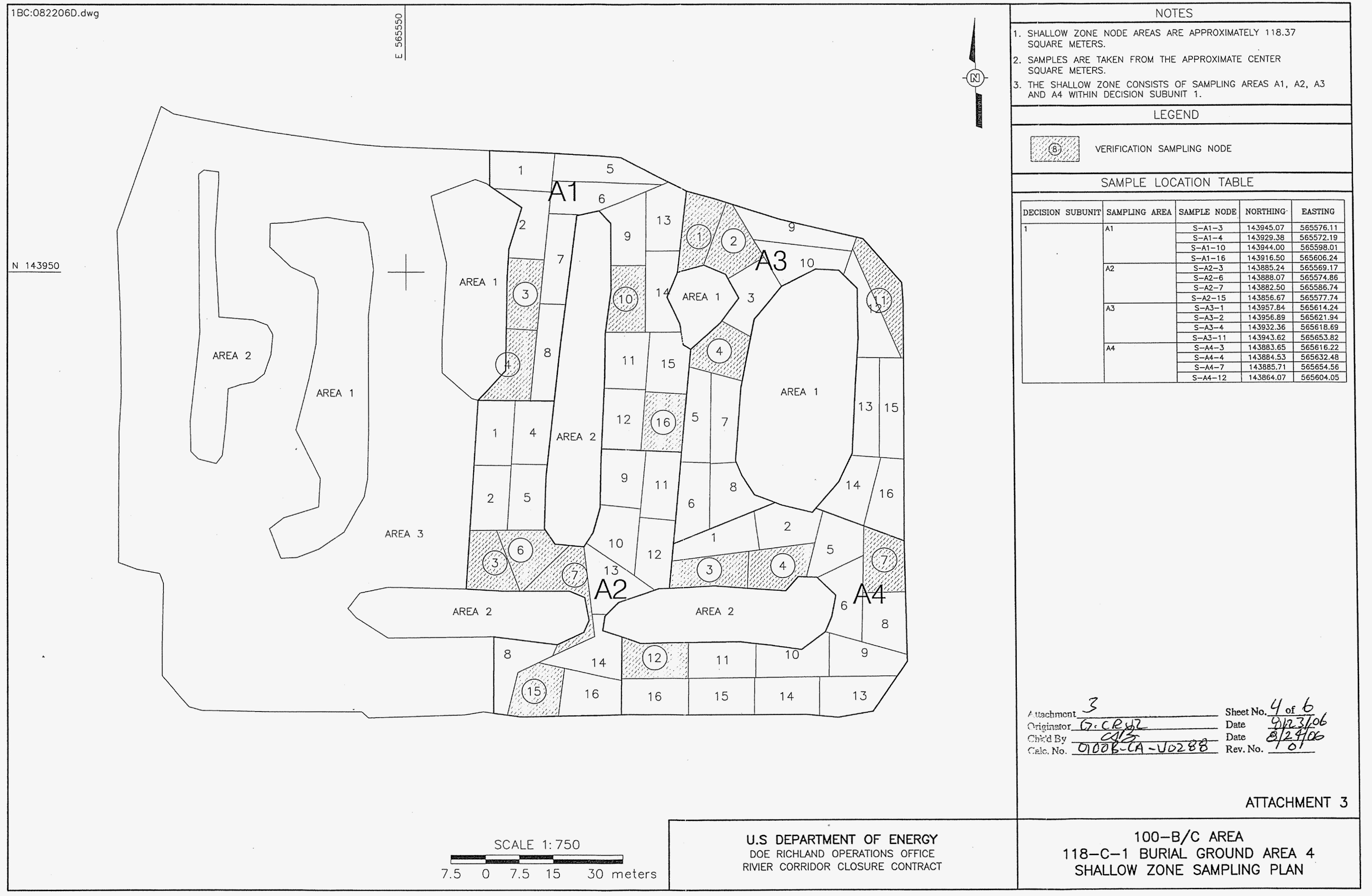




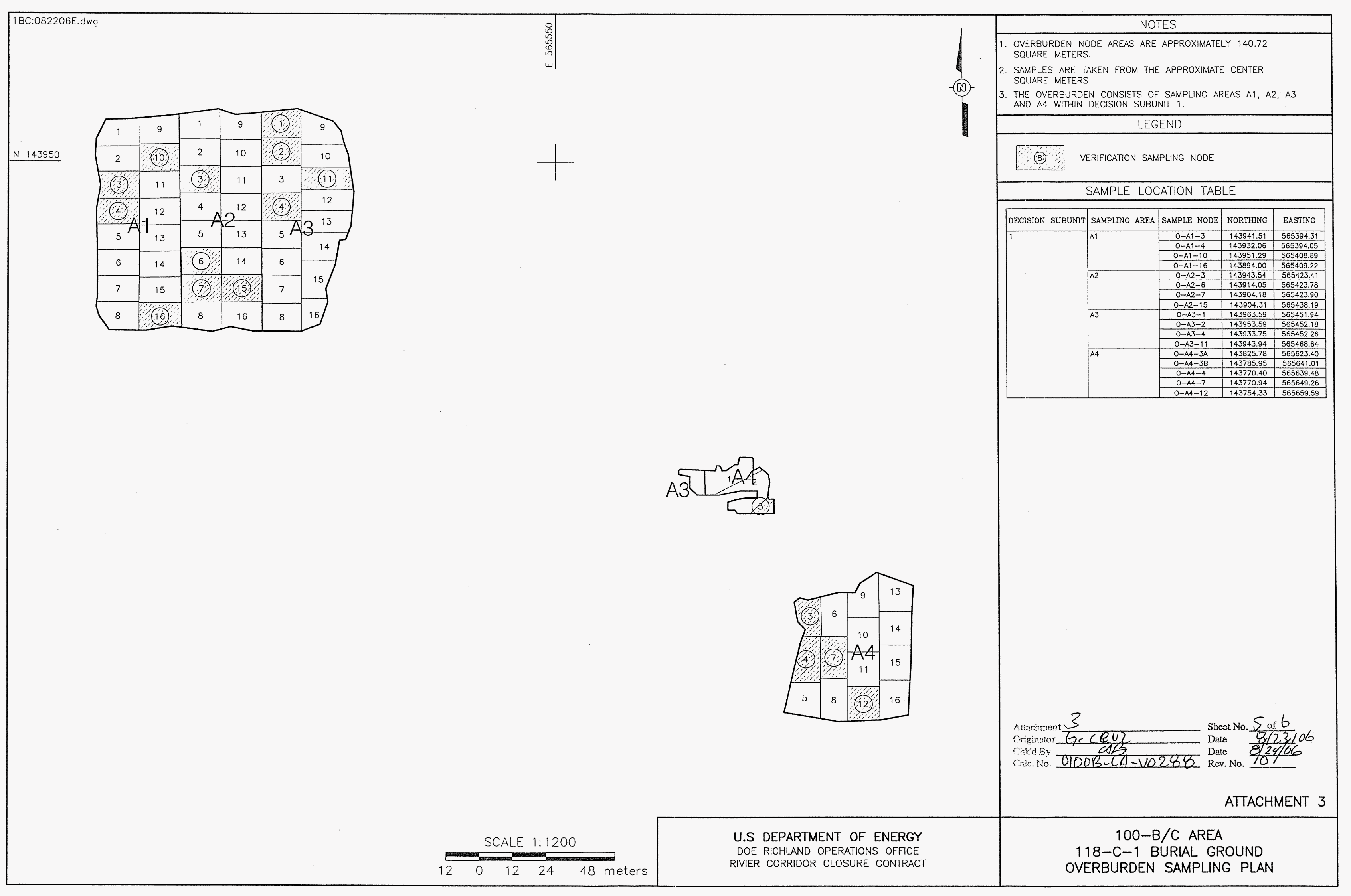




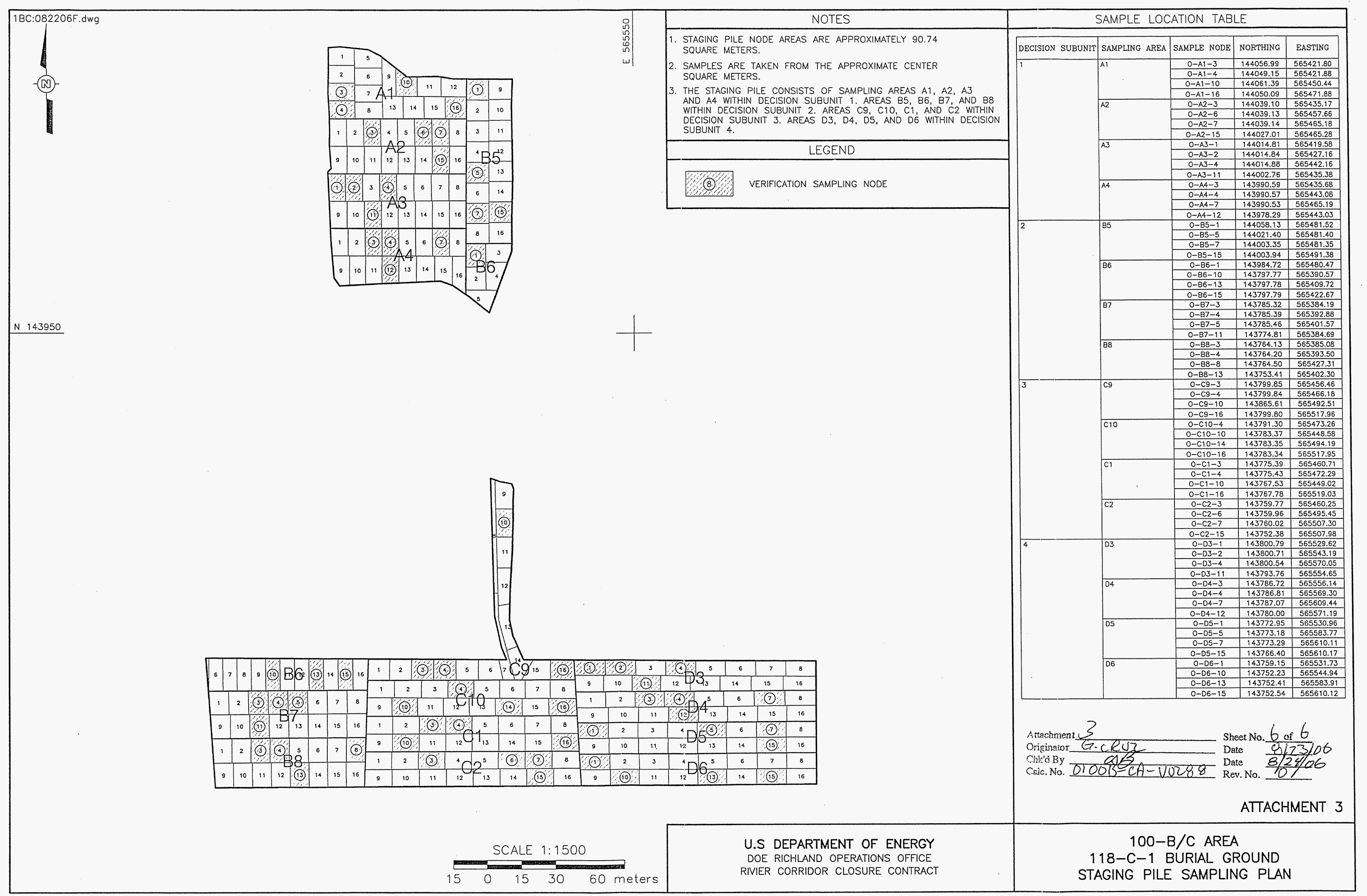




\section{CALCULATION COVER SHEET}

Project Title $100-\mathrm{B} / \mathrm{C}$ Field Remediation

Job No. 14655

Area $100-B / C$

Discipline Environmental

*Calc. No. 0100C-CA-V0034

Subject $118-\mathrm{C}-1$ Burial Ground Hazard Quotient and Carcinogenic Risk Calculations Computer Program Excel Program No. Excel 2003

The attached calculations have been generated to document compliance with established cleanup levels. These calculations should be used in conjunction with other relevant documents in the administrative record.

Committed Calculation $\otimes \quad$ Preliminary $\square \quad$ Superseded $\square \quad$ Voided

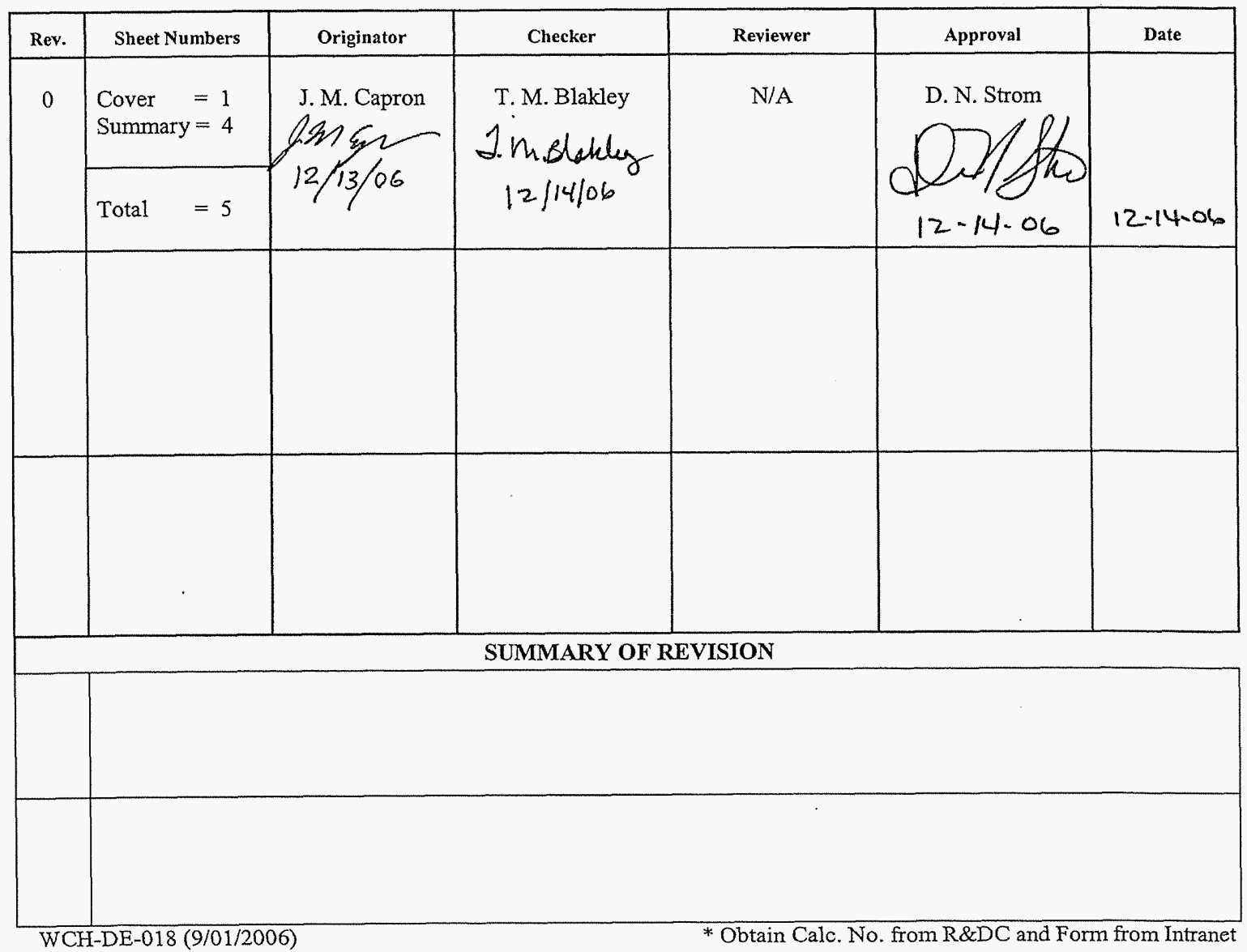


Washington Closure Hanford CALCULATION SHEET

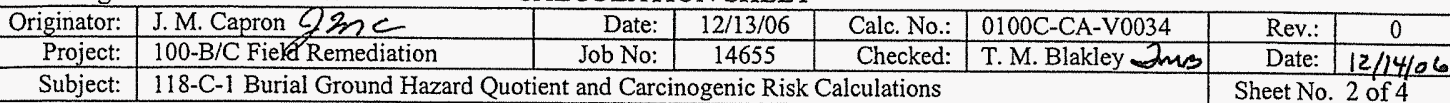

\section{METHODOLOGY:}

Hazard quotient and carcinogenic risk calculations were performed for the 118-C-1 burial ground using the highest statistical value from all sampling areas or individual detections in focused samples (locations 1 through 7). Of the contaminants of concern (COCs) and contaminants of potential concern (COPCs) for the site, boron and molybdenum require the HQ calculations because they were detected and Washington State or Hanford Site background values are not available. Barium, copper, lead, selenium, and zinc are included because they were quantified above their respective Hanford Site background values. Hexavalent chromium, aroclor-1254, aroclor-1260, and multiple volatile and semivolatile organic compounds (as identified in Table 1) are included because they were detected by laboratory analysis and cannot be attributed to natural occurrence. Polycyclic aromatic hydrocarbons (PAHs) detected in the verification samples from the staging pile area are not included because they are the result of asphalt cross-contamination in the sample matrix from a former overlying road and paved area. Asphalt that has been used for structural and construction purposes is excluded from consideration as a dangerous waste by WAC 173-303-071(3)(e), is listed as an inert waste in WAC 173-350990(2)(b), and its constituents are therefore not considered in attainment of soil RAGs (PAH concentrations in the soil matrix are assumed to be negligible). Residual PAH concentrations detected in other sampling areas were considered for the risk calculations. All other site nonradionuclide COCs and COPCs were not detected or were detected below background levels. An example of the HQ and risk calculations is presented below:

1) For example, the maximum value for boron is $3.4 \mathrm{mg} / \mathrm{kg}$, divided by the noncarcinogenic RAG value of $16,000 \mathrm{mg} / \mathrm{kg}$ (calculated in accordance with the noncarcinogenic toxics effects formula in WAC 173-340-740[3]), is $2.1 \times 10^{-4}$. Comparing this value, and all other individual values, to the requirement of $<1.0$, this criterion is met.

2) After the HQ calculations are completed for the appropriate analytes, the cumulative $H Q$ is obtained by summing the individual values. (To avoid errors due to intermediate rounding, the individual HQ values prior to rounding are used for this calculation.) The sum of the HQ values is $1.6 \times 10^{-1}$. Comparing this value to the requirement of $<1.0$, this criterion is met.

3) To calculate the excess carcinogenic risk, the $95 \%$ upper confidence limit or maximum value is divided by the carcinogenic RAG value, then multiplied by $1 \times 10^{-6}$. For example, the maximum value for hexavalent chromium is $0.5 \mathrm{mg} / \mathrm{kg}$; divided by $2.1 \mathrm{mg} / \mathrm{kg}$ and multiplied as indicated is $2.4 \times 10^{-7}$. Comparing this value, and all other individual values, to the requirement of $<1 \times 10^{-6}$, this criterion is met.

4) After these calculations are completed for the carcinogenic analytes, the cumulative excess carcinogenic risk is obtained by summing the individual values. (To avoid errors due to intermediate rounding, the individual values prior to rounding are used for this calculation.) The sum of the excess carcinogenic risk values is $1.6 \times 10^{-6}$. Comparing this value to the requirement of $<1 \times 10^{-5}$, this criterion is met.

\section{RESULTS:}

1) List individual noncarcinogens and corresponding HQs $>1.0$ : None 
CVP-2006-00011

Rev. 0

Washington Closure Hanford CALCULATION SHEET

Originator: J.M. Capron 9 亿 Project: $100-\mathrm{B} / \mathrm{C}$ Fiele Remediation

\begin{tabular}{|r|r|r|r}
\hline Date: & $12 / 13 / 06$ & Calc. No.: & $0100 \mathrm{C}-\mathrm{CA}-\mathrm{V} 0034$
\end{tabular}

Checked: T. M. Blakley 2 m 3

Subject: $118-\mathrm{C}-1$ Burial Ground Hazard Quotient and Carcinogenic Risk Calculations

\begin{tabular}{l|l} 
Rev.: & 0 \\
\hline
\end{tabular}

Date: $12 / 14 / 06$

Sheet No. 3 of 4

1 2) List the cumulative noncarcinogenic $H Q>1.0$ : None

3) List individual carcinogens and corresponding excess cancer risk $>1 \times 10^{-6}$ : None

4) List the cumulative excess cancer risk for carcinogens $>1 \times 10^{-5}$ : None.

Table 1 shows the results of the calculations for the 118-C-1 burial ground.

\section{CONCLUSION:}

This calculation demonstrates that the 118-C-1 waste site meets the requirements for hazard quotient and excess carcinogenic risk as identified in the RDR/RAWP (DOE-RL 2005). 
Washington Closure Hanford

CALCULATION SHEET

Originator: J. M. Capron g/n

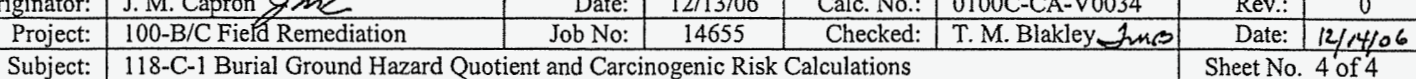

Table 1. Hazard Quotient and Excess Cancer Risk Results for the 100-B-14:2 (Area 4) Subsite.

\begin{tabular}{|l|c|c|c|c|c|}
\hline $\begin{array}{c}\text { Contaminants of Concern/ } \\
\text { Contaminants of Potential Concern }\end{array}$ & $\begin{array}{c}\text { Maximum or } \\
\text { Statistical Value } \\
(\mathrm{mg} / \mathrm{kg})\end{array}$ & $\begin{array}{c}\text { Noncarcinogen } \\
\mathrm{RAG}^{\mathrm{b}} \\
(\mathrm{mg} / \mathrm{kg})\end{array}$ & $\begin{array}{c}\text { Hazard } \\
\text { Quotient }\end{array}$ & $\begin{array}{c}\text { Carcinogen } \\
\mathrm{RAG}^{\mathrm{b}} \\
(\mathrm{mg} / \mathrm{kg})\end{array}$ & $\begin{array}{c}\text { Carcinogen } \\
\text { Risk }\end{array}$ \\
\hline Metals
\end{tabular}

Barium

Boron

\begin{tabular}{|l|r|}
\hline Chromium, hexavalent $^{c}$ & 0.5 \\
\hline Copper & 38.6 \\
\hline
\end{tabular}

\begin{tabular}{l|r}
\hline 206 & 5,600 \\
\hline
\end{tabular}

Lead $^{\text {d }}$

Molybdenum

Selenium

\begin{tabular}{c|c}
3.4 & 16,000 \\
\hline 0.5 & 240
\end{tabular}

240

\begin{tabular}{l|c|c}
\hline 38.6 & 2,960 & $1.3 \mathrm{E}-02$ \\
\hline 18.6 & 353 & $5.3 \mathrm{E}-02$ \\
\hline
\end{tabular}

\begin{tabular}{l|r}
38.6 & 2,960 \\
\hline 18.6 & 353
\end{tabular}

Zinc

\begin{tabular}{|l|l}
\hline 4.5 & 400 \\
\hline 0.80 & 400 \\
\hline
\end{tabular}

400

$1.1 \mathrm{E}-02$

\begin{tabular}{l|c|c}
\hline 74.8 & 400 & $2.0 \mathrm{E}-03$ \\
\hline
\end{tabular}

\begin{tabular}{l|l}
\hline $3.7 \mathrm{E}-02$ & \\
\hline $2.1 \mathrm{E}-04$ & \\
\hline $2.1 \mathrm{E}-03$ & 2. \\
\hline $1.3 \mathrm{E}-02$ & \\
\hline $5.3 \mathrm{E}-02$ & \\
\hline $1.1 \mathrm{E}-02$ & \\
\hline $2.0 \mathrm{E}-03$ & \\
\hline $3.1 \mathrm{E}-03$ & \\
\hline
\end{tabular}

\begin{tabular}{|c|c|}
\hline- & - \\
\hline- & -- \\
\hline 2.1 & $2.4 \mathrm{E}-07$ \\
\hline- & -- \\
\hline- & -- \\
\hline- & -- \\
\hline- & -- \\
\hline- & - \\
\hline
\end{tabular}

Semivolatiles

Benzo(a)anthracene

Benzo(a)pyrene

Benzo(b)fluoranthene

0.18

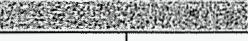

$-$

Benzo(k)fluoranthene

Benzo(g,h,i)perylene ${ }^{c}$

Bis(2-ethylhexyl) phthalate

Butylbenzylphthalate

Chrysene

Diethylphthalate

Di-n-butylphthalate

Fluoranthene

Indeno(1,2,3-cd) pyrene

Phenanthrene $^{e}$

Phenol

Pyrene

0.12

\begin{tabular}{|r|r}
\hline & - \\
\hline & - \\
\hline
\end{tabular}

Polychlorinated Biphenvis

$\frac{0.14}{0.051}$

\begin{tabular}{l|r}
0.28 & 2,400 \\
\hline & 1,600 \\
\hline
\end{tabular}

\begin{tabular}{l|l}
- & \\
\hline- & \\
\hline- & \\
\hline
\end{tabular}

\begin{tabular}{r|r}
1.37 \\
\hline
\end{tabular}

7.

Aroclor-1254

\begin{tabular}{|l|c|}
\hline Aroclor -1260 & 0.061 \\
\hline Wolatile Organic Compounds: & \\
\hline
\end{tabular}

\begin{tabular}{c|c|c|c}
\hline 0.038 & 16,000 & $2.4 \mathrm{E}-06$ & - \\
\hline 0.21 & -- & - & 137 \\
\hline 0.022 & 64,000 & $3.4 \mathrm{E}-07$ & - \\
\hline
\end{tabular}

\begin{tabular}{l|l}
8,000 & $3.4 \mathrm{E}-07$ \\
\hline
\end{tabular}

\begin{tabular}{l|l}
0.13 & 8,000 \\
\hline 0.33 & 3,200 \\
\hline
\end{tabular}

\begin{tabular}{l|l}
\hline 200 & $1.6 \mathrm{E}-05$ \\
\hline
\end{tabular}

0.046

0.036

0.32

24,000

24,000

2,400

4.0E-06

$-$

$-1$.

Acetone

Benzene

Butanone; 2-

Carbon tetrachloride

Methylene chloride

Styrene

Toluene

Xylenes (total)

0.024
0.001

\begin{tabular}{l|l}
0.024 & 320 \\
\hline 0.004 & 48,000
\end{tabular}

\begin{tabular}{c|c}
0.004 & 48,000 \\
\hline 0.039 & 56
\end{tabular}

\begin{tabular}{c|c}
0.039 & 56 \\
\hline 0.023 & 4,800 \\
\hline
\end{tabular}

\begin{tabular}{l|l}
0.004 & 16,000 \\
\hline 0.002 & 6,400
\end{tabular}

$1.5 \mathrm{E}-06$

\begin{tabular}{c|c}
1.37 & $1.3 \mathrm{E}-07$ \\
\hline 0.137 & $8.8 \mathrm{E}-07$ \\
\hline 1.37 & $1.0 \mathrm{E}-07$ \\
\hline 13.7 & $1.0 \mathrm{E}-08$ \\
\hline
\end{tabular}

\begin{tabular}{l|r}
0.001 & 16,000
\end{tabular}

\begin{tabular}{|c|c|c|}
\hline .6 & $3.4 \mathrm{E}-02$ \\
\hline & -
\end{tabular}

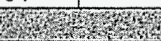

$--$

\begin{tabular}{|c|c|c|}
\hline $6.3 \mathrm{E}-08$ & - & - \\
\hline
\end{tabular}

Cumulative Hazard Quotient:

Cumulative Excess Cancer Risk:

$1.6 \mathrm{E}-06$

Notes:

${ }^{a}=$ From WCH (2006).

$b=$ Value obtained from Washington Administrative Code (WAC) 173-340-740(3), Method B, 1996.

$c=$ Value for the carcinogen RAG calculated based on the inhalation exposure pathway (WAC) 173-340-750(3), 1996.

${ }^{d}=$ Value for the noncarcinogen RAG obtained from EPA (1994).

: = Toxicity data are not available for this constituent. RAGs for benzo $(\mathrm{g}, \mathrm{h}, \mathrm{i})$ perylene and phenanthrene are based

on the surrogate chemicals pyrene and anthracene, respectively.

$\ldots=$ not applicable

$\mathrm{RAG}=$ remedial action goal 


\section{CALCULATION COVER SHEET}

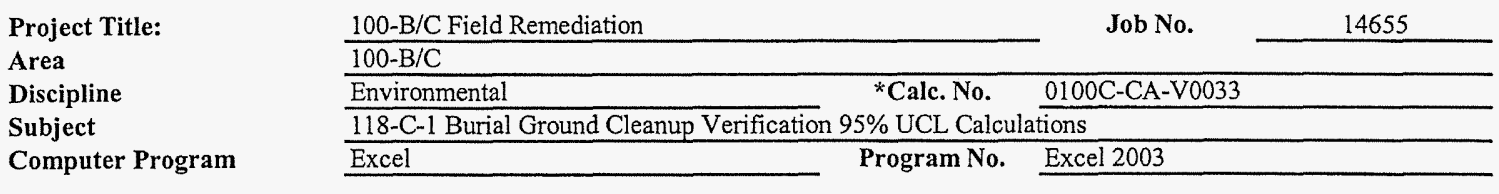

The attached calculations have been generated to document compliance with established cleanup levels. These calculations should be used in conjunction with other relevant documents in the administrative record.

$\begin{array}{llll}\text { Committed Calculation } & \text { Preliminary }\end{array} \quad$ Superseded $\square \quad$ Voided $\square$

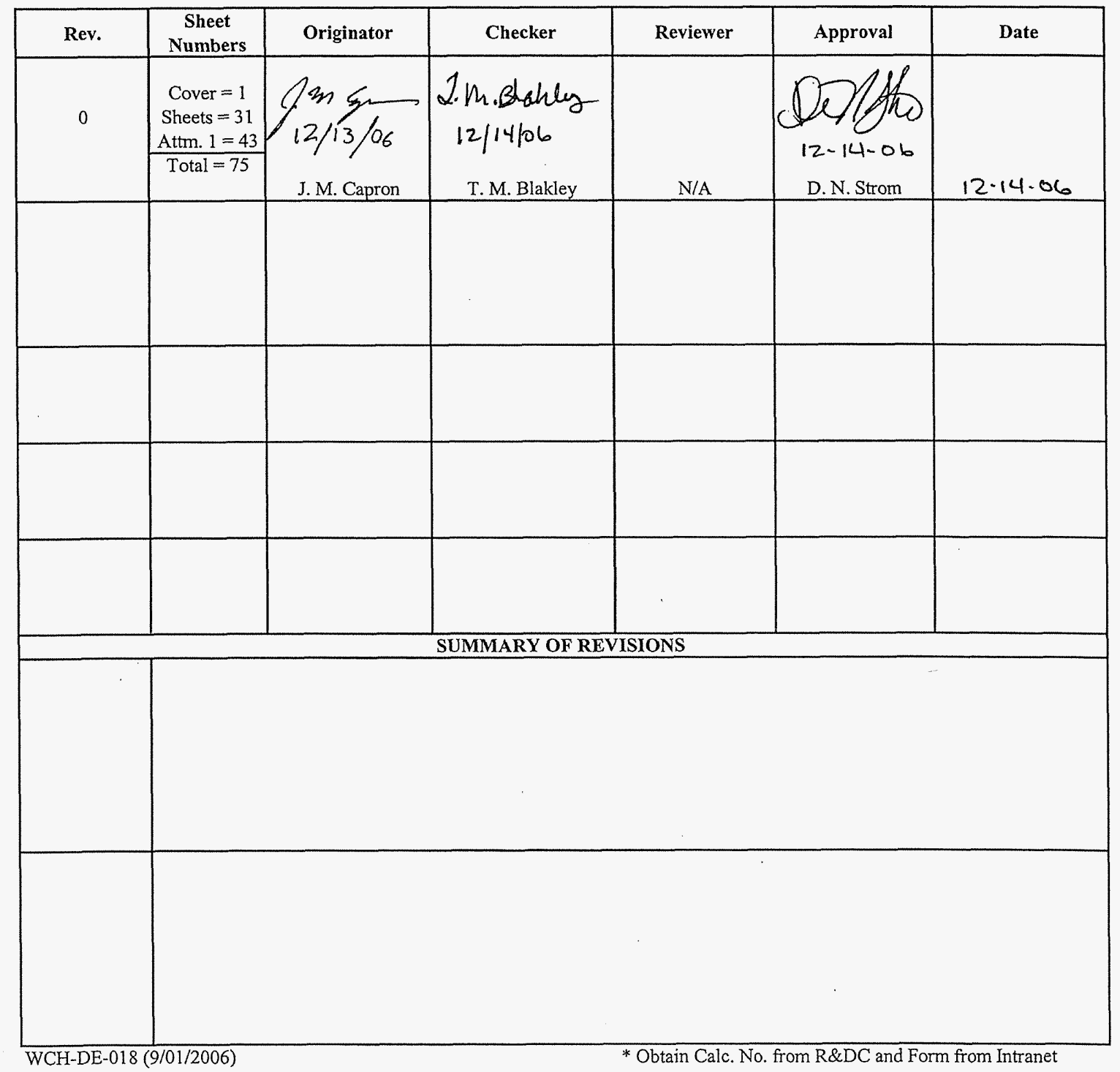




\section{Washington Closure Hanford}

Originator J.M. Capron 2 .

Project 100-B/C Field Remediation

Subject $\overline{118-C-1 \text { Burial Ground Cleanup Verification 95\% UCL Calculations }}$
Date $12 / 13 / 06$ Job No. 14655 Job No
Calc. No. 0100C-CA-V0033 Rev. No. 0 Checked T.M. Blakley $\sin B$ Date $\frac{0}{12 / 14 / 06}$ Sheet No. 1 of 31

Summary

1 Purpose:

2 Calculate the 95\% upper confidence limit (UCL) values to evaluate compliance with cleanup standards for the subject site. Also, 3 perform the Washington Administrative Code (WAC) 173-340-740(7)(e) 3-part test for each nonradioactive contaminant of concern 4 (COC) and contaminant of potential concern (COPC) and calculate the relative percent difference (RPD) for primary-duplicate and 6 primary-split sample pairs, as necessary.

Table of Contents:

Sheets 1 to 4 - Summary

Sheets 5 to $6-118-\mathrm{C}-1$ Area 1 Statistical Calculations

Sheets 7 to $9-118-C-1$ Area 2 Statistical Calculations

Sheets 10 to 12 - 118-C-1 Area 3 Statistical Calculations

Sheets 13 to $15-118-\mathrm{C}-1$ Area 4 Statistical Calculations

Sheets 16 to $18-118-C-1$ Overburden/BCL Material Statistical Calculations

Sheets 19 to 22 - 118-C-1 Staging Pile Area Statistical Calculations

Sheets 23 to 28 - Ecology Software (MTCAStat) Results (Staging Pile Area)

Sheets 29 to 31 - Split/Duplicate Analysis

Attachment 1 - 118-C-1 Verification Sampling Results (43 sheets)

Given/References:

1) Sample Results (Attachment 1).

2) Background values and remedial action goals (RAGs) are taken from DOE-RL (2005b), DOE-RL (2001), and Ecology (2005).

3) DOE-RL, 2001a, 100 Area Burial Grounds Remedial Action Sampling and Analysis Plan (SAP), DOE/RL-2001-35, Rev. 0, U.S. Department of Energy, Richland Operations Office, Richland, Washington.

4) DOE-RL, 2001b, Hanford Site Background: Part 1, Soil Background for Nonradioactive Analytes, DOE/RL-92-24, Rev. 4, U.S. Department of Energy, Richland Operations Office, Richland, Washington.

5) DOE-RL, 2005, Remedial Design Report/Remedial Action Work Plan for the 100 Area (RDR/RAWP), DOE/RL-96-17, Rev. 5, U.S. Department of Energy, Richland Operations Office, Richland, Washington.

6) Ecology, 1992, Statistical Guidance for Ecology Site Managers, Publication \#92-54, Washington Department of Ecology, Olympia, Washington.

7) Ecology, 1993, Statistical Guidance for Ecology Site Managers, Supplement S-6, Analyzing Site or Background Data with Below-detection Limit or Below-PQL Values (Censored Data Sets), Publication \#92-54, Washington Department of Ecology, Olympia, Washington.

8) Ecology, 2005, Cleanup Levels and Risk Calculations (CLARC) Database, Washington State Department of Ecology, Olympia, Washington, <https://fortress.wa.gov/ecy/clarc/CLARCHome.aspx>.

9) EPA, 1994, USEPA Contract Laboratory Program National Functional Guidelines for Inorganic Data Review, EPA 540/R-94/013. U.S. Environmental Protection Agency, Washington, D.C.

10) WAC 173-340, 1996, "Model Toxic Control Act - Cleanup," Washington Administrative Code.

Solution:

Calculation methodology is described in Ecology Pub. \#92-54 (Ecology 1992, 1993), below, and in the RDR/RAWP (DOE-RL $2005 \mathrm{~b})$. Use data from attached worksheets to perform the $95 \%$ UCL calculation for each analyte, the

WAC 173-340-740(7)(e) 3-part test for nonradionuclides, and the RPD calculations, as required. The hazard quotient and carcinogenic risk calculations are located in a separate calculation brief as an appendix to the Cleanup Verification Package (CVP).

Calculation Description:

2 The subject calculations were performed on data from soil verification samples from the subject waste site. The data were entered into an EXCEL 2003 spreadsheet and calculations performed by using the built-in spreadsheet functions and/or creating formulae within the cells. The statistical evaluation of data for use in accordance with the RDR/RAWP (DOE-RL 2005b) is documented by this calculation. Duplicate and split RPD results are used in evaluation of data quality within the CVP for this site.

\section{Methodology:}

For nonradioactive analytes with $\leq 50 \%$ of the data below detection limits and all detected radionuclide analytes, the statistical value calculated to evaluate the effectiveness of cleanup is the $95 \%$ UCL. For nonradioactive analytes with $>50 \%$ of the data below detection limits, the maximum detected value for the data set is used instead of the $95 \% \mathrm{UCL}$. The $95 \% \mathrm{UCL}$ is not calculated for 2 data sets with no reported detections. The evaluation of the portion of each analyte's data set below detection limits was 3 performed by direct inspection of the attached sample results, and no further calculations were performed for those data sets where $>50 \%$ of the data was below detection limits. The $95 \%$ UCL values were not calculated for radium- 226 , radium-228, 


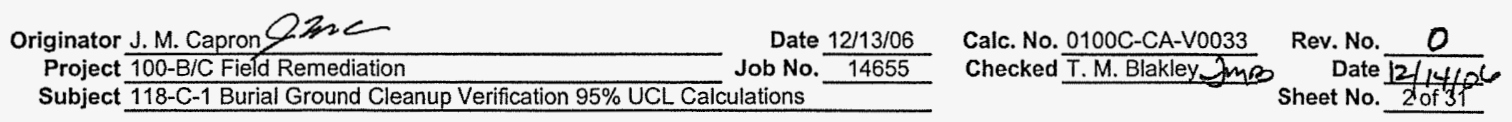

Summary (continued)

1 thorium-228, thorium-232, and potassium-40, as these isotopes are excluded from consideration as COCs based on natural

2 occurrence and analogous site information. The $95 \%$ UCL values were also not calculated for aluminum, calcium, iron,

3 magnesium, potassium, silicon, and sodium, as no parameters to calculate cleanup values under WAC 173-340-740(3) are

4 available in Ecology (2005) or other reference databases, and these constituents are thus not considered site COCs/COPCs.

All calculations for uranium isotopes were performed using data from isotope-specific analysis rather than data resulting from

8 gamma spectroscopy. Due to data quality deficiencies, a portion of the staging pile area (SPA) data for fluoranthene were rejected;

9 therefore, no statistical calculations were performed for this analyte. The result reported in the results summary is the maximum

accepted result from the SPA; additional discussion is provided in the CVP.

1

12 Initial data results from the SPA indicated the presence of arsenic and total petroleum hydrocarbons (TPH) at concentrations

13 exceeding cleanup criteria in sampling areas A2 and A4, respectively. Therefore, additional material removal was performed and

14 new samples collected and submitted for metals and TPH analysis, respectively. Further evaluation and calculations for affected

analytes were performed using the replacement sample data. Initial evaluation of radionuclide data from the SPA indicated that

17 statistical evaluation and dose modeling would result in an overly conservative dose estimate due to the presence of carbon-14 in

18 one sample (J135P7). Therefore, this sample was not considered in statistical calculations for residual radionuclide activities at the

19 SPA, and a sample-specific dose evaluation was performed. Additional discussion is provided in the CVP.

20

21 All nonradionuclide data reported as being undetected are set to $1 / 2$ the detection limit value for calculation of the statistics (Ecology 22 1993). For radionuclide data, calculation of the statistics is done using the reported value. In cases where the laboratory does not

report a value below the minimum detectable activity (MDA), half of the MDA is used in the calculation. For the statistical

evaluation of duplicate sample pairs, the samples are averaged before being included in the data set, after adjustments for censored data as described above.

For nonradionuclides, the WAC 173-340 statistical guidance suggests that a test for distributional form be performed on the data and the $95 \%$ UCL calculated on the appropriate distribution using Ecology software. For nonradionuclide small data sets (n < 10) and all radionuclide data sets, the calculations are performed assuming nonparametric distribution, so no tests for distribution are 1 performed. For nonradionuclide data sets of ten or greater, distributional testing and calculation of the $95 \%$ UCL is done using Ecology's MTCAStat software (Ecology 1993). Due to differences in addressing censored data between the RDR/RAWP (DOE-RL 3 2005b) and MTCAStat coding and due to a limitation in the MTCAStat coding (no direct capability to address variable quantitation 4 limits within a data set), substitutions for censored data are performed before software input and the resulting input set treated as uncensored.

The WAC 173-340-740(7)(e) 3-part test is performed for nonradionuclide analytes only and determines if:

1) the $95 \%$ UCL exceeds the most stringent cleanup limit for each $C O P C / C O C$,

2) greater than $10 \%$ of the raw data exceed the most stringent cleanup limit for each COPC/COC,

3) the maximum value of the raw data set exceeds two times the most stringent cleanup limit for each $\mathrm{COPC/COC.}$

The WAC 173-340-740(7)(e) 3-part test is not performed for COPCs/COCs where the statistical value defaults to the maximum value in the data set. Instead, direct comparison of the maximum value against site RAGs (within the CVP) is used as the compliance basis.

The RPD values are evaluated for analytes detected in a primary-duplicate or primary-split sample pair for the purposes of data quality assessment within the RSVP (where direct evaluation of the attached data showed that a given analyte was undetected in both the primary and duplicate sample, no further calculations were performed). The RPD is calculated when both the primary value and the duplicate value for a given analyte are above detection limits and are greater than 5 times the target detection limit (TDL). The TDL is a laboratory detection limit pre-determined for each analytical method, listed in Table II-1 of the SAP (DOE-RL 2005a). The RPD calculations use the following formula:

$$
R P D=[|M-S| /((M+S) / 2)]^{*} 100
$$

$$
\text { where, } \quad M=\text { main sample value } \quad S=\text { split (or duplicate) sample value }
$$

For quality assurance/quality control (QAVQ) split and duplicate RPD calculations, a value less than $30 \%$ indicates the data compare favorably. For regulatory splits, a threshold of $35 \%$ is used (EPA 1994). If the RPD is greater than $30 \%$ (or $35 \%$ for regulatory split data), further investigation regarding the usability of the data is performed. To assist in the identification of anomalous sample pairs, when an analyte is detected in the primary or duplicate/split sample, but was quantified at less than 5 4 times the TDL in one or both samples, an additional parameter is evaluated. In this case, if the difference between the primary and 5 duplicate/split results exceeds a control limit of 2 times the TDL, further assessment regarding the usability of the data is 
CVP-2006-00011

Rev. 0

\section{CALCULATION SHEET}

\section{Washington Closure Hanford}

Originator J.M. Capron QRe

Project 100 -B/C Field Remediation

Date $12 / 13 / 06$

Subject 118-C-1 Burial Ground Cleanup Verification $95 \%$ UCL Calculations

Calc. No. 0100C-CA-V0033 Checked T.M. Blakley Lmp

\section{Rev. No. \\ Date $12 / 410$ \\ Sheet No 3 of}

Summary (continued)

1 performed. Additional discussion is provided in the data quality assessment section of the applicable CVP, as necessary.

2

3 The results presented in the summary tables that follow are for use in risk analysis and the CVP for this site. In addition to the

statistical samples collected at the subject site, focused samples were collected from selected locations based on observations

made during site remediation. Statistical methodology is not applicable to non-statistical sampling, and direct evaluation of detected

values will be used as the compliance basis. The maximum detected value for each analyte from all focused samples (locations 1

through 7) is presented in the results summary for use in the CVP.

10

11 Area 1 - WAC 173-340 3-Part Test for most stringent RAG:

12 95\% UCL > Cleanup Limit?

$13>10 \%$ above Cleanup Limit?

14 Any sample $>2 \times$ Cleanup Limit?

NO

NO

16 All data sets evaluated meet the 3-part test criteria when compared

17 to the most stringent cleanup limit

18

19 Area 3 - WAC 173-340 3-Part Test for most stringent RAG:

$2095 \%$ UCL > Cleanup Limit? YES

$21>10 \%$ above Cleanup Limit? YES

22 Any sample $>2 x$ Cleanup Limit? YES

23

24 Because of the "yes" answer to the 3-part test for copper, lead,

25 zinc, and aroclor-1254, additional evaluation of the attainment of

19 cleanup criteria will be performed.

20

21 OB/BCL - WAC 173-340 3-Part Test for most stringent RAG:

$2295 \%$ UCL > Cleanup Limit? NO

$23>10 \%$ above Cleanup Limit? NO

24 Any sample $>2 x$ Cleanup Limit? $\quad$ NO

25

26 All data sets evaluated meet the 3-part test criteria when compared

27 to the most stringent cleanup limit

28

29

$31 \quad$ Relative Percent Difference Results ${ }^{2}$ -

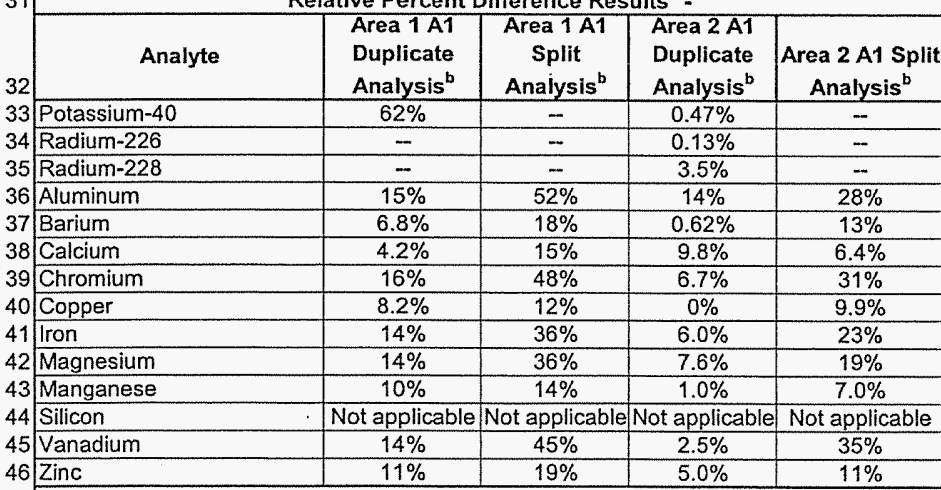

47 Quantitated results for cesium-137, radium-226, radium-228, and cobalt (metal) in

48 one or more sample pairs did not exceed the relative percent difference quality control

49 threshold of $5 \times T D L$, but the difference between the results exceeded the 2 XTDL control

50 threshold.

51 Relative percent difference evaluation was not required for analytes not included in this table.

$52{ }^{\mathrm{b}}$ The significance of relative percent difference values are discussed within the cleanup verification package for the subject site.

$53 \ldots$ - $=$ analysis not required

$54 \mathrm{QAVC}=$ quality assurance/quality control

\begin{tabular}{ll} 
Area 2 -WAC 173-340 3-Part Test for most stringent RAG: \\
\hline $95 \%$ UCL > Cleanup Limit? & NO \\
$>10 \%$ above Cleanup Limit? & YES \\
Any sample $>2 x$ Cleanup Limit? & NO
\end{tabular}

Because of the "yes" answer to the 3-part test for bis(2-ehtylhexyl)phthalate, additional evaluation of the attainment of cleanup criteria will be performed.

\begin{tabular}{ll} 
& Area \\
\hline $95 \%$ UCL $>$ Cleanup Limit? & YES \\
$>10 \%$ above Cleanup Limit? & YES \\
Any sample $>2 x$ Cleanup Limit? & YES
\end{tabular}

Because of the "yes" answer to the 3-part test for barium, additional evaluation of the attainment of cleanup criteria will be performed.

SPA - WAC 173-340 3-Part Test for most stringent RAG:

Cleanup Limit?

Any sample $>2 x$ Cleanup Limit?

Because of the "yes" answer to the 3-part test for barium, copper, lead, aroclor-1254, and multiple polycyclic aromatic hydrocarbons, additional evaluation of the attainment of cleanup criteria will be performed. 
CVP-2006-00011

Rev. 0

Washington Closure Hanford

UMLUULMIIUYY OTEE!

Originator J.M. Capron Gye Date 12/13/06 Checked T.M. Blakley Jum Date $12 / 14106$

Project 100-B/C Field Remediation Calc. No. 0100C-CA-V0033 Rev. No. 0

Subject 118-C-1 Burial Ground Cleanup Verification 95\% UCL Calculations $\quad$ Job No. $\frac{14655}{1}$ Sheet No. 4 of 31

1 Summary (continued)

\begin{tabular}{|c|c|c|c|c|c|c|c|c|c|}
\hline \multirow[t]{2}{*}{2} & \multicolumn{9}{|c|}{ Results Summary ${ }^{a}$} \\
\hline & Analyte & Area $1^{b}$ & Area $2^{b}$ & Area $3^{b}$ & Area $4^{b}$ & $O B-B C L^{b}$ & $\mathrm{SPA}^{\mathrm{b}, \mathrm{c}}$ & $\begin{array}{l}\text { Focused } \\
\text { Samples }\end{array}$ & Units \\
\hline 4 & Carbon-14 & - & 8.0 & - & - & - & $--^{c}$ & - & $\mathrm{pCl/g}$ \\
\hline 5 & Cesium-137 & 0.185 & 0.140 & 0.709 & 0.81 & $0(<B G)$ & 1.03 & - & $\mathrm{pCi} / \mathrm{g}$ \\
\hline 6 & Cobalt-60 & - & 0.99 & 0.037 & - & - & 0.029 & - & $\mathrm{pCi} / \mathrm{g}$ \\
\hline 7 & Europium-152 & 0.121 & 0.199 & 0.140 & 0.114 & - & 0.177 & - & $\mathrm{pCi} / \mathrm{g}$ \\
\hline 8 & Nickel-63 & - & 26.1 & - & - & - & - & - & $\mathrm{pCi} / \mathrm{g}$ \\
\hline 9 & Strontium-90 & - & - & 0.268 & - & 1.23 & 0.334 & - & $\mathrm{pCi} / \mathrm{g}$ \\
\hline 0 & Tritium & - & 4.76 & - & - & - & - & - & $\mathrm{pCilg}$ \\
\hline 1 & Uranium-233/234 & $0(<B G)$ & - & $0(<8 G)$ & $O(<B G)$ & $0(<B G)$ & $O(<B G)$ & - & $\mathrm{pCi} / \mathrm{g}$ \\
\hline 2 & Uranium-235 & $O(<B G)$ & - & - & - & - & $O(<B G)$ & - & $\mathrm{pCi} / \mathrm{g}$ \\
\hline 3 & Uranium-238 & $0(<B G)$ & - & $0(<B G)$ & $0(<B G)$ & $0(<B G)$ & $O(<B G)$ & - & $\mathrm{pCi} / \mathrm{g}$ \\
\hline 4 & Arsenic & 3.0 & 2.7 & 4.5 & 2.4 & 2.4 & 2.8 & 5.4 & $\mathrm{mg} / \mathrm{kg}$ \\
\hline 5 & Barium & 54.3 & 51.9 & 75.3 & 206 & 55.2 & 130 & 80.2 & $\mathrm{mg} / \mathrm{kg}$ \\
\hline 6 & Beryllium & 0.84 & 0.88 & 0.64 & 0.58 & 0.68 & 0.43 & 0.34 & $\mathrm{mg} / \mathrm{kg}$ \\
\hline 7 & Boron & 1.9 & 2.1 & 2.7 & 3.4 & - & 2.8 & 3.2 & $\mathrm{mg} / \mathrm{kg}$ \\
\hline 8 & Cadmium & - & - & 0.12 & - & - & - & - & $\mathrm{mg} / \mathrm{kg}$ \\
\hline 9 & Chromium (total) & 7.9 & 6.1 & 8.7 & 6.5 & 6.7 & 8.2 & 10.2 & $\mathrm{mg} / \mathrm{kg}$ \\
\hline 0 & Cobalt & 7.9 & 7.7 & 8.8 & 8.7 & 7.7 & 7.0 & 10.5 & $\mathrm{mg} / \mathrm{kg}$ \\
\hline 1 & Copper & 13.7 & 13.6 & 38.6 & 15.0 & 15.4 & 36 & 23.3 & $\mathrm{mg} / \mathrm{kg}$ \\
\hline 2 & Hexavalent chromium & - & 0.24 & - & - & 0.24 & 0.5 & - & $\mathrm{mg} / \mathrm{kg}$ \\
\hline 3 & Lead & 4.7 & 4.1 & 18.6 & 5.0 & 4.9 & 10.7 & 7.2 & $\mathrm{mg} / \mathrm{kg}$ \\
\hline 4 & Manganese & 339 & 340 & 349 & 340 & 325 & 323 & 446 & $\mathrm{mg} / \mathrm{kg}$ \\
\hline 5 & Mercury & - & - & - & - & - & 0.06 & 0.04 & $\mathrm{mg} / \mathrm{kg}$ \\
\hline 6 & Molybdenum & - & - & 4.5 & - & - & 0.62 & - & $\mathrm{mg} / \mathrm{kg}$ \\
\hline 7 & Nickel & 10.3 & 9.8 & 12.0 & 9.9 & 9.5 & 10.6 & 14.1 & $\mathrm{mg} / \mathrm{kg}$ \\
\hline 8 & Selenium & - & - & - & - & - & 0.80 & - & $\mathrm{mg} / \mathrm{kg}$ \\
\hline 9 & Vanadium & 39.8 & 46.2 & 50.6 & 51.6 & 44.7 & 46.6 & 46.4 & $\mathrm{mg} / \mathrm{kg}$ \\
\hline 0 & Zinc & 37.3 & 39.3 & 74.8 & 41.0 & 37.5 & 46.1 & 45.1 & $\mathrm{mg} / \mathrm{kg}$ \\
\hline 11 & TPH & - & - & - & - & - & 146 & - & $\mathrm{mg} / \mathrm{kg}$ \\
\hline 2 & Aroclor-1254 & - & - & 0.054 & 0.013 & 0.0065 & 0.015 & 0.022 & $\mathrm{mg} / \mathrm{kg}$ \\
\hline 3 & Aroclor-1260 & - & - & - & - & - & 0.061 & - & $\mathrm{mg} / \mathrm{kg}$ \\
\hline 4 & Acenaphthene & - & - & - & - & - & 0.041 & - & $\mathrm{mg} / \mathrm{kg}$ \\
\hline 5 & Anthracene & - & - & - & - & - & 0.26 & - & $\mathrm{mg} / \mathrm{kg}$ \\
\hline 6 & Benzo(a)anthracene & - & - & 0.18 & 0.13 & 0.038 & 0.5 & - & $\mathrm{mg} / \mathrm{kg}$ \\
\hline 7 & Benzo(a)pyrene & - & - & 0.12 & 0.083 & 0.024 & 0.48 & - & $\mathrm{mg} / \mathrm{kg}$ \\
\hline 8 & Benzo(b)fluoranthene & - & - & 0.14 & 0.11 & 0.033 & 0.21 & - & $\mathrm{mg} / \mathrm{kg}$ \\
\hline 9 & Benzo(g,h,i)perylene & - & - & 0.051 & 0.037 & - & 0.26 & - & $\mathrm{mg} / \mathrm{kg}$ \\
\hline 0 & Benzo(k)fluoranthene & - & - & 0.14 & 0.092 & 0.029 & 0.20 & - & $\mathrm{mg} / \mathrm{kg}$ \\
\hline 11 & bis(2-Ethylhexyl)phthalate & - & 0.28 & 0.22 & 0.017 & - & 0.052 & 0.067 & $\mathrm{mg} / \mathrm{kg}$ \\
\hline 12 & Butylbenzylphthalate & - & - & 0.038 & - & - & 0.017 & - & $\mathrm{mg} / \mathrm{kg}$ \\
\hline 3 & Carbazole & - & - & - & - & - & 0.24 & - & $\mathrm{mg} / \mathrm{kg}$ \\
\hline 4 & Chrysene & - & - & 0.21 & 0.17 & 0.052 & 0.4 & - & $\mathrm{mg} / \mathrm{kg}$ \\
\hline 5 & Di-n-butylphthalate & - & 0.035 & 0.13 & 0.026 & - & 0.11 & 0.055 & $\mathrm{mg} / \mathrm{kg}$ \\
\hline 6 & Dibenz $(a, h)$ anthracene & - & - & - & - & - & 0.18 & - & $\mathrm{mg} / \mathrm{kg}$ \\
\hline 17) & Dibenzofuran & - & - & - & - & - & 0.034 & - & $\mathrm{mg} / \mathrm{kg}$ \\
\hline 18 & Diethylphthalate & - & - & - & - & - & - & 0.022 & $\mathrm{mg} / \mathrm{kg}$ \\
\hline 19 & Fluoranthene & - & - & 0.32 & 0.33 & 0.11 & $0.19^{2}$ & - & $\mathrm{mg} / \mathrm{kg}$ \\
\hline 50 & Fluorene & - & - & $m$ & - & - & 0.043 & - & $\mathrm{mg} / \mathrm{kg}$ \\
\hline ;1 & Indeno $(1,2,3$-cd)pyrene & - & - & 0.046 & 0.035 & - & 0.25 & - & $\mathrm{mg} / \mathrm{kg}$ \\
\hline 2 & Phenanthrene & - & - & 0.055 & 0.097 & 0.037 & 1.3 & - & $\mathrm{mg} / \mathrm{kg}$ \\
\hline 3 & Phenol & - & - & - & - & - & 0.024 & 0.036 & $\mathrm{mg} / \mathrm{kg}$ \\
\hline 84 & Pyrene & - & - & 0.32 & 0.35 & 0.057 & 0.5 & - & $\mathrm{mg} / \mathrm{kg}$ \\
\hline 55 & 2-Butanone & - & - & - & - & - & 0.004 & - & $\mathrm{mg} / \mathrm{kg}$ \\
\hline 6 & Acetone & - & - & 0.011 & 0.012 & 0.000 & 0.015 & 0.024 & $\mathrm{mg} / \mathrm{kg}$ \\
\hline 7 & Benzene & - & - & - & 0.001 & - & - & - & $\mathrm{mg} / \mathrm{kg}$ \\
\hline 88 & Carbon tetrachlordie & - & - & - & - & - & - & 0.039 & $\mathrm{mg} / \mathrm{kg}$ \\
\hline 9 & Methylene chloride & - & 0.012 & 0.011 & 0.014 & 0.012 & 0.023 & 0.019 & $\mathrm{mg} / \mathrm{kg}$ \\
\hline 0 & Styrene & - & - & - & - & - & 0.004 & - & $\mathrm{mg} / \mathrm{kg}$ \\
\hline 81 & Toluene & - & - & - & -- & - & 0.002 & - & $\mathrm{mg} / \mathrm{kg}$ \\
\hline 62 & Xylenes (total) & - & - & - & 0.001 & - & 0.001 & - & $\mathrm{mg} / \mathrm{kg}$ \\
\hline
\end{tabular}

$63^{2} \mathrm{No}$ detections were reported in any data set for COCs/COPCs not listed in this table.

$64{ }^{\circ}$ Maximum or $95 \%$ UCL result, depending on data censorship, as described in the calculation methodology.

$65^{\circ}$ One sample (J135P7) was excluded from radionuclide evaluation for the SPA, and will be considered separately in subsequent evaluation.

$66^{\mathrm{d}}$ Maximum detected result from focused samples 1 through 7.

$67^{\circ}$ Maximum accepted result.

$68 \ldots=$ not evaluated by laboratory analysis or not detected (for all samples in the data set)

$69 \mathrm{BCL}=$ below cleanup levels

$70 \mathrm{COC}=$ contaminant of concern

$71 \mathrm{COPC}=$ contaminant of potential concern

72 OB goverburden

$73 \mathrm{SPA}=$ staging pile area

$74 \mathrm{TPH}=$ total petroleum hydrocarbons

$75 \mathrm{UCL}=$ upper confidence limit 
CVP-2006-00011

Rev. 0

E-108 
Washington Closure Hanford

$$
\text { Originator J.M. Capron Q }
$$

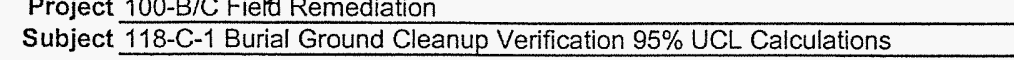

CALCULATION SHEET

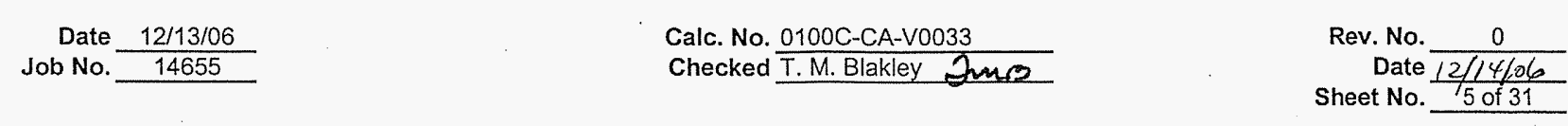

1118-C-1 Area 1 Statistical Calculations

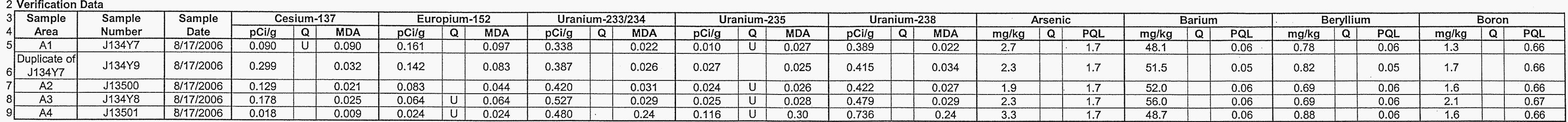

\section{Statistical Computation Input Data}

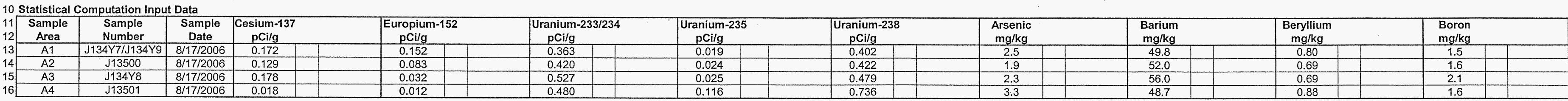

\section{Statistical Computations}

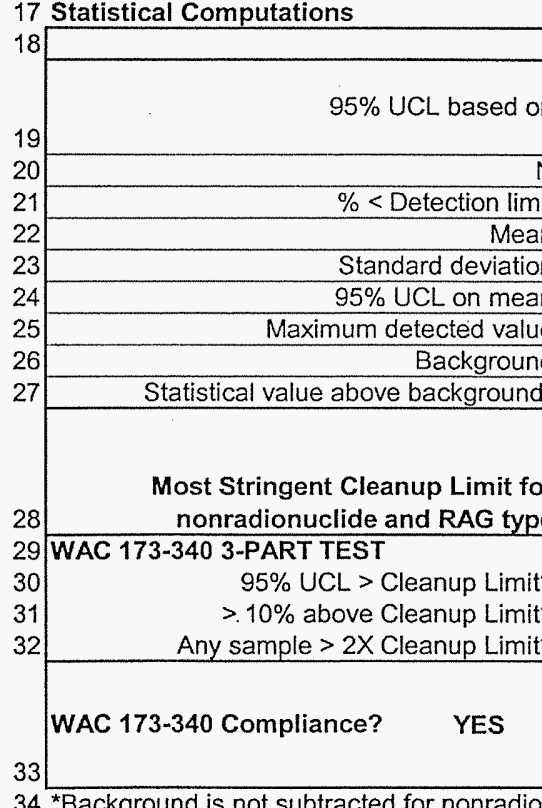

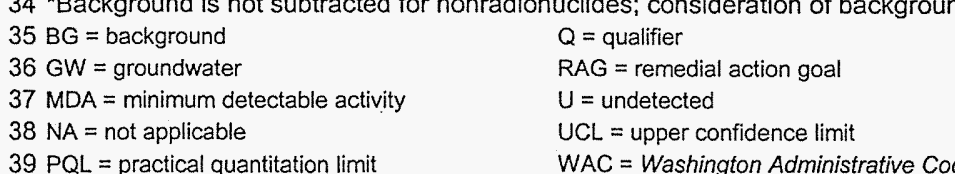

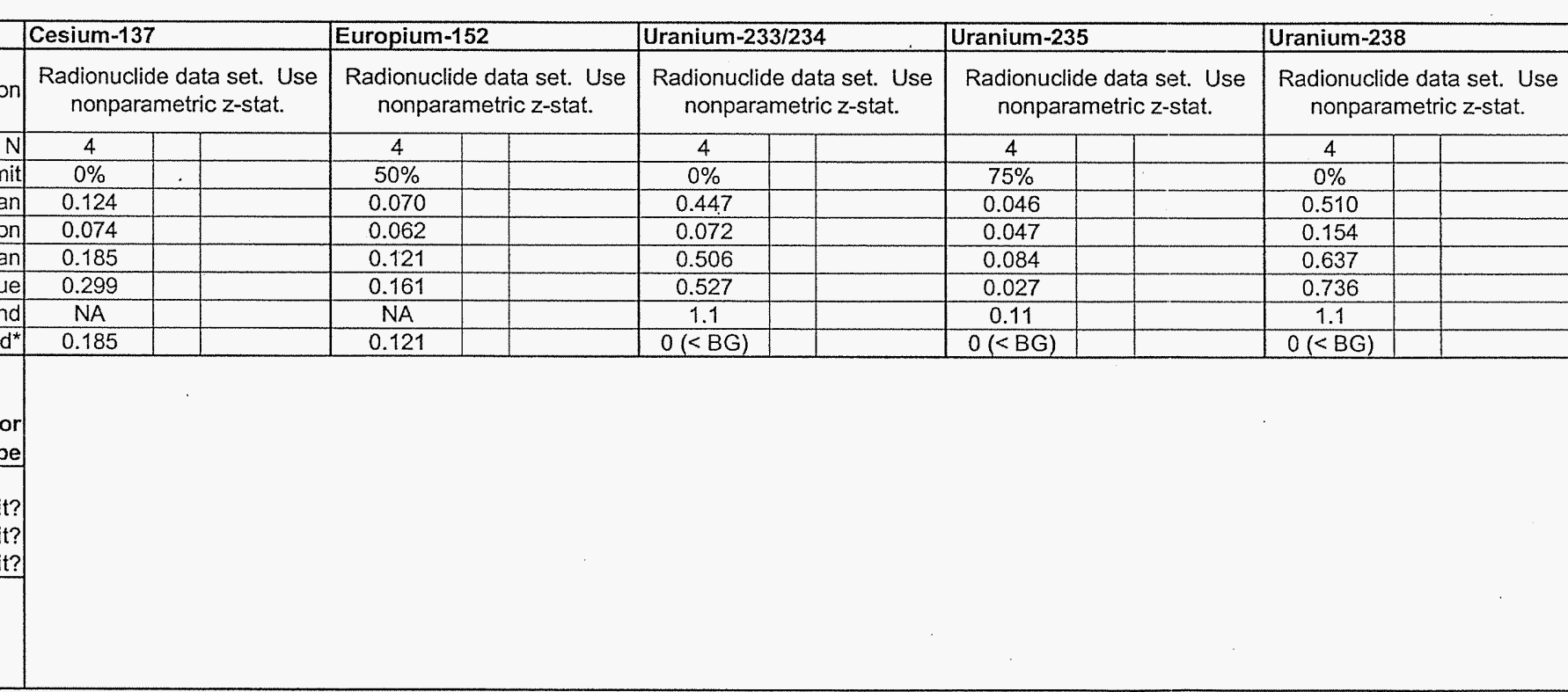

W = upper conifidence limit

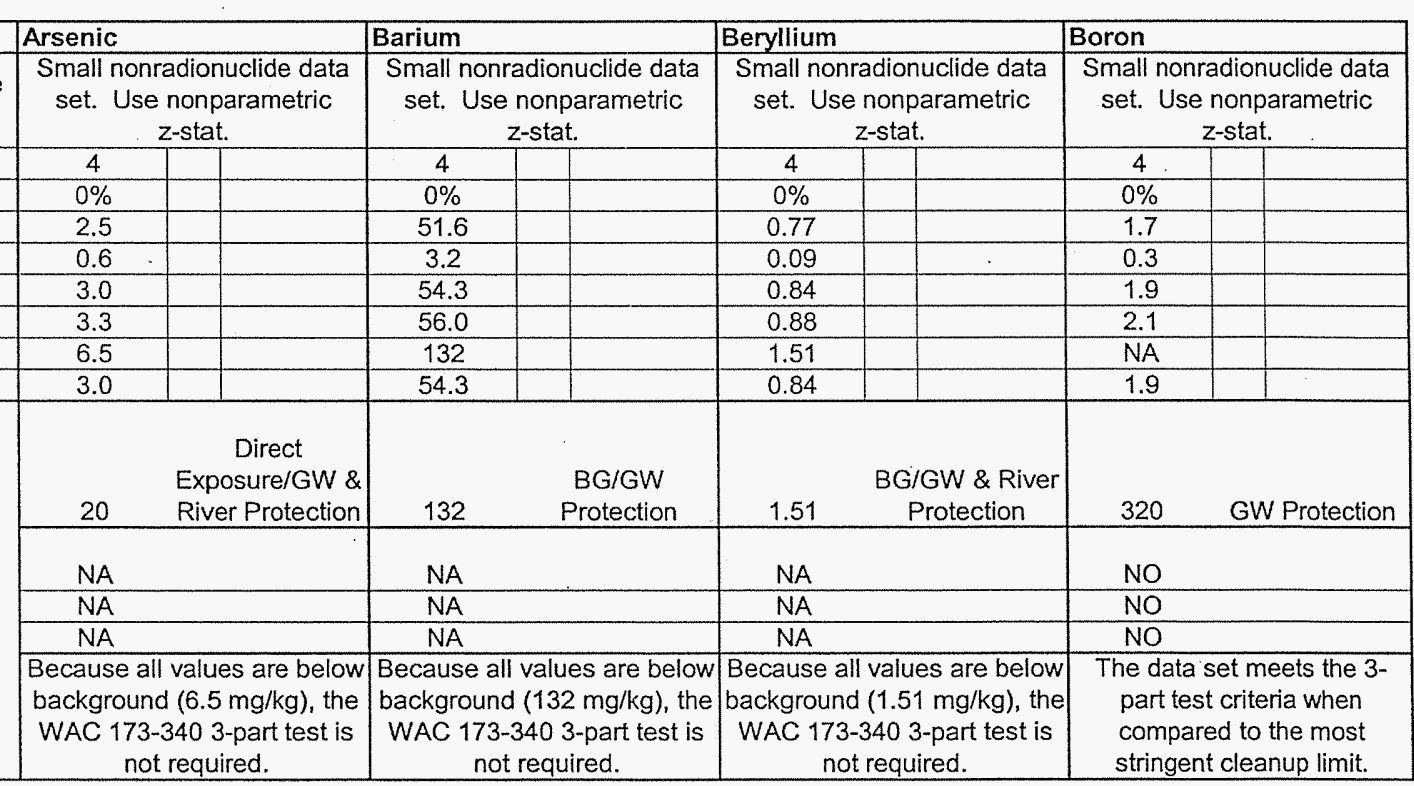

173-340 3-part test is 
1 118-C-1 Area 1 Statistical Calculations (continued)

\begin{tabular}{|c|c|c|c|c|c|c|c|c|c|c|c|c|c|c|c|c|c|c|c|c|c|c|c|c|c|c|}
\hline \multirow{2}{*}{\begin{tabular}{l|c} 
S Sample \\
4 & Area \\
5 & A1 \\
5
\end{tabular}} & \multirow{2}{*}{$\begin{array}{l}\text { Sample } \\
\text { Number } \\
\end{array}$} & \multirow{2}{*}{$\begin{array}{c}\begin{array}{c}\text { Sample } \\
\text { Date }\end{array} \\
8 / 17 / 2006\end{array}$} & \multicolumn{3}{|c|}{ Chromium } & \multicolumn{3}{|c|}{ Cobalt } & \multicolumn{3}{|c|}{ Copper } & \multicolumn{3}{|c|}{ Lead } & \multicolumn{3}{|c|}{ Manganese } & \multicolumn{3}{|c|}{ Nickel } & \multicolumn{3}{|c|}{ Vanadium } & \multicolumn{3}{|c|}{ Zinc } \\
\hline & & & $\frac{\mathrm{mg} / \mathrm{kg}}{56}$ & $\frac{Q}{C}$ & $\begin{array}{l}P Q L \\
036\end{array}$ & $\frac{\mathrm{mg} / \mathrm{kg}}{7 \mathrm{~g}}$ & $Q$ & $P Q L$ & $\frac{\mathrm{mg} / \mathrm{kg}}{117}$ & Q & $P Q L$ & $\mathrm{mg} / \mathrm{kg}$ & $Q$ & $\frac{P Q L}{086}$ & $\frac{\mathrm{mg} / \mathrm{kg}}{20 \mathrm{~g}}$ & $Q$ & $P Q L$ & $\mathrm{mg} / \mathrm{kg}$ & $Q$ & $P Q L$ & $\frac{\mathrm{mg} / \mathrm{kg}}{362}$ & $Q$ & $\begin{array}{l}P Q L \\
025\end{array}$ & $\frac{\mathrm{mg} / \mathrm{kg}}{330}$ & & $\begin{array}{l}P Q L \\
044\end{array}$ \\
\hline 5 & $\begin{array}{l}J 134 Y 7 \\
J 134 Y 9\end{array}$ & $\begin{array}{l}8 / 17 / 2006 \\
8 / 17 / 2006\end{array}$ & $\begin{array}{l}5.6 \\
6.6\end{array}$ & c & $\begin{array}{l}0.36 \\
0.36\end{array}$ & $\begin{array}{l}7.0 \\
7.9\end{array}$ & & $\begin{array}{l}0.39 \\
0.38\end{array}$ & 11.7 & & $\begin{array}{l}0.33 \\
0.33\end{array}$ & $\begin{array}{l}4.5 \\
4.5\end{array}$ & & $\begin{array}{l}0.80 \\
0.85\end{array}$ & 328 & & 0.08 & 9.5 & & $\begin{array}{l}0.00 \\
0.66\end{array}$ & 41.6 & & $\begin{array}{l}0.25 \\
0.25\end{array}$ & 33.0 & & 0.44 \\
\hline A2 & $J 13500$ & $8 / 17 / 2006$ & 8.6 & C & 0.36 & 5.9 & & 0.39 & 9.9 & & & 4.1 & & 0.86 & & & & & & & 31.1 & & 0.25 & 30.1 & & 0.44 \\
\hline & J134Y8 & $8 / 17 / 2006$ & 6.8 & C & 0.36 & 5.7 & & 0.39 & 11.3 & & 0.33 & 3.5 & & 0.86 & 259 & & 0.08 & 9.9 & & 0.67 & 31.4 & & 0.25 & 32.2 & & 0.45 \\
\hline A4 & $J 13501$ & $8 / 17 / 2006$ & 6.0 & C & 0.36 & 8.4 & & 0.39 & 14.7 & & 0.33 & 4.9 & & 0.86 & 362 & & 0.08 & 10.2 & & 0.66 & 41.1 & & 0.25 & 39.1 & & 0.44 \\
\hline
\end{tabular}

\begin{tabular}{c|c|c|c}
\hline A4 & J13501 & $8 / 17 / 2$ \\
\hline
\end{tabular}

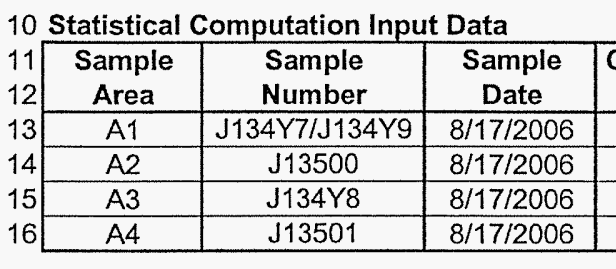

17 Statistical Computations

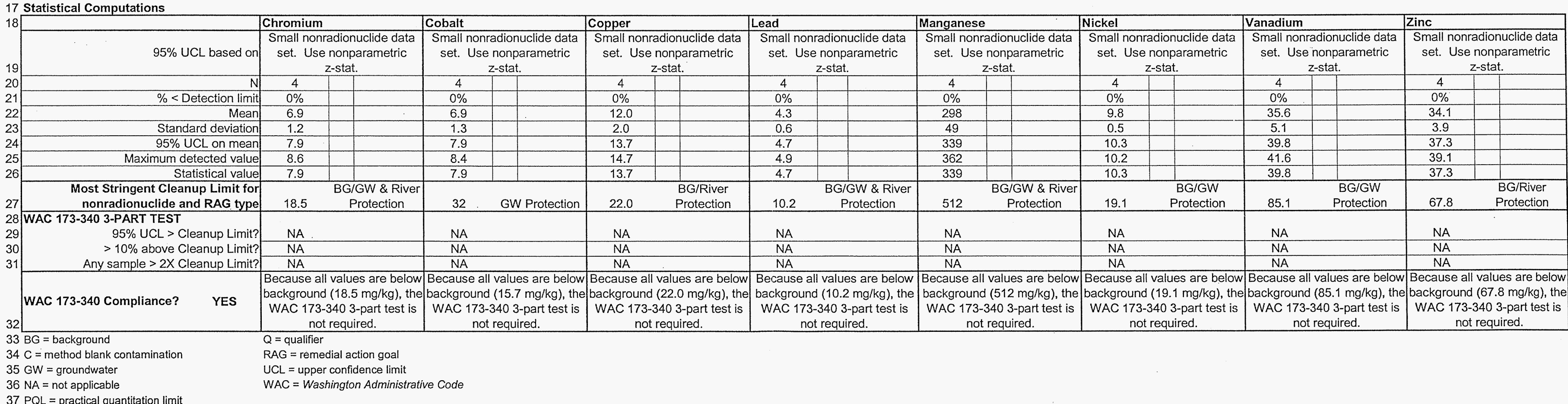


Washington Closure Hanford

Originator J.M. Capron $f m c$

Project $100-B / C$ Field Remediation
Subject $118-C-1$ Burial Ground Cleanup Verification 95\% UCL Calculations
CALCULATION SHEET

Job No. $\stackrel{12 / 13106}{14655}$
Calc. No. 0100C-CA-V0033
Checked T. M. Blakley $2 \mathrm{mBs}$
Rev. No. $\frac{0}{\text { Date }} \frac{12 / 14 / 06}{7 / 06}$
Sheet No. 31

\section{8-C-1 Area 2 Statistical Calculations}

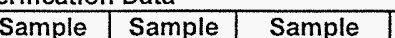

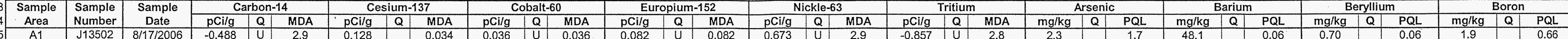

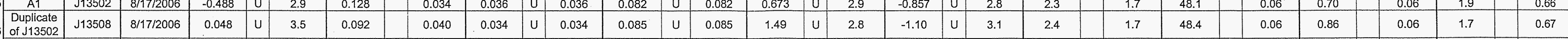

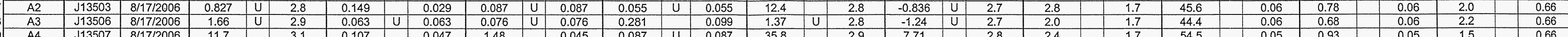

\begin{tabular}{|c|c|c|c|c|c|c|c|c|c|c|c|c|}
\hline $\begin{array}{lll}11 & \text { Sample } \\
12 & \text { Area } \\
\end{array}$ & $\begin{array}{l}\text { Sample } \\
\text { Number } \\
\text { umber }\end{array}$ & $\begin{array}{c}\text { Sample } \\
\text { Date }\end{array}$ & $\begin{array}{c}\text { Carbon-14 } \\
\text { pcilg }\end{array}$ & \begin{tabular}{|c} 
Cesium-1 \\
pCilg
\end{tabular} & $\begin{array}{c}\text { Cobalt-60 } \\
\text { pCilg } \\
\end{array}$ & \begin{tabular}{|} 
Europiur \\
pcilg
\end{tabular} & $\begin{array}{c}\text { Nickle-6: } \\
\text { pCilgg }\end{array}$ & $\begin{array}{c}\begin{array}{c}\text { Tritium } \\
\text { pcilg }\end{array} \\
\text { s. }\end{array}$ & $\begin{array}{c}\text { Arseni } \\
\mathrm{mg} / \mathrm{kg}\end{array}$ & $\begin{array}{l}\text { Barium } \\
\text { mglkg }\end{array}$ & $\begin{array}{c}\text { Berylliur } \\
\text { mglkg }\end{array}$ & $\begin{array}{l}\text { Boron } \\
\text { mglkg }\end{array}$ \\
\hline $\mathrm{A} 1$ & $\begin{array}{l}\mathrm{J} 13502 / \\
\mathrm{J} 13508\end{array}$ & $8 / 17 / 2006$ & -0.220 & 0.110 & 0.018 & 0.042 & 1.08 & -0.98 & 2.4 & 48.3 & 0.78 & 1.8 \\
\hline$A 2$ & \begin{tabular}{|l|l|}
113503 \\
13503
\end{tabular} & $8 / 17 / 2006$ & 0.827 & \begin{tabular}{|l|l|}
0.149 \\
\end{tabular} & \begin{tabular}{|l|l|} 
\\
\end{tabular} & $\begin{array}{l}.028 \\
\end{array}$ & $\begin{array}{l}12.4 \\
1.27 \\
\end{array}$ & -0.836 & 2.8 & 45.6 & 0.78 & $\begin{array}{l}2.0 \\
.2\end{array}$ \\
\hline$\frac{A_{3}}{A_{4}}$ & \begin{tabular}{|l|}
13506 \\
13507
\end{tabular} & $\begin{array}{l}881 / 1712006 \\
811 / 2006\end{array}$ & $\begin{array}{l}1.66 \\
11.7\end{array}$ & \begin{tabular}{|l|}
0.032 \\
0.107 \\
\end{tabular} & \begin{tabular}{|l}
.038 \\
1.48 \\
\end{tabular} & $\begin{array}{l}0.281 \\
0.044 \\
\end{array}$ & $\begin{array}{r}1.31 \\
35.8 \\
\end{array}$ & $\frac{-1.24}{7.71}$ & 2.0 & $\frac{44.4}{54.5}$ & $\begin{array}{l}0.68 \\
0.93 \\
\end{array}$ & $\begin{array}{l}2.2 \\
1.5\end{array}$ \\
\hline
\end{tabular}

17 Statistical Computations

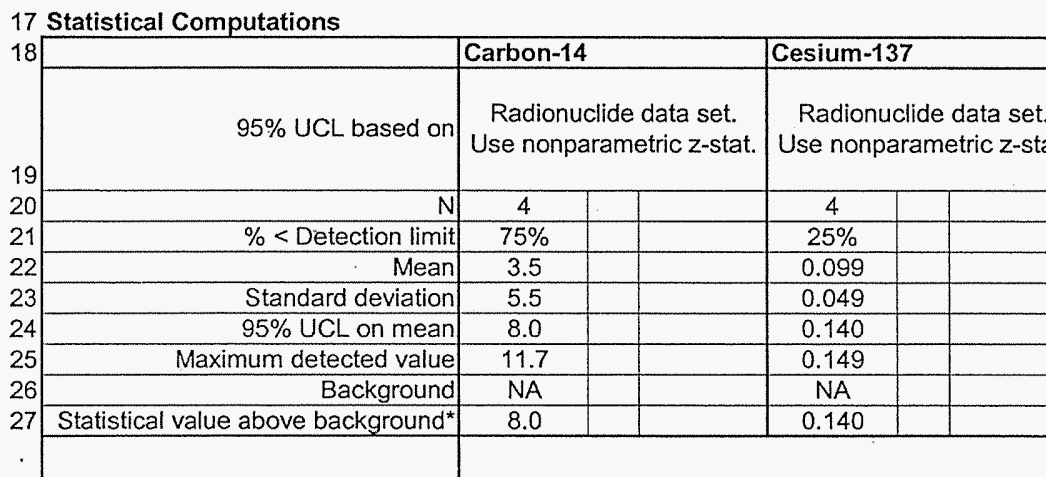

Cobalt-60
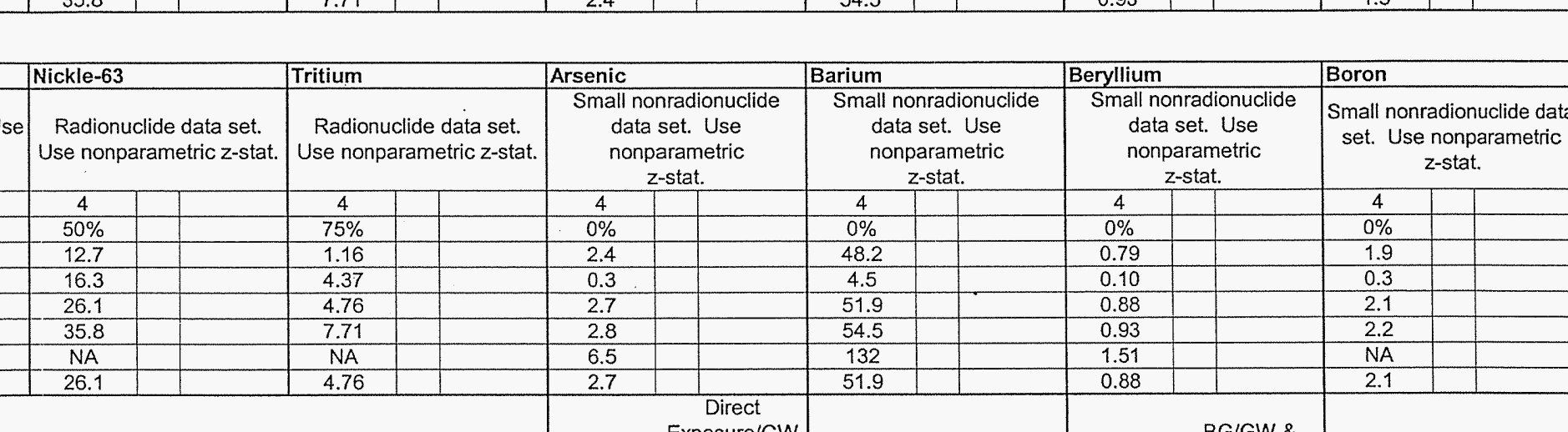

\section{Most Stringent Cleanup Limit for
nonradionuclide and RAG type}

28 nonradionuclide and RAG tyP

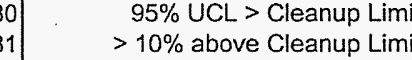

Any sample $2 \times$ Cleanup Limit?

WAC 173-340

YES

${ }_{34}{ }^{*}$ Background is not subtracted for no

$35 \mathrm{BG}=$ background
$36 \mathrm{GW}=$ groundwater

$36 \mathrm{GW}=$ groundwater

$38 \mathrm{NA}=$ not applicable Q
$Q=$ qualifier
RAs

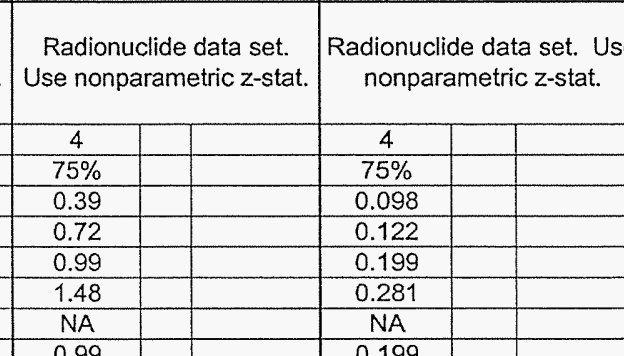

Small nonradionuclide dat

$39 \mathrm{PQL}=$ p practical quantitation limit $Q=$ qualifier
$\mathrm{RAG}=$ remedial action goal
$U=$ undetetected

UCL = upper confidence limit
WAC $=$ Washington Administrative Code

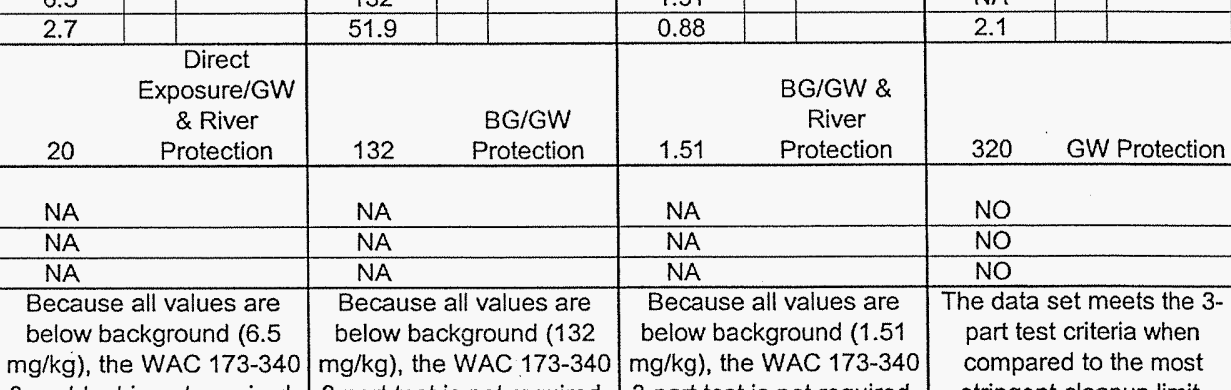


Washington Closure Hanford

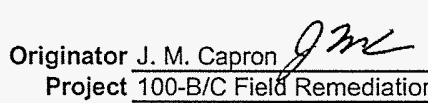

Project 100-B/C Fiell Remediation
CALCULATION SHEET
Date $\frac{12 / 13 / 06}{14655}$
Job No.
Calc. No. $\frac{0100 C-C A-V 0033}{}$
Checked T.M. Blakley $2 \mathrm{~mm}$
Rev. No. $\frac{0}{\text { Date }}$
Sheet No. $\frac{18 / 14 / 06}{80 / 31}$

1 118-C- 1 Area 2 Statistical Calculations (continued)
2 Verification Data

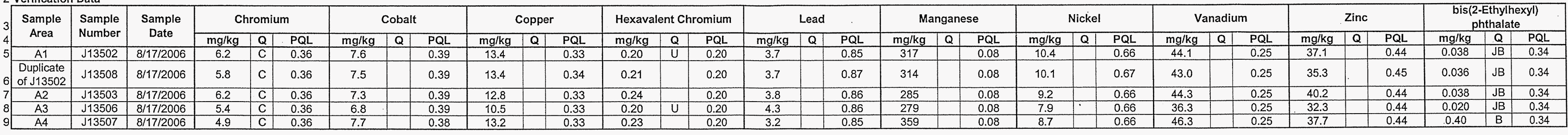

10 Statistical Computation Input Data

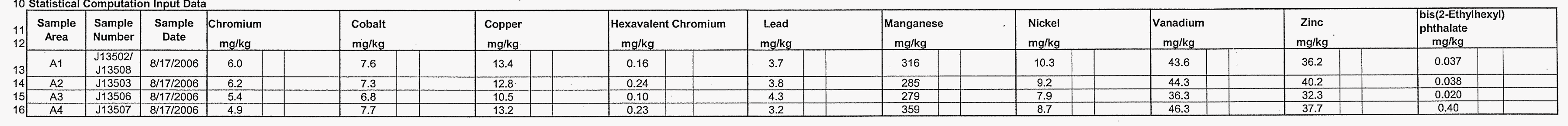

17 Statistical Computations

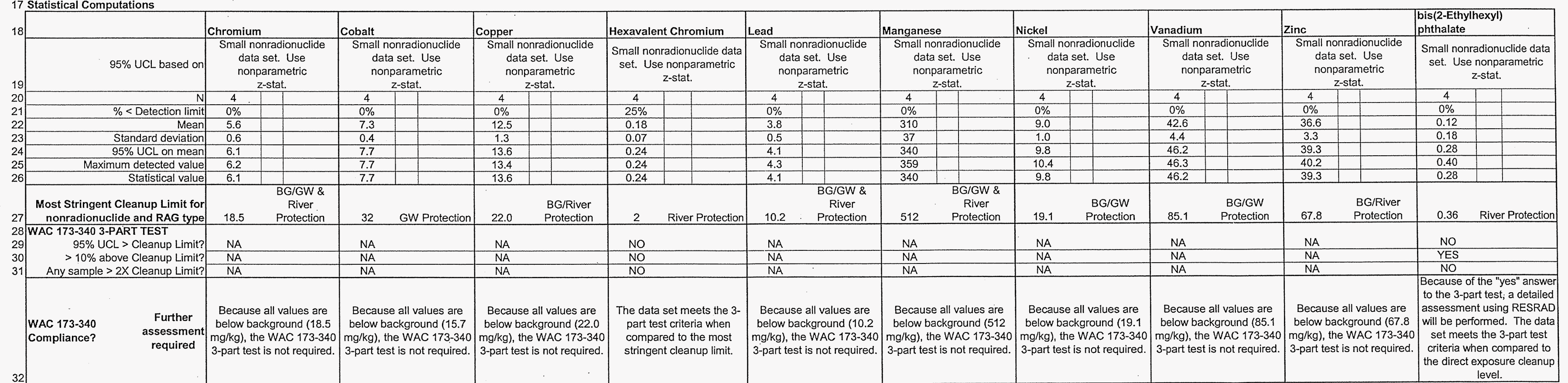

${ }_{33}^{32} \mathrm{C}=$ method blank contamination (inorganic constitutents)

RAG = remedial action goal exposure cond
level.

$34 \mathrm{BG}=$ background
$35 \mathrm{GW}=$ groundwater

$36 \mathrm{NA}=$ not applicable
$37 \mathrm{PQL}=$ practical quantitition limit
$38 \mathrm{Q}=$ qualfier

RESRAD $=$ RES
$U=$ undetected

UCL $=$ upper confidence limit
WAC $=$ Washington Administrative Code 
Washington Closure Hanford

Originator J. M. Capron Zson

Project $100-\mathrm{B} / \mathrm{C}$ Field Remediation

118-C-1 Area 2 Statistical Calculations (continued)

2 Verification Data

\begin{tabular}{|c|c|c|c|c|c|c|}
\hline & \multirow{2}{*}{$\begin{array}{c}\text { Sample } \\
\text { Area }\end{array}$} & \multirow{2}{*}{$\begin{array}{l}\text { Sample } \\
\text { Number }\end{array}$} & \multirow{2}{*}{$\begin{array}{c}\text { Sample } \\
\text { Date }\end{array}$} & \multicolumn{3}{|c|}{ Methylene Chloride } \\
\hline & & & & $\mathrm{mg} / \mathrm{kg}$ & $\mathbf{Q}$ & $\mathrm{PQL}$ \\
\hline & $\mathrm{A} 1$ & J135R0 & $8 / 28 / 2006$ & 0.005 & $\bar{B}$ & 0.005 \\
\hline & $\begin{array}{l}\text { Duplicate } \\
\text { of } \mathrm{J} 135 \mathrm{R} 0\end{array}$ & J135T6 & $8 / 28 / 2006$ & 0.011 & B & 0.005 \\
\hline & $\mathrm{A} 2$ & J135R1 & $8 / 28 / 2006$ & 0.006 & $B$ & 0.005 \\
\hline & $\mathrm{A} 3$ & J135R2 & $8 / 28 / 2006$ & 0.009 & $B$ & 0.006 \\
\hline & A4 & J135R3 & $8 / 28 / 2006$ & 0.014 & B & 0.006 \\
\hline
\end{tabular}

10 Statistical Computation Input Data

\begin{tabular}{|c|c|c|c|c|c|}
\hline & $\begin{array}{c}\text { Sample } \\
\text { Area }\end{array}$ & $\begin{array}{l}\text { Sample } \\
\text { Number }\end{array}$ & $\begin{array}{c}\text { Sample } \\
\text { Date }\end{array}$ & \multicolumn{2}{|c|}{$\begin{array}{l}\text { Methylene Chloride } \\
\mathrm{mg} / \mathrm{kg}\end{array}$} \\
\hline & $\mathrm{A} 1$ & $\begin{array}{l}\mathrm{J} 13502 / \\
\mathrm{J} 13508\end{array}$ & $8 / 28 / 2006$ & 0.008 & \\
\hline & A2 & J135R1 & $8 / 28 / 2006$ & 0.006 & \\
\hline & A3 & J135R2 & $8 / 28 / 2006$ & 0.009 & \\
\hline & A4 & J135R3 & $8 / 28 / 2006$ & 0.014 & \\
\hline
\end{tabular}

17 Statistical Computations

\begin{tabular}{|c|c|c|c|}
\hline 8 & & \multirow{2}{*}{\multicolumn{2}{|c|}{$\begin{array}{c}\text { Methylene Chloride } \\
\text { Small nonradionuclide } \\
\text { data set. Use } \\
\text { nonparametric } \\
\text { z-stat. }\end{array}$}} \\
\hline 19 & $95 \%$ UCL based on & & \\
\hline 20 & $N$ & 4 & \\
\hline 21 & $\%<$ Detection limit & $0 \%$ & \\
\hline 22 & Mean & 0.009 & \\
\hline 23 & Standard deviation & 0.003 & \\
\hline 24 & $95 \%$ UCL on mean & 0.012 & \\
\hline 25 & Maximum detected value & 0.014 & \\
\hline 26 & Statistical value & 0.012 & \\
\hline 27 & $\begin{array}{l}\text { Most Stringent Cleanup Limit for } \\
\text { nonradionuclide and RAG type }\end{array}$ & 0.5 & GW Protection \\
\hline 28 & WAC $173-340$ 3-PART TEST & & \\
\hline 29 & $95 \%$ UCL > Cleanup Limit? & No & \\
\hline 30 & $>10 \%$ above Cleanup Limit? & NO & \\
\hline 31 & Any sample $>2 \times$ Cleanup Limit? & NO & \\
\hline & $\begin{array}{l}\text { WAC 173-340 } \\
\text { Compliance? }\end{array}$ & $\begin{array}{l}\text { The data } \\
\text { part tes } \\
\text { compar } \\
\text { stringer }\end{array}$ & $\begin{array}{l}\text { meets the } 3- \\
\text { criteria when } \\
d \text { to the most } \\
\text { cleanup limit. }\end{array}$ \\
\hline
\end{tabular}

$33 \mathrm{~B}=$ method blank contamination (organic constitutents)

$34 \mathrm{GW}=$ groundwater

$35 \mathrm{PQL}=$ practical quantitation limit

$36 \mathrm{Q}=$ qualifier

37 RAG = remedial action goal

$38 \mathrm{UCL}=$ upper confidence limit

39 WAC $=$ Washington Administrative Code

\section{CALCULATION SHEET}

Date $12 / 13 / 06$ 95\% UCL Calculations
Calc. No. 0100C-CA-V0033 Rev. No. 0 Checked T. M. Blakley $2 \mathrm{~mm}$ Date $12 / 14 / 06$ Sheet No. 9 of 31 
CVP-2006-00011

Rev. 0

E-114 
Washington Closure Hanford

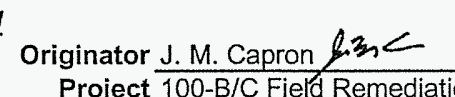
Sroject $\frac{1 \text { 100-B/C Fiela Remediation }}{118-C-1 \text { Burial Ground Cleanup Verification } 95 \% \text { UCL Calculations }}$
CALCULATION SHEET
\begin{aligned} & Date $12 / 13 / 06 \\ &$ Job No. 14655 \\ & \hline\end{aligned}
Calc. No. 0100 C-CA-V0033
Checked T.M. Blakley Jmps
Rev. No.
Sheet No. $\frac{12 / 4 / 06}{10 \text { of } 31}$

1118-C-1 Area 3 Statistical Calculation

\begin{tabular}{|c|c|c|c|c|c|c|c|c|c|c|c|c|c|c|c|c|c|c|c|c|c|c|c|c|c|}
\hline \multirow{3}{*}{\begin{tabular}{|c|c|}
3 & Sample \\
4 & Area \\
5 & A1 \\
\end{tabular}} & \multirow{3}{*}{$\begin{array}{l}\text { Satample } \\
\text { Sumber } \\
\text { Number }\end{array}$} & \multirow{2}{*}{$\begin{array}{c}\text { Sample } \\
\text { Date }\end{array}$} & \multirow{2}{*}{\multicolumn{2}{|c|}{ Cesium-137 }} & \multirow{2}{*}{\multicolumn{3}{|c|}{ Cobalt-60 }} & \multirow{2}{*}{\multicolumn{3}{|c|}{ Europium-152 }} & \multicolumn{3}{|c|}{ Strontium-90 } & \multirow{2}{*}{\multicolumn{3}{|c|}{ Uranium-233/234 }} & \multicolumn{3}{|c|}{ Uranium-238 } & \multirow{2}{*}{$\begin{array}{c}\begin{array}{c}\text { Sampling } \\
\text { Area }\end{array} \\
\end{array}$} & \multirow{2}{*}{$\begin{array}{c}\text { Sample } \\
\text { Number }\end{array}$} & \multirow{2}{*}{$\begin{array}{c}\text { Sample } \\
\text { Date }\end{array}$} & \multicolumn{3}{|c|}{ Methylene Chloride } \\
\hline & & & & \begin{tabular}{|l|l|}
$Q$ & MDA \\
\end{tabular} & & & & & & & 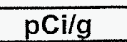 & Q & MDA & & & & pcilg & $\mathbf{Q}$ & MDA & & & & $\mathrm{mg} / \mathrm{kg}$ & & \\
\hline & & & 0.195 & 0.03 & 0.034 & U & 0.034 & & U & 0.069 & & u & 0.27 & 0.364 & & 0.25 & 0.265 & & 0.25 & A1 & & & 0.012 & B & s. \\
\hline$A^{2}$ & J13511 & $8 / 21 / 2006$ & 0.240 & 0.020 & 0.042 & +1 & 0.025 & 0.138 & & 0.053 & 0.087 & $\mathrm{u}$ & 0.26 & 1.09 & & 0.20 & 0.825 & & & $\mathrm{~A} 2$ & & & 0.008 & B & 0.005 \\
\hline & $\mathrm{J} 13512$ & $8 / 21 / 2006$ & 0.958 & 0.049 & 0.061 & U & 0.061 & 0.140 & & 0.097 & 0.279 & & 0.19 & 0.235 & $u$ & & & & & & $135 \mathrm{R} 6$ & 06 & 0.009 & & 0.005 \\
\hline A4 & $J 13513$ & $8 / 21 / 2006$ & 0.227 & 0.027 & 0.024 & $U$ & 0.024 & 0.082 & & 0.046 & 0.263 & & 0.21 & 0.328 & - & 0. & 0.438 & & 0.21 & $\mathrm{~A} 4$ & J135R7 & & 0.010 & & .005 \\
\hline
\end{tabular}

9 Statistical Computation Input Data

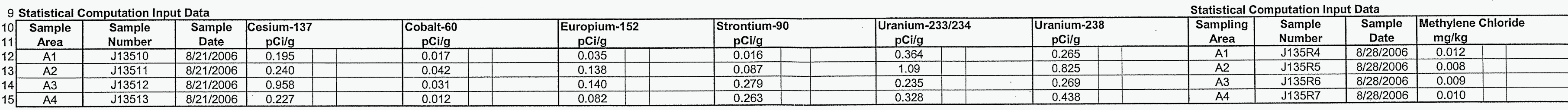

16 Statistical Computations

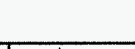

Statistical Computations

\begin{tabular}{|c|c|c|c|c|c|c|c|c|c|c|c|c|c|c|c|c|}
\hline \multirow{2}{*}{17 | } & s & \multicolumn{2}{|c|}{ Tesium-137 } & \multicolumn{2}{|l|}{ Cobalt-60 } & \multicolumn{2}{|c|}{ Europium-152 } & \multicolumn{2}{|c|}{ Strontium-90 } & \multicolumn{4}{|c|}{\begin{tabular}{|l|l|} 
Uranium-233/234 & Uranium-238 \\
\end{tabular}} & \multicolumn{3}{|c|}{\begin{tabular}{|l|l|} 
Methylene Chloride \\
\end{tabular}} \\
\hline & $95 \%$ UCL based on & \multicolumn{2}{|c|}{$\begin{array}{l}\text { Radionuclide data set. Use } \\
\text { nonparametric } z \text {-stat. }\end{array}$} & \multicolumn{2}{|c|}{$\begin{array}{l}\text { Radionuclide data set. Use } \\
\text { nonparametric z-stat. }\end{array}$} & \multicolumn{2}{|c|}{$\begin{array}{l}\text { Radionuclide data set. Use } \\
\text { nonparametric z-stat. }\end{array}$} & \multicolumn{2}{|c|}{$\begin{array}{l}\text { Radionuclide data set. Use } \\
\text { nonparametric z-stat. }\end{array}$} & \multicolumn{2}{|c|}{$\begin{array}{l}\text { Radionuclide data set. Use } \\
\text { nonparametric z-stat. }\end{array}$} & \multicolumn{2}{|c|}{$\begin{array}{l}\text { Radionuclide data set. Use } \\
\text { nonparametric z-stat. }\end{array}$} & \multirow[t]{2}{*}{$95 \%$ UCL based on } & \multicolumn{2}{|c|}{$\begin{array}{l}\text { Small nonradionuclide data } \\
\text { set. Use nonparametric } \\
\text { z-stat }\end{array}$} \\
\hline \multirow{2}{*}{$\begin{array}{l}18 \\
19 \\
20 \\
21\end{array}$} & & 4 & & 4 & & 4 & & 4 & & 4 & & 4 & & & 4 & \\
\hline & $\%<$ Detection limit & $0 \%$ & & $75 \%$ & & $25 \%$ & & $50 \%$ & 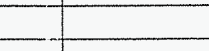 & $25 \%$ & & $0 \%$ & & $\%<$ Detection limit & $0 \%$ & \\
\hline \multirow{2}{*}{$\begin{array}{l}21 \\
22 \\
23\end{array}$} & $\begin{array}{r}\text { Mean } \\
\text { Standard devititan }\end{array}$ & 0.405 & & 0.025 & & 0.099 & & 0.161 & & 0.50 & & 0.449 & & $\begin{aligned} \text { Mean } \\
\text { Standard deviation }\end{aligned}$ & 0.010 & \\
\hline & $\begin{array}{l}\text { Standard deviation } \\
95 \% \text { UCL on mean }\end{array}$ & 0.369 & & 0.014 & & $\frac{0.050}{0.140}$ & & 0.130 & 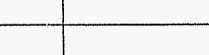 & 0.39 & & 0.2663 & & $\begin{array}{l}\text { Standara deviation } \\
95 \% \text { UCL on mean }\end{array}$ & 0.011 & \\
\hline \multirow{2}{*}{24} & Maximum detected value & 0.958 & & 0.042 & & 0.140 & & 0.279 & & 1.09 & & 0.825 & & Maximum detected value & e 0.012 & \\
\hline & Background & NA & & NA & & NA & & NA & & 1.1 & & 1.1 & & Background & NA & \\
\hline \multirow[t]{2}{*}{26} & Statistical value above background* & 0.709 & & 0.037 & & 0.140 & & 0.268 & & $0(<B G)$ & & $0(<\mathrm{BG})$ & & Statistical value above background* & \multirow{2}{*}{\multicolumn{2}{|c|}{$0.5 \quad$ GW Protection }} \\
\hline & & & & & & & & & & & & & & $\begin{array}{c}\text { Most Stringent Cleanup Limit tor } \\
\text { nonradionuclide and RAG type }\end{array}$ & & \\
\hline \multirow{4}{*}{$\begin{array}{l}27 \\
28 \\
29 \\
30 \\
31\end{array}$} & & & & & & & & & & & & & & WAC 173-340 3-PART TEST & \multirow{3}{*}{\multicolumn{2}{|c|}{$\begin{array}{l}\text { NO } \\
\text { NO } \\
\text { NO }\end{array}$}} \\
\hline & & & & & & & & & & & & & & $>10 \%$ above Cleanup Limit? & & \\
\hline & & & & & & & & & & & & & & Any sample $>2 X$ Cleanup Limit? & & \\
\hline & & & & & & & & & & & & & & WAC 173-340 Compliance? & \multicolumn{2}{|c|}{$\begin{array}{l}\text { NO } \\
\text { The data set meets the 3- } \\
\text { part test criteria when } \\
\text { compared to the most } \\
\text { stringent cleanup limit. }\end{array}$} \\
\hline
\end{tabular}

$33^{\star}$ Background is not subtracted for nonradionuclides; consideration of background is given by direct comparison against background values.

$34 \mathrm{~B}=$ method blank contamination $\quad Q=$ qualifier

$35 \mathrm{BG}=$ background

$R A G=$ remedial action goal

$36 \mathrm{GW}=$ groundwater
$37 \mathrm{MDA}=$ minimum detectable activity

$38 \mathrm{NA}=$ not applicable
$39 \mathrm{PQL}=$ practical quantitation limit

UCL $=$ upper confidence limit
WAC $=$ Washington Administrative Code 
Originator J.M. Capron 2 और

Project $\frac{100-\mathrm{B} / \mathrm{C} \text { Field Remediation }}{118-\mathrm{C}-1 \text { Burial Ground Cleanup Verification } 95 \% \text { UCL Calculations }}$
CALCULATION SHEET
Calc. No. $0100 C-$ CA-V0033
Checked T. M. Blakley $2 \mathrm{mB}$
Rev. No. $\frac{0}{12 / 14 / 06}$
Date
Sheet No. 11 of 31

1.118-C-1 Area 3 Statistical Calculations (continued)

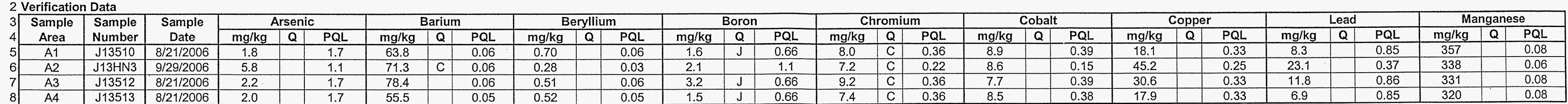

\section{Statistical Computation

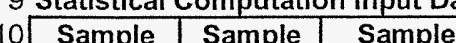

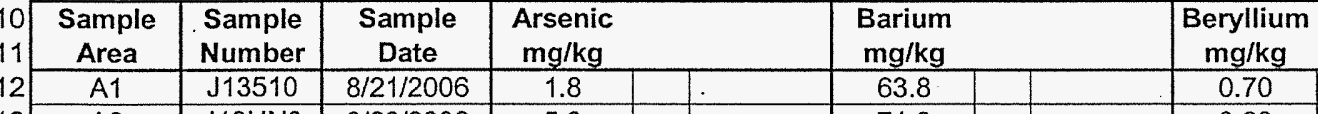

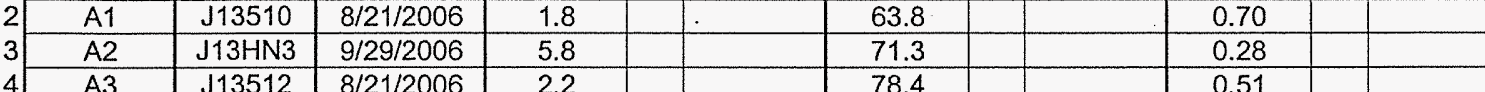

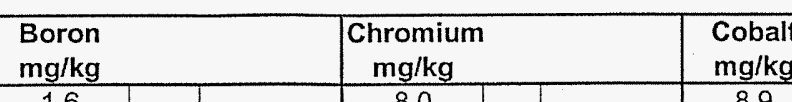

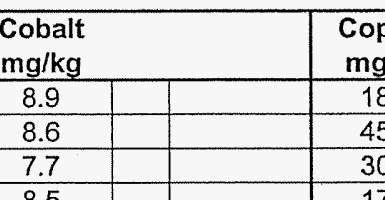

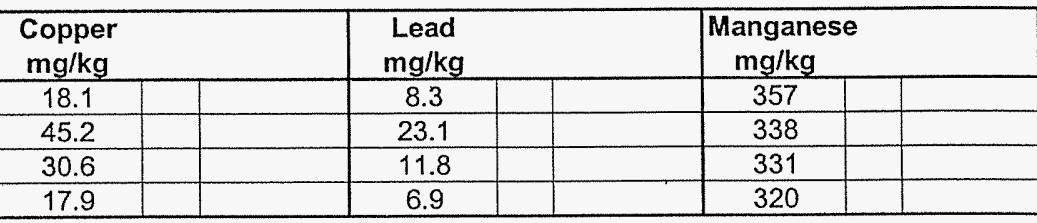

16 Statistical Computations
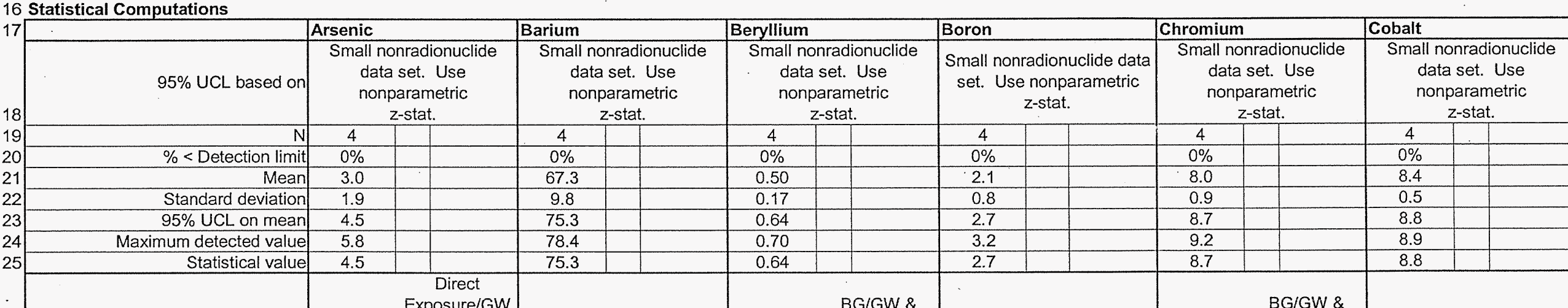

Most Stringent Cleanup Limit for $20 \quad$ \& River \begin{tabular}{lll}
\hline WAG 173-340 3-PART TEST & Protection \\
\hline
\end{tabular} $95 \%$ UCL > Cleanup Limit? NA

$29>10 \%$ above Cleanup Lim Any sample $>2 X$ Cleanup Limit? $N A$

WAC 173-340 Fur

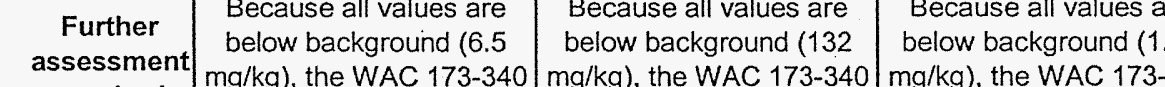

$\mathrm{mg} / \mathrm{kg}$ ), the WAC 173-340 $\mathrm{mg} / \mathrm{kg}$ ), the WAC

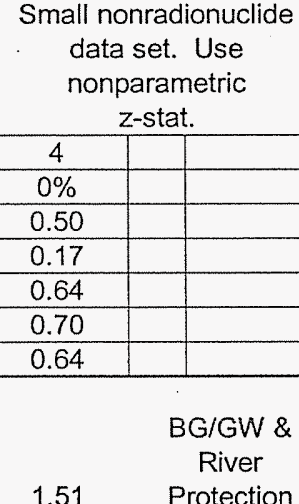

320 aw protion

$B G / G W$ \&

132 Protection

$32 \mathrm{C}=$ method blank contamination (inorganic constitutents)

$33 \mathrm{BG}=$ background

$34 \mathrm{GW}=$ groundwater
$35 \mathrm{NA}=$ not applicable

$36 \mathrm{PQL}=$ practical quantitation limit

$Q=$ qualifier
$R A G=$ remedial action goal

Copper

Small nonradionuclide

\begin{tabular}{c|c|c}
$\begin{array}{c}\text { Small nonradionuclide data } \\
\text { set. Use nonparametric }\end{array}$ & $\begin{array}{c}\text { Sinall nonradionuclide data } \\
\text { set. Use nonparametric }\end{array}$ & $\begin{array}{r}\text { Small nonradionuch } \\
\text { data set. Use } \\
\text { nonparametric }\end{array}$ \\
\hline
\end{tabular}

\begin{tabular}{|l|l|l|l|l|l|}
\multicolumn{2}{c|}{ z-stat. } & \multicolumn{2}{|c|}{ z-stat. } & \multicolumn{2}{|c}{ z-stat. } \\
\hline 4 & & 4 & & 4 & \\
\hline
\end{tabular}

28.0
12.9

\begin{tabular}{l|l}
38.6 \\
45.2
\end{tabular}

BG/River

1.51 Protection

NO
NO
NO

\begin{tabular}{|l|l} 
NA & NA \\
\hline
\end{tabular}

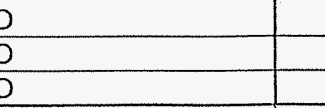

18.5 Protection

32 GW Protection

\begin{tabular}{ll} 
BG/River \\
$22.0 \quad$ Protection \\
\hline
\end{tabular}

The data set meets the

part test criteria when

stringent cleanup limit

Because all values are

NA
NA
NA

\begin{tabular}{l|ll} 
& 10.2 & Protection \\
\hline YES & &
\end{tabular}

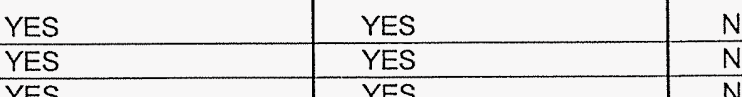

\begin{tabular}{l|l} 
Because of the "yes" & Because of the "yes" \\
\hline
\end{tabular}

detailed assessment using detailed assessment using

Because all values are
below background (15.7 RESRAD will be performed. RESRAD will be perform $\mathrm{mg} / \mathrm{kg}$ ), the WAC 173-340

below background (18.5

$\mathrm{mg} / \mathrm{kg}$ ), the WAC 173-340

The data set meets the 3- The data set meets the
part test criteria when

compared to the direct

compared to the direct
exposure cleanup level.

3-part test is not required.

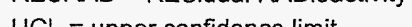

WAC $=$ Washington Adminisitrative code 
Originator J. M. Capron $\mathrm{y}^{2}$

Subject 1 118-C-1 Burial Ground Cleanup Verification 95\% UCL Calculations
Date $\frac{12 / 13 / 06}{14655}$
Job No.
Calc. No. $0100 \mathrm{C}-\mathrm{CA}-\mathrm{V} 0033$ Rev. No. $\frac{0}{12 / 140 \mathrm{C}}$

1118-C-1 Area 3 Statistical Calculations (continued)

\begin{tabular}{|c|c|c|c|c|c|c|c|c|c|c|c|c|c|c|c|c|c|c|c|c|c|c|c|}
\hline Sample & Sample & Sample & & jicke & & & nadit & & & Zinc & & Sampling & Sample & Sample & & clor- & & $\mathrm{bis}(2-\mathrm{E}$ th & hexy & phthalate & Di-n-b & tylph & thalate \\
\hline Area & Number & Date & $\mathrm{mg} / \mathrm{kg}$ & $Q$ & $\mathrm{PQL}$ & $\mathrm{mg} / \mathrm{kg}$ & Q & $\mathrm{PQL}$ & $\mathrm{mg} / \mathrm{kg}$ & Q & $P Q L$ & Area & Number & Date & $\mathrm{mg} / \mathrm{kg}$ & Q & $\mathrm{PQL}$ & $\mathrm{mg} / \mathrm{kg}$ & Q & $P Q L$ & $\mathrm{mg} / \mathrm{kg}$ & Q & PQL \\
\hline$\frac{A 1}{A 2}$ & $J 13510$ & $\frac{8 / 21 / 2006}{9120000}$ & $\begin{array}{l}11.0 \\
10 ?\end{array}$ & & 0.66 & $\begin{array}{r}53.2 \\
157\end{array}$ & & 0.25 & 47.4 & & 0.44 & A1 & $\mathrm{J} 13510$ & $8 / 21 / 2006$ & 0.014 & & 0.0 & 0.33 & $U$ & 0.33 & 0.051 & $J$ & 0.33 \\
\hline A2 & J13HN3 & $9 / 29 / 2006$ & 10.2 & & 0.40 & 45.7 & & 0.15 & 77.1 & & 0. & A2 & & $8 / 21 /$ & 0.046 & & & 0.030 & $\mathrm{~J}$ & & & U & 0.33 \\
\hline A3 & $\begin{array}{l}J 13512 \\
11512\end{array}$ & $8 / 21 / 2006$ & $\frac{12.7}{0.9}$ & & 0.66 & $\begin{array}{l}44.7 \\
15.7\end{array}$ & & 0. & 73.8 & & 0.44 & $\mathrm{A3}$ & & $8 / 21 /$ & 0.062 & & & 0.04 & $U$ & 0. & 0.02 & J & 0.34 \\
\hline $\mathrm{A} 4$ & J13513 & $8 / 21 / 2006$ & 9.6 & & 0.66 & 45.7 & & 0.25 & 42.9 & & 0.44 & $\mathrm{~A} 4$ & $J 1351$ & $8 / 21 / 2006$ & 0.021 & & 0.01 & 0.24 & IJ & 0. & 0.063 & $J$ & 0.33 \\
\hline
\end{tabular}

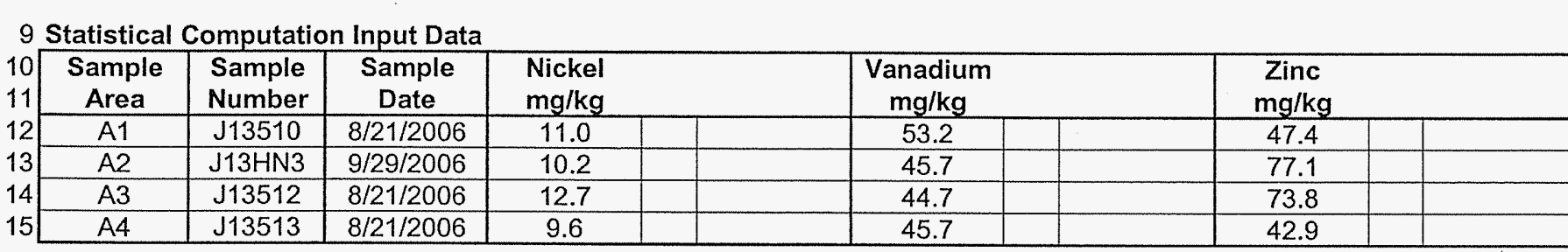

\section{Statistical Computations}

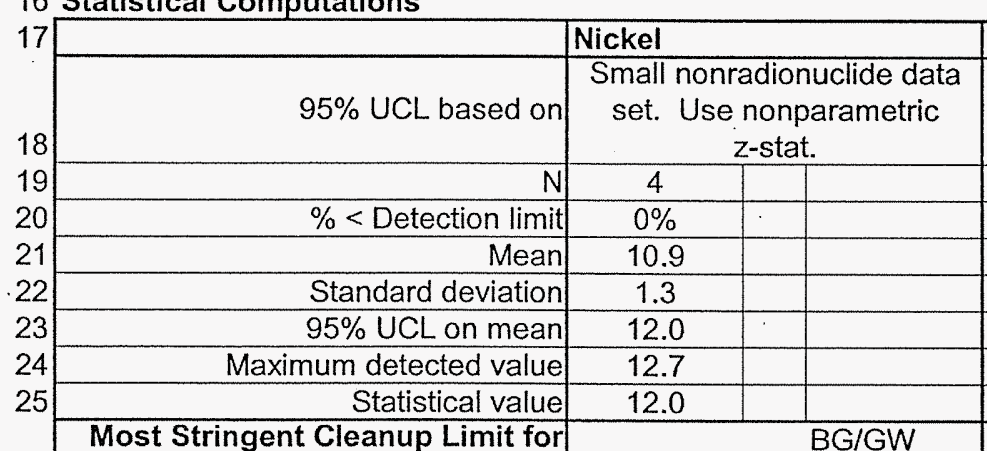

Most Stringent Cleanup Limit fo 27 WAC 173-340 3-PART TEST $5 \%$ UCL > Cleanup Lim

Any $>10 \%$ abo $>$ C WAC 173-340
Compliance?

Further

Because all values are below Because all values are below required WAC 173-340 3-part test is WAC 173-340 3-part test 31 $32 \mathrm{BG}=$ background
$33 \mathrm{GW}=$ groundwate $33 \mathrm{GW}=$ groundwater

$34 \mathrm{~J}=$ estimate

$36 \mathrm{PQL}=$ practical quantitation linit

$37 \mathrm{Q}=$ qualifier

RAG = remedial action goal
$R D L=$ required detection limit

$U=$ undetected
Statistical Computation Input Data

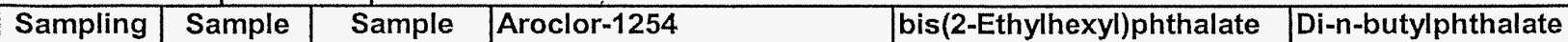

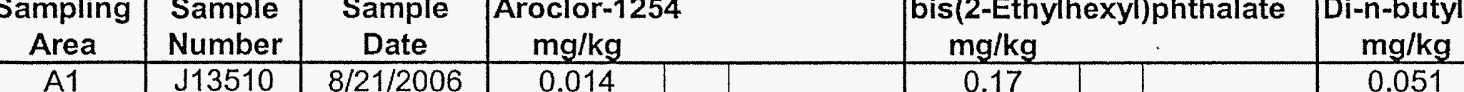
\begin{tabular}{l|l|l|l|} 
A2 & $J 13511$ & $8 / 21 / 2006$ & 0.014 \\
A3 & $J 13512$ & $8 / 212006$ & 0.046 \\
A4 & J13513 & $821 / 2006$ & 0.021 \\
\hline
\end{tabular} \begin{tabular}{l|l|l|l|l} 
A3 & $J 13512$ & $8 / 21 / 2006$ & 0.062 \\
A4 & $\mathrm{J} 13513$ & $8 / 21 / 2006$ & 0.021 \\
\hline
\end{tabular}

Statistical Computations

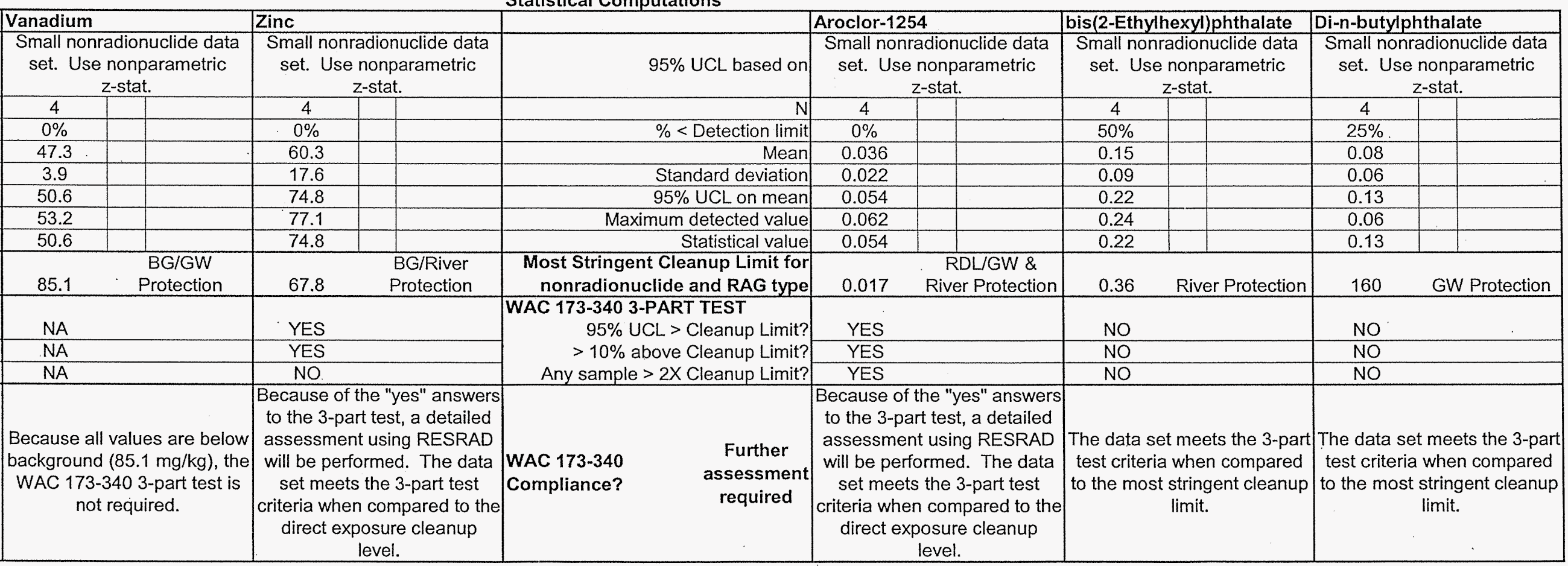

RESRAD = RESidual RADioactivity (dose assessment model) 
Washington Closure Hanford

Originator J. M. Capron gze

Project $100-B / C$ Field Remediation
CALCULATION SHEET

$$
\begin{array}{r}
\text { Date } \frac{12 / 13 / 06}{14655} \\
\text { Job No. }
\end{array}
$$

Rev. No. $\frac{0}{1.406}$

1118-C-1 Area 4 Statistical Calculations

\begin{tabular}{|c|c|c|c|c|c|c|c|c|c|c|c|c|c|c|c|c|c|c|c|c|c|c|c|c|c|c|c|c|}
\hline \multirow{3}{*}{\begin{tabular}{|c|c|} 
& Seritication \\
3 & Sample \\
4 & Area \\
5 & A1
\end{tabular}} & \multirow{3}{*}{$\begin{array}{l}\text { Sample } \\
\text { Number } \\
13514\end{array}$} & \multirow{3}{*}{$\begin{array}{c}\text { Sample } \\
\text { Date }\end{array}$} & \multirow{2}{*}{\multicolumn{2}{|c|}{ Cesium-137 }} & \multirow{2}{*}{\multicolumn{3}{|c|}{ Europium-152 }} & \multirow{2}{*}{\multicolumn{3}{|c|}{ Uranium-233/234 }} & \multirow{2}{*}{\multicolumn{3}{|c|}{ Uranium-238 }} & \multirow{2}{*}{\multicolumn{3}{|c|}{ Arsenic }} & \multirow{2}{*}{\multicolumn{3}{|c|}{ Barium }} & \multirow{2}{*}{\multicolumn{3}{|c|}{ Beryllium }} & \multirow{2}{*}{\multicolumn{3}{|c|}{ Boron }} & \multirow{2}{*}{\multicolumn{3}{|c|}{ Chromium }} \\
\hline & & & & & & & & & & & & & & & & & & & & & & & & & & & & \\
\hline & & & pCilg & MDA & & \begin{tabular}{|l|} 
Q \\
$U$
\end{tabular} & $\frac{\text { MDA }}{0.070}$ & $\frac{\mathrm{pCi} / \mathrm{g}}{0^{231}}$ & $Q$ & $\begin{array}{l}\text { MDA } \\
0 ? 2\end{array}$ & $p \mathrm{Ci} / \mathrm{g}$ & Q & MDA & & $Q$ & $\frac{P Q L}{17}$ & $\frac{\mathrm{mg} / \mathrm{kg}}{486}$ & Q & $\frac{P C}{0 . C}$ & $\mathrm{mg} / \mathrm{kg}$ & Q & $\frac{P Q L}{005}$ & $\frac{\mathrm{mg} / \mathrm{kg}}{10}$ & Q & $\frac{P Q L}{066}$ & $\frac{\mathrm{mg} / \mathrm{kg}}{66}$ & \begin{tabular}{|l|}
$Q$ \\
$C$ \\
\end{tabular} & $P Q L$ \\
\hline A2 & \begin{tabular}{|l|l|}
$J 13515$ \\
\end{tabular} & $8 / 21 / 2006$ & 1.19 & & 0.159 & 4 & & & U & & & & & $\frac{2}{2}$ & & & & & & & & & 4.9 & & & 6.0 & \begin{tabular}{|l|l|l|}
$c$ & \\
\end{tabular} & 0.00 \\
\hline $\mathrm{A} 3$ & J13516 & $8 / 21 / 2006$ & & 0.020 & 0.038 & $u$ & 0.038 & & & 0.26 & & & & 2.4 & & & & & & & & & 0.65 & |UJ & 0. & 6.0 & c & 0.35 \\
\hline A4 & \begin{tabular}{|l|l|}
$J 13517$ \\
\end{tabular} & $8 / 21 / 2006$ & 0.082 & 0.024 & 0.048 & U & 0.048 & 0.422 & & 0.29 & 0.268 & & 0.29 & 2.2 & & 1.7 & 49.2 & & 0.06 & 0.43 & & 0.06 & 0.66 & & 0.66 & 5.3 & c & 0.36 \\
\hline
\end{tabular}

\section{Statistical Computation In}

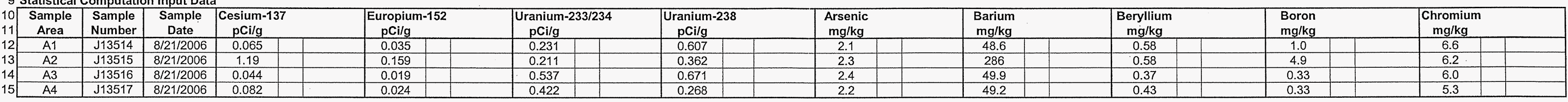

16 Statistical Computations

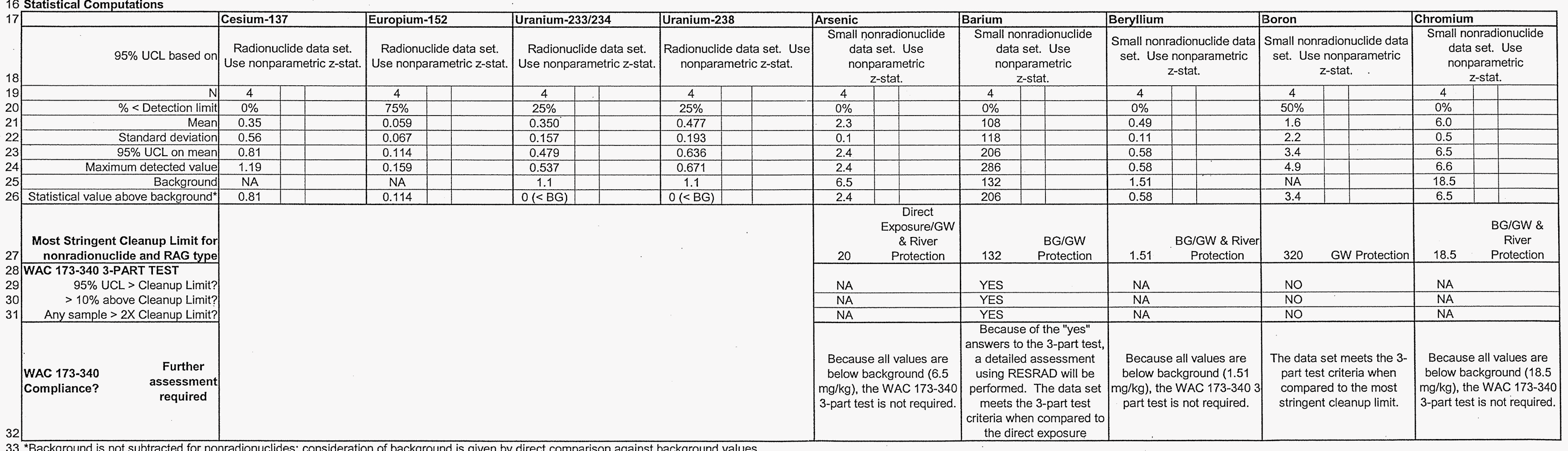

$34 \mathrm{C}=$ method blank contamination (inorganic constitutents)

$35 \mathrm{BG}=$ background
$36 \mathrm{GW}=$ groundwater

$Q=$ qualifier
$R A G=$ remedial action goa

$37 \mathrm{MDA}=$ miniwater

$38 \mathrm{NA}=$ not applicable

RESRAD = RESidual RADioactivity (dose assessment mode)

$39 \mathrm{PQL}=$ practical quantitation limit

UCL = upper confidence lim

WAC $=$ Washington Administrative Code 
1 118-C-1 Area 4 Statistical Calculations (continued)

\begin{tabular}{|c|c|c|c|c|c|c|c|c|c|c|c|c|c|c|c|c|c|c|c|c|c|c|c|c|c|c|}
\hline \multirow{2}{*}{$\begin{array}{c}\text { Sample } \\
\text { Area }\end{array}$} & \multirow{2}{*}{$\begin{array}{l}\text { Sample } \\
\text { Number }\end{array}$} & \multirow{2}{*}{$\begin{array}{c}\text { Sample } \\
\text { Date }\end{array}$} & \multicolumn{3}{|c|}{ Cobalt } & \multicolumn{3}{|c|}{ Copper } & \multicolumn{3}{|c|}{ Lead } & \multicolumn{3}{|c|}{ Manganese } & \multicolumn{3}{|c|}{ Nickel } & \multicolumn{3}{|c|}{ Vanadium } & \multicolumn{3}{|c|}{ Zinc } & \multicolumn{3}{|c|}{ Aroclor-1254 } \\
\hline & & & $\mathrm{mg} / \mathrm{kg}$ & Q & $\overline{P Q L}$ & $\mathrm{mg} / \mathrm{kg}$ & $Q$ & PQL & $\mathrm{mg} / \mathrm{kg}$ & Q & PQL & $\mathrm{mg} / \mathrm{kg}$ & Q & PQL & $\mathrm{mg} / \mathrm{kg}$ & & $\overline{P Q L}$ & $\mathrm{mg} / \mathrm{kg}$ & Q & PQL & $\mathrm{mg} / \mathrm{kg}$ & & $\overline{P Q L}$ & $\mathrm{mg} / \mathrm{kg}$ & $Q$ & $\frac{P Q L}{0.013}$ \\
\hline$\frac{A 1}{A 2}$ & $\frac{J 13514}{J 13515}$ & $8 / 21 / 2006$ & $\frac{8.8}{8.5}$ & & $\frac{0.38}{0.38}$ & 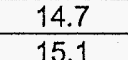 & & 0.33 & 5.0 & & 0.85 & 323 & & $\begin{array}{l}0.08 \\
0.08\end{array}$ & $\frac{10.1}{9.3}$ & & $\begin{array}{l}0.66 \\
0.66\end{array}$ & $\begin{array}{l}55.4 \\
46.4\end{array}$ & & 0.25 & $\frac{42.2}{38.8}$ & & 0.44 & $\frac{0.013}{0.0064}$ & $\frac{U}{J}$ & \\
\hline $\mathrm{A3}$ & J13516 & $8 / 21 / 2006$ & 7.2 & & 0.38 & 14.2 & & 0.00 & $\frac{4.1}{3.1}$ & & 0.05 & 269 & & 0.08 & 0.0 & & 0.05 & 38.7 & & 0.25 & 33.7 & & 0.44 & & u & \\
\hline$\frac{\mathrm{As}}{\mathrm{A} 4}$ & J13517 & $8 / 21 / 2006$ & $\frac{7.2}{8.1}$ & & 0.39 & $\frac{14.2}{14.8}$ & & 0.33 & $\frac{3.1}{4.5}$ & & $\begin{array}{l}0.05 \\
0.85\end{array}$ & 209 & & 0.08 & $\frac{9.1}{87}$ & & 0.05 & $\frac{30.1}{42.4}$ & & 0.25 & $\frac{3.1}{37.7}$ & & 0.44 & 0.016 & & \\
\hline
\end{tabular}

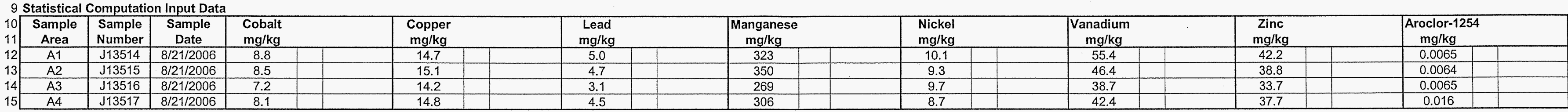

16 Statistical Computations

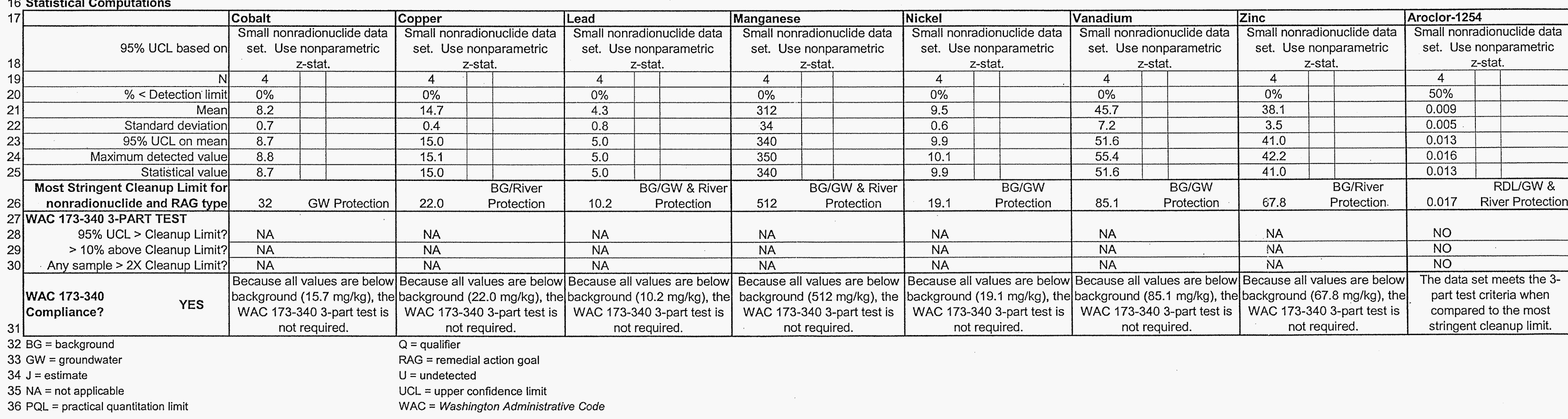


Washington Closure Hanford

Originator J.M. Capron 23 C

Project 100-B/C Field Remediation

Subject 118-C-1 Burial Ground Cleanup Verification 95\% UCL Calculations

Date $12 / 13 / 06$ Job No. $\frac{12 / 13 / 06}{14655}$

\section{CALCULATION SHEET}

Calc. No. 0100C-CA-V0033 Rev. No. 0 Checked T.M. Blakley 2mes Date $\overline{12 / 4 / 06}$ Sheet No. 15 of 31

1118-C-1 Area 4 Statistical Calculations (continued)

2 Verification Data

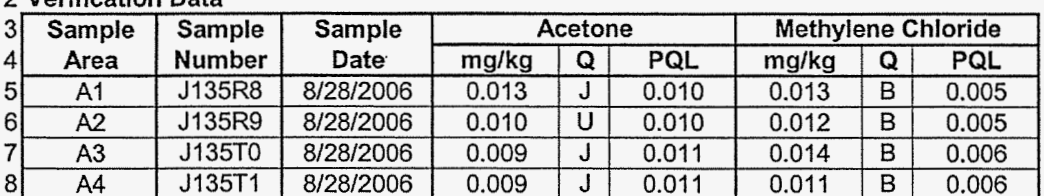

9 Statistical Computation Input Data

\begin{tabular}{|c|c|c|c|c|c|c|}
\hline $\begin{array}{l}10 \\
11\end{array}$ & $\begin{array}{c}\text { Sample } \\
\text { Area }\end{array}$ & $\begin{array}{l}\text { Sample } \\
\text { Number }\end{array}$ & $\begin{array}{c}\text { Sample } \\
\text { Date }\end{array}$ & $\begin{array}{c}\text { Acetone } \\
\mathrm{mg} / \mathrm{kg}\end{array}$ & $\begin{array}{r}\text { Methyler } \\
\text { mg/kg }\end{array}$ & oride \\
\hline 12 & $\mathrm{A1}$ & J135R8 & $8 / 28 / 2006$ & 0.013 & 0.013 & \\
\hline 13 & A2 & J135R9 & $8 / 28 / 2006$ & 0.0050 & 0.012 & \\
\hline 14 & A3 & J135T0 & $8 / 28 / 2006$ & 0.009 & 0.014 & \\
\hline 15 & A4 & J135T1 & $8 / 28 / 2006$ & 0.009 & 0.011 & \\
\hline
\end{tabular}

16 Statistical Computations

\begin{tabular}{|c|c|c|c|c|c|}
\hline \multirow[b]{2}{*}{18} & & \multicolumn{2}{|l|}{ Acetone } & \multicolumn{2}{|c|}{ Methylene Chloride } \\
\hline & $95 \%$ UCL based on & \multicolumn{2}{|c|}{$\begin{array}{l}\text { Small nonradionuclide } \\
\text { data set. Use } \\
\text { nonparametric } \\
\text { z-stat. }\end{array}$} & \multicolumn{2}{|c|}{$\begin{array}{c}\text { Small nonradionuclide } \\
\text { data set. Use } \\
\text { nonparametric } \\
\text { z-stat. }\end{array}$} \\
\hline 19 & $\mathrm{~N}$ & 4 & & 4 & \\
\hline 20 & $\%<$ Detection limit & $25 \%$ & & $0 \%$ & \\
\hline 21 & Mean & 0.009 & & 0.013 & \\
\hline 22 & Standard deviation & 0.003 & & 0.001 & \\
\hline 23 & $95 \%$ UCL on mean & 0.012 & & 0.014 & \\
\hline 24 & Maximum detected value & 0.013 & & 0.014 & \\
\hline 25 & Statistical value & 0.012 & & 0.014 & \\
\hline 2 & $\begin{array}{l}\text { Most Stringent Cleanup Limit for } \\
\text { nonradionuclide and RAG type }\end{array}$ & 720 & GW Protection & 0.5 & GW Protection \\
\hline & WAC 173-340 3-PART TEST & & & & \\
\hline 28 & $95 \%$ UCL > Cleanup Limit? & NO & & NO & \\
\hline 2 & $>10 \%$ above Cleanup Limit? & $\mathrm{NO}$ & & NO & \\
\hline & Any sample $>2 \times$ Cleanup Limit? & NO & & NO & \\
\hline & $\begin{array}{l}\text { WAC 173-340 } \\
\text { Compliance? }\end{array}$ & $\begin{array}{l}\text { The data } \\
\text { part te } \\
\text { compa } \\
\text { stringe }\end{array}$ & $\begin{array}{l}\text { et meets the 3- } \\
\text { criteria when } \\
\text { d to the most } \\
\text { cleanup limit. }\end{array}$ & $\begin{array}{l}\text { The date } \\
\text { part te } \\
\text { compe } \\
\text { stringe }\end{array}$ & $\begin{array}{l}\text { et meets the 3- } \\
\text { criteria when } \\
\text { d to the most } \\
\text { cleanup limit. }\end{array}$ \\
\hline
\end{tabular}

$32 \mathrm{~B}=$ method blank contamination (organic constitutents)

$33 \mathrm{GW}=$ groundwater

$34 \mathrm{~J}=$ estimated

$35 \mathrm{PQL}=$ practical quantitation limit

$36 \mathrm{Q}=$ qualifier

37 RAG = remedial action goal

$38 \mathrm{UCL}=$ upper confidence limit

39 WAC $=$ Washington Administrative Code 
CVP-2006-00011

Rev. 0

E-122 
Originator J. M. Capron GMnC
Project
$100-B / C$ Field Remediation

Project $\frac{100-B / C \text { C Fiel\& Remediation }}{118-C-1 \text { Burial Ground Cleanup Verification } 95 \% \text { UCL Calculations }}$

1118-C-1 Overburden/BCL Material Statistical Calculations

\begin{tabular}{|c|c|c|c|c|c|c|c|c|c|c|c|c|c|c|c|c|c|c|c|c|c|c|c|c|c|c|}
\hline sample & Sample & Sample & & ium- & & & ntiun & & Urani & $m-2$ & $3 / 234$ & & ium- & & & rsenic & & & Sarium & & & rylliul & & & romi & \\
\hline Area & Number & $\mathrm{Da}$ & $\mathrm{i} / \mathrm{g}$ & $Q$ & MDA & $\mathrm{OCi} / \mathrm{g}$ & \begin{tabular}{l|l} 
& \\
\end{tabular} & MDA & $\mathrm{pCi} / \mathrm{g}$ & \begin{tabular}{|l|l|l|} 
& \\
\end{tabular} & MDA & $\overline{\mathrm{pCi} / \mathrm{g}}$ & Q & MDA & $\mathrm{mg} / \mathrm{kg}$ & $\mid$\begin{tabular}{l|}
$\mid$ \\
\end{tabular} & $\overline{P Q L}$ & $\mathrm{mg} / \mathrm{kg}$ & Tि & QQL & $\mathrm{mg} / \mathrm{kg}$ & & & $\mathrm{mg} / \mathrm{kg}$ & & PQL \\
\hline A1 & $J 13518$ & $8 / 22 / 2006$ & 0.22 & & 0.035 & 0.080 & U & 0.26 & 0.430 & & 0.13 & 0.480 & & 0.13 & 2.2 & & 1.8 & 48.7 & & 0.06 & 0.65 & & 0.06 & 6.1 & & 0.38 \\
\hline $\mathrm{A} 2$ & J13519 & 2006 & 0.059 & & 0.033 & 0.052 & $U$ & 0.29 & 0.428 & & 0.13 & 0.478 & & 0.13 & 2.2 & & 1.8 & 50.0 & & 0.06 & 0.69 & & 0.06 & 6.4 & & 0.38 \\
\hline A3 & $J 13520$ & $8 / 22 / 2006$ & 0.43 & & 0.018 & 2.10 & & 0.25 & & & 0.1 & 0.508 & & 0.13 & 1.8 & U & 1.8 & 46.0 & & 0.06 & 0.60 & & 0.06 & 5.3 & & 38 \\
\hline A4 & J135N5 & $8 / 22 / 2006$ & 0.172 & & 0.046 & 0.089 & $U$ & 0.27 & 0.383 & & 0.12 & 0.399 & & 0.12 & 2.3 & & 1.8 & 58.4 & & 0.06 & 0.52 & & 0.06 & 6.8 & & 0.38 \\
\hline
\end{tabular}

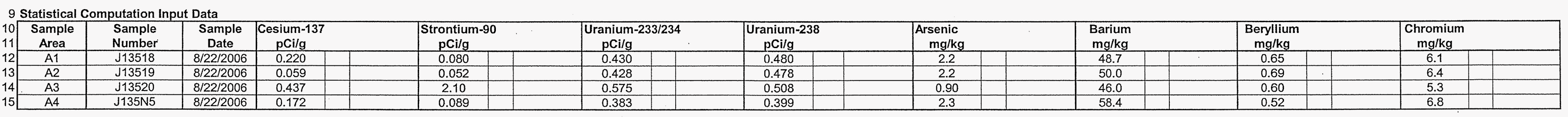

16 Statistical Computation

\begin{tabular}{|c|c|}
\hline \multicolumn{2}{|l|}{17} \\
\hline $18 \mid$ & $95 \% \cup C L b$ \\
\hline & etection limit \\
\hline & Mean \\
\hline & Standard deviation \\
\hline & $\begin{array}{l}95 \% \text { UCL on mean } \\
\text { Maximum detected value }\end{array}$ \\
\hline & Background \\
\hline & Statistical value above backgro \\
\hline 7 & $\begin{array}{l}\text { Most Stringent Cleanup Limit for } \\
\text { nonradionuclide and RAG type }\end{array}$ \\
\hline & WAC 173-340 3-PART TEST \\
\hline & $95 \%$ UCL > Cleanup Limit? \\
\hline & $\begin{array}{l}>10 \% \text { above Cleanup Limit? } \\
\text { Anv sample }>2 \times \text { Cleanup Limit? }\end{array}$ \\
\hline & 73-340 Compliance? \\
\hline
\end{tabular}

$34 \mathrm{BCL}=$ below-cleanup-levels

$35 \mathrm{BG}=$ background

$37 \mathrm{MDA}=$ minimum detectable activity

$38 \mathrm{NA}=$ not applicable

$39 \mathrm{PQL}=$ practical quantitation lim

\section{\begin{tabular}{l|l|l|l}
\hline Cesium-137 & Strontium-90 & Uranium-233/234 & Uranium-238
\end{tabular}}

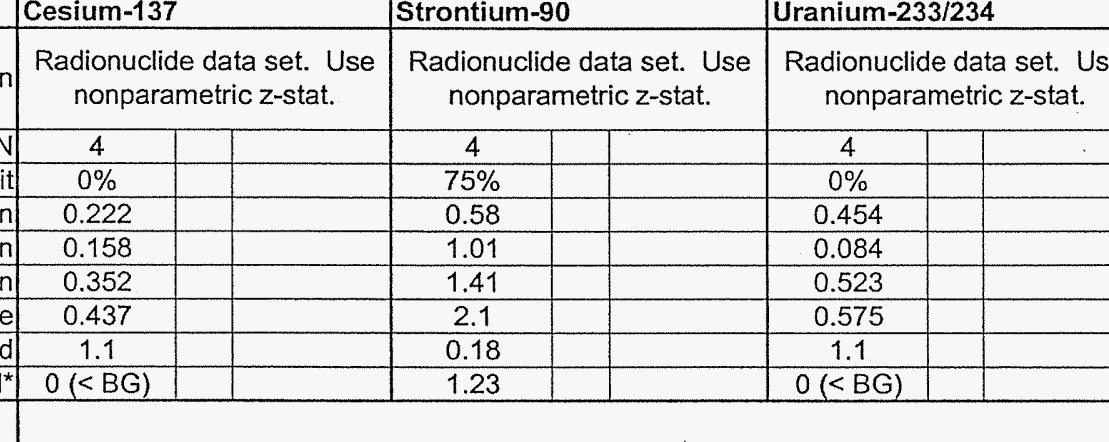

\begin{tabular}{c|c|c|}
\hline se & \multicolumn{2}{|c|}{$\begin{array}{c}\text { Radionuclide data set. Use } \\
\text { nonparametric z-stat. }\end{array}$} \\
\hline 4 & & \\
\hline $0 \%$ & & \\
\hline 0.466 & & \\
0.047 & & \\
0.505 & & \\
0.508 & & \\
1.1 & & \\
& &
\end{tabular}

\begin{tabular}{c|c}
1.1 \\
$0(<B G)$ \\
\hline
\end{tabular} $Q=$ qualifier

$Q=$ qualfifier

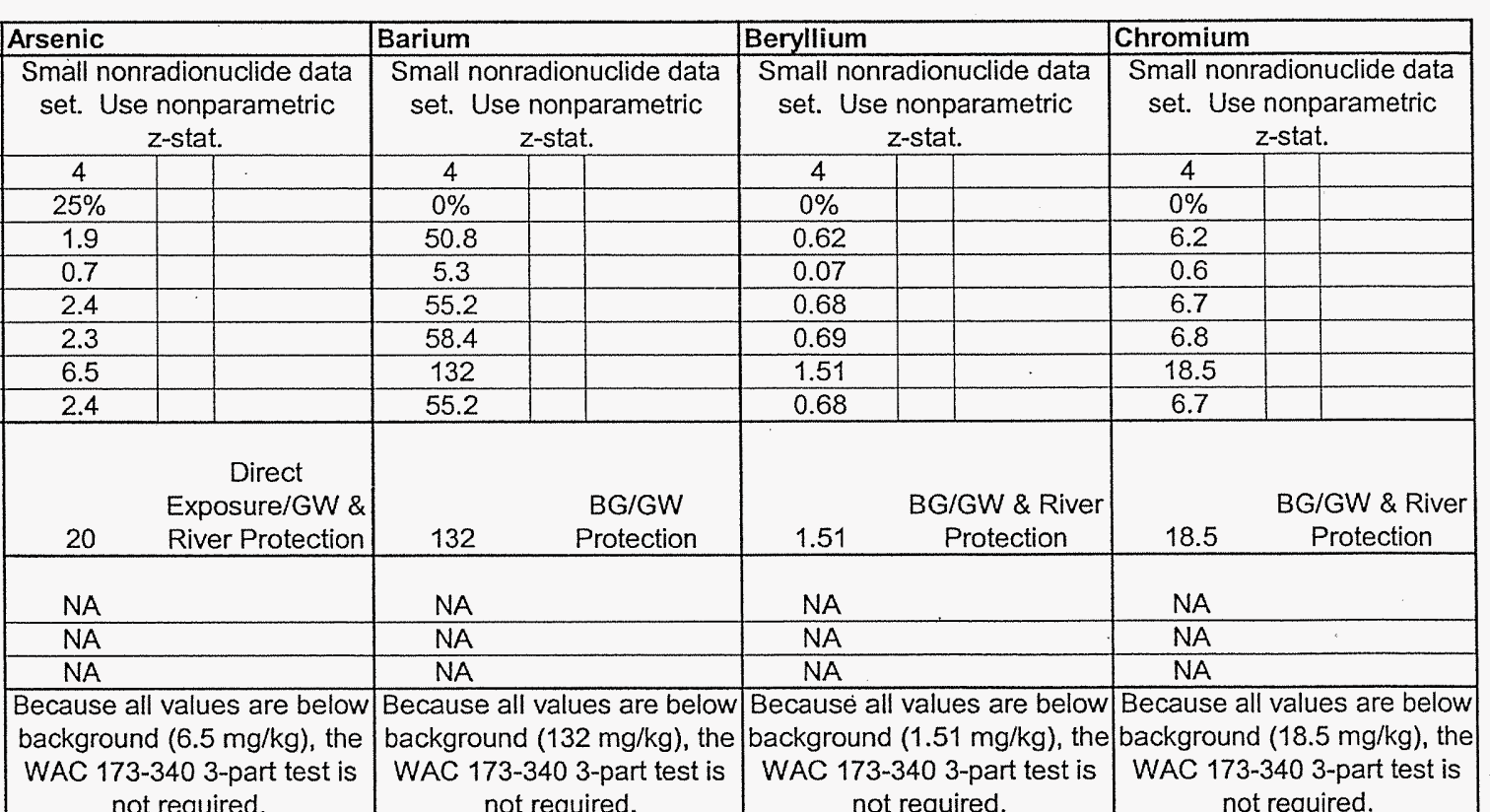

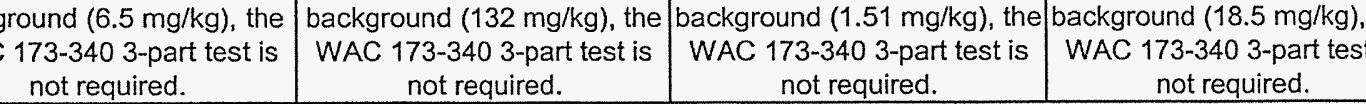


1 118-C-1 Overburde

2 Verification Data

\begin{tabular}{|c|c|c|c|c|c|c|c|c|c|c|c|c|c|c|c|c|c|c|c|c|c|c|c|c|c|c|}
\hline Sample & Sample & Sample & & 5 oba & & & oppe & & & Lead & & & ingan & & & Nicke & & & Vanadii & & & Zinc & & Aroc & clor-12 & \\
\hline & Number & Date & $\mathrm{mg} / \mathrm{kg}$ & Q & PQL & $\mathrm{mg} / \mathrm{kg}$ & Q & PQL & $\mathrm{mg} / \mathrm{kg}$ & $Q$ & PQL & $\mathrm{mg} / \mathrm{kg}$ & $Q$ & PQL & $\mathrm{mg} / \mathrm{kg}$ & $Q$ & PQL & $\mathrm{mg} / \mathrm{kg}$ & \begin{tabular}{l|l|}
9 & $Q$ \\
\end{tabular} & $\mathrm{PQL}$ & $\mathrm{mg} / \mathrm{kg}$ & Q & $\mathrm{PQL}$ & $\mathrm{mg} / \mathrm{kg}$ & $Q$ & $\mathrm{PQL}$ \\
\hline $\mathrm{A1}$ & $\begin{array}{l}13518 \\
12510\end{array}$ & $8 / 22 / 2006$ & & & 0.40 & 15.8 & & 0.35 & 5.2 & & 0.90 & 311 & & 0.09 & 9.0 & & 0.69 & & & 0.26 & 37.3 & & 0.46 & 0.013 & U & 0.013 \\
\hline$\frac{A 2}{A 3}$ & $\frac{J 13519}{J 13520}$ & $8 / 22 / 20006$ & $\frac{7.8}{6.9}$ & & $\frac{0.41}{0.44}$ & $\frac{15.0}{12.8}$ & & $\begin{array}{l}0.35 \\
0.35\end{array}$ & $\frac{4.5}{3.8}$ & & $\begin{array}{l}0.91 \\
0.91\end{array}$ & $\frac{329}{272}$ & & 0.09 & $\frac{9.3}{96}$ & & 0.70 & $\begin{array}{l}45.7 \\
36.2\end{array}$ & & $\begin{array}{l}0.26 \\
026\end{array}$ & $\begin{array}{l}37.5 \\
31.5\end{array}$ & & 0.47 & $\begin{array}{l}0.0049 \\
0.0053\end{array}$ & & $\begin{array}{l}0.013 \\
0.013\end{array}$ \\
\hline$\frac{\mathrm{As}}{\mathrm{A} 4}$ & J135N5 & $8 / 22 / 2006$ & 7.1 & & 0.41 & $\frac{12.0}{11.9}$ & & 0.35 & $\frac{3.8}{4.3}$ & & 0.90 & $\frac{272}{308}$ & & 0.099 & $\begin{array}{l}9.0 \\
9.0\end{array}$ & & 0.70 & $\frac{30.2}{39.6}$ & & 0.26 & $\begin{array}{l}31.5 \\
34.6 \\
\end{array}$ & & 0.47 & $\frac{0.0050}{0.013}$ & U & 0.013 \\
\hline
\end{tabular}

\section{Statistical Computation Input Data}

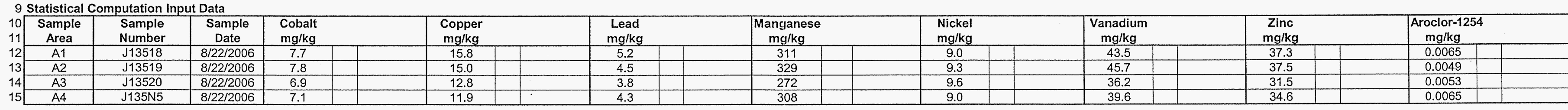

16 Statistical Computations

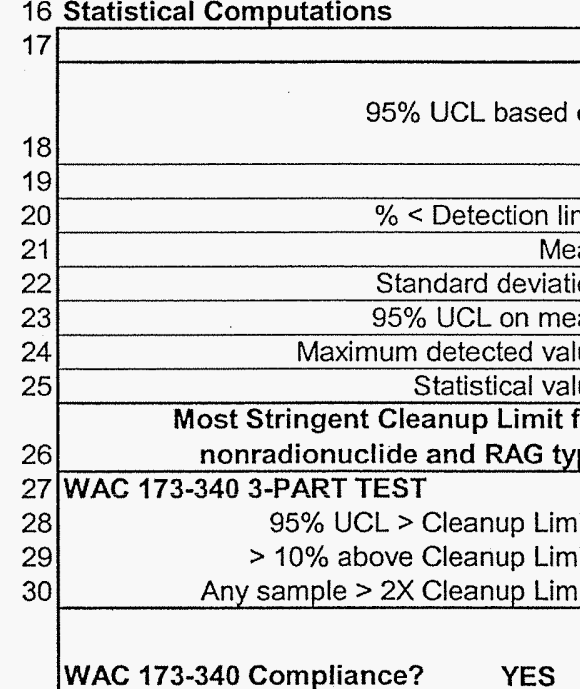$$
\text { pliance? YES }
$$

$33 \mathrm{BG}=$ below-cleanup-levels

$33 \mathrm{BG}=$ background
$34 \mathrm{GW}=$ groundwater

$36 \mathrm{NA}=$ not applica

$36 \mathrm{NA}=$ not applicable
$37 \mathrm{PQL}=$ practical quantitation limit

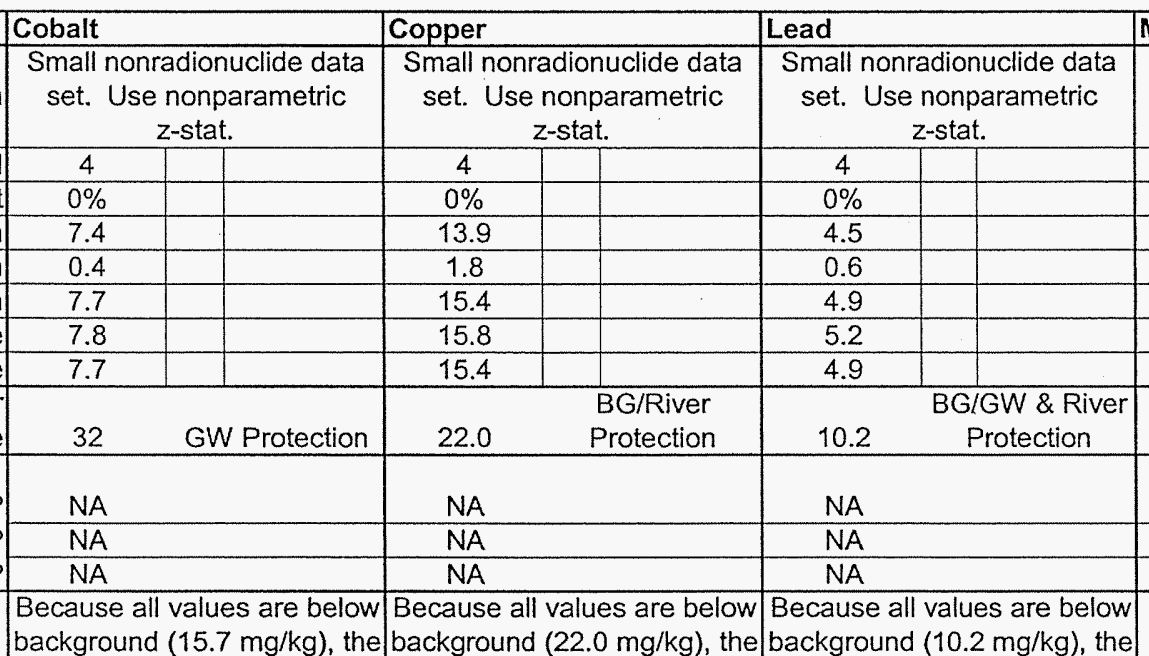

Because all values are below Because all values are below Because all valu \begin{tabular}{l|l} 
WAC 173-340 3-part test is & WAC 173-340 3-part test is \\
\hline
\end{tabular} not required.
$Q=$ Wualfier $R A G=$ remedial action goal

$\mathrm{RDL}=$ required
$U=$ undetected
$U$

UCL $=$ upper confidence lim

$\mathrm{UCL}=$ upper confidence limit
$\mathrm{WAC}=$ Washington Administrative Cood

not required.

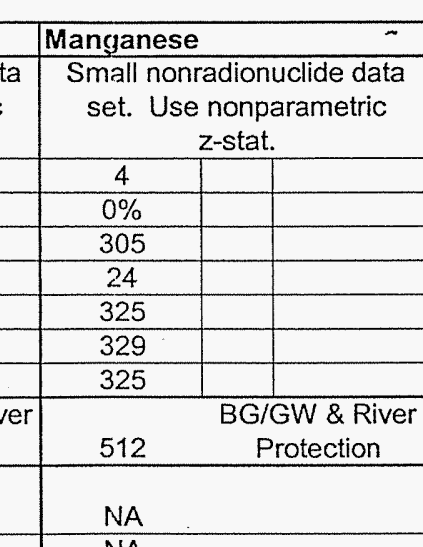

NA

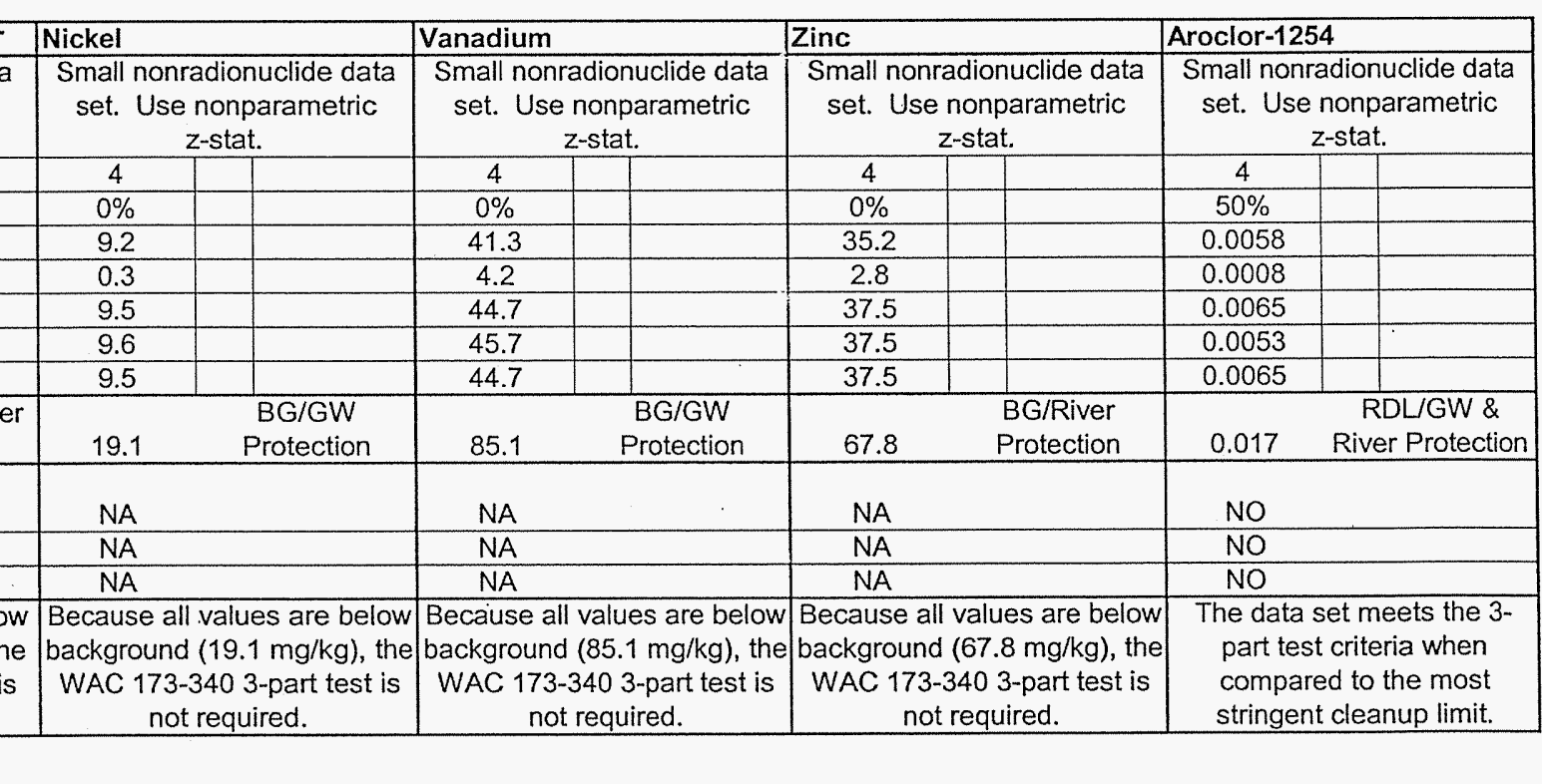

173-340 3-part test is

not required.

not required.

not required.
not

compared to the most 
CALCULATION SHEET

Project $\frac{100-B / C \text { Field Remediation }}{100}$
Subject $118-C-1$ Burial Ground Cleanup Verification $95 \%$ UCL Calculation

$$
\underset{\text { Date }}{\text { Job No. }} \frac{12 / 13 / 06}{14655}
$$

\begin{tabular}{|c|c|c|c|c|c|c|c|c|c|c|c|c|c|c|c|c|c|c|c|c|c|c|c|c|}
\hline \multirow{2}{*}{\multicolumn{2}{|c|}{ 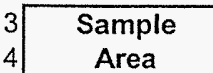 }} & \multirow{2}{*}{$\begin{array}{l}\text { Sample } \\
\text { Number }\end{array}$} & \multirow{2}{*}{$\begin{array}{c}\text { Sample } \\
\text { Date }\end{array}$} & \multirow{2}{*}{\multicolumn{3}{|c|}{ Cesium-137 }} & \multicolumn{3}{|c|}{ Cobalt-60 } & \multirow{2}{*}{\multicolumn{3}{|c|}{ Europium-152 }} & \multirow{2}{*}{\multicolumn{3}{|c|}{ Strontium-90 }} & \multirow{2}{*}{\multicolumn{3}{|c|}{ Uranium-233/234 }} & \multirow{2}{*}{\multicolumn{3}{|c|}{ Uranium-235 }} & \multirow{2}{*}{\multicolumn{3}{|c|}{ Uranium-238 }} \\
\hline & & & & & \begin{tabular}{|l|l|l|l|l} 
& \\
\end{tabular} & MDA & $\mathrm{pCi} / \mathrm{g}$ & $Q$ & MDA & & & & & & & & & & & & & & & \\
\hline & $\overline{\mathrm{A} 1}$ & J13522 & $9 / 11 / 06$ & 0.424 & & 0.050 & 0.040 & $\bar{U}$ & 0.040 & 0.11 & $\bar{U}$ & 0.11 & 0.211 & ut & 0.22 & 0,415 & & & $\frac{0.19}{0.067}$ & $\frac{\pi}{4}$ & 026 & & & NDA \\
\hline & A2 & J13523 & $9 / 11 / 106$ & 0.141 & & 0.035 & 0.031 & 4 & & 0.075 & is & 0075 & 0.6178 & 11 & 0.21 & 0 & & 0 & 0.00 & II & 0.20 & 0.0008 & & 0.45 \\
\hline & A3 & J135P1 & 9/12/106 & 3.00 & & 0.041 & 0.036 & U & 0.036 & 0.483 & & 0.11 & 0.855 & & 021 & 0.0706 & & & 0 & it & & $\frac{0.390}{0.541}$ & & $\frac{0.25}{0.26}$ \\
\hline 8 & A4 & J135PO & 9/12/06 & 0.826 & & 0.039 & 0.029 & U & 0.029 & 0.214 & & 0.085 & 0.315 & & 0.25 & 0.387 & & & 0.039 & 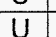 & & $\frac{0.041}{0.646}$ & & 0.20 \\
\hline & B5 & $\mathrm{J13524}$ & & 0.494 & & 0.028 & 0.026 & u & 0.0 & 0.612 & & 0.0064 & 0.235 & & 0.21 & & & 0.4 & & u & & 0.811 & & 0.21 \\
\hline & B6 & & $9 / 11$ & & & 0.0 & 0.044 & $u$ & & & $u$ & & & $U$ & 0.2 & & & & 0 & ut & & 0.237 & & 0.23 \\
\hline & $B 7$ & & & 0.160 & & 0.024 & & $u$ & & 0.046 & u & 0.046 & 0.057 & $\mathrm{u}$ & 0.21 & 0.276 & & & 0.042 & $u$ & 0.32 & 0.518 & & 0.26 \\
\hline & B8 & & $9 / 11$ & & & 0.0 & 0.034 & $u$ & 0.0 & 0.093 & u & 0.093 & 0.115 & $\mathrm{u}$ & 0.25 & 0.320 & & 0.22 & 0.035 & $u$ & 0.27 & 0.640 & & 0.22 \\
\hline & $\mathrm{C9}$ & & $9 / 12$ & 0.290 & & & & $u$ & & 0.049 & U & & 0.203 & $\mathrm{U}$ & 0.2 & 0.626 & & 0.039 & 0.045 & & 0.0 & 0.498 & & 0.039 \\
\hline & C10 & & & 0.979 & & & 0.055 & & 0.0 & 0.363 & & 0.079 & 0.327 & & 0.2 & & & 0.0 & 0.047 & & 0.0 & 0.554 & & 0.033 \\
\hline & C1 & & $9 / 12$ & 1.16 & & 0.04 & 0.050 & U & 0.05 & 0.156 & & 0.12 & 0.333 & & 0.21 & 0.467 & & 0.042 & 0.011 & U & & 0.370 & & 0.034 \\
\hline & $\frac{\mathrm{C} 2}{\mathrm{D} 2}$ & & $9 / 12$ & 0.644 & & 0.055 & 0.055 & u & 0.055 & 0.16 & U & 0.16 & 0.191 & $u$ & 0.2 & 0.467 & & 0.03 & 0.035 & U & 0.0 & 0.396 & & 0.032 \\
\hline & D3 & $\begin{array}{l}J 135 P 6 \\
11250\end{array}$ & $9 / 12 / 0$ & 0.071 & & 0.033 & 0.034 & $u$ & & 0.077 & U & 0.077 & 0.117 & U & 0.23 & 0.552 & & 0.047 & 0.036 & $\mathrm{U}$ & 0.045 & 0.489 & & 0.047 \\
\hline & DS & J135P8 & $9 / 12 / 06$ & 1.18 & & 0.053 & 0.063 & & 0.051 & 0.14 & $u$ & 0.14 & 0.475 & & 0.22 & 0.484 & & 0.066 & 0.042 & & 40 & 0.510 & & 033 \\
\hline & & & & & & & & & & & & & & & & & & & & & & 0.426 & & \\
\hline
\end{tabular}

1 118-C-1 Staging Pile Area Statistical Calculations

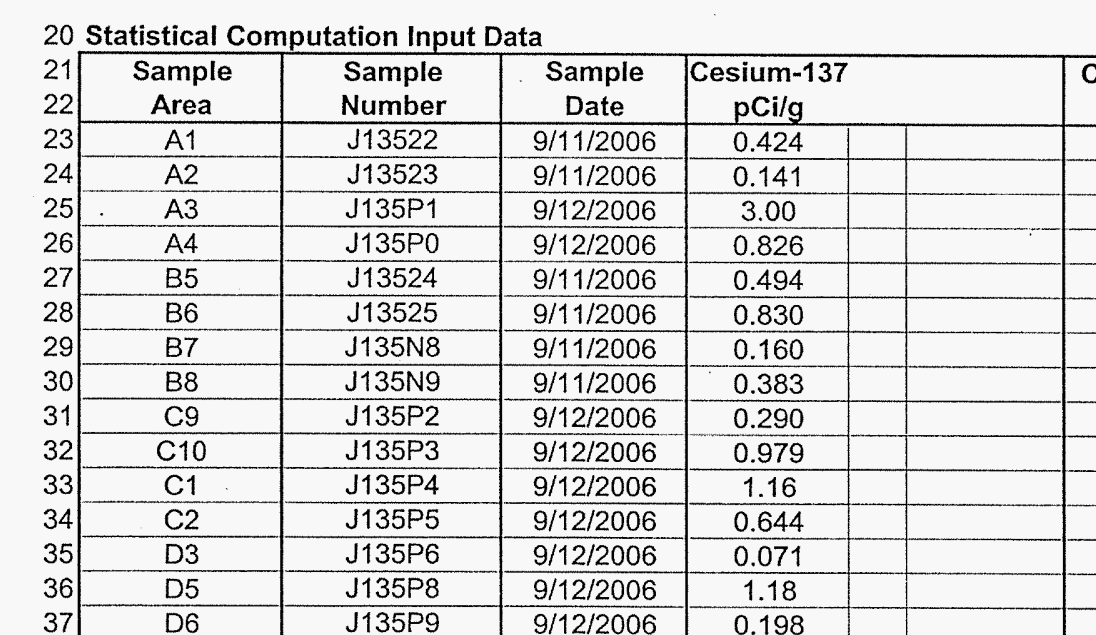

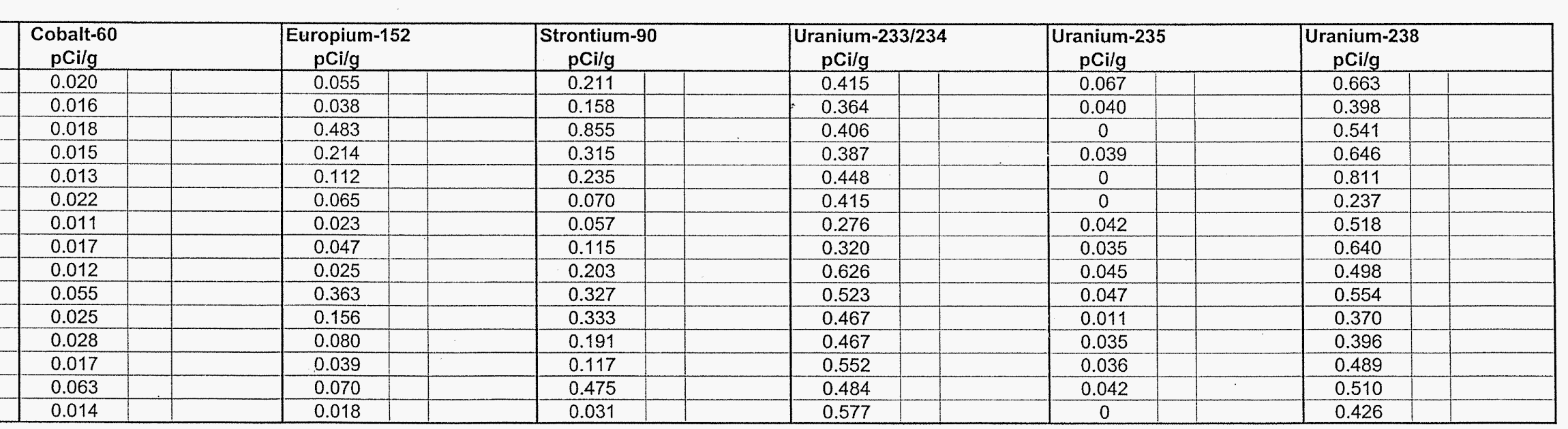

\section{Statistical Computations}

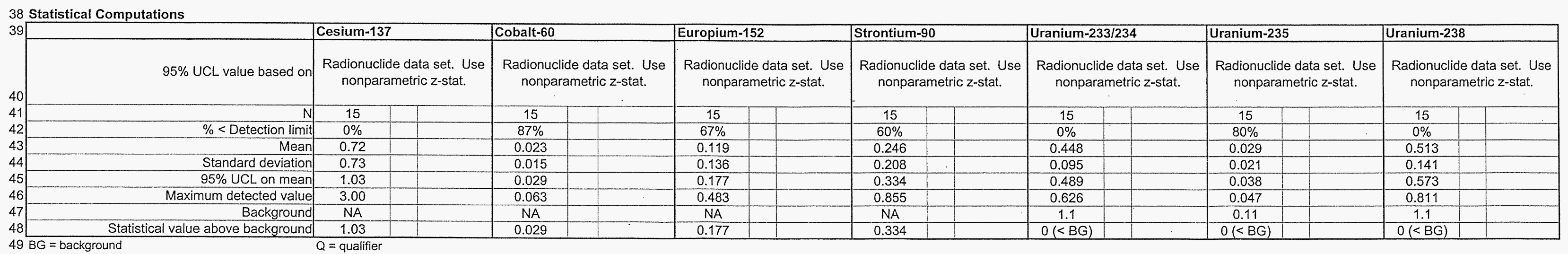


$1118-C-1$ Staging Pile Area Statistical Calculations (continued)
2 Verification Data
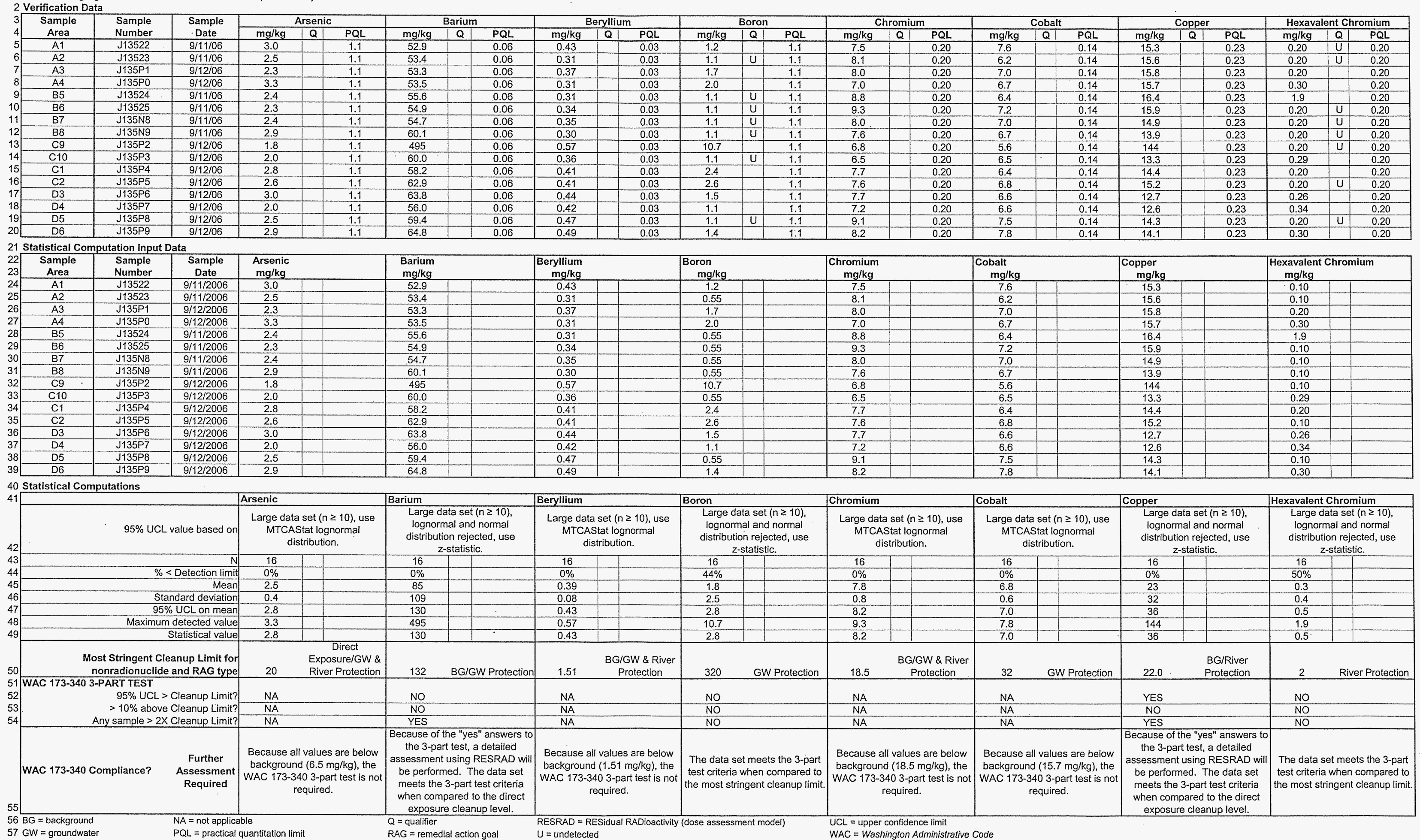

56 BG background
$57 \mathrm{GW}=$ groundwater NA $=$ not applicable
PQL $=$ pracical ouantitation lim

$Q=$ qualifier
$R A G=$ remedial action goal RESRAD $=$ RES

WCL = upper confidence limit 
118-C-1 Staging Pile Area Statistical Calculations (continued)
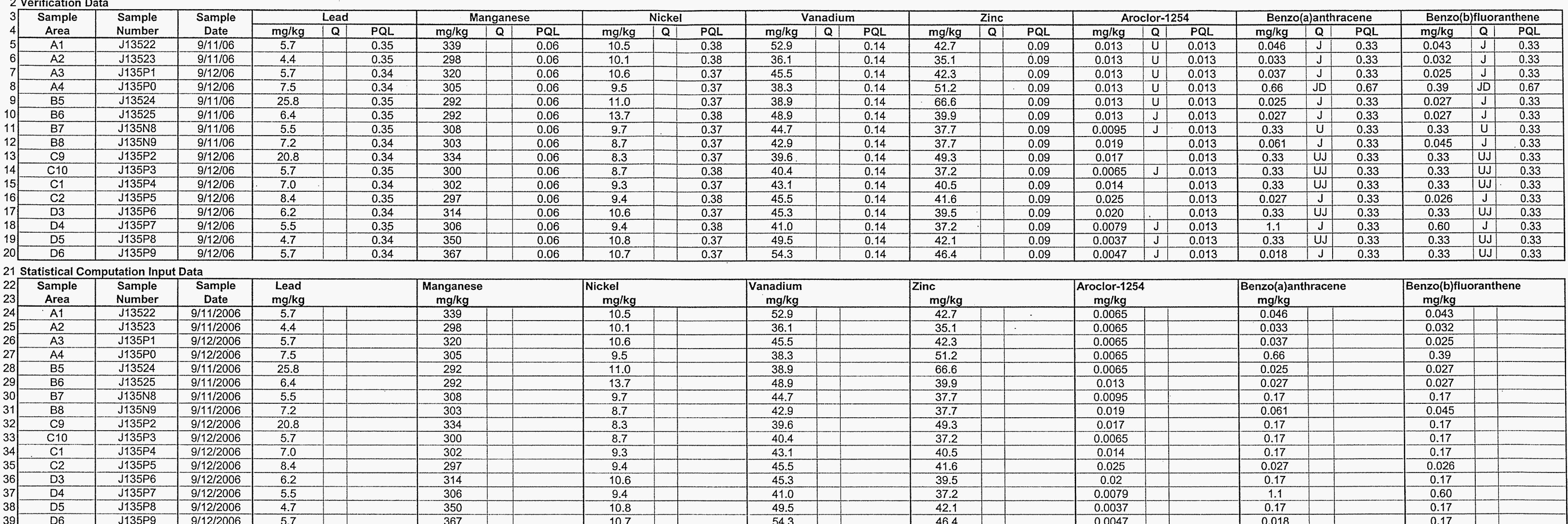

\begin{tabular}{|c|c|c|}
38 & $D 5$ & $135 \mathrm{PP}$ \\
\hline 30 & $\mathrm{D} 6$ & $\mathrm{J135 \textrm {P } 9}$ \\
\hline
\end{tabular}
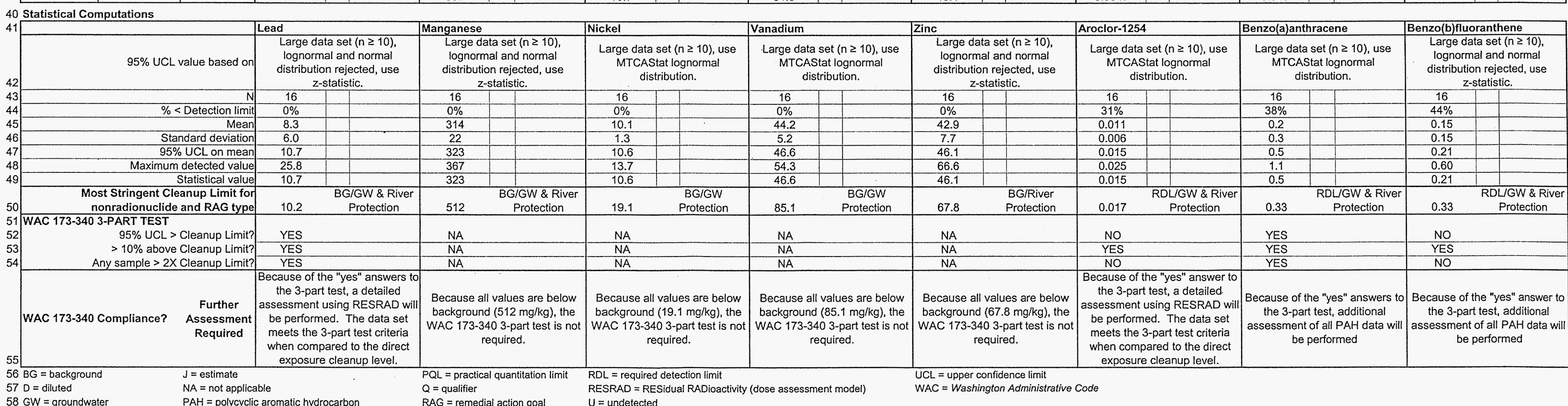
1 118-C-1 Staging Pile Area Statistical Calculations (continued)

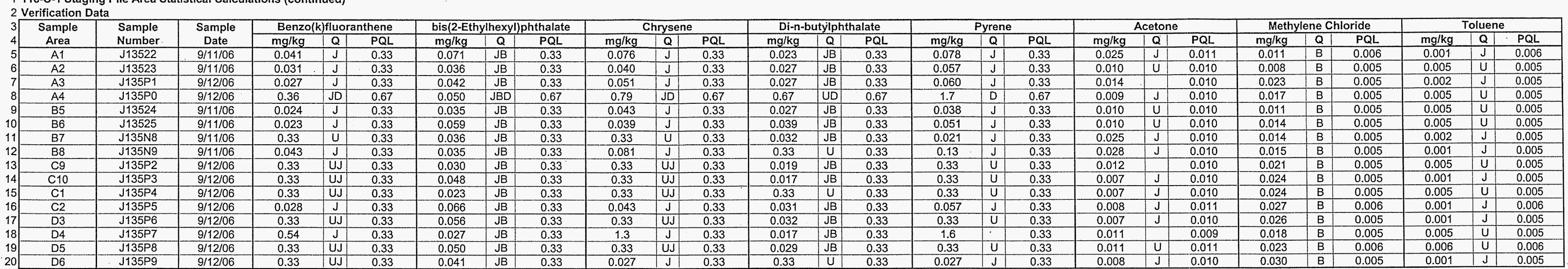

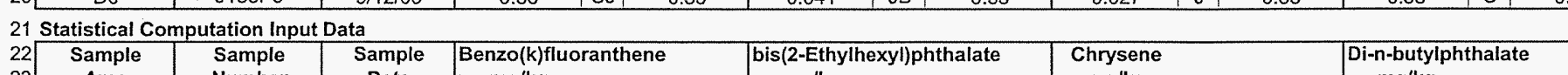

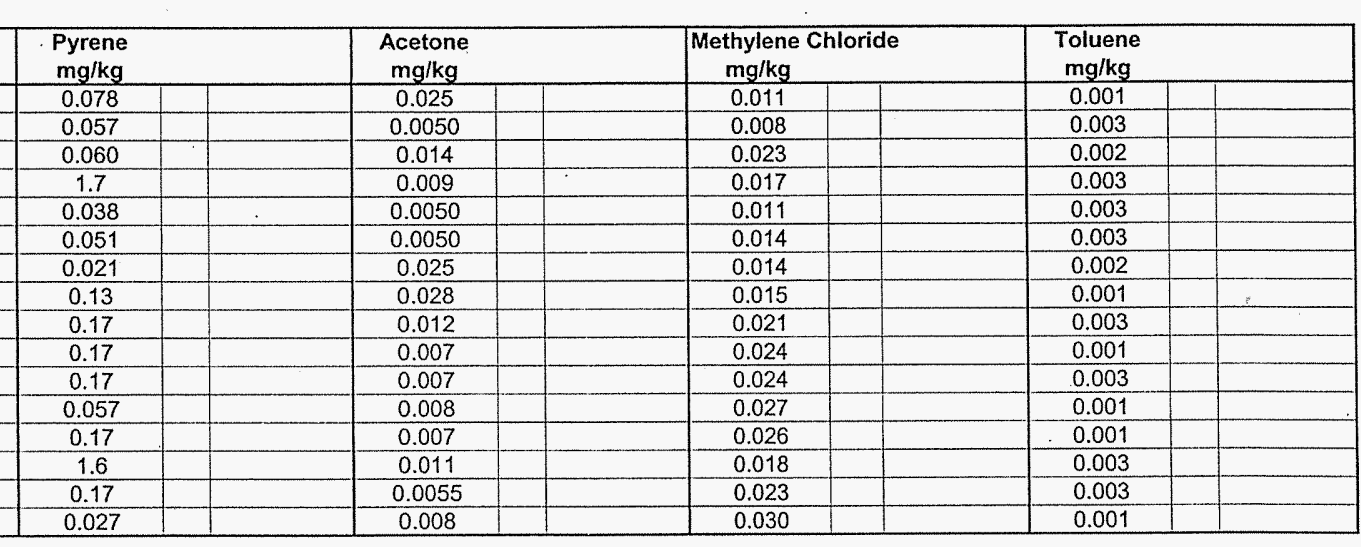

\begin{tabular}{c|c|c}
338 & $\mathrm{D} 5$ & $\mathrm{~J}$ \\
$\mathrm{D}$ & $\mathrm{J} 135$ \\
\hline
\end{tabular}

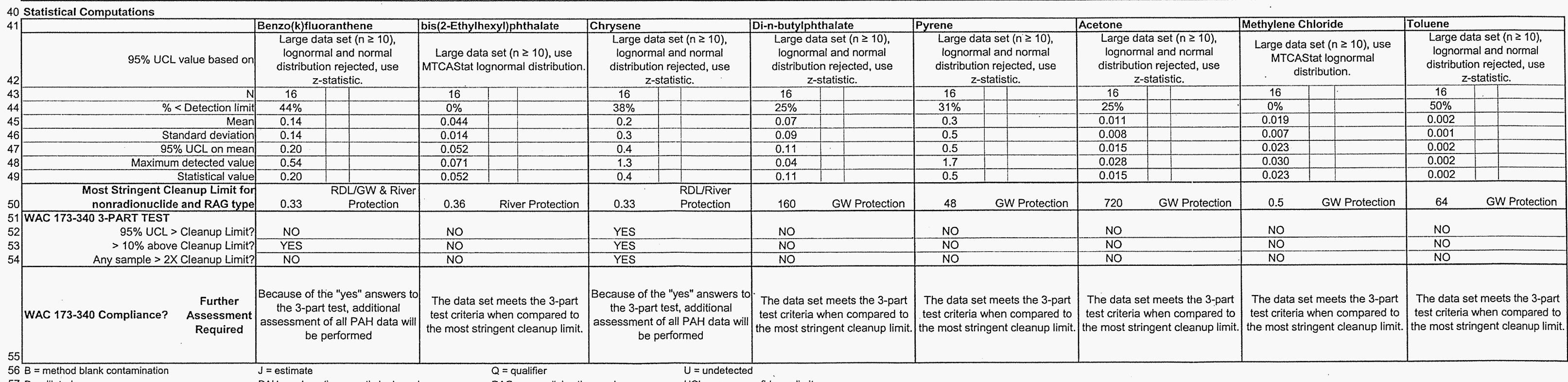

$56 \mathrm{~B}=$ method blank
$57 \mathrm{D}=$ diluted
$58 \mathrm{GW}=$ oroundwater

$J=$ estimate
PAH = polycyclic aromatic hydrocarbon
$P Q L=$ practical quantitation limit

$\mathrm{RAG}=$ remedial action goal
$\mathrm{RDL}=$ = required detection limit

UCL $=$ upper confidenes limit
WAC = Washington Administrative Code 


\section{CALCULATION SHEET}

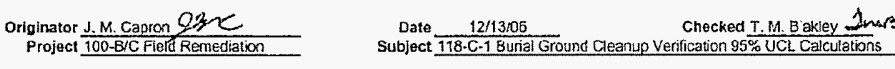

Date $12 / 4 \mid$ ol

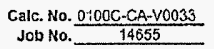

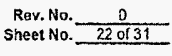

\section{8-C-1 Staging Pilo Aroa Statisticas Caiculations (continued}

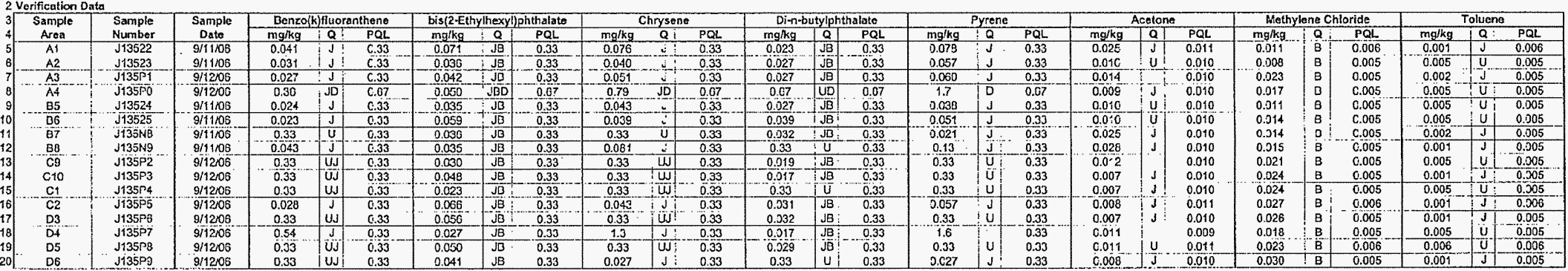

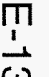

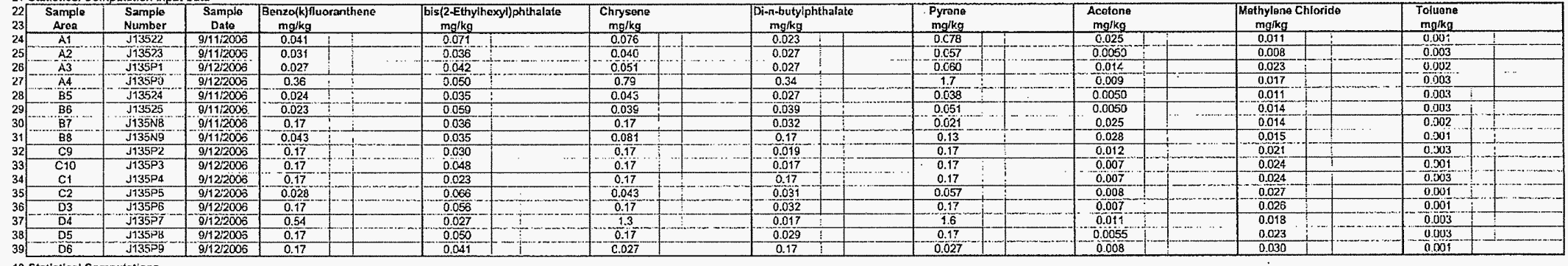

40 Statistical Computations

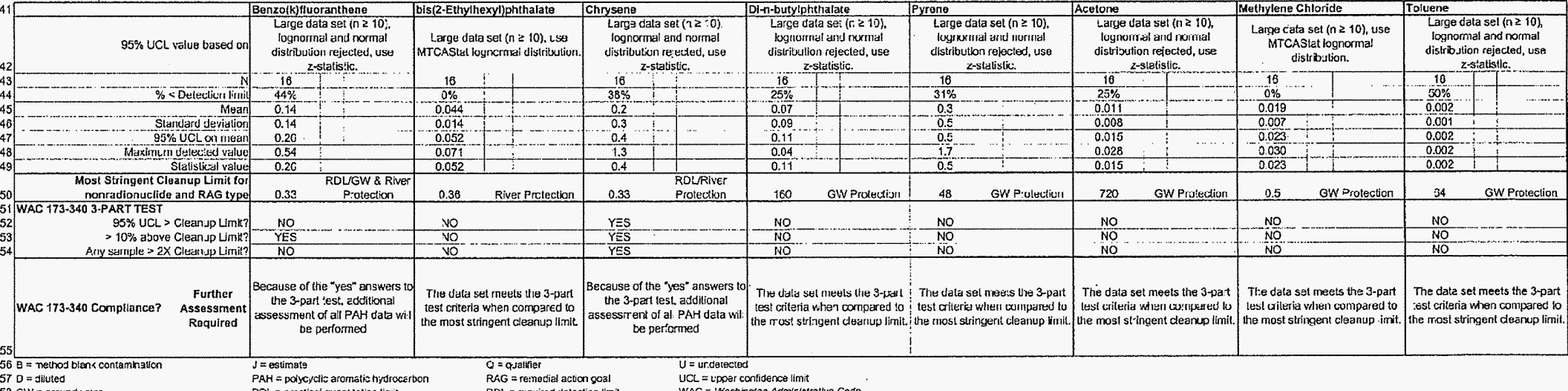


CALCULATION SHEET

Washington Closure Hanford
Originator J. M. Capron $92 \mathrm{~m} C$
Project 100-B/C Field Remediation
Subject $118-\mathrm{C}-1$ Burial Ground Cleanup Verification 95\% UCL Calculation

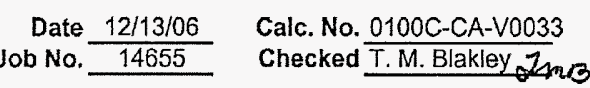

Rev. No. $\frac{0}{\text { Date } \frac{12 / 4 / 06}{23 \text { of } 31}}$
Sheet No.

Ecology Software (MTCAStat) Results (Staging Pile Area)

\begin{tabular}{|c|c|c|c|c|c|c|c|c|c|c|c|c|}
\hline \multirow{3}{*}{$\begin{array}{l}1 \\
2 \\
3\end{array}$} & \multirow{3}{*}{$\begin{array}{c}\text { DATA } \\
3.0 \\
2.5\end{array}$} & \multirow{3}{*}{$\begin{array}{c}\text { ID } \\
\mathrm{J} 13522 \\
\mathrm{~J} 13523\end{array}$} & \multicolumn{3}{|c|}{ Arsenic $95 \%$ UCL Calculation } & & \multirow{3}{*}{$\begin{array}{c}\text { DATA } \\
52.9 \\
53.4\end{array}$} & \multirow{3}{*}{$\begin{array}{c}\text { ID } \\
\mathrm{J} 13522 \\
\mathrm{~J} 13523\end{array}$} & \multicolumn{3}{|c|}{ Barium 95\% UCL Calculation } & \multirow[b]{5}{*}{85} \\
\hline & & & & & & & & & & & & \\
\hline & & & & & & & & & & & & \\
\hline 4 & 2.3 & J135P1 & Number of samples & & Uncensored values & & 53.3 & J135P1 & Number of samples & & Uncensored values & \\
\hline 5 & 3.3 & J135P0 & Uncensored & 16 & Mean & 2.5 & 53.5 & J135P0 & Uncensored & 16 & Mean & \\
\hline 6 & 2.4 & $\mathrm{~J} 13524$ & Censored & & Lognormal mean & 2.5 & 55.6 & $J 13524$ & Censored & & Lognormal mean & 76 \\
\hline 7 & 2.3 & J13525 & Detection limit or $\mathrm{PQL}$ & & Std. devn. & 0.4 & 54.9 & J13525 & Detection limit or PQL & & Std, devn. & 109 \\
\hline 8 & 2.4 & J135N8 & Method detection limit & & Median & 2.5 & 54.7 & J135N8 & Methad detection limit & & Median & 57 \\
\hline 9 & 2.9 & J135N9 & TOTAL & 16 & Min. & 1.8 & 60.1 & J135N9 & TOTAL & 16 & Min. & 52.9 \\
\hline $10 \mid$ & 1.8 & $\mathrm{~J} 135 \mathrm{P} 2$ & & & Max. & 3.3 & 495 & J135P2 & & & Max. & 495 \\
\hline 11 & 2.0 & J135P3 & & & & & 60.0 & J135P3 & & & & \\
\hline 12 & 2.8 & $\mathrm{~J} 135 \mathrm{P} 4$ & & & & & 58.2 & J135P4 & & & & \\
\hline 13 & 2.6 & J135P5 & Lognormal distribution? & & Normal distribution? & & 62.9 & J135P5 & Lognormal distribution? & & Normal distribution? & \\
\hline 14 & 3.0 & J135P6 & r-squared is: $\quad 0.967$ & & r-squared is: $\quad 0.976$ & & 63.8 & J135P6 & r-squared is: $\quad 0.351$ & & r-squared is: $\quad 0.273$ & \\
\hline 15 & 2.0 & J135P7 & Recommendations: & & & & 56.0 & J135P7 & Recommendations: & & & \\
\hline 16. & 2.5 & J135P8 & Use lognormal distributio & & & & 59.4 & J135P8 & Reject BOTH lognormal & and $n$ & nal distributions. & \\
\hline 17 & 2.9 & J135P9 & & & & & 64.8 & J135P9 & & & & \\
\hline 19 & & & UCL (Land's method) is & & 2.8 & & & & UCL (based on Z-statisti & c) is & 130 & \\
\hline 21 & DATA & ID & Beryllium 95\% & $\widehat{\mathrm{UCl}}$ & Calculation & & DATA & TD & Boron 95\% & $\overline{\mathrm{UCL}}$ & Calculation & \\
\hline 22 & 0.43 & J13522 & & & & & 1.2 & $J 13522$ & & & & \\
\hline 23 & 0.31 & J13523 & & & & & 0.55 & $J 13523$ & & & & \\
\hline 24 & 0.37 & J135P1 & Number of samples & & Uncensored values & & 1.7 & J135P1 & Number of samples & & Uncensored values & \\
\hline 25 & 0.31 & $\mathrm{~J} 135 \mathrm{P} 0$ & Uncensored & 16 & Mean & 0.39 & 2.0 & J135P0 & Uncensored & 16 & Mean & 1.8 \\
\hline $26 \mid$ & 0.31 & J13524 & Censored & & Lognormal mean & 0.39 & 0.55 & $J 13524$ & Censored & & Lognormal mean & 1.6 \\
\hline 27) & 0.34 & $J 13525$ & Detection limit or $P Q L$ & & Std. devn. & 0.08 & 0.55 & J13525 & Detection limit or PQL & & Std. devn. & 2.5 \\
\hline 28 & 0.35 & J135N8 & Method detection limit & & Median & 0.39 & 0.55 & J135N8 & Method detection limit & & Median & 1.2 \\
\hline 29 & 0.30 & J135N9 & TOTAL & 16 & Min. & 0.30 & 0.55 & J135N9 & TOTAL & 16 & Min. & 0.55 \\
\hline 30 & 0.57 & J135P2 & & & Max. & 0.57 & 10.7 & $\mathrm{~J} 135 \mathrm{P} 2$ & & & Max. & 10.7 \\
\hline 31 & 0.36 & J135P3 & & & & & 0.55 & J135P3 & & & & \\
\hline 32 & 0.41 & $\mathrm{~J} 135 \mathrm{P} 4$ & & & & & 2.4 & $\mathrm{~J} 135 \mathrm{P} 4$ & & & & \\
\hline 33 & 0.41 & J135P5 & Lognormal distribution? & & Normal distribution? & & 2.6 & J135P5 & Lognormal distribution? & & Normal distribution? & \\
\hline 34 & 0.44 & J135P6 & r-squared is: $\quad 0.959$ & & r-squared is: $\quad 0.937$ & & 1.5 & J135P6 & r-squared is: 0.825 & & $r$-squared is: 0.483 & \\
\hline 35 & 0.42 & J135P7 & Recommendations: & & & & 1.1 & J135P7 & Recommendations: & & & \\
\hline 36 & 0.47 & J135P8 & Use lognormal distributio & & & & 0.55 & J135P8 & Reject BOTH lognormal & and $n$ & ormal distributions. & \\
\hline 37 & 0.49 & J135P9 & & & & & 1.4 & $\mathrm{~J} 135 \mathrm{P9}$ & & & & \\
\hline 39 & & & UCL (Land's method) is & & 0.43 & & & & UCL (based on Z-statistic & & 2.8 & \\
\hline
\end{tabular}


Washington Closure Hanford

CALCULATION SHEET

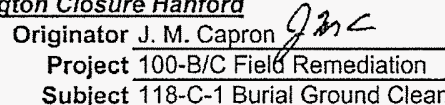

Perification 95\% UCL Calculations

Date $12 / 13 / 06$ Calc. No. 0100C-CA-V0033

Checked T.M. Blakley JmB

Rev. No.

Date $12 / 14 / 0$

Sheet No. 24 of 31

Ecology Software (MTCAStat) Results (Staging Pile Area)

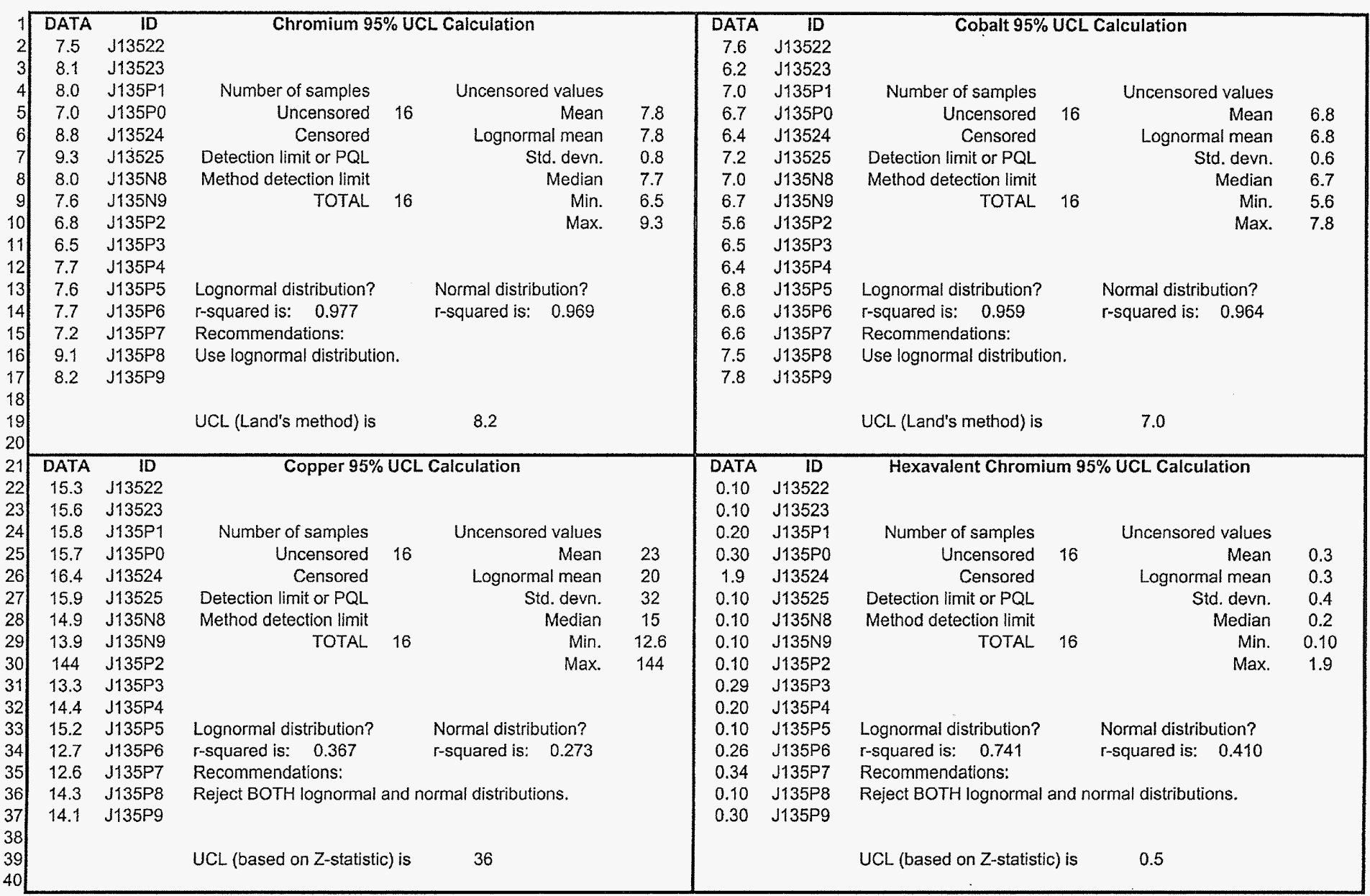

$41 \mathrm{PQL}=$ practical quantitation limit

42 UCL = upper confidence limit 
Washington Closure Hanford

Originator J.M. Capron $\ln C$

Project $100-B / C$ Fiele Remediation
CALCULATION SHEET

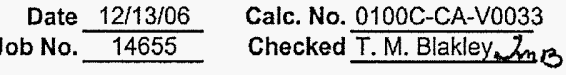

Rev. No.

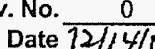

Ecology Software (MTCAStat) Results (Staging Pile Area)

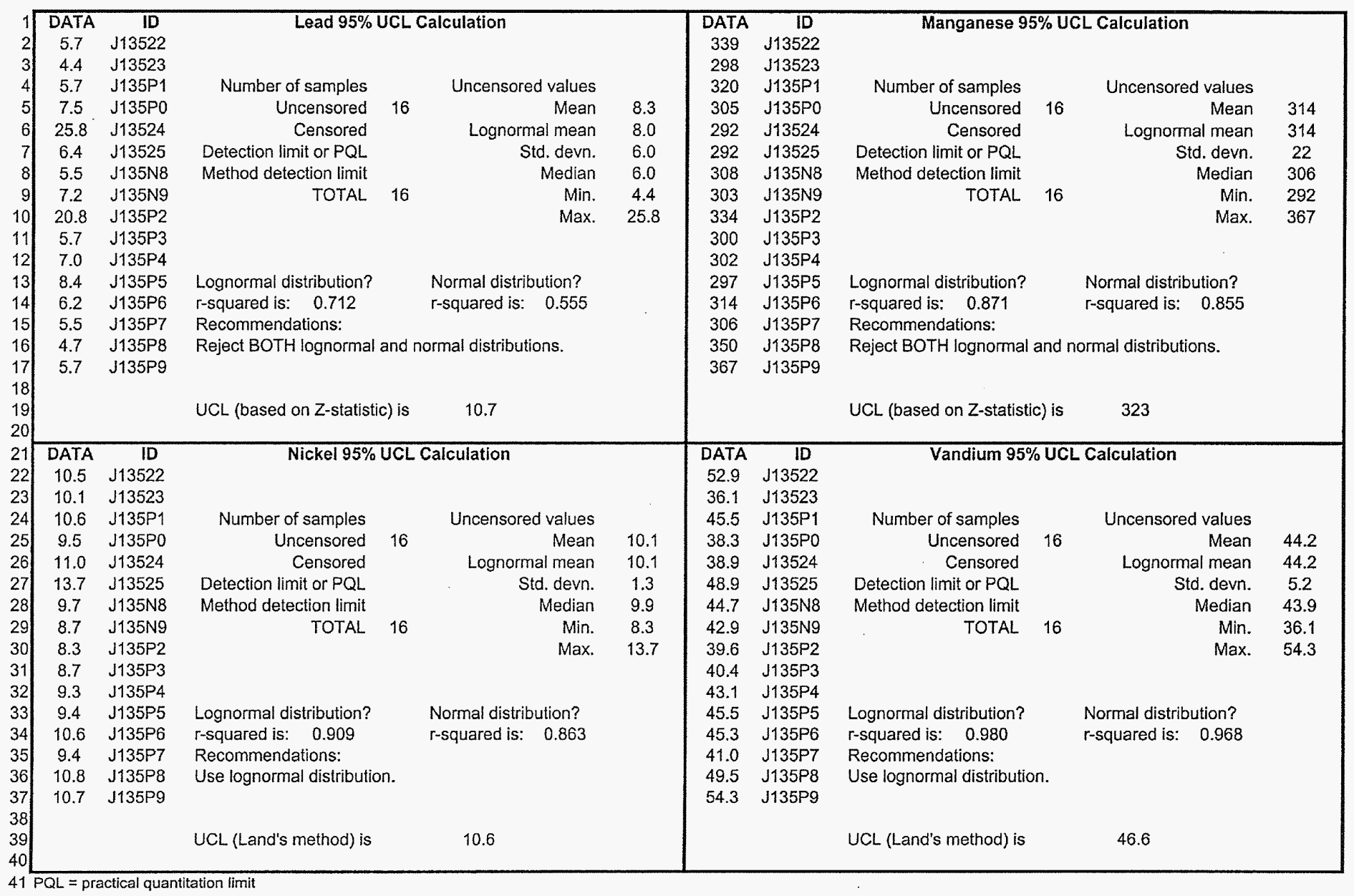




\section{CALCULATION SHEET}

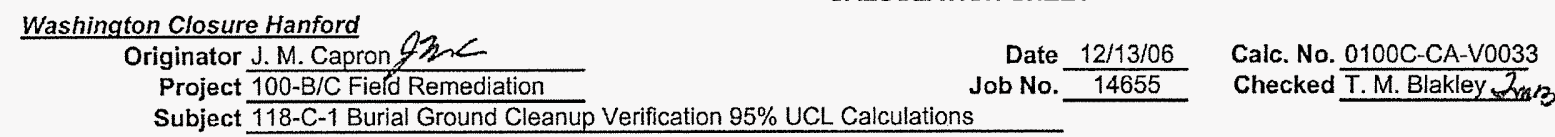

Rev. No. $\frac{0}{\text { Date } 12 / 14 / 06}$ Date $\frac{12 / 14 / 06}{26 \text { of } 31}$

Ecology Software (MTCAStat) Results (Staging Pile Area)

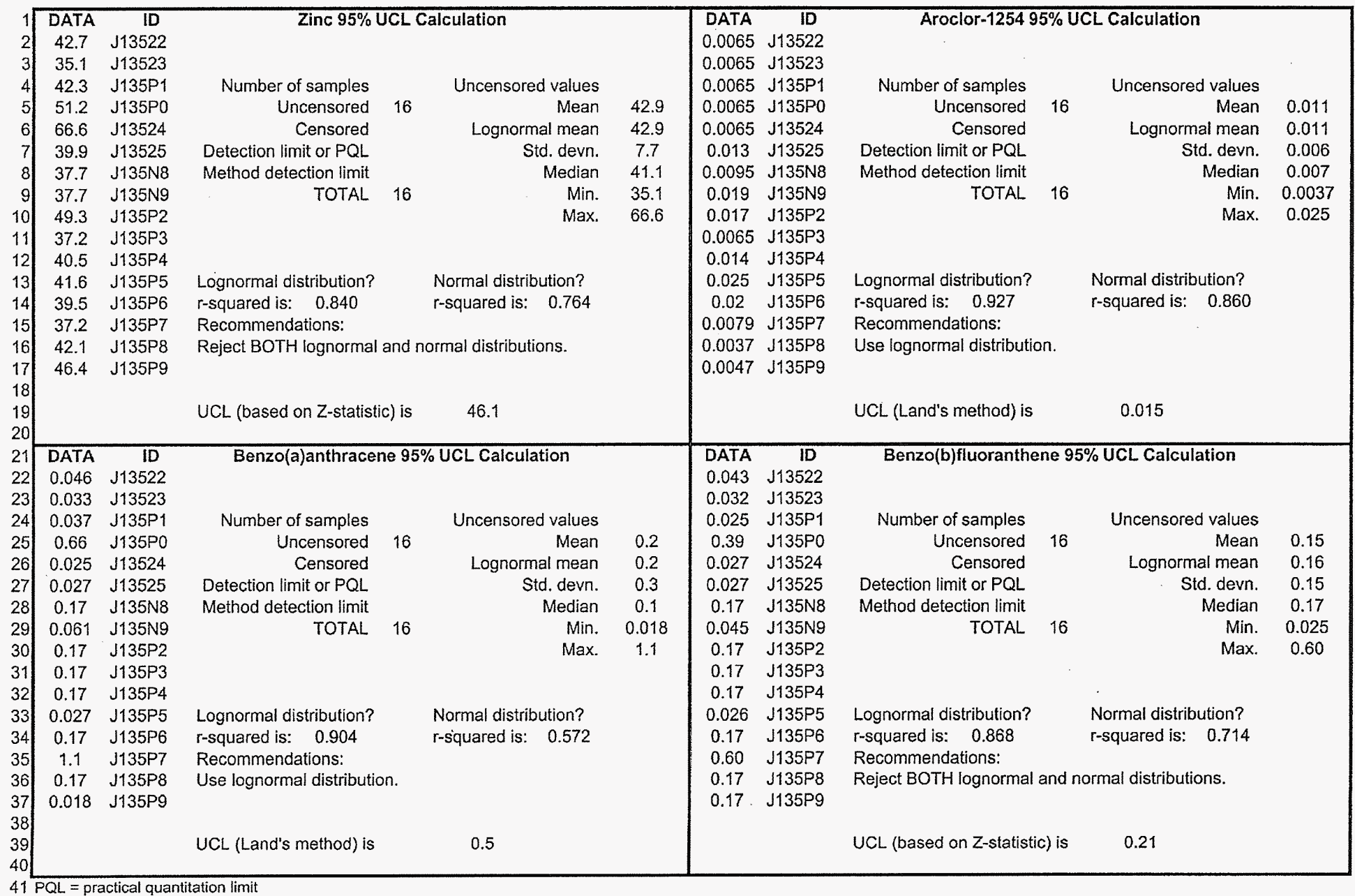




\section{CALCULATION SHEET}

\section{Washington Closure Hanford}

Originator J. M. Capron Shse

Project 100-B/C Field Remediation

Subject $118-\mathrm{C}-1$ Burial Ground Cleanup Verification $95 \%$ UCL Calculations
Date $\frac{12 / 13 / 06}{14655}$ Calc. No. 0100C-CA-V0033
Rev. No.

heet No $\frac{12 / 14 / 06}{270 / 31}$

Ecology Software (MTCAStat) Results (Staging Pile Area)

\begin{tabular}{|c|c|c|c|c|c|c|c|c|c|c|c|c|}
\hline 1[ & DATA & ID & \multicolumn{4}{|c|}{ Benzo(k)fluoranthene $95 \%$ UCL Calculation } & DATA & ID & \multicolumn{4}{|c|}{ bis(2-Ethy|hexyl)phthalate $95 \%$ UCL Calculation } \\
\hline 2 & 0.041 & J13522 & & & & & 0.071 & J13522 & & & & \\
\hline 3 & 0.031 & J13523 & & & & & 0.036 & J13523 & & & & \\
\hline 4 & 0.027 & J135P1 & Number of samples & & Uncensored values & & 0.042 & J135P1 & Number of samples & & Uncensored values & \\
\hline 5 & 0.36 & J135P0 & Uncensored & 16 & Mean & 0.14 & 0.050 & $\mathrm{~J} 135 \mathrm{PO}$ & Uncensored & 16 & Mean & 0.044 \\
\hline 6 & 0.024 & J13524 & Censored & & Lognormal mean & 0.15 & 0.035 & J13524 & Censored & & Lognormal mean & 0.044 \\
\hline 7 & 0.023 & J13525 & Detection limit or $\mathrm{PQL}$ & & Std. devn. & 0.14 & 0.059 & J13525 & Detection limit or PQL & & Std. devn. & 0.014 \\
\hline 8 & 0.17 & J135N8 & Method detection limit & & Median & 0.17 & 0.036 & J135N8 & Method detection limit & & Median & 0.042 \\
\hline 9 & 0.043 & J135NG & TOTAL & 16 & Min. & 0.023 & 0.035 & J135N9 & TOTAL & 16 & Min. & 0.023 \\
\hline 10 & 0.17 & $\mathrm{~J} 135 \mathrm{P} 2$ & & & Max. & 0.54 & 0.030 & J135P2 & & & Max. & 0.071 \\
\hline 11) & 0.17 & J135P3 & & & & & 0.048 & J135P3 & & & & \\
\hline 12 & 0.17 & J135P4 & & & & & 0.023 & J135P4 & & & & \\
\hline 13 & 0.028 & J135P5 & Lognormal distribution? & & Normal distribution? & & 0.066 & J135P5 & Lognormal distribution? & & Normal distribution? & \\
\hline 14) & 0.17 & $\mathrm{~J} 135 \mathrm{P} 6$ & $r$-squared is: 0.870 & & r-squared is: 0.744 & & 0.056 & J135P6 & $r$-squared is: 0.985 & & r-squared is: 0.969 & \\
\hline 15 & 0.54 & J135P7 & Recommendations: & & & & 0.027 & J135P7 & Recommendations: & & & \\
\hline 16) & 0.17 & J135P8 & Reject BOTH lognormal & and $t$ & ormal distributions. & & 0.050 & J135P8 & Use lognormal distributior & & & \\
\hline 47) & 0.17 & J135P9 & & & & & 0.041 & J135P9 & & & & \\
\hline 19 & & & UCL (based on Z-statisti & c) is & 0.20 & & & & UCL (Land's method) is & & 0.052 & \\
\hline 21 & $\overline{\text { DATA }}$ & ID & Chrysene $95^{\circ}$ & $6 \mathrm{UC}$ & Calculation & & DATA & ID & Di-n-butylphthalate & e 95\% & GUCL Calculation & \\
\hline 22 & 0.076 & J13522 & & & & & 0.023 & $J 13522$ & & & & \\
\hline 23. & 0.040 & J13523 & & & & & 0.027 & J13523 & & & & \\
\hline 24 & 0.051 & J135P1 & Number of samples & & Uncensored values & & 0.027 & J135P1 & Number of samples & & Uncensored values & \\
\hline 25 & 0.79 & J135P0 & Uncensored & 16 & Mean & 0.2 & 0.34 & J135P0 & Uncensored & 16 & Mean & 0.07 \\
\hline 26 & 0.043 & J13524 & Censored & & Lognormal mean & 0.2 & 0.027 & $\mathrm{~J} 13524$ & Censored & & Lognormal mean & 0.07 \\
\hline 27) & 0.039 & $J 13525$ & Detection limit or PQL & & Std. devn. & 0.3 & 0.039 & $\mathrm{~J} 13525$ & Detection limit or $\mathrm{PQL}$ & & Std. devn. & 0.09 \\
\hline 28 & 0.17 & J135N8 & Method detection limit & & Median & 0.1 & 0.032 & J135N8 & Method detection limit & & Median & 0.03 \\
\hline 29 & 0.081 & J135N9 & TOTAL & 16 & Min. & 0.027 & 0.17 & J135N9 & TOTAL & 16 & Min. & 0.017 \\
\hline 30) & 0.17 & $\mathrm{~J} 135 \mathrm{P} 2$ & & & Max. & 1.3 & 0.019 & J135P2 & & & Max. & 0.34 \\
\hline 31 & 0.17 & J135P3 & & & & & 0.017 & J135P3 & & & & \\
\hline 32 & 0.17 & J135P4 & & & & & 0.17 & J135P4 & & & & \\
\hline 33 & 0.043 & J135P5 & Lognormal distribution? & & Normal distribution? & & 0.031 & J135P5 & Lognormal distribution? & & Normal distribution? & \\
\hline 34 & 0.17 & J135P6 & $r$-squared is: 0.880 & & r-squared is: $\quad 0.533$ & & 0.032 & J135P6 & r-squared is: 0.794 & & r-squared is: 0.623 & \\
\hline 35 & 1.3 & J135P7 & Recommendations: & & & & 0.017 & J135P7 & Recommendations: & & & \\
\hline 36 & 0.17 & $\mathrm{~J} 135 \mathrm{P} 8$ & Reject BOTH lognormal & and $r$ & ormal distributions. & & 0.029 & J135P8 & Reject BOTH lognormal a & and $n$ & ormal distributions. & \\
\hline & 0.027 & J135P9 & & & & & 0.17 & J135P9 & & & & \\
\hline & & & UCL (based on Z-statisti & & 0.4 & & & & UCL (based on Z-statistic & c) is & 0.11 & \\
\hline
\end{tabular}




\section{CALCULATION SHEET}

\begin{tabular}{|c|c|c|}
\hline Originator J. M. Capron $/ 3 \mathrm{C}$ & $\begin{aligned} \text { Date } & 12 / 13 / 06 \\
\text { Job No. } & 14655\end{aligned}$ & $\begin{array}{l}\text { Calc. No. 0100C-CA-V0033 } \\
\text { Checked T. M. Blakley } 27 \text { re }\end{array}$ \\
\hline
\end{tabular}

Subject 118-C-1 Burial Ground Cleanup Verification $95 \%$ UCL Calculations

Ecology Software (MTCAStat) Results (Staging Pile Area)

\begin{tabular}{|c|c|c|c|c|c|c|c|c|c|c|c|c|}
\hline $1 \Gamma$ & DATA & ID & \multicolumn{4}{|c|}{ Pyrene $95 \%$ UCL Calculation } & DATA & & \multicolumn{3}{|c|}{ Acetone $95 \%$ UCL Calculation } & \\
\hline 3 & & & & & & & & & & & & \\
\hline 4 & 0.057 & $J 13523$ & & \multirow{2}{*}{\multicolumn{3}{|c|}{ Uncensored values }} & 0.0050 & $J 13523$ & & \\
\hline 5 & 0.060 & J135P1 & Number of samples & & & & 0.014 & $J 135 P 1$ & Number of samples & \multicolumn{3}{|c|}{ Uncensored values } \\
\hline 6 & 1.7 & J135P0 & Uncensored & 16 & Mean & 0.3 & 0.009 & J135P0 & Uncensored & \multirow{2}{*}{16} & Mean & 0.011 \\
\hline & 0.038 & J13524 & Censored & & Lognormal mean & 0.2 & 0.0050 & J13524 & Censored & & Lognormal mean & 0.011 \\
\hline 7 & 0.051 & J13525 & Detection limit or $P Q L$ & & Std. devn. & 0.5 & 0.0050 & $J 13525$ & Detection limit or $\mathrm{PQL}$ & & Std. devn. & 0.008 \\
\hline 8 & 0.021 & J135N8 & Method detection limit & & Median & 0.1 & 0.025 & J135N8 & Method detection limit & \multirow{4}{*}{16} & Median & 0.008 \\
\hline 9 & 0.13 & J135N9 & TOTAL & 16 & Min. & 0.021 & 0.028 & J135N9 & TOTAL & & Min. & 0.0050 \\
\hline 10 & 0.17 & $\mathrm{~J} 135 \mathrm{P} 2$ & & & Max. & 1.7 & 0.012 & J135P2 & & & Max. & 0.028 \\
\hline 11 & 0.17 & J135P3 & & & & & 0.007 & J135P3 & & & & \\
\hline 12 & 0.17 & J135P4 & & & & & 0.007 & J135P4 & & & & \\
\hline 13. & 0.057 & J135P5 & Lognormal distribution? & & Normal distribution? & & 0.008 & J135P5 & Lognormal distribution? & & Normal distribution? & \\
\hline 14 & 0.17 & J135P6 & r-squared is: $\quad 0.867$ & & $r$-squared is: 0.484 & & 0.007 & J135P6 & $r$-squared is: $\quad 0.896$ & & \multirow[t]{2}{*}{$r$-squared is: $\quad 0.770$} & \\
\hline 15 & 1.6 & J135P7 & \multirow{2}{*}{\multicolumn{4}{|c|}{$\begin{array}{l}\text { Recommendations: } \\
\text { Reject BOTH lognormal and normal distributions. }\end{array}$}} & 0.011 & J135P7 & & & & \\
\hline 16 & 0.17 & J135P8 & & & & & 0.0055 & $J 135 P 8$ & \multicolumn{3}{|c|}{ Reject BOTH lognormal and normal distributions. } & \\
\hline 177] & 0.027 & J135P9 & & & & & 0.008 & J135P9 & & & & \\
\hline 9 & & & \multicolumn{3}{|c|}{ UCL (based on Z-statistic) is } & & & & \multicolumn{2}{|c|}{ UCL (based on Z-statistic) is } & 0.015 & \\
\hline & DATA & ID & Methylene Chloride & e 95\% & UCL Calculation & & DATA & ID & Toluene $95 \%$ & $\overline{U C L}$ & Calculation & \\
\hline 22 & 0.011 & $J 13522$ & & & 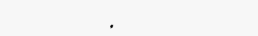 & & 0.001 & $J 13522$ & & & & \\
\hline 23 & 0.008 & $J 13523$ & & & & & 0.003 & $J 13523$ & & & & \\
\hline 24 & 0.023 & J135P1 & Number of samples & & Uncensored values & & 0.002 & J135P1 & Number of samples & & Uncensored values & \\
\hline 5 & 0.017 & $J 135 \mathrm{P0}$ & Uncensored & 16 & Mean & 0.019 & 0.003 & J135PO & Uncensored & 16 & Mean & 0.002 \\
\hline 26 & 0.011 & J13524 & Censored & & Lognormal mean & 0.019 & 0.003 & $\mathrm{~J} 13524$ & Censored & & Lognormal mean & 0.002 \\
\hline 27 & 0.014 & $\mathrm{~J} 13525$ & Detection limit or $P Q L$ & & Std. devn. & 0.007 & 0.003 & $J 13525$ & Detection limit or $P Q L$ & & Std. devn. & 0.001 \\
\hline 8 & 0.014 & J135N8 & Method detection limit & & Median & 0.020 & 0.002 & J135N8 & Method detection limit & & Median & 0.002 \\
\hline 29 & 0.015 & J135N9 & TOTAL & 16 & Min. & 0.008 & 0.001 & J135N9 & TOTAL & 16 & Min. & 0.001 \\
\hline 30 & 0.021 & $\mathrm{~J} 135 \mathrm{P} 2$ & & & Max. & 0.030 & 0.003 & J135P2 & & & Max. & 0.003 \\
\hline & 0.024 & J135P3 & & & & & 0.001 & J135P3 & & & & \\
\hline 32 & 0.024 & $\mathrm{~J} 135 \mathrm{P} 4$ & & & & & 0.003 & $\mathrm{~J} 135 \mathrm{P} 4$ & & & & \\
\hline 33 & 0.027 & J135P5 & Lognormal distribution? & & Normal distribution? & & 0.001 & J135P5 & Lognormal distribution? & & Normal distribution? & \\
\hline & 0.026 & J135P6 & $r$-squared is: 0.943 & & r-squared is: 0.970 & & 0.001 & J135P6 & r-squared is: $\quad 0.757$ & & r-squared is: 0.793 & \\
\hline 5 & 0.018 & J135P7 & Recommendations: & & & & 0.003 & $\mathrm{~J} 135 \mathrm{P} 7$ & Recommendations: & & & \\
\hline 36 & 0.023 & J135P8 & Use lognormal distribution & & & & 0.003 & J135P8 & Reject BOTH lognormal a & and $n$ & ormal distributions. & \\
\hline & 0.030 & J135P9 & & & & & 0.001 & J135P9 & & & & \\
\hline & & & UCL (Land's method) is & & 0.023 & & & & UCL (based on Z-statistic & & 0.002 & \\
\hline
\end{tabular}


CVP-2006-00011

Rev. 0

E-138 
Washington Closure Hanford

Originator J. M. Capron 2 \& 2

Project 100-B/C Field Remediation
CALCULATION SHEET

$$
\begin{aligned}
& \text { Date } \frac{12 / 13 / 06}{14655} \\
& \text { Job No. }
\end{aligned}
$$

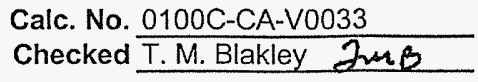

Rev. No. $\frac{0}{\text { Date } \frac{12 / 14 / 06}{29031}}$

1 Split/Duplicate Analysis

\begin{tabular}{|c|c|c|c|c|c|c|c|c|c|c|c|c|c|c|c|c|c|c|c|c|c|c|c|c|}
\hline \multirow{2}{*}{$3 \begin{array}{c}\text { Sample } \\
\text { Area } \\
\end{array}$} & \multirow{2}{*}{$\begin{array}{l}\text { Sample } \\
\text { Number } \\
114 Y 7\end{array}$} & \multirow{2}{*}{$\begin{array}{c}\begin{array}{c}\text { Sample } \\
\text { Date }\end{array} \\
\end{array}$} & \multicolumn{3}{|c|}{ Cesium-137 } & \multicolumn{2}{|c|}{ Europium-152 } & \multicolumn{3}{|c|}{ Nickel-63 } & \multicolumn{3}{|c|}{ Plutonium-239/240 } & \multicolumn{2}{|c|}{ Potassium-40 } & \multicolumn{3}{|c|}{ Radium-226 } & \multicolumn{3}{|c|}{ Radium-228 } & \multicolumn{3}{|c|}{ Thorium-228 } \\
\hline & & & pCi/g & Q & MDA & $\mathrm{pCilg}$ & MDA & $\mathrm{pCi} / \mathrm{g}$ & & $\mathrm{MDA}$ & pCilg & & MDA & & \begin{tabular}{|l|l|}
$\mathrm{Q}$ & $\mathrm{MDA}$ \\
\end{tabular} & $\mathrm{pCi/g}$ & $Q$ & MDA & & & MDA & $\mathrm{pCi} / \mathrm{g}$ & & MDA \\
\hline & $\frac{J 134 Y 7}{1349}$ & & $\begin{array}{l}0.090 \\
0.299\end{array}$ & U & 0.090 & $\begin{array}{l}0.161 \\
0.142\end{array}$ & 0.097 & 2.49 & u & $\frac{2.9}{2 .}$ & 0.025 & $\mathrm{U}$ & 0.19 & 21.6 & 0.37 & 0.754 & & 0.082 & $\frac{1.04}{0.520}-x-3$ & & 0.19 & $\frac{1.09}{0.022}$ & & 0.063 \\
\hline $\begin{array}{l}\text { Duplicate of } J 134 Y Y 7 \\
\text { Split of } \mathrm{J} 134 \mathrm{Y}\end{array}$ & $\frac{J 13499}{113504}$ & $8817 / 106$ & $\frac{0.299}{0.391}$ & & $\frac{0.032}{0.0162}$ & $\frac{0.142}{0.128}$ & 1) $\begin{array}{l}0.083 \\
0.0519\end{array}$ & $\frac{1.42}{770}$ & $u$ & $\begin{array}{r}3.1 \\
573\end{array}$ & $\frac{0}{0.0461}$ & & 0.57 & 11.4 & 0.28 & 0.401 & & 0.063 & 0.530 & & 0.15 & 0.622 & & \\
\hline \multirow{2}{*}{\multicolumn{25}{|c|}{$\begin{array}{ll}\text { Sppltis of } & \\
\text { Analysis: } & \text { TDL } \\
\end{array}$}} \\
\hline & & & \multirow{2}{*}{\multicolumn{3}{|c|}{$\begin{array}{l}0.1 \\
\text { No - evaluate difference }\end{array}$}} & \multirow{2}{*}{\multicolumn{2}{|c|}{$\frac{0.1}{1 \text { es (continue) }}$}} & \multirow{2}{*}{\multicolumn{3}{|c|}{ No palugto differance }} & & \multirow{2}{*}{\multicolumn{2}{|c|}{0.5}} & \multirow{2}{*}{\multicolumn{3}{|c|}{$\frac{0.1}{\text { Yes (continue) }}$}} & \multirow{2}{*}{\multicolumn{3}{|c|}{$\frac{0.2}{\text { continue) }}$}} & \multirow{2}{*}{\multicolumn{3}{|c|}{$\frac{1}{1}$}} \\
\hline \multirow{3}{*}{ Duplicate Analysis } & \multirow{2}{*}{\multicolumn{2}{|c|}{$\begin{array}{l}\text { Both > MDA? } \\
\text { Both }>5 \times T D \mid ?\end{array}$}} & & & & & & & & & & & & \multirow{3}{*}{\multicolumn{2}{|c|}{$\begin{array}{l}\text { Yes (continue) } \\
\text { Yes (calc RPD) } \\
62 \%\end{array}$}} & & & & & & & & & \\
\hline & \multirow{2}{*}{\multicolumn{2}{|c|}{ RPD }} & \multirow{2}{*}{\multicolumn{3}{|c|}{ (5) }} & \multicolumn{2}{|c|}{ No - evaluate difference } & \multicolumn{3}{|c|}{ No-evaluate difference } & \multicolumn{3}{|c|}{ No- evaluate difference } & & & \multicolumn{3}{|c|}{ No - evaluate difference } & \multicolumn{3}{|c|}{ No-evaluate difference } & \multirow{2}{*}{\multicolumn{3}{|c|}{ No - evaluate difference }} \\
\hline & & & & & & & ac & & & & & & & & & Yes- & ssess & further & Yes & ss & further & & & \\
\hline & & DDA? & No-eva & uate & ference & & & No-eval & luate $d$ & difference & No-e & & lifference & & applicalole & & & & & & & & & Itavle \\
\hline Split Analysis & Both & TDL? & & & & No-eva & uate difference & & & & & & & & & & & & & & & & & \\
\hline & Differen & TDL? & Yes & ses: & ther & & ble & & & ble & & & & & & & & & & & & & & \\
\hline
\end{tabular}

\section{6}

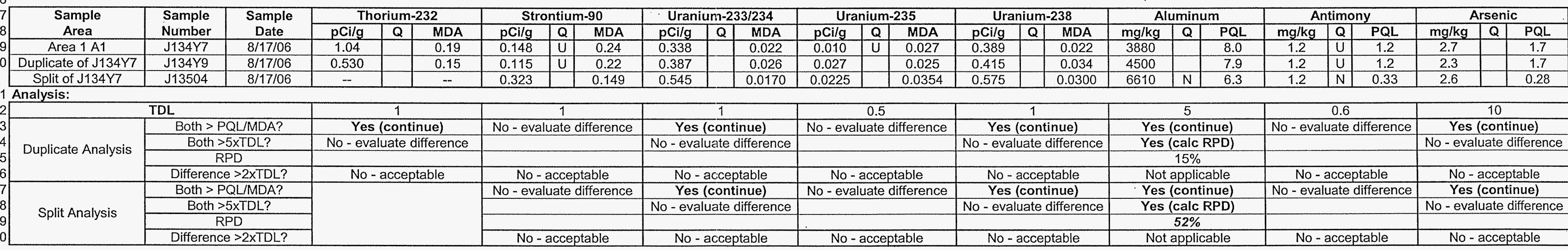

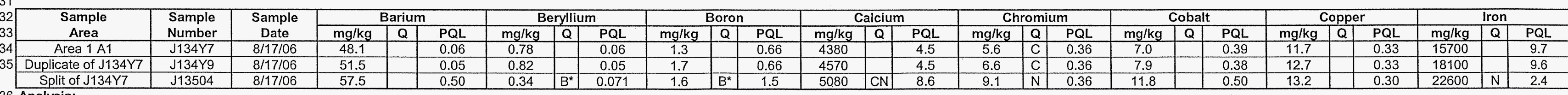

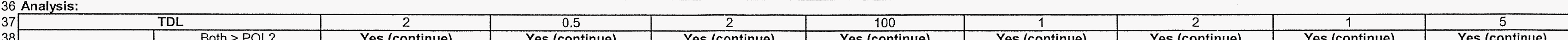
Duplicate Analysis

Split Analysis Yes (continue)

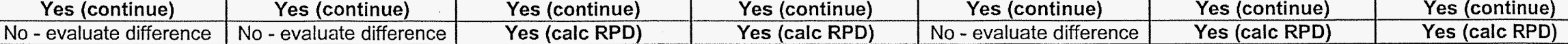

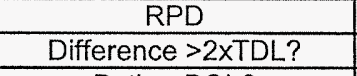
$6.8 \%$ $46-=$ not reported $47 \mathrm{~B}^{*}$ estimated

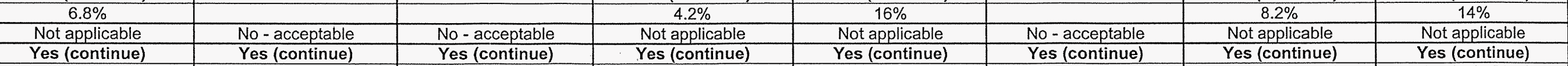

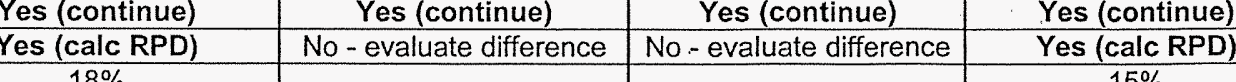
th > PQL? Not applicable Not applicable
Yes (continue)

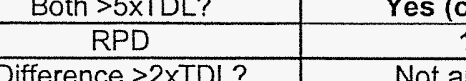
PQL $=$ practica $P Q L=$ practical quantitation limit
$Q=$ qualifier $\begin{array}{ll}\mathrm{R}=\text { method blank contamination } & R P D=\text { relative percent differen } \\ \mathrm{TDL}=\text { = target detection limit } \\ \mathrm{U}=\text { minimum detectable activity } & U=\text { undetected }\end{array}$ 


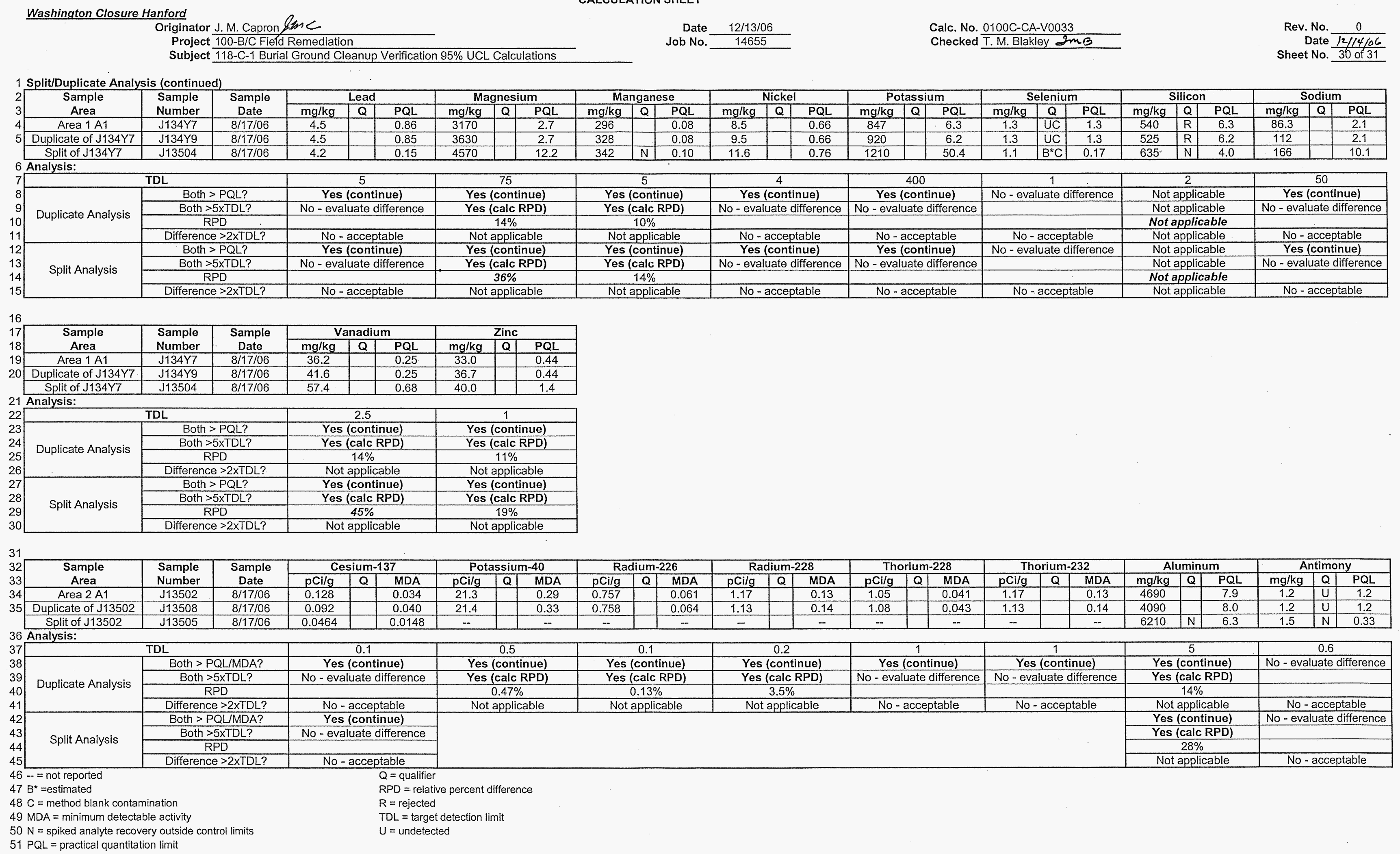

$51 \mathrm{PQL}=$ practical quantitation limit 
Washington Closure Hanford

Originator J. M. Capron Qme

Subject 118 - 118 -C-1 Burrial Ground Cleanup Verification $95 \%$ UCL Calculations
CALCULATION SHEET

$\begin{aligned} & \text { Date } \frac{12113 / 06}{14655} \\ & \text { Job No. }\end{aligned}$
Calc. No. $\frac{0100 C-C A-V 0033}{\text { Checked }}$ T. M. Blakley 2 mo
C.
Rev. No. 0

Sheet No. $\frac{12 / 14 / 06}{31 \text { of } 31}$

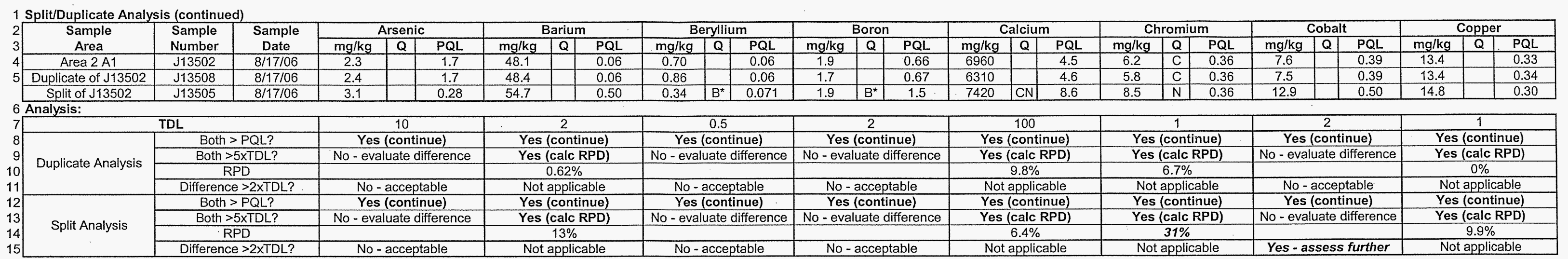

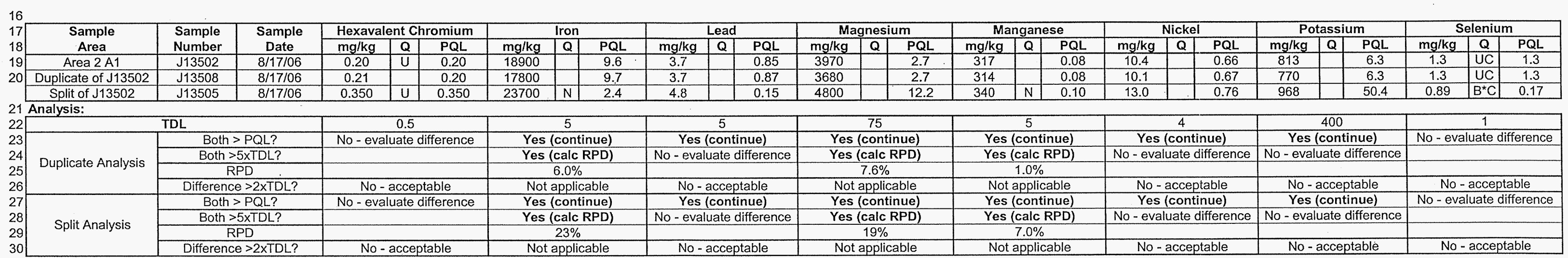

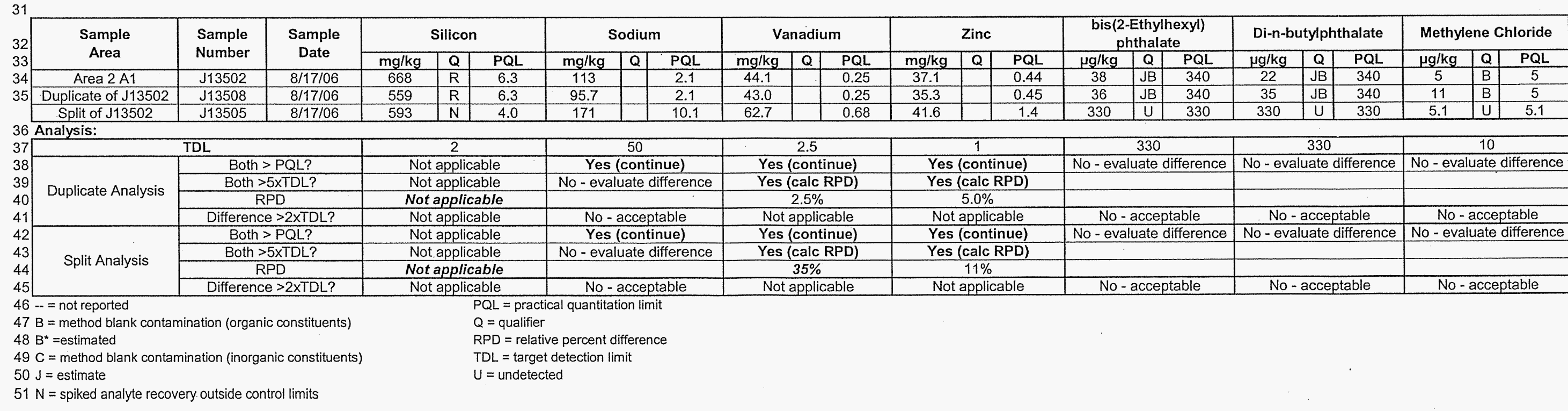


Attachment 1. 118-C-1 Verification Sampling Results.

\begin{tabular}{|c|c|c|c|c|c|c|c|c|c|c|c|c|c|c|c|c|c|}
\hline \multirow{2}{*}{ Sample Location } & \multirow{2}{*}{$\begin{array}{l}\text { Sample } \\
\text { Number }\end{array}$} & \multirow{2}{*}{$\begin{array}{c}\text { Sample } \\
\text { Date }\end{array}$} & \multicolumn{3}{|c|}{ Americium-241 } & \multicolumn{3}{|c|}{ Americium-241 GEA } & \multicolumn{3}{|c|}{ Carbon-14 } & \multicolumn{3}{|c|}{ Cesium-137 } & \multicolumn{3}{|c|}{ Cobalt-60 } \\
\hline & & & $\mathrm{pCi} / \mathrm{g}$ & $\mathbf{Q}$ & MDA & $\mathrm{pCi} / \mathrm{g}$ & $Q$ & MDA & $\mathrm{pCi} / \mathrm{g}$ & $\mathbf{Q}$ & MDA & $\mathrm{pCi} / \mathrm{g}$ & Q & MDA & $\mathrm{pCi} / \mathrm{g}$ & $\mathbf{Q}$ & MDA \\
\hline Area $1 \mathrm{~A} 1$ & $\mathrm{J134Y7}$ & $8 / 17 / 06$ & 0.073 & $\mathrm{U}$ & 0.28 & 0.42 & $\mathrm{U}$ & 0.42 & -0.292 & $\mathrm{U}$ & 3.0 & 0.090 & $\mathrm{U}$ & 0.090 & 0.048 & U & 0.048 \\
\hline Duplicate of J134Y7 & $\mathrm{J} 134 \mathrm{Y9}$ & $8 / 17 / 06$ & -0.036 & $\mathrm{U}$ & 0.27 & 0.24 & $\mathrm{U}$ & 0.24 & -0.395 & $\mathrm{U}$ & 3.0 & 0.299 & & 0.032 & 0.046 & $U$ & 0.046 \\
\hline Split of J134Y7 & $\mathrm{J} 13504$ & $8 / 17 / 06$ & 0.0311 & $\mathrm{U}$ & 0.0211 & & & & 0.418 & $\mathrm{U}$ & 0.720 & 0.391 & & 0.0162 & 0.00841 & $\mathrm{U}$ & 0.0217 \\
\hline Area $1 \mathrm{~A} 2$ & $\mathrm{~J} 13500$ & $8 / 17 / 06$ & 0 & $\mathrm{U}$ & 0.34 & 0.18 & $\mathrm{U}$ & 0.18 & 1.24 & $\mathrm{U}$ & 3.4 & 0.129 & & 0.021 & 0.020 & $\mathrm{U}$ & 0.020 \\
\hline Area $1 \mathrm{~A} 3$ & J134Y8 & $8 / 17 / 06$ & -0.041 & $\mathrm{U}$ & 0.32 & 0.15 & $\mathrm{U}$ & 0.15 & -1.60 & $\mathrm{U}$ & 3.2 & 0.178 & & 0.025 & 0.026 & $\mathrm{U}$ & 0.026 \\
\hline Area $1 \mathrm{~A} 4$ & $J 13501$ & $8 / 17 / 06$ & 0.036 & $\mathrm{U}$ & 0.28 & 0.056 & $\mathrm{U}$ & 0.056 & 1.64 & $\mathrm{U}$ & 2.9 & 0.018 & & 0.009 & 0.009 & $\mathrm{U}$ & 0.009 \\
\hline Area $2 \mathrm{Al}$ & $\mathrm{J} 13502$ & $8 / 17 / 06$ & & & & 0.32 & $\mathrm{U}$ & 0.32 & -0.488 & $\mathrm{U}$ & 2.9 & 0.128 & & 0.034 & 0.036 & $\mathrm{U}$ & 0.036 \\
\hline Duplicate of J13502 & $\mathrm{J} 13508$ & $8 / 17 / 06$ & & & & 0.32 & $\mathrm{U}$ & 0.32 & 0.048 & $\mathrm{U}$ & 3.5 & 0.092 & & 0.040 & 0.034 & $\mathrm{U}$ & 0.034 \\
\hline Split of J13502 & $\mathrm{J} 13505$ & $8 / 17 / 06$ & & & & & & & 0.407 & $\mathrm{U}$ & 0.719 & 0.0464 & & 0.0148 & 0.00506 & $\mathrm{U}$ & 0.0174 \\
\hline Area 2 A2 & $\mathrm{J} 13503$ & $8 / 17 / 06$ & & & & 0.16 & $\mathrm{U}$ & 0.16 & 0.827 & $\mathrm{U}$ & 2.8 & 0.149 & & 0.029 & 0.087 & $\mathrm{U}$ & 0.087 \\
\hline Area $2 \mathrm{~A} 3$ & $\mathrm{~J} 13506$ & $8 / 17 / 06$ & & & & 0.43 & $\mathrm{U}$ & 0.43 & 1.66 & $\mathrm{U}$ & 2.9 & 0.063 & $\mathrm{U}$ & 0.063 & 0.076 & $\mathrm{U}$ & 0.076 \\
\hline Area 2 A4 & $\mathrm{J} 13507$ & $8 / 17 / 06$ & & & & 0.24 & $\mathrm{U}$ & 0.24 & 11.7 & & 3.1 & 0.107 & & 0.047 & 1.48 & & 0.045 \\
\hline Area $3 \mathrm{~A} 1$ & $\mathrm{J13510}$ & $8 / 21 / 06$ & -0.009 & $U$ & 0.064 & 0.20 & $\mathrm{U}$ & 0.20 & 0.660 & $\mathrm{U}$ & 2.8 & 0.195 & & 0.033 & 0.034 & $\mathrm{U}$ & 0.034 \\
\hline Area $3 \mathrm{A2}$ & J13511 & $8 / 21 / 06$ & 0.007 & $\mathrm{U}$ & 0.041 & & & & 0.636 & $\mathrm{U}$ & 2.9 & 0.240 & & 0.020 & 0.042 & & 0.025 \\
\hline Area $3 \mathrm{A3}$ & $\mathrm{J13512}$ & $8 / 21 / 06$ & 0.027 & $\mathrm{U}$ & 0.042 & 0.44 & $\mathrm{U}$ & 0.44 & -0.369 & $\mathrm{U}$ & 3.0 & 0.958 & & 0.049 & 0.061 & $\mathrm{U}$ & 0.061 \\
\hline Area 3 A4 & $\mathrm{J13513}$ & $8 / 21 / 06$ & 0.039 & $\mathrm{U}$ & 0.048 & & & & -0.866 & $\mathrm{U}$ & 2.8 & 0.227 & & 0.027 & 0.024 & $\mathrm{U}$ & 0.024 \\
\hline Area 4 A1 & $\mathrm{J} 13514$ & $8 / 21 / 06$ & -0.031 & $\mathrm{U}$ & 0.063 & 0.22 & $\mathrm{U}$ & 0.22 & 1.72 & $\mathrm{U}$ & 2.8 & 0.065 & & 0.031 & 0.035 & $\mathrm{U}$ & 0.035 \\
\hline Area 4 A2 & $\mathrm{J} 13515$ & $8 / 21 / 06$ & -0.014 & $\mathrm{U}$ & 0.068 & 1.7 & $\mathrm{U}$ & 1.7 & 0.560 & $\mathrm{U}$ & 2.8 & 1.19 & & 0.036 & 0.050 & $\mathrm{U}$ & 0.050 \\
\hline Area $4 \mathrm{~A} 3$ & $\mathrm{~J} 13516$ & $8 / 21 / 06$ & -0.008 & $\mathrm{U}$ & 0.050 & & & & 0.360 & $\mathrm{U}$ & 3.0 & 0.044 & & 0.020 & 0.016 & $\mathrm{U}$ & 0.016 \\
\hline Area 4 A4 & $\mathrm{J13517}$ & $8 / 21 / 06$ & 0.053 & $\mathrm{U}$ & 0.13 & 0.14 & $\mathrm{U}$ & 0.14 & -0.307 & $\mathrm{U}$ & 2.9 & 0.082 & & 0.024 & 0.022 & $\mathrm{U}$ & 0.022 \\
\hline $\mathrm{OB} / \mathrm{BCL} \mathrm{A} 1$ & $J 13518$ & $8 / 22 / 06$ & 0.123 & $\mathrm{U}$ & 0.31 & 0.25 & $\mathrm{U}$ & 0.25 & -0.903 & $\mathrm{U}$ & 2.9 & 0.220 & & 0.035 & 0.030 & $\mathrm{U}$ & 0.030 \\
\hline $\mathrm{OB} / \mathrm{BCL} \mathrm{A} 2$ & $J 13519$ & $8 / 22 / 06$ & 0.036 & $\mathrm{U}$ & 0.28 & 0.20 & $\mathrm{U}$ & 0.20 & -0.592 & $\mathrm{U}$ & 2.9 & 0.059 & & 0.033 & 0.030 & $\mathrm{U}$ & 0.030 \\
\hline $\mathrm{OB} / \mathrm{BCL} \mathrm{A} 3$ & $J 13520$ & $8 / 22 / 06$ & 0.169 & $\mathrm{U}$ & 0.43 & 0.13 & $\mathrm{U}$ & 0.13 & -0.345 & $\mathrm{U}$ & 2.9 & 0.437 & & 0.018 & 0.015 & $\mathrm{U}$ & 0.015 \\
\hline $\mathrm{OB} / \mathrm{BCL} \mathrm{A4}$ & J135N5 & $8 / 22 / 06$ & 0.007 & $\mathrm{U}$ & 0.11 & 0.37 & $\mathrm{U}$ & 0.37 & 0.472 & $\mathrm{U}$ & 3.3 & 0.172 & & 0.046 & 0.040 & $\mathrm{U}$ & 0.040 \\
\hline
\end{tabular}

Note: The following abbreviations apply to all Attachment 1 tables.

Note: Data qualified with B, B*, C, D, N, or J are considered acceptable values.

$\mathrm{B}=$ method blank contamination

$\mathrm{OB}=$ overburden

$\mathrm{B}^{*}=$ estimated

$\mathrm{MDA}=$ minimum detectable activity

$\mathrm{BCL}=$ below cleanup levels

$\mathrm{N}=$ spiked analyte recovery outside control limits

$\mathrm{C}=$ method blank contamination

$\mathrm{PQL}=$ practical quantitation limit

$\mathrm{D}=$ diluted

$\mathrm{Q}=$ qualifier

FS $=$ focused sample

$\mathrm{GEA}=$ gamma energy analysis

$\mathrm{R}=$ rejected

$\mathrm{SPA}=$ staging pile area

$\mathrm{J}=$ estimated

$\mathrm{U}=$ undetected

\begin{tabular}{|c|c|c|c|}
\hline Attachment & 1 & et No. & 1 of 43 \\
\hline Originator & J.M. Capron Lage & Date & $12 / 13 / 06$ \\
\hline Cheo & T. M. Blakley Jum & Date & $12 / 14 / 06$ \\
\hline Calc. No. & $0100 \mathrm{C}-\mathrm{CA}-\mathrm{V} 0033$ & Rev. No & 0 \\
\hline
\end{tabular}


Attachment 1. 118-C-1 Verification Sampling Results.

\begin{tabular}{|c|c|c|c|c|c|c|c|c|c|c|c|c|c|c|c|c|c|}
\hline \multirow{2}{*}{ Sample Location } & \multirow{2}{*}{$\begin{array}{c}\text { Sample } \\
\text { Number }\end{array}$} & \multirow{2}{*}{$\begin{array}{c}\text { Sample } \\
\text { Date }\end{array}$} & \multicolumn{3}{|c|}{ Americium-241 } & \multicolumn{3}{|c|}{ Americium-241 GEA } & \multicolumn{3}{|c|}{ Carbon-14 } & \multicolumn{3}{|c|}{ Cesium-137 } & \multicolumn{3}{|c|}{ Cobalt-60 } \\
\hline & & & $\mathrm{pCi} / \mathrm{g}$ & $\mathbf{Q}$ & MDA & $\mathrm{pCi} / \mathrm{g}$ & $Q$ & MDA & $\mathrm{pCi} / \mathrm{g}$ & $\mathbf{Q}$ & MDA & $\mathrm{pCi} / \mathrm{g}$ & $Q$ & MDA & $\mathrm{pCi} / \mathrm{g}$ & $\mathbf{Q}$ & MDA \\
\hline SPAA1 & $\mathrm{J} 13522$ & $9 / 11 / 06$ & 0 & $\mathrm{U}$ & 0.24 & 0.056 & $\mathrm{U}$ & 0.056 & -1.24 & $\mathrm{U}$ & 3.3 & 0.424 & & 0.050 & 0.040 & $U$ & 0.040 \\
\hline SPA A2 & $\mathrm{J} 13523$ & $9 / 11 / 06$ & 0.056 & $\mathrm{U}$ & 0.21 & 0.26 & $\mathrm{U}$ & 0.26 & -1.19 & $\mathrm{U}$ & 2.8 & 0.141 & & 0.035 & 0.031 & $U$ & 0.031 \\
\hline SPA A3 & J135P1 & $9 / 12 / 06$ & 0 & UJ & 1.1 & 0.39 & $\mathrm{U}$ & 0.39 & -0.524 & $\mathrm{U}$ & 2.1 & 3.00 & & 0.041 & 0.036 & U & 0.036 \\
\hline SPA A4 & $\mathrm{J} 135 \mathrm{PO}$ & $9 / 12 / 06$ & -0.068 & $\mathrm{U}$ & 0.22 & 0.045 & $\mathrm{U}$ & 0.045 & -0.403 & $\mathrm{U}$ & 2.5 & 0.826 & & 0.039 & 0.029 & $\mathrm{U}$ & 0.029 \\
\hline SPAB5 & $\mathrm{J} 13524$ & $9 / 11 / 06$ & 0.034 & $\mathrm{U}$ & 0.26 & 0.15 & $\mathrm{U}$ & 0.15 & -1.58 & $U$ & 2.9 & 0.494 & & 0.028 & 0.026 & $\mathrm{U}$ & 0.026 \\
\hline SPAB6 & $\mathrm{J13525}$ & $9 / 11 / 06$ & 0 & $\mathrm{U}$ & 0.22 & 0.47 & $\bar{U}$ & 0.47 & -1.05 & $\mathrm{U}$ & 3.1 & 0.830 & & 0.049 & 0.044 & $\mathrm{U}$ & 0.044 \\
\hline SPA B7 & $3135 \mathrm{NS}$ & $9 / 11 / 06$ & 0 & $\mathrm{U}$ & 0.23 & 0.025 & $\mathrm{U}$ & 0.025 & -1.27 & $\mathrm{U}$ & 2.7 & 0.160 & & 0.024 & 0.022 & $\mathrm{U}$ & 0.022 \\
\hline SPAB8 & J135N9 & $9 / 11 / 06$ & 0.060 & $\mathrm{U}$ & 0.23 & 0.051 & $\mathrm{U}$ & 0.051 & -1.53 & $\mathrm{U}$ & 2.8 & 0.383 & & 0.048 & 0.034 & $U$ & 0.034 \\
\hline SPAC9 & $\mathrm{J} 135 \mathrm{P} 2$ & $9 / 12 / 06$ & 0.030 & $\mathrm{U}$ & 0.23 & 0.11 & $\mathrm{U}$ & 0.11 & -0.600 & U & 2.6 & 0.290 & & 0.020 & 0.023 & $\mathrm{U}$ & 0.023 \\
\hline SPA C10 & J135P3 & $9 / 12 / 06$ & 0.271 & $\mathrm{U}$ & 0.52 & 0.042 & $\mathrm{U}$ & 0.042 & -0.647 & $\mathrm{U}$ & 2.1 & 0.979 & & 0.035 & 0.055 & & 0.033 \\
\hline SPA CI & $\mathrm{J} 135 \mathrm{P} 4$ & $9 / 12 / 06$ & 0 & $U$ & 0.23 & 0.49 & $U$ & 0.49 & -0.487 & $\mathrm{U}$ & 2.4 & 1.16 & & 0.046 & 0.050 & $\mathrm{U}$ & 0.050 \\
\hline SPA C2 & J135P5 & $9 / 12 / 06$ & -0.033 & $\mathrm{U}$ & 0.25 & 0.57 & $\mathrm{U}$ & 0.57 & -0.935 & $\mathrm{U}$ & 2.1 & 0.644 & & 0.055 & 0.055 & $\mathrm{U}$ & 0.055 \\
\hline SPAD3 & J135P6 & $9 / 12 / 06$ & 0.063 & $\mathrm{U}$ & 0.24 & 0.27 & $\mathrm{U}$ & 0.27 & -0.356 & $\mathrm{U}$ & 2.1 & 0.071 & & 0.033 & 0.034 & $\mathrm{U}$ & 0.034 \\
\hline SPA D4 & J135P7 & $9 / 12 / 06$ & 0.085 & $\mathrm{U}$ & 0.22 & 0.16 & $\mathrm{U}$ & 0.16 & 182 & & 2.1 & 0.091 & & 0.033 & 0.030 & $\mathrm{U}$ & 0.030 \\
\hline SPAD5 & J135P8 & $9 / 12 / 06$ & 0 & $\mathrm{U}$ & 0.59 & 0.067 & $\mathrm{U}$ & 0.067 & 0.362 & $\mathrm{U}$ & 2.2 & 1.18 & & 0.053 & 0.063 & & 0.051 \\
\hline SPAD6 & J135P9 & $9 / 12 / 06$ & 0.059 & $U$ & 0.23 & 0.020 & $\mathrm{U}$ & 0.020 & -1.06 & $\mathrm{U}$ & 2.3 & 0.198 & & 0.017 & 0.014 & $\mathrm{U}$ & 0.015 \\
\hline FS-7 & $\mathrm{J} 12 \mathrm{XM} 4$ & $7 / 26 / 06$ & & & & 0.040 & $\mathrm{U}$ & 0.040 & & & & 0.045 & $\mathrm{U}$ & 0.045 & 0.062 & $\mathrm{U}$ & 0.062 \\
\hline
\end{tabular}

$\frac{\prod_{1}}{\frac{1}{A}}$

\begin{tabular}{|c|c|c|c|c|c|c|c|c|c|c|c|c|c|c|c|c|c|}
\hline \multirow{2}{*}{ Sample Location } & \multirow{2}{*}{$\begin{array}{c}\text { Sample } \\
\text { Number }\end{array}$} & \multirow{2}{*}{$\begin{array}{c}\text { Sample } \\
\text { Date }\end{array}$} & \multicolumn{3}{|c|}{ Europium-152 } & \multicolumn{3}{|c|}{ Europium-154 } & \multicolumn{3}{|c|}{ Europium-155 } & \multicolumn{3}{|c|}{ Nickel-63 } & \multicolumn{3}{|c|}{ Plutonium-238 } \\
\hline & & & $\mathrm{pCi} / \mathrm{g}$ & $\mathbf{Q}$ & MDA & $\mathrm{pCi} / \mathrm{g}$ & $Q$ & MDA & $\mathrm{pCi} / \mathrm{g}$ & $\mathbf{Q}$ & MDA & $\mathrm{pCi} / \mathrm{g}$ & $Q$ & MDA & $\mathrm{pCi} / \mathrm{g}$ & $\mathbf{Q}$ & MDA \\
\hline Area $1 \mathrm{~A} 1$ & $\mathrm{~J} 134 \mathrm{Y} 7$ & $8 / 17 / 06$ & 0.161 & & 0.097 & 0.15 & $\mathrm{U}$ & 0.15 & 0.16 & $\overrightarrow{\mathrm{U}}$ & 0.16 & 2.49 & $\mathrm{U}$ & 2.9 & -0.025 & $\overrightarrow{\mathrm{U}}$ & 0.30 \\
\hline Duplicate of J134Y7 & J134Y9 & $8 / 17 / 06$ & 0.142 & & 0.083 & 0.16 & $\mathrm{U}$ & 0.16 & 0.12 & $U$ & 0.12 & 1.42 & $\mathrm{U}$ & 3.1 & -0.074 & $\mathrm{U}$ & 0.57 \\
\hline Split of J134Y7 & $\mathrm{J13504}$ & $8 / 17 / 06$ & 0.128 & $\mathrm{U}$ & 0.0519 & 0.0360 & $U$ & 0.0648 & 0.0388 & $\mathrm{U}$ & 0.0437 & 7.70 & & 5.73 & 0.00793 & $\mathrm{U}$ & 0.0442 \\
\hline Area $1 \mathrm{~A} 2$ & $\mathrm{~J} 13500$ & $8 / 17 / 06$ & 0.083 & & 0.044 & 0.069 & $\mathrm{U}$ & 0.069 & 0.068 & $\mathrm{U}$ & 0.068 & 0.441 & $\mathrm{U}$ & 2.9 & 0.186 & $\mathrm{U}$ & 0.71 \\
\hline Area $1 \mathrm{~A} 3$ & $\mathrm{~J} 134 \mathrm{Y8}$ & $8 / 17 / 06$ & 0.064 & $\mathrm{U}$ & 0.064 & 0.082 & $\mathrm{U}$ & 0.082 & 0.092 & $\mathrm{U}$ & 0.092 & -0.194 & $\mathrm{U}$ & 3.1 & -0.110 & $\mathrm{U}$ & 0.84 \\
\hline Area $1 \mathrm{~A} 4$ & $\mathrm{~J} 13501$ & $8 / 17 / 06$ & 0.024 & $\mathrm{U}$ & 0.024 & 0.032 & $\mathrm{U}$ & 0.032 & 0.051 & $\mathrm{U}$ & 0.051 & 0.086 & $\mathrm{U}$ & 2.8 & 0 & $\mathrm{U}$ & 0.56 \\
\hline Area $2 \mathrm{Al}$ & $\mathrm{J} 13502$ & $8 / 17 / 06$ & 0.082 & $\mathrm{U}$ & 0.082 & 0.11 & $\mathrm{U}$ & 0.11 & 0.092 & $U$ & 0.13 & 0.673 & 0 & 2.9 & & & \\
\hline Duplicate of $\mathrm{J} 13502$ & $\mathrm{~J} 13508$ & $8 / 17 / 06$ & 0.085 & $\mathrm{U}$ & 0.085 & 0.11 & $\mathrm{U}$ & 0.11 & 0.12 & $\mathrm{U}$ & 0.12 & 1.49 & $U$ & 2.8 & & & \\
\hline Split of J13502 & $\mathrm{J} 13505$ & $8 / 17 / 06$ & 0.00852 & $\mathrm{U}$ & 0.0396 & 0.0175 & U & 0.0522 & 0.0255 & $\mathrm{U}$ & 0.0411 & 3.57 & $\mathrm{U}$ & 5.58 & & & \\
\hline Area $2 \mathrm{~A} 2$ & $\mathrm{~J} 13503$ & $8 / 17 / 06$ & 0.055 & $\mathrm{U}$ & 0.055 & 0.075 & $U$ & 0.075 & 0.073 & $\mathrm{U}$ & 0.073 & 12.4 & & 2.8 & & & \\
\hline Area $2 \mathrm{~A} 3$. & J13506 & $8 / 17 / 06$ & 0.281 & & 0.099 & 0.15 & $\mathrm{U}$ & 0.15 & 0.14 & $\mathrm{U}$ & 0.14 & 1.37 & $\mathrm{U}$ & 2.8 & & & \\
\hline Area $2 \mathrm{~A} 4$ & $\mathrm{~J} 13507$ & $8 / 17 / 06$ & 0.087 & $\bar{U}$ & 0.087 & 0.11 & $U$ & 0.11 & 0.11 & $\mathrm{U}$ & 0.11 & 35.8 & & 2.9 & & & \\
\hline
\end{tabular}

\begin{tabular}{lll} 
Attachment & \multicolumn{1}{c}{ Sheet No. } & $\frac{2 \text { of } 43}{12 / 13 / 06}$ \\
Originator & Date & J.M. Capron \\
Checked & T.M. Blakley & Date \\
Calc. No. & Rev. No. No. &
\end{tabular}


Attachment 1. 118-C-1 Verification Sampling Results.

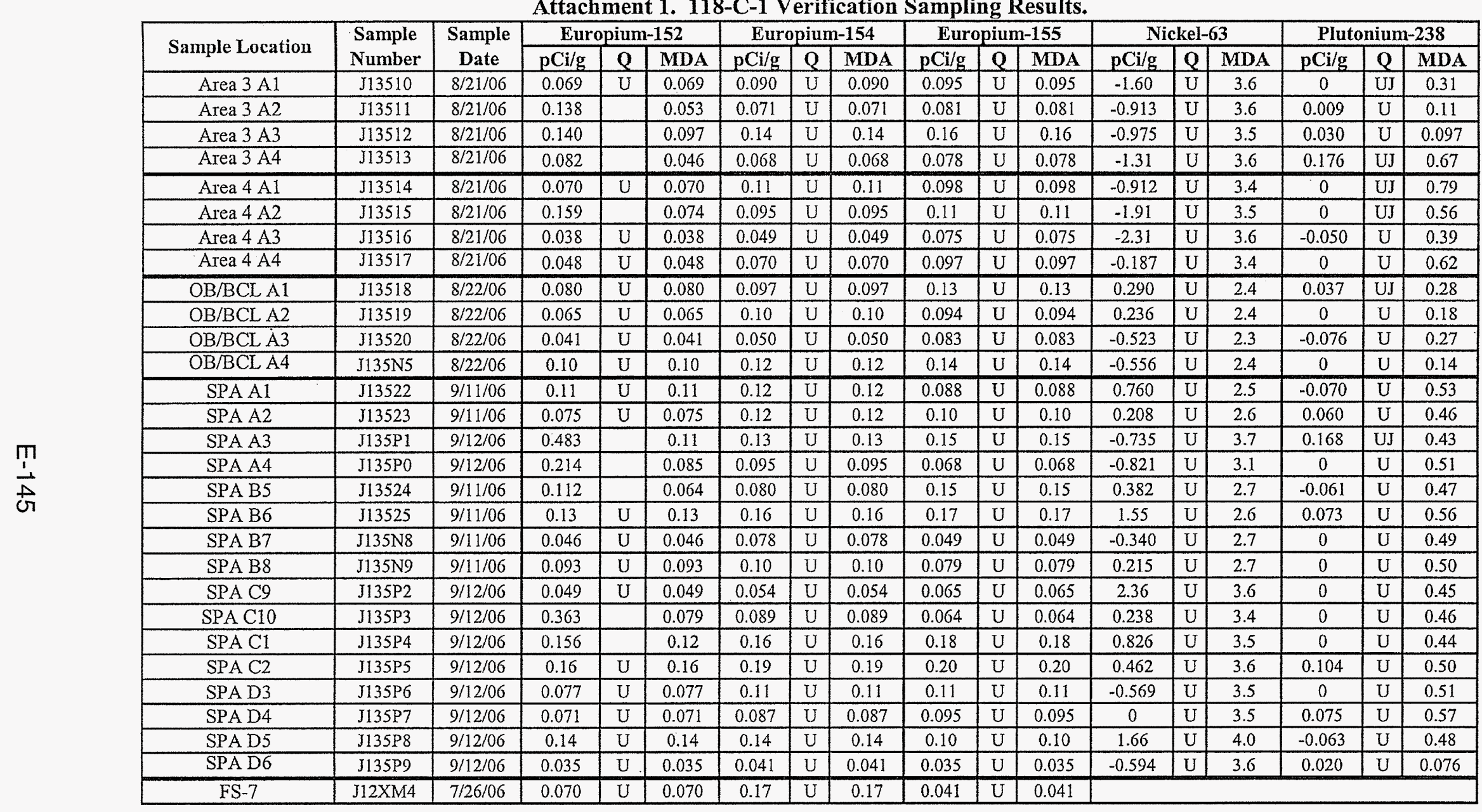

\begin{tabular}{|c|c|c|c|}
\hline Attachment & 1 & Sheet No & 3 of 43 \\
\hline Originator & J.M. Capron & Date & $12 / 13 / 06$ \\
\hline Checked & T. M. Blakley & Date & \\
\hline Calc. No. & $0100 \mathrm{C}-\mathrm{CA}-\mathrm{V} 0033$ & Rev. No. & 0 \\
\hline
\end{tabular}


Attachment 1. 118-C-1 Verification Sampling Results.

\begin{tabular}{|c|c|c|c|c|c|c|c|c|c|c|c|c|c|c|c|c|c|}
\hline \multirow{2}{*}{ Sample Location } & \multirow{2}{*}{$\begin{array}{c}\text { Sample } \\
\text { Number }\end{array}$} & \multirow{2}{*}{$\begin{array}{c}\text { Sample } \\
\text { Date }\end{array}$} & \multicolumn{3}{|c|}{ Plutonium-239/240 } & \multicolumn{3}{|c|}{ Plutonium-241 } & \multicolumn{3}{|c|}{ Potassium-40 } & \multicolumn{3}{|c|}{ Radium-226 } & \multicolumn{3}{|c|}{ Radium-228 } \\
\hline & & & $\mathrm{pCi} / \mathrm{g}$ & $\mathbf{Q}$ & MDA & $\mathrm{pCi} / \mathrm{g}$ & $Q$ & MDA & $\mathrm{pCi} / \mathrm{g}$ & $\mathbf{Q}$ & MDA & $\mathrm{pCi} / \mathrm{g}$ & $Q$ & MDA & $\mathrm{pCi} / \mathrm{g}$ & $\mathbf{Q}$ & MDA \\
\hline Area $1 \mathrm{Al}$ & $5134 \mathrm{Y} 7$ & $8 / 17 / 06$ & 0.025 & $\mathrm{U}$ & 0.19 & 1.16 & $\mathrm{U}$ & 24 & 21.6 & & 0.37 & 0.754 & & 0.082 & 1.04 & & 0.19 \\
\hline Duplicate of J134Y7 & $\$ 134 Y 9$ & $8 / 17 / 06$ & 0 & $\mathrm{U}$ & 0.57 & 4.91 & $\mathrm{U}$ & 21 & 11.4 & & 0.28 & 0.401 & & 0.063 & 0.530 & & 0.15 \\
\hline Split of J134Y7 & $\mathrm{J} 13504$ & $8 / 17 / 06$ & 0.0461 & & 0.0250 & 1.26 & $\mathrm{U}$ & 2.87 & & & & & & & & & \\
\hline Area 1 A2 & $\mathrm{J} 13500$ & $8 / 17 / 06$ & 0 & $\mathrm{U}$ & 0.71 & 14.1 & $\mathrm{U}$ & 21 & 23.8 & & 0.18 & 0.843 & & 0.032 & 1.34 & & 0.080 \\
\hline Area $1 \mathrm{~A} 3$ & $\mathrm{~J} 134 \mathrm{Y} 8$ & $8 / 17 / 06$ & 0 & $\mathrm{U}$ & 0.84 & 6.40 & $\mathrm{U}$ & 22 & 13.1 & & 0.27 & 0.566 & & 0.048 & 0.873 & & 0.11 \\
\hline Area $1 \mathrm{~A} 4$ & $\mathrm{~J} 13501$ & $8 / 17 / 06$ & 0 & $\mathrm{U}$ & 0.56 & 8.14 & $\mathrm{U}$ & 22 & 11.5 & & 0.10 & 0.409 & & 0.021 & 0.638 & & 0.040 \\
\hline Area $2 \mathrm{~A} 1$ & $\mathrm{~J} 13502$ & $8 / 17 / 06$ & & & & & & & 21.3 & & 0.29 & 0.757 & & 0.061 & 1.17 & & 0.13 \\
\hline Duplicate of $J 13502$ & $\mathrm{~J} 13508$ & $8 / 17 / 06$ & & & & & & & 21.4 & & 0.33 & 0.758 & & 0.064 & 1.13 & & 0.14 \\
\hline Split of $J 13502$ & $\mathrm{~J} 13505$ & $8 / 17 / 06$ & & & & & & & & & & & & & & & \\
\hline Area 2 A2 & $\mathrm{J13503}$ & $8 / 17 / 06$ & & & & & & & 10.7 & & 0.26 & 0.414 & & 0.046 & 0.656 & & 0.096 \\
\hline Area $2 \mathrm{A3}$ & $\mathrm{J} 13506$ & $8 / 17 / 06$ & & & & & & & 21.9 & & 0.38 & 0.832 & & 0.089 & 1.15 & & 0.17 \\
\hline Area $2 \mathrm{A4}$ & $\mathrm{J} 13507$ & $8 / 17 / 06$ & & & & & & & 10.5 & & 0.32 & 0.382 & & 0.064 & 0.680 & & 0.25 \\
\hline Area $3 \mathrm{~A} 1$ & $\mathrm{J13510}$ & $8 / 21 / 06$ & -0.023 & $\mathrm{UJ}$ & 0.22 & -0.542 & $\mathrm{U}$ & 23 & 11.3 & & 0.23 & 0.524 & & 0.059 & 0.781 & & 0.14 \\
\hline Area 3 A2 & $\mathrm{J} 13511$ & $8 / 21 / 06$ & 0.036 & $\mathrm{U}$ & 0.068 & -1.91 & U & 22 & 12.3 & & 0.21 & 0.833 & & 0.042 & 0.844 & & 0.094 \\
\hline Area $3 \mathrm{~A} 3$ & $\mathrm{~J} 13512$ & $8 / 21 / 06$ & 0 & $\mathrm{U}$ & 0.077 & -10.6 & $\mathrm{U}$ & 18 & 20.2 & & 0.41 & 0.827 & & 0.086 & 1.14 & & 0.18 \\
\hline Area 3 A4 & $\mathrm{J} 13513$ & $8 / 21 / 06$ & 0 & $\mathrm{UJ}$ & 0.67 & -10.6 & $U$ & 20 & 11.7 & & 0.22 & 0.411 & & 0.042 & 0.644 & & 0.097 \\
\hline Area $4 \mathrm{Al}$ & $\mathrm{J} 13514$ & $8 / 21 / 06$ & 0 & UJ & 0.79 & -4.03 & $\mathrm{U}$ & 24 & 10.5 & & 0.32 & 0.441 & & 0.051 & 0.607 & & 0.15 \\
\hline Area $4 \mathrm{A2}$ & $\mathrm{J} 13515$ & $8 / 21 / 06$ & 0.073 & $\mathrm{UJ}$ & 0.56 & -7.73 & $\mathrm{U}$ & 27 & 20.2 & & 0.32 & 0.981 & & 0.056 & 1.25 & & 0.13 \\
\hline Area $4 \mathrm{A3}$ & $\mathrm{J} 13516$ & $8 / 21 / 06$ & 0.050 & $\mathrm{U}$ & 0.39 & -3.48 & $\mathrm{U}$ & 22 & 12.0 & & 0.16 & 0.434 & & 0.032 & 0.649 & & 0.076 \\
\hline Area 4 A4 & $\mathrm{J} 13517$ & $8 / 21 / 06$ & 0.081 & $\mathrm{U}$ & 0.62 & -5.31 & $\mathrm{U}$ & 21 & 10.4 & & 0.19 & 0.399 & & 0.036 & 0.596 & & 0.085 \\
\hline $\mathrm{OB} / \mathrm{BCL} \mathrm{Al}$ & $\mathrm{J13518}$ & $8 / 22 / 06$ & 0.037 & UJ & 0.14 & 14.2 & $U$ & 28 & 22.0 & & 0.36 & 0.724 & & 0.062 & 1.31 & & 0.15 \\
\hline $\mathrm{OB} / \mathrm{BCL} \mathrm{A} 2$ & $\mathrm{J13519}$ & $8 / 22 / 06$ & 0 & $\mathrm{U}$ & 0.11 & 7.02 & $\mathrm{U}$ & 28 & 11.1 & & 0.25 & 0.417 & & 0.052 & 0.670 & & 0.13 \\
\hline $\mathrm{OB} / \mathrm{BCL} \mathrm{A} 3$ & $\mathrm{~J} 13520$ & $8 / 22 / 06$ & 0.045 & $\mathrm{U}$ & 0.12 & 6.27 & $\mathrm{U}$ & 27 & 21.7 & & 0.15 & 0.710 & & 0.030 & 1.11 & & 0.068 \\
\hline $\mathrm{OB} / \mathrm{BCL} \mathrm{A4}$ & J135N5 & $8 / 22 / 06$ & 0 & $\mathrm{U}$ & 0.069 & 6.19 & $\mathrm{U}$ & 27 & 25.8 & & 0.35 & 0.911 & & 0.076 & 1.34 & & 0.14 \\
\hline
\end{tabular}

1

Checked T.M. Blakley

Sheet No. 4 of 43

$\begin{array}{lll}\text { Calc. No. } & \text { Date } & \\ \text { O100C-CA-V0033 Rev. No. } & \text { Ro }\end{array}$ 
Attachment 1. 118-C-1 Verification Sampling Results.

\begin{tabular}{|c|c|c|c|c|c|c|c|c|c|c|c|c|c|c|c|c|c|}
\hline \multirow{2}{*}{ Sample Location } & \multirow{2}{*}{$\begin{array}{c}\text { Sample } \\
\text { Number }\end{array}$} & \multirow{2}{*}{$\begin{array}{c}\text { Sample } \\
\text { Date }\end{array}$} & \multicolumn{3}{|c|}{ Plutonium-239/240 } & \multicolumn{3}{|c|}{ Plutonium-241 } & \multicolumn{3}{|c|}{ Potassium-40 } & \multicolumn{3}{|c|}{ Radium-226 } & \multicolumn{3}{|c|}{ Radium-228 } \\
\hline & & & $\mathrm{pCi} / \mathrm{g}$ & $Q$ & MDA & $\mathrm{pCi} / \mathrm{g}$ & $Q$ & MDA & $\mathrm{pCi} / \mathrm{g}$ & $Q$ & MDA & $\mathrm{pCi} / \mathrm{g}$ & $Q$ & MDA & $\mathrm{pCi} / \mathrm{g}$ & $Q$ & MDA \\
\hline SPA A1 & $\mathrm{J} 13522$ & $9 / 11 / 06$ & 0 & $\mathrm{U}$ & 0.53 & 13.7 & $\mathrm{U}$ & 19 & 12.4 & & 0.38 & 0.503 & & 0.078 & 0.824 & & 0.17 \\
\hline SPA A2 & J13523 & $9 / 11 / 06$ & 0 & $\mathrm{U}$ & 0.46 & 5.29 & $\mathrm{U}$ & 19 & 12.0 & & 0.34 & 0.376 & & 0.063 & 0.598 & & 0.15 \\
\hline SPA A3 & J135PI & $9 / 12 / 06$ & 0.279 & UJ & 0.43 & 5.82 & $\mathrm{U}$ & 18 & 1.3 & $\mathrm{U}$ & 1.3 & 0.740 & & 0.062 & 1.08 & & 0.15 \\
\hline SPA A4 & $\mathrm{J} 135 \mathrm{P} 0$ & $9 / 12 / 06$ & 0 & $\mathrm{U}$ & 0.51 & 1.71 & $\mathrm{U}$ & 21 & 12.5 & & 0.26 & 0.438 & & 0.068 & 0.765 & & 0.12 \\
\hline SPA B5 & $\mathrm{J} 13524$ & $9 / 11 / 06$ & 0.061 & $U$ & 0.47 & 11.9 & $\mathrm{U}$ & 18 & 14.1 & & 0.26 & 0.412 & & 0.053 & 0.822 & & 0.10 \\
\hline SPA B6 & $\mathrm{J} 13525$ & 9/11/06 & 0.145 & $\mathrm{U}$ & 0.56 & 2.92 & $\mathrm{U}$ & 18 & 22.7 & & 0.42 & 0.799 & & 0.076 & 1.33 & & 0.18 \\
\hline SPA B7 & J135N8 & $9 / 11 / 06$ & 0.064 & $\mathrm{U}$ & 0.49 & 7.08 & $\mathrm{U}$ & 16 & 13.0 & & 0.18 & 0.446 & & 0.043 & 0.728 & & 0.10 \\
\hline SPA B8 & J135N9 & $9 / 11 / 06$ & 0 & $\mathrm{U}$ & 0.50 & 9.76 & $\mathrm{U}$ & 21 & 13.6 & & 0.33 & 0.561 & & 0.072 & 1.09 & & 0.16 \\
\hline SPAC9 & $\mathrm{J} 135 \mathrm{P} 2$ & $9 / 12 / 06$ & 0 & $\mathrm{U}$ & 0.45 & -1.19 & $\mathrm{U}$ & 18 & 13.0 & & 0.17 & 0.670 & & 0.036 & 0.927 & & 0.082 \\
\hline SPA C10 & J135P3 & $9 / 12 / 06$ & 0.179 & $\mathrm{U}$ & 0.46 & 4.53 & U & 21 & 12.6 & & 0.25 & 0.501 & & 0.061 & 0.698 & & 0.14 \\
\hline SPAC1 & J135P4 & $9 / 12 / 06$ & 0.115 & $\mathrm{U}$ & 0.44 & 6.88 & $\mathrm{U}$ & 22 & 27.4 & & 1.7 & 0.835 & & 0.078 & 1.37 & & 0.21 \\
\hline SPA C2 & J135P5 & $9 / 12 / 06$ & 0.157 & $\mathrm{U}$ & 0.40 & 6.23 & $\mathrm{U}$ & 20 & 23.1 & & 0.34 & 0.878 & & 0.100 & 1.17 & & 0.24 \\
\hline SPAD3 & J135P6 & $9 / 12 / 06$ & 0.106 & $\mathrm{U}$ & 0.41 & 5.04 & $\mathrm{U}$ & 19 & 11.4 & & 0.34 & 0.499 & & 0.069 & 0.729 & & 0.13 \\
\hline SPA D4 & J135P7 & $9 / 12 / 06$ & 0 & $\mathrm{U}$ & 0.57 & 12.5 & $U$ & 20 & 13.1 & & 0.30 & 0.491 & & 0.053 & 0.769 & & 0.10 \\
\hline SPA D5 & J135P8 & $9 / 12 / 06$ & 0 & $\mathrm{U}$ & 0.48 & 0.953 & $\mathrm{U}$ & 20 & 13.1 & & 0.42 & 0.547 & & 0.100 & 0.779 & & 0.20 \\
\hline SPAD6 & J135P9 & $9 / 12 / 06$ & 0.010 & $\mathrm{U}$ & 0.076 & 4.26 & $\mathrm{U}$ & 19 & 13.1 & & 0.13 & 0.563 & & 0.027 & 0.824 & & 0.062 \\
\hline FS-7 & J12XM4 & $7 / 26 / 06$ & & & & & & & 7.49 & & 0.58 & 0.256 & & 0.078 & 0.357 & & 0.21 \\
\hline
\end{tabular}

\begin{tabular}{|c|c|c|c|c|c|c|c|c|c|c|c|c|c|c|c|c|c|}
\hline \multirow[t]{2}{*}{ Sample Location } & \multirow{2}{*}{$\begin{array}{l}\text { Sample } \\
\text { Number }\end{array}$} & \multirow{2}{*}{$\begin{array}{l}\text { Sample } \\
\text { Date }\end{array}$} & \multicolumn{3}{|c|}{ Silver-108m } & \multicolumn{3}{|c|}{ Thorium-228 } & \multicolumn{3}{|c|}{ Thorium-232 } & \multicolumn{3}{|c|}{$\begin{array}{c}\text { Total beta } \\
\text { Radiostrontium }\end{array}$} & \multicolumn{3}{|c|}{ Tritium } \\
\hline & & & $\mathrm{pCi} / \mathrm{g}$ & $\mathbf{Q}$ & MDA & $\mathrm{pCi} / \mathrm{g}$ & $Q$ & MDA & $\mathrm{pCi} / \mathrm{g}$ & $\mathbf{Q}$ & MDA & $\mathrm{pCi} / \mathrm{g}$ & $\mathbf{Q}$ & MDA & $\mathrm{pCi} / \mathrm{g}$ & $\mathbf{Q}$ & MDA \\
\hline Area $1 \mathrm{~A} 1$ & $\mathrm{~J} 134 \mathrm{Y} 7$ & $8 / 17 / 06$ & 0.034 & $\mathrm{U}$ & 0.034 & 1.09 & & 0.063 & 1.04 & & 0.19 & 0.148 & $\mathrm{U}$ & 0.24 & -2.44 & $\mathrm{U}$ & 2.7 \\
\hline Duplicate of J134Y7 & $\mathrm{J} 134 \mathrm{Y9}$ & $8 / 17 / 06$ & 0.025 & $\mathrm{U}$ & 0.025 & 0.622 & & 0.049 & 0.530 & & 0.15 & 0.115 & $U$ & 0.22 & -1.10 & $\mathrm{U}$ & 2.7 \\
\hline Split of J134Y7 & $\mathrm{J} 13504$ & $8 / 17 / 06$ & -0.00909 & $\mathrm{U}$ & 0.0130 & & & & & & & 0.323 & & 0.149 & 0.00726 & $\mathrm{U}$ & 0.044 \\
\hline Area $1 \mathrm{~A} 2$ & $\mathrm{~J} 13500$ & $8 / 17 / 06$ & 0.014 & $\mathrm{U}$ & 0.014 & 1.26 & & 0.025 & 1.34 & & 0.080 & 0.048 & $\mathrm{U}$ & 0.27 & -1.42 & $\mathrm{U}$ & 3.1 \\
\hline Area $1 \mathrm{A3}$ & J134Y8 & $8 / 17 / 06$ & 0.018 & $\mathrm{U}$ & 0.018 & 0.705 & & 0.034 & 0.873 & & 0.11 & 0.136 & $U$ & 0.21 & -1.21 & $\mathrm{U}$ & 2.9 \\
\hline Area $1 \mathrm{~A} 4$ & $J 13501$ & $8 / 17 / 06$ & 0.006 & $\mathrm{U}$ & 0.006 & 0.549 & & 0.012 & 0.638 & & 0.040 & 0.049 & $U$ & 0.24 & -1.56 & $U$ & 2.7 \\
\hline Area $2 \mathrm{~A} 1$ & $\mathrm{J13502}$ & $8 / 17 / 06$ & 0.024 & $\mathrm{U}$ & 0.024 & 1.05 & & 0.041 & 1.17 & & 0.13 & 0.009 & $U$ & 0.27 & -0.857 & $\mathrm{U}$ & 2.8 \\
\hline Duplicate of $\mathrm{J} 13502$ & $\mathrm{J13508}$ & $8 / 17 / 06$ & 0.023 & $\mathrm{U}$ & 0.023 & 1.08 & & 0.043 & 1.13 & & 0.14 & 0.065 & $\mathrm{U}$ & 0.22 & -1.10 & $\mathrm{U}$ & 3.1 \\
\hline Split of J13502 & $\mathrm{J} 13505$ & $8 / 17 / 06$ & 0.00243 & $\mathrm{U}$ & 0.0112 & & & & & & & 0.0128 & $\mathrm{U}$ & 0.157 & -0.0323 & $\mathrm{U}$ & 0.0492 \\
\hline Area $2 \mathrm{~A} 2$ & $\mathrm{J13503}$ & $8 / 17 / 06$ & 0.017 & $\mathrm{U}$ & 0.017 & 0.646 & & 0.043 & 0.656 & & 0.096 & 0.114 & $\mathrm{U}$ & 0.22 & -0.836 & $\mathrm{U}$ & 2.7 \\
\hline Area $2 \mathrm{A3}$ & $\mathrm{J13506}$ & $8 / 17 / 06$ & 0.029 & $\mathrm{U}$ & 0.029 & 1.10 & & 0.055 & 1.15 & & 0.17 & 0.035 & $\mathrm{U}$ & 0.21 & -1.24 & $\mathrm{U}$ & 2.7 \\
\hline Area $2 \mathrm{~A} 4$ & $\mathrm{~J} 13507$ & $8 / 17 / 06$ & 0.026 & $\mathrm{U}$ & 0.026 & 0.647 & & 0.073 & 0.680 & & 0.25 & 0.094 & $\mathrm{U}$ & 0.18 & 7.71 & & 2.8 \\
\hline
\end{tabular}

\begin{tabular}{|c|c|c|c|}
\hline Attachment & 1 & Sheet No. & 5 of 43 \\
\hline Originator & J.M. Capron & Date & $12 / 13 / 06$ \\
\hline Checked & T. M. Blakley & Date & \\
\hline Calc. No. & $0100 \mathrm{C}-\mathrm{CA}-\mathrm{V} 0033$ & Rev. No. & 0 \\
\hline
\end{tabular}


Attachment 1. 118-C-1 Verification Sampling Results.

\begin{tabular}{|c|c|c|c|c|c|c|c|c|c|c|c|c|c|c|c|c|c|}
\hline \multirow[t]{2}{*}{ Sample Location } & \multirow{2}{*}{$\begin{array}{l}\text { Sample } \\
\text { Number }\end{array}$} & \multirow{2}{*}{$\begin{array}{c}\text { Sample } \\
\text { Date }\end{array}$} & \multicolumn{3}{|c|}{ Silver-108m } & \multicolumn{3}{|c|}{ Thorium-228 } & \multicolumn{3}{|c|}{ Thorium-232 } & \multicolumn{3}{|c|}{$\begin{array}{c}\text { Total beta } \\
\text { Radiostrontium }\end{array}$} & \multicolumn{3}{|c|}{ Tritium } \\
\hline & & & $\mathrm{pCi} / \mathrm{g}$ & $\mathbf{Q}$ & MDA & $\mathrm{pCi} / \mathrm{g}$ & $\mathbf{Q}$ & MDA & $\mathrm{pCi} / \mathrm{g}$ & $Q$ & MDA & $\mathrm{pCi} / \mathrm{g}$ & $\mathbf{Q}$ & MDA & $\mathrm{pCi} / \mathrm{g}$ & $\mathbf{Q}$ & $\overline{\mathrm{MDA}}$ \\
\hline Area $3 \mathrm{~A} 1$ & $\mathrm{~J} 13510$ & $8 / 21 / 06$ & 0.021 & $\vec{U}$ & 0.021 & 0.548 & & 0.036 & 0.781 & & 0.14 & 0.016 & $\mathrm{U}$ & 0.27 & 0.488 & $U$ & 2.9 \\
\hline Area 3 A2 & $\mathrm{J} 13511$ & $8 / 21 / 06$ & 0.016 & $\mathrm{U}$ & 0.016 & 0.693 & & 0.030 & 0.844 & & 0.094 & 0.087 & $\mathrm{U}$ & 0.26 & -1.16 & $\mathrm{U}$ & 3.0 \\
\hline Area $3 \mathrm{~A} 3$ & $\mathrm{~J} 13512$ & $8 / 21 / 06$ & 0.032 & $\mathrm{U}$ & 0.032 & 1.03 & & 0.058 & 1.14 & & 0.18 & 0.279 & & 0.19 & -0.361 & $\mathrm{U}$ & 3.1 \\
\hline Area $3 \mathrm{~A} 4$ & J13513 & $8 / 21 / 06$ & 0.016 & $\bar{U}$ & 0.016 & 0.534 & & 0.028 & 0.644 & & 0.097 & 0.263 & & 0.21 & -1.58 & $U$ & 2.9 \\
\hline Area $4 \mathrm{Al}$ & $\mathrm{J} 13514$ & $8 / 21 / 06$ & 0.022 & $\bar{U}$ & 0.022 & 0.551 & & 0.038 & 0.607 & & 0.15 & -0.008 & $\overline{\mathrm{U}}$ & 0.22 & -0.966 & $\mathrm{U}$ & 2.9 \\
\hline Area $4 \mathrm{A2}$ & $\mathrm{J} 13515$ & $8 / 21 / 06$ & 0.023 & U & 0.023 & 1.22 & & 0.040 & 1.25 & & 0.13 & 0.139 & $U$ & 0.19 & -1.45 & $\mathrm{U}$ & 2.8 \\
\hline Area $4 \mathrm{A3}$ & $\mathrm{J} 13516$ & $8 / 21 / 06$ & 0.010 & $\mathrm{U}$ & 0.010 & 0.573 & & 0.019 & 0.649 & & 0.076 & 0.110 & $\mathrm{U}$ & 0.20 & -1.53 & $\mathrm{U}$ & 3.0 \\
\hline Area 4 A4 & $\mathrm{J} 13517$ & $8 / 21 / 06$ & 0.014 & $\mathrm{U}$ & 0.014 & 0.515 & & 0.023 & 0.596 & & 0.085 & -0.048 & $\mathrm{U}$ & 0.20 & -2.33 & $\mathrm{U}$ & 2.9 \\
\hline $\mathrm{OB} / \mathrm{BCL} \mathrm{A} 1$ & $J 13518$ & $8 / 22 / 06$ & 0.021 & $\mathrm{U}$ & 0.021 & 1.05 & & 0.044 & 1.31 & & 0.15 & 0.080 & $\mathrm{U}$ & 0.26 & -2.09 & $\overline{\mathrm{UJ}}$ & 2.8 \\
\hline $\mathrm{OB} / \mathrm{BCL} \mathrm{A} 2$ & $J 13519$ & $8 / 22 / 06$ & 0.019 & $\mathrm{U}$ & 0.019 & 0.550 & & 0.035 & 0.670 & & 0.13 & 0.052 & $\mathrm{U}$ & 0.29 & -1.49 & UJ & 2.7 \\
\hline $\mathrm{OB} / \mathrm{BCL} \mathrm{A3}$ & $\mathrm{J} 13520$ & $8 / 22 / 06$ & 0.011 & $\bar{U}$ & 0.011 & 1.06 & & 0.022 & 1.11 & & 0.068 & 2.10 & & 0.25 & -0.518 & $\overline{U J}$ & 2.7 \\
\hline $\mathrm{OB} / \mathrm{BCL} \mathrm{A} 4$ & J135N5 & $8 / 22 / 06$ & 0.027 & $\mathrm{U}$ & 0.027 & 1.20 & & 0.052 & 1.34 & & 0.14 & 0.089 & $\mathrm{U}$ & 0.27 & -2.00 & UJ & 3.1 \\
\hline SPAA1 & $J 13522$ & $9 / 11 / 06$ & 0.032 & $\bar{U}$ & 0.032 & 0.807 & & 0.075 & 0.824 & & 0.17 & 0.211 & $\mathrm{U}$ & 0.22 & -0.913 & $\bar{U}$ & 3.0 \\
\hline SPA A2 & $J 13523$ & $9 / 11 / 06$ & 0.022 & $\mathrm{U}$ & 0.022 & 0.496 & & 0.036 & 0.598 & & 0.15 & 0.158 & $\mathrm{U}$ & 0.21 & 0 & $\mathrm{U}$ & 2.5 \\
\hline SPA A3 & $\mathrm{J} 135 \mathrm{P} 1$ & $9 / 12 / 06$ & 0.033 & $\mathrm{U}$ & 0.033 & 1.36 & & 0.073 & 1.08 & & 0.15 & 0.855 & & 0.21 & -1.33 & $\mathrm{U}$ & 2.5 \\
\hline SPA A4 & $\mathrm{J} 135 \mathrm{P0}$ & $9 / 12 / 06$ & 0.025 & $\mathrm{U}$ & 0.025 & 0.886 & & 0.057 & 0.765 & & 0.12 & 0.315 & & 0.25 & 0.253 & $u$ & 3.0 \\
\hline SPA B5 & $\mathrm{J} 13524$ & $9 / 11 / 06$ & 0.022 & $\mathrm{U}$ & 0.022 & 0.678 & & 0.036 & 0.822 & & 0.10 & 0.235 & & 0.21 & -0.768 & $\mathrm{U}$ & 2.6 \\
\hline SPA B6 & $J 13525$ & $9 / 11 / 06$ & 0.035 & $\mathrm{U}$ & 0.035 & 1.41 & & 0.075 & 1.33 & & 0.18 & 0.070 & $U$ & 0.23 & -0.821 & $U$ & 2.8 \\
\hline SPA B7 & $\mathrm{J} 135 \mathrm{~N} 8$ & $9 / 11 / 06$ & 0.014 & $\mathrm{U}$ & 0.014 & 0.644 & & 0.022 & 0.728 & & 0.10 & 0.057 & $\mathrm{U}$ & 0.21 & 0.189 & $\mathrm{U}$ & 2.5 \\
\hline SPAB8 & J135N9 & $9 / 11 / 06$ & 0.028 & $\mathrm{U}$ & 0.028 & 0.965 & & 0.062 & 1.09 & & 0.16 & 0.115 & $U$ & 0.25 & -0.757 & $U$ & 2.6 \\
\hline SPA C9 & $\mathrm{J} 135 \mathrm{P} 2$ & $9 / 12 / 06$ & 0.014 & $\mathrm{U}$ & 0.014 & 0.771 & & 0.025 & 0.927 & & 0.082 & 0.203 & $\mathrm{U}$ & 0.21 & -0.566 & $\mathrm{U}$ & 3.0 \\
\hline SPA C10 & $\mathrm{J} 135 \mathrm{P} 3$ & $9 / 12 / 06$ & 0.025 & $\mathrm{U}$ & 0.025 & 0.898 & & 0.054 & 0.698 & & 0.14 & 0.327 & & 0.21 & -0.214 & $\mathrm{U}$ & 2.6 \\
\hline SPAC1 & $\mathrm{J} 135 \mathrm{P} 4$ & $9 / 12 / 06$ & 0.037 & $U$ & 0.037 & 1.50 & & 0.076 & 1.37 & & 0.21 & 0.333 & & 0.21 & -0.952 & $\mathrm{U}$ & 3.0 \\
\hline SPA C2 & J135P5 & $9 / 12 / 06$ & 0.040 & $\mathrm{U}$ & 0.040 & 1.35 & & 0.095 & 1.17 & & 0.24 & 0.191 & $\bar{U}$ & 0.22 & -0.933 & $\mathrm{U}$ & 2.6 \\
\hline SPAD3 & $\mathrm{J} 135 \mathrm{P} 6$ & $9 / 12 / 06$ & 0.022 & $\mathrm{U}$ & 0.022 & 0.558 & & 0.038 & 0.729 & & 0.13 & 0.117 & $U$ & 0.23 & -1.60 & $\mathrm{U}$ & 2.6 \\
\hline SPA D4 & $\mathrm{J} 135 \mathrm{P} 7$ & $9 / 12 / 06$ & 0.020 & $\mathrm{U}$ & 0.020 & 0.703 & & 0.037 & 0.769 & & 0.10 & -0.019 & $\mathrm{U}$ & 0.22 & -0.272 & $\mathrm{U}$ & 2.5 \\
\hline SPA D5 & $\mathrm{J} 135 \mathrm{P} 8$ & $9 / 12 / 06$ & 0.040 & $\mathrm{U}$ & 0.040 & 0.865 & & 0.084 & 0.779 & & 0.20 & 0.475 & & 0.22 & -0.322 & $\mathrm{U}$ & 2.7 \\
\hline SPA D6 & $\mathrm{J} 135 \mathrm{P9}$ & $9 / 12 / 06$ & 0.011 & U & 0.011 & 0.926 & & 0.025 & 0.824 & & 0.062 & 0.031 & $\mathrm{U}$ & 0.25 & -0.406 & U & 2.8 \\
\hline FS-7 & J12XM4 & $7 / 26 / 06$ & 0.025 & $\mathrm{U}$ & 0.025 & 0.226 & & 0.033 & 0.357 & & 0.21 & & & & & & \\
\hline
\end{tabular}

\begin{tabular}{|c|c|c|c|}
\hline Attachment & 1 & Sheet No. & 6 of 43 \\
\hline Originator & J. M. Capron & Date & $12 / 13 / 06$ \\
\hline Checked & T.M. Blakley & Date & \\
\hline Calc. No. & $0100 \mathrm{C}-\mathrm{CA}-\mathrm{V} 0033$ & Rev. No. & 0 \\
\hline
\end{tabular}


Attachment 1. 118-C-1 Verification Sampling Results.

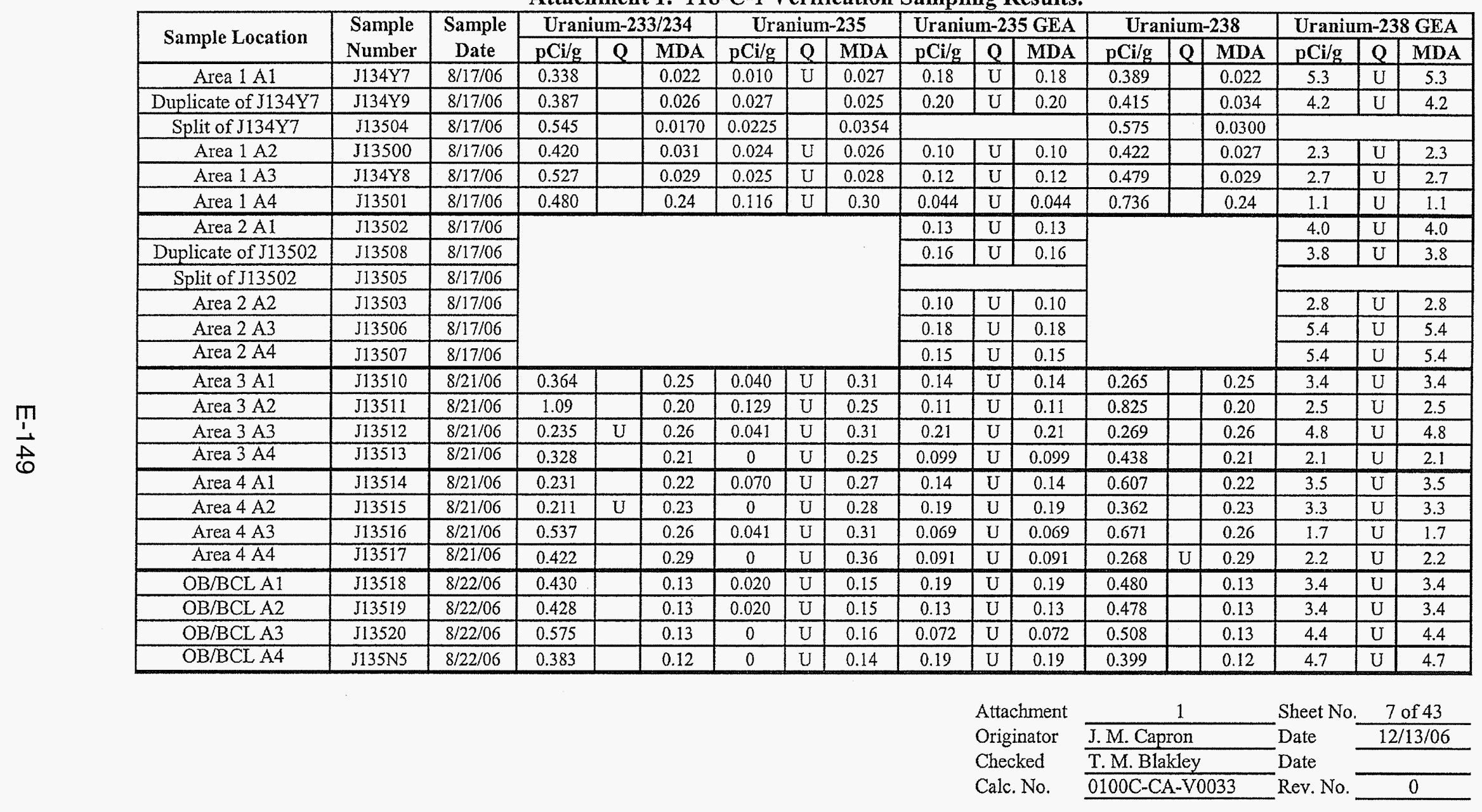


Attachment 1. 118-C-1 Verification Sampling Results.

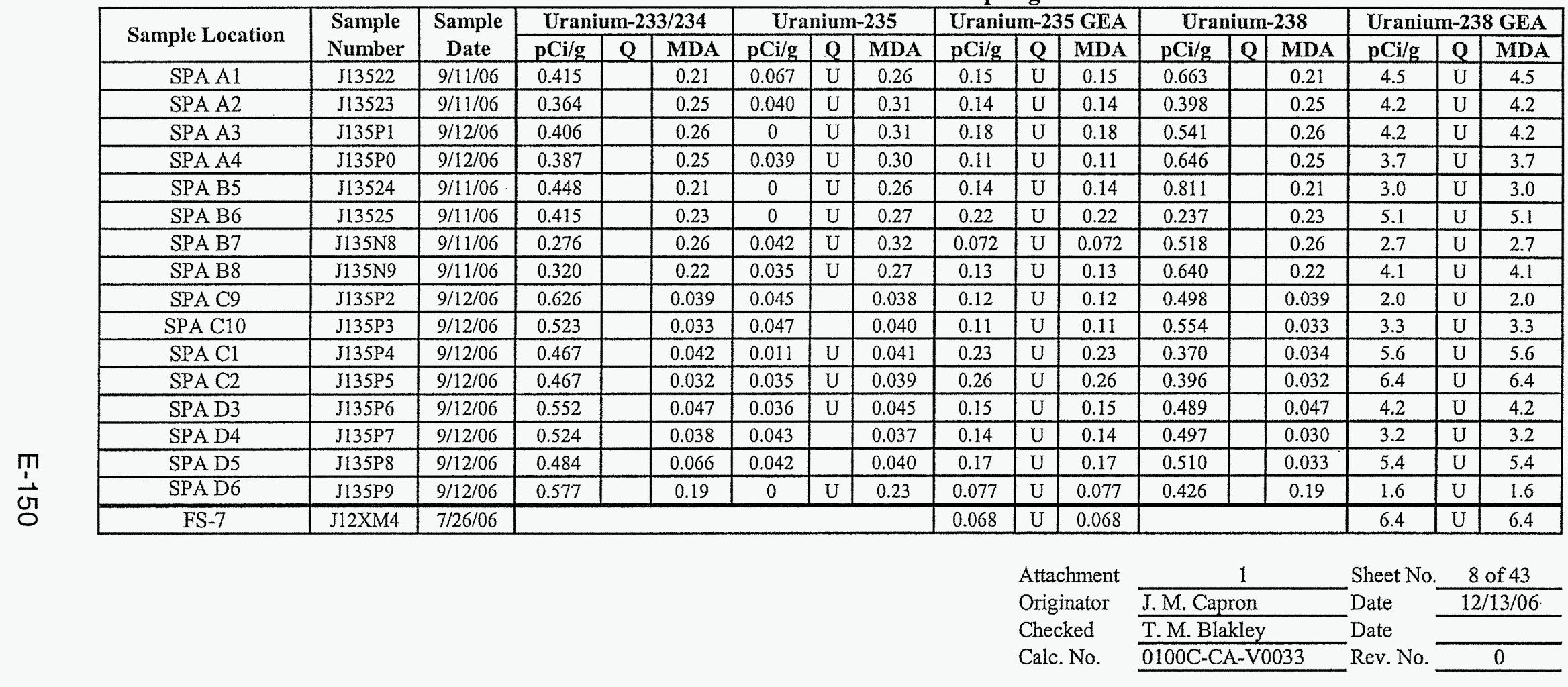


Attachment 1. 118-C-1 Verification Sampling Results.

\begin{tabular}{|c|c|c|c|c|c|c|c|c|c|c|c|c|c|c|c|c|c|c|c|c|}
\hline \multirow{2}{*}{ Sample Location } & \multirow{2}{*}{$\begin{array}{c}\text { Sample } \\
\text { Number }\end{array}$} & \multirow{2}{*}{$\begin{array}{c}\text { Sample } \\
\text { Date }\end{array}$} & \multicolumn{3}{|c|}{ Aluminum } & \multicolumn{3}{|c|}{ Antimony } & \multicolumn{3}{|c|}{ Arsenic } & \multicolumn{3}{|c|}{ Barium } & \multicolumn{3}{|c|}{ Beryllium } & \multicolumn{3}{|c|}{ Boron } \\
\hline & & & $\mathrm{mg} / \mathrm{kg}$ & $Q$ & PQL & $\mathrm{mg} / \mathrm{kg}$ & $Q$ & $\mathrm{PQL}$ & $\mathrm{mg} / \mathrm{kg}$ & $Q$ & PQL & $\mathrm{mg} / \mathrm{kg}$ & $Q$ & PQL & $\mathrm{mg} / \mathrm{kg}$ & $Q$ & PQL & $\mathrm{mg} / \mathrm{kg}$ & Q & PQL \\
\hline Area $1 \mathrm{~A} 1$ & J134Y7 & $8 / 17 / 06$ & 3880 & & 8.0 & 1.2 & $\mathrm{U}$ & 1.2 & 2.7 & & 1.7 & 48.1 & & 0.06 & 0.78 & & 0.06 & 1.3 & & 0.66 \\
\hline Duplicate of J134Y7 & J134Y9 & $8 / 17 / 06$ & 4500 & & 7.9 & 1.2 & $\mathrm{U}$ & 1.2 & 2.3 & & 1.7 & 51.5 & & 0.05 & 0.82 & & 0.05 & 1.7 & & 0.66 \\
\hline Split of J134Y7 & $\mathrm{J} 13504$ & $8 / 17 / 06$ & 6610 & $\mathrm{~N}$ & 6.3 & 1.2 & $\mathrm{~N}$ & 0.33 & 2.6 & & 0.28 & 57.5 & & 0.50 & 0.34 & $\mathrm{~B}^{*}$ & 0.071 & 1.6 & $\mathrm{~B}^{*}$ & 1.5 \\
\hline Area $1 \mathrm{~A} 2$ & $\mathrm{J13500}$ & $8 / 17 / 06$ & 4130 & & 8.0 & 1.2 & $\mathrm{U}$ & 1.2 & 1.9 & & 1.7 & 52.0 & & 0.06 & 0.69 & & 0.06 & 1.6 & & 0.66 \\
\hline Area $1 \mathrm{~A} 3$ & J134Y8 & $8 / 17 / 06$ & 4570 & & 8.0 & 1.2 & $\mathrm{U}$ & 1.2 & 2.3 & & 1.7 & 56.0 & & 0.06 & 0.69 & & 0.06 & 2.1 & & 0.67 \\
\hline Area $1 \mathrm{~A} 4$ & $\mathrm{~J} 13501$ & $8 / 17 / 06$ & 4460 & & 7.9 & 1.2 & $\mathrm{U}$ & 1.2 & 3.3 & & 1.7 & 48.7 & & 0.06 & 0.88 & & 0.06 & 1.6 & & 0.66 \\
\hline Area 2 A1 & $\mathrm{J} 13502$ & $8 / 17 / 06$ & 4690 & & 7.9 & 1.2 & $\mathrm{U}$ & 1.2 & 2.3 & & 1.7 & 48.1 & & 0.06 & 0.70 & & 0.06 & 1.9 & & 0.66 \\
\hline Duplicate of $\mathrm{J} 13502$ & $\mathrm{~J} 13508$ & $8 / 17 / 06$ & 4090 & & 8.0 & 1.2 & $\mathrm{U}$ & 1.2 & 2.4 & & 1.7 & 48.4 & & 0.06 & 0.86 & & 0.06 & 1.7 & & 0.67 \\
\hline Split of J13502 & $\mathrm{J13505}$ & $8 / 17 / 06$ & 6210 & $\mathrm{~N}$ & 6.3 & 1.5 & $\mathrm{~N}$ & 0.33 & 3.1 & & 0.28 & 54.7 & & 0.50 & 0.34 & $\mathrm{~B}^{*}$ & 0.071 & 1.9 & $B^{*}$ & 1.5 \\
\hline Area $2 \mathrm{~A} 2$ & $\mathrm{~J} 13503$ & $8 / 17 / 06$ & 4310 & & 8.0 & 1.2 & $\mathrm{U}$ & 1.2 & 2.8 & & 1.7 & 45.6 & & 0.06 & 0.78 & & 0.06 & 2.0 & & 0.66 \\
\hline Area $2 \mathrm{A3}$ & $\mathrm{J} 13506$ & $8 / 17 / 06$ & 4050 & & 8.0 & 1.2 & $\mathrm{U}$ & 1.2 & 2.0 & & 1.7 & 44.4 & & 0.06 & 0.68 & & 0.06 & 2.2 & & 0.66 \\
\hline Area 2 A4 & $\mathrm{J} 13507$ & $8 / 17 / 06$ & 3990 & & 7.9 & 1.2 & $\mathrm{U}$ & 1.2 & 2.4 & & 1.7 & 54.5 & & 0.05 & 0.93 & & 0.05 & 1.5 & & 0.66 \\
\hline Area $3 \mathrm{Al}$ & $\mathrm{J} 13510$ & $8 / 21 / 06$ & 5540 & & 7.9 & 1.2 & $\mathrm{U}$ & 1.2 & 1.8 & & 1.7 & 63.8 & & 0.06 & 0.70 & & 0.06 & 1.6 & $\mathrm{~J}$ & 0.66 \\
\hline Area $3 \mathrm{~A} 2$ & $\mathrm{J13511}$ & $8 / 21 / 06$ & 4550 & & 8.0 & 4.3 & & 1.2 & 33.9 & & 1.7 & 132 & & 0.06 & 0.50 & & 0.06 & 4.6 & $\mathrm{~J}$ & 0.66 \\
\hline Arca 3 A2 resample & J13HN3 & 9/29/06 & 4950 & $\mathrm{C}$ & 7.0 & 1.1 & $\mathrm{u}$ & 1.1 & 5.8 & & 1.1 & 71.3 & C & 0.06 & 0.28 & & 0.03 & 2.1 & & 1.1 \\
\hline Area $3 \mathrm{~A} 3$ & $\mathrm{~J} 13512$ & $8 / 21 / 06$ & 5040 & & 7.9 & 1.2 & $\mathrm{U}$ & 1.2 & 2.2 & & 1.7 & 78.4 & & 0.06 & 0.51 & & 0.06 & 3.2 & $\mathrm{~J}$ & 0.66 \\
\hline Area 3 A4 & $\mathrm{J} 13513$ & $8 / 21 / 06$ & 4700 & & 7.9 & 1.2 & U & 1.2 & 2.0 & & 1.7 & 55.5 & & 0.05 & 0.52 & & 0.05 & 1.5 & $\mathrm{~J}$ & 0.66 \\
\hline Area 4 Al & $\mathrm{J} 13514$ & $8 / 21 / 06$ & 4880 & & 7.9 & 1.2 & $\mathrm{U}$ & 1.2 & 2.1 & & 1.7 & 48.6 & & 0.05 & 0.58 & & 0.05 & 1.0 & $\mathrm{~J}$ & 0.66 \\
\hline Area 4 A2 & $\mathrm{J} 13515$ & $8 / 21 / 06$ & 5100 & & 7.9 & 1.2 & $\mathrm{U}$ & 1.2 & 2.3 & & 1.7 & 286 & & 0.05 & 0.58 & & 0.05 & 4.9 & $\mathrm{~J}$ & 0.66 \\
\hline Area $4 \mathrm{~A} 3$ & $\mathrm{J13516}$ & $8 / 21 / 06$ & 4080 & & 7.9 & 1.2 & $\mathrm{U}$ & 1.2 & 2.4 & & 1.7 & 49.9 & & 0.05 & 0.37 & & 0.05 & 0.65 & Uu & 0.65 \\
\hline Area 4 A4 & J13517 & $8 / 21 / 06$ & 4070 & & 7.9 & 1.2 & $\mathrm{U}$ & 1.2 & 2.2 & & 1.7 & 49.2 & & 0.06 & 0.43 & & 0.06 & 0.66 & UJ & 0.66 \\
\hline $\mathrm{OB} / \mathrm{BCL} \mathrm{Al}$ & $\mathrm{J} 13518$ & $8 / 22 / 06$ & 4660 & & 8.3 & 1.3 & UJ & 1.3 & 2.2 & & 1.8 & 48.7 & & 0.06 & 0.65 & & 0.06 & 2.1 & $\mathrm{UJ}$ & 0.69 \\
\hline OB/BCL A2 & $\mathrm{J} 13519$ & $8 / 22 / 06$ & 4740 & & 8.4 & 1.3 & $\mathrm{UJ}$ & 1.3 & 2.2 & & 1.8 & 50.0 & & 0.06 & 0.69 & & 0.06 & 1.8 & UI & 0.70 \\
\hline $\mathrm{OB} / \mathrm{BCL} \mathrm{A} 3$ & $\mathrm{~J} 13520$ & $8 / 22 / 06$ & 3690 & & 8.4 & 1.3 & $\mathrm{UJ}$ & 1.3 & 1.8 & $\mathrm{U}$ & 1.8 & 46.0 & & 0.06 & 0.60 & & 0.06 & 1.6 & $\mathrm{UJ}$ & 0.70 \\
\hline OB/BCLA4 & J135N5 & $8 / 22 / 06$ & 4760 & & 8.4 & 1.3 & $\mathrm{UJ}$ & 1.3 & 2.3 & & 1.8 & 58.4 & & 0.06 & 0.52 & & 0.06 & 2.0 & UJ & 0.70 \\
\hline SPA Al & $\mathrm{J} 13522$ & $9 / 11 / 06$ & 5120 & & 6.6 & 1.0 & $\mathrm{U}$ & 1.0 & 3.0 & & 1.1 & 52.9 & & 0.06 & 0.43 & & 0.03 & 1.2 & & 1.1 \\
\hline SPA A2 & $\mathrm{J} 13523$ & $9 / 11 / 06$ & 5020 & & 6.6 & 1.0 & $\mathrm{U}$ & 1.0 & 2.5 & & 1.1 & 53.4 & & 0.06 & 0.31 & & 0.03 & 1.1 & $\mathrm{U}$ & 1.1 \\
\hline SPA A3 & J135P1 & $9 / 12 / 06$ & 5300 & & 6.5 & 1.0 & $\mathrm{U}$ & 1.0 & 2.3 & & 1.1 & 53.3 & & 0.06 & 0.37 & & 0.03 & 1.7 & & 1.1 \\
\hline SPA A4 & $\mathrm{J} 135 \mathrm{PO}$ & $9 / 12 / 06$ & 4600 & & 6.5 & 1.0 & $\mathrm{U}$ & 1.0 & 3.3 & & 1.1 & 53.5 & & 0.06 & 0.31 & & 0.03 & 2.0 & & 1.1 \\
\hline SPAB5 & J13524 & $9 / 11 / 06$ & 5130 & & 6.6 & 1.0 & $\mathrm{U}$ & 1.0 & 2.4 & & 1.1 & 55.6 & & 0.06 & 0.31 & & 0.03 & 1.1 & $\mathrm{U}$ & 1.1 \\
\hline SPA BG & $\mathrm{J13525}$ & $9 / 11 / 06$ & 4830 & & 6.6 & 1.0 & $\mathrm{U}$ & 1.0 & 2.3 & & 1.1 & 54.9 & & 0.06 & 0.34 & & 0.03 & 1.1 & \begin{tabular}{|l|}
$\mathrm{U}$ \\
\end{tabular} & 1.1 \\
\hline SPAB7 & J135N8 & $9 / 11 / 06$ & 5310 & & 6.6 & 1.0 & $\mathrm{U}$ & 1.0 & 2.4 & & 1.1 & 54.7 & & 0.06 & 0.35 & & 0.03 & 1.1 & $\mathrm{U}$ & 1.1 \\
\hline SPA B8 & J135N9 & $9 / 11 / 06$ & 4900 & & 6.5 & 1.0 & $\mathrm{U}$ & 1.0 & 2.9 & & 1.1 & 60.1 & & 0.06 & 0.30 & & 0.03 & 1.1 & U & 1.1 \\
\hline SPAC9 & $\mathrm{J} 135 \mathrm{P} 2$ & $9 / 12 / 06$ & 6390 & & 6.5 & 1.0 & $\mathrm{U}$ & 1.0 & 1.8 & & 1.1 & 495 & & 0.06 & 0.57 & & 0.03 & 10.7 & & 1.1 \\
\hline SPACIO & J135P3 & $9 / 12 / 06$ & 4580 & & 6.6 & 1.0 & $\mathrm{U}$ & 1.0 & 2.0 & & 1.1 & 60.0 & & 0.06 & 0.36 & & 0.03 & 1.1 & U & 1.1 \\
\hline SPACl & $\mathrm{J135P4}$ & $9 / 12 / 06$ & 5100 & & 6.5 & 1.0 & $\mathrm{U}$ & 1.0 & 2.8 & & 1.1 & 58.2 & & 0.06 & 0.41 & & 0.03 & 2.4 & & 1.1 \\
\hline $\mathrm{SPAC2}$ & J135P5 & 9/12/06 & 4840 & & 6.6 & 1.0 & $\mathrm{U}$ & 1.0 & 2.6 & & 1.1 & 62.9 & & 0.06 & 0.41 & & 0.03 & 2.6 & & 1.1 \\
\hline SPAD3 & J135P6 & $9 / 12 / 06$ & 5000 & & 6.5 & 1.0 & $U$ & 1.0 & 3.0 & & 1.1 & 63.8 & & 0.06 & 0.44 & & 0.03 & 1.5 & & 1.1 \\
\hline SPA D4 & J135P7 & $9 / 12 / 06$ & 4870 & & 6.6 & 1.0 & $U$ & 1.0 & 2.0 & & 1.1 & 56.0 & & 0.06 & 0.42 & & 0.03 & 1.1 & & 1.1 \\
\hline SPADS & $\mathrm{J} 135 \mathrm{P} 8$ & $9 / 12 / 06$ & 5540 & & 6.5 & 1.0 & $\mathrm{U}$ & 1.0 & 2.5 & & 1.1 & 59.4 & & 0.06 & 0.47 & & 0.03 & 1.1 & U & 1.1 \\
\hline SPA D6 & J135P9 & $9 / 12 / 06$ & 5540 & & 6.5 & 1.0 & $\mathrm{U}$ & 1.0 & 2.9 & & 1.1 & 64.8 & & 0.06 & 0.49 & & 0.03 & 1.4 & & 1.1 \\
\hline
\end{tabular}

\begin{tabular}{|c|c|c|c|}
\hline Attachment & 1 & Sheet No & 9 of 43 \\
\hline Originator & J.M. Capron & Date & $12 / 13 / 06$ \\
\hline Checked & T.M. Blakley & Date & \\
\hline Calc. No. & $0100 \mathrm{C}-\mathrm{CA}-\mathrm{V} 0033$ & Rev. No. & 0 \\
\hline
\end{tabular}


Attachment 1. 118-C-1 Verification Sampling Results.

\begin{tabular}{|c|c|c|c|c|c|c|c|c|c|c|c|c|c|c|c|c|c|c|c|c|}
\hline \multirow{2}{*}{ Sample Location } & \multirow{2}{*}{$\begin{array}{c}\text { Sample } \\
\text { Number }\end{array}$} & \multirow{2}{*}{$\begin{array}{c}\text { Sample } \\
\text { Date }\end{array}$} & \multicolumn{3}{|c|}{ Aluminum } & \multicolumn{3}{|c|}{ Antimony } & \multicolumn{3}{|c|}{ Arsenic } & \multicolumn{3}{|c|}{ Barium } & \multicolumn{3}{|c|}{ Beryllium } & \multicolumn{3}{|c|}{ Boron } \\
\hline & & & $\mathrm{mg} / \mathrm{kg}$ & $Q$ & PQL & $\mathrm{mg} / \mathrm{kg}$ & $Q$ & $\mathrm{PQL}$ & $\mathrm{mg} / \mathrm{kg}$ & $Q$ & $\mathrm{PQL}$ & $\mathrm{mg} / \mathrm{kg}$ & $Q$ & $\mathrm{PQL}$ & $\mathrm{mg} / \mathrm{kg}$ & $Q$ & PQL & $\mathrm{mg} / \mathrm{kg}$ & $Q$ & PQL \\
\hline FS-1 & $\mathrm{J} 12 \mathrm{XL8}$ & $7 / 26 / 06$ & 5510 & & 8.3 & 1.3 & $\overrightarrow{\mathrm{U}}$ & 1.3 & 2.2 & & 1.8 & 59.6 & $\mathrm{C}$ & 0.06 & 0.23 & & 0.06 & 1.9 & & 0.69 \\
\hline FS-2 & J12XL9 & $7 / 26 / 06$ & 4040 & & 8.3 & 1.3 & $\mathrm{U}$ & 1.3 & 3.5 & & 1.8 & 40.5 & C & 0.06 & 0.18 & & 0.06 & 1.4 & & 0.69 \\
\hline FS-3 & $\mathrm{J} 12 \mathrm{XM0}$ & $7 / 26 / 06$ & 3630 & & 8.2 & 1.3 & $\mathrm{U}$ & 1.3 & 2.5 & & 1.7 & 41.8 & $\mathrm{C}$ & 0.06 & 0.16 & & 0.06 & 1.2 & & 0.69 \\
\hline FS-4 & $\mathrm{J} 12 \mathrm{XM1}$ & $7 / 26 / 06$ & 5670 & & 8.4 & 1.3 & $\mathrm{U}$ & 1.3 & 5.4 & & 1.8 & 47.7 & $\mathrm{C}$ & 0.06 & 0.19 & & 0.06 & 1.9 & & 0.70 \\
\hline FS-5 & $\mathrm{J} 12 \mathrm{XM} 2$ & $7 / 26 / 06$ & 7520 & & 8.3 & 1.3 & $\mathrm{U}$ & 1.3 & 3.4 & & 1.8 & 80.2 & $\mathrm{C}$ & 0.06 & 0.34 & & 0.06 & 3.2 & & 0.69 \\
\hline FS-7 & J12XM14 & $7 / 26 / 06$ & 3160 & & 8.4 & 1.3 & $\mathrm{U}$ & 1.3 & 1.8 & $\mathrm{U}$ & 1.8 & 34.3 & $\mathrm{C}$ & 0.06 & 0.12 & & 0.06 & 2.5 & & 0.70 \\
\hline Equipment blank & $\mathrm{J} 13509$ & $8 / 17 / 06$ & 39.4 & & 2.6 & 0.40 & $\mathrm{U}$ & 0.40 & 0.56 & U & 0.56 & 1.1 & & 0.02 & 0.02 & & 0.02 & 0.42 & & 0.22 \\
\hline
\end{tabular}

\begin{tabular}{|c|c|c|c|c|c|c|c|c|c|c|c|c|c|c|c|c|c|c|c|c|}
\hline \multirow{2}{*}{ Sample Location } & \multirow{2}{*}{$\begin{array}{l}\text { Sample } \\
\text { Number }\end{array}$} & \multirow{2}{*}{$\begin{array}{c}\text { Sample } \\
\text { Date }\end{array}$} & \multicolumn{3}{|c|}{ Cadmium } & \multicolumn{3}{|c|}{ Calcium } & \multicolumn{3}{|c|}{ Chromium } & \multicolumn{3}{|c|}{ Cobalt } & \multicolumn{3}{|c|}{ Copper } & \multicolumn{3}{|c|}{$\begin{array}{l}\text { Hexavalent } \\
\text { Chromium }\end{array}$} \\
\hline & & & $\mathrm{mg} / \mathrm{kg}$ & $Q$ & PQL & $\mathrm{mg} / \mathrm{kg}$ & $Q$ & PQL & $\mathrm{mg} / \mathrm{kg}$ & $Q$ & $\mathrm{PQL}$ & $\mathrm{mg} / \mathrm{kg}$ & $Q$ & PQL & $\mathrm{mg} / \mathrm{kg}$ & $Q$ & PQL & $\mathrm{mg} / \mathrm{kg}$ & $Q$ & $\mathrm{PQL}$ \\
\hline Area $1 \mathrm{Al}$ & J134Y7 & $8 / 17 / 06$ & 0.19 & $\mathrm{U}$ & 0.19 & 4380 & & 4.5 & 5.6 & $\mathrm{C}$ & 0.36 & 7.0 & & 0.39 & 11.7 & & 0.33 & 0.20 & $\overrightarrow{\mathrm{U}}$ & 0.20 \\
\hline Duplicate of J134Y7 & J134Y9 & $8 / 17 / 06$ & 0.19 & $\mathrm{U}$ & 0.19 & 4570 & & 4.5 & 6.6 & $\mathrm{C}$ & 0.36 & 7.9 & & 0.38 & 12.7 & & 0.33 & 0.20 & $\bar{U}$ & 0.20 \\
\hline Split of J134Y7 & $\mathrm{J} 13504$ & $8 / 17 / 06$ & 0.14 & UN & 0.14 & 5080 & $\mathrm{CN}$ & 8.6 & 9.1 & $\mathrm{~N}$ & 0.36 & 11.8 & & 0.50 & 13.2 & & 0.30 & 0.350 & $\mathrm{U}$ & 0.350 \\
\hline Area 1 A2 & $\mathrm{J} 13500$ & $8 / 17 / 06$ & 0.19 & $\mathrm{U}$ & 0.19 & 3820 & & 4.5 & 8.6 & $\mathrm{C}$ & 0.36 & 5.9 & & 0.39 & 9.9 & & 0.33 & 0.20 & $U$ & 0.20 \\
\hline Area $1 \mathrm{~A} 3$ & J134Y8 & $8 / 17 / 06$ & 0.20 & $\mathrm{U}$ & 0.20 & 6890 & & 4.6 & 6.8 & $\mathrm{C}$ & 0.36 & 5.7 & & 0.39 & 11.3 & & 0.33 & 0.20 & $\mathrm{U}$ & 0.20 \\
\hline Area $1 \mathrm{A4}$ & $\mathrm{J13501}$ & $8 / 17 / 06$ & 0.19 & $\mathrm{U}$ & 0.19 & 8720 & & 4.5 & 6.0 & $\mathrm{C}$ & 0.36 & 8.4 & & 0.39 & 14.7 & & 0.33 & 0.20 & $\mathrm{U}$ & 0.20 \\
\hline Area $2 \mathrm{Al}$ & $\mathrm{J} 13502$ & $8 / 17 / 06$ & 0.19 & $\mathrm{U}$ & 0.19 & 6960 & & 4.5 & 6.2 & C & 0.36 & 7.6 & & 0.39 & 13.4 & & 0.33 & 0.20 & U & 0.20 \\
\hline Duplicate of J13502 & $\mathrm{J} 13508$ & $8 / 17 / 06$ & 0.20 & $\mathrm{U}$ & 0.20 & 6310 & & 4.6 & 5.8 & $\mathrm{C}$ & 0.36 & 7.5 & & 0.39 & 13.4 & & 0.34 & 0.21 & & 0.20 \\
\hline Split of J13502 & $\mathrm{J} 13505$ & $8 / 17 / 06$ & 0.14 & UN & 0.14 & 7420 & $\mathrm{CN}$ & 8.6 & 8.5 & $\mathrm{~N}$ & 0.36 & 12.9 & & 0.50 & 14.8 & & 0.30 & 0.350 & $U$ & 0.350 \\
\hline Area $2 \mathrm{~A} 2$ & $\mathrm{~J} 13503$ & $8 / 17 / 06$ & 0.19 & $\mathrm{U}$ & 0.09 & 6810 & & 4.5 & 6.2 & $\mathrm{C}$ & 0.36 & 7.3 & & 0.39 & 12.8 & & 0.33 & 0.24 & & 0.20 \\
\hline Area $2 \mathrm{A3}$ & $\mathrm{J13506}$ & $8 / 17 / 06$ & 0.19 & $\mathrm{U}$ & 0.19 & 3560 & & 4.5 & 5.4 & C & 0.36 & 6.8 & & 0.39 & 10.5 & & 0.33 & 0.20 & $\mathrm{U}$ & 0.20 \\
\hline Area $2 \mathrm{A4}$ & $\sqrt{13507}$ & $8 / 17 / 06$ & 0.19 & $\mathrm{U}$ & 0.19 & 5160 & & 4.5 & 4.9 & $\mathrm{C}$ & 0.36 & 7.7 & & 0.38 & 13.2 & & 0.33 & 0.23 & & 0.20 \\
\hline Area $3 \mathrm{Al}$ & J13510 & $8 / 21 / 06$ & 0.19 & $U$ & 0.19 & 4550 & & 4.5 & 8.0 & $\mathrm{C}$ & 0.36 & 8.9 & & 0.39 & 18.1 & & 0.33 & 0.20 & $\bar{U}$ & 0.20 \\
\hline Area $3 \mathrm{~A} 2$ & $\mathrm{~J} 13511$ & $8 / 21 / 06$ & 0.19 & $\mathrm{U}$ & 0.19 & 5450 & & 4.5 & 8.1 & $\mathrm{C}$ & 0.36 & 13.1 & & 0.39 & 286 & & 0.33 & 0.20 & $\mathrm{U}$ & 0.20 \\
\hline Area $3 \mathrm{~A} 2$ resample & J13HN3 & $9 / 29 / 06$ & 0.12 & & 0.09 & 5080 & $\mathrm{C}$ & 2.6 & 7.2 & $\mathrm{C}$ & 0.22 & 8.6 & & 0.15 & 45.2 & & 0.25 & & & \\
\hline Area $3 \mathrm{~A} 3$ & $\mathrm{~J} 13512$ & $8 / 21 / 06$ & 0.19 & $\mathrm{U}$ & 0.19 & 14100 & & 4.5 & 9.2 & $\mathrm{C}$ & 0.36 & 7.7 & & 0.39 & 30.6 & & 0.33 & 0.20 & $\mathrm{U}$ & 0.20 \\
\hline Area 3 A4 & $J 13513$ & $8 / 21 / 06$ & 0.19 & $\mathrm{U}$ & 0.19 & 5580 & & 4.5 & 7.4 & $\mathrm{C}$ & 0.36 & 8.5 & & 0.38 & 17.9 & & 0.33 & 0.20 & $\mathrm{U}$ & 0.20 \\
\hline Area $4 \mathrm{AI}$ & $\mathrm{J13514}$ & $8 / 21 / 06$ & 0.19 & $\mathrm{U}$ & 0.19 & 5600 & & 4.5 & 6.6 & $\mathrm{C}$ & 0.36 & 8.8 & & 0.38 & 14.7 & & 0.33 & 0.20 & $U$ & 0.20 \\
\hline Area 4 A2 & $\mathrm{J} 13515$ & $8 / 21 / 06$ & 0.19 & $\mathrm{U}$ & 0.19 & 7040 & & 4.5 & 6.2 & $\mathrm{C}$ & 0.35 & 8.5 & & 0.38 & 15.1 & & 0.33 & 0.20 & $\mathrm{U}$ & 0.20 \\
\hline Area $4 \mathrm{A3}$ & $\mathrm{J} 13516$ & $8 / 21 / 06$ & 0.19 & $\mathrm{U}$ & 0.19 & 8360 & & 4.5 & 6.0 & $\mathrm{C}$ & 0.35 & 7.2 & & 0.38 & 14.2 & & 0.33 & 0.20 & $\mathrm{U}$ & 0.20 \\
\hline Area 4 A4 & $J 13517$ & $8 / 21 / 06$ & 0.19 & $\mathrm{U}$ & 0.19 & 5450 & & 4.5 & 5.3 & $\mathrm{C}$ & 0.36 & 8.1 & & 0.39 & 14.8 & & 0.33 & 0.20 & $\mathrm{U}$ & 0.20 \\
\hline $\mathrm{OB} / \mathrm{BCL} \mathrm{Al}$ & $J 13518$ & $8 / 22 / 06$ & 0.20 & $\mathrm{U}$ & 0.20 & 5120 & & 4.7 & 6.1 & & 0.38 & 7.7 & & 0.40 & 15.8 & & 0.35 & 0.20 & $\mathrm{U}$ & 0.20 \\
\hline $\mathrm{OB} / \mathrm{BCL} \mathrm{A2}$ & $\mathrm{J13519}$ & $8 / 22 / 06$ & 0.21 & $\mathrm{U}$ & 0.21 & 5110 & & 4.8 & 6.4 & & 0.38 & 7.8 & & 0.41 & 15.0 & & 0.35 & 0.20 & $\mathrm{U}$ & 0.20 \\
\hline $\mathrm{OB} / \mathrm{BCL} \mathrm{A} 3$ & $\mathrm{~J} 13520$ & $8 / 22 / 06$ & 0.20 & $\mathrm{U}$ & 0.20 & 4200 & & 4.8 & 5,3 & & 0.38 & 6.9 & & 0.41 & 12.8 & & 0.35 & 0.24 & & 0.20 \\
\hline $\mathrm{OB} / \mathrm{BCL} \mathrm{A} 4$ & J135N5 & $8 / 22 / 06$ & 0.20 & $\mathrm{U}$ & 0.20 & 4710 & & 4.8 & 6.8 & & 0.38 & 7.1 & & 0.41 & 11.9 & & 0.35 & 0.20 & $\mathrm{U}$ & 0.20 \\
\hline
\end{tabular}

\begin{tabular}{lll} 
Attachment & \multicolumn{1}{c}{1} & Sheet No. 10 of 43 \\
Originator & J.M. Capron & $12 / 13 / 06$ \\
Checked & Date & 1. M. Blakley \\
Calc. No. & Date & \\
O100C-CA-V0033 Rev. No. &
\end{tabular}


Attachment 1. 118-C-1 Verification Sampling Results.

\begin{tabular}{|c|c|c|c|c|c|c|c|c|c|c|c|c|c|c|c|c|c|c|c|c|}
\hline \multirow[t]{2}{*}{ Sample Location } & \multirow{2}{*}{$\begin{array}{l}\text { Sample } \\
\text { Number }\end{array}$} & \multirow{2}{*}{$\begin{array}{c}\text { Sample } \\
\text { Date }\end{array}$} & \multicolumn{3}{|c|}{ Cadmium } & \multicolumn{3}{|c|}{ Calcium } & \multicolumn{3}{|c|}{ Chromium } & \multicolumn{3}{|c|}{ Cobalt } & \multicolumn{3}{|c|}{ Copper } & \multicolumn{3}{|c|}{$\begin{array}{l}\text { Hexavalent } \\
\text { Chromium }\end{array}$} \\
\hline & & & $\mathrm{mg} / \mathrm{kg}$ & $Q$ & PQL & $\mathrm{mg} / \mathrm{kg}$ & $Q$ & $\mathrm{PQL}$ & $\mathrm{mg} / \mathrm{kg}$ & $Q$ & $\mathrm{PQL}$ & $\mathrm{mg} / \mathrm{kg}$ & $Q$ & $\mathbf{P Q L}$ & $\mathrm{mg} / \mathrm{kg}$ & $Q$ & $\mathrm{PQL}$ & $\mathrm{mg} / \mathrm{kg}$ & $Q$ & $\mathrm{PQL}$ \\
\hline SPAAl & $\mathrm{J} 13522$ & $9 / 11 / 06$ & 0.09 & $\mathrm{U}$ & 0.09 & 5340 & & 2.5 & 7.5 & & 0.20 & 7.6 & & 0.14 & 15.3 & & 0.23 & 0.20 & $\mathrm{U}$ & 0.20 \\
\hline SPA A2 & J13523 & $9 / 11 / 06$ & 0.09 & $\mathrm{U}$ & 0.09 & 4730 & & 2.5 & 8.1 & & 0.20 & 6.2 & & 0.14 & 15.6 & & 0.23 & 0.20 & $\mathrm{U}$ & 0.20 \\
\hline SPA A3 & J135P1 & $9 / 12 / 06$ & 0.09 & $\mathrm{U}$ & 0.09 & 6010 & & 2.5 & 8.0 & & 0.20 & 7.0 & & 0.14 & 15.8 & & 0.23 & 0.20 & & 0.20 \\
\hline SPA A4 & $\mathrm{J} 135 \mathrm{PO}$ & $9 / 12 / 06$ & 0.09 & $\mathrm{U}$ & 0.09 & 5390 & & 2.5 & 7.0 & & 0.20 & 6.7 & & 0.14 & 15.7 & & 0.23 & 0.30 & & 0.20 \\
\hline SPA B5 & $J 13524$ & $9 / 11 / 06$ & 0.09 & $\mathrm{U}$ & 0.09 & 4420 & & 2.5 & 8.8 & & 0.20 & 6.4 & & 0.14 & 16.4 & & 0.23 & 1.9 & & 0.20 \\
\hline SPAB6 & $\mathrm{J} 13525$ & $9 / 11 / 06$ & 0.09 & $\mathrm{U}$ & 0.09 & 4330 & & 2.5 & 9.3 & & 0.20 & 7.2 & & 0.14 & 15.9 & & 0.23 & 0.20 & $\mathrm{U}$ & 0.20 \\
\hline SPAB7 & J135N8 & $9 / 11 / 06$ & 0.09 & $\mathrm{U}$ & 0.09 & 4610 & & 2.5 & 8.0 & & 0.20 & 7.0 & & 0.14 & 14.9 & & 0.23 & 0.20 & $\mathrm{u}$ & 0.20 \\
\hline SPA B 8 & J135N9 & $9 / 111 / 06$ & 0.09 & $\mathrm{U}$ & 0.09 & 4090 & & 2.5 & 7.6 & & 0.20 & 6.7 & & 0.14 & 13.9 & & 0.23 & 0.20 & $\mathrm{U}$ & 0.20 \\
\hline SPAC9 & J135P2 & $9 / 12 / 06$ & 0.09 & $\mathrm{U}$ & 0.09 & 8710 & & 2.5 & 6.8 & & 0.20 & 5.6 & & 0.14 & 144 & & 0.23 & 0.20 & $\mathrm{U}$ & 0.20 \\
\hline SPA C10 & $\mathrm{J135P3}$ & $9 / 12 / 06$ & 0.09 & $\mathrm{U}$ & 0.09 & 4870 & & 2.5 & 6.5 & & 0.20 & 6.5 & & 0.14 & 13.3 & & 0.23 & 0.29 & & 0.20 \\
\hline SPACl & $\mathrm{J} 135 \mathrm{P} 4$ & $9 / 12 / 06$ & 0.09 & $\mathrm{U}$ & 0.09 & 3800 & & 2.5 & 7.7 & & 0.20 & 6.4 & & 0.14 & 14.4 & & 0.23 & 0.20 & & 0.20 \\
\hline SPAC2 & J135P5 & $9 / 12 / 06$ & 0.09 & $\mathrm{U}$ & 0.09 & 4920 & & 2.5 & 7.6 & & 0.20 & 6.8 & & 0.14 & 15.2 & & 0.23 & 0.20 & $\mathrm{U}$ & 0.20 \\
\hline SPA. D3 & J135P6 & $9 / 12 / 06$ & 0.09 & $\mathrm{U}$ & 0.09 & 5000 & & 2.5 & 7.7 & & 0.20 & 6.6 & & 0.14 & 12.7 & & 0.23 & 0.26 & & 0.20 \\
\hline SPA D4 & J135P7 & $9 / 12 / 06$ & 0.09 & $\bar{U}$ & 0.09 & 5170 & & 2.5 & 7.2 & & 0.20 & 6.6 & & 0.14 & 12.6 & & 0.23 & 0.34 & & 0.20 \\
\hline SPAD5 & $\mathrm{J135 \textrm {PB }}$ & $9 / 12 / 06$ & 0.09 & $\mathrm{U}$ & 0.09 & 4860 & & 2.5 & 9.1 & & 0.20 & 7.5 & & 0.14 & 14.3 & & 0.23 & 0.20 & $\mathrm{U}$ & 0.20 \\
\hline SPAD6 & J135P9 & $9 / 12 / 06$ & 0.09 & $\mathrm{U}$ & 0.09 & 5180 & & 2.5 & 8.2 & & 0.20 & 7.8 & & 0.14 & 14.1 & & 0.23 & 0.30 & & 0.20 \\
\hline FS-1 & J12XL8 & $7 / 26 / 06$ & 0.20 & $\mathrm{U}$ & 0.20 & 3880 & & 4.7 & 8.6 & & 0.38 & 7.3 & & 0.41 & 13.8 & & 0.35 & & & \\
\hline FS-2 & J12XL9 & $7 / 26 / 06$ & 0.20 & $\mathrm{U}$ & 0.20 & 9250 & & 4.7 & 6.3 & & 0.38 & 7.9 & & 0.40 & 13.6 & & 0.35 & & & \\
\hline FS-3 & J12XM0 & $7 / 26 / 06$ & 0.20 & $\mathrm{U}$ & 0.20 & 6320 & & 4.7 & 4.8 & & 0.37 & 6.1 & & 0.40 & 10.5 & & 0.34 & & & \\
\hline FS-4 & $\mathrm{J} 12 \mathrm{XM1}$ & $7 / 26 / 06$ & 0.20 & $\mathrm{U}$ & 0.20 & 11400 & & 4.8 & 7.2 & & 0.38 & 8.6 & & 0.41 & 17.4 & & 0.35 & & & \\
\hline FS-5 & $\mathrm{J} 12 \mathrm{XM} 2$ & $7 / 26 / 06$ & 0.20 & $\mathrm{U}$ & 0.20 & 6130 & & 4.7 & 10.2 & & 0.38 & 10.5 & & 0.40 & 23.3 & & 0.35 & & & \\
\hline FS-7 & $\mathrm{J} 12 \mathrm{XM} 4$ & $7 / 26 / 06$ & 0.20 & $\mathrm{U}$ & 0.20 & 13900 & & 4.8 & 4.0 & & 0.38 & 7.0 & & 0.41 & 16.7 & & 0.35 & & & \\
\hline Equipment blank & $\mathrm{J13509}$ & $8 / 17 / 06$ & 0.06 & $\mathrm{U}$ & 0.06 & 22.1 & & 1.5 & 0.70 & $\mathrm{C}$ & 0.12 & 0.13 & $U$ & 0.13 & 0.11 & $\mathrm{U}$ & 0.11 & & & \\
\hline
\end{tabular}

\begin{tabular}{|c|c|c|c|c|c|c|c|c|c|c|c|c|c|c|c|c|c|c|c|c|}
\hline \multirow{2}{*}{ Sample Location } & \multirow{2}{*}{$\begin{array}{l}\text { Sample } \\
\text { Number }\end{array}$} & \multirow{2}{*}{$\begin{array}{c}\text { Sample } \\
\text { Date }\end{array}$} & \multicolumn{3}{|c|}{ Iron } & \multicolumn{3}{|c|}{ Lead } & \multicolumn{3}{|c|}{ Magnesium } & \multicolumn{3}{|c|}{ Manganese } & \multicolumn{3}{|c|}{ Mercury } & \multicolumn{3}{|c|}{ Molybdenum } \\
\hline & & & $\mathrm{mg} / \mathrm{kg}$ & $Q$ & $\mathrm{PQL}$ & $\mathrm{mg} / \mathrm{kg}$ & $Q$ & $\mathrm{PQL}$ & $\mathrm{mg} / \mathrm{kg}$ & $Q$ & PQL & $\mathrm{mg} / \mathrm{kg}$ & $Q$ & $\mathrm{PQL}$ & $\mathrm{mg} / \mathrm{kg}$ & $Q$ & PQL & $\mathrm{mg} / \mathrm{kg}$ & $Q$ & PQL \\
\hline Area $1 \mathrm{Al}$ & $\mathrm{J} 134 \mathrm{Y7}$ & $8 / 17 / 06$ & 15700 & & 9.7 & 4.5 & & 0.86 & 3170 & & 2.7 & 296 & & 0.08 & 0.02 & $\mathrm{U}$ & 0.02 & 0.80 & $\mathrm{U}$ & 0.80 \\
\hline Duplicate of $J 134 \mathrm{Y7}$ & $\mathrm{J134Y9}$ & $8 / 17 / 06$ & 18100 & & 9.6 & 4.5 & & 0.85 & 3630 & & 2.7 & 328 & & 0.08 & 0.01 & $\mathrm{U}$ & 0.01 & 0.80 & $\mathrm{U}$ & 0.80 \\
\hline Split of J134Y7 & $\mathrm{J} 13504$ & $8 / 17 / 06$ & 22600 & $\mathrm{~N}$ & 2.4 & 4.2 & & 0.15 & 4570 & & 12.2 & 342 & $\mathrm{~N}$ & 0.10 & 0.0067 & $\mathrm{U}$ & 0.0067 & 1.2 & $\bar{U}$ & 1.2 \\
\hline Area 1 A2 & $\mathrm{J} 13500$ & $8 / 17 / 06$ & 13900 & & 9.6 & 4.1 & & 0.86 & 3270 & & 2.7 & 260 & & 0.08 & 0.02 & $\mathrm{U}$ & 0.02 & 0.80 & $\mathrm{U}$ & 0.80 \\
\hline Area $1 \mathrm{~A} 3$ & $\mathrm{~J} 134 \mathrm{Y} 8$ & $8 / 17 / 06$ & 14000 & & 9.7 & 3.5 & & 0.86 & 3250 & & 2.7 & 259 & & 0.08 & 0.02 & $\mathrm{U}$ & 0.02 & 0.81 & $\mathrm{U}$ & 0.81 \\
\hline Area 1 A4 & $\mathrm{J} 13501$ & $8 / 17 / 06$ & 19100 & & 9.6 & 4.9 & & 0.86 & 4620 & & 2.7 & 362 & & 0.08 & 0.02 & $\mathrm{U}$ & 0.02 & 0.80 & $U$ & 0.80 \\
\hline Area $2 \mathrm{Al}$ & $\mathrm{J} 13502$ & $8 / 17 / 06$ & 18900 & & 9.6 & 3.7 & & 0.85 & 3970 & & 2.7 & 317 & & 0.08 & 0.01 & $\mathrm{U}$ & 0.01 & 0.80 & $\mathrm{U}$ & 0.80 \\
\hline Duplicate of J13502 & $\mathrm{J} 13508$ & $8 / 17 / 06$ & 17800 & & 9.7 & 3.7 & & 0.87 & 3680 & & 2.7 & 314 & & 0.08 & 0.01 & $\mathrm{U}$ & 0.01 & 0.81 & $\mathrm{U}$ & 0.81 \\
\hline Split of $J 13502$ & $J 13505$ & $8 / 17 / 06$ & 23700 & $\mathrm{~N}$ & 2.4 & 4.8 & & 0.15 & 4800 & & 12.2 & 340 & $\mathrm{~N}$ & 0.10 & 0.0067 & $\mathrm{U}$ & 0.0067 & 1.2 & $\mathrm{U}$ & 1.2 \\
\hline Area $2 \mathrm{~A} 2$ & $\mathrm{~J} 13503$ & $8 / 17 / 06$ & 18400 & & 9.7 & 3.8 & & 0.86 & 3760 & & 2.7 & 285 & & 0.08 & 0.01 & $\mathrm{U}$ & 0.01 & 0.80 & $\mathrm{U}$ & 0.80 \\
\hline Area $2 \mathrm{~A} 3$ & $\mathrm{~J} 13506$ & $8 / 17106$ & 15900 & & 9.7 & 4.3 & & 0.86 & 3150 & & 2.7 & 279 & & 0.08 & 0.02 & $\mathrm{U}$ & 0.02 & 0.80 & $\mathrm{U}$ & 0.80 \\
\hline Area 2 A4 & $\mathrm{J} 13507$ & $8 / 17 / 06$ & 19000 & & 9.6 & 3.2 & & 0.85 & 3660 & & 2.7 & 359 & & 0.08 & 0.02 & $\mathrm{U}$ & 0.02 & 0.80 & $\mathrm{U}$ & 0.80 \\
\hline
\end{tabular}


Attachment 1. 118-C-1 Verification Sampling Results.

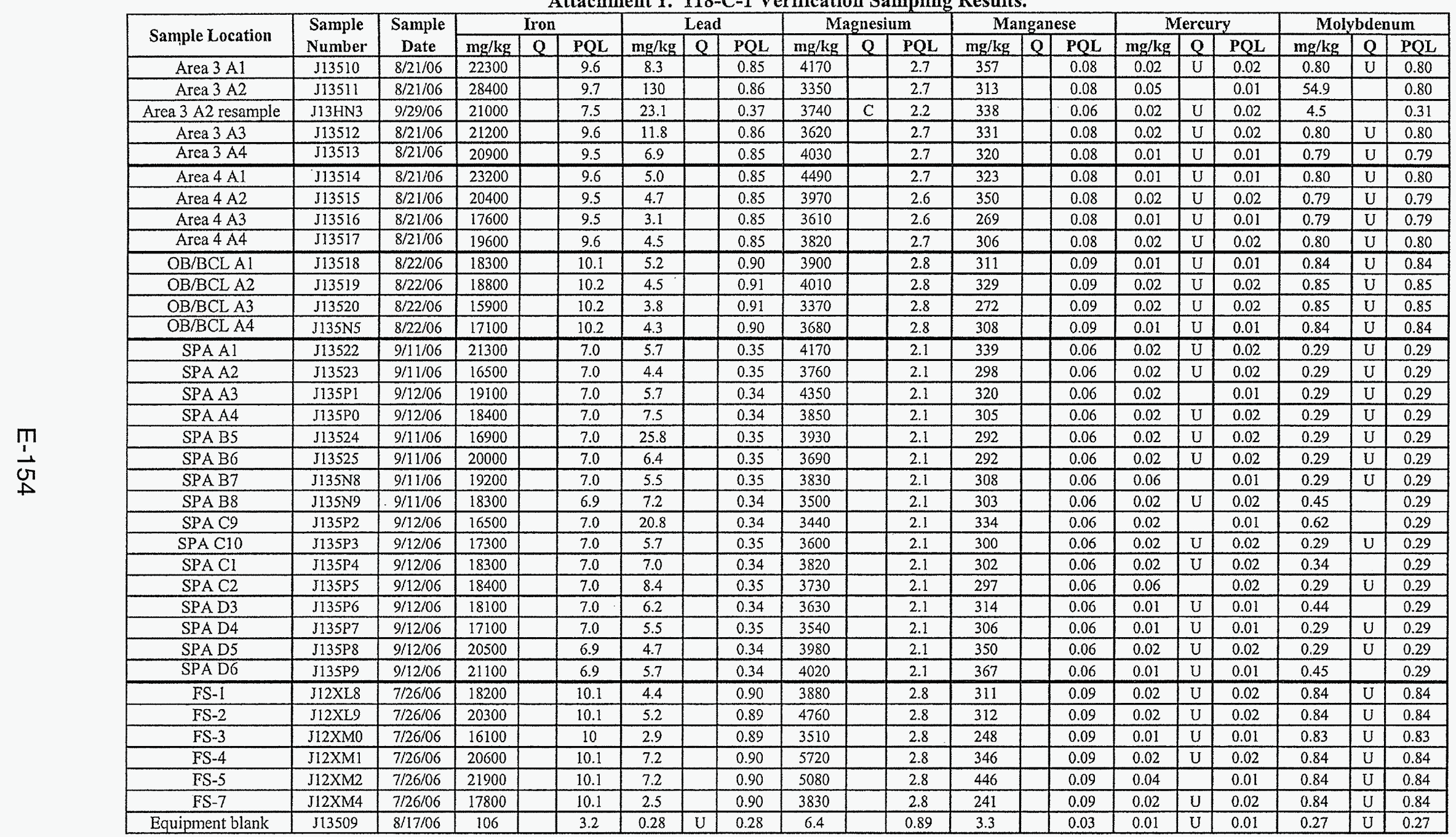

$$
\begin{aligned}
& \text { Attachment } 1 \text { Sheet No. } 12 \text { of } 43 \\
& \begin{array}{lll}
\text { Originator } & & 1 \\
\text { Checked } & \text { Date Capron } & 12 / 13 / 06 \\
\text { T. M. Blakley } & \text { Date } &
\end{array} \\
& \text { Rev. No. }
\end{aligned}
$$


Attachment 1. 118-C-1 Verification Sampling Results.

\begin{tabular}{|c|c|c|c|c|c|c|c|c|c|c|c|c|c|c|c|c|c|c|c|c|}
\hline \multirow{2}{*}{ Sample Location } & \multirow{2}{*}{$\begin{array}{c}\text { Sample } \\
\text { Number }\end{array}$} & \multirow{2}{*}{$\begin{array}{c}\text { Sample } \\
\text { Date }\end{array}$} & \multicolumn{3}{|c|}{ Nickel } & \multicolumn{3}{|c|}{ Potassium } & \multicolumn{3}{|c|}{ Selenium } & \multicolumn{3}{|c|}{ Silicon } & \multicolumn{3}{|c|}{ Silver } & \multicolumn{3}{|c|}{ Sodium } \\
\hline & & & $\mathrm{mg} / \mathrm{kg}$ & $Q$ & PQL & $\mathrm{mg} / \mathrm{kg}$ & $\mathrm{Q}$ & $\mathrm{PQL}$ & $\mathrm{mg} / \mathrm{kg}$ & $Q$ & $\mathbf{P Q L}$ & $\mathrm{mg} / \mathrm{kg}$ & Q & PQL & $\mathrm{mg} / \mathrm{kg}$ & $Q$ & PQL & $\mathrm{mg} / \mathrm{kg}$ & $Q$ & $\mathrm{PQL}$ \\
\hline Area $1 \mathrm{Al}$ & $\mathrm{J} 134 \mathrm{YY}$ & $8 / 17106$ & 8.5 & & 0.66 & 847 & & 6.3 & 1.3 & $\mathrm{UC}$ & 1.3 & 540 & R & 6.3 & 0.19 & $\mathrm{U}$ & 0.19 & 86.3 & & 2.1 \\
\hline Duplicate of J134Y7 & $\mathrm{J} 134 \mathrm{Y9}$ & $8 / 17 / 06$ & 9.5 & & 0.66 & 920 & & 6.2 & 1.3 & UC & 1.3 & 525 & $\mathrm{R}$ & 6.2 & 0.19 & $\mathrm{U}$ & 0.19 & 112 & & 2.1 \\
\hline Split of J134Y7 & $\mathrm{J} 13504$ & $8 / 17 / 06$ & 11.6 & & 0.76 & 1210 & & 50.4 & 1.1 & $\mathrm{~B}^{* \mathrm{C}}$ & 0.17 & 635 & $\mathrm{~N}$ & 4.0 & 0.20 & UN & 0.20 & 166 & & 10.1 \\
\hline Area $1 \mathrm{A2}$ & $\mathrm{J13500}$ & $8 / 17 / 06$ & 10.1 & & 0.66 & 858 & & 6.3 & 1.3 & UC & 1.3 & 550 & $\mathrm{R}$ & 6.3 & 0.19 & $\mathrm{U}$ & 0.19 & 85.3 & & 2.1 \\
\hline Area $1 \mathrm{~A} 3$ & J134Y8 & $8 / 17 / 06$ & 9.9 & & 0.67 & 916 & & 6.3 & 1.3 & $\mathrm{UC}$ & 1.3 & 560 & $\mathrm{R}$ & 6.3 & 0.20 & $\mathrm{U}$ & 0.20 & 122 & & 2.1 \\
\hline Area $1 \mathrm{~A} 4$ & $\mathrm{~J} 13501$ & $8 / 17 / 06$ & 10.2 & & 0.66 & 957 & & 6.3 & 1.3 & $\mathrm{UC}$ & 1.3 & 544 & $\mathrm{R}$ & 6.3 & 0.19 & $\mathrm{U}$ & 0.19 & 143 & & 2.1 \\
\hline Area $2 \mathrm{Al}$ & $\mathrm{J} 13502$ & $8 / 17 / 06$ & 10.4 & & 0.66 & 813 & & 6.3 & 1.3 & UC & 1.3 & 668 & $\mathrm{R}$ & 6.3 & 0.19 & $\mathrm{U}$ & 0.19 & 113 & & 2.1 \\
\hline Duplicate of J13502 & $\mathrm{J} 13508$ & $8 / 17 / 06$ & 10.1 & & 0.67 & 770 & & 6.3 & 1.3 & UC & 1.3 & 559 & $\mathrm{R}$ & 6.3 & 0.20 & $\mathrm{U}$ & 0.20 & 95.7 & & 2.1 \\
\hline Split of J13502 & .113505 & $8 / 17 / 06$ & 13.0 & & 0.76 & 968 & & 50.4 & 0.89 & $\mathrm{~B}^{*} \mathrm{C}$ & 0.17 & 593 & $\mathrm{~N}$ & 4.0 & 0.20 & UN & 0.20 & 171 & & 10.1 \\
\hline Area $2 \mathrm{~A} 2$ & $\mathrm{~J} 13503$ & $8 / 17 / 06$ & 9.2 & & 0.66 & 805 & & 6.3 & 1.3 & UC & 1.3 & 506 & $\mathrm{R}$ & 6.3 & 0.19 & $\mathrm{U}$ & 0.19 & 141 & & 2.1 \\
\hline Area $2 \mathrm{A3}$ & $\mathrm{J} 13506$ & $8 / 17 / 06$ & 7.9 & & 0.66 & 862 & & 6.3 & 1.3 & $\mathrm{UC}$ & 1.3 & 557 & $\mathrm{R}$ & 6.3 & 0.19 & $\mathrm{U}$ & 0.19 & 126 & & 2.1 \\
\hline Area $2 \mathrm{~A} 4$ & $\mathrm{J13507}$ & $8 / 17 / 06$ & 8.7 & & 0.66 & 803 & & 6.2 & 1.3 & $\mathrm{UC}$ & 1.3 & 530 & $\mathrm{R}$ & 6.2 & 0.19 & $\mathrm{U}$ & 0.19 & 127 & & 2.1 \\
\hline Area $3 \mathrm{~A} 1$ & $\mathrm{~J} 13510$ & $8 / 21 / 06$ & 11.0 & & 0.66 & 1120 & & 6.3 & 1.3 & UC & 1.3 & 593 & $\mathrm{R}$ & 6.3 & 0.19 & $\mathrm{U}$ & 0.19 & 141 & & 2.1 \\
\hline Area $3 \mathrm{~A} 2$ & $\mathrm{J13511}$ & $8 / 21 / 06$ & 9.3 & & 0.66 & 1050 & & 6.3 & 1.3 & UC & 1.3 & 612 & \begin{tabular}{l|l}
$R$ \\
\end{tabular} & 6.3 & 0.19 & $\mathrm{U}$ & 0.19 & 190 & & 2.1 \\
\hline Area 3 A2 resample & J13HN3 & $9 / 29 / 06$ & 10.2 & & 0.40 & 1080 & $\mathrm{C}$ & 6.5 & 0.80 & $\mathrm{U}$ & 0.80 & 550 & CI & 1.2 & 0.34 & $\mathrm{U}$ & 0.34 & 123 & $\mathrm{C}$ & 0.65 \\
\hline Area $3 \mathrm{A3}$ & $\mathrm{J} 13512$ & $8 / 21 / 06$ & 12.7 & & 0.66 & 1140 & & 6.3 & 1.3 & $\mathrm{UC}$ & 1.3 & 660 & $R$ & 6.3 & 0.19 & $\mathrm{U}$ & 0.19 & 228 & & 2.1 \\
\hline Area 3 A4 & $J 13513$ & $8 / 21 / 06$ & 9.6 & & 0.66 & 919 & & 6.2 & 1.3 & UC & 1.3 & 465 & $R$ & 6.2 & 0.19 & $\mathrm{U}$ & 0.19 & 143 & & 2.1 \\
\hline Area 4Al & $\mathrm{J} 13514$ & $8 / 21 / 06$ & 10.1 & & 0.66 & 933 & & 6.2 & 1.3 & UC & 1.3 & 439 & $\mathrm{R}$ & 6.2 & 0.19 & $\mathrm{U}$ & 0.19 & 147 & & 2.1 \\
\hline Area 4 A2 & $\mathrm{J13515}$ & $8 / 21 / 06$ & 9.3 & & 0.66 & 873 & & 6.2 & 1.3 & $\mathrm{UC}$ & 1.3 & 516 & $\mathrm{R}$ & 6.2 & 0.19 & $\mathrm{U}$ & 0.19 & 160 & & 2.1 \\
\hline Area 4A3 & $\mathrm{J} 13516$ & $8 / 21 / 06$ & 9.7 & & 0.65 & 774 & & 6.2 & 1.3 & $\mathrm{UC}$ & 1.3 & 527 & R & 6.2 & 0.19 & $\mathrm{U}$ & 0.19 & 113 & & 2.1 \\
\hline Area 4A4 & $J 13517$ & $8 / 21 / 06$ & 8.7 & & 0.66 & 853 & & 6.3 & 1.3 & UC & 1.3 & 534 & $R$ & 6.3 & 0.19 & $\mathrm{U}$ & 0.19 & 114 & & 2.1 \\
\hline $\mathrm{OB} / \mathrm{BCL} \mathrm{Al}$ & $\mathrm{J13518}$ & $8 / 22 / 06$ & 9.0 & & 0.69 & 917 & & 6.6 & 1.4 & $\mathrm{U}$ & 1.4 & 527 & $\mathrm{~J}$ & 6.6 & 0.20 & $\mathrm{U}$ & 0.20 & 110 & & 2.2 \\
\hline $\mathrm{OB} / \mathrm{BCL} \mathrm{A} 2$ & $J 13519$ & $8 / 22 / 06$ & 9.3 & & 0.70 & 960 & & 6.7 & 1.4 & $\mathrm{U}$ & 1.4 & 510 & $\mathrm{~J}$ & 6.7 & 0.21 & $\mathrm{U}$ & 0.21 & 113 & & 2.2 \\
\hline$O B / B C L A 3$ & $\mathrm{J13520}$ & $8 / 22 / 06$ & 9.6 & & 0.70 & 763 & & 6.6 & 1.4 & $\mathrm{U}$ & 1.4 & 540 & $\mathrm{~J}$ & 6.6 & 0.20 & U & 0.20 & 89.3 & & 2.2 \\
\hline $\mathrm{OB} / \mathrm{BCL} \mathrm{A} 4$ & J135N5 & $8 / 22 / 06$ & 9.0 & & 0.70 & 1080 & & 6.6 & 1.4 & $\mathrm{U}$ & 1.4 & 568 & $\mathrm{~J}$ & 6.6 & 0.20 & $\mathrm{U}$ & 0.20 & 104 & & 2.2 \\
\hline SPA Al & $\mathrm{J} 13522$ & $9 / 11 / 06$ & 10.5 & & 0.38 & 886 & & 6.1 & 0.75 & $\mathrm{U}$ & 0.75 & 450 & $\mathrm{~J}$ & 1.2 & 0.32 & $\mathrm{U}$ & 0.32 & 148 & & 0.61 \\
\hline SPA A2 & $\mathrm{J} 13523$ & $9 / 11 / 06$ & 10.1 & & 0.38 & 776 & & 6.1 & 0.75 & $U$ & 0.75 & 373 & $\mathrm{~J}$ & 1.2 & 0.32 & $\mathrm{U}$ & 0.32 & 138 & & 0.61 \\
\hline SPA A3 & $\mathrm{J} 135 \mathrm{P} 1$ & $9 / 12 / 06$ & 10.6 & & 0.37 & 967 & & 6.1 & 0.74 & $\mathrm{U}$ & 0.74 & 366 & $\mathrm{~J}$ & 1.1 & 0.32 & $\mathrm{U}$ & 0.32 & 182 & & 0.60 \\
\hline SPA A4 & $\mathrm{J} 135 \mathrm{PO}$ & $9 / 12 / 06$ & 9.5 & & 0.37 & 932 & & 6.1 & 0.75 & $\mathrm{U}$ & 0.75 & 389 & $\mathrm{~J}$ & 1.1 & 0.32 & $\mathrm{U}$ & 0.32 & 145 & & 0.60 \\
\hline SPA BS & $\mathrm{J} 13524$ & $9 / 11 / 06$ & 11.0 & & 0.37 & 836 & & 6.1 & 0.75 & $\mathrm{U}$ & 0.75 & 417 & $\mathrm{~J}$ & 1.2 & 0.32 & $\mathrm{U}$ & 0.32 & 128 & & 0.61 \\
\hline SPAB6 & $\mathrm{J} 13525$ & $9 / 11 / 06$ & 13.7 & & 0.38 & 991 & & 6.1 & 0.75 & $\mathrm{U}$ & 0.75 & 377 & $\mathrm{~J}$ & 1.2 & 0.32 & $\mathrm{U}$ & 0.32 & 156 & & 0.61 \\
\hline SPA B7 & J135N8 & $9 / 11 / 06$ & 9.7 & & 0.37 & 1100 & & 6.1 & 0.75 & U & 0.75 & 461 & $\mathrm{~J}$ & 1.2 & 0.32 & $U$ & 0.32 & 148 & & 0.61 \\
\hline SPA B8 & J135N9 & $9 / 11 / 06$ & 8.7 & & 0.37 & 1130 & & 6.1 & 0.74 & $\mathrm{U}$ & 0.74 & 415 & $\mathrm{~J}$ & 1.1 & 0.31 & $\mathrm{U}$ & 0.31 & 139 & & 0.60 \\
\hline SPAC9 & $\sqrt{1135 \mathrm{P} 2}$ & $9 / 12 / 06$ & 8.3 & & 0.37 & 1060 & & 6.1 & 0.74 & $\mathrm{U}$ & 0.74 & 632 & $\mathrm{~J}$ & 1.1 & 0.31 & $\mathrm{U}$ & 0.31 & 274 & & 0.60 \\
\hline SPA C10 & $\mathrm{J} 135 \mathrm{P} 3$ & $9 / 12 / 06$ & 8.7 & & 0.38 & 923 & & 6.1 & 0.75 & $\mathrm{U}$ & 0.75 & 406 & $\mathrm{~J}$ & 1.2 & 0.32 & $\mathrm{U}$ & 0.32 & 139 & & 0.61 \\
\hline SPA Cl & $\mathrm{J} 135 \mathrm{P} 4$ & $9 / 12 / 06$ & 9.3 & & 0.37 & 1030 & & 6.1 & 0.74 & $\mathrm{U}$ & 0.74 & 472 & $\mathrm{~J}$ & 1.1 & 0.31 & $\mathrm{U}$ & 0.31 & 111 & & 0.60 \\
\hline SPAC2 & J135P5 & $9 / 12 / 06$ & 9.4 & & 0.38 & 943 & & 6.1 & 0.75 & $\mathrm{U}$ & 0.75 & 435 & $\mathrm{~J}$ & 1.2 & 0.32 & $\mathrm{U}$ & 0.32 & 170 & & 0.61 \\
\hline SPA D3 & J135P6 & $9 / 12 / 06$ & 10.6 & & 0.37 & 997 & & 6.1 & 0.80 & & 0.74 & 530 & $\mathrm{~J}$ & 1.1 & 0.31 & $\mathrm{U}$ & 0.31 & 142 & & 0.60 \\
\hline SPA D4 & J135P7 & $9 / 12 / 06$ & 9.4 & & 0.38 & 967 & & 6.1 & 0.75 & $\mathrm{U}$ & 0.75 & 459 & $\mathrm{~J}$ & 1.2 & 0.32 & $\mathrm{U}$ & 0.32 & 115 & & 0.61 \\
\hline SPA D5 & $\mathrm{J} 135 \mathrm{P} 8$ & $9 / 12 / 06$ & 10.8 & & 0.37 & 1050 & & 6.1 & 0.74 & $\mathrm{U}$ & 0.74 & 584 & $\mathrm{~J}$ & 1.1 & 0.31 & $\mathrm{U}$ & 0.31 & 126 & & 0.60 \\
\hline SPA D6 & ग135P9 & $9 / 12 / 06$ & 10.7 & & 0.37 & 1070 & & 6.1 & 0.74 & $\mathrm{U}$ & 0.74 & 449 & $\mathrm{~J}$ & 1.1 & 0.31 & $\mathrm{U}$ & 0.31 & 165 & & 0.60 \\
\hline
\end{tabular}

\begin{tabular}{|c|c|c|c|}
\hline Attachment & 1 & Sheet No & 13 of 43 \\
\hline Originator & J.M. Capron & Date & $12 / 13 / 06$ \\
\hline Checked & T.M. Blakley & Date & \\
\hline Calc. No. & $0100 \mathrm{C}-\mathrm{CA}-\mathrm{V} 0033$ & Rev. No. & 0 \\
\hline
\end{tabular}


Attachment 1. 118-C-1 Verification Sampling Results.

\begin{tabular}{|c|c|c|c|c|c|c|c|c|c|c|c|c|c|c|c|c|c|c|c|c|}
\hline \multirow{2}{*}{ Sample Location } & \multirow{2}{*}{$\begin{array}{c}\text { Sample } \\
\text { Number }\end{array}$} & \multirow{2}{*}{$\begin{array}{c}\text { Sample } \\
\text { Date }\end{array}$} & \multicolumn{3}{|c|}{ Nickel } & \multicolumn{3}{|c|}{ Potassium } & \multicolumn{3}{|c|}{ Selenium } & \multicolumn{3}{|c|}{ Silicon } & \multicolumn{3}{|c|}{ Silver } & \multicolumn{3}{|c|}{ Sodium } \\
\hline & & & $\mathrm{mg} / \mathrm{kg}$ & $Q$ & PQL & $\mathrm{mg} / \mathrm{kg}$ & $Q$ & PQL & $\mathrm{mg} / \mathrm{kg}$ & $Q$ & PQL & $\mathrm{mg} / \mathrm{kg}$ & $Q$ & PQL & $\mathrm{mg} / \mathrm{kg}$ & $Q$ & PQL & $\mathrm{mg} / \mathrm{kg}$ & $Q$ & PQL \\
\hline FS-1 & J12XL8 & $7 / 26 / 06$ & 10.7 & & 0.69 & 1180 & & 6.6 & 1.4 & $\mathrm{U}$ & 1.4 & 597 & $\mathrm{~J}$ & 6.6 & 0.20 & $\mathrm{U}$ & 0.20 & 105 & & 2.2 \\
\hline FS-2 & J12XL9 & $7 / 26 / 06$ & 10.1 & & 0.69 & 828 & & 6.6 & 1.4 & $\mathrm{U}$ & 1.4 & 564 & $\mathrm{~J}$ & 6.6 & 0.20 & $\mathrm{U}$ & 0.20 & 122 & & 2.2 \\
\hline FS-3 & J12XMO & $7 / 26 / 06$ & 9.6 & & 0.69 & 619 & & 6.5 & 1.3 & $\mathrm{U}$ & 1.3 & 618 & $\mathrm{~J}$ & 6.5 & 0.20 & $\mathrm{U}$ & 0.20 & 101 & & 2.2 \\
\hline FS-4 & $\mathrm{J} 12 \mathrm{XM1}$ & $7 / 26 / 06$ & 11.1 & & 0.70 & 1120 & & 6.6 & 1.4 & $\mathrm{U}$ & 1.4 & 670 & $\mathrm{~J}$ & 6.6 & 0.20 & $\mathrm{U}$ & 0.20 & 185 & & 2.2 \\
\hline FS-5 & $\mathrm{J} 12 \mathrm{XM2}$ & $7 / 26 / 06$ & 14.1 & & 0.69 & 1640 & & 6.6 & 1.4 & $\mathrm{U}$ & 1.4 & 697 & $\mathrm{~J}$ & 6.6 & 0.20 & $\mathrm{U}$ & 0.20 & 153 & & 2.2 \\
\hline FS-7 & J12XM4 & $7 / 26 / 06$ & 9.9 & & 0.70 & 573 & & 6.6 & 1.4 & $\mathrm{U}$ & 1.4 & 533 & $\mathrm{~J}$ & 6.6 & 0.20 & $\mathrm{U}$ & 0.20 & 135 & & 2.2 \\
\hline Equipment blank & $J 13509$ & $8 / 17 / 06$ & 0.26 & & 0.22 & 16.6 & & 2.1 & 0.43 & $\mathrm{UC}$ & 0.43 & 30.7 & $\mathrm{R}$ & 2.1 & 0.06 & $\mathrm{U}$ & 0.06 & 7.1 & & 0.70 \\
\hline
\end{tabular}

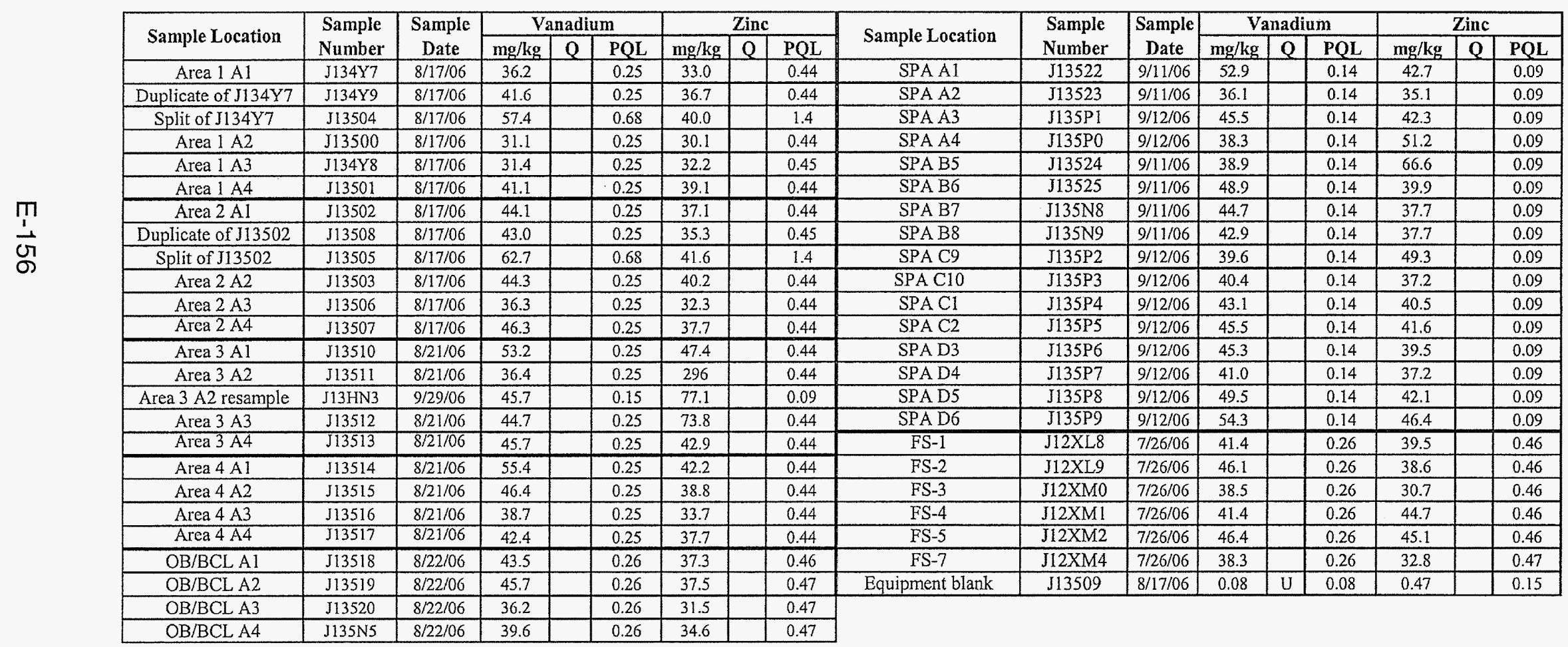

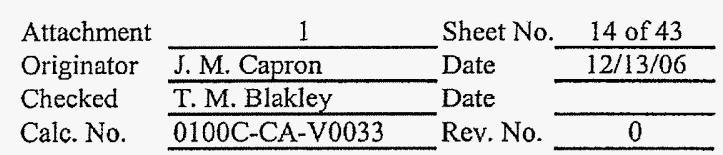


Attachment 1. 118-C-1 Verification Sampling Results.

\begin{tabular}{|c|c|c|c|c|c|}
\hline \multirow[t]{2}{*}{ Sample Location } & \multirow{2}{*}{$\begin{array}{l}\text { Sample } \\
\text { Number }\end{array}$} & \multirow{2}{*}{$\begin{array}{c}\text { Sample } \\
\text { Date }\end{array}$} & \multicolumn{3}{|c|}{$\begin{array}{l}\text { Total Petroleum } \\
\text { Hydrocarbons }\end{array}$} \\
\hline & & & $\mathrm{mg} / \mathrm{kg}$ & $Q$ & $\mathrm{PQL}$ \\
\hline Area $2 \mathrm{A1}$ & J135R0 & $8 / 28 / 06$ & 137 & $\mathrm{U}$ & 137 \\
\hline Duplicate of J135R0 & $\mathrm{J} 135 \mathrm{~T} 6$ & $8 / 28 / 06$ & 141 & $\mathrm{U}$ & 141 \\
\hline Split of J135R0 & J135T7 & $8 / 28 / 06$ & 173 & UN & 173 \\
\hline Area $2 \mathrm{~A} 2$ & $\mathrm{~J} 135 \mathrm{R} 1$ & $8 / 28 / 06$ & 137 & $\mathrm{U}$ & 137 \\
\hline Area $2 \mathrm{~A}^{3}$ & $\mathrm{~J} 135 \mathrm{R} 2$ & $8 / 28 / 06$ & 139 & $\mathrm{U}$ & 139 \\
\hline Area 2 A4 & $\mathrm{J} 135 \mathrm{R} 3$ & $8 / 28 / 06$ & 149 & $\mathrm{U}$ & 149 \\
\hline Area 3 A1 & $\mathrm{J} 135 \mathrm{R} 4$ & $8 / 28 / 06$ & 135 & $U$ & 135 \\
\hline Area 3 A2 & J135R5 & $8 / 28 / 06$ & 133 & $\mathrm{U}$ & 133 \\
\hline Area $3 \mathrm{~A} 3$ & J135R6 & $8 / 28 / 06$ & 135 & $\mathrm{U}$ & 135 \\
\hline Area 3 A4 & J135R7 & $8 / 28 / 06$ & 348 & & 133 \\
\hline Area 3 A4 resample & J13HN4 & $9 / 29 / 06$ & 135 & $U$ & 135 \\
\hline Area 4Al & J135R8 & $8 / 28 / 06$ & 143 & $\mathrm{U}$ & 143 \\
\hline Area 4 A2 & $\mathrm{J} 135 \mathrm{R} 9$ & $8 / 28 / 06$ & 133 & $\mathrm{U}$ & 133 \\
\hline Area 4 A3 & $\mathrm{J} 135 \mathrm{TO}$ & $8 / 28 / 06$ & 144 & $\mathrm{U}$ & 144 \\
\hline Area 4A4 & $\mathrm{J} 135 \mathrm{~T} 1$ & $8 / 28 / 06$ & 142 & $\mathrm{U}$ & 142 \\
\hline OB/BCL $A 1$ & J135T2 & $8 / 28 / 06$ & 133 & $\mathrm{U}$ & 133 \\
\hline $\mathrm{OB} / \mathrm{BCL} \mathrm{A} 2$ & $\mathrm{J135T3}$ & $8 / 28 / 06$ & 146 & $\mathrm{U}$ & 146 \\
\hline $\mathrm{OB} / \mathrm{BCL} \mathrm{A3}$ & $\mathrm{J} 135 \mathrm{~T} 4$ & $8 / 28 / 06$ & 138 & $\mathrm{U}$ & 138 \\
\hline$\overline{\mathrm{OB} / \mathrm{BCL} \mathrm{A} 4}$ & J135T5 & $8 / 28 / 06$ & 133 & $\mathrm{U}$ & 133 \\
\hline
\end{tabular}

\begin{tabular}{|c|c|c|c|c|c|}
\hline \multirow[t]{2}{*}{ Sample Location } & \multirow{2}{*}{$\begin{array}{l}\text { Sample } \\
\text { Number }\end{array}$} & \multirow{2}{*}{$\begin{array}{c}\text { Sample } \\
\text { Date }\end{array}$} & \multicolumn{3}{|c|}{$\begin{array}{l}\text { Total Petroleum } \\
\text { Hydrocarbons }\end{array}$} \\
\hline & & & $\mathrm{mg} / \mathrm{kg}$ & $Q$ & PQL \\
\hline SPA Al & $\mathrm{J} 13522$ & $9 / 11 / 06$ & 134 & $\mathrm{U}$ & 134 \\
\hline SPAA2 & $\mathrm{J13523}$ & $9 / 11 / 06$ & 132 & $\mathrm{U}$ & 132 \\
\hline SPA A3 & $\mathrm{J} 135 \mathrm{P} 1$ & $9 / 12 / 06$ & 133 & UC & 133 \\
\hline SPA A4 & J135P0 & $9 / 12 / 06$ & 135 & $\mathrm{CJ}$ & 134 \\
\hline SPA B5 & $\mathrm{J} 13524$ & $9 / 11 / 06$ & 132 & U & 132 \\
\hline SPA B6 & $\mathrm{J} 13525$ & $9 / 11 / 06$ & 133 & $\mathrm{U}$ & 133 \\
\hline SPAB7 & $\mathrm{J135N8}$ & $9 / 11106$ & 133 & $\mathrm{U}$ & 133 \\
\hline SPAB 8 & J135N9 & $9 / 11 / 06$ & 133 & $\mathrm{U}$ & 133 \\
\hline SPA C9 & $\mathrm{J} 135 \mathrm{P} 2$ & $9 / 12 / 06$ & 132 & UC & 132 \\
\hline$\overline{S P A C l 0}$ & $\mathrm{~J} 135 \mathrm{P} 3$ & $9 / 12 / 06$ & 133 & UC & 133 \\
\hline SPACl & J135P4 & $9 / 12 / 06$ & 133 & UC & 133 \\
\hline SPA C2 & J135P5 & $9 / 12 / 06$ & 133 & $\mathrm{UC}$ & 133 \\
\hline SPAD3 & $\mathrm{J} 135 \mathrm{P} 6$ & $9 / 12 / 06$ & 133 & $\mathrm{UC}$ & 133 \\
\hline SPAD4 & $\mathrm{J135 \textrm {P } 7}$ & $9 / 12 / 06$ & 146 & $\mathrm{CJ}$ & 134 \\
\hline SPA D5 & J135P8 & $9 / 12 / 06$ & 133 & UC & 133 \\
\hline SPAD6 & J135P9 & $9 / 12 / 06$ & 133 & UC & 133 \\
\hline FS-5 & J12XM2 & $7 / 26 / 06$ & 133 & $\mathrm{U}$ & 133 \\
\hline FS-6 & J12XM3 & $7 / 26 / 06$ & 135 & $\mathrm{U}$ & 135 \\
\hline
\end{tabular}

\begin{tabular}{lll} 
Attachment & \multicolumn{1}{c}{ Sheet No. } & 15 of 43 \\
Originator & Date & $12 / 13 / 06$ \\
Checked & T.M. Capron &
\end{tabular}

$\begin{array}{ll}\text { Checked } & \text { T.M. Blakley } \\ \text { Calc. No. } & \text { 0100C-CA-V0033 Rev. No. }\end{array}$ 
Attachment 1. 118-C-1 Verification Sampling Results.

\begin{tabular}{|c|c|c|c|c|c|c|c|c|c|c|c|c|}
\hline \multirow[t]{2}{*}{ Constituents } & \multicolumn{3}{|c|}{\begin{tabular}{|c|}
$\mathrm{J13502}$ \\
Area $2 \mathrm{A1}$ \\
Sample Date 8/17/06 \\
\end{tabular}} & \multicolumn{3}{|c|}{\begin{tabular}{|c|}
$\mathbf{J 1 3 5 0 8}$ \\
Duplicate of $\mathbf{J 1 3 5 0 2}$ \\
Sample Date 8/17/06 \\
\end{tabular}} & \multicolumn{3}{|c|}{$\begin{array}{c}\mathrm{J13505} \\
\text { Split of } \mathbf{J 1 3 5 0 2} \\
\text { Sample Date 8/17/06 } \\
\end{array}$} & \multicolumn{3}{|c|}{$\begin{array}{c}\mathbf{J 1 3 5 0 3} \\
\text { Area } 2 \text { A2 } \\
\text { Sample Date 8/17/06 }\end{array}$} \\
\hline & $\mu \mathrm{g} / \mathrm{kg}$ & $\mathbf{Q}$ & PQL & $\mu \mathrm{g} / \mathrm{kg}$ & $\mathbf{Q}$ & PQL & $\mu \mathrm{g} / \mathrm{kg}$ & $\mathbf{Q}$ & PQL & $\mu \mathrm{g} / \mathrm{kg}$ & $\mathbf{Q}$ & PQL \\
\hline \multicolumn{13}{|c|}{ Polychlorinated Biphenyls } \\
\hline Aroclor-1016 & 13 & $\mathrm{U}$ & 13 & 14 & $\mathrm{U}$ & 14 & 17 & $\mathrm{U}$ & 17 & 13 & $\mathrm{U}$ & 13 \\
\hline Aroclor-1221 & 13 & $\mathrm{U}$ & 13 & 14 & $\mathrm{U}$ & 14 & 17 & $\mathrm{U}$ & 17 & 13 & $\mathrm{U}$ & 13 \\
\hline Aroclor-1232 & 13 & $\mathrm{U}$ & 13 & 14 & $\bar{U}$ & 14 & 17 & $\mathrm{U}$ & 17 & 13 & $\mathrm{U}$ & 13 \\
\hline Aroclor 1242 & 13 & $\mathrm{U}$ & 13 & 14 & $\mathrm{U}$ & 14 & 17 & U & 17 & 13 & $\mathrm{U}$ & 13 \\
\hline Aroclor-1248 & 13 & $\mathrm{U}$ & 13 & 14 & $\mathrm{U}$ & 14 & 17 & $\mathrm{U}$ & 17 & 13 & $\mathrm{U}$ & 13 \\
\hline Aroclor- 1254 & 13 & $\mathrm{U}$ & 13 & 14 & $\mathrm{U}$ & 14 & 17 & $\mathrm{U}$ & 17 & 13 & $\mathrm{U}$ & 13 \\
\hline Aroclor- 1260 & 13 & $\mathrm{U}$ & 13 & 14 & $\mathrm{U}$ & 14 & 17 & U & 17 & 13 & U & 13 \\
\hline \multicolumn{13}{|c|}{ Semivolatile Organic Compounds } \\
\hline 1,2,4-Trichlorobenzene & 340 & $\mathrm{U}$ & 340 & 340 & $\mathrm{U}$ & 340 & 330 & $\bar{U}$ & 330 & 340 & $\bar{U}$ & 340 \\
\hline 1,2-Dichlorobenzene & 340 & $\bar{U}$ & 340 & 340 & $\bar{U}$ & 340 & 330 & $\bar{U}$ & 330 & 340 & $U$ & 340 \\
\hline 1,3-Dichlorobenzene & 340 & $\mathrm{U}$ & 340 & 340 & $\mathrm{U}$ & 340 & 330 & $\mathrm{U}$ & 330 & 340 & $\mathrm{U}$ & 340 \\
\hline 1,4-Dichlorobenzene & 340 & $\mathrm{U}$ & 340 & 340 & $\mathrm{U}$ & 340 & 330 & $\mathrm{U}$ & 330 & 340 & $\mathrm{U}$ & 340 \\
\hline 2,4,5-Trichlorophenol & 840 & $\mathrm{U}$ & 840 & 840 & $\mathrm{U}$ & 840 & 330 & $\mathrm{U}$ & 330 & 840 & $\mathrm{U}$ & 840 \\
\hline 2,4,6-Trichlorophenol & 340 & $\mathrm{U}$ & 340 & 340 & $\mathrm{U}$ & 340 & 330 & $\mathrm{U}$ & 330 & 340 & $\mathrm{U}$ & 340 \\
\hline 2,4-Dichlorophenol & 340 & $\mathrm{U}$ & 340 & 340 & $\mathrm{U}$ & 340 & 330 & $\mathrm{U}$ & 330 & 340 & $\mathrm{U}$ & 340 \\
\hline 2,4-Dimethylphenol & 340 & $\mathrm{U}$ & 340 & 340 & $\mathrm{U}$ & 340 & 330 & $\mathrm{U}$ & 330 & 340 & $\mathrm{U}$ & 340 \\
\hline 2,4-Dinitrophenol & 840 & $\mathrm{U}$ & 840 & 840 & $\mathrm{U}$ & 840 & 1600 & UNR & 1600 & 840 & U & 840 \\
\hline 2,4-Dinitrotoluene & 340 & $\mathrm{U}$ & 340 & 340 & $\mathrm{U}$ & 340 & 330 & $\mathrm{U}$ & 330 & 340 & $\mathrm{U}$ & 340 \\
\hline 2,6-Dinitrotoluene & 340 & $\mathrm{U}$ & 340 & 340 & $\mathrm{U}$ & 340 & 330 & $\mathrm{U}$ & 330 & 340 & $\mathrm{U}$ & 340 \\
\hline 2-Chloronaphthalene & 340 & $\mathrm{U}$ & 340 & 340 & $\mathrm{U}$ & 340 & 330 & $\mathrm{U}$ & 330 & 340 & $\mathrm{U}$ & 340 \\
\hline 2-Chlorophenol & 340 & U & 340 & 340 & $\mathrm{U}$ & 340 & 330 & $\mathrm{U}$ & 330 & 340 & $\mathrm{U}$ & 340 \\
\hline 2-Methylnaphthalene & 340 & $\mathrm{U}$ & 340 & 340 & $\mathrm{U}$ & 340 & 330 & $\mathrm{U}$ & 330 & 340 & $\mathrm{U}$ & 340 \\
\hline 2-Methylphenol (cresol, o-) & 340 & $\mathrm{U}$ & 340 & 340 & $\mathrm{U}$ & 340 & 330 & $\mathrm{U}$ & 330 & 340 & $\mathrm{U}$ & 340 \\
\hline 2-Nitroaniline & 840 & $\mathrm{U}$ & 840 & 840 & U & 840 & 1600 & $\mathrm{U}$ & 1600 & 840 & $\mathrm{U}$ & 840 \\
\hline 2-Nitrophenol & 340 & $\mathrm{U}$ & 340 & 340 & U & 340 & 330 & $U$ & 330 & 340 & $\mathrm{U}$ & 340 \\
\hline 3,3'-Dichlorobenzidine & 340 & $\mathrm{U}$ & 340 & 340 & $\mathrm{U}$ & 340 & 1600 & $\mathrm{U}$ & 1600 & 340 & $\mathrm{U}$ & 340 \\
\hline 3-Nitroaniline & 840 & $\mathrm{U}$ & 840 & 840 & $\mathrm{U}$ & 840 & 1600 & $\mathrm{U}$ & 1600 & 840 & $\mathrm{U}$ & 840 \\
\hline 4,6-Dinitro-2-methylphenol & 840 & $\mathrm{U}$ & 840 & 840 & $\mathrm{U}$ & 840 & 1600 & $\mathrm{U}$ & 1600 & 840 & $\mathrm{U}$ & 840 \\
\hline 4-Bromophenyl-phenylether & 340 & $\mathrm{U}$ & 340 & 340 & $\mathrm{U}$ & 340 & 330 & $U$ & 330 & 340 & $\mathrm{U}$ & 340 \\
\hline 4-Chloro-3-methylphenol & 340 & $\mathrm{U}$ & 340 & 340 & $\mathrm{U}$ & 340 & 330 & $\mathrm{U}$ & 330 & 340 & $\mathrm{U}$ & 340 \\
\hline 4-Chloroaniline & 340 & U & 340 & 340 & $\mathrm{U}$ & 340 & 330 & $\mathrm{U}$ & 330 & 340 & $\mathrm{U}$ & 340 \\
\hline 4-Chlorophenyl-phenylether & 340 & $\mathrm{U}$ & 340 & 340 & $\mathrm{U}$ & 340 & 330 & $\mathrm{U}$ & 330 & 340 & $\mathrm{U}$ & 340 \\
\hline 4-Methylphenol (p-cresol) & 340 & $\mathrm{U}$ & 340 & 340 & $\mathrm{U}$ & 340 & 670 & $\mathrm{U}$ & 670 & 340 & $U$ & 340 \\
\hline 4-Nitroaniline & 840 & $\mathrm{U}$ & 840 & 840 & $\mathrm{U}$ & 840 & 1600 & $\mathrm{U}$ & 1600 & 840 & $\mathrm{U}$ & 840 \\
\hline 4-Nitrophenol & 840 & $\mathrm{U}$ & 840 & 840 & $\mathrm{U}$ & 840 & 1600 & $\mathrm{U}$ & 1600 & 840 & U & 840 \\
\hline Acenaphthene & 340 & $\mathrm{U}$ & 340 & 340 & $\mathrm{U}$ & 340 & 330 & $\mathrm{U}$ & 330 & 340 & $\mathrm{U}$ & 340 \\
\hline Acenaphthylene & 340 & $\mathrm{U}$ & 340 & 340 & $\mathrm{U}$ & 340 & 330 & $\mathrm{U}$ & 330 & 340 & $\mathrm{U}$ & 340 \\
\hline Anthracene & 340 & $\mathrm{U}$ & 340 & 340 & $\mathrm{U}$ & 340 & 330 & $\mathrm{U}$ & 330 & 340 & $\mathrm{U}$ & 340 \\
\hline Benzo(a)anthracene & 340 & $\mathrm{U}$ & 340 & 340 & $\mathrm{U}$ & 340 & 330 & $\mathrm{U}$ & 330 & 340 & $\mathrm{U}$ & 340 \\
\hline Benzo(a)pyrene & 340 & $\mathrm{U}$ & 340 & 340 & $\mathrm{U}$ & 340 & 330 & $\mathrm{U}$ & 330 & 340 & $\mathrm{U}$ & 340 \\
\hline Benzo(b)fluoranthene & 340 & $\mathrm{U}$ & 340 & 340 & $\mathrm{U}$ & 340 & 330 & $\mathrm{U}$ & 330 & 340 & $\mathrm{U}$ & 340 \\
\hline Benzo(g,h,i)perylene & 340 & $\mathrm{U}$ & 340 & 340 & $\mathrm{U}$ & 340 & 330 & $\mathrm{U}$ & 330 & 340 & $\mathrm{U}$ & 340 \\
\hline Benzo(k)fluoranthene & 340 & $\mathrm{U}$ & 340 & 340 & $\mathrm{U}$ & 340 & 330 & $\mathrm{U}$ & 330 & 340 & $\mathrm{U}$ & 340 \\
\hline bis(2-Chloro-1-methylethyl)ether & 340 & $\mathrm{U}$ & 340 & 340 & $\mathrm{U}$ & 340 & 330 & $\mathrm{U}$ & 330 & 340 & $\mathrm{U}$ & 340 \\
\hline bis(2-Chloroethoxy)methane & 340 & $\mathrm{U}$ & 340 & 340 & $\mathrm{U}$ & 340 & 330 & $\mathrm{U}$ & 330 & 340 & $\mathrm{U}$ & 340 \\
\hline bis(2-Chloroethyl)ether & 340 & $\mathrm{U}$ & 340 & 340 & $\mathrm{U}$ & 340 & 330 & $\mathrm{U}$ & 330 & 340 & $\mathrm{U}$ & 340 \\
\hline bis(2-Ethylhexyl)phthalate & 38 & $\mathrm{JB}$ & 340 & 36 & JB & 340 & 330 & $\mathrm{U}$ & 330 & 38 & $\sqrt{B}$ & 340 \\
\hline
\end{tabular}

Attachment

Originator

Checked

Calc. No.

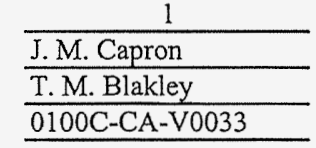

Sheet No. 16 of 43

Date $12 / 13 / 06$

Date

Rev. No. 0 
Attachment 1. 118-C-1 Verification Sampling Results.

\begin{tabular}{|c|c|c|c|c|c|c|c|c|c|c|c|c|}
\hline \multirow[t]{2}{*}{ Constituents } & \multicolumn{3}{|c|}{$\begin{array}{c}\mathrm{J} 13502 \\
\text { Area } 2 \text { A1 } \\
\text { Sample Date 8/17/06 }\end{array}$} & \multicolumn{3}{|c|}{$\begin{array}{c}\mathrm{J13508} \\
\text { Duplicate of } \mathrm{J13502} \\
\text { Sample Date 8/17/06 }\end{array}$} & \multicolumn{3}{|c|}{$\begin{array}{c}\mathrm{J13505} \\
\text { Split of } \mathbf{J 1 3 5 0 2} \\
\text { Sample Date 8/17/06 }\end{array}$} & \multicolumn{3}{|c|}{\begin{tabular}{|c|}
$\mathrm{J13503}$ \\
Area $2 \mathrm{A2}$ \\
Sample Date 8/17/06 \\
\end{tabular}} \\
\hline & $\mu \mathrm{g} / \mathrm{kg}$ & $\mathbf{Q}$ & PQL & $\mu \mathrm{g} / \mathrm{kg}$ & $Q$ & PQL & $\mu \mathrm{g} / \mathrm{kg}$ & $\mathbf{Q}$ & MDL & $\mu \mathrm{g} / \mathrm{kg}$ & $\mathbf{Q}$ & PQL \\
\hline \multicolumn{13}{|c|}{ Semivolatile Organic Compounds (continued) } \\
\hline Butylbenzylphthalate & 340 & $\mathrm{U}$ & 340 & 340 & $\mathrm{U}$ & 340 & 330 & $\mathrm{U}$ & 330 & 340 & $\mathrm{U}$ & 340 \\
\hline Carbazole & 340 & $\mathrm{U}$ & 340 & 340 & $\mathrm{U}$ & 340 & 330 & $\mathrm{U}$ & 330 & 340 & $\mathrm{U}$ & 340 \\
\hline Chrysene & 340 & $\mathrm{U}$ & 340 & 340 & $\mathrm{U}$ & 340 & 330 & $\mathrm{U}$ & 330 & 340 & $\bar{U}$ & 340 \\
\hline Di-n-butylphthalate & 22 & $\mathrm{JB}$ & 340 & 35 & $\sqrt{\mathrm{B}}$ & 340 & 330 & $\mathrm{U}$ & 330 & 340 & $\mathrm{U}$ & 340 \\
\hline Di-n-octylphthalate & 340 & $\mathrm{U}$ & 340 & 340 & $\mathrm{U}$ & 340 & 330 & $\mathrm{U}$ & 330 & 340 & $\mathrm{U}$ & 340 \\
\hline Dibenz(a,h)anthracene & 340 & $\mathrm{U}$ & 340 & 340 & $\mathrm{U}$ & 340 & 330 & $\mathrm{U}$ & 330 & 340 & $\mathrm{U}$ & 340 \\
\hline Dibenzofuran & 340 & $\mathrm{U}$ & 340 & 340 & $\mathrm{U}$ & 340 & 330 & $\mathrm{U}$ & 330 & 340 & $\mathrm{U}$ & 340 \\
\hline Diethylphthalate & 340 & $\mathrm{U}$ & 340 & 340 & $\mathrm{U}$ & 340 & 330 & $U$ & 330 & 340 & $\mathrm{U}$ & 340 \\
\hline Dimethylphthalate & 340 & $\mathrm{U}$ & 340 & 340 & $\mathrm{U}$ & 340 & 330 & $\mathrm{U}$ & 330 & 340 & $\mathrm{U}$ & 340 \\
\hline Fluoranthene & 340 & $\mathrm{U}$ & 340 & 340 & $\mathrm{U}$ & 340 & 330 & $\mathrm{U}$ & 330 & 340 & $\mathrm{U}$ & 340 \\
\hline Fluorene & 340 & $\mathrm{U}$ & 340 & 340 & $\mathrm{U}$ & 340 & 330 & $\mathrm{U}$ & 330 & 340 & $\mathrm{U}$ & 340 \\
\hline Hexachlorobenzene & 340 & $\mathrm{U}$ & 340 & 340 & $\mathrm{U}$ & 340 & 330 & $\mathrm{U}$ & 330 & 340 & $\mathrm{U}$ & 340 \\
\hline Hexachlorobutadiene & 340 & $\mathrm{U}$ & 340 & 340 & $\mathrm{U}$ & 340 & 330 & $\mathrm{U}$ & 330 & 340 & $\mathrm{U}$ & 340 \\
\hline Hexachlorocyclopentadiene & 340 & $\mathrm{U}$ & 340 & 340 & $\mathrm{U}$ & 340 & 1600 & $\mathrm{U}$ & 1600 & 340 & $\mathrm{U}$ & 340 \\
\hline Hexachloroethane & 340 & $\mathrm{U}$ & 340 & 340 & $\mathrm{U}$ & 340 & 330 & $\mathrm{U}$ & 330 & 340 & $\mathrm{U}$ & 340 \\
\hline Indeno(1,2,3-cd)pyrene & 340 & $\mathrm{U}$ & 340 & 340 & $\mathrm{U}$ & 340 & 330 & $\mathrm{U}$ & 330 & 340 & $\mathrm{U}$ & 340 \\
\hline Isophorone & 340 & $\mathrm{U}$ & 340 & 340 & $\mathrm{U}$ & 340 & 330 & $\mathrm{U}$ & 330 & 340 & $\mathrm{U}$ & 340 \\
\hline N-Nitroso-di-n-dipropylamine & 340 & $\mathrm{U}$ & 340 & 340 & $\mathrm{U}$ & 340 & 330 & $\mathrm{U}$ & 330 & 340 & $\mathrm{U}$ & 340 \\
\hline N-Nitrosodiphenylamine & 340 & $\mathrm{U}$ & 340 & 340 & $\mathrm{U}$ & 340 & 330 & $\mathrm{U}$ & 330 & 340 & $\mathrm{U}$ & 340 \\
\hline Naphthalene & 340 & $\mathrm{U}$ & 340 & 340 & $\mathrm{U}$ & 340 & 330 & $\mathrm{U}$ & 330 & 340 & $\mathrm{U}$ & 340 \\
\hline Nitrobenzene & 340 & $\mathrm{U}$ & 340 & 340 & $\mathrm{U}$ & 340 & 330 & $\mathrm{U}$ & 330 & 340 & $\mathrm{U}$ & 340 \\
\hline Pentachlorophenol & 840 & $\mathrm{U}$ & 840 & 840 & $\mathrm{U}$ & 840 & 1600 & $\mathrm{U}$ & 1600 & 840 & $\mathrm{U}$ & 840 \\
\hline Phenanthrene & 340 & $\mathrm{U}$ & 340 & 340 & $\mathrm{U}$ & 340 & 330 & $\mathrm{U}$ & 330 & 340 & $\mathrm{U}$ & 340 \\
\hline Phenol & 340 & $\mathrm{U}$ & 340 & 340 & $\mathrm{U}$ & 340 & 330 & $\mathrm{U}$ & 330 & 340 & $\mathrm{U}$ & 340 \\
\hline Pyrene & 340 & $\mathrm{U}$ & 340 & 340 & $\mathrm{U}$ & 340 & 330 & $\mathrm{U}$ & 330 & 340 & $\mathrm{U}$ & 340 \\
\hline & & & & Attachm & & & 1 & & & et No. & & of 43 \\
\hline & & & & Originat & & J.M. Ca & ron & & & Date & & $13 / 06$ \\
\hline & & & & Checked & & T.M.BI & kley & & & Date & & \\
\hline & & & & Calc. Nc & & $0100 \mathrm{C}-\mathrm{C}$ & $\mathrm{A}-\mathrm{V} 0033$ & & & Rev. No. & & 0 \\
\hline
\end{tabular}


Attachment 1. 118-C-1 Verification Sampling Results.

\begin{tabular}{|c|c|c|c|c|c|c|c|c|c|c|c|c|}
\hline \multirow[t]{2}{*}{ Constituents } & \multicolumn{3}{|c|}{$\begin{array}{c}\text { J135R0 } \\
\text { Area } 2 \text { A1 } \\
\text { Sample Date } 8 / 28 / 06\end{array}$} & \multicolumn{3}{|c|}{$\begin{array}{c}\text { J135T6 } \\
\text { Duplicate of J135R0 } \\
\text { Sample Date } 8 / 28 / 06\end{array}$} & \multicolumn{3}{|c|}{$\begin{array}{c}\text { J135T7 } \\
\text { Split of J135R0 } \\
\text { Sample Date 8/28/06 }\end{array}$} & \multicolumn{3}{|c|}{$\begin{array}{c}\text { J135R1 } \\
\text { Area } 2 \text { A2 } \\
\text { Sample Date } 8 / 28 / 06\end{array}$} \\
\hline & $\mu \mathrm{g} / \mathrm{kg}$ & $\mathbf{Q}$ & PQL & $\mu \mathrm{g} / \mathrm{kg}$ & $\mathbf{Q}$ & PQL & $\mu \mathrm{g} / \mathrm{kg}$ & $\mathbf{Q}$ & MDL & $\mu \mathrm{g} / \mathrm{kg}$ & $\mathbf{Q}$ & PQL \\
\hline \multicolumn{13}{|c|}{ Volatile Organic Compounds } \\
\hline 1,1,1-Trichloroethane & 5 & $\mathrm{U}$ & 5 & 5 & $\mathrm{U}$ & 5 & 5.1 & $\mathrm{U}$ & 5.1 & 5 & $\mathrm{U}$ & 5 \\
\hline 1,1,2,2-Tetrachloroethane & 5 & UJ & 5 & 5 & UJ & 5 & 5.1 & U & 5.1 & 5 & UJ & 5 \\
\hline 1,1,2-Trichloroethane & 5 & $\mathrm{U}$ & 5 & 5 & $\mathrm{U}$ & 5 & 5.1 & U & 5.1 & 5 & $\mathrm{U}$ & 5 \\
\hline 1,1-Dichloroethane & 5 & $\mathrm{U}$ & 5 & 5 & $U$ & 5 & 5.1 & $\mathrm{U}$ & 5.1 & 5 & $\mathrm{U}$ & 5 \\
\hline 1,1-Dichloroethene & 5 & $\mathrm{U}$ & 5 & 5 & $\mathrm{U}$ & 5 & 5.1 & $\mathrm{U}$ & 5.1 & 5 & $\mathrm{U}$ & 5 \\
\hline 1,2-Dichloroethane & 5 & $\mathrm{U}$ & 5 & 5 & $\mathrm{U}$ & 5 & 5.1 & $\mathrm{U}$ & 5.1 & 5 & U & 5 \\
\hline 1,2-Dichloroethene (total) & 5 & $\mathrm{U}$ & 5 & 5 & $\mathrm{U}$ & 5 & 10 & $\mathrm{U}$ & 10 & 5 & $\mathrm{U}$ & 5 \\
\hline 1,2-Dichloropropane & 5 & $\mathrm{U}$ & 5 & 5 & $\mathrm{U}$ & 5 & 5.1 & $\mathrm{U}$ & 5.1 & 5 & $\mathrm{U}$ & 5 \\
\hline 2-Butanone & 9 & $\mathrm{U}$ & 9 & 10 & $\mathrm{U}$ & 10 & 10 & $\mathrm{U}$ & 10 & 10 & $\mathrm{U}$ & 10 \\
\hline 2-Hexanone & 9 & $\mathrm{U}$ & 9 & 10 & $\mathrm{U}$ & 10 & 20 & $\mathrm{U}$ & 20 & 10 & $\mathrm{U}$ & 10 \\
\hline 4-Methyl-2-pentanone & 9 & $\mathrm{U}$ & 9 & 10 & $\mathrm{U}$ & 10 & 10 & $\mathrm{U}$ & 10 & 10 & $\mathrm{U}$ & 10 \\
\hline Acetone & 9 & $\mathrm{U}$ & 9 & 10 & $\mathrm{U}$ & 10 & 20 & $\mathrm{U}$ & 20 & 10 & $\mathrm{U}$ & 10 \\
\hline Benzene & 5 & $\mathrm{U}$ & 5 & 5 & $\mathrm{U}$ & 5 & 5.1 & $\mathrm{U}$ & 5.1 & 5 & $\mathrm{U}$ & 5 \\
\hline Bromodichloromethane & 5 & $\mathrm{U}$ & 5 & 5 & $\mathrm{U}$ & 5 & 5.1 & $\mathrm{U}$ & 5.1 & 5 & $\mathrm{U}$ & 5 \\
\hline Bromoform & 5 & $\mathrm{U}$ & 5 & 5 & $\mathrm{U}$ & 5 & 5.1 & $\mathrm{U}$ & 5.1 & 5 & $\mathrm{U}$ & 5 \\
\hline Bromomethane & 9 & $\mathrm{U}$ & 9 & 10 & $\mathrm{U}$ & 10 & 10 & $\mathrm{U}$ & 10 & 10 & $\mathrm{U}$ & 10 \\
\hline Carbon disulfide & 5 & $\mathrm{U}$ & 5 & 5 & $\mathrm{U}$ & 5 & 5.1 & $\mathrm{U}$ & 5.1 & 5 & $\mathrm{U}$ & 5 \\
\hline Carbon tetrachloride & 5 & $\mathrm{U}$ & 5 & 5 & $\mathrm{U}$ & 5 & 5.1 & $\mathrm{U}$ & 5.1 & 5 & $\mathrm{U}$ & 5 \\
\hline Chlorobenzene & 5 & $\mathrm{U}$ & 5 & 5 & $\mathrm{U}$ & 5 & 5.1 & $U$ & 5.1 & 5 & $\mathrm{U}$ & 5 \\
\hline Chloroethane & 9 & $\mathrm{U}$ & 9 & 10 & U & 10 & 10 & U & 10 & 10 & $\mathrm{U}$ & 10 \\
\hline Chloroform & 5 & U & 5 & 5 & $\mathrm{U}$ & 5 & 5.1 & $\mathrm{U}$ & 5.1 & 5 & $\mathrm{U}$ & 5 \\
\hline Chloromethane & 9 & U & 9 & 10 & $\mathrm{U}$ & 10 & 10 & $\mathrm{U}$ & 10 & 10 & $\mathrm{U}$ & 10 \\
\hline cis-1,2-Dichloroethylene & 5 & $\mathrm{U}$ & 5 & 5 & $\mathrm{U}$ & 5 & & & & 5 & $\mathrm{U}$ & 5 \\
\hline cis-1,3-Dichloropropene & 5 & $\mathrm{U}$ & 5 & 5 & $\mathrm{U}$ & 5 & 5.1 & $\mathrm{U}$ & 5.1 & 5 & $\mathrm{U}$ & 5 \\
\hline Dibromochloromethane & 5 & $\mathrm{U}$ & 5 & 5 & $\mathrm{U}$ & 5 & 5.1 & $\mathrm{U}$ & 5.1 & 5 & $\mathrm{U}$ & 5 \\
\hline Ethylbenzene & 5 & $\mathrm{U}$ & 5 & 5 & $\mathrm{U}$ & 5 & 5.1 & $\mathrm{U}$ & 5.1 & 5 & $\mathrm{U}$ & 5 \\
\hline Methylene chloride & 5 & $\mathrm{~B}$ & 5 & 11 & $\mathrm{~B}$ & 5 & 5.1 & $\mathrm{U}$ & 5.1 & 6 & $\mathrm{~B}$ & 5 \\
\hline Styrene & 5 & $\mathrm{U}$ & 5 & 5 & $\mathrm{U}$ & 5 & 5.1 & $\mathrm{U}$ & 5.1 & 5 & $\mathrm{U}$ & 5 \\
\hline Tetrachloroethene & 5 & $\mathrm{U}$ & 5 & 5 & $\mathrm{U}$ & 5 & 5.1 & UN & 5.1 & 5 & $\mathrm{U}$ & 5 \\
\hline Toluene & 5 & $\mathrm{U}$ & 5 & 5 & $\mathrm{U}$ & 5 & 5.1 & U & 5.1 & 5 & $\mathrm{U}$ & 5 \\
\hline trans-1,2-Dichloroethylene & 5 & $\mathrm{U}$ & 5 & 5 & $\mathrm{U}$ & 5 & & & 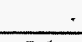 & 5 & $\mathrm{U}$ & 5 \\
\hline trans-1,3-Dichloropropene & 5 & $\mathrm{U}$ & 5 & 5 & $\mathrm{U}$ & 5 & 5.1 & $\mathrm{U}$ & 5.1 & 5 & $\mathrm{U}$ & 5 \\
\hline Trichloroethene & 5 & $\mathrm{U}$ & 5 & 5 & $\mathrm{U}$ & 5 & 5.1 & $\mathrm{U}$ & 5.1 & 5 & $\mathrm{U}$ & 5 \\
\hline Vinyl chloride & 9 & $\mathrm{U}$ & 9 & 10 & $\mathrm{U}$ & 10 & 5.1 & $\mathrm{U}$ & 5.1 & 10 & $\mathrm{U}$ & 10 \\
\hline Xylenes (total) & 5 & $\mathrm{U}$ & 5 & 5 & $\mathrm{U}$ & 5 & 10 & $\mathrm{U}$ & 10 & 5 & $\mathrm{U}$ & 5 \\
\hline
\end{tabular}

Attachment Originator

Checked

Calc. No.

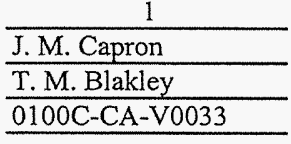

Sheet No. $\quad 18$ of 43

Date $12 / 13 / 06$

Date

Rev. No. 0 
Attachment 1. 118-C-1 Verification Sampling Results.

\begin{tabular}{|c|c|c|c|c|c|c|c|c|c|c|c|c|}
\hline \multirow[t]{2}{*}{ Constituents } & \multicolumn{3}{|c|}{$\begin{array}{c}\mathrm{J} 13506 \\
\text { Area } 2 \mathrm{A3} \\
\text { Sample Date } 8 / 17 / 06 \\
\end{array}$} & \multicolumn{3}{|c|}{$\begin{array}{c}\text { J13507 } \\
\text { Area } 2 \text { A4 } \\
\text { Sample Date } 8 / 17 / 06 \\
\end{array}$} & \multicolumn{3}{|c|}{$\begin{array}{c}\text { J13510 } \\
\text { Area } 3 \text { A1 } \\
\text { Sample Date 8/21/06 }\end{array}$} & \multicolumn{3}{|c|}{$\begin{array}{c}\text { J13511 } \\
\text { Area } 3 \mathrm{A2} \\
\text { Sample Date 8/21/06 }\end{array}$} \\
\hline & $\mu \mathrm{g} / \mathrm{kg}$ & $\mathbf{Q}$ & PQL & $\mu \mathrm{g} / \mathrm{kg}$ & $\mathbf{Q}$ & PQL & $\mu \mathrm{g} / \mathrm{kg}$ & $Q$ & PQL & $\mu \mathrm{g} / \mathrm{kg}$ & $Q$ & PQL \\
\hline \multicolumn{13}{|c|}{ Polychlorinated Biphenyls } \\
\hline Aroclor-1016 & 13 & $\mathrm{U}$ & 13 & 13 & $\mathrm{U}$ & 13 & 13 & $\mathrm{U}$ & 13 & 13 & $\mathrm{U}$ & 13 \\
\hline Aroclor-1221 & 13 & $\mathrm{U}$ & 13 & 13 & $\mathrm{U}$ & 13 & 13 & $\mathrm{U}$ & 13 & 13 & $\mathrm{U}$ & 13 \\
\hline Aroclor-1232 & 13 & $\mathrm{U}$ & 13 & 13 & $\mathrm{U}$ & 13 & 13 & $\mathrm{U}$ & 13 & 13 & $\mathrm{U}$ & 13 \\
\hline Aroclor-1242 & 13 & $\mathrm{U}$ & 13 & 13 & $\mathrm{U}$ & 13 & 13 & $\mathrm{U}$ & 13 & 13 & $\mathrm{U}$ & 13 \\
\hline Aroclor- 1248 & 13 & $\mathrm{U}$ & 13 & 13 & $\mathrm{U}$ & 13 & 13 & $\mathrm{U}$ & 13 & 13 & $\mathrm{U}$ & 13 \\
\hline Aroclor-1254 & 13 & $\mathrm{U}$ & 13 & 13 & $\mathrm{U}$ & 13 & 14 & & 13 & 46 & & 13 \\
\hline Aroclor -1260 & 13 & $\mathrm{U}$ & 13 & 13 & $\mathrm{U}$ & 13 & 13 & $\mathrm{U}$ & 13 & 13 & $\mathrm{U}$ & 13 \\
\hline \multicolumn{13}{|c|}{ Semivolatile Organic Compounds } \\
\hline 1,2,4-Trichlorobenzene & 340 & $\mathrm{U}$ & 340 & 340 & $\mathrm{U}$ & 340 & 330 & $\mathrm{U}$ & 330 & 330 & $\mathrm{U}$ & 330 \\
\hline 1,2-Dichlorobenzene & 340 & $\mathrm{U}$ & 340 & 340 & $\mathrm{U}$ & 340 & 330 & $\mathrm{U}$ & 330 & 330 & $\mathrm{U}$ & 330 \\
\hline 1,3-Dichlorobenzene & 340 & $\mathrm{U}$ & 340 & 340 & $\mathrm{U}$ & 340 & 330 & $\mathrm{U}$ & 330 & 330 & $\mathrm{U}$ & 330 \\
\hline 1,4-Dichlorobenzene & 340 & $\mathrm{U}$ & 340 & 340 & $\mathrm{U}$ & 340 & 330 & $\mathrm{U}$ & 330 & 330 & $\mathrm{U}$ & 330 \\
\hline 2,4,5-Trichlorophenol & 840 & $\mathrm{U}$ & 840 & 840 & $\mathrm{U}$ & 840 & 840 & $\mathrm{U}$ & 840 & 840 & $\mathrm{U}$ & 840 \\
\hline 2,4,6-Trichlorophenol & 340 & $\mathrm{U}$ & 340 & 340 & $\mathrm{U}$ & 340 & 330 & $\mathrm{U}$ & 330 & 330 & $\mathrm{U}$ & 330 \\
\hline 2,4-Dichlorophenol & 340 & $\mathrm{U}$ & 340 & 340 & $\mathrm{U}$ & 340 & 330 & $\mathrm{U}$ & 330 & 330 & $\mathrm{U}$ & 330 \\
\hline 2,4-Dimethylphenol & 340 & $\mathrm{U}$ & 340 & 340 & $\mathrm{U}$ & 340 & 330 & $\mathrm{U}$ & 330 & 330 & $\mathrm{U}$ & 330 \\
\hline 2,4-Dinitrophenol & 840 & $\mathrm{U}$ & 840 & 840 & $\mathrm{U}$ & 840 & 840 & UJ & 840 & 840 & UJ & 840 \\
\hline 2,4-Dinitrotoluene & 340 & $\mathrm{U}$ & 340 & 340 & $\mathrm{U}$ & 340 & 330 & $\mathrm{U}$ & 330 & 330 & $\mathrm{U}$ & 330 \\
\hline 2,6-Dinitrotoluene & 340 & $\mathrm{U}$ & 340 & 340 & $\mathrm{U}$ & 340 & 330 & $\mathrm{U}$ & 330 & 330 & $\mathrm{U}$ & 330 \\
\hline 2-Chloronaphthalene & 340 & $\mathrm{U}$ & 340 & 340 & $\mathrm{U}$ & 340 & 330 & $\mathrm{U}$ & 330 & 330 & $\mathrm{U}$ & 330 \\
\hline 2-Chlorophenol & 340 & $\mathrm{U}$ & 340 & 340 & $\mathrm{U}$ & 340 & 330 & $\mathrm{U}$ & 330 & 330 & $\mathrm{U}$ & 330 \\
\hline 2-Methylnaphthalene & 340 & $\mathrm{U}$ & 340 & 340 & $\mathrm{U}$ & 340 & 330 & $\mathrm{U}$ & 330 & 330 & $\mathrm{U}$ & 330 \\
\hline 2-Methylphenol (cresol, o-) & 340 & $\mathrm{U}$ & 340 & 340 & $\mathrm{U}$ & 340 & 330 & $\mathrm{U}$ & 330 & 330 & $\mathrm{U}$ & 330 \\
\hline 2-Nitroaniline & 840 & $\mathrm{U}$ & 840 & 840 & $\mathrm{U}$ & 840 & 840 & $\mathrm{U}$ & 840 & 840 & U & 840 \\
\hline 2-Nitrophenol & 340 & $\mathrm{U}$ & 340 & 340 & $\mathrm{U}$ & 340 & 330 & $\mathrm{U}$ & 330 & 330 & $\mathrm{U}$ & 330 \\
\hline 3,3'-Dichlorobenzidine & 340 & $\mathrm{U}$ & 340 & 340 & $\mathrm{U}$ & 340 & 330 & U & 330 & 330 & $\mathrm{U}$ & 330 \\
\hline 3-Nitroaniline & 840 & $\mathrm{U}$ & 840 & 840 & $\mathrm{U}$ & 840 & 840 & $\mathrm{U}$ & 840 & 840 & $\mathrm{U}$ & 840 \\
\hline 4,6-Dinitro-2-methylphenol & 840 & $\mathrm{U}$ & 840 & 840 & $\mathrm{U}$ & 840 & 840 & $\mathrm{U}$ & 840 & 840 & $\bar{U}$ & 840 \\
\hline 4-Bromophenyl-phenylether & 340 & $\mathrm{U}$ & 340 & 340 & $\mathrm{U}$ & 340 & 330 & $\mathrm{U}$ & 330 & 330 & $\mathrm{U}$ & 330 \\
\hline 4-Chloro-3-methylphenol & 340 & $\mathrm{U}$ & 340 & 340 & $\mathrm{U}$ & 340 & 330 & $\mathrm{U}$ & 330 & 330 & $\mathrm{U}$ & 330 \\
\hline 4-Chloroaniline & 340 & $\mathrm{U}$ & 340 & 340 & $\mathrm{U}$ & 340 & 330 & $\mathrm{U}$ & 330 & 330 & $\mathrm{U}$ & 330 \\
\hline 4-Chlorophenyl-phenylether & 340 & $\mathrm{U}$ & 340 & 340 & $\mathrm{U}$ & 340 & 330 & $\mathrm{U}$ & 330 & 330 & $\mathrm{U}$ & 330 \\
\hline 4-Methylphenol (p-cresol) & 340 & $\mathrm{U}$ & 340 & 340 & $\mathrm{U}$ & 340 & 330 & $\mathrm{U}$ & 330 & 330 & $\mathrm{U}$ & 330 \\
\hline 4-Nitroaniline & 840 & $\mathrm{U}$ & 840 & 840 & $\mathrm{U}$ & 840 & 840 & $\mathrm{U}$ & 840 & 840 & $\mathrm{U}$ & 840 \\
\hline 4-Nitrophenol & 840 & $\mathrm{U}$ & 840 & 840 & $\mathrm{U}$ & 840 & 840 & $\mathrm{U}$ & 840 & 840 & $\mathrm{U}$ & 840 \\
\hline Acenaphthene & 340 & $\mathrm{U}$ & 340 & 340 & $\mathrm{U}$ & 340 & 330 & $\mathrm{U}$ & 330 & 330 & $\mathrm{U}$ & 330 \\
\hline Acenaphthylene & 340 & $\mathrm{U}$ & 340 & 340 & $\mathrm{U}$ & 340 & 330 & $\mathrm{U}$ & 330 & 330 & $\mathrm{U}$ & 330 \\
\hline Anthracene & 340 & $\mathrm{U}$ & 340 & 340 & $\mathrm{U}$ & 340 & 330 & $\mathrm{U}$ & 330 & 330 & $\mathrm{U}$ & 330 \\
\hline Benzo(a)anthracene & 340 & $\mathrm{U}$ & 340 & 340 & $\mathrm{U}$ & 340 & 180 & $\mathrm{~J}$ & 330 & 330 & U & 330 \\
\hline Benzo(a)pyrene & 340 & $\mathrm{U}$ & 340 & 340 & $\mathrm{U}$ & 340 & 120 & $\mathrm{~J}$ & 330 & 330 & $\mathrm{U}$ & 330 \\
\hline Benzo(b)fluoranthene & 340 & $\mathrm{U}$ & 340 & 340 & $\mathrm{U}$ & 340 & 140 & $\mathbf{J}$ & 330 & 330 & $\mathrm{U}$ & 330 \\
\hline Benzo(g,h,i)perylene & 340 & $\mathrm{U}$ & 340 & 340 & $\mathrm{U}$ & 340 & 51 & $\mathrm{~J}$ & 330 & 330 & U & 330 \\
\hline Benzo(k)fluoranthene & 340 & $\mathrm{U}$ & 340 & 340 & $\mathrm{U}$ & 340 & 140 & $\mathrm{~J}$ & 330 & 330 & $\mathrm{U}$ & 330 \\
\hline bis(2-Chloro-1-methylethyl)ether & 340 & $\mathrm{U}$ & 340 & 340 & $\mathrm{U}$ & 340 & 330 & $\mathrm{U}$ & 330 & 330 & $\mathrm{U}$ & 330 \\
\hline bis(2-Chloroethoxy)methane & 340 & $\mathrm{U}$ & 340 & 340 & $\mathrm{U}$ & 340 & 330 & $\mathrm{U}$ & 330 & 330 & $\mathrm{U}$ & 330 \\
\hline bis(2-Chloroethyl)ether & 340 & $\mathrm{U}$ & 340 & 340 & $\mathrm{U}$ & 340 & 330 & U & 330 & 330 & $\mathrm{U}$ & 330 \\
\hline bis(2-Ethylhexyl)phthalate & 20 & $\sqrt{B}$ & 340 & 400 & $\mathrm{~B}$ & 340 & 330 & $\mathrm{U}$ & 330 & 30 & $\mathrm{~J}$ & 330 \\
\hline
\end{tabular}

Attachment

Originator

Checked

J. M. Capron

Calc. No.
T. M. Blakley

0100C-CA-V0033
Sheet No. 19 of 43

Date $\frac{19}{12 / 13 / 06}$

Date

Rev. No. 
Attachment 1. 118-C-1 Verification Sampling Results.

\begin{tabular}{|c|c|c|c|c|c|c|c|c|c|c|c|c|}
\hline \multirow[t]{2}{*}{ Constituents } & \multicolumn{3}{|c|}{\begin{tabular}{|c|}
$\mathbf{J 1 3 5 0 6}$ \\
Area $2 \mathrm{A3}$ \\
Sample Date 8/17/06
\end{tabular}} & \multicolumn{3}{|c|}{$\begin{array}{c}\text { J13507 } \\
\text { Area } 2 \text { A4 } \\
\text { Sample Date 8/17/06 } \\
\end{array}$} & \multicolumn{3}{|c|}{$\begin{array}{c}\text { J13510 } \\
\text { Area } 3 \text { A1 } \\
\text { Sample Date 8/21/06 }\end{array}$} & \multicolumn{3}{|c|}{$\begin{array}{c}\text { J13511 } \\
\text { Area } 3 \mathrm{A2} \\
\text { Sample Date 8/21/0 } \\
\end{array}$} \\
\hline & $\mu \mathrm{g} / \mathrm{kg}$ & $\mathbf{Q}$ & PQL & $\mu \mathrm{g} / \mathrm{kg}$ & $\mathbf{Q}$ & PQL & $\mu \mathrm{g} / \mathrm{kg}$ & $\mathbf{Q}$ & PQL & $\mu \mathrm{g} / \mathrm{kg}$ & $Q$ & PQL \\
\hline \multicolumn{13}{|c|}{ Semivolatile Organic Compounds (continued) } \\
\hline Butylbenzylphthalate & 340 & $\mathrm{U}$ & 340 & 340 & $\mathrm{U}$ & 340 & 330 & $\mathrm{U}$ & 330 & 330 & $\mathrm{U}$ & 330 \\
\hline Carbazole & 340 & $\mathrm{U}$ & 340 & 340 & $\mathrm{U}$ & 340 & 330 & $\mathrm{U}$ & 330 & 330 & $\mathrm{U}$ & 330 \\
\hline Chrysene & 340 & $\mathrm{U}$ & 340 & 340 & $\mathrm{U}$ & 340 & 210 & $\mathrm{~J}$ & 330 & 330 & $\mathrm{U}$ & 330 \\
\hline Di-n-butylphthalate & 340 & $\mathrm{U}$ & 340 & 340 & $\mathrm{U}$ & 340 & 51 & $\mathrm{~J}$ & 330 & 330 & $U$ & 330 \\
\hline Di-n-octylphthalate & 340 & $\mathrm{U}$ & 340 & 340 & $\mathrm{U}$ & 340 & 330 & $\mathrm{U}$ & 330 & 330 & $\mathrm{U}$ & 330 \\
\hline Dibenz $(\mathrm{a}, \mathrm{h})$ anthracene & 340 & $\mathrm{U}$ & 340 & 340 & $\mathrm{U}$ & 340 & 330 & $\mathrm{U}$ & 330 & 330 & $U$ & 330 \\
\hline Dibenzofuran & 340 & $\mathrm{U}$ & 340 & 340 & $\mathrm{U}$ & 340 & 330 & $\mathrm{U}$ & 330 & 330 & $\mathrm{U}$ & 330 \\
\hline Diethylphthalate & 340 & $\mathrm{U}$ & 340 & 340 & $\mathrm{U}$ & 340 & 330 & $\mathrm{U}$ & 330 & 330 & U & 330 \\
\hline Dimethylphthalate & 340 & $\mathrm{U}$ & 340 & 340 & $\mathrm{U}$ & 340 & 330 & $\mathrm{U}$ & 330 & 330 & $\mathrm{U}$ & 330 \\
\hline Fluoranthene & 340 & $\mathrm{U}$ & 340 & 340 & $\mathrm{U}$ & 340 & 320 & $\mathrm{~J}$ & 330 & 330 & $\mathrm{U}$ & 330 \\
\hline Fluorene & 340 & $\mathrm{U}$ & 340 & 340 & $\mathrm{U}$ & 340 & 330 & $\mathrm{U}$ & 330 & 330 & $\mathrm{U}$ & 330 \\
\hline Hexachlorobenzene & 340 & $\mathrm{U}$ & 340 & 340 & $\mathrm{U}$ & 340 & 330 & $\mathrm{U}$ & 330 & 330 & $\mathrm{U}$ & 330 \\
\hline Hexachlorobutadiene & 340 & $\mathrm{U}$ & 340 & 340 & $\mathrm{U}$ & 340 & 330 & $\mathrm{U}$ & 330 & 330 & $\mathrm{U}$ & 330 \\
\hline Hexachlorocyclopentadiene & 340 & $\mathrm{U}$ & 340 & 340 & $\mathrm{U}$ & 340 & 330 & $\mathrm{U}$ & 330 & 330 & $\mathrm{U}$ & 330 \\
\hline Hexachloroethane & 340 & $\mathrm{U}$ & 340 & 340 & $\mathrm{U}$ & 340 & 330 & $\mathrm{U}$ & 330 & 330 & $\mathrm{U}$ & 330 \\
\hline Indeno(1,2,3-cd)pyrene & 340 & $\mathrm{U}$ & 340 & 340 & $\mathrm{U}$ & 340 & 46 & $\mathrm{~J}$ & 330 & 330 & $\mathrm{U}$ & 330 \\
\hline Isophorone & 340 & U & 340 & 340 & $\mathrm{U}$ & 340 & 330 & $\mathrm{U}$ & 330 & 330 & $\mathrm{U}$ & 330 \\
\hline N-Nitroso-di-n-dipropylamine & 340 & $\mathrm{U}$ & 340 & 340 & $\mathrm{U}$ & 340 & 330 & $\mathrm{U}$ & 330 & 330 & $\mathrm{U}$ & 330 \\
\hline N-Nitrosodiphenylamine & 340 & $\mathrm{U}$ & 340 & 340 & $\mathrm{U}$ & 340 & 330 & $\mathrm{U}$ & 330 & 330 & $\mathrm{U}$ & 330 \\
\hline Naphthalene & 340 & $\mathrm{U}$ & 340 & 340 & $\mathrm{U}$ & 340 & 330 & $\mathrm{U}$ & 330 & 330 & U & 330 \\
\hline Nitrobenzene & 340 & $\mathrm{U}$ & 340 & 340 & $\mathrm{U}$ & 340 & 330 & $\mathrm{U}$ & 330 & 330 & $\mathrm{U}$ & 330 \\
\hline Pentachlorophenol & 840 & $\mathrm{U}$ & 840 & 840 & $\mathrm{U}$ & 840 & 840 & $\mathrm{U}$ & 840 & 840 & $\mathrm{U}$ & 840 \\
\hline Phenanthrene & 340 & $\mathrm{U}$ & 340 & 340 & $\mathrm{U}$ & 340 & 55 & $\mathrm{~J}$ & 330 & 330 & $\mathrm{U}$ & 330 \\
\hline Phenol & 340 & $\mathrm{U}$ & 340 & 340 & $\mathrm{U}$ & 340 & 330 & $\mathrm{U}$ & 330 & 330 & $\mathrm{U}$ & 330 \\
\hline Pyrene & 340 & $\mathrm{U}$ & 340 & 340 & $\mathrm{U}$ & 340 & 320 & $\mathrm{~J}$ & 330 & 330 & $\mathrm{U}$ & 330 \\
\hline & & & & Attachn & & & 1 & & & Sheet No. & & of 43 \\
\hline & & & & Origina & & J.M.Ca & oron & & & Date & & $/ 13 / 06$ \\
\hline & & & & Checke & & T.M. B & akley & & & Date & & \\
\hline & & & & Calc. $\mathrm{N}$ & & $0100 \mathrm{C}-\mathrm{C}$ & $\mathrm{A}-\mathrm{V003}$ & & & Rev. No & & 0 \\
\hline
\end{tabular}


Attachment 1. 118-C-1 Verification Sampling Results.

\begin{tabular}{|c|c|c|c|c|c|c|c|c|c|c|c|c|}
\hline \multirow[t]{2}{*}{ Constituents } & \multicolumn{3}{|c|}{$\begin{array}{c}\text { J135R2 } \\
\text { Area } 2 \mathrm{A3} \\
\text { Sample Date 8/28/06 }\end{array}$} & \multicolumn{3}{|c|}{$\begin{array}{c}\text { J135R3 } \\
\text { Area } 2 \text { A4 } \\
\text { Sample Date 8/28/06 } \\
\end{array}$} & \multicolumn{3}{|c|}{$\begin{array}{c}\text { J135R4 } \\
\text { Area } 3 \mathrm{~A} 1 \\
\text { Sample Date 8/21/06 }\end{array}$} & \multicolumn{3}{|c|}{$\begin{array}{c}\text { J135R5 } \\
\text { Area } 3 \text { A2 } \\
\text { Sample Date 8/28/06 } \\
\end{array}$} \\
\hline & $\mu \mathrm{g} / \mathrm{kg}$ & $\mathbf{Q}$ & PQL & $\mu \mathrm{g} / \mathrm{kg}$ & $Q$ & $\mathrm{PQL}$ & $\mu \mathrm{g} / \mathrm{kg}$ & $\mathbf{Q}$ & PQL & $\mu \mathrm{g} / \mathrm{kg}$ & Q & PQL \\
\hline \multicolumn{13}{|c|}{ Volatile Organic Compounds } \\
\hline 1,1,1-Trichloroethane & 6 & $\mathrm{U}$ & 6 & 6 & $\mathrm{U}$ & 6 & 5 & $\mathrm{U}$ & 5 & 5 & $\mathrm{U}$ & 5 \\
\hline 1,1,2,2-Tetrachloroethane & 6 & UJ & 6 & 6 & $\mathrm{UJ}$ & 6 & 5 & UJ & 5 & 5 & $\mathrm{UJ}$ & 5 \\
\hline 1,1,2-Trichloroethane & 6 & $\mathrm{U}$ & 6 & 6 & $\mathrm{U}$ & 6 & 5 & $\mathrm{U}$ & 5 & 5 & $\mathrm{U}$ & 5 \\
\hline 1,1-Dichloroethane & 6 & $\mathrm{U}$ & 6 & 6 & $\mathrm{U}$ & 6 & 5 & $\mathrm{U}$ & 5 & 5 & $\mathrm{U}$ & 5 \\
\hline 1,1-Dichloroethene & 6 & $\mathrm{U}$ & 6 & 6 & $\mathrm{U}$ & 6 & 5 & $\mathrm{U}$ & 5 & 5 & $\mathrm{U}$ & 5 \\
\hline 1,2-Dichloroethane & 6 & $\mathrm{U}$ & 6 & 6 & $\mathrm{U}$ & 6 & 5 & $\mathrm{U}$ & 5 & 5 & $\mathrm{U}$ & 5 \\
\hline 1,2-Dichloroethene (total) & 6 & $\mathrm{U}$ & 6 & 6 & $\mathrm{U}$ & 6 & 5 & $\mathrm{U}$ & 5 & 5 & $\mathrm{U}$ & 5 \\
\hline 1,2-Dichloropropane & 6 & $\mathrm{U}$ & 6 & 6 & $\mathrm{U}$ & 6 & 5 & $\mathrm{U}$ & 5 & 5 & $\mathrm{U}$ & 5 \\
\hline 2-Butanone & 11 & $\mathrm{U}$ & 11 & 11 & $\mathrm{U}$ & 11 & 10 & $\mathrm{U}$ & 10 & 10 & $\mathrm{U}$ & 10 \\
\hline 2-Hexanone & 11 & $\mathrm{U}$ & 11 & 11 & $\mathrm{U}$ & 11 & 10 & $\mathrm{U}$ & 10 & 10 & $\mathrm{U}$ & 10 \\
\hline 4-Methyl-2-pentanone & 11 & $\mathrm{U}$ & 11 & 11 & $\mathrm{U}$ & 11 & 10 & $\mathrm{U}$ & 10 & 10 & $\mathrm{U}$ & 10 \\
\hline Acetone & 11 & $\mathrm{U}$ & 11 & 11 & $U$ & 11 & 10 & $\mathrm{U}$ & 10 & 10 & $\mathrm{U}$ & 10 \\
\hline Benzene & 6 & $\mathrm{U}$ & 6 & 6 & $\mathrm{U}$ & 6 & 5 & $\mathrm{U}$ & 5 & 5 & $\mathrm{U}$ & 5 \\
\hline Bromodichloromethane & 6 & $\mathrm{U}$ & 6 & 6 & $\mathrm{U}$ & 6 & 5 & $\mathrm{U}$ & 5 & 5 & $\mathrm{U}$ & 5 \\
\hline Bromoform & 6 & $\mathrm{U}$ & 6 & 6 & $\mathrm{U}$ & 6 & 5 & $\mathrm{U}$ & 5 & 5 & $U$ & 5 \\
\hline Bromomethane & 11 & $\mathrm{U}$ & 11 & 11 & $\mathrm{U}$ & 11 & 10 & $\mathrm{U}$ & 10 & 10 & $\mathrm{U}$ & 10 \\
\hline Carbon disulfide & 6 & $\mathrm{U}$ & 6 & 6 & $\mathrm{U}$ & 6 & 5 & $\mathrm{U}$ & 5 & 5 & $\mathrm{U}$ & 5 \\
\hline Carbon tetrachloride & 6 & $\mathrm{U}$ & 6 & 6 & $\mathrm{U}$ & 6 & 5 & $U$ & 5 & 5 & $\mathrm{U}$ & 5 \\
\hline Chlorobenzene & 6 & $\mathrm{U}$ & 6 & 6 & $\mathrm{U}$ & 6 & 5 & $\mathrm{U}$ & 5 & 5 & $\mathrm{U}$ & 5 \\
\hline Chloroethane & 11 & $\mathrm{U}$ & 11 & 11 & $\mathrm{U}$ & 11 & 10 & $\mathrm{U}$ & 10 & 10 & $\mathrm{U}$ & 10 \\
\hline Chloroform & 6 & $\mathrm{U}$ & 6 & 6 & $\mathrm{U}$ & 6 & 5 & $\mathrm{U}$ & 5 & 5 & $\mathrm{U}$ & 5 \\
\hline Chloromethane & 11 & $\mathrm{U}$ & 11 & 11 & $\mathrm{U}$ & 11 & 10 & $\mathrm{U}$ & 10 & 10 & $\mathrm{U}$ & 10 \\
\hline cis-1,2-Dichloroethylene & 6 & $\mathrm{U}$ & 6 & 6 & $\mathrm{U}$ & 6 & 5 & $\mathrm{U}$ & 5 & 5 & $\mathrm{U}$ & 5 \\
\hline cis-1,3-Dichloropropene & 6 & $\mathrm{U}$ & 6 & 6 & $\mathrm{U}$ & 6 & 5 & $\mathrm{U}$ & 5 & 5 & $\mathrm{U}$ & 5 \\
\hline Dibromochloromethane & 6 & $\mathrm{U}$ & 6 & 6 & $\mathrm{U}$ & 6 & 5 & $\mathrm{U}$ & 5 & 5 & $U$ & 5 \\
\hline Ethylbenzene & 6 & $\mathrm{U}$ & 6 & 6 & $\mathrm{U}$ & 6 & 5 & $\mathrm{U}$ & 5 & 5 & $\mathrm{U}$ & 5 \\
\hline Methylene chloride & 9 & $\mathrm{~B}$ & 6 & 14 & $\mathrm{~B}$ & 6 & 12 & $\mathrm{~B}$ & 5 & 8 & $\mathrm{~B}$ & 5 \\
\hline Styrene & 6 & $\mathrm{U}$ & 6 & 6 & $\mathrm{U}$ & 6 & 5 & $\mathrm{U}$ & 5 & 5 & $\mathrm{U}$ & 5 \\
\hline Tetrachloroethene & 6 & $U$ & 6 & 6 & $\mathrm{U}$ & 6 & 5 & $\mathrm{U}$ & 5 & 5 & $\mathrm{U}$ & 5 \\
\hline Toluene & 6 & $\mathrm{U}$ & 6 & 6 & $\mathrm{U}$ & 6 & 5 & $\mathrm{U}$ & 5 & 5 & $\mathrm{U}$ & 5 \\
\hline trans-1,2-Dichloroethylene & 6 & U & 6 & 6 & $\mathrm{U}$ & 6 & 5 & $\mathrm{U}$ & 5 & 5 & $\mathrm{U}$ & 5 \\
\hline trans-1,3-Dichloropropene & 6 & $\mathrm{U}$ & 6 & 6 & $\mathrm{U}$ & 6 & 5 & $\mathrm{U}$ & 5 & 5 & $\mathrm{U}$ & 5 \\
\hline Trichloroethene & 6 & $U$ & 6 & 6 & $\mathrm{U}$ & 6 & 5 & $\mathrm{U}$ & 5 & 5 & $\mathrm{U}$ & 5 \\
\hline Vinyl chloride & 11 & $\mathrm{U}$ & 11 & 11 & $\mathrm{U}$ & 11 & 10 & $\mathrm{U}$ & 10 & 10 & $\mathrm{U}$ & 10 \\
\hline Xylenes (total) & 6 & $\mathrm{U}$ & 6 & 6 & $\mathrm{U}$ & 6 & 5 & $\mathrm{U}$ & 5 & 5 & $\mathrm{U}$ & 5 \\
\hline & & & & Attachm & & & 1 & & & heet No. & & of 43 \\
\hline & & & & Originat & & J.M. Car & ron & & & Date & & $13 / 06$ \\
\hline & & & & Checked & & T.M. Bl & kley & & & Date & & \\
\hline & & & & Calc. No & & $0100 \mathrm{C}-\mathrm{C}$ & $\mathrm{A}-\mathrm{V} 0033$ & & & Rev. No. & & 0 \\
\hline
\end{tabular}


Attachment 1. 118-C-1 Verification Sampling Results.

\begin{tabular}{|c|c|c|c|c|c|c|c|c|c|c|c|c|}
\hline \multirow[t]{2}{*}{ Constituents } & \multicolumn{3}{|c|}{$\begin{array}{c}\text { J13512 } \\
\text { Area } 3 \text { A3 } \\
\text { Sample Date 8/21/06 } \\
\end{array}$} & \multicolumn{3}{|c|}{$\begin{array}{c}\mathrm{J13513} \\
\text { Area } 3 \mathrm{A4} \\
\text { Sample Date } 8 / 21 / 06\end{array}$} & \multicolumn{3}{|c|}{$\begin{array}{c}\text { J13514 } \\
\text { Area } 4 \text { A1 } \\
\text { Sample Date } 8 / 21 / 06\end{array}$} & \multicolumn{3}{|c|}{$\begin{array}{c}\text { J13515 } \\
\text { Area } 4 \text { A2 } \\
\text { Sample Date } 8 / 21 / 06\end{array}$} \\
\hline & $\mu \mathrm{g} / \mathrm{kg}$ & $\mathbf{Q}$ & PQL & $\mu \mathrm{g} / \mathrm{kg}$ & $\mathbf{Q}$ & PQL & $\mu \mathrm{g} / \mathrm{kg}$ & $\mathbf{Q}$ & PQL & $\mu \mathrm{g} / \mathrm{kg}$ & $\mathbf{Q}$ & PQL \\
\hline \multicolumn{13}{|c|}{ Polychlorinated Biphenyls } \\
\hline Aroclor-1016 & 13 & $\mathrm{U}$ & 13 & 13 & $\mathrm{U}$ & 13 & 13 & $\mathrm{U}$ & 13 & 13 & $\mathrm{U}$ & 13 \\
\hline Aroclor-1221 & 13 & $\mathrm{U}$ & 13 & 13 & $\mathrm{U}$ & 13 & 13 & $\mathrm{U}$ & 13 & 13 & $\mathrm{U}$ & 13 \\
\hline Aroclor-1232 & 13 & $\mathrm{U}$ & 13 & 13 & $\mathrm{U}$ & 13 & 13 & $\mathrm{U}$ & 13 & 13 & $\mathrm{U}$ & 13 \\
\hline Aroclor-1242 & 13. & $\mathrm{U}$ & 13 & 13 & $\mathrm{U}$ & 13 & 13 & $\mathrm{U}$ & 13 & 13 & $\mathrm{U}$ & 13 \\
\hline Aroclor-1248 & 13 & $\mathrm{U}$ & 13 & 13 & $\mathrm{U}$ & 13 & 13 & $\mathrm{U}$ & 13 & 13 & $\mathrm{U}$ & 13 \\
\hline Aroclor-1254 & 62 & & 13 & 21 & & 13 & 13 & $\mathrm{U}$ & 13 & 6.4 & $\mathrm{~J}$ & 13 \\
\hline Aroclor-1260 & 13 & $\mathrm{U}$ & 13 & 13 & $\mathrm{U}$ & 13 & 13 & $U$ & 13 & 13 & $\mathrm{U}$ & 13 \\
\hline \multicolumn{13}{|c|}{ Semivolatile Organic Compounds } \\
\hline 1,2,4-Trichlorobenzene & 340 & $\mathrm{U}$ & 340 & 330 & $\mathrm{U}$ & 330 & 330 & $\mathrm{U}$ & 330 & 330 & $\mathrm{U}$ & 330 \\
\hline 1,2-Dichlorobenzene & 340 & $\mathrm{U}$ & 340 & 330 & $\mathrm{U}$ & 330 & 330 & $\mathrm{U}$ & 330 & 330 & $\mathrm{U}$ & 330 \\
\hline 1,3-Dichlorobenzene & 340 & $\mathrm{U}$ & 340 & 330 & $\mathrm{U}$ & 330 & 330 & $\mathrm{U}$ & 330 & 330 & $\mathrm{U}$ & 330 \\
\hline 1,4-Dichlorobenzene & 340 & $\mathrm{U}$ & 340 & 330 & $\mathrm{U}$ & 330 & 330 & $\mathrm{U}$ & 330 & 330 & $\mathrm{U}$ & 330 \\
\hline 2,4,5-Trichlorophenol & 840 & $\bar{U}$ & 840 & 840 & $\mathrm{U}$ & 840 & 840 & $\mathrm{U}$ & 840 & 840 & $\mathrm{U}$ & 840 \\
\hline 2,4,6-Trichlorophenol & 340 & $\mathrm{U}$ & 340 & 330 & $\mathrm{U}$ & 330 & 330 & $\mathrm{U}$ & 330 & 330 & $\mathrm{U}$ & 330 \\
\hline 2,4-Dichlorophenol & 340 & $\mathrm{U}$ & 340 & 330 & $\mathrm{U}$ & 330 & 330 & $\mathrm{U}$ & 330 & 330 & $\mathrm{U}$ & 330 \\
\hline 2,4-Dimethylphenol & 340 & $\mathrm{U}$ & 340 & 330 & $U$ & 330 & 330 & $\mathrm{U}$ & 330 & 330 & $\mathrm{U}$ & 330 \\
\hline 2,4-Dinitrophenol & 840 & UJ & 840 & 840 & $\mathrm{UJ}$ & 840 & 840 & $\mathrm{UJ}$ & 840 & 840 & UJ & 840 \\
\hline 2,4-Dinitrotoluene & 340 & $\mathrm{U}$ & 340 & 330 & $\mathrm{U}$ & 330 & 330 & $\mathrm{U}$ & 330 & 330 & $\mathrm{U}$ & 330 \\
\hline 2,6-Dinitrotoluene & 340 & $\mathrm{U}$ & 340 & 330 & $\mathrm{U}$ & 330 & 330 & $\mathrm{U}$ & 330 & 330 & $\mathrm{U}$ & 330 \\
\hline 2-Chloronaphthalene & 340 & $\mathrm{U}$ & 340 & 330 & $\mathrm{U}$ & 330 & 330 & $\mathrm{U}$ & 330 & 330 & $\mathrm{U}$ & 330 \\
\hline 2-Chlorophenol & 340 & $\mathrm{U}$ & 340 & 330 & $\mathrm{U}$ & 330 & 330 & $\mathrm{U}$ & 330 & 330 & $\mathrm{U}$ & 330 \\
\hline 2-Methylnaphthalene & 340 & $\mathrm{U}$ & 340 & 330 & $\mathrm{U}$ & 330 & 330 & $\mathrm{U}$ & 330 & 330 & $\mathrm{U}$ & 330 \\
\hline 2-Methylphenol (cresol, o-) & 340 & $U$ & 340 & 330 & $\mathrm{U}$ & 330 & 330 & $\mathrm{U}$ & 330 & 330 & $\mathrm{U}$ & 330 \\
\hline 2-Nitroaniline & 840 & $\mathrm{U}$ & 840 & 840 & $\mathrm{U}$ & 840 & 840 & $\mathrm{U}$ & 840 & 840 & $\mathrm{U}$ & 840 \\
\hline 2-Nitrophenol & 340 & $\mathrm{U}$ & 340 & 330 & $\mathrm{U}$ & 330 & 330 & $\mathrm{U}$ & 330 & 330 & $\mathrm{U}$ & 330 \\
\hline 3,3'-Dichlorobenzidine & 340 & $\mathrm{U}$ & 340 & 330 & $\mathrm{U}$ & 330 & 330 & $\mathrm{U}$ & 330 & 330 & U & 330 \\
\hline 3-Nitroaniline & 840 & $U$ & 840 & 840 & $\mathrm{U}$ & 840 & 840 & $\mathrm{U}$ & 840 & 840 & $\mathrm{U}$ & 840 \\
\hline 4,6-Dinitro-2-methylphenol & 840 & $\mathrm{U}$ & 840 & 840 & $\mathrm{U}$ & 840 & 840 & $\mathrm{U}$ & 840 & 840 & $\bar{U}$ & 840 \\
\hline 4-Bromophenyl-phenylether & 340 & $U$ & 340 & 330 & $\mathrm{U}$ & 330 & 330 & $\mathrm{U}$ & 330 & 330 & $\mathrm{U}$ & 330 \\
\hline 4-Chloro-3-methylphenol & 340 & $\mathrm{U}$ & 340 & 330 & $\mathrm{U}$ & 330 & 330 & $\mathrm{U}$ & 330 & 330 & $\mathrm{U}$ & 330 \\
\hline 4-Chloroaniline & 340 & U & 340 & 330 & $\mathrm{U}$ & 330 & 330 & $\mathrm{U}$ & 330 & 330 & $\mathrm{U}$ & 330 \\
\hline 4-Chlorophenyl-phenylether & 340 & $\mathrm{U}$ & 340 & 330 & $\mathrm{U}$ & 330 & 330 & $\mathrm{U}$ & 330 & 330 & $\mathrm{U}$ & 330 \\
\hline 4-Methylphenol (p-cresol) & 340 & $\mathrm{U}$ & 340 & 330 & $\mathrm{U}$ & 330 & 330 & $\mathrm{U}$ & 330 & 330 & $\mathrm{U}$ & 330 \\
\hline 4-Nitroaniline & 840 & U & 840 & 840 & $\mathrm{U}$ & 840 & 840 & $\mathrm{U}$ & 840 & 840 & $\mathrm{U}$ & 840 \\
\hline 4-Nitrophenol & 840 & $U$ & 840 & 840 & $\mathrm{U}$ & 840 & 840 & $\mathrm{U}$ & 840 & 840 & $\mathrm{U}$ & 840 \\
\hline Acenaphthene & 340 & $\mathrm{U}$ & 340 & 330 & $\mathrm{U}$ & 330 & 330 & $\mathrm{U}$ & 330 & 330 & $\mathrm{U}$ & 330 \\
\hline Acenaphthylene & 340 & $\mathrm{U}$ & 340 & 330 & $\mathrm{U}$ & 330 & 330 & $\mathrm{U}$ & 330 & 330 & $\mathrm{U}$ & 330 \\
\hline Anthracene & 340 & $\mathrm{U}$ & 340 & 330 & $\mathrm{U}$ & 330 & 330 & $\mathrm{U}$ & 330 & 330 & $\mathrm{U}$ & 330 \\
\hline Benzo(a)anthracene & 340 & $\mathrm{U}$ & 340 & 330 & $\mathrm{U}$ & 330 & 330 & $\mathrm{U}$ & 330 & 130 & $\mathrm{~J}$ & 330 \\
\hline Benzo(a)pyrene & 340 & $\mathrm{U}$ & 340 & 330 & $\mathrm{U}$ & 330 & 330 & $\mathrm{U}$ & 330 & 83 & $\mathrm{~J}$ & 330 \\
\hline Benzo(b)fluoranthene & 340 & $\mathrm{U}$ & 340 & 330 & $\mathrm{U}$ & 330 & 330 & $\mathrm{U}$ & 330 & 110 & $\mathrm{~J}$ & 330 \\
\hline Benzo $(\mathrm{g}, \mathrm{h}, \mathrm{i})$ perylene & 340 & $\mathrm{U}$ & 340 & 330 & $\mathrm{U}$ & 330 & 330 & $\mathrm{U}$ & 330 & 37 & $\mathrm{~J}$ & 330 \\
\hline Benzo(k)fluoranthene & 340 & $\mathrm{U}$ & 340 & 330 & $\mathrm{U}$ & 330 & 330 & $\mathrm{U}$ & 330 & 92 & $\mathrm{~J}$ & 330 \\
\hline bis(2-Chloro-1-methylethyl)ether & 340 & $\mathrm{U}$ & 340 & 330 & $\mathrm{U}$ & 330 & 330 & $\mathrm{U}$ & 330 & 330 & $\mathrm{U}$ & 330 \\
\hline bis(2-Chloroethoxy)methane & 340 & $U$ & 340 & 330 & $\mathrm{U}$ & 330 & 330 & U & 330 & 330 & $\mathrm{U}$ & 330 \\
\hline bis(2-Chloroethyl)ether & 340 & $\mathrm{U}$ & 340 & 330 & $\mathrm{U}$ & 330 & 330 & $\mathrm{U}$ & 330 & 330 & $\mathrm{U}$ & 330 \\
\hline bis(2-Ethylhexyl)phthalate & 340 & $\mathrm{U}$ & 340 & 240 & $\mathrm{~J}$ & 330 & 17 & $\mathrm{~J}$ & 330 & 330 & $\mathrm{U}$ & 330 \\
\hline & & & & Attachm & & & 1 & & & $t \mathrm{~N}$ & & f 43 \\
\hline & & & & Originat & & J.M. C & on & & & Dat & & $13 / 06$ \\
\hline & & & & Checked & & T.M. Bl & kley & & & Date & & \\
\hline & & & & Calc. N & & $0100 \mathrm{C}-\mathrm{C}$ & A-V003 & & & Rev. No. & & 0 \\
\hline
\end{tabular}


Attachment 1. 118-C-1 Verification Sampling Results.

\begin{tabular}{|c|c|c|c|c|c|c|c|c|c|c|c|c|}
\hline \multirow[t]{2}{*}{ Constituents } & \multicolumn{3}{|c|}{$\begin{array}{c}\text { J13512 } \\
\text { Area } 3 \mathrm{A3} \\
\text { Sample Date 8/21/06 }\end{array}$} & \multicolumn{3}{|c|}{$\begin{array}{c}\mathrm{J13513} \\
\text { Area } 3 \mathrm{A4} \\
\text { Sample Date 8/21/06 } \\
\end{array}$} & \multicolumn{3}{|c|}{$\begin{array}{c}\text { J13514 } \\
\text { Area } 4 \text { A1 } \\
\text { Sample Date 8/21/06 } \\
\end{array}$} & \multicolumn{3}{|c|}{\begin{tabular}{|c|} 
J13515 \\
Area 4 A2 \\
Sample Date 8/21/06 \\
\end{tabular}} \\
\hline & $\mu \mathrm{g} / \mathrm{kg}$ & $\mathbf{Q}$ & PQL & $\mu \mathrm{g} / \mathrm{kg}$ & $\mathbf{Q}$ & PQL & $\mu \mathrm{g} / \mathrm{kg}$ & $\mathbf{Q}$ & PQL & $\mu \mathrm{g} / \mathrm{kg}$ & $\mathbf{Q}$ & PQL \\
\hline \multicolumn{13}{|c|}{ Semivolatile Organic Compounds (continued) } \\
\hline Butylbenzylphthalate & 340 & $\mathrm{U}$ & 340 & 38 & $\mathrm{~J}$ & 330 & 330 & $\mathrm{U}$ & 330 & 330 & $\mathrm{U}$ & 330 \\
\hline Carbazole & 340 & $\mathrm{U}$ & 340 & 330 & $\mathrm{U}$ & 330 & 330 & $\mathrm{U}$ & 330 & 330 & $\mathrm{U}$ & 330 \\
\hline Chrysene & 340 & $\mathrm{U}$ & 340 & 330 & $\mathrm{U}$ & 330 & 330 & $\mathrm{U}$ & 330 & 170 & $\mathrm{~J}$ & 330 \\
\hline Di-n-butylphthalate & 25 & $\mathrm{~J}$ & 340 & 63 & $\mathrm{~J}$ & 330 & 330 & $\mathrm{U}$ & 330 & 330 & $\mathrm{U}$ & 330 \\
\hline \begin{tabular}{|l} 
Di-n-octylphthalate \\
\end{tabular} & 340 & $\mathrm{U}$ & 340 & 330 & $\mathrm{U}$ & 330 & 330 & $\mathrm{U}$ & 330 & 330 & $\mathrm{U}$ & 330 \\
\hline Dibenz $(\mathrm{a}, \mathrm{h})$ anthracene & 340 & $\mathrm{U}$ & 340 & 330 & $\mathrm{U}$ & 330 & 330 & $\mathrm{U}$ & 330 & 330 & $\mathrm{U}$ & 330 \\
\hline \begin{tabular}{|l|} 
Dibenzofuran \\
\end{tabular} & 340 & $\mathrm{U}$ & 340 & 330 & $\mathrm{U}$ & 330 & 330 & $\mathrm{U}$ & 330 & 330 & $\mathrm{U}$ & 330 \\
\hline Diethylphthalate & 340 & $\mathrm{U}$ & 340 & 330 & $\mathrm{U}$ & 330 & 330 & $\mathrm{U}$ & 330 & 330 & $\mathrm{U}$ & 330 \\
\hline Dimethylphthalate & 340 & $\mathrm{U}$ & 340 & 330 & $\mathrm{U}$ & 330 & 330 & $\mathrm{U}$ & 330 & 330 & $\mathrm{U}$ & 330 \\
\hline Fluoranthene & 340 & $\mathrm{U}$ & 340 & 330 & $\mathrm{U}$ & 330 & 330 & $\mathrm{U}$ & 330 & 330 & & 330 \\
\hline Fluorene & 340 & $\mathrm{U}$ & 340 & 330 & $\mathrm{U}$ & 330 & 330 & $\mathrm{U}$ & 330 & 330 & $\mathrm{U}$ & 330 \\
\hline Hexachlorobenzene & 340 & $\mathrm{U}$ & 340 & 330 & $\mathrm{U}$ & 330 & 330 & $\mathrm{U}$ & 330 & 330 & $\mathrm{U}$ & 330 \\
\hline Hexachlorobutadiene & 340 & $\mathrm{U}$ & 340 & 330 & $\mathrm{U}$ & 330 & 330 & $\mathrm{U}$ & 330 & 330 & $\mathrm{U}$ & 330 \\
\hline Hexachlorocyclopentadiene & 340 & $\mathrm{U}$ & 340 & 330 & $\mathrm{U}$ & 330 & 330 & $\mathrm{U}$ & 330 & 330 & $\mathrm{U}$ & 330 \\
\hline Hexachloroethane & 340 & $\mathrm{U}$ & 340 & 330 & $\mathrm{U}$ & 330 & 330 & $\mathrm{U}$ & 330 & 330 & $\mathrm{U}$ & 330 \\
\hline Indeno(1,2,3-cd)pyrene & 340 & $\mathrm{U}$ & 340 & 330 & $\mathrm{U}$ & 330 & 330 & $\mathrm{U}$ & 330 & 35 & $\mathrm{~J}$ & 330 \\
\hline Isophorone & 340 & $\mathrm{U}$ & 340 & 330 & $\mathrm{U}$ & 330 & 330 & $\mathrm{U}$ & 330 & 330 & $\mathrm{U}$ & 330 \\
\hline N-Nitroso-di-n-dipropylamine & 340 & $\mathrm{U}$ & 340 & 330 & $\mathrm{U}$ & 330 & 330 & $\mathrm{U}$ & 330 & 330 & $\mathrm{U}$ & 330 \\
\hline \begin{tabular}{|l|} 
N-Nitrosodiphenylamine \\
\end{tabular} & 340 & $\bar{U}$ & 340 & 330 & $\mathrm{U}$ & 330 & 330 & $\mathrm{U}$ & 330 & 330 & $\mathrm{U}$ & 330 \\
\hline Naphthalene & 340 & $\mathrm{U}$ & 340 & 330 & $\mathrm{U}$ & 330 & 330 & U & 330 & 330 & $\mathrm{U}$ & 330 \\
\hline Nitrobenzene & 340 & $\mathrm{U}$ & 340 & 330 & $\mathrm{U}$ & 330 & 330 & $\mathrm{U}$ & 330 & 330 & $U$ & 330 \\
\hline Pentachlorophenol & 840 & $\mathrm{U}$ & 840 & 840 & $\mathrm{U}$ & 840 & 840 & $\mathrm{U}$ & 840 & 840 & $\mathrm{U}$ & 840 \\
\hline Phenanthrene & 340 & $\mathrm{U}$ & 340 & 330 & $\mathrm{U}$ & 330 & 330 & $\mathrm{U}$ & 330 & 97 & $\mathrm{~J}$ & 330 \\
\hline Phenol & 340 & $\mathrm{U}$ & 340 & 330 & $\mathrm{U}$ & 330 & 330 & $\mathrm{U}$ & 330 & 330 & $\mathrm{U}$ & 330 \\
\hline Pyrene & 340 & $\mathrm{U}$ & 340 & 330 & $\mathrm{U}$ & 330 & 330 & $\mathrm{U}$ & 330 & 350 & & 330 \\
\hline & & & & Attachm & & & 1 & & & heet No. & & of 43 \\
\hline & & & & Originat & & J.M.Ca & ron & & & Date & & $13 / 06$ \\
\hline & & & & Checker & & T.M. B1 & kley & & & Date & & \\
\hline & & & & Calc. $\mathrm{N}$ & & $0100 \mathrm{C}$ & A-V0033 & & & Rev. No. & & 0 \\
\hline
\end{tabular}


Attachment 1. 118-C-1 Verification Sampling Results.

\begin{tabular}{|c|c|c|c|c|c|c|c|c|c|c|c|c|}
\hline \multirow[t]{2}{*}{ Constituents } & \multicolumn{3}{|c|}{$\begin{array}{c}\text { J135R6 } \\
\text { Area } 3 \mathrm{A3} \\
\text { Sample Date } 8 / 28 / 06\end{array}$} & \multicolumn{3}{|c|}{$\begin{array}{c}\text { J135R7 } \\
\text { Area } 3 \text { A4 } \\
\text { Sample Date 8/28/06 }\end{array}$} & \multicolumn{3}{|c|}{$\begin{array}{c}\text { J135R8 } \\
\text { Area } 4 \text { A1 } \\
\text { Sample Date 8/28/06 }\end{array}$} & \multicolumn{3}{|c|}{$\begin{array}{c}\text { J135R9 } \\
\text { Area 4 A2 } \\
\text { Sample Date 8/28/06 } \\
\end{array}$} \\
\hline & $\mu \mathrm{g} / \mathrm{kg}$ & Q & PQL & $\mu \mathrm{g} / \mathrm{kg}$ & $\mathbf{Q}$ & PQL & $\mu \mathrm{g} / \mathrm{kg}$ & $\mathbf{Q}$ & PQL & $\mu \mathrm{g} / \mathrm{kg}$ & $Q$ & PQL \\
\hline \multicolumn{13}{|c|}{ Volatile Organic Compounds } \\
\hline 1,1,1-Trichloroethane & 5 & $\mathrm{U}$ & 5 & 5 & $\mathrm{U}$ & 5 & 5 & $\mathrm{U}$ & 5 & 5 & $\mathrm{U}$ & 5 \\
\hline 1,1,2,2-Tetrachloroethane & 5 & UJ & 5 & 5 & $\mathrm{UJ}$ & 5 & 5 & $\mathrm{UJ}$ & 5 & 5 & UJ & 5 \\
\hline 1,1,2-Trichloroethane & 5 & $\mathrm{U}$ & 5 & 5 & $\mathrm{U}$ & 5 & 5 & $\mathrm{U}$ & 5 & 5 & $\mathrm{U}$ & 5 \\
\hline 1,1-Dichloroethane & 5 & $\mathrm{U}$ & 5 & 5 & $\mathrm{U}$ & 5 & 5 & $\mathrm{U}$ & 5 & 5 & $\mathrm{U}$ & 5 \\
\hline 1,1-Dichloroethene & 5 & $\mathrm{U}$ & 5 & 5 & $\mathrm{U}$ & 5 & 5 & $\mathrm{U}$ & 5 & 5 & $\mathrm{U}$ & 5 \\
\hline 1,2-Dichloroethane & 5 & $\mathrm{U}$ & 5 & 5 & $\mathrm{U}$ & 5 & 5 & $\mathrm{U}$ & 5 & 5 & $\mathrm{U}$ & 5 \\
\hline 1,2-Dichloroethene (total) & 5 & $U$ & 5 & 5 & $\mathrm{U}$ & 5 & 5 & $\mathrm{U}$ & 5 & 5 & $\mathrm{U}$ & 5 \\
\hline 1,2-Dichloropropane & 5 & $\mathrm{U}$ & 5 & 5 & $\mathrm{U}$ & 5 & 5 & $\mathrm{U}$ & 5 & 5 & $\mathrm{U}$ & 5 \\
\hline 2-Butanone & 10 & $\mathrm{U}$ & 10 & 10 & $\mathrm{U}$ & 10 & 10 & $\mathrm{U}$ & 10 & 10 & $\mathrm{U}$ & 10 \\
\hline 2-Hexanone & 10 & $U$ & 10 & 10 & $\mathrm{U}$ & 10 & 10 & $\mathrm{U}$ & 10 & 10 & $\mathrm{U}$ & 10 \\
\hline 4-Methyl-2-pentanone & 10 & $\mathrm{U}$ & 10 & 10 & $\mathrm{U}$ & 10 & 10 & $\mathrm{U}$ & 10 & 10 & $\mathrm{U}$ & 10 \\
\hline Acetone & 10 & $\mathrm{U}$ & 10 & 11 & $\mathrm{~J}$ & 10 & 13 & $\mathrm{~J}$ & 10 & 10 & $\mathrm{U}$ & 10 \\
\hline Benzene & 5 & $\mathrm{U}$ & 5 & 5 & $\mathrm{U}$ & 5 & 5 & $\mathrm{U}$ & 5 & 1 & $\mathrm{~J}$ & 5 \\
\hline Bromodichloromethane & 5 & $\mathrm{U}$ & 5 & 5 & $\mathrm{U}$ & 5 & 5 & $\mathrm{U}$ & 5 & 5 & $\mathrm{U}$ & 5 \\
\hline Bromoform & 5 & U & 5 & 5 & $\mathrm{U}$ & 5 & 5 & $\mathrm{U}$ & 5 & 5 & $\mathrm{U}$ & 5 \\
\hline Bromomethane & 10 & $\mathrm{U}$ & 10 & 10 & $\mathrm{U}$ & 10 & 10 & $\mathrm{U}$ & 10 & 10 & $\mathrm{U}$ & 10 \\
\hline Carbon disulfide & 5 & $\mathrm{U}$ & 5 & 5 & $\mathrm{U}$ & 5 & 5 & $\mathrm{U}$ & 5 & 5 & $\mathrm{U}$ & 5 \\
\hline Carbon tetrachloride & 5 & $\mathrm{U}$ & 5 & 5 & $\mathrm{U}$ & 5 & 5 & $\mathrm{U}$ & 5 & 5 & $\mathrm{U}$ & 5 \\
\hline Chlorobenzene & 5 & $\mathrm{U}$ & 5 & 5 & $\mathrm{U}$ & 5 & 5 & $\mathrm{U}$ & 5 & 5 & $\mathrm{U}$ & 5 \\
\hline Chloroethane & 10 & $\mathrm{U}$ & 10 & 10 & $\mathrm{U}$ & 10 & 10 & $\mathrm{U}$ & 10 & 10 & $\mathrm{U}$ & 10 \\
\hline Chloroform & 5 & $\mathrm{U}$ & 5 & 5 & $\mathrm{U}$ & 5 & 5 & $\mathrm{U}$ & 5 & 5 & $\mathrm{U}$ & 5 \\
\hline Chloromethane & 10 & $\mathrm{U}$ & 10 & 10 & $U$ & 10 & 10 & $\mathrm{U}$ & 10 & 10 & $\mathrm{U}$ & 10 \\
\hline cis-1,2-Dichloroethylene & 5 & $\mathrm{U}$ & 5 & 5 & $\mathrm{U}$ & 5 & 5 & $\mathrm{U}$ & 5 & 5 & $\mathrm{U}$ & 5 \\
\hline cis-1,3-Dichloropropene & 5 & $\mathrm{U}$ & 5 & 5 & $\mathrm{U}$ & 5 & 5 & $\mathrm{U}$ & 5 & 5 & $\mathrm{U}$ & 5 \\
\hline Dibromochloromethane & 5 & $\mathrm{U}$ & 5 & 5 & $\mathrm{U}$ & 5 & 5 & $\mathrm{U}$ & 5 & 5 & $\mathrm{U}$ & 5 \\
\hline Ethylbenzene & 5 & $\mathrm{U}$ & 5 & 5 & $\mathrm{U}$ & 5 & 5 & $\mathrm{U}$ & 5 & 5 & $\mathrm{U}$ & 5 \\
\hline Methylene chloride & 9 & $\mathrm{~B}$ & 5 & 10 & $\mathrm{~B}$ & 5 & 13 & $\mathrm{~B}$ & 5 & 12 & $\mathrm{~B}$ & 5 \\
\hline Styrene & 5 & $\mathrm{U}$ & 5 & 5 & $\mathrm{U}$ & 5 & 5 & $\mathrm{U}$ & 5 & 5 & $\mathrm{U}$ & 5 \\
\hline Tetrachloroethene & 5 & $\mathrm{U}$ & 5 & 5 & $\mathrm{U}$ & 5 & 5 & $\mathrm{U}$ & 5 & 5 & $\mathrm{U}$ & 5 \\
\hline Toluene & 5 & $\mathrm{U}$ & 5 & 5 & $\mathrm{U}$ & 5 & 5 & $\mathrm{U}$ & 5 & 5 & U & 5 \\
\hline trans-1,2-Dichloroethylene & 5 & $\mathrm{U}$ & 5 & 5 & $\mathrm{U}$ & 5 & 5 & $\mathrm{U}$ & 5 & 5 & $\mathrm{U}$ & 5 \\
\hline trans-1,3-Dichloropropene & 5 & $\mathrm{U}$ & 5 & 5 & $\mathrm{U}$ & 5 & 5 & $\mathrm{U}$ & 5 & 5 & U & 5 \\
\hline Trichloroethene & 5 & $\mathrm{U}$ & 5 & 5 & $\mathrm{U}$ & 5 & 5 & $\mathrm{U}$ & 5 & 5 & $\mathrm{U}$ & 5 \\
\hline Vinyl chloride & 10 & $\mathrm{U}$ & 10 & 10 & $\mathrm{U}$ & 10 & 10 & $\mathrm{U}$ & 10 & 10 & $\mathrm{U}$ & 10 \\
\hline Xylenes (total) & 5 & $\mathrm{U}$ & 5 & 5 & $\mathrm{U}$ & 5 & 5 & $\mathrm{U}$ & 5 & 1 & $\mathrm{~J}$ & 5 \\
\hline & & & & Attachm & & & 1 & & & heet No. & & of 43 \\
\hline & & & & Originat & & J. M. Cap & ron & & & Date & & $/ 13 / 06$ \\
\hline & & & & Checked & & T. M. Bla & kley & & & Date & & \\
\hline & & & & Calc. No & & $0100 \mathrm{C}-\mathrm{C}$ & $\mathrm{A}-\mathrm{V} 0033$ & & & Rev. No. & & 0 \\
\hline
\end{tabular}


Attachment 1. 118-C-1 Verification Sampling Results.

\begin{tabular}{|c|c|c|c|c|c|c|c|c|c|c|c|c|}
\hline \multirow[t]{2}{*}{ Constituents } & \multicolumn{3}{|c|}{$\begin{array}{c}\text { J13516 } \\
\text { Area } 4 \text { A3 } \\
\text { Sample Date 8/21/06 } \\
\end{array}$} & \multicolumn{3}{|c|}{$\begin{array}{c}\mathrm{J13517} \\
\text { Area } 4 \mathrm{A4} \\
\text { Sample Date 8/21/06 } \\
\end{array}$} & \multicolumn{3}{|c|}{$\begin{array}{c}\text { J13518 } \\
\text { OB/BCL A1 } \\
\text { Sample Date } 8 / 22 / 06\end{array}$} & \multicolumn{3}{|c|}{$\begin{array}{c}\text { J13519 } \\
\text { OB/BCL A2 } \\
\text { Sample Date } 8 / 22 / 06\end{array}$} \\
\hline & $\mu \mathrm{g} / \mathrm{kg}$ & $Q$ & PQL & $\mu \mathrm{g} / \mathrm{kg}$ & $\mathbf{Q}$ & PQL & $\mu \mathrm{g} / \mathrm{kg}$ & Q & PQL & $\mu \mathrm{g} / \mathrm{kg}$ & $Q$ & PQL \\
\hline \multicolumn{13}{|c|}{ Polychlorinated Biphenyls } \\
\hline Aroclor-1016 & 13 & $\mathrm{U}$ & 13 & 13 & $\mathrm{U}$ & 13 & 13 & $\mathrm{U}$ & 13 & 13 & $\mathrm{U}$ & 13 \\
\hline Aroclor-1221 & 13 & $\mathrm{U}$ & 13 & 13 & $\mathrm{U}$ & 13 & 13 & $\mathrm{U}$ & 13 & 13 & $\mathrm{U}$ & 13 \\
\hline Aroclor-1232 & 13 & $\mathrm{U}$ & 13 & 13 & $\mathrm{U}$ & 13 & 13 & $\mathrm{U}$ & 13 & 13 & $\mathrm{U}$ & 13 \\
\hline Aroclor- 1242 & 13 & $\mathrm{U}$ & 13 & 13 & $\mathrm{U}$ & 13 & 13 & $\mathrm{U}$ & 13 & 13 & $U$ & 13 \\
\hline Aroclor-1248 & 13 & $\mathrm{U}$ & 13 & 13 & $\mathrm{U}$ & 13 & 13 & $\mathrm{U}$ & 13 & 13 & $\mathrm{U}$ & 13 \\
\hline \begin{tabular}{|l|} 
Aroclor-1254 \\
\end{tabular} & 13 & $\mathrm{U}$ & 13 & 16 & & 13 & 13 & $\mathrm{U}$ & 13 & 4.9 & $\mathrm{~J}$ & 13 \\
\hline Aroclor-1260 & 13 & $\mathrm{U}$ & 13 & 13 & $\mathrm{U}$ & 13 & 13 & $\mathrm{U}$ & 13 & 13 & $\mathrm{U}$ & 13 \\
\hline \multicolumn{13}{|c|}{ Semivolatile Organic Compounds } \\
\hline 1,2,4-Trichlorobenzene & 330 & $\mathrm{U}$ & 330 & 330 & $\mathrm{U}$ & 330 & 330 & $\mathrm{U}$ & 330 & 340 & $\mathrm{U}$ & 340 \\
\hline 1,2-Dichlorobenzene & 330 & $\mathrm{U}$ & 330 & 330 & $\mathrm{U}$ & 330 & 330 & $\mathrm{U}$ & 330 & 340 & $\mathrm{U}$ & 340 \\
\hline 1,3-Dichlorobenzene & 330 & $\mathrm{U}$ & 330 & 330 & $\mathrm{U}$ & 330 & 330 & $\mathrm{U}$ & 330 & 340 & $\mathrm{U}$ & 340 \\
\hline 1,4-Dichlorobenzene & 330 & $\mathrm{U}$ & 330 & 330 & $\mathrm{U}$ & 330 & 330 & $\mathrm{U}$ & 330 & 340 & $\mathrm{U}$ & 340 \\
\hline 2,4,5-Trichlorophenol & 840 & $\mathrm{U}$ & 840 & 840 & $\mathrm{U}$ & 840 & 840 & $\mathrm{U}$ & 840 & 840 & $\mathrm{U}$ & 840 \\
\hline 2,4,6-Trichlorophenol & 330 & $\mathrm{U}$ & 330 & 330 & $\mathrm{U}$ & 330 & 330 & $\mathrm{U}$ & 330 & 340 & $\mathrm{U}$ & 340 \\
\hline 2,4-Dichlorophenol & 330 & $\mathrm{U}$ & 330 & 330 & $\mathrm{U}$ & 330 & 330 & $\mathrm{U}$ & 330 & 340 & $\mathrm{U}$ & 340 \\
\hline 2,4-Dimethylphenol & 330 & $\mathrm{U}$ & 330 & 330 & $\mathrm{U}$ & 330 & 330 & $\mathrm{U}$ & 330 & 340 & U & 340 \\
\hline 2,4-Dinitrophenol & 840 & UJ & 840 & 840 & UJ & 840 & 840 & $\mathrm{U}$ & 840 & 840 & $\mathrm{U}$ & 840 \\
\hline 2,4-Dinitrotoluene & 330 & $\mathrm{U}$ & 330 & 330 & $\mathrm{U}$ & 330 & 330 & $\mathrm{U}$ & 330 & 340 & $\mathrm{U}$ & 340 \\
\hline 2,6-Dinitrotoluene & 330 & $\mathrm{U}$ & 330 & 330 & $\mathrm{U}$ & 330 & 330 & $\mathrm{U}$ & 330 & 340 & $\mathrm{U}$ & 340 \\
\hline 2-Chloronaphthalene & 330 & $\mathrm{U}$ & 330 & 330 & $\mathrm{U}$ & 330 & 330 & $\mathrm{U}$ & 330 & 340 & $\mathrm{U}$ & 340 \\
\hline 2-Chlorophenol & 330 & $\mathrm{U}$ & 330 & 330 & $\mathrm{U}$ & 330 & 330 & $\mathrm{U}$ & 330 & 340 & $\mathrm{U}$ & 340 \\
\hline 2-Methylnaphthalene & 330 & $\mathrm{U}$ & 330 & 330 & $\mathrm{U}$ & 330 & 330 & $\mathrm{U}$ & 330 & 340 & $\mathrm{U}$ & 340 \\
\hline 2-Methylphenol (cresol, o-) & 330 & $\mathrm{U}$ & 330 & 330 & $\mathrm{U}$ & 330 & 330 & $\mathrm{U}$ & 330 & 340 & $\mathrm{U}$ & 340 \\
\hline 2-Nitroaniline & 840 & $\mathrm{U}$ & 840 & 840 & $\mathrm{U}$ & 840 & 840 & $\mathrm{U}$ & 840 & 840 & $\mathrm{U}$ & 840 \\
\hline 2-Nitrophenol & 330 & $\mathrm{U}$ & 330 & 330 & $\mathrm{U}$ & 330 & 330 & $\mathrm{U}$ & 330 & 340 & $\mathrm{U}$ & 340 \\
\hline 3,3'-Dichlorobenzidine & 330 & $\mathrm{U}$ & 330 & 330 & $\mathrm{U}$ & 330 & 330 & U & 330 & 340 & $\mathrm{U}$ & 340 \\
\hline 3-Nitroaniline & 840 & $\mathrm{U}$ & 840 & 840 & $\mathrm{U}$ & 840 & 840 & $\mathrm{U}$ & 840 & 840 & $\mathrm{U}$ & 840 \\
\hline 4,6-Dinitro-2-methylphenol & 840 & $\mathrm{U}$ & 840 & 840 & $\mathrm{U}$ & 840 & 840 & $\mathrm{U}$ & 840 & 840 & $\mathrm{U}$ & 840 \\
\hline 4-Bromophenyl-phenylether & 330 & $\mathrm{U}$ & 330 & 330 & $\mathrm{U}$ & 330 & 330 & $U$ & 330 & 340 & $\mathrm{U}$ & 340 \\
\hline 4-Chloro-3-methylphenol & 330 & $\mathrm{U}$ & 330 & 330 & $\mathrm{U}$ & 330 & 330 & $\mathrm{U}$ & 330 & 340 & $\mathrm{U}$ & 340 \\
\hline 4-Chloroaniline & 330 & $\mathrm{U}$ & 330 & 330 & $\mathrm{U}$ & 330 & 330 & $\mathrm{U}$ & 330 & 340 & $\mathrm{U}$ & 340 \\
\hline 4-Chlorophenyl-phenylether & 330 & $\mathrm{U}$ & 330 & 330 & $\mathrm{U}$ & 330 & 330 & $\mathrm{U}$ & 330 & 340 & $\mathrm{U}$ & 340 \\
\hline 4-Methylphenol (p-cresol) & 330 & $\mathrm{U}$ & 330 & 330 & $\mathrm{U}$ & 330 & 330 & $\mathrm{U}$ & 330 & 340 & $\mathrm{U}$ & 340 \\
\hline 4-Nitroaniline & 840 & $\mathrm{U}$ & 840 & 840 & $U$ & 840 & 840 & $\mathrm{U}$ & 840 & 840 & $\mathrm{U}$ & 840 \\
\hline 4-Nitrophenol & 840 & $\mathrm{U}$ & 840 & 840 & $\mathrm{U}$ & 840 & 840 & $\mathrm{U}$ & 840 & 840 & $\mathrm{U}$ & 840 \\
\hline Acenaphthene & 330 & $\mathrm{U}$ & 330 & 330 & $\mathrm{U}$ & 330 & 330 & $\mathrm{U}$ & 330 & 340 & $\mathrm{U}$ & 340 \\
\hline Acenaphthylene & 330 & $\mathrm{U}$ & 330 & 330 & $\mathrm{U}$ & 330 & 330 & $\mathrm{U}$ & 330 & 340 & $\mathrm{U}$ & 340 \\
\hline Anthracene & 330 & $\mathrm{U}$ & 330 & 330 & $\mathrm{U}$ & 330 & 330 & $\mathrm{U}$ & 330 & 340 & $\mathrm{U}$ & 340 \\
\hline Benzo(a)anthracene & 330 & $\mathrm{U}$ & 330 & 330 & $\mathrm{U}$ & 330 & 330 & $\mathrm{U}$ & 330 & 340 & $\mathrm{U}$ & 340 \\
\hline Benzo(a)pyrene & 330 & $\mathrm{U}$ & 330 & 330 & $\mathrm{U}$ & 330 & 330 & U & 330 & 340 & $\mathrm{U}$ & 340 \\
\hline Benzo(b)fluoranthene & 330 & $\mathrm{U}$ & 330 & 330 & $\mathrm{U}$ & 330 & 330 & $\mathrm{U}$ & 330 & 340 & $\mathrm{U}$ & 340 \\
\hline Benzo $(\mathrm{g}, \mathrm{h}, \mathrm{i})$ perylene & 330 & $\mathrm{U}$ & 330 & 330 & $\mathrm{U}$ & 330 & 330 & $\mathrm{U}$ & 330 & 340 & $\mathrm{U}$ & 340 \\
\hline Benzo(k)fluoranthene & 330 & $\mathrm{U}$ & 330 & 330 & $\mathrm{U}$ & 330 & 330 & $\mathrm{U}$ & 330 & 340 & $\mathrm{U}$ & 340 \\
\hline bis(2-Chloro-1-methylethyl)ether & 330 & $\mathrm{U}$ & 330 & 330 & $\mathrm{U}$ & 330 & 330 & $\mathrm{U}$ & 330 & 340 & $\mathrm{U}$ & 340 \\
\hline bis(2-Chloroethoxy)methane & 330 & $\mathrm{U}$ & 330 & 330 & $\mathrm{U}$ & 330 & 330 & $\mathrm{U}$ & 330 & 340 & $\mathrm{U}$ & 340 \\
\hline bis(2-Chloroethyl)ether & 330 & $\mathrm{U}$ & 330 & 330 & $\mathrm{U}$ & 330 & 330 & $\mathrm{U}$ & 330 & 340 & $\mathrm{U}$ & 340 \\
\hline bis(2-Ethylhexyl)phthalate & 330 & $\mathrm{U}$ & 330 & 330 & $\mathrm{U}$ & 330 & 330 & $\mathrm{U}$ & 330 & 330 & $\mathrm{U}$ & 340 \\
\hline & & & & Attachm & & & 1 & & & et No. & & of 43 \\
\hline & & & & Originat & & J.M. Ca & & & & Date & & $113 / 06$ \\
\hline & & & & Checked & & T.M.BI & kley & & & Date & & \\
\hline & & & & Calc. No & & $0100 \mathrm{C}-\mathrm{C}$ & $\mathrm{A}-\mathrm{V} 0033$ & & & Rev. No. & & 0 \\
\hline
\end{tabular}


Attachment 1. 118-C-1 Verification Sampling Results.

\begin{tabular}{|c|c|c|c|c|c|c|c|c|c|c|c|c|}
\hline \multirow[t]{2}{*}{ Constituents } & \multicolumn{3}{|c|}{$\begin{array}{c}\text { J13516 } \\
\text { Area } 4 \text { A3 } \\
\text { Sample Date 8/21/06 } \\
\end{array}$} & \multicolumn{3}{|c|}{\begin{tabular}{|c|}
$\mathbf{J 1 3 5 1 7}$ \\
Area 4 A4 \\
Sample Date 8/21/06 \\
\end{tabular}} & \multicolumn{3}{|c|}{$\begin{array}{c}\text { J13518 } \\
\text { OB/BCL A1 } \\
\text { Sample Date 8/22/06 }\end{array}$} & \multicolumn{3}{|c|}{$\begin{array}{c}\text { J13519 } \\
\text { OB/BCL A2 } \\
\text { Sample Date 8/22/06 }\end{array}$} \\
\hline & $\mu \mathrm{g} / \mathrm{kg}$ & $\mathbf{Q}$ & PQL & $\mu \mathrm{g} / \mathrm{kg}$ & $\mathbf{Q}$ & PQL & $\mu \mathrm{g} / \mathrm{kg}$ & Q & PQL & $\mu \mathrm{g} / \mathrm{kg}$ & $Q$ & $\mathbf{P Q L}$ \\
\hline \multicolumn{13}{|c|}{ Semivolatile Organic Compounds (continued) } \\
\hline Butylbenzylphthalate & 330 & $\mathrm{U}$ & 330 & 330 & $\mathrm{U}$ & 330 & 330 & $\mathrm{U}$ & 330 & 340 & $\mathrm{U}$ & 340 \\
\hline Carbazole & 330 & $\mathrm{U}$ & 330 & 330 & $\mathrm{U}$ & 330 & 330 & $\mathrm{U}$ & 330 & 340 & $\mathrm{U}$ & 340 \\
\hline Chrysene & 330 & $\mathrm{U}$ & 330 & 330 & $\mathrm{U}$ & 330 & 330 & $\mathrm{U}$ & 330 & 340 & $\mathrm{U}$ & 340 \\
\hline Di-n-butylphthalate & 330 & $\mathrm{U}$ & 330 & 26 & $\mathrm{~J}$ & 330 & 330 & $\mathrm{U}$ & 330 & 330 & $\mathrm{U}$ & 340 \\
\hline Di-n-octylphthalate & 330 & U & 330 & 330 & $\mathrm{U}$ & 330 & 330 & $\mathrm{U}$ & 330 & 340 & $\mathrm{U}$ & 340 \\
\hline Dibenz $(\mathrm{a}, \mathrm{h})$ anthracene & 330 & $U$ & 330 & 330 & $\mathrm{U}$ & 330 & 330 & $\mathrm{U}$ & 330 & 340 & $\mathrm{U}$ & 340 \\
\hline Dibenzofuran & 330 & $\mathrm{U}$ & 330 & 330 & $\mathrm{U}$ & 330 & 330 & $\mathrm{U}$ & 330 & 340 & $\mathrm{U}$ & 340 \\
\hline Diethylphthalate & 330 & $\mathrm{U}$ & 330 & 330 & $\mathrm{U}$ & 330 & 330 & $\mathrm{U}$ & 330 & 340 & $\mathrm{U}$ & 340 \\
\hline Dimethylphthalate & 330 & $\mathrm{U}$ & 330 & 330 & $\mathrm{U}$ & 330 & 330 & $\mathrm{U}$ & 330 & 340 & $U$ & 340 \\
\hline Fluoranthene & 330 & $\mathrm{U}$ & 330 & 330 & $\mathrm{U}$ & 330 & 330 & UJ & 330 & 340 & UJ & 340 \\
\hline Fluorene & 330 & $\mathrm{U}$ & 330 & 330 & $\mathrm{U}$ & 330 & 330 & $\bar{U}$ & 330 & 340 & $\mathrm{U}$ & 340 \\
\hline Hexachlorobenzene & 330 & $\mathrm{U}$ & 330 & 330 & $\mathrm{U}$ & 330 & 330 & $\mathrm{U}$ & 330 & 340 & $\mathrm{U}$ & 340 \\
\hline Hexachlorobutadiene & 330 & $\mathrm{U}$ & 330 & 330 & $\mathrm{U}$ & 330 & 330 & $\mathrm{U}$ & 330 & 340 & U & 340 \\
\hline Hexachlorocyclopentadiene & 330 & $\mathrm{U}$ & 330 & 330 & $\mathrm{U}$ & 330 & 330 & $\mathrm{U}$ & 330 & 340 & $\mathrm{U}$ & 340 \\
\hline Hexachloroethane & 330 & $\mathrm{U}$ & 330 & 330 & $\mathrm{U}$ & 330 & 330 & $\mathrm{U}$ & 330 & 340 & $\mathrm{U}$ & 340 \\
\hline Indeno( $1,2,3$-cd)pyrene & 330 & $\mathrm{U}$ & 330 & 330 & $\mathrm{U}$ & 330 & 330 & $\mathrm{U}$ & 330 & 340 & $\mathrm{U}$ & 340 \\
\hline Isophorone & 330 & $\mathrm{U}$ & 330 & 330 & U & 330 & 330 & $\mathrm{U}$ & 330 & 340 & $\mathrm{U}$ & 340 \\
\hline N-Nitroso-di-n-dipropylamine & 330 & $\mathrm{U}$ & 330 & 330 & $\mathrm{U}$ & 330 & 330 & $\mathrm{U}$ & 330 & 340 & $U$ & 340 \\
\hline N-Nitrosodiphenylamine & 330 & $\mathrm{U}$ & 330 & 330 & $\mathrm{U}$ & 330 & 330 & $\mathrm{U}$ & 330 & 340 & $\mathrm{U}$ & 340 \\
\hline Naphthalene & 330 & $\mathrm{U}$ & 330 & 330 & $\mathrm{U}$ & 330 & 330 & $\mathrm{U}$ & 330 & 340 & $\mathrm{U}$ & 340 \\
\hline Nitrobenzene & 330 & $\mathrm{U}$ & 330 & 330 & $\mathrm{U}$ & 330 & 330 & $\mathrm{U}$ & 330 & 340 & $\mathrm{U}$ & 340 \\
\hline Pentachlorophenol & 840 & $\mathrm{U}$ & 840 & 840 & $\mathrm{U}$ & 840 & 840 & $\mathrm{U}$ & 840 & 840 & U & 840 \\
\hline Phenanthrene & 330 & $\mathrm{U}$ & 330 & 330 & $\mathrm{U}$ & 330 & 330 & $\mathrm{UJ}$ & 330 & 340 & UJ & 340 \\
\hline Phenol & 330 & $\mathrm{U}$ & 330 & 330 & $\mathrm{U}$ & 330 & 330 & $\mathrm{U}$ & 330 & 340 & U & 340 \\
\hline Pyrene & 330 & U & 330 & 330 & $\mathrm{U}$ & 330 & 330 & UJ & 330 & 340 & UJ & 340 \\
\hline
\end{tabular}

Attachment Originator

Checked
Sheet No. 26 of 43

Date $\frac{26 / 12 / 06}{12 / 06}$

Date

Rev. No. 
Attachment 1. 118-C-1 Verification Sampling Results.

\begin{tabular}{|c|c|c|c|c|c|c|c|c|c|c|c|c|}
\hline \multirow[t]{2}{*}{ Constituents } & \multicolumn{3}{|c|}{$\begin{array}{c}\text { J135T0 } \\
\text { Area } 4 \text { A3 } \\
\text { Sample Date 8/28/06 } \\
\end{array}$} & \multicolumn{3}{|c|}{$\begin{array}{c}\text { J135T1 } \\
\text { Area } 4 \text { A4 } \\
\text { Sample Date } 8 / 28 / 06 \\
\end{array}$} & \multicolumn{3}{|c|}{$\begin{array}{c}\text { J135T2 } \\
\text { OB/BCL A1 } \\
\text { Sample Date } 8 / 28 / 06\end{array}$} & \multicolumn{3}{|c|}{$\begin{array}{c}\text { J135T3 } \\
\text { OB/BCL A2 } \\
\text { Sample Date } 8 / 28 / 06\end{array}$} \\
\hline & $\mu \mathrm{g} / \mathrm{kg}$ & $\mathbf{Q}$ & PQL & $\mu \mathrm{g} / \mathrm{kg}$ & $\mathbf{Q}$ & PQL & $\mu \mathrm{g} / \mathrm{kg}$ & $\mathbf{Q}$ & PQL & $\mu \mathrm{g} / \mathrm{kg}$ & $Q$ & PQL \\
\hline \multicolumn{13}{|c|}{ Volatile Organic Compounds } \\
\hline 1,1,1-Trichloroethane & 6 & $\mathrm{U}$ & 6 & 6 & $\mathrm{U}$ & 6 & 5 & $\mathrm{U}$ & 5 & 6 & $\mathrm{U}$ & 6 \\
\hline 1,1,2,2-Tetrachloroethane & 6 & $\mathrm{UJ}$ & 6 & 6 & UJ & 6 & 5 & UJ & 5 & 6 & UJ & 6 \\
\hline 1,1,2-Trichloroethane & 6 & U & 6 & 6 & $\mathrm{U}$ & 6 & 5 & $\mathrm{U}$ & 5 & 6 & $\mathrm{U}$ & 6 \\
\hline 1,1-Dichloroethane & 6 & $\mathrm{U}$ & 6 & 6 & $\mathrm{U}$ & 6 & 5 & $\mathrm{U}$ & 5 & 6 & $\mathrm{U}$ & 6 \\
\hline 1,1-Dichloroethene & 6 & $\mathrm{U}$ & 6 & 6 & $\mathrm{U}$ & 6 & 5 & $\mathrm{U}$ & 5 & 6 & $\mathrm{U}$ & 6 \\
\hline 1,2-Dichloroethane & 6 & $\mathrm{U}$ & 6 & 6 & $\mathrm{U}$ & 6 & 5 & $\mathrm{U}$ & 5 & 6 & $\mathrm{U}$ & 6 \\
\hline 1,2-Dichloroethene (total) & 6 & $\mathrm{U}$ & 6 & 6 & $\mathrm{U}$ & 6 & 5 & $\mathrm{U}$ & 5 & 6 & $\mathrm{U}$ & 6 \\
\hline 1,2-Dichloropropane & 6 & $\mathrm{U}$ & 6 & 6 & $\mathrm{U}$ & 6 & 5 & $\mathrm{U}$ & 5 & 6 & $\mathrm{U}$ & 6 \\
\hline 2-Butanone & 11 & $\mathrm{U}$ & 11 & 11 & $\mathrm{U}$ & 11 & 10 & $\mathrm{U}$ & 10 & 11 & $U$ & 11 \\
\hline 2-Hexanone & 11 & $\mathrm{U}$ & 11 & 11 & $\mathrm{U}$ & 11 & 10 & $\mathrm{U}$ & 10 & 11 & $\mathrm{U}$ & 11 \\
\hline 4-Methyl-2-pentanone & 11 & $\mathrm{U}$ & 11 & 11 & $\mathrm{U}$ & 11 & 10 & $\mathrm{U}$ & 10 & 11 & $\mathrm{U}$ & 11 \\
\hline Acetone & 9 & $\mathrm{~J}$ & 11 & 9 & $\mathrm{~J}$ & 11 & 10 & $U$ & 10 & 11 & $\mathrm{U}$ & 11 \\
\hline Benzene & 6 & $\mathrm{U}$ & 6 & 6 & $\mathrm{U}$ & 6 & 5 & $\mathrm{U}$ & 5 & 6 & $\mathrm{U}$ & 6 \\
\hline Bromodichloromethane & 6 & $\mathrm{U}$ & 6 & 6 & $\mathrm{U}$ & 6 & 5 & $U$ & 5 & 6 & $\mathrm{U}$ & 6 \\
\hline Bromoform & 6 & $\mathrm{U}$ & 6 & 6 & $\mathrm{U}$ & 6 & 5 & $\mathrm{U}$ & 5 & 6 & $U$ & 6 \\
\hline Bromomethane & 11 & $\mathrm{U}$ & 11 & 11 & $\mathrm{U}$ & 11 & 10 & $\mathrm{U}$ & 10 & 11 & $\mathrm{U}$ & 11 \\
\hline Carbon disulfide & 6 & $\mathrm{U}$ & 6 & 6 & $\mathrm{U}$ & 6 & 5 & $\mathrm{U}$ & 5 & 6 & $\mathrm{U}$ & 6 \\
\hline Carbon tetrachloride & 6 & $\mathrm{U}$ & 6 & 6 & $\mathrm{U}$ & 6 & 5 & $\mathrm{U}$ & 5 & 6 & $\mathrm{U}$ & 6 \\
\hline Chlorobenzene & 6 & $\mathrm{U}$ & 6 & 6 & $\mathrm{U}$ & 6 & 5 & $\mathrm{U}$ & 5 & 6 & $\mathrm{U}$ & 6 \\
\hline Chloroethane & 11 & $\mathrm{U}$ & 11 & 11 & $\mathrm{U}$ & 11 & 10 & $\bar{U}$ & 10 & 11 & $U$ & 11 \\
\hline \begin{tabular}{|l} 
Chloroform \\
\end{tabular} & 6 & $\mathrm{U}$ & 6 & 6 & $\mathrm{U}$ & 6 & 5 & $\mathrm{U}$ & 5 & 6 & $\mathrm{U}$ & 6 \\
\hline Chloromethane & 11 & $U$ & 11 & 11 & $\mathrm{U}$ & 11 & 10 & $\mathrm{U}$ & 10 & 11 & $\mathrm{U}$ & 11 \\
\hline cis-1,2-Dichloroethylene & 6 & $\mathrm{U}$ & 6 & 6 & $\mathrm{U}$ & 6 & 5 & $\mathrm{U}$ & 5 & 6 & $\mathrm{U}$ & 6 \\
\hline cis-1,3-Dichloropropene & 6 & $\mathrm{U}$ & 6 & 6 & $\mathrm{U}$ & 6 & 5 & $\mathrm{U}$ & 5 & 6 & $\mathrm{U}$ & 6 \\
\hline Dibromochloromethane & 6 & $\mathrm{U}$ & 6 & 6 & $\mathrm{U}$ & 6 & 5 & $\mathrm{U}$ & 5 & 6 & $\mathrm{U}$ & 6 \\
\hline \begin{tabular}{|l|} 
Ethylbenzene \\
\end{tabular} & 6 & 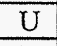 & 6 & 6 & $\mathrm{U}$ & 6 & 5 & $\mathrm{U}$ & 5 & 6 & $\mathrm{U}$ & 6 \\
\hline Methylene chloride & 14 & $\mathrm{~B}$ & 6 & 11 & $\mathrm{~B}$ & 6 & 9 & $\mathrm{~B}$ & 5 & 8 & $\mathrm{~B}$ & 6 \\
\hline Styrene & 6 & $\mathrm{U}$ & 6 & 6 & $\mathrm{U}$ & 6 & 5 & $\mathrm{U}$ & 5 & 6 & $\mathrm{U}$ & 6 \\
\hline Tetrachloroethene & 6 & $\mathrm{U}$ & 6 & 6 & $\mathrm{U}$ & 6 & 5 & $\mathrm{U}$ & 5 & 6 & $\mathrm{U}$ & 6 \\
\hline Toluene & 6 & $\mathrm{U}$ & 6 & 6 & $\mathrm{U}$ & 6 & 5 & $\mathrm{U}$ & 5 & 6 & $\mathrm{U}$ & 6 \\
\hline trans-1,2-Dichloroethylene & 6 & $\mathrm{U}$ & 6 & 6 & $\mathrm{U}$ & 6 & 5 & $\mathrm{U}$ & 5 & 6 & $\bar{U}$ & 6 \\
\hline trans-1,3-Dichloropropene & 6 & $\mathrm{U}$ & 6 & 6 & $\mathrm{U}$ & 6 & 5 & $\mathrm{U}$ & 5 & 6 & $\mathrm{U}$ & 6 \\
\hline Trichloroethene & 6 & $\mathrm{U}$ & 6 & 6 & $\mathrm{U}$ & 6 & 5 & $\mathrm{U}$ & 5 & 6 & $\mathrm{U}$ & 6 \\
\hline Vinyl chloride & 11 & $\mathrm{U}$ & 11 & 11 & $\mathrm{U}$ & 11 & 10 & $\mathrm{U}$ & 10 & 11 & $\mathrm{U}$ & 11 \\
\hline Xylenes (total) & 6 & $\mathrm{U}$ & 6 & 6 & $\mathrm{U}$ & 6 & 5 & $\mathrm{U}$ & 5 & 6 & $U$ & 6 \\
\hline
\end{tabular}

Attachment

Originator

Checked

Calc. No.

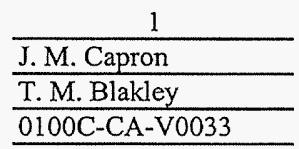

Sheet No. $\frac{27 \text { of } 43}{12 / 13 / 06}$
Date
Date
Rev. No. 
Attachment 1. 118-C-1 Verification Sampling Results.

\begin{tabular}{|c|c|c|c|c|c|c|c|c|c|c|c|c|}
\hline \multirow[t]{2}{*}{ Constituents } & \multicolumn{3}{|c|}{$\begin{array}{c}\text { J13520 } \\
\text { OB/BCL A3 } \\
\text { Sample Date 8/22/06 }\end{array}$} & \multicolumn{3}{|c|}{$\begin{array}{c}\text { J135N5 } \\
\text { OB/BCL A4 } \\
\text { Sample Date 8/22/06 }\end{array}$} & \multicolumn{3}{|c|}{$\begin{array}{c}\text { J13522 } \\
\text { SPA A1 } \\
\text { Sample Date } 9 / 11 / 06\end{array}$} & \multicolumn{3}{|c|}{$\begin{array}{c}\mathbf{J 1 3 5 2 3} \\
\text { SPA A2 } \\
\text { Sample Date } 9 / 11 / 06 \\
\end{array}$} \\
\hline & $\mu \mathrm{g} / \mathrm{kg}$ & $\mathbf{Q}$ & PQL & $\mu \mathrm{g} / \mathrm{kg}$ & $\mathbf{Q}$ & PQL & $\mu \mathrm{g} / \mathrm{kg}$ & $\mathbf{Q}$ & PQL & $\mu \mathrm{g} / \mathrm{kg}$ & $\mathbf{Q}$ & PQL \\
\hline \multicolumn{13}{|c|}{ Polychlorinated Biphenyls } \\
\hline Aroclor-1016 & 13 & $\mathrm{U}$ & 13 & 13 & $\mathrm{U}$ & 13 & 13 & $\mathrm{U}$ & 13 & 13 & $\mathrm{U}$ & 13 \\
\hline Aroclor-1221 & 13 & $\mathrm{U}$ & 13 & 13 & $\mathrm{U}$ & 13 & 13 & $\mathrm{U}$ & 13 & 13 & $\mathrm{U}$ & 13 \\
\hline \begin{tabular}{|l|} 
Aroclor- 1232 \\
\end{tabular} & 13 & $\mathrm{U}$ & 13 & 13 & $\mathrm{U}$ & 13 & 13 & $\mathrm{U}$ & 13 & 13 & $\mathrm{U}$ & 13 \\
\hline Aroclor -1242 & 13 & $\mathrm{U}$ & 13 & 13 & $\mathrm{U}$ & 13 & 13 & $\mathrm{U}$ & 13 & 13 & $\mathrm{U}$ & 13 \\
\hline Aroclor- 1248 & 13 & $\mathrm{U}$ & 13 & 13 & $\mathrm{U}$ & 13 & 13 & $\mathrm{U}$ & 13 & 13 & $\mathrm{U}$ & 13 \\
\hline Aroclor- 1254 & 5.3 & $\mathrm{~J}$ & 13 & 13 & $\mathrm{U}$ & 13 & 13 & $\mathrm{U}$ & 13 & 13 & $\mathrm{U}$ & 13 \\
\hline Aroclor-1260 & 13 & $\mathrm{U}$ & 13 & 13 & $\mathrm{U}$ & 13 & 3.7 & $\mathrm{~J}$ & 13 & 13 & $\mathrm{U}$ & 13 \\
\hline \multicolumn{13}{|c|}{ Semivolatile Organic Compounds } \\
\hline 1,2,4-Trichlorobenzene & 330 & $\mathrm{U}$ & 330 & 330 & $\mathrm{U}$ & 330 & 330 & UJ & 330 & 330 & UJ & 330 \\
\hline 1,2-Dichlorobenzene & 330 & U & 330 & 330 & $\mathrm{U}$ & 330 & 330 & $\mathrm{U}$ & 330 & 330 & $\mathrm{U}$ & 330 \\
\hline 1,3-Dichlorobenzene & 330 & $\mathrm{U}$ & 330 & 330 & $\mathrm{U}$ & 330 & 330 & $\mathrm{U}$ & 330 & 330 & $\mathrm{U}$ & 330 \\
\hline 1,4-Dichlorobenzene & 330 & $\mathrm{U}$ & 330 & 330 & $\mathrm{U}$ & 330 & 330 & $\mathrm{U}$ & 330 & 330 & $\mathrm{U}$ & 330 \\
\hline 2,4,5-Trichlorophenol & 840 & $\mathrm{U}$ & 840 & 830 & $\mathrm{U}$ & 830 & 840 & $\mathrm{U}$ & 840 & 840 & $\mathrm{U}$ & 840 \\
\hline 2,4,6-Trichlorophenol & 330 & $\mathrm{U}$ & 330 & 330 & $\mathrm{U}$ & 330 & 330 & $\mathrm{U}$ & 330 & 330 & $\mathrm{U}$ & 330 \\
\hline 2,4-Dichlorophenol & 330 & $\mathrm{U}$ & 330 & 330 & $\mathrm{U}$ & 330 & 330 & UJ & 330 & 330 & UJ & 330 \\
\hline 2,4-Dimethylphenol & 330 & $\mathrm{U}$ & 330 & 330 & U & 330 & 330 & UJ & 330 & 330 & UJ & 330 \\
\hline 2,4-Dinitrophenol & 840 & $\mathrm{U}$ & 840 & 830 & $U$ & 830 & 840 & UR & 840 & 840 & UR & 840 \\
\hline 2,4-Dinitrotoluene & 330 & $\mathrm{U}$ & 330 & 330 & $\mathrm{U}$ & 330 & 330 & $\mathrm{U}$ & 330 & 330 & $\mathrm{U}$ & 330 \\
\hline 2,6-Dinitrotoluene & 330 & $\mathrm{U}$ & 330 & 330 & $\mathrm{U}$ & 330 & 330 & U & 330 & 330 & $\mathrm{U}$ & 330 \\
\hline 2-Chloronaphthalene & 330 & $\mathrm{U}$ & 330 & 330 & $\mathrm{U}$ & 330 & 330 & $\mathrm{U}$ & 330 & 330 & $\mathrm{U}$ & 330 \\
\hline 2-Chlorophenol & 330 & $\mathrm{U}$ & 330 & 330 & $\mathrm{U}$ & 330 & 330 & $\mathrm{U}$ & 330 & 330 & $\mathrm{U}$ & 330 \\
\hline 2-Methylnaphthalene & 330 & U & 330 & 330 & U & 330 & 330 & UJ & 330 & 330 & UJ & 330 \\
\hline 2-Methylphenol (cresol, o-) & 330 & U & 330 & 330 & $\mathrm{U}$ & 330 & 330 & UJ & 330 & 330 & UJ & 330 \\
\hline 2-Nitroaniline & 840 & $\mathrm{U}$ & 840 & 830 & $\mathrm{U}$ & 830 & 840 & U & 840 & 840 & $\mathrm{U}$ & 840 \\
\hline 2-Nitrophenol & 330 & $\mathrm{U}$ & 330 & 330 & $\mathrm{U}$ & 330 & 330 & UJ & 330 & 330 & UJ & 330 \\
\hline 3,3'-Dichlorobenzidine & 330 & $\mathrm{U}$ & 330 & 330 & $\mathrm{U}$ & 330 & 330 & \begin{tabular}{|l}
$U$ \\
\end{tabular} & 330 & 330 & $\mathrm{U}$ & 330 \\
\hline 3-Nitroaniline & 840 & $\mathrm{U}$ & 840 & 830 & $\mathrm{U}$ & 830 & 840 & $\mathrm{U}$ & 840 & 840 & $\mathrm{U}$ & 840 \\
\hline 4,6-Dinitro-2-methylphenol & 840 & $\mathrm{U}$ & 840 & 830 & $\mathrm{U}$ & 830 & 840 & $\mathrm{U}$ & 840 & 840 & $\mathrm{U}$ & 840 \\
\hline 4-Bromophenyl-phenylether & 330 & $\mathrm{U}$ & 330 & 330 & $\mathrm{U}$ & 330 & 330 & $\mathrm{U}$ & 330 & 330 & U & 330 \\
\hline 4-Chloro-3-methylphenol & 330 & $\mathrm{U}$ & 330 & 330 & U & 330 & 330 & UJ & 330 & 330 & UJ & 330 \\
\hline 4-Chloroaniline & 330 & $\mathrm{U}$ & 330 & 330 & $\mathrm{U}$ & 330 & 330 & $\mathrm{U}$ & 330 & 330 & $\mathrm{U}$ & 330 \\
\hline 4-Chlorophenyl-phenylether & 330 & $\mathrm{U}$ & 330 & 330 & $\mathrm{U}$ & 330 & 330 & $\mathrm{U}$ & 330 & 330 & $\mathrm{U}$ & 330 \\
\hline 4-Methylphenol (p-cresol) & 330 & $\mathrm{U}$ & 330 & 330 & $\mathrm{U}$ & 330 & 330 & UJ & 330 & 330 & UJ & 330 \\
\hline 4-Nitroaniline & 840 & $\mathrm{U}$ & 840 & 830 & $\mathrm{U}$ & 830 & 840 & $\mathrm{U}$ & 840 & 840 & $\mathrm{U}$ & 840 \\
\hline 4-Nitrophenol & 840 & $\mathrm{U}$ & 840 & 830 & $\mathrm{U}$ & 830 & 840 & $\mathrm{U}$ & 840 & 840 & $\mathrm{U}$ & 840 \\
\hline Acenaphthene & 330 & $\mathrm{U}$ & 330 & 330 & $\mathrm{U}$ & 330 & 330 & $\mathrm{U}$ & 330 & 330 & $\mathrm{U}$ & 330 \\
\hline Acenaphthylene & 330 & $\mathrm{U}$ & 330 & 330 & $\mathrm{U}$ & 330 & 330 & $\mathrm{U}$ & 330 & 330 & $\mathrm{U}$ & 330 \\
\hline Anthracene & 330 & $\mathrm{U}$ & 330 & 330 & $\mathrm{U}$ & 330 & 330 & $\mathrm{U}$ & 330 & 330 & $\mathrm{U}$ & 330 \\
\hline Benzo(a)anthracene & 330 & $\mathrm{U}$ & 330 & 38 & $\mathrm{~J}$ & 330 & 46 & $\mathrm{~J}$ & 330 & 33 & $\mathrm{~J}$ & 330 \\
\hline Benzo(a)pyrene & 330 & $\mathrm{U}$ & 330 & 24 & $\mathrm{~J}$ & 330 & 19 & $\mathrm{~J}$ & 330 & 30 & $\mathrm{~J}$ & 330 \\
\hline Benzo(b)fluoranthene & 330 & $\mathrm{U}$ & 330 & 33 & $\mathrm{~J}$ & 330 & 43 & $\mathrm{~J}$ & 330 & 32 & $\bar{J}$ & 330 \\
\hline Benzo(g,h,i)perylene & 330 & $\mathrm{U}$ & 330 & 330 & $\mathrm{U}$ & 330 & 19 & $\mathrm{~J}$ & 330 & 22 & & 330 \\
\hline Benzo(k)fluoranthene & 330 & $\mathrm{U}$ & 330 & 29 & $\mathrm{~J}$ & 330 & 41 & $\mathrm{~J}$ & 330 & 31 & $\mathrm{~J}$ & 330 \\
\hline bis(2-Chloro-1-methylethyl)ether & 330 & $\mathrm{U}$ & 330 & 330 & $\mathrm{U}$ & 330 & 330 & $\mathrm{U}$ & 330 & 330 & $\mathrm{U}$ & 330 \\
\hline bis(2-Chloroethoxy)methane & 330 & U & 330 & 330 & $\mathrm{U}$ & 330 & 330 & $\mathrm{U}$ & 330 & 330 & $\mathrm{U}$ & 330 \\
\hline \begin{tabular}{|l|} 
bis(2-Chloroethyl)ether \\
\end{tabular} & 330 & $\mathrm{U}$ & 330 & 330 & $\mathrm{U}$ & 330 & 330 & $\mathrm{U}$ & 330 & 330 & $\mathrm{U}$ & 330 \\
\hline bis(2-Ethylhexyl)phthalate & 330 & $\mathrm{U}$ & 330 & 330 & $\mathrm{U}$ & 330 & 71 & $\mathrm{JB}$ & 330 & 36 & $\mathrm{JB}$ & 330 \\
\hline
\end{tabular}

\begin{tabular}{|c|c|c|c|}
\hline Attachment & 1 & Sheet No. & 28 of 43 \\
\hline Originator & J.M. Capron & Date & $12 / 13 / 06$ \\
\hline Checked & T.M. Blakley & Date & \\
\hline Calc. No. & $0100 \mathrm{C}-\mathrm{CA}-\mathrm{V} 0033$ & Rev. No. & 0 \\
\hline
\end{tabular}


Attachment 1. 118-C-1 Verification Sampling Results.

\begin{tabular}{|c|c|c|c|c|c|c|c|c|c|c|c|c|}
\hline \multirow[t]{2}{*}{ Constituents } & \multicolumn{3}{|c|}{$\begin{array}{c}\text { J13520 } \\
\text { OB/BCL A3 } \\
\text { Sample Date 8/22/06 } \\
\end{array}$} & \multicolumn{3}{|c|}{\begin{tabular}{|c|} 
J135N5 \\
OB/BCL A4 \\
Sample Date $8 / 22 / 06$ \\
\end{tabular}} & \multicolumn{3}{|c|}{$\begin{array}{c}\text { J13522 } \\
\text { SPA A1 } \\
\text { Sample Date } 9 / 11 / 06 \\
\end{array}$} & \multicolumn{3}{|c|}{\begin{tabular}{|c|} 
J13523 \\
SPA A2 \\
Sample Date 9/11/06 \\
\end{tabular}} \\
\hline & $\mu \mathrm{g} / \mathrm{kg}$ & $\mathbf{Q}$ & PQL & $\mu \mathrm{g} / \mathrm{kg}$ & $\mathbf{Q}$ & PQL & $\mu \mathrm{g} / \mathrm{kg}$ & $Q$ & PQL & $\mu \mathrm{g} / \mathrm{kg}$ & $\mathbf{Q}$ & PQL \\
\hline \multicolumn{13}{|c|}{ Semivolatile Organic Compounds (continued) } \\
\hline Butylbenzylphthalate & 330 & $\mathrm{U}$ & 330 & 330 & $\mathrm{U}$ & 330 & 330 & $\mathrm{U}$ & 330 & 330 & $\mathrm{U}$ & 330 \\
\hline Carbazole & 330 & $\mathrm{U}$ & 330 & 330 & $\mathrm{U}$ & 330 & 330 & $\mathrm{U}$ & 330 & 330 & $\mathrm{U}$ & 330 \\
\hline Chrysene & 330 & $\mathrm{U}$ & 330 & 52 & $J$ & 330 & 76 & $\mathrm{~J}$ & 330 & 40 & $\mathrm{~J}$ & 330 \\
\hline Di-n-butylphthalate & 330 & $\mathrm{U}$ & 330 & 330 & $\mathrm{U}$ & 330 & 23 & $\mathrm{JB}$ & 330 & 27 & $\mathrm{JB}$ & 330 \\
\hline Di-n-octylphthalate & 330 & $\mathrm{U}$ & 330 & 330 & $\mathrm{U}$ & 330 & 330 & $\mathrm{U}$ & 330 & 330 & $\mathrm{U}$ & 330 \\
\hline Dibenz(a,h)anthracene & 330 & $\mathrm{U}$ & 330 & 330 & $\mathrm{U}$ & 330 & 330 & $\mathrm{U}$ & 330 & 330 & $\mathrm{U}$ & 330 \\
\hline Dibenzofuran & 330 & $\mathrm{U}$ & 330 & 330 & $\mathrm{U}$ & 330 & 330 & $\mathrm{U}$ & 330 & 330 & $U$ & 330 \\
\hline Diethylphthalate & 330 & $\mathrm{U}$ & 330 & 330 & $\mathrm{U}$ & 330 & 330 & $\mathrm{U}$ & 330 & 330 & $\mathrm{U}$ & 330 \\
\hline Dimethylphthalate & 330 & $\mathrm{U}$ & 330 & 330 & $\mathrm{U}$ & 330 & 330 & $\mathrm{U}$ & 330 & 330 & $\mathrm{U}$ & 330 \\
\hline Fluoranthene & 330 & $\mathrm{UJ}$ & 330 & 110 & $\mathrm{~J}$ & 330 & 110 & $\mathrm{~J}$ & 330 & 56 & $\mathrm{~J}$ & 330 \\
\hline Fluorene & 330 & $\mathrm{U}$ & 330 & 330 & $\mathrm{U}$ & 330 & 330 & $\mathrm{U}$ & 330 & 330 & $\mathrm{U}$ & 330 \\
\hline Hexachlorobenzene & 330 & $\mathrm{U}$ & 330 & 330 & $\mathrm{U}$ & 330 & 330 & $\mathrm{U}$ & 330 & 330 & $\mathrm{U}$ & 330 \\
\hline Hexachlorobutadiene & 330 & $\mathrm{U}$ & 330 & 330 & $\mathrm{U}$ & 330 & 330 & $\mathrm{U}$ & 330 & 330 & $\mathrm{U}$ & 330 \\
\hline Hexachlorocyclopentadiene & 330 & $\mathrm{U}$ & 330 & 330 & $\mathrm{U}$ & 330 & 330 & $\mathrm{U}$ & 330 & 330 & $\mathrm{U}$ & 330 \\
\hline Hexachloroethane & 330 & $\mathrm{U}$ & 330 & 330 & $\mathrm{U}$ & 330 & 330 & $\mathrm{U}$ & 330 & 330 & $\mathrm{U}$ & 330 \\
\hline Indeno(1,2,3-cd)pyrene & 330 & $\mathrm{U}$ & 330 & 330 & $\mathrm{U}$ & 330 & 17 & $\mathrm{~J}$ & 330 & 330 & $\mathrm{U}$ & 330 \\
\hline Isophorone & 330 & $\mathrm{U}$ & 330 & 330 & $\mathrm{U}$ & 330 & 330 & $\mathrm{UJ}$ & 330 & 330 & $\overline{U J}$ & 330 \\
\hline N-Nitroso-di-n-dipropylamine & 330 & $\mathrm{U}$ & 330 & 330 & $\mathrm{U}$ & 330 & 330 & $\mathrm{U}$ & 330 & 330 & $\mathrm{U}$ & 330 \\
\hline N-Nitrosodiphenylamine & 330 & $\mathrm{U}$ & 330 & 330 & $\mathrm{U}$ & 330 & 330 & $\mathrm{U}$ & 330 & 330 & $\mathrm{U}$ & 330 \\
\hline Naphthalene & 330 & $\mathrm{U}$ & 330 & 330 & $\mathrm{U}$ & 330 & 330 & $\mathrm{U}$ & 330 & 330 & $\mathrm{U}$ & 330 \\
\hline Nitrobenzene & 330 & $\mathrm{U}$ & 330 & 330 & $\mathrm{U}$ & 330 & 330 & $\mathrm{UJ}$ & 330 & 330 & $\mathrm{UJ}$ & 330 \\
\hline Pentachlorophenol & 840 & $\mathrm{U}$ & 840 & 830 & $\mathrm{U}$ & 830 & 840 & $\mathrm{U}$ & 840 & 840 & $\mathrm{U}$ & 840 \\
\hline Phenanthrene & 330 & $\mathrm{UJ}$ & 330 & 37 & $\mathrm{~J}$ & 330 & 27 & $\mathrm{~J}$ & 330 & 330 & $\mathrm{U}$ & 330 \\
\hline Phenol & 330 & $\mathrm{U}$ & 330 & 330 & $\mathrm{U}$ & 330 & 330 & $\mathrm{U}$ & 330 & 330 & $\mathrm{U}$ & 330 \\
\hline Pyrene & 330 & UJ & 330 & 57 & $\mathrm{~J}$ & 330 & 78 & $\mathrm{~J}$ & 330 & 57 & $\mathrm{~J}$ & 330 \\
\hline & & & & Attachm & & & 1 & & & heet No. & & of 43 \\
\hline & & & & Originat & & J.M. Ca & ron & & & Date & & $13 / 06$ \\
\hline & & & & Checked & & T.M. Bl & kley & & & Date & & \\
\hline & & & & Calc. No & & $0100 \mathrm{C}-\mathrm{C}$ & $\mathrm{A}-\mathrm{V} 0033$ & & & Rev. No. & & 0 \\
\hline
\end{tabular}


Attachment 1. 118-C-1 Verification Sampling Results.

\begin{tabular}{|c|c|c|c|c|c|c|c|c|c|c|c|c|}
\hline \multirow[t]{2}{*}{ Constituents } & \multicolumn{3}{|c|}{$\begin{array}{c}\text { J135T4 } \\
\text { OB/BCL A3 } \\
\text { Sample Date 8/28/06 }\end{array}$} & \multicolumn{3}{|c|}{$\begin{array}{c}\text { J135T5 } \\
\text { OB/BCL A4 } \\
\text { Sample Date } 8 / 28 / 06\end{array}$} & \multicolumn{3}{|c|}{$\begin{array}{c}\text { J13522 } \\
\text { SPA A1 } \\
\text { Sample Date } 9 / 11 / 06\end{array}$} & \multicolumn{3}{|c|}{$\begin{array}{c}\text { J13523 } \\
\text { SPA A2 } \\
\text { Sample Date } 9 / 11 / 06\end{array}$} \\
\hline & $\mu \mathrm{g} / \mathrm{kg}$ & $\mathbf{Q}$ & PQL & $\mu \mathrm{g} / \mathrm{kg}$ & $\mathbf{Q}$ & PQL & $\mu \mathrm{g} / \mathrm{kg}$ & $\mathbf{Q}$ & PQL & $\mu \mathrm{g} / \mathrm{kg}$ & $\mathbf{Q}$ & PQL \\
\hline \multicolumn{13}{|c|}{ Volatile Organic Compounds } \\
\hline 1,1,1-Trichloroethane & 6 & $\mathrm{U}$ & 6 & 5 & $\mathrm{U}$ & 5 & 6 & $\mathrm{U}$ & 6 & 5 & $\mathrm{U}$ & 5 \\
\hline 1,1,2,2-Tetrachloroethane & 6 & UJ & 6 & 5 & UJ & 5 & 6 & UJ & 6 & 5 & $\mathrm{UJ}$ & 5 \\
\hline 1,1,2-Trichloroethane & 6 & $\mathrm{U}$ & 6 & 5 & $\mathrm{U}$ & 5 & 6 & $\mathrm{U}$ & 6 & 5 & $\mathrm{U}$ & 5 \\
\hline 1,1-Dichloroethane & 6 & $\mathrm{U}$ & 6 & 5 & $\mathrm{U}$ & 5 & 6 & $\mathrm{U}$ & 6 & 5 & $\mathrm{U}$ & 5 \\
\hline 1,1-Dichloroethene & 6 & $\mathrm{U}$ & 6 & 5 & $\mathrm{U}$ & 5 & 6 & $\mathrm{U}$ & 6 & 5 & $\mathrm{U}$ & 5 \\
\hline 1,2-Dichloroethane & 6 & $\mathrm{U}$ & 6 & 5 & $\mathrm{U}$ & 5 & 6 & $\mathrm{U}$ & 6 & 5 & $\mathrm{U}$ & 5 \\
\hline 1,2-Dichloroethene (total) & 6 & $\mathrm{U}$ & 6 & 5 & $\mathrm{U}$ & 5 & 6 & $\mathrm{U}$ & 6 & 5 & $\mathrm{U}$ & 5 \\
\hline 1,2-Dichloropropane & 6 & $\mathrm{U}$ & 6 & 5 & $\mathrm{U}$ & 5 & 6 & $\mathrm{U}$ & 6 & 5 & $\mathrm{U}$ & 5 \\
\hline 2-Butanone & 11 & $\mathrm{U}$ & 11 & 10 & $\mathrm{U}$ & 10 & 11 & $\mathrm{U}$ & 11 & 10 & $\mathrm{U}$ & 10 \\
\hline 2-Hexanone & 11 & $\mathrm{U}$ & 11 & 10 & $U$ & 10 & 11 & $\mathrm{U}$ & 11 & 10 & $\mathrm{U}$ & 10 \\
\hline 4-Methyl-2-pentanone & 11 & $\mathrm{U}$ & 11 & 10 & $\mathrm{U}$ & 10 & 11 & $\mathrm{U}$ & 11 & 10 & $U$ & 10 \\
\hline Acetone & 12 & $\mathrm{~J}$ & 11 & 11 & $\mathrm{~J}$ & 10 & 25 & $\mathrm{~J}$ & 11 & 10 & $\mathrm{U}$ & 10 \\
\hline Benzene & 6 & $\mathrm{U}$ & 6 & 5 & $\mathrm{U}$ & 5 & 6 & $\mathrm{U}$ & 6 & 5 & $\mathrm{U}$ & 5 \\
\hline Bromodichloromethane & 6 & $\mathrm{U}$ & 6 & 5 & $\mathrm{U}$ & 5 & 6 & $\mathrm{U}$ & 6 & 5 & $\mathrm{U}$ & 5 \\
\hline Bromoform & 6 & $\mathrm{U}$ & 6 & 5 & $\mathrm{U}$ & 5 & 6 & $\mathrm{U}$ & 6 & 5 & $\mathrm{U}$ & 5 \\
\hline Bromomethane & 11 & $\mathrm{U}$ & 11 & 10 & $\mathrm{U}$ & 10 & 11 & $\mathrm{U}$ & 11 & 10 & $\mathrm{U}$ & 10 \\
\hline Carbon disulfide & 6 & $\mathrm{U}$ & 6 & 5 & $\mathrm{U}$ & 5 & 6 & $\mathrm{U}$ & 6 & 5 & $\mathrm{U}$ & 5 \\
\hline Carbon tetrachloride & 6 & $\mathrm{U}$ & 6 & 5 & $\mathrm{U}$ & 5 & 6 & $\mathrm{U}$ & 6 & 5 & $\mathrm{U}$ & 5 \\
\hline Chlorobenzene & 6 & $\mathrm{U}$ & 6 & 5 & $\mathrm{U}$ & 5 & 6 & $\mathrm{U}$ & 6 & 5 & $\mathrm{U}$ & 5 \\
\hline Chloroethane & 11 & $\mathrm{U}$ & 11 & 10 & $\mathrm{U}$ & 10 & 11 & $\mathrm{U}$ & 11 & 10 & $\mathrm{U}$ & 10 \\
\hline Chloroform & 6 & $\mathrm{U}$ & 6 & 5 & $\mathrm{U}$ & 5 & 6 & $\mathrm{U}$ & 6 & 5 & $\mathrm{U}$ & 5 \\
\hline Chloromethane & 11 & $\mathrm{U}$ & 11 & 10 & $\mathrm{U}$ & 10 & 11 & $\mathrm{U}$ & 11 & 10 & $\mathrm{U}$ & 10 \\
\hline cis-1,2-Dichloroethylene & 6 & $\mathrm{U}$ & 6 & 5 & $\mathrm{U}$ & 5 & 6 & $\mathrm{U}$ & 6 & 5 & $\mathrm{U}$ & 5 \\
\hline cis-1,3-Dichloropropene & 6 & $\mathrm{U}$ & 6 & 5 & $U$ & 5 & 6 & $\mathrm{U}$ & 6 & 5 & $\mathrm{U}$ & 5 \\
\hline Dibromochloromethane & 6 & $\mathrm{U}$ & 6 & 5 & $\mathrm{U}$ & 5 & 6 & $\mathrm{U}$ & 6 & 5 & $\mathrm{U}$ & 5 \\
\hline Ethylbenzene & 6 & $\mathrm{U}$ & 6 & 5 & $\mathrm{U}$ & 5 & 6 & $\mathrm{U}$ & 6 & 5 & $\mathrm{U}$ & 5 \\
\hline Methylene chloride & 12 & $\mathrm{~B}$ & 6 & 13 & $\mathrm{~B}$ & 5 & 11 & $\mathrm{~B}$ & 6 & 8 & $\mathrm{~B}$ & 5 \\
\hline Styrene & 6 & $\mathrm{U}$ & 6 & 5 & $\mathrm{U}$ & 5 & 6 & $\mathrm{U}$ & 6 & 5 & $\mathrm{U}$ & 5 \\
\hline Tetrachloroethene & 6 & $\mathrm{U}$ & 6 & 5 & U & 5 & 6 & $\mathrm{U}$ & 6 & 5 & $\mathrm{U}$ & 5 \\
\hline Toluene & 6 & $\mathrm{U}$ & 6 & 5 & $\mathrm{U}$ & 5 & 1 & $J$ & 6 & 5 & $\mathrm{U}$ & 5 \\
\hline trans-1,2-Dichloroethylene & 6 & $\mathrm{U}$ & 6 & 5 & $\mathrm{U}$ & 5 & 6 & $\mathrm{U}$ & 6 & 5 & $\mathrm{U}$ & 5 \\
\hline trans-1,3-Dichloropropene & 6 & $\mathrm{U}$ & 6 & 5 & $\mathrm{U}$ & 5 & 6 & $\mathrm{U}$ & 6 & 5 & $U$ & 5 \\
\hline Trichloroethene & 6 & $\mathrm{U}$ & 6 & 5 & $\mathrm{U}$ & 5 & 6 & $\mathrm{U}$ & 6 & 5 & $\mathrm{U}$ & 5 \\
\hline Vinyl chloride & 11 & $\mathrm{U}$ & 11 & 10 & $\mathrm{U}$ & 10 & 11 & U & 11 & 10 & $\mathrm{U}$ & 10 \\
\hline Xylenes (total) & 6 & $\mathrm{U}$ & 6 & 5 & $\mathrm{U}$ & 5 & 6 & $\mathrm{U}$ & 6 & 5 & $\mathrm{U}$ & 5 \\
\hline & & & & Attachm & & & 1 & & & sheet No. & & of 43 \\
\hline & & & & Originat & & J. M. Ca & oron & & & Date & & $113 / 06$ \\
\hline & & & & Checkec & & T. M. BI & akley & & & Date & & \\
\hline & & & & Calc. Nc & & $0100 \mathrm{C}-\mathrm{C}$ & $A-V 003$ & & & Rev. No. & & 0 \\
\hline
\end{tabular}


Attachment 1. 118-C-1 Verification Sampling Results.

\begin{tabular}{|c|c|c|c|c|c|c|c|c|c|c|c|c|}
\hline \multirow[t]{2}{*}{ Constituents } & \multicolumn{3}{|c|}{$\begin{array}{c}\text { J135P1 } \\
\text { SPA A3 } \\
\text { Sample Date } 9 / 12 / 06 \\
\end{array}$} & \multicolumn{3}{|c|}{$\begin{array}{c}\text { J135P0 } \\
\text { SPA A4 } \\
\text { Sample Date 9/12/06 } \\
\end{array}$} & \multicolumn{3}{|c|}{$\begin{array}{c}\text { J13524 } \\
\text { SPA B5 } \\
\text { Sample Date 9/11/06 } \\
\end{array}$} & \multicolumn{3}{|c|}{$\begin{array}{c}\text { J13525 } \\
\text { SPA B6 } \\
\text { Sample Date 9/11/06 } \\
\end{array}$} \\
\hline & $\mu \mathrm{g} / \mathrm{kg}$ & $\mathbf{Q}$ & PQL & $\mu \mathrm{g} / \mathrm{kg}$ & Q & PQL & $\mu \mathrm{g} / \mathrm{kg}$ & $\mathbf{Q}$ & PQL & $\mu \mathrm{g} / \mathrm{kg}$ & $Q$ & PQL \\
\hline \multicolumn{13}{|c|}{ Polychlorinated Biphenyls } \\
\hline Aroclor -1016 & 13 & $U$ & 13 & 13 & $\mathrm{U}$ & 13 & 13 & $\mathrm{U}$ & 13 & 13 & $\mathrm{U}$ & 13 \\
\hline Aroclor-1221 & 13 & $\mathrm{U}$ & 13 & 13 & $\mathrm{U}$ & 13 & 13 & $\mathrm{U}$ & 13 & 13 & $\mathrm{U}$ & 13 \\
\hline Aroclor -1232 & 13 & $\mathrm{U}$ & 13 & 13 & $\mathrm{U}$ & 13 & 13 & $U$ & 13 & 13 & $\mathrm{U}$ & 13 \\
\hline Aroclor-1242 & 13 & $\mathrm{U}$ & 13 & 13 & $\mathrm{U}$ & 13 & 13 & $\mathrm{U}$ & 13 & 13 & $\mathrm{U}$ & 13 \\
\hline Aroclor- 1248 & 13 & $\mathrm{U}$ & 13 & 13 & $\mathrm{U}$ & 13 & 13 & U & 13 & 13 & $\mathrm{U}$ & 13 \\
\hline Aroclor-1254 & 13 & $\mathrm{U}$ & 13 & 13 & $\mathrm{U}$ & 13 & 13 & $\mathrm{U}$ & 13 & 13 & $\mathrm{~J}$ & 13 \\
\hline Aroclor -1260 & 13 & $\bar{U}$ & 13 & 31 & & 13 & 61 & & 13 & 13 & $\mathrm{U}$ & 13 \\
\hline \multicolumn{13}{|c|}{ Semivolatile Organic Compounds } \\
\hline 1,2,4-Trichlorobenzene & 330 & $\mathrm{UJ}$ & 330 & 670 & UDJ & 670 & 330 & $\mathrm{UJ}$ & 330 & 330 & UI & 330 \\
\hline 1,2-Dichlorobenzene & 330 & UJ & 330 & 670 & UDJ & 670 & 330 & $\mathrm{U}$ & 330 & 330 & $\mathrm{U}$ & 330 \\
\hline 1,3-Dichlorobenzene & 330 & UJ & 330 & 670 & UDJ & 670 & 330 & $\mathrm{U}$ & 330 & 330 & $\mathrm{U}$ & 330 \\
\hline 1,4-Dichlorobenzene & 330 & UJ & 330 & 670 & UDJ & 670 & 330 & $\mathrm{U}$ & 330 & 330 & $\mathrm{U}$ & 330 \\
\hline 2,4,5-Trichlorophenol & 840 & $\mathrm{U}$ & 840 & 1700 & UD & 1700 & 840 & $\mathrm{U}$ & 840 & 840 & $\mathrm{U}$ & 840 \\
\hline 2,4,6-Trichlorophenol & 330 & $\mathrm{U}$ & 330 & 670 & UD & 670 & 330 & $\mathrm{U}$ & 330 & 330 & $\mathrm{U}$ & 330 \\
\hline 2,4-Dichlorophenol & 330 & $\mathrm{U}$ & 330 & 670 & UD & 670 & 330 & UJ & 330 & 330 & UJ & 330 \\
\hline 2,4-Dimethylphenol & 330 & $\mathrm{UJ}$ & 330 & 670 & UDJ & 670 & 330 & $\mathrm{UJ}$ & 330 & 330 & UJ & 330 \\
\hline 2,4-Dinitrophenol & 840 & UJ & 840 & 1700 & UDJ & 1700 & 840 & UR & 840 & 840 & UR & 840 \\
\hline 2,4-Dinitrotoluene & 330 & $\mathrm{U}$ & 330 & 670 & UD & 670 & 330 & $\mathrm{U}$ & 330 & 330 & $\bar{U}$ & 330 \\
\hline 2,6-Dinitrotoluene & 330 & $\mathrm{U}$ & 330 & 670 & UD & 670 & 330 & $\mathrm{U}$ & 330 & 330 & $U$ & 330 \\
\hline 2-Chloronaphthalene & 330 & $\mathrm{U}$ & 330 & 670 & UD & 670 & 330 & $\bar{U}$ & 330 & 330 & $\bar{U}$ & 330 \\
\hline 2-Chlorophenol & 330 & UJ & 330 & 670 & UDJ & 670 & 330 & $\mathrm{U}$ & 330 & 330 & $\mathrm{U}$ & 330 \\
\hline 2-Methylnaphthalene & 330 & UJ & 330 & 670 & UDJ & 670 & 330 & UI & 330 & 330 & UJ & 330 \\
\hline 2-Methylphenol (cresol, o-) & 330 & UJ & 330 & 670 & UDJ & 670 & 330 & UJ & 330 & 330 & UJ & 330 \\
\hline 2-Nitroaniline & 840 & $\mathrm{U}$ & 840 & 1700 & UD & 1700 & 840 & $\mathrm{U}$ & 840 & 840 & $\mathrm{U}$ & 840 \\
\hline 2-Nitrophenol & 330 & UJ & 330 & 670 & UDJ & 670 & 330 & UJ & 330 & 330 & $\mathrm{UJ}$ & 330 \\
\hline 3,3'-Dichlorobenzidine & 330 & $U$ & 330 & 670 & UD & 670 & 330 & $\mathrm{U}$ & 330 & 330 & $\mathrm{U}$ & 330 \\
\hline 3-Nitroaniline & 840 & U & 840 & 1700 & UD & 1700 & 840 & $\mathrm{U}$ & 840 & 840 & $\mathrm{U}$ & 840 \\
\hline 4,6-Dinitro-2-methylphenol & 840 & $\mathrm{U}$ & 840 & 1700 & UD & 1700 & 840 & $\mathrm{U}$ & 840 & 840 & $\mathrm{U}$ & 840 \\
\hline 4-Bromophenyl-phenylether & 330 & U & 330 & 670 & UD & 670 & 330 & $\mathrm{U}$ & 330 & 330 & $\mathrm{U}$ & 330 \\
\hline 4-Chloro-3-methylphenol & 330 & UJ & 330 & 670 & UDJ & 670 & 330 & UJ & 330 & 330 & UJ & 330 \\
\hline 4-Chloroaniline & 330 & $\mathrm{U}$ & 330 & 670 & UD & 670 & 330 & $\mathrm{U}$ & 330 & 330 & $\mathrm{U}$ & 330 \\
\hline 4-Chlorophenyl-phenylether & 330 & $\mathrm{U}$ & 330 & 670 & UD & 670 & 330 & $\mathrm{U}$ & 330 & 330 & $\mathrm{U}$ & 330 \\
\hline 4-Methylphenol (p-cresol) & 330 & US & 330 & 670 & UDJ & 670 & 330 & UJ & 330 & 330 & $\mathrm{UJ}$ & 330 \\
\hline 4-Nitroaniline & 840 & $\mathrm{U}$ & 840 & 1700 & UD & 1700 & 840 & $\mathrm{U}$ & 840 & 840 & $\mathrm{U}$ & 840 \\
\hline 4-Nitrophenol & 840 & $\mathrm{U}$ & 840 & 1700 & UD & 1700 & 840 & $\mathrm{U}$ & 840 & 840 & $\mathrm{U}$ & 840 \\
\hline Acenaphthene & 330 & $\mathrm{U}$ & 330 & 670 & UD & 670 & 330 & $\mathrm{U}$ & 330 & 330 & $\mathrm{U}$ & 330 \\
\hline \begin{tabular}{|l|} 
Acenaphthylene \\
\end{tabular} & 330 & $\mathrm{U}$ & 330 & 670 & UD & 670 & 330 & $\mathrm{U}$ & 330 & 330 & $\mathrm{U}$ & 330 \\
\hline Anthracene & 330 & UJ & 330 & 170 & $\mathrm{~J}$ & 670 & 330 & $\mathrm{U}$ & 330 & 330 & $\mathrm{U}$ & 330 \\
\hline Benzo(a)anthracene & 37 & $\mathrm{~J}$ & 330 & 660 & $\mathrm{JD}$ & 670 & 25 & $\mathrm{~J}$ & 330 & 27 & $\mathrm{~J}$ & 330 \\
\hline Benzo(a)pyrene & 330 & UJ & 330 & 320 & $\mathrm{JD}$ & 670 & 330 & $\mathrm{U}$ & 330 & 20 & $\mathrm{~J}$ & 330 \\
\hline Benzo(b)fluoranthene & 25 & $\mathrm{~J}$ & 330 & 390 & $\mathrm{JD}$ & 670 & 27 & $\mathrm{~J}$ & 330 & 27 & $\mathrm{~J}$ & 330 \\
\hline Benzo(g,h,i)perylene & 330 & $\mathrm{U}$ & 330 & 140 & $\mathrm{JD}$ & 670 & 330 & $\mathrm{U}$ & 330 & 330 & $\mathrm{U}$ & 330 \\
\hline Benzo(k)fluoranthene & 27 & $\mathrm{~J}$ & 330 & 360 & $\mathrm{JD}$ & 670 & 24 & $\mathrm{~J}$ & 330 & 23 & $\mathrm{~J}$ & 330 \\
\hline bis(2-Chloro-1-methylethyl)ether & 330 & $\mathrm{U}$ & 330 & 670 & UD & 670 & 330 & $\mathrm{U}$ & 330 & 330 & $\mathrm{U}$ & 330 \\
\hline bis(2-Chloroethoxy)methane & 330 & $\mathrm{U}$ & 330 & 670 & UD & 670 & 330 & $\mathrm{U}$ & 330 & 330 & $\mathrm{U}$ & 330 \\
\hline bis(2-Chloroethyl)ether & 330 & $\mathrm{U}$ & 330 & 670 & UD & 670 & 330 & $\mathrm{U}$ & 330 & 330 & $\mathrm{U}$ & 330 \\
\hline bis(2-Ethylhexyl)phthalate & 42 & $\mathrm{JB}$ & 330 & 50 & $\mathrm{JBD}$ & 670 & 35 & JB & 330 & 59 & JB & 330 \\
\hline
\end{tabular}

Attachment

Originator

Checked

Calc. No.

$\frac{1}{\frac{1}{\text { J.M. Capron }}}$

Sheet No. $\quad 31$ of 43

Date $12 / 13 / 06$

Date

Rev. No. 
Attachment 1. 118-C-1 Verification Sampling Results.

\begin{tabular}{|c|c|c|c|c|c|c|c|c|c|c|c|c|}
\hline \multirow[t]{2}{*}{ Constituents } & \multicolumn{3}{|c|}{$\begin{array}{c}\text { J135P1 } \\
\text { SPA A3 } \\
\text { Sample Date 9/12/06 }\end{array}$} & \multicolumn{3}{|c|}{$\begin{array}{c}\text { J135P0 } \\
\text { SPA A4 } \\
\text { Sample Date } 9 / 12 / 06\end{array}$} & \multicolumn{3}{|c|}{$\begin{array}{c}\text { J13524 } \\
\text { SPA B5 } \\
\text { Sample Date } 9 / 11 / 06\end{array}$} & \multicolumn{3}{|c|}{\begin{tabular}{|c|} 
J13525 \\
SPA B6 \\
Sample Date 9/11/06 \\
\end{tabular}} \\
\hline & $\mu \mathrm{g} / \mathrm{kg}$ & Q & PQL & $\mu \mathrm{g} / \mathrm{kg}$ & $\mathbf{Q}$ & PQL & $\mu \mathrm{g} / \mathrm{kg}$ & $\mathbf{Q}$ & PQL & $\mu \mathrm{g} / \mathrm{kg}$ & Q & PQL \\
\hline \multicolumn{13}{|c|}{ Semivolatile Organic Compounds (continued) } \\
\hline Butylbenzylphthalate & 17 & $\mathrm{~J}$ & 330 & 670 & UD & 670 & 330 & $\bar{U}$ & 330 & 330 & $\mathrm{U}$ & 330 \\
\hline Carbazole & 330 & UJ & 330 & 47 & J & 670 & 330 & $\mathrm{U}$ & 330 & 330 & $\mathrm{U}$ & 330 \\
\hline Chrysene & 51 & $\mathrm{~J}$ & 330 & 790 & $\mathrm{JD}$ & 670 & 43 & $\mathrm{~J}$ & 330 & 39 & $\mathrm{~J}$ & 330 \\
\hline Di-n-butylphthalate & 27 & $\mathrm{JB}$ & 330 & 670 & UD & 670 & 27 & $\mathrm{JB}$ & 330 & 39 & $\mathrm{JB}$ & 330 \\
\hline Di-n-octylphthalate & 330 & $\mathrm{U}$ & 330 & 670 & UD & 670 & 330 & $\mathrm{U}$ & 330 & 330 & $\mathrm{U}$ & 330 \\
\hline Dibenz $(a, h)$ anthracene & 330 & $\mathrm{U}$ & 330 & 75 & JD & 670 & 330 & $\mathrm{U}$ & 330 & 330 & $\mathrm{U}$ & 330 \\
\hline Dibenzofuran & 330 & UJ & 330 & 670 & UDJ & 670 & 330 & U & 330 & 330 & $\mathrm{U}$ & 330 \\
\hline Diethylphthalate & 330 & $\mathrm{U}$ & 330 & 670 & UD & 670 & 330 & $\mathrm{U}$ & 330 & 330 & $\mathrm{U}$ & 330 \\
\hline Dimethylphthalate & 330 & $\mathrm{U}$ & 330 & 670 & UD & 670 & 330 & $\mathrm{U}$ & 330 & 330 & $\mathrm{U}$ & 330 \\
\hline Fluoranthene & 73 & $\mathrm{JR}$ & 330 & 1400 & $\mathrm{DR}$ & 670 & 48 & $\mathrm{~J}$ & 330 & 59 & $\mathrm{~J}$ & 330 \\
\hline Fluorene & 330 & UJ & 330 & 670 & UDJ & 670 & 330 & $\mathrm{U}$ & 330 & 330 & $\mathrm{U}$ & 330 \\
\hline Hexachlorobenzene & 330 & $\mathrm{U}$ & 330 & 670 & UD & 670 & 330 & $\mathrm{U}$ & 330 & 330 & $\mathrm{U}$ & 330 \\
\hline Hexachlorobutadiene & 330 & $\mathrm{U}$ & 330 & 670 & UD & 670 & 330 & $\mathrm{U}$ & 330 & 330 & $\mathrm{U}$ & 330 \\
\hline Hexachlorocyclopentadiene & 330 & $\mathrm{U}$ & 330 & 670 & UD & 670 & 330 & $\mathrm{U}$ & 330 & 330 & $\mathrm{U}$ & 330 \\
\hline Hexachloroethane & 330 & $\mathrm{UJ}$ & 330 & 670 & UDJ & 670 & 330 & U & 330 & 330 & $\mathrm{U}$ & 330 \\
\hline Indeno( $1,2,3-$-cd)pyrene & 330 & UJ & 330 & 150 & $\mathrm{JD}$ & 670 & 330 & $\mathrm{U}$ & 330 & 330 & $\mathrm{U}$ & 330 \\
\hline Isophorone & 330 & UJ & 330 & 670 & UDJ & 670 & 330 & UJ & 330 & 330 & UJ & 330 \\
\hline N-Nitroso-di-n-dipropylamine & 330 & UJ & 330 & 670 & UDJ & 670 & 330 & $\mathrm{U}$ & 330 & 330 & $\mathrm{U}$ & 330 \\
\hline N-Nitrosodiphenylamine & 330 & $\mathrm{U}$ & 330 & 670 & UD & 670 & 330 & $U$ & 330 & 330 & $\mathrm{U}$ & 330 \\
\hline Naphthalene & 330 & $\mathrm{U}$ & 330 & 670 & UD & 670 & 330 & $\mathrm{U}$ & 330 & 330 & $\mathrm{U}$ & 330 \\
\hline Nitrobenzene & 330 & UJ & 330 & 670 & UDJ & 670 & 330 & UJ & 330 & 330 & UJ & 330 \\
\hline Pentachlorophenol & 840 & $\mathrm{U}$ & 840 & 1700 & UD & 1700 & 840 & $\mathrm{U}$ & 840 & 840 & $\mathrm{U}$ & 840 \\
\hline Phenanthrene & 26 & $\mathrm{~J}$ & 330 & 710 & $\mathrm{D}$ & 670 & 330 & $\mathrm{U}$ & 330 & 19 & $\mathrm{~J}$ & 330 \\
\hline Phenol & 330 & UJ & 330 & 670 & UDJ & 670 & 24 & $\mathrm{~J}$ & 330 & 330 & $\mathrm{U}$ & 330 \\
\hline Pyrene & 60 & $\mathrm{~J}$ & 330 & 1700 & $\mathrm{D}$ & 670 & 38 & $\mathrm{~J}$ & 330 & 51 & $\mathrm{~J}$ & 330 \\
\hline & & & & Attachm & & & 1 & & & eet No. & & of 43 \\
\hline & & & & Originat & & J. M. Ca & ron & & & Date & & $13 / 06$ \\
\hline & & & & Checked & & T.M. Bl & kley & & & Date & & \\
\hline & & & & Calc. Nc & & $0100 \mathrm{C}-$ & $\mathrm{A}-\mathrm{V} 00^{3}$ & & & Rev. No. & & 0 \\
\hline
\end{tabular}


Attachment 1. 118-C-1 Verification Sampling Results.

\begin{tabular}{|c|c|c|c|c|c|c|c|c|c|c|c|c|}
\hline \multirow[t]{2}{*}{ Constituents } & \multicolumn{3}{|c|}{\begin{tabular}{c|} 
J135P1 \\
SPA A3 \\
Sample Date 9/12/06
\end{tabular}} & \multicolumn{3}{|c|}{$\begin{array}{c}\text { J135P0 } \\
\text { SPA A4 } \\
\text { Sample Date } 9 / 12 / 06 \\
\end{array}$} & \multicolumn{3}{|c|}{$\begin{array}{c}\text { J13524 } \\
\text { SPA B5 } \\
\text { Sample Date } 9 / 11 / 06 \\
\end{array}$} & \multicolumn{3}{|c|}{\begin{tabular}{|c|} 
J13525 \\
SPA B6 \\
Sample Date 9/11/06
\end{tabular}} \\
\hline & $\mu \mathrm{g} / \mathrm{kg}$ & $\mathbf{Q}$ & PQL & $\mu \mathrm{g} / \mathrm{kg}$ & $\mathbf{Q}$ & PQL & $\mu \mathrm{g} / \mathrm{kg}$ & $\mathbf{Q}$ & PQL & $\mu \mathrm{g} / \mathrm{kg}$ & $\mathbf{Q}$ & PQL \\
\hline \multicolumn{13}{|c|}{ Volatile Organic Compounds } \\
\hline 1,1,1-Trichloroethane & 5 & $\mathrm{U}$ & 5 & 5 & $\mathrm{U}$ & 5 & 5 & $\mathrm{U}$ & 5 & 5 & $\mathrm{U}$ & 5 \\
\hline 1,1,2,2-Tetrachloroethane & 5 & $\mathrm{U}$ & 5 & 5 & $\mathrm{U}$ & 5 & 5 & UJ & 5 & 5 & UJ & 5 \\
\hline 1,1,2-Trichloroethane & 5 & $\mathrm{U}$ & 5 & 5 & $\mathrm{U}$ & 5 & 5 & $\mathrm{U}$ & 5 & 5 & $\mathrm{U}$ & 5 \\
\hline 1,1-Dichloroethane & 5 & $\mathrm{U}$ & 5 & 5 & $\mathrm{U}$ & 5 & 5 & $\mathrm{U}$ & 5 & 5 & $\mathrm{U}$ & 5 \\
\hline 1,1-Dichloroethene & 5 & $\mathrm{U}$ & 5 & 5 & $\mathrm{U}$ & 5 & 5 & $\mathrm{U}$ & 5 & 5 & $\mathrm{U}$ & 5 \\
\hline 1,2-Dichloroethane & 5 & $\mathrm{U}$ & 5 & 5 & $\mathrm{U}$ & 5 & 5 & $\mathrm{U}$ & 5 & 5 & U & 5 \\
\hline 1,2-Dichloroethene (total) & 5 & $\mathrm{U}$ & 5 & 5 & U & 5 & 5 & $\mathrm{U}$ & 5 & 5 & $\mathrm{U}$ & 5 \\
\hline 1,2-Dichloropropane & 5 & $\mathrm{U}$ & 5 & 5 & $\mathrm{U}$ & 5 & 5 & $\mathrm{U}$ & 5 & 5 & $\mathrm{U}$ & 5 \\
\hline 2-Butanone & 4 & $\mathrm{~J}$ & 10 & 10 & $\mathrm{U}$ & 10 & 10 & $\mathrm{U}$ & 10 & 10 & $\mathrm{U}$ & 10 \\
\hline 2-Hexanone & 10 & $\mathrm{U}$ & 10 & 10 & $\mathrm{U}$ & 10 & 10 & $\mathrm{U}$ & 10 & 10 & $\mathrm{U}$ & 10 \\
\hline 4-Methyl-2-pentanone & 10 & $\mathrm{U}$ & 10 & 10 & $\mathrm{U}$ & 10 & 10 & $\mathrm{U}$ & 10 & 10 & $\mathrm{U}$ & 10 \\
\hline Acetone & 14 & & 10 & 9 & $J$ & 10 & 10 & $\mathrm{U}$ & 10 & 10 & $\mathrm{U}$ & 10 \\
\hline Benzene & 5 & $U$ & 5 & 5 & $\mathrm{U}$ & 5 & 5 & $\mathrm{U}$ & 5 & 5 & $\mathrm{U}$ & 5 \\
\hline Bromodichloromethane & 5 & $\mathrm{U}$ & 5 & 5 & $\mathrm{U}$ & 5 & 5 & $\mathrm{U}$ & 5 & 5 & $\mathrm{U}$ & 5 \\
\hline Bromoform & 5 & $\mathrm{U}$ & 5 & 5 & $\mathrm{U}$ & 5 & 5 & $\mathrm{U}$ & 5 & 5 & $\mathrm{U}$ & 5 \\
\hline Bromomethane & 10 & $\mathrm{U}$ & 10 & 10 & $U$ & 10 & 10 & $\mathrm{U}$ & 10 & 10 & U & 10 \\
\hline Carbon disulfide & 5 & $\mathrm{U}$ & 5 & 5 & $\mathrm{U}$ & 5 & 5 & $\mathrm{U}$ & 5 & 5 & U & 5 \\
\hline Carbon tetrachloride & 5 & $\mathrm{U}$ & 5 & 5 & U & 5 & 5 & $\mathrm{U}$ & 5 & 5 & $\mathrm{U}$ & 5 \\
\hline Chlorobenzene & 5 & $\mathrm{U}$ & 5 & 5 & $\mathrm{U}$ & 5 & 5 & $\mathrm{U}$ & 5 & 5 & $\mathrm{U}$ & 5 \\
\hline Chloroethane & 10 & $\mathrm{U}$ & 10 & 10 & $\mathrm{U}$ & 10 & 10 & $\mathrm{U}$ & 10 & 10 & $\mathrm{U}$ & 10 \\
\hline Chloroform & 5 & $\mathrm{U}$ & 5 & 5 & $\mathrm{U}$ & 5 & 5 & $\mathrm{U}$ & 5 & 5 & $\mathrm{U}$ & 5 \\
\hline Chloromethane & 10 & $\mathrm{U}$ & 10 & 10 & $\mathrm{U}$ & 10 & 10 & $\mathrm{U}$ & 10 & 10 & $\mathrm{U}$ & 10 \\
\hline cis-1,2-Dichloroethylene & 5 & $\mathrm{U}$ & 5 & 5 & $\mathrm{U}$ & 5 & 5 & $\mathrm{U}$ & 5 & 5 & $\mathrm{U}$ & 5 \\
\hline cis-1,3-Dichloropropene & 5 & $\mathrm{U}$ & 5 & 5 & $\mathrm{U}$ & 5 & 5 & $\mathrm{U}$ & 5 & 5 & $\mathrm{U}$ & 5 \\
\hline Dibromochloromethane & 5 & $\mathrm{U}$ & 5 & 5 & $\mathrm{U}$ & 5 & 5 & $\mathrm{U}$ & 5 & 5 & $U$ & 5 \\
\hline Ethylbenzene & 5 & $\mathrm{U}$ & 5 & 5 & $\mathrm{U}$ & 5 & 5 & $\mathrm{U}$ & 5 & 5 & $\mathrm{U}$ & 5 \\
\hline Methylene chloride & 23 & $\mathrm{~B}$ & 5 & 17 & $\mathrm{~B}$ & 5 & 11 & $\mathrm{~B}$ & 5 & 14 & $\mathrm{~B}$ & 5 \\
\hline Styrene & 5 & $\mathrm{U}$ & 5 & 5 & $\mathrm{U}$ & 5 & 5 & $\mathrm{U}$ & 5 & 5 & $\mathrm{U}$ & 5 \\
\hline Tetrachloroethene & 5 & $\mathrm{U}$ & 5 & 5 & $\mathrm{U}$ & 5 & 5 & $\mathrm{U}$ & 5 & 5 & $\mathrm{U}$ & 5 \\
\hline Toluene & 2 & $\mathrm{~J}$ & 5 & 5 & $\mathrm{U}$ & 5 & 5 & $\mathrm{U}$ & 5 & 5 & $U$ & 5 \\
\hline trans-1,2-Dichloroethylene & 5 & U & 5 & 5 & $\mathrm{U}$ & 5 & 5 & $\mathrm{U}$ & 5 & 5 & $U$ & 5 \\
\hline trans-1,3-Dichloropropene & 5 & $U$ & 5 & 5 & U & 5 & 5 & $\mathrm{U}$ & 5 & 5 & $\mathrm{U}$ & 5 \\
\hline Trichloroethene & 5 & $\mathrm{U}$ & 5 & 5 & $\mathrm{U}$ & 5 & 5 & $\mathrm{U}$ & 5 & 5 & $\mathrm{U}$ & 5 \\
\hline Vinyl chloride & 10 & $\mathrm{U}$ & 10 & 10 & $\mathrm{U}$ & 10 & 10 & $\mathrm{U}$ & 10 & 10 & $\mathrm{U}$ & 10 \\
\hline Xylenes (total) & 5 & $\mathrm{U}$ & 5 & 5 & $\mathrm{U}$ & 5 & 5 & $\mathrm{U}$ & 5 & 5 & $\mathrm{U}$ & 5 \\
\hline & & & & Attachn & & & 1 & & & Sheet No. & & of 43 \\
\hline & & & & Origina & & J.M.Ca & ron & & & Date & & $13 / 06$ \\
\hline & & & & Checke & & T. M. Bl & kley & & & Date & & \\
\hline & & & & Calc. $\mathrm{N}$ & & $0100 \mathrm{C}-\mathrm{C}$ & $\mathrm{A}-\mathrm{V} 0033$ & & & Rev. No. & & 0 \\
\hline
\end{tabular}


Attachment 1. 118-C-1 Verification Sampling Results.

\begin{tabular}{|c|c|c|c|c|c|c|c|c|c|c|c|c|}
\hline \multirow[t]{2}{*}{ Constituents } & \multicolumn{3}{|c|}{\begin{tabular}{|c|} 
J135N8 \\
SPA B7 \\
Sample Date 9/11/06 \\
\end{tabular}} & \multicolumn{3}{|c|}{$\begin{array}{c}\text { J135N9 } \\
\text { SPA B8 } \\
\text { Sample Date 9/11/06 } \\
\end{array}$} & \multicolumn{3}{|c|}{$\begin{array}{c}\text { J135P2 } \\
\text { SPA C9 } \\
\text { Sample Date 9/12/06 } \\
\end{array}$} & \multicolumn{3}{|c|}{$\begin{array}{c}\text { J135P3 } \\
\text { SPA C10 } \\
\text { Sample Date } 9 / 12 / 0 \\
\end{array}$} \\
\hline & $\mu \mathrm{g} / \mathrm{kg}$ & $Q$ & PQL & $\mu \mathrm{g} / \mathrm{kg}$ & $Q$ & PQL & $\mu \mathrm{g} / \mathrm{kg}$ & $\mathbf{Q}$ & PQL & $\mu \mathrm{g} / \mathrm{kg}$ & $Q$ & PQL \\
\hline \multicolumn{13}{|c|}{ Polychlorinated Biphenyls } \\
\hline Aroclor -1016 & 13 & $\mathrm{U}$ & 13 & 13 & $\mathrm{U}$ & 13 & 13 & $\mathrm{U}$ & 13 & 13 & $\mathrm{U}$ & 13 \\
\hline Aroclor-1221 & 13 & $\mathrm{U}$ & 13 & 13 & $\mathrm{U}$ & 13 & 13 & $\mathrm{U}$ & 13 & 13 & $\mathrm{U}$ & 13 \\
\hline Aroclor -1232 & 13 & $\mathrm{U}$ & 13 & 13 & $\mathrm{U}$ & 13 & 13 & $\mathrm{U}$ & 13 & 13 & $\mathrm{U}$ & 13 \\
\hline Aroclor- 1242 & 13 & $\mathrm{U}$ & 13 & 13 & $\mathrm{U}$ & 13 & 13 & $\mathrm{U}$ & 13 & 13 & $\mathrm{U}$ & 13 \\
\hline Aroclor -1248 & 13 & $\mathrm{U}$ & 13 & 13 & $\mathrm{U}$ & 13 & 13 & $\mathrm{U}$ & 13 & 13 & $\mathrm{U}$ & 13 \\
\hline Aroclor- 1254 & 9.5 & $\mathrm{~J}$ & 13 & 19 & & 13 & 17 & & 13 & 6.5 & $\mathrm{~J}$ & 13 \\
\hline Aroclor- 1260 & 13 & $\mathrm{U}$ & 13 & 13 & $\mathrm{U}$ & 13 & 13 & $\mathrm{U}$ & 13 & 13 & $\mathrm{U}$ & 13 \\
\hline \multicolumn{13}{|c|}{ Semivolatile Organic Compounds } \\
\hline 1,2,4-Trichlorobenzene & 330 & $\mathrm{UJ}$ & 330 & 330 & UJ & 330 & 330 & $\mathrm{UJ}$ & 330 & 330 & UJ & 330 \\
\hline 1,2-Dichlorobenzene & 330 & $\mathrm{U}$ & 330 & 330 & $\mathrm{U}$ & 330 & 330 & UJ & 330 & 330 & UJ & 330 \\
\hline 1,3-Dichlorobenzene & 330 & $\mathrm{U}$ & 330 & 330 & $\mathrm{U}$ & 330 & 330 & UJ & 330 & 330 & UJ & 330 \\
\hline 1,4-Dichlorobenzene & 330 & $\mathrm{U}$ & 330 & 330 & $\mathrm{U}$ & 330 & 330 & UJ & 330 & 330 & UJ & 330 \\
\hline 2,4,5-Trichlorophenol & 840 & $\mathrm{U}$ & 840 & 840 & $\mathrm{U}$ & 840 & 840 & $\mathrm{U}$ & 840 & 840 & U & 840 \\
\hline 2,4,6-Trichlorophenol & 330 & $\mathrm{U}$ & 330 & 330 & $\mathrm{U}$ & 330 & 330 & $\mathrm{U}$ & 330 & 330 & $\mathrm{U}$ & 330 \\
\hline 2,4-Dichlorophenol & 330 & UJ & 330 & 330 & UJ & 330 & 330 & $\mathrm{U}$ & 330 & 330 & $U$ & 330 \\
\hline 2,4-Dimethylphenol & 330 & $\mathrm{UJ}$ & 330 & 330 & UJ & 330 & 330 & UJ & 330 & 330 & UJ & 330 \\
\hline 2,4-Dinitrophenol & 840 & UR & 840 & 840 & UR & 840 & 840 & $\mathrm{UJ}$ & 840 & 840 & UJ & 840 \\
\hline 2,4-Dinitrotoluene & 330 & $\mathrm{U}$ & 330 & 330 & $\mathrm{U}$ & 330 & 330 & $\mathrm{U}$ & 330 & 330 & $\mathrm{U}$ & 330 \\
\hline 2,6-Dinitrotoluene & 330 & $U$ & 330 & 330 & $\mathrm{U}$ & 330 & 330 & $\mathrm{U}$ & 330 & 330 & $\mathrm{U}$ & 330 \\
\hline 2-Chloronaphthalene & 330 & $\mathrm{U}$ & 330 & 330 & $\mathrm{U}$ & 330 & 330 & $\mathrm{U}$ & 330 & 330 & $U$ & 330 \\
\hline 2-Chlorophenol & 330 & $\mathrm{U}$ & 330 & 330 & $U$ & 330 & 330 & $\mathrm{UJ}$ & 330 & 330 & UJ & 330 \\
\hline 2-Methylnaphthalene & 330 & UJ & 330 & 330 & UJ & 330 & 330 & UJ & 330 & 330 & UJ & 330 \\
\hline 2-Methylphenol (cresol, o-) & 330 & UJ & 330 & 330 & UJ & 330 & 330 & UJ & 330 & 330 & UJ & 330 \\
\hline 2-Nitroaniline & 840 & $\mathrm{U}$ & 840 & 840 & $\mathrm{U}$ & 840 & 840 & $\mathrm{U}$ & 840 & 840 & $\mathrm{U}$ & 840 \\
\hline 2-Nitrophenol & 330 & UJ & 330 & 330 & UJ & 330 & 330 & UJ & 330 & 330 & UJ & 330 \\
\hline 3,3'-Dichlorobenzidine & 330 & U & 330 & 330 & $\mathrm{U}$ & 330 & 330 & $\mathrm{U}$ & 330 & 330 & $\mathrm{U}$ & 330 \\
\hline 3-Nitroaniline & 840 & $\mathrm{U}$ & 840 & 840 & $\mathrm{U}$ & 840 & 840 & $\mathrm{U}$ & 840 & 840 & $\mathrm{U}$ & 840 \\
\hline 4,6-Dinitro-2-methylphenol & 840 & $\mathrm{U}$ & 840 & 840 & $U$ & 840 & 840 & $\mathrm{U}$ & 840 & 840 & $\mathrm{U}$ & 840 \\
\hline 4-Bromophenyl-phenylether & 330 & $\mathrm{U}$ & 330 & 330 & $\mathrm{U}$ & 330 & 330 & $\mathrm{U}$ & 330 & 330 & $\mathrm{U}$ & 330 \\
\hline 4-Chloro-3-methylphenol & 330 & UJ & 330 & 330 & UJ & 330 & 330 & UJ & 330 & 330 & UJ & 330 \\
\hline 4-Chloroaniline & 330 & $\mathrm{U}$ & 330 & 330 & $\mathrm{U}$ & 330 & 330 & $\mathrm{U}$ & 330 & 330 & $\mathrm{U}$ & 330 \\
\hline 4-Chlorophenyl-phenylether & 330 & $\mathrm{U}$ & 330 & 330 & $\mathrm{U}$ & 330 & 330 & $\mathrm{U}$ & 330 & 330 & $\mathrm{U}$ & 330 \\
\hline 4-Methylphenol (p-cresol) & 330 & UJ & 330 & 330 & UJ & 330 & 330 & UJ & 330 & 330 & UJ & 330 \\
\hline 4-Nitroaniline & 840 & $\mathrm{U}$ & 840 & 840 & $\mathrm{U}$ & 840 & 840 & $\mathrm{U}$ & 840 & 840 & $\mathrm{U}$ & 840 \\
\hline 4-Nitrophenol & 840 & $\mathrm{U}$ & 840 & 840 & $U$ & 840 & 840 & $\mathrm{U}$ & 840 & 840 & $\mathrm{U}$ & 840 \\
\hline Acenaphthene & 330 & $\mathrm{U}$ & 330 & 330 & $\mathrm{U}$ & 330 & 330 & $\mathrm{U}$ & 330 & 330 & $\mathrm{U}$ & 330 \\
\hline Acenaphthylene & 330 & $\mathrm{U}$ & 330 & 330 & $\mathrm{U}$ & 330 & 330 & U & 330 & 330 & $\mathrm{U}$ & 330 \\
\hline \begin{tabular}{|l} 
Anthracene \\
\end{tabular} & 330 & $\mathrm{U}$ & 330 & 330 & $\mathrm{U}$ & 330 & 330 & UJ & 330 & 330 & UJ & 330 \\
\hline Benzo(a)anthracene & 330 & $\mathrm{U}$ & 330 & 61 & $\mathrm{~J}$ & 330 & 330 & UJ & 330 & 330 & UJ & 330 \\
\hline Benzo(a)pyrene & 330 & $\mathrm{U}$ & 330 & 38 & $\mathrm{~J}$ & 330 & 330 & UJ & 330 & 330 & UJ & 330 \\
\hline Benzo(b)fluoranthene & 330 & $\mathrm{U}$ & 330 & 45 & $\mathrm{~J}$ & 330 & 330 & UJ & 330 & 330 & $\mathrm{U}$ & 330 \\
\hline Benzo(g,h,i)perylene & 330 & $\mathrm{U}$ & 330 & 22 & $\mathrm{~J}$ & 330 & 330 & $U$ & 330 & 330 & $\mathrm{U}$ & 330 \\
\hline Benzo(k)fluoranthene & 330 & $\mathrm{U}$ & 330 & 43 & $\mathrm{~J}$ & 330 & 330 & UJ & 330 & 330 & UJ & 330 \\
\hline bis(2-Chloro-1-methylethyl)ether & 330 & $\mathrm{U}$ & 330 & 330 & $\mathrm{U}$ & 330 & 330 & $\mathrm{U}$ & 330 & 330 & $\mathrm{U}$ & 330 \\
\hline bis(2-Chloroethoxy)methane & 330 & $\mathrm{U}$ & 330 & 330 & $\mathrm{U}$ & 330 & 330 & $\mathrm{U}$ & 330 & 330 & $\mathrm{U}$ & 330 \\
\hline bis(2-Chloroethyl)ether & 330 & $\mathrm{U}$ & 330 & 330 & $\mathrm{U}$ & 330 & 330 & $\mathrm{U}$ & 330 & 330 & $U$ & 330 \\
\hline bis(2-Ethylhexyl)phthalate & 36 & $\sqrt{B}$ & 330 & 35 & JB & 330 & 30 & JB & 330 & 48 & $\mathrm{JB}$ & 330 \\
\hline
\end{tabular}

Attachment

Originator

Checked

Calc. No.

\begin{tabular}{|c|}
\hline J.M. Capron \\
\hline T. M. Blakley \\
\hline $0100 \mathrm{C}-\mathrm{CA}-\mathrm{V} 0033$ \\
\hline
\end{tabular}

Sheet No. 34 of 43 Date 12/13/06 Date

Rev. No. 
Attachment 1. 118-C-1 Verification Sampling Results.

\begin{tabular}{|c|c|c|c|c|c|c|c|c|c|c|c|c|}
\hline \multirow[t]{2}{*}{ Constituents } & \multicolumn{3}{|c|}{$\begin{array}{c}\text { J135N8 } \\
\text { SPA B7 } \\
\text { Sample Date 9/11/06 }\end{array}$} & \multicolumn{3}{|c|}{\begin{tabular}{|c|} 
J135N9 \\
SPA B8 \\
Sample Date 9/11/06 \\
\end{tabular}} & \multicolumn{3}{|c|}{$\begin{array}{c}\text { J135P2 } \\
\text { SPA C9 } \\
\text { Sample Date 9/12/06 }\end{array}$} & \multicolumn{3}{|c|}{$\begin{array}{c}\text { J135P3 } \\
\text { SPA C10 } \\
\text { Sample Date } 9 / 12 / 06\end{array}$} \\
\hline & $\mu \mathrm{g} / \mathrm{kg}$ & $\mathbf{Q}$ & PQL & $\mu \mathrm{g} / \mathrm{kg}$ & $Q$ & PQL & $\mu \mathrm{g} / \mathrm{kg}$ & $\mathbf{Q}$ & PQL & $\mu \mathrm{g} / \mathrm{kg}$ & $Q$ & PQL \\
\hline \multicolumn{13}{|c|}{ Semivolatile Organic Compounds (continued) } \\
\hline Butylbenzylphthalate & 330 & $\mathrm{U}$ & 330 & 330 & $\mathrm{U}$ & 330 & 330 & $\mathrm{U}$ & 330 & 330 & $\mathrm{U}$ & 330 \\
\hline Carbazole & 330 & $\mathrm{U}$ & 330 & 330 & U & 330 & 330 & UJ & 330 & 330 & UJ & 330 \\
\hline Chrysene & 330 & $\mathrm{U}$ & 330 & 81 & $\mathrm{~J}$ & 330 & 330 & UJ & 330 & 330 & UJ & 330 \\
\hline Di-n-butylphthalate & 32 & $\mathrm{JB}$ & 330 & 330 & $\mathrm{U}$ & 330 & 19 & $\mathrm{JB}$ & 330 & 17 & $\mathrm{JB}$ & 330 \\
\hline Di-n-octylphthalate & 330 & $\mathrm{U}$ & 330 & 330 & $\mathrm{U}$ & 330 & 330 & $\mathrm{U}$ & 330 & 330 & $\mathrm{U}$ & 330 \\
\hline Dibenz(a,h)anthracene & 330 & $\mathrm{U}$ & 330 & 330 & $\mathrm{U}$ & 330 & 330 & $\mathrm{U}$ & 330 & 330 & $\mathrm{U}$ & 330 \\
\hline Dibenzofuran & 330 & $\mathrm{U}$ & 330 & 330 & U & 330 & 330 & UJ & 330 & 330 & UJ & 330 \\
\hline Diethylphthalate & 330 & $\mathrm{U}$ & 330 & 330 & $\mathrm{U}$ & 330 & 330 & $\mathrm{U}$ & 330 & 330 & $\mathrm{U}$ & 330 \\
\hline Dimethylphthalate & 330 & $\mathrm{U}$ & 330 & 330 & $\bar{U}$ & 330 & 330 & $\bar{U}$ & 330 & 330 & $\bar{U}$ & 330 \\
\hline Fluoranthene & 24 & $\mathrm{~J}$ & 330 & 190 & $\mathrm{~J}$ & 330 & 330 & $\mathrm{U}$ & 330 & 330 & $\mathrm{U}$ & 330 \\
\hline Fluorene & 330 & $\mathrm{U}$ & 330 & 330 & $U$ & 330 & 330 & UJ & 330 & 330 & UJ & 330 \\
\hline Hexachlorobenzene & 330 & $\mathrm{U}$ & 330 & 330 & $U$ & 330 & 330 & $\mathrm{U}$ & 330 & 330 & $\bar{U}$ & 330 \\
\hline Hexachlorobutadiene & 330 & $\mathrm{U}$ & 330 & 330 & $\mathrm{U}$ & 330 & 330 & $\bar{U}$ & 330 & 330 & $\mathrm{U}$ & 330 \\
\hline Hexachlorocyclopentadiene & 330 & $\mathrm{U}$ & 330 & 330 & $\mathrm{U}$ & 330 & 330 & $\mathrm{U}$ & 330 & 330 & $\mathrm{U}$ & 330 \\
\hline Hexachloroethane & 330 & $\mathrm{U}$ & 330 & 330 & $\mathrm{U}$ & 330 & 330 & UJ & 330 & 330 & UJ & 330 \\
\hline Indeno(1,2,3-cd)pyrene & 330 & $\mathrm{U}$ & 330 & 19 & $\mathrm{~J}$ & 330 & 330 & UJ & 330 & 330 & $\mathrm{UJ}$ & 330 \\
\hline Isophorone & 330 & UJ & 330 & 330 & UJ & 330 & 330 & UJ & 330 & 330 & \begin{tabular}{|l|} 
UJ \\
\end{tabular} & 330 \\
\hline $\mathrm{N}$-Nitroso-di-n-dipropylamine & 330 & $\mathrm{U}$ & 330 & 330 & $\bar{U}$ & 330 & 330 & UJ & 330 & 330 & UJ & 330 \\
\hline $\mathrm{N}$-Nitrosodiphenylamine & 330 & $\mathrm{U}$ & 330 & 330 & $\mathrm{U}$ & 330 & 330 & $\mathrm{U}$ & 330 & 330 & $\mathrm{U}$ & 330 \\
\hline Naphthalene & 330 & $\mathrm{U}$ & 330 & 330 & $\mathrm{U}$ & 330 & 330 & $\mathrm{U}$ & 330 & 330 & $\mathrm{U}$ & 330 \\
\hline Nitrobenzene & 330 & $\mathrm{UJ}$ & 330 & 330 & $\overline{U J}$ & 330 & 330 & UJ & 330 & 330 & UJ & 330 \\
\hline Pentachlorophenol & 840 & $\mathrm{U}$ & 840 & 840 & $\mathrm{U}$ & 840 & 840 & $\mathrm{U}$ & 840 & 840 & $\mathrm{U}$ & 840 \\
\hline Phenanthrene & 330 & $\mathrm{U}$ & 330 & 71 & $\mathrm{~J}$ & 330 & 330 & UJ & 330 & 330 & $\mathrm{UJ}$ & 330 \\
\hline Phenol & 330 & $U$ & 330 & 330 & $\mathrm{U}$ & 330 & 330 & $\mathrm{U}$ & 330 & 330 & $\mathrm{U}$ & 330 \\
\hline Pyrene & 21 & $\mathrm{~J}$ & 330 & 130 & $\mathrm{~J}$ & 330 & 330 & $\mathrm{U}$ & 330 & 330 & $\mathrm{U}$ & 330 \\
\hline & & & & Attachm & & & 1 & & & Sheet No. & & of 43 \\
\hline & & & & Origina & & J.M. Ca & ron & & & Date & & $13 / 06$ \\
\hline & & & & Checke & & T.M. Bl & kley & & & Date & & \\
\hline & & & & Calc. $\mathrm{N}$ & & $0100 \mathrm{C}-\mathrm{C}$ & A-V0033 & & & Rev. No. & & 0 \\
\hline
\end{tabular}


Attachment 1. 118-C-1 Verification Sampling Results.

\begin{tabular}{|c|c|c|c|c|c|c|c|c|c|c|c|c|}
\hline \multirow[t]{2}{*}{ Constituents } & \multicolumn{3}{|c|}{$\begin{array}{c}\text { J135N8 } \\
\text { SPA B7 } \\
\text { Sample Date 9/11/06 }\end{array}$} & \multicolumn{3}{|c|}{$\begin{array}{c}\text { J135N9 } \\
\text { SPA B8 } \\
\text { Sample Date 9/11/06 }\end{array}$} & \multicolumn{3}{|c|}{$\begin{array}{c}\text { J135P2 } \\
\text { SPA C9 } \\
\text { Sample Date } 9 / 12 / 06 \\
\end{array}$} & \multicolumn{3}{|c|}{$\begin{array}{c}\text { J135P3 } \\
\text { SPA C10 } \\
\text { Sample Date } 9 / 12 / 06 \\
\end{array}$} \\
\hline & $\mu \mathrm{g} / \mathrm{kg}$ & $\mathbf{Q}$ & PQL & $\mu \mathrm{g} / \mathrm{kg}$ & $\mathbf{Q}$ & PQL & $\mu \mathrm{g} / \mathrm{kg}$ & $\mathbf{Q}$ & PQL & $\mu \mathrm{g} / \mathrm{kg}$ & $Q$ & PQL \\
\hline \multicolumn{13}{|c|}{ Volatile Organic Compounds } \\
\hline 1,1,1-Trichloroethane & 5 & $\mathrm{U}$ & 5 & 5 & $\mathrm{U}$ & 5 & 5 & $\mathrm{U}$ & 5 & 5 & $\mathrm{U}$ & 5 \\
\hline 1,1,2,2-Tetrachloroethane & 5 & $\mathrm{UJ}$ & 5 & 5 & UJ & 5 & 5 & $\bar{U}$ & 5 & 5 & $\mathrm{U}$ & 5 \\
\hline 1,1,2-Trichloroethane & 5 & $\mathrm{U}$ & 5 & 5 & $\mathrm{U}$ & 5 & 5 & $\mathrm{U}$ & 5 & 5 & $\mathrm{U}$ & 5 \\
\hline 1,1-Dichloroethane & 5 & $\mathrm{U}$ & 5 & 5 & $\mathrm{U}$ & 5 & 5 & $\mathrm{U}$ & 5 & 5 & $\mathrm{U}$ & 5 \\
\hline 1,1-Dichloroethene & 5 & $\mathrm{U}$ & 5 & 5 & $\mathrm{U}$ & 5 & 5 & $\mathrm{U}$ & 5 & 5 & $\mathrm{U}$ & 5 \\
\hline 1,2-Dichloroethane & 5 & $\mathrm{U}$ & 5 & 5 & $\mathrm{U}$ & 5 & 5 & $\mathrm{U}$ & 5 & 5 & $\mathrm{U}$ & 5 \\
\hline 1,2-Dichloroethene (total) & 5 & $\mathrm{U}$ & 5 & 5 & $\mathrm{U}$ & 5 & 5 & $\mathrm{U}$ & 5 & 5 & $\mathrm{U}$ & 5 \\
\hline 1,2-Dichloropropane & 5 & $\mathrm{U}$ & 5 & 5 & $\mathrm{U}$ & 5 & 5 & $\mathrm{U}$ & 5 & 5 & $\mathrm{U}$ & 5 \\
\hline 2-Butanone & 10 & $\mathrm{U}$ & 10 & 10 & $\mathrm{U}$ & 10 & 4 & $\mathrm{~J}$ & 10 & 10 & $\mathrm{U}$ & 10 \\
\hline 2-Hexanone & 10 & $\mathrm{U}$ & 10 & 10 & $\mathrm{U}$ & 10 & 10 & $\mathrm{U}$ & 10 & 10 & $\mathrm{U}$ & 10 \\
\hline 4-Methyl-2-pentanone & 10 & $\mathrm{U}$ & 10 & 10 & $\mathrm{U}$ & 10 & 10 & $\mathrm{U}$ & 10 & 10 & $\mathrm{U}$ & 10 \\
\hline Acetone & 25 & $\mathrm{~J}$ & 10 & 28 & $\mathrm{~J}$ & 10 & 12 & & 10 & 7 & $\mathrm{~J}$ & 10 \\
\hline Benzene & 5 & $\mathrm{U}$ & 5 & 5 & $\mathrm{U}$ & 5 & 5 & $\mathrm{U}$ & 5 & 5 & $\mathrm{U}$ & 5 \\
\hline Bromodichloromethane & 5 & $\mathrm{U}$ & 5 & 5 & $\mathrm{U}$ & 5 & 5 & $\bar{U}$ & 5 & 5 & $\mathrm{U}$ & 5 \\
\hline Bromoform & 5 & $\mathrm{U}$ & 5 & 5 & $\mathrm{U}$ & 5 & 5 & $\mathrm{U}$ & 5 & 5 & $\mathrm{U}$ & 5 \\
\hline Bromomethane & 10 & $\mathrm{U}$ & 10 & 10 & $\mathrm{U}$ & 10 & 10 & $\mathrm{U}$ & 10 & 10 & $\mathrm{U}$ & 10 \\
\hline Carbon disulfide & 5 & $\mathrm{U}$ & 5 & 5 & $\mathrm{U}$ & 5 & 5 & $\mathrm{U}$ & 5 & 5 & $\mathrm{U}$ & 5 \\
\hline Carbon tetrachloride & 5 & $\mathrm{U}$ & 5 & 5 & $\mathrm{U}$ & 5 & 5 & $\mathrm{U}$ & 5 & 5 & $\mathrm{U}$ & 5 \\
\hline Chlorobenzene & 5 & $\mathrm{U}$ & 5 & 5 & $\mathrm{U}$ & 5 & 5 & $\mathrm{U}$ & 5 & 5 & $\mathrm{U}$ & 5 \\
\hline Chloroethane & 10 & $\mathrm{U}$ & 10 & 10 & $\mathrm{U}$ & 10 & 10 & $\mathrm{U}$ & 10 & 10 & $\mathrm{U}$ & 10 \\
\hline Chloroform & 5 & $\mathrm{U}$ & 5 & 5 & $\mathrm{U}$ & 5 & 5 & $\bar{U}$ & 5 & 5 & $\mathrm{U}$ & 5 \\
\hline Chloromethane & 10 & $\mathrm{U}$ & 10 & 10 & $\mathrm{U}$ & 10 & 10 & $\mathrm{U}$ & 10 & 10 & $\mathrm{U}$ & 10 \\
\hline cis-1,2-Dichloroethylene & 5 & $\mathrm{U}$ & 5 & 5 & $\mathrm{U}$ & 5 & 5 & $\mathrm{U}$ & 5 & 5 & $\mathrm{U}$ & 5 \\
\hline cis-1,3-Dichloropropene & 5 & $\mathrm{U}$ & 5 & 5 & $\mathrm{U}$ & 5 & 5 & $\mathrm{U}$ & 5 & 5 & $\mathrm{U}$ & 5 \\
\hline Dibromochloromethane & 5 & $\mathrm{U}$ & 5 & 5 & $\mathrm{U}$ & 5 & 5 & U & 5 & 5 & $\mathrm{U}$ & 5 \\
\hline Ethylbenzene & 5 & $\mathrm{U}$ & 5 & 5 & $\mathrm{U}$ & 5 & 5 & $\mathrm{U}$ & 5 & 5 & $\mathrm{U}$ & 5 \\
\hline Methylene chloride & 14 & $\mathrm{~B}$ & 5 & 15 & $\mathrm{~B}$ & 5 & 21 & $\mathrm{~B}$ & 5 & 24 & $\mathrm{~B}$ & 5 \\
\hline Styrene & 5 & $\mathrm{U}$ & 5 & 5 & $\mathrm{U}$ & 5 & 5 & $\mathrm{U}$ & 5 & 5 & $\mathrm{U}$ & 5 \\
\hline Tetrachloroethene & 5 & $\mathrm{U}$ & 5 & 5 & $\mathrm{U}$ & 5 & 5 & $\mathrm{U}$ & 5 & 5 & $\mathrm{U}$ & 5 \\
\hline Toluene & 2 & $\mathrm{~J}$ & 5 & 1 & $\mathrm{~J}$ & 5 & 5 & $\mathrm{U}$ & 5 & 1 & $\mathrm{~J}$ & 5 \\
\hline trans-1,2-Dichloroethylene & 5 & $\mathrm{U}$ & 5 & 5 & U & 5 & 5 & $\mathrm{U}$ & 5 & 5 & $\mathrm{U}$ & 5 \\
\hline trans-1,3-Dichloropropene & 5 & $\mathrm{U}$ & 5 & 5 & $\mathrm{U}$ & 5 & 5 & $\mathrm{U}$ & 5 & 5 & $\mathrm{U}$ & 5 \\
\hline Trichloroethene & 5 & $\mathrm{U}$ & 5 & 5 & $\mathrm{U}$ & 5 & 5 & $\mathrm{U}$ & 5 & 5 & $\mathrm{U}$ & 5 \\
\hline Vinyl chloride & 10 & $\mathrm{U}$ & 10 & 10 & $\mathrm{U}$ & 10 & 10 & $\mathrm{U}$ & 10 & 10 & $\mathrm{U}$ & 10 \\
\hline Xylenes (total) & 5 & $\mathrm{U}$ & 5 & 5 & $\mathrm{U}$ & 5 & 5 & $\mathrm{U}$ & 5 & 5 & $\mathrm{U}$ & 5 \\
\hline & & & & Attachm & & & 1 & & & heet No. & & of 43 \\
\hline & & & & Originat & & J.M. Car & ron & & & Date & & $13 / 06$ \\
\hline & & & & Checked & & T.M. Bla & kley & & & Date & & \\
\hline & & & & Calc. No & & $0100 \mathrm{C}-\mathrm{C}$ & $A-V 0033$ & & & Rev. No. & & 0 \\
\hline
\end{tabular}


Attachment 1. 118-C-1 Verification Sampling Results.

\begin{tabular}{|c|c|c|c|c|c|c|c|c|c|c|c|c|}
\hline \multirow[t]{2}{*}{ Constituents } & \multicolumn{3}{|c|}{\begin{tabular}{|c|} 
J135P4 \\
SPA C1 \\
Sample Date $9 / 12 / 06$
\end{tabular}} & \multicolumn{3}{|c|}{\begin{tabular}{|c|} 
J135P5 \\
SPA C2 \\
Sample Date 9/12/06 \\
\end{tabular}} & \multicolumn{3}{|c|}{$\begin{array}{c}\text { J135P6 } \\
\text { SPA D3 } \\
\text { Sample Date 9/12/06 } \\
\end{array}$} & \multicolumn{3}{|c|}{$\begin{array}{c}\text { J135P7 } \\
\text { SPA D4 } \\
\text { Sample Date 9/12/06 }\end{array}$} \\
\hline & $\mu \mathrm{g} / \mathrm{kg}$ & Q & PQL & $\mu \mathrm{g} / \mathrm{kg}$ & $\mathbf{Q}$ & PQL & $\mu \mathrm{g} / \mathrm{kg}$ & $Q$ & PQL & $\mu \mathrm{g} / \mathrm{kg}$ & $Q$ & PQL \\
\hline \multicolumn{13}{|c|}{ Polychlorinated Biphenyls } \\
\hline Aroclor-1016 & 13 & $\mathrm{U}$ & 13 & 13 & $\mathrm{U}$ & 13 & 13 & $\mathrm{U}$ & 13 & 13 & $U$ & 13 \\
\hline Aroclor-1221 & 13 & $\mathrm{U}$ & 13 & 13 & $\mathrm{U}$ & 13 & 13 & $\mathrm{U}$ & 13 & 13 & $\mathrm{U}$ & 13 \\
\hline Aroclor-1232 & 13 & $\mathrm{U}$ & 13 & 13 & $U$ & 13 & 13 & $\mathrm{U}$ & 13 & 13 & $U$ & 13 \\
\hline Aroclor-1242 & 13 & $\mathrm{U}$ & 13 & 13 & $\mathrm{U}$ & 13 & 13 & $\mathrm{U}$ & 13 & 13 & $\mathrm{U}$ & 13 \\
\hline Aroclor- 1248 & 13 & $\mathrm{U}$ & 13 & 13 & $\mathrm{U}$ & 13 & 13 & $\mathrm{U}$ & 13 & 13 & $\mathrm{U}$ & 13 \\
\hline Aroclor-1254 & 14 & & 13 & 25 & & 13 & 20 & & 13 & 7.9 & $\mathrm{~J}$ & 13 \\
\hline Aroclor- 1260 & 13 & $\mathrm{U}$ & 13 & 13 & $\mathrm{U}$ & 13 & 13 & $\overline{\mathrm{U}}$ & 13 & 13 & $\mathrm{U}$ & 13 \\
\hline \multicolumn{13}{|c|}{ Semivolatile Organic Compounds } \\
\hline 1,2,4-Trichlorobenzene & 330 & UJ & 330 & 330 & UJ & 330 & 330 & $\mathrm{UJ}$ & 330 & 330 & UJ & 330 \\
\hline 1,2-Dichlorobenzene & 330 & $\mathrm{UJ}$ & 330 & 330 & UJ & 330 & 330 & UJ & 330 & 330 & $\mathrm{UJ}$ & 330 \\
\hline 1,3-Dichlorobenzene & 330 & UJ & 330 & 330 & UJ & 330 & 330 & UJ & 330 & 330 & UJ & 330 \\
\hline 1,4-Dichlorobenzene & 330 & UJ & 330 & 330 & UJ & 330 & 330 & UJ & 330 & 330 & UJ & 330 \\
\hline 2,4,5-Trichlorophenol & 840 & $\mathrm{U}$ & 840 & 840 & $\mathrm{U}$ & 840 & 840 & $\mathrm{U}$ & 840 & 840 & $\mathrm{U}$ & 840 \\
\hline 2,4,6-Trichlorophenol & 330 & $\mathrm{U}$ & 330 & 330 & $\mathrm{U}$ & 330 & 330 & $\mathrm{U}$ & 330 & 330 & $\mathrm{U}$ & 330 \\
\hline 2,4-Dichlorophenol & 330 & $\mathrm{U}$ & 330 & 330 & $\mathrm{U}$ & 330 & 330 & $\mathrm{U}$ & 330 & 330 & $\mathrm{U}$ & 330 \\
\hline 2,4-Dimethylphenol & 330 & $\mathrm{UJ}$ & 330 & 330 & UJ & 330 & 330 & UJ & 330 & 330 & UJ & 330 \\
\hline 2,4-Dinitrophenol & 840 & UY & 840 & 840 & UJ & 840 & 840 & UJ & 840 & 840 & UJ & 840 \\
\hline 2,4-Dinitrotoluene & 330 & $U$ & 330 & 330 & U & 330 & 330 & $U$ & 330 & 330 & $\mathrm{U}$ & 330 \\
\hline 2,6-Dinitrotoluene & 330 & $\mathrm{U}$ & 330 & 330 & $\mathrm{U}$ & 330 & 330 & $\mathrm{U}$ & 330 & 330 & $\mathrm{U}$ & 330 \\
\hline 2-Chloronaphthalene & 330 & $\mathrm{U}$ & 330 & 330 & $\mathrm{U}$ & 330 & 330 & $\mathrm{U}$ & 330 & 330 & $\mathrm{U}$ & 330 \\
\hline 2-Chlorophenol & 330 & UJ & 330 & 330 & UJ & 330 & 330 & UJ & 330 & 330 & UJ & 330 \\
\hline 2-Methylnaphthalene & 330 & UJ & 330 & 330 & $\mathrm{UJ}$ & 330 & 330 & UJ & 330 & 330 & UJ & 330 \\
\hline 2-Methylphenol (cresol, o-) & 330 & UJ & 330 & 330 & UJ & 330 & 330 & UJ & 330 & 330 & UJ & 330 \\
\hline 2-Nitroaniline & 840 & $U$ & 840 & 840 & $\mathrm{U}$ & 840 & 840 & $U$ & 840 & 840 & $\mathrm{U}$ & 840 \\
\hline 2-Nitrophenol & 330 & UJ & 330 & 330 & UJ & 330 & 330 & UJ & 330 & 330 & UJ & 330 \\
\hline 3,3'-Dichlorobenzidine & 330 & $U$ & 330 & 330 & $\mathrm{U}$ & 330 & 330 & $\mathrm{U}$ & 330 & 330 & $\mathrm{U}$ & 330 \\
\hline 3-Nitroaniline & 840 & $\mathrm{U}$ & 840 & 840 & $\mathrm{U}$ & 840 & 840 & $\mathrm{U}$ & 840 & 840 & $\mathrm{U}$ & 840 \\
\hline 4,6-Dinitro-2-methylphenol & 840 & $U$ & 840 & 840 & $\bar{U}$ & 840 & 840 & $\mathrm{U}$ & 840 & 840 & $\overline{\mathrm{U}}$ & 840 \\
\hline 4-Bromophenyl-phenylether & 330 & $\mathrm{U}$ & 330 & 330 & $\mathrm{U}$ & 330 & 330 & $\mathrm{U}$ & 330 & 330 & $\mathrm{U}$ & 330 \\
\hline 4-Chloro-3-methylphenol & 330 & UJ & 330 & 330 & UJ & 330 & 330 & UJ & 330 & 330 & UJ & 330 \\
\hline 4-Chloroaniline & 330 & $\mathrm{U}$ & 330 & 330 & $\mathrm{U}$ & 330 & 330 & $U$ & 330 & 330 & $\mathrm{U}$ & 330 \\
\hline 4-Chlorophenyl-phenylether & 330 & $U$ & 330 & 330 & $\mathrm{U}$ & 330 & 330 & $\mathrm{U}$ & 330 & 330 & $\mathrm{U}$ & 330 \\
\hline 4-Methylphenol (p-cresol) & 330 & UJ & 330 & 330 & UJ & 330 & 330 & UJ & 330 & 330 & UJ & 330 \\
\hline 4-Nitroaniline & 840 & $\mathrm{U}$ & 840 & 840 & $U$ & 840 & 840 & $\mathrm{U}$ & 840 & 840 & $\mathrm{U}$ & 840 \\
\hline 4-Nitrophenol & 840 & $\mathrm{U}$ & 840 & 840 & $\mathrm{U}$ & 840 & 840 & $\mathrm{U}$ & 840 & 840 & $\mathrm{U}$ & 840 \\
\hline Acenaphthene & 330 & $\mathrm{U}$ & 330 & 330 & $\mathrm{U}$ & 330 & 330 & $\mathrm{U}$ & 330 & 41 & $\mathrm{~J}$ & 330 \\
\hline Acenaphthylene & 330 & $\mathrm{U}$ & 330 & 330 & $\mathrm{U}$ & 330 & 330 & $\mathrm{U}$ & 330 & 330 & $\mathrm{U}$ & 330 \\
\hline Anthracene & 330 & UJ & 330 & 330 & UJ & 330 & 330 & UJ & 330 & 260 & $\mathrm{~J}$ & 330 \\
\hline Benzo(a)anthracene & 330 & UJ & 330 & 27 & $\mathrm{~J}$ & 330 & 330 & UJ & 330 & 1100 & $\mathrm{~J}$ & 330 \\
\hline Benzo(a)pyrene & 330 & UJ & 330 & 28 & $\mathrm{~J}$ & 330 & 330 & UJ & 330 & 480 & $\mathrm{~J}$ & 330 \\
\hline Benzo(b)fluoranthene & 330 & UJ & 330 & 26 & $\mathrm{~J}$ & 330 & 330 & UJ & 330 & 600 & $\mathrm{~J}$ & 330 \\
\hline Benzo $(\mathrm{g}, \mathrm{h}, \mathrm{i})$ perylene & 330 & $\mathrm{U}$ & 330 & 21 & $\mathrm{~J}$ & 330 & 330 & $\mathrm{U}$ & 330 & 260 & $\mathrm{~J}$ & 330 \\
\hline Benzo(k)fluoranthene & 330 & UJ & 330 & 28 & $\mathrm{~J}$ & 330 & 330 & UJ & 330 & 540 & $\mathrm{~J}$ & 330 \\
\hline bis(2-Chloro-1-methylethyl)ether & 330 & $\mathrm{U}$ & 330 & 330 & $\mathrm{U}$ & 330 & 330 & $\mathrm{U}$ & 330 & 330 & $\mathrm{U}$ & 330 \\
\hline bis(2-Chloroethoxy)methane & 330 & $\mathrm{U}$ & 330 & 330 & $\mathrm{U}$ & 330 & 330 & $\mathrm{U}$ & 330 & 330 & U & 330 \\
\hline bis(2-Chloroethyl)ether & 330 & $\mathrm{U}$ & 330 & 330 & $\mathrm{U}$ & 330 & 330 & $\mathrm{U}$ & 330 & 330 & $\mathrm{U}$ & 330 \\
\hline bis(2-Ethylhexyl)phthalate & 23 & $\mathrm{JB}$ & 330 & 66 & $\mathrm{JB}$ & 330 & 56 & $\mathrm{JB}$ & 330 & 27 & $\mathrm{JB}$ & 330 \\
\hline
\end{tabular}

Attachment

Originator

Checked

Caic. No.

\begin{tabular}{|c|}
\hline 1 \\
\hline J.M. Capron \\
\hline T. M. Blakley \\
\hline $0100 \mathrm{C}-\mathrm{CA}-\mathrm{V} 0033$ \\
\hline
\end{tabular}

Sheet No. 37 of 43

Date $\frac{37 / 12 / 13 / 06}{6}$ Date

Rev. No. -0 
Attachment 1. 118-C-1 Verification Sampling Results.

\begin{tabular}{|c|c|c|c|c|c|c|c|c|c|c|c|c|}
\hline \multirow[t]{2}{*}{ Constituents } & \multicolumn{3}{|c|}{\begin{tabular}{|c|} 
J135P4 \\
SPA C1 \\
Sample Date 9/12/06
\end{tabular}} & \multicolumn{3}{|c|}{$\begin{array}{c}\text { J135P5 } \\
\text { SPA C2 } \\
\text { Sample Date 9/12/06 }\end{array}$} & \multicolumn{3}{|c|}{$\begin{array}{c}\text { J135P6 } \\
\text { SPA D3 } \\
\text { Sample Date 9/12/06 }\end{array}$} & \multicolumn{3}{|c|}{\begin{tabular}{|c|} 
J135P7 \\
SPA D4 \\
Sample Date 9/12/06 \\
\end{tabular}} \\
\hline & $\mu \mathrm{g} / \mathrm{kg}$ & $\mathbf{Q}$ & PQL & $\mu \mathrm{g} / \mathrm{kg}$ & $\mathbf{Q}$ & PQL & $\mu \mathrm{g} / \mathrm{kg}$ & $\mathbf{Q}$ & PQL & $\mu \mathrm{g} / \mathrm{kg}$ & $\mathbf{Q}$ & PQL \\
\hline \multicolumn{13}{|c|}{ Semivolatile Organic Compounds (continued) } \\
\hline Butylbenzylphthalate & 330 & $\mathrm{U}$ & 330 & 330 & $\mathrm{U}$ & 330 & 330 & $\mathrm{U}$ & 330 & 330 & $\mathrm{U}$ & 330 \\
\hline Carbazole & 330 & UJ & 330 & 330 & $\mathrm{UJ}$ & 330 & 330 & UJ & 330 & 240 & $\mathrm{~J}$ & 330 \\
\hline Chrysene & 330 & UJ & 330 & 43 & $\mathrm{~J}$ & 330 & 330 & $\mathrm{UJ}$ & 330 & 1300 & $J$ & 330 \\
\hline Di-n-butylphthalate & 330 & $\mathrm{U}$ & 330 & 31 & $\mathrm{JB}$ & 330 & 32 & $\mathrm{JB}$ & 32 & 17 & $\mathrm{JB}$ & 330 \\
\hline Di-n-octylphthalate & 330 & $\mathrm{U}$ & 330 & 330 & $\mathrm{U}$ & 330 & 330 & $\mathrm{U}$ & 330 & 330 & $\mathrm{U}$ & 330 \\
\hline Dibenz(a,h)anthracene & 330 & $\mathrm{U}$ & 330 & 330 & $\mathrm{U}$ & 330 & 330 & $\mathrm{U}$ & 330 & 180 & $\mathrm{~J}$ & 330 \\
\hline \begin{tabular}{|l|} 
Dibenzofuran \\
\end{tabular} & 330 & UJ & 330 & 330 & $\mathrm{UJ}$ & 330 & 330 & UJ & 330 & 34 & $\mathrm{~J}$ & 330 \\
\hline Diethylphthalate & 330 & $\mathrm{U}$ & 330 & 330 & $\mathrm{U}$ & 330 & 330 & $\mathrm{U}$ & 330 & 330 & $\mathrm{U}$ & 330 \\
\hline Dimethylphthalate & 330 & $\mathrm{U}$ & 330 & 330 & $U$ & 330 & 330 & $\mathrm{U}$ & 330 & 330 & $\mathrm{U}$ & 330 \\
\hline \begin{tabular}{|l|} 
Fluoranthene \\
\end{tabular} & 330 & $\mathrm{U}$ & 330 & 52 & $\mathrm{JR}$ & 330 & .330 & UR & 330 & 2100 & $\mathrm{R}$ & 330 \\
\hline Fluorene & 330 & UJ & 330 & 330 & $\mathrm{UJ}$ & 330 & 330 & $\mathrm{UJ}$ & 330 & 43 & $\mathbf{J}$ & 330 \\
\hline Hexachlorobenzene & 330 & $\mathrm{U}$ & 330 & 330 & $\mathrm{U}$ & 330 & 330 & $\mathrm{U}$ & 330 & 330 & $\mathrm{U}$ & 330 \\
\hline Hexachlorobutadiene & 330 & $\mathrm{U}$ & 330 & 330 & $\mathrm{U}$ & 330 & 330 & $\mathrm{U}$ & 330 & 330 & $\mathrm{U}$ & 330 \\
\hline Hexachlorocyclopentadiene & 330 & $\mathrm{U}$ & 330 & 330 & $\mathrm{U}$ & 330 & 330 & $\mathrm{U}$ & 330 & 330 & $\mathrm{U}$ & 330 \\
\hline Hexachloroethane & 330 & $\mathrm{UJ}$ & 330 & 330 & $\mathrm{UJ}$ & 330 & 330 & UJ & 330 & 330 & $\mathrm{UJ}$ & 330 \\
\hline Indeno(1,2,3-cd)pyrene & 330 & $\mathrm{UJ}$ & 330 & 18 & $\mathrm{~J}$ & 330 & 330 & $\mathrm{UJ}$ & 330 & 250 & $\mathrm{~J}$ & 330 \\
\hline Isophorone & 330 & UJ & 330 & 330 & $\mathrm{UJ}$ & 330 & 330 & UJ & 330 & 330 & UJ & 330 \\
\hline N-Nitroso-di-n-dipropylamine & 330 & UJ & 330 & 330 & $\mathrm{UJ}$ & 330 & 330 & UJ & 330 & 330 & $\mathrm{UJ}$ & 330 \\
\hline N-Nitrosodiphenylamine & 330 & $\mathrm{U}$ & 330 & 330 & $\mathrm{U}$ & 330 & 330 & $\mathrm{U}$ & 330 & 330 & $\mathrm{U}$ & 330 \\
\hline Naphthalene & 330 & $\mathrm{U}$ & 330 & 330 & $\mathrm{U}$ & 330 & 330 & $\mathrm{U}$ & 330 & 330 & $\mathrm{U}$ & 330 \\
\hline Nitrobenzene & 330 & UJ & 330 & 330 & UJ & 330 & 330 & UJ & 330 & 330 & $\mathrm{UJ}$ & 330 \\
\hline Pentachlorophenol & 840 & $\mathrm{U}$ & 840 & 840 & $\mathrm{U}$ & 840 & 840 & $\mathrm{U}$ & 840 & 840 & $\mathrm{U}$ & 840 \\
\hline Phenanthrene & 330 & $\mathrm{UJ}$ & 330 & 19 & $\mathrm{~J}$ & 330 & 330 & UJ & 330 & 1300 & $\mathrm{~J}$ & 330 \\
\hline Phenol & 330 & $\mathrm{U}$ & 330 & 330 & $\mathrm{U}$ & 330 & 330 & $\mathrm{U}$ & 330 & 330 & $\mathrm{U}$ & 330 \\
\hline Pyrene & 330 & $\mathrm{U}$ & 330 & 57 & $\mathrm{~J}$ & 330 & 330 & $\mathrm{U}$ & 330 & 1600 & & 330 \\
\hline
\end{tabular}

Attachment

Originator

Checked

Calc. No.

\begin{tabular}{|c|}
\hline$\frac{1}{\text { J. M. Capron }}$ \\
\hline T. M. Blakley \\
\hline $0100 \mathrm{C}-\mathrm{CA}-\mathrm{V} 0033$ \\
\hline
\end{tabular}

Sheet No. 38 of 43

Date $12 / 13 / 06$

Date

Rev. No. 
Attachment 1. 118-C-1 Verification Sampling Results.

\begin{tabular}{|c|c|c|c|c|c|c|c|c|c|c|c|c|}
\hline \multirow[t]{2}{*}{ Constituents } & \multicolumn{3}{|c|}{\begin{tabular}{|c|} 
J135P4 \\
SPA C1 \\
Sample Date 9/12/06 \\
\end{tabular}} & \multicolumn{3}{|c|}{\begin{tabular}{|c|} 
J135P5 \\
SPA C2 \\
Sample Date 9/12/06 \\
\end{tabular}} & \multicolumn{3}{|c|}{$\begin{array}{c}\text { J135P6 } \\
\text { SPA D3 } \\
\text { Sample Date 9/12/06 } \\
\end{array}$} & \multicolumn{3}{|c|}{$\begin{array}{c}\text { J135P7 } \\
\text { SPA D4 } \\
\text { Sample Date 9/12/06 } \\
\end{array}$} \\
\hline & $\mu \mathrm{g} / \mathrm{kg}$ & $\mathbf{Q}$ & PQL & $\mu \mathrm{g} / \mathrm{kg}$ & $\mathbf{Q}$ & PQL & $\mu \mathrm{g} / \mathrm{kg}$ & $Q$ & PQL & $\mu \mathrm{g} / \mathrm{kg}$ & Q & PQL \\
\hline \multicolumn{13}{|c|}{ Volatile Organic Compounds } \\
\hline 1,1,1-Trichloroethane & 5 & $\mathrm{U}$ & 5 & 6 & U & 6 & 5 & $\mathrm{U}$ & 5 & 5 & $U$ & 5 \\
\hline 1,1,2,2-Tetrachloroethane & 5 & $\mathrm{U}$ & 5 & 6 & $\mathrm{U}$ & 6 & 5 & $\mathrm{U}$ & 5 & 5 & $\mathrm{U}$ & 5 \\
\hline 1,1,2-Trichloroethane & 5 & $\mathrm{U}$ & 5 & 6 & $\mathrm{U}$ & 6 & 5 & $\mathrm{U}$ & 5 & 5 & $\mathrm{U}$ & 5 \\
\hline 1,1-Dichloroethane & 5 & $\mathrm{U}$ & 5 & 6 & $U$ & 6 & 5 & $\mathrm{U}$ & 5 & 5 & $U$ & 5 \\
\hline 1,1-Dichloroethene & 5 & $\mathrm{U}$ & 5 & 6 & $\mathrm{U}$ & 6 & 5 & $\mathrm{U}$ & 5 & 5 & $\mathrm{U}$ & 5 \\
\hline 1,2-Dichloroethane & 5 & $\mathrm{U}$ & 5 & 6 & $\mathrm{U}$ & 6 & 5 & U & 5 & 5 & $\mathrm{U}$ & 5 \\
\hline 1,2-Dichloroethene (total) & 5 & $\mathrm{U}$ & 5 & 6 & U & 6 & 5 & $\mathrm{U}$ & 5 & 5 & $\mathrm{U}$ & 5 \\
\hline \begin{tabular}{|l} 
1,2-Dichloropropane \\
\end{tabular} & 5 & $\mathrm{U}$ & 5 & 6 & $\mathrm{U}$ & 6 & 5 & $\mathrm{U}$ & 5 & 5 & $\mathrm{U}$ & 5 \\
\hline 2-Butanone & 10 & $\mathrm{U}$ & 10 & 11 & $\mathrm{U}$ & 11 & 10 & $\mathrm{U}$ & 10 & 9 & $\bar{U}$ & 9 \\
\hline 2-Hexanone & 10 & $\mathrm{U}$ & 10 & 11 & $\mathrm{U}$ & 11 & 10 & $\mathrm{U}$ & 10 & 9 & $\mathrm{U}$ & 9 \\
\hline 4-Methyl-2-pentanone & 10 & $\mathrm{U}$ & 10 & 11 & $U$ & 11 & 10 & $\mathrm{U}$ & 10 & 9 & $\mathrm{U}$ & 9 \\
\hline Acetone & 7 & $\mathrm{~J}$ & 10 & 8 & $\mathrm{~J}$ & 11 & 7 & $\mathrm{~J}$ & 10 & 11 & & 9 \\
\hline Benzene & 5 & $\overline{\mathrm{U}}$ & 5 & 6 & $\mathrm{U}$ & 6 & 5 & U & 5 & 5 & $\mathrm{U}$ & 5 \\
\hline Bromodichloromethane & 5 & $\mathrm{U}$ & 5 & 6 & $\mathrm{U}$ & 6 & 5 & U & 5 & 5 & $\mathrm{U}$ & 5 \\
\hline Bromoform & 5 & $\mathrm{U}$ & 5 & 6 & U & 6 & 5 & $\mathrm{U}$ & 5 & 5 & $\mathrm{U}$ & 5 \\
\hline Bromomethane & 10 & $\mathrm{U}$ & 10 & 11 & $\mathrm{U}$ & 11 & 10 & $\mathrm{U}$ & 10 & 9 & $\mathrm{U}$ & 9 \\
\hline Carbon disulfide & 5 & $\bar{U}$ & 5 & 6 & $\mathrm{U}$ & 6 & 5 & $\mathrm{U}$ & 5 & 5 & $\bar{U}$ & 5 \\
\hline Carbon tetrachloride & 5 & $\mathrm{U}$ & 5 & 6 & $\mathrm{U}$ & 6 & 5 & $\mathrm{U}$ & 5 & 5 & $\mathrm{U}$ & 5 \\
\hline \begin{tabular}{|l|} 
Chlorobenzene \\
\end{tabular} & 5 & $\mathrm{U}$ & 5 & 6 & $\mathrm{U}$ & 6 & 5 & $\mathrm{U}$ & 5 & 5 & $\mathrm{U}$ & 5 \\
\hline Chloroethane & 10 & $\mathrm{U}$ & 10 & 11 & $\mathrm{U}$ & 11 & 10 & $\mathrm{U}$ & 10 & 9 & $\mathrm{U}$ & 9 \\
\hline Chloroform & 5 & $\mathrm{U}$ & 5 & 6 & $\mathrm{U}$ & 6 & 5 & $\bar{U}$ & 5 & 5 & $\mathrm{U}$ & 5 \\
\hline Chloromethane & 10 & $\mathrm{U}$ & 10 & 11 & $\mathrm{U}$ & 11 & 10 & $\mathrm{U}$ & 10 & 9 & $U$ & 9 \\
\hline cis-1,2-Dichloroethylene & 5 & $\mathrm{U}$ & 5 & 6 & $\mathrm{U}$ & 6 & 5 & U & 5 & 5 & $\mathrm{U}$ & 5 \\
\hline cis-1,3-Dichloropropene & 5 & $\mathrm{U}$ & 5 & 6 & $\mathrm{U}$ & 6 & 5 & $\mathrm{U}$ & 5 & 5 & $U$ & 5 \\
\hline Dibromochloromethane & 5 & $\mathrm{U}$ & 5 & 6 & $\mathrm{U}$ & 6 & 5 & $\mathrm{U}$ & 5 & 5 & $\mathrm{U}$ & 5 \\
\hline Ethylbenzene & 5 & $\mathrm{U}$ & 5 & 6 & $\mathrm{U}$ & 6 & 5 & $\mathrm{U}$ & 5 & 5 & $\bar{U}$ & 5 \\
\hline Methylene chloride & 24 & $\mathrm{~B}$ & 5 & 27 & $B$ & 6 & 26 & $\mathrm{~B}$ & 5 & 18 & $\mathrm{~B}$ & 5 \\
\hline Styrene & 5 & $\mathrm{U}$ & 5 & 4 & $\mathrm{~J}$ & 6 & 5 & $\mathrm{U}$ & 5 & 5 & U & 5 \\
\hline Tetrachloroethene & 5 & $\mathrm{U}$ & 5 & 6 & $\mathrm{U}$ & 6 & 5 & $\mathrm{U}$ & 5 & 5 & $U$ & 5 \\
\hline \begin{tabular}{|l} 
Toluene \\
\end{tabular} & 5 & $\mathrm{U}$ & 5 & 1 & $\mathrm{~J}$ & 6 & 1 & $\mathrm{~J}$ & 5 & 5 & $U$ & 5 \\
\hline trans-1,2-Dichloroethylene & 5 & $\mathrm{U}$ & 5 & 6 & $\mathrm{U}$ & 6 & 5 & $\mathrm{U}$ & 5 & 5 & $U$ & 5 \\
\hline trans-1,3-Dichloropropene & 5 & $\mathrm{U}$ & 5 & 6 & $\mathrm{U}$ & 6 & 5 & $\mathrm{U}$ & 5 & 5 & $U$ & 5 \\
\hline Trichloroethene & 5 & $\mathrm{U}$ & 5 & 6 & $U$ & 6 & 5 & $\mathrm{U}$ & 5 & 5 & $\mathrm{U}$ & 5 \\
\hline Vinyl chloride & 10 & $\mathrm{U}$ & 10 & 11 & $\mathrm{U}$ & 11 & 10 & $\mathrm{U}$ & 10 & 9 & $\mathrm{U}$ & 9 \\
\hline Xylenes (total) & 5 & $\mathrm{U}$ & 5 & 6 & $\mathrm{U}$ & 6 & 5 & $\mathrm{U}$ & 5 & 5 & $\mathrm{U}$ & 5 \\
\hline & & & & Attachm & & & 1 & & & heet No. & & of 43 \\
\hline & & & & Originat & & J.M. Cap & oron & & & Date & & $13 / 06$ \\
\hline & & & & Checked & & T.M. Bla & kley & & & Date & & \\
\hline & & & & Calc. No & & $0100 \mathrm{C}-\mathrm{C}$ & $\mathrm{A}-\mathrm{V} 0033$ & & & Rev. No. & & 0 \\
\hline
\end{tabular}


Attachment 1. 118-C-1 Verification Sampling Results.

\begin{tabular}{|c|c|c|c|c|c|c|c|c|c|c|c|c|}
\hline \multirow[t]{2}{*}{ Constituents } & \multicolumn{3}{|c|}{$\begin{array}{c}\text { J135P8 } \\
\text { SPA D5 } \\
\text { Sample Date 9/12/06 } \\
\end{array}$} & \multicolumn{3}{|c|}{$\begin{array}{c}\text { J135P9 } \\
\text { SPA D6 } \\
\text { Sample Date 9/12/06 } \\
\end{array}$} & \multicolumn{3}{|c|}{$\begin{array}{c}\text { J12XM2 } \\
\text { FS-5 } \\
\text { Sample Date } 7 / 26 / 06 \\
\end{array}$} & \multicolumn{3}{|c|}{$\begin{array}{c}\text { J12XM3 } \\
\text { FS-6 } \\
\text { Sample Date } 7 / 26 / 06 \\
\end{array}$} \\
\hline & $\mu \mathrm{g} / \mathrm{kg}$ & $Q$ & PQL & $\mu \mathrm{g} / \mathrm{kg}$ & $\mathbf{Q}$ & PQL & $\mu \mathrm{g} / \mathrm{kg}$ & $\mathbf{Q}$ & PQL & $\mu \mathrm{g} / \mathrm{kg}$ & Q & $\mathrm{PQL}$ \\
\hline \multicolumn{13}{|c|}{ Polychlorinated Biphenyls } \\
\hline Aroclor-1016 & 13 & $\mathrm{U}$ & 13 & 13 & $\mathrm{U}$ & 13 & 13 & $\mathrm{U}$ & 13 & 14 & $\mathrm{U}$ & 14 \\
\hline Aroclor- 1221 & 13 & $\mathrm{U}$ & 13 & 13 & $\mathrm{U}$ & 13 & 13 & $\mathrm{U}$ & 13 & 14 & $\mathrm{U}$ & 14 \\
\hline Aroclor-1232 & 13 & $U$ & 13 & 13 & $\mathrm{U}$ & 13 & 13 & $\mathrm{U}$ & 13 & 14 & $\mathrm{U}$ & 14 \\
\hline Aroclor- 1242 & 13 & $\mathrm{U}$ & 13 & 13 & $\mathrm{U}$ & 13 & 13 & $\bar{U}$ & 13 & 14 & $\mathrm{U}$ & 14 \\
\hline Aroclor-1248 & 13 & $\mathrm{U}$ & 13 & 13 & $\mathrm{U}$ & 13 & 13 & $\mathrm{U}$ & 13 & 14 & $\mathrm{U}$ & 14 \\
\hline Aroclor -1254 & 3.7 & $\mathrm{~J}$ & 13 & 4.7 & $\mathrm{~J}$ & 13 & 22 & & 13 & 14 & $\mathrm{U}$ & 14 \\
\hline Aroclor- 1260 & 13 & $\mathrm{U}$ & 13 & 13 & $\mathrm{U}$ & 13 & 13 & $\mathrm{U}$ & 13 & 14 & $\mathrm{U}$ & 14 \\
\hline \multicolumn{13}{|c|}{ Semivolatile Organic Compounds } \\
\hline 1,2,4-Trichlorobenzene & 330 & $\mathrm{UJ}$ & 330 & 330 & UJ & 330 & 330 & $\mathrm{U}$ & 330 & 340 & $\mathrm{U}$ & 340 \\
\hline 1,2-Dichlorobenzene & 330 & $\mathrm{UJ}$ & 330 & 330 & UJ & 330 & 330 & $\mathrm{U}$ & 330 & 340 & $\mathrm{U}$ & 340 \\
\hline 1,3-Dichlorobenzene & 330 & UJ & 330 & 330 & $\mathrm{UJ}$ & 330 & 330 & $\mathrm{U}$ & 330 & 340 & $\mathrm{U}$ & 340 \\
\hline 1,4-Dichlorobenzene & 330 & UJ & 330 & 330 & UJ & 330 & 330 & $\mathrm{U}$ & 330 & 340 & $\mathrm{U}$ & 340 \\
\hline 2,4,5-Trichlorophenol & 840 & $\mathrm{U}$ & 840 & 840 & $\mathrm{U}$ & 840 & 840 & $\mathrm{U}$ & 840 & 850 & $\mathrm{U}$ & 850 \\
\hline 2,4,6-Trichlorophenol & 330 & $\mathrm{U}$ & 330 & 330 & $\bar{U}$ & 330 & 330 & $\mathrm{U}$ & 330 & 340 & $\mathrm{U}$ & 340 \\
\hline 2,4-Dichlorophenol & 330 & $U$ & 330 & 330 & $\mathrm{U}$ & 330 & 330 & $\mathrm{U}$ & 330 & 340 & $\mathrm{U}$ & 340 \\
\hline 2,4-Dimethylphenol & 330 & UI & 330 & 330 & UJ & 330 & 330 & $\mathrm{U}$ & 330 & 340 & $\mathrm{U}$ & 340 \\
\hline 2,4-Dinitrophenol & 840 & $\mathrm{UJ}$ & 840 & 840 & UJ & 840 & 840 & $\mathrm{U}$ & 840 & 850 & $\mathrm{U}$ & 850 \\
\hline 2,4-Dinitrotoluene & 330 & $\mathrm{U}$ & 330 & 330 & $\mathrm{U}$ & 330 & 330 & $\mathrm{U}$ & 330 & 340 & $\mathrm{U}$ & 340 \\
\hline 2,6-Dinitrotoluene & 330 & $\mathrm{U}$ & 330 & 330 & $\mathrm{U}$ & 330 & 330 & U & 330 & 340 & $\mathrm{U}$ & 340 \\
\hline 2-Chloronaphthalene & 330 & $\mathrm{U}$ & 330 & 330 & $\mathrm{U}$ & 330 & 330 & $\mathrm{U}$ & 330 & 340 & $\mathrm{U}$ & 340 \\
\hline 2-Chlorophenol & 330 & $\mathrm{UJ}$ & 330 & 330 & UJ & 330 & 330 & $\mathrm{U}$ & 330 & 340 & $\mathrm{U}$ & 340 \\
\hline 2-Methylnaphthalene & 330 & $\mathrm{UJ}$ & 330 & 330 & $\mathrm{U}$ & 330 & 330 & $\mathrm{U}$ & 330 & 340 & $\bar{U}$ & 340 \\
\hline 2-Methylphenol (cresol, o-) & 330 & US & 330 & 330 & UJ & 330 & 330 & $\mathrm{U}$ & 330 & 340 & $\mathrm{U}$ & 340 \\
\hline 2-Nitroaniline & 840 & $\mathrm{U}$ & 840 & 840 & $\mathrm{U}$ & 840 & 840 & $\mathrm{U}$ & 840 & 850 & $\mathrm{U}$ & 850 \\
\hline 2-Nitrophenol & 330 & UJ & 330 & 330 & $\mathrm{UJ}$ & 330 & 330 & $\mathrm{U}$ & 330 & 340 & $\mathrm{U}$ & 340 \\
\hline 3,3'-Dichlorobenzidine & 330 & $\mathrm{U}$ & 330 & 330 & $\mathrm{U}$ & 330 & 330 & $\mathrm{U}$ & 330 & 340 & $\mathrm{U}$ & 340 \\
\hline 3-Nitroaniline & 840 & $U$ & 840 & 840 & $\mathrm{U}$ & 840 & 840 & $\mathrm{U}$ & 840 & 850 & $\bar{U}$ & 850 \\
\hline 4,6-Dinitro-2-methylphenol & 840 & $\mathrm{U}$ & 840 & 840 & $\mathrm{U}$ & 840 & 840 & $\mathrm{U}$ & 840 & 850 & $\mathrm{U}$ & 850 \\
\hline 4-Bromophenyl-phenylether & 330 & $\mathrm{U}$ & 330 & 330 & $\mathrm{U}$ & 330 & 330 & $\mathrm{U}$ & 330 & 340 & $\mathrm{U}$ & 340 \\
\hline 4-Chloro-3-methylphenol & 330 & $\mathrm{UJ}$ & 330 & 330 & $\mathrm{UJ}$ & 330 & 330 & $\mathrm{U}$ & 330 & 340 & $\mathrm{U}$ & 340 \\
\hline 4-Chloroaniline & 330 & $\mathrm{U}$ & 330 & 330 & $\mathrm{U}$ & 330 & 330 & $\mathrm{U}$ & 330 & 340 & $\mathrm{U}$ & 340 \\
\hline 4-Chlorophenyl-phenylether & 330 & $\mathrm{U}$ & 330 & 330 & $\mathrm{U}$ & 330 & 330 & $\mathrm{U}$ & 330 & 340 & $\mathrm{U}$ & 340 \\
\hline 4-Methylphenol (p-cresol) & 330 & UJ & 330 & 330 & $\mathrm{UJ}$ & 330 & 330 & $\mathrm{U}$ & 330 & 340 & U & 340 \\
\hline 4-Nitroaniline & 840 & $\mathrm{U}$ & 840 & 840 & $\mathrm{U}$ & 840 & 840 & $\mathrm{U}$ & 840 & 850 & $\mathrm{U}$ & 850 \\
\hline 4-Nitrophenol & 840 & $U$ & 840 & 840 & $\mathrm{U}$ & 840 & 840 & $\mathrm{U}$ & 840 & 850 & $\mathrm{U}$ & 850 \\
\hline Acenaphthene & 330 & $\mathrm{U}$ & 330 & 330 & $\mathrm{U}$ & 330 & 330 & $\mathrm{U}$ & 330 & 340 & $\mathrm{U}$ & 340 \\
\hline Acenaphthylene & 330 & $\mathrm{U}$ & 330 & 330 & $\mathrm{U}$ & 330 & 330 & $\mathrm{U}$ & 330 & 340 & $\mathrm{U}$ & 340 \\
\hline Anthracene & 330 & UJ & 330 & 330 & UJ & 330 & 330 & $\mathrm{U}$ & 330 & 340 & $\mathrm{U}$ & 340 \\
\hline Benzo(a)anthracene & 330 & US & 330 & 18 & $\mathrm{~J}$ & 330 & 330 & $\mathrm{U}$ & 330 & 340 & $\mathrm{U}$ & 340 \\
\hline Benzo(a)pyrene & 330 & $\mathrm{UJ}$ & 330 & 330 & UJ & 330 & 330 & $\mathrm{U}$ & 330 & 340 & $\mathrm{U}$ & 340 \\
\hline Benzo(b)fluoranthene & 330 & $\mathrm{UJ}$ & 330 & 330 & $\mathrm{UJ}$ & 330 & 330 & $\mathrm{U}$ & 330 & 340 & $\mathrm{U}$ & 340 \\
\hline Benzo(g,h,i)perylene & 330 & $\mathrm{U}$ & 330 & 330 & $\mathrm{U}$ & 330 & 330 & $\mathrm{U}$ & 330 & 340 & $\mathrm{U}$ & 340 \\
\hline Benzo(k)fluoranthene & 330 & $\mathrm{UJ}$ & 330 & 330 & UJ & 330 & 330 & $\mathrm{U}$ & 330 & 340 & $\mathrm{U}$ & 340 \\
\hline bis(2-Chloro-1-methylethyl)ether & 330 & $\mathrm{U}$ & 330 & 330 & $\mathrm{U}$ & 330 & 330 & $\mathrm{U}$ & 330 & 340 & $\mathrm{U}$ & 340 \\
\hline bis(2-Chloroethoxy)methane & 330 & $\mathrm{U}$ & 330 & 330 & $\mathrm{U}$ & 330 & 330 & $\mathrm{U}$ & 330 & 340 & $\mathrm{U}$ & 340 \\
\hline bis(2-Chloroethyl)ether & 330 & $\mathrm{U}$ & 330 & 330 & $\mathrm{U}$ & 330 & 330 & $\mathrm{U}$ & 330 & 340 & $\mathrm{U}$ & 340 \\
\hline bis(2-Ethylhexyl)phthalate & 50 & JB & 330 & 41 & JB & 330 & 39 & $\mathrm{JB}$ & 330 & 67 & $\mathrm{JB}$ & 340 \\
\hline
\end{tabular}

Attachment

Originator

Checked

Calc. No.

$\frac{1}{\frac{1}{\text { J.M. Capron }}} \frac{}{\text { T.M. Blakley }}$

Sheet No. 40 of 43

Date $12 / 13 / 06$

Date

Rev. No. $\overline{0}$ 
Attachment 1. 118-C-1 Verification Sampling Results.

\begin{tabular}{|c|c|c|c|c|c|c|c|c|c|c|c|c|}
\hline \multirow[t]{2}{*}{ Constituents } & \multicolumn{3}{|c|}{$\begin{array}{c}\text { J135P8 } \\
\text { SPA D5 } \\
\text { Sample Date 9/12/06 } \\
\end{array}$} & \multicolumn{3}{|c|}{$\begin{array}{c}\text { J135P9 } \\
\text { SPA D6 } \\
\text { Sample Date 9/12/06 } \\
\end{array}$} & \multicolumn{3}{|c|}{$\begin{array}{c}\text { J12XM2 } \\
\text { FS-5 } \\
\text { Sample Date } 7 / 26 / 06 \\
\end{array}$} & \multicolumn{3}{|c|}{$\begin{array}{c}\text { J12XM3 } \\
\text { FS-6 } \\
\text { Sample Date } 7 / 26 / 06\end{array}$} \\
\hline & $\mu \mathrm{g} / \mathrm{kg}$ & $\mathbf{Q}$ & PQL & $\mu \mathrm{g} / \mathrm{kg}$ & $\mathbf{Q}$ & PQL & $\mu \mathrm{g} / \mathrm{kg}$ & $Q$ & PQL & $\mu \mathrm{g} / \mathrm{kg}$ & $\mathbf{Q}$ & PQL \\
\hline \multicolumn{13}{|c|}{ Semivolatile Organic Compounds (continued) } \\
\hline Butylbenzylphthalate & 330 & $\mathrm{U}$ & 330 & 330 & $\mathrm{U}$ & 330 & 330 & $\mathrm{U}$ & 330 & 340 & $\mathrm{U}$ & 340 \\
\hline Carbazole & 330 & $\mathrm{UJ}$ & 330 & 330 & $\mathrm{UJ}$ & 330 & 330 & $U$ & 330 & 340 & $\mathrm{U}$ & 340 \\
\hline Chrysene & 330 & $\mathrm{UJ}$ & 330 & 27 & $\mathrm{~J}$ & 330 & 330 & $\mathrm{U}$ & 330 & 340 & $\mathrm{U}$ & 340 \\
\hline Di-n-butylphthalate & 29 & JB & 330 & 330 & $\mathrm{U}$ & 330 & 55 & $\mathrm{JB}$ & 330 & 49 & $\mathrm{JB}$ & 340 \\
\hline Di-n-octylphthalate & 330 & $\mathrm{U}$ & 330 & 330 & $\mathrm{U}$ & 330 & 330 & $\mathrm{U}$ & 330 & 340 & $\mathrm{U}$ & 340 \\
\hline Dibenz(a,h)anthracene & 330 & $\mathrm{U}$ & 330 & 330 & $\mathrm{U}$ & 330 & 330 & $\mathrm{U}$ & 330 & 340 & $\mathrm{U}$ & 340 \\
\hline Dibenzofuran & 330 & $\mathrm{UJ}$ & 330 & 330 & $\mathrm{UJ}$ & 330 & 330 & $\mathrm{U}$ & 330 & 340 & $\mathrm{U}$ & 340 \\
\hline Diethylphthalate & 330 & $\mathrm{U}$ & 330 & 330 & $\mathrm{U}$ & 330 & 22 & $\mathrm{~J}$ & 330 & 340 & $\mathrm{U}$ & 340 \\
\hline Dimethylphthalate & 330 & $\mathrm{U}$ & 330 & 330 & $\mathrm{U}$ & 330 & 330 & $\mathrm{U}$ & 330 & 340 & $\mathrm{U}$ & 340 \\
\hline Fluoranthene & 18 & $\mathrm{JR}$ & 330 & 34 & $\mathrm{JR}$ & 330 & 330 & $\mathrm{U}$ & 330 & 340 & $\mathrm{U}$ & 340 \\
\hline Fluorene & 330 & UJ & 330 & 330 & UJ & 330 & 330 & $\mathrm{U}$ & 330 & 340 & $\mathrm{U}$ & 340 \\
\hline Hexachlorobenzene & 330 & $\mathrm{U}$ & 330 & 330 & $\mathrm{U}$ & 330 & 330 & $\mathrm{U}$ & 330 & 340 & $\mathrm{U}$ & 340 \\
\hline Hexachlorobutadiene & 330 & $\mathrm{U}$ & 330 & 330 & $\mathrm{U}$ & 330 & 330 & $\mathrm{U}$ & 330 & 340 & $\mathrm{U}$ & 340 \\
\hline Hexachlorocyclopentadiene & 330 & $\mathrm{U}$ & 330 & 330 & $\mathrm{U}$ & 330 & 330 & $\mathrm{U}$ & 330 & 340 & $\mathrm{U}$ & 340 \\
\hline Hexachloroethane & 330 & $\mathrm{UJ}$ & 330 & 330 & UJ & 330 & 330 & $\mathrm{U}$ & 330 & 340 & $\mathrm{U}$ & 340 \\
\hline Indeno $(1,2,3$-cd)pyrene & 330 & UJ & 330 & 330 & $\overline{U J}$ & 330 & 330 & $\bar{U}$ & 330 & 340 & $\mathrm{U}$ & 340 \\
\hline Isophorone & 330 & $\mathrm{UJ}$ & 330 & 330 & $\mathrm{UJ}$ & 330 & 330 & $\mathrm{U}$ & 330 & 340 & $\mathrm{U}$ & 340 \\
\hline $\mathrm{N}$-Nitroso-di-n-dipropylamine & 330 & UJ & 330 & 330 & $\mathrm{UJ}$ & 330 & 330 & $\mathrm{U}$ & 330 & 340 & $\mathrm{U}$ & 340 \\
\hline $\mathrm{N}$-Nitrosodiphenylamine & 330 & $U$ & 330 & 330 & $\mathrm{U}$ & 330 & 330 & $\mathrm{U}$ & 330 & 340 & $\mathrm{U}$ & 340 \\
\hline Naphthalene & 330 & $\mathrm{U}$ & 330 & 330 & $\mathrm{U}$ & 330 & 330 & $\mathrm{U}$ & 330 & 340 & $\mathrm{U}$ & 340 \\
\hline Nitrobenzene & 330 & UY & 330 & 330 & $\mathrm{UJ}$ & 330 & 330 & $\mathrm{U}$ & 330 & 340 & $\mathrm{U}$ & 340 \\
\hline Pentachlorophenol & 840 & $\mathrm{U}$ & 840 & 840 & $\mathrm{U}$ & 840 & 840 & $\mathrm{U}$ & 840 & 850 & $\mathrm{U}$ & 850 \\
\hline Phenanthrene & 330 & UJ & 330 & 330 & UJ & 330 & 330 & $\mathrm{U}$ & 330 & 340 & $\mathrm{U}$ & 340 \\
\hline Phenol & 330 & $\mathrm{U}$ & 330 & 330 & $\mathrm{U}$ & 330 & 36 & $\mathrm{JB}$ & 330 & 33 & $\mathrm{JB}$ & 340 \\
\hline Pyrene & 330 & $\mathrm{U}$ & 330 & 27 & $\mathrm{~J}$ & 330 & 330 & $\mathrm{U}$ & 330 & 340 & $\mathrm{U}$ & 340 \\
\hline & & & & Attachm & & & 1 & & & et No. & & of 43 \\
\hline & & & & Originat & & J. M. Ca & ron & & & Date & & $13 / 06$ \\
\hline & & & & Checked & & T. M. Bl & kley & & & Date & & \\
\hline & & & & Calc. No & & $0100 \mathrm{C}-\mathrm{C}$ & A-V0033 & & & Rev. No. & & 0 \\
\hline
\end{tabular}


Attachment 1. 118-C-1 Verification Sampling Results.

\begin{tabular}{|c|c|c|c|c|c|c|c|c|c|c|c|c|}
\hline \multirow[t]{2}{*}{ Constituents } & \multicolumn{3}{|c|}{$\begin{array}{c}\text { J135P8 } \\
\text { SPA D5 } \\
\text { Sample Date 9/12/06 }\end{array}$} & \multicolumn{3}{|c|}{\begin{tabular}{|c|} 
J135P9 \\
SPA D6 \\
Sample Date 9/12/06
\end{tabular}} & \multicolumn{3}{|c|}{$\begin{array}{c}\text { J12XM2 } \\
\text { FS-5 } \\
\text { Sample Date 7/26/06 }\end{array}$} & \multicolumn{3}{|c|}{\begin{tabular}{|c|} 
J12XM3 \\
FS-6 \\
Sample Date $7 / 26 / 06$ \\
\end{tabular}} \\
\hline & $\mu \mathrm{g} / \mathrm{kg}$ & $\mathbf{Q}$ & PQL & $\mu \mathrm{g} / \mathrm{kg}$ & $\mathbf{Q}$ & PQL & $\mu \mathrm{g} / \mathrm{kg}$ & Q & PQL & $\mu \mathrm{g} / \mathrm{kg}$ & $Q$ & PQL \\
\hline \multicolumn{13}{|c|}{ Volatile Organic Compounds } \\
\hline 1,1,1-Trichloroethane & 6 & $\mathrm{U}$ & 6 & 5 & $\mathrm{U}$ & 5 & 5 & $\mathrm{U}$ & 5 & 5 & $\mathrm{U}$ & 5 \\
\hline 1,1,2,2-Tetrachloroethane & 6 & $\mathrm{U}$ & 6 & 5 & $\mathrm{U}$ & 5 & 5 & $\bar{U}$ & 5 & 5 & $\mathrm{U}$ & 5 \\
\hline 1,1,2-Trichloroethane & 6 & $\mathrm{U}$ & 6 & 5 & $\mathrm{U}$ & 5 & 5 & $\mathrm{U}$ & 5 & 5 & $\mathrm{U}$ & 5 \\
\hline 1,1-Dichloroethane & 6 & $\bar{U}$ & 6 & 5 & $\mathrm{U}$ & 5 & 5 & $\mathrm{U}$ & 5 & 5 & $\mathrm{U}$ & 5 \\
\hline 1,1-Dichloroethene & 6 & $\mathrm{U}$ & 6 & 5 & $\mathrm{U}$ & 5 & 5 & $\mathrm{U}$ & 5 & 5 & $\mathrm{U}$ & 5 \\
\hline 1,2-Dichloroethane & 6 & $\mathrm{U}$ & 6 & 5 & $\mathrm{U}$ & 5 & 5 & $\mathrm{U}$ & 5 & 5 & $\mathrm{U}$ & 5 \\
\hline 1,2-Dichloroethene (total) & 6 & $\mathrm{U}$ & 6 & 5 & $\mathrm{U}$ & 5 & 5 & $\mathrm{U}$ & 5 & 5 & $\mathrm{U}$ & 5 \\
\hline 1,2-Dichloropropane & 6 & $\mathrm{U}$ & 6 & 5 & $\mathrm{U}$ & 5 & 5 & $\bar{U}$ & 5 & 5 & $\mathrm{U}$ & 5 \\
\hline 2-Butanone & 11 & $\mathrm{U}$ & 11 & 10 & $\mathrm{U}$ & 10 & 10 & $\mathrm{U}$ & 10 & 10 & $\mathrm{U}$ & 10 \\
\hline 2-Hexanone & 11 & $\mathrm{U}$ & 11 & 10 & $\mathrm{U}$ & 10 & 10 & $\mathrm{U}$ & 10 & 10 & $\mathrm{U}$ & 10 \\
\hline 4-Methyl-2-pentanone & 11 & $\mathrm{U}$ & 11 & 10 & $\mathrm{U}$ & 10 & 10 & $\mathrm{U}$ & 10 & 10 & $U$ & 10 \\
\hline Acetone & 11 & $\mathrm{U}$ & 11 & 8 & $\mathrm{~J}$ & 10 & 24 & & 10 & 10 & $\mathrm{~J}$ & 10 \\
\hline Benzene & 6 & $\mathrm{U}$ & 6 & 5 & $\mathrm{U}$ & 5 & 5 & $\mathrm{U}$ & 5 & 5 & $\mathrm{U}$ & 5 \\
\hline Bromodichloromethane & 6 & $\mathrm{U}$ & 6 & 5 & $\mathrm{U}$ & 5 & 5 & $\mathrm{U}$ & 5 & 5 & $\mathrm{U}$ & 5 \\
\hline Bromoform & 6 & $\mathrm{U}$ & 6 & 5 & $\mathrm{U}$ & 5 & 5 & $\mathrm{U}$ & 5 & 5 & $\mathrm{U}$ & 5 \\
\hline Bromomethane & 11 & $\mathrm{U}$ & 11 & 10 & $\mathrm{U}$ & 10 & 10 & $\mathrm{U}$ & 10 & 10 & $\mathrm{U}$ & 10 \\
\hline Cárbon disulfide & 6 & $\mathrm{U}$ & 6 & 5 & $\mathrm{U}$ & 5 & 5 & $\mathrm{U}$ & 5 & 5 & $\mathrm{U}$ & 5 \\
\hline Carbon tetrachloride & 6 & U & 6 & 5 & $\mathrm{U}$ & 5 & 39 & & 5 & 10 & & 5 \\
\hline \begin{tabular}{|l|} 
Chlorobenzene \\
\end{tabular} & 6 & $\mathrm{U}$ & 6 & 5 & $\mathrm{U}$ & 5 & 5 & $\mathrm{U}$ & 5 & 5 & $\mathrm{U}$ & 5 \\
\hline Chloroethane & 11 & $\mathrm{U}$ & 11 & 10 & $\mathrm{U}$ & 10 & 10 & $\mathrm{U}$ & 10 & 10 & $\mathrm{U}$ & 10 \\
\hline Chloroform & 6 & $\mathrm{U}$ & 6 & 5 & $\mathrm{U}$ & 5 & 5 & $\mathrm{U}$ & 5 & 5 & $\mathrm{U}$ & 5 \\
\hline Chloromethane & 11 & $U$ & 11 & 10 & $\mathrm{U}$ & 10 & 10 & $\mathrm{U}$ & 10 & 10 & $\mathrm{U}$ & 10 \\
\hline cis-1,2-Dichloroethylene & 6 & $\mathrm{U}$ & 6 & 5 & $\mathrm{U}$ & 5 & 5 & $\bar{U}$ & 5 & 5 & $\mathrm{U}$ & 5 \\
\hline cis-1,3-Dichloropropene & 6 & $\mathrm{U}$ & 6 & 5 & $\mathrm{U}$ & 5 & 5 & $\bar{U}$ & 5 & 5 & U & 5 \\
\hline Dibromochloromethane & 6 & $\mathrm{U}$ & 6 & 5 & U & 5 & 5 & $\mathrm{U}$ & 5 & 5 & $\mathrm{U}$ & 5 \\
\hline Ethylbenzene & 6 & $\mathrm{U}$ & 6 & 5 & $\mathrm{U}$ & 5 & 5 & $\mathrm{U}$ & 5 & 5 & $\mathrm{U}$ & 5 \\
\hline Methylene chloride & 23 & $\mathrm{~B}$ & 6 & 30 & $\mathrm{~B}$ & 5 & 19 & & 5 & 5 & $\mathrm{U}$ & 5 \\
\hline Styrene & 6 & $\mathrm{U}$ & 6 & 1 & $\mathrm{~J}$ & 5 & 5 & $\mathrm{U}$ & 5 & 5 & $\mathrm{U}$ & 5 \\
\hline Tetrachloroethene & 6 & $\mathrm{U}$ & 6 & 5 & $\mathrm{U}$ & 5 & 5 & $\mathrm{U}$ & 5 & 5 & $\mathrm{U}$ & 5 \\
\hline Toluene & 6 & $\mathrm{U}$ & 6 & 1 & $\mathrm{~J}$ & 5 & 5 & $\mathrm{U}$ & 5 & 5 & $\mathrm{U}$ & 5 \\
\hline trans-1,2-Dichloroethylene & 6 & $\mathrm{U}$ & 6 & 5 & $\mathrm{U}$ & 5 & 5 & $\mathrm{U}$ & 5 & 5 & $\mathrm{U}$ & 5 \\
\hline trans-1,3-Dichloropropene & 6 & $\mathrm{U}$ & 6 & 5 & $\mathrm{U}$ & 5 & 5 & $\mathrm{U}$ & 5 & 5 & $\mathrm{U}$ & 5 \\
\hline Trichloroethene & 6 & $\mathrm{U}$ & 6 & 5 & $\mathrm{U}$ & 5 & 5 & $\mathrm{U}$ & 5 & 5 & $\mathrm{U}$ & 5 \\
\hline Vinyl chloride & 11 & $\mathrm{U}$ & 11 & 10 & $\mathrm{U}$ & 10 & 10 & $\mathrm{U}$ & 10 & 10 & $\mathrm{U}$ & 10 \\
\hline Xylenes (total) & 6 & $\mathrm{U}$ & 6 & 1 & $\mathrm{~J}$ & 5 & 5 & $\mathrm{U}$ & 5 & 5 & $\mathrm{U}$ & 5 \\
\hline & & & & Attachm & & & 1 & & & heet No. & & of 43 \\
\hline & & & & Originat & & J.M. Ca & ron & & & Date & & $13 / 06$ \\
\hline & & & & Checked & & T.M. BI & kley & & & Date & & \\
\hline & & & & Calc. Nc & & $0100 \mathrm{C}-\mathrm{C}$ & $\mathrm{A}-\mathrm{V} 0033$ & & & Rev. No. & & 0 \\
\hline
\end{tabular}


Attachment 1. 100-C-9:2 Verification Sampling Results.

\begin{tabular}{|c|c|c|c|c|c|c|c|}
\hline \multirow[t]{2}{*}{ Constituents } & \multicolumn{3}{|c|}{$\begin{array}{c}\text { J13509 } \\
\text { Equipment Blank } \\
\text { Sample Date 8/17/06 } \\
\end{array}$} & \multirow[t]{2}{*}{ Constituents } & \multicolumn{3}{|c|}{$\begin{array}{c}\mathbf{J 1 3 5 0 9} \\
\text { Equipment Blank } \\
\text { Sample Date 8/17/06 }\end{array}$} \\
\hline & $\mu \mathrm{g} / \mathrm{kg}$ & $\mathbf{Q}$ & PQL & & $\mu \mathrm{g} / \mathrm{kg}$ & $\mathbf{Q}$ & PQL \\
\hline \multicolumn{4}{|c|}{ Semivolatile Organic Compounds } & \multicolumn{4}{|c|}{ Semivolatile Organic Compounds (continued) } \\
\hline 1,2,4-Trichlorobenzene & 330 & $\mathrm{U}$ & 330 & Dimethylphthalate & 330 & $\mathrm{U}$ & 330 \\
\hline 1,2-Dichlorobenzene & 330 & $\mathrm{U}$ & 330 & \begin{tabular}{|l|} 
Fluoranthene \\
\end{tabular} & 330 & $\mathrm{U}$ & 330 \\
\hline 1,3-Dichlorobenzene & 330 & $\mathrm{U}$ & 330 & Fluorene & 330 & $\mathrm{U}$ & 330 \\
\hline 1,4-Dichlorobenzene & 330 & $\mathrm{U}$ & 330 & Hexachlorobenzene & 330 & $\mathrm{U}$ & 330 \\
\hline 2,4,5-Trichlorophenol & 840 & $\mathrm{U}$ & 840 & Hexachlorobutadiene & 330 & $\mathrm{U}$ & 330 \\
\hline 2,4,6-Trichlorophenol & 330 & $\mathrm{U}$ & 330 & Hexachlorocyclopentadiene & 330 & $\mathrm{U}$ & 330 \\
\hline 2,4-Dichlorophenol & 330 & $\mathrm{U}$ & 330 & Hexachloroethane & 330 & $\mathrm{U}$ & 330 \\
\hline 2,4-Dimethylphenol & 330 & $\bar{U}$ & 330 & Indeno(1,2,3-cd)pyrene & 330 & $\mathrm{U}$ & 330 \\
\hline 2,4-Dinitrophenol & 840 & $\mathrm{U}$ & 840 & Isophorone & 330 & $\mathrm{U}$ & 330 \\
\hline 2,4-Dinitrotoluene & 330 & $\mathrm{U}$ & 330 & N-Nitroso-di-n-dipropylamine & 330 & $\bar{U}$ & 330 \\
\hline 2,6-Dinitrotoluene & 330 & $\mathrm{U}$ & 330 & N-Nitrosodiphenylamine & 330 & $\mathrm{U}$ & 330 \\
\hline 2-Chloronaphthalene & 330 & $\mathrm{U}$ & 330 & \begin{tabular}{|l|} 
Naphthalene \\
\end{tabular} & 330 & $\mathrm{U}$ & 330 \\
\hline 2-Chlorophenol & 330 & $\mathrm{U}$ & 330 & \begin{tabular}{|l} 
Nitrobenzene \\
\end{tabular} & 330 & $\mathrm{U}$ & 330 \\
\hline 2-Methylnaphthalene & 330 & $\mathrm{U}$ & 330 & Pentachlorophenol & 840 & $\mathrm{U}$ & 840 \\
\hline 2-Methylphenol (cresol, o-) & 330 & $\mathrm{U}$ & 330 & Phenanthrene & 330 & $\mathrm{U}$ & 330 \\
\hline 2-Nitroaniline & 840 & $\mathrm{U}$ & 840 & Phenol & 330 & $\mathrm{U}$ & 330 \\
\hline 2-Nitrophenol & 330 & $\mathrm{U}$ & 330 & Pyrene & 330 & $\mathrm{U}$ & 330 \\
\hline
\end{tabular}

\begin{tabular}{|l|c|c|c|}
\hline 3,3'-Dichlorobenzidine & 330 & $\mathrm{U}$ & 330 \\
\hline 3-Nitroaniline & 840 & $\mathrm{U}$ & 840 \\
\hline 4,6-Dinitro-2-methylphenol & 840 & $\mathrm{U}$ & 840 \\
\hline 4-Bromophenyl-phenylether & 330 & $\mathrm{U}$ & 330 \\
\hline 4-Chloro-3-methylphenol & 330 & $\mathrm{U}$ & 330 \\
\hline 4-Chloroaniline & 330 & $\mathrm{U}$ & 330 \\
\hline 4-Chlorophenyl-phenylether & 330 & $\mathrm{U}$ & 330 \\
\hline 4-Methylphenol (p-cresol) & 330 & $\mathrm{U}$ & 330 \\
\hline 4-Nitroaniline & 840 & $\mathrm{U}$ & 840 \\
\hline 4-Nitrophenol & 840 & $\mathrm{U}$ & 840 \\
\hline Acenaphthene & 330 & $\mathrm{U}$ & 330 \\
\hline Acenaphthylene & 330 & $\mathrm{U}$ & 330 \\
\hline Anthracene & 330 & $\mathrm{U}$ & 330 \\
\hline Benzo(a)anthracene & 330 & $\mathrm{U}$ & 330 \\
\hline Benzo(a)pyrene & 330 & $\mathrm{U}$ & 330 \\
\hline Benzo(b)fluoranthene & 330 & $\mathrm{U}$ & 330 \\
\hline Benzo(g,h,i)perylene & 330 & $\mathrm{U}$ & 330 \\
\hline Benzo(k)fluoranthene & 330 & $\mathrm{U}$ & 330 \\
\hline bis(2-Chloro-1-methylethyl)ether & 330 & $\mathrm{U}$ & 330 \\
\hline bis(2-Chloroethoxy)methane & 330 & $\mathrm{U}$ & 330 \\
\hline bis(2-Chloroethyl)ether & 330 & $\mathrm{U}$ & 330 \\
\hline bis(2-Ethylhexyl)phthalate & 27 & $\mathrm{JB}$ & 330 \\
\hline Butylbenzylphthalate & 330 & $\mathrm{U}$ & 330 \\
\hline Carbazole & 330 & $\mathrm{U}$ & 330 \\
\hline Chrysene & 330 & $\mathrm{U}$ & 330 \\
\hline Di-n-butylphthalate & 33 & $\mathrm{JB}$ & 330 \\
\hline Di-n-octylphthalate & 330 & $\mathrm{U}$ & 330 \\
\hline Dibenz(a,h)anthracene & 330 & $\mathrm{U}$ & 330 \\
\hline Dibenzofuran & 330 & $\mathrm{U}$ & 330 \\
\hline Diethylphthalate & 330 & $\mathrm{U}$ & 330 \\
\hline
\end{tabular}

Attachment Originator Checked Calc. No.

\begin{tabular}{l}
$\frac{1}{\text { J. M. Capron }}$ \\
\hline T. M. Blakley \\
\hline 0100 C-CA-V0033
\end{tabular}

Sheet No.

Date $12 / 13 / 06$

Rev. No. 0 


\section{CALCULATION COVER SHEET}

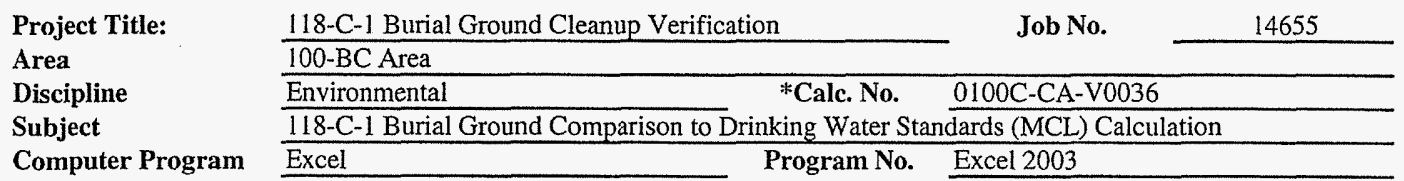

The attached calculations have been generated to document compliance with established cleanup levels. These calculations should be used in conjunction with other relevant documents in the administrative record.

Committed Calculatior $X$

Preliminary

Superseded

Voided

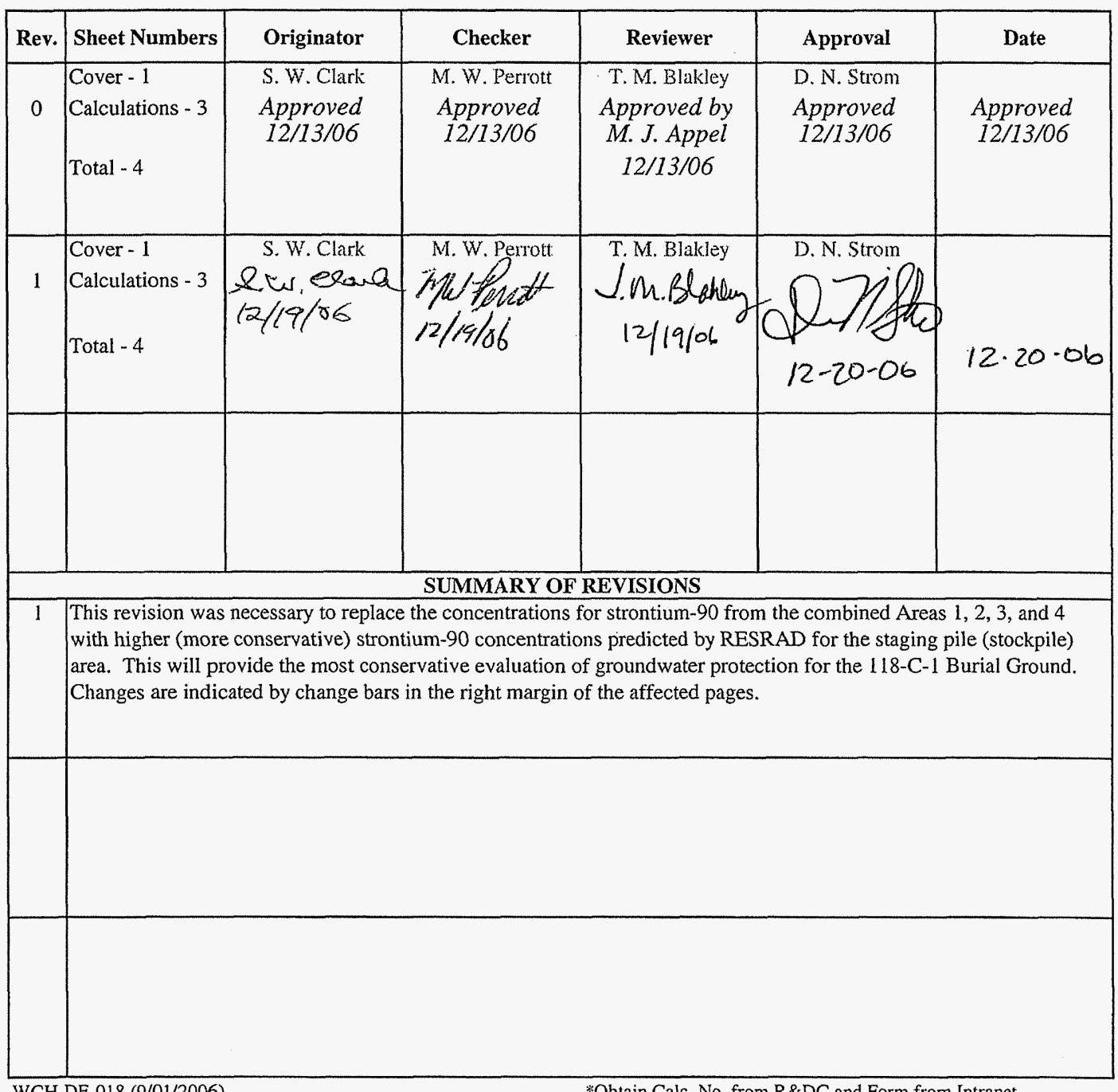




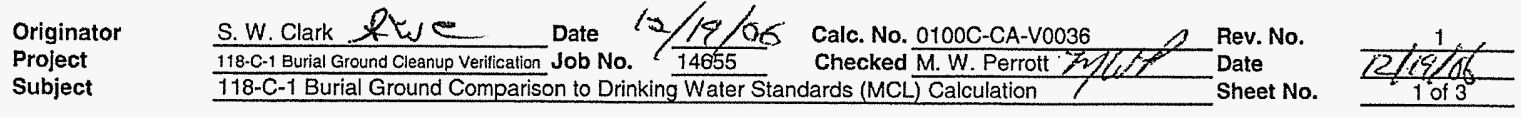

\section{Purpose:}

Compare RESRAD derived groundwater radionuclide concentrations to remedial action goals and maximum contaminant levels (MCLs) for groundwater. Compare beta/gamma emitter dose contributions to the maximum allowable dose of $4 \mathrm{mrem} / \mathrm{yr}$. Compare alpha emitter dose contributions to the maximum allowable gross particle activity of $15 \mathrm{pCi} / \mathrm{L}$ or $1 / 25$ th of the derived concentration guide (DCG).

Table of Contents:

1. Calculation Summan

2. Comparison to $\mathrm{MCl}$

3. Cumulative Dose Comparison

Given/References:

1) RESRAD derived groundwater radionuclide concentrations from Calculation 0100C-CA-V0035, 118-C-1 Burial Ground Cleanup Verification RESRAD

Calculation Brief. To be conservative, the highest predicted groundwater concentrations of the individual radionuclides from the combined Areas $1,2,3$, and 4

and from the overburden/below cleanup level soil and the staging pile (stockpile) soil are evaluated to determine if the maximum allowable dose of $4 \mathrm{mrem} / \mathrm{yr}$

may be exceeded.

2) Remedial action goals for groundwater and MCLs are summarized in Table 2-3 of the Remedial Design Report/Remedial Action Work Plan for the 100 Area (RDR/RAWP), DOE/RL-96-17, Rev. 5, U.S. Department of Energy, Richland Operations office, Richland, WA.

3) Maximum allowable dose of $4 \mathrm{mrem} / \mathrm{yr}$ for beta/gamma emitters and maximum allowable gross particle activity of $15 \mathrm{pCi} / \mathrm{L}$ for alpha emitters from: $40 \mathrm{CFR}$

141, "National Primary Drinking Water Regulation," Code of Federal Regulations, as amended (40 CFR Part 141, Subpart G, 141.66).

4) $1 / 25$ th of the derived concentration guide values from Radiation Protection of the Public and Environment, DOE Order 5400.5, U.S. Department of Energy, Washington D.C.

5) Individual organ-dose caclulation methodology for beta/gamma emitter dose contribution to the maximum allowable dose of $4 \mathrm{mrem} / \mathrm{yr}$ from: Maximum Permissible Body Burdens and Maximum Permissible Concetrations of Radionuclides in Air or Water for Occupational Exposure, National Bureau of Standards (NBS) Handbook 69, as amended, U.S. Department of Commerce, Washington D.C., and National Primary Drinking Water Regulations, EPA-570/9-76-003, U.S. Environmental Protection Agency, Office of Water Supply, Washington D.C.

Solution:

1. The site data for the calculation are the groundwater concentrations for the contaminants of concern (COCs) (daughter products are not considered) over time from the RESRAD groundwater concentration file.

2. Where the site conceptual model breaks the contamination into multiple layers with differing concentrations, then the groundwater concentrations from the various model runs are added (for each time interval) to provide the concentration data for comparison to the individual remedial action goals and MCLS and the dose calculation for the beta and gamma emitters.

3. Compare the summed concentrations for each radionuclide to the groundwater remedial action goal and MCL given in the RDR/RAWP, Table 2-3.

4. The cumulative dose for each organ for all beta and gamma emitting COCs (C-14, Co-60, Cs-137, Eu-152, Eu-154, Eu-155, H-3, Ni-63, and Sr-90) at time t is calculated separately using the concentration corresponding to $4 \mathrm{mrem} / \mathrm{year}$ dose (C4) and the sum of fractions equation shown below (from EPA-570/9-76-

003). The organs for which doses need to be computed are total body, bone, liver and gastrointestinal tract [lower large intestine] (Gl(LLI)). The individual organ doses are compared to $4 \mathrm{mrem} / \mathrm{yr}$.

Doseorgan $x(t)=[\operatorname{Conc} A(t) / C 4 A(x)+\operatorname{Conc} B(t) / C 4 B(x)+\ldots] \times.(4 \mathrm{mrem} / \mathrm{yr})$

where:

Doseorgan $x(t)$ is the total dose to organ $x$ in $m r e m / y r$

ConcA(t) is the concentration of isotope $A$ at time $t$ in $p C i / L$

$C 4 A(x)$ is the $4 \mathrm{mrem} / \mathrm{yr}$ dose equivalent concentration for organ $x$ of isotope $A$ at time $t$ in $p C i / L$

If the dose for organ $x<4 \mathrm{mrem} / \mathrm{yr}$, then the standard is met.

Conclusions:

1. The summed concentrations for each radionuclide $\mathrm{COC}$ are less than the groundwater remedial action goals and MCLS given in the RDR/RAWP. Table 2-3.

2. The cumulative dose for each total body, bone, liver and gastrointestinal tract for all beta and gamma emitting COCs is less than $4 \mathrm{mrem} / \mathrm{yr}$

3. RESRAD modeling indicates that the alpha emitting COCs (Am-241, Pu-238, Pu-239, Pu-240, and Pu-241) will not impact groundwater. Therefore,

concentrations for the alpha emitting COCs are less than the maximum allowable gross particle activity of $15 \mathrm{pCi} / \mathrm{L}$ and the $1 / 25$ th of the DCG.

4. The total uranium COCs $(\mathrm{U}-233 / 234, \mathrm{U}-235$, and $\mathrm{U}-238)$ are present at concentrations less than natural background. 


\section{CALCULATION SHEET}

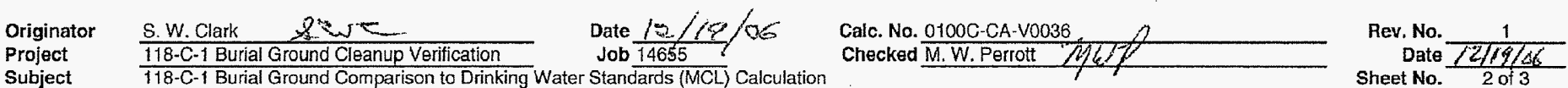

1 Comparison of the summed concentrations for each radionuclide to the GW MCL

Data derived from RESRAD modelling, units are $\mathrm{pCi} / \mathrm{L}$

\begin{tabular}{|c|c|c|c|c|c|c|c|c|c|c|c|c|c|}
\hline Radionuclide & \multicolumn{9}{|c|}{ Time (years) } & $\mathrm{MCL}$ & $\begin{array}{l}\text { Exceeds } \\
\text { MCL? }\end{array}$ & $\begin{array}{l}\text { Peak } \\
\text { Conc. }\end{array}$ & $\begin{array}{c}\text { Year of Peak } \\
\text { Conc. }\end{array}$ \\
\hline & & & & & & & & & & & & & \\
\hline C-14 & 0 & 0 & 0 & 0 & 0 & 0 & 0 & 0 & 0 & 2,000 & No & 0 & 0 \\
\hline Cs-137 & 0 & $1.78 \mathrm{E}-03$ & $5.11 \mathrm{E}-03$ & $1.21 E-02$ & $1,66 \mathrm{E}-02$ & $2.90 \mathrm{E}-02$ & $1.16 \mathrm{E}-02$ & $5.33 \mathrm{E}-54$ & 0 & 60 & No & $2.90 \mathrm{E}-02$ & 43 \\
\hline & & & & & & & & & & & & & \\
\hline$C 0-60$ & 0 & $2.24 \mathrm{E}-03$ & $5.15 \mathrm{E}-03$ & $7.12 \mathrm{E}-03$ & $6.31 \mathrm{E}-03$ & $3.84 E-144$ & $1.10 \mathrm{E}-08$ & $5.60 \mathrm{E}-18$ & $2.24 \mathrm{E}-03$ & 100 & No & $7.12 \mathrm{E}-03$ & 8 \\
\hline Eu-152 & 0 & 0 & 0 & 0 & 0 & $0^{-}$ & 0 & 0 & 0 & 200 & No & 0 & $\begin{array}{l}0 \\
0\end{array}$ \\
\hline & & & & & & & & & & & & & \\
\hline$E u-154$ & 0 & 0 & 0 & 0 & 0 & 0 & 0 & 0 & 0 & 60 & No & 0 & 0 \\
\hline & & & & & & & & & & & & & \\
\hline Eu-155 & 0 & 0 & 0 & 0 & 0 & 0 & 0 & 0 & 0 & 600 & No & 0 & 0 \\
\hline $\mathrm{H}-3$ & 0 & $237 \mathrm{E}+03$ & $5.43 \mathrm{E}+03$ & $402 \mathrm{E}+03$ & $164 \mathrm{E}+03$ & $153 \mathrm{E}+00$ & $362 \mathrm{E}-09$ & $768 \mathrm{E}-26$ & 0 & 20000 & No & $5.43 F+03$ & 3 \\
\hline & & & & & & & & & 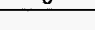 & & & & 3 \\
\hline $\mathrm{N} 1-63$ & 0 & $3.08 \mathrm{E}-01$ & $9.11 \mathrm{E}-01$ & $2.34 \mathrm{E}+00$ & $3.41 \mathrm{E}+60$ & $9.77 E+00$ & $1.57 E+01$ & $1.06 \mathrm{E}+01$ & $2.20 \mathrm{E}-01$ & 50 & No & $1.57 \mathrm{E}+01$ & 131 \\
\hline Sr-90 & $\begin{array}{ll}0 \\
0\end{array}$ & $2.26 \mathrm{E}-02$ & $6.46 \mathrm{E}-02$ & $1.53 \mathrm{E}-01$ & $208 \mathrm{E}-01$ & $3.57 \mathrm{E}-01$ & $133 \mathrm{E}-0 \mathrm{~L}$ & $54 \mathrm{~F}_{-13}$ & $5.10 \mathrm{E}-10$ & 8 & No & $3.57 \mathrm{E}-01$ & 43 \\
\hline
\end{tabular}




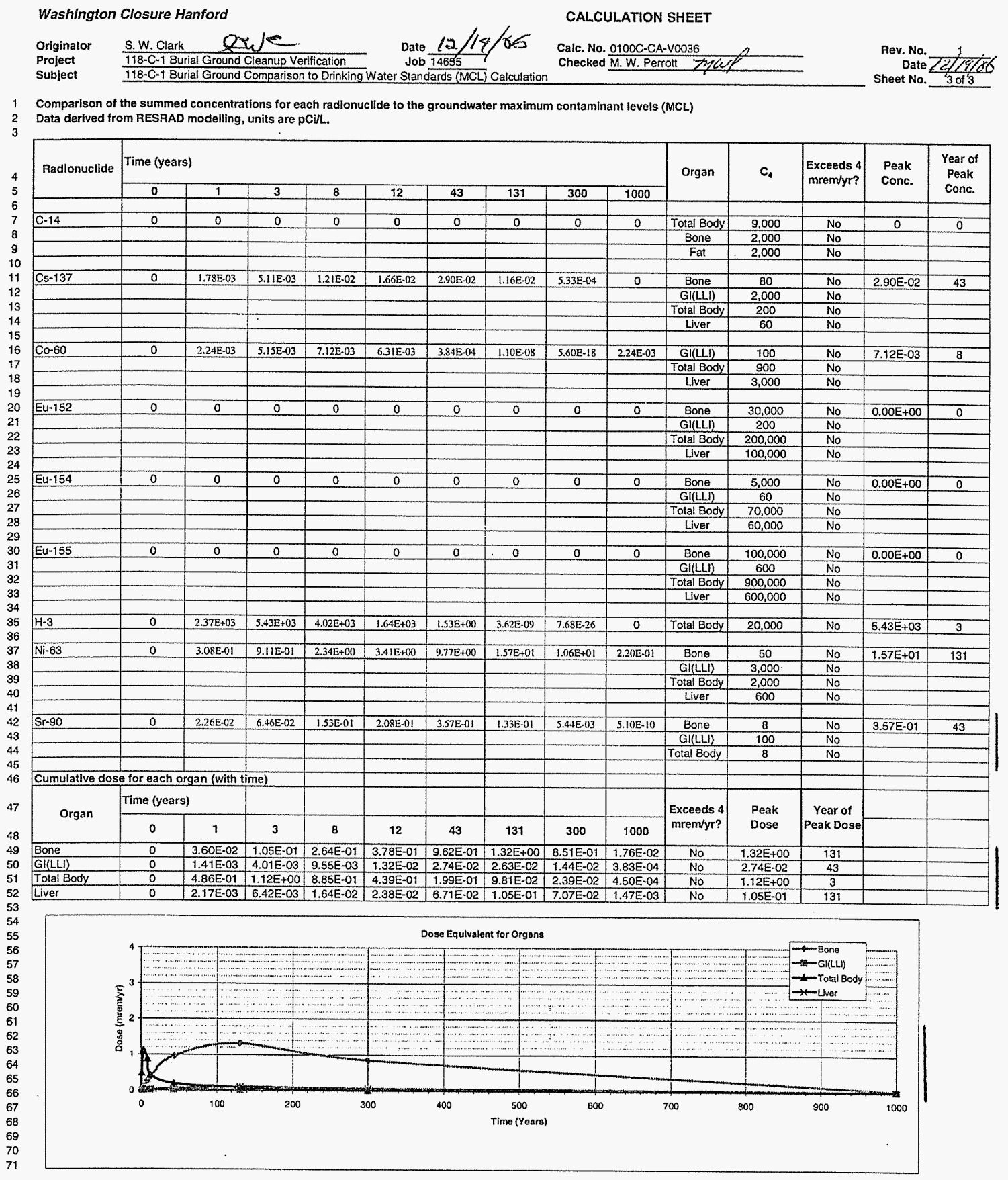

118-C-1 MCL (0100C-CA-V0036) Rev. 1 


\section{CALCULATION COVER SHEET}

Project Title 118-C-1 Burial Ground Cleanup Verification Job No.

Area $100 \mathrm{~B} / \mathrm{C}$ Area

Discipline Environmental

*Calc. No. 0100C-CA-V0035

Subject 118-C-1 Burial Ground Cleanup Verification RESRAD Calculation Brief

\section{Computer Program}

RESRAD

Program No. Version 6.30

The attached calculations have been generated to document compliance with established cleanup levels.

These calculations should be used in conjunction with other relevant documents in the administrative record.

\section{Committed Calculation $\square \quad$ Preliminary $\square \quad$ Superseded $\square$ Voided}

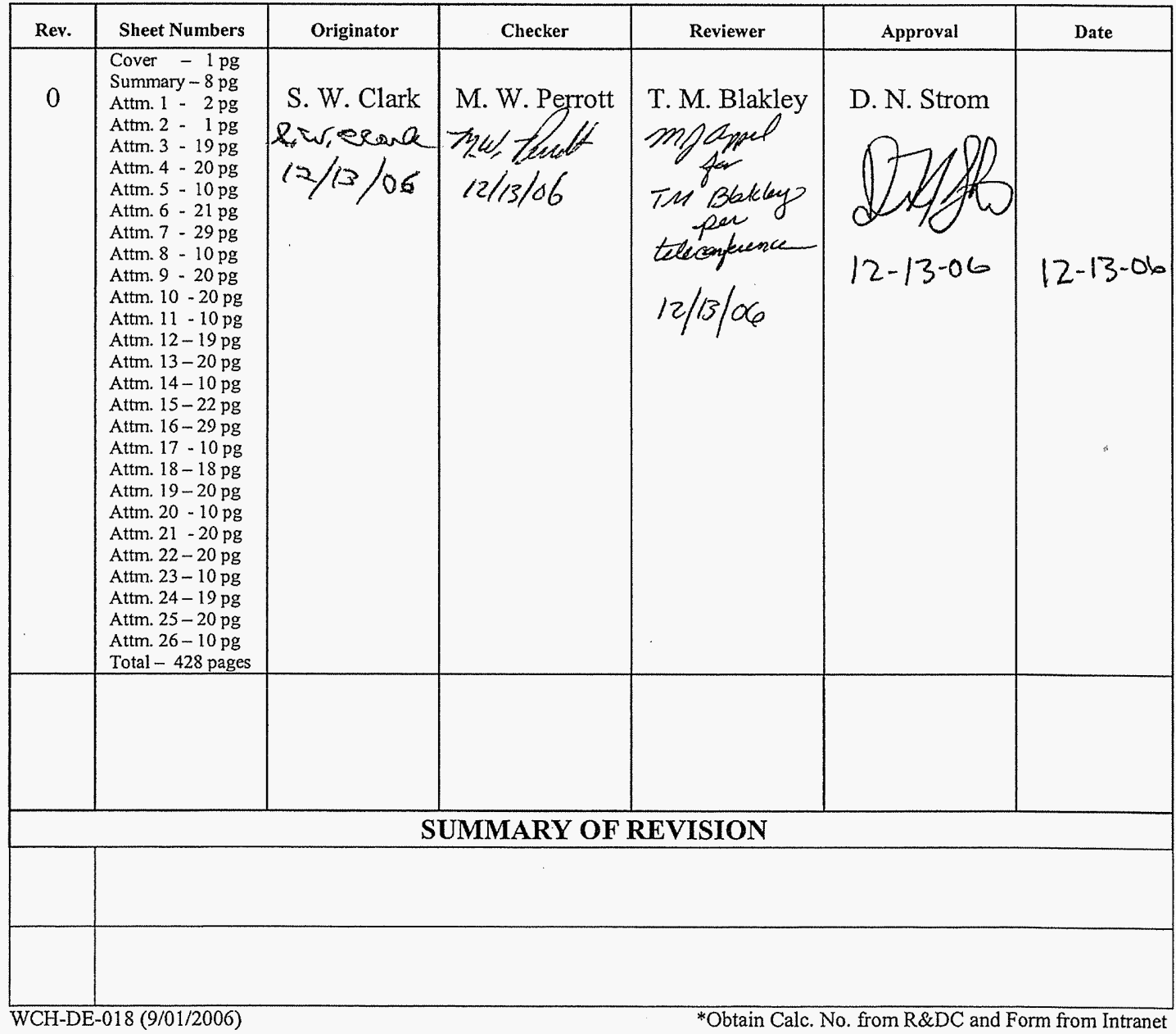




\begin{tabular}{|c|c|c|c|c|c|c|}
\hline Urigl & S. W. Clark & Date: & $1 / 2 / 13 /$ GeCalc. N & 0100 & $\operatorname{Re}$ & 0 \\
\hline & 118-C-1 Bur & & Checked: & M. W. Pe & : & 2 \\
\hline subjec & \multicolumn{6}{|c|}{ 118-C-1 Burial Ground Cleanup Verification RESRAD Calculation Brief } \\
\hline
\end{tabular}

\section{PURPOSE:}

Calculate the soil and groundwater concentrations, dose, and risk contributions from remaining radionuclide contaminants in remediated 118-C-1 Burial Ground areas, in overburden/below cleanup level soil, and in staging pile (stockpile) areas over a period of 1,000 years.

\section{GIVEN/REFERENCES:}

1) Cleanup verification data from 118-C-1 Burial Ground 95\% UCL Calculation, Calculation No. 0100C-CA-V0033, Rev. 0, Washington Closure Hanford, Richland, Washington.

2) Remedial Design Report/Remedial Action Work Plan for the 100 Area (RDR/RAWP), DOE/RL-96-17, Rev. 5, U.S. Department of Energy, Richland Operations Office, Richland, Washington.

3) Radioactive and nonradioactive contaminants of concern from the 100 Area Burial Grounds Remedial Action Sampling and Analysis Plan, DOE/RL-2001-35, Rev. 0, U.S. Department of Energy, Richland Operations Office, Richland, Washington. For the purpose of these RESRAD calculations, the radioactive contaminants of potential concern (COPCs) are americium-241, carbon-14, cesium-137, cobalt-60, europium-152, europium-154, europium-155, nickel-63, plutonium-238, plutonium-239/240, plutonium-241, silver-108m, strontium-90, tritium (H-3), uranium-233/234, uranium-235, and uranium-238. The nonradionuclide contaminants of concern are cadmium, hexavalent chromium, mercury, PCBs, SVOAs, TPH, and VOAs.

4) RESidual RADioactivity (RESRAD) computer code, version 6.30, to calculate compliance with residual radioactivity guidelines, developed for the U.S. Department of Energy by the Environmental Assessment Division of Argonne National Laboratory, Argonne, Illinois.

5) Sample design data from the 118-C-1 Burial Ground Sampling Plan, Calculation No. 0100B-CA-V0288, Rev. 0, Washington Closure Hanford, Richland, Washington..

6) Groundwater elevation from Hanford Groundwater Monitoring for Fiscal Year 2005, PNNL-15670, Pacific Northwest National Laboratory, Richland, Washington.

\section{SOLUTION:}

1) Separate RESRAD runs were performed for the 118-C-1 Burial Ground trenches with spent nuclear fuel (Area 1), other trenches (Area 2), west interstitial area (Area 3), east interstitial area (Area 4), combined Areas 1,2, 3, and 4, overburden/below cleanup level (OB/BCL) material, and staging pile (stockpile) area (SPA). The RESRAD run for the combined Areas $1,2,3$, and 4 was done using using the maximum $95 \%$ UCL values from the individual Areas. Table 1 shows the elevations (NAVD88) and thickness of each soil horizon. The average surrounding grade elevation and the maximum excavation depth are used to represent the burial ground excavation. Attachment 1 shows representative dimensions of soil horizons and contaminant pathways considered for dose, risk, and groundwater protection. Attachment 2 shows a schematic of the 118-C-1 Burial Ground area with the pieces making up the individual units labeled. Input factors for each run are shown in the "Summary" section of the RESRAD "Mixture Sums and Single Radionuclide Guidelines" printouts in the Attachments to this Calculation Summary. 


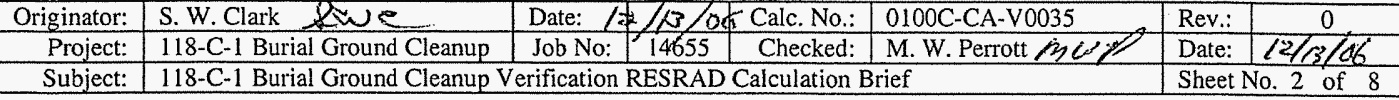

1

\begin{tabular}{|c|c|c|c|c|c|c|c|c|c|}
\hline Parameter & Units & Area 1 & Area 2 & Area 3 & Area 4 & $\begin{array}{c}\text { Combined } \\
\text { Areas }\end{array}$ & $\mathrm{OB} / \mathrm{BCL}$ & \begin{tabular}{|c|} 
Shallow \\
Zone SPA \\
\end{tabular} & \begin{tabular}{|c} 
Deep \\
Zone SPA
\end{tabular} \\
\hline \multicolumn{10}{|c|}{ Contaminated Zone Dimensions } \\
\hline Cover Depth & $\mathrm{m}$ & 0 & 0 & 0 & 0 & 0 & 0 & 0 & 4.6 \\
\hline $\begin{array}{l}\text { Area of Contaminated } \\
\text { Zone (CZ) }\end{array}$ & $\mathrm{m}^{2}$ & 3,018 & 2,339 & 7,576 & 7,576 & 20,509 & 9,006 & 23,230 & 23,230 \\
\hline $\begin{array}{l}\text { Length Parallel to } \\
\text { Aquifer Flow } \\
\end{array}$ & $\mathrm{m}$ & 75 & 85 & 130 & 130 & 150 & 80 & 240 & 240 \\
\hline \multicolumn{10}{|c|}{ Elevations of Vadose Zone Horizons } \\
\hline Elevation: Surface & $\mathrm{m}$ & 150.0 & 149.0 & 151.0 & 151.0 & 151.0 & 151.5 & 148.5 & 148.5 \\
\hline $\begin{array}{l}\text { Elevation: Bottom of } \\
\text { Excavation }\end{array}$ & $\mathrm{m}$ & 145.5 & 146.0 & 146.0 & 146.0 & 146.0 & -- & -- & -- \\
\hline Elevation: Groundwater & $\mathrm{m}$ & 121.8 & 121.8 & 121.8 & 121.8 & 121.8 & 121.8 & 121.8 & 121.8 \\
\hline $\begin{array}{l}\text { Thickness: } \\
\text { Contaminated Zone }\end{array}$ & $\mathrm{m}$ & 28.2 & 27.2 & 29.2 & 29.2 & 29.2 & 29.7 & 4.6 & 22.1 \\
\hline $\begin{array}{l}\text { Thickness: Unsaturated } \\
\text { Zone }\end{array}$ & $\mathrm{m}$ & 0 & 0 & 0 & 0 & 0 & 0 & 22.1 & 0 \\
\hline
\end{tabular}

2) The year where the peak dose (or concentration) occurs from each individual radionuclide $\mathrm{COC}$ and layer is determined by a preliminary run. This year is then added for all horizons for the final RESRAD runs. For the direct exposure pathway (i.e. soil ingestion and inhalation and external radiation), the peak year occurred at year zero (year 2006) for all COCs. For the water pathways (i.e. drinking water and food ingestion) the peak year was year 3 for tritium, year 8 for cobalt-60, year 43 for cesium-137 and strontium-90, and year 131 for nickel-63. The 3,8,43, and 131-year time periods were added to all RESRAD runs.

To be conservative, shallow zone evaluations were performed for the 118-C-1 Burial Ground areas assuming that the residual contamination levels from the shallow zone sample data sets extend uniformly from the surface to groundwater (Attachment 1). For Area 2 and the combined Areas 1, 2, 3, and 4 this evaluation is too conservative and carbon-14 in the pathways affected by plant ingestion caused the total all pathways dose to be greater than 15 mrem/yr. Because the residual carbon-14 is deeper than the root penetration zone (10 ft), and not accessible to plants, the RESRAD inputs for "contaminated fractions" in the plant ingestion input parameters for Area 2 and the combined Areas 1, 2, 3, and 4 are set equal to zero.

For the staging pile area (SPA) the maximum carbon-14 concentration $(182 \mathrm{pCi} / \mathrm{g})$ is above the direct exposure lookup value ( $5.16 \mathrm{pCi} / \mathrm{g}$ per the 100 Area RDR/RAWP) while carbon-14 in all other SPA sampling grid areas was undetected. The soil that contains carbon-14 above lookup values will be used as backfill in deep zone areas and has been evaluated in RESRAD

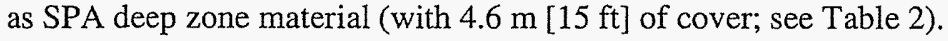




\begin{tabular}{|c|c|c|c|c|c|c|}
\hline $011 \mathrm{~g}$ & R & $1 \%$ & $\mathrm{~N}$ & 01 & Rev.: & 0 \\
\hline & 11 & Job No: 14655 & & M. W. Perroty 360 & Date: & $12 / 13 / 26$ \\
\hline Subject: & \multicolumn{6}{|c|}{ 118-C-1 Burial Ground Cleanup Verification RESRAD Calculation Brief } \\
\hline
\end{tabular}

\section{METHODOLOGY:}

1) Runs of RESRAD version 6.30 were completed for the 118-C-1 Burial Ground trenches with spent nuclear fuel (Area 1), other trenches (Area 2), west interstitial area (Area 3), east interstitial area (Area 4), the combined Areas 1,2,3, and 4, overburden/below cleanup level $(\mathrm{OB} / \mathrm{BCL})$ material, and staging pile area (SPA) soils using the radionuclide concentrations shown in Table 2. RESRAD numerical output reports for dose, risk, and concentration for the shallow zone, deep zone, and overburden are presented in the Attachments to this calculation summary.

\begin{tabular}{|c|c|c|c|c|c|c|c|c|}
\hline \multicolumn{9}{|c|}{ Table 2. Cleanup Verification Data Set ${ }^{a}$} \\
\hline COPCs & Area 1 & Area 2 & Area 3 & Area 4 & $\begin{array}{c}\text { Combined } \\
\text { Areas }\end{array}$ & $\mathrm{OB} / \mathrm{BCL}$ & $\begin{array}{c}\text { Shallow } \\
\text { Zone SPA }\end{array}$ & $\begin{array}{c}\text { Deep } \\
\text { Zone SPA }\end{array}$ \\
\hline \multicolumn{9}{|c|}{ Radionuclide Activity ( $p$ Ci $/ g$ ) } \\
\hline $\mathrm{Am}-241$ & -- & -- & -- & - & -.. & - & -- & - \\
\hline C-14 & - & 8.0 & -- & -. & 8.0 & -- & -- & 182 \\
\hline Cs-137 & 0.185 & 0.140 & 0.709 & 0.81 & 0.140 & $0(<B G)$ & 1.03 & 0.091 \\
\hline $\mathrm{Co}-60$ & & 0.99 & 0.037 & & 0.99 & - & 0.029 & -- \\
\hline Eu-152 & 0.121 & 0.199 & 0.140 & 0.114 & 0.199 & - & 0.177 & -- \\
\hline Eu-154 & -- & - & -- & -- & -- & -- & - & -- \\
\hline Eu-155 & -- & -- & -- & - & -- & -- & -- & -- \\
\hline $\mathrm{Ni}-63$ & & 26.1 & & & 26.1 & -- & & .. \\
\hline $\mathrm{Pu}-238$ & -- & -- & -- & - & - & - & -- & -- \\
\hline Pu-239/240 & - & -- & - & -- & -- & -- & -- & -- \\
\hline Pu-241 & -- & - & - & -- & -- & -- & -- & $\sim$ \\
\hline Silver $(\mathrm{Ag}-108 \mathrm{~m})$ & -- & -- & - & -- & -- & - & -- & - \\
\hline Sr-90 & -- & - & 0.268 & - & 0.268 & 1.23 & 0.334 & -- \\
\hline Tritium (H-3) & & 4.76 & -- & - & 4.76 & - & $\ldots$ & $\ldots$ \\
\hline U-233/234 & $O(<B G)$ & $\therefore$ & $0(<\mathrm{BG})$ & $O(<B G)$ & $0(<\mathrm{BG})$ & $0(<B G)$ & $0(<\mathrm{BG})$ & -- \\
\hline $\mathrm{U}-235$ & $0(<\mathrm{BG})$ & $\ldots$ & -- & -- & $0(<B G)$ & $\ldots$ & $0(<B G)$ & - \\
\hline $\mathrm{U}-238$ & $0(<B G)$ & - & $\mathrm{O}(<\mathrm{BG})$ & $\mathrm{O}(<\mathrm{BG})$ & $0(<\mathrm{BG})$ & $O(<B G)$ & $0(<\mathrm{BG})$ & -- \\
\hline
\end{tabular}

$--=$ Not evaluated by laboratory analysis or not detected (for all samples in the data set).

$\mathrm{OB} / \mathrm{BCL}=$ Overburden/Below Cleanup Levels

SPA $=$ Staging Pile (Stockpile) Area

${ }^{\text {a }}$ Soil concentration values are from 118-C-1 Burial Ground 95\% UCL Calculation, Calculation No. 0100C-CA-V0033, Rev. 0, Washington Closure Hanford, Richland, Washington. Refer to the $95 \%$ UCL for nonradionuclide concentrations. Nonradionuclides are not evaluated in this 


\section{RESULTS:}

2

10

\section{1) Radionuclide "All Pathways" Dose Rate}

The "all pathways" (maximum) dose rates are shown in Table 3. The maximum all pathways dose rate from the 118-C-1 Burial Ground combined Areas 1, 2, 3, and 4 is $12.8 \mathrm{mrem} / \mathrm{yr}$ at year zero (2006). The maximum all-pathways dose rate for the overburden (OB/BCL) soil is 4.01 $\mathrm{mrem} / \mathrm{yr}$ at year zero (2006). For the staging pile area (SPA) the total maximum all-pathways dose rate for the shallow and deep zones is $12.4 \mathrm{mrem} / \mathrm{yr}$ at year zero (2006).

\begin{tabular}{|c|c|c|c|c|c|c|c|c|c|c|}
\hline \multicolumn{8}{|c|}{ Table 3. All Pathways Dose Rate (mrem/yr) } \\
\hline $\begin{array}{c}\text { RESRAD } \\
\text { Run }\end{array}$ & $\begin{array}{c}\text { Vadose Zone } \\
\text { Horizons }\end{array}$ & \multicolumn{7}{c|}{ "All Pathways" Dose Contributions in mrem/yr at Each Time Slice (yr) } \\
\cline { 3 - 11 }$y$ & 0 & 1 & 3 & 8 & 12 & 43 & 131 & 300 & 1000 \\
\hline Area 1 & Shallow Zone & $9.45 \mathrm{E}-01$ & $9.09 \mathrm{E}-01$ & $8.41 \mathrm{E}-01$ & $6.95 \mathrm{E}-01$ & $6.00 \mathrm{E}-01$ & $2.12 \mathrm{E}-01$ & $2.10 \mathrm{E}-02$ & $4.13 \mathrm{E}-04$ & $4.01 \mathrm{E}-11$ \\
\hline Area 2 & Shallow Zone & $1.11 \mathrm{E}+01$ & $9.91 \mathrm{E}+00$ & $7.95 \mathrm{E}+00$ & $4.36 \mathrm{E}+00$ & $2.77 \mathrm{E}+00$ & $2.40 \mathrm{E}-01$ & $2.23 \mathrm{E}-02$ & $4.91 \mathrm{E}-03$ & $5.61 \mathrm{E}-05$ \\
\hline Area 3 & Shallow Zone & $3.54 \mathrm{E}+00$ & $3.41 \mathrm{E}+00$ & $3.16 \mathrm{E}+00$ & $2.65 \mathrm{E}+00$ & $2.33 \mathrm{E}+00$ & $1.01 \mathrm{E}+00$ & $1.24 \mathrm{E}-01$ & $2.50 \mathrm{E}-03$ & $2.22 \mathrm{E}-10$ \\
\hline Area 4 & Shallow Zone & $2.43 \mathrm{E}+00$ & $2.36 \mathrm{E}+00$ & $2.23 \mathrm{E}+00$ & $1.94 \mathrm{E}+00$ & $1.73 \mathrm{E}+00$ & $7.69 \mathrm{E}-01$ & $9.46 \mathrm{E}-02$ & $1.91 \mathrm{E}-03$ & $1.88 \mathrm{E}-10$ \\
\hline \multicolumn{2}{|c|}{ Combined Areas } & $1.28 \mathrm{E}+01$ & $1.16 \mathrm{E}+01$ & $9.46 \mathrm{E}+00$ & $5.70 \mathrm{E}+00$ & $3.89 \mathrm{E}+00$ & $7.26 \mathrm{E}-01$ & $8.82 \mathrm{E}-02$ & $6.59 \mathrm{E}-03$ & $1.01 \mathrm{E}-04$ \\
\hline OB/BCL & Shallow Zone & $4.01 \mathrm{E}+00$ & $3.92 \mathrm{E}+00$ & $3.74 \mathrm{E}+00$ & $3.34 \mathrm{E}+00$ & $3.04 \mathrm{E}+00$ & $1.49 \mathrm{E}+00$ & $1.95 \mathrm{E}-01$ & $3.91 \mathrm{E}-03$ & $2.47 \mathrm{E}-10$ \\
\hline SPA & Shallow Zone & $1.24 \mathrm{E}+01$ & $1.18 \mathrm{E}+01$ & $1.08 \mathrm{E}+01$ & $8.72 \mathrm{E}+00$ & $7.38 \mathrm{E}+00$ & $2.31 \mathrm{E}+00$ & $1.91 \mathrm{E}-01$ & $3.39 \mathrm{E}-03$ & $2.39 \mathrm{E}-10$ \\
\hline SPA & Deep Zone & $0.00 \mathrm{E}+00$ & $2.94 \mathrm{E}-05$ & $9.38 \mathrm{E}-05$ & $2.30 \mathrm{E}-04$ & $3.16 \mathrm{E}-04$ & $5.58 \mathrm{E}-04$ & $2.23 \mathrm{E}-04$ & $1.03 \mathrm{E}-05$ & $3.22 \mathrm{E}-12$ \\
\hline $\begin{array}{c}\text { SPA Total All Pathway } \\
\text { Dose Rate (mrem/yr) }\end{array}$ & $1.24 \mathrm{E}+01$ & $1.18 \mathrm{E}+01$ & $1.08 \mathrm{E}+01$ & $8.72 \mathrm{E}+00$ & $7.38 \mathrm{E}+00$ & $2.31 \mathrm{E}+00$ & $1.91 \mathrm{E}-01$ & $3.40 \mathrm{E}-03$ & $2.42 \mathrm{E}-10$ \\
\hline
\end{tabular}

$\mathrm{OB} / \mathrm{BCL}=$ Overburden/Below Cleanup Levels

SPA $=$ Staging Pile (Stockpile) Area

\section{2) Radionuclide Excess Lifetime Cancer Risk}

The radionuclide excess lifetime cancer risk (ELCR) results are shown in Table 4. The maximum ELCR for the 118-C-1 Burial Ground combined Areas 1, 2, 3, and 4 is $9.95 \times 10^{-5}$ at year zero (2006). The maximum ELCR for the overburden (OB/BCL) soil is $5.42 \times 10^{-5}$ which also occurs at year zero (2006). For the staging pile area (SPA) the total maximum all-pathways ELRC for the shallow and deep zones is $1.55 \times 10^{-4}$ at year zero (2006).

\begin{tabular}{|c|c|c|c|c|c|c|c|c|c|c|}
\hline \multicolumn{11}{|c|}{ Table 4. Radionuclide Excess Lifetime Cancer Risk } \\
\hline \multirow{2}{*}{$\begin{array}{c}\text { RESRAD } \\
\text { Run }\end{array}$} & \multirow{2}{*}{$\begin{array}{c}\text { Vadose Zone } \\
\text { Horizons }\end{array}$} & \multicolumn{9}{|c|}{ Excess Cancer Risk at Each Time Slice (yr) } \\
\hline & & 0 & 1 & 3 & 8 & 12 & 43 & 131 & 300 & 1000 \\
\hline Area 1 & Shallow Zone & $1.28 \mathrm{E}-05$ & $1.24 \mathrm{E}-05$ & $1.15 \mathrm{E}-05$ & $9.63 \mathrm{E}-06$ & $8.38 \mathrm{E}-06$ & $3.16 \mathrm{E}-06$ & $3.36 \mathrm{E}-07$ & $6.68 \mathrm{E}-09$ & $6.45 \mathrm{E}-16$ \\
\hline Area 2 & Shallow Zone & $7.35 \mathrm{E}-05$ & $6.60 \mathrm{E}-05$ & $5.34 \mathrm{E}-05$ & $3.12 \mathrm{E}-05$ & $2.11 \mathrm{E}-05$ & $3.24 \mathrm{E}-06$ & 5.05E-07 & $1.62 \mathrm{E}-07$ & $1.80 \mathrm{E}-09$ \\
\hline Area 3 & Shallow Zone & 4.81E-05 & $4.66 \mathrm{E}-05$ & $4.38 \mathrm{E}-05$ & $3.77 \mathrm{E}-05$ & 3.37E-05 & $1.51 \mathrm{E}-05$ & $1.89 \mathrm{E}-06$ & $3.78 \mathrm{E}-08$ & $3.37 \mathrm{E}-15$ \\
\hline Area 4 & Shallow Zone & $3.70 \mathrm{E}-05$ & 3.60E-05 & $3.41 \mathrm{E}-05$ & $2.98 \mathrm{E}-05$ & $2.68 \mathrm{E}-05$ & $1.22 \mathrm{E}-05$ & $1.53 \mathrm{E}-06$ & $3.09 \mathrm{E}-08$ & $3.02 \mathrm{E}-15$ \\
\hline \multicolumn{2}{|c|}{ Combined Areas } & $9.95 \mathrm{E}-05$ & 9.07E-05 & $7.60 \mathrm{E}-05$ & $5.04 \mathrm{E}-05$ & $3.79 \mathrm{E}-05$ & $1.11 \mathrm{E}-05$ & $1.57 \mathrm{E}-06$ & $1.97 \mathrm{E}-07$ & $3.32 \mathrm{E}-09$ \\
\hline $\mathrm{OB} / \mathrm{BCL}$ & Shallow Zone & $5.42 \mathrm{E}-05$ & $5.29 \mathrm{E}-05$ & $5.05 \mathrm{E}-05$ & $4.50 \mathrm{E}-05$ & $4.10 \mathrm{E}-05$ & $2.00 \mathrm{E}-05$ & $2.59 \mathrm{E}-06$ & \begin{tabular}{|l|}
$5.09 \mathrm{E}-08$ \\
\end{tabular} & $3.14 \mathrm{E}-15$ \\
\hline SPA & Shallow Zone & $1.55 \mathrm{E}-04$ & 1.49E-04 & 1.37E-04 & $1.12 \mathrm{E}-04$ & $9.57 \mathrm{E}-05$ & $3.20 \mathrm{E}-05$ & $2.88 \mathrm{E}-06$ & $5.20 \mathrm{E}-08$ & $3.74 \mathrm{E}-15$ \\
\hline SPA & Deep Zone & 7.15E-09 & $7.51 \mathrm{E}-09$ & $8.18 \mathrm{E}-09$ & $9.55 \mathrm{E}-09$ & $1.04 \mathrm{E}-08$ & $1.13 \mathrm{E}-08$ & $3.80 \mathrm{E}-09$ & $1.66 \mathrm{E}-10$ & $5.05 \mathrm{E}-17$ \\
\hline \multicolumn{2}{|c|}{ SPA Total ELCR } & $1.55 \mathrm{E}-04$ & 1.49E-04 & 1.37E-04 & $1.12 \mathrm{E}-04$ & $9.57 \mathrm{E}-05$ & $3.20 \mathrm{E}-05$ & $2.89 \mathrm{E}-06$ & $5.21 \mathrm{E}-08$ & $3.79 \mathrm{E}-15$ \\
\hline
\end{tabular}


Washington Closure Hanford

CALCULATION SHEET

\begin{tabular}{|c|c|c|c|c|c|c|}
\hline Originator: & S.W. Clark Sinje & Date: $A>>>0$ & Calc. No.: & $0100 \mathrm{C}-\mathrm{CA}-\mathrm{V} 0035$ & Rev.: & 0 \\
\hline Project: & 118-C-1 Burial Ground Cleanup & Job No: 14655 & Checked: & M.W. Perrott Gqla/p & Date: & 121706 \\
\hline Subject: & \multicolumn{4}{|c|}{ 118-C-1 Burial Ground Cleanup Verification RESRAD Calculation Brief } & \multicolumn{2}{|c|}{ Sheet No. 5 of 8} \\
\hline
\end{tabular}

1

2 3) Radionuclide Groundwater Protection

3 The radionuclide concentrations in groundwater calculated by the RESRAD model are

4 summarized in Table 5. Among the radionuclide contaminants of concern only cesium-137,

5 cobalt-60, nickel-63, strontium-90, and tritium are calculated to reach groundwater in the 1,000

6 years of the RESRAD model run. The organ specific dose via the groundwater (and river)

7 pathway is presented in a separate calculation brief [118-C-1 Burial Ground Comparison To

8 Drinking Water Standards (MCL) Calculation, Calculation No. 0100C-CA-V0036, Rev. 0,

9 Washington Closure Hanford, Richland, Washington]. Only concentrations are presented here. 10

Table 5. Predicted Groundwater (Well Water/Drinking Water) Concentrations (2 pages)

\begin{tabular}{|c|c|c|c|c|c|c|c|c|c|c|c|}
\hline \multirow{2}{*}{$\begin{array}{l}\text { Radio- } \\
\text { nuclides }\end{array}$} & \multirow{2}{*}{$\begin{array}{c}\text { Vadose Zone } \\
\text { Horizon }\end{array}$} & \multicolumn{9}{|c|}{ Groundwater Concentrations in $\mathrm{pCi} / \mathrm{L}$ at Each Time Slice (yr) } & \multirow{2}{*}{$\begin{array}{l}\text { RAGs, } \\
\mathrm{pCi} / \mathrm{L}\end{array}$} \\
\hline & & 0 & 1 & 3 & 8 & 12 & 43 & 131 & 300 & 1000 & \\
\hline \multirow{9}{*}{ Cs- 137} & Area $1 \mathrm{SZ}$ & 0 & 4.65E-04 & $1.33 \mathrm{E}-03$ & 3.17E-03 & 4.33E-03 & $7.58 \mathrm{E}-03$ & $3.02 \mathrm{E}-03$ & $1.39 \mathrm{E}-04$ & $4.34 \mathrm{E}-11$ & \multirow{9}{*}{60} \\
\hline & Area $2 \mathrm{SZ}$ & 0 & $3.52 \mathrm{E}-04$ & $1.01 \mathrm{E}-03$ & $2.40 \mathrm{E}-03$ & $3.28 \mathrm{E}-03$ & $5.74 \mathrm{E}-03$ & $2.29 \mathrm{E}-03$ & 1.05E-04 & $3.29 \mathrm{E}-11$ & \\
\hline & Area $3 \mathrm{SZ}$ & 0 & $1.78 \mathrm{E}-03$ & $5.11 \mathrm{E}-03$ & $1.21 \mathrm{E}-02$ & $1.66 \mathrm{E}-02$ & $2.91 \mathrm{E}-02$ & $1.16 \mathrm{E}-02$ & 5.33E-04 & $1.67 \mathrm{E}-10$ & \\
\hline & Area $4 \mathrm{SZ}$ & 0 & 2.04E-03 & $5.84 \mathrm{E}-03$ & 1.39E-02 & $1.90 \mathrm{E}-02$ & $3.32 \mathrm{E}-02$ & 1.32E-02 & $6.09 \mathrm{E}-04$ & $1.90 \mathrm{E}-10$ & \\
\hline & Comb. Areas & 0 & 1.78E-03 & $5.11 \mathrm{E}-03$ & $1.21 \mathrm{E}-02$ & $1.66 \mathrm{E}-02$ & $2.90 \mathrm{E}-02$ & $1.16 \mathrm{E}-02$ & 5.33E-04 & $1.66 \mathrm{E}-10$ & \\
\hline & $\mathrm{OB} / \mathrm{BCL}$ & 0 & 0 & 0 & 0 & 0 & 0 & 0 & 0 & 0 & \\
\hline & SPA SZ & 0 & 0 & 0 & 0 & 0 & 0 & 0 & 0 & 0 & \\
\hline & SPA DZ & 0 & 2.29E-04 & $6.56 \mathrm{E}-04$ & $1.56 \mathrm{E}-03$ & $2.13 \mathrm{E}-03$ & 3.73E-03 & $1.49 \mathrm{E}-03$ & $6.85 \mathrm{E}-05$ & $2.15 \mathrm{E}-11$ & \\
\hline & SPA Total & 0 & $2.29 \mathrm{E}-04$ & $6.56 \mathrm{E}-04$ & $1.56 \mathrm{E}-03$ & $2.13 \mathrm{E}-03$ & $3.73 \mathrm{E}-03$ & $1.49 \mathrm{E}-03$ & $6.85 \mathrm{E}-05$ & $2.15 \mathrm{E}-11$ & \\
\hline \multirow{9}{*}{ Co-60 } & Area $1 \mathrm{SZ}$ & 0 & 0 & 0 & 0 & 0 & 0 & 0 & 0 & 0 & \multirow{9}{*}{100} \\
\hline & Area $2 \mathrm{SZ}$ & 0 & $2.24 \mathrm{E}-03$ & $5.15 \mathrm{E}-03$ & 7.12E-03 & $6.31 \mathrm{E}-03$ & $3.84 \mathrm{E}-04$ & $1.10 \mathrm{E}-08$ & $5.60 \mathrm{E}-18$ & 0 & \\
\hline & Area $3 \mathrm{SZ}$ & 0 & $8.35 \mathrm{E}-05$ & $1.93 \mathrm{E}-04$ & 2.66E-04 & $2.36 \mathrm{E}-04$ & $1.43 \mathrm{E}-05$ & 4.11E-10 & $2.10 \mathrm{E}-19$ & 0 & \\
\hline & Area $4 \mathrm{SZ}$ & 0 & 0 & 0 & 0 & 0 & 0 & 0 & 0 & 0 & \\
\hline & Comb. Areas & 0 & $2.24 \mathrm{E}-03$ & $5.15 \mathrm{E}-03$ & $7.12 \mathrm{E}-03$ & $6.31 \mathrm{E}-03$ & 3.84E-04 & $1.10 \mathrm{E}-08$ & $5.60 \mathrm{E}-18$ & $2.24 \mathrm{E}-03$ & \\
\hline & $\mathrm{OB} / \mathrm{BCL}$ & 0 & 0 & 0 & 0 & 0 & 0 & 0 & 0 & 0 & \\
\hline & SPA SZ & 0 & 0 & 0 & 0 & 0 & 0 & 0 & 0 & 0 & \\
\hline & SPA DZ & 0 & 0 & 0 & 0 & 0 & 0 & 0 & 0 & 0 & \\
\hline & SPA Total & 0 & 0 & 0 & 0 & 0 & 0 & 0 & 0 & 0 & \\
\hline \multirow{9}{*}{$\mathrm{Ni}-63$} & Area $1 \mathrm{SZ}$ & 0 & 0 & 0 & 0 & 0 & 0 & 0 & 0 & 0 & \multirow{9}{*}{50} \\
\hline & Area $2 \mathrm{SZ}$ & 0 & $3.08 \mathrm{E}-01$ & $9.11 \mathrm{E}-01$ & $2.34 \mathrm{E}+00$ & $3.41 \mathrm{E}+00$ & $9.77 \mathrm{E}+00$ & $1.57 \mathrm{E}+01$ & $1.06 E+01$ & 1.30E-01 & \\
\hline & Area $3 \mathrm{SZ}$ & 0 & 0 & 0 & 0 & 0 & 0 & 0 & 0 & 0 & \\
\hline & Area $4 \mathrm{SZ}$ & 0 & 0 & 0 & 0 & 0 & 0 & 0 & 0 & 0 & \\
\hline & Comb. Areas & 0 & $3.08 \mathrm{E}-01$ & $9.11 \mathrm{E}-01$ & $2.34 \mathrm{E}+00$ & $3.41 \mathrm{E}+00$ & $9.77 \mathrm{E}+00$ & $1.57 \mathrm{E}+01$ & $1.06 \mathrm{E}+01$ & $2.20 \mathrm{E}-01$ & \\
\hline & $\mathrm{OB} / \mathrm{BCL}$ & 0 & 0 & 0 & 0 & 0 & 0 & 0 & 0 & 0 & \\
\hline & SPA SZ & 0 & 0 & 0 & 0 & 0 & 0 & 0 & 0 & 0 & \\
\hline & SPA DZ & 0 & 0 & 0 & 0 & 0 & 0 & 0 & 0 & 0 & \\
\hline & SPA Total & 0 & 0 & 0 & 0 & 0 & 0 & 0 & 0 & 0 & \\
\hline
\end{tabular}


Washington Closure Hanford

CALCULATION SHEET

\begin{tabular}{|c|c|c|c|c|c|c|}
\hline Originator: & S. W. Clark X Qwe & Date: $14 / 2 / 86$ & Calc. No.: & $0100 \mathrm{C}-\mathrm{CA}-\mathrm{V} 0035$ & Rev.: & 0 \\
\hline Project: & 118-C-1 Burial Ground Cleanup & Job No: 14655 & Checked: & M. W. Perrott Zyere & Date: & 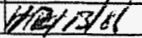 \\
\hline Subject: & \multicolumn{4}{|c|}{ 118-C-1 Burial Ground Cleanup Verification RESRAD Calculation Brief } & Sheet & No. 6 of 8 \\
\hline
\end{tabular}

Table 5. Predicted Groundwater (Well Water/Drinking Water) Concentrations ( 2 pages)

\begin{tabular}{|c|c|c|c|c|c|c|c|c|c|c|c|}
\hline \multirow{2}{*}{$\begin{array}{l}\text { Radio- } \\
\text { nuclides }\end{array}$} & \multirow{2}{*}{$\begin{array}{c}\text { Vadose Zone } \\
\text { Horizon }\end{array}$} & \multicolumn{9}{|c|}{ Groundwater Concentrations in $\mathrm{pCi} / \mathrm{L}$ at Each Time Slice (yr) } & \multirow{2}{*}{$\begin{array}{l}\text { RAGs, } \\
\mathrm{pCi} / \mathrm{L}\end{array}$} \\
\hline & & 0 & 1 & 3 & 8 & 12 & 43 & 131 & 300 & 1000 & \\
\hline \multirow{9}{*}{ Sr-90 } & Area $1 \mathrm{SZ}$ & 0 & 0 & 0 & 0 & 0 & 0 & 0 & 0 & 0 & \multirow{9}{*}{8} \\
\hline & Area $2 \mathrm{SZ}$ & 0 & 0 & 0 & 0 & 0 & 0 & 0 & 0 & 0 & \\
\hline & Area $3 \mathrm{SZ}$ & 0 & $4.92 \mathrm{E}-03$ & $1.41 \mathrm{E}-02$ & 3.33E-02 & $4.54 \mathrm{E}-02$ & $7.77 \mathrm{E}-02$ & $2.91 \mathrm{E}-02$ & $1.18 \mathrm{E}-03$ & $1.53 \mathrm{E}-10$ & \\
\hline & Area $4 \mathrm{SZ}$ & 0 & 0 & 0 & 0 & 0 & 0 & 0 & 0 & 0 & \\
\hline & Comb. Areas & 0 & $4.92 \mathrm{E}-03$ & $1.41 \mathrm{E}-02$ & 3.33E-02 & $4.54 \mathrm{E}-02$ & 7.77E-02 & $2.90 \mathrm{E}-02$ & $1.18 \mathrm{E}-03$ & $1.77 \mathrm{E}-10$ & \\
\hline & $\mathrm{OB} / \mathrm{BCL}$ & 0 & $2.26 \mathrm{E}-02$ & $6.46 \mathrm{E}-02$ & $1.53 \mathrm{E}-01$ & $2.08 \mathrm{E}-01$ & 3.57E-01 & $1.33 \mathrm{E}-01$ & $5.44 \mathrm{E}-03$ & $5.10 \mathrm{E}-10$ & \\
\hline & SPA SZ & 0 & 0 & 0 & 0 & 0 & 0 & 0 & 0 & 0 & \\
\hline & SPADZ & 0 & 0 & 0 & 0 & 0 & 0 & 0 & 0 & 0 & \\
\hline & SPA Total & 0 & 0 & 0 & 0 & 0 & 0 & 0 & 0 & 0 & \\
\hline \multirow{9}{*}{ Tritium (H-3) } & Area $1 \mathrm{SZ}$ & 0 & 0 & 0 & 0 & 0 & 0 & 0 & 0 & 0 & \multirow{9}{*}{20,000} \\
\hline & Area $2 \mathrm{SZ}$ & 0 & $2.37 \mathrm{E}+03$ & $5.43 E+03$ & $1.82 \mathrm{E}+03$ & $7.42 \mathrm{E}+02$ & $6.93 \mathrm{E}-01$ & 1.64E-09 & $3.46 \mathrm{E}-26$ & 0 & \\
\hline & Area $3 \mathrm{SZ}$ & 0 & 0 & 0 & 0 & 0 & 0 & 0 & 0 & 0 & \\
\hline & Area $4 \mathrm{SZ}$ & 0 & 0 & 0 & 0 & 0 & 0 & 0 & 0 & 0 & \\
\hline & Comb. Areas & 0 & $2.37 \mathrm{E}+03$ & $5.43 E+03$ & $4.02 E+03$ & $1.64 \mathrm{E}+03$ & $1.53 E+00$ & $3.62 \mathrm{E}-09$ & $7.68 \mathrm{E}-26$ & 0 & \\
\hline & $\mathrm{OB} / \mathrm{BCL}$ & 0 & 0 & 0 & 0 & 0 & 0 & 0 & 0 & 0 & \\
\hline & SPA SZ & 0 & 0 & 0 & 0 & 0 & 0 & 0 & 0 & 0 & \\
\hline & SPADZ & 0 & 0 & 0 & 0 & 0 & 0 & 0 & 0 & 0 & \\
\hline & SPA Total & 0 & 0 & 0 & 0 & 0 & 0 & 0 & 0 & 0 & \\
\hline
\end{tabular}

RAGs $=$ Remedial action goals from the 100 Area RDR/RAWP $\mathrm{OB} / \mathrm{BCL}=$ Overburden/Below Cleanup Levels SPA $=$ Staging Pile (Stockpile) Area

1

2

\section{CONCLUSIONS:}

- The "all pathways" (maximum) dose rates are shown in Table 3. The maximum all pathways dose rate from the 118-C-1 Burial Ground excavated combined Areas 1, 2, 3, and 4 is 12.8 $\mathrm{mrem} / \mathrm{yr}$ at year zero (2006). The maximum all-pathways dose rate for the overburden $(\mathrm{OB} / \mathrm{BCL}$ ) soil is $4.01 \mathrm{mrem} / \mathrm{yr}$ at year zero (2006). For the staging pile area (SPA) the total maximum all-pathways dose rate for the shallow and deep zones is $12.4 \mathrm{mrem} / \mathrm{yr}$ at year zero (2006).

- The dominant pathway for the dose rate for most of the RESRAD evaluations is direct external exposure (external gamma). See Table 6, below.

- The primary radionuclide contributing to the direct exposure pathway for the majority of the evaluations is cesium-137. The primary radionuclide contributing to the direct exposure pathway for the combined Areas 1,2,3, and 4 is cobalt-60. See Table 6, below.

- None of the site COCs are projected to exceed remedial action goals (RAGs). 


\begin{tabular}{|c|c|c|c|c|c|c|}
\hline Ori & S. W. Clark $<\infty$ & 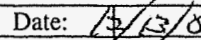 & 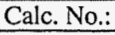 & 01 & & 0 \\
\hline & 118-C-1 Burial Ground Cleanup & Job No: 414655 & Checked: & M. W. Perrott Zyiof & Date: & $\mid / 2 / / 3$ \\
\hline & \multicolumn{6}{|c|}{ 118-C-1 Burial Ground Cleanup Verification RESRAD Calculation Brief } \\
\hline
\end{tabular}

1

- The radionuclide excess lifetime cancer risk (ELCR) results are shown in Table 4. The maximum ELCR from the 118-C-1 Burial Ground areas combined Areas 1, 2, 3, and 4 is $9.95 \times 10^{-5}$ at year zero (2006). The maximum ELCR for the overburden (OB/BCL) soil $\left(5.42 \times 10^{-5}\right)$ also occurs at year zero (2006). The total maximum all-pathways ELRC for the staging pile area (SPA) shallow and deep zones is $1.55 \times 10^{-4}$ at year zero (2006).

\begin{tabular}{|c|c|c|c|}
\hline \multicolumn{5}{|c|}{ Table 6. Dominant Pathways and Primary Radionuclides for RESRAD Evaluations } \\
\hline RESRAD Run & Vadose Zone Horizon & Dominant Pathway & Primary Radionuclide \\
\hline Area 1 & Shallow Zone & External Gamma & Eu-152 \\
\hline Area 2 & Shallow Zone & External Gamma & Co-60 \\
\hline Area 3 & Shallow Zone & External Gamma & Cs-137 \\
\hline Area 4 & Shallow Zone & External Gamma & Cs-137 \\
\hline Combined Areas & Shallow Zone & External Gamma & Co-60 \\
\hline OB/BCL & Shallow Zone & Plant Ingestion & Sr-90 \\
\hline SPA & Shallow Zone & External Gamma & Eu-152 \\
\hline SPA & Deep Zone & Fish & Cs-137 \\
\hline
\end{tabular}

$\mathrm{OB} / \mathrm{BCL}=$ Overburden/Below Cleanup Levels $\mathrm{SPA}=$ Staging Pile (Stockpile) Area

- Among the radionuclide contaminants of concern, cesium-137, cobalt-60, nickel-63, strontium-90, and tritium are calculated to reach groundwater in the 1,000 years of the RESRAD model run. These radionuclides are predicted to reach groundwater at concentrations significantly below the RAGs.

\section{ATTACHMENTS:}

1. Graphic showing 118-C-1 Cleanup Verification Model (2 pages)

2. Schematic of the 118-C-1 Burial Ground Remediation Areas (1 page)

3. RESRAD Output: 118-C-1 Area 1 Shallow Zone Radionuclides - Mixture Sums and Single Radionuclide Guidelines (19 pages)

4. RESRAD Output: 118-C-1 Area 1 Shallow Zone Radionuclides - Intake Quantities and Health Risk Factors (20 pages)

5. RESRAD Output: 118-C-1 Area 1 Shallow Zone Radionuclides - Concentration of Radionuclides, (10 pages)

6. RESRAD Output: 118-C-1 Area 2 Shallow Zone Radionuclides - Mixture Sums and Single Radionuclide Guidelines (21 pages)

7. RESRAD Output: 118-C-1 Area 2 Shallow Zone Radionuclides - Intake Quantities and Health Risk Factors (29 pages)

8. RESRAD Output: 118-C-1 Area 2 Shallow Zone Radionuclides - Concentration of Radionuclides (10 pages)

9. RESRAD Output: 118-C-1 Area 3 Shallow Zone Radionuclides - Mixture Sums and Single Radionuclide Guidelines (20 pages) 


\begin{tabular}{|c|c|c|c|c|c|c|}
\hline Originator: & S.W. Clark $D \leq 0$ & Date: $t=1 / 3 / 06$ & Calc. No.: & $0100 \mathrm{C}-\mathrm{CA}-\mathrm{V} 0035$ & Rev.: & 0 \\
\hline
\end{tabular}

10. RESRAD Output: 118-C-1 Area 3 Shallow Zone Radionuclides - Intake Quantities and Health Risk Factors (20 pages)

11. RESRAD Output: 118-C-1 Area 3 Shallow Zone Radionuclides - Concentration of Radionuclides (10 pages)

12. RESRAD Output: 118-C-1 Area 4 Shallow Zone Radionuclides - Mixture Sums and Single Radionuclide Guidelines (19 pages)

13. RESRAD Output: 118-C-1 Area 4 Shallow Zone Radionuclides - Intake Quantities and Health Risk Factors (20 pages)

14. RESRAD Output: 118-C-1 Area 4 Shallow Zone Radionuclides - Concentration of Radionuclides (10 pages)

15. RESRAD Output: 118-C-1 Combined Areas 1, 2, 3, and 4 Shallow Zone Radionuclides Mixture Sums and Single Radionuclide Guidelines (22 pages)

16. RESRAD Output: 118-C-1 Combined Areas 1, 2, 3, and 4 Shallow Zone Radionuclides Intake Quantities and Health Risk Factors (29 pages)

17. RESRAD Output: 118-C-1 Combined Areas 1, 2, 3, and 4 Shallow Zone Radionuclides Concentration of Radionuclides (10 pages)

18. RESRAD Output: 118-C-1 Overburden/Below Cleanup Levels Radionuclides - Mixture Sums and Single Radionuclide Guidelines (18 pages)

19. RESRAD Output: 118-C-1 Overburden/Below Cleanup Levels Radionuclides - Intake Quantities and Health Risk Factors (20 pages)

20. RESRAD Output: 118-C-1 Overburden/Below Cleanup Levels Radionuclides Concentration of Radionuclides (10 pages)

21. RESRAD Output: 118-C-1 Staging Pile (Stockpile) Area Shallow Zone Radionuclides Mixture Sums and Single Radionuclide Guidelines (20 pages)

22. RESRAD Output: 118-C-1 Staging Pile (Stockpile) Area Shallow Zone Radionuclides Intake Quantities and Health Risk Factors (20 pages)

23. RESRAD Output: 118-C-1 Staging Pile (Stockpile) Area Shallow Zone Radionuclides Concentration of Radionuclides (10 pages)

24. RESRAD Output: 118-C-1 Staging Pile (Stockpile) Area Deep Zone Radionuclides Mixture Sums and Single Radionuclide Guidelines (19 pages)

25. RESRAD Output: 118-C-1 Staging Pile (Stockpile) Area Deep Zone Radionuclides - Intake Quantities and Health Risk Factors (20 pages)

26. RESRAD Output: 118-C-1 Staging Pile (Stockpile) Area Deep Zone Radionuclides Concentration of Radionuclides (10 pages) 


\section{DISTRIBUTION}

U.S. Department of Energy

Richland Operations Office

D. C. Smith (5) A3-04

DOE-RL Public Reading Room

$\mathrm{H} 2-53$

Fluor Hanford, Inc.

J. P. Shearer (WIDS) E6-35

Washington Closure Hanford

R. A. Carlson X4-08

S. W. Clark

$\mathrm{H} 4-23$

J. S. Decker

$\mathrm{H} 4-08$

L. A. Dietz

$\mathrm{H} 4-22$

L. M. Dittmer

$\mathrm{H} 4-23$

M. T. Hughes

$\mathrm{H} 4-23$

L. R. Miller

X4-08

D. N. Strom

X3-40

Records and Document Control

H4-11

Hanford Technical Library

P8-55 
CVP-2006-00011

Rev. 0

Distr-2 